\title{
Lawrence Livermore National Laboratory East Avenue/Emergency Response Planning Traffic Study
}

T. W. Schmiegel

February 15, 2002

U.S. Department of Energy

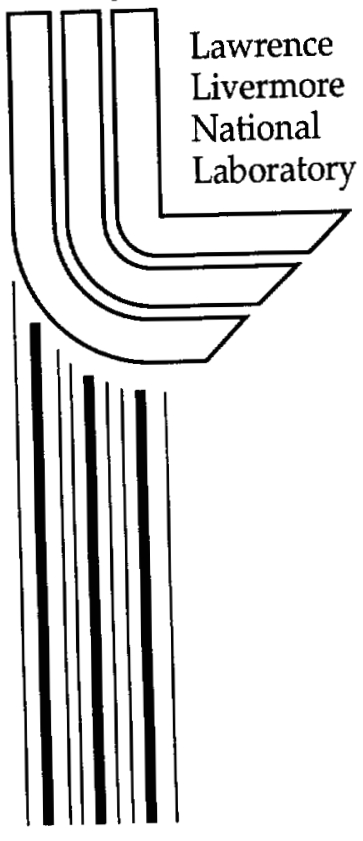




\section{DISCLAIMER}

This document was prepared as an account of work sponsored by an agency of the United States Government. Neither the United States Government nor the University of California nor any of their employees, makes any warranty, express or implied, or assumes any legal liability or responsibility for the accuracy, completeness, or usefulness of any information, apparatus, product, or process disclosed, or represents that its use would not infringe privately owned rights. Reference herein to any specific commercial product, process, or service by trade name, trademark, manufacturer, or otherwise, does not necessarily constitute or imply its endorsement, recommendation, or favoring by the United States Government or the University of California. The views and opinions of authors expressed herein do not necessarily state or reflect those of the United States Government or the University of California, and shall not be used for advertising or product endorsement purposes.

This work was performed under the auspices of the U. S. Department of Energy by the University of California, Lawrence Livermore National Laboratory under Contract No. W-7405-Eng-48.

This report has been reproduced directly from the best available copy.

Available electronically at http://www.doe.gov/bridge

Available for a processing fee to U.S. Department of Energy

and its contractors in paper from

U.S. Department of Energy

Office of Scientific and Technical Information

$$
\text { P.O. Box } 62
$$

Oak Ridge, TN 37831-0062

Telephone: (865) 576-8401

Facsimile: (865) 576-5728

E-mail: reports@adonis.osti.gov

Available for the sale to the public from

U.S. Department of Commerce

National Technical Information Service

5285 Port Royal Road

Springfield, VA 22161

Telephone: (800) 553-6847

Facsimile: (703) 605-6900

E-mail: orders@ntis.fedworld.gov

Online ordering: http://www.ntis.gov/ordering.htm

OR

Lawrence Livermore National Laboratory

Technical Information Department's Digital Library

http://www.llnl.gov/tid/Library.html 


\title{
Lawrence Livermore National Laboratory
}

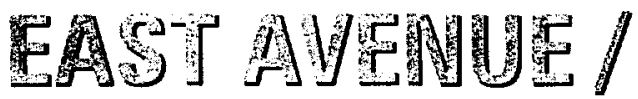

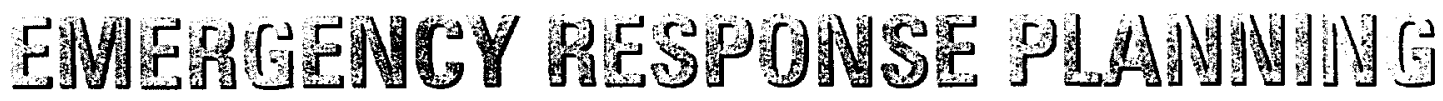

T

Prepared for

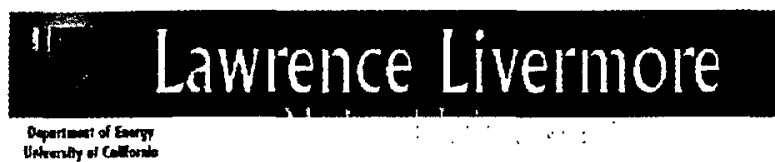

Livermore, California

February 15, 2002 


\section{Table of Contents}

1.0 INTRODUCTION......................................................................................................... 1

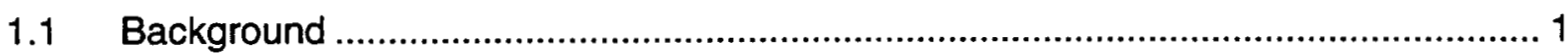

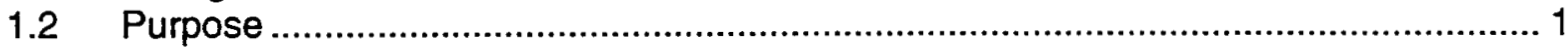

2.0 EXISTING CONDITIONS ....................................................................................... 1

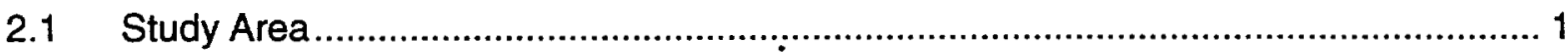

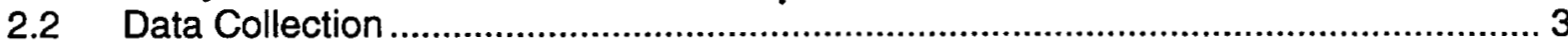

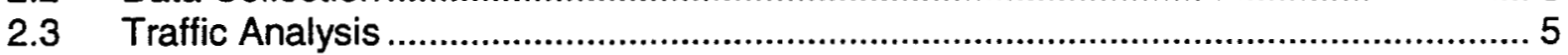

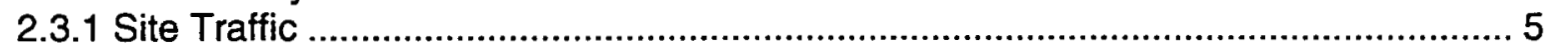

2.3.2 Levels of Service ................................................................................... 13

2.3.3 Required Left-turn Storage Lengths ......................................................... 16

2.3.4 Environmental Documentation Review....................................................... 16

3.0 ADMINISTRATIVE CONTROL OF EAST AVENUE ................................................ 17

3.1 New East Avenue Security Control Point at South Vasco Road .............................. 18

3.2 New East Avenue Security Control Point at Greenville Road ................................... 18

3.3 New Truck Inspection Facility - East Avenue at Greenville Road ......................... 19

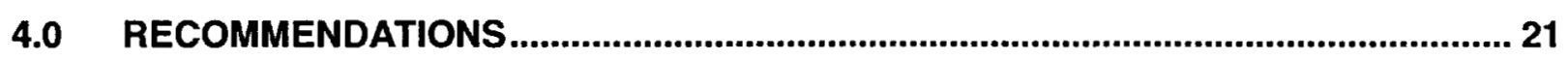




\section{List of Tables}

Table 1 LLNL Entrances: 24-Hour Counts (5-Day Weekday Average) ............................. 8

Table 2 LLNL Entrances Peak Hour Volumes (5-Day Weekday Average) .......................... 8

Table 3 SNL Entrances 24-Hour Counts (5-Day Weekday Average) ................................. 12

Table 4 SNL Entrances Peak Hour Volumes (5-Day Weekday Average) ......................... 12

Table 5 East Avenue 24-Hour Counts (5-Day Weekday Average) ..................................... 12

Table 6 East Avenue Peak Hour Volumes (5-Day Weekday Average) ............................ 13

Table 7 Intersection Level of Service Definitions........................................................ 13

Table $8 \quad$ Existing Conditions Level of Service - Signalized Intersection ............................. 14

Table 9 Existing Conditions Level of Service - Unsignalized Intersections ...................... 14

Table $10 \quad 2020$ Conditions Level of Service - Signalized Intersection................................ 15

Table 112020 Conditions Level of Service - Unsignalized Intersections .......................... 15

Table 12 Left-Turn Storage Length Requirements ...................................................... 16

Table 13 East Avenue Traffic Volume Comparison...................................................... 17

Table 14 Calculation of Weekday Non Laboratory Traffic (5-Day Average) ........................ 17

Table 15 Existing Total Trucks - Location 7 (5-Day Weekday Average) ............................ 19

Table 16 Existing Total Trucks - Location 8 (5-Day Weekday Average) ........................... 19

Table 17 Combined Total Trucks - Locations 7 and 8 (5-Day Weekday Average)............... 20

Table 18 Truck Size Summary.................................................................................... 20

\section{List of Figures}

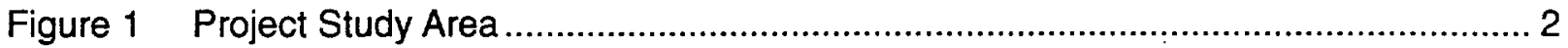

Figure 2 Count Locations and Movements Counted.................................................... 4

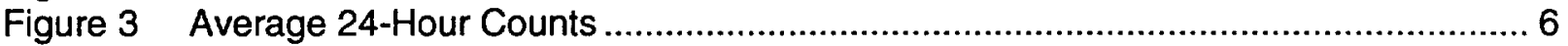

Figure 4 Average Weekday Peak Hour Traffic Volumes ........................................... 7

Figure 5 LLNL-Combined Entrances (5-Day Weekday Average) ................................... 9

Figure 6 LLNL - Westgate (\#17) (5-Day Weekday Average) ........................................ 10

Figure 7 SNL Combined Entrances (5-Day Weekday Average) ....................................... 11

Figure 8 East Avenue Conceptual Plan - Recommendations....................................... 22

\section{Technical Appendix}
A. 24-Hour Traffic Counts
B. Intersection Traffic Counts
C. Truck Counts
D. Traffic Analysis - Existing Conditions
E. Traffic Analysis - Future Conditions - Year 2020 


\subsection{INTRODUCTION}

\section{$1.1 \quad$ Background}

The Lawrence Livermore National Laboratory (LLNL) and the Sandia National Laboratory (SNL) are located at the eastern end of the City of Livermore. Recently the research facilities have been placed on heightened security alerts due to the events of September 11. To respond to the security concerns, LLNL and SNL have proposed to place East Avenue between South Vasco Road and Greenville Road under administrative control. This type of control would require security check points at both ends of this segment of East Avenue, including a truck inspection facility west of the Greenville Road intersection. In this configuration, East Avenue would be closed to general public traffic. Figure 1 illustrates the project study area.

\subsection{Purpose}

The purpose of this traffic study is to determine the potential traffic impact of placing East Avenue under administrative control. The primary focus of the traffic study is to evaluate the proposed modifications to the geometry and operation of East Avenue between South Vasco Road and Greenville Road. The study also includes a review of a traffic study prepared for the closure of East Avenue in1989 to determine if the assumptions and conclusions of that study remain valid.

\subsection{EXISTING CONDITIONS}

\subsection{Study Area}

The Lawrence Livermore National Laboratory (LLNL) is bounded by Patterson Pass Road to the north, Greenville Road to the east, East Avenue to the south, and Vasco Road to the west. As shown in Figure 1, there are currently five gated entrances to the LLNL, controlled by uniformed Protective Service Officers (PSO):

1. Westgate;

2. Eastgate;

3. Southwestgate;

4. Southgate; and

5. LLNL Shipping and Receiving.

The primary entrance is the Westgate at the signalized intersection of Vasco Road/Westgate Drive, which is also where the badge office is located. All visitors must obtain a visitor badge to enter the laboratory. The Eastgate is located west of the signalized intersection of Greenville Road and Eastgate Drive. The Visitors Center and Public Affairs Center are located next to the Eastgate. The other three LLNL entrances are located off of East Avenue at unsignalized intersections. The Southwestgate is located at the West Perimeter Road and the Southgate is situated at the Southgate Drive. The LLNL Shipping and Receiving entry point is located east of Southgate Drive and is restricted to delivery trucks only. 


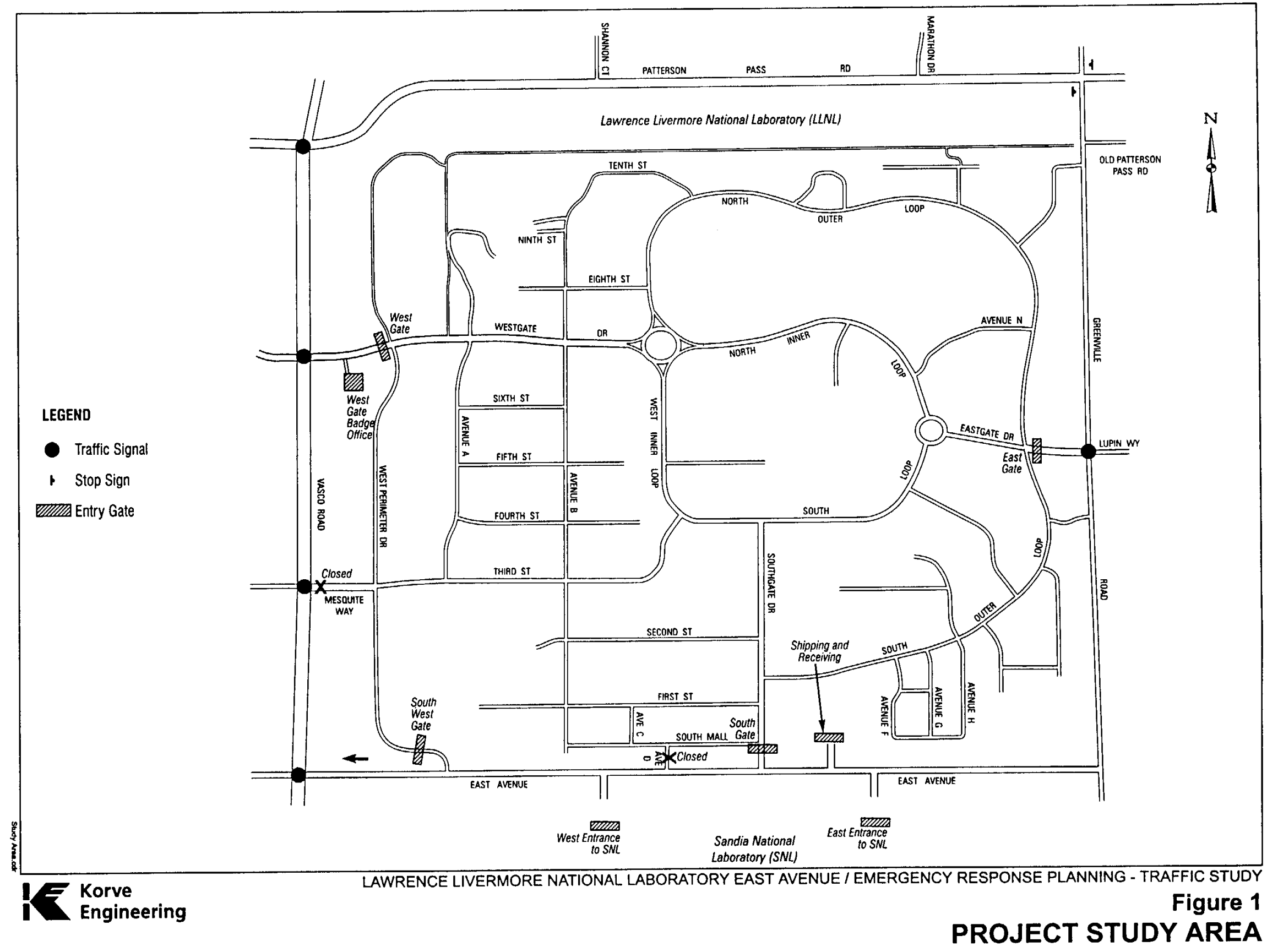


The Sandia National Laboratory (SNL) is located across from the LLNL south of East Avenue. Two gated entry points off East Avenue are available for the SNL- the West Entrance and the East Entrance. Deliveries to SNL are required to use the East Entrance.

\subsection{Data Collection}

In order to better assess the circulation of traffic in and out of the two Laboratories, vehicle counts passing through given points of access have been collected and reviewed. Twenty-four hour, seven-day hose counts were conducted at the following locations:

1. Southbound left turn from South Vasco Road to East Avenue;

2. Northbound right turn from South Vasco Road to East Avenue;

3. Eastbound and westbound East Avenue, east of South Vasco Road;

4. LLNL Southwest Entrance - single inbound lane, outbound right turn and outbound left turn (counted separately);

5. SNL West Entrance - single inbound lane, outbound right turn and outbound left turn (counted separately);

6. LLNL Southgate Drive - two lanes inbound, outbound right turn and outbound left turn (counted separately);

7. LLNL post $P-411$ - one lane inbound, outbound right turn and outbound left turn (counted separately). Also, conduct five-day, 7 AM to 5 PM manual vehicle classification counts for inbound and outbound traffic (automobiles, single-unit trucks, 3axle trucks, 4-axle trucks and 5+axle trucks);

8. SNL East Entrance - one lane inbound, outbound right turn and outbound left turn (counted separately). Also, conduct five-day, 7 AM to 4 PM manual vehicle classification counts for inbound and outbound traffic (automobiles, single-unit trucks, 3-axle trucks, 4-axle trucks and 5+axle trucks);

9. Southbound Greenville Road at East Avenue;

10. Northbound Greenville Road at East Avenue;

11. East Avenue west of Greenville Road (eastbound and westbound counted separately);

12. Greenville Road/LLNL Eastgate Drive - Southbound inbound right tum and northbound inbound left turn from Greenville Road;

13. LLNL Eastgate Drive west of Greenville Road at gate entrance, inbound - single left turn lane, single through lane and single right turn lane (counted separately);

14. LLNL Eastgate Drive west of Greenville Road - two outbound lanes;

15. Southbound left turn from South Vasco Road to Westgate Drive;

16. Northbound right turn from South Vasco Road to Westgate Drive; and

17. Westgate Drive east of South Vasco Road - inbound and outbound (counted separately).

Figure 2 depicts the counting locations. The traffic counts were summarized into fifteen-minute intervals and have been attached in the Technical Appendix. Weekday morning and evening peak hour turning movement counts were also collected at the intersections of South Vasco Road/East Avenue and Greenville Road/East Avenue. The traffic counts were collected during the week of December $6^{\text {th }}$ to December $12^{\text {th }}, 2001$. 
The average 2-day weekend and 5-day weekday 24-hour traffic volumes at these key locations and critical turning movements are illustrated in Figure 3. The average 5-day weekday peak hour volume for the main entrances and turning movements are depicted in Figure 4. As shown in Figure 3 , since the weekend traffic volumes are extremely low compared to the weekday volumes, the traffic analysis is focused on the more critical weekday traffic volumes. Section 3.3 describes the analysis of truck data that was collected at locations 7 and 8.

\subsection{Traffic Analysis}

Using the data collected, the proposed modifications to East Avenue between South Vasco Road and Greenville Road were evaluated. Detailed technical analyses included the following:

- East Avenue/South Vasco Road Intersection - Existing and future levels of service and vehicular queue lengths.

- East Avenue/Greenville Road Intersection - Existing and future levels of service and vehicular queue lengths.

- New East Avenue Security Control Point at South Vasco Road-Required inbound and outbound lanes and lane widths and anticipated queue lengths.

- . New East Avenue Security Control Point at Greenville Road - Required inbound and outbound lanes and lane widths and anticipated queue lengths.

- New Truck Inspection Facility - East Avenue at Greenville Road-Performance, required lanes and anticipated queue lengths.

- $\quad$ East Avenue Intersections - Levels of service and queue lengths at intersections along East Avenue - LLNL Southwest Entrance, LLNL South Entrance, SNL West Entrance and SNL Main Entrance.

For each study facility, geometry currently under consideration has been evaluated. An evaluation of future traffic conditions was also conducted. The evaluation took into account planned residential and commercial projects south of East Avenue along South Vasco Road, along with other developments under consideration throughout Livermore, through the use of the Alameda County Congestion Management Agency's travel demand model.

\subsubsection{Site Traffic}

\section{LLNL Traffic}

Table 1 summarizes the traffic volumes collected at the five entrances to LLNL. A total of 21,975 vehicles enter and exit the LLNL on a typical day. The counts indicate that the Westgate is the most utilized at 36.4 percent $(8,005 \mathrm{veh} /$ day $)$, and the Southwestgate is the least used $(4,000$ veh/day or 18.2 percent). This is to be expected since the Westgate is the primary entrance to the facility. The Westgate and Eastgate combined for 57.4 percent of the traffic at LLNL while the East Avenue entrances constitute $\mathbf{4 2 . 6}$ percent of LLNL traffic. 


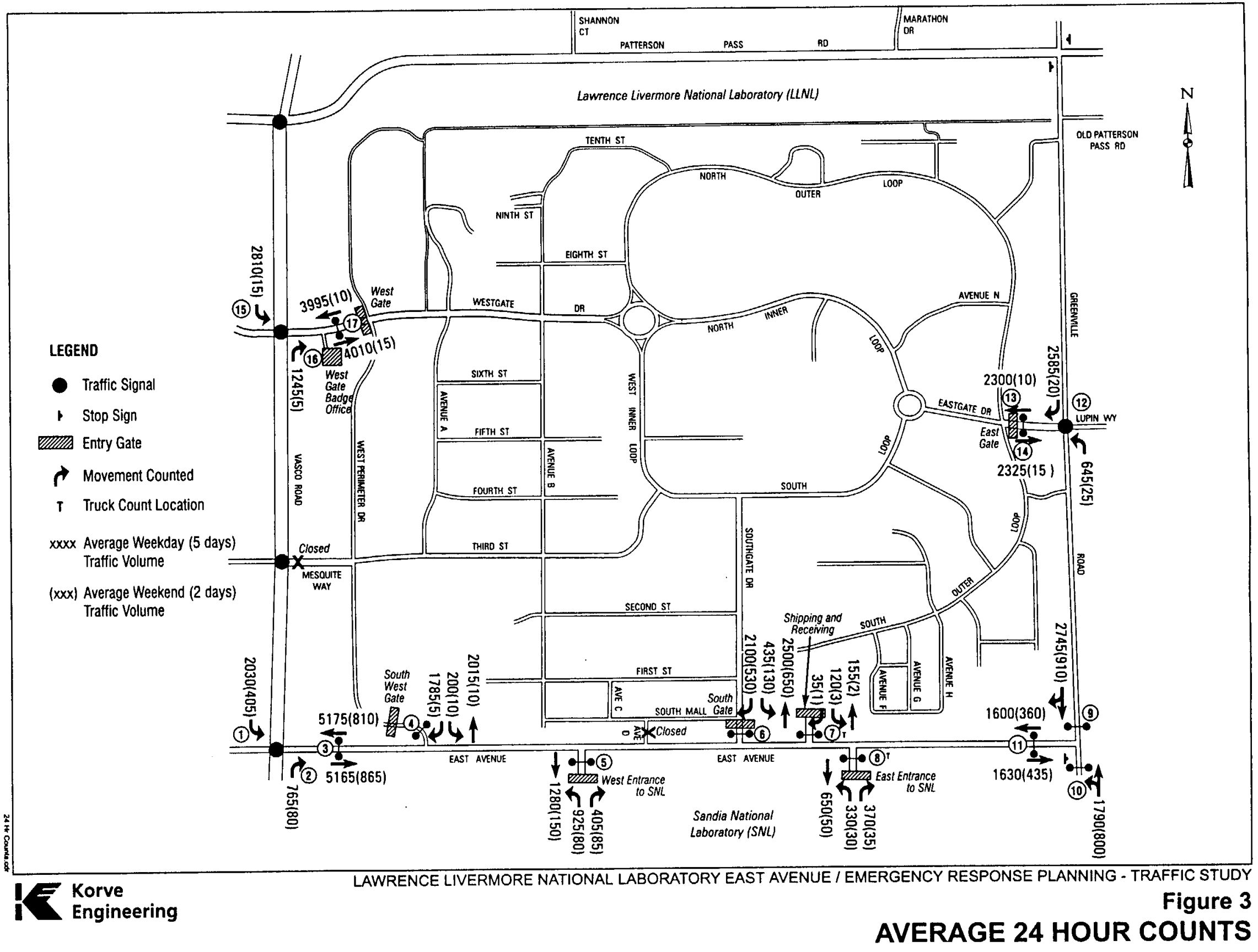




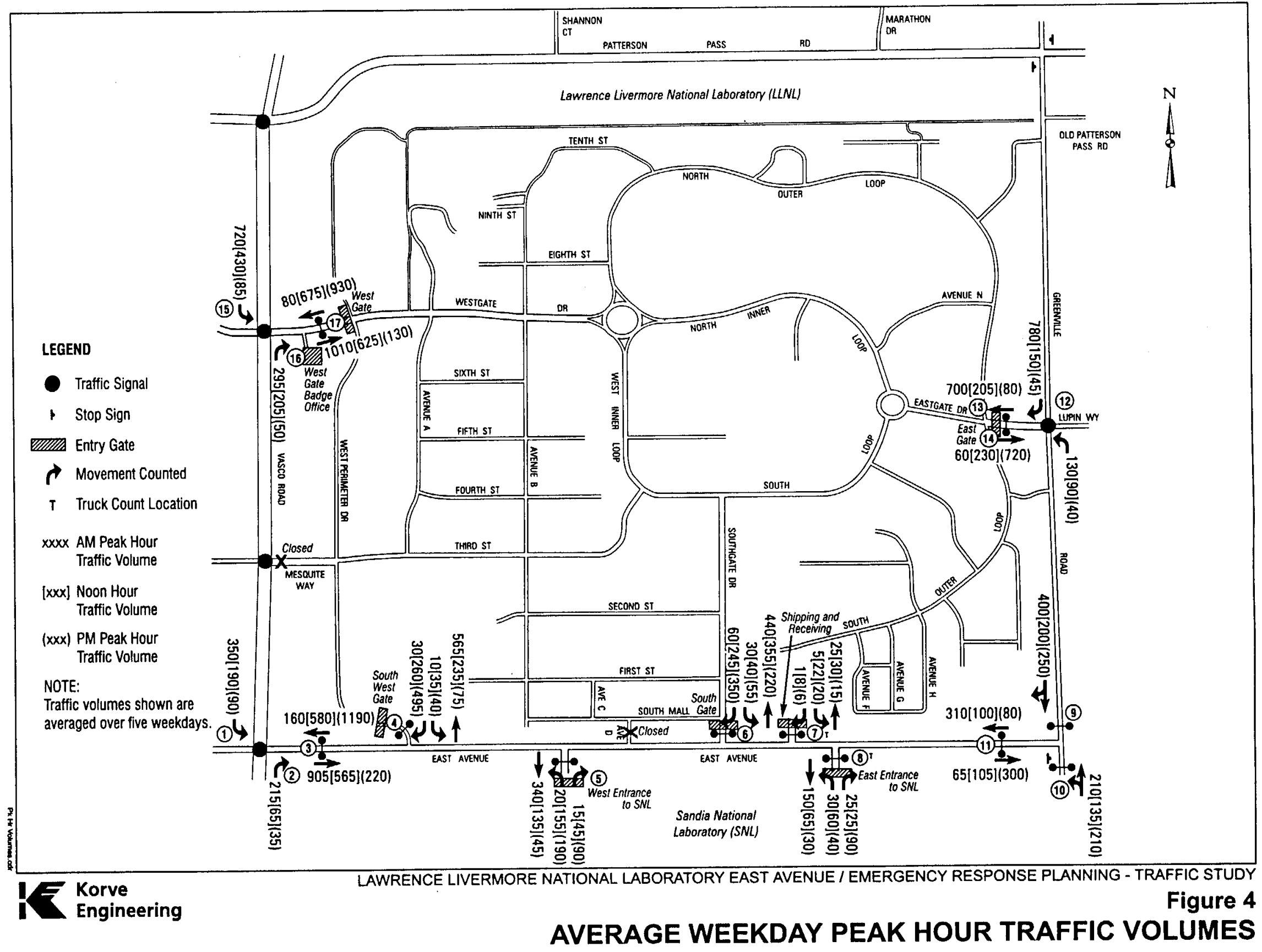


Table 1 LLNL Entrances: 24-Hour Counts (5-Day Weekday Average)

\begin{tabular}{|l|c|c|c|c|c|c|}
\hline Daily Total & $\begin{array}{c}\text { Westgate } \\
(\# 17)\end{array}$ & $\begin{array}{c}\text { Eastgate } \\
(\# 13 / 14)\end{array}$ & $\begin{array}{c}\text { Southwest- } \\
\text { gate (\# 4) }\end{array}$ & $\begin{array}{c}\text { Southgate } \\
(\# 6)\end{array}$ & $\begin{array}{c}\text { Shipping } \\
(\# 7)\end{array}$ & Total \\
\hline In & 4,010 & 2,300 & 2,015 & 2,500 & 155 & 10,980 \\
\hline Out & 3,995 & 2,325 & 1,985 & 2,535 & 155 & 10,995 \\
\hline Total & 8,005 & 4,625 & 4,000 & 5,035 & 310 & 21,975 \\
\hline Percentage & $36.4 \%$ & $21.0 \%$ & $18.2 \%$ & $23 \%$ & $1.4 \%$ & $100 \%$ \\
\hline
\end{tabular}

The hourly distribution of total traffic entering and exiting the LLNL is illustrated in Figure 5 . As shown in Figure 5 the total in-bound traffic peaks between 7:00 AM and 8:00 AM and the out-bound traffic peaks between 5:00 PM and 6:00 PM. The graph reflects a typical work day traffic pattern with distinctive inbound and outbound peaks in the morning and afternoon. The large spike in traffic volumes in the morning causes a surge in demand at the entry gates. The Westgate has the highest morning peak hour volume $(1,010$ vehicles per hour) and has been observed to queue traffic into the signalized intersection of Vasco Road regularly, causing delays and congestion. Figure 6 illustrates this sharp peaking characteristic of the Westgate as the traffic volume jumps sharply from $680 \mathrm{vph}$ (7 to 8 AM) to $1,010 \mathrm{vph}$ (8-9 AM).

Mid-day or non peak periods serve relatively low traffic levels and the entry gates are often reduced to one entry lane. Table 2 summarizes the average weekday peak hour volumes at the LLNL entrances.

Table 2 LLNL Entrances Peak Hour Volumes (5-Day Weekday Average)

\begin{tabular}{|c|c|c|c|c|c|c|c|c|c|c|c|c|}
\hline & \multicolumn{2}{|c|}{$\begin{array}{c}\text { Westgate } \\
(\# ~ 17)\end{array}$} & \multicolumn{2}{c|}{$\begin{array}{c}\text { Eastgate } \\
(\# 13 / 14)\end{array}$} & \multicolumn{2}{c|}{$\begin{array}{c}\text { Southwest- } \\
\text { gate (\# 4) }\end{array}$} & \multicolumn{2}{c|}{$\begin{array}{c}\text { Southgate } \\
(\# \text { 6) }\end{array}$} & \multicolumn{2}{c|}{$\begin{array}{c}\text { Shipping } \\
(\# ~ 7)\end{array}$} & \multicolumn{2}{c|}{$\begin{array}{c}\text { Total LLNL } \\
\text { Entrance }\end{array}$} \\
\hline $\begin{array}{c}\text { Peak } \\
\text { Hour }\end{array}$ & In & Out & In & Out & In & Out & In & Out & In & Out & In & Out \\
\hline 7-8 AM & 1,010 & 80 & 700 & 60 & 565 & 40 & 440 & 90 & 25 & 6 & 2,740 & 276 \\
\hline $\begin{array}{c}\text { Mid-day } \\
(12-1 \text { PM })\end{array}$ & 625 & 675 & 205 & 230 & 235 & 295 & 355 & 285 & 30 & 30 & 1,450 & 1,515 \\
\hline 5-6 PM & 130 & 930 & 80 & 720 & 75 & 535 & 220 & 405 & 15 & 26 & 520 & 2,616 \\
\hline
\end{tabular}

1. Shipping and Receiving Traffic counts include passenger vehicles and trucks.

2. Peak Hour for specific movements fluctuates. Peak hour noted is for the entire LLNL facility.

\section{SNL Traffic}

Table 3 summarizes the average 5-day weekday 24-hour traffic volumes at the two entrances to SNL. A total of 3,960 vehicles enter and exit the SNL in a typical day. For comparison purposes this volume represents about 15 percent of the combined LLNL and SNL daily traffic volume. The results also indicate that the West Entrance to SNL is the most utilized out of the two at 66 percent (2610 veh/day). Figure 7 shows the traffic pattern for the SNL entrances. Table 4 summarizes the average 5-day weekday peak hour volumes at the SNL entrances. 


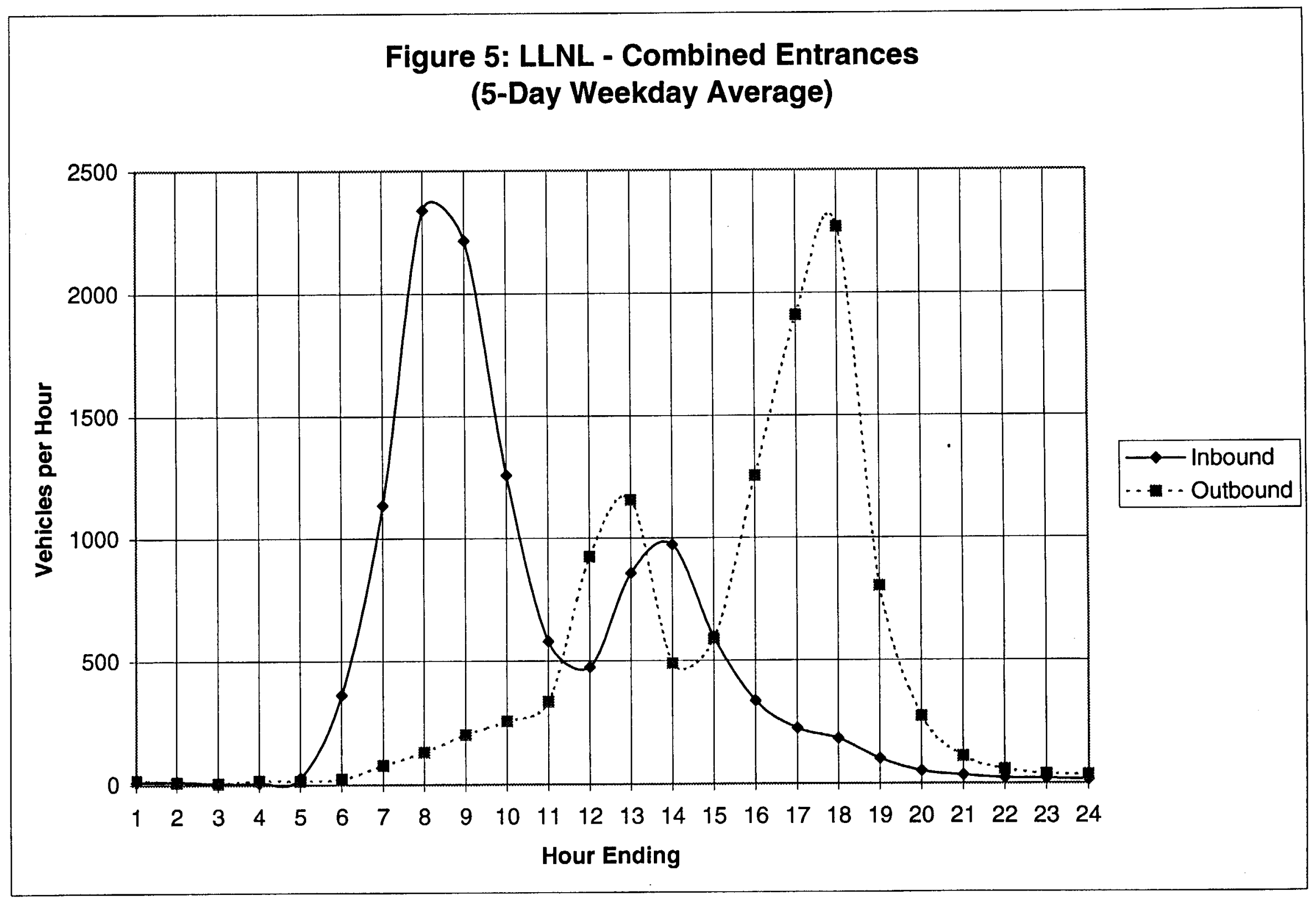




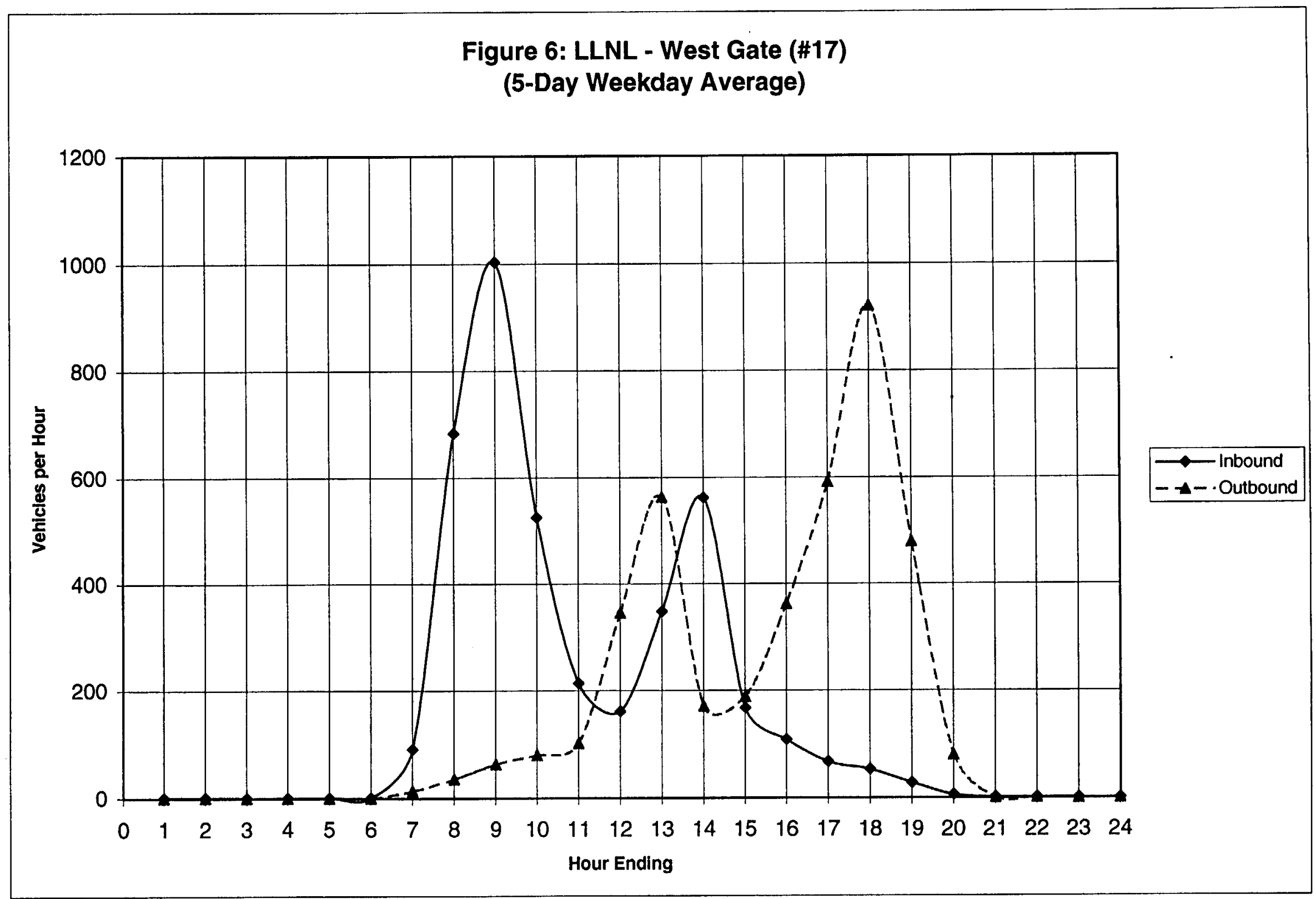

Korve Engineering 


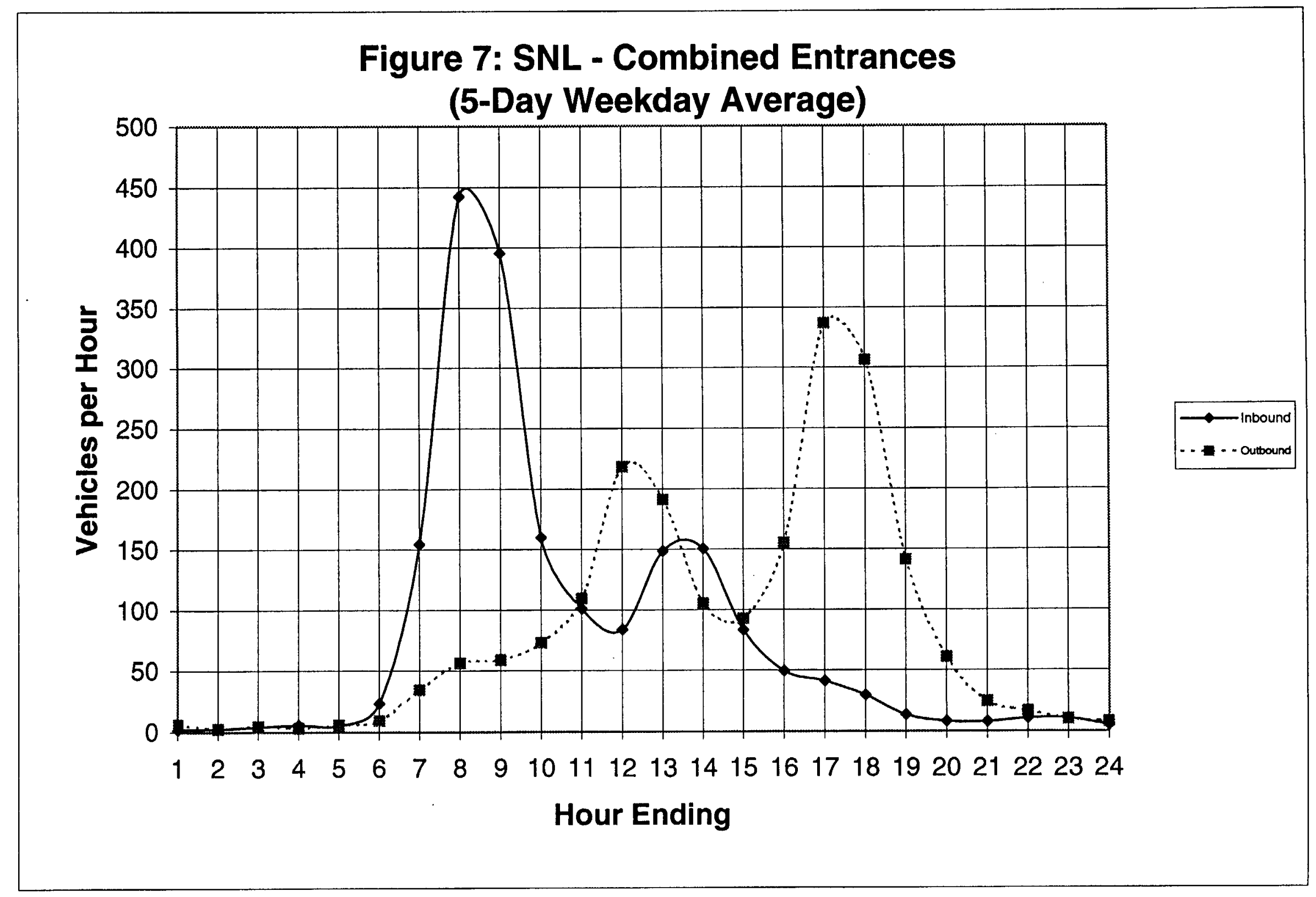


Table 3

SNL Entrances 24-Hour Counts (5-Day Weekday Average)

\begin{tabular}{|l|c|c|c|}
\hline Daily Total & $\begin{array}{c}\text { West Entrance } \\
(\# 5)\end{array}$ & $\begin{array}{c}\text { East Entrance } \\
(\# 8)\end{array}$ & Total \\
\hline In & 1,280 & 650 & 1,930 \\
\hline Out & 1,330 & 700 & 2,030 \\
\hline Total & 2,610 & 1,350 & 3,960 \\
\hline Percentage & $66 \%$ & $34 \%$ & $100 \%$ \\
\hline
\end{tabular}

Table 4 SNL Entrances Peak Hour Volumes (5-Day Weekday Average)

\begin{tabular}{|c|c|c|c|c|c|c|}
\hline & \multicolumn{2}{|c|}{$\begin{array}{c}\text { West Entrance } \\
\text { (\# 5) }\end{array}$} & \multicolumn{2}{c|}{$\begin{array}{c}\text { East Entrance } \\
\text { (\# 8) }\end{array}$} & \multicolumn{2}{c|}{ Total SNL Entrance } \\
\hline Peak Hour & In & Out & In & Out & In & Out \\
\hline $7-8$ AM & 340 & 35 & 150 & 55 & 490 & 90 \\
\hline $\begin{array}{c}\text { Mid-day } \\
(12-1 \text { PM) }\end{array}$ & 135 & 200 & 65 & 85 & 200 & 285 \\
\hline $4-5$ PM & 45 & 280 & 30 & 130 & 75 & 410 \\
\hline
\end{tabular}

1. East Entrance traffic counts include passenger vehicles and trucks.

2. Peak Hour for specific movements fluctuates. Peak hour noted is for the SNL facility.

\section{East Avenue Traffic}

Table 5 summarizes the traffic volumes on East Avenue. A total of 13,570 vehicles use East Avenue on a daily basis. The traffic counts indicate that 76 percent of the traffic on East Avenue is from the west. Table 6 summarizes the average weekday peak hour volumes at the western and eastern ends of East Avenue. The peak hour volumes are used to determine the vehicle storage length requirements for the new entry gates on East Avenue, as desribed in Section 3.0.

Table 5 East Avenue 24-Hour Counts (5-Day Weekday Average)

\begin{tabular}{|l|c|c|c|}
\hline Daily Total & $\begin{array}{c}\text { East Ave (Vasco Rd) } \\
\text { Location \# 3 }\end{array}$ & $\begin{array}{c}\text { East Ave } \\
\text { (Greenville Rd) } \\
\text { Location \# 11 }\end{array}$ & Total \\
\hline In & 5,165 & 1,600 & 6,765 \\
\hline Out & 5,175 & 1,630 & 6,805 \\
\hline Total & 10,340 & 3,230 & 13,570 \\
\hline Percentage & $76 \%$ & $24 \%$ & $100 \%$ \\
\hline
\end{tabular}


Table 6 East Avenue Peak Hour Volumes (5-Day Weekday Average)

\begin{tabular}{|c|c|c|c|c|c|c|}
\hline & \multicolumn{2}{|c|}{$\begin{array}{c}\text { East Ave } \\
\text { (Vasco Rd) } \\
\text { Location \# 3 }\end{array}$} & \multicolumn{2}{c|}{$\begin{array}{c}\text { East Ave } \\
\text { (Greenville Rd) } \\
\text { Location \# 11 }\end{array}$} & \multicolumn{2}{c|}{ Ootal on East Avenue } \\
\hline Peak Hour & In & Out & In & Out & In & 225 \\
\hline 8-9 AM & 905 & 160 & 310 & 65 & 1,215 & 685 \\
\hline $\begin{array}{c}\text { Mid-day } \\
\text { (12-1 PM) }\end{array}$ & 565 & 580 & 100 & 105 & 665 & 1,490 \\
\hline 4-5 PM & 220 & 1,190 & 80 & 300 & 300 & \\
\hline
\end{tabular}

1. Peak Hour for specific movements fluctuates. Peak hour noted is for East Avenue.

\subsubsection{Levels of Service}

\section{Existing Traffic Conditions}

Traffic conditions in the study area are assessed through the evaluation of peak hour Levels of Service (LOS) at critical intersections. The LOS concept qualitatively characterizes traffic conditions associated with varying levels of traffic. A LOS determination is a measure of congestion, which is the principal measure of roadway service. Levels of Service for signalized and unsignalized intersections are illustrated in Table 7. Level of Service ranges from LOS A which indicates a free-flow condition to LOS F, which indicates a jammed condition. LOS A, B and C are generally considered to be satisfactory service levels while LOS D is marginally acceptable, LOSE is undesirable and LOS F conditions are unacceptable.

Table 7 Intersection Level of Service Definitions

\begin{tabular}{|c|l|c|c|}
\hline \multirow{2}{*}{$\begin{array}{c}\text { Level of } \\
\text { Service }\end{array}$} & \multicolumn{1}{|c|}{ Description } & \multicolumn{2}{|c|}{ Average Total Delay (seconds/vehicle) } \\
\cline { 3 - 4 } & & Unsignalized Intersections & Signalized Intersections \\
\hline A & Little or no delay & $\leq 10.0$ & $\leq 10.0$ \\
\hline B & Short traffic delay & $>10.0$ and $\leq 15.0$ & $>10.0$ and $\leq 20.0$ \\
\hline C & Average traffic delay & $>15.0$ and $\leq 25.0$ & $>20.0$ and $\leq 35.0$ \\
\hline D & Long traffic delay & $>25.0$ and $\leq 35.0$ & $>35.0$ and $\leq 55.0$ \\
\hline E & Very long traffic delay & $>35.0$ and $\leq 50.0$ & $>55.0$ and $\leq 80.0$ \\
\hline F & Extreme traffic delay & $>50.0$ & $>80.0$ \\
\hline
\end{tabular}

Source: Highway Capacity Manual, Special Report 209, Transportation Research Board, 2000.

Traffic conditions at study intersections are evaluated for the morning and evening peak hours using the methodology of the Transportation Research Board's 2000 Highway Capacity Manual. With this methodology a level of service is assigned based on average total vehicle delay.

The following six intersections in the study area were evaluated:

1. East Avenue and Vasco Road (signalized intersection);

2. East Avenue and Southwest Entrance/West Perimeter Drive (stop control on West Perimeter 
Road);

3. East Avenue and West Entrance to Sandia Labs (stop control on West Entrance);

4. East Avenue and Southgate (stop control on Southgate);

5. East Avenue and East Entrance to Sandia Labs (stop control on East Entrance); and

6. East Avenue and Greenville Road (stop control on East Avenue).

Traffic counts were conducted at two of the study intersections (the East Avenue and Vasco Road and the East Avenue and Greenville Road intersections) during the morning and evening peak hours on December $6^{\text {th }}, 2001$. Based on 24-hour counts, the turning movements were calculated for the other intersections. Summaries of these counts are attached in the report's technical appendix. Using these traffic counts along with lane geometry and signal phasing collected as part of this analysis, each of the six study intersections has been assessed using the methodology of the 2000 Highway Capacity Manual. The results of this analysis are presented in Tables 8 and 9. Level of Service calculation worksheets for each intersection are also attached in the technical appendix.

Table 8 Existing Conditions Level of Service - Signalized Intersection

\begin{tabular}{|l|c|c|}
\hline Signalized Intersection & Peak Hour & Level of Service (Seconds $/$ Vehicle) \\
\cline { 3 - 3 } & & Overall Intersection \\
\hline 1. East Avenue \& Vasco Road & AM & B (17.5) \\
\cline { 2 - 3 } & PM & $\mathrm{C} \mathrm{(21.9)}$ \\
\hline
\end{tabular}

1. For signalized intersections, the overall intersection LOS and intersection delay in seconds per vehicle are presented.

Table 9 Existing Conditions Level of Service - Unsignalized Intersections

\begin{tabular}{|c|c|c|c|}
\hline \multirow[t]{2}{*}{ Unsignalized Intersection $^{1}$} & \multirow[t]{2}{*}{ Peak Hour } & \multicolumn{2}{|c|}{ Level of Service (Seconds/Nehicle) } \\
\hline & & $\begin{array}{l}\text { Minor Street } \\
\text { Approach }\end{array}$ & $\begin{array}{c}\text { Worst Minor Street } \\
\text { Movement }\end{array}$ \\
\hline \multirow{2}{*}{$\begin{array}{l}\text { 1. East Avenue \& SW Entrance } \\
\text { / West Perimeter Drive }\end{array}$} & AM & $\mathrm{C}(18.3)$ & $E(45.9)$ \\
\hline & $\mathrm{PM}$ & $\mathrm{D}(28.1)$ & $\mathrm{D}(28.8)$ \\
\hline \multirow{2}{*}{$\begin{array}{l}\text { 2. East Avenue \& West } \\
\text { Entrance to Sandia Labs }\end{array}$} & $\overline{A M}$ & $\mathrm{~B}(12.6)$ & $C(16.4)$ \\
\hline & PM & $\mathrm{B}(10.3)$ & $C(12.6)$ \\
\hline \multirow[t]{2}{*}{ 3. East Avenue \& Southgate } & $\overline{A M}$ & $B(12.6)$ & $\mathrm{B}(16.4)$ \\
\hline & $\overline{P M}$ & $C(15.6)$ & $C(17.3)$ \\
\hline \multirow{2}{*}{$\begin{array}{l}\text { 4. East Avenue \& East } \\
\text { Entrance to Sandia Labs }\end{array}$} & AM & $\mathrm{B}(10.7)$ & $\mathrm{B}(12.4)$ \\
\hline & $\overline{P M}$ & $B(10.2)$ & $\mathrm{B}(10.7)$ \\
\hline \multirow{2}{*}{$\begin{array}{l}\text { 5. East Avenue \& Greenville } \\
\text { Road }\end{array}$} & AM & $\mathrm{B}(12.9)$ & $\mathrm{B}(12.9)$ \\
\hline & PM & $C(16.3)$ & $C(16.3)$ \\
\hline
\end{tabular}

1. For unsignalized intersections, the LOS and delays in seconds per vehicle for the minor street approach and the worst minor street movement are presented.

Tables 8 and 9 presents summary of level of services at the six study intersections. LOS A through $D$ are generally considered to be satisfactory service levels. The East Avenue/Southwest Entrance was found to be operating at LOS E during the morning peak hour for the minor street movement. 


\section{Future Traffic Conditions}

To assess future traffic conditions in the Study Area, growth rates were obtained from the Alameda County Congestion Management Agency's travel demand model. This model predicts a 0.65 percent annual increase in traffic in the study area to the year 2020.

Tables 10 and 11 present the results of the existing and 2020 LOS analysis at the six study intersections. Level of Service calculation worksheets for each of the two scenarios are attached in the technical appendix.

Table $10 \quad 2020$ Conditions Level of Service - Signalized Intersection

\begin{tabular}{|c|c|c|c|}
\hline \multirow[t]{3}{*}{ Signalized Intersection ${ }^{1}$} & \multirow{3}{*}{$\begin{array}{l}\text { Peak } \\
\text { Hour }\end{array}$} & \multicolumn{2}{|c|}{ Level of Service (Seconds/Vehicle) } \\
\hline & & Existing 2001 & Year 2020 \\
\hline & & Overall Intersection & Overall Intersection \\
\hline \multirow{2}{*}{$\begin{array}{l}\text { 1. East Avenue \& } \\
\text { Vasco Road }\end{array}$} & AM & $\mathrm{B}(17.5)$ & $B(19.0)$ \\
\hline & PM & $C(21.9)$ & $C(28.4)$ \\
\hline
\end{tabular}

1. For signalized intersections, the overall intersection LOS and intersection delay in seconds per vehicle are presented.

Table 112020 Conditions Level of Service - Unsignalized Intersections

\begin{tabular}{|c|c|c|c|c|c|}
\hline \multirow{3}{*}{$\begin{array}{l}\text { Unsignalized } \\
\text { Intersections }\end{array}$} & \multirow{3}{*}{$\begin{array}{l}\text { Peak } \\
\text { Hour }\end{array}$} & \multicolumn{4}{|c|}{ Level of Service (Seconds/Nehicle) } \\
\hline & & \multicolumn{2}{|c|}{ Existing 2001} & \multicolumn{2}{|c|}{ Year 2020} \\
\hline & & $\begin{array}{c}\text { Minor Street } \\
\text { Approach }\end{array}$ & $\begin{array}{c}\text { Worst Minor } \\
\text { Street } \\
\text { Movement }\end{array}$ & $\begin{array}{c}\text { Minor Street } \\
\text { Approach }\end{array}$ & $\begin{array}{l}\text { Worst Minor } \\
\text { Street } \\
\text { Movement }\end{array}$ \\
\hline \multirow{2}{*}{$\begin{array}{l}\text { 1. East Ave \& SW } \\
\text { Entrance }\end{array}$} & AM & $C(18.3)$ & $E(45.9)$ & $\mathrm{C}(22.8)$ & $F(64.9)$ \\
\hline & $P M$ & $\mathrm{D}(28.1)$ & $\mathrm{D}(28.8)$ & $F(61.0)$ & $F(64.1)$ \\
\hline \multirow{2}{*}{$\begin{array}{l}\text { 2. East Avenue \& W } \\
\text { Entrance to SNL }\end{array}$} & AM & $\mathrm{B}(12.6)$ & $C(16.4)$ & $\mathrm{B}(14.2)$ & $C(19.6)$ \\
\hline & PM & $\mathrm{B}(10.3)$ & $C(12.6)$ & $B(13.2)$ & $C(21.2)$ \\
\hline \multirow{2}{*}{$\begin{array}{l}\text { East Avenue \& } \\
\text { Southgate }\end{array}$} & AM & $\mathrm{B}(12.6)$ & $\mathrm{B}(16.4)$ & $C(15.3)$ & $C(24.1)$ \\
\hline & PM & $\mathrm{C}(15.6)$ & $C(17.3)$ & $C(18.6)$ & $C(20.1)$ \\
\hline \multirow{2}{*}{$\begin{array}{l}\text { 4. East Avenue \& E } \\
\text { Entrance to SNL }\end{array}$} & AM & $\mathrm{B}(10.7)$ & $\mathrm{B}(12.4)$ & $\mathrm{B}(11.2)$ & $\mathrm{B}(13.1)$ \\
\hline & PM & $B(10.2)$ & $\mathrm{B}(10.7)$ & $B(10.5)$ & $\mathrm{B}(11.1)$ \\
\hline \multirow{2}{*}{$\begin{array}{l}\text { 5. East Avenue \& } \\
\text { Greenville Road }\end{array}$} & $\overline{A M}$ & $\mathrm{~B}(12.9)$ & $\mathrm{B}(12.9)$ & $B(14.2)$ & $B(14.2)$ \\
\hline & PM & $C(16.3)$ & $C(16.3)$ & $C(19.9)$ & $C(19.9)$ \\
\hline
\end{tabular}

1. For unsignalized intersections, the LOS and delays in seconds per vehicle for the minor street approach and the worst minor street movement are presented.

As shown in Table 10, the signalized intersection of East Avenue and Vasco Road continues to perform at LOS B and C in 2020.

Minor approach movements have longer delays than the major approach movements since the major street approach does not have to stop; therefore, only the minor street approach and the worst minor street movements calculations are presented. As shown in Table 11, level of service for many of the minor street approaches and worst minor street movements is identified as LOS B or C. In 2020 , the intersection of East Avenue and the Southwestgate was found to function at 
LOS F for the minor street approach and minor street movement. The intersection was tested for signalization and the intersection would improve to LOS B during the AM and PM peak hours with a signal. Future monitoring of this intersection is required to determine when/if signalization is needed.

\subsubsection{Required Left-turn Storage Lengths}

At selected locations on East Avenue, left-turn lanes should be provided. Appropriate storage lengths at unsignalized intersections were determined based on the existing heaviest hour of traffic. Table 12 presents the calculated left turn storage requirements.

\section{Table 12 Left-Turn Storage Length Requirements}

\begin{tabular}{|l|c|}
\hline Location & Storage Length (feet) $^{1}$ \\
\hline $\begin{array}{l}\text { Left-turn lane on East Avenue in westbound direction at Vasco } \\
\text { Road }\end{array}$ & 350 \\
\hline $\begin{array}{l}\text { Left-turn lane on East Avenue in eastbound direction at the } \\
\text { South West Entrance }\end{array}$ & 125 \\
\hline $\begin{array}{l}\text { Left turn lane on East Avenue in westbound direction at West } \\
\text { Entrance to Sandia Lab }\end{array}$ & 150 \\
\hline $\begin{array}{l}\text { Left-turn lane on East Avenue in eastbound direction at } \\
\text { Southgate }\end{array}$ & \\
\hline
\end{tabular}

1. Left turn storage lengths are calculated based on existing 5-day weekday average peak hour volume

Three additional entrances to the SNL between Avenue D and Southgate are currently closed. When the LLNL and SNL do decide to reopen these entrances, new traffic counts and analysis are required to determine appropriate left-turn storage lengths for these intersections.

\subsubsection{Environmental Documentation Review}

In 1989, a detailed traffic study was prepared for the closure of East Avenue between South Vasco Road and Greenville Road. This study was prepared as environmental documentation for CEQA/NEPA clearance. Using the data collected in Section 2.2 and the analysis conducted in Section 2.3, the results of the 1989 study were reviewed to determine if its assumptions and conclusions remain valid. Since the combined LLNL and SNL employment has declined from approximately 11,000 in 1989 to a current level of approximately 10,000 (or 9 percent reduction), the previous study is likely conservative in its analysis. Table 13 compares the traffic volumes collected for the westbound movement at East Avenue and Vasco Road between the 1989 Study and this study. The reduction in the peak hour traffic volume is consistent with the reduction in the number of employees. The traffic westbound traffic volume on East Avenue is expected to increase to $1,337 \mathrm{vph}$. The current employment level is expected to be stable through year 2020 . 
Table 13 East Avenue Traffic Volume Comparison

\begin{tabular}{|l|c|c|}
\hline Source & $\begin{array}{c}\text { PM Peak Hour (vph) } \\
\text { Westbound at } \\
\text { East Ave/Nasco Rd }\end{array}$ & $\begin{array}{c}\text { Employees } \\
\text { (LLNL+SNL) }\end{array}$ \\
\hline 1989 East Avenue Report (1-Day Count) & 1,420 & 11,000 \\
\hline 2001 Traffic Count by KORVE (5-Day Average) & 1,190 & 9,990 \\
\hline Percentage Reduction (Existing) & $-16 \%$ & $-9 \%$ \\
\hline 2020 Projection (CMA Model Growth Rate) & 1,337 & $9,990^{1}$ \\
\hline
\end{tabular}

1. The current employment level of 9,990 is expected to be stable through year 2020 .

The non-laboratory traffic volumes were also calculated in order to determine the amount of traffic that would be impacted by the closure of East Avenue. As shown in Table 14, the non-laboratory traffic currently using East Avenue is only two percent. This small percentage of traffic will be distributed to other roadways in the study area, such as Vasco Road, Greenville Road, Patterson Pass Road and Tesla Road to the south, with the closure of East Avenue.

Table 14 Calculation of Weekday Non Laboratory Traffic (5-Day Average)

\begin{tabular}{|c|l|c|c|c|c|c|}
\hline & Location & Location \# & Inbound & Outbound & TOTAL & $\begin{array}{c}\% \text { of East } \\
\text { Ave }\end{array}$ \\
\hline (A) & East Avenue & $3+11$ & 6,765 & 6,805 & 13,570 & $100 \%$ \\
\hline (B) & LLNL Entrances on East Ave & $4+6+7$ & 4,670 & 4,675 & 9,345 & $69 \%$ \\
\hline (C) & SNL Entrances on East Ave & $5+8$ & 1,930 & 2,030 & 3,960 & $29 \%$ \\
\hline & & & & & & \\
\hline B + C & LLNL + SNL & & 6,600 & 6,705 & 13,305 & $98 \%$ \\
\hline A-(B+C) & Non Lab Traffic & & 165 & 100 & 265 & $2 \%$ \\
\hline
\end{tabular}

\subsection{Administrative Control of East Avenue}

This section describes the analysis of implementing the following controls to support the administrative control of East Avenue:

- New East Avenue Security Control Point at South Vasco Road;

- New East Avenue Security Control Point at Greenville Road; and

- New Truck Inspection Facility - East Avenue at Greenville Road.

The required inbound and outbound lanes and lane widths and anticipated queue lengths for the three new facilities were determined. 


\subsection{New East Avenue Security Control Point at South Vasco Road}

In order to determine the storage length required for the entry gate, the average service rate at the existing Westgate was measured. The Westgate was selected because it is currently congested and provides the maximum service rate at peak flow. The service rate was measured on Tuesday, January 22, 2002 and determined to be approximately 7.25 seconds/vehicle/lane or 497 vehicle/hour/lane (vphpl). The current eastbound AM peak hour demand on East Avenue is 905 vph. Assuming random arrivals (Poisson Distribution) a factor of 1.5 is applied to the demand to account for random arrival and peaking characteristics, which translates to a demand or arrival rate of $1,358 \mathrm{vph}$. Since the arrival rate is greater than the service rate, additional entry lanes are required to avoid traffic backing into Vasco Road, similar to the existing condition at the Westgate. The number of lanes required is determined by dividing the arrival rate by the service rate $(1,358$ $\mathrm{vph} / 497 \mathrm{vphpl}=2.73$ lanes or 3 lanes (rounded up). With three entry lanes, the new service rate would be 1,491 vph ( 3 lanes $\times 497$ vphpl).

The queue length is determined using the following formulas:

Given: $\lambda=$ Arrival rate

$\mu=$ Service rate

And arrival rate is less than service rate: $\lambda<\mu$

Equation 1 to determine the ratio of arrival rate over the service rate, $r: \quad r=\lambda / \mu$

Equation 2 to determine the number of vehicles in the queue, $L q: \quad L q=r^{2} /(1-r)$

Source: Fundamentals of Transportation Engineering, C.S. Papacostas, 1987

Using the above equations, the number of vehicles expected in the queue at the East Avenue/Nasco Road Gate is approximately nine vehicles per lane. Assuming an average vehicle length of 25 feet, the storage length required is approximately 225 feet per lane. According to the preliminary design sketch (SK-1-11-14-01) provided by the LLNL staff, the available storage length is 360 feet per lane for only two lanes. Based on the above analysis, it is recommended that three entry lanes be provided with a minimum storage length of 225 feet per lane.

\subsection{New East Avenue Security Control Point at Greenville Road}

A new entry gate is also proposed for the westbound traffic on East Avenue at Greenville Road. The current westbound traffic demand at this location is $310 \mathrm{vph}$. Using the same methodology to determine the number of lanes required, which is described above, the potential maximum arrival rate is $465 \mathrm{vph}$ ( $1.5 \times 310 \mathrm{vph})$ and the estimated service rate remains the same at $497 \mathrm{vphpl}$. Since the service rate is greater that the arrival rate, one westbound lane is sufficient to serve the forecast demand.

Using equations 1 and 2 above, the number of vehicles expected in the queue is approximately 15 vehicles per lane. Assuming an average vehicle length of 25 feet, the storage length required is 
approximately 375 feet per lane. Based on the preliminary design sketch (SK-1-11-14-01) provided by the LLNL staff, the proposed storage length was 315 feet. At this location the distance between the gate portal and the pop-up barrier is 454 feet, while at the Vasco Road, the distance is require only 300 feet. Therefore, the storage length could be gained by reducing the distance from the gate portal to the pop-up barrier.

\subsection{New Truck Inspection Facility - East Avenue at Greenville Road}

Weekday truck classification counts were conducted at Gate location \#7 for LLNL and \#8 for SNL concurrently as the other counts. Tables 15 and 16 summarize the average weekday trucks counted at locations 7 and 8 respectively. Table 17 summarizes the average combined trucks for the two gate locations. Tables 15, 16 and 17 show the average trucks counted for the peak period between $9 \mathrm{AM}$ and $3 \mathrm{PM}$. Other non-peak hour truck counts are shown in the appendix. The combined maximum total trucks measured were 16 trucks per hour. Majority of the trucks are the smaller 2-axle type delivery trucks such as FedEx trucks. All the trucks entering the LLNL and SNL will be required to be inspected at the new truck inspection facility west of the intersection of East Avenue and Greenville Road.

Table 15 Existing Total Trucks - Location 7 (5-Day Weekday Average)

\begin{tabular}{|l|c|c|c|c|c|c|}
\hline Time & 2 Axle & 3 Axle & 4 Axle & 5 Axle & 6 Axle & Total Trucks \\
\hline 9am-10am & 2 & 1 & 1 & 2 & 0 & 5 \\
\hline $10 \mathrm{am}-11 \mathrm{am}$ & 3 & 1 & 1 & 3 & 0 & 7 \\
\hline 11am-12pm & 4 & 0 & 0 & 2 & 0 & 7 \\
\hline $12 \mathrm{pm}-1 \mathrm{pm}$ & 5 & 0 & 0 & 2 & 0 & 7 \\
\hline $1 \mathrm{pm}-2 \mathrm{pm}$ & 4 & 0 & 0 & 2 & 0 & 6 \\
\hline 2pm-3pm & 2 & 0 & 0 & 1 & 0 & 3 \\
\hline
\end{tabular}

Table 16 Existing Total Trucks - Location 8 (5-Day Weekday Average)

\begin{tabular}{|l|c|c|c|c|c|c|}
\hline Time & 2 Axle & 3 Axle & 4 Axle & 5 Axle & 6 Axle & Total Trucks \\
\hline $9 a \mathrm{~m}-10 \mathrm{am}$ & 4 & 0 & 1 & 1 & 0 & 6 \\
\hline $10 \mathrm{am}-11 \mathrm{am}$ & 7 & 1 & 0 & 1 & 0 & 9 \\
\hline $11 \mathrm{am}-12 \mathrm{pm}$ & 7 & 0 & 0 & 1 & 0 & 9 \\
\hline $12 \mathrm{pm}-1 \mathrm{pm}$ & 6 & 0 & 0 & 1 & 0 & 7 \\
\hline $1 \mathrm{pm}-2 \mathrm{pm}$ & 3 & 0 & 0 & 0 & 0 & 4 \\
\hline $2 \mathrm{pm}-3 \mathrm{pm}$ & 4 & 0 & 0 & 0 & 0 & 4 \\
\hline
\end{tabular}


Table 17 Combined Total Trucks - Locations 7 and 8 (5-Day Weekday Average)

\begin{tabular}{|l|c|c|c|c|c|c|}
\hline Time & 2 Axle & 3 Axle & 4 Axle & 5 Axle & 6 Axle & Total Trucks \\
\hline $9 a m-10 a m$ & 6 & 1 & 2 & 3 & 0 & 11 \\
\hline $10 \mathrm{am}-11 \mathrm{am}$ & 10 & 1 & 1 & 4 & 0 & 16 \\
\hline 11 am-12pm & 11 & 0 & 1 & 3 & 0 & 16 \\
\hline $12 \mathrm{pm}-1 \mathrm{pm}$ & 10 & 0 & 0 & 3 & 0 & 14 \\
\hline $1 \mathrm{pm}-2 \mathrm{pm}$ & 8 & 0 & 0 & 2 & 0 & 10 \\
\hline $2 \mathrm{pm}-3 \mathrm{pm}$ & 6 & 0 & 0 & 1 & 0 & 7 \\
\hline
\end{tabular}

In order to determine the storage length required for the entry gate, the average service rate at the existing truck gate was measured. The average truck service rate was measured on Tuesday, January $22^{\text {nd }}, 2002$ at the LLNL Shipping and Receiving Gate and found to be approximately 3 minutes/truck/lane or $20 \mathrm{tphpl}$. The current westbound truck arrival rate is 16 trucks per hour.

In order to use equations 1 and 2 above, the arrival rate must be less than the service rate. Using an arrival rate of 16 trucks per hour and a factor (Poisson) of 2 to account for random arrivals, the maximum queue is expected to be six trucks total or three trucks per lane in two lanes. The weighted average truck length was determined to be 42 feet using the truck data collected shown in Table 17 and the design truck length for a given truck size as shown in Table 18. Since there is only one inspection lane available, storage lanes are required to avoid traffic spilling back onto East Avenue, which would block the new westbound entry at Greenville Road. If two lanes were provided, the storage length would be 135 feet/lane. This would accommodate one-5axle trucks and two-2axle trucks per lane $\left(130\right.$ feet per lane $\left.=70^{\prime}+30^{\prime}+30^{\prime}\right)$, which is consistent with field observations.

Table 18 Truck Size Summary

\begin{tabular}{|c|c|c|l|}
\hline Truck Type & $\begin{array}{c}\text { AASHTO } \\
\text { (feet) }\end{array}$ & $\begin{array}{c}\text { CALTRANS }^{2} \\
\text { (feet) }\end{array}$ & \multicolumn{1}{|c|}{ Description } \\
\hline $2-$ Axle & 30 & - & Single unit trucks \\
\hline $3-$ Axle & - & 40 & N/A \\
\hline $4-$ Axle & 50 & 65 & N/A \\
\hline $5-$ Axle & $55-65$ & 68 & N/A \\
\hline $6-$ Axle & $102-118$ & - & Three-trailers \\
\hline
\end{tabular}

Source:

1. A Policy on Geometric Design of Highway Streets, 1990, American Association of State Highway and Transportation Officials (ASSTHO), Chapter 2

2. Caltrans Highway Design Manual, Figure 404.2

\section{Future Queue Lengths}

The queue lengths calculated for the entry lanes and left turn lanes for East Avenue were based on existing traffic volumes. Although in Section 2, the future traffic growth in the general area was reported using the Alameda County Congestion Management Agency's Model, the design of East Avenue is contingent primarily on the size of LLNL and SNL. 


\subsection{RECOMMENDATIONS}

Based on the traffic analysis described in Sections 2 and 3, KORVE has determined the geometric improvements required to facilitate the administrative control of East Avenue between Vasco Road and Greenville Road. Figure 8 illustrates the conceptual layout of East Avenue with the recommended geometric improvements. This includes the location of left turn lanes, required number of entry lanes, expected queue lengths, truck inspection area storage and laning requirements, bike lanes, and pedestrian crosswalks.

\section{Other Improvements}

- Flexible Work Hours - As discussed in the traffic pattern analysis in Section 2.3.1, and as shown in Figure 6, the sharp spike in traffic arriving to work at the same time can create congestion and delays at the entry gates. One effective Traffic Demand Management tool (TDM) is to implement a flexible work schedule so that not all the employees are arriving at 8-9 AM. This would reduce the peak hour congestion and would 'flatten" the sharp curve, which would spread the peak hour to more than one hour at a less intensive level. The current spike at LLNL and SNL is much more severe than those typically observed at other private facilities.

- Changeable Message Sign (CMS) - A CMS is recommended at the new entrance at the intersection of East Avenue and Vasco Road. Since East Avenue west of Vasco Road is a public street, the potential for vehicles mistakenly entering the laboratory is high. A CMS will minimize this occurrence, which can increase delay for laboratory-bound vehicles. The CMS would display informational messages, such as, welcoming messages, visitors must visit badge office, special events messages, and trucks must enter at Greenville Road.

- Signal at East Avenue and South West Entrance - Should traffic volumes rise, a signal may be necessary at this location to provide an acceptable service level. 

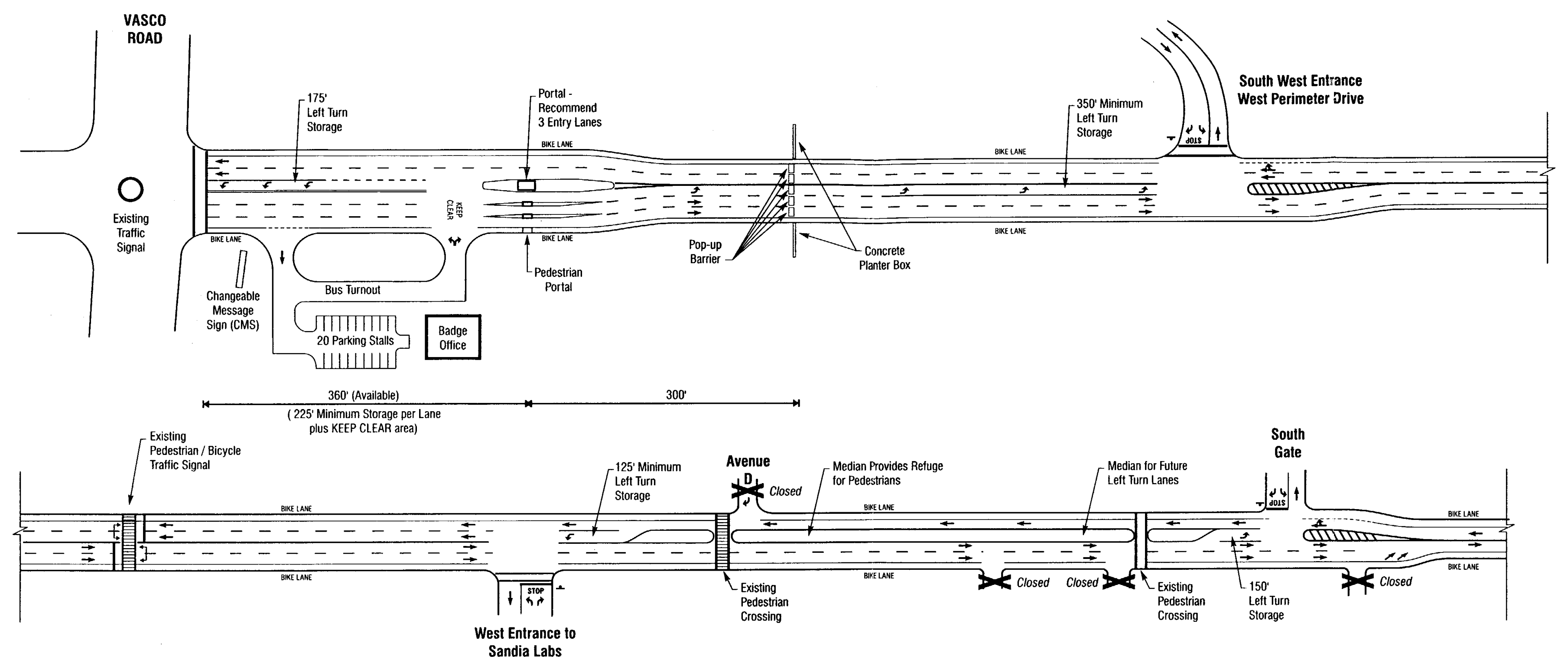

NOT TOSGALE

Existing LLNL Shipping and Receiving

LAWRENCE LIVERMORE NATIONAL LABORATORY
EAST AVENUE / TMERGENCY RESPONSE PLANNING - TRAFFIC STUDY Figure 8

EAST AVENUE CONCEPTUAL PLAN - RECOMMENDATIONS

$\mathrm{Z}=$ Korve

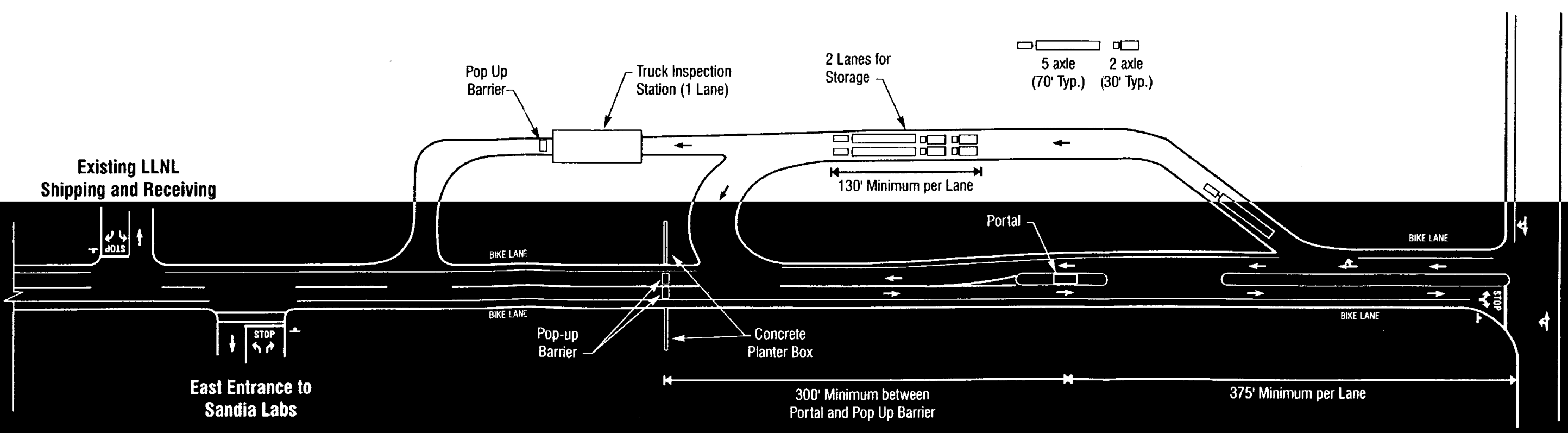

Rev 2.1251402 
TECHNICAL APPENDIX 



\section{BAYMETRICS TRAFFIC RESOURCES Location 1: Southbound Left-Turn On Vasco North of East}

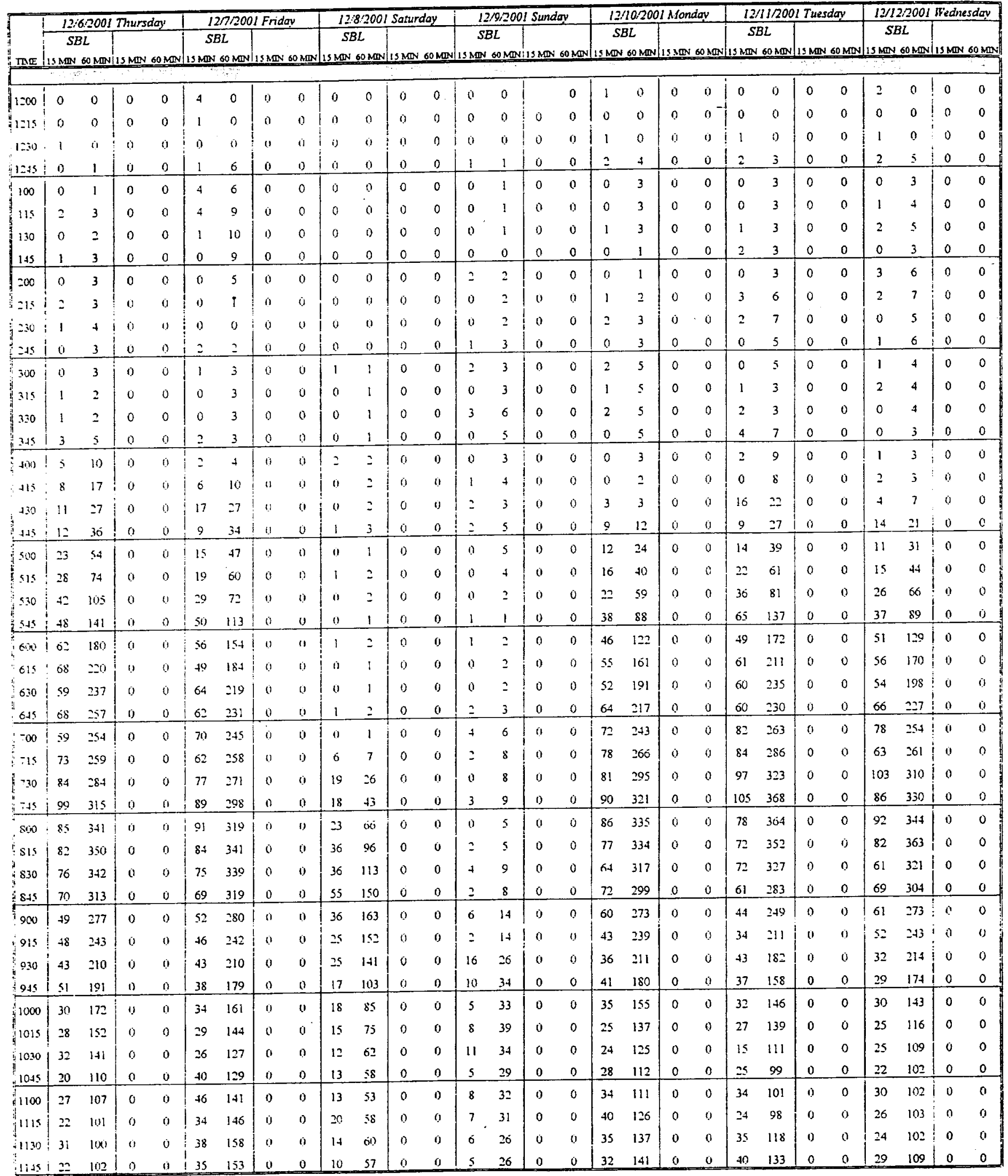




\section{BAYMETRICS TRAFFIC RESOURCES Location 1: Southbound Left-Turn On Vasco North of East}

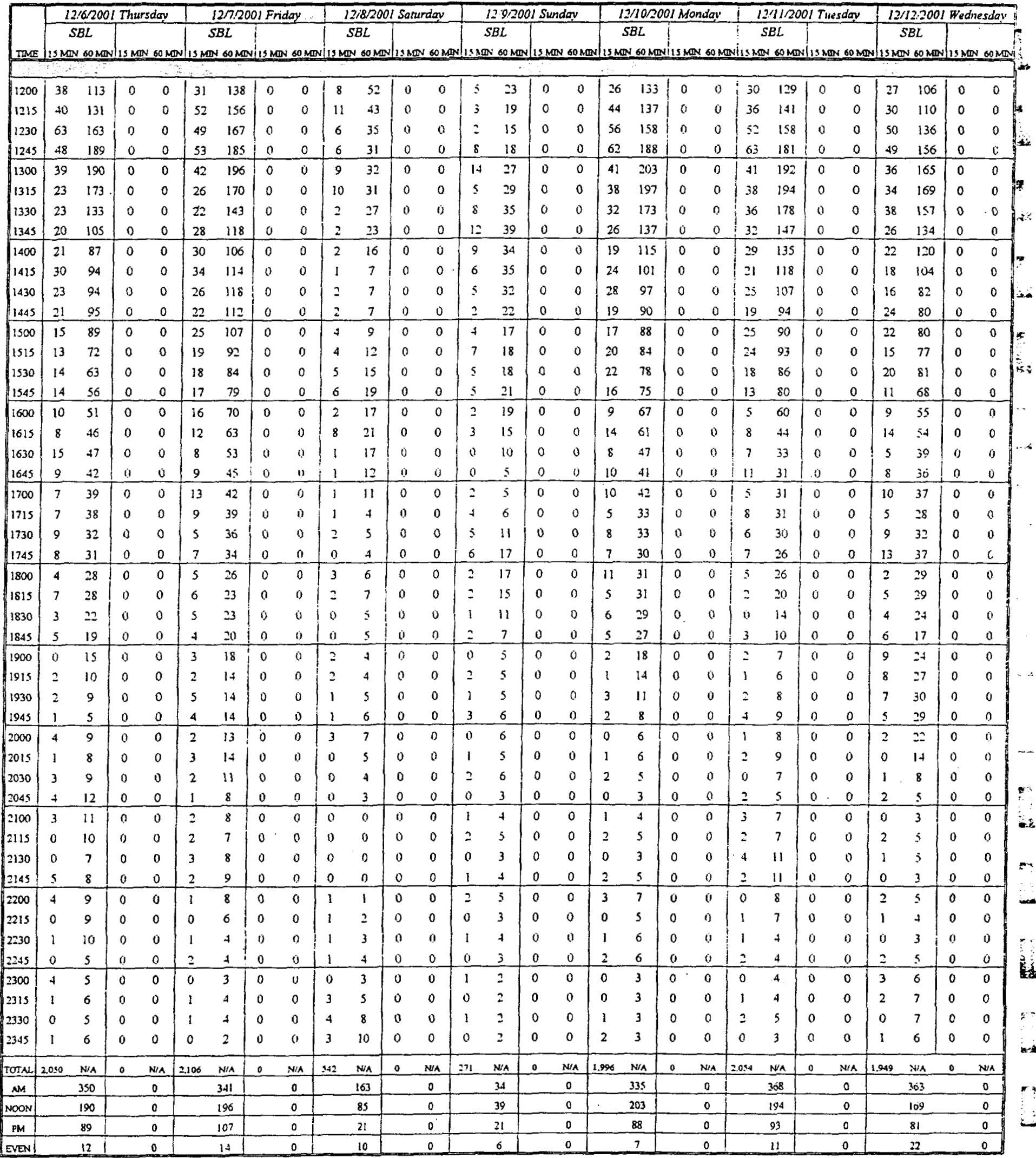




\section{BAYMETRICS TRAFFIC RESOURCES Location 2: Northbound Right-Turn On Vasco South of East}

\begin{tabular}{|c|c|c|c|c|c|c|c|c|c|c|c|c|c|c|c|c|c|c|c|c|c|c|c|c|c|c|c|c|c|}
\hline \multirow{2}{*}{ TAE } & \multirow{2}{*}{\multicolumn{4}{|c|}{$12 / 6 / 2001$ Thursday }} & \multicolumn{4}{|c|}{$12 \pi n 2001$ Friday } & \multicolumn{4}{|c|}{ 12782001 Saturdoy } & \multicolumn{5}{|c|}{ 13/9/2001 Sunday } & \multicolumn{4}{|c|}{ 13/10/2001 Mondiay } & \multicolumn{4}{|c|}{ 12:11/2001 Tiuesdav } & \multicolumn{4}{|c|}{$12 / 12 / 2001$ Weinesday } \\
\hline & & $8 R$ & & & & $B R$ & & & & & & & & $N B$ & & & & & $N B R$ & & & & $B R$ & & & & $B R$ & & \\
\hline 1200 & 1 & 0 & 0 & 0 & 1 & 0 & 0 & 0 & 1 & 0 & 0 & 0 & & 0 & 0 & 0 & 0 & 0 & 0 & 0 & 0 & 1 & & 0 & 0 & 2 & 0 & 0 & 0 \\
\hline 1215 & 0 & 0 & 0 & 0 & 0 & 0 & 0 & 0 & 0 & 0 & 0 & 0 & & i & 0 & 0 & 0 & 0 & 0 & 0 & 0 & 0 & 0 & 0 & 0 & 1 & 0 & 0 & 0 \\
\hline 1230 & 3 & 0 & 0 & 0 & 0 & $\dot{0}$ & $i$ & 0 & 1 & 0 & 0 & 0 & & !! & 0 & 0 & 0 & 1 & i) & 9 & $\dot{0}$ & 0 & 9 & 0 & 0 & 0 & 0 & 0 & 0 \\
\hline 1245 & 1 & 5 & 0 & 0 & 0 & 1 & 0 & 0 & 8 & 2 & 0 & 0 & & $=$ & 3 & 0 & 0 & 0 & 1 & 0 & 0 & 0 & 1 & 0 & 0 & 0 & 3 & 0 & 0 \\
\hline 100 & 0 & 4 & 0 & 0 & 1 & 1 & 0 & 0 & $\mathrm{c}$ & 1 & 0 & 0 & & 1) & 3 & 0 & 0 & 1 & 2 & 0 & 0 & 1 & 1 & 0 & 0 & 0 & 1 & 0 & 0 \\
\hline 115 & 1 & 5 & 0 & 0 & 0 & 1 & 0 & 0 & 1 & 2 & 0 & 0 & & 9 & $=$ & 0 & 0 & 0 & 2 & 0 & $\dot{0}$ & 0 & 1 & 0 & 0 & 1 & 1 & 0 & 0 \\
\hline 130 & 0 & 2 & 0 & 0 & 0 & I & 0 & 0 & 0 & 1 & 0 & 0 & & 0 & 2 & 0 & 0 & 1 & 2 & 0 & 0 & 1 & 2 & 0 & 0 & 0 & 1 & 0 & 0 \\
\hline 145 & 2 & 3 & 0 & 0 & 0 & 1 & 0 & 0 & 0 & 1 & 0 & 0 & & 0 & 0 & 0 & 0 & 1 & 3 & 0 & 0 & 0 & 2 & 0 & 0 & 0 & 1 & 0 & 0 \\
\hline 200 & 1 & 4 & 0 & 0 & 0 & 0 & 0 & 0 & 1 & 2 & 0 & 0 & & 0 & 0 & 0 & 0 & 0 & 2 & 0 & 0 & 1 & 2 & 0 & 0 & 1 & 2 & 0 & 0 \\
\hline 215 & 1 & 4 & 0 & 0 & 0 & 0 & 0 & 0 & 0 & 1 & 0 & 0 & & 1 & $i$ & 0 & 0 & 0 & 2 & 0 & 0 & 0 & 2 & 0 & 0 & 1 & 2 & 0 & 0 \\
\hline 230 & 0 & 4 & 0 & 0 & 0 & 0 & 0 & 0 & 1 & 2 & 0 & 0 & & 0 & 1 & 0 & 0 & 2 & 3 & 0 & 0 & 0 & 1 & 0 & 0 & 0 & 2 & 0 & 0 \\
\hline 245 & 0 & 2 & 0 & 0 & 1 & 1 & 0 & 0 & 0 & 2 & 0 & 0 & & 0 & 1 & ij & 0 & 1 & 3 & 0 & 0 & 1 & 2 & 0 & 0 & 2 & 4 & 0 & 0 \\
\hline 300 & 1 & 2 & 0 & 0 & 0 & 1 & 0 & 0 & 1 & 2 & 0 & 0 & & 0 & 1 & 0 & 0 & $\dot{0}$ & 3 & 0 & 0 & 1 & 2 & 0 & 0 & 0 & 3 & 0 & 0 \\
\hline 315 & 1 & 2 & $\dot{0}$ & 0 & 0 & 1 & 0 & 0 & 0 & 2 & 0 & 0 & & 1 & 1 & 0 & 0 & 1 & 4 & 0 & 0 & 0 & 2 & 0 & 0 & 0 & 2 & 0 & 0 \\
\hline 330 & 0 & 2 & 0 & 0 & 2 & 3 & 0 & 0 & 3 & 4 & 0 & 0 & & 0 & 1 & 0 & 0 & 0 & 2 & 0 & 0 & 2 & 4 & 0 & 0 & 0 & 2 & 0 & 0 \\
\hline 345 & 0 & 2 & 0 & 0 & 0 & 2 & 0 & 0 & 0 & 4 & 0 & 0 & & 0 & 1 & 0 & 0 & 0 & 1 & 0 & 0 & 1 & 4 & 0 & 0 & 1 & 1 & 0 & 0 \\
\hline 400 & 3 & 4 & 0 & 0 & 2 & 4 & 0 & 0 & 1 & 4 & 0 & 0 & & 0 & 1 & 0 & 0 & 2 & 3 & 0 & 0 & 1 & 4 & 0 & 0 & 2 & 3 & 0 & 0 \\
\hline 415 & 0 & 3 & 0 & 0 & 1 & 5 & (1) & 0 & 1) & 4 & 0 & $\dot{v}$ & & 0 & 0 & 0 & 0 & 0 & 2 & 0 & 0 & 0 & 4 & 0 & 0 & 0 & 3 & 0 & 0 \\
\hline 430 & 2 & 5 & 0 & 0 & 1 & 4 & is & 0 & 1 & $=$ & 0 & 0 & & 0 & 0 & 0 & 0 & 2 & 4 & 0 & 0 & 2 & 4 & 0 & 0 & $=$ & 5 & 0 & 0 \\
\hline 415 & 3 & 8 & 0 & 0 & 0 & $t$ & 19 & 0 & 0 & 2 & 0 & 0 & & 0 & 0 & 0 & 0 & 0 & 4 & 0 & 0 & 0 & 3 & 0 & 0 & 0 & 4 & 0 & 0 \\
\hline 500 & 3 & 8 & 0 & 0 & $=$ & 4 & $u$ & 0 & 1 & 2 & 0 & 0 & & 0 & 0 & 0 & (1) & I & 3 & 0 & 0 & 1 & 3 & 0 & 0 & 2 & 4 & 0 & 0 \\
\hline 515 & 3 & 11 & 0 & 0 & 1 & 4 & 0 & 0 & 0 & 2 & $\dot{0}$ & 0 & & 1 & 1 & 0 & 0 & 2 & $s$ & 10 & 0 & 2 & 5 & 0 & 0 & 3 & 7 & 0 & 0 \\
\hline 530 & 2 & 11 & 0 & 0 & 6 & 9 & 0 & " & 2 & 3 & 0 & 0 & & 1 & 1 & "1) & 0 & $\varsigma$ & 8 & 0 & 0 & 7 & 10 & 0 & 0 & 2 & 7 & 0 & 0 \\
\hline 545 & 8 & 16 & 0 & 0 & 4 & 13 & 0 & 0 & 2 & 5 & 0 & 0 & & $z$ & 3 & 0 & 0 & 6 & 14 & 0 & 0 & 6 & 16 & 0 & 0 & 5 & 12 & 0 & 0 \\
\hline 600 & 8 & & 0 & 0 & 8 & 10 & 0 & 0 & 0 & 4 & 0 & 0 & & 1 & 4 & 0 & 0 & 6 & 19 & 0 & 0 & 11 & 26 & 0 & 0 & 7 & 17 & 0 & 0 \\
\hline 615 & 12 & 30 & 0 & 0 & $1=$ & 30 & 0 & 0 & 0 & 4 & 0 & 0 & & $=$ & 5 & 0 & $u$ & 10) & 27 & 0 & 0 & 14 & 38 & 0 & 0 & 7 & 31 & 0 & 0 \\
\hline 630 & 14 & 42 & $\dot{0}$ & 0 & 16 & 40 & 0 & 0 & 0 & $=$ & 0 & 0 & & 0 & 5 & 0 & 0 & 13 & 35 & 0 & 0 & 13 & 44 & 0 & $j$ & 16 & 35 & 0 & 0 \\
\hline 645 & 24 & 58 & 0 & 0 & 21 & 57 & 0 & 0 & -1 & 1 & 0 & 0 & & $z$ & 5 & 0 & 0 & 25 & 54 & 0 & 0 & 21 & 59 & 0 & 0 & 22 & 52 & 0 & 0 \\
\hline 700 & 31 & 81 & 0 & 0 & 29 & 78 & 11 & " & 3 & 4 & 0 & 0 & & 1 & 5 & 0 & 0 & 27 & 75 & 0 & 0 & 35 & 8.3 & 0 & $\dot{0}$ & 33 & 78 & 0 & 0 \\
\hline 715 & 35 & 104 & 0 & 0 & 35 & 101 & 0 & 0 & 1 & 5 & 0 & 0 & & 1 & 4 & 0 & 0 & 33 & 98 & 0 & 0 & 36 & 105 & 0 & 0 & 27 & 98 & 0 & 0 \\
\hline 730 & 49 & 139 & 0 & 0 & 16 & 131 & 0 & 0 & 0 & $s$ & 0 & 0 & & $=$ & 6 & 0 & 0 & 36 & 121 & 0 & 0 & 44 & 136 & 0 & 0 & 45 & 127 & 0 & 0 \\
\hline 745 & 52 & 167 & 0 & 0 & 50 & 160 & 0 & 0 & 4 & 8 & 0 & 0 & & 1 & 5 & 0 & 0 & 56 & 152 & 0 & 0 & 48 & 163 & 0 & 0 & 58 & 363 & 0 & 0 \\
\hline 800 & 46 & 182 & 0 & 0 & $\$ 8$ & 189 & 0 & 0 & 2 & 7 & 0 & 0 & & 2 & 6 & 0 & 0 & 70 & 195 & 0 & 0 & 74 & 202 & 0 & 0 & 65 & 195 & 0 & $\mathrm{c}$ \\
\hline 815 & 43 & 190 & 0 & 0 & 42 & 196 & 0 & 0 & 1 & 7 & 0 & 0 & & 3 & 8 & 0 & 0 & 61 & 223 & 0 & 0 & 55 & 221 & 0 & 0 & 60 & 228 & 0 & 0 \\
\hline 830 & 38 & 179 & 0 & 0 & 41 & 191 & 0 & 0 & 2 & 9 & 0 & 0 & & 0 & 6 & 0 & 0 & 42 & 229 & 0 & 0 & 46 & 223 & 0 & 0 & 45 & 208 & 0 & 0 \\
\hline 845 & 36 & 163 & 0 & 0 & 35 & 176 & 0 & 0 & 1 & 6 & 0 & 0 & & 3 & 8 & 0 & 0 & 39 & 212 & 0 & 0 & 34 & 309 & 0 & 0 & 31 & 201 & 0 & 0 \\
\hline 900 & 23 & 140 & 0 & 0 & 28 & $1+6$ & 0 & 0 & I & 0 & 0 & 0 & & 1 & 7 & 0 & 0 & 27 & 169 & 0 & 0 & 25 & 160 & ij & 0 & 27 & 163 & 0 & 0 \\
\hline 915 & 20 & 117 & 0 & 0 & 15 & 119 & 0 & 0 & 1 & 6 & 0 & 0 & & $=$ & $\dot{0}$ & 0 & 0 & 11 & 119 & 0 & 0 & 15 & 120 & $\dot{0}$ & 0 & 11 & 114 & 0 & 0 \\
\hline 930 & 15 & 94 & 0 & 0 & 14 & 92 & 0 & 0 & 2 & 6 & 0 & 0 & & $=$ & 8 & 0 & 0 & 13 & 90 & 0 & 0 & 13 & 87 & 0 & 0 & 18 & 87 & 0 & 0 \\
\hline 945 & 17 & 75 & 0 & 0 & 14 & 71 & 0 & 0 & 3 & 8 & 0 & 0 & & 1 & 6 & 0 & 0 & 12 & 63 & 0 & 0 & 10 & 63 & 0 & 0 & 11 & 67 & 0 & 0 \\
\hline 1000 & 19 & 71 & 0 & 0 & 10 & 53 & 0 & 0 & 1 & 7 & 0 & 0 & & $=$ & 7 & 0 & 0 & $s$ & 41 & 0 & 0 & 9 & 47 & 0 & 0 & 13 & $\$ 3$ & 0 & 0 \\
\hline 1015 & 6 & 57 & 0 & 0 & 11 & 19 & 0 & 0 & 2 & 8 & 0 & 0 & & 1 & 6 & 0 & 0 & 8 & 38 & 0 & 0 & 10 & 42 & 0 & 0 & 8 & so & 0 & 0 \\
\hline 1030 & 7 & 49 & 0 & 0 & $s$ & 40 & 0 & 0 & 2 & 8 & 0 & 0 & $c$ & 0 & 4 & 0 & 0 & 5 & 30 & 0 & 0 & 5 & 34 & 0 & 0 & 4 & 36 & 0 & 0 \\
\hline 1045 & 5 & 37 & 0 & 0 & 3 & 29 & 0 & 0 & 2 & 7 & 0 & 0 & 3 & & 5 & 0 & 0 & 7 & 25 & 0 & 0 & 1 & 25 & 0 & 0 & 11 & 36 & 0 & 0 \\
\hline 1100 & 3 & 21 & 0 & 0 & 2 & 21 & 0 & 0 & 1 & 7 & 0 & 0 & 0 & 0 & 3 & 0 & 0 & 6 & 26 & 0 & 0 & 4 & 20 & 0 & 0 & 2 & 25 & 0 & 0 \\
\hline 1115 & 3 & 18 & 0 & 0 & 1 & 11 & 0 & 0 & 1 & 6 & 0 & 0 & 0 & & 2 & 0 & 0 & 5 & 23 & 0 & 0 & 4 & 14 & 0 & 0 & 4 & 21 & 0 & 0 \\
\hline 1130 & 6 & 17 & 0 & 0 & 7 & 13 & 0 & 0 & 0 & 4 & 0 & 0 & 1 & & 3 & 0 & 0 & 5 & 23 & 0 & 0 & 3 & 12 & 0 & 0 & 5 & 22 & 0 & 0 \\
\hline 1145 & 7 & 19 & 0 & 0 & 10 & 20 & 0 & 0 & 2 & 4 & 0 & 0 & 1 & 1 & 2 & 0 & 0 & 6 & 22 & 0 & 0 & 4 & 15 & 0 & 0 & 6 & 17 & 0 & 0 \\
\hline
\end{tabular}




\section{BAYMETRICS TRAFFIC RESOURCES Location 2: Northbound Right-Turn On Vasco South of East}

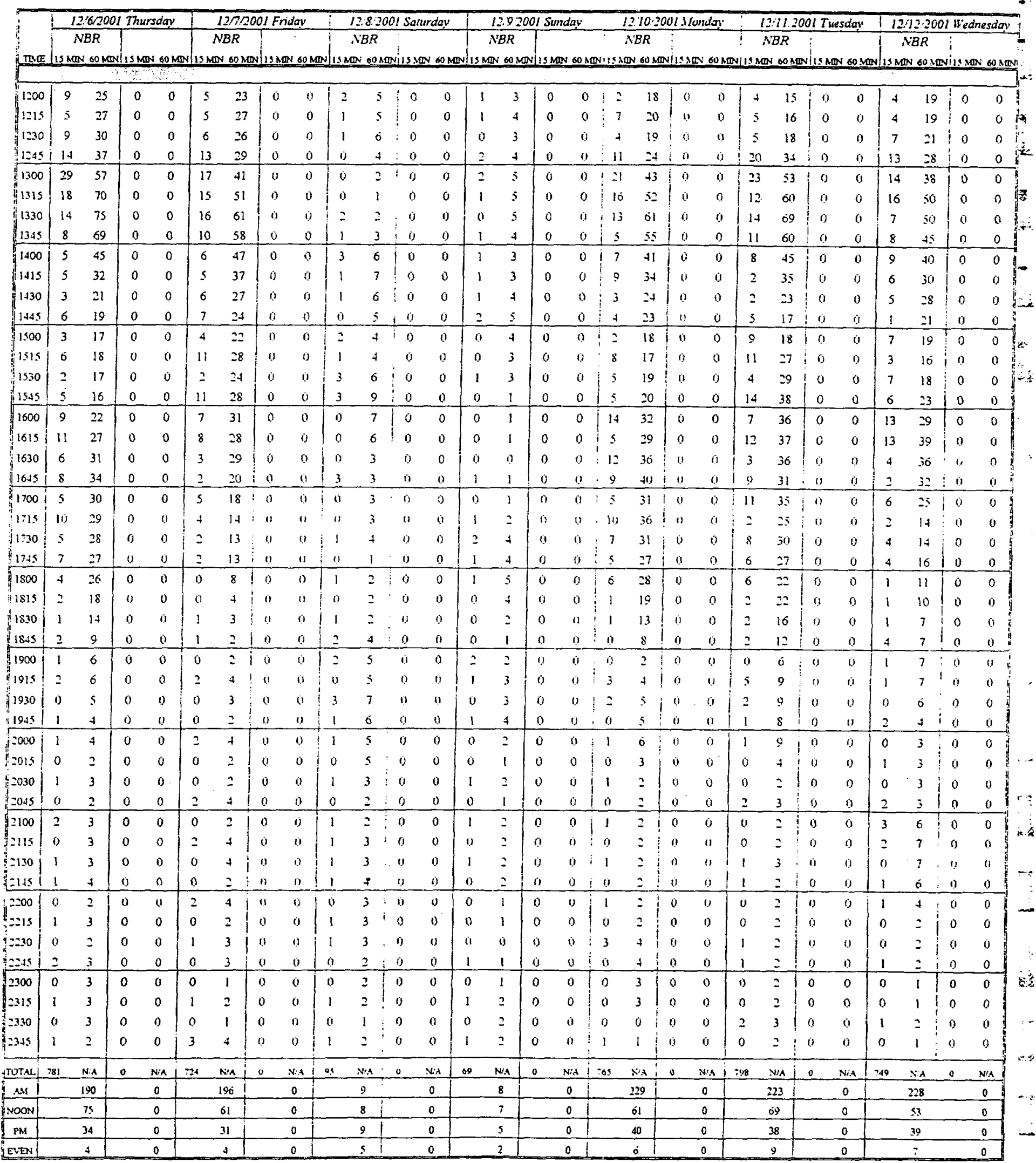




\section{BAYMETRICS TRAFFIC RESOURCES Location 3: East Avenue East of Vasco Road}

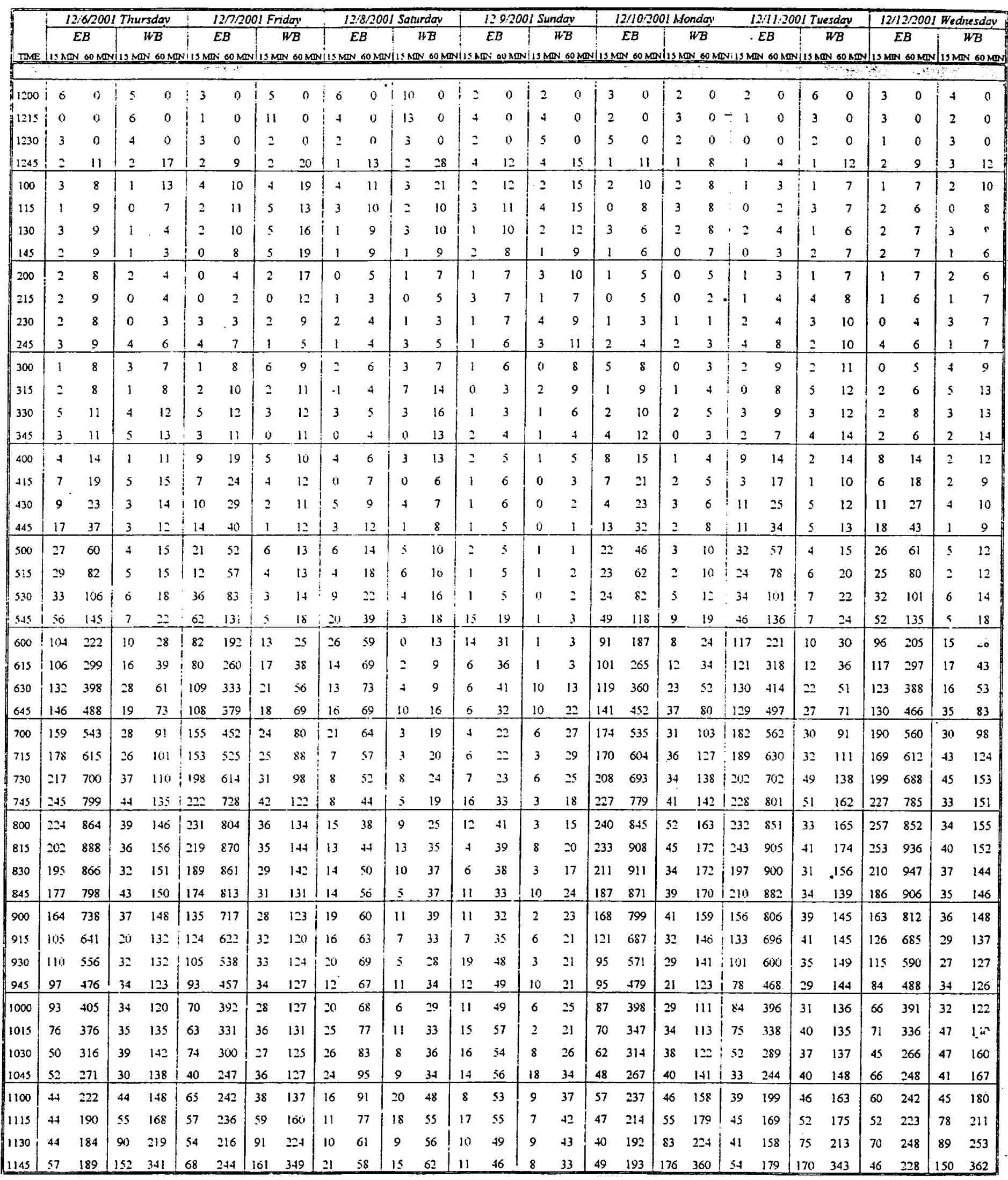




\section{BAYMETRICS TRAFFIC RESOURCES Location 3: East Avenue East of Vasco Road}

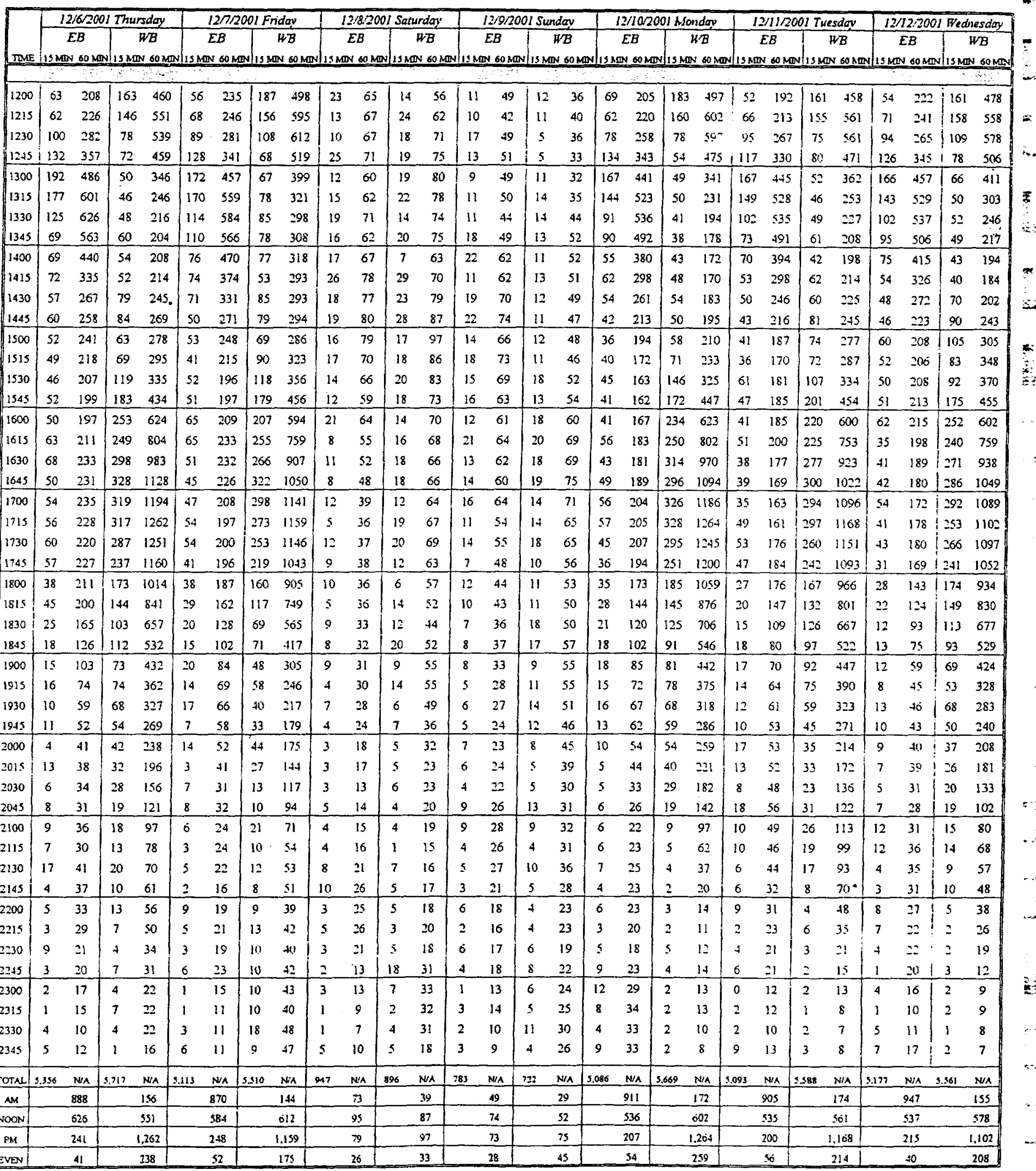




\section{BAYMETRICS TRAFFIC RESOURCES Location 4: Driveway North of East Aevnue (NB - Inbounds)}

\begin{tabular}{|c|c|c|c|c|c|c|c|c|c|c|c|c|c|c|c|c|c|c|c|c|c|c|c|c|c|c|c|c|}
\hline \multirow{2}{*}{ max } & \multicolumn{4}{|c|}{ 12.6:2001 Thursdn } & \multicolumn{4}{|c|}{$12 / 72001$ Frida: } & \multicolumn{4}{|c|}{ 12:82001 Saturdan } & \multicolumn{4}{|c|}{1292001 Sundtat } & \multicolumn{4}{|c|}{ 1270 200! Monday } & \multicolumn{4}{|c|}{ 12i1l/2001 Tuesdav } & \multicolumn{4}{|c|}{$12 / 122001$ Wednesdav } \\
\hline & \multicolumn{2}{|c|}{$\bar{N}(\bar{N})$} & 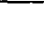 & 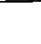 & & $(n N)$ & & & & $(N)$ & & & & & & & & (AN) & & & & (aN) & & & & $a N)$ & & \\
\hline 1200 & 0 & 0 & 0 & 0 & 0 & 0 & 1) & 0 & 0 & 0 & 0 & 0 & 0 & 0 & 0 & 0 & 0 & 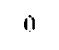 & 0 & 0 & 0 & 0 & 0 & 0 & 0 & 0 & 0 & 0 \\
\hline 1215 & 0 & 0 & 0 & 0 & 0 & 0 & 0 & 0 & 0 & 0 & 0 & 0 & 1. & 0 & $\dot{0}$ & 0 & 0 & 0 & 0 & 0 & 0 & 0 & 0 & 0 & 0 & 0 & 0 & 0 \\
\hline 1230 & 0 & 0 & 0 & 0 & 1 & 0 & a & 0 & 0 & is & 0 & 0 & 9 & 0 & 0 & a & 0 & $a$ & 0 & 0 & 0 & 0 & 0 & 0 & 0 & 0 & 0 & 0 \\
\hline 1245 & 0 & 0 & 0 & 0 & 1 & 2 & 0 & 0 & 0 & 0 & 0 & 0 & 9 & 0 & 0 & 0 & 0 & 0 & 0 & 0 & 0 & 0 & 0 & 0 & 0 & 0 & 0 & 0 \\
\hline 100 & 0 & 0 & 0 & 0 & 0 & 2 & 0 & 0 & 0 & 1) & 9 & 0 & 0 & 0 & 0 & 0 & $\dot{0}$ & 0 & 0 & 0 & 0 & 0 & 0 & 0 & 0 & 0 & 0 & 0 \\
\hline 115 & 0 & 0 & 0 & 0 & 0 & 2 & 0 & 0 & $\dot{a}$ & 0 & 0 & i) & 0 & 0 & 9 & 0 & 0 & 0 & i) & 0 & 0 & 0 & 0 & 0 & 0 & 0 & 0 & $\dot{0}$ \\
\hline 130 & $\dot{0}$ & 0 & 0 & 0 & 0 & 1 & 0 & 0 & 0 & 0 & 0 & 0 & 0 & 0 & 0 & 0 & 0 & 0 & 0 & 0 & 0 & 0 & 0 & 0 & 0 & 0 & 0 & 0 \\
\hline 145 & 0 & 0 & 0 & 0 & 0 & 0 & 0 & 0 & 0 & 0 & 0 & $\dot{0}$ & 0 & 0 & 0 & 0 & 0 & 0 & 0 & 0 & 0 & 0 & 0 & 0 & 0 & 0 & 0 & 0 \\
\hline 200 & 0 & 0 & 0 & 0 & 0 & 0 & 0 & 0 & 0 & 0 & 0 & 0 & 0 & 0 & 0 & 0 & 0 & 0 & 0 & 0 & 0 & 0 & 0 & 0 & 0 & 0 & 0 & 0 \\
\hline 215 & 0 & 0 & 0 & 0 & 0 & 0 & 0 & 0 & 0 & 0 & 0 & 0 & 0 & 0 & 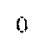 & 0 & 0 & 0 & 0 & 0 & 0 & 0 & 0 & 0 & 0 & 0 & 0 & 0 \\
\hline 230 & 0 & 0 & 0 & 0 & 0 & 0 & 0 & 0 & 0 & 0 & 0 & 0 & 0 & 0 & 0 & 0 & 0 & 0 & 0 & 0 & 0 & 0 & 0 & 0 & 0 & 0 & 0 & 0 \\
\hline$=45$ & 0 & 0 & D) & 0 & (1) & 0 & 9 & 0 & 0 & (1) & $\underline{\square}$ & 0 & 0 & 0 & 0 & 0 & 9 & 0 & 0 & 0 & 0 & 0 & 0 & 0 & 0 & 0 & 0 & 0 \\
\hline 300 & 0 & 0 & 0 & 0 & 0 & 0 & i & 0 & 0 & 0 & 0 & 0 & 0 & 0 & i) & 0 & 0 & 0 & 0 & 0 & 0 & 0 & 0 & 0 & 0 & 0 & 0 & 0 \\
\hline 315 & 1 & 1 & 0 & 0 & 0 & 0 & 0 & ij & 0 & 0 & 0 & 0 & 0 & 0 & 0 & 0 & 1 & 1 & 0 & 0 & 0 & 0 & 0 & 0 & 0 & 0 & 0 & 0 \\
\hline 330 & 0 & 1 & 0 & 0 & 0 & 0 & $n$ & 0 & 0 & 0 & 0 & 0 & 0 & 0 & 0 & 0 & 0 & 1 & 0 & 0 & 0 & 0 & 0 & 0 & 0 & 0 & 0 & 0 \\
\hline 345 & 0 & 1 & 0 & 0 & 0 & 0 & 11 & 10 & 0 & 0 & 0 & 0 & 0 & 0 & 0 & 0 & 0 & 1 & 0 & 0 & 0 & 1) & 0 & 0 & 0 & 0 & 0 & 0 \\
\hline 400 & 0 & 1 & 0 & 0 & 0 & 0 & 0 & 0 & 0 & 0 & 0 & 0 & 0 & 0 & 0 & 0 & 1 & 2 & 0 & 0 & 0 & 0 & 0 & 0 & 0 & 0 & 0 & 0 \\
\hline 415 & 2 & 2 & 0 & 0 & 1 & 1 & 9 & 0 & 0 & 0 & 0 & 0 & 0 & 0 & a & $"$ & 3 & + & 0 & 0 & 4 & 4 & 0 & 0 & 2 & 2 & 0 & 0 \\
\hline 430 & 4 & 6 & 0 & 0 & 3 & 4 & 0 & " & 1 & 1 & $\theta$ & 0 & 0 & () & is & 0 & 7 & 11 & 0 & 0 & 1 & 5 & 0 & 0 & 10 & 12 & 0 & 0 \\
\hline 145 & 5 & 11 & 0 & 0 & 6 & 10 & - & 0 & 1 & $\underline{Z}$ & 0 & 0 & 0 & 9 & 0 & 0 & 5 & 16 & 0 & 0 & 8 & 13 & 0 & 0 & 3 & 15 & 0 & 0 \\
\hline 500 & 2 & 13 &.. & 0 & 3 & 13 & 0 & 0 & 0 & 2 & 0 & 0 & 0 & i & 0 & 0 & 4 & 19 & 0 & 0 & 3 & 16 & 0 & 0 & 1 & & 0 & \\
\hline 515 & $s$ & 19 & 0 & 0 & 11 & 23 & 0 & 0 & 0 & 2 & $a$ & 0 & a & 0 & 0 & 0 & 7 & 23 & 0 & 0 & 1 & 13 & 0 & 0 & 12 & 26 & 0 & 0 \\
\hline 5310 & 12 & 27 & 0 & 0 & 11 & 31 & $a$ & 0 & $n$ & $i$ & 0 & 0 & 0 & 0 & 0 & 0 & 11 & 27 & 0 & 0 & 12 & 24 & 0 & 0 & 12 & 28 & 0 & .1 \\
\hline 545 & 16 & 38 & 0 & 0 & 7 & 32 & 0 & 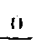 & 0 & 0 & 0 & 0 & 0 & (a) & 0 & -9 & 10 & 32 & $10^{\circ}$ & 0 & 11 & 27 & i & 0 & 12 & 37 & 0 & 0 \\
\hline 600 & 17 & 53 & 0 & $\mathrm{u}$ & 25 & 54 & " & 0 & 1 & 1 & 0 & 0 & " & $a$ & 0 & 0 & 22 & 50 & 0 & ") & 15 & 39 & 0 & 0 & 25 & ol & 0 & 0 \\
\hline 615 & 25 & 70 & 0 & a & 29 & 72 & 0 & 0 & 0 & 1 & 0 & 0 & 2 & 3 & 0 & 0 & 35 & $7 s$ & 0 & 0 & 21 & 59 & 0 & $v$ & 26 & 75 & 0 & 0 \\
\hline 630 & 42 & 100 & 0 & 0 & 32 & 93 & 0 & 0 & D) & 1 & 0 & 0 & 0 & 2 & 0 & 0 & 26 & 93 & 0 & 0 & 34 & 81 & 0 & 0 & 40 & 103 & 0 & 0 \\
\hline 645 & 68 & 152 & 0 & 0 & 60 & $1+6$ & $i$ & (1) & 0 & 1 & 0 & (i) & (1) & 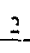 & 0 & 0 & 63 & $1+6$ & 0 & 0 & 48 & 118 & 0 & 0 & 52 & 143 & 0 & 0 \\
\hline.$\infty 0$ & 70 & 205 & 0 & 0 & 62 & 183 & 0 & a & 0 & 0 & 9 & 0 & 0 & $=$ & c) & 0 & 83 & 7 & 7 & 0 & 57 & 160 & 0 & 9 & 83 & & & \\
\hline 715 & 116 & 296 & 0 & 0 & 102 & 25 & $\dot{0}$ & 0 & 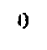 & 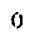 & $\dot{0}$ & $\dot{0}$ & 0 & 0 & 0 & 0 & 100 & 272 & 0 & 0 & 9.4 & 33 & 0 & 0 & 119 & 294 & 0 & 0 \\
\hline 730 & 140 & 394 & 0 & 0 & 140 & 370 & 0 & 1) & 0 & 0 & 0 & 0 & 0 & 0 & 0 & 0 & 154 & +100 & 0 & 0 & 119 & 318 & 0 & 0 & 146 & $+\infty 0$ & 0 & 0 \\
\hline 745 & 151 & +777 & 0 & 0 & 210 & 520 & ii & 0 & a & $\therefore$ & 0 & ii & 0 & D & 0 & 0 & 133 & +70 & 0 & 0 & 185 & +55 & 0 & 0 & 150 & 198 & 0 & 0 \\
\hline 800 & 118 & 525 & 0 & ij & 126 & 584 & 0 & 0 & 0 & 1) & 0 & 0 & 0 & 1) & 0 & 0 & 150 & 537 & 0 & 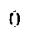 & 145 & 2.43 & 0 & $a$ & 130 & $5+5$ & 0 & 0 \\
\hline 815 & 110 & 519 & 0 & 0 & 121 & 603 & 0 & 0 & 0 & 0 & 0 & 0 & 0 & a & 0 & 0 & 111 & $\$ 48$ & 0 & 0 & 137 & 586 & 0 & 0 & 94 & 520 & 0 & 0 \\
\hline 830 & 101 & 480 & 0 & 0 & 97 & 554 & 0 & 9 & 0 & 0 & 0 & 0 & i & a & 0 & 0 & 94 & 488 & 0 & 0 & 126 & 593 & 11 & 0 & $\$ 8$ & +62 & 0 & 0 \\
\hline 845 & 69 & 398 & 0 & (]) & 79 & 423 & 0 & 0 & 0 & 0 & 0 & i) & (1) & 0 & 0 & 0 & 50 & 405 & 0 & (i) & 72 & 480 & 0 & 0 & 62 & 374 & $\underline{0}$ & 0 \\
\hline $9(0)$ & 99 & 339 & 0 & 0 & 54 & 351 & $\hat{0}$ & 0 & 0 & 0 & 0 & i) & ( & 0 & 0 & 0 & 52 & 307 & 0 & 0 & 63 & 398 & 0 & 0 & $\$ 3$ & 297 & 0 & \\
\hline 915 & 53 & 282 & " & a & 56 & 286 & 0 & 0 & 1) & 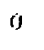 & 0 & 0 & 0 & 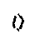 & 0 & 0 & 59 & 255 & 0 & 0 & 54 & 315 & 0 & 0 & 45 & 248 & 0 & 0 \\
\hline 930 & 48 & 229 & 0 & 0 & 46 & 235 & 0 & 0 & 0 & 0 & 0 & 0 & 0 & 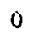 & 0 & 0 & 43 & 204 & 0 & 0 & 36 & 225 & 0 & 0 & 42 & 202 & . & 0 \\
\hline 9.45 & 35 & 195 & 0 & 0 & 35 & 191 & 0 & $\underline{0}$ & 0 & 0 & 0 & 0 & (1) & 0 & 0 & 0 & 28 & 182 & i) & 0 & 4 & 197 & 0 & 0 & 25 & 165 & 9 & 1 \\
\hline 000 & 27 & 163 & 0 & 0 & 26 & 163 & 0 & i) & 0 & 0 & a & 0 & 0 & 0 & 0 & 0 & 23 & 153 & 0 & 0 & 33 & 167 & 0 & 0 & 32 & 144 & 0 & 0 \\
\hline 015 & 19 & 129 & 0 & 0 & 22 & 129 & $"$ & 0 & 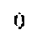 & ij & 0 & (1) & 0 & $y$ & 0 & 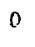 & 23 & 117 & 0 & 0 & 19 & 132 & 0 & 0 & 25 & 124 & D) & 0 \\
\hline 030 & 17 & 98 & 0 & 0 & 14 & 97 & 0 & 0 & 1 & 1 & 0 & 0 & 0 & 0 & $\dot{0}$ & 0 & 18 & 92 & 0 & 0 & 22 & 118 & 0 & 0 & 22 & 104 & 0 & 0 \\
\hline 045 & 12 & 75 & 0 & 0 & 18 & 80 & 0 & 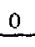 & 0 & 1 & 0 & 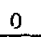 & c & & & & 16 & 80 & 0 & & 16 & 90 & 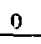 & 0 & 13 & 92 & 0 & 0 \\
\hline 00 & 19 & 67 & 0 & 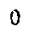 & 15 & 09 & 0 & 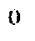 & 0 & 1 & 0 & 0 & 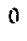 & 0 & 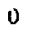 & 0 & 30 & 87 & 0 & 0 & 18 & 75 & 0 & 0 & 29 & 89 & D & 0 \\
\hline 15 & 32 & 80 & 0 & $\theta$ & 26 & 73 & 0 & 0 & 0 & 1 & 0 & 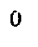 & 0 & 0 & 0 & 0 & 31 & 95 & 0 & 0 & 22 & 78 & 0 & 0 & 28 & 92 & 0 & 0 \\
\hline 30) & 35 & 98 & 0 & 0 & 31 & 90 & 0 & 0 & 1 & 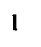 & 0 & 0 & 0 & 0 & 0 & 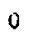 & 36 & 113 & 0 & 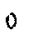 & 29 & 85 & 0 & 0 & 27 & 97 & 0 & 0 \\
\hline 45 & 26 & 112 & 0 & 0 & 25 & 97 & 0 & 0 & 0 & 1 & 0 & 0 & 0 & 0 & 0 & (1) & 24 & 121 & 0 & 0 & 27 & 96 & 0 & 0 & 38 & 122 & 0 & 0 \\
\hline
\end{tabular}




\section{BAYMETRICS TRAFFIC RESOURCES Location 4: Driveway North of East Aevnue (NB - Inbounds)}

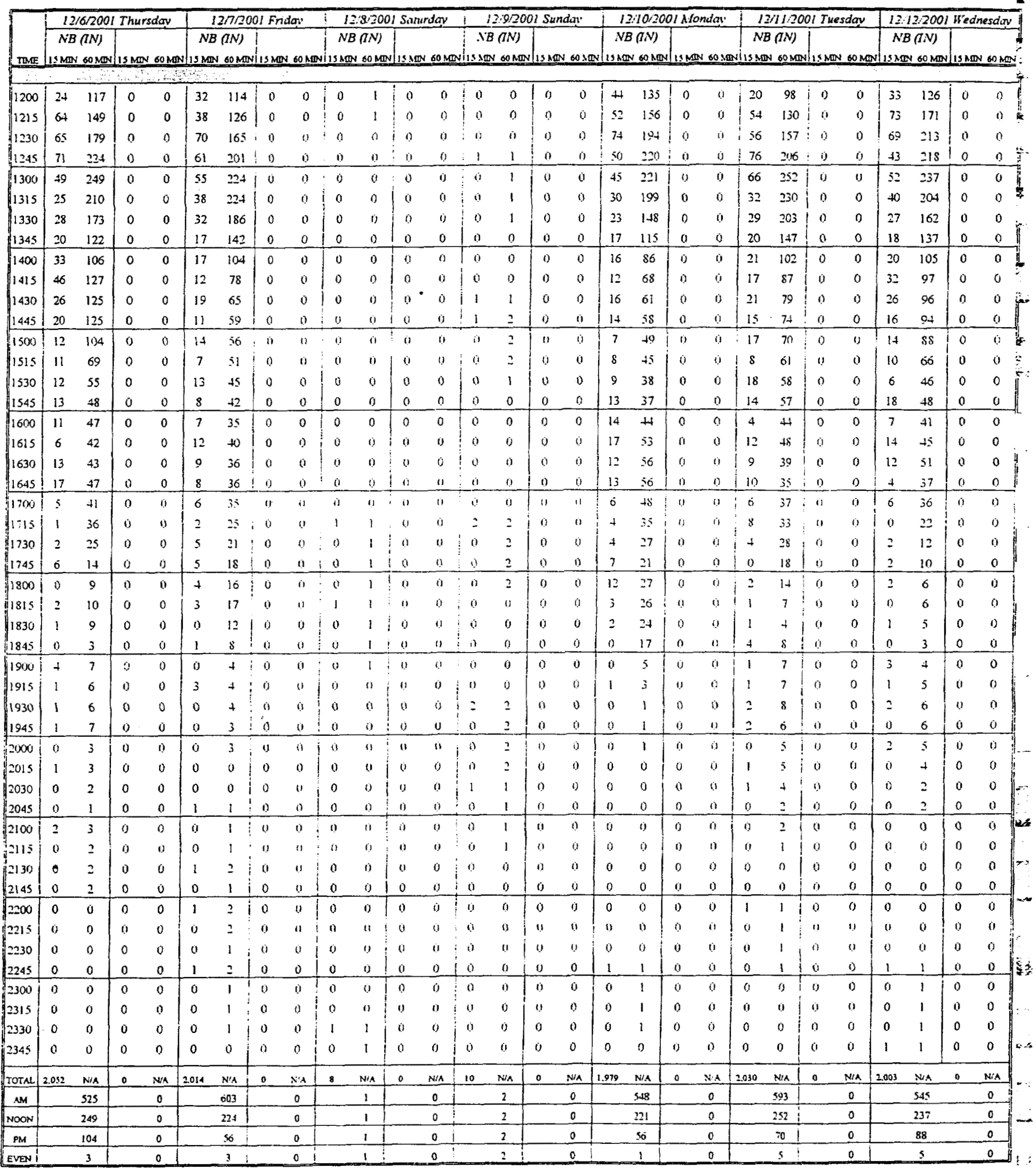




\section{BAYMETRICS TRAFFIC RESOURCES Location 4B: Driveway North of East Aevnue (SB - Outbounds)}

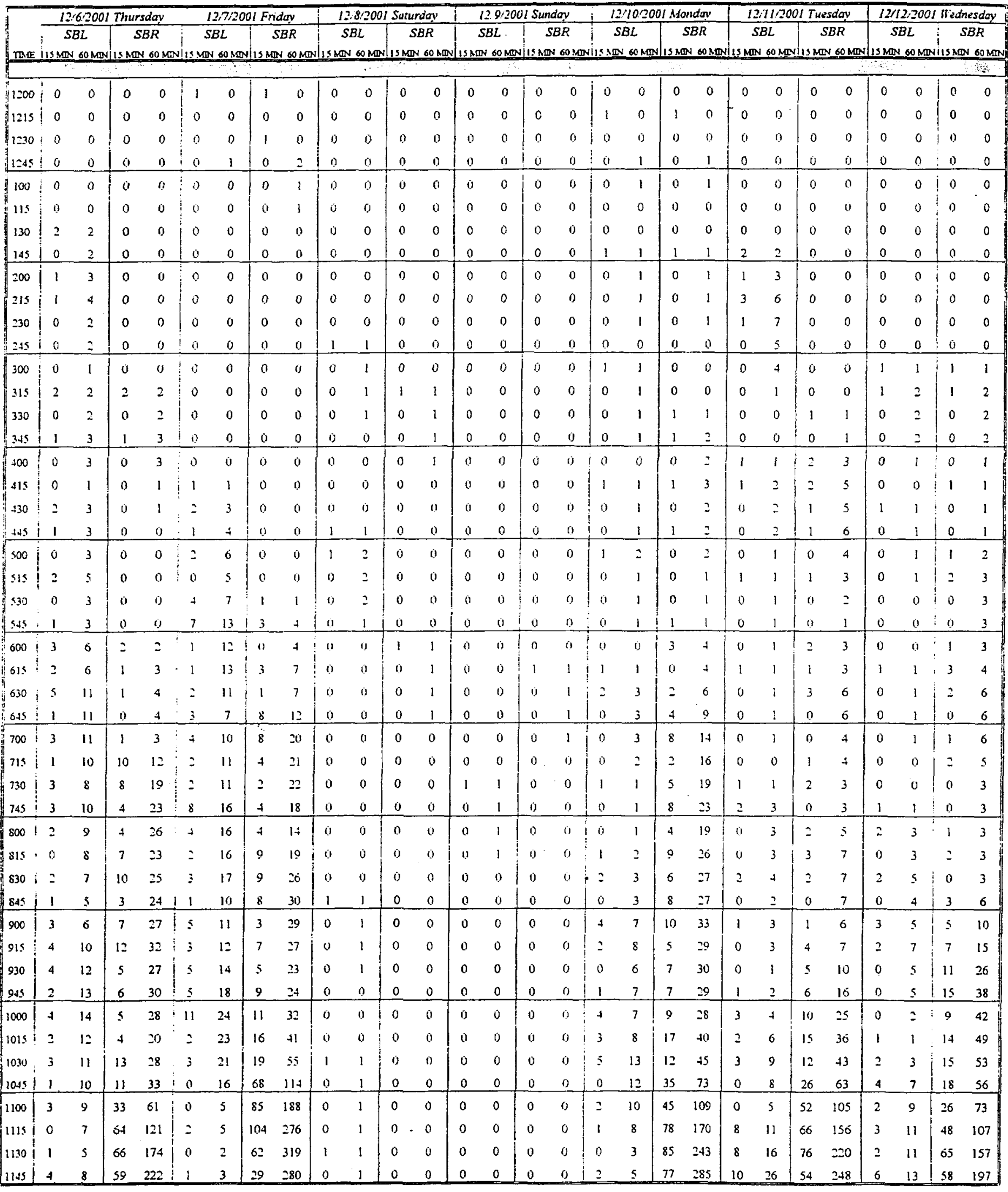




\section{BAYMETRICS TRAFFIC RESOURCES Location 4B: Driveway North of East Aevnue (SB - Outbounds)}

\begin{tabular}{|c|c|c|c|c|c|c|c|c|c|c|c|c|c|c|c|c|c|c|c|c|c|c|c|c|c|c|c|c|}
\hline & \multicolumn{4}{|c|}{ 12:602001 Thursday } & \multicolumn{4}{|c|}{$12 \cap / 2001$ Friday } & \multicolumn{4}{|c|}{ 12/82001 Samurday } & \multicolumn{4}{|c|}{1792001 Sundov } & \multicolumn{4}{|c|}{$12 / 10,2001$ Monday } & \multicolumn{4}{|c|}{ 12/1L2001 Tuesday } & $12 / 12$ & 2001 & Yednesd & \\
\hline & $S E$ & & & $B R$ & & $B L$ & $S E$ & & & & & & & $B L$ & $S E$ & & $\overline{S E}$ & & $S E$ & & & $\overline{S L}$ & & $R$ & $S B$ & & $S B R$ & \\
\hline 1200 & 0 & 5 & 39 & 228 & 0 & 3 & 22 & 217 & 0 & 1 & $a$ & 0 & 0 & 0 & 0 & 0 & 5 & 8 & 56 & 296 & 15 & 41 & 43 & 239 & 15 & 36 & 50 & 221 \\
\hline 1215 & $s$ & 10 & 16 & 180 & 5 & 6 & 45 & 158 & 1 & 2 & 0 & 0 & 0 & 0 & 0 & 0 & 5 & 12 & 43 & 261 & 9 & 42 & 45 & 218 & 12 & 35 & 26 & 199 \\
\hline 1230 & 8 & 17 & 13 & 127 & 7 & 13 & 47 & 143 & 0 & 1 & 0 & 0 & 0 & 0 & 0 & 0 & 1 & 13 & 32 & 208 & 4 & 38 & 26 & 168 & 9 & 42 & 29 & 163 \\
\hline 1245 & 5 & 18 & 21 & 89 & 18 & 30 & 13 & 127 & 0 & 1 & 0 & 0 & 1 & 1 & 0 & 0 & 5 & 16 & 18 & $1+9$ & 6 & 34 & 32 & 146 & 12 & 48 & 22 & 127 \\
\hline 1300 & 5 & 23 & 19 & 69 & 4 & 34 & 30 & 135 & 0 & 1 & 0 & 0 & 0 & 1 & 0 & 0 & 2 & 13 & 15 & 108 & $=$ & 21 & 28 & 131 & 5 & 38 & 18 & 95 \\
\hline 1315 & 0 & 18 & 21 & 74 & 5 & 34 & 24 & 114 & 0 & 0 & 0 & 0 & 0 & 1 & 0 & 0 & 2 & 10 & 24 & 89 & 0 & 12 & 29 & 115 & 4 & 30 & 25 & 94 \\
\hline 1330 & 4 & 14 & 12 & 73 & 4 & 31 & 20 & 87 & 0 & 0 & 0 & 0 & 0 & 1 & 0 & 0 & 6 & 15 & 14 & 71 & 1 & 9 & 25 & 114 & 2 & 23 & 13 & 78 \\
\hline 1345 & 5 & 14 & 23 & 75 & 1 & 14 & 19 & 93 & 0 & 0 & 0 & 0 & 0 & 0 & 0 & 0 & 3 & 13 & 13 & $60^{\circ}$ & 2 & 5 & 22 & 104 & 6 & 17 & 37 & 93 \\
\hline 1400 & 4 & 13 & 14 & 70 & 2 & 12 & 23 & 86 & 0 & 0 & 0 & 0 & 0 & 0 & 0 & 0 & 3 & 14 & 25 & 76 & 4 & 7 & 19 & 95 & 2 & 14 & 26 & 101 \\
\hline 1415 & 4 & 17 & 20 & 69 & 3 & 10 & 35 & 97 & 0 & 0 & 0 & 0 & 0 & 0 & 0 & 0 & 0 & .12 & 24 & 76 & 2 & 9 & 32 & 98 & 2 & 12 & 12 & 88 \\
\hline 1430 & 5 & 18 & 17 & 74 & 2 & 8 & 32 & 109 & 0 & 0 & 0 & 0 & 0 & 0 & 0 & 0 & 0 & .6 & 35 & 97 & 3 & 11 & 45 & 118 & 1 & 11 & 14 & 89 \\
\hline 1445 & 0 & 13 & 29 & 80 & 2 & 9 & 28 & 118 & 0 & 0 & 0 & 0 & 1 & 1 & 1 & 1 & 1 & 4 & 43 & 127 & 2 & 11 & 30 & 126 & 0 & 5 & 15 & 67 \\
\hline 1500 & 2 & 11 & 34 & 100 & 0 & 7 & 40 & 135 & 0 & 0 & 0 & 0 & 1 & 2 & 0 & 1 & 2 & 3 & 29 & 131 & 0 & 7 & 29 & 136 & 0 & 3 & 30 & 71 \\
\hline 1515 & 9 & 16 & 54 & 134 & 3 & 7 & 59 & 159 & 0 & 0 & 0 & 0 & 0 & 2 & 0 & 1 & 0 & 3 & 53 & 160 & 1 & 6 & 45 & 149 & 4 & 5 & 38 & 97 \\
\hline 1530 & 2 & 13 & 84 & 201 & $s$ & 10 & 69 & 196 & 0 & 0 & 0 & 0 & 0 & 2 & 0 & 1 & 3 & 6 & 49 & 174 & 4 & 7 & 59 & 163 & 5 & 9 & 56 & 139 \\
\hline 1545 & 6 & 19 & 92 & 264 & 2 & 10 & 78 & 246 & 0 & 0 & 0 & 0 & 0 & 1 & 0 & 0 & 2 & 7 & 83 & 214 & 2 & 7 & 77 & 210 & 0 & 9 & 84 & 208 \\
\hline 1600 & 10 & 27 & 119 & 349 & 9 & 19 & 95 & 301 & 0 & 0 & 0 & 0 & 0 & 0 & 0 & 0 & 4 & 9 & 95 & 280 & 3 & 10 & 104 & 285 & 2 & 11 & 115 & 993 \\
\hline 1615 & 6 & 24 & 135 & 430 & 7 & 23 & 102 & 344 & 0 & 0 & 0 & 0 & 0 & 0 & 0 & 0 & 2 & 11 & 112 & 339 & 16 & 25 & 95 & 335 & 4 & 11 & 126 & 381 \\
\hline 1630 & 7 & 29 & 117 & 463 & 5 & 23 & 126 & 401 & 0 & 0 & 0 & 0 & 0 & 0 & 0 & 0 & 16 & 24 & 136 & 426 & 22 & 43 & $! 46$ & 422 & 3 & 9 & 135 & 460 \\
\hline 1645 & 6 & 29 & 172 & 543 & 0 & 21 & 145 & 468 & 0 & 0 & 0 & 0 & 0 & 0 & 0 & 0 & 11 & 33 & 125 & 468 & 10 & 51 & 137 & 482 & 11 & 20 & 114 & 490 \\
\hline 1700 & 0 & 19 & 97 & 521 & 2 & 14 & 113 & 486 & 0 & 0 & 0 & 0 & 0 & 0 & 0 & 0 & 12 & 41 & 98 & 471 & 15 & 63 & 105 & 483 & 8 & 26 & $1 / 4$ & 489 \\
\hline 1715 & 2 & 15 & 73 & 459 & 0 & 7 & 82 & 466 & 2 & 2 & 0 & 0 & 0 & 0 & 0 & 0 & 5 & 44 & 44 & 403 & 18 & 65 & 73 & 461 & 12 & 34 & $1: 2$ & 475 \\
\hline 1730 & 3 & 11 & 58 & 400 & 2 & 4 & 66 & 406 & 0 & $z$ & 0 & 0 & 2 & $=$ & 1 & I & 8 & 36 & 31 & 298 & 11 & $\$ 4$ & 36 & 351 & 5 & 36 & 70 & 430 \\
\hline 1745 & 1 & 6 & 34 & 262 & 0 & 4 & 40 & 301 & 0 & 2 & 0 & 0 & 0 & 2 & 0 & 1 & 7 & 32 & 24 & 197 & 5 & 49 & 22 & 236 & 4 & 29 & 38 & 334 \\
\hline 1800 & 0 & 6 & 32 & 197 & 1 & 3 & 26 & 214 & 0 & 2 & 0 & 0 & 0 & 2 & 0 & 1 & 5 & 25 & 17 & 116 & 4 & 38 & 15 & 146 & 6 & 27 & 22 & 242 \\
\hline 1815 & 0 & 4 & 24 & 148 & 0 & 3 & 22 & 154 & 1 & 1 & 0 & 0 & 0 & 2 & 0 & 1 & 6 & 26 & 11 & 83 & 6 & 26 & 13 & $86 \dot{ }$ & 5 & 20 & 12 & $1+2$ \\
\hline 1830 & 2 & 3 & 8 & 98 & 2 & 3 & 15 & 103 & 1 & 2 & 0 & 0 & 0 & 0 & 0 & 0 & 5 & 23 & 6 & 58 & 5 & 20 & 9 & 59 & 2 & 17 & 5 & 77 \\
\hline 1845 & 3 & 5 & 15 & 79 & 0 & 3 & 7 & 70 & 0 & 2 & 0 & 0 & 0 & 0 & 0 & 0 & 2 & 18 & 7 & 41 & 2 & 17 & 5 & 42 & 9 & $\therefore$ & 8 & $\therefore$ \\
\hline 1900 & 3 & 8 & 13 & 00 & 1 & 3 & 9 & 53 & 0 & 2 & 0 & 0 & 0 & 0 & 0 & 0 & 3 & 16 & 5 & 29 & 2 & 15 & 4 & 31 & 5 & 21 & 10 & 35 \\
\hline 1915 & 1 & 9 & 10 & 46 & 0 & 3 & 3 & 34 & 0 & 1 & 0 & 0 & 0 & 1) & 0 & 0 & 3 & 13 & 7 & 25 & 1 & 10 & 7 & 25 & 4 & 20 & 5 & 29 \\
\hline 1930 & 2 & 9 & 8 & 46 & 0 & 1 & 2 & 21 & 0 & 0 & 0 & 0 & 2 & 2 & 1 & 1 & 1 & 9 & 1 & 20 & 0 & 5 & 2 & 18 & 1 & 19 & 4 & 27 \\
\hline 1945 & 0 & 6 & 11 & 42 & 0 & 1 & 5 & 19 & 0 & 0 & 0 & 0 & 0 & 2 & 0 & 1 & 3 & 10 & 3 & 16 & 0 & 3 & 11 & 24 & 1 & 11 & 2 & $2:$ \\
\hline 2000 & 1 & 4 & 2 & 31 & 1 & I & $I$ & 12 & 0 & 0 & 0 & 0 & 0 & 2 & 0 & 1 & 0 & 7 & 3 & 14 & 0 & 1 & 5 & 25 & 0 & 6 & 3 & 14 \\
\hline 2015 & 1 & 4 & 3 & 24 & 1 & 2 & 1 & 10 & 0 & 0 & 0 & 0 & 0 & 2 & 0 & 1 & 0 & 4 & 0 & 7 & 0 & 0 & 4 & 22 & 0 & $=$ & 2 & 1i \\
\hline 2030 & 0 & 2 & 1 & 17 & 0 & 2 & 0 & 8 & 0 & 0 & 0 & 0 & 2 & 2 & 0 & 0 & 2 & 5 & 0 & 6 & 0 & 0 & 6 & 26 & 3 & $=$ & 2 & 9 \\
\hline 2045 & 0 & 2 & 0 & 6 & 0 & 2 & 0 & 3 & 0 & 0 & 0 & 0 & 0 & 2 & 0 & 0 & 0 & 2 & 0 & 3 & 0 & 0 & 2 & 17 & 0 & 1 & 4 & 11 \\
\hline 2100 & 2 & 3 & 2 & 6 & 2 & 3 & 0 & 1 & 0 & ) & 0 & 0 & 0 & 2 & 0 & 0 & 0 & 2 & 0 & 0 & 0 & 0 & 3 & 15 & 1 & 2 & 2 & 10 \\
\hline 2115 & 1 & 3 & 0 & 3 & 0 & 2 & 0 & 0 & 0 & 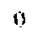 & 0 & 0 & 0 & 2 & 1 & 1 & 1 & 3 & 0 & 0 & 0 & 0 & 2 & 13 & 2 & 4 & 3 & 11 \\
\hline 2130 & 0 & 3 & 0 & 2 & 0 & 2 & 2 & 2 & 0 & 0 & 0 & 0 & 0 & 0 & 0 & 1 & 0 & 1 & 0 & 0 & 0 & 1) & 2 & 9 & 4 & 7 & 2 & 11 \\
\hline 2145 & 0 & 3 & 0 & 2 & 0 & 2 & 0 & 2 & 0 & 0 & 0 & 0 & 0 & 0 & 0 & 1 & 0 & 1 & 0 & 0 & 0 & 0 & 1 & 8 & 2 & 9 & 2 & 9 \\
\hline 2200 & 0 & 1 & 0 & 0 & 0 & 0 & 0 & 2 & 0 & 0 & 0 & 0 & 0 & 0 & 0 & 1 & 0 & 1 & 0 & 0 & 0 & 0 & $=$ & 7 & 0 & 8 & 0 & 7 \\
\hline 2215 & 0 & 0 & 0 & 0 & 1 & 1 & 0 & 2 & 0 & 0 & 0 & 0 & 0 & 0 & 0 & 0 & 0 & 0 & 0 & 0 & 0 & 0 & 2 & 7 & 1 & 7 & 1 & 5 \\
\hline 2230 & 0 & 0 & $\dot{v}$ & 0 & 0 & 1 & 0 & 0 & 0 & 0 & 0 & 0 & 0 & 0 & 0 & 0 & 1 & 1 & 1 & 1 & 0 & 0 & 3 & 8 & 2 & 5 & 2 & 5 \\
\hline 2245 & 0 & 0 & 0 & 0 & 0 & 1 & 0 & 0 & 0 & 0 & 0 & 0 & 0 & 0 & 0 & 0 & 2 & 3 & 0 & 1 & 0 & 0 & 2 & 9 & 0 & 3 & 0 & 3 \\
\hline 2300 & 0 & 0 & 0 & 0 & 1 & 2 & 1 & 1 & 0 & 0 & 0 & 0 & 0 & 0 & 0 & 0 & 0 & 3 & 0 & 1 & 0 & 0 & 1 & 8 & 1 & 4 & 2 & 5 \\
\hline 2315 & 0 & 0 & 0 & 0 & 0 & 1 & 0 & I & 0 & 0 & 0 & 0 & 0 & 0 & 0 & 0 & 0 & 3 & 0 & 1 & 0 & 0 & 0 & $\sigma$ & 0 & 3 & 1 & 5 \\
\hline 2330 & 0 & 0 & 0 & 0 & 0 & 1 & 0 & 1 & 0 & 0 & 1 & 1 & 0 & 0 & 0 & 0 & 0 & 2 & 1 & 1 & 0 & 0 & 0 & 3 & 0 & 1 & 0 & 3 \\
\hline 2345 & 0 & 0 & 0 & 0 & 0 & 1 & 0 & 1 & 0 & 0 & 0 & 1 & 0 & 0 & 0 & 0 & 0 & 0 & 0 & 1 & 0 & 0 & 0 & 1 & 0 & 1 & 0 & 3 \\
\hline TOTAL & 183 & $\mathrm{NAA}$ & $1.78 \mathrm{~s}$ & $\mathrm{~N} / \mathrm{A}$ & 186 & $N / A$ & 1.932 & $N / A$ & 11 & N:A & 3 & $N / A$ & 10 & $N / A$ & 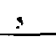 & NA & 175 & $N / A$ & 1.736 & $N / A$ & 929 & N/A & 1.786 & $\mathrm{~N} / \mathrm{A}$ & 209 & $N: A$ & $m$ & $\mathrm{~N} / \mathrm{A}$ \\
\hline$M M$ & & 13 & & 32 & & 18 & & 30 & & 1 & & 1 & & 1 & & 1 & & 8 & & 33 & & 4 & & $10^{\circ}$ & & 2 & & 38 \\
\hline YOON & & 23 & & 228 & & 34 & & 319 & & 2 & & 0 & & 1 & & 1 & & 16 & & 290 & & 42 & & 248 & & 48 & & 221 \\
\hline PM & & 29 & & 543 & & 23 & & 486 & & 2 & & 0 & & 2 & & 1 & & 44 & & $\$ 71$ & & 69 & & .883 & & 36 & & 490 \\
\hline ENEN & & 4 & & 31 & & 3 & & 12 & & 0 & & 1 & & 2 & & 1 & & 3 & & 14 & & 1 & & 20 & & 9 & & 14 \\
\hline
\end{tabular}




\section{BAYMETRICS TRAFFIC RESOURCES Location 5: Driveway North of East Aevnue (NB - Inbounds)}

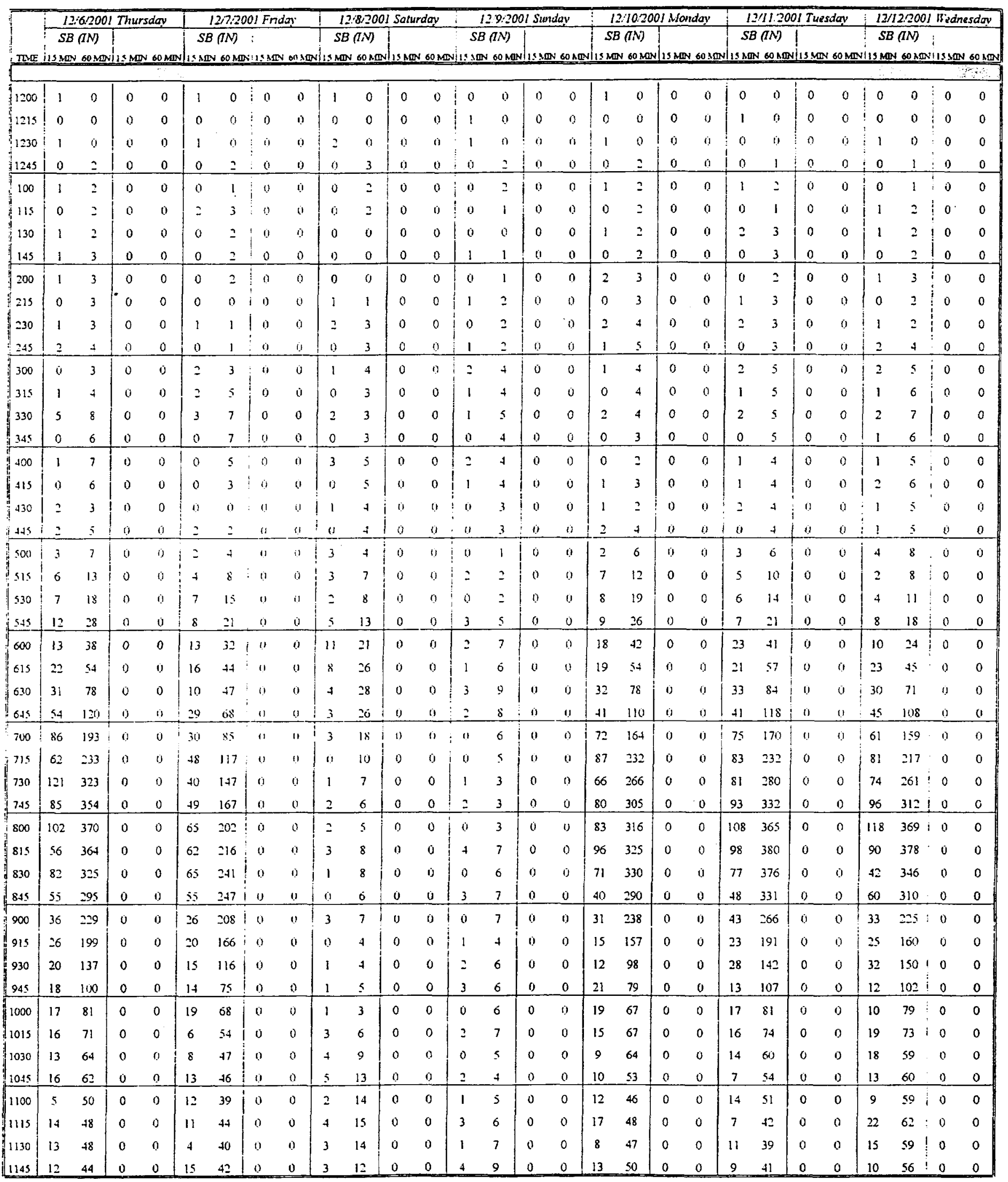




\section{BAYMETRICS TRAFFIC RESOURCES Location 5: Driveway North of East Aevnue (NB - Inbounds)}

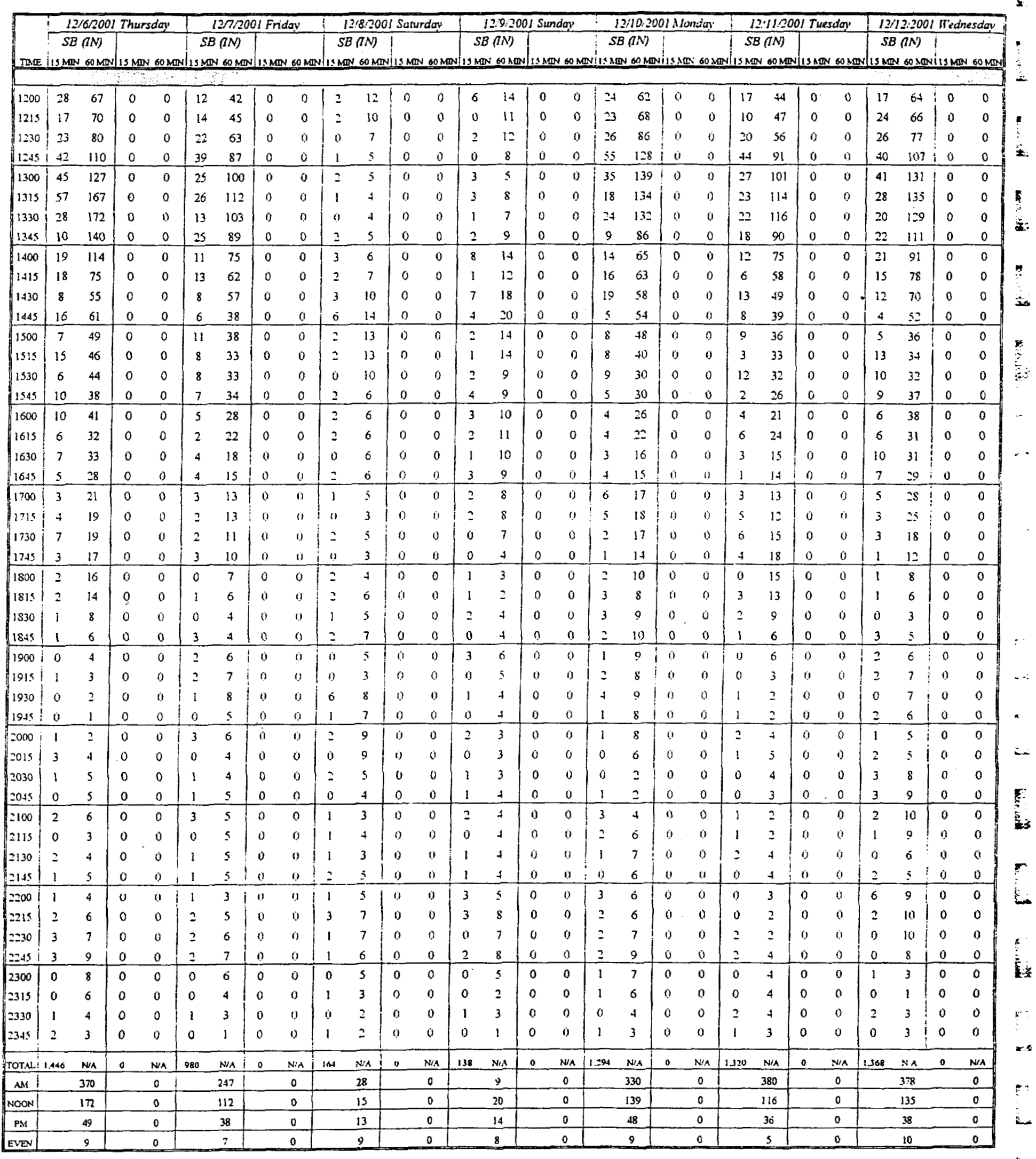




\section{BAYMETRICS TRAFFIC RESOURCES Location 5B: Driveway North of East Aevnue (SB - Outbounds)}

\begin{tabular}{|c|c|c|c|c|c|c|c|c|c|c|c|c|c|c|c|c|c|c|c|c|c|c|c|c|c|c|c|c|c|c|}
\hline \multirow{2}{*}{$\mathrm{ME}$} & \multicolumn{4}{|c|}{ 12/6/2001 Thursday } & \multicolumn{4}{|c|}{$12 / 7 / 2001$ Friday } & \multicolumn{5}{|c|}{ 12/8/2001 Suturday } & & \multicolumn{4}{|c|}{ 129:2001 Sundoy } & \multicolumn{4}{|c|}{ 12/10:2001 Mondtay } & \multicolumn{4}{|c|}{ 12/11/3001 Tuesday } & \multicolumn{4}{|c|}{ 12/12:2001 We:inesday } \\
\hline & & & & & & $B$ & & $B R$ & & NB L & & & & & $A B$ & & & & & $B L$ & & & & $B L$ & & & & & & $3 R$ \\
\hline 1200 & 4 & 0 & 5 & 0 & 0 & 0 & 0 & 0 & & 0 & 0 & 0 & 0 & & 1 & 0 & 0 & 0 & 0 & 0 & 0 & 0 & 1 & 0 & 1 & 0 & 3 & 0 & 0 & 0 \\
\hline 1215 & 1 & 0 & 0 & 0 & 1 & 0 & 2 & 0 & & 0 & 0 & 0 & 0 & & () & 0 & 1 & 0 & 0 & 0 & 2 & 0 & 0 & 0 & 0 & 0 & 1 & 0 & 1 & 0 \\
\hline 1230 & 0 & 0 & 2 & 0 & 1 & 0 & 0 & 0 & & y & j & 0 & 0 & & 0 & 0 & 0 & 0 & 0 & 0 & 1 & 0 & 0 & 0 & 1 & 0 & 0 & 0 & 0 & 0 \\
\hline 1245 & 0 & 5 & a & 7 & 0 & 2 & 0 & 2 & & $n$ & 0 & 1 & 1 & & 1 & 2 & 1 & 2 & 1 & 1 & 0 & 3 & 0 & 1 & 1 & 3 & 0 & 4 & 2 & 3 \\
\hline 100 & 0 & 1 & 1 & 3 & 2 & 4 & 0 & 2 & & ) & 0 & 0 & 1 & & 1 & 2 & 0 & 2 & 1 & 2 & 1 & 4 & 0 & 0 & ) & 2 & 0 & 1 & 1 & 4 \\
\hline 115 & 1 & 1 & 0 & 3 & 0 & 3 & 1 & 1 & & i & 9 & 1 & 2 & & 0 & $=$ & 1 & 2 & 0 & 2 & 0 & 2 & 1 & 1 & 1 & 3 & 0 & 0 & 1 & 4 \\
\hline 130 & 0 & 1 & 1 & 2 & 0 & 2 & 0 & 1 & & 0 & to & 1 & 3 & & $=$ & 4 & 0 & $=$ & 0 & 2 & 0 & 1 & 0 & 1 & 0 & 2 & 0 & 0 & 0 & 4 \\
\hline 145 & 0 & 1 & 0 & $=$ & 0 & $=$ & 0 & 1 & & $a$ & $\dot{u}$ & 0 & 2 & & 0 & 3 & 1 & 2 & 0 & 1 & 0 & 1 & 0 & 1 & 1 & 2 & 0 & 0 & 0 & 2 \\
\hline 200 & 0 & 1 & 2 & 3 & 1 & 1 & 0 & 1 & & 0 & 0 & I & 3 & & 0 & 2 & 0 & 2 & I & 1 & 0 & 0 & 0 & 1 & 1 & 3 & 0 & 0 & 1 & 2 \\
\hline 215 & 0 & 0 & 0 & 3 & 2 & 3 & 0 & 0 & 2 & 2 & & 0 & 2 & & 0 & 2 & 2 & 3 & 0 & 1 & 1 & I & 0 & 0 & 0 & 2 & 0 & 0 & 0 & 1 \\
\hline 230 & 0 & 0 & 2 & 4 & 0 & 3 & 3 & 3 & 0 & 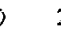 & 2 & 0 & 1 & & 0 & 0 & 0 & 3 & 0 & 1 & 0 & 1 & 0 & 0 & 1 & 3 & 0 & 0 & 0 & 1 \\
\hline 245 & 0 & 0 & 0 & 4 & 0 & 3 & 0 & 3 & 0 & 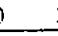 & ? & 1 & 2 & & 0 & 0 & 0 & 2 & 0 & 1 & 0 & 1 & 1 & 1 & 0 & 2 & 1 & 1 & 0 & 1 \\
\hline 300 & -1 & -1 & 3 & 5 & 0 & 2 & 0 & 3 & - & 1 & 1 & 2 & 3 & & 0 & 0 & 0 & 2 & 0 & 0 & 1 & 2 & 1 & 2 & 1 & 2 & 0 & 1 & 2 & 2 \\
\hline 315 & 2 & I & 0 & 5 & 0 & 0 & 0 & 3 & 0 & D. & 1 & 0 & 3 & & 1 & 1 & 0 & 0 & 1 & 1 & 1 & 2 & 0 & 2 & 0 & 2 & 0 & 1 & 0 & 2 \\
\hline 330 & 0 & 1 & 1 & 4 & 0 & 0 & 1 & 1 & - & 1 & 2 & $=$ & 5 & & $=$ & 3 & 0 & 0 & 2 & 3 & 0 & 2 & 0 & 2 & 0 & 1 & 0 & 1 & 0 & 2 \\
\hline 345 & 1 & 2 & 0 & 4 & 0 & 0 & 0 & 1 & 1 & 1 & 1 & 3 & 7 & & 0 & 3 & 0 & 0 & 0 & 3 & 0 & 2 & 0 & 1 & 0 & 1 & 0 & 0 & 0 & 2 \\
\hline 400 & 1 & 4 & 1 & 2 & 1 & 1 & 0 & 1 & ? & : & 2 & 1 & 6 & & 2 & 5 & 3 & 3 & 0 & 3 & 0 & 1 & 0 & 0 & 1 & 1 & 0 & 0 & $i$ & 1 \\
\hline 415 & 0 & 2 & 4 & 6 & 0 & 1 & 0 & 1 & 0 & 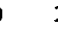 & $=$ & 1 & 7 & & 1 & 5 & 2 & 5 & 1 & 3 & 1 & 1 & 0 & 0 & 0 & 1 & 0 & 0 & 2 & 3 \\
\hline 430 & 0 & $=$ & 0 & 5 & 1 & 2 & 1 & 1 & 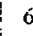 & 5 & 9 & $=$ & 7 & & 1 & 4 & 0 & 5 & 2 & 3 & 1 & $=$ & 1 & 1 & 1 & 2 & 1 & 1 & 0 & 3 \\
\hline 445 & 0 & 1 & 1 & 6 & 2 & 4 & 0 & 1 & & 3 & 11 & 1 & 5 & & 4 & 8 & 0 & 5 & 0 & 3 & 0 & 2 & 2 & 3 & 1 & 3 & 0 & 1 & 0 & 3 \\
\hline 500 & l & 1 & 0 & 5 & 0 & 3 & 3 & 4 & & 1 & 10 & 3 & 7 & & 0 & 0 & 0 & 2 & 0 & 3 & 1 & 3 & 0 & 3 & $\dot{0}$ & 2 & 0 & 1 & 0 & 2 \\
\hline$\$ 15$ & 0 & 1 & 2 & 3 & 3 & 6 & 0 & 4 & & 3 & 13 & 9 & 6 & & $\dot{0}$ & 5 & 0 & 0 & 1 & 3 & 0 & 2 & 0 & 3 & 0 & 2 & 0 & 1 & 0 & 0 \\
\hline 530 & $=$ & 3 & 19 & 3 & 4 & 9 & 0 & 3 & & 0 & 7 & 1 & 5 & & 1 & 5 & () & (1) & 1 & $=$ & 4 & 1 & 1 & 3 & 0 & 1 & 2 & 2 & 0 & 0 \\
\hline 545 & 4 & 7 & a & 2 & 2 & 9 & 2 & 5 & 1 & 0 & 4 & 0 & 4 & & $=$ & 3 & 5 & 5 & 1 & 3 & 0 & 1 & 2 & 3 & 1 & 1 & 1 & 3 & 1 & 1 \\
\hline 600 & 4 & 10 & 1) & 2 & 0 & 9 & 0 & 2 & 3 & 3 & 6 & 1 & $z$ & & (i) & 3 & 3 & 8 & 0 & 3 & 0 & 0 & (i) & 3 & 0 & 1 & 1 & 4 & 0 & 1 \\
\hline 615 & 3 & 13 & 3 & 3 & 0 & 6 & 3 & 5 & 1 & 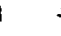 & 4 & 1 & 3 & & 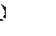 & 3 & 1) & 8 & 0 & 2 & 1 & 1 & 0 & 3 & 0 & 1 & 2 & 6 & 0 & 1 \\
\hline 630 & 7 & 18 & D) & 3 & 1 & 3 & 5 & 10 & 1) & 1) & 4 & 3 & 5 & & 1 & 3 & 1 & 9 & 0 & 1 & 2 & 3 & 1 & 3 & 0 & 1 & 0 & 4 & 1 & 2 \\
\hline 645 & 6 & 20 & 0 & 3 & 2 & 3 & 4 & 12 & 1) & 0 & 4 & 1 & 0 & a & a & 1 & 0 & 4 & 0 & 0 & 0 & 3 & 2 & 3 & 1 & 1 & 3 & 0 & $\therefore$ & 3 \\
\hline 700 & 2 & 18 & 7 & 10 & $=$ & 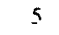 & 3 & 15 & & 0 & 1 & 1 & ${ }^{\circ}$ & 1 & 1 & $=$ & 1 & 2 & (1) & 0 & 0 & 3 & 0 & 3 & 0 & 1 & 2 & 7 & 0 & 3 \\
\hline 715 & 7 & 22 & 7 & 14 & 3 & 8 & 2 & 14 & & 0 & 0 & 0 & 5 & & ji & 2 & 0 & 2 & 2 & 2 & 1 & 3 & 3 & 6 & 2 & 3 & 2 & 7 & 3 & 6 \\
\hline 730 & 3 & 18 & 9 & 93 & 2 & 9 & 4 & 13 & & 2 & $=$ & 2 & 4 & 1 & 1 & 2 & 0 & 1 & 0 & 2 & 2 & 3 & 0 & 5 & 0 & 3 & 0 & 7 & 0 & 5 \\
\hline 245 & 3 & 15 & 4 & 27 & 7 & 14 & 3 & 12 & & 1) & 2 & 0 & 3 & 0 & 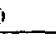 & 2 & 2 & 3 & 3 & 5 & 0 & 3 & 0 & 3 & 0 & 2 & 0 & 4 & 0 & 3 \\
\hline 800 & 6 & 19 & 0 & 20 & 10 & 22 & 3 & 13 & & 0 & $=$ & 2 & 4 & $=$ & 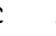 & 3 & 0 & 2 & 2 & 7 & 3 & 6 & 1 & 4 & 1 & 3 & 1 & 3 & 2 & 5 \\
\hline 815 & 6 & 18 & 1 & 14 & 3 & $=2$ & 4 & 14 & & 2 & 4 & 1 & 5 & ( & & 3 & 0 & 2 & 7 & 12 & 2 & 7 & 1 & 2 & $=$ & 3 & 2 & 3 & 4 & 6 \\
\hline 830 & 8 & 23 & 3 & 8 & 2 & $\because 2$ & 6 & 16 & & 1 & 3 & 1 & 4 & (i) & & 2 & 0 & 2 & 7 & 16 & 0 & 5 & 0 & 2 & 0 & 3 & 0 & 3 & 2 & 8 \\
\hline 845 & 4 & 24 & 2 & 6 & 6 & 21 & 4 & 17 & & 0 & 3 & 3 & 7 & 0 & & 2 & 0 & 0 & 2 & 15 & 1 & $\dot{0}$ & 2 & 4 & 0 & 3 & 5 & 8 & 6 & 14 \\
\hline 900 & $s$ & 23 & $\leq$ & 11 & $s$ & 16 & 4 & 18 & 0 & 0 & 3 & 1 & 0 & $=$ & & 2 & 0 & 0 & 7 & 20 & 2 & 5 & 1 & 4 & 2 & 4 & 4 & 11 & 6 & 18 \\
\hline 915 & 3 & 20 & 7 & 17 & 4 & 17 & 2 & 16 & $=$ & $=$ & 3 & 7 & 12 & a & & $\geq$ & 0 & 0 & 5 & 18 & 4 & 7 & 2 & 5 & 0 & 2 & 2 & 11 & 9 & 23 \\
\hline 930 & 8 & 20 & 1 & 15 & 2 & 17 & 5 & 15 & & 2 & 4 & 0 & 11 & 1 & & 3 & 2 & 2 & 6 & 20 & 2 & 9 & 3 & 8 & 1 & 3 & 6 & 17 & 2 & 23 \\
\hline 945 & 2 & 18 & 5 & 18 & 5 & 16 & 1 & 12 & & 1 & 5 & 6 & 14 & 1 & & 4. & 1 & 3 & 9 & 27 & 5 & 13 & 10 & 16 & 2 & 5 & 10 & 22 & 4 & 21 \\
\hline 1000 & 8 & 21 & 1 & 14 & 7 & 18 & 1 & 9 & 3 & 3 & 8 & 0 & 13 & 3 & & 5 & 2 & 5 & 11 & 31 & 9 & 20 & 17 & 32 & 0 & 3 & 12 & 30 & 5 & 20 \\
\hline 101.5 & 14 & 32 & 8 & & 10 & 24 & 5 & 12 & 0 & 0 & 6 & 0 & 6 & 0 & 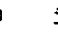 & 5 & 0 & 5 & 15 & 41 & 11 & 37 & 20 & 50 & 3 & 6 & 15 & 43 & 8 & 19 \\
\hline 1030 & 6 & 30 & 11 & 25 & 12 & 34 & 4 & 11 & 2 & 2 & 6 & $=$ & 8 & o & 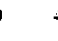 & 4 & o & 3 & 12 & 47 & 15 & 40) & 14 & 61 & 8 & 13 & 19 & 56 & 4 & 21 \\
\hline 1045 & 9 & 37 & 4 & 24 & 16 & 45 & 9 & 19 & 1 & 1 & 6 & 0 & 2 & a & 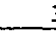 & 3 & 0 & 2 & 20 & 58 & 8 & $\$ 3$ & 13 & 64 & 7 & 18 & 20 & 66 & 7 & 24 \\
\hline 1100 & 19 & 48 & 11 & 34 & 20 & 58 & 4 & $\because 2$ & 1 & 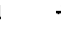 & 4 & 3 & 5 & $=$ & & 8 & 1 & 1 & 25 & 73 & 10 & 44 & 15 & 62 & $s$ & 23 & 11 & 65 & 10 & 29 \\
\hline 1115 & 20 & 54 & 11 & 37 & 25 & 73 & 12 & 29 & 0 & 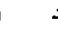 & 4 & 0 & 5 & 1 & & 3 & 1 & 2 & 19 & 76 & 14 & 47 & 18 & 60 & 7 & 27 & 17 & 67 & 5 & 26 \\
\hline 1130 & 33 & 81 & 8 & 34 & 38 & & 9 & 34 & 2 & 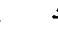 & 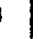 & 0 & 3 & 0 & 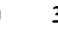 & 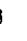 & 2 & 4 & 34 & 98 & 5 & 37 & 39 & 75 & $\$ 1$ & 30 & 22 & 70 & 4 & 26 \\
\hline 1145 & 53 & 125 & 6 & 36 & 48 & 131 & 3 & 28 & 0 & 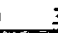 & 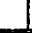 & 1 & 4 & 1 & 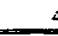 & 4 & 1 & 5 & 45 & 123 & 8 & 37 & 52 & 114 & 16 & 39 & 38 & 88 & 7 & 26 \\
\hline
\end{tabular}




\section{BAYMETRICS TRAFFIC RESOURCES Location 5B: Driveway North of East Aevnue (SB - Outbounds)}

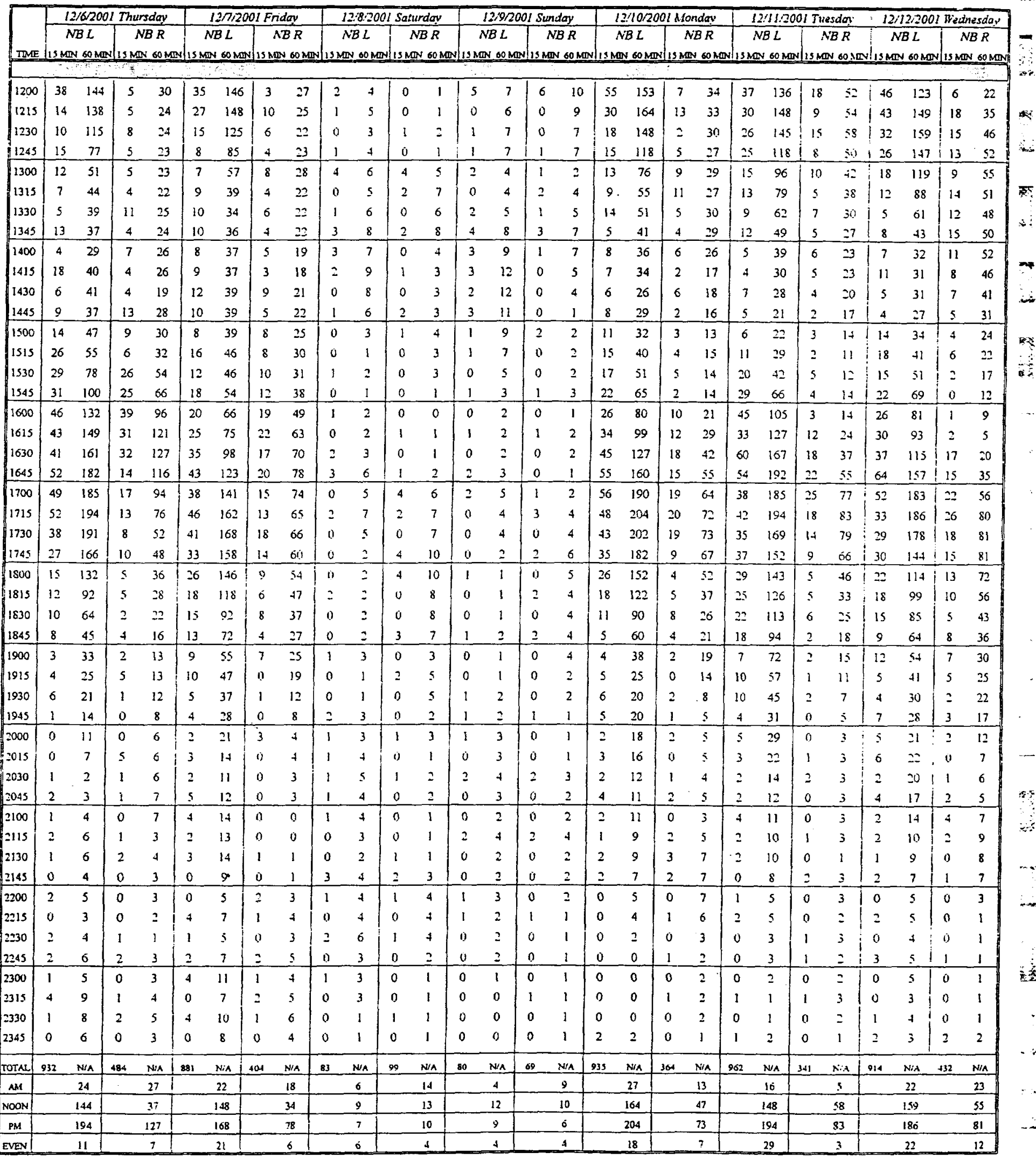




\begin{tabular}{|c|c|c|c|c|c|c|c|c|c|c|c|c|c|c|c|c|c|c|c|c|c|c|c|c|c|c|c|c|}
\hline 0 & & $8 E$ & & 0 & & $\varepsilon \varepsilon$ & & 0 & & IE & & 0 & & IE & & 0 & & 02 & & 0 & & 82 & & 0 & & $8 i$ & & NGA \\
\hline 0 & & $\angle \pi$ & & 0 & & $2 \pi t$ & & 0 & & $z z \bar{z}$ & & 0 & & $s t$ & & 0 & & FP & & 0 & & $80 t$ & & 0 & & 902 & & Wd \\
\hline 0 & & $6 L E$ & & 0 & & $\angle Q \Phi$ & & 0 & & $99 \varepsilon$ & & 0 & & $6 P$ & & 0 & & 09 & & 0 & & $8 \%$ & & 0 & & 062 & & NOON \\
\hline 0 & & $5 D$ & & 0 & & 296 & & 0 & & EEF & & 0 & & 45 & & 0 & & SII & & 0 & & $96 E$ & & 0 & & $\varepsilon 9$ & & $w v$ \\
\hline $\overrightarrow{v N}$ & $D$ & V:N & Fist & $V N$ & 0 & $\mathrm{VNN}$ & 1657 & $V / N$ & $\circ$ & $\overline{V i N}$ & $1+n z$ & $\sqrt{v i s}$ & 0 & $V N$ & 955 & $\overline{V I N}$ & 0 & VNN & $\overline{B S C}$ & $\overline{Y N N}$ & 0 & vini & BLE2 & $\overline{V N N}$ & 0 & VIN & 9856 & ivial \\
\hline 0 & 0 & 21 & 5 & 0 & 0 & 91 & 1 & 0 & 0 & 01 & 1 & 0 & 0 & 11 & $\tau$ & 0 & 0 & 91 & $\varepsilon$ & 0 & 0 & हl & $\tau$ & 0 & 0 & El & 9 & stzz \\
\hline 0 & 0 & 91 & $\xi$ & 0 & 0 & 41 & 0 & 0 & 0 & 81 & $\varepsilon$ & 0 & 0 & HI & I & 0 & 0 & $a$ & 5 & 0 & 0 & चl & 5 & 0 & 0 & 9 & $\mathfrak{E}$ & Oø£z \\
\hline 0 & 0 & $\kappa$ & + & 0 & 0 & 61 & 8 & D) & 0 & ¿l & $\varepsilon$ & 0 & 0 & 51 & 1 & 0 & 0 & 61 & 5 & 0 & 0 & II & $\varepsilon$ & 0 & 0 & $L$ & $i$ & siet \\
\hline 0 & 0 & $i$ & $\varepsilon$ & 0 & D) & 51 & $L$ & 0 & 0 & 91 & $\mathcal{E}$ & 0 & 0 & 91 & $l$ & 0 & 0 & $\angle 1$ & E & 0 & 0 & $\because$ & $\varepsilon$ & 0 & 0 & $L$ & i & oorc \\
\hline 0 & 0 & $F$ & $b$ & 0 & 0 & $\pi$ & $\bar{i}$ & 0 & 0 & $\angle 1$ & $F$ & 0 & 0 & 51 & 5 & 0 & 0 & 61 & $L$ & 0 & 0 & 11 & 1 & 0 & 0 & 6 & 0 & sbza \\
\hline 0 & $\dot{0}$ & I & 01 & 0 & 0 & $t i$ & 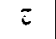 & 0 & 0 & $8 !$ & $\tau$ & 0 & 0 & s1 & $\Sigma$ & 0 & 0 & EI & $r$ & 0 & 6 & $\S$ & 5 & 0 & 0 & EI & 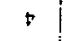 & o $0 \pi \overline{\mid}$ \\
\hline 0 & 0 & $H$ & $b$ & 0 & 0 & $\sigma \overline{\tilde{\epsilon}}$ & $\hbar$ & 0 & 0 & 61 & $L$ & 0 & 0 & $\$ 1$ & $\approx$ & 0 & 0 & 11 & $\varepsilon$ & 0 & 0 & 51 & $\nabla$ & 0 & 0 & $\angle I$ & $i$ & 5120 \\
\hline 0 & 0 & 51 & 9 & 0 & 0 & EE & +1 & 0 & 0 & $\angle I$ & 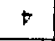 & 0 & 0 & 91 & 9 & 0 & 0 & 11 & $\varepsilon$ & n) & 0 & 91 & $i$ & 0 & 0 & $0 \tilde{\varepsilon}$ & $\varepsilon$ & | $00 \pi$ \\
\hline 0 & 0 & 51 & 1 & 0 & .0 & $5 \bar{i}$ & $r$ & 0 & 0 & $\alpha_{2}^{2}$ & 5 & 0 & 0 & +1 & 5 & 0 & 0 & il & $\varepsilon$ & 0 & 0 & 61 & $\mathfrak{E}$ & 0 & 0 & 61 & 5 & 5012 \\
\hline 0 & 0 & $t=$ & $\varepsilon$ & 0 & 0 & I؟ & 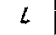 & 0 & 0 & $\alpha$ & $\varepsilon$ & 0 & 0 & ¿l & $i$ & 0 & 0 & 31 & $\Sigma$ & 0 & 11 & に & $s$ & 0 & 0 & $\bar{\tau} \tau$ & 8 & orta \\
\hline 0 & 0 & $t=$ & 5 & 0 & 0 & $\tau E$ & 8 & 0 & 0 & $\varsigma 5$ & 5 & 0 & 0 & 51 & $\mathfrak{E}$ & 0 & 0 & 11 & $\varepsilon$ & 0 & 0 & $5 i$ & 9 & 0 & 0 & 61 & 5 & st1i \\
\hline 0 & 0 & $\hbar$ & 5 & 0 & 0 & $\tau \varepsilon$ & 9 & 0 & 0 & 95 & $L$ & 0 & 0 & 61 & 5 & 0 & 0 & §॥ & 5 & 0 & 0 & $t$ & 5 & 0 & 0 & $\pi$ & $z$ & $001=1$ \\
\hline 0 & 0 & $L$ & 11 & 0 & 0 & 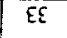 & 01 & 0 & 0 & $\div 5$ & 5 & 0 & 0 & $\bar{\sigma}$ & 5 & 0 & 0 & 51 & 1 & $n$ & 0 & $L$ & 5 & 0 & 0 & $\overline{b i}$ & l & SPOE \\
\hline 0 & 0 & $\sqrt{i}$ & $\varepsilon$ & 0 & 0 & $6 i$ & 8 & 0 & 0 & $t$ & 6 & 0 & 0 & $9 \bar{z}$ & 5 & 0 & 0 & $9 !$ & $\varepsilon$ & 0 & (1) & 85 & 8 & 0 & 0 & $8 \pi$ & 5 & OEOZ \\
\hline 0 & 0 & IE & 5 & 0 & 0 & $8 \bar{c}$ & 8 & 0 & 0 & $\tau \xi$ & 9 & 0 & 0 & II & $L$ & 0 & 0 & $n$ & 5 & 0 & 0 & $\vec{L}$ & 9 & 0 & 0 & $8 i$ & 8 & sloz \\
\hline 0 & 0 & $8 \xi$ & 8 & 0 & 0 & $5 \bar{c}$ & L & 0 & 0 & $L \varepsilon$ & 9 & 0 & 0 & 87 & 2 & 0 & 0 & 61 & 9 & 0 & is & $s$ & 8 & 0 & 0 & $8 \varepsilon$ & $t$ & $1000=$ \\
\hline 0 & 0. & $O T$ & 5 & 1) & 0 & $9 \varepsilon$ & 9 & 0 & 0 & $s t$ & $\varepsilon$ & 0 & 0 & 82 & $i$ & 0 & 0 & 81 & $\bar{i}$ & i & 0 & of & 9 & 0 & 0 & $\varepsilon t$ & 11 & 5760 \\
\hline 0 & 0 & $H$ & $\mathfrak{E l}$ & 0 & 0 & It & $L$ & 0 & 0 & $9 i$ & $\angle I$ & 0 & 0 & $s$ & 01 & 0 & $\dot{0}$ & $\varepsilon$ & 1 & ! & 0 & צִ & L & 0 & 0 & $6 \varepsilon$ & 5 & o£6t \\
\hline 0 & 0 & it & $i 1$ & 0 & 0 & $\$ 5$ & 5 & 0 & 0 & $\therefore$ & 11 & 0 & 0 & $\hbar$ & $t$ & 0 & 0 & $q_{2}^{-}$ & 01 & 0 & (1) & $0 t$ & 5 & 0 & 0 & It & 81 & 5161 \\
\hline 0 & 0 & 15 & 01 & 0 & 0 & 19 & 81 & 0 & 0 & 19 & +1 & 0 & 0 & OE & 1 & 0 & 0 & $\pi$ & $\xi$ & 0 & 0 & 95 & El & 0 & 0 & $\mathfrak{E} \varepsilon$ & 6 & 0061 \\
\hline 0 & 0 & 19 & 6 & 0 & $\bar{v}$ & 019 & 11 & 0 & 0 & 79 & 51 & 0 & 0 & ๕؟ & 5 & 0 & 0 & 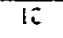 & L & 0 & i) & 0 & 11 & 0 & 0 & $5 t$ & $L$ & $5+81$ \\
\hline 0 & 0 & 69 & II & 0 & 0 & 19 & E & 0 & 0 & os & EI & 0 & 0 & $L S$ & $\dot{0}$ & 0 & 0 & $\$ i$ & $t$ & n & 0 & $r$ & $\approx I$ & 0 & 0 & is & $L$ & oisi \\
\hline 0 & 0 & $L L$ & $i$ & 0 & 0 & $6 s$ & 11 & 0 & 0 & 99 & 92 & 0 & (1) & 55 & al & 0 & 0 & 81 & $?$ & i & 0 & 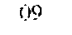 & $a$ & 0 & 0 & $\approx$ & 01 & 5181 \\
\hline 0 & 0 & $00 !$ & $0 \bar{c}$ & 0 & 0 & 16 & $\angle I$ & 0 & 0 & is & 6 & 0 & 0 & $\angle \varepsilon$ & of & 0 & 0 & $E$ & $t$ & 0 & $n$ & $S L$ & 6 & 0 & 0 & $L L$ & $i$ & 0081 \\
\hline 0 & 0 & 96 & 41 & 0 & 0 & 101 & $\pi$ & 0 & 0 & 16 & 11 & 1 & 0 & 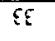 & 8 & $\dot{0}$ & 0 & LE & $r$ & 11 & 0 & $\angle 8$ & 51 & 0 & 0 & 56 & 51 & $5+\angle 1$ \\
\hline 0 & 0 & 56 & $6 \mathrm{I}$ & 0 & 0 & 111 & 61 & 0 & 0 & 501 & $\alpha z$ & 0 & 0 & It & $L$ & 0 & 0 & $u \varepsilon$ & $t$ & 1) & 0 & of & 81 & 0 & 0 & 86 & 41 & $0 E \angle I$ \\
\hline 0 & 0 & na & $*$ & 0 & 0 & 501 & $E t$ & 0 & 0 & $90^{\circ}$ & 15 & 0 & 0 & $5 t$ & $\because 1$ & 0 & 0 & $\check{\varepsilon}$ & al & $"$ & 0 & $\angle 6$ & $9 \mathcal{E}$ & ) & 0 & 56 & $\varsigma$ & sill \\
\hline 0 & 0 & 59 & 91 & 0 & 0 & $\mathscr{L}$ & $L$ & 0 & 0 & 16 & 61 & 0 & 0 & It & 9 & 0 & 0 & 5 & $L$ & 0 & 0 & $\angle L$ & 81 & 0 & 0 & $\angle 8$ &.$\angle E$ & $00<1$ \\
\hline 0 & 0 & 69 & SI & 0 & 0 & 99 & $\pi$ & 0 & 0 & $6 L$ & $\pi$ & 0 & 0 & Er & 91 & 0 & 0 & 65 & l & 0 & $\dot{0}$ & $B L$ & 41 & 0 & 0 & 99 & $6 I$ & sp91 \\
\hline 0 & 0 & 82 & s! & 0 & 0 & $0 L$ & $\approx 1$ & i) & 0 & $\angle 8$ & 11 & 0 & 0 & 65 & 11 & 0 & 0 & 05 & 6 & 0 & 0 & 88 & $9 i$ & $\dot{u}$ & 0 & $\angle 9$ & $\# 1$ & $0 \varepsilon 91$ \\
\hline 0 & 0 & $\varepsilon 8$ & 61 & 0 & 0 & 28 & \I & 0 & 0 & 501 & 61 & 0 & 0 & 55 & 8 & 0 & 0 & $\xi$ & 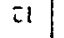 & 0 & 0 & 96 & 91 & 0 & 0 & $u$ & $\angle 1$ & 5191 \\
\hline 0 & 0 & 68 & $0 \bar{z}$ & 0 & 0 & 56 & $\angle I$ & 0 & 0 & $00^{\circ}$ & $t i$ & 0 & 0 & $D \varepsilon$ & 8 & 0 & 0 & ib & 11 & 0 & 0 & 801 & 61 & 0 & 0 & 68 & 91 & 0091 \\
\hline 0 & 0 & 06 & $t i$ & 0 & 0 & $\angle 01$ & 96 & 0 & 0 & 111 & $\overline{E \xi}$ & 0 & 0 & $\overline{\Phi \varepsilon}$ & EI & 0 & 0 & $\angle \varepsilon$ & 8 & 0 & 0 & 501 & $L \tau$ & 0 & 0 & 001 & $0 \bar{\varepsilon}$ & srsi \\
\hline 0 & 0 & $0 \geq 1$ & $\alpha \tilde{\tau}$ & 0 & 0 & OEI & $5 i$ & 0 & 0 & $\$ 11$ & $8 \pi$ & 0 & 0 & is & l & 0 & 0 & th & $\pi$ & 0 & 0 & $8 E 1$ & $t \varepsilon$ & 0 & 0 & 011 & $t \bar{\imath}$ & oesilf \\
\hline 0 & 0 & $\pi \angle I$ & 5 & 0 & 0 & $16 i$ & 85 & 0 & 0 & 651 & 51 & 1) & 0 & $n \varepsilon$ & $l$ & 0 & 0 & $i 5$ & II & 0 & 0 & $9 L 1$ & $8 \bar{i}$ & 0 & 0 & 51 & $6 \bar{\tau}$ & 5151 \\
\hline 0 & 0 & $\angle I \pi$ & 12 & 0 & 0 & $i t i$ & $6 i$ & 0 & 0 & こ & $9 \varepsilon$ & 0 & 0 & $L$ & 0 & 0 & 0 & $t$ & 9 & 0 & 0 & sot & $9 \varepsilon$ & 0 & 0 & 902 & $L \vec{c}$ & 0051 \\
\hline 0 & 0 & $S F i$ & $b \varsigma$ & 0 & 0 & 592 & 65 & 0 & 0 & $6 \pi$ & ऽร & 0 & 0 & $\bar{E}$ & 0 & 0 & 0 & SS & SI & 0 & 0 & Liడ & $0 p$ & 0 & 0 & $8 \mathrm{si}$ & OE & 5001 \\
\hline 0 & 0 & $i \pi \bar{i}$ & $u$ & 0 & 0 & ZSi & $\$ 8$ & 0 & 0 & に & $\because L$ & 0 & 0 & $i$ & 5 & 0 & 0 & 85 & $a$ & 0 & 0 & $T E i$ & IL & 0 & 0 & $06 \tau$ & 85 & $0 \mathrm{ert}$ \\
\hline 0 & 0 & $i L i$ & $0 L$ & 0 & 0 & $56 \mathrm{I}$ & $6 l$ & 0 & 0 & $\mathfrak{\varepsilon} \angle \mathbb{I}$ & $U$ & 0 & 0 & $\varepsilon$ & $t$ & 0 & 0 & $\angle 5$ & $\varepsilon I$ & 0 & 0 & $\varepsilon 81$ & 09 & 0 & 0 & $08 i$ & 16 & sidis \\
\hline 0 & 0 & $t \subseteq I$ & $6 t$ & 0 & 0 & $9 \varepsilon 1$ & $\tau$ & 0 & 0 & 811 & \&b & 0 & 0 & $8 i$ & 5 & 0 & 0 & 05 & $\angle L$ & 0 & 0 & 151 & 55 & 0 & 0 & $\| z$ & $6 L$ & 0011 \\
\hline 0 & 0 & 801 & os & 0 & 0 & 011 & $9 \varepsilon$ & 0 & 0 & cor & LZ & 0 & 0 & $9 \bar{i}$ & $L$ & 0 & 0 & is & 8 & 0 & 0 & IEI & $L t$ & 0 & 0 & 891 & 29 & 57E \\
\hline 0 & 0 & EII & $\tilde{\Sigma}$ & 0 & 0 & 101 & $8 i$ & 0 & 0 & (x)1 & $9 \bar{c}$ & 0 & 0 & IS & 6 & 10 & 0 & 85 & 6 & 0 & i & 56 & $i \tau$ & 0 & 0 & $\operatorname{tEI}$ & $8 t$ & oระ1: \\
\hline 0 & 0 & 111 & $\tau \varepsilon$ & 0 & ) & 20 & $a$ & 0 & 0 & $\therefore 6$ & $\pi$ & 0 & 0 & 85 & 6 & 0 & 0 & st & 91! & 0 & ) & $6 S$ & $8 \pi$ & 0 & 0 & $\angle O L$ & $\pi i$ & 5161 \\
\hline 0 & 0 & 201 & $\varepsilon$ & 0 & 0 & £8 & $9 i$ & 0 & 0 & 100 & $L i$ & : & 0 & $6 \varepsilon$ & 5 & 0 & i & ur & 81 & 0 & 0 & $8 L$ & $5 i$ & 0 & 0 & $\angle 01$ & $9 \varepsilon$ & 0021 \\
\hline 0 & 0 & 011 & 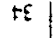 & D & 0 & 08 & LE & 0 & 0 & OS & $\leqslant$ & 0 & 0 & $\angle t$ & $\therefore 1$ & 0 & 0 & $\varepsilon \varsubsetneqq$ & 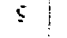 & 0 & 0 & 59 & $\sigma i$ & 0 & 0 & $60^{\circ}$ & $8 \bar{i}$ & Srat \\
\hline 0 & 0 & 16 & $\bar{i}$ & 0 & 0 & UL & 61 & i) & i & $Q L$ & $\$ 1$ & 0 & 0 & $6 t$ & $n$ & 0 & 0 & 5 & 9 & $\dot{0}$ & 0 & $u$ & 91 & 0 & 0 & $\varepsilon 6$ & $\pi$ & | $0 \varepsilon=1$ \\
\hline 0 & 0 & $\mathfrak{\varepsilon 8}$ & $\bar{\varepsilon}$ & 0 & 0 & 166 & 11 & 0 & 0 & ol & $t \bar{\varepsilon}$ & 0 & 0 & $L t$ & 01 & 0 & 0 & 55 & 11 & 0 & 0 & $\sigma L$ & 21 & 0 & 0 & 06 & $\pi i$ & StE1 \\
\hline 0 & 0 & 06 & IE & 0 & 0 & 811 & $\pi$ & 0 & 0 & $B L$ & $\varepsilon$ & 0 & 0 & $\varepsilon t$ & 11 & 0 & 0 & 85 & 11 & 0 & $0:$ & $\angle 8$ & 21 & 0 & 0 & 96 & $8 \bar{C}$ & | 0021 \\
\hline \multicolumn{28}{|c|}{ 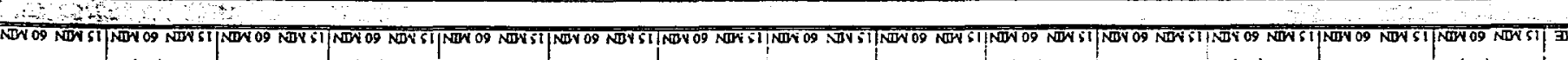 } & \\
\hline & & $(N D)$ & & & & $(N D)$ & & & & $(\Delta: D)$ & & & & \multirow{2}{*}{\multicolumn{2}{|c|}{$\frac{(N D) g N}{00 i \cdot 6: l}$}} & & & \multirow{2}{*}{\multicolumn{2}{|c|}{ 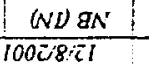 }} & & & \multirow{2}{*}{\multicolumn{2}{|c|}{$\frac{(N) g N}{002-2 i}$}} & & & & & \\
\hline \multicolumn{4}{|c|}{ Kopsatpa } & \multicolumn{4}{|c|}{ nopsen $\perp$ looz/ll $: C l$} & \multicolumn{4}{|c|}{ iopuofy $100 \overline{0} 01 \mathrm{Cl}$} & & $\mathrm{Nin}_{3}$ & & & & & & & & $1+100$ & & & & & $02 \%$ & & \\
\hline
\end{tabular}




\section{BAYMETRICS TRAFFIC RESOURCES Location 6: Southgate Drive North of East Aevnue (NB - Inbounds)}

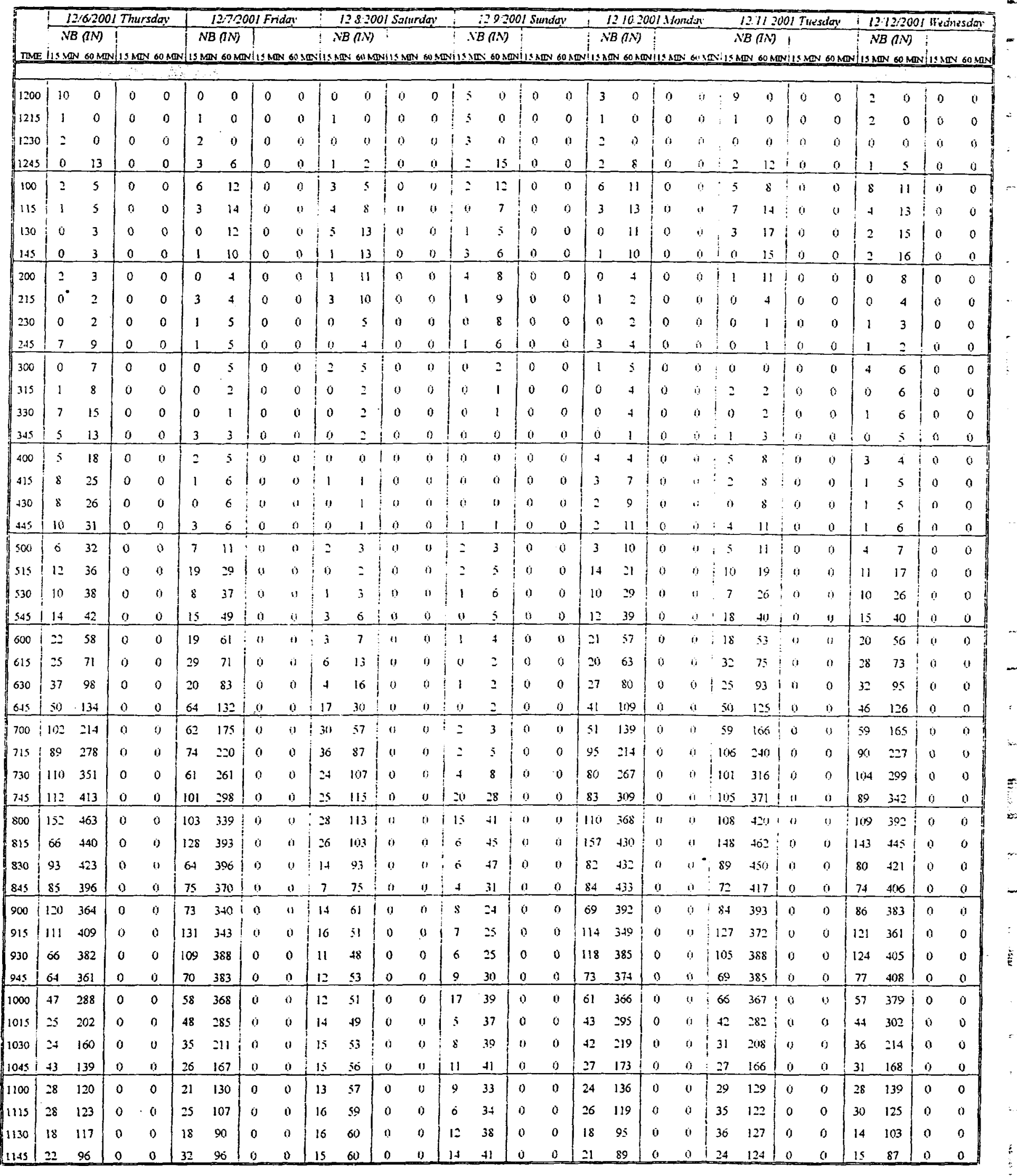




\section{BAYMETRICS TRAFFIC RESOURCES}

Location 6B: Southgate Drive North of East Aevnue (SB - Outbounds)

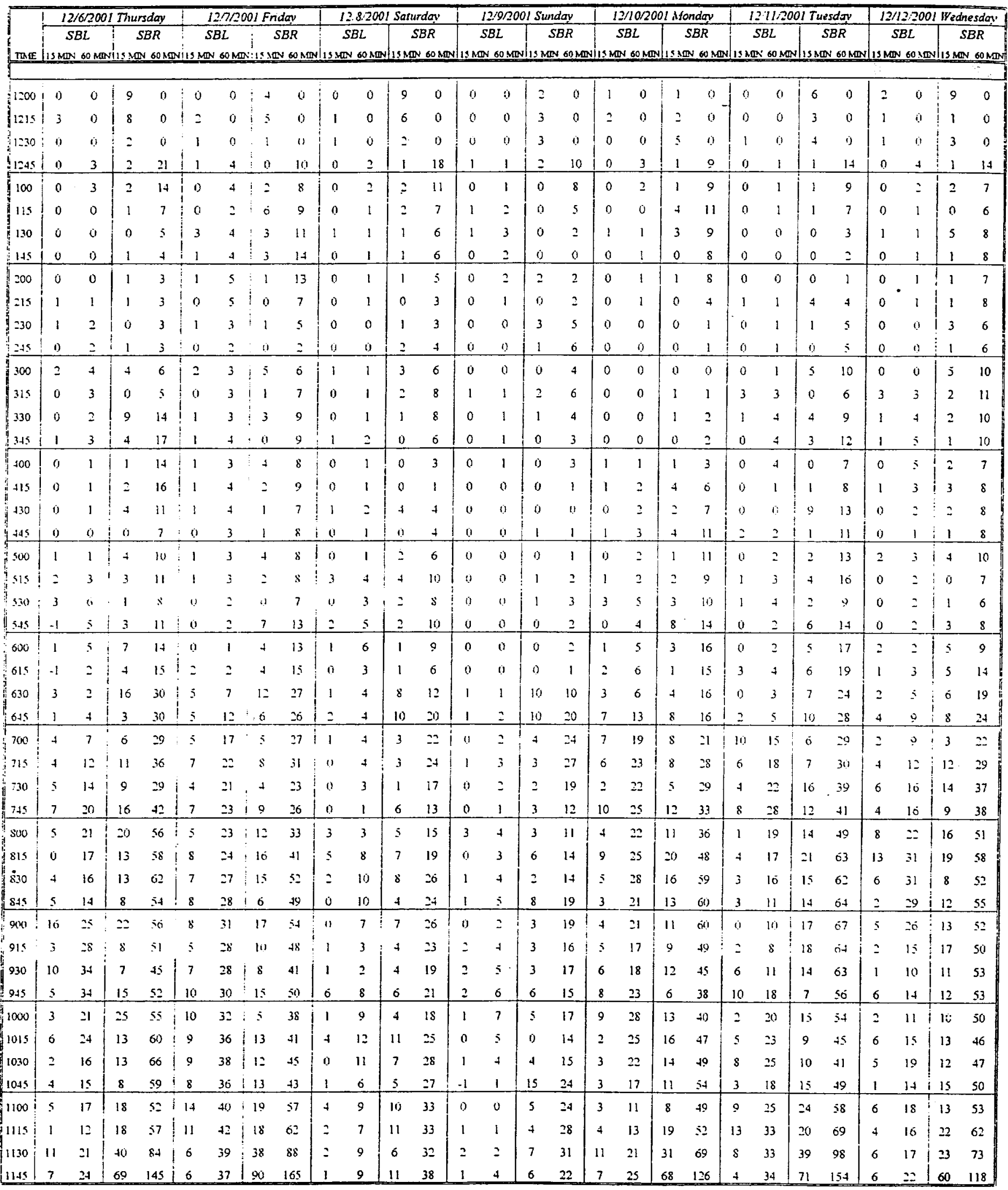




\section{BAYMETRICS TRAFFIC RESOURCES Location 6B: Southgate Drive North of East Aevnue (SB - Outbounds)}

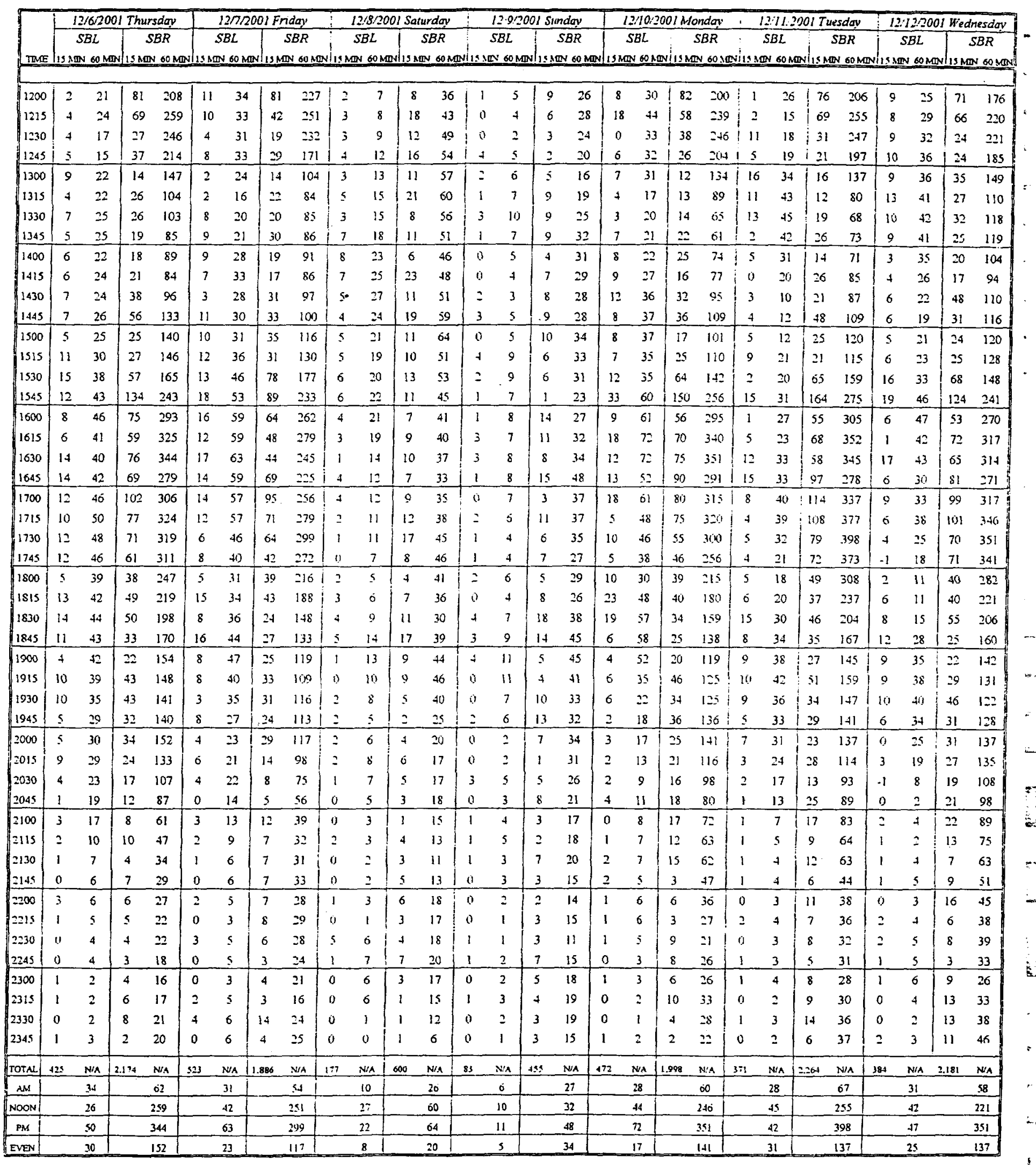




\section{BAYMETRICS TRAFFIC RESOURCES Location 7: Shipping Entrance North of East Aevnue (NB - Inbounds)}

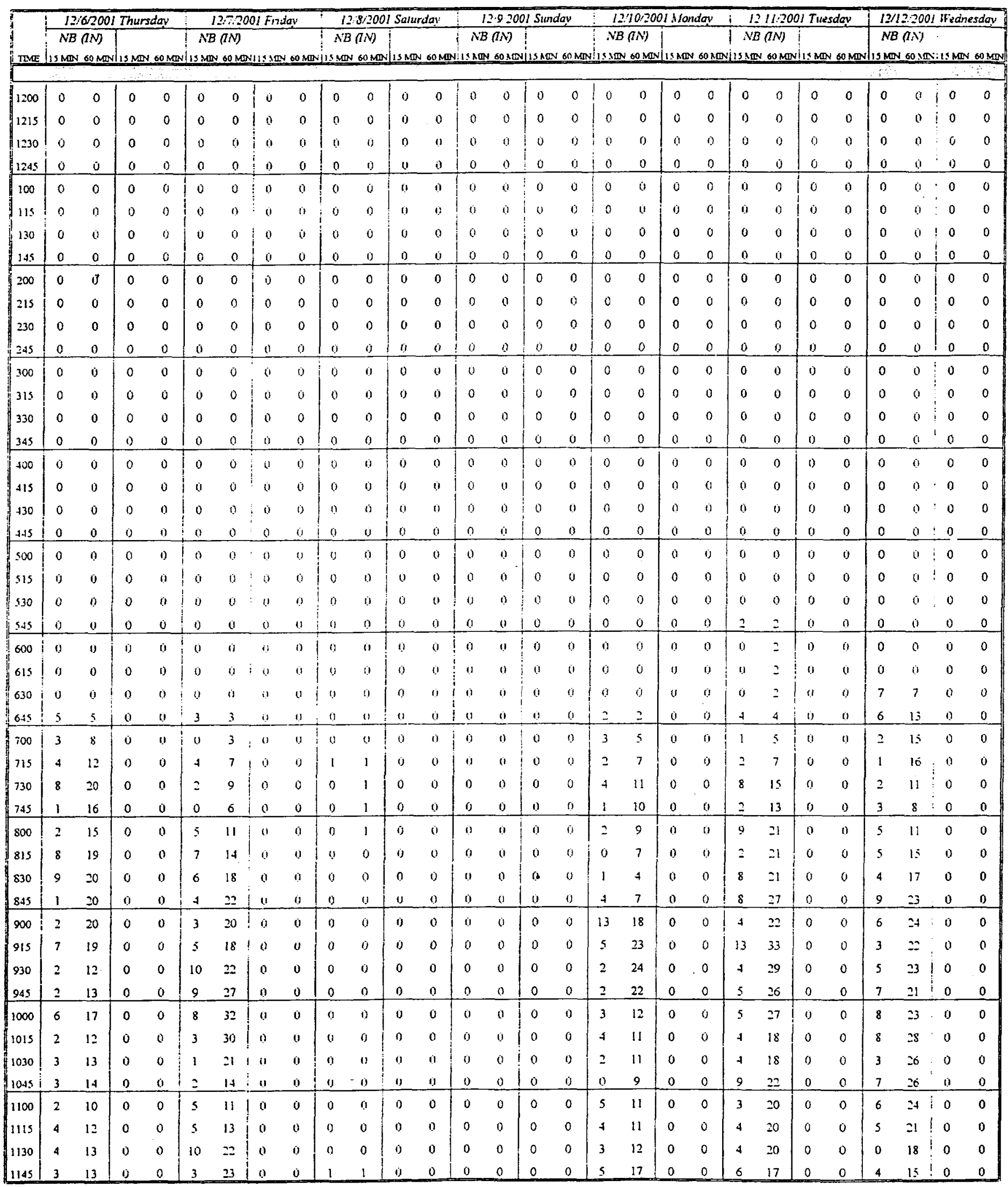




\section{BAYMETRICS TRAFFIC RESOURCES Location 7: Shipping Entrance North of East Aevnue (NB - Inbounds)}

\begin{tabular}{|c|c|c|c|c|c|c|c|c|c|c|c|c|c|c|c|c|c|c|c|c|c|c|c|c|c|c|c|c|}
\hline & 120 & $5 / 2001$ & Thurse & & & 7200 & I Frie & & & 2001 & Seutura & & & 2.2001 & Sunday & & & 20011 & & & & & & & 12123 & & Wedne & \\
\hline & $N B$ & & & & $N B$ & & & & $N B$ & $\mathrm{Ni}$ & & & $N^{\prime} B I$ & & & & $N B \cap$ & & & & $N B$ & & & & $N B a A$ & & & \\
\hline$\frac{M E}{4}$ & $13 \mathrm{Mm}$ & $0 \mathrm{x}$ & 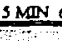 & & $\mathrm{MON}$ & & & & & & & & & & & & & & & & & - & & & & & - & \\
\hline 1200 & 3 & 14 & 0 & 0 & 13 & 31 & 0 & 0 & ) & 1 & 1) & 0 & 0 & 0 & 0 & 0 & $\underline{s}$ & 17 & 0 & 0 & ᄀ & 16 & 0 & 0 & & & & \\
\hline 1215 & 5 & 15 & 0 & 0 & 4 & 30 & 0 & 0 & 0 & 1 & 0 & 0 & 0 & 0 & 0 & 0 & 2 & 15 & 0 & 1) & 6 & 18 & 0 & 0 & 3 & 11 & 0 & 0 \\
\hline 1230 & $=$ & 13 & 0 & 0 & 2 & 22 & 0 & 0 & 0 & 1 & 0 & 0 & 0 & 0 & 0 & 0 & 3 & 15 & 0 & 0 & : & 16 & 0 & 0 & 6 & 17 & 0 & 0 \\
\hline 1245 & 7 & 17 & 0 & 0 & 0 & 191 & 0 & 0 & 0 & 9 & 0 & 0 & 0 & 0 & 0 & 0 & 4 & 14 & 9 & a & 2 & 12 & 0 & 0 & 2 & 15 & 0 & 0 \\
\hline 1300 & 11 & 25 & 0 & 0 & 2 & 81 & 0 & 0 & 0 & 0 & 0 & 1) & 0 & 0 & 0 & 0 & 3 & 12 & 0 & 0 & 5 & 15 & (j) & 0 & 5 & $10^{\circ}$ & ) & 0 \\
\hline :1315 & $s$ & 25 & 0 & 0 & 5 & 9 & 0 & 0 & 0 & i) & 0 & $"$ & i) & 0 & 0 & 0 & $=$ & 12 & 0 & 0 & 4 & 13 & 0 & 0 & 3 & 16 & 0 & 0 \\
\hline 1330 & 4 & 27 & 0 & 0 & 11 & 18 & 0 & 0 & 0 & 0 & 0 & 0 & 19 & 0 & 0 & 0 & 10 & 19 & 0 & 0 & $=$ & 13 & 0 & 0 & 3 & 13 & 0 & 0 \\
\hline 1345 & 7 & 27 & 0 & 0 & 3 & 21 & 0 & 0 & 0 & 0 & 0 & 0 & 0 & 0 & 0 & 0 & 3 & 18 & 0 & 0 & 1 & 12 & 0 & 0 & 3 & 14 & 0 & 0 \\
\hline 1400 & 7 & 23 & 0 & 0 & 5 & 24 & 0 & 0 & 0 & 0 & 0 & 0 & 0 & 0 & 0 & 0 & 3 & 18 & 0 & 0 & 1 & 8 & 0 & 0 & 12 & 21 & 0 & 0 \\
\hline 1415 & 5 & 23 & 0 & 0 & 5 & 24 & 0 & 0 & 0 & 0 & 0 & 0 & 0 & 0 & 0 & 0 & 2 & 18 & 0 & 0 & 0 & 4 & 0 & 0 & 4 & $\because 2$ & 0 & 0 \\
\hline 1430 & 5 & 24 & 0 & 0 & 6 & 19 & 0 & 0 & 0 & 0 & 0 & 0 & 0 & 0 & $a$ & 0 & 4 & 12 & 0 & 0 & 6 & 8 & 0 & 0 & 3 & $\therefore 2$ & 0 & 0 \\
\hline 1445 & 5 & 22 & 0 & 0 & 2 & 18 & $\dot{0}$ & 0 & 0 & 0 & 0 & 0 & 0 & 0 & 0 & 0 & 3 & 12 & 0 & 0 & 2 & 9 & 0 & 0 & 4 & 23 & 0 & 0 \\
\hline 1500 & 1 & 16 & 0 & 0 & 1 & 14 & 0 & 0 & 0 & 0 & 0 & 0 & 0 & 0 & 0 & 0 & 4 & 13 & 0 & 0 & 7 & 15 & 0 & 0 & 4 & 15 & 0 & 0 \\
\hline 1515 & 2 & 13 & 0 & $\dot{0}$ & 5 & 14 & 0 & 0 & 0 & 1) & 0 & 0 & 0 & 0 & 0 & 0 & $\vdots$ & $16=$ & 0 & 0 & 3 & 18 & 0 & 0 & 2 & 13 & 0 & 0 \\
\hline 1530 & 2 & 10 & 0 & 0 & 1 & 9 & 3 & 11 & 0 & 0 & 0 & 1) & 0 & 0 & 0 & 0 & $=$ & 14 & 0 & 9 & 1 & 13 & 0 & 0 & 1 & 11 & 1) & 0 \\
\hline 1545 & 2 & 7 & 0 & 0 & 1 & 8 & 0 & 0 & 0 & i) & 0 & 0 & 0 & 0 & 0 & 0 & 2 & 13 & 0 & 0 & 0 & 11 & 9 & 0 & 1 & 8 & 0 & 0 \\
\hline 1600 & 1 & 7 & 0 & 0 & 1 & 8 & 0 & 0 & 0 & 0 & 0 & 0 & 0 & 0 & 0 & 0 & 3 & 12 & 0 & 0 & 0 & 4 & $\dot{0}$ & 0 & 0 & 4 & 0 & 0 \\
\hline 1615 & 0 & 5 & 0 & 0 & 0 & 3 & 0 & c) & 0 & 0 & 0 & 0 & 0 & 0 & 0 & 0 & 0 & 7 & $a$ & 0 & 0 & 1 & 0 & 0 & 0 & $=$ & 0 & 0 \\
\hline 1630 & 0 & 3 & 0 & 0 & 2 & 4 & 0 & 0 & 0 & 0 & 0 & 0 & 0 & 0 & 0 & 0 & 0 & $s$ & 0 & 0 & D & 0 & 0 & 0 & 0 & 1 & 0 & 0 \\
\hline 1645 & 0 & 1 & 0 & 0 & 0 & 3 & 0 & 0 & 0 & 0 & 0 & 0 & 0 & 0 & u & 0 & 0 & 3 & 0 & $a$ & 0 & 0 & 0 & 0 & 0 & 0 & 0 & 0 \\
\hline 1700 & 0 & 0 & 0 & 0 & 0 & 21 & (9) & 0 & 0 & 0 & 0 & 0 & 1 & 1 & 0 & 0 & 0 & 0 & 0 & 0 & 0 & 0 & 0 & 0 & 0 & 01 & 0 & 0 \\
\hline 1715 & 0 & 0 & 0 & 0 & 0 & 2 & 11 & " & a & " & a & 0 & 0 & 1 & i) & (1) & 0 & 3 & y & $n$ & 0 & 0 & 0 & 0 & 0 & 0 & 0 & 0 \\
\hline 1730 & 0 & 0 & 0 & 0 & 0 & 0 & 0 & 0 & 0 & o & (1) & 0 & 0 & 1 & 0 & 0 & 0 & 0 & i) & 0 & 0 & 0 & 0 & 0 & 0 & ) & D) & G \\
\hline 1745 & 0 & 0 & 0 & 0 & (1) & 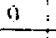 & $a$ & $1 i$ & a) & 0 & 0 & 0 & 0 & 1 & 0 & 0 & $\dot{0}$ & 9 & 0 & 0 & 0 & 0 & 0 & (3) & 0 & 0 & 0 & 0 \\
\hline 1800 & 0 & 0 & 0 & 0 & 0 & 0 & 0 & 0 & 0 & 0 & 0 & () & 0 & 0 & 0 & 0 & 0 & 0 & a & 0 & 0 & 0 & 0 & 0 & 0 & 0 & 0 & 0 \\
\hline 1815 & 0 & 0 & 0 & 0 & 0 & 0 & $a$ & ") & 0 & 0 & 0 & 0 & 0 & 0 & 0 & 0 & 1) & 0 & $\dot{0}$ & (I) & 0 & 0 & $"$ & 0 & 0 & $\theta$ & 0 & 0 \\
\hline 1830 & 0 & 0 & 0 & 0 & 0 & 0 & in & $" 1$ & " & i) & 0 & 0 & 0 & 0 & 0 & 0 & 0 & 0 & 0 & 0 & 0 & 0 & 0 & $\dot{0}$ & 0 & 0 & 1) & 0 \\
\hline 1845 & 0 & 0 & 0 & 0 & 0 & 0 & 0 & 0 & 0 & 0 & 0 & 0 & 0 & 0 & 9 & 0 & 0 & 0 & 0 & 0 & 0 & 0 & 0 & 0 & 0 & 0 & 0 & 0 \\
\hline 1900 & ij & 0 & 0 & 0 & 0 & 0 & 0 & " & 11 & 0 & 0 & 0 & 0 & (1 & 0 & 0 & 0 & 0 & 0 & 0 & 0 & 0 & 0 & 0 & 0 & 9 & 0 & 0 \\
\hline 1915 & 0 & 0 & 0 & 0 & 0 & 01 & 0 & 11 & 0 & 0 & 0 & 0 & 0 & 0 & 0 & 0 & 0 & 01 & 0 & 0 & 0 & 0 & 0 & 0 & 0 & 0 & 0 & 0 \\
\hline 1930 & i) & 0 & 0 & 0 & 0 & 0 & 0 & 0 & " & $u$ & 0 & 0 & 0 & 0 & ij & $\dot{0}$ & 0 & $0 \quad:$ & 0 & a & 0 & 0 & 0 & 0 & 0 & 0 & 0 & 0 \\
\hline 19.45 & 11 & 0 & 0 & 0 & 0 & 0 & 0 & 0 & 4 & 0 & 9 & 0 & 0 & (1) & 0 & 0 & 0 & 0 & 0 & 0 & 9 & 0 & 0 & () & 0 & 0 & ()) & 0 \\
\hline 2000 & 0 & 0 & 0 & 0 & 0 & i) & 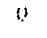 & 0 & ) & 0 & i & 0 & 0 & 11 & ij & 0 & 0 & 0 & (1) & 0 & 0 & 0 & 0 & i) & 0 & 0 & 0 & 0 \\
\hline 2015 & 0 & 0 & 0 & 0 & 0 & 0 & 0 & 0 & () & 0 & 0 & 0 & 0 & 0 & ) & 0 & 0 & 0 & 0 & 0 & 0 & 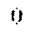 & 0 & 0 & 0 & 0 & 0 & 0 \\
\hline 030 & 0 & 0 & 0 & 0 & 0 & $\dot{\mathrm{d}}$ & 0 & 0 & 0 & is & 0 & $a$ & 0 & 0 & $\dot{0}$ & 0 & 0 & 0 & 0 & 0 & 0 & 0 & $D$ & 0 & 0 & 0 & 0 & 0 \\
\hline 2045 & 0 & 0 & 0 & 0 & 0 & 0 & $v$ & 0 & 0 & 0 & (1) & 0 & 0 & 0 & 0 & 0 & 0 & 0 & 0 & 0 & 0 & 0 & 0 & 0 & 0 & is & 0 & 0 \\
\hline 2100 & 0 & 0 & 0 & 0 & 0 & $0 i$ & 9 & 4 & 0 & 0 & 0 & 0 & 0 & 0 & (1) & 0 & 0 & 0 & 0 & 0 & 0 & 0 & 0 & 0 & (j) & 0 & ) & 0 \\
\hline$=115$ & 0 & 0 & 0 & 0 & 0 & 0 & 0 & ") & 0 & 0 & 0 & 0 & 0 & 0 & () & 0 & 0 & a & 0 & 0 & 0 & 0 & 0 & 0 & 0 & 0 & 0 & 0 \\
\hline$=130$ & 9 & 0 & 0 & 0 & 0 & 0 & 0 & 0 & () & 1) & 0 & 0 & 0 & 0 & o & 0 & 0 & 0 & 0 & 0 & 0 & 0 & 0 & 0 & 0 & 0 & 0 & 0 \\
\hline 2145 & 0 & 0 & 0 & 0 & 0 & 0 & 0 & qu & 0 & 0 & 0 & 0 & 0 & 0 & 0 & 0 & 0 & $0 \quad$ & 0 & (1) & 0 & 0 & 0 & 0 & 0 & 0 & 0 & 0 \\
\hline 2200 & 0 & 0 & 0 & 0 & 0 & 0 & 0 & 0 & 0 & 0 & 0 & 0 & $a$ & 0 & 0 & 0 & 0 & $\dot{0}$ & 0 & 0 & 0 & 0 & 0 & 0 & 0 & 0 & 0 & 0 \\
\hline 215 & 0 & 0 & 0 & 0 & 0 & 0 & 0 & 0 & 0 & 0 & 0 & 0 & 0 & 0 & 0 & 0 & 0 & 0 & 0 & 0 & 0 & 0 & 0 & 0 & 0 & 0 & 0 & 0 \\
\hline$=230$ & i) & 0 & 0 & 0 & 0 & 0 & 0 & " & $1)$ & 0 & 1) & 0 & 0 & 0 & 0 & 0 & 0 & 0 & 0 & 0 & 0 & 9 & i) & 0 & 0 & 4 & 0 & $\dot{u}$ \\
\hline 245 & 0 & 0 & 0 & 0 & 0 & i) & 0 & 0 & (1) & 0 & 0 & 0 & 0 & 0 & () & 19 & 0 & 0 & 0 & 0 & 0 & 0 & a & $a$ & 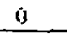 & 9 & 0 & 0 \\
\hline$=300$ & 0 & 0 & 0 & 0 & 0 & 0 & 0 & 0 & 0 & 0 & 0 & 0 & 0 & 0 & 0 & 0 & 0 & 0 & 0 & 0 & 0 & 0 & 0 & 1) & 0 & 0 & 0 & 0 \\
\hline 315 & 0 & 0 & 0 & 0 & 0 & 0 & 0 & 0 & 0 & 0 & 1) & 0 & 0 & 0 & i) & 0 & 0 & 0 & 1) & 0 & 0 & 0 & 0 & 0 & 0 & 0 & 0 & 0 \\
\hline 330 & 0 & 0 & 0 & 0 & 0 & 0 & 0 & 0 & 0 & 0 & i) & 0 & 0 & 0 & 0 & 0 & 0 & 0 & 0 & 0 & 0 & ) & 0 & 0 & 0 & 0 & 0 & 0 \\
\hline 2345 & 0 & 0 & 0 & 0 & 0 & 0 & $\dot{0}$ & 0 & 0 & $"$ & 0 & 0 & 0 & 0 & 0 & 0 & 0 & a & 0 & 0 & 0 & 0 & 0 & 0 & 0 & $0:$ & 0 & 0 \\
\hline TOTN & 195 & $N / \Lambda$ & 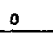 & $\mathrm{N} / \mathrm{A}$ & 164 & $\mathrm{NiA} I$ & 0 & $N / A$ & 2 & $\mathrm{~N} / \mathrm{A}$ & 0 & $\therefore / A$ & & N:A & 0 & N/A & 137 & $\underline{N} \mathrm{~A}$ & 0 & $\mathrm{~N} / \mathrm{A}$ & $m$ & $\mathrm{~N} / \mathrm{A}$ & 0 & $\mathrm{~N} / \mathrm{A}$ & 166 & $\therefore *$ & 0 & $\mathrm{~N} / \mathbf{A}$ \\
\hline$i M$ & & 20 & & 0 & & 27 & & 0 & & 1 & & 0 & & 0 & & 0 & & 24 & & 0 & & 33 & & 0 & & 21 & & 0 \\
\hline NOON & & 27 & & 0 & & 32 & & 0 & & 1 & & 0 & & - & & 0 & & 19 & & 0 & & 27 & & 0 & & 38 & & 0 \\
\hline$M$ & & 10 & & 0 & & 14 & & 0 & & 0 & & 0 & & 1 & & 0 & & 10 & & 0 & & 18 & & 0 & & 15 & & 0 \\
\hline EN & & 0 & & 0 & & 0 & & 0 & & 0 & & 0 & & 0 & & 0 & & 0 & & 0 & & 0 & & 0 & & 0 & & 0 \\
\hline
\end{tabular}




\section{BAYMETRICS TRAFFIC RESOURCES Location 7B: Shipping Entrance North of East Aevnue (SB - Outbounds)}

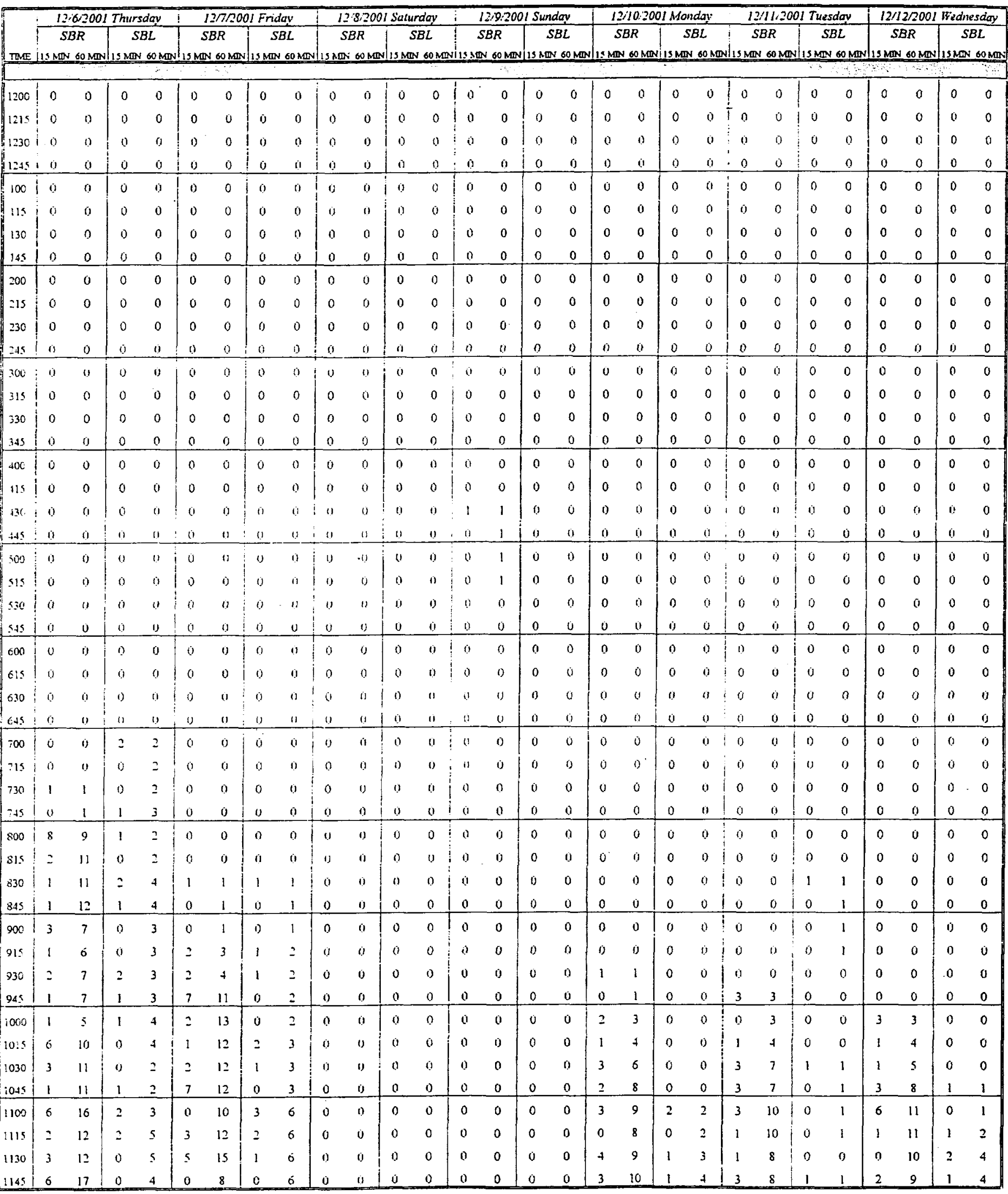




\section{BAYMETRICS TRAFFIC RESOURCES}

Location 7B: Shipping Entrance North of East Aevnue (SB - Outbounds)

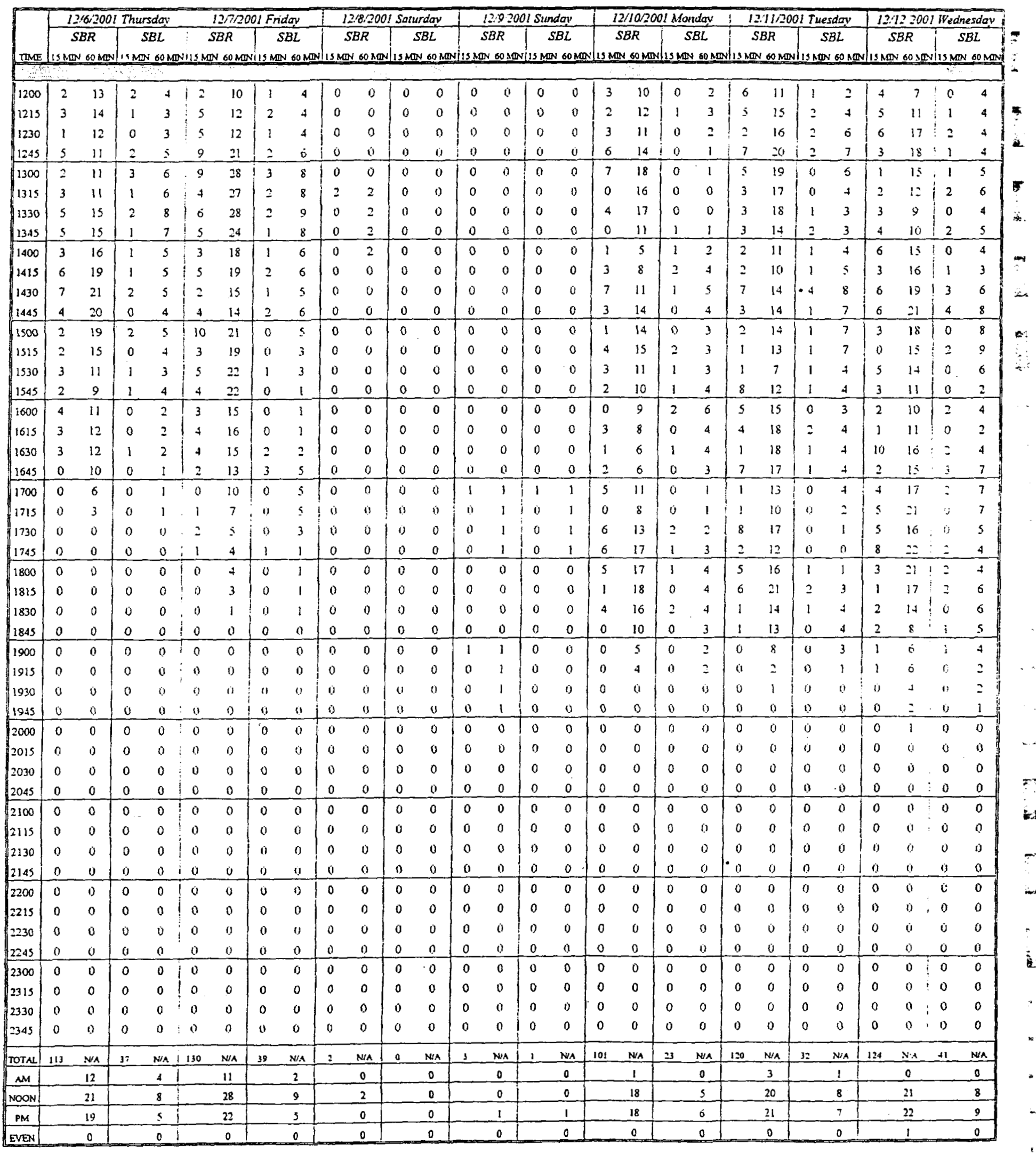




\section{BAYMETRICS TRAFFIC RESOURCES Location 8: Southbound Onto Driveway East To Sandia Lab}

\begin{tabular}{|c|c|c|c|c|c|c|c|c|c|c|c|c|c|c|c|c|c|c|c|c|c|c|c|c|c|c|c|c|}
\hline \multirow{2}{*}{ TNE } & \multicolumn{4}{|c|}{ 12/62001 Thursday } & \multicolumn{4}{|c|}{ 13/2/2001 Friday } & \multicolumn{4}{|c|}{ 12:2:2001 Salurday } & \multicolumn{4}{|c|}{ 12:9/2001 Sunday } & \multicolumn{4}{|c|}{12.102001 Monday } & \multicolumn{4}{|c|}{ 12/112001 Tuesday } & \multicolumn{4}{|c|}{ 12,12/2001 Hednesioy } \\
\hline & \multicolumn{2}{|c|}{$S B(I N)$} & & & & & & & & $B(N)$ & & & & $S B A N$ & & & & $3(N)$ & & & & & & & & $(I N)$ & & \\
\hline $1200 \mid$ & 0 & & 0 & 0 & 0 & 0 & 0 & 0 & 8 & ) & & 0 & & c & 0 & 0 & 0 & 0 & 0 & 0 & 0 & 0 & 0 & 0 & 0 & 0 & 0 & 0 \\
\hline 1215 & 0 & 0 & 0 & 0 & 0 & 0 & 0 & 0 & 1 & 0 & & 0 & & 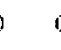 & 0 & 0 & 0 & 0 & 0 & 0 & 0 & 0 & 0 & 0 & 0 & 0 & 0 & 0 \\
\hline 1230 & 0 & 0 & 0 & a & 0 & 0 & 0 & 9 & 1 & $" 1$ & & 0 & & 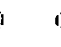 & 0 & 0 & 0 & 0 & 0 & $i$ & 0 & 0 & 0 & 0 & 0 & ) & 0 & 0 \\
\hline 1245 & 0 & 0 & 0 & 0 & 0 & 0 & g & 0 & 1 & $\because$ & & 0 & & 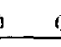 & 0 & 0 & $\underline{0}$ & 0 & a & 0 & 0 & () & 0 & 0 & 0 & 0 & 0 & 0 \\
\hline 100 & 0 & 0 & 0 & 0 & 0 & 0 & 0 & 0 & c & 0 & & 0 & & $c$ & 0 & 0 & 0 & 0 & 0 & 0 & 0 & 0 & jo & 0 & 0 & 0 & 0 & 0 \\
\hline 115 & 0 & 0 & 0 & 0 & 0 & 0 & 9 & 0 & 1 & 1 & & i) & & 1 & 0 & 0 & 0 & 0 & 0 & i) & 0 & 0 & 0 & 0 & 0 & 0 & 0 & 0 \\
\hline 130 & 0 & 0 & 0 & 0 & 0 & 0 & 0 & 0 & 6 & 1 & & 0 & & 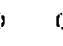 & 0 & 0 & $\dot{0}$ & 0 & 0 & 0 & 0 & 0 & 0 & 0 & 0 & 0 & 0 & 0 \\
\hline 145 & 0 & 0 & 0 & 0 & 0 & 0 & 0 & 0 & $i$ & 1 & & () & & 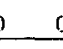 & 0 & 0 & 0 & 0 & 0 & 0 & 0 & 0 & 0 & 0 & 0 & 0 & 0 & 0 \\
\hline 200 & 0 & 0 & 0 & 0 & 0 & 0 & 0 & 0 & i & 1 & & 0 & & 0 & 0 & 0 & 0 & 0 & 0 & 0 & 0 & 0 & 0 & 0 & 0 & 0 & 0 & 0 \\
\hline 215 & 0 & 0 & 0 & 0 & 0 & 0 & 0 & 0 & 0 & 0 & & 0 & & 0 & 0 & 0 & 0 & 0 & 0 & 0 & 0 & 0 & 0 & 0 & 0 & 0 & 0 & 0 \\
\hline 230 & 0 & 0 & 0 & 0 & 0 & j & 0 & 0 & 0 & 0 & & 0 & ( & 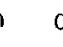 & 0 & 0 & 0 & 0 & 0 & 0 & 0 & 0 & 0 & 0 & 0 & 0 & 0 & 0 \\
\hline 245 & 0 & 0 & 0 & 0 & 0 & 0 & $\underline{0}$ & 0 & 4 & 0 & & 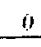 & & 8 & 0 & 0 & 3 & 3 & 0 & 0 & 0 & $\dot{0}$ & 0 & 0 & 0 & 0 & 0 & 0 \\
\hline 300 & 0 & 0 & 0 & $\dot{0}$ & 0 & 0 & 0 & 0 & ij & 0 & & 0 & se & 3 & 0 & 0 & 0 & 3 & 0 & 0 & 0 & 0 & 0 & 0 & 0 & 0 & 0 & 0 \\
\hline 315 & 0 & 0 & 0 & 0 & 0 & 0 & 0 & 0 & 0 & 0 & & 0 & ( & 3 & 0 & 0 & 0 & 3 & 0 & 0 & 0 & 0 & 0 & 0 & 0 & 0 & 0 & 0 \\
\hline 330 & 0 & 0 & 0 & 0 & 0 & 0 & 0 & 0 & 0 & 0 & ( & 0 & ( & & 0 & 0 & 0 & 3 & 0 & 0 & 0 & 0 & 0 & 0 & 0 & 0 & 0 & 0 \\
\hline 345 & 0 & 1) & 0 & 0 & 0 & 0 & 0 & 0 & 0 & 0 & s & 0 & c & 3 & 0 & ()) & 0 & 0 & 0 & 0 & 0 & 0 & 0 & 0 & 0 & 0 & 0 & 0 \\
\hline 400 & 0 & 0 & 0 & 0 & 0 & 0 & 0 & 0 & 0 & 0 & se & 0 & 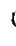 & 0 & 0 & 0 & 2 & $=$ & 0 & 0 & 0 & 0 & 0 & 0 & 0 & 0 & 0 & 0 \\
\hline 415 & 2 & 2 & 0 & 0 & 0 & 0 & 0 & 0 & 0 & 0 & t & 0 & 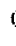 & 0 & 0 & 0 & 0 & $=$ & 0 & 0 & 0 & 0 & 0 & 0 & 0 & 0 & 0 & 0 \\
\hline .130 & 1 & 3 & 0 & $\dot{0}$ & $\dot{v}$ & 0 & " & " & 4 & $u$ & $t$ & 0) & 1 & 0 & 0 & 0 & 0 & $=$ & 13 & 0 & 0 & 0 & 0 & 0 & 0 & 0 & 0 & 0 \\
\hline 445 & 0) & 3 & 0 & 0 & 0 & 0 & $\underline{\underline{\theta}}$ & 9 & II & a & 1 & 0 & 1 & $n$ & 0 & 0 & 0 & $=$ & 0 & 0 & 0 & 0 & a & 0 & 0 & 0 & 0 & 0 \\
\hline 500 & 0 & 3 & 0 & 0 & 0 & 0 & 0 & 0 & a & 1.1 & & 0 & i & 0 & 0 & 0 & (1) & 0 & 0 & 0 & 0 & 0 & 0 & 0 & 0 & 0 & 0 & 0 \\
\hline 515 & $\mathrm{u}$ & 1 & 0 & 0 & 0 & 0 & 0 & 0 & "1 & a & i & 0 & 6 & 0 & 0 & 0 & 0 & 0 & 0 & 0 & 0 & 0 & 0 & 0 & 0 & 0 & 0 & 0 \\
\hline 530 & 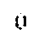 & 0 & 0 & 0 & 0 & a & "1 & $"$ & 1 & 1 & 1 & 0 & 1 & 0 & 0 & 0 & 0 & 0 & 0 & 0 & 0 & 0 & 0 & 0 & 0 & 0 & 0 & 0 \\
\hline 545 & 0 & 0 & 0 & 0 & 0 & 0 & 0 & 0 & 1 & 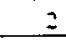 & 0 & 0 & - & 0 & 0 & 0 & 0 & $\stackrel{0}{0}$ & 0 & 0 & 2 & 2 & 0 & 0 & 0 & 0 & 0 & 0 \\
\hline 600 & $s$ & $\varsigma$ & 0 & 0 & 0 & 0 & 0 & 0 & 0 & $=$ & 0 & 0 & 0 & 0 & 0 & 0 & 6 & 6 & 0 & 0 & 3 & 5 & 0 & 0 & 7 & 7 & 0 & 0 \\
\hline 615 & 12 & 17 & 0 & 0 & 10 & 10 & 0 & 0 & 3 & $\underline{s}$ & 0 & 0 & t. & 0 & 0 & 0 & 7 & 13 & 0 & 0 & 6 & 11 & 0 & 0 & 10 & 17 & 0 & 0 \\
\hline 630 & 8 & 25 & 0 & 0 & 11 & \pm 1 & 0 & 11 & a & 4 & 0 & () & 9 & $u$ & 0 & 0 & 14 & 27 & 0 & 0 & 25 & 36 & 0 & $\dot{0}$ & 21 & 38 & ij & 0 \\
\hline 645 & .23 & 48 & 0 & 0 & 17 & 38 & 0 & 11 & 11 & 3 & 1 & (1) & 9 & 19 & 0 & 0 & 23 & 50 & (1) & 0 & 15 & 49 & 0 & 0 & 24 & $5=$ & 0 & 0 \\
\hline 700 & 37 & 80 & 0 & 1 & 27 & 65 & " & 11 & 0 & 3 & 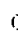 & 0 & $i$ & a & 0 & (3) & 37 & 81 & 0 & 0 & $\$ 4$ & 100 & i) & 0 & 41 & 96 & ij & 0 \\
\hline 715 & 46 & 114 & 0 & 0 & 37 & 9 & " & 0 & 4 & 0 & 0 & 1) & 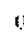 & 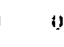 & 0 & 0 & 38 & 112 & 0 & 0 & 96 & 140 & 0 & 0 & 33 & 119 & $"$ & 0 \\
\hline 730 & 35 & 141 & 0 & 0 & 30 & 111 & 0 & 9 & o & 0 & 0 & 0 & 0 & 0 & 0 & 0 & 44 & 142 & 0 & 0 & ง0 & 155 & 0 & 0 & 41 & 139 & 0 & 0 \\
\hline 745 & 32 & 150 & 0 & 0 & 31 & 125 & 0 & 11 & 0 & 0 & 0 & 0 & $=$ & 2 & 0 & 0 & 38 & 157 & 0 & 0 & 35 & 175 & 0 & 0 & 19 & 134 & 0 & 0 \\
\hline 800 & 32 & & 0 & 0 & 27 & 125 & 0 & 0 & 4 & 0 & 0 & 0 & -1 & 1 & 0 & 0 & 44 & 164 & 0 & 0 & 38 & 159 & 0 & 0 & 39 & 132 & 0 & 0 \\
\hline 815 & 27 & 123 & 0 & 0 & 23 & 111 & 0 & 0 & $=$ & 2 & 0 & 0 & 0 & 1 & 0 & 0 & 25 & 151 & 0 & 0 & 34 & 147 & 0 & 0 & 28 & 127 & 0 & 0 \\
\hline 830 & 15 & 103 & 0 & 0 & 22 & 103 & 0 & 0 & 0 & $=$ & 0 & 0 & 0 & 1 & 0 & 0 & $=4$ & 131 & 0 & 0 & 13 & 120 & 0 & i) & 27 & 113 & 0 & 0 \\
\hline 845 & 18 & 89 & 0 & 0 & 17 & 89 & 0 & 0 & $u$ & 2 & 0 & 0 & 0 & -1 & 0 & 9 & 24 & 117 & 0 & 0 & 19 & 114 & 0 & 0 & 10 & 104 & 0 & 0 \\
\hline 900 & $=4$ & 81 & 0 & 0 & 22 & 84 & 11 & 0 & 1) & 2 & . & 0 & $=$ & 2 & 0 & 0 & 26 & 99 & 0 & 0 & 14 & 80 & 0 & 0 & 26 & 91 & 0 & 0 \\
\hline gis & 11 & 58 & 0 & 0 & 22 & 83 & ij & 0 & () & 0 & 0 & ij & 0 & $=$ & (1) & (1) & 9 & 83 & 0 & 0 & 14 & 60 & 0 & 0 & 13 & 76 & $\dot{0}$ & 0 \\
\hline 930 & 22 & 75 & 0 & 0 & 20 & 81 & 0 & 0 & 0 & 0 & 0 & 0 & 0 & 2 & 0 & $\dot{0}$ & 10 & 69 & 0 & 0 & 17 & 64 & 0 & 0 & 16 & 65 & 0 & 0 \\
\hline 945 & 17 & 74 & 0 & 0 & 12 & 76 & 0 & 0 & 0 & 0 & 0 & 0 & - & 2 & 0 & $\underline{0}$ & 8 & 53 & 0 & 0 & 16 & 61 & 0 & 0 & i7 & 72 & 0 & 0 \\
\hline 1000 & 16 & 66 & 0 & 0 & 13 & 67 & 0 & 0 & 1 & 1 & 0 & i) & 3 & 3 & 0 & 0 & 12 & 39 & 0 & 0 & 12 & 59 & 0 & 0 & 10 & 56 & 0 & 0 \\
\hline 1015 & 17 & 72 & 0 & 0 & 8 & $\$ 3$ & 0 & 0 & 0) & 1 & 0 & 0 & 0 & 3 & 0 & 0 & 10 & 40 & 0 & 0 & 14 & 59 & 0 & 0 & n & 54 & 0 & 0 \\
\hline 1030 & 10 & 60 & 0 & 0 & 13 & 46 & 0 & 0 & 2 & 3 & 0 & 0 & 0 & 3 & 0 & 0 & 10 & 40 & 0 & 0 & 3 & 45 & 0 & 0 & 7 & 45 & 0 & 0 \\
\hline 104s & 15 & 58 & 0 & 0 & 16 & 50 & 0 & 0 & 0 & 3 & 0 & 0 & 0 & 3 & 0 & 0 & 11 & 43 & 0 & 0 & 12 & 41 & 0 & 1) & 9 & 37 & 0 & 0 \\
\hline 1100 & 13 & 55 & 0 & 0 & 14 & $\$ 1$ & 0 & 0 & 0 & 2 & 0 & 0 & $=$ & 2 & 0 & ") & 10 & 41 & 0 & 0 & 10 & 39 & $\dot{0}$ & 0 & $s$ & 32 & 0 & 0 \\
\hline 1115 & 9 & 47 & 0 & 0 & 12 & 55 & 0 & 0 & 1 & 3 & D & 0 & o & 2 & 0 & 0 & 6 & 37 & 0 & 0 & 6 & 31 & 0 & D & 4 & 25 & 0 & 0 \\
\hline ?130 & 13 & 50 & 0 & 0 & 11 & 53 & 0 & 0 & 0 & 1 & 0 & 0 & 0 & 2 & 0 & 0 & 8 & 35 & 0 & 0 & 5 & 33 & 0 & D & 7 & 25 & 0 & 0 \\
\hline 1145 & 10 & 45 & 0 & 0 & 10 & 47 & () & 0 & 0 & 1 & 0 & 0 & 2 & 4 & 0 & 0 & 8 & 32 & 0 & 0 & 12 & 33 & 0 & 0 & 11 & 27 & 0 & 0 \\
\hline
\end{tabular}




\section{BAYMETRICS TRAFFIC RESOURCES}

Location 8: Southbound Onto Driveway East To Sandia Lab

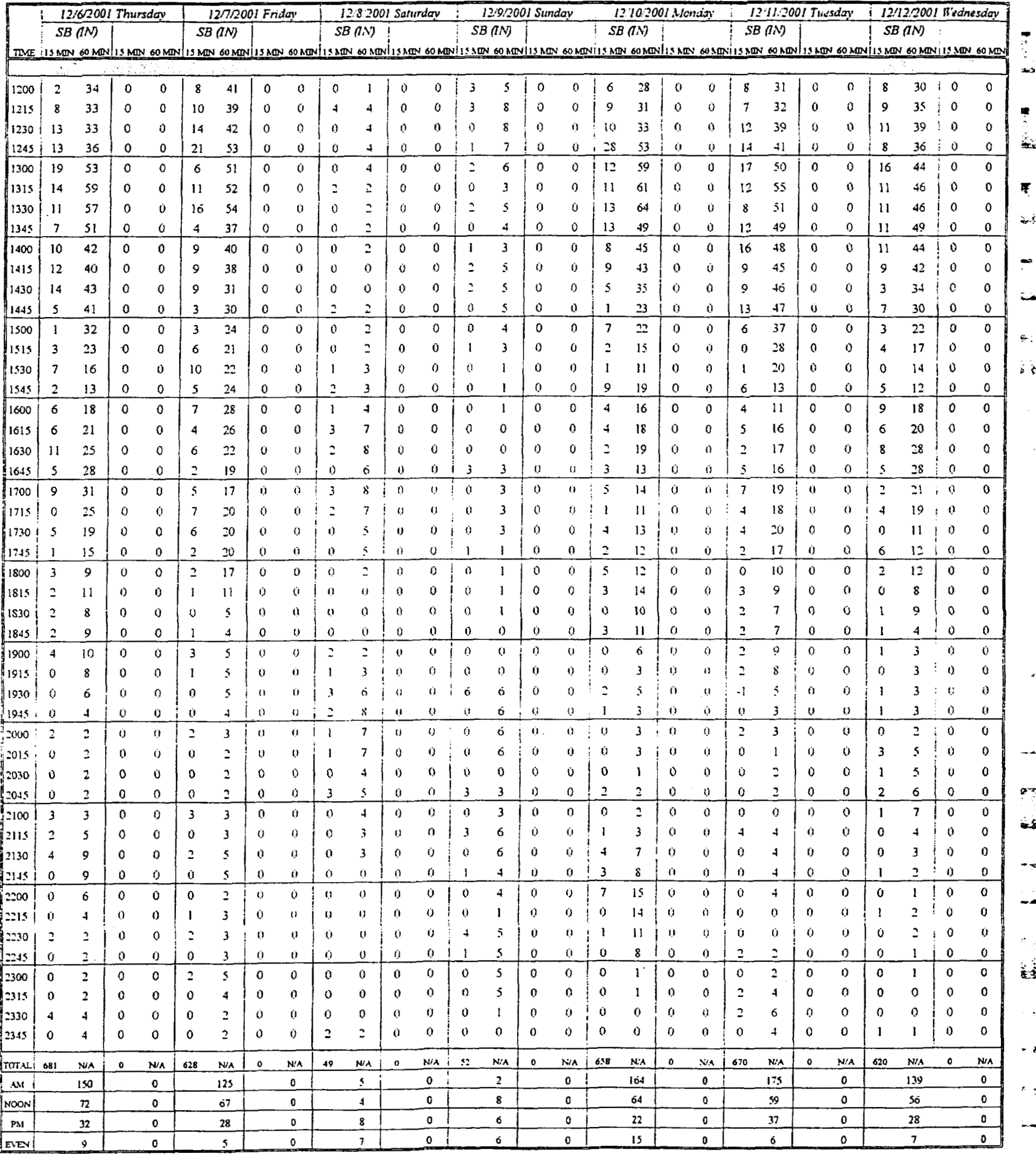




\section{BAYMETRICS TRAFFIC RESOURCES Location 8b: Northbound Onto East Street From Sandia Lab}

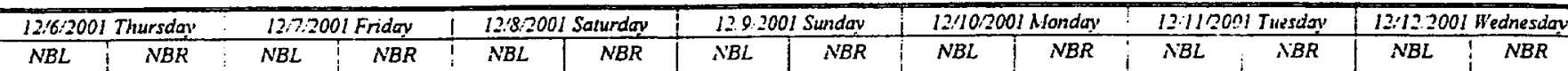

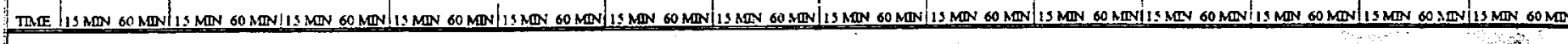

\begin{tabular}{|l|ll|lllll|lllll|lll|ll|lll|ll|l|l|l|l|l|l|l|l|lll|l}
\hline 1200 & 0 & 0 & 0 & 0 & 0 & 0 & 0 & 0 & 0 & 0 & 0 & 0 & 0 & 0 & 0 & 0 & 0 & 0 & 0 & 0 & 0 & 0 & 0 & 0 & 0 & 0 & 0 & 0
\end{tabular}

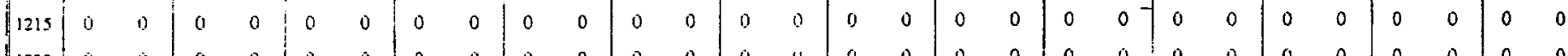
\begin{tabular}{lll|lllll|ll|ll|ll|lllll|ll|ll|ll|llllllllll}
1230 & 0 & 0 & 0 & 0 & 0 & 0 & 0 & 0 & 0 & 0 & 0 & 0 & 0 & 0 & 0 & 0 & 0 & 0 & 0 & 0 & 0 & 0 & 0 & 0 & 0 & 0 & 0 & 0
\end{tabular} \begin{tabular}{|l|ll|lllllll|ll|lllll|lllllllllllllllll}
1245 & 0 & 0 & 0 & 0 & 0 & 0 & 0 & 0 & 0 & 0 & 0 & 0 & 0 & 0 & 0 & 0 & 0 & 0 & 0 & 0 & 0 & 0 & 0 & 0 & 0 & 0 & 0 & 0 \\
\hline 100 & 0 & 0 & 0 & 0 & 0 & 0 & 0 & 0 & 1 & 1 & 0 & 0 & 0 & 0 & 0 & 0 & 0 & 0 & 0 & 0 & 0 & 0 & 0 & 0 & 0 & 0 & 0 & 0
\end{tabular}

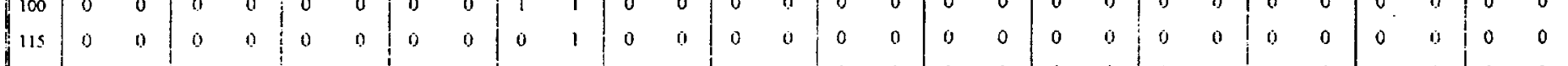
\begin{tabular}{|l|lllllll|ll|ll|ll|lllllll|lllll|llllllll}
130 & 0 & 0 & 0 & 0 & 0 & 0 & 0 & 0 & 0 & 1 & 0 & 0 & 0 & 0 & 0 & 0 & 0 & 0 & 0 & 0 & 0 & 0 & 0 & 0 & 0 & 0 & 0 & 0
\end{tabular} \begin{tabular}{|l|lllllll|ll|ll|lllllllllll|lllllllllll}
115 & 0 & 0 & 0 & 0 & 0 & 0 & 0 & 0 & 0 & 1 & 0 & 0 & 0 & 0 & 0 & 0 & 0 & 0 & 0 & 0 & 0 & 0 & 0 & 0 & 0 & 0 & 0 & 0 \\
\hline 200 & 0 & 0 & 0 & 0 & 0 & 0 & 0 & 0 & 0 & 0 & 0 & 0 & 0 & 0 & 0 & 0 & 0 & 0 & 0 & 0 & 0 & 0 & 0 & 0 & 0 & 0 & 0 & 0
\end{tabular} \begin{tabular}{|l|lllllllll|ll|lllll|ll|ll|lllllllllllllll}
215 & 0 & 0 & 0 & 0 & 0 & 0 & 0 & 0 & 0 & 0 & 0 & 0 & 0 & 0 & 0 & 0 & 0 & 0 & 0 & 0 & 0 & 0 & 0 & 0 & 0 & 0 & 0 & 0
\end{tabular} \begin{tabular}{|l|lllllll|ll|ll|ll|ll|llllllll|lllll}
230 & 0 & 0 & 0 & 0 & 0 & 0 & 0 & 0 & 0 & 0 & 0 & 0 & 0 & 0 & 0 & 0 & 0 & 0 & 0 & 0 & 0 & 0 & 0 & 0 & 0 & 0 & 0 & 0 \\
245 & 4 & 4 & 1 & 1 & 0 & 0 & 0 & 0 & 0 & 0 & 0 & 0 & 0 & 0 & 0 & 0 & 0 & 0 & 1 & 1 & 0 & 0 & 0 & 0 & 0 & 0 & 0 & 0 \\
\hline
\end{tabular} \begin{tabular}{|l|l|ll|ll|ll|ll|lllll|ll|ll|ll|lllll|ll|lll|l}
\hline 300 & 0 & 0 & 0 & 1 & 0 & 0 & 0 & 0 & 0 & 0 & 0 & 0 & 0 & 0 & 0 & 0 & 0 & 0 & 0 & 1 & 0 & 0 & 0 & 0 & 0 & 0 & 0 & 0
\end{tabular} \begin{tabular}{|lllllll|ll|ll|ll|ll|ll|llllllllllllll}
315 & 0 & 4 & 0 & 1 & 0 & 0 & 0 & 0 & 0 & 0 & 0 & 0 & 0 & 0 & 0 & 0 & 0 & 0 & 0 & 1 & 0 & 0 & 0 & 0 & 0 & 0 & 0 & 0
\end{tabular}

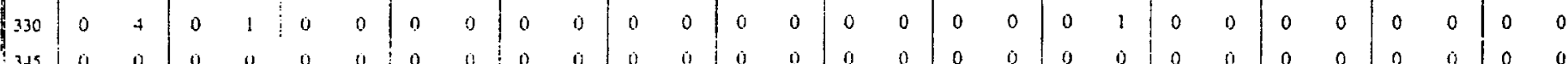

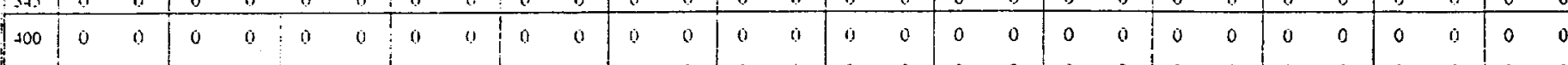
\begin{tabular}{llllllllll|ll|lllll|ll|lllll|llllllllll}
+15 & 0 & 0 & 0 & 0 & 0 & 0 & 0 & 0 & 0 & 0 & 0 & 0 & 0 & 0 & 0 & 0 & 0 & 0 & 0 & 0 & 0 & 0 & 0 & 0 & 0 & 0 & 0 & 0
\end{tabular} \begin{tabular}{l|ll|lllllll:ll:ll|ll|ll|llllllllllllllllll}
$1+30$ & 1 & 1 & 0 & 0 & 0 & 0 & 0 & 0 & 0 & 0 & 0 & 0 & 0 & 0 & 0 & 0 & 0 & 0 & 0 & 0 & 0 & 0 & 0 & 0 & 0 & 0 & 0 & 0
\end{tabular} \begin{tabular}{|l|ll|llllllllll|llllllll|ll|ll|llllllll}
\hline 15 & 0 & 1 & 0 & 0 & 0 & 0 & 0 & 0 & 0 & 0 & 0 & 0 & 0 & 0 & 0 & 0 & 0 & 0 & 0 & 0 & 0 & 0 & 0 & 0 & 0 & 0 & 0 & 0 \\
\hline 500 & 0 & 1 & 0 & 0 & 0 & 0 & 0 & 0 & 0 & 0 & 0 & 0 & 0 & 0 & 0 & 0 & 0 & 0 & 0 & 0 & 0 & 0 & 0 & 0 & 0 & 0 & 0 & 0 &
\end{tabular} \begin{tabular}{lll|llllllllll|ll|ll|ll|ll|lllll|llllllll}
515 & 0 & 1 & 0 & 0 & 0 & 0 & 0 & 0 & 0 & 0 & 0 & 0 & 0 & 0 & 0 & 0 & 0 & 0 & 0 & 0 & 0 & 0 & 0 & 0 & 0 & 0 & 0 & 0
\end{tabular}

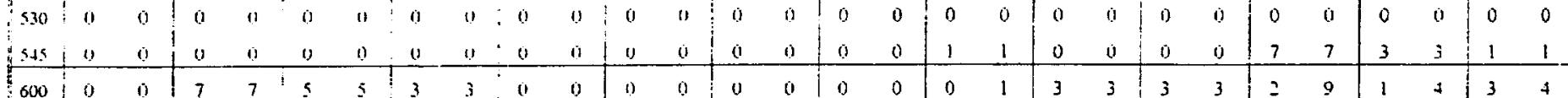

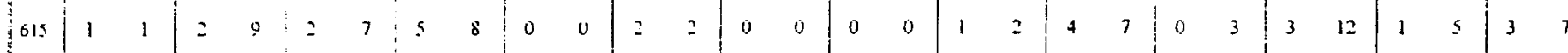
\begin{tabular}{lll|lllllllllll|ll|ll|ll|ll|ll|ll|l|l|l|l}
630 & 1 & 2 & 0 & 9 & 0 & 7 & 3 & 11 & 0 & 0 & 0 & 2 & 0 & 0 & 0 & 0 & 4 & 6 & 2 & 9 & 1 & 4 & 4 & 16 & 1 & 0 & 2 & 9 \\
\hline
\end{tabular}

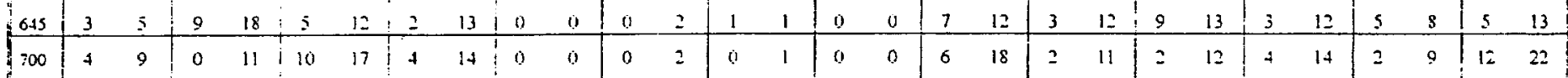

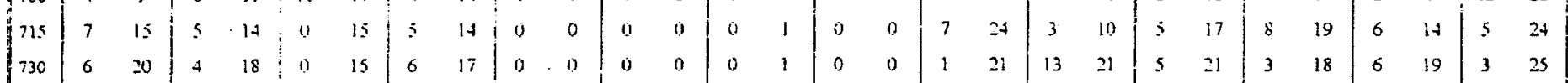

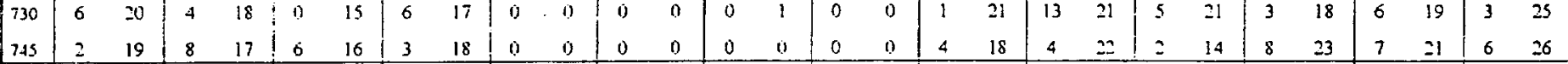

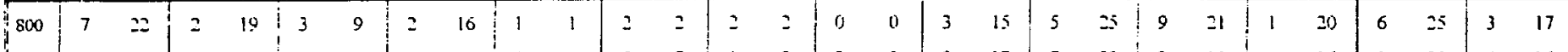

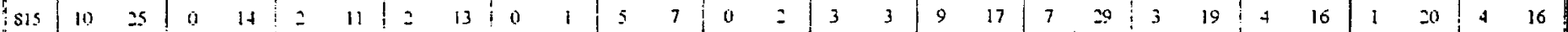

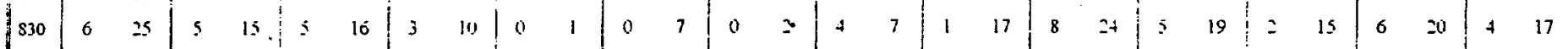
\begin{tabular}{|l|ll|ll|ll|ll|ll|ll|lllll|ll|ll|ll|lllll}
845 & 3 & 26 & 7 & 14 & 5 & 15 & 7 & 14 & 0 & 1 & 0 & 7 & 0 & 2 & 0 & 7 & 2 & 15 & 7 & 27 & 3 & 20 & 7 & 14 & 4 & 17 & 4 & 15 \\
\hline 900 & 3 & 32 & 3 & 15 & 1 & 13 & 6 & 18 & 0 & 0 & 0 & 5 & 0 & 0 & 0 & 7 & 6 & 18 & 5 & 27 & 2 & 13 & 4 & 17 & 4 & 15 & 5 & 17 \\
\hline
\end{tabular}

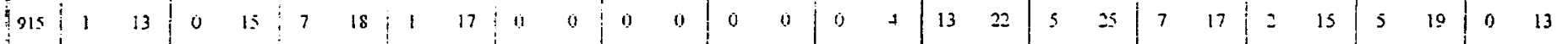

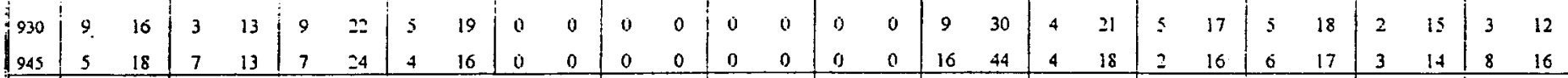

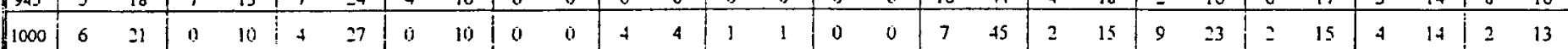
\begin{tabular}{|l|ll|ll|ll|ll|ll|ll|ll|ll|ll|llllllllllllll}
1015 & 3 & 23 & 9 & 19 & 5 & 25 & 7 & 16 & 0 & 0 & 0 & 4 & 0 & 1 & 0 & 0 & 4 & 36 & 7 & 17 & 1 & 17 & 5 & 18 & 3 & 12 & 6 & 19
\end{tabular}

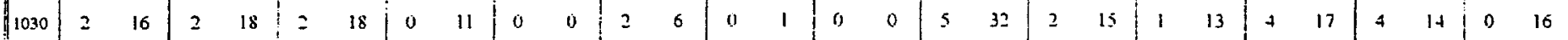
\begin{tabular}{|l|ll|ll|ll|llll|llll|llllllllllllll}
1045 & 3 & 14 & 7 & 18 & 2 & 13 & 5 & 12 & 0 & 0 & 2 & 8 & 1 & 2 & 1 & 1 & 3 & 19 & 8 & 19 & 4 & 15 & 3 & 14 & 2 & 13 & 5 & 13 \\
\hline
\end{tabular}

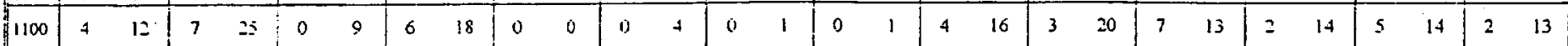

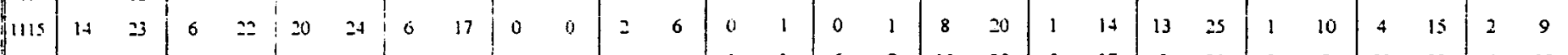

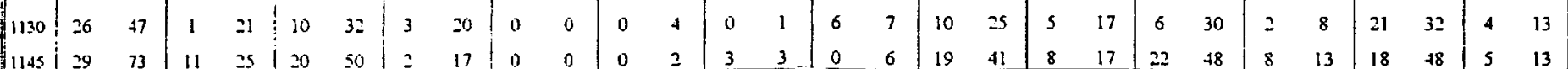




\section{BAYMETRICS TRAFFIC RESOURCES Location 8b: Northbound Onto East Street From Sandia Lab}

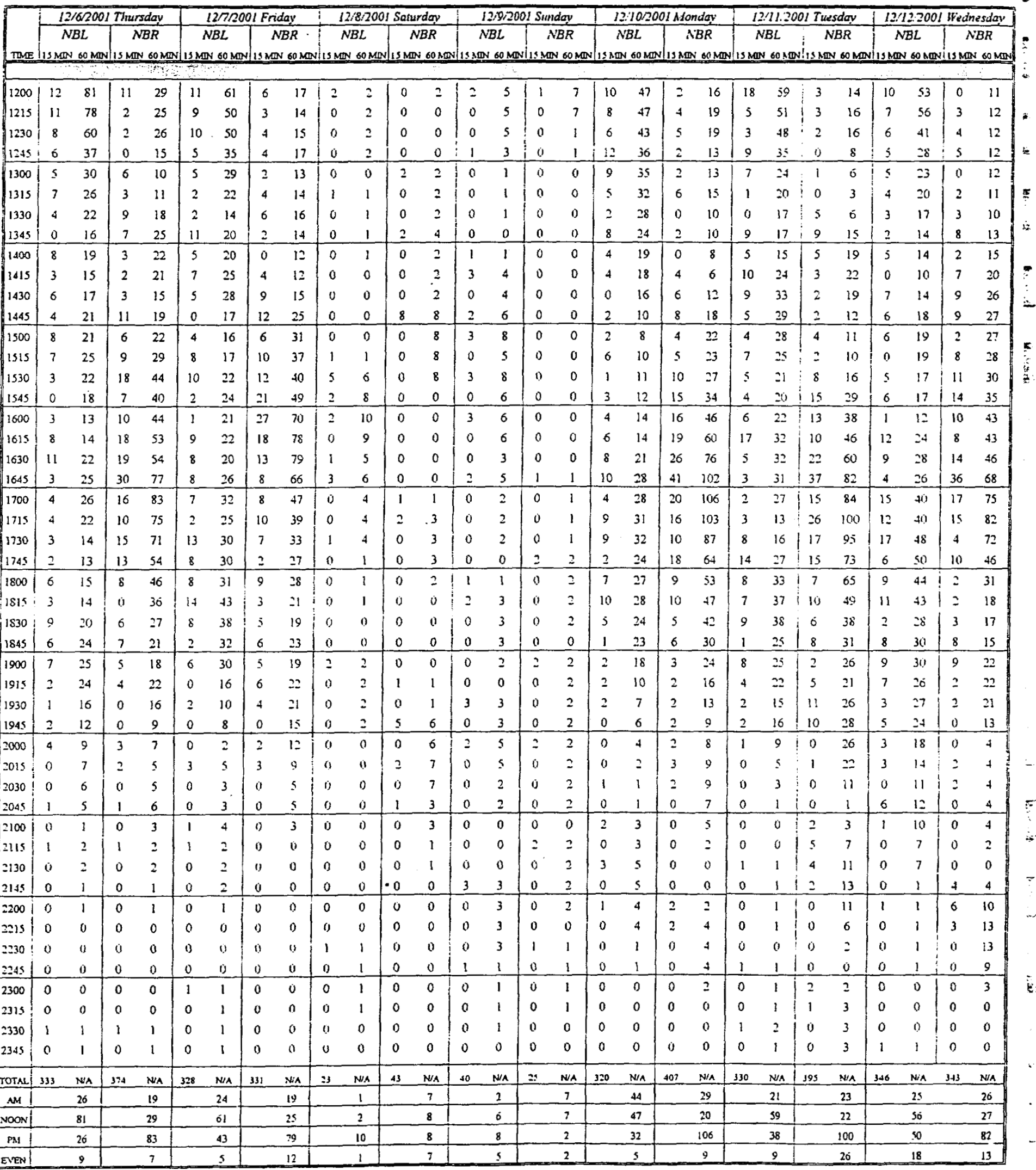




\section{BAYMETRICS TRAFFIC RESOURCES Location 9: SBTR At Greenville Road Onto WB East Avenue}

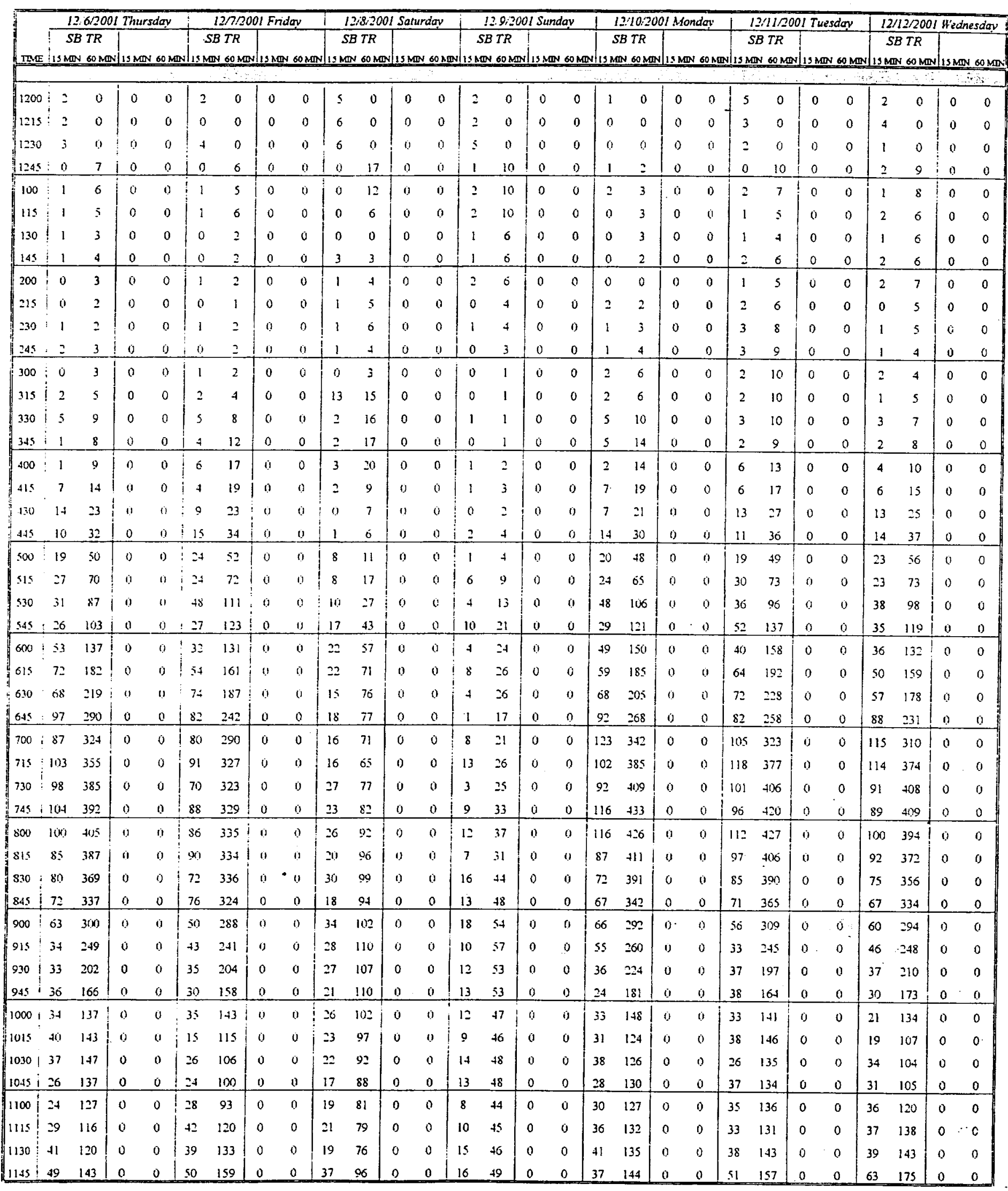




\section{BAYMETRICS TRAFFIC RESOURCES Location 9: SBTR At Greenville Road Onto WB East Avenue}

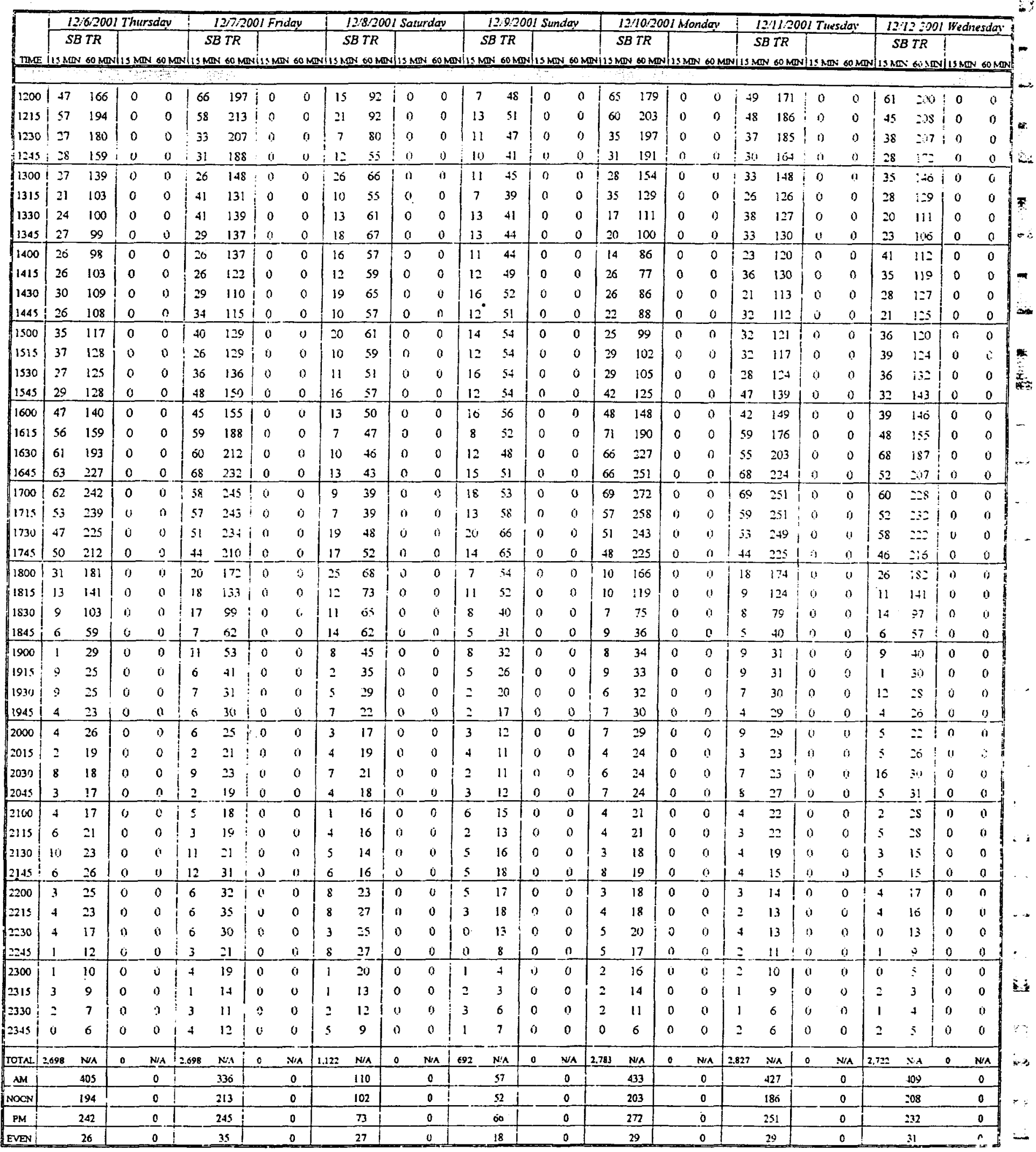




\section{BAYMETRICS TRAFFIC RESOURCES Location 10: NB Greenville Rond South of East Avenue}

\begin{tabular}{|c|c|c|c|c|c|}
\hline 12:62001 Thursciar. & 1272001 Fridar & 128,2001 Saturday & 12:9/2001 Suiday & 12102001 Monday & 12:112001 Tuesdoy \\
\hline & & $N B L T$ & $\therefore B \angle T$ & $N B L T$ & $L T$ \\
\hline
\end{tabular}

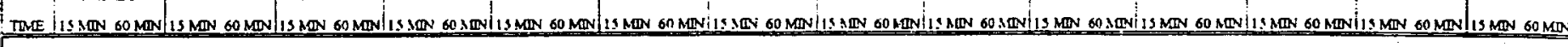

\begin{tabular}{|c|c|c|c|c|c|c|c|c|c|c|c|c|c|c|c|c|c|c|c|c|c|c|c|c|c|c|c|c|}
\hline 1200 & 0 & 0 & 0 & 0 & 0 & 0 & 0 & 0 & 1 & 0 & 0 & 0 & 1 & 0 & 0 & 0 & 1 & 0 & 0 & 0 & 2 & 0 & 0 & 0 & 1 & 0 & 0 & 0 \\
\hline 1215 & 0 & 0 & 0 & 0 & 1 & 0 & 0 & 0 & 2 & 0 & 0 & 0 & i & 0 & 0 & i) & $=$ & 0 & 0 & 0 & 0 & 0 & 0 & 0 & 1 & 0 & 0 & 0 \\
\hline $1=30$ & 2 & 0 & 0 & 0 & 1 & 0 & 0 & 0 & 1 & 0 & 1) & 0 & 3 & 0 & 0 & ) & 0 & 0 & 0 & 0 & 0 & 0 & 0 & 0 & 1 & 0 & 0 & 0 \\
\hline $1=15$ & 0 & 2 & 0 & 0 & $\dot{0}$ & 2 & 0 & 0 & 1 & 5 & i) & 0 & 1 & 8 & 0 & 0 & 1 & 4 & 0 & 0 & $\dot{0}$ & $=$ & 0 & 0 & 1 & 4 & 0 & 0 \\
\hline 100 & 1 & 3 & 0 & 0 & 1 & 3 & 0 & 0 & 0 & 4 & 0 & 0 & 1 & 8 & 0 & 0 & 1 & 4 & 0 & 0 & 0 & 0 & 0 & 0 & 2 & s & 0 & 0 \\
\hline 115 & 2 & $s$ & 0 & 0 & 0 & 2 & 0 & a & 1 & 3 & 0 & 0 & $:$ & 7 & 0 & 0 & 0 & 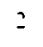 & 0 & 0 & 0 & 0 & ij & 0 & 0 & 4 & 0 & 0 \\
\hline 130 & 0 & 3 & 0 & 0 & 0 & 1 & 1) & 0 & 0 & 2 & 0 & 0 & 0 & 4 & 0 & 0 & 1 & 3 & 0 & 1) & 0 & 0 & 0 & 0 & 0 & 3 & 0 & 0 \\
\hline 145 & 0 & 3 & 0 & 0 & 0 & 1 & 0 & 0 & 1 & 2 & 0 & 0 & $!$ & 4 & 0 & 0 & 1 & 3 & 0 & 0 & 0 & 0 & 0 & i) & 1 & 3 & 0 & 0 \\
\hline 200 & 1 & 3 & 0 & 0 & 0 & 0 & 0 & 0 & 0 & 2 & 0 & 0 & 1 & 4 & a & 0 & 0 & $z$ & 0 & 0 & 0 & 0 & 0 & 0 & 2 & 3 & 0 & 0 \\
\hline 215 & 0 & 1 & 0 & 0 & 0 & 0 & 0 & 0 & 2 & 3 & 0 & 0 & 1 & 3 & 0 & 0 & 0 & 2 & 0 & 0 & 1 & 1 & 0 & 0 & 0 & 3 & 0 & 0 \\
\hline 230 & 0 & 1 & 0 & 0 & 0 & 0 & 9 & 0 & $\dot{0}$ & 3 & !) & 0 & 0 & 3 & $D$ & 0 & 0 & 1 & 0 & 0 & 1 & $=$ & 0 & 0 & 1 & 4 & 0 & 0 \\
\hline 245 & 0 & 1 & 0 & $\dot{0}$ & 0 & 0 & $\dot{0}$ & $u$ & 2 & \pm & 0 & 0 & 1 & 3 & $\dot{0}$ & 0 & 0 & 0 & 0 & 0 & 0 & 2 & 0 & 0 & 0 & 3 & 0 & 0 \\
\hline 300 & 0 & 0 & 0 & 0 & 0 & 0 & 0 & 0 & 0 & 4 & 0 & 0 & $=$ & 4 & 0 & 0 & 0 & 0 & 0 & 0 & 0 & 2 & 0 & 0 & 0 & 1 & 0 & 0 \\
\hline 315 & 1 & $i$ & 0 & 0 & 2 & 2 & 0 & i & 1 & 3 & 0 & 0 & 0 & 3 & 0 & 0 & 0 & 0 & 0 & 0 & 1 & 2 & 0 & 0 & 2 & 3 & 0 & 0 \\
\hline 330 & 0 & 1 & 0 & 0 & 0 & 2 & 0 & 0 & 0 & 3 & 0 & 0 & 1 & 4 & 0 & 0 & 1 & 1 & 0 & 0 & 0 & 1 & 0 & 0 & 0 & 2 & 0 & 0 \\
\hline 345 & 0 & 1 & 0 & 0 & 0 & 2 & 0 & i & 0 & 1 & 0 & () & 0 & 3 & 0 & 0 & 0 & 1 & 0 & 0 & 0 & 1 & 0 & 0 & 2 & 4 & 0 & 0 \\
\hline 100 & 0 & 1 & 0 & 0 & 0 & 2 & 0 & 0 & 2 & 3 & 0 & 0 & a & 1 & $a$ & 0 & 1 & $=$ & 0 & 0 & 1 & 2 & 9 & 0 & 0 & 4 & 0 & 0 \\
\hline tis & 1 & 1 & 0 & 0 & 1 & 1 & 0 & $" 1$ & 0 & $=$ & 0 & 0 & 0 & 1 & 0 & 0 & 0 & $=$ & 0 & 0 & 6 & 7 & 0 & 0 & 0 & 2 & 0 & 0 \\
\hline 430 & 1 & $=$ & 0 & 0 & 1 & $=$ & "1 & $" 1$ & 1 & 3 & a & 9 & 0 & 0 & 0 & 0 & i) & 1 & is & 0 & 1 & 8 & 9 & 0 & 4 & 6 & 0 & 0 \\
\hline 445 & 3 & 5 & 0 & 0 & 0 & 2 & 11 & i] & 0 & 3 & 0 & 0 & 0 & 0 & 0 & 0 & $z$ & 3 & () & 0 & 2 & 10 & 0 & 0 & 1 & 5 & 0 & 0 \\
\hline 500 & 4 & 9 & 0 & 0 & 0 & 2 & 0 & $a$ & 1 & 2 & 0 & 9 & : & 2 & 0 & 0 & 0 & 2 & 1) & 0 & 4 & 13 & 0 & 0 & 6 & 11 & 0 & 0 \\
\hline 515 & 3 & 11 & 0 & 0 & 3 & 4 & 0 & 9 & 4 & 6 & 0 & 0 & 0 & 2 & 0 & is & 2 & 4 & 0 & 0 & 3 & 10 & 0 & 0 & 3 & 17 & 0 & 0 \\
\hline 530 & $s$ & is & 0 & 0 & $s$ & 8 & 0 & 9 & 1 & 6 & 0 & 0 & 1 & 3 & 0 & 0 & 6 & 10 & 0 & 0 & 6 & 15 & 0 & 0 & 7 & 17 & 0 & 0 \\
\hline 545 & 1 & 13 & 0 & 0 & 4 & 12 & 0 & 0 & 1 & 7 & 0 & 0 & 0 & 3 & 0 & 0 & 5 & 13 & 0 & (i) & 6 & 19 & 0 & 0 & 6 & $\because 2$ & 0 & 0 \\
\hline 600 & 8 & 17 & 0 & 0 & 9 & 21 & 0 & 0 & 2 & 8 & 0 & 9 & $i$ & 2 & " & 0 & 8 & 21 & 0 & (1) & 0 & 21 & $"$ & 0 & 5 & $=1$ & 0 & 0 \\
\hline 615 & 10 & 24 & 0 & 0 & 0 & 24 & " & 11 & 1 & 5 & 0 & a) & 1 & 3 & 0 & 0 & 13 & $\vdots 2$ & $\theta$ & i) & 16 & 34 & 11 & 0 & 18 & 36 & 0 & 0 \\
\hline 630 & 20 & $4 B$ & 0 & 0 & 16 & 35 & 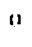 & " & 2 & 0 & 0 & $"$ & $=$ & 4 & 0 & 0 & 20 & 45 & 0 & 0 & 19 & 47 & 9 & 0 & 21 & 50 & " & i \\
\hline ous & 3 & 70 & 0 & 0 & 35 & 66 & 0 & 11 & 1 & 6 & 0 & i & $\therefore$ & 6 & 0 & 0 & 260 & 67 & $\dot{0}$ & $\dot{\theta}$ & 35 & 70 & 0 & 0 & 30 & 64 & 0 & 0 \\
\hline 700 & 37 & 99 & 0 & 0 & 33 & 90 & 0 & $"$ & 0 & 4 & i) & 0 & $a$ & 5 & 0 & 9 & 34 & 93 & 0 & (1) & 37 & 107 & 0 & 0 & 28 & 87 & 0 & 0 \\
\hline 715 & 48 & 137 & 0 & 0 & 50 & 134 & 0 & (1) & 3 & 6 & 0 & 0 & 0 & 4 & 0 & 0 & 54 & 134 & D) & 0 & 45 & 136 & 0 & 0 & 57 & 126 & 0 & 0 \\
\hline 730 & 53 & 161 & 0 & 0 & 32 & 150 & 0 & 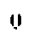 & 6 & 10 & 0 & $\dot{0}$ & 0 & 2 & 0 & 0 & 48 & 102 & 0 & 0 & 55 & 172 & 0 & 0 & II & 157 & 0 & 0 \\
\hline 745 & 56 & 194 & 0 & 0 & 41 & 156 & 0 & 0 & 4 & 13 & 0 & 0 & 1 & 1 & 0 & 0 & 50 & 186 & 0 & 0 & 49 & 186 & 0 & 0 & 57 & 194 & 0 & 0 \\
\hline 800 & 51 & 208 & 0 & 0 & 52 & 175 & 0 & $"$ & 6 & 19 & 0 & 0 & 0 & 1 & 0 & 0 & 58 & 210 & 0 & 0 & 58 & 207 & 0 & 0 & 49 & 215 & 0 & 0 \\
\hline 815 & 45 & 205 & 0 & 0 & 51 & 176 & 0 & $" 1$ & 8 & 24 & 0 & 0 & 7 & 8 & 0 & 0 & 65 & $\therefore 1$ & 0 & 0 & 65 & $\because 27$ & 0 & 0 & 57 & 215 & 0 & 0 \\
\hline 830 & 41 & 193 & $\dot{0}$ & 0 & 4 & 185 & 0 & $"$ & 4 & 22 & u & 0 & 4 & 12 & 0 & 0 & 46 & $=19$ & 0 & 0 & $40^{\circ}$ & 218 & 4 & 0 & 45 & 208 & 0 & 0 \\
\hline 845 & 38 & 175 & 0 & 0 & 33 & 177 & 0 & 0 & 9 & 27 & $\underline{0}$ & 0 & 5 & 16 & 0 & 0 & 33 & 202 & 0 & 0 & 35 & 204 & 0 & 0 & 35 & $180^{\circ}$ & 0 & 0 \\
\hline$\infty 00$ & 23 & 147 & 0 & 0 & 26 & 151 & 0 & : & 8 & 29 & 0 & 0 & 4 & 20 & 0 & 0 & 27 & 171 & 0 & 0 & 38 & 184 & 0 & 0 & 30 & 167 & 0 & 0 \\
\hline 915 & 21 & 123 & 0 & 0 & 27 & 127 & 0 & 11 & 10 & 31 & 0 & 0 & 6 & 19 & 0 & 0 & 17 & 123 & 0 & 1) & 27 & 146 & 0 & 9 & $\because$ & 132 & 0 & 0 \\
\hline 930 & 17 & $99^{\circ}$ & 0 & 0 & 32 & 118 & 0 & 0 & 12 & 39 & 0 & 0 & 0 & 15 & 0 & 0 & 14 & 91 & 0 & 0 & 22 & 122 & 0 & 0 & 19 & 106 & 0 & 0 \\
\hline 945 & 17 & 78 & 0 & 0 & 18 & 103 & 0 & 0 & 5 & 35 & 0 & 0 & 6 & 16 & 0 & 0 & 23 & 81 & 0 & 0 & 15 & 102 & 0 & 0 & 21 & 92 & 0 & 0 \\
\hline 1000 & 13 & 68 & 0 & 0 & 16 & 93 & 0 & (1) & 11 & 38 & 0 & 0 & 10 & $\because$ & ) & 0 & 15 & 69 & 0 & 0 & 17 & 81 & 0 & 0 & 17 & 79 & 0 & 0 \\
\hline 1015 & 8 & 55 & 0 & 0 & 21 & 87 & " & $v$ & 9 & 37 & i) & 0 & 10 & 26 & 0 & 0 & 15 & 67 & 0 & 0 & 23 & 77 & 0 & 0 & 19 & 76 & 0 & 0 \\
\hline 1030 & 14 & 52 & 0 & 0 & 17 & 72 & 0 & 0 & 16 & 41 & 19 & 0 & 12 & 38 & 0 & 0 & 9 & 62 & 0 & 0 & 18 & 73 & 0 & 0 & 15 & $7=$ & 0 & 0 \\
\hline 1045 & 24 & 59 & 0 & 0 & 7 & 61 & 0 & 0 & 8 & 44 & 0 & 0 & 11 & 43 & 0 & 0 & 12 & 51 & 0 & $\dot{0}$ & 19 & 77 & 0 & 0 & 7 & 58 & - & 0 \\
\hline 1100 & 12 & $\leq 8$ & 0 & 0 & 21 & 66 & 0 & 9 & 15 & 48 & 0 & 0 & 10 & 43 & 0 & 0 & 16 & 52 & 0 & 0 & 22 & 82 & 0 & 0 & 20 & 61 & 0 & $\dot{0}$ \\
\hline 1115 & 16 & 66 & 0 & 0 & 11 & 56 & 0 & 0 & 0 & 45 & 0 & 0 & $\delta$ & 41 & 0 & 0 & 18 & 55 & 0 & 0 & 10 & 69 & 0 & 0 & 9 & 51 & 0 & 0 \\
\hline 1130 & 13 & 65 & 0 & 0 & 13 & 52 & 0 & i & 15 & +4 & 0 & 0 & 19 & 48 & 0 & 0 & 13 & 59 & 0 & 0 & 13 & 64 & 0 & 0 & 16 & $\vdots 2$ & 0 & 0 \\
\hline 1145 & 15 & 56 & 0 & 0 & 13 & 58 & 0 & 0 & 16 & $\$ 2$ & 0 & 0 & 6 & 43 & 0 & 0 & 12 & $\$ 9$ & 0 & 0 & 14 & 59 & 0 & 0 & 11 & 56 & 0 & 0 \\
\hline
\end{tabular}




\section{BAYMETRICS TRAFFIC RESOURCES Location 10: NB Greenville Road South of East Avenue}

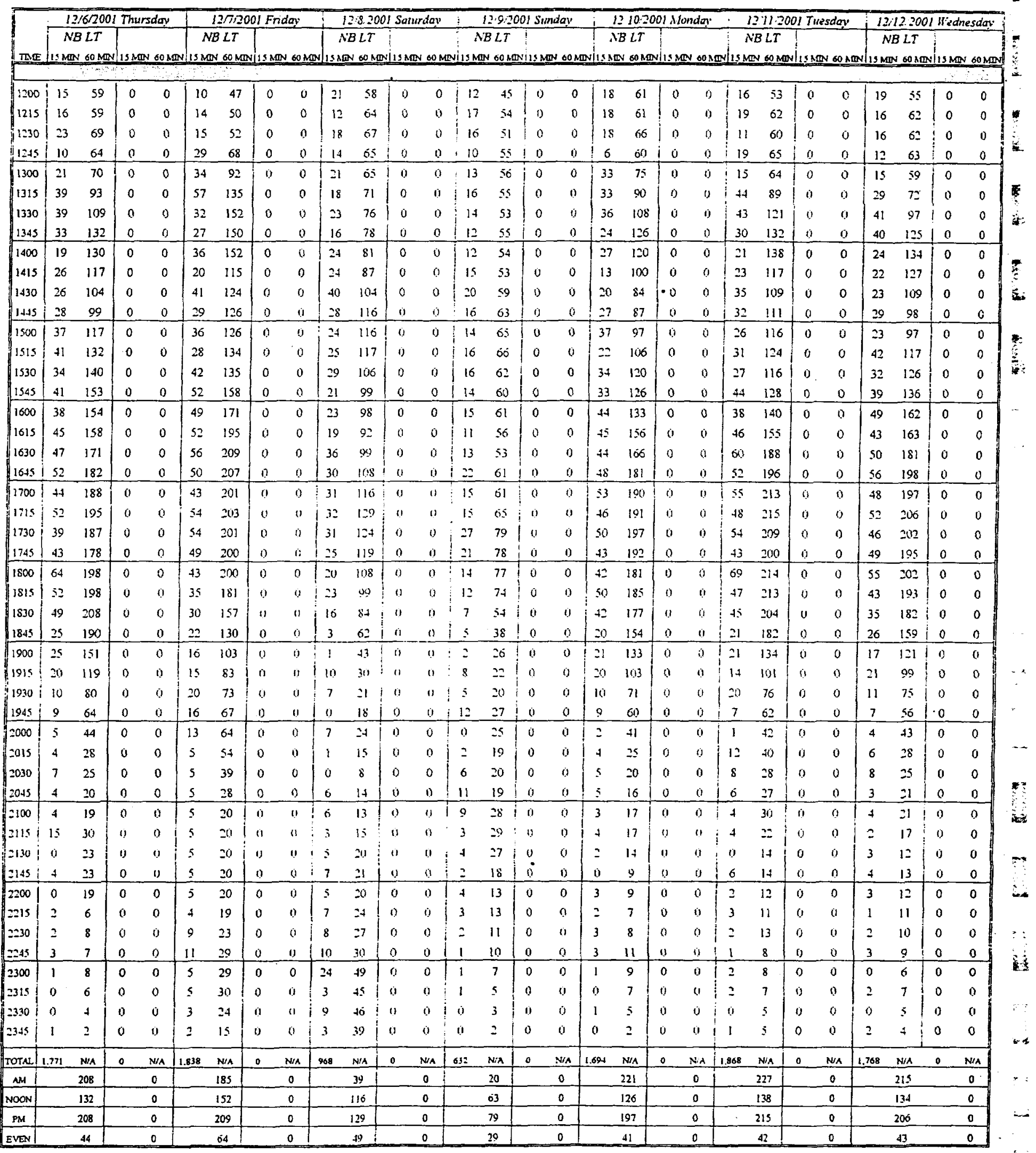




\section{BAYMETRICS TRAFFIC RESOURCES Location 11: East Avenue West of Greenville Road}

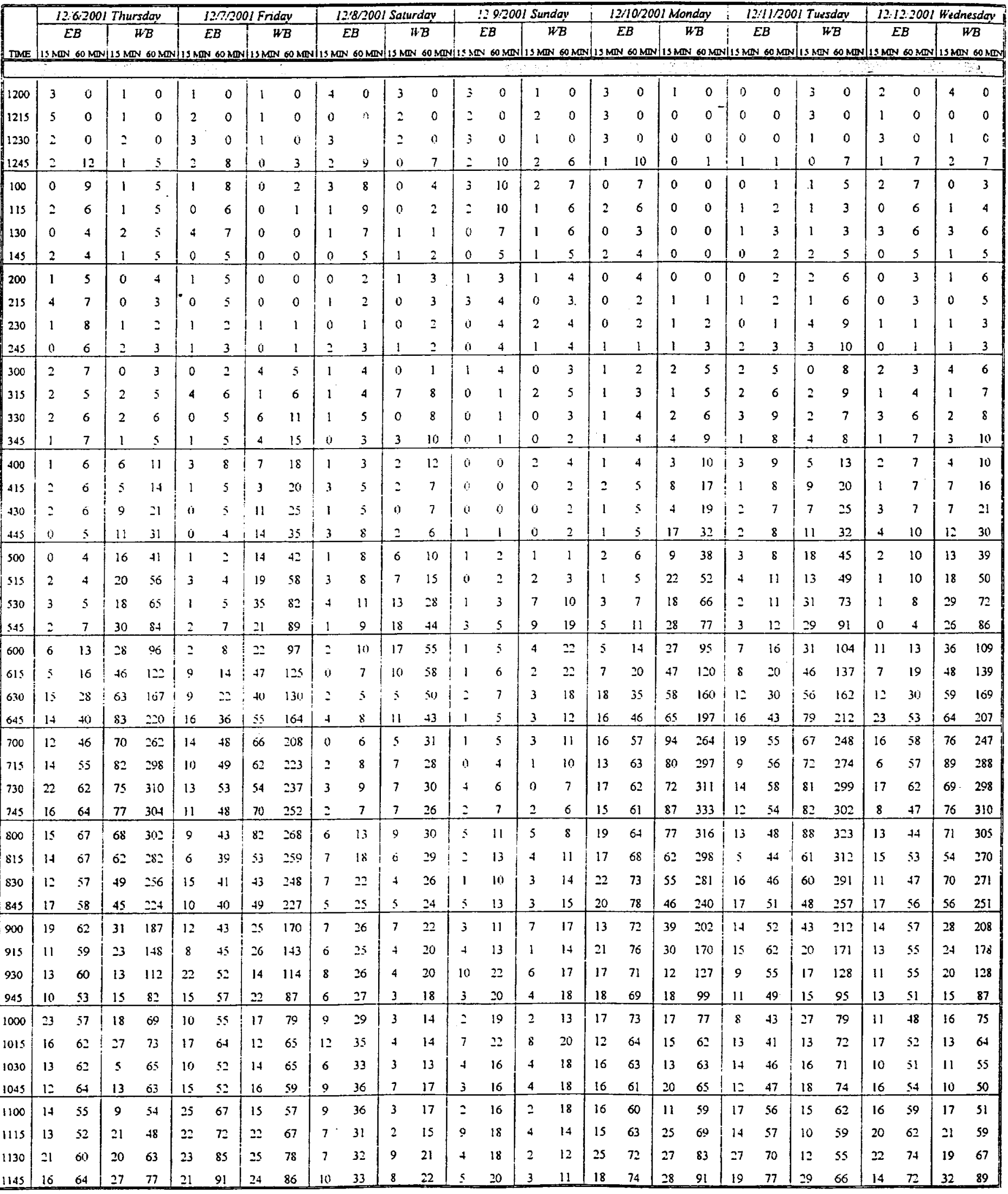




\section{BAYMETRICS TRAFFIC RESOURCES Location 11: East Avenue West of Greenville Road}

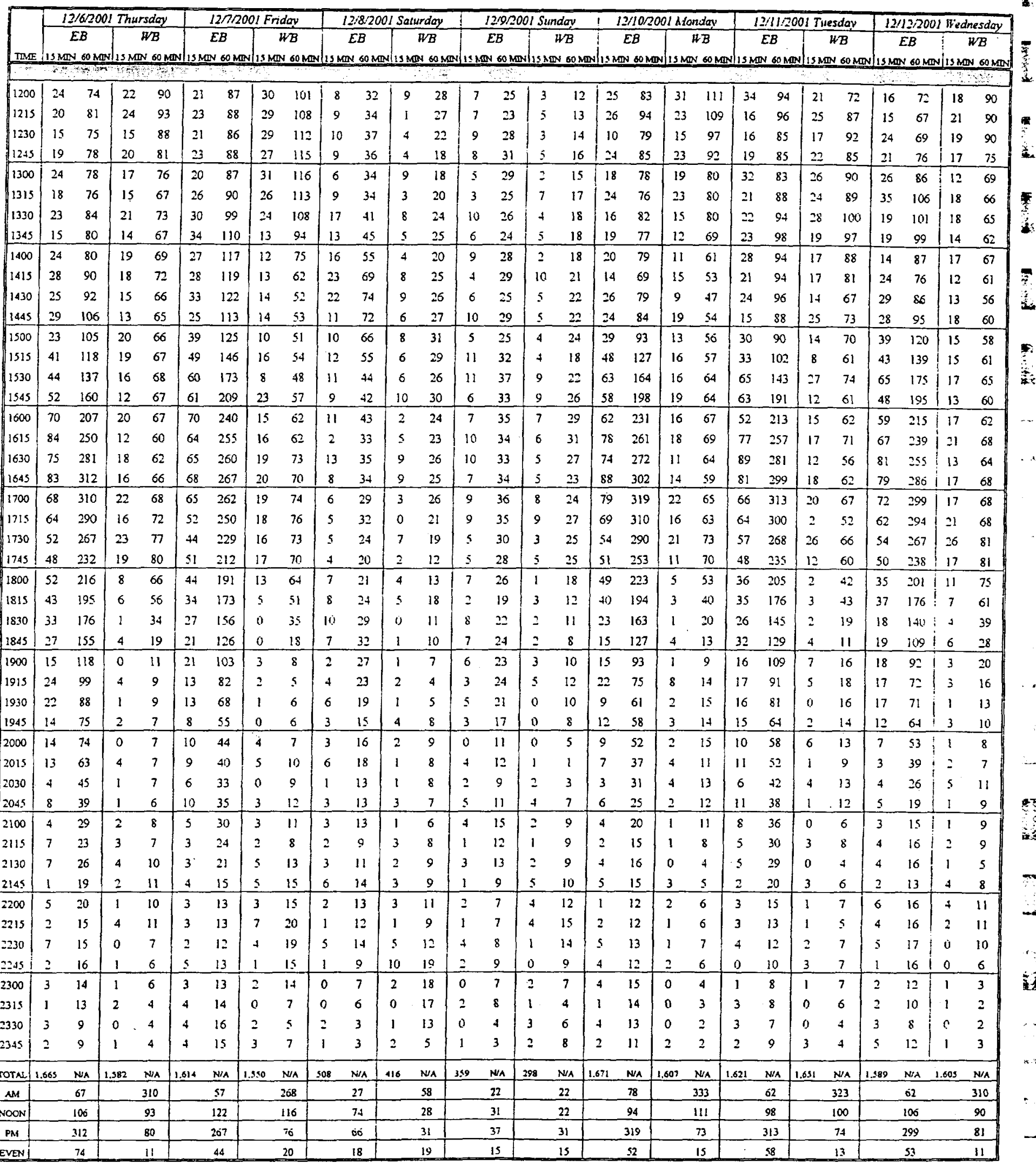




\section{BAYMETRICS TRAFFIC RESOURCES Location 12: NB LT\& SB RT Onto Eastgate From Greenville}

\begin{tabular}{|c|c|c|c|c|c|c|c|c|c|c|c|c|c|c|c|c|c|c|c|c|c|c|c|c|c|c|c|c|}
\hline & \multicolumn{4}{|c|}{ 12/6/2001 Thursday } & \multicolumn{4}{|c|}{$12 / 7 / 2001$ Fridoy } & \multicolumn{4}{|c|}{ 12:\&2001 Saturday } & \multicolumn{3}{|c|}{ 12900001 Sunday } & & \multicolumn{4}{|c|}{$12 / 102001$ Monday } & \multicolumn{4}{|c|}{$12 / 1 / 2001$ Tuesday } & \multicolumn{4}{|c|}{ 12/12/3001 Wednesdon: } \\
\hline & \multicolumn{2}{|c|}{$N B L$} & & $B R$. & & VBL & & $S B R$ & & $N B L$ & & & & $Y B L$ & & & & $N B L$ & & $B R$ & & & & & & & & \\
\hline & & & & & & & & & & & & & & & & & & & & & & & & & & & & \\
\hline 1200 & 1 & 0 & 0 & 0 & 0 & 0 & 0 & 0 & 1 & 0 & 0 & 0 & 0 & 0 & 0 & 0 & & 0 & 0 & 0 & 0 & 0 & 0 & 0 & 0 & 0 & 0 & 0 \\
\hline 1215 & 1 & 0 & 0 & 0 & 0 & 0 & 0 & 0 & 0 & 0 & 0 & 0 & 0 & 0 & 0 & 0 & & 0 & 0 & 0 & 0 & 0 & 0 & 0 & 1 & 0 & 0 & 0 \\
\hline 1230 & 0 & 0 & 0 & 0 & 0 & 0 & 0 & 0 & 0 & 0 & 0 & i) & 0 & 0 & 0 & 0 & 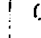 & 0 & 0 & 0 & 0 & 0 & 0 & 0 & 0 & 0 & 0 & 0 \\
\hline 1245 & 0 & 2 & 0 & 0 & 0 & 0 & 0 & 0 & $\ddot{0}$ & 1 & 0 & 0 & 0 & 1 & ? & 0 & 0 & 0 & 0 & $\stackrel{0}{?}$ & 0 & 0 & 0 & 0 & 0 & 1 & 0 & 0 \\
\hline 100 & 0 & 1 & 0 & 0 & 0 & 0 & 0 & 0 & 1 & 1 & 0 & 0 & (1) & 0 & 1) & 0 & $c$ & 0 & 0 & 0 & 0 & 0 & 0 & 0 & 0 & 1 & 0 & 0 \\
\hline 115 & 0 & 0 & 0 & 0 & 0 & 0 & 0 & 0 & 0 & 1 & 0 & 0 & 0 & 0 & 0 & 0 & 0 & 0 & 0 & 0 & 0 & 0 & 0 & 0 & 0 & 0 & 0 & 0 \\
\hline 130 & 0 & 0 & 0 & 0 & 0 & 0 & ) & 0 & 0 & 1 & 0 & 0 & 0 & 0 & 0 & 0 & 0 & 0 & 0 & 0 & 0 & 0 & 0 & 0 & 0 & 0 & 0 & 0 \\
\hline 145 & 0 & 0 & 0 & 0 & $\underline{0}$ & 0 & 0 & 0 & 0 & 1 & 0 & 0 & o & 0 & 0 & 0 & 0 & 0 & 0 & 0 & 0 & 0 & 0 & 0 & 0 & 0 & 0 & 0 \\
\hline 200 & 0 & 0 & 0 & 0 & 0 & 0 & 0 & 0 & 0 & 0 & 0 & 0 & 0 & 0 & 0 & 0 & 0 & 0 & 0 & 0 & 0 & 0 & 0 & 0 & 0 & 0 & 0 & \\
\hline 215 & 0 & 0 & 0 & 0 & 0 & 0 & 0 & 0 & 0 & 0 & 0 & 0 & 0 & 0 & 0 & 9 & 0 & 0 & 0 & 0 & 0 & 0 & 0 & 0 & 0 & 0 & 0 & 0 \\
\hline 230 & 0 & 0 & 0 & $\dot{0}$ & 0 & 0 & $\dot{0}$ & 0 & 0 & 0 & 0 & 0 & 0 & 0 & 0 & 0 & 0 & 0 & 0 & 0 & 0 & 0 & 0 & 0 & 0 & 0 & 0 & 0 \\
\hline 245 & 0 & 0 & 0 & 0 & 0 & 0 & 0 & 0 & $\underline{0}$ & 0 & 0 & 0 & 0 & 0 & 0 & 0 & 0 & 0 & 0 & 0 & 0 & 0 & 0 & 0 & 0 & 0 & 0 & 0 \\
\hline 300 & 0 & 0 & 0 & 0 & 0 & 0 & 0 & 0 & 0 & 0 & 0 & $\dot{0}$ & 0 & 0 & 0 & 0 & 0 & 0 & 0 & 0 & 0 & 0 & 0 & 0 & 0 & 0 & 0 & 0 \\
\hline 315 & 0 & 0 & 0 & 0 & 0 & 0 & 0 & 0 & 0 & 0 & 0 & 0 & 0 & 0 & 0 & 0 & 0 & 0 & 0 & 0 & 0 & 0 & 0 & 0 & 0 & 0 & 0 & 0 \\
\hline 330 & 0 & 0 & 0 & 0 & 0 & 0 & 0 & 0 & 0 & 0 & 0 & 0 & 0 & 0 & 0 & 0 & 0 & 0 & 0 & 0 & 0 & 0 & 0 & 0 & 0 & 0 & 0 & 0 \\
\hline 345 & 0 & 0 & 0 & 0 & 2 & 2 & 0 & 0 & 1 & 1 & 0 & 0 & 0 & 0 & 0 & 0 & 0 & 0 & 0 & 0 & 0 & 0 & 0 & 0 & 0 & 0 & 0 & 0 \\
\hline 400 & $c$ & 0 & 0 & 0 & 1 & 3 & 0 & 0 & 0 & 1 & 0 & 0 & 0 & 0 & 0 & 0 & 0 & 0 & 0 & 0 & 0 & 0 & 0 & 0 & 0 & 0 & 0 & 0 \\
\hline 415 & 0 & 0 & 0 & 9 & 0 & 3 & 0 & 0 & 0 & 1 & 1 & $i$ & 0 & 0 & 0 & 0 & 0 & 0 & 0 & 0 & 0 & 0 & 3 & 3 & 0 & 0 & 0 & 0 \\
\hline 430 & 1 & 1 & 0 & 0 & 0 & 3 & 2 & $=$ & 0 & 1 & 0 & 1 & 0 & 0 & 0 & 0 & 0 & 0 & 1 & 1 & 0 & 0 & 0 & 3 & 0 & 0 & 0 & 0 \\
\hline 145 & $\therefore$ & 1 & 0 & 4 & 0 & 1 & 0 & 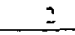 & a & 0 & 0 & 1 & 9 & 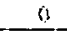 & 9 & 0 & 0 & 0 & 0 & 1 & 0 & (1) & i) & 3 & 0 & 0 & 0 & 0 \\
\hline 500 & $a$ & 1 & 10 & 10 & 0 & 0 & 6 & 8 & 0 & 0 & 0 & 1 & 0 & 0 & i) & 0 & 1 & 1 & 13 & 14 & 1 & 1 & 11 & 14 & 1 & 1 & 6 & 6 \\
\hline 515 & 1 & 2 & 62 & 72 & 0 & 0 & 5 & 63 & 1 & 1 & 0 & 0 & 0 & 0 & 0 & 0 & 1 & 2 & 41 & 55 & 0 & 1 & 46 & 57 & 0 & 1 & 55 & 61 \\
\hline$\$ 30$ & 1 & 2 & 101 & 173 & 0 & 0 & 77 & 138 & 0 & 1 & 0 & ) & 0 & 0 & 0 & 0 & 1 & 3 & 92 & 146 & 1 & 2 & 111 & 168 & 1 & $=$ & 119 & 180 \\
\hline 545 & 1 & 3 & 120 & 293 & 0 & 0 & 109 & 247 & 0 & 1 & $\dot{0}$ & 0 & 1 & 1 & 2 & $=$ & 2 & 5 & 120 & 266 & 0 & 2 & 149 & 317 & 0 & $=$ & 163 & 343 \\
\hline 600 & 5 & 8 & 154 & 437 & 2 & 2 & 149 & 390 & 0 & 1 & 4 & 4 & 0 & I & 0 & 2 & 4 & 8 & 183 & 436 & 8 & 9 & 194 & 500 & 6 & 7 & 164 & 501 \\
\hline 615 & 12 & 19 & 218 & 593 & 3 & 5 & 230 & 565 & 1 & 1 & 1 & 5 & 0 & 1 & 0 & 2 & 6 & 13 & 211 & 606 & 11 & 20 & 234 & 688 & 9 & 16 & $23 i$ & 677 \\
\hline 630 & 16 & 34 & 195 & 687 & 4 & 9 & 205 & 693 & 0 & 1 & 2 & 7 & 0 & 1 & ) & 2 & 13 & 25 & 226 & 740 & 19 & 38 & 222 & 799 & 13 & 28 & 244 & soz \\
\hline 645 & 27 & 60 & 115 & 682 & 12 & 21 & 142 & 726 & 0 & 1 & $\therefore$ & 9 & 0 & i & 0 & 0 & 25 & 48 & 120 & 740 & 21 & 59 & 130 & 780 & 24 & $\Xi$ & 142 & 781 \\
\hline 700 & 35 & 90 & 140 & 668 & 10 & 29 & $1+0$ & 717 & 0 & 1 & 0 & 5 & 0 & 0 & 2 & $=$ & 34 & 78 & 145 & 702 & 37 & 88 & 151 & 737 & 31 & & 135 & 752 \\
\hline 715 & 11 & 89 & 189 & 639 & 22 & 48 & 176 & 663 & ) & 0 & 0 & 4 & i & 0 & 0 & $=$ & 19 & 91 & 180 & 671 & 16 & 93 & 172 & 675 & 25 & 93 & {$[4]$} & 602 \\
\hline 730 & 29 & 102 & 220 & 664 & 17 & 61 & 255 & 713 & 0 & 0 & 0 & 2 & 0 & 0 & 0 & 2 & 34 & 112 & 196 & 641 & 19 & 93 & 201 & 654 & 20 & 100 & 233 & 651 \\
\hline 745 & 22 & 97 & 202 & 751 & 29 & 78 & 220 & 791 & 1 & 1 & () & 0 & 0 & 0 & 0 & 2 & 25 & 112 & 215 & 736 & 27 & 99 & 174 & 698 & 22 & 98 & 191 & 700 \\
\hline 800 & \pm 0 & 102 & 101 & 712 & 24 & 92 & 110 & 761 & 0 & l & 0 & 0 & 1 & 1 & 0 & 0 & 42 & 120 & 106 & 697 & 44 & 106 & 106 & 653 & 41 & 108 & 112 & 677 \\
\hline 815 & 42 & 133 & 75 & 598 & 34 & 104 & 79 & 604 & 0 & 1 & 0 & 0 & 1 & 2 & 0 & 0 & 33 & 134 & 72 & 589 & 35 & 125 & 74 & 555 & 32 & 115 & 63 & 599 \\
\hline 830 & 25 & 129 & so & 428 & 35 & 122 & 47 & 456 & 1 & 2 & 0 & 0 & 1 & 3 & 0 & 0 & 22 & 122 & 48 & & 19 & 125 & 61 & 415 & 26 & 121 & 51 & 417 \\
\hline 845 & 0 & 116 & 49 & 275 & 16 & 109 & 29 & 255 & 0 & 1 & 0 & 0 & 2 & $s$ & 2 & 2 & 16 & 113 & 46 & 272 & 26 & 124 & 28 & 269 & 7 & 106 & 47 & 273 \\
\hline 900 & $=0$ & 96 & 30 & 204 & 11 & 96 & 37 & 192 & 0 & 1 & 0 & 0 & 0 & 4 & 2 & 4 & 19 & 90 & 21 & 187 & 14 & 94 & 35 & 198 & 9 & 74 & 29 & 190 \\
\hline 915 & 14 & 68 & 15 & 144 & 15 & 77 & 28 & 141 & 0 & 1 & i) & 0 & 0 & 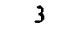 & 3 & 7 & 19 & 76 & 32 & 147 & 9 & 68 & 26 & 150 & 9 & $\$ 1$ & 30 & 147 \\
\hline 930 & 14 & 57 & 17 & 111 & 11 & 53 & 25 & 119 & 1 & 1 & 0 & 0 & 0 & 2 & 2 & 9 & 16 & 70 & 23 & 122 & 9 & 58 & 13 & 102 & 13 & 38 & 19 & 115 \\
\hline 945 & 15 & 63 & 13 & 75 & 16 & 53 & 10 & 100 & 1 & 2 & 2 & 2 & 0 & 0 & 0 & 7 & 12 & 66 & 23 & 99 & 10 & 42 & 16 & 90 & 9 & 40 & 24 & $s=1$ \\
\hline 1000 & 11 & $\$ 4$ & 10 & 55 & 17 & 59 & 18 & 81 & 0 & 2 & 0 & 2 & 0 & 0 & 0 & 5 & 15 & 62 & 19 & 97 & 11 & 39 & 17 & 72 & 7 & 38 & 15 & 78 \\
\hline 1015 & 13 & 53 & 11 & $\leqq$ & 18 & 62 & 16 & 69 & 0 & 2 & 0 & 2 & 0 & 0 & 0 & 2 & 16 & 59 & 16 & 81 & 13 & 43 & 27 & 73 & 16 & 45 & 14 & 72 \\
\hline 1030 & 8 & 47 & 18 & 52 & 6 & 57 & $10^{\circ}$ & 60 & 9 & 10) & () & 2 & 1 & 1 & 0 & 0 & 17 & 60 & 13 & 71 & 11 & 45 & 7 & 67 & 15 & 47 & 15 & 68 \\
\hline 1045 & $\varepsilon$ & 40 & 11 & so & 9 & 50 & 17 & 67 & 1 & 10) & 0 & 0 & 0 & 1 & 0 & 0 & 18 & 66 & 14 & 62 & 7 & 42 & 12 & 63 & 10 & 48 & 12 & 56 \\
\hline 1100 & 13 & 42 & 12 & 52 & 17 & 50 & 12 & 61 & 0 & 10 & 0 & 0 & 0 & 1 & 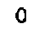 & 0 & 12 & 63 & 12 & 55 & 4 & 35 & 3 & 49 & 10 & 51 & 14 & 35 \\
\hline 115 & 8 & 37 & 13 & 54 & 13 & 45 & 14 & 59 & (I) & 10 & 0 & 0 & 1 & 2 & 0 & 0 & 11 & 58 & 14 & 53 & 14 & 36 & 12 & 34 & 13 & 48 & 6 & 47 \\
\hline 130 & 11 & 40 & 14 & 50 & 6 & 45 & 3 & 46 & 0 & 1 & 0 & 0 & 0 & 1 & 0 & 0 & 13 & 54 & 26 & 66 & 9 & 34 & 15 & 42 & 12 & 45 & 5 & 37 \\
\hline 145 & 13 & 45 & 21 & 60 & 5 & 41 & 8 & 37 & 2 & 2 & 0 & 0 & 1 & 2 & 0 & 0 & 8 & 44 & 15 & 67 & 13 & 40 & 13 & 43 & 8 & 43 & 10 & 35 \\
\hline
\end{tabular}




\section{BAYMETRICS TRAFFIC RESOURCES Location 12: NB LT\& SB RT Onto Eastgate From Greenville}

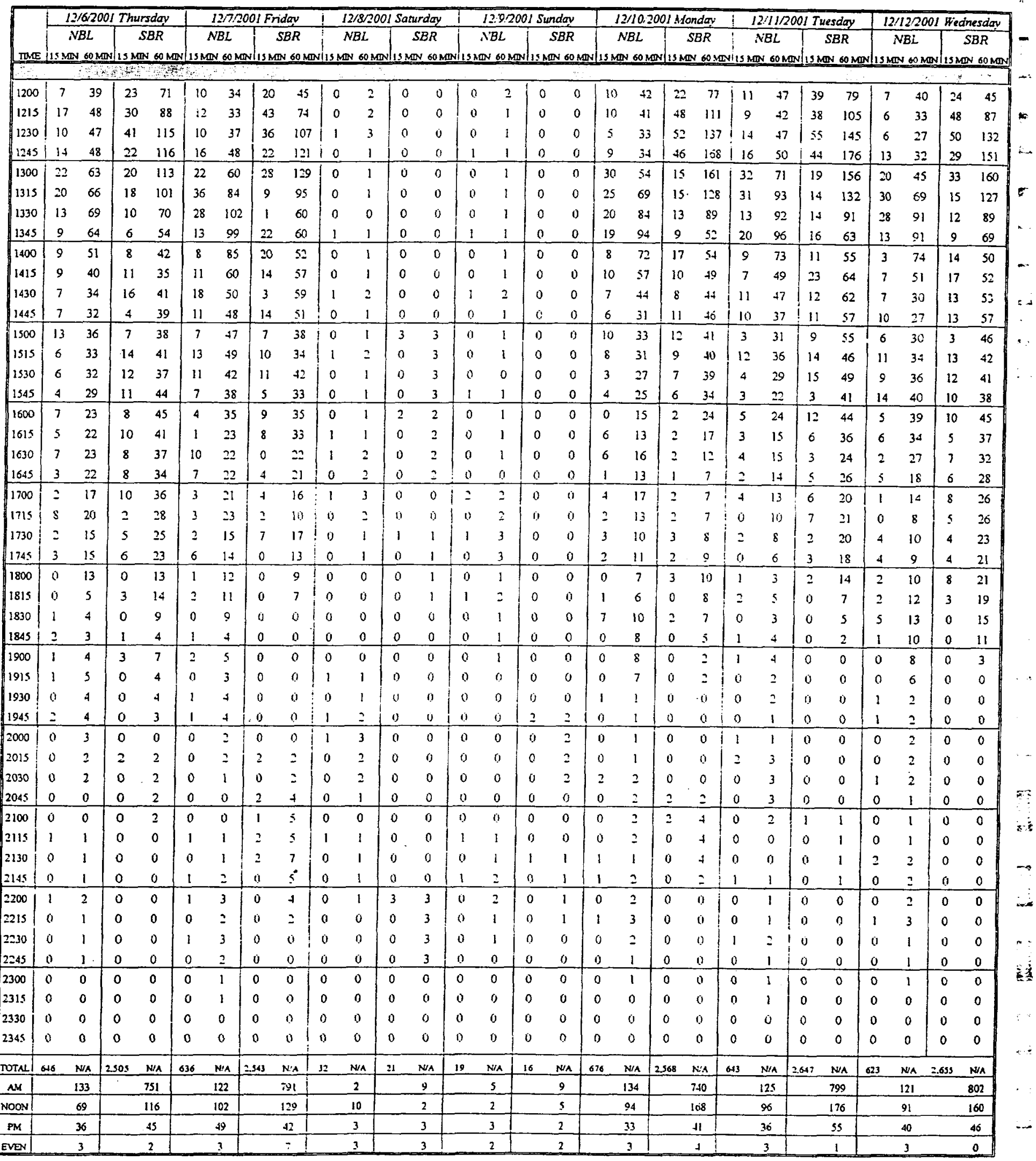




\section{BAYMETRICS TRAFFIC RESOURCES Location 13: WBLT \& WBR On Eastgate From Greenville}

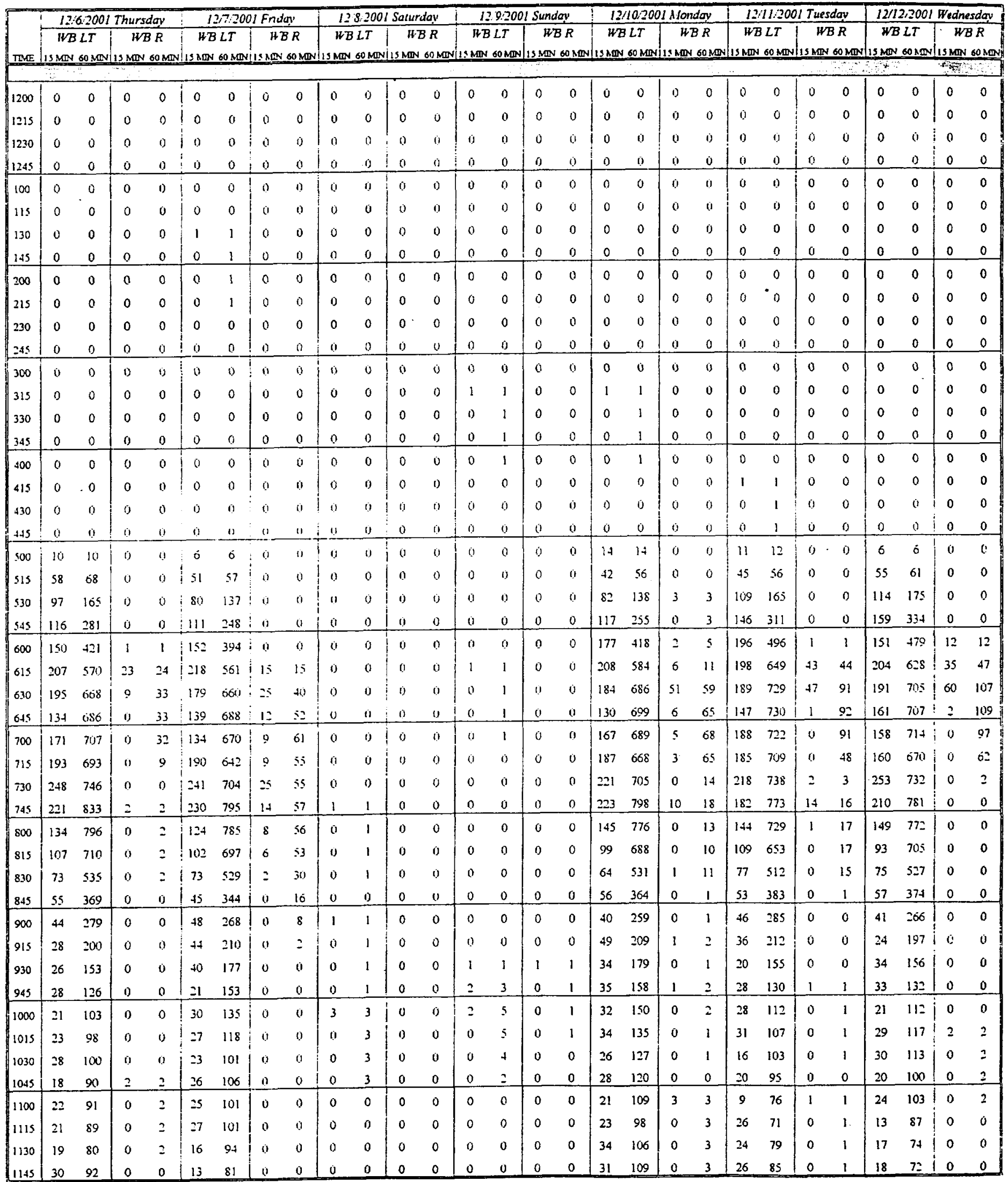




\section{BAYMETRICS TRAFFIC RESOURCES Location 13: WBLT \& WBR On Eastgate From Greenville}

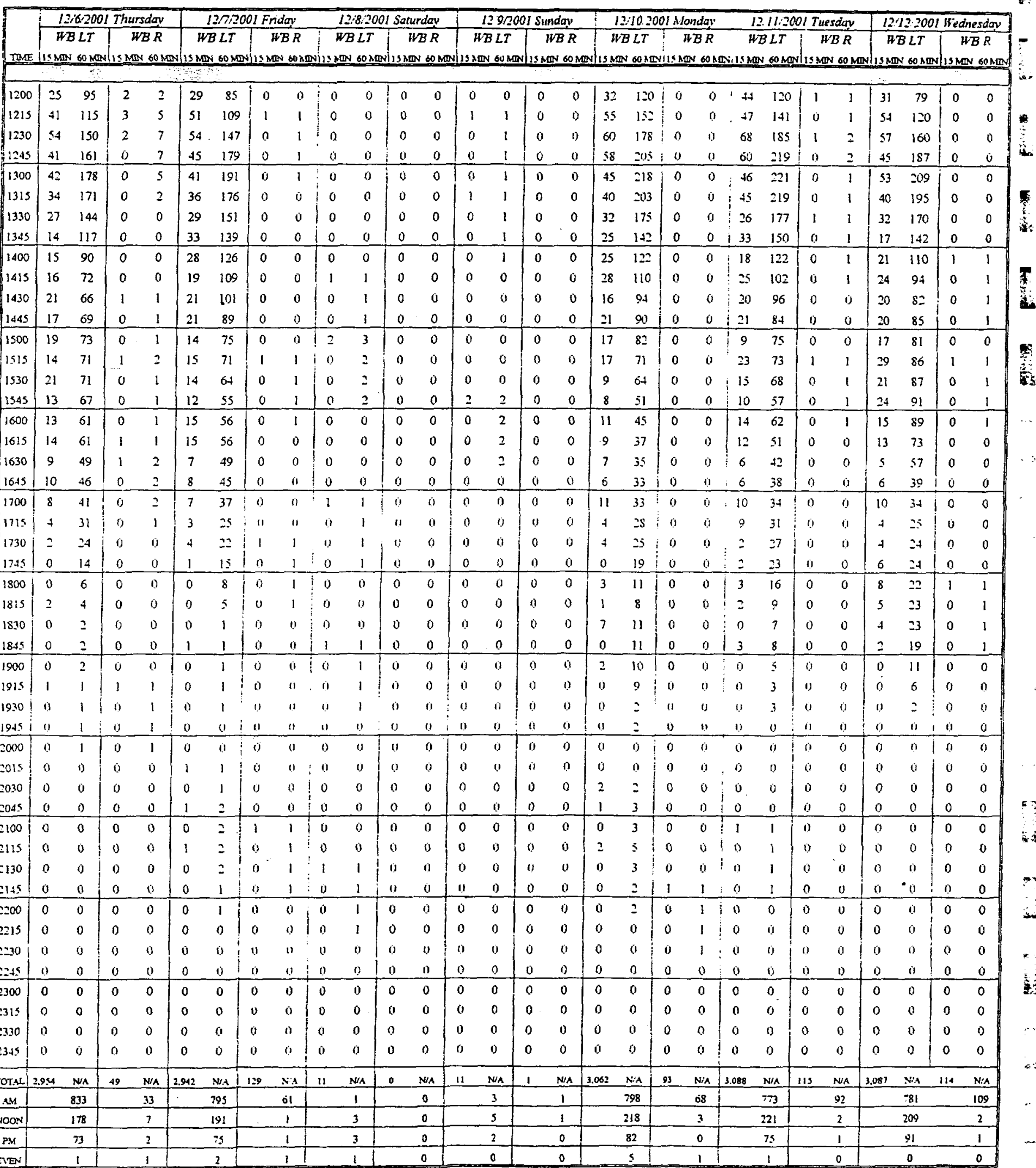




\section{BAYMETRICS TRAFFIC RESOURCES Location 14: Eastbound On Eastgate To Greenville}

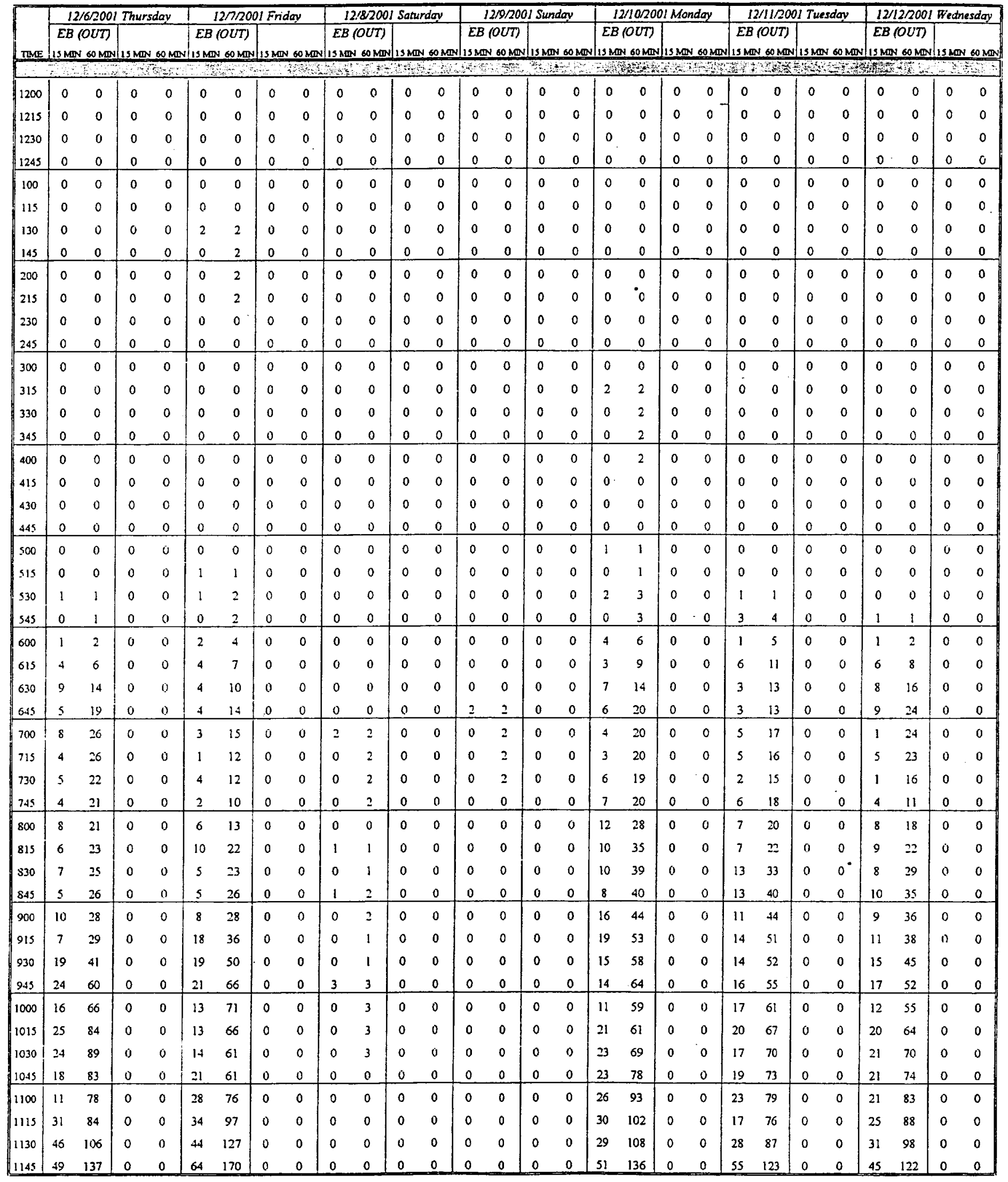




\section{BAYMETRICS TRAFFIC RESOURCES Location 14: Eastbound On Eastgate To Greenville}

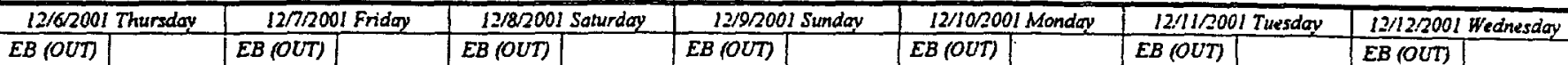

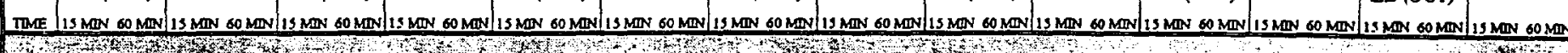

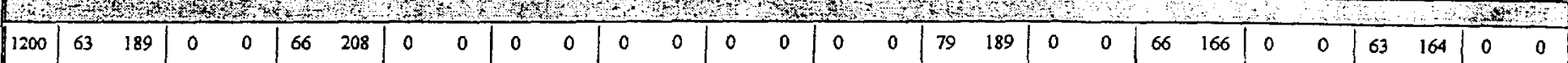

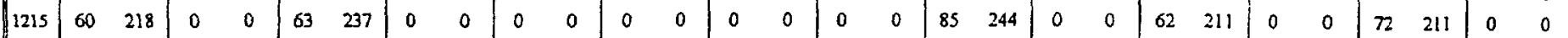
\begin{tabular}{lll|ll|llll|ll|ll|ll|ll|llllllllllllll}
1230 & 27 & 199 & 0 & 0 & 38 & 231 & 0 & 0 & 0 & 0 & 0 & 0 & 0 & 0 & 0 & 0 & 19 & 234 & 0 & 0 & 33 & 216 & 0 & 0 & 40 & 220 & 0 & 0 & 3
\end{tabular} \begin{tabular}{|l|llllllll|ll|ll|lllll|lllllllllll}
1245 & 24 & 174 & 0 & 0 & 23 & 190 & 0 & 0 & 2 & 2 & 0 & 0 & 0 & 0 & 0 & 0 & 30 & 213 & 0 & 0 & 28 & 189 & 0 & 0 & 22 & 197 & 0 & 0 \\
\hline 1300 & 15 & 126 & 0 & 0 & 17 & 141 & 0 & 0 & 0 & 2 & 0 & 0 & 0 & 0 & 0 & 0 & 24 & 158 & 0 & 0 & 20 & 143 & 0 & 0 & 23 & 156 & 0
\end{tabular} \begin{tabular}{l|ll|ll|ll|ll|ll|llllllllll|lllllllll}
1300 & 15 & 126 & 0 & 0 & 17 & 141 & 0 & 0 & 0 & 2 & 0 & 0 & 0 & 0 & 0 & 0 & 24 & 158 & 0 & 0 & 20 & 143 & 0 & 0 & 22 & 156 & 0 & 0 \\
1315 & 16 & 82 & 0 & 0 & 29 & 107 & 0 & 0 & 0 & 2 & 0 & 0 & 0 & 0 & 0 & 0 & 21 & 94 & 0 & 0 & 21 & 102 & 0 & 0 & 17 & 101 & 0 & 0 &
\end{tabular} \begin{tabular}{lll|ll|ll|ll|ll|ll|ll|ll|llllllllllllll}
1330 & 13 & 68 & 0 & 0 & 23 & 92 & 0 & 0 & 0 & 2 & 0 & 0 & 0 & 0 & 0 & 0 & 24 & 99 & 0 & 0 & 23 & 92 & 0 & 0 & 18 & 79 & 0 & 0 & 3
\end{tabular} \begin{tabular}{|l|ll|ll|llll|llllllllllllllllllll}
1345 & 27 & 71 & 0 & 0 & 30 & 99 & 0 & 0 & 0 & 0 & 0 & 0 & 0 & 0 & 0 & 0 & 14 & 83 & 0 & 0 & 25 & 89 & 0 & 0 & 29 & 86 & 0 & 0 \\
\hline
\end{tabular} \begin{tabular}{|l|ll|ll|ll|ll|ll|ll|ll|ll|ll|ll|ll|ll|ll|ll|}
\hline 1400 & 18 & 74 & 0 & 0 & 24 & 106 & 0 & 0 & 0 & 0 & 0 & 0 & 0 & 0 & 0 & 0 & 21 & 80 & 0 & 0 & 19 & 88 & 0 & 0 & 29 & 93 & 0 & 0 \\
\hline
\end{tabular} \begin{tabular}{l|ll|ll|ll|ll|ll|ll|lllll|ll|llllllllllll}
1415 & 25 & 83 & 0 & 0 & 35 & 112 & 0 & 0 & 0 & 0 & 0 & 0 & 0 & 0 & 0 & 0 & 23 & 82 & 0 & 0 & 28 & 95 & 0 & 0 & 25 & 101 & 0 & 0 & 5
\end{tabular}

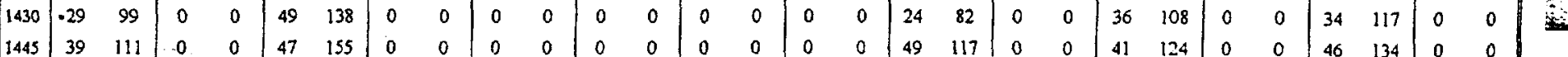
\begin{tabular}{|l|llllllllll|llllllll|llllllllll}
1445 & 39 & 111 & 0 & 0 & 47 & 155 & 0 & 0 & 0 & 0 & 0 & 0 & 0 & 0 & 0 & 0 & 49 & 117 & 0 & 0 & 41 & 124 & 0 & 0 & 46 & 134 & 0 & 0 \\
\hline 1500 & 43 & 136 & 0 & 0 & 56 & 187 & 0 & 0 & 0 & 0 & 0 & 0 & 0 & 0 & 0 & 0 & 30 & 126 & 0 & 0 & 26 & 131 & 0 & 0
\end{tabular}

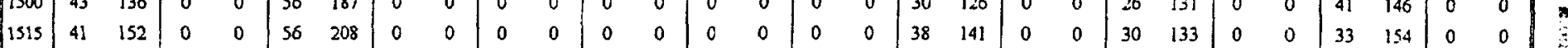
\begin{tabular}{lllll|llll|ll|ll|ll|ll|ll|lllllllllll}
1530 & 75 & 198 & 0 & 0 & 117 & 276 & 0 & 0 & 0 & 0 & 0 & 0 & 0 & 0 & 0 & 0 & 102 & 219 & 0 & 0 & 102 & 199 & 0 & 0 & 93 & 213 & 0 & 0 & -3
\end{tabular} \begin{tabular}{l|llllllllllllllllllllllllllllll}
1545 & 172 & 331 & 0 & 0 & 166 & 395 & 0 & 0 & 0 & 0 & 0 & 0 & 0 & 0 & 0 & 0 & 139 & 309 & 0 & 0 & 172 & 330 & 0 & 0 & 165 & 332 & 0 & 0 \\
\hline
\end{tabular}

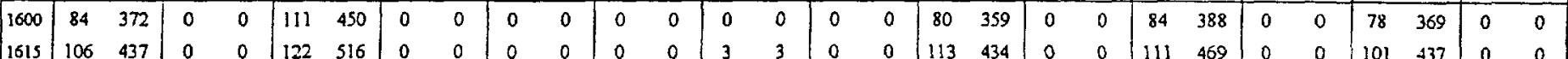
$\begin{array}{lllllllllllllllllllllllllllllll}1630 & 99 & 461 & 0 & 0 & 127 & 526 & 0 & 0 & 0 & 0 & 0 & 0 & 0 & 3 & 0 & 0 & 97 & 429 & 0 & 0 & 128 & 495 & 0 & 0 & 112 & 450 & 0 & 0\end{array}$ \begin{tabular}{l|llll|ll|llllllllllllllllllllll}
1645 & 143 & 432 & 0 & 0 & 161 & 521 & 0 & 0 & 0 & 0 & 0 & 0 & 0 & 3 & 0 & 0 & 178 & 468 & 0 & 0 & 162 & 485 & 0 & 0 & 150 & 441 & 0 & 0 \\
\hline
\end{tabular}

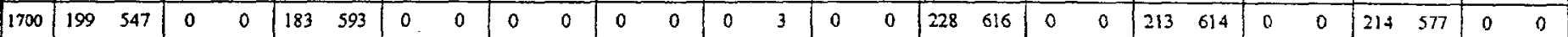

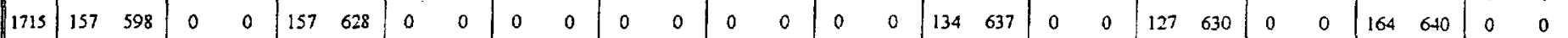

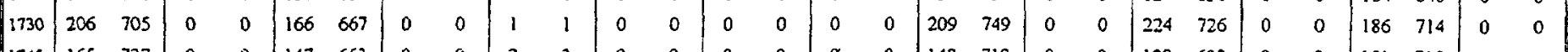

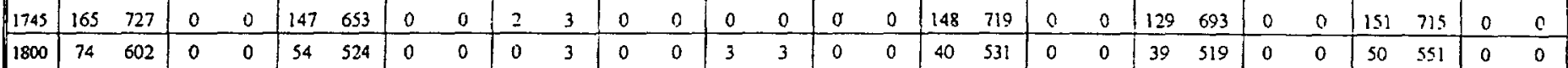

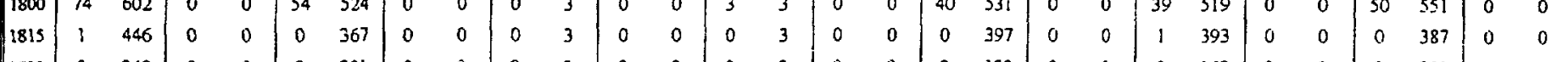

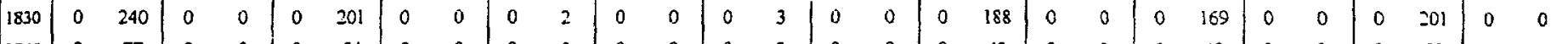
\begin{tabular}{|l|llll|ll|ll|ll|ll|lllll|llllllllllll}
1845 & 2 & 77 & 0 & 0 & 0 & 54 & 0 & 0 & 0 & 0 & 0 & 0 & 0 & 3 & 0 & 0 & 0 & 40 & 0 & 0 & 0 & 40 & 0 & 0 & 0 & 50 & 0 & 0 \\
\hline 1900 & 0 & 3 & 0 & 0 & 0 & 0 & 0 & 0 & 0 & 0 & 0 & 0 & 0 & 0 & 0 & 0 & 0 & 0 & 0 & 0 & 0 & 1 & 0 & 0 & 1 & 1 & 0 & 0
\end{tabular}

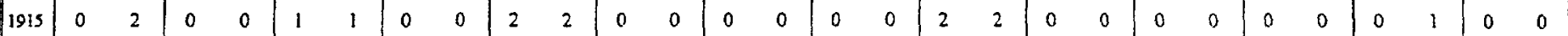
\begin{tabular}{lllll|ll|ll|ll|ll|ll|ll|ll|ll|llllllllll}
1930 & 0 & 2 & 0 & 0 & 0 & 1 & 0 & 0 & 0 & 2 & 0 & 0 & 0 & 0 & 0 & 0 & 3 & 5 & 0 & 0 & 0 & 0 & 0 & 0 & 0 & 1 & 0 & 0 \\
1945 & 2 & 2 & 0 & 0 & 0 & 1 & 0 & 0 & 0 & 2 & 0 & 0 & 0 & 0 & 0 & 0 & 0 & 5 & 0 & 0 & 0 & 0 & 0 & 0 & 0 & 1 & 0 & 0 \\
\hline
\end{tabular}

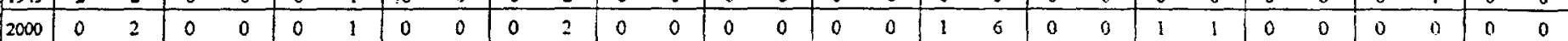

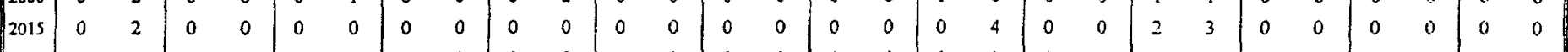
\begin{tabular}{l|ll|ll|ll|ll|ll|ll|ll|ll|lll|llllllllll}
2030 & 0 & 2 & 0 & 0 & 0 & 0 & 0 & 0 & 0 & 0 & 0 & 0 & 0 & 0 & 0 & 0 & 0 & 1 & 0 & 0 & 2 & 5 & 0 & 0 & 7 & 7 & 0 & 0 \\
2045 & 0 & 0 & 0 & 0 & 0 & 0 & 0 & 0 & 0 & 0 & 0 & 0 & 0 & 0 & 0 & 0 & 13 & 14 & 0 & 0 & 0 & 5 & 0 & 0 & 5 & 12 & 0 & 0 \\
\hline
\end{tabular} \begin{tabular}{|l|llllll|lllll|lllll|ll|lll|lllllllll}
2045 & 0 & 0 & 0 & 0 & 0 & 0 & 0 & 0 & 0 & 0 & 0 & 0 & 0 & 0 & 0 & 0 & 13 & 14 & 0 & 0 & 0 & 5 & 0 & 0 & 5 & 12 & 0 & 0 \\
\hline 2100 & 2 & 2 & 0 & 0 & 0 & 0 & 0 & 0 & 0 & 0 & 0 & 0 & 0 & 0 & 0 & 0 & 0 & 13 & 0 & 0 & 0 & 4 & 0 & 0 & 0 & 12 & 0 & 0 &
\end{tabular}

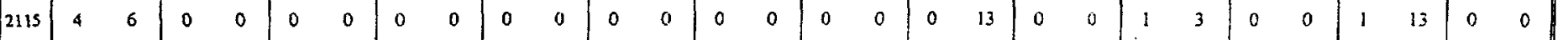
\begin{tabular}{lllll|ll|ll|ll|lllll|ll|ll|lllll|llllll}
2130 & 7 & 13 & 0 & 0 & 0 & 0 & 0 & 0 & 0 & 0 & 0 & 0 & 0 & 0 & 0 & 0 & 1 & 14 & 0 & 0 & 0 & 1 & 0 & 0 & 0 & 6 & 0 & 0 & 0 \\
2145 & 0 & 13 & 0 & 0 & 0 & 0 & 0 & 0 & 0 & 0 & 0 & 0 & 0 & 0 & 0 & 0 & 1 & 2 & 0 & 0 & 0 & 1 & 0 & 0 & 0 & 1 & 0 & 0 \\
\hline
\end{tabular}

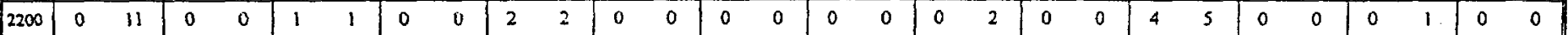

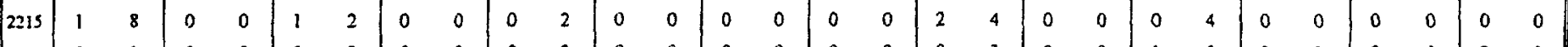

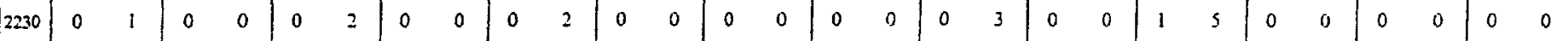

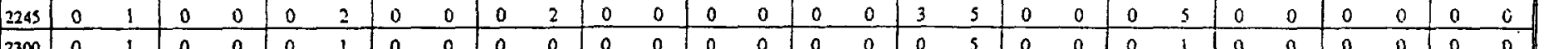

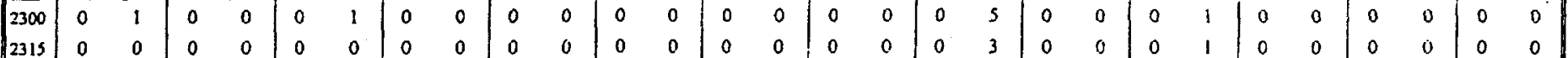

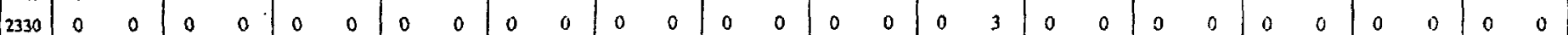
\begin{tabular}{|l|ll|ll|ll|ll|llllllllllllll|llllllllll}
2345 & 0 & 0 & 0 & 0 & 0 & 0 & 0 & 0 & 0 & 0 & 0 & 0 & 0 & 0 & 0 & 0 & 0 & 0 & 0 & 0 & 0 & 0 & 0 & 0 & 0 & 0 & 0 & 0 \\
\hline
\end{tabular}

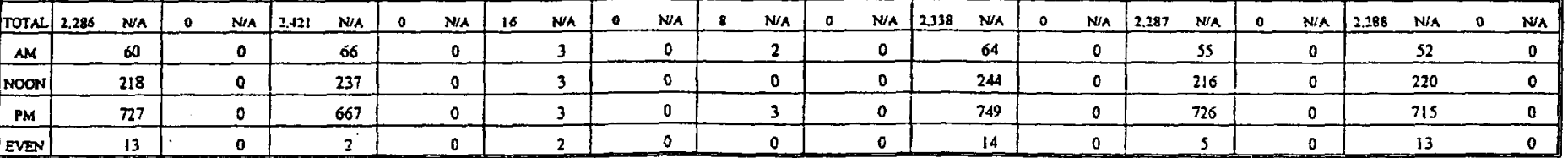




\section{BAYMETRICS TRAFFIC RESOURCES Location 15: SBL on Vasco Road North of Westgate Drive}

\begin{tabular}{|c|c|c|c|c|c|c|c|c|c|c|c|c|c|c|c|c|c|c|c|c|c|c|c|c|c|c|c|c|}
\hline \multirow{3}{*}{ TME } & \multicolumn{4}{|c|}{ 12/6/2001 Thursday } & \multicolumn{4}{|c|}{$127 n 001$ Fridoy } & \multicolumn{4}{|c|}{ 12/8/2001 Saturday } & \multicolumn{4}{|c|}{ 12/9/2001 Sundoy } & \multicolumn{4}{|c|}{ 12/10/2001 Monday } & \multicolumn{4}{|c|}{ 12/11/2001 Tuesday } & & 2200 & Wed & sday \\
\hline & $s$ & & & & & & & & & & & & & & & & & & & & & & & & & & & \\
\hline & & 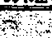 & & & & 2 & & & & $\frac{\pi}{2}$ & 4 & 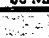 & & 4 & 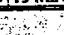 & & $\because$ & & & & 8 & & 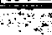 & 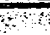 & 48 & $2+8$ & & Ft \\
\hline 1200 & 47 & 126 & 0 & 0 & 35 & 116 & 0 & 0 & 0 & 4 & 0 & 0 & 0 & 2 & 0 & 0 & 33 & 122 & 0 & 0 & so & 131 & 0 & 0 & 43 & 121 & 0 & 0 \\
\hline 1215 & 39 & 132 & 0 & 0 & 37 & 130 & 0 & 0 & 0 & 3 & 0 & 0 & 0 & 0 & 0 & 0 & 49 & 146 & 0 & 0 & 39 & 142 & 0 & 0 & 46 & 147 & 0 & 0 \\
\hline 1230 & 60 & 161 & 0 & 0 & 57 & 163 & 0 & 0 & c & 0 & 0 & 0 & 0 & 0 & 0 & 0 & 91 & 207 & 0 & 0 & 76 & 189 & 0 & 0 & 75 & 196 & 0 & 0 \\
\hline 1245 & 84 & 230 & 0 & $\dot{0}$ & 88 & 217 & 0 & i & 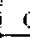 & 0 & 0 & 0 & 0 & 0 & 0 & 0 & 110 & 283 & 0 & 0 & 115 & 280 & 0 & 0 & 91 & 255 & 0 & 0 \\
\hline 1300 & 147 & 330 & 0 & 0 & 108 & 290 & 0 & 0 & 1 & 1 & 0 & 0 & 0 & 0 & 0 & 0 & 140 & 390 & 0 & 0 & 145 & 375 & 0 & 0 & 136 & 348 & 0 & 0 \\
\hline 1315 & 121 & 412 & 0 & 0 & 126 & 379 & 0 & 0 & 0 & 1 & 0 & 0 & 0 & 0 & 0 & 0 & 84 & 425 & 0 & 0 & 113 & 449 & 0 & 0 & 106 & 408 & 0 & 0 \\
\hline 1330 & 86 & 438 & 0 & 0 & 95 & 417 & 0 & 0 & 0 & 1 & 0 & 0 & 0 & 0 & 0 & 0 & 55 & 389 & 0 & 0 & 62 & 435 & 0 & 0 & 79 & 412 & 0 & 0 \\
\hline 1345 & 70 & 424 & 0 & 0 & so & 379 & 0 & 0 & 0 & 1 & 0 & 0 & 0 & 0 & 0 & 0 & 32 & 311 & 0 & 0 & 44 & 364 & 0 & 0 & 64 & 385 & 0 & 0 \\
\hline 1400 & 43 & 320 & 0 & 0 & 47 & 318 & 0 & 0 & 0 & 0 & 0 & 0 & 1 & 1 & 0 & 0 & 37 & 208 & 0 & 0 & 36 & 255 & 0 & 0 & 40 & 289 & 0 & 0 \\
\hline 1415 & 31 & 230 & 0 & 0 & 38 & 230 & 0 & 0 & 0 & 0 & 0 & 0 & 0 & I & 0 & 0 & 13 & 137 & 0 & 0 & 20 & 162 & 0 & 0 & 26 & 209 & 0 & 0 \\
\hline 1430 & 27 & 171 & 0 & 0 & 18 & 153 & 0 & 0 & 0 & 0 & 0 & 0 & 0 & 1 & 0 & 0 & 15 & 97 & 0 & 0 & 19 & 119 & 0. & 0 & 24 & 154 & 0 & 0 \\
\hline 1445 & 24 & 125 & 0 & 0 & 18 & 121 & 0 & 0 & 0 & 0 & 0 & 0 & 0 & 1 & 0 & 0 & 21 & 86 & 0 & 0 & 18 & 93 & 0 & 0 & 14 & 104 & 0 & 0 \\
\hline 1500 & 26 & 108 & 0 & 0 & 24 & 98 & 0 & 0 & i) & 0 & 0 & 0 & 0 & 0 & 0 & 0 & 17 & 66 & 0 & 0 & 16 & 73 & 0 & 0 & 19 & 83 & 0 & 0 \\
\hline 1515 & 24 & 101 & 0 & 0 & 28 & 88 & 0 & 0 & 1 & 1 & 0 & 0 & 1 & 1 & 0 & 0 & 14 & 67 & 0 & 0 & 15 & 68 & 0 & 0 & 18 & 75 & 0 & 0 \\
\hline 1530 & 12 & 86 & 0 & 0 & 13 & 83 & 0 & 0 & 0 & 1 & 0 & 0 & 0 & 1 & 0 & 0 & 11 & 63 & 0 & 0 & 25 & 74 & 0 & 0 & 23 & 74 & 0 & 0 \\
\hline 1545 & 17 & 79 & 0 & 0 & 10 & 75 & 0 & 0 & 0 & 1 & 0 & 0 & 1 & 2 & 0 & 0 & 10 & 52 & 0 & 0 & 9 & 65 & 0 & 0 & {$[3$} & 73 & 0 & 0 \\
\hline 1600 & 12 & 65 & 0 & 0 & 13 & 64 & 0 & 0 & 0 & 1 & 0 & 0 & 0 & 2 & 0 & 0 & 7 & 42 & 0 & 0 & 10 & 59 & 0 & 0 & 17 & 71 & 0 & 0 \\
\hline 1615 & 9 & 50 & 0 & 0 & 9 & 45 & 0 & 0 & 0 & 0 & 0 & 0 & 0 & 1 & 0 & 0 & 8 & 36 & 0 & 0 & 12 & 56 & 0 & 0 & 10 & 63 & 0 & 0 \\
\hline 1630 & 6 & 44 & 0 & 0 & 5 & 37 & 0 & 0 & 0 & 0 & 0 & 0 & 0 & 1 & 0 & 0 & 4 & 39 & 0 & 0 & 6 & 37 & 0 & 0 & 8 & 48 & 0 & 0 \\
\hline 1645 & 10 & 37 & 0 & 0 & 6 & 33 & 0 & 0 & 0 & 0 & 0 & 0 & 0 & 0 & 0 & 0 & 8 & 27 & 0 & 0 & 10 & 38 & 0 & 0 & 7 & 42 & 0 & 0 \\
\hline 1700 & 8 & 33 & 0 & 0 & 11 & 31 & 0 & 0 & 0 & 0 & 0 & 0 & 0 & 0 & 0 & 0 & 7 & 27 & 0 & 0 & 13 & 41 & 0 & 0 & 6 & 31 & 0 & 0 \\
\hline 1715 & 10 & 34 & 0 & 0 & 8 & 30 & 0 & 0 & 0 & $\dot{0}$ & 0 & 0 & 1 & 1 & 0 & 0 & 8 & 27 & 0 & 0 & 8 & 37 & 0 & 0 & 6 & 27 & 0 & 0 \\
\hline 1730 & 9 & 37 & 0 & 0 & 6 & 31 & 0 & 0 & 4 & 4 & 0 & 0 & 0 & 1 & 0 & 0 & 4 & 27 & 0 & 0 & 5 & 36 & 0 & 0 & 15 & 34 & 0 & 0 \\
\hline 1745 & 7 & 34 & 0 & 0 & 5 & 30 & 0 & 0 & 0 & 4 & 0 & 0 & 0 & 1 & 0 & 0 & 7 & 26 & 0 & 0 & 8 & 34 & 0 & 0 & 9 & 36 & 0 & 0 \\
\hline 1800 & 3 & 29 & 0 & 0 & 14 & 33 & 0 & 0 & 0 & 4 & 0 & 0 & 0 & 1 & 0 & 0 & 7 & 26 & 0 & 0 & 6 & 27 & 0 & 0 & 3 & 33 & 0 & 0 \\
\hline 1815 & 6 & 25 & 0 & 0 & 7 & 32 & 0 & ¿ & 0 & 4 & 0 & 0 & 0 & 0 & 0 & 0 & 0 & 18 & 0 & 0 & 1 & 20 & 0 & 0 & 5 & 32 & 0 & 0 \\
\hline 1830 & 6 & 32 & 0 & 0 & 5 & 31 & 0 & 0 & 0 & 0 & 0 & 0 & 0 & 0 & 0 & 0 & 0 & 14 & 0 & 0 & 2 & 17 & 0 & 0 & 3 & 20 & 0 & 0 \\
\hline 1845 & 4 & 19 & 0 & 0 & 4 & 30 & 0 & 0 & 0 & 0 & 0 & 0 & 0 & 0 & 0 & 0 & 1 & 8 & 0 & 0 & 3 & 12 & 0 & 0 & 6 & 17 & 0 & 0 \\
\hline 1900 & 3 & 19 & 0 & 0 & 0 & 16 & 0 & $\dot{0}$ & 0 & 0 & 0 & 0 & $\overline{0}$ & 0 & 0 & 0 & 0 & 1 & 0 & 0 & 2 & 8 & 0 & 0 & 1 & 15 & 0 & 0 \\
\hline 1915 & 2 & 15 & 0 & 0 & 0 & 9 & 0 & 0 & 0 & 0 & 0 & 0 & 2 & 2 & 0 & 0 & 0 & 1 & 0 & 0 & 0 & 7 & 0 & 0 & 0 & 10 & 0 & 0 \\
\hline 1930 & 2 & 11 & 0 & 0 & 0 & 4 & 0 & 0 & 0 & 0 & 0 & 0 & 0 & 2 & 0 & 0 & 0 & 1 & 0 & 0 & 0 & 5 & 0 & 0 & 0 & 7 & 0 & 0 \\
\hline 1945 & 1 & 8 & 0 & 0 & 1 & 1 & 0 & 0 & 0 & 0 & 0 & 0 & 0 & 2 & 0 & 0 & 0 & 0 & 0 & 0 & 1 & 3 & 0 & 0 & 0 & 1 & 0 & 0 \\
\hline 2000 & 0 & 5 & 0 & 0 & 0 & 1 & 0 & 0 & 0 & 0 & 0 & 0 & 0 & 2 & 0 & 0 & 0 & 0 & 0 & 0 & 0 & 1 & 0 & 0 & 0 & 0 & 0 & 0 \\
\hline 2015 & 0 & 3 & 0 & 0 & 0 & 1 & 0 & 0 & 0 & 0 & 0 & 0 & 0 & 0 & 0 & 0 & 0 & 0 & 0 & i) & 0 & 1 & 0 & 0 & 0 & 0 & D) & 0 \\
\hline 2030 & 0 & 1 & 0 & 0 & 0 & 1 & 0 & 0 & 0 & 0 & 0 & 0 & 0 & 0 & 0 & 0 & 0 & 0 & 0 & 0 & 0 & 1 & 0 & 0 & 0 & 0 & 0 & 0 \\
\hline 2045 & 0 & 0 & 0 & 0 & 0 & 0 & 0 & 0 & 0 & 0 & 0 & 0 & 0 & 0 & 0 & 0 & 0 & 0 & 0 & 0 & 0 & 0 & 0 & 0 & 0 & 0 & 0 & 0 \\
\hline 2100 & 0 & 0 & 0 & 0 & 0 & 0 & 0 & 0 & 0 & 0 & 0 & 0 & 0 & 0 & 0 & 0 & 0 & 0 & 0 & 0 & 0 & 0 & 0 & 0 & 0 & 0 & 0 & 0 \\
\hline 2115 & 0 & 0 & 0 & 0 & 0 & 0 & 0 & 0 & 0 & 0 & 0 & 0 & 0 & 0 & 0 & 0 & 0 & 0 & 0 & 0 & 0 & 0 & 0 & 0 & 0 & 0 & 0 & 0 \\
\hline 2130 & 0 & 0 & 0 & 0 & 0 & 0 & 0 & 0 & 0 & 0 & 0 & 0 & 0 & 0 & 0 & 0 & 0 & 0 & 0 & 0 & 0 & 0 & 0 & 0 & 0 & 0 & 0 & 0 \\
\hline 2145 & .0 & 0 & 0 & 0 & 0 & 0 & 0 & 0 & 0 & 0 & 0 & 0 & 0 & 0 & 0 & 0 & 0 & 0 & 0 & 0 & 0 & 0 & 0 & 0 & 0 & 0 & 0 & 0 \\
\hline 2200 & 0 & 0 & 0 & 0 & 0 & 0 & 0 & 0 & 0 & 0 & 0 & 0 & 0 & 0 & 0 & 0 & 0 & 0 & 0 & 0 & 1 & 1 & 0 & 0 & 0 & 0 & 0 & 0 \\
\hline 2215 & 0 & 0 & 0 & 0 & 0 & 0 & 0 & 0 & 0 & 0 & 0 & 0 & 0 & 0 & 0 & 0 & 0 & 0 & 0 & 0 & 0 & 1 & 0 & 0 & 0 & 0 & 0 & 0 \\
\hline 2230 & 0 & 0 & 0 & 0 & 0 & 0 & $\dot{0}$ & 0 & 0 & 0 & o & 0 & 0 & 0 & 0 & 0 & 0 & 0 & 0 & 0 & 0 & 1 & 0 & 0 & 0 & 0 & 0 & 0 \\
\hline 2245 & 0 & 0 & 0 & 0 & 0 & 0 & 0 & 0 & 0 & 0 & 0 & 0 & 0 & 0 & 0 & 0 & 0 & 0 & 0 & 0 & 0 & 1 & 0 & 0 & 0 & 0 & 0 & 0 \\
\hline 2300 & 0 & 0 & 0 & 0 & 0 & 0 & 0 & 0 & 0 & 0 & 0 & $\theta$ & 0 & 0 & 0 & 0 & 0 & 0 & 0 & 0 & 0 & 0 & 0 & 0 & 0 & 0 & 0 & 0 \\
\hline 2315 & 0 & 0 & 0 & 0 & 0 & 0 & 0 & 0 & 0 & 0 & 0 & 0 & 0 & 0 & 0 & 0 & 0 & 0 & 0 & 0 & 0 & 0 & 0 & 0 & 0 & 0 & 0 & 0 \\
\hline 2330 & 1 & 1 & 0 & 0 & 0 & 0 & 0 & 0 & 0 & 0 & 0 & 0 & 0 & 0 & 0 & 0 & 0 & 0 & 0 & 0 & 0 & 0 & 0 & 0 & 0 & 0 & 0 & 0 \\
\hline 2345 & 0 & 1 & 0 & 0 & 0 & 0 & 0 & 0 & 0 & 0 & 0 & 0 & 0 & 0 & 0 & 0 & 0 & 0 & 0 & 0 & 0 & 0 & 0 & 0 & 0 & 0 & 0 & 0 \\
\hline TOTAL & 2886 & NA & 0 & N/A & 2.315 & $\mathrm{~N} / \mathrm{A}$ & 0 & $N / n$ & 17 & $N / A$ & 0 & $\mathrm{~N} / \mathrm{A}$ & 10 & $\mathrm{~N} t$ & 0 & $N_{A}$ & 2.666 & $\mathrm{~N} / \mathrm{A}$ & & N/A & 2.868 & $N / \Lambda$ & & $\mathrm{N} / \mathrm{A}$ & \begin{tabular}{|l}
2.880 \\
\end{tabular} & $N / A$ & 0 & $\mathrm{~N} / \mathrm{A}$ \\
\hline AM & & 733 & & 8 & & 673 & & 0 & & 4 & & 0 & & 1 & & D & & 6.58 & & 0 & & 756 & & 0 & & 766 & & 0 \\
\hline NOON & & 438 & & 0 & & 417 & & 0 & & 4 & & 0 & & 2 & & 0 & & 425 & & 0 & & 449 & & 0 & & 412 & & 0 \\
\hline PM & & 108 & & 0 & & 98 & & 0 & & 4 & & 0 & & 2 & & 0 & & 67 & & 0 & & 74 & & 0 & & 83 & & 0 \\
\hline EVEN & & 3 & & 0 & & 1 & & 0 & & 0 & & 0 & & 2 & & 0 & & 0 & & 0 & & 1 & & 0 & & 0 & & 0 \\
\hline
\end{tabular}




\section{BAYMETRICS TRAFFIC RESOURCES Location 15: SBL on Vasco Road North of Westgate Drive}

\begin{tabular}{|c|c|c|c|c|c|c|c|c|c|c|c|c|c|c|c|c|c|c|c|c|c|c|c|c|c|c|c|c|}
\hline \multirow{3}{*}{ RME } & \multicolumn{4}{|c|}{$12 / 6 / 2001$ Thursday } & \multicolumn{4}{|c|}{$12 n 12001$ Friday } & \multicolumn{4}{|c|}{ 12/8/2001 Saturdoy } & \multicolumn{4}{|c|}{ 12/9:2001 Sunday } & \multicolumn{4}{|c|}{ 12/102001 Monday } & \multicolumn{4}{|c|}{ 12/11/2001 Tuesday } & \multicolumn{4}{|c|}{ 12/12/2001 Wednesdoy } \\
\hline & & & $15 \mathrm{MO}$ & $60 \mathrm{MN}$ & & $\begin{array}{l}B B L \\
60 \mathrm{MB}\end{array}$ & & $60 \mathrm{MTN}$ & & $\begin{array}{l}S B L \\
\text { an } 60 \mathrm{MmN}\end{array}$ & & & & $\begin{array}{l}S B L \\
\text { N } 60 \mathrm{MON}\end{array}$ & & & & $\begin{array}{l}S B L \\
O N G 0 \mathrm{MN}\end{array}$ & & & & $\begin{array}{l}S B L \\
\mathrm{~N} 60 \mathrm{MOD}\end{array}$ & & & & & & \\
\hline & & 2 & & & & & & 2 & & & & & & & & & & 5, & & & & & & & & & & \\
\hline 1200 & 0 & 0 & 0 & 0 & 1 & 0 & 0 & 0 & 0 & 0 & 0 & 0 & 0 & 0 & 0 & 0 & 0 & 0 & 0 & 0 & 0 & 0 & 0 & 0 & 0 & 0 & 0 & 0 \\
\hline 1215 & 0 & 0 & 0 & 0 & 0 & 0 & 0 & 0 & 0 & 0 & 0 & 0 & 0 & 0 & 0 & 0 & 0 & 0 & 0 & 0 & 0 & 0 & 0 & 0 & 0 & 0 & 0 & 0 \\
\hline $1230 !$ & 0 & 0 & 0 & 0 & 0 & 0 & 0 & 0 & 0 & 0 & 0 & 0 & 0 & 0 & 0 & 0 & 0 & 0 & 0 & 0 & 0 & 0 & 0 & 0 & 0 & 0 & 0 & 0 \\
\hline 12451 & 0 & 0 & 0 & 0 & 0 & 1 & 0 & 0 & 0 & 0 & 0 & 0 & 0 & 0 & 0 & 0 & 0 & 0 & 0 & 0 & 0 & 0 & 0 & 0 & 0 & 0 & 0 & 0 \\
\hline 100 & 0 & 0 & 0 & 0 & 0 & 0 & 0 & 0 & 0 & 0 & 0 & 0 & 0 & 0 & 0 & 0 & 0 & 0 & 0 & 0 & 0 & 0 & 0 & 0 & 0 & 0 & 0 & 0 \\
\hline 115 & 1 & 1 & 0 & 0 & 0 & 0 & 0 & 0 & 0 & 0 & 0 & 0 & 0 & 0 & 0 & 0 & 0 & 0 & 0 & 0 & 0 & 0 & 0 & 0 & 0 & 0 & 0 & 0 \\
\hline 130 & 0 & 1 & 0 & 0 & 0 & 0 & 0 & 0 & 0 & 0 & 0 & 0 & 1 & 1 & 0 & 0 & 0 & 0 & 0 & 0 & 0 & 0 & 0 & 0 & 0 & 0 & 0 & 0 \\
\hline 145 & 0 & 1 & 0 & 0 & 0 & 0 & 0 & 0 & 0 & 0 & 0 & 0 & 0 & 1 & 0 & 0 & 0 & 0 & 0 & 0 & 0 & 0 & 0 & 0 & 0 & 0 & 0 & 0 \\
\hline 200 & 0 & 1 & 0 & 0 & 0 & 0 & 0 & 0 & 0 & 0 & 0 & 0 & 0 & 1 & 0 & 0 & 0 & 0 & 0 & 0 & 0 & 0 & 0 & 0 & 0 & 0 & 0 & 0 \\
\hline 215 & 0 & 0 & 0 & 0 & 0 & 0 & 0 & 0. & 0 & 0 & 0 & 0 & 0 & 1 & 0 & 0 & 0 & 0 & 0 & 0 & 0 & 0 & 0 & 0 & 0 & 0 & 0 & 0 \\
\hline 230 & 0 & 0 & 0 & 0 & 0 & 0 & 0 & 0 & 0 & 0 & 0 & 0 & 0 & 0 & 0 & 0 & 0 & 0 . & 0 & 0 & 0 & 0 & 0 & 0 & 0 & 0 & 0 & 0 \\
\hline 245 & 0 & 0 & 0 & 0 & 0 & 0 & 0 & 0 & 0 & 0 & 0 & 0 & 0 & 0 & 0 & 0 & 0 & 0 & 0 & 0 & 0 & 0 & 0 & 0 & 0 & 0 & 0 & 0 \\
\hline 300 & 0 & 0 & 0 & 0 & 0 & 0 & 0 & 0 & 0 & 0 & 0 & 0 & 0 & 0 & 0 & 0 & 0 & 0 & 0 & 0 & 0 & 0 & 0 & 0 & 0 & 0 & 0 & 0 \\
\hline 315 & 0 & 0 & 0 & 0 & 0 & 0 & 0 & 0 & 0 & 0 & 0 & 0 & 0 & 0 & 0 & 0 & 0 & 0 & 0 & 0 & 0 & 0 & 0 & 0 & 0 & 0 & 0 & 0 \\
\hline 330 & 0 & 0 & 0 & 0 & 0 & 0 & 0 & 0 & 0 & 0 & 0 & 0 & 0 & 0 & 0 & 0 & 0 & 0 & 0 & 0 & 0 & 0 & 0 & 0 & 0 & 0 & 0 & 0 \\
\hline 345 & 0 & 0 & 0 & 0 & 0 & 0 & 0 & 0 & 0 & 0 & 0 & 0 & 0 & 0 & 0 & 0 & 0 & 0 & 0 & 0 & 0 & 0 & 0 & 0 & 0 & 0 & 0 & 0 \\
\hline 400 & 0 & 0 & 0 & 0 & 0 & 0 & 0 & 0 & 0 & 0 & 0 & 0 & 0 & 0 & 0 & 0 & 0 & 0 & 0 & 0 & 0 & 0 & 0 & 0 & 0 & 0 & 0 & 0 \\
\hline 415 & 0 & 0 & 0 & 0 & 0 & 0 & 0 & 0 & 0 & 0 & 0 & 0 & 0 & 0 & 0 & 0 & 0 & 0 & 0 & 0 & 0 & 0 & 0 & 0 & 0 & 0 & 0 & c \\
\hline 430 & 0 & 0 & 0 & 0 & 0 & 0 & 0 & 0 & 0 & 0 & 0 & 0 & 0 & 0 & 0 & 0 & 0 & 0 & 0 & 0 & 0 & 0 & 0 & 0 & 0 & 0 & 0 & 0 \\
\hline 445 & 0 & 0 & 0 & 0 & 0 & 0 & 0 & 0 & 0 & 0 & 0 & 0 & 0 & 0 & 0 & 0 & 0 & 0 & 0 & 0 & 0 & 0 & 0 & 0 & 0 & 0 & 0 & 0 \\
\hline 500 & 0 & 0 & 0 & 0 & 0 & 0 & 0 & 0 & 0 & 0 & 0 & 0 & 0 & 0 & 0 & 0 & 0 & 0 & 0 & 0 & 0 & 0 & 0 & 0 & 0 & 0 & 0 & 0 \\
\hline 515 & 0 & 0 & 0 & 0 & 0 & 0 & 0 & 0 & 0 & 0 & 0 & 0 & 1 & 1 & 0 & 0 & 0 & 0 & 0 & 0 & 0 & 0 & 0 & 0 & 0 & 0 & 0 & 0 \\
\hline$\$ 30$ & 0 & 0 & 0 & 0 & 0 & 0 & 0 & 0 & 0 & 0 & 0 & 0 & 0 & 1 & 0 & 0 & 0 & 0 & 0 & 0 & 0 & 0 & 0 & 0 & 0 & 0 & 0 & 0 \\
\hline 545 & 0 & 0 & 0 & 0 & 0 & 0 & 0 & y & 0 & 0 & 0 & 0 & 0 & 1 & 0 & 0 & 0 & 0 & 0 & 0 & 0 & 0 & 1) & 0 & 0 & 0 & 0 & 0 \\
\hline 600 & 0 & 0 & 0 & 0 & 0 & 0 & 0 & 0 & 1) & 0 & 0 & 0 & 0 & 1 & 0 & 0 & 1 & 1 & 0 & 0 & 0 & 0 & 0 & 0 & 1 & 1 & 0 & 0 \\
\hline 615 & 0 & 0 & 0 & 0 & 0 & 0 & 0 & 0 & 0 & 0 & 0 & 0 & 0 & 0 & 0 & 0 & 1 & 2 & 0 & 0 & 7 & 7 & 0 & 0 & 2 & 3 & 0 & 0 \\
\hline 630 & 4 & 4 & 0 & 0 & 0 & 0 & 0 & 0 & $\dot{v}$ & 0 & 0 & 0 & 0 & 0 & 0 & 0 & 12 & 14 & 0 & 0 & 13 & 20 & 0 & 0 & 11 & 14 & 0 & 0 \\
\hline 645 & 40 & 44 & 0 & 0 & 27 & 27 & 0 & 0 & 0 & 0 & 0 & () & 0 & 0 & 0 & 0 & 68 & 82 & 0 & 0 & 75 & 92 & 0 & 0 & 57 & 71 & 0 & 0 \\
\hline 700 & 97 & $14 !$ & 0 & 0 & 98 & 125 & 0 & 0 & 0 & 0 & 0 & 0 & 0 & 0 & 0 & 0 & 114 & 195 & 0 & 0 & 107 & 199 & 0 & 0 & 125 & & 0 & \\
\hline 715 & 126 & 267 & 0 & 0 & 111 & 236 & 0 & 0 & 2 & 2 & 0 & 0 & 0 & 0 & 0 & 0 & 110 & 304 & 0 & 0 & 128 & 320 & 0 & 0 & 99 & 292 & 0 & 0 \\
\hline 730 & 112 & 375 & 0 & 0 & 123 & 359 & 0 & 0 & 0 & 2 & 0 & 0 & 0 & 0 & 0 & 0 & 118 & 410 & 0 & 0 & 111 & 418 & 0 & 0 & 120 & 401 & 0 & 0 \\
\hline 745 & 145 & 480 & 0 & 0 & 127 & 459 & 0 & 0 & 0 & 2 & 0 & 0 & 0 & 0 & 0 & 0 & 139 & 481 & 0 & 0 & 133 & 479 & 0 & 0 & 172 & 516 & D & 0 \\
\hline 800 & 169 & 552 & 0 & 0 & 153 & 514 & 0 & 0 & $=$ & 4 & 0 & 0 & 0 & 0 & 0 & 0 & 197 & $\$ 64$ & 0 & 0 & 206 & 578 & 0 & 0 & 209 & & 0 & 0 \\
\hline 815 & 215 & $0+1$ & 0 & 0 & 199 & 602 & 0 & 0 & 0 & 2 & 0 & 0 & 0 & 0 & 0 & 0 & 164 & 018 & i) & 0 & 219 & 659 & 0 & 0 & 205 & & 0 & 0 \\
\hline 830 & 194 & 723 & 0 & 0 & 166 & 645 & 0 & 0 & 0 & 2 & 0 & 0 & 0 & 0 & 0 & 0 & 158 & 658 & 0 & 0 & 188 & 746 & 0 & 0 & 180 & 766 & 0 & 0 \\
\hline 845 & 155 & 733 & 0 & 0 & 155 & 673 & 0 & 0 & 0 & 2 & 0 & 0 & 0 & 0 & 0 & 0 & 131 & 650 & 0 & 0 & 143 & 756 & 0 & 0 & 151 & 745 & 0 & 0 \\
\hline 900 & 151 & 715 & 0 & 0 & 119 & 639 & 0 & 0 & 0 & 0 & 0 & 0 & 0 & 0 & 0 & 0 & 117 & 570 & 0 & 0 & 132 & 682 & 0 & 0 & 139 & 675 & 0 & 0 \\
\hline 915 & 97 & 597 & 0 & 0 & 105 & 545 & 0 & 0 & 0 & 0 & 0 & 0 & 0 & 0 & 0 & 0 & 99 & 505 & 0 & 0 & 100 & 563 & 0 & 0 & 101 & 571 & 0 & 0 \\
\hline 930 & 103 & 506 & 0 & 0 & 105 & 484 & 0 & 0 & 1 & 1 & 0 & 0 & 0 & 0 & 0 & 0 & 81 & 428 & 0 & 0 & 85 & 460 & 0 & 0 & 78 & 469 & 0 & 0 \\
\hline 945 & 59 & 410 & 0 & 0 & 79 & 408 & 0 & 0 & 2 & 3 & 0 & 0 & 0 & 0 & 0 & 0 & 59 & 356 & 0 & 0 & 71 & 388 & 0 & 0 & 59 & 377 & 0 & 0 \\
\hline 1000 & 67 & 326 & 0 & 0 & 60 & 349 & 0 & $a$ & 0 & 3 & 0 & 0 & 0 & 0 & 0 & 0 & 45 & 284 & 0 & 0 & 29 & 285 & 0 & 0 & 51 & & 0 & 0 \\
\hline 1015 & 39 & 268 & 0 & 0 & 48 & 292 & 0 & 0 & 0 & 3 & $\dot{u}$ & 0 & 0 & 0 & 0 & 0 & 52 & 237 & 0 & 0 & 33 & 218 & 0 & $a$ & 34 & 222 & 0 & 0 \\
\hline 1030 & 37 & 202 & 0 & 0 & 39 & 226 & 0 & 0 & 0 & 2 & 0 & 0 & 0 & 0 & 0 & 0 & 37 & 193 & 0 & 0 & 43 & 176 & 0 & 0 & 37 & 181 & 0 & 0 \\
\hline 1045 & 25 & 168 & 0 & 0 & 32 & 179 & 0 & 0 & 0 & 0 & 0 & 0 & 0 & 0 & 0 & 0 & 38 & 172 & 0 & 0 & 30 & 135 & 0 & 0 & 23 & 145 & 0 & 0 \\
\hline 1100 & 14 & 115 & 0 & 0 & 21 & 140 & 0 & 0 & 0 & 0 & 0 & 0 & 0 & 0 & 0 & 0 & 43 & 170 & 0 & 0 & 47 & 153 & 0 & 0 & 35 & 129 & 0 & 0 \\
\hline 1115 & 33 & 109 & 0 & 0 & 23 & 115 & 0 & 0 & 1 & 1 & 0 & 0 & 2 & 2 & 0 & 0 & 25 & 143 & 0 & 0 & 28 & 148 & 0 & 0 & 20 & 115 & 0 & 0 \\
\hline 1130 & 31 & 103 & 0 & 0 & 24 & 100 & 0 & 0 & 3 & 4 & 0 & 0 & 0 & 2 & 0 & 0 & 30 & 136 & 0 & 0 & 20 & 134 & 0 & 0 & 26 & 104 & 0 & 0 \\
\hline 1145 & 15 & 93 & 0 & 0 & 34 & 102 & 0 & 0 & 0 & 4 & 0 & 0 & 0 & 2 & 0 & 0 & 34 & 132 & 0 & 0 & 24 & 128 & 0 & 0 & 32 & 113 & 0 & 0 \\
\hline
\end{tabular}




\section{BAYMETRICS TRAFFIC RESOURCES Location 16: Northbound Right-Turn On Vasco South of Westgate}

\begin{tabular}{|c|c|c|c|c|c|c|c|c|c|c|c|c|c|c|c|c|c|c|c|c|c|c|c|c|c|c|c|c|}
\hline & & 6,20 & Thu & & & $2 \pi / 2$ & $F$ & & & $8 / 20$ & Sa & & & $12 / 9$ & & & & $12 / 10120$ & Mo & & & $8 / 11 / 2$ & $T$ & & & $2 / 200$ & Wec & \\
\hline & & $B R$ & & & & $B R$ & & & & & & & & $\mathrm{NBR}$ & & & & $N B R$ & & & & /BR & & & & $B R$ & & \\
\hline & & & & & & & & & & & & & & & & & & & & & & & & & & & & \\
\hline 1200 & 0 & 0 & 0 & 0 & 0 & 0 & 0 & 0 & 0 & 0 & 0 & 0 & & & & 0 & 0 & 0 & 0 & 0 & 0 & 0 & 0 & 0 & 0 & 0 & 0 & 0 \\
\hline 1215 & 0 & 0 & 0 & 0 & 0 & 0 & 0 & 0 & 0 & 0 & 0 & 0 & & & & 0 & 0 & 0 & 0 & 0 & 0 & 0 & 0 & 0 & 0 & 0 & 0 & 0 \\
\hline 1230 & 0 & 0 & 0 & 0 & 0 & 0 & 0 & 0 & 0 & 0 & 0 & 0 & & 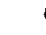 & & 0 & 0 & 0 & 0 & 0 & 0 & 0 & 0 & 0 & 0 & 0 & 0 & 0 \\
\hline 1245 & 0 & 0 & 0 & 0 & 0 & 0 & 0 & 0 & 0 & 0 & 0 & 0 & & & & 0 & 0 & 0 & 0 & 0 & 0 & 0 & 0 & 0 & 0 & 0 & 0 & 0 \\
\hline 100 & 0 & 0 & 0 & 0 & 0 & 0 & 0 & 0 & 0 & 0 & 0 & 0 & & 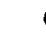 & & 0 & 0 & 0 & 0 & 0 & 0 & 0 & 0 & 0 & 0 & 0 & 0 & 0 \\
\hline 115 & 0 & 0 & 0 & 0 & 0 & 0 & 0 & 0 & 0 & 0 & 0 & 0 & & 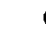 & & 0 & 0 & 0 & 0 & 0 & 0 & 0 & 0 & 0 & 0 & 0 & 0 & 0 \\
\hline 130 & 0 & 0 & 0 & 0 & 0 & 0 & 0 & 0 & 0 & 0 & 0 & 0 & & 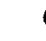 & & 0 & 0 & 0 & 0 & 0 & 0 & 0 & 0 & 0 & 0 & 0 & 0 & 0 \\
\hline 145 & 0 & 0 & 0 & 0 & 0 & 0 & 0 & 0 & 0 & 0 & 0 & 0 & & ( & & 0 & 0 & 0 & 0 & 0 & 0 & 0 & 0 & 0 & 0 & 0 & 0 & 0 \\
\hline 200 & 0 & 0 & 0 & 0 & 0 & 0 & 0 & 0 & 0 & 0 & 0 & 0 & 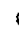 & 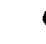 & & 0 & 0 & 0 & 0 & 0 & 0 & 0 & 0 & 0 & 0 & 0 & 0 & 0 \\
\hline 215 & 0 & 0 & 0 & 0 & 0 & 0 & 0 & 0 & 0 & 0 & 0 & 0 & & ( & & 0 & 0 & 0 & 0 & 0 & 0 & 0 & 0 & 0 & 0 & 0 & 0 & 0 \\
\hline 230 & 0 & 0 & 0 & 0 & 0 & 0 & 0 & 0 & 0 & 0 & 0 & 0 & & 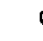 & & 0 & 0 & 0 & 0 & 0 & 0 & 0 & 0 & $\cdot 0$ & 0 & 0 & 0 & 0 \\
\hline 245 & 0 & 0 & 0 & 0 & 0 & 0 & 0 & 0 & 0 & 0 & 0 & 0 & & I & & $\underline{0}$ & 0 & 0 & 0 & 0 & 0 & 0 & 0 & 0 & 0 & 0 & 0 & 0 \\
\hline 300 & 0 & 0 & 0 & 0 & 0 & 0 & 0 & 0 & 0 & 0 & 0 & 0 & & 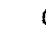 & & 0 & 0 & 0 & 0 & 0 & 0 & 0 & 0 & 0 & 0 & 0 & 0 & 0 \\
\hline 315 & 0 & 0 & 0 & 0 & 0 & 0 & 0 & 0 & 1 & 1 & 0 & 0 & & 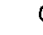 & & 0 & 0 & 0 & 0 & 0 & 0 & 0 & 0 & 0 & 0 & 0 & 0 & 0 \\
\hline 330 & 0 & 0 & 0 & 0 & 0 & 0 & 0 & 0 & 0 & 1 & 0 & 0 & 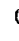 & 0 & & 0 & 0 & 0 & 0 & 0 & 0 & 0 & 0 & 0 & 0 & 0 & 0 & 0 \\
\hline 3.45 & 1 & 1 & 0 & 0 & 0 & 0 & 0 & 0 & 0 & 1 & 0 & 0 & 1 & 0 & & 0 & 0 & 0 & 0 & 0 & 0 & 0 & 0 & 0 & 0 & 0 & 0 & 0 \\
\hline 400 & 0 & 1 & 0 & 0 & 0 & 0 & 0 & a & 0 & 1 & 0 & 0 & 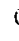 & 0 & & 0 & 0 & 0 & 0 & 0 & 0 & 0 & 0 & 0 & 0 & 0 & 0 & 0 \\
\hline 415 & 0 & 1 & 0 & 0 & 0 & 0 & 0 & 0 & 0 & 0 & 0 & 0 & 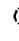 & i & & 0 & 0 & 0 & 0 & 0 & 0 & 0 & 0 & 0 & 0 & 0 & 0 & 0 \\
\hline 430 & 0 & 1 & 0 & 0 & 0 & 0 & 0 & 0 & 0 & 0 & 0 & 0 & 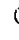 & 0 & & 0 & 0 & 0 & 0 & 0 & 0 & 0 & 0 & 0 & 0 & 0 & 0 & 0 \\
\hline 445 & 0 & 0 & 0 & 0 & 0 & 0 & 0 & 0 & 0 & 0 & 0 & 0 & 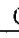 & $\underline{0}$ & & 0 & 0 & 0 & 0 & 0 & 0 & 0 & 0 & 0 & 0 & 0 & 0 & 0 \\
\hline 500 & 0 & 0 & 0 & 0 & 0 & 0 & 0 & 0 & 0 & 0 & 0 & 0 & 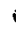 & 1 & & 0 & 0 & 0 & 0 & 0 & 0 & 0 & 0 & 0 & 0 & 0 & 0 & 0 \\
\hline 515 & 0 & 0 & 0 & 0 & 0 & 0 & 0 & 0 & 0 & 0 & 0 & 0 & 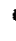 & 0 & & 0 & 0 & 0 & 0 & 0 & 0 & 0 & 0 & 0 & 0 & 0 & 0 & 0 \\
\hline$\leq 30$ & 0 & 0 & 0 & 0 & 0 & 0 & 0 & 0 & 0 & 0 & 0 & 0 & & 0 & & 0 & 0 & 0 & 0 & 0 & 0 & 0 & 0 & 0 & 0 & 0 & 0 & 0 \\
\hline 545 & 1 & 1 & 0 & 0 & 0 & 0 & 0 & 0 & 0 & $\mathfrak{j}$ & 0 & 0 & $i$ & 0 & & 0 & 0 & 0 & 0 & 0 & 1 & 1 & 0 & 0 & 0 & 0 & 0 & 0 \\
\hline 600 & 0 & 1 & 0 & i & 0 & 0 & 0 & $\dot{0}$ & 0 & 0 & 0 & () & 1 & 0 & & 0 & 1 & 1 & 0 & 0 & 0 & 1 & 0 & 0 & 0 & 0 & 0 & 0 \\
\hline 615 & 3 & 4 & 0 & 0 & ij & 0 & 0 & 0 & 0 & 0 & 0 & 0 & 4 & 0 & & 0 & 2 & 3 & 0 & 0 & 1 & 2 & 0 & 0 & 1 & 1 & 0 & 0 \\
\hline 630 & 6 & 10 & 0 & 0 & 5 & $s$ & 0 & 0 & 0 & 0 & 0 & 0 & 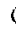 & 0 & & 0 & 6 & 9 & 0 & 0 & 6 & 8 & 0 & 0 & 5 & 6 & 0 & 0 \\
\hline 645 & 25 & 34 & 0 & 0 & 23 & 28 & 0 & 0 & ) & 0 & 0 & 0 & 0 & 0 & & 0 & 22 & 31 & 0 & 0 & 22 & 29 & 0 & 0 & 25 & 31 & 0 & 0 \\
\hline 700 & 48 & 82 & 0 & 0 & 50 & 78 & 0 & 0 & I & 1 & 0 & 0 & 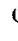 & 0 & & 0 & 37 & 67 & 0 & 0 & 43 & 72 & 0 & 0 & 47 & 78 & 0 & 0 \\
\hline 715 & 49 & 128 & 0 & 0 & 39 & 117 & 0 & 0 & 0 & 1 & 0 & 0 & 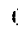 & 0 & & 0 & 48 & 113 & 0 & 0 & 64 & $i 35$ & 0 & 0 & 48 & 125 & 0 & $c$ \\
\hline 730 & 65 & 187 & 0 & $\tilde{0}$ & 48 & 160 & 0 & 0 & 0 & 1 & 0 & 0 & 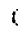 & 0 & & 0 & 43 & 150 & 0 & 0 & 56 & 185 & 0 & 0 & so & 170 & 0 & 0 \\
\hline 745 & 64 & 226 & 0 & 0 & 60 & 197 & 0 & 0 & 0 & 1 & (1) & 0 & 1 & 0 & I & 0 & 58 & 186 & 0 & 0 & 59 & 222 & 0 & 0 & 64 & 209 & 0 & 0 \\
\hline 800 & 82 & 260 & 0 & 0 & 76 & 223 & 0 & 0 & 0 & 0 & 1) & 0 & 0 & 0 & 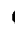 & 0 & 84 & 233 & 0 & 0 & 92 & 271 & 0 & 0 & 81 & 243 & 0 & 0 \\
\hline 815 & 72 & 283 & 0 & 0 & 87 & 271 & 0 & 0 & 0 & 0 & 0 & 0 & 0 & 0 & 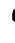 & 0 & 77 & 262 & 0 & 0 & 80 & 287 & 0 & 0 & 71 & 260 & 0 & 0 \\
\hline 830 & 63 & 281 & 0 & 0 & 54 & 277 & 0 & 0 & 1 & 1 & 0 & 0 & $?$ & 0 & 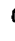 & 0 & 62 & $\cdot 281$ & 0 & 0 & 76 & 307 & 0 & 0 & 69 & 285 & 0 & 0 \\
\hline 845 & 71 & 288 & 0 & 0 & 67 & 284 & 0 & 0 & 1 & $=$ & 0 & 0 & $i$ & 0 & 1 & 0 & 79 & 302 & 0 & 0 & 80 & 328 & 0 & 0 & 63 & 284 & 0 & $a$ \\
\hline 900 & 49 & 255 & 0 & 0 & 41 & 249 & 0 & 0 & 0 & 2 & 0 & 0 & 1) & 0 & 8 & 0 & 50 & 268 & 1) & 0 & 53 & 289 & 0 & 0 & $\subseteq 2$ & 255 & 0 & 0 \\
\hline 915 & 36 & 219 & 0 & 0 & 44 & 206 & 0 & 0 & 0 & 2 & 0 & 0 & 0 & 0 & $c$ & 0 & 38 & 299 & 0 & 0 & 26 & 235 & 0 & 0 & 41 & 225 & 0 & 0 \\
\hline 930 & 38 & 194 & 0 & 0 & 32 & 184 & 0 & 0 & 0 & 1 & 0 & 0 & 0 & 0 & 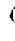 & 0 & 21 & 188 & 0 & 0 & 30 & 189 & 0 & 0 & 28 & 184 & 0 & 0 \\
\hline 945 & 20 & 143 & 0 & 0 & 16 & 133 & 0 & $\underline{0}$ & 0 & (1) & 0 & 0 & 0 & 0 & 0 & 0 & 27 & 136 & 0 & 0 & 25 & 134 & 0 & 0 & 26 & 147 & 0 & 0 \\
\hline 1000 & 8 & 102 & 0 & 0 & 15 & 107 & 0 & 0 & 0 & () & 0 & 0 & 0 & 0 & c & 0 & 19 & 105 & 0 & 0 & 13 & 94 & 0 & 0 & 20 & 115 & 0 & 0 \\
\hline 1015 & 15 & 81 & 0 & 0 & 14 & 77 & 0 & 0 & 0 & 0 & 0 & 0 & 0 & 0 & 1 & 0 & 16 & 83 & 0 & 0 & 20 & 88 & 0 & 0 & 24 & 98 & 0 & 0 \\
\hline 1030 & 15 & 58 & 0 & 0 & 17 & 62 & 0 & 0 & 0 & D) & 0 & 0 & 0 & ) & $r$ & 0 & 9 & 71 & 0 & 0 & 18 & 76 & 0 & 0 & $s$ & 75 & 0 & 0 \\
\hline 1045 & 8 & +6 & 0 & $\dot{0}$ & 8 & $\$ 4$ & 0 & 0 & 1) & 0 & 0 & 1) & a & 0 & 1 & D) & 10 & $\$ .4$ & 0 & 0 & 18 & 69 & 0 & 0 & 11 & 60 & 0 & 0 \\
\hline 1100 & 8 & 46 & 0 & 0 & 14 & 53 & 0 & 0 & 0 & 0 & 0 & 0 & 0 & 0 & $s$ & 0 & 15 & 50 & 0 & 0 & 12 & 68 & 0 & 0 & 17 & 57 & 0 & 0 \\
\hline 1115 & 9 & 40 & 0 & 0 & 8 & 47 & 0 & 0 & 0 & 0 & 0 & 0 & 0 & 0 & $c$ & 0 & 9 & 43 & 0 & 0 & 14 & 62 & 0 & 0 & 12 & 45 & 0 & 0 \\
\hline 1130 & 12 & 37 & 0 & 0 & 12 & 42 & 0 & 0 & 0 & 0 & 0 & 0 & 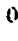 & 0 & 0 & 0 & 19 & 53 & 0 & 0 & 9 & 53 & 0 & 0 & 12 & $\$ 2$ & 0 & 0 \\
\hline 1845 & 10 & 39 & 0 & 0 & 14 & 48 & 0 & 0 & 0 & 0 & 0 & 0 & 0 & 0 & 0 & 0 & 20 & 63 & 0 & 0 & 12 & 47 & 0 & 0 & 13 & $\$ 4$ & 0 & 0 \\
\hline
\end{tabular}




\section{BAYMETRICS TRAFFIC RESOURCES Location 16: Northbound Right-Turn On Vasco South of Westgate}

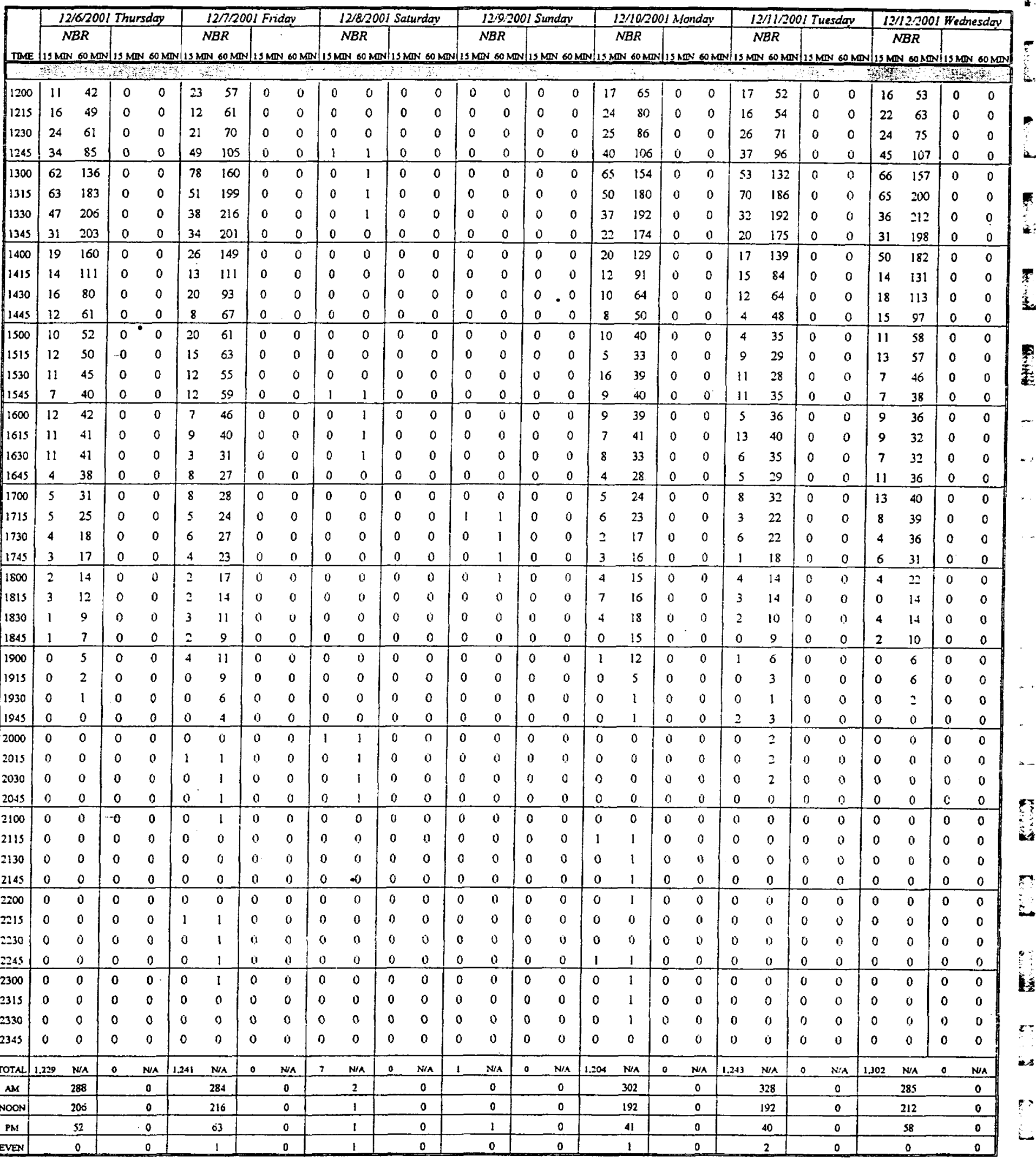




\section{BAYMETRICS TRAFFIC RESOURCES Location 17: Westgate Drive East of Vasco Road}

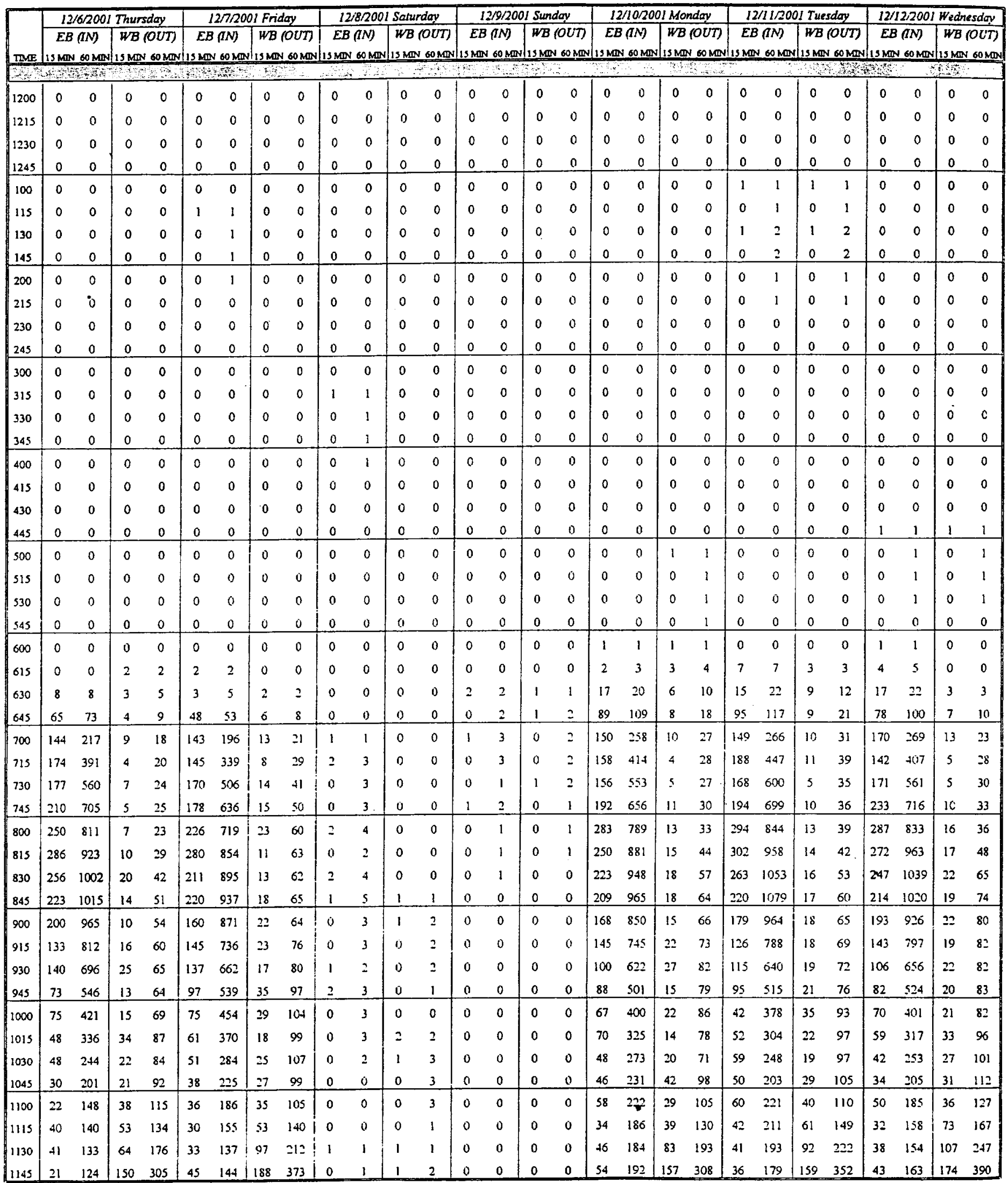




\section{BAYMETRICS TRAFFIC RESOURCES Location 17: Westgate Drive East of Vasco Road}

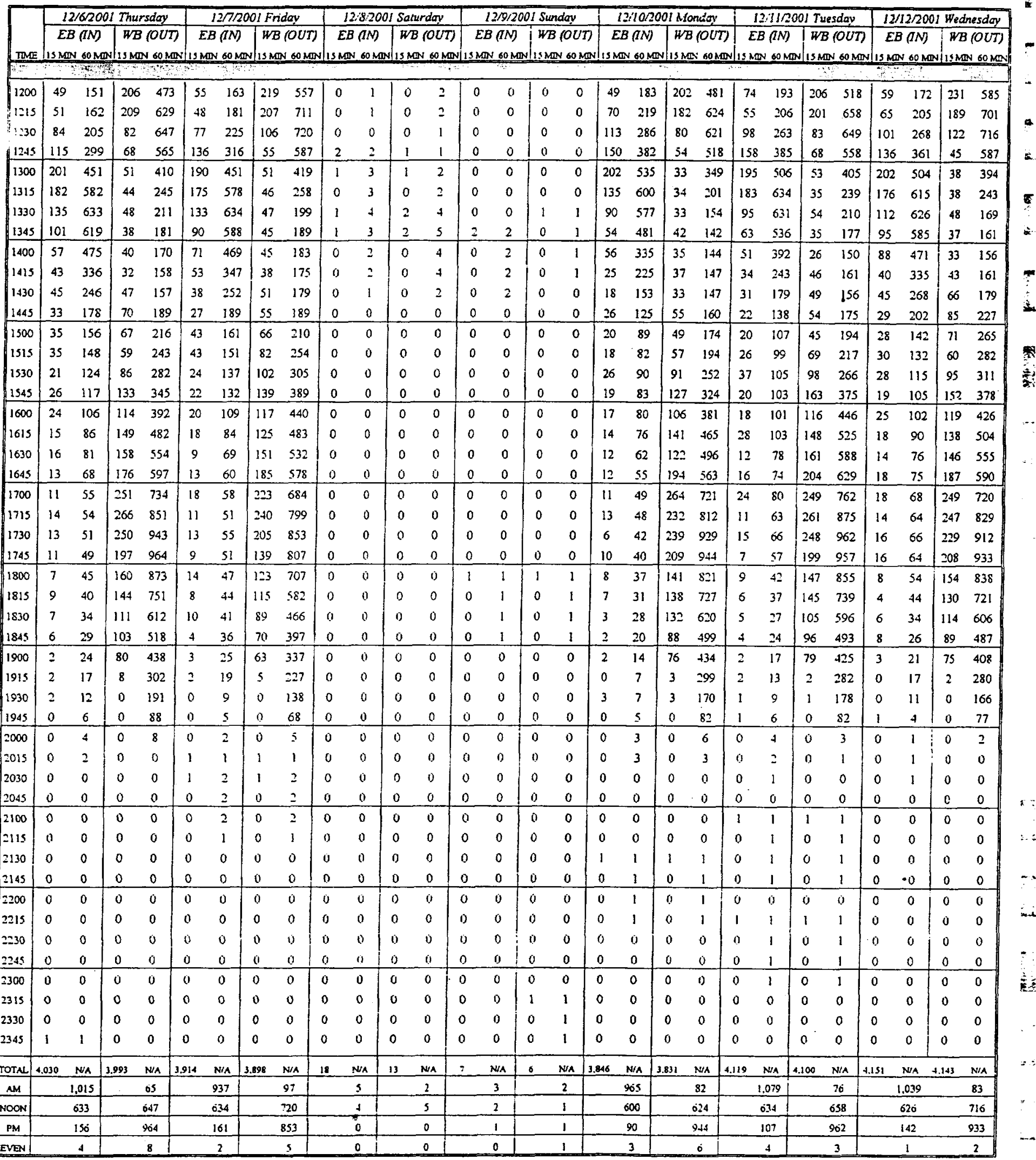




\section{B. INTERSECTION TRAFFIC COUNTS}

- East Avenue and Vasco Road

- East Avenue and Greenville Road

December 6, 2001 



\section{BAYMETRICS TRAFFIC RESOURCES INTERSECTION TURNING MOVEMENT SUMMARY}

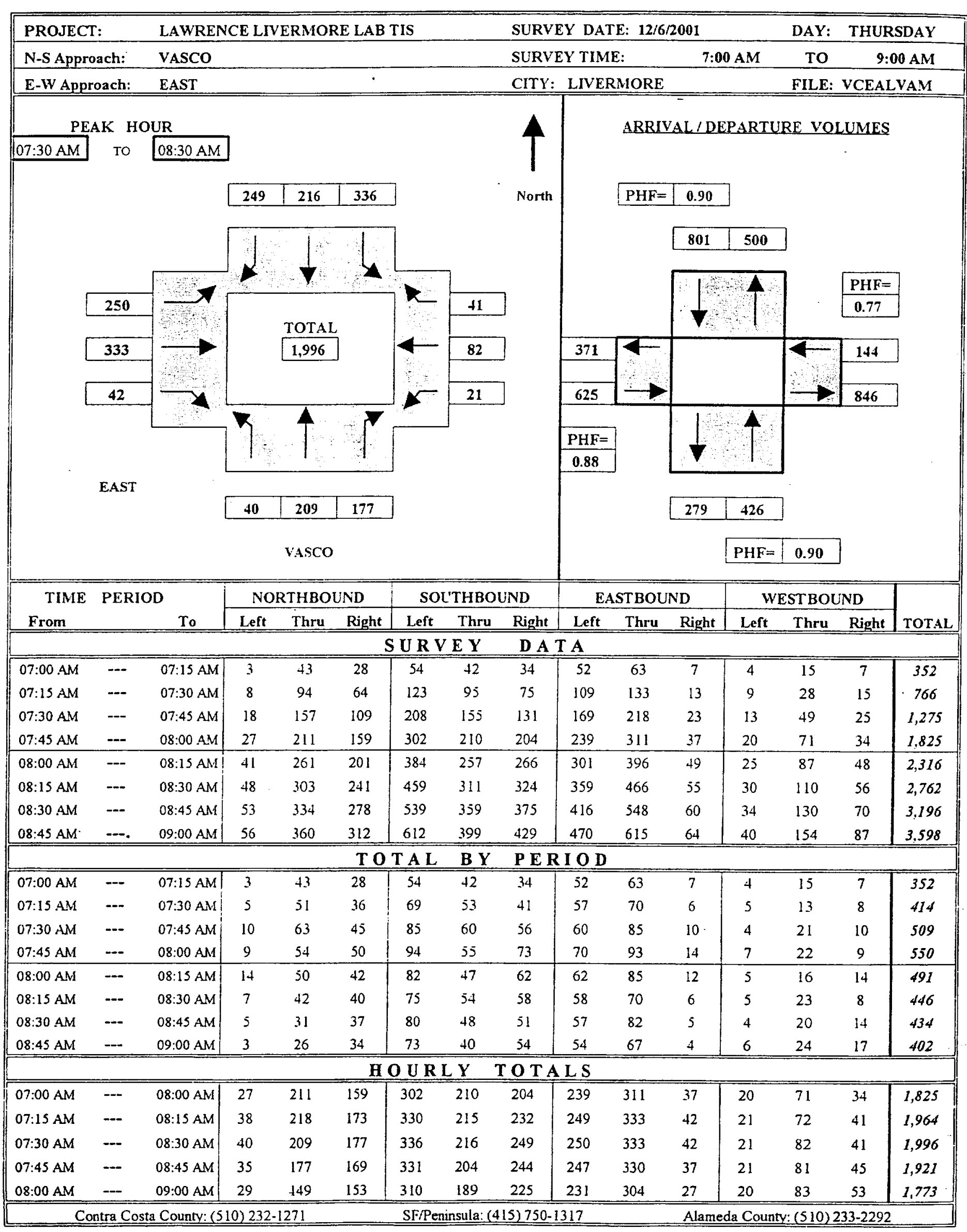




\section{BAYMETRICS TRAFFIC RESOURCES INTERSECTION TURNING MOVEMENT SUMMARY}

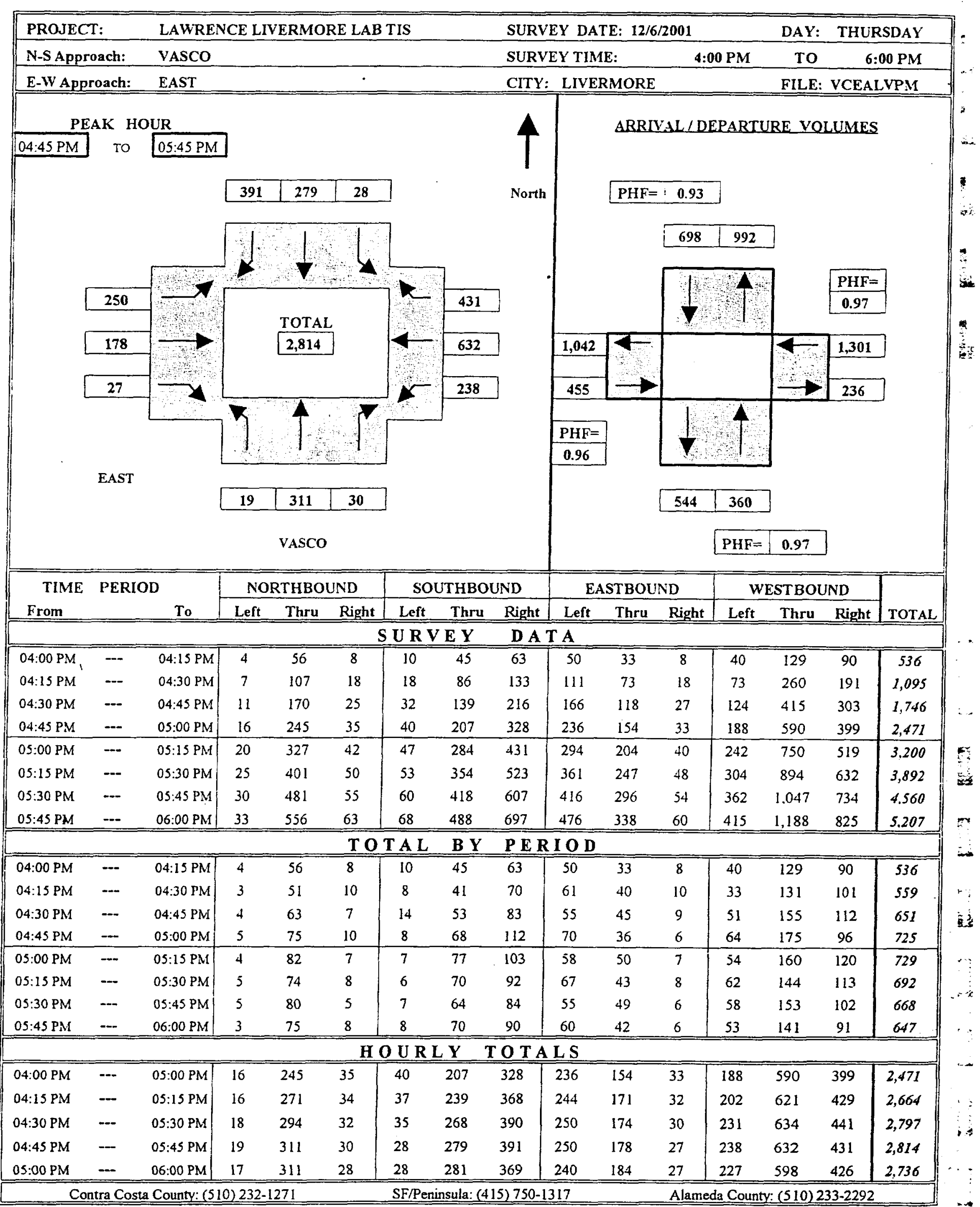




\section{BAYMETRICS TRAFFIC RESOURCES}

INTERSECTION TURNING MOVEMENT SUMMARY

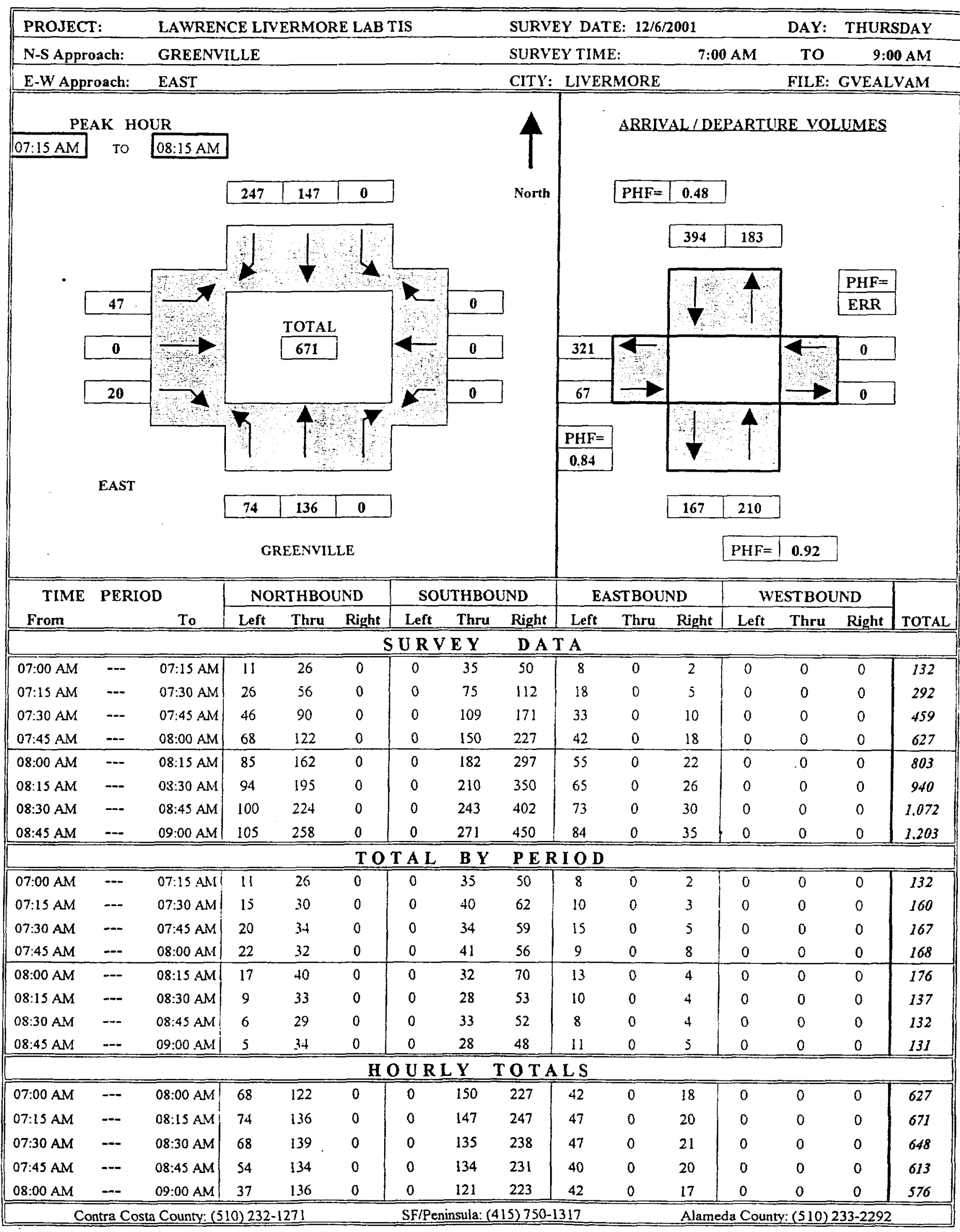




\section{BAYMETRICS TRAFFIC RESOURCES INTERSECTION TURNING MOVEMENT SUMMARY}

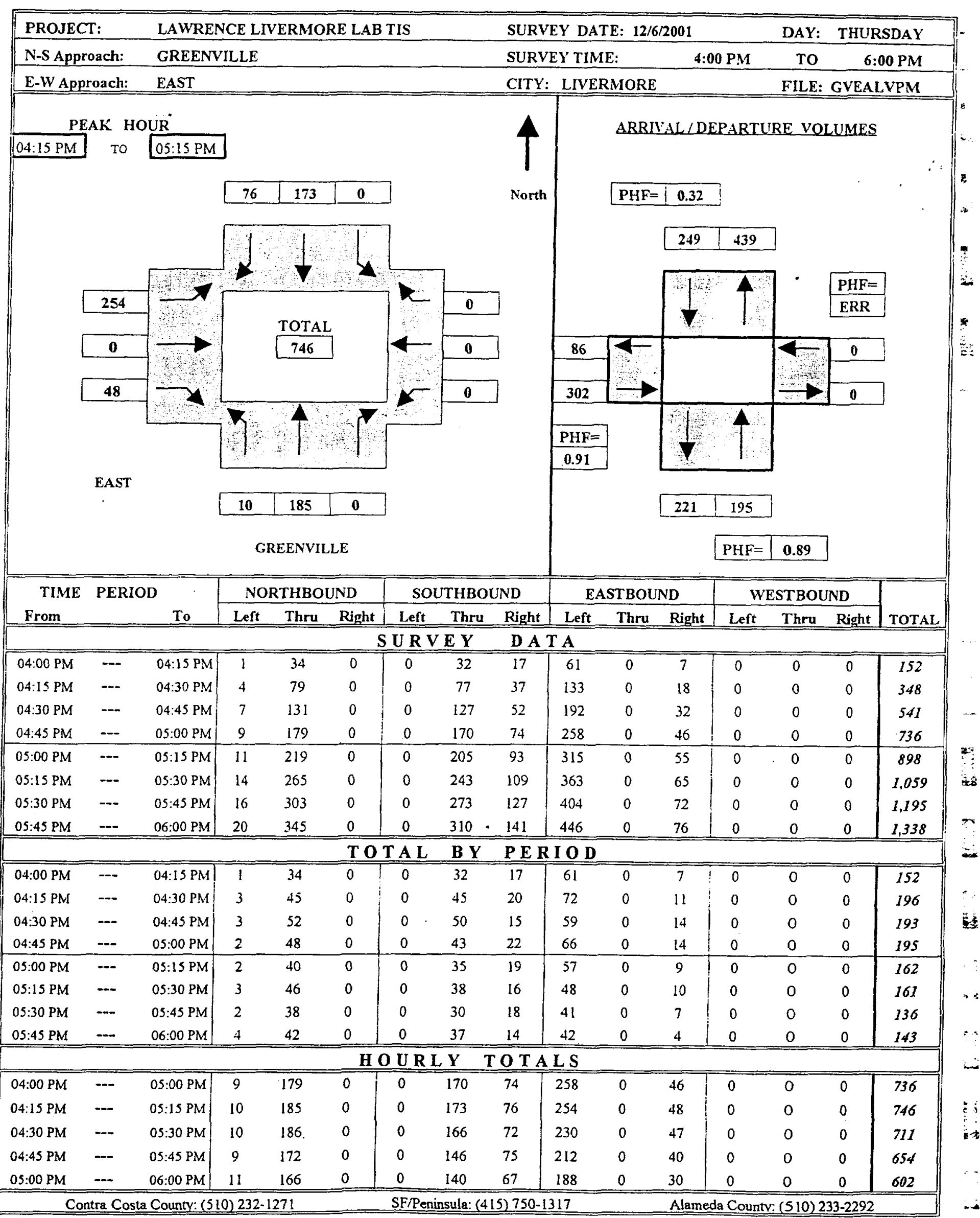


C. TRUCK COUNTS 



\section{Truck Counts}

Locations 7 and 8

Thursday, December 6, 2001 



\section{Baymetrics Traffic Resources INTERSECTION TURNING MOVEMENT SUMMARY}

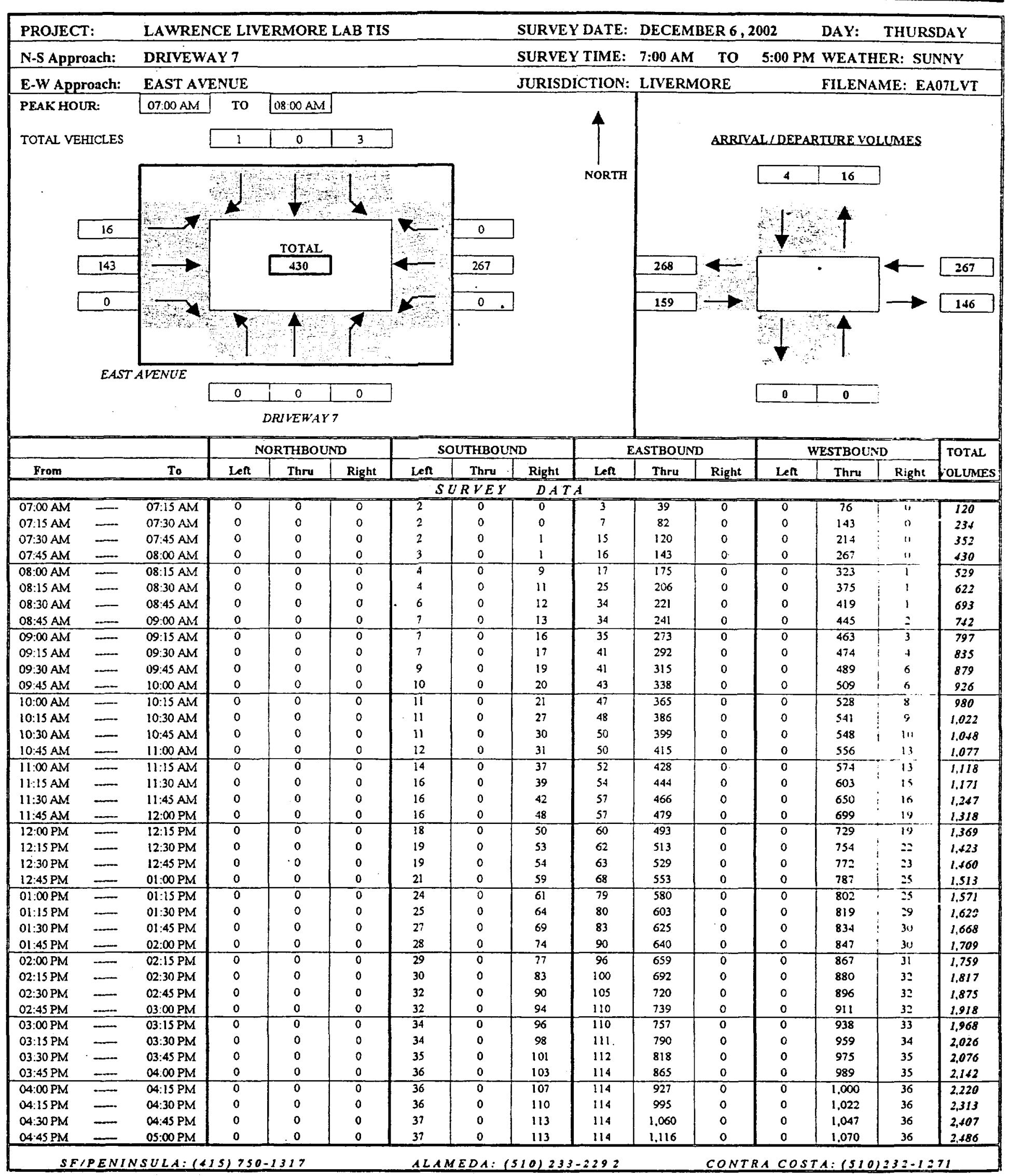




\section{Baymetrics Traffic Resources INTERSECTION TURNING MOVEMENT SUMMARY}

\begin{tabular}{|c|c|c|c|c|c|c|c|c|c|c|c|c|c|c|c|}
\hline \multirow{3}{*}{\multicolumn{2}{|c|}{$\begin{array}{l}\text { PLzo.Jic:T: } \\
\text { N-S Apprnach: } \\
\text { J-W Apprnach: }\end{array}$}} & \multirow{2}{*}{\multicolumn{6}{|c|}{$\begin{array}{l}\text { IAWRJNCE LIVRMORE LAB TIS } \\
\text { IJIVIEWAY } 7\end{array}$}} & \multirow{2}{*}{\multicolumn{2}{|c|}{$\begin{array}{l}\text { SURVEY DATKS: } \\
\text { SLRRVY TIME: }\end{array}$}} & \multicolumn{3}{|c|}{ DIXCK:MIBFK G, 20012} & DAY: & THusks & DAY \\
\hline & & & & & & & & & & $7: 00 \mathrm{A.M}$ & TO & 5:01 IM & WEATH & [E,R: SU] & NNY \\
\hline & & LAST AT & YUE & & & & & IUKSSI) & TIOSN & : $1.1 \mathrm{VHIR.1}$ & ORE: & & ITIJIS.XA & ME: $\mathrm{ZA}$ & $07 \mathrm{LVT}$ \\
\hline & & & & Timot & & & minot & & & EASTHOUN & & & vestruoun & & Total, \\
\hline Prons & & Iu & $1 \leq a$ & Thru. & Righte & ien & Thru: & Rlght & Len & Thmi & 12uith & Len & Ileru & Kigint & yor.un:rs:s \\
\hline & & & & & & To & $L B$ & $P E R I$ & & & & & & & \\
\hline $\begin{array}{l}07.00 \mathrm{AM} \\
07: 15 \mathrm{AM}\end{array}$ & $\ldots$ & n7 $3 \wedge \mathrm{MM}$ & 0 & $0^{\circ}$ & $0^{0}$ & 2 & 0 & a & 7 & 3 & 5 & 6 & 26 & 0 & $m$ \\
\hline $00: 30 \mathrm{AM}$ & - & $07: 15 \cap M$ & $\begin{array}{l}0 \\
n\end{array}$ & $\because$ & i & 0 & $a$ & 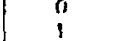 & $\hat{x}$ & $\begin{array}{l}43 \\
38\end{array}$ & $\begin{array}{l}0 \\
0\end{array}$ & $\begin{array}{l}0 \\
0\end{array}$ & $\begin{array}{l}6 i \\
31\end{array}$ & 0 & 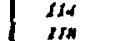 \\
\hline $09: 49 M$ & $=$ & $0 \times 00 \wedge M$ & $n$ & a & 6 & $i$ & 0 & -0 & 1 & 23 & 0 & i) & 53 & 0 & $7 \pi$ \\
\hline OK: & $\cdots$ & वसं ISAM & 0 & 0 & 0 & $i$ & $n$ & $x$ & i & -32 & 0 & 0 & So & 1 & $\phi$ \\
\hline $\begin{array}{l}\text { O8: ISAM } \\
\text { OR:30AM }\end{array}$ & $\ldots$ & $\begin{array}{l}\text { 08:10 } \mathrm{AM} \\
08: 45 \wedge \mathrm{M}\end{array}$ & $?$ & n & 0 & $\frac{3}{3}$ & u & $i$ & $\underset{g}{k}$ & 315 & 8 & $\begin{array}{l}0 \\
11\end{array}$ & $\frac{52}{44}$ & 0 & $\begin{array}{l}y z \\
y x\end{array}$ \\
\hline cos:as $1 \mathrm{M}$ & $\ldots$ & argon $A M$ & 0 & $\begin{array}{l}0 \\
0\end{array}$ & 0 & $i$ & $a$ & 1 & $\stackrel{9}{0}$ & 2i) & 0 & 0 & $\begin{array}{l}44 \\
26\end{array}$ & $\stackrel{0}{1}$ & sy \\
\hline N1.00AM & - & $-0015 \mathrm{Mm}$ & 0 & $a$ & 5 & 0 & 0 & 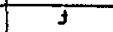 & 7 & 32 & 0 & 0 & is & 1 & -5 \\
\hline $0 \mathrm{Y}: 1: \mathrm{AM}$ & ...... & 0):10 AM & ij & v & 0 & 0 & 0 & $i$ & $i$ & 19 & y) & 0 & 11 & $i$ & st \\
\hline $\begin{array}{l}09: 10 \mathrm{AM} \\
00: 15 \mathrm{AM}\end{array}$ & $\cdots$ & $09.15 \mathrm{AM}$ & $n$ & $i$ & 0 & 2 & 0 & 2 & $\because$ & $\frac{23}{72}$ & 9 & 8 & 15 & 2 & $s$ \\
\hline Totis & $\cdots$ & $-10000 \mathrm{ng}$ & $-\because 0^{\prime}$ & $\begin{array}{l}9 \\
0\end{array}$ & 6. & 1 & $\frac{0}{6}$ & -1 & $=$ & -23 & -0 & 3 & 21) & 0 & 27 \\
\hline $10.15 \mathrm{NK}$ & $\cdots \cdot$. & $100 \mathrm{MAM}$ & i & 0 & 0 & 0 & 0 & 6 & $i$ & $\begin{array}{l}27 \\
21\end{array}$ & $\begin{array}{l}u \\
0\end{array}$ & $\dot{0}$ & $\begin{array}{l}19 \\
13\end{array}$ & $i$ & 48 \\
\hline 10:Nun & $\cdots$ & 10:14AM & v & 0 & 0 & 0 & 0 & 3 & 2 & 13 & o) & 0 & $\because$ & $i$ & 30 \\
\hline $10.5 S \cap M$ & $-\cdots$ & H:pans & $n$ & $\therefore$ & -3 & 1 & 0 & $i$ & $n$ & iii & $n$ & $n$ & $\dot{x}$ & 3 & 30 \\
\hline 11:0unu & $\ldots . . .$. & $11 \overline{A M}$ & iv & 0 & 0 & 2 & 0 & $i$ & 2 & 13 & ij & ij & IH & 6 & $\pi$ \\
\hline "1SAM & -- & 11:30 AMP & 0 & $n$ & o & 2 & 0 & 2 & 2 & 10 & 0 & 0 & 20 & 2 & 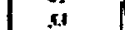 \\
\hline 11:30 A.M & $\ldots-$ & $11: 4 \times A M$ & u & 0 & 0 & 0 & a & 3 & 3 & $z$ & 0 & 0 & 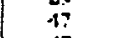 & $i$ & 90 \\
\hline IISS nM & $\because$ & $1200 \mathrm{PM}$ & 0 & 0 & $a$ & $a$ & a. & 6 & ij & 13 & 8 & $n$ & 49 & 3 & $7 t$ \\
\hline 12:00 $\mathrm{s} \cdot \mathrm{M}$ & $\ldots$ & -12015 & 71 & 0 & 0 & $=$ & - & 2 & 3 & 10 & 7 & a & 30 & 0 & 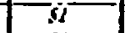 \\
\hline $\begin{array}{l}12: 19 \mathrm{MM} \\
1=3 \mathrm{nMM}\end{array}$ & $=$ & $\begin{array}{l}12 \cdot 3 \mathrm{mmM} \\
12.45 \mathrm{mM}\end{array}$ & 0 & 0 & 0 & ! & ? & 3 & 2 & 20 & 0 & 0 & 25 & 3 & 54 \\
\hline $12: 45 \mathrm{IPM}$ & $\ldots . .$. & D1.00 PPM & 0 & a & 0 & $\because$ & $a$ & 5 & 5 & 0 & i & y & 18 & 1 & 37 \\
\hline "W1 $00^{\circ} 12 M$ & $\ldots$ & D.1.15M & 0 & 0 & 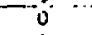 & 3 & 0 & -2 & $T \Gamma$ & 27 & 6 & 0 & -15 & jo & 30 \\
\hline $0.15 \mathrm{PM}$ & -- & $01.301 ' \mathrm{~K}$ & $a$ & 3 & i1 & 1 & 0 & 3 & 1 & 23 & $n$ & 0 & if & 4 & 9 \\
\hline 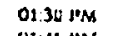 & $\ldots . .$. & 01:45 PM & 0 & 0 & u & 2 & 0 & 5 & 3 & 22 & u & u & 1.5 & 1 & se \\
\hline $0: \Delta 4, \mu M$ & $\cdots$ & O2:Da PM & -0 & -0 & 0 & 1 & 0 & 5 & - & $1:$ & a & 0 & ii & 0 & $A$ \\
\hline $02: 00 \mathrm{~ms}$ & $\ldots$ & $02: 15 \ln M$ & 67 & 6 & i & 1 & 0 & 3 & 8 & 19 & 0 & 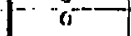 & 20 & $T$ & is \\
\hline O2: 15 PM & $\cdots$ & $02.3019 \mathrm{M}$ & 0 & 0 & 0 & I & 0 & ii & 4 & 3 & 0 & 0 & 13 & $i$ & st \\
\hline $02: 30 \mathrm{rm}$ & -- & $02: 45 \mathrm{ll} \mathrm{M}$ & 0 & 0 & it & 2 & u & 7 & 5 & 3 & $n$ & a & if. & $i$ & sn \\
\hline $02: 15 \mathrm{PM}$ & $\cdots$ & -09:0018M & -6 & i & (1 & 0 & 0 & 4 & 5 & 18 & $\therefore$ & 0 & 13 & i & 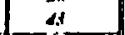 \\
\hline $03.000 \mathrm{BN}$ & $\ldots$ & $03: 151 \mathrm{~min}$ & 0 & $\dot{0}$ & 11 & -2 & 4 & 2 & 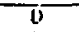 & 18 & 0 & 0 & $2 i$ & -1 & \\
\hline $03: 15$ PM & $\cdots$ & L.:T: & 0 & 0 & a & 0 & 0 & 2 & $i$ & 33 & 0 & $v$ & 21 & i & $s$ \\
\hline U3:Jo Pas & -..- & axdi I & 0 & u & u & 1 & 0 & 3 & i & $g$ & v & 0 & 16 & i & 30 \\
\hline $0119 P M$ & $-\cdots$ & an:00 IM & 0 & $\therefore$ & 8 & $i$ & 0 & 2 & $=$ & 47 & 0 & $\therefore$ & 14 & 0 & 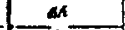 \\
\hline जo & $\cdots$ & $0.111 M$ & 0 & 10 & 0 & $a$ & 67 & 4 & 5 & 32 & 0 & $0^{-}$ & 11 & 1 & -78 \\
\hline OAIIS SM & $\cdots$ & Or:Su PMA & 0 & $\dot{y}$ & 0 & 0 & 0 & 3 & 0 & rin & 0 & 0 & $2 z$ & $v$ & 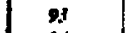 \\
\hline OA:J0 PM & ....... & 0A:45 IN & 0 & 0 & 0 & 1 & 0 & 3 & $n$ & 65 & " & 0 & 25 & 0 & N \\
\hline $01: 45 Y_{M}$ & $=$ & Q5000M & ? & 0 & $\because$ & 0 & 0 & $\frac{0}{T 0 T}$ & 0 & 56 & 0 & -4 & 23 & 2 & 20 \\
\hline & & & & & & & $R t . Y$ & $T O T A N$ & & & & & & & \\
\hline M7.15AM & $\cdots$ & $\begin{array}{l}\text { ORODAM } \\
\text { OS:15 AM }\end{array}$ & $n$ & 0 & 0 & 3 & 0 & $\therefore$ & 76 & 113 & 0 & 0 & 201 & 2 & 250 \\
\hline $100 \mathrm{AM}$ & - & - & . & is & 0 & $\underline{z}$ & $\begin{array}{l}0 \\
0\end{array}$ & $\stackrel{?}{11}$ & $\begin{array}{l}11 \\
18\end{array}$ & $\begin{array}{l}1.36 \\
194\end{array}$ & $a$ & $\begin{array}{l}a \\
0\end{array}$ & $\begin{array}{l}247 \\
237\end{array}$ & 1 & sen \\
\hline $07 \sqrt{1 \overline{A M}}$ & $=$ & OQ 15 AM & 0 & $a$ & 0 & $-\bar{n}$ & i & ii & $\frac{\pi}{19}$ & (101) & -8 & 0 & 205 & $\frac{1}{1}$ & $-1 \dot{1}$ \\
\hline $\begin{array}{l}\text { OB.00 AN } \\
\text { ax:11.AM }\end{array}$ & $=$ & $\begin{array}{l}05: 001 A M \\
00.15 A M\end{array}$ & $"$ & $y$ & 0 & i & 0 & 12 & $1 x$ & ox & $n$ & 0 & 124 & 2 & 312 \\
\hline Ot:J0 $\cap k$ & $\ldots$ & QW:JOAM & $-i$ & $\pi$ & 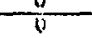 & -3 & -0 & $e$ & $\begin{array}{l}18 \\
16\end{array}$ & 86 & -6 & $\frac{0}{0}$ & -140 & $-\frac{2}{3}$ & $-26 \pi$ \\
\hline ossins nes & $\cdots$ & Or:A: AM & $y$ & o & 0 & 3 & 0 & $?$ & $?$ & 94 & 0 & o & 70 & 5 & 306 \\
\hline 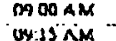 & $=$ & $-10: 00 \wedge y$ & $\frac{0}{11}$ & $\frac{D}{D}$ & 0 & -3 & 0 & 7 & $-\because$ & 37 & 0 & 0 & 6 & 4 & int. \\
\hline 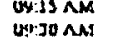 & $\ldots$ & 10:70 A & u & 0 & 0 & 4 & 0 & $\stackrel{5}{10}$ & $\frac{12}{7}$ & 2 & 0 & 0 & 63 & 5 & -103 \\
\hline mod $13 \cap \geq 1$ & $\cdots$ & $10: 15 \wedge \mathrm{M}$ & 0 & $a$ & 0 & 2 & $\ddot{0}$ & 11 & 9 & kn & $\begin{array}{l}n \\
n\end{array}$ & 0 & $\begin{array}{l}67 \\
59\end{array}$ & $\begin{array}{l}5 \\
4\end{array}$ & 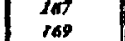 \\
\hline ivesine & $\cdots$ & $11002 \mathrm{M}$ & 0 & 0 & $0^{-}$ & 2 & 0 & in & $?$ & $m$ & 0 & $\because$ & is & 7 & Ist \\
\hline $\begin{array}{l}1019 \mathrm{AM} \\
10.30 \mathrm{AM}\end{array}$ & $=$ & $\begin{array}{l}11: 13 \mathrm{AM} \\
11: 30 \mathrm{NM1}\end{array}$ & 0 & 0 & $\begin{array}{l}0 \\
0\end{array}$ & 3 & $\begin{array}{l}0 \\
0\end{array}$ & if & 5 & 80 & $\begin{array}{l}u \\
0\end{array}$ & $\begin{array}{l}0 \\
0\end{array}$ & $\begin{array}{l}46 \\
t^{3}\end{array}$ & 5 & sst \\
\hline $10.43 \cap \mathrm{M}$ & $\ldots$ & $11 \overline{A N A M}$ & 0 & 0 & 0 & 5 & -0 & II & $\because$ & -6 & 0 & 0 & 70 & -6 & \\
\hline IIMAM & $\cdots$ & $12: 00 \mathrm{rm}$ & 0 & 0 & 0 & 4 & 0 & 17 & 7 & G & 0 & 0 & 14.7 & $f_{i}$ & XII \\
\hline $11: 13 \mathrm{nM}$ & $\ldots$ & $12: 19 \mathrm{PM}$ & 0 & o) & 0 & 1. & 0 & in & $x$ & ris & i. & 0 & 155 & 6 & 351 \\
\hline$\sqrt{1.3 n л M}$ & $\cdots$ & 12.0019 & 0 & 0 & c & 3 & 0 & 74 & 8 & 6 & 0 & u & 1.51 & 7 & נונד \\
\hline $\begin{array}{l}11: 15 \mathrm{AM} \\
1200 \mathrm{PM}\end{array}$ & 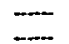 & 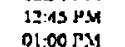 & 0 & $\ddot{a}$ & $\begin{array}{l}u \\
n\end{array}$ & 3 & $\begin{array}{l}u \\
n\end{array}$ & 12 & $\stackrel{5}{\circ}$ & 63 & 0 & 0 & 120 & 7 & 23 \\
\hline - & - & $01: 15 \% \mathrm{YM}$ & 0 & $\because-$ & ii & a & $\pi$ & 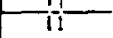 & 13 & -47 & 0 & $\cdot b$ & $\frac{8 x}{7.3}$ & 6 & $\frac{\beta Y S}{262}$ \\
\hline $\begin{array}{l}12: 00 \mathrm{PAS} \\
1 \geq A .1 \mathrm{PM}\end{array}$ & $=$ & 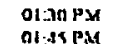 & $\begin{array}{l}0 \\
0\end{array}$ & 0 & 0 & i & 0 & 11 & $1 x$ & נit & 0 & 0 & 65 & i & 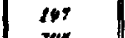 \\
\hline $\begin{array}{l}12: 4.3 \mathrm{PM} \\
01: 00 \mathrm{rMM}\end{array}$ & & 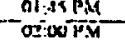 & -6 & $\frac{0}{10}$ & $\frac{0}{11}$ & $\frac{k}{3}$ & -5 & is & 20 & -96 & $\stackrel{0}{8}$ & $\frac{0}{6}$ & $\frac{62}{6 i 1}$ & 7 & 304 \\
\hline at:os ins & - & $0211 \mathrm{PM}$ & 0 & 0 & 0 & $\dot{s}$ & 0 & 16 & 17 & iy & 0 & 0 & 65 & 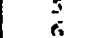 & 19x \\
\hline $0120 \mathrm{mM}$ & $-\infty$ & $02 \cdot 30 \mathrm{PM}$ & 0 & 0 & $\dot{0}$ & 5 & u & y & 20 & 80 & 0 & $\therefore$ & (i) & 3 & son \\
\hline an:Aspis & $-\infty$ & $02: 15$ & $0^{-}$ & D & 0 & 5 & 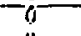 & 21 & 22 & w: & 0 & $0^{-}$ & 62 & 2 & 2047 \\
\hline $\begin{array}{l}02.00 \mathrm{~lm} M \\
\mathrm{u2:13} \mathrm{km}\end{array}$ & $\cdots$ & $0300 \mathrm{HM}$ & 0 & 0 & 0 & 1 & 0 & 20 & $3 n$ & 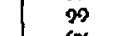 & a & 0 & Ga & 2 & 309 \\
\hline 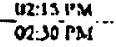 & $\ldots$ & 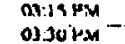 & -0 & $\pi$ & 0 & $\dot{5}$ & $\frac{a}{4}$ & $\frac{19}{13}$ & 19 & s & 3 & $-\sigma$ & $\frac{71}{71}$ & 2 & 30 \\
\hline 09:15 JPA & $\cdots$ & a].45 $19 \mathrm{PM}$ & i) & 0 & 0 & 3 & 0 & ii & 7 & 策 & $\mathfrak{a}$ & 0 & $y$ & 3 & 201 \\
\hline OXMn & $\ldots$ & $0900 \mathrm{PM}$ & 0 & 0 & 0 & 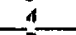 & $n$ & $\therefore$ & 4 & 126 & 0 & 0 & 78 & 3 & 23 \\
\hline 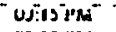 & $=\cdots$ & $041510 \mathrm{~ms}$ & 0 & 0 & 0 & 2 & 0 & 11 & 4 & $9-170$ & 6 & & & 3 & 25 \\
\hline Oa:so & $\ldots$ & OAl:30 1'AS & 0 & 0 & 0 & $\sum$ & 0 & 12 & $x$ & - $2 n 5$ & i & 0 & 6.3 & 2 & $3 n$ \\
\hline $03.4 \mathrm{MM}$ & $\longrightarrow$ & Q $1.9 S P M$ & 0 & 0 & 0 & -2 & $n$ & .12 & $\Sigma$ & 212 & 0 & 0 & 72 & $i$ & III \\
\hline 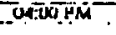 & $\ldots$ & Orwo & 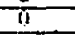 & a & 0 & 1 & 0 & in & 0 & 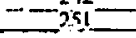 & 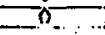 & 6 & HI & $i$ & iss \\
\hline$s$ & $N$ & $2 i 21:$ & 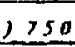 & 17 & & A & $D A$ & o) & & & 0. & $+c$ & 15 & $z-$ & \\
\hline
\end{tabular}




\section{Baymetrics Traffic Resources INTERSECTION TURNING MOVEMENT SUMMARY}

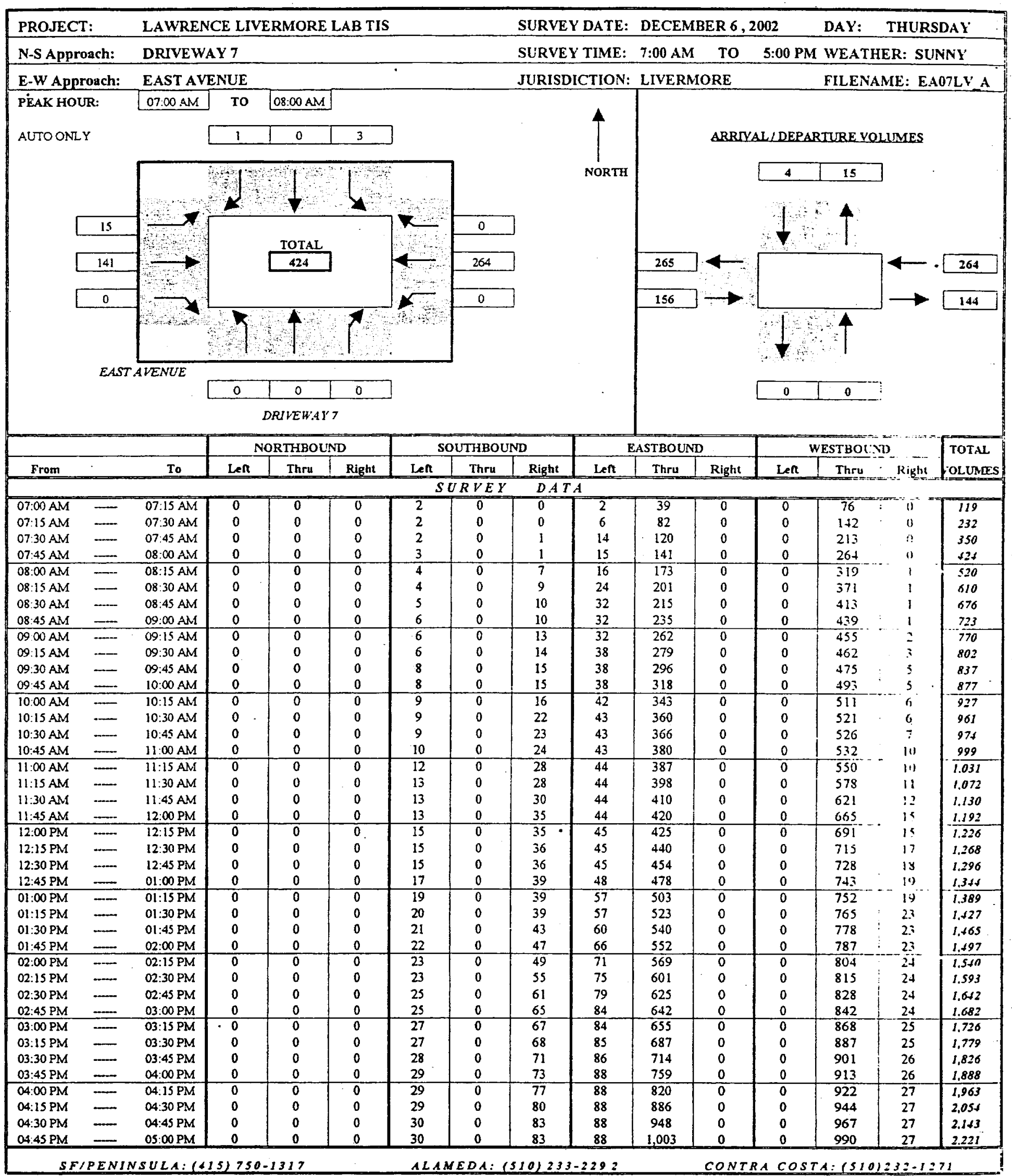




\section{Baymetrics Traffic Resources INTERSECTION TURNING MOVEMENT SUMMARY}

\begin{tabular}{|c|c|c|c|c|c|c|c|c|c|c|c|c|c|c|c|}
\hline \multirow{2}{*}{\multicolumn{2}{|c|}{$\begin{array}{l}\text { PROJECT: } \\
\text { N.S A pproach: }\end{array}$}} & \multicolumn{6}{|c|}{ LAWRENCE LIVERMORE LAB TIS } & \multicolumn{5}{|c|}{ SURVEY DATE: DECEMBER 6,2002 } & DAY: & THIIRS & $\mathrm{DAY}$ \\
\hline & & DRIVEW & & & & & & SURVE & IIME: & 7:00 AM & To & 5:00 PM & WEATI & IER: SU & NY \\
\hline E-W Ap & ach: & EAST AV & NUE & & & & & JLRISD & TION: & LIVERA & RE & & FILEN & ME: EA & $37 \mathrm{LV}$ A \\
\hline & & & & THBO & & & THBO & & & ASTBOUN & & & ESTBOU & & TOTAL \\
\hline From & & To & Left & Thru & Right & Left & Thru & Right & Left & Thru & Right & Len & Thru & Right & YOLUMES \\
\hline & & & & & & & $L \quad B$ & $P E R I$ & & & & & & & \\
\hline $\begin{array}{l}07: 00 \text { AM } \\
07: 15 \text { AM }\end{array}$ & $\bar{z}$ & $\begin{array}{l}07: 15 \mathrm{AM} \\
07: 30 \mathrm{AM}\end{array}$ & 0 & $\begin{array}{l}0 \\
0\end{array}$ & $\begin{array}{l}0 \\
0\end{array}$ & $\begin{array}{l}2 \\
0\end{array}$ & $\begin{array}{l}0 \\
0\end{array}$ & $\begin{array}{l}0 \\
0\end{array}$ & $\begin{array}{l}2 \\
4\end{array}$ & $\begin{array}{l}39 \\
43\end{array}$ & $\begin{array}{l}0 \\
0\end{array}$ & 0 & $\begin{array}{l}16 \\
66\end{array}$ & 0 & 119 \\
\hline $\begin{array}{l}07: 30 \mathrm{AM} \\
07: 45 \mathrm{AM}\end{array}$ & $=$ & $\begin{array}{l}07: 45 \mathrm{AM} \\
08.00 \mathrm{AM}\end{array}$ & $\begin{array}{l}0 \\
0\end{array}$ & $\begin{array}{l}0 \\
0\end{array}$ & $\begin{array}{l}0 \\
0\end{array}$ & $\begin{array}{l}0 \\
1\end{array}$ & $\begin{array}{l}0 \\
0\end{array}$ & 1 & $\begin{array}{l}8 \\
1\end{array}$ & $\begin{array}{l}38 \\
21\end{array}$ & $\begin{array}{l}0 \\
0\end{array}$ & $\begin{array}{l}0 \\
0\end{array}$ & $\begin{array}{l}7 ! \\
51\end{array}$ & $\begin{array}{l}0 \\
0\end{array}$ & 118 \\
\hline $08: 00 \mathrm{AM}$ & - & $08: 15 \mathrm{AM}$ & 0 & 0 & 0 & $\frac{T}{1}$ & 0 & $\frac{0}{6}$ & $\frac{1}{1}$ & $-\frac{1}{32}$ & 0 & $\frac{\pi}{0}$ & $\frac{31}{55}$ & $\frac{1}{1}$ & 96 \\
\hline 08:15 AM & - & 08:30 AM & 0 & 0 & 0 & 0 & 0 & 2 & 8 & 28 & 0 & 0 & 52 & 0 & 90 \\
\hline $\begin{array}{l}08: 30 \mathrm{AM} \\
08: 45 \mathrm{AM}\end{array}$ & $\ldots$ & $\begin{array}{l}08: 45 \mathrm{AM} \\
09.00 \mathrm{AM}\end{array}$ & $\begin{array}{l}0 \\
0\end{array}$ & $\begin{array}{l}0 \\
0\end{array}$ & $\begin{array}{l}0 \\
0\end{array}$ & 1 & $\begin{array}{l}0 \\
0\end{array}$ & 1 & $\begin{array}{l}8 \\
0\end{array}$ & $\begin{array}{l}14 \\
20\end{array}$ & $\begin{array}{l}0 \\
0\end{array}$ & 0 & $\begin{array}{l}42 \\
26\end{array}$ & 0 & 80 \\
\hline $09.00 \mathrm{AM}$ & $\ldots$ & $09: 15 \mathrm{AM}$ & 0 & 0 & 0 & $\frac{1}{0}$ & 0 & $\frac{0}{3}$ & 0 & $\frac{27}{27}$ & $\frac{0}{0}$ & $\frac{0}{0}$ & $\frac{20}{16}$ & $\frac{1}{1}$ & $\frac{11}{47}$ \\
\hline 09:15 AM & $\cdots$ & $09: 30 \mathrm{AM}$ & 0 & 0 & 0. & 0 & 0 & $i$ & 6 & 17 & 0 & 0 & 7 & $i$ & 32 \\
\hline $09: 30 \mathrm{AM}$ & - & $09: 45 \mathrm{AM}$ & 0 & 0 & 0 & 2 & 0 & i & 0 & 17 & 0 & 0 & 13 & 2 & 35 \\
\hline $09: 45 \mathrm{AM}$ & $\mp$ & $10.00 \mathrm{AM}$ & 0 & 0 & 0 & 0 & 0 & 0 & 0 & 22 & 0 & 0 & 18 & $\bar{j}$ & $\$ 0$ \\
\hline $10: 00$ AM & $\ldots$ & $10: 15 \mathrm{AM}$ & 0 & $\overline{0}$ & 0 & $T$ & 0 & 1 & 4 & 25 & 0 & 0 & 18 & 1 & 50 \\
\hline $10: 15 A M$ & $\ldots$ & $10: 30 \mathrm{AM}$ & 0 & 0 & 0 & 0 & 0 & 6 & 1 & 17 & 0 & 0 & 10 & $\therefore$ & $3 s$ \\
\hline $10: 30 \mathrm{AM}$ & $\ldots$ & $10: 45 \mathrm{AM}$ & 0 & 0 & 0 & 0 & 0 & 1 & 0 & 6 & 0 & 0 & 5 & $i$ & I3 \\
\hline $10: 45 \mathrm{AM}$ & $\ldots$ & $11: 00 \mathrm{AM}$ & 0 & 0 & 0 & 1 & 0 & $i$ & 0 & 14 & 0 & 0 & 6 & 3 & 23 \\
\hline $11: 00 \mathrm{AM}$ & $=$ & $11: 15 \mathrm{AM}$ & 0 & 0 & 0 & 2 & 0 & 4 & $T$ & 7 & $\bar{\sigma}$ & 0 & 18 & $\because$ & 32 \\
\hline 11:15 AM & - & $11: 30 \mathrm{AM}$ & 0 & 0 & 0 & 1 & 0 & 0 & 0 & 11 & 0 & 0 & 28 & 1 & $\pi$ \\
\hline $\begin{array}{l}11: 30 \mathrm{AM} \\
11.45 \mathrm{AM}\end{array}$ & $=$ & $\begin{array}{l}11: 49 . \mathrm{AM} \\
12.00 \mathrm{PM}\end{array}$ & $\begin{array}{l}0 \\
0\end{array}$ & 0 & 0 & $\begin{array}{l}0 \\
0\end{array}$ & 0 & $\frac{2}{5}$ & $\begin{array}{l}0 \\
0\end{array}$ & 12 & 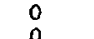 & ${ }_{0}^{0}$ & 43 & $!$ & 58 \\
\hline$\frac{11: 44 \mathrm{AM}}{12: 00 \mathrm{PM}}$ & $=$ & $\frac{12.00 \mathrm{PM}}{12: 15 \mathrm{PM}}$ & $\frac{0}{0}$ & $\frac{0}{0}$ & 0 & $\frac{0}{2}$ & $\frac{0}{0}$ & $\frac{3}{0}$ & 0 & $\frac{10}{5}$ & $\frac{0}{0}$ & $\frac{0}{0}$ & $\frac{44}{36}$ & $\therefore$ & $6 ?$ \\
\hline $12: 15 \mathrm{PM}$ & $\ldots+$. & 12:30 PM & 0 & 0 & 0 & 0 & 0 & 1 & $\begin{array}{l}1 \\
0\end{array}$ & 15 & 0 & 0 & 26 & $\because$ & 31 \\
\hline 12:30 PM & $\ldots$ & $12: 45 \mathrm{PM}$ & 0 & 0 & 0 & 0 & 0 & 0 & 0 & 14 & 0 & 0 & 13 & $\bar{i}$ & 28 \\
\hline $12: 45 \mathrm{PM}$ & - & $01: 00 \mathrm{PM}$ & 0 & 0 & 0 & 2 & 0 & 3 & 3. & 24 & 0 & 0 & 15 & 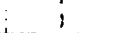 & 18 \\
\hline $01: 00 \mathrm{PM}$ & - & $01: 15 \mathrm{PM}$ & 0 & 0 & 0 & 2 & 0 & 0 & 9 & 25 & 0 & 0 & 9 & "i & is \\
\hline $01: 15$ PM & $\cdots$ & 01:30 PM & 0 & 0 & 0 & 1 & 0 & 0 & 0 & 20 & 0 & 0 & 13 & 1 & 18 \\
\hline $01: 30 \mathrm{PM}$ & - & $08: 49 \mathrm{PM}$ & 0 & 0 & 0 & 1 & 0 & 4 & 3 & 17 & 0 & 0 & 13 & :! & 38 \\
\hline 01:45 PM & $\ldots$ & $\begin{array}{l}\text { 02:00 PM } \\
\text {. }\end{array}$ & 0 & 0 & 0 & $i$ & 0 & 4 & 6 & 12 & 0 & 0 & 9 & $\because$ & 年 \\
\hline $02: 00 \mathrm{PM}$ & $\cdots$ & $02: 15 \mathrm{PM}$ & $\%$ & 0 & 0 & 1 & 0 & 2 & 5 & 17 & 0 & 0. & 17 & 1 & $\sqrt{3}$ \\
\hline $02: 15$ PM & $\cdots$ & $02: 30 \mathrm{PM}$ & 0 & 0 & 0 & 0 & 0 & 6 & 4 & 32 & 0 & 0 & i1 & $\therefore$ & 53 \\
\hline 02:30 PM & $\ldots$ & $\begin{array}{l}02: 15 \mathrm{PM} \\
03: 00 \mathrm{PM}\end{array}$ & $\begin{array}{l}0 \\
0\end{array}$ & 0 & 0 & 2 & 0 & 6 & 4 & 24 & 0 & 0 & 13 & $\because$ & 19 \\
\hline $02: 40$ PM & $=$ & $\begin{array}{l}03: 00 \mathrm{PM} \\
03: 15 \mathrm{PM}\end{array}$ & $\frac{0}{0}$ & $\frac{0}{0}$ & $\frac{0}{0}$ & $\frac{2}{2}$ & $\frac{0}{0}$ & $\frac{4}{2}$ & $\frac{5}{0}$ & -17 & 0 & $\frac{0}{n}$ & $\frac{14}{26}$ & $\vdots$ & $\frac{11}{11}$ \\
\hline 03:15 PM & $\cdots$ & 03:30 PM & 0 & 0 & 0 & 0 & 0 & i & 1. & 32 & 0 & 0 & 19 & $\therefore$ & 33 \\
\hline $03: 30 \mathrm{PM}$ & $\ldots$ & 03:45 PM & 0 & 0 & 0 & 1 & 0 & 3 & $i$ & 27 & 0 & 0 & is & $!$ & $\therefore$ \\
\hline $03: 459 \mathrm{PM}$ & $-\ldots$ & 04:00 PM & 0 & 0 & 0 & 1 & 0 & 2 & 2 & 45 & 0 & 0 & 12 & ." & 6 \\
\hline $04.00 \mathrm{PM}$ & $\cdots$ & $04: 15 \mathrm{PM}$ & 0 & 0 & 0 & 0 & 0 & 4 & 0 & 61 & 0 & 0 & 9 & 1 & 5 \\
\hline OA:15 PM & $\cdots$ & 04:30 PM & 0 & 0 & 0 & 0 & 0 & 3 & 0 & 66 & 0 & 0 & 22 & $\because$ & 91 \\
\hline $\begin{array}{l}04: 30 \mathrm{PM} \\
04: 45 \mathrm{PM}\end{array}$ & $\ldots$ & $\begin{array}{l}04: 45 \mathrm{PM} \\
\text { OS:00 PM }\end{array}$ & $\begin{array}{l}0 \\
0\end{array}$ & $\begin{array}{l}0 \\
0\end{array}$ & $\begin{array}{l}0 \\
0\end{array}$ & $\begin{array}{l}1 \\
0\end{array}$ & $\begin{array}{l}0 \\
0\end{array}$ & $\begin{array}{l}3 \\
0\end{array}$ & $\begin{array}{l}0 \\
0\end{array}$ & $\begin{array}{l}62 \\
55\end{array}$ & $\begin{array}{l}0 \\
0\end{array}$ & $\begin{array}{l}0 \\
0\end{array}$ & $\begin{array}{l}23 \\
23\end{array}$ & .1 & $\begin{array}{l}89 \\
-89\end{array}$ \\
\hline & & & & & & & $R L Y$ & TOTA & & & & & & & \\
\hline $07: 00 \mathrm{AM}$ & $=$ & 08:00AM & $\overline{0}$ & 0 & $\sigma$ & 3 & 0 & $T$ & 5 & T4I & $\overline{0}$ & 0 & 264 & $\because$ & $\sqrt{51}$ \\
\hline $07: 15 \mathrm{AM}$ & $\cdots$ & 08:15 AM & 0 & 0 & 0 & 2 & 0 & $?$ & 14 & 134 & ${ }_{0}^{0}$ & 0 & $2+3$ & $:$ & sot \\
\hline & $=$ & $08: 30 \mathrm{AM}$ & 0 & $\frac{0}{0}$ & $\frac{0}{0}$ & $\frac{2}{3}$ & $\frac{0}{0}$ & $\frac{9}{9}$ & $\frac{18}{18}$ & $\frac{119}{95}$ & $\frac{0}{0}$ & $\frac{0}{0}$ & $2=9$ & $\vdots$ & 18 \\
\hline $\begin{array}{l}07: 45 \mathrm{AM} \\
08: 00 \mathrm{AM}\end{array}$ & $\bar{I}$ & $\begin{array}{l}08: 45 \mathrm{AM} \\
09: 00 \mathrm{AM}\end{array}$ & o & 0 & $\begin{array}{l}0 \\
0\end{array}$ & 3 & 0 & 9 & 17 & 94 & 0 & $\begin{array}{l}0 \\
0\end{array}$ & $20 \%$ & 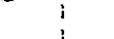 & $\sqrt{126}$ \\
\hline $08: 15$ AM & $\cdots$ & 09:15 AM & 0 & 0 & 0 & 2 & 0 & 6 & 16 & 89 & 0 & 0 & 135 & $\vdots$ & 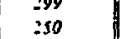 \\
\hline $08: 30 \overline{A M}$ & - & $09: 30 \mathrm{AM}$ & 0 & 0 & 0 & $\frac{2}{2}$ & 0 & 5 & 14 & 78 & 0 & 0 & $9 !$ & $\vdots$ & 192 \\
\hline $08: 45 . \mathrm{AM}$ & $-\infty$ & $09: 45$ AMI & $a$ & 0 & 0 & 3 & 0 & 5 & 6 & 81 & 0 & 0 & 62 & $i$ & $16 t$ \\
\hline $09: 00 \mathrm{AM}$ & $\ldots$. & $10.00 \mathrm{AM}$ & 0 & 0 & 0 & 2 & 0 & 5 & 6 & 83 & 0 & 0 & 5.8 & $i$ & iss \\
\hline $09: 15 \mathrm{AM}$ & $=$ & $10: 15 \mathrm{AM}$ & 0 & 0 & 0 & 3 & 0 & 3 & 10 & 81 & 0 & 0 & 56 & $i$ & 157 \\
\hline $09: 30 \mathrm{AM}$ & - & 10:30 AM & 0 & 0 & 0 & 3 & 0 & 8 & 5 & 81 & 0 & 0 & 59 & 3 & 159 \\
\hline $09: 45 \mathrm{AM}$ & $\ldots$ & 10:45 AM & 0 & 0 & 0 & 1 & 0 & 8 & 5 & 70 & 0 & 0 & 51 & $=$ & $13^{\circ}$ \\
\hline $10: 00 \mathrm{AM}$ & $\ldots$ & $11: 00 \mathrm{AM}$ & 0 & 0 & 0 & $\frac{2}{3}$ & 0 & 9 & 5 & 62 & 0 & 0 & 39 & $i$ & inz \\
\hline 10:15 AM & - & $11: 15 \mathrm{AM}$ & $\begin{array}{l}0 \\
0\end{array}$ & 0 & $\begin{array}{l}0 \\
0\end{array}$ & $\begin{array}{l}3 \\
4\end{array}$ & $\begin{array}{l}0 \\
0\end{array}$ & $\begin{array}{c}12 \\
6\end{array}$ & $\frac{2}{1}$ & $\begin{array}{l}44 \\
38\end{array}$ & $\begin{array}{l}0 \\
0\end{array}$ & $: 0$ & $\begin{array}{l}39 \\
57\end{array}$ & $\vdots$ & ios \\
\hline$\frac{10: 30 \mathrm{AM}}{10.45 \mathrm{AM}}$ & $\bar{E}$ & $\frac{1130 \mathrm{AM}}{11: 45 \mathrm{AM}}$ & $\frac{0}{0}$ & $\frac{1}{0}$ & $\frac{0}{0}$ & $\frac{4}{4}$ & 0 & $\frac{0}{1}$ & $\frac{1}{1}$ & $\frac{30}{44}$ & $\frac{1}{0}$ & 0 & $95-$ & $\because$ & 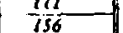 \\
\hline $1100 \mathrm{AM}$ & $\ldots$ & 12:00 PM & 0 & 0 & 0 & 3 & 0 & ii & $i$ & 40 & 0 & 0 & 133 & s & 193 \\
\hline $11: 13 \mathrm{AM}$ & $=$ & $12: 15 \mathrm{PM}$ & 0 & 0 & 0 & 3 & 0 & 7 & 1 & 38 & 0 & 0 & 141 & ; & 199 \\
\hline $11: 30 \mathrm{AM}$ & - & $12: 30 \mathrm{PM}$ & 0 & $\%$ & 0 & 2 & 0 & 8 & 1 & 42 & $\ddot{0}$ & 0 & 137 & 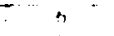 & 196 \\
\hline $11: 45 \mathrm{AM}$ & - & 32:45 PM & 0 & 0 & 0 & 2 & 0 & 6 & 1 & $\begin{array}{cc}A 4 \\
50\end{array}$ & 0 & 0 & 107 & $n$ & 166 \\
\hline $12: 00 \mathrm{PM}$ & $\ldots$ & $01: 00 \mathrm{PM}$ & 0 & 0 & 0 & 4 & 0 & 4 & 4 & 58 & 0 & 0 & 78 & 1 & 152 \\
\hline $12: 15 \mathrm{PM}$ & $\ldots$ & $01: 15 \mathrm{PM}$ & 0 & 0 & 0 & 4 & 0 & 4 & 12 & 38 & 0 & 0 & 61 & 1 & 783 \\
\hline $12: 30 \mathrm{gM}$ & $\cdots$ & $01: 30 \mathrm{PM}$ & 0 & 0 & 0 & 5 & 0 & 3 & 12 & 83 & 0 & 0 & 50 & th & 159 \\
\hline $12: 45 \mathrm{PM}$ & $\cdots$ & $01: 45 \mathrm{PM}$ & 0 & 0 & 0 & 6 & 0 & 7 & 15 & 86 & 0 & 0 & so & 5 & 169 \\
\hline $01: 00 \mathrm{PM}$ & $=$ & $02: 00 \mathrm{PM}$ & 0 & 0 & 0 & 5 & 0 & 8 & 18 & 34 & 0 & 0 & 44 & 4 & 153 \\
\hline 01:15 PM & 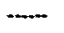 & 02:15 PM & 0 & 0 & 0 & 4 & 0 & 10 & 14 & 66 & 0 & 0 & 52 & 5 & $15 I$ \\
\hline 01:30 PM & $\ldots$ & $02: 30 \mathrm{PM}$ & 0 & 0 & 0 & 3 & 0 & 16 & 18 & 18 & 0 & 0 & 50 & $i$ & 166 \\
\hline $01: 45 \mathrm{PM}$ & $=$ & $02: 45$ PM & 0 & 0 & 0 & 4 & 0 & 18 & 19 & 85 & 0 & 0 & 50 & $T$ & 179 \\
\hline 02:00 PM & - & 03:00 PM & 0 & 0 & 0 & 3 & 0 & 18 & 18 & 90 & 0 & 0 & 55 & 1 & 185 \\
\hline $02: 15$ PM & $=$ & $03: 15 \mathrm{PM}$ & 0 & 0 & 0 & 4 & 0 & 18 & 13 & 86 & 0 & 0 & 64 & & 186 \\
\hline $02: 30 \mathrm{PM}$ & $=$ & $03: 30 \mathrm{PM}$ & 0 & 0 & 0 & 4 & 0 & 13 & To & 86 & 0 & $\sigma$ & 72 & $T$ & 186 \\
\hline $02: 45 \mathrm{PM}$ & $\ldots$ & 03:45 PM & 0 & 0 & 0 & 3 & 0 & 10 & 7 & 89 & 0 & 0 & 73 & $=$ & IS \\
\hline $03: 00 \mathrm{PM}$ & $\dot{\square}$ & $04: 00 \mathrm{PM}$ & 0 & 0 & 0 & 4 & 0 & 8 & 4 & 117 & 0 & 0 & 71 & & 306 \\
\hline $03: 15$ PM & & $0 A 15$ PM & 0 & 0 & 0 & 2 & 0 & 10 & 4 & 165 & 0 & 0 & 54 & 2 & $23^{7}$ \\
\hline 03:30 PM & 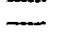 & $0 \times 30 \mathrm{PM}$ & 0 & 0 & 0 & 2 & 0 & 12 & 3 & 199. & 0 & 0 & 57 & 5 & 375 \\
\hline 03:45 PM & $\ldots$ & Q1:45 PM & 0 & 0 & 0 & 2 & 0 & 12 & 2 & 234 & 0 & 0 & 66 & 1 & $3 ! ?$ \\
\hline $04: 00 \mathrm{PM}$ & $=$ & $05: 00 \mathrm{PM}$ & 0 & 0 & 0 & & 0 & 10 & 0 & 244 & 0 & 0 & 77 & $I$ & $3 B S$ \\
\hline$S t$ & $E N I$ & ULA: & 列 & 7 & & $A L$ & $D A$ & a) 2 & 292 & & OA & 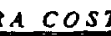 & $:(5$ & 32 & \\
\hline
\end{tabular}




\section{Baymetrics Traffic Resources INTERSECTION TURNING MOVEMENT SUMMARY}

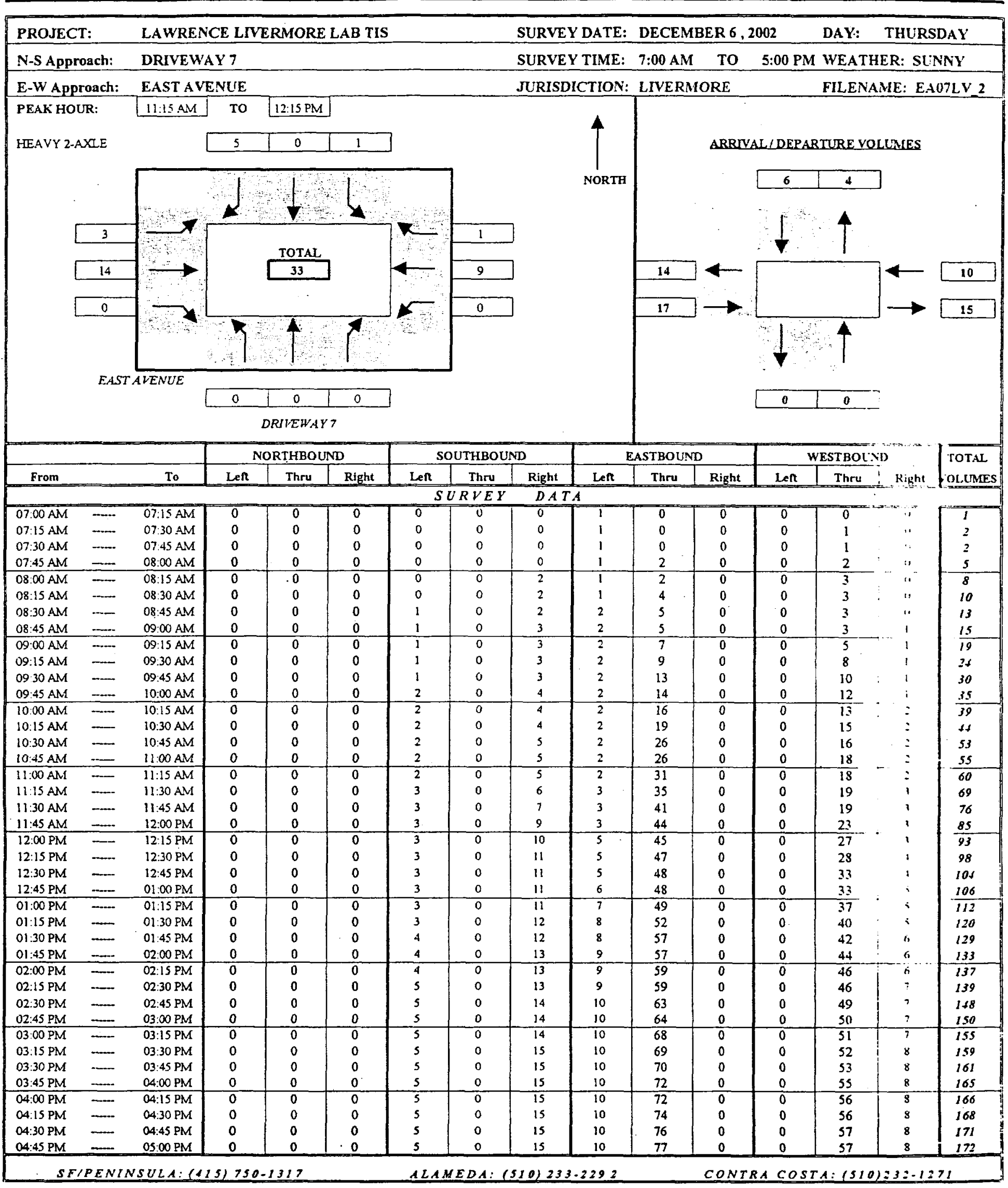




\section{Baymetrics Traffic Resources INTERSECTION TURNING MOVEMENT SUMMARY}

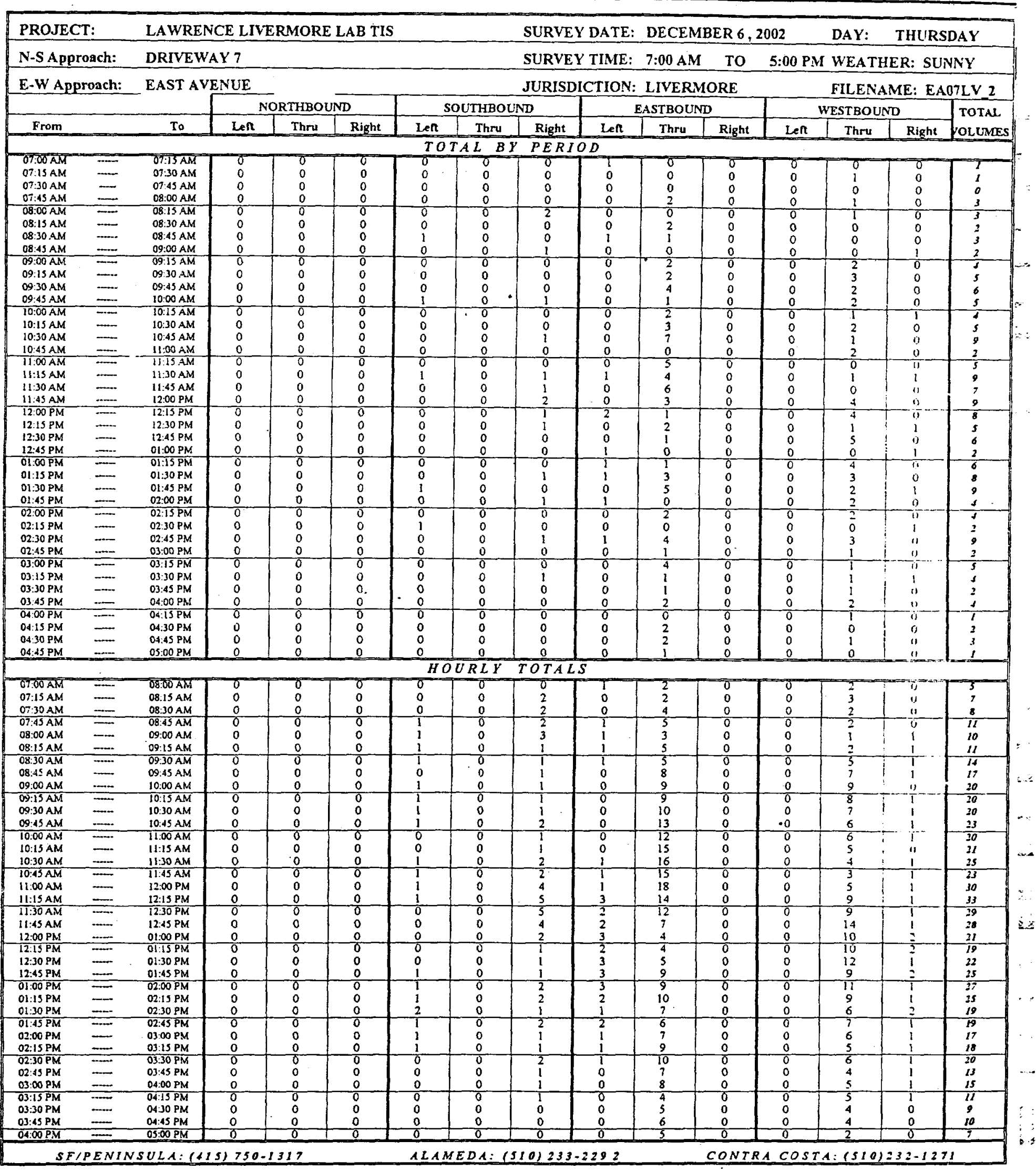




\section{Baymetrics Traffic Resources INTERSECTION TURNING MOVEMENT SUMMARY}

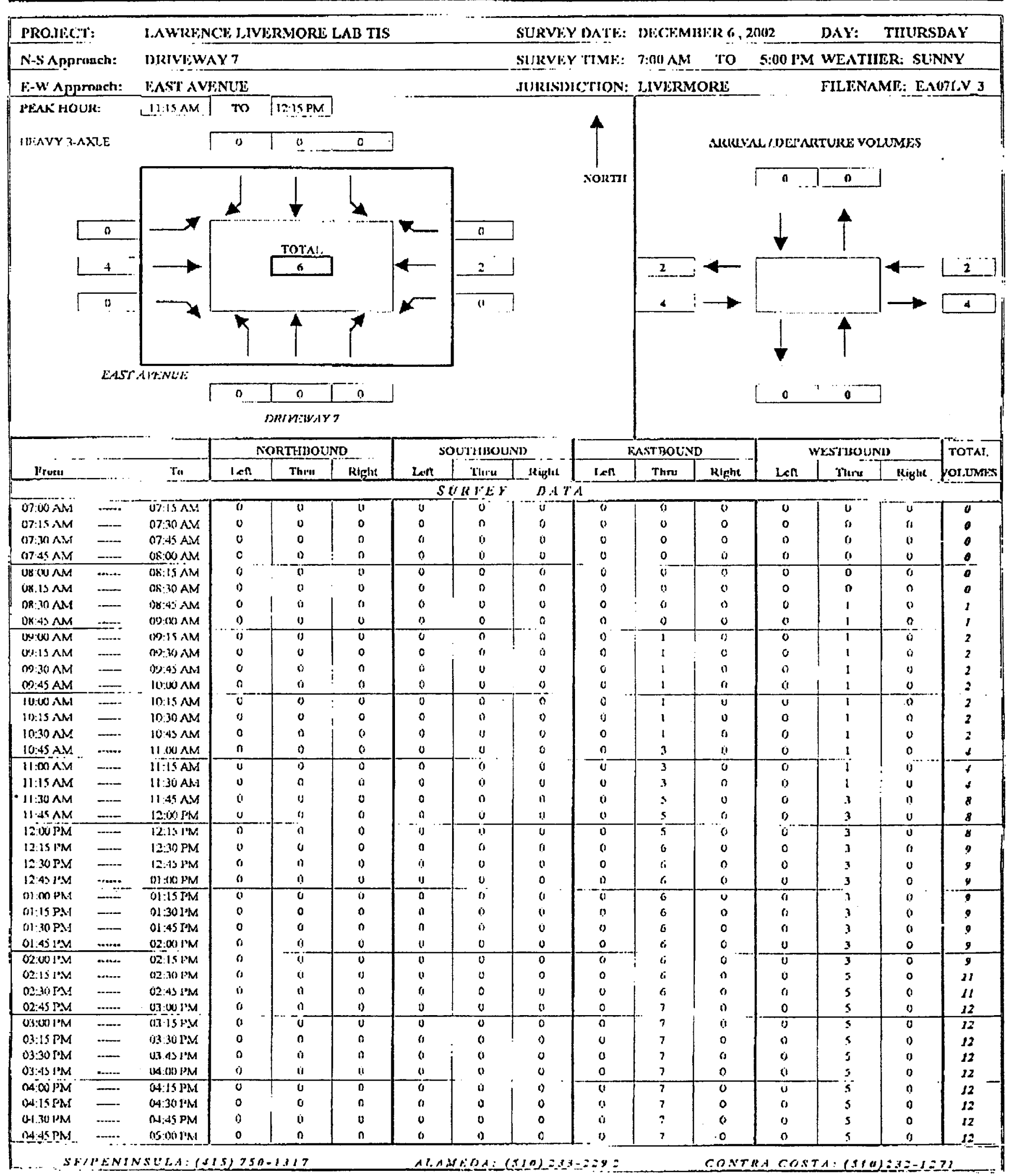




\section{Baymetrics Traffic Resources: INTERSECTION TURNING MOVEMENT SUMMARY}

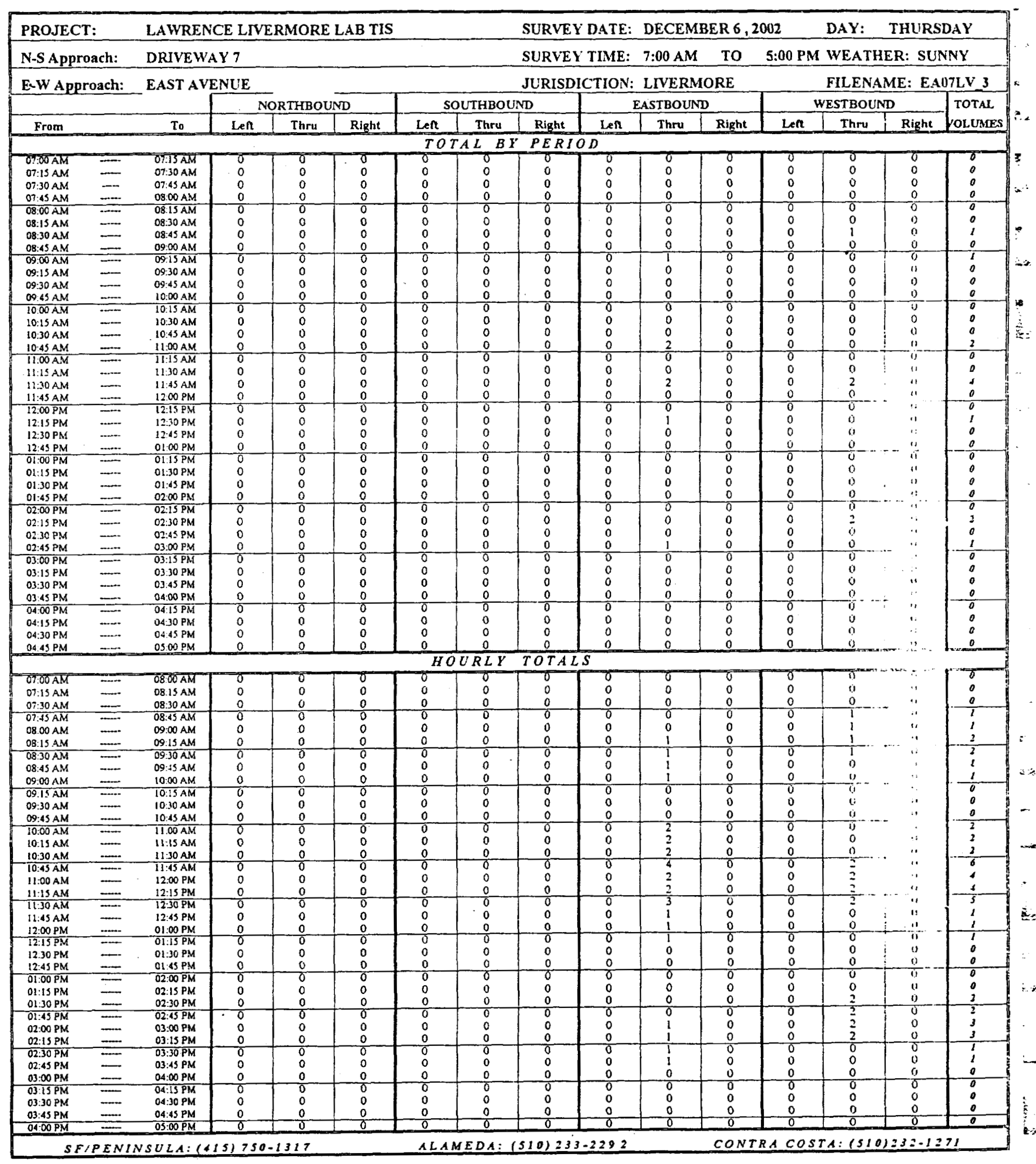




\section{Baymetrics Traffic Resources INTERSECTION TURNING MOVEMENT SUMMARY}

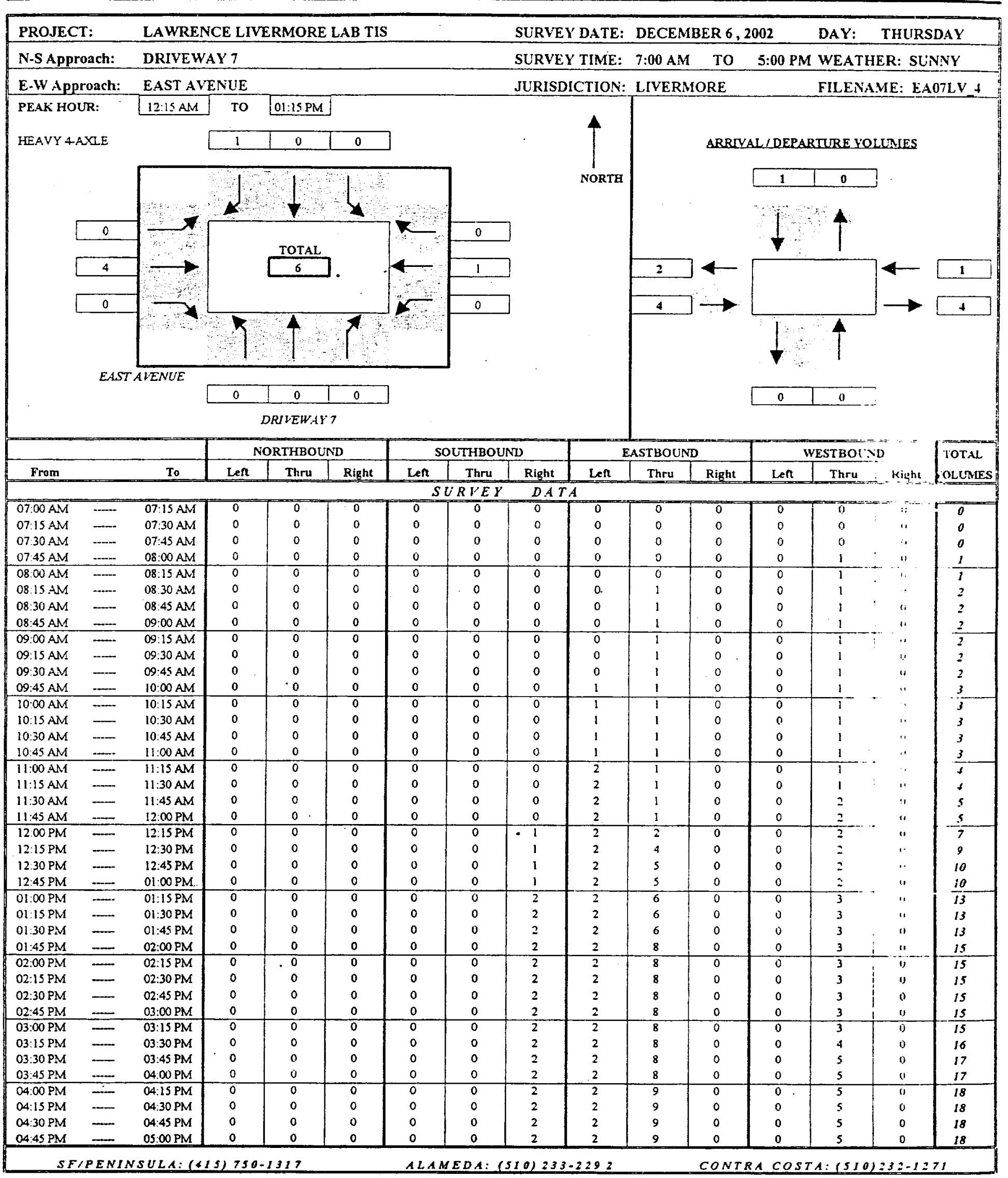




\section{Baymetrics Traffic Resources INTERSECTION TURNING MOVEMENT SUMMARY}

\begin{tabular}{|c|c|c|c|c|c|c|c|c|c|c|c|c|c|c|c|}
\hline \multirow{2}{*}{\multicolumn{2}{|c|}{$\begin{array}{l}\text { PROJECT: } \\
\text { N-S Approach: }\end{array}$}} & LAWRE & E LIV & MOR & AB TIS & & & SURVEY & DATE: & DECEM & ER 6,2 & 2002 & DAY: & THURS & DAY \\
\hline & & DRIVEW & & & & & & SURVEY & TIME: & 7:00 AM & To & 5:00 PM & WEATH & IER: SLis & NY \\
\hline E-W API & oach: & EAST A & UE & & & & & JURISD & TION: & LIVERN & RE & & FILENA & ME: EA & $67 \mathrm{LV} 44$ \\
\hline & & & & THBO & & & THBOO & & & ASTBOUN & & & ESTBOUN & & TOTAL \\
\hline From & & To & Left & Thru & Right & Len & Thru & Right & Lent & Thru & Right & Len & Thru & Right & KoLUMES \\
\hline & & & & & & & $L \quad B$ & $P E R I$ & & & & & & & \\
\hline $0700 \mathrm{AM}$ & $\cdots$ & TPISAM & 0 & 0 & 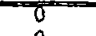 & $\overline{0}$ & 0 & 0 & 0 & 0 & 0 & $\overline{0}$ & $\overline{0}$ & i & 6 \\
\hline $07: 15 \mathrm{AM}$ & $\cdots$ & 07:30 AM & 0 & 0 & 0 & 0 & 0 & 0 & 0 & 0 & 0 & 0 & 0 & 0 & 0 \\
\hline $\begin{array}{l}07: 30 \mathrm{AM} \\
07: 45 \mathrm{AM}\end{array}$ & $=$ & $\begin{array}{l}\text { 07:45 AM } \\
08: 00 \mathrm{AM}\end{array}$ & $\begin{array}{l}0 \\
0\end{array}$ & $\begin{array}{l}0 \\
0\end{array}$ & $\begin{array}{l}0 \\
0\end{array}$ & $\begin{array}{l}0 \\
0\end{array}$ & $\begin{array}{l}0 \\
0\end{array}$ & $\begin{array}{l}0 \\
0\end{array}$ & $\begin{array}{l}0 \\
0\end{array}$ & $\begin{array}{l}0 \\
0\end{array}$ & $\begin{array}{l}0 \\
0\end{array}$ & $\begin{array}{l}0 \\
0\end{array}$ & $\begin{array}{l}0 \\
1\end{array}$ & $\begin{array}{l}0 \\
0\end{array}$ & $i$ \\
\hline $08: 00 \mathrm{AM}$ & $=$ & $08: 15 \mathrm{AM}$ & 0 & 0 & 0 & 0 & 0 & 0 & 0 & 0 & 0 & 0 & $\frac{1}{0}$ & $\frac{0}{0}$ & $\frac{1}{0}$ \\
\hline 08:15 AM & $\ldots$ & 08: $30 \mathrm{AM}$ & 0 & 0 & 0 & 0 & 0 & 0 & 0 & 1 & 0 & 0 & 0 & 0 & 1 \\
\hline $08: 30 \mathrm{AM}$ & $\cdots$ & 08:45 AM & 0 & 0 & 0 & 0 & 0 & 0 & 0 & 0 & 0 & 0 & 0 & 0 & 0 \\
\hline 08:43 AM & $\ldots$ & $09: 00 \mathrm{AM}$ & 0 & 0 & 0 & 0 & 0 & 0 & 0 & 0 & 0 & 0 & 0 & 0 & 0 \\
\hline $09: 00 \mathrm{AM}$ & $=$ & $09: 15 \mathrm{AM}$ & 0 & 0 & 0 & 0 & 0 & 0 & 0 & 0 & 0 & 0 & 0 & 0 & 0 \\
\hline $09: 15 \mathrm{AM}$ & - & $09: 30 \mathrm{AM}$ & 0 & 0 & 0 & 0 & 0 & 0 & 0 & 0 & 0 & 0 & 0 & 0 & 0 \\
\hline $\begin{array}{l}09: 30 \mathrm{AM} \\
09: 45 \mathrm{AM}\end{array}$ & $=$ & $09: 45 \mathrm{AM}$ & 0 & 0. & 0 & 0 & 0 & 0 & 0 & 0 & 0 & 0 & 0 & o & 0 \\
\hline$\frac{10.45 \mathrm{AM}}{10: 00 \mathrm{AM}}$ & $\equiv$ & $10: 15 \mathrm{AM}$ & $\frac{0}{0}$ & $\frac{0}{0}$ & $\frac{0}{0}$ & 0 & 0 & & 1 & 0 & 0 & 0 & 0 & 0 & 1 \\
\hline $10: 15 \mathrm{AM}$ & $\ldots$ & $10: 30 \mathrm{AM}$ & 0 & $\begin{array}{l}0 \\
0\end{array}$ & 0 & 0 & 0 & 0 & 0 & 0 & 0 & 0 & $\overline{0}$ & $\pi$ & 0 \\
\hline $10: 30 \mathrm{AM}$ & - & $10: 45 \mathrm{AM}$ & 0 & 0 & $\begin{array}{l}0 \\
0\end{array}$ & 0 & 0 & 0 & 0 & 0 & 0 & 0 & 0 & o & 0 \\
\hline $10: 45 \mathrm{AM}$ & - & $11: 00 \mathrm{AM}$ & 0 & 0 & $\begin{array}{l}0 \\
0\end{array}$ & $\begin{array}{l}0 \\
0\end{array}$ & 0 & & 0 & $\begin{array}{l}0 \\
0\end{array}$ & 0 & 0 & $\begin{array}{l}0 \\
0\end{array}$ & $" 1$ & 0 \\
\hline $11: 00 \mathrm{AM}$ & $\cdots$ & $11: 15 \mathrm{AM}$ & 0 & 0 & 0 & 0 & 0 & $\frac{0}{0}$ & $\frac{0}{1}$ & $\frac{0}{0}$ & $\frac{0}{0}$ & $\frac{0}{0}$ & $\frac{0}{0}$ & & $\frac{0}{1}$ \\
\hline $11: 15 \mathrm{AM}$ & $\ldots$ & $11: 30 \mathrm{AM}$ & 0 & 0 & 0 & 0 & 0 & 0 & 0 & 0 & 0 & 0 & 0 & $" 1$ & 0 \\
\hline $11: 30 \mathrm{AM}$ & $\ldots$ & $11: 45 \mathrm{AM}$ & 0 & 0 & 0 & 0 & 0 & 0 & 0 & 0 & 0 & 0 & 1 & $\because$ & $i$ \\
\hline $11: 45 \mathrm{AM}$ & $\ldots$ & $12: 00 \mathrm{PM}$ & 0 & 0 & 0 & 0 & 0 & 0 & 0 & 0 & 0 & 0 & $\dot{0}$ & $\because$ & 0 \\
\hline $12: 00 \overline{\mathrm{PM}}$ & & $12: 15 \mathrm{PM}$ & 0 & 0 & 0 & 0 & 0 & 1 & 0 & $\mathrm{~T}$ & 0 & 0 & 0 & " & 2 \\
\hline 12:15 PM & - & 12:30 PM & 0 & 0 & 0 & 0 & 0 & 0 & 0 & 2 & 0 & 0 & $\dot{0}$ & $\because$ & 2 \\
\hline $12: 30 \mathrm{PM}$ & $\bar{m}$ & 12:45 PM & 0 & 0 & 0 & 0 & $\begin{array}{l}0 \\
0\end{array}$ & $\begin{array}{l}0 \\
0\end{array}$ & $\begin{array}{l}0 \\
0\end{array}$ & $\begin{array}{l}1 \\
0\end{array}$ & $:$ & $\begin{array}{l}0 \\
0 \\
0\end{array}$ & 0 & "1 & 1 \\
\hline$\frac{12.45 \mathrm{PM}}{01.00 \mathrm{PM}}$ & $=$ & $\frac{01: 00 \mathrm{PM}}{01: 15 \mathrm{PM}}$ & $\frac{0}{0}$ & $\frac{0}{.0}$ & $\frac{0}{0}$ & $\frac{0}{0}$ & $\frac{0}{0}$ & $\frac{0}{1}$ & $\frac{0}{0}$ & $\frac{0}{1}$ & $\frac{0}{0}$ & $\frac{0}{0}$ & $\frac{0}{1}$ & $\frac{11}{41}$ & 0 \\
\hline $01: 15 \mathrm{PM}$ & $=$ & $01: 30 \mathrm{PM}$ & 0 & 0 & 0 & 0 & 0 & $\begin{array}{l}1 \\
0\end{array}$ & $\begin{array}{l}0 \\
0\end{array}$ & 0 & 0 & 0 & 1 & 11 & 0 \\
\hline 01:30 PM & $\cdots$ & 01:45 PM & 0 & 0 & 0 & 0 & 0 & 0 & 0 & 0 & 0 & 0 & 0 & $"$ & 0 \\
\hline $01: 45 \mathrm{PM}$ & $=$ & $02: 00 \mathrm{PM}$ & 0 & 0 & $=\frac{a}{a}$ & $\frac{0}{0}$ & 0 & 0 & 0 & 2 & 0 & 0 & 0 & $\therefore$ & 2 \\
\hline 02:00 PM & $=$ & $02: 15$ PM & 0 & 0 & 0 & $\overline{0}$ & 0 & 0 & 0 & 0 & 0 & 0 & 0 & $i$ & 0 \\
\hline 02:15 PM & - & 02:30 PM & 0 & 0 & 0 & 0 & 0 & 0 & 0 & 0 & 0 & 0 & $\dot{0}$ & $\because$ & 0 \\
\hline 02:30 PM & $\cdots$ & $02: 45$ PM & 0 & 0 & 0 & 0 & 0 & 0 & 0 & 0 & 0 & 0 & 0 & $\because$ & 0 \\
\hline $02: 45$ PM & $-\cdots$ & 03:00 PM & 0 & 0 & 0 & 0 & 0 & 0 & 0 & 0 & 0 & 0 & $u$ & $\therefore$ & 0 \\
\hline 03:00 PM & $\ldots$ & $03: 15 \mathrm{PM}$ & 0 & 0 & 0 & $\overline{0}$ & 0 & 0 & 0 & 0 & 0 & 0 & 0 & 11 & 0 \\
\hline $03: 15 \mathrm{PM}$ & $\ldots$ & $03: 30 \mathrm{PM}$ & 0 & 0 & 0 & 0 & 0 & 0 & 0 & 0 & 0 & 0 & 1 & $" 1$ & I \\
\hline $03: 30 \mathrm{PM}$ & $\cdots$ & 03:45 PM & 0 & 0 & 0 & 0 & 0 & 0 & 0 & 0 & 0 & 0 & $i$ & . & $i$ \\
\hline 03:45 PM & $=$ & $04: 00 \mathrm{PM}$ & 0 & 0 & 0 & 0 & 0 & 0 & 0 & 0 & 0 & 0 & 0 & " & 0 \\
\hline $04: 00 \mathrm{PM}$ & $=$ & $04: 15 \mathrm{PM}$ & 0 & 0 & 0 & 0 & 0 & 0 & 0 & $T$ & 0 & $\overline{0}$ & 0 & 1 & 7 \\
\hline 04:15 PM & + & $04: 30 \mathrm{PM}$ & 0 & 0 & 0 & 0 & 0 & 0 & 0 & 0 & 0 & 0 & 0 & $"$ & 0 \\
\hline $\begin{array}{l}\text { 04:30 PM } \\
08.45 M\end{array}$ & $\overline{0}$ & 04:45 PM & ${ }_{0}^{0}$ & 0 & 0 & 0 & ${ }_{0}^{0}$ & 0 & $0_{0}^{0}$ & 0 & 0 & 0 & 0 & $"$ & 0 \\
\hline $04: 45$ PM & $\ldots$ & $05: 00 \mathrm{PM}$ & 0 & 0 & 0 & $\frac{O}{H}$ & $\frac{0}{R L Y}$ & $\frac{0}{T O T A R}$ & 0 & 0 & 0 & 0 & 0 & $\because-$ & 0 \\
\hline & & & & & & & $R L Y$ & TOTAL & & & & & & & \\
\hline T.00AM & - & 68:00 AM & 0 & 0 & $\overline{0}$ & 0 & 0 & 0 & 0 & o & 8 & $\overline{0}$ & $T$ & $\pi$ & $T$ \\
\hline $\begin{array}{l}07: 15 \text { AM } \\
07: 30 \text { AM }\end{array}$ & $=$ & $\begin{array}{l}\text { 08:15 AM } \\
08: 30 \mathrm{AM}\end{array}$ & $\begin{array}{l}0 \\
0\end{array}$ & $\begin{array}{l}0 \\
0\end{array}$ & $\begin{array}{l}0 \\
0\end{array}$ & $\begin{array}{l}0 \\
0\end{array}$ & $\begin{array}{l}0 \\
0\end{array}$ & $\begin{array}{l}0 \\
0\end{array}$ & $\begin{array}{l}0 \\
0\end{array}$ & $\begin{array}{l}0 \\
1\end{array}$ & $\begin{array}{l}0 \\
0\end{array}$ & $\begin{array}{l}0 \\
0\end{array}$ & 1 & $\because$ & 1 \\
\hline $07: 45 \mathrm{AM}$ & - & $08: 45 A M$ & 0 & 0 & 0 & 0 & $\frac{1}{0}$ & 0 & 0 & 1 & 0 & 0 & 1 & ". & 2 \\
\hline $08: 00 \mathrm{AM}$ & $\ldots$ & $09: 00 \mathrm{AM}$ & 0 & 0 & 0 & 0 & 0 & 0 & 0 & 1 & 0 & 0 & 0 & ". & 1 \\
\hline 08:15 AM & $=$ & $09: 15 \mathrm{AM}$ & 0 & 0 & 0 & 0 & 0 & 0 & 0 & $\frac{1}{0}$ & 0 & 0 & 0 & $\cdot$ & 1 \\
\hline $08: 30 \mathrm{AM}$ & $=$ & $09: 30 \mathrm{AM}$ & 0 & 0 & 0 & 0 & 0 & 0 & 0 & 0 & 0. & 0 & 0 & $\because$ & 0 \\
\hline 08:45 AM & - & OS:A5 AM & $\begin{array}{l}0 \\
0\end{array}$ & $\begin{array}{l}0 \\
0\end{array}$ & $\begin{array}{l}0 \\
0\end{array}$ & $\begin{array}{l}0 \\
0\end{array}$ & $\begin{array}{l}0 \\
0\end{array}$ & $\begin{array}{l}0 \\
0\end{array}$ & $\begin{array}{l}0 \\
1\end{array}$ & $\begin{array}{l}0 \\
0\end{array}$ & $\begin{array}{l}0 \\
0\end{array}$ & 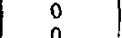 & 0 & $\because$ & 0 \\
\hline $09 ; 00 \mathrm{AM}$ & $=$ & $\frac{10: 00 \mathrm{AM}}{10: 15 \mathrm{AM}}$ & $-\frac{0}{0}$ & 0 & 0 & 0 & 0 & 0 & $\frac{1}{1}$ & $\frac{0}{0}$ & $\frac{0}{0}$ & $\frac{0}{0}$ & 0 & $: 1$ & 1 \\
\hline $09: 15 \overline{A M}$ & $\bar{E}$ & $\begin{array}{l}10: 15 \mathrm{AM} \\
10: 30 \mathrm{AM}\end{array}$ & 0 & 0 & 0 & 0 & 0 & 0 & 1 & 0 & & $\begin{array}{l}0 \\
0\end{array}$ & 0 & $\ddot{~}$ & 1 \\
\hline 09:30 AM & $=$ & $\begin{array}{l}10: 30 \mathrm{AM} \\
10: 45 \mathrm{AM}\end{array}$ & 0 & 0 & 0 & 0 & 0 & 0 & i & 0 & 0 & $\begin{array}{l}0 \\
0\end{array}$ & $\begin{array}{l}0 \\
0\end{array}$ & $\because "$ & $i$ \\
\hline$\frac{09: 45 \mathrm{AM}}{10,00 \mathrm{AM}}$ & $=$ & $\frac{10: 45 \mathrm{AM}}{11: 00 \mathrm{AM}}$ & 0 & 0 & 0 & 0 & क & 0 & 0 & 0 & 0 & $\frac{0}{0}$ & $\frac{0}{10}$ & $\because$ & $\frac{1}{0}$ \\
\hline $\begin{array}{l}10: 00 \mathrm{AM} \\
10: 15 \mathrm{AM}\end{array}$ & $\overline{-}$ & $11: 15 \mathrm{AM}$ & 0 & 0 & 0 & 0 & 0 & 0 & 1 & 0 & 0 & 0 & 0 & "1 & 1 \\
\hline $10: 30 \mathrm{AM}$ & $=$ & $11: 30 \mathrm{AM}$ & 0 & 0 & 0 & 0 & 0 & 0 & $i$ & 0 & 0 & 0 & 0 &.. & $i$ \\
\hline $10.45 \mathrm{AM}$ & - & $11.45 \mathrm{AM}$ & 0 & 0 & 0 & 0 & 0 & 0 & 1 & 0 & 0 & 0 & $\frac{T}{1}$ & ". & 2 \\
\hline $11: 00 \mathrm{AM}$ & $\cdots$ & $17.00 \mathrm{PM}$ & 0 & 0 & 0 & 0 & 0 & 0 & 1 & 0 & 0 & 0 & i & $"$ & 3 \\
\hline I1:ISAM & - & 12:15 PM & 0 & 0 & 0 & 0 & 0 & 1 & 0 & 1 & 0 & 0 & $i$ & $" 1$ & 3 \\
\hline $1130 \mathrm{AM}$ & - & $12: 30 \mathrm{PM}$ & 0 & 0 & 0 & 0 & 0 & 1 & 0 & 3 & 0 & 0 & $T$ & "1 & 5 \\
\hline $11: 45 \mathrm{AM}$ & - & $12: 45$ PM & 0 & 0 & 0 & 0 & $\stackrel{0}{0}$ & 1 & 0 & 4 & 0 & 0 & 0 & $" 1$ & s \\
\hline $12: 00$ PM & $=$ & $01: 00$ PM & 0 & 0 & 0 & 0 & 0 & 1 & 0 & 4 & 0 & 0 & 0 & $\therefore$ & 3 \\
\hline $12: 13 \mathrm{PM}$ & $=$ & $0115 \mathrm{PM}$ & 0 & 0 & 0 & 0 & 0 & 1 & 0 & 4 & 0 & 0 & 1 & $\bar{n}$ & 6 \\
\hline 12:30 PM & 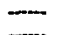 & $01: 30$ PM & 0 & 0 & 0 & 0 & 0 & 1 & 0 & 2 & 0 & 0 & 1 & 3 & \pm \\
\hline $12: 45$ PM & $=$ & 01:45 PM & 0 & 0 & 0 & 0 & $\frac{0}{0}$ & 1 & $\frac{0}{0}$ & $\frac{1}{2}$ & $\frac{0}{1}$ & 0 & 1 & $\therefore$ & 3 \\
\hline $01: 00 \overline{\mathrm{PM}}$ & - & $02: 00^{\prime} \mathrm{PM}$ & 0 & 0 & $\gamma$ & 0 & 0 & $T$ & 0 & 3 & 0 & 0 & 1 & 5 & 5 \\
\hline $01: 15$ PM & - & $02: 15$ PM & 0 & 0 & 0 & 0 & 0 & 0 & 0 & 2 & 0 & 0 & 0 & $\because$ & 2 \\
\hline $0130 \mathrm{PM}$ & $=$ & $02: 30 \mathrm{PM}$ & 0 & 0 & 0 & 0 & 0 & 0 & 0 & 2 & 0 & 0 & 0 & 9 & 3 \\
\hline $01: 45$ PM & $\ldots$ & $02: 45 \mathrm{PM}$ & 0 & 0 & 0 & 0 & 0 & 0 & 0 & 2 & 0 & 0 & 0 & 0 & 2 \\
\hline $02: 00 \mathrm{PM}$ & $\ldots$ & $03: 00 \mathrm{PM}$ & 0 & 0 & 0 & 0 & 0 & 0 & 0 & 0 & 0 & 0 & 0 & 0 & 0 \\
\hline $02.15 P M$ & $\ldots$ & $03: 15 \mathrm{PM}$ & 0 & 0 & 0 & 0 & 0 & 0 & 0 & 0 & 0 & 0 & 0 & 0 & 0 \\
\hline $02.30 \mathrm{PM}$ & $=$ & $03: 30 \mathrm{PM}$ & 0 & 0 & $\overline{0}$ & 0 & 0 & $\gamma$ & 0 & 0 & 0 & 0 & 1 & 0 & $I$ \\
\hline $02: 45$ PM & $\ldots$ & 03:45 PM & 0 & 0 & 0 & 0 & 0 & 0 & 0 & 0 & 0 & 0 & 2 & D & 2 \\
\hline $03: 00 \mathrm{PM}$ & 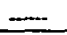 & $04: 00 \mathrm{PM}$ & 0 & 0 & 0 & 0 & 0 & 0 & 0 & 0 & 0 & 0 & 2 & 0 & 2 \\
\hline $03: 15$ PM & $=$ & $04: 15 \mathrm{PM}$ & 0 & 0 & 0 & 0 & 0 & 0 & 0 & 1 & 0 & 0 & 2 & 0 & 3 \\
\hline 03:10 PM & $\cdots$ & $04: 30 \mathrm{PM}$ & 0 & 0 & 0 & 0 & 0 & 0 & 0 & 1 & 0 & 0 & 1 & 0 & 2 \\
\hline 03:45 PM & $\ldots$ & $04: 45 \mathrm{PM}$ & 0 & 0 & 0 & 0 & 0 & 0 & 0 & 1 & 0 & 0 & 0 & 0 & 1 \\
\hline $04: 00 \mathrm{PM}$ & $=$ & $05: 00 \mathrm{PM}$ & 0 & 0 & 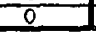 & 0 & 0 & 0 & 0 & & 0 & 0 & 0 & 0 & $I$ \\
\hline$S F$ & $E N I$ & $U L A: 1$ & 17 & 7 & & 11 & DA: & 0) 2 & 292 & & $0 \Omega$ & Co & 15 & $=32-$ & \\
\hline
\end{tabular}




\section{Baymetrics Traffic Resources INTERSECTION TURNING MOVEMENT SUMMARY}

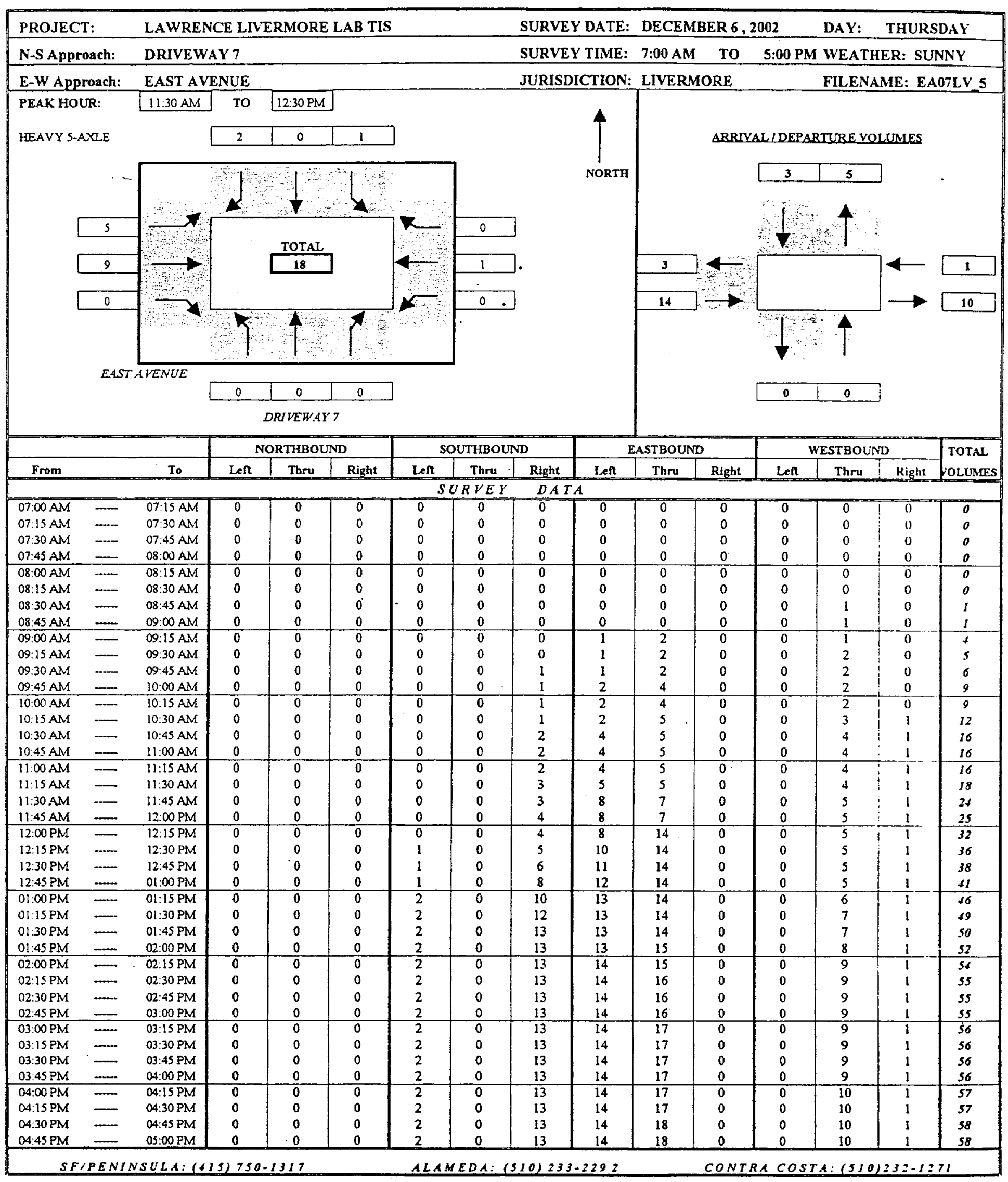




\section{Baymetrics Traffic Resources INTERSECTION TURNING MOVEMENT SUMMARY}

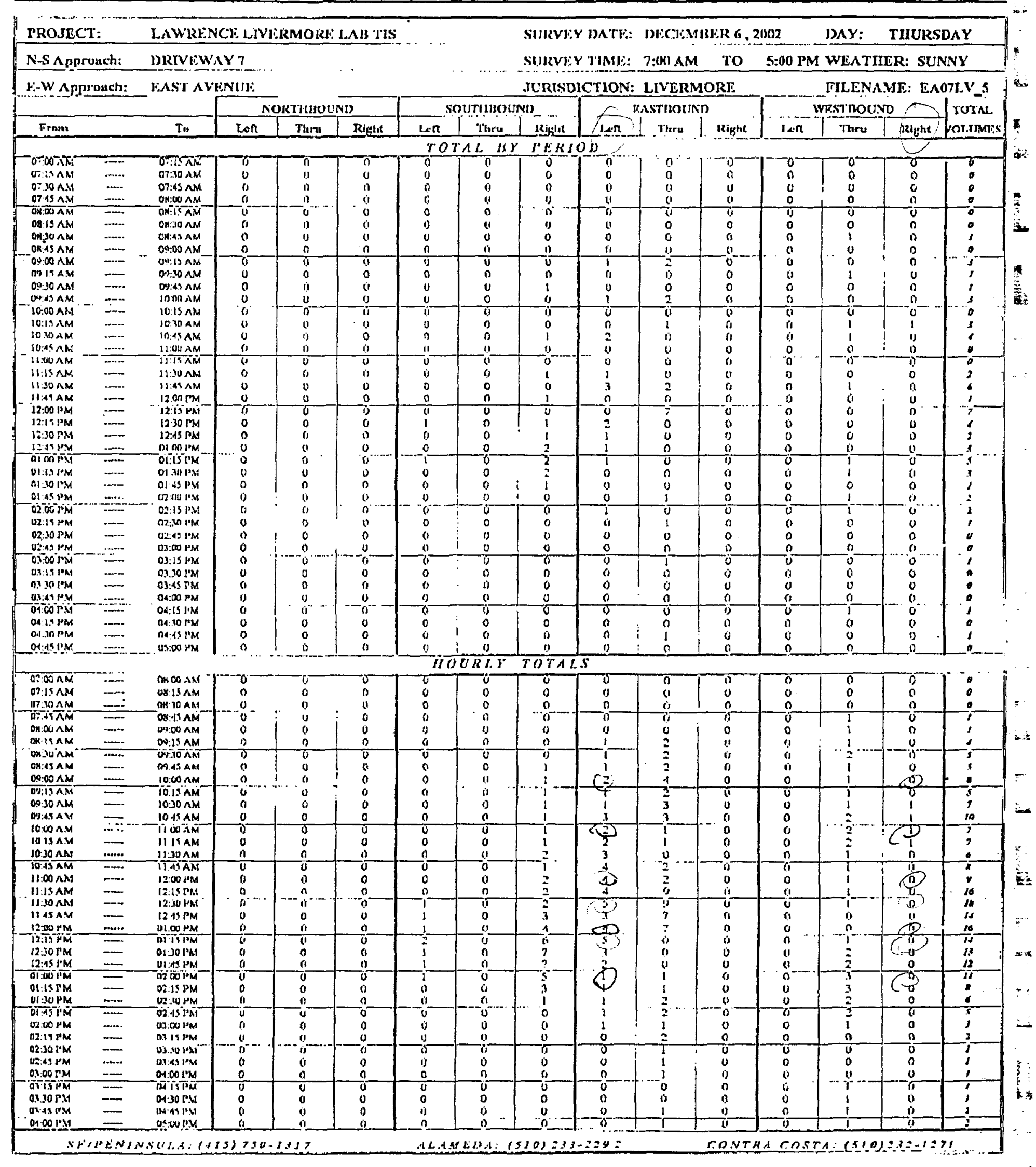




\section{Baymetrics Traffic Resources INTERSECTION TURNING MOVEMENT SUMMARY}

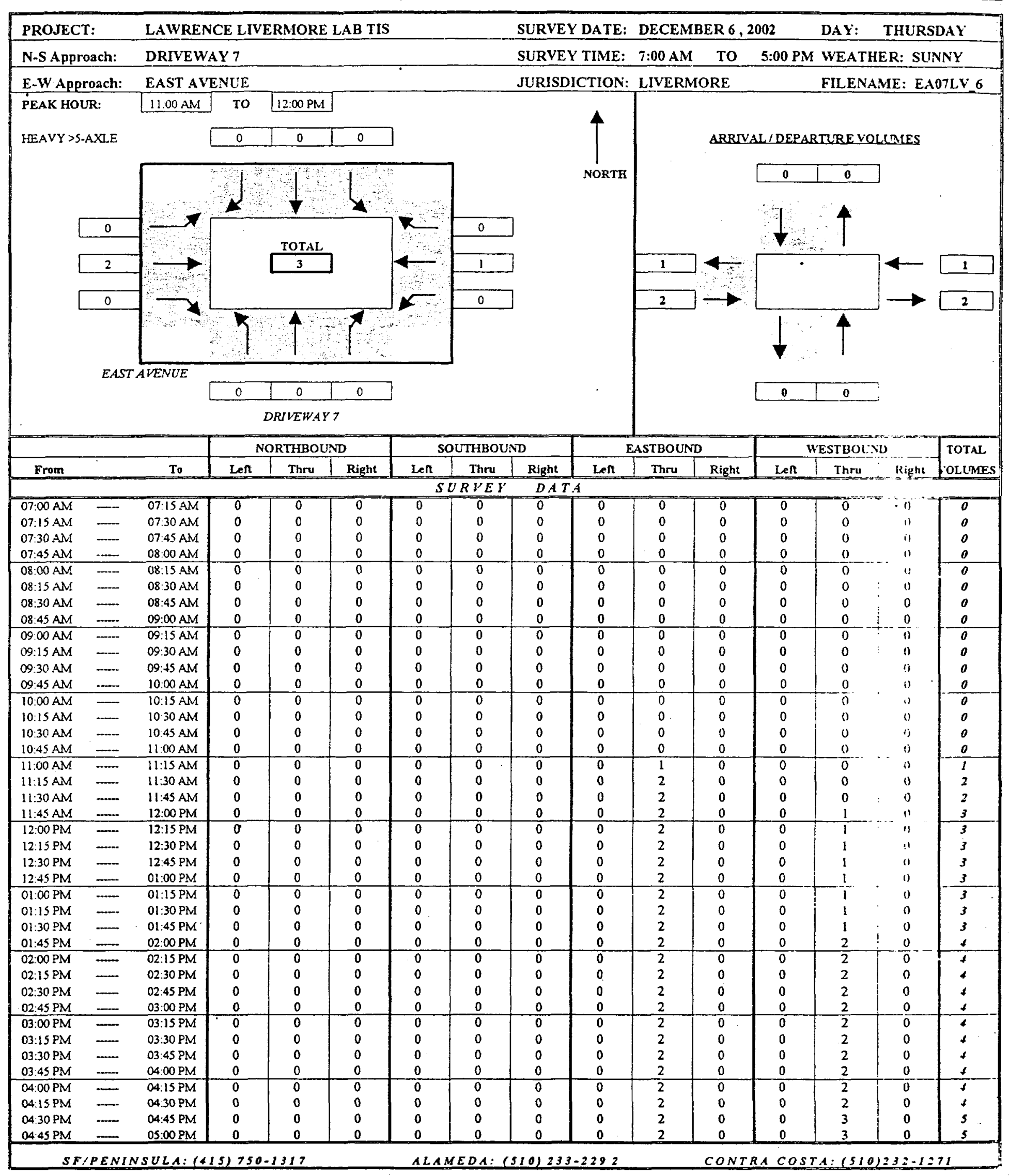




\section{Baymetrics Traffic Resources INTERSECTION TURNING MOVEMENT SUMMARY}

\begin{tabular}{|c|c|c|c|c|c|c|c|c|c|c|c|c|c|c|c|}
\hline \multirow{2}{*}{\multicolumn{2}{|c|}{$\begin{array}{l}\text { PROJECT: } \\
\text { N-S Approach: }\end{array}$}} & \multicolumn{6}{|c|}{ LAWRENCE LIVERMORE LAB TIS } & SURVE & DATE: & DECEM & ER 6, & 002 & DAY: & THURS & DAY \\
\hline & & DRIVE H & & & & & & SURVE & IIME: & $7: 00 \mathrm{AM}$ & TO & 5:00 PI & WEAT & ER: SU & NY \\
\hline E-W AP & oach: & EAST AT & IUE & & & & & JURISD & TION: & LIVERN & ORE & & FILEN & ME: EA & $07 \mathrm{LV} 6$ \\
\hline & & & & THBO & & & THBO & & & ASTBOUN & & & ESTBOU & & TOTAL \\
\hline From & & To & Len & Thru & Right & Lent & Thru & Right & Left & Thru & Right & Len & Thru & Right & younares \\
\hline & & & & & & & $L \quad B$ & $P E R I$ & & & & & & & \\
\hline $07.00 \mathrm{AM}$ & $=$ & ग:डडAM & 0 & 0 & 8 & 0 & 8 & 0 & 0 & 0 & $\sigma$ & 0 & 0 & $\overline{0}$ & 0 \\
\hline $\begin{array}{l}07: 15 \mathrm{AM} \\
07: 30 \mathrm{AM}\end{array}$ & $\bar{m}$ & $\begin{array}{l}07: 30 \mathrm{AM} \\
07: 43 \mathrm{AM}\end{array}$ & $\begin{array}{l}0 \\
0\end{array}$ & $\begin{array}{l}0 \\
0\end{array}$ & $\begin{array}{l}0 \\
0\end{array}$ & $\begin{array}{l}0 \\
0\end{array}$ & $\begin{array}{l}0 \\
0\end{array}$ & $\begin{array}{l}0 \\
0\end{array}$ & $\begin{array}{l}0 \\
0\end{array}$ & $\begin{array}{l}0 \\
0\end{array}$ & $\begin{array}{l}0 \\
0\end{array}$ & $\begin{array}{l}0 \\
0\end{array}$ & $\begin{array}{l}0 \\
0\end{array}$ & $?$ & 0 \\
\hline 07:45 AM & $\overline{-}$ & $\begin{array}{r}08.00 \mathrm{AM} \\
\end{array}$ & 0 & 0 & 0 & 0 & $\begin{array}{l}0 \\
0 \\
\end{array}$ & $\begin{array}{l}0 \\
0 \\
\end{array}$ & $\begin{array}{l}0 \\
0 \\
\end{array}$ & $\begin{array}{l}0 \\
0 \\
\end{array}$ & $\begin{array}{l}0 \\
0 \\
\end{array}$ & $\begin{array}{l}0 \\
0 \\
\end{array}$ & $\begin{array}{l}0 \\
0 \\
\end{array}$ & $\begin{array}{l}0 \\
0 \\
\end{array}$ & $:$ \\
\hline $08.00 \mathrm{AM}$ & $\ldots$ & $08: 15 \mathrm{AM}$ & 0 & 0 & 0 & 0 & 0 & 0 & 0 & 0 & 0 & 0 & 0 & 0 & 0 \\
\hline $\begin{array}{l}\text { 08:15 AM } \\
08: 30 \text { AM }\end{array}$ & 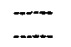 & $\begin{array}{l}08: 30 \text { AM } \\
08: 45 \text { AM }\end{array}$ & $\begin{array}{l}0 \\
0\end{array}$ & $\begin{array}{l}0 \\
0\end{array}$ & $\begin{array}{l}0 \\
0\end{array}$ & $\begin{array}{l}0 \\
0\end{array}$ & $\begin{array}{l}0 \\
0\end{array}$ & $\begin{array}{l}0 \\
0\end{array}$ & $\begin{array}{l}0 \\
0\end{array}$ & $\begin{array}{l}0 \\
0\end{array}$ & $\begin{array}{l}0 \\
0\end{array}$ & $\begin{array}{l}0 \\
0\end{array}$ & $\begin{array}{l}0 \\
0\end{array}$ & $\hat{\imath}$ & $:$ \\
\hline 08:45 AM & $\ldots$ & $09: 00 \mathrm{AM}$ & $\begin{array}{l}0 \\
0\end{array}$ & 0 & $\begin{array}{l}0 \\
0 \\
\end{array}$ & 0 & 0 & 0 & $\begin{array}{l}0 \\
0 \\
\end{array}$ & $\begin{array}{l}0 \\
0\end{array}$ & $\begin{array}{l}0 \\
0 \\
\end{array}$ & 0 & 0 & 0 & 0 \\
\hline $09: 00 \mathrm{AM}$ & $\cdots$ & $09: 15 \mathrm{AM}$ & 0. & 0 & 0 & 0 & 0 & 8 & 0 & 0 & 0 & 0 & 0 & 6 & 0 \\
\hline $09: 15$ AMS & - & $09.30 \mathrm{AM}$ & 0 & 0 & 0 & 0 & 0 & 0 & 0 & 0 & 0 & 0 & 0 & i & 0 \\
\hline 09:30 AM & $\cdots$ & $09: 45 \mathrm{AM}$ & 0 & 0 & 0 & 0 & 0 & 0 & 0 & 0 & 0 & 0 & 0 & 0 & 0 \\
\hline $09: 45 \mathrm{AM}$ & $=$ & $10: 00 \mathrm{AM}$ & 0 & 0 & 0 & 0 & 0 & 0 & 0 & 0 & 0 & 0 & 0 & 0 & 0 \\
\hline $10: 00 \mathrm{AM}$ & $=$ & $10: 15 \mathrm{AM}$ & 0 & 0 & 0 & 0 & 0 & 0 & 0 & 0 & 0 & 0 & 0 & is & $\theta$ \\
\hline $10: 15 \mathrm{AM}$ & $\ldots$ & $10: 30 \mathrm{AM}$ & 0 & 0 & 0 & 0 & 0 & 0 & 0 & 0 & 0 & 0 & 0 & " & 0 \\
\hline $10: 30 \mathrm{AM}$ & - & $10: 45 \mathrm{AM}$ & 0 & 0 & 0 & 0 & 0 & 0 & 0 & 0 & 0 & 0 & 0 & a & 0 \\
\hline $10: 45 \mathrm{AM}$ & - & $11: 00 \mathrm{AM}$ & 0 & 0 & 0 & 0 & 0 & 0 & 0 & 0 & 0 & 0 & 0 & $\because$ & 0 \\
\hline $11: 00 \mathrm{AM}$ & $=$ & $11: 19 \mathrm{AM}$ & 0 & 0 & 0 & 0 & 0 & 0 & $\overline{0}$ & 1 & \% & 0 & & is & I \\
\hline 11:15 AM & $=$ & $11: 30 \mathrm{AM}$ & 0 & 0 & 0 & 0 & 0 & 0 & 0 & 1 & 0 & 0 & 0 & " & 1 \\
\hline $\begin{array}{l}11: 30 \mathrm{AM} \\
11.45 \mathrm{AM}\end{array}$ & $\ldots$ & $\begin{array}{l}11: 45 \mathrm{AM} \\
1300 \mathrm{PM}\end{array}$ & $\begin{array}{l}0 \\
0\end{array}$ & $\begin{array}{l}0 \\
0\end{array}$ & $\begin{array}{l}0 \\
0\end{array}$ & $\begin{array}{l}0 \\
0 \\
0\end{array}$ & $\begin{array}{l}0 \\
0\end{array}$ & 0 & 0 & 0 & 0 & $\begin{array}{l}0 \\
0\end{array}$ & 0 & $\ddot{a}$ & 0 \\
\hline$\frac{11.43 \mathrm{AM}}{12: 00 \mathrm{PM}}$ & $=$ & $\frac{12.00 \mathrm{PM}}{12: 15 \mathrm{PM}}$ & 0 & 0 & 0 & $\frac{0}{0}$ & 0 & 0 & $\frac{0}{0}$ & $\frac{0}{0}$ & $\frac{0}{0}$ & 0 & $\frac{1}{0}$ & $"-$ & $\frac{1}{0}$ \\
\hline 12:15 PM & - & $12: 30 \mathrm{PM}$ & 0 & 0 & 0 & 0 & 0 & 0 & 0 & 0 & 0 & 0 & 0 & $\therefore$ & 0 \\
\hline 12:30 PM & 一 & $12: 45$ PM & 0 & 0 & 0 & 0 & 0 & 0 & 0 & 0 & 0 & 0 & 0 & . & 0 \\
\hline 12:45 PM & $=$ & $01: 00$ PM & 0 & 0 & 0 & 0 & 0 & 0 & 0 & 0 & 0 & 0 & 0 & $\therefore$ & 0 \\
\hline $01: 00 \mathrm{PM}$ & 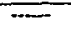 & $01: 15 \mathrm{PM}$ & $\overline{0}$ & 0 & 0 & 0 & 0 & 0 & 0 & 0 & 0 & 0 & 0 & 11 & 0 \\
\hline $0: 15$ PM & $\ldots$ & $06.30 \mathrm{PM}$ & 0 & 0 & 0 & 0 & 0 & 0 & 0 & 0 & 0 & 0 & 0 & $" 1$ & 0 \\
\hline $01: 30 \mathrm{PM}$ & $\square$ & $01: 45 \mathrm{PM}$ & 0 & 0 & 0 & 0 & 0 & 0 & 0 & 0 & 0 & 0 & 0 & "s & 0 \\
\hline Ol:45 PM & $\ldots$ & $02: 00 \mathrm{PM}$ & 0 & 0 & 0 & 0 & 0 & 0 & 0 & 0 & 0 & 0 & 1 & $\because$ & 1 \\
\hline $02: 00 \mathrm{PM}$ & - & $02: 15 \mathrm{PM}$ & 0 & 0 & 0 & 0 & 0 & 0 & 0 & 0 & 0 & 0 & 0 & $\because$ & 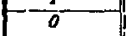 \\
\hline 02:15 PM & $\cdots$ & 02:30 PM & 0 & 0 & 0 & 0 & 0 & 0 & 0 & 0 & 0 & 0 & 0 & $\therefore$ & 0 \\
\hline $02: 30 \mathrm{PM}$ & $一$ & $02: 45 \mathrm{PM}$ & 0 & 0 & 0 & 0 & 0 & 0 & 0 & 0 & 0 & & 0 & .' & 0 \\
\hline $02: 45$ PM & $\ldots$ & $03: 00 \mathrm{PM}$ & 0 & 0 & 0 & 0 & 0 & 0 & 0 & 0 & 0 & 0 & 0 & $\because$ & $\div$ \\
\hline $03: 00$ PM & $\infty$ & $03: 15 \mathrm{PM}$ & 0 & 0 & 0 & 0 & 0 & 0 & 0 & 0 & 0 & 0 & $\overline{0}$ & $\because$ & 0 \\
\hline 03:15 PM & $\cdots$ & $03: 30$ PM & 0 & 0 & 0 & 0 & 0 & 0 & $a$ & 0 & 0 & 0 & 0 &. & 0 \\
\hline 03:30 PM & - & 03:45 PM & 0 & 0 & 0 & 0 & 0 & 0 & 0 & 0 & 0 & 0 & 0 & "1 & 0 \\
\hline 03:45 PM & $\ldots$ & 04:00 PM & 0 & 0 & 0 & 0 & 0 & 0 & 0 & 0 & 0 & 0 & 0 & 11 & 0 \\
\hline $04: 00 \mathrm{PM}$ & $\ldots$ & OA:15 PM & 0 & 0 & 0 & 0 & 0 & 0 & 0 & 0 & 0 & 0 & 0 & $\because$ & $\overline{0}$ \\
\hline $04: 15$ PM & $\ldots$ & $04: 30 \mathrm{PM}$ & 0 & 0 & 0 & 0 & 0 & 0 & 0 & 0 & 0 & 0 & 0 & " & 0 \\
\hline 04:30 PM & $\ldots$ & $04: 45 \mathrm{PM}$ & 0 & 0 & 0 & 0 & 0 & 0 & 0 & 0 & 0 & 0 & 1 & $\because$ & 1 \\
\hline $04: 45 \mathrm{PM}$ & $\ldots$ & $05: 00 \mathrm{PM}$ & 0 & 0 & 0 & 0 & 0 & 0 & 0 & 0 & 0 & 0 & i & $\therefore$ & 0 \\
\hline & & & & & & & $R L Y$ & TOTA & & & & & & & \\
\hline क7:00मM & 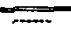 & O8:00AM & $\pi$ & $\gamma$ & 0 & 0 & 8 & $\sigma$ & $\sigma$ & 0 & 0 & 0 & $\overline{1}$ & $\therefore$ & 0 \\
\hline $07: 15 \mathrm{AM}$ & - & $08: 15$ AM & 0 & 0 & 0 & 0 & 0 & 0 & 0 & 0 & 0 & 0 & 0 & $\therefore$ & 0 \\
\hline 07:30 AM & $\ldots$ & $08: 30 \mathrm{AM}$ & 0 & 0 & $\frac{0}{0}$ & 0 & 0 & $\frac{0}{0}$ & $\frac{0}{0}$ & $\frac{0}{0}$ & 0 & 0 & 0 & $\because$ & 0 \\
\hline $07.45 \mathrm{AM}$ & $\infty$ & $08.45 \mathrm{AM}$ & 0 & $\begin{array}{l}0 \\
0\end{array}$ & 0 & 0 & $\begin{array}{l}0 \\
0\end{array}$ & $\begin{array}{l}0 \\
0\end{array}$ & $\begin{array}{l}0 \\
0\end{array}$ & $\begin{array}{l}0 \\
0\end{array}$ & $\begin{array}{l}0 \\
0\end{array}$ & $\begin{array}{l}0 \\
0\end{array}$ & 0 & $\because$ & 0 \\
\hline $\begin{array}{l}08.00 \mathrm{AM} \\
08: 15 \mathrm{AM}\end{array}$ & - & $09: 00 \mathrm{AM}$ & $\begin{array}{l}0 \\
0\end{array}$ & $\begin{array}{l}0 \\
0\end{array}$ & $\begin{array}{l}0 \\
0\end{array}$ & $\begin{array}{l}0 \\
0\end{array}$ & $\begin{array}{l}0 \\
0\end{array}$ & $\begin{array}{l}0 \\
0\end{array}$ & 0 & $\begin{array}{l}0 \\
0\end{array}$ & $\begin{array}{l}0 \\
0\end{array}$ & $\begin{array}{l}0 \\
0\end{array}$ & $\begin{array}{l}0 \\
0\end{array}$ & $\because$ & 0 \\
\hline$\frac{08: 15 \mathrm{AM}}{-08: 30 \mathrm{AM}}$ & 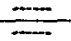 & $\frac{09: 19 \text { AM }}{09: 30 \text { AM }}$ & $\frac{0}{0}$ & 0 & $\frac{0}{0}$ & $-\frac{0}{0}$ & $\frac{0}{0}$ & 0 & 0 & 0 & 0 & 0 & $\frac{0}{0}$ & $\because "$ & $\frac{1}{0}$ \\
\hline 08:45 AM & $\ldots$ & $09: 49 \mathrm{AM}$ & 0 & 0 & 0 & 0 & 0 & 0 & 0 & 0 & 0 & 0 & 0 & .". & 0 \\
\hline $09: 00 \mathrm{AM}$ & $\ldots$ & $10: 00 \mathrm{AM}$ & 0 & 0 & 0 & 0 & 0 & 0 & 0 & 0 & 0 & 0 & 0 & .1 & $a$ \\
\hline $09: 15 \mathrm{AM}$ & $\infty$ & $1015 \mathrm{AM}$ & 0 & 0 & 0 & 0 & 0 & 0 & 0 & 0 & 0 & 0 & 0 & ., & 0 \\
\hline $09: 30 \mathrm{AM}$ & - & 10:30 AM & 0 & 0 & 0 & 0 & 0 & 0 & 0 & 0 & 0 & 0 & 0 & . & 0 \\
\hline 09:05 AM & $=$ & $10: 45 \mathrm{AM}$ & 0 & 0 & 0 & 0 & 0 & 0 & 0 & 0 & 0 & 0 & 0 & . & 0 \\
\hline $10: 00 \mathrm{AM}$ & $\ldots$ & $11: 00 \mathrm{AM}$ & 0 & 0 & 0 & 0 & 0 & 8 & 0 & 0 & 0 & $\overline{0}$ & $\dot{0}$ & & 0 \\
\hline 10:15 AM & - & 11:15 AM & $\stackrel{0}{0}$ & $\begin{array}{l}0 \\
0\end{array}$ & $\begin{array}{l}0 \\
0\end{array}$ & $\begin{array}{l}0 \\
0\end{array}$ & $\begin{array}{l}0 \\
0\end{array}$ & 0 & $\begin{array}{l}0 \\
0\end{array}$ & $\frac{1}{2}$ & $\begin{array}{l}0 \\
0\end{array}$ & $\begin{array}{l}0 \\
0\end{array}$ & $\begin{array}{l}0 \\
0\end{array}$ & $\because$ & 1 \\
\hline $10: 30 \mathrm{AM}$ & $\ldots$ & 11:30 AM & $\frac{0}{0}$ & $-\frac{0}{0}$ & $\frac{0}{0}$ & $\frac{0}{0}$ & 0 & $\frac{0}{0}$ & $\frac{0}{0}$ & $\frac{2}{2}$ & $\frac{0}{0}$ & $\frac{0}{0}$ & $\frac{0}{0}$ & $\therefore$ & $\frac{2}{2}$ \\
\hline $10: 45 \mathrm{AM}$ & 5 & $\begin{array}{l}11: 45 \mathrm{AM} \\
12: 00 \mathrm{PM}\end{array}$ & $\begin{array}{l}0 \\
0\end{array}$ & $\begin{array}{l}0 \\
0\end{array}$ & $\begin{array}{l}0 \\
0\end{array}$ & 0 & 0 & 0 & 0 & $\frac{1}{2}$ & 0 & 0 & 1 & $\because$ & j \\
\hline $\begin{array}{l}11: 00 \mathrm{AM} \\
11: 15 \mathrm{AM}\end{array}$ & $\bar{F}$ & $\begin{array}{l}12: 00 \mathrm{PM} \\
12: 15 \mathrm{PM}\end{array}$ & $\begin{array}{l}0 \\
0\end{array}$ & 0 & 0 & 0 & 0 & 0 & 0 & $i$ & 0 & 0 & $i$ & $\because 4$ & 3 \\
\hline $11: 30 \mathrm{AM}$ & $=$ & $12: 30 \mathrm{PM}$ & 0 & $\%$ & 0 & 0 & 0 & 0 & 0 & 0 & 0 & 0 & 1 & iii & $T$ \\
\hline $11: 45$ AM & $\cdots$ & 12:19 PM & 0 & 0 & 0 & 0 & 0 & 0 & 0 & 0 & 0 & $\circ$ & $i$ & $"$ & 1 \\
\hline $12: 00 \mathrm{PM}$ & $\ldots$ & $01: 00 \mathrm{PM}$ & 0 & 0 & 0 & 0 & 0 & 0 & 0 & 0 & 0 & 0 & 0 & at & $a$ \\
\hline $12: 15 \mathrm{PM}$ & $\cdots$ & $01.15 \mathrm{PM}$ & 0 & -0 & 0 & 0 & 0 & 0 & 0 & 0 & 0 & 0 & 0 & $7 i$ & 0 \\
\hline 12:30 PM & $\ldots$ & $01: 30 \mathrm{PM}$ & 0 & 0 & 0 & 0 & 0 & 0 & 0 & 0 & 0 & 0 & 0 & 0 & 0 \\
\hline $12: 45 \mathrm{PM}$ & $\ldots$ & $01: 45 \mathrm{PM}$ & 0 & 0 & 0 & 0 & 0 & 0 & 0 & 0 & 0 & 0 & 0 & it & 0 \\
\hline $01: 00 \mathrm{PM}$ & $-\infty$ & $02: 00 \mathrm{PM}$ & 0 & 0 & 0 & 0 & 0 & 0 & 0 & 0 & 0 & 0 & $T$ & 3 & 8 \\
\hline 01:15 PM & $\ldots$ & $02: 15 \mathrm{PM}$ & 0 & 0 & 0 & 0 & 0 & 0 & 0 & 0 & 0 & 0 & $i$ & 0 & 1 \\
\hline $01: 30$ PM & $=$ & $02: 30 \mathrm{PM}$ & 0 & 0 & 0 & 0 & 0 & 0 & 0 & 0 & 0 & 0 & i & 0 & 1 \\
\hline $01: 45 \mathrm{PM}$ & $=$ & $02: 45 \mathrm{PM}$ & 0 & 0 & 0 & 0 & 0 & 0 & 0 & 0 & 0 & 0 & $i$ & i1 & $\pi$ \\
\hline 02:00 PM & - & $03: 00$ PM & 0 & $\begin{array}{l}0 \\
0\end{array}$ & 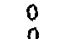 & 0 & $\begin{array}{l}0 \\
0 \\
0\end{array}$ & 0 & 0 & 0 & 0 & 0 & 0 & 0 & 0 \\
\hline 02:15 PM & $\cdots$ & $03: 15$ PM & 0 & 0 & 0 & 0 & 0 & 0 & 2 & 0 & 0 & $\frac{0}{0}$ & 0 & $\frac{0}{10}$ & 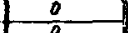 \\
\hline $02: 30 \mathrm{PM}$ & $\ldots$ & $03: 309 \mathrm{M}$ & 0 & 0 & $\begin{array}{l}0 \\
0\end{array}$ & 0 & 0 & 0 & $\begin{array}{l}0 \\
0\end{array}$ & 0 & $\begin{array}{l}0 \\
0\end{array}$ & $\begin{array}{l}0 \\
0 \\
0\end{array}$ & $\begin{array}{l}0 \\
0 \\
0\end{array}$ & 8 & 0 \\
\hline $02: 45 \mathrm{PM}$ & $\div$ & 03:45 PM & $\begin{array}{l}0 \\
0\end{array}$ & $\begin{array}{l}0 \\
0\end{array}$ & $\begin{array}{l}0 \\
0\end{array}$ & $\begin{array}{l}0 \\
0 \\
0\end{array}$ & $\begin{array}{l}0 \\
0\end{array}$ & $\begin{array}{l}0 \\
0\end{array}$ & $\begin{array}{l}0 \\
0\end{array}$ & $\begin{array}{l}0 \\
0\end{array}$ & $\begin{array}{l}0 \\
0 \\
0\end{array}$ & $\begin{array}{l}0 \\
0\end{array}$ & $\begin{array}{l}0 \\
0\end{array}$ & $\begin{array}{l}0 \\
0\end{array}$ & $\vdots$ \\
\hline$\frac{03: 00 \mathrm{PM}}{0.15 \mathrm{PM}}$ & 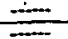 & $\frac{04: 00 \mathrm{PM}}{04: 15 \mathrm{PM}}$ & $\frac{0}{0}$ & $\frac{0}{0}$. & $\frac{0}{0}$ & $\frac{0}{0}$ & $\frac{0}{0}$ & 0 & 0 & 0 & 0 & 0 & 0 & 0 & $\div$ \\
\hline $\begin{array}{l}03: 13 \mathrm{PMM} \\
00.3 \mathrm{PM}\end{array}$ & $\ldots$ & $\begin{array}{l}04: 15 \text { PM } \\
04: 30 \text { PM }\end{array}$ & 0 & 0 & 0 & 0 & 0 & 0 & 0 & 0 & 0 & 0 & 0 & 0 & 0 \\
\hline $03: 45 \mathrm{PM}$ & $\ldots$ & 04:45 PM & 0 & 0 & 0 & 0 & 0 & 0 & 0 & 0 & 0 & 0 & 1 & 0 & $I$ \\
\hline $04: 00 \mathrm{PM}$ & $=$ & $05: 00 \mathrm{PM}$ & 0 & 0 & 0 & 0 & 0 & 0 & 0 & 0 & 0 & 0 & 7 & 0 & 7 \\
\hline$s$ & $E N I$ & $U L A:$ & 7 & 17 & & $A L$ & $D A$ & 1012 & 292 & & $: 01$ & $\triangle C D$ & 15 & $3=-$ & \\
\hline
\end{tabular}




\section{Baymetrics Traffic Resources INTERSECTION TURNING MOVEMENT SUMMARY}

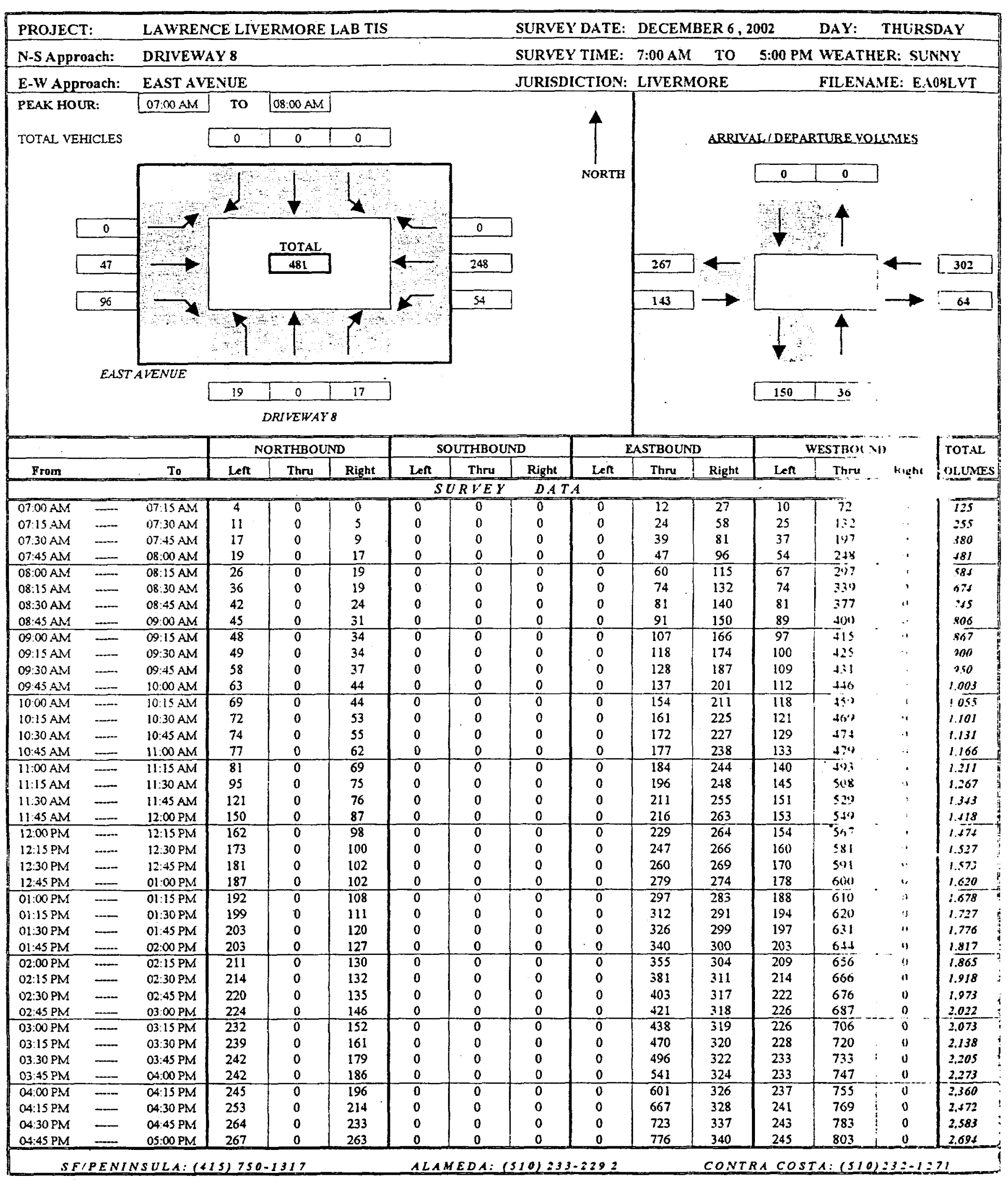




\section{Baymetrics Traffic Resources INTERSECTION TURNING MOVEMENT SUMMARY}

\begin{tabular}{|c|c|c|c|c|c|c|c|c|c|c|c|c|c|c|c|}
\hline \multirow{2}{*}{\multicolumn{2}{|c|}{$\begin{array}{l}\text { PROJECT: } \\
\text { N-S Approach: }\end{array}$}} & \multicolumn{6}{|c|}{ LAWRENCE LIVERMORE LAB TIS } & \multicolumn{5}{|c|}{ SURVEY DATE: DECEMBER 6, 2002} & DAY: & THURS & DAY \\
\hline & & DRIVE & & & & & & SURVE & IME: & 7:00 AM & To & 5:00 PM & WEATH & IER: SUP & NNY \\
\hline E-W AP & oach: & EAST AT & IUE & & & & & JURISD & TION: & LIVERM & RE & & FILENA & ME: EA & O8LVT \\
\hline & & & & THBO & & & THBOI & & & ASTBOUN & & & ESTBOU & $\mathrm{D}$ & TOTAL \\
\hline From & & To & Let & Thru & Right & Len & Thru & Right & Len & Thru & Right & Len & Thru & Right & LOLUNES \\
\hline & & & & & & & $L B$ & $P E R I$ & & & & & & & \\
\hline O7:0AM & 5 & O7:15 AM & 4 & 0 & v & 7 & 0 & o & 0 & 12 & $2 T$ & 70 & 72 & $\overline{0}$ & TIS \\
\hline $07.15 \mathrm{AM}$ & $\overline{-}$ & 07:30 AM & 7 & 0 & 5 & 0 & 0 & 0 & 0 & 12 & 31 & 15 & 60 & 0 & 130 \\
\hline $\begin{array}{l}07,30 \mathrm{AM} \\
07: 45 \mathrm{AM}\end{array}$ & 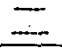 & $\begin{array}{l}07.45 \mathrm{AM} \\
08: 00 \mathrm{AM} \\
\end{array}$ & $\begin{array}{l}6 \\
2 \\
\end{array}$ & $\begin{array}{l}0 \\
0 \\
0\end{array}$ & $\begin{array}{l}4 \\
8 \\
\end{array}$ & $\begin{array}{l}0 \\
0 \\
0\end{array}$ & $\begin{array}{l}0 \\
0\end{array}$ & $\begin{array}{l}0 \\
0\end{array}$ & $\begin{array}{l}0 \\
0\end{array}$ & $\begin{array}{l}15 \\
8\end{array}$ & $\begin{array}{l}23 \\
15\end{array}$ & $\begin{array}{l}12 \\
17\end{array}$ & $\begin{array}{l}65 \\
51\end{array}$ & $\begin{array}{l}0 \\
0\end{array}$ & $\begin{array}{l}125 \\
101\end{array}$ \\
\hline 08:00 AM & $\cdots$ & $08: 15 \mathrm{AM}$ & 7 & 0 & 2 & 0 & 0 & 0 & 0 & $\frac{15}{13}$ & 19 & $\frac{11}{13}$ & $\frac{18}{49}$ & 0 & 103 \\
\hline 08:15 AM & - & 08:30 AM & 10 & 0 & 0 & 0 & 0 & 0 & 0 & 14 & 17 & 7 & 42 & 0 & $\infty$ \\
\hline $\begin{array}{l}\text { 08:30 AM } \\
\text { 08:45 AM }\end{array}$ & - & 08:45 AM & 6 & 0 & 5 & 0 & 0 & 0 & 0 & 7 & 8 & $?$ & 38 & 0 & $n$ \\
\hline$\frac{08: 45 \mathrm{AM}}{09: 00 \mathrm{AM}}$ & $\equiv$ & $\frac{09: 00 \mathrm{AM}}{09: 15 \mathrm{AN}}$ & $-\frac{3}{3}$ & $\frac{0}{0}$ & $\frac{7}{3}$ & 0 & 0 & $\frac{0}{0}$ & 0 & $\frac{10}{16}$ & $\frac{10}{16}$ & $\frac{8}{8}$ & $\frac{23}{15}$ & $\frac{1}{i}$ & $6 i$ \\
\hline $09: 15$ AM & - & $09: 30 \mathrm{AM}$ & $\hat{1}$ & 0 & 0 & 0 & 0 & 0 & 0 & 11 & 8 & 3 & 10 & i) & 33 \\
\hline $09.30 \mathrm{AM}$ & $\ldots$ & $09: 43$ AM & 9 & 0 & 3 & 0 & 0 & 0 & 0 & 10 & 13 & 9 & 6 & 0 & so \\
\hline$\frac{09: 45 \mathrm{AM}}{10: 00 \mathrm{AM}}$ & $=$ & $10: 00 \mathrm{AM}$ & 5 & 0 & 7 & 0 & 0 & 0 & 0 & 9 & 14 & 3 & 15 & 0 & 53 \\
\hline $\begin{array}{l}10: 00 \mathrm{AM} \\
10: 15 \mathrm{AM}\end{array}$ & 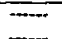 & $10: 15 \mathrm{AM}$ & 6 & 0 & 0 & 0 & 0 & $\%$ & 8 & 17 & 10 & 6 & 13 & $\overline{0}$ & 52 \\
\hline $\begin{array}{l}10: 15 \mathrm{AM} \\
10: 30 \mathrm{AM}\end{array}$ & $=$ & 10:30 AM & $\frac{3}{2}$ & 0 & 9 & 0 & 0 & 0 & ${ }_{0}^{0}$ & 7 & 14 & 3 & 10 & 9 & 46 \\
\hline $10: 45 \mathrm{AM}$ & $\overline{-}$ & $\begin{array}{l}10: 45 \mathrm{AM} \\
11: 00 \mathrm{AM}\end{array}$ & $\begin{array}{l}2 \\
3\end{array}$ & $\begin{array}{l}0 \\
0\end{array}$ & $\frac{2}{7}$ & $\stackrel{0}{0}$ & 0 & 0 & $\begin{array}{l}0 \\
0\end{array}$ & $\begin{array}{c}11 \\
5\end{array}$ & 2 & 8 & 5 & si & 30 \\
\hline $11: 00 \mathrm{AM}$ & $=$ & $\frac{1000 \mathrm{AM}}{11: 15 \mathrm{AM}}$ & $\frac{3}{4}$ & 0 & $\frac{1}{3}$ & 0 & $\frac{0}{0}$ & $\frac{0}{0}$ & $\frac{0}{0}$ & $\frac{5}{9}$ & $\frac{11}{6}$ & $\frac{4}{7}$ & $\frac{5}{14}$ & $\frac{11}{3}$ & $\frac{35}{15}$ \\
\hline 11:15 AM & - & 11:30 AM & 14 & 0 & 6 & 0 & 0 & 0 & 0 & 12 & 4 & 5 & is & 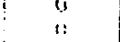 & $\begin{array}{l}15 \\
56\end{array}$ \\
\hline $11: 30$ AM & - & $11: 45$ AM & 26 & 0 & 1 & 0 & 0 & 0 & 0 & 15 & 1 & 6 & $=i$ & $\therefore$ & $76^{\circ}$ \\
\hline 11:45.AM & $=$ & $12: 00 \mathrm{PM}$ & $\frac{29}{19}$ & 0 & 11 & 0 & 0 & 0 & 0 & 5 & 8 & 2 & 30 & "t & -5 \\
\hline $12: 00 \mathrm{PM}$ & $=$ & $12: 15 \overline{\mathrm{PM}}$ & 12 & 0 & 11 & 0 & 0 & 0 & 0 & 13 & 1 & $T$ & & i" & 56 \\
\hline 12:13 PM & $\ldots$ & 12:30 PM & 11 & 0 & 2 & 0 & 0 & 0 & 0 & 18 & 2 & 6 & i. & $\therefore$ & 53 \\
\hline 12:30 PM & - & $12: 45 \mathrm{PM}$ & 8 & 0 & 2 & 0 & 0 & 0 & 0 & 13 & 3 & 10 & 10 & $\because$ & $\$ 6$ \\
\hline 12:45 PM & $=$ & $01: 00 \mathrm{PM}$ & 6 & 0 & 0 & 0 & 0 & 0 & 0 & 19 & 5 & 8 & 9 & $\because$ & 17 \\
\hline $\begin{array}{l}01.00 \mathrm{PM} \\
01.15 \mathrm{PM}\end{array}$ & $=$ & $01: 15 \mathrm{PM}$ & 5 & 0 & 6 & 0 & 6 & 0 & 0 & 18 & 9 & 10 & 10 & $\bar{a}$ & 58 \\
\hline $\begin{array}{l}01.15 \mathrm{PM} \\
01: 30 \mathrm{PM}\end{array}$ & $\bar{\ldots}$ & $\begin{array}{l}01: 30 \mathrm{PM} \\
01: 45 \mathrm{PM}\end{array}$ & $\begin{array}{l}7 \\
4\end{array}$ & 0 & 3 & 0 & $\stackrel{0}{0}$ & 0 & 0 & 15 & 8 & 6 & 10 & !" & 19. \\
\hline $01: 45$ PM & $\ldots$ & $02.00 \mathrm{PM}$ & 0 & 0 & $\frac{9}{7}$ & 0 & $\begin{array}{l}0 \\
0\end{array}$ & $\begin{array}{l}0 \\
0\end{array}$ & $\begin{array}{l}0 \\
0\end{array}$ & $\begin{array}{l}14 \\
14\end{array}$ & 8 & $\begin{array}{l}3 \\
6\end{array}$ & 11 & $\because$ & $s 9$ \\
\hline $02: 00$ PM & $\cdots$ & $02: 15$ PM & 8 & 0 & 3 & 0 & $\overline{0}$ & 0 & 0 & $\frac{14}{15}$ & $\frac{1}{4}$ & $\frac{0}{6}$ & $\frac{13}{12}-$ & $\because$ & $-\frac{\sqrt{1}}{\sqrt{8}}$ \\
\hline 02:15 PM & - & 0:30 PM & 3 & 0 & 2 & 0 & 0 & 0 & 0 & 26 & 7 & 5 & 10 & .. & s3 \\
\hline $02: 30 \mathrm{PM}$ & $\cdots$ & 02:45 PM & 6 & 0 & 3 & 0 & 0 & 0 & 0 & 22 & 6 & 8 & is & . & is \\
\hline $02: 45$ PM & - & $03: 00$ PM & 4 & 0 & i1 & 0 & 0 & 0 & 0 & 18 & 1 & 4 & 11 & $\because$ & 69 \\
\hline $03: 00 \mathrm{PM}$ & $=$ & $03: 15 \mathrm{PM}$ & 8 & 0 & 6 & $\overline{0}$ & 0 & 0 & 0 & 17 & $T$ & 0 & 10 & $\because$ & SI \\
\hline 03:15 PM & $\cdots$ & 03:30 PM & 7 & 0 & 9 & 0 & 0 & 0 & 0 & 32 & 1 & 2 & 14 & .. & os \\
\hline 03:30 PM & $\cdots$ & $03: 45 \mathrm{PM}$ & 3 & 0 & 18 & 0 & 0 & 0 & 0 & 26 & 2 & $\underline{s}$ & 13 & " & 67 \\
\hline 03:45 PM & $\ldots$ & $0400 \mathrm{PM}$ & 0 & 0 & 7 & 0 & 0 & 0 & 0 & 45 & 2 & 0 & 1.4 & $\therefore$ & 68 \\
\hline $04: 00 \mathrm{PM}$ & $=$ & $04: 15 \mathrm{PM}$ & 3 & 0 & 10 & 0 & 0 & 0 & 0 & 60 & 2 & 4 & 8 & $"$ & 67 \\
\hline OA:IS PM & - & $04: 30 \mathrm{PM}$ & 8 & 0 & 18 & 0 & 0 & 0 & 0 & 66 & 2 & 4 & 14 & . & 182 \\
\hline 04:30 PM & $-\cdots$ & 04:45 PM & 11 & 0 & 19 & 0 & 0 & 0 & 0 & $\begin{array}{l}56 \\
53\end{array}$ & $\begin{array}{l}9 \\
3\end{array}$ & 2 & 14 & . & 111 \\
\hline D4:45 PM & $=$ & $05: 00 \mathrm{PM}$ & 3 & 0 & 30 & $\frac{a}{h}$ & $\frac{0}{R L Y}$ & $\frac{0}{T O T A L}$ & 0 & 53 & 3 & 2 & $\approx$ & $\because$ & 111 \\
\hline $07: 00 \mathrm{AM}$ & $\Longrightarrow$ & $\overline{C 8: \overline{A M}}$ & T9 & $\sigma$ & $\pi$ & 0 & $\sigma$ & 0 & 0 & 47 & 96 & 54 & 7.4. & : & ग8I \\
\hline 07:15 AM & - & 08:15 AM & 22 & 0 & 19 & 0 & 0 & 0 & 0 & 48 & 88 & 59 & 225 & $\therefore$ & 459 \\
\hline$\frac{07: 30 \mathrm{AM}}{07.15 \mathrm{AM}}$ & - & 08:30 AM & 25 & 0 & 14 & 0 & 0 & 0 & 0 & 50 & 74 & 49 & $20:$ & "1" & sig \\
\hline $07: 45 \mathrm{AM}$ & $=$ & $08.45 \mathrm{AM}$ & 25 & 0 & 15 & 0 & 0 & 0 & 0 & 42 & 59 & 44 & 1811 & ii & 365 \\
\hline & - & $09: 00 \mathrm{AM}$ & 26 & 0 & 14 & 0 & 0 & 0 & 0 & 44 & 54 & 35 & $15=$ & $\because$ & 325 \\
\hline$\frac{08: 15 \mathrm{AM}}{08: 30 \mathrm{AM}}$ & $\equiv$ & $09: 15 \mathrm{AM}$ & 22 & 0 & 15 & 0 & 0 & 0 & 0 & 47 & 51 & 30 & 118 & ." & 383 \\
\hline & $=$ & $\begin{array}{l}09 \cdot 30 \mathrm{AM} \\
09: 45 \mathrm{AM}\end{array}$ & 13 & $\begin{array}{l}0 \\
0\end{array}$ & 15 & $\begin{array}{l}0 \\
0\end{array}$ & $\begin{array}{l}0 \\
0 \\
0\end{array}$ & 0 & 0 & 44 & 42 & 26 & 86 & ' & 226 \\
\hline 09:00 AM & $\ldots$ & $\begin{array}{l}09: 45 \mathrm{AM} \\
10: 00 \mathrm{AM}\end{array}$ & $\begin{array}{l}16 \\
18 \\
\end{array}$ & $\begin{array}{l}0 \\
0 \\
\end{array}$ & $\begin{array}{l}13 \\
13 \\
\end{array}$ & 0 & 0 & $\begin{array}{l}0 \\
0 \\
\end{array}$ & $\begin{array}{l}0 \\
0\end{array}$ & $\begin{array}{r}47 \\
46 \\
\end{array}$ & $\begin{array}{l}47 \\
51\end{array}$ & $\begin{array}{l}28 \\
23\end{array}$ & $\begin{array}{l}5.1 \\
46\end{array}$ & $\because "$ & 205 \\
\hline 09:15.AM & $=$ & $10: 15 \overline{A M}$ & 21 & 0 & 10 & 0 & 0 & 0 & 0 & 47 & $4 \overline{5}$ & $\frac{21}{21}$ & 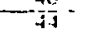 & $\because$ & $\frac{197}{188}$ \\
\hline $09: 30 \mathrm{AM}$ & - & $10: 30 \mathrm{AM}$ & 23 & 0 & 19 & 0 & 0 & 0 & 0 & 43 & si & $2 i$ & \pm 4 & .. & $20 t$ \\
\hline 09:45 AM & $\ldots$ & $10: 45 \mathrm{AM}$ & 16 & 0 & 18 & 0 & 0 & 0 & 0. & 47 & 40 & 20 & 43 & $\therefore$ & 18 \\
\hline $10.00 \mathrm{AM}$ & $\cdots$ & $11: 00 \mathrm{AM}$ & 14 & 0 & 18 & 0 & 0 & 0 & 0 & 40 & 37 & 21 & -33 & . & 163 \\
\hline $10: 15 \mathrm{AM}$ & $\ldots$ & $11: 15 \mathrm{AM}$ & 12 & 0 & 25 & 0 & 0 & 0 & 0 & 30 & 33 & 22 & 3.1 & $\therefore$ & 250 \\
\hline $10: 30 \mathrm{AM}$ & $=$ & $11-30 \mathrm{AM}$ & 23 & 0 & 22 & 0 & 0 & 0 & 0 & 35 & 23 & $2 \pi$ & 39 & $\therefore$ & 166 \\
\hline $10.45 \mathrm{AM}$ & $=$ & $11: 45 \mathrm{AM}$ & 47 & 0 & 21 & $\delta$ & $\overline{0}$ & 0 & 0 & 39 & 28 & 22 & 55 & .. & $2 / 2$ \\
\hline $11: 00 \mathrm{AM}$ & $\ldots$ & $12.00 \mathrm{PM}$ & 73 & 0 & 25 & 0 & 0 & 0 & 0 & 39 & 25 & 20 & $=0$ & $،$ & 252 \\
\hline $11: 15 \mathrm{AM}$ & $\ldots$ & 12:15 PM & 81 & 0 & 29 & 0 & 0 & 0 & 0 & 45 & 20 & 14 & 24 & ." & 263 \\
\hline $11: 30 \mathrm{AM}$ & $=$ & $12: 30 \mathrm{PM}$ & 78 & 0 & 25 & 0 & 0 & 0 & 0 & 51 & 18 & 15 & 3 & $\therefore$ & 260 \\
\hline $11: 45$ AM & - & $12: 45 \mathrm{PM}$ & 60 & 0 & 26 & 0 & 0 & 0 & 0 & 49 & 14 & 19 & $6=$ & $\because$ & 230 \\
\hline $12: 00 \mathrm{PM}$ & - & $01.00 \mathrm{PM}$ & $\frac{37}{30}$ & 0 & 15 & 0 & 0 & 0 & 0 & $\frac{63}{69}$ & 11 & 25 & 51 & 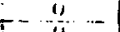 & 202 \\
\hline $12: 15 \mathrm{PM}$ & $=$ & $0115 \mathrm{PM}$ & 30 & 0 & 10 & 0 & 0 & 0 & 0 & 68 & 19 & 34 & 43 & 11 & 204 \\
\hline $\begin{array}{l}12: 30 \mathrm{PM} \\
12.45 \mathrm{PM}\end{array}$ & $=$ & $\begin{array}{l}01: 30 \mathrm{PM} \\
01: 45 \mathrm{PM}\end{array}$ & $\begin{array}{l}26 \\
22\end{array}$ & $\begin{array}{l}0 \\
0\end{array}$ & 11 & $\begin{array}{l}0 \\
0\end{array}$ & $\begin{array}{l}0 \\
0\end{array}$ & $\begin{array}{l}0 \\
0\end{array}$ & $\begin{array}{l}0 \\
0\end{array}$ & $\begin{array}{l}65 \\
66\end{array}$ & $\begin{array}{l}25 \\
30\end{array}$ & $\begin{array}{l}34 \\
27\end{array}$ & $\begin{array}{l}39 \\
40\end{array}$ & 0 & $\begin{array}{l}200 \\
305\end{array}$ \\
\hline $01: 00 \mathrm{PM}$ & $=$ & $02: 00 \mathrm{PM}$ & $\frac{2 t}{16}$ & 0 & $\frac{15}{25}$ & 0 & 0 & 0 & 0 & $\frac{\infty}{61}$ & $\frac{30}{26}$ & $-\frac{21}{25}$ & $\frac{4}{44}$ & $\frac{1}{0}$ & $\frac{50}{199}$ \\
\hline $01: 15$ PM & $=$ & 02:15 PM & 19 & 0 & 22 & 0 & 0 & 0 & 0 & 58 & 21 & 21 & 46 & $y$ & 187 \\
\hline $01: 30 \mathrm{PM}$ & $=$ & 02:30 PM & 15 & $\frac{0}{0}$ & 21 & $\frac{0}{0}$ & $\frac{0}{0}$ & $\frac{0}{0}$ & $\frac{0}{0}$ & $\frac{69}{77}$ & $\frac{20}{18}$ & $\frac{20}{25}$ & $\frac{46}{15}$ & 0 & 191 \\
\hline $01: 45 \mathrm{PM}$ & - & $02: 45 \mathrm{PM}$ & T7 & 0 & 15 & 0 & 0 & 0 & 0 & 77 & 18 & 25 & 45 & u & $19=$ \\
\hline 02:00 PM & - & 03:00 PM & 21 & $\begin{array}{l}0 \\
0\end{array}$ & 19 & $\begin{array}{l}0 \\
0\end{array}$ & $\begin{array}{l}0 \\
0\end{array}$ & $\begin{array}{l}0 \\
0\end{array}$ & $\begin{array}{l}0 \\
0\end{array}$ & $\begin{array}{l}81 \\
83\end{array}$ & 18 & 23 & 43 & 0 & 305 \\
\hline 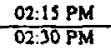 & $=$ & $\frac{03.15 \mathrm{PM}}{03: 30 \mathrm{PM}}$ & $-\frac{21}{25}$ & $-\frac{0}{0}$ & $-\frac{22}{29}$ & 0 & 0 & -0 & $\frac{0}{0}$ & $\frac{83}{89}$ & $\frac{15}{9}$ & $\frac{17}{14}$ & $\frac{50}{54}$ & $\frac{0}{0}$ & $\frac{208}{320}$ \\
\hline 02:45 PM & - & $03: 45$ PM & 22 & 0 & 44 & 0 & 0 & 0 & 0 & 93 & 5 & ii & 57 & 0 & 232 \\
\hline $03: 00 \mathrm{PM}$ & $=$ & 04:00 PM & 18 & 0 & 40 & 0 & 0 & 0 & 0 & 120 & 6 & 7 & 60 & 0 & 251 \\
\hline $03: 15$ PM & $=$ & $04: 15 \mathrm{PM}$ & 13 & 0 & 44 & 0 & 0 & 0 & 0 & 163 & 7 & $\pi$ & 49 & 0 & 287 \\
\hline 03:10 PM & - & $04: 30 \mathrm{PM}$ & 14 & 0 & 53 & 0 & 0 & 0 & 0 & 197 & 8 & $i 3$ & 49 & 0 & Jjs \\
\hline 03:45 PM & $\ldots$ & $04: 45 \mathrm{PM}$ & 22 & 0 & 54 & 0 & 0 & 0 & 0 & 227 & 15 & 10 & 50 & 0 & 378 \\
\hline $04: 00 \mathrm{PM}$ & $=$ & $05: 00 \mathrm{PM}$ & 25 & 0 & 77 & 0 & 0 & 0 & 0 & 235 & 6 & 2 & 56 & 0 & 221 \\
\hline$s$ & $E N$ & ULA: & 75 & & & $A I$ & 0 & i) & $g$ & & $0 \Omega$ & Co & 1 & $=-$ & \\
\hline
\end{tabular}




\section{Baymetrics Traffic Resources INTERSECTION TURNING MOVEMENT SUMMARY}

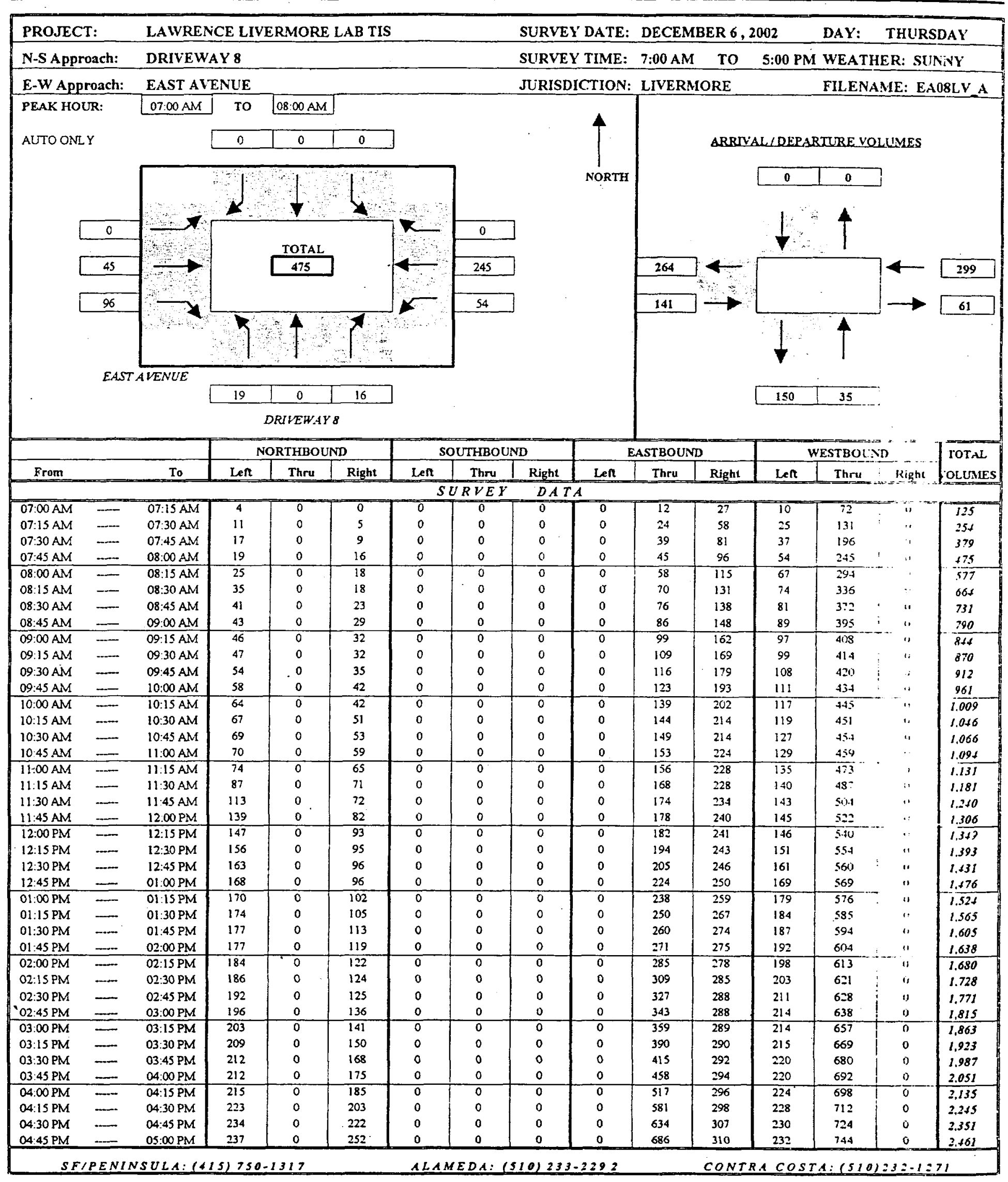




\section{Baymetrics Traffic Resources INTERSECTION TURNING MOVEMENT SUMMARY}

\begin{tabular}{|c|c|c|c|c|c|c|c|c|c|c|c|c|c|c|c|}
\hline \multirow{2}{*}{\multicolumn{2}{|c|}{$\begin{array}{l}\text { PROJECT: } \\
\text { N-S Approach: }\end{array}$}} & \multicolumn{6}{|c|}{ LAWRENCE LIVERMORE LAB TIS } & SURVE & DATE: & DECEM & ER 6, 2 & 002 & DAY: & THURS & DAY \\
\hline & & DRIVEV & & & & & & SURVE & IIME: & 7:00 AM & To & 5:00 PN & WEATI & IER: SUI & NNY \\
\hline E-W AP & oach: & EAST A & UE & & & & & JURISD & TION: & LIVERI & $\mathrm{RE}$ & & FILEN & ME: EA & $08 \mathrm{LV}$ A \\
\hline & & & & THBO & & & THBOL & & & ASTBOUN & & & ESTBOU & & TOTAL \\
\hline From & & To & Len & Thru & Right & Left & Thru & Right & Len & Thru & Right & Len & Thru & Right & LoLUMES \\
\hline & & & & & & & $\begin{array}{ll}L \\
\end{array}$ & $P E R I$ & & & & & & & \\
\hline $8700 \mathrm{AM}$ & -7 & $87: 15 \mathrm{AM}$ & 4 & 8 & \% & $\overline{0}$ & 0 & 0 & 8 & $T 2$ & 27 & $\pi$ & 72 & $\sigma$ & 73 \\
\hline 07:15 AM & $\cdots$ & 07:30 AM & 7 & 0 & 5 & 0 & 0 & 0 & 0 & 12 & 31 & 15 & 59 & 0 & 129 \\
\hline $\begin{array}{l}07.30 \mathrm{AM} \\
07: 45 \mathrm{AM}\end{array}$ & $=$ & $07.45 \mathrm{AM}$ & 6 & 0 & 4 & 0 & 0 & $\begin{array}{l}0 \\
0 \\
0\end{array}$ & 0 & $\begin{array}{c}15 \\
6\end{array}$ & $\begin{array}{l}23 \\
15\end{array}$ & 12 & 65 & 0 & 125 \\
\hline $08.00 \mathrm{AM}$ & $=$ & $\frac{08: 00 \mathrm{AM}}{08: 15 \mathrm{AM}}$ & $-\frac{2}{6}$ & 0 & $-\frac{1}{2}$ & 0 & $\frac{0}{0}$ & 0 & $\frac{0}{0}$ & $\frac{6}{13}$ & $\frac{15}{19}$ & $\frac{17}{13}$ & $\frac{49}{49}$ & $\frac{0}{0}$ & $\frac{86}{102}$ \\
\hline 08:15 AM & - & $08: 30 \mathrm{AM}$ & 10 & 0 & 0 & 0 & 0 & 0 & 0 & 12 & 16 & 7 & 42 & 0 & 87 \\
\hline $\begin{array}{l}08: 30 \mathrm{AM} \\
08: 45 \mathrm{AM}\end{array}$ & $\cdots$ & 08:45 AM & 6 & 0 & 5 & 0 & 0 & 0 & 0 & 6 & 7 & 7 & 36 & 0 & 67 \\
\hline$\frac{08: 45 \text { AM }}{09: 00 \text { AM }}$ & $\ldots$ & $\frac{09: 00 A M}{09: 15 A M}$ & $-\frac{2}{3}$ & $\frac{0}{0}$ & $-\frac{6}{3}$ & $\begin{array}{l}0 \\
0\end{array}$ & $\frac{0}{0}$ & $\frac{0}{0}$ & $\frac{0}{0}$ & $\frac{10}{13}$ & $\frac{10}{14}$ & $\frac{8}{8}$ & 23 & $\begin{array}{l}0 \\
0\end{array}$ & 59 \\
\hline $09: 15 \mathrm{AM}$ & - & $09: 30 \mathrm{AM}$ & 1 & 0 & 0 & 0 & 0 & 0 & 0 & 10 & $\begin{array}{l}14 \\
7\end{array}$ & $\begin{array}{l}8 \\
2\end{array}$ & $\begin{array}{l}13 \\
6\end{array}$ & 0 & 26 \\
\hline $09: 30 \mathrm{AM}$ & - & $09.45 \mathrm{AM}$ & 7 & 0. & 3 & 0 & 0 & 0 & 0 & 7 & 10 & 9 & 6 & 0 & 42 \\
\hline $09: 45$ AM & $=$ & $10: 00 \mathrm{AM}$ & 4 & 0 & 7 & 0 & 0 & 0 & 0 & 7 & 14 & 3 & 14 & 0 & $s 9$ \\
\hline $10.00 \mathrm{AM}$ & $\ldots$ & $10.15 \mathrm{AM}$ & 6 & 0 & 0 & 0 & 0 & 0 & 0 & 16 & 9 & 6 & 11 & ii & $\sqrt{8}$ \\
\hline 10:15 AM & $\cdots$ & 10:30 AM & 3 & 0 & 9 & 0 & 0 & 0 & 0 & 5 & 12 & 2 & 6 & a & 37 \\
\hline 10:30 AM & ..... & $10: 45 \mathrm{AM}$ & 2 & 0 & 2 & 0 & 0 & 0 & 0 & 5 & 0 & 8 & 3 & a & 20 \\
\hline $10.49 \mathrm{AM}$ & $\cdots$ & 11:00 AM & 1 & 0 & 6 & o & 0 & 0 & 0 & 4 & 10 & 2 & 5 & $" 1$ & 28 \\
\hline $1100 \mathrm{AM}$ & $\ldots$ & $11: 15 \overline{\mathrm{AM}}$ & 4 & 0 & 6 & 0 & 0 & 0 & 0 & 3 & 4 & 6 & 14 & $\pi$ & 37 \\
\hline $11: 15$ AM & $\cdots$ & $11: 30 \mathrm{AM}$ & 13 & 0 & 6 & 0 & 0 & 0 & 0 & 12 & 0 & 5 & 14 & $" 1$ & so \\
\hline $11: 30 \mathrm{AM}$ & - & $11: 45 \mathrm{AM}$ & 26 & 0 & 1 & 0 & 0 & 0 & 0 & 6 & 6 & 3 & 17 & $"$ & $s 9$ \\
\hline 11.45 AM & $\cdots$ & $12: 00 \mathrm{PM}$ & 26 & 0 & 10 & 0 & 0 & 0 & 0 & 4 & 6 & 2 & 18 & :1 & $\phi 6$ \\
\hline $12: 00 \mathrm{PM}$ & 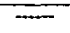 & $12: 19 \mathrm{PM}$ & 8 & 0 & i1 & 0 & 0 & 0 & 0 & 4 & 1 & 1 & 18 & $1{ }^{-}$ & $\sqrt{3}$ \\
\hline $12: 15$ PM & 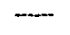 & 12:30 PM & 9 & 0 & 2 & 0 & 0 & 0 & 0 & 12 & 2 & 5 & 14 & 11 & $d$ \\
\hline $\begin{array}{l}\text { 12:30 PM } \\
12: 45 \mathrm{PM}\end{array}$ & $\ldots$ & 12:45 PM & 7 & 0 & 1 & 0 & $\begin{array}{l}0 \\
0\end{array}$ & 0 & ${ }_{0}^{0}$ & 11 & 3 & 10 & 6 & 11 & 38 \\
\hline$\frac{12: 45 \text { PM }}{01: 00 \mathrm{PM}}$ & $\overline{-1}$ & $\frac{01: 00 \mathrm{PM}}{01: 15 \mathrm{PM}}$ & $-\frac{5}{2}$ & $\frac{0}{0}$ & $\frac{0}{6}$ & $\frac{0}{0}$ & $\frac{0}{0}$ & 0 & $\frac{0}{0}$ & $\frac{19}{14}$ & $\frac{4}{9}$ & 8 & & $\frac{13}{11}$ & vs \\
\hline $0 \mathrm{t} 15 \mathrm{PM}$ & - & $01: 30 \mathrm{PM}$ & 4 & 0 & 3 & 0 & 0 & 0 & 0 & $\begin{array}{l}14 \\
12\end{array}$ & $\begin{array}{l}9 \\
8\end{array}$ & $\begin{array}{c}10 \\
5\end{array}$ & $\begin{array}{l}7 \\
9\end{array}$ & $" 1$ & 98 \\
\hline $01: 30$ PM & $\cdots$ & $01: 45$ PM & 3 & 0 & 8 & 0 & 0 & 0 & 0 & 10 & 7 & 3 & 9 & $"$ & so \\
\hline $01: 45$ PM & - & 02:00 PM & 0 & 0 & 6 & 0 & 0 & 0 & 0 & ii & $i$ & 5 & 10 & $\because$ & J3 \\
\hline $02: 00 \mathrm{PM}$ & $\cdots$ & $02: 15 \mathrm{PM}$ & 7 & 0 & $\overline{3}$ & $\overline{0}$ & 0 & 0 & 0 & 14 & 3 & 6 & 9 & ii & $\sqrt{2}$ \\
\hline 02:15 PM & -- & 02:30 PM & 2 & 0 & 2 & 0 & 0 & 0 & 0 & 24 & 9 & 5 & 8 & ، & 48 \\
\hline 02:30 PM & $\cdots$ & $02: 45 \mathrm{PM}$ & 6 & 0 & 1 & 0 & 0 & 0 & 0 & 18 & 3 & 8 & 7 & $\because$ & a \\
\hline 02:45 PM & $\ldots$ & $03: 00 \mathrm{PMM}$ & 4 & 0 & 11 & 0 & 0 & 0 & 0 & 16 & 0 & 3 & 10 & ". & $\Delta$ \\
\hline $03: 00 \mathrm{PM}$ & 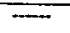 & $03: 15 \mathrm{PM}$ & 7 & 0 & 5 & 0 & 0 & 0 & 0 & 16 & $i$ & 0 & 19 & " & 18 \\
\hline 03:15 PM & -- & $03: 30 \mathrm{PM}$ & 6 & 0 & 9 & 0 & 0 & 0 & 0 & 31 & 1 & 1 & 12 & .1 & 60 \\
\hline 03:30 PM & $\cdots$ & 03:45 PM. & 3 & 0 & 18 & 0 & 0 & 0 & 0 & 25 & 2 & 5 & 11 & $"$ & 64 \\
\hline 03:45 PM & $\cdots$ & $04: 00 \mathrm{PM}$ & 0 & 0 & 7 & 0 & 0 & 0 & 0 & 43 & 2 & 0 & 12 & $\because$ & or \\
\hline $04.00 \mathrm{PM}$ & $-\infty$ & $04: 15 \mathrm{PM}$ & 3 & 0 & 10 & 0 & 0 & $\overline{0}$ & 0 & 59 & 2 & 4 & 6 & 1 & 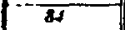 \\
\hline 04:15 PM & $\cdots$ & $04: 30$ PM & 8 & 0 & 18 & 0 & 0 & 0 & 0 & 64 & 2 & 4 & 14 & " & 110 \\
\hline $04: 30$ PM & $\ldots$ & $04: 43$ PM & 11 & 0 & 19 & 0 & 0 & 0 & 0 & 53 & 9 & 2 & 12 & " & 106 \\
\hline $04: 45$ PM & $-\cdots$ & $05: 00 \mathrm{PM}$ & 3 & 0 & 30 & 0 & 0 & 0 & 0 & 52 & 3 & 2 & 20 & . & 110 \\
\hline & & & & & & & $R L Y$ & $T O T A L$ & & & & & & & \\
\hline $07.01 \mathrm{AM}$ & - & 08:00 AM & 79 & 7 & 16 & 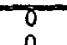 & 0 & 0 & $\%$ & 45 & 96 & 54 & 35 & $\pi$ & 7,5 \\
\hline $\begin{array}{l}07: 15 \mathrm{AM} \\
07.30 \mathrm{AM}\end{array}$ & - & $08: 19 \mathrm{AM}$ & 21 & $\begin{array}{l}0 \\
0\end{array}$ & 18 & 0 & 0 & $\circ$ & 8 & 46 & 88 & $\begin{array}{l}57 \\
49\end{array}$ & 202 & $"$ ". & 552 \\
\hline$\frac{0,70 \mathrm{AM}}{07: 45 \mathrm{AM}}$ & $=$ & $\begin{array}{l}08: 30 \mathrm{AM} \\
08: 45 \mathrm{AM}\end{array}$ & $-\frac{24}{24}$ & -0 & $\frac{13}{14}$ & $\frac{0}{0}$ & 0 & 0 & 0 & $\frac{46}{37}$ & $\frac{73}{57}$ & $\frac{49}{44}$ & $\frac{207}{176}$ & $\because$ & $-\frac{10}{352}$ \\
\hline 08:00 AM & - & $09.00 \mathrm{AM}$ & 24 & 0 & 13 & 0 & 0 & 0 & 0 & 4) & 52 & 35 & $i 56$ & . & 315 \\
\hline $08: 15 \mathrm{AM}$ & $\ldots$ & $09: 15 \mathrm{AM}$ & 21 & 0 & 14 & 0 & 0 & 0 & 0 & $4 !$ & 47 & 30 & 114 & $"$ & 267 \\
\hline $08: 30 \mathrm{AM}$ & $=$ & $09: 30 \mathrm{AM}$ & 12 & 0 & 14 & 0 & 0 & 0 & 0 & 39 & 38 & $\frac{55}{25}$ & $\frac{18}{38}$ & $"$ & $\frac{-106}{206}$ \\
\hline 08:45 AM & $\ldots$ & $09: 45$ AM & 13 & 0 & 12 & 0 & 0 & 0 & 0 & 40 & 41 & 27 & 48 & $\therefore$ & 181 \\
\hline $09: 00 \mathrm{AM}$ & $\ldots$ & $1000 \mathrm{AM}$ & 15 & 0 & 13 & 0 & 0 & 0 & 0 & 37 & 45 & 22 & 39 &. & $a$ \\
\hline $09.15 \mathrm{AM}$ & - & $10.15 \mathrm{AM}$ & 18 & 0 & 10 & 0 & 0 & 0 & 0 & 40 & 40 & $\frac{20}{20}$ & $3 ?$ & .. & 165 \\
\hline $09: 30 \mathrm{AM}$ & $\cdots$ & $10: 30 \mathrm{AM}$ & 20 & 0 & 19 & 0 & 0 & 0 & 0 & 35 & 45 & 20 & 37 & .. & 178 \\
\hline $08.45 \mathrm{AM}$ & $=$ & $10: 45 \mathrm{AM}$ & 15 & 0 & 18 & 0 & 0 & 0 & 0 & 33 & 35 & 19 & 34 & $\because$ & iss \\
\hline $10.00 \mathrm{AM}$ & $\ldots$ & $11: 00 \mathrm{AM}$ & $i 2$ & 0 & 19 & 0 & 0 & 0 & 0 & 30 & 31 & 18 & 25 & $\because i^{-}$ & 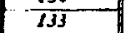 \\
\hline $10: 15 \mathrm{AM}$ & $\cdots$ & 11:15 AM & 10 & 0 & 23 & $\begin{array}{l}0 \\
0\end{array}$ & 0 & $\begin{array}{l}0 \\
0\end{array}$ & $\begin{array}{l}0 \\
0\end{array}$ & $\begin{array}{l}17 \\
24\end{array}$ & 26 & 18 & 28 & " & 132 \\
\hline$\frac{10: 30 \mathrm{AM}}{10: 45 \mathrm{AM}}$ & $=$ & $\frac{11: 30 \mathrm{AM}}{11: 45 \mathrm{AM}}$ & $-\frac{20}{44}$ & $-\frac{0}{0}$ & $\frac{20}{19}$ & $\frac{0}{0}$ & $\frac{0}{0}$ & $\frac{0}{0}$ & $\frac{0}{0}$ & $-\frac{24}{35}$ & $\frac{14}{30}$ & -21 & $\frac{36}{60}$ & 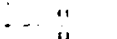 & 135 \\
\hline $\begin{array}{l}10: 45 \mathrm{AM} \\
1100 \mathrm{AM}\end{array}$ & $\bar{E}$ & $12: 00 \mathrm{PM}$ & $\begin{array}{l}44 \\
69\end{array}$ & 0 & 23 & 0 & 0 & 0 & 0 & 25 & 20 & 16 & 50 & $"$ & 18 \\
\hline $\begin{array}{l}1100 \mathrm{AM} \\
11: 15 \mathrm{AM}\end{array}$ & $\ldots$ & $12: 15 \mathrm{PM}$ & 73 & 0 & 28 & 0 & 0 & 0 & 0 & 26 & $\begin{array}{l}16 \\
13\end{array}$ & 16 & 63 & "“ & 212 \\
\hline$\frac{11: 15 \mathrm{AM}}{11: 30 \mathrm{AM}}$ & - & $12.30 \mathrm{PM}$ & 69 & 0 & 24 & 0 & 0 & 0 & -0 & 26 & $\frac{13}{15}$ & 11 & 6: & $"$ & $2 / 8$ \\
\hline & & & & & & & & & & & 15 & II & 67 & is & 212 \\
\hline $11: 45 \mathrm{AM}$ & - & $12: 45 \mathrm{PM}$ & 50 & 0 & 24 & 0 & 0 & 0 & 0 & 31 & 12 & 18 & 56 & $" 1$ & 191 \\
\hline $12: 00 \mathrm{PM}$ & $\ldots$ & $01: 00 \mathrm{PM}$ & 29 & 0 & 14 & 0 & 0 & 0 & 0 & 46 & 10 & 24 & 4 & $\ddot{\prime \prime}$ & 100 \\
\hline $12: 15 \mathrm{PM}$ & 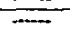 & $01: 15 \mathrm{PM}$ & 23 & 0 & 9 & 0 & 0 & 0 & 0 & $-\frac{-76}{56}$ & 18 & 33 & $\frac{7}{36}$ & ii & 175 \\
\hline 12:30 PM & $\cdots$ & $01 \cdot 30 \mathrm{PM}$ & 18 & 0 & 10 & 0 & 0 & 0 & 0 & 56 & 24 & 33 & 31 & (3) & 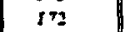 \\
\hline $12: 45 \mathrm{PM}$ & $\ldots$ & O1:AS.PM & 14 & 0 & 17 & 0 & 0 & 0 & 0 & 55 & 28 & 26 & 34 & $u$ & 17 \\
\hline $01: 00 \mathrm{PM}$ & $\cdots$ & $02: 00 \mathrm{PM}$ & 9 & 0 & 23 & 0 & 0 & 0 & 0 & 47 & 25 & 23 & 35 & 0 & 102 \\
\hline $0: 115 \mathrm{PM}$ & $\overline{-}$ & $02: 15 \mathrm{PM}$ & 14 & $\begin{array}{l}0 \\
0\end{array}$ & $\begin{array}{l}20 \\
19\end{array}$ & $\begin{array}{l}0 \\
0\end{array}$ & $\begin{array}{l}0 \\
0\end{array}$ & $\begin{array}{l}0 \\
0\end{array}$ & $\begin{array}{l}0 \\
0\end{array}$ & $\begin{array}{l}47 \\
59\end{array}$ & $\begin{array}{l}19 \\
18\end{array}$ & 19 & $\begin{array}{l}37 \\
36\end{array}$ & 0 & 156 \\
\hline$\frac{01: 30 \mathrm{PM}}{01: 45 \mathrm{PM}}$ & $=$ & $\begin{array}{l}02: 30 \mathrm{PM} \\
02: 45 \mathrm{PM}\end{array}$ & $\frac{12}{15}$ & 0 & $\frac{12}{12}$ & 0 & $\frac{\pi}{0}$ & 0 & 0 & $\frac{39}{67}$ & $\frac{18}{14}$ & 24 & $\frac{30}{34}$ & 0 & $\frac{200}{160}$ \\
\hline $02: 00 \mathrm{PM}$ & - & $03: 00 \mathrm{PM}$ & 19 & $\stackrel{0}{0}$ & 17 & 0 & 0 & $\begin{array}{l}0 \\
0\end{array}$ & $\begin{array}{l}0 \\
0\end{array}$ & 72 & 13 & 22 & 34 & $\begin{array}{l}0 \\
0\end{array}$ & 77 \\
\hline $02: 15$ PM & $=$ & 03:15 PM & $\frac{19}{97}$ & $\frac{0}{0}$ & $\frac{19}{36}$ & 0 & $\frac{0}{0}$ & $\frac{0}{0}$ & $\frac{0}{n}$ & $\frac{74}{81}$ & & $\frac{16}{17}$ & 44 & 0 & $\frac{183}{108}$ \\
\hline $02: 30 \mathrm{PM}$ & 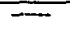 & $03: 30 \mathrm{PM}$ & 23 & 0 & 26 & 0 & 0 & 0 & $\begin{array}{l}0 \\
0\end{array}$ & 81 & 3 & 12 & 48 & 0 & 195 \\
\hline 02:43 PM & - & 03:45 PM & 20 & 0 & 43 & & $\begin{array}{l}0 \\
0\end{array}$ & 0 & $\begin{array}{l}0 \\
0\end{array}$ & 115 & $\begin{array}{l}4 \\
6\end{array}$ & 9 & 52 & $0_{0}^{0}$ & 216 \\
\hline $03.00 \mathrm{PM}$ & $=$ & $04: 00$ PM & 16 & $\frac{0}{0}$ & $\frac{39}{44}$ & $\frac{0}{0}$ & 0 & 0 & 0 & $\frac{115}{158}$ & $\frac{0}{7}$ & $\frac{6}{10}$ & $\frac{54}{41}$ & $\frac{a}{a}$ & $\frac{236}{372}$ \\
\hline $03: 15 \mathrm{PM}$ & - & 04:15 PM & 12 & $\begin{array}{l}0 \\
0\end{array}$ & $\begin{array}{l}44 \\
53\end{array}$ & 0 & 0 & 0 & 0 & & 8 & 13 & 43 & 0 & 332 \\
\hline $\begin{array}{l}03: 30 \mathrm{PM} \\
003: 5 \mathrm{PM}\end{array}$ & $=$ & $\begin{array}{l}04.30 \mathrm{PM} \\
04.45 \mathrm{PM}\end{array}$ & $\begin{array}{l}14 \\
22\end{array}$ & 0 & 54 & 0 & 0 & 0 & 0 & 219 & is & 10 & 14 & 0 & 36.1 \\
\hline$\frac{03: 45 \mathrm{PM}}{04: 00 \mathrm{PM}}$ & $=$ & $-\frac{04: 45 \mathrm{PM}}{05: 00 \mathrm{PM}}$ & 25 & 0 & 77 & 0 & 0 & 0 & 0 & 228 & 16 & 12 & 52 & 0 & 510 \\
\hline$s$ & $E N I$ & $U_{L A}:$ & 7 & & & $A L$ & $D A$ & $0) 2$ & 222 & & $O N$ & $c 0$ & (s & $3=-1$ & \\
\hline
\end{tabular}




\section{Baymetrics Traffic Resources INTERSECTION TURNING MOVEMENT SUMMARY}

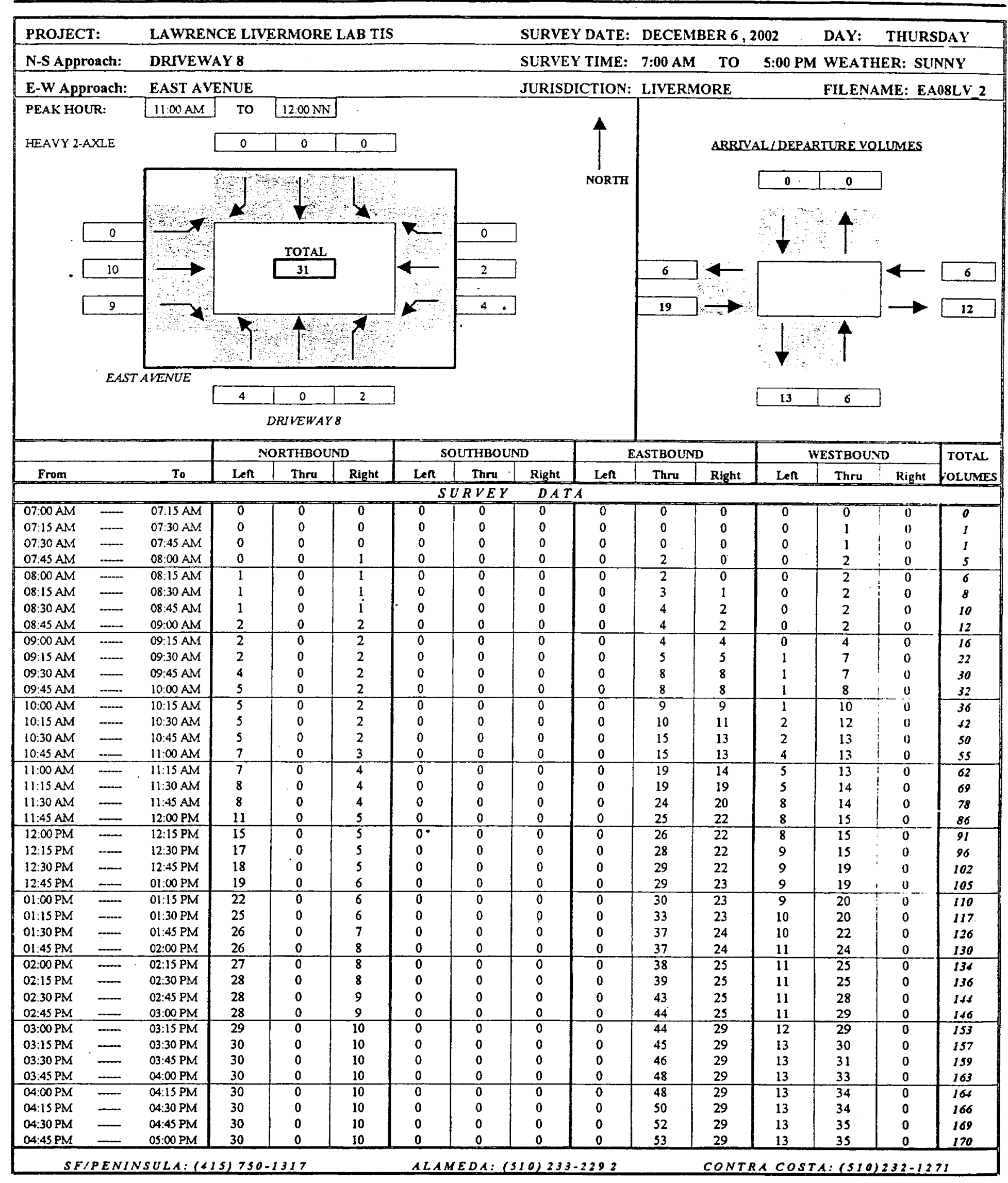




\section{Baymetrics Traffic Resources INTERSECTION TURNING MOVEMENT SUMMARY}

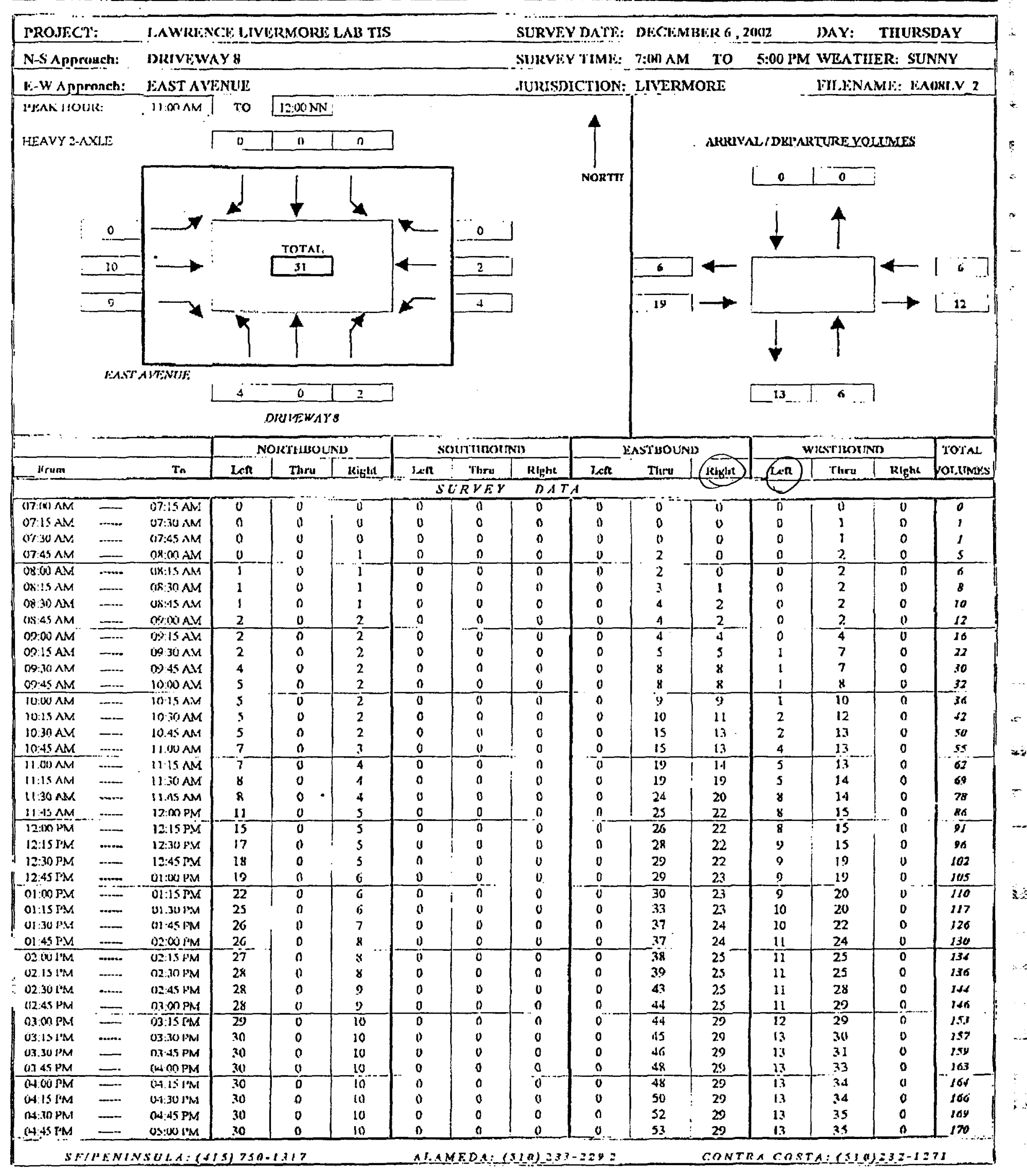




\section{Baymetrics Traffic Resources INTERSECTION TURNING MOVEMENT SUMMARY}

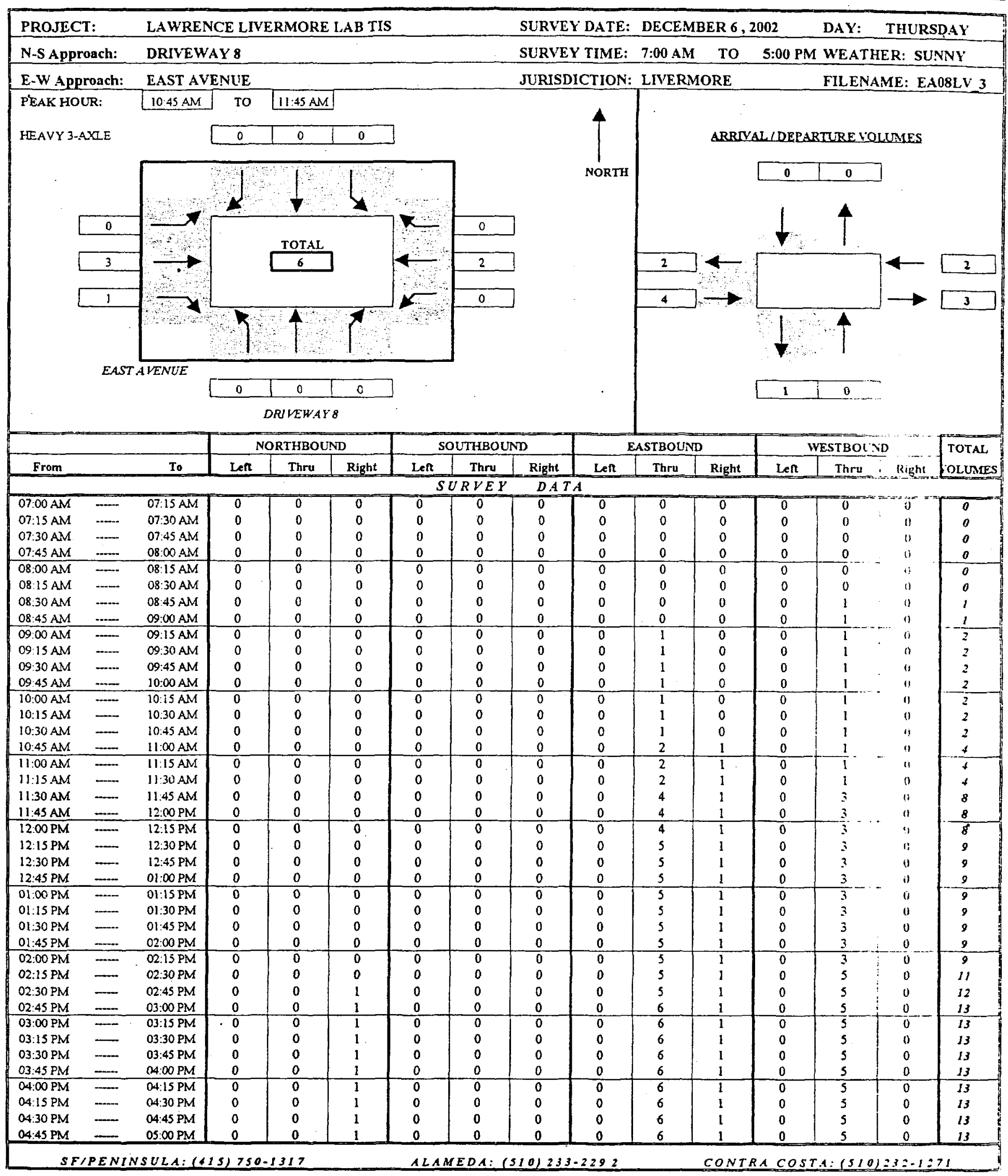




\section{Baymetrics Traffic Resources INTERSECTION TURNING MOVEMENT SUMMARY}

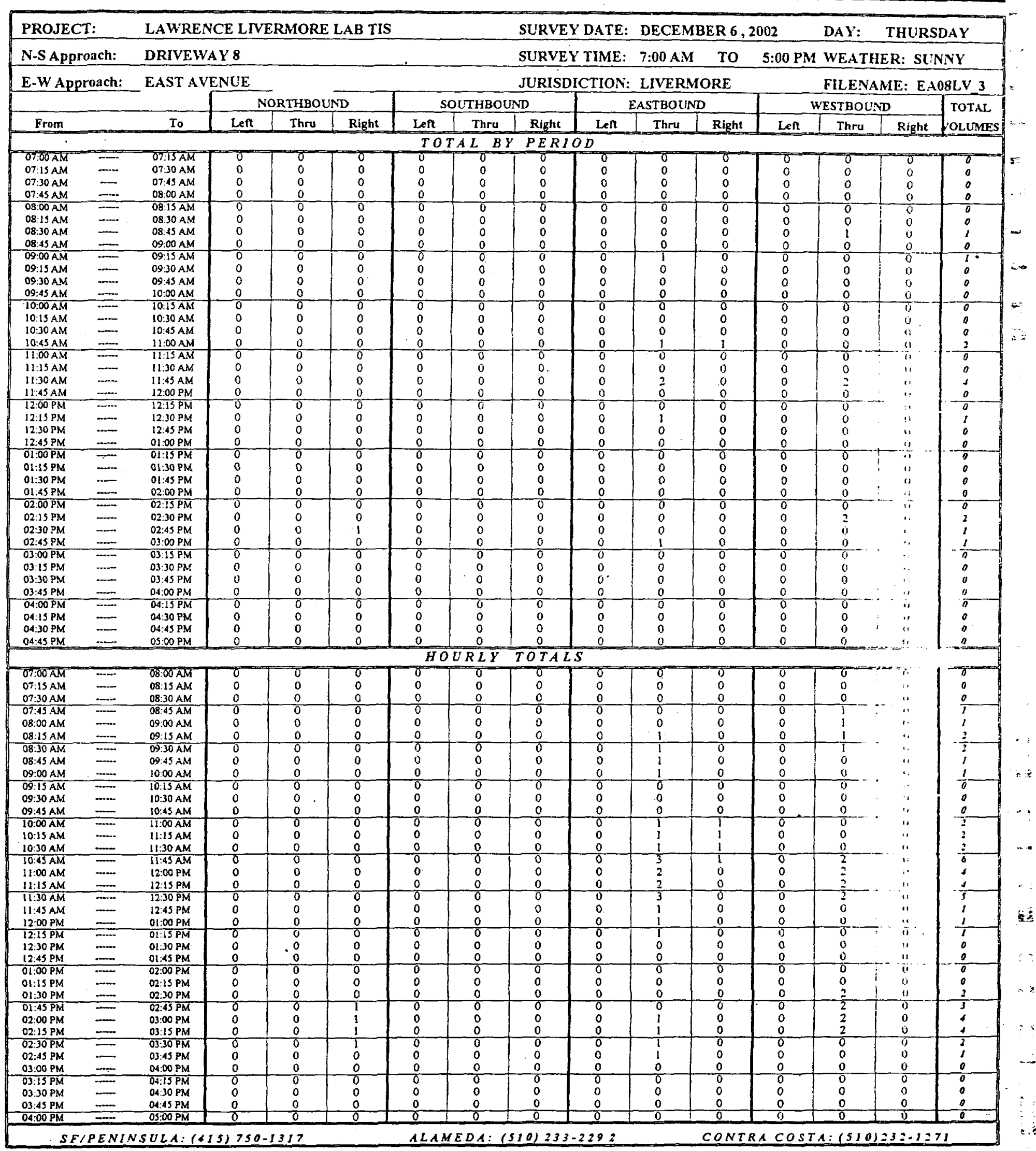




\section{Baymetrics Traffic Resources INTERSECTION TURNING MOVEMENT SUMMARY}

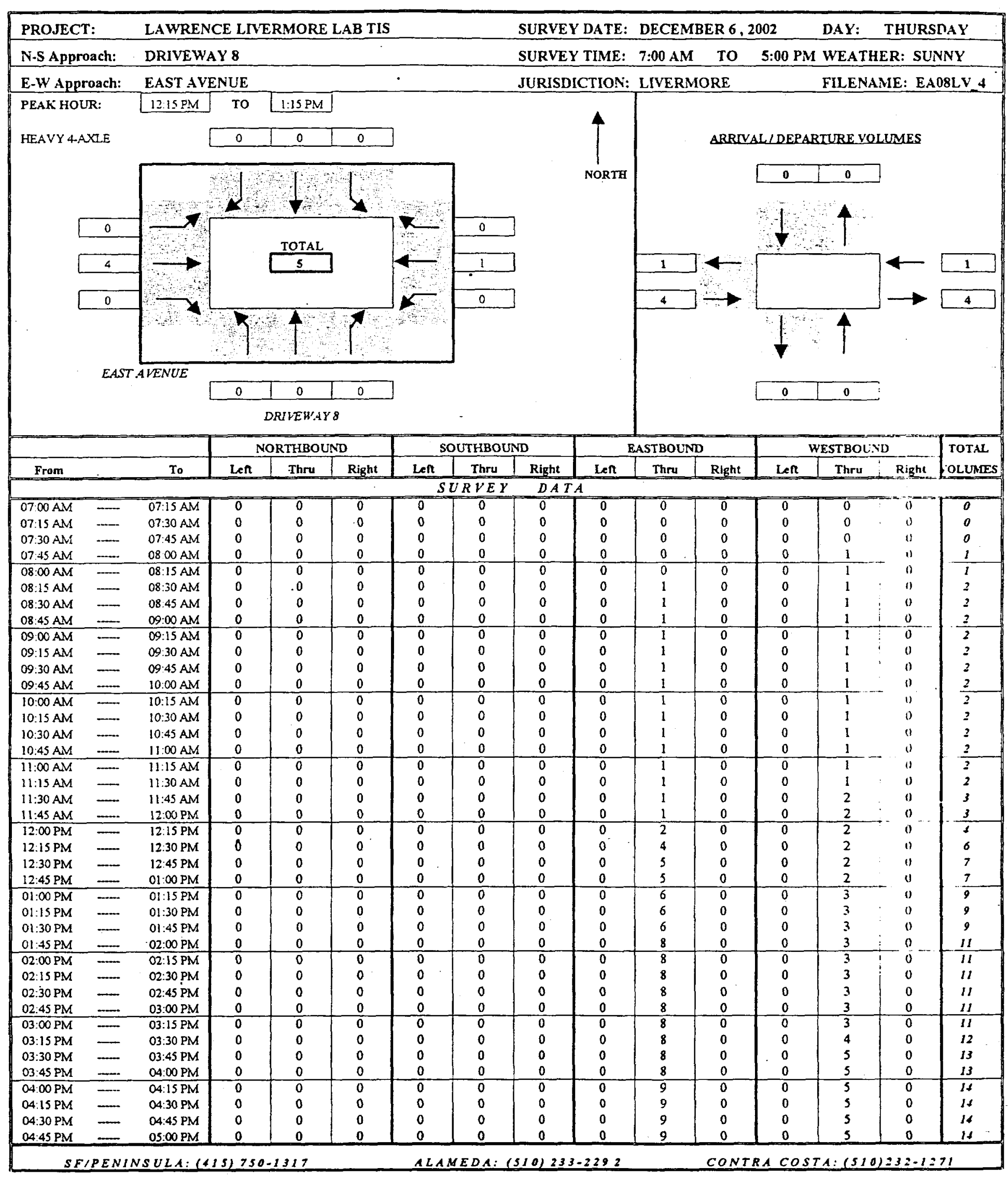




\section{Baymetrics Traffic Resources INTERSECTION TURNING MOVEMENT SUMMARY}

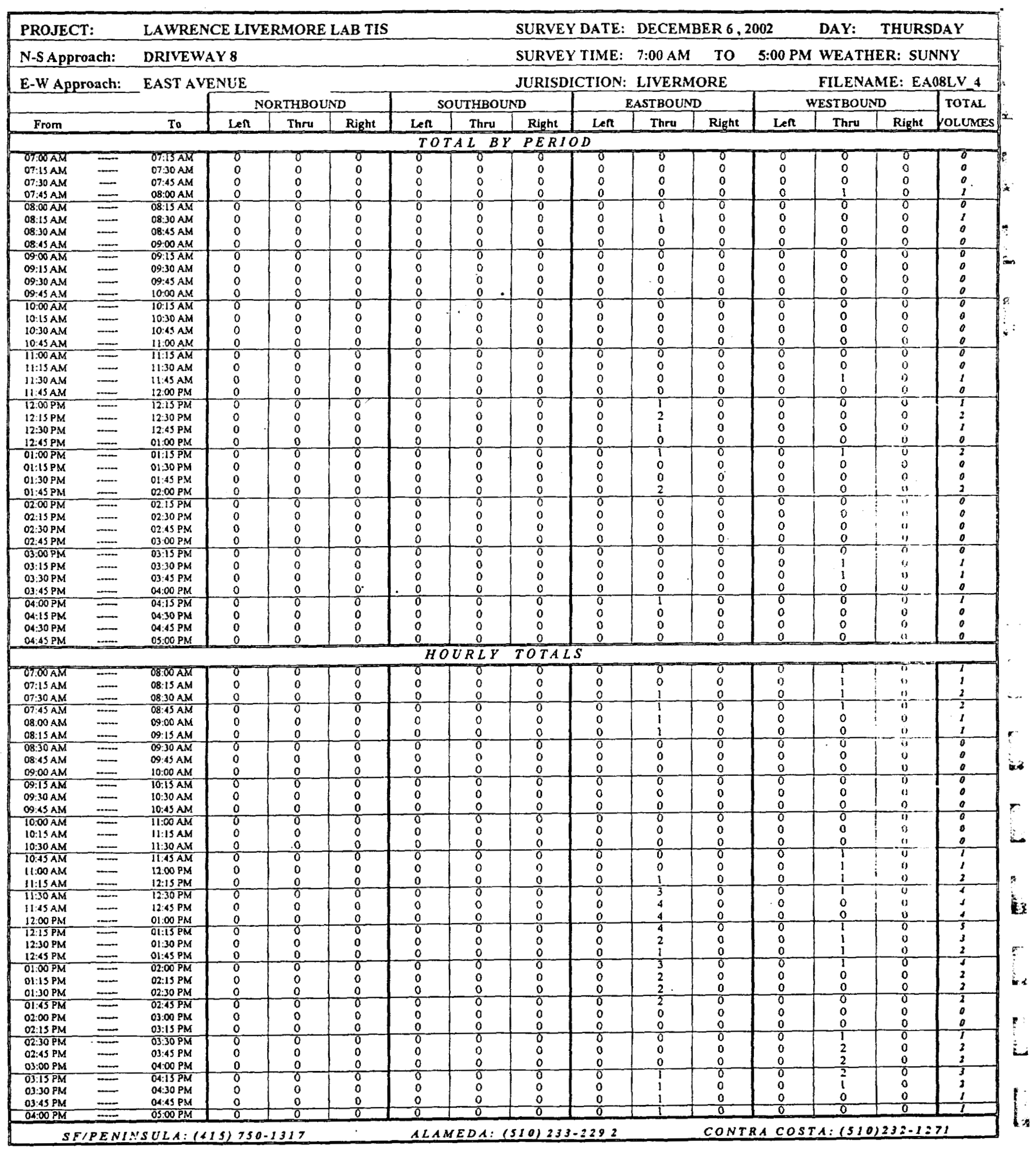




\section{Baymetrics Traffic Resources INTERSECTION TURNING MOVEMENT SUMMARY}

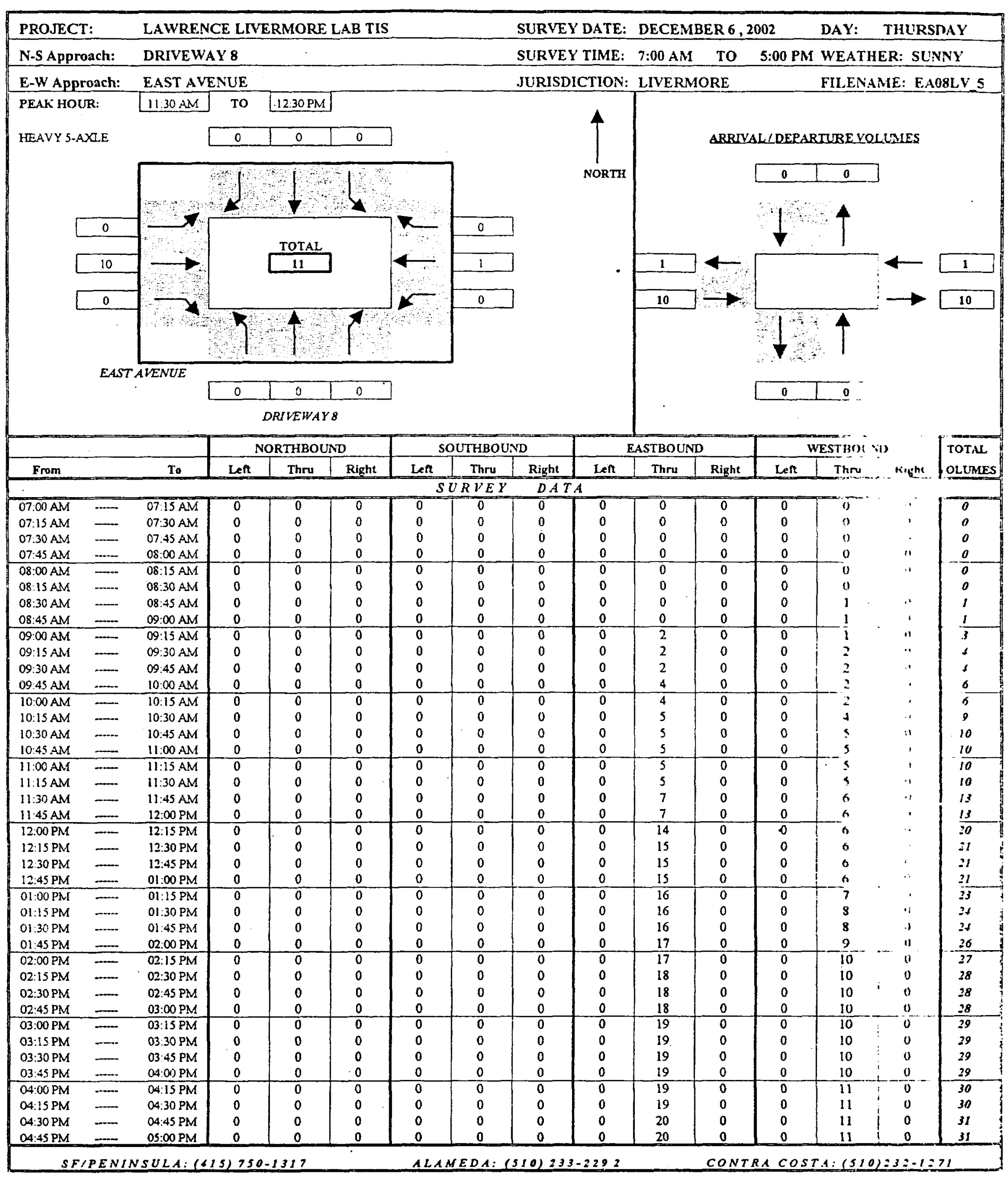




\section{Baymetrics Traffic Resources INTERSECTION TURNING MOVEMENT SUMMARY}

\begin{tabular}{|c|c|c|c|c|c|c|c|c|c|c|c|c|c|c|c|}
\hline \multirow{2}{*}{\multicolumn{2}{|c|}{$\begin{array}{l}\text { PROJECT: } \\
\text { N-S Approach: }\end{array}$}} & \multicolumn{6}{|c|}{ LAWRENCE LIVERMORE LAB TIS } & SURVEY & DATE: & DECEM & ER 6,2 & 002 & DAY: & THURS & DAY \\
\hline & & DRIVEK & & & & & & SURVEY & TIME: & 7:00 AM & To & 5:00 PM & WEATH & ER: SLIN & NY \\
\hline E-W Ap & oach: & EAST A & VUE & & & & & JURISDI & TION: & LIVERM & RE & & FILENA & ME: EAO & $8 \mathrm{LV} 5$ \\
\hline & & & & THBO & & & THBOL & & & ASTBOUN & & & ESTBOUN & & TOTAL \\
\hline From & & To & Len & Thru & Right & Len & Thru & Right & Len & Thru & Right & Len & Thru & Right & KoLURES \\
\hline & & & & & & TO & $L B$ & $P E R I C$ & & & & & & & \\
\hline $07: 00 \mathrm{AM}$ & $=$ & $6715 \mathrm{AM}$ & 0 & $\sigma$ & 0 & $\bar{\sigma}$ & $\sigma$ & 0 & 0 & o & $\sigma$ & 0 & $\pi$ & 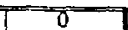 & J \\
\hline 07:15 AM & $\cdots$ & $07: 30 \mathrm{AM}$ & 0 & 0 & 0 & 0 & 0 & 0 & 0 & 0 & 0 & 0 & 0 & 0 & 0 \\
\hline $\begin{array}{l}07: 30 \mathrm{AM} \\
07: 45 \mathrm{AM}\end{array}$ & $=$ & $\begin{array}{l}07: 45 \mathrm{AM} \\
08: 00 \mathrm{AM}\end{array}$ & $\begin{array}{l}0 \\
0\end{array}$ & $\begin{array}{l}0 \\
0\end{array}$ & $\begin{array}{l}0 \\
0\end{array}$ & $\begin{array}{l}0 \\
0\end{array}$ & $\begin{array}{l}0 \\
0\end{array}$ & $\begin{array}{l}0 \\
0\end{array}$ & $\begin{array}{l}0 \\
0\end{array}$ & $\begin{array}{l}0 \\
0\end{array}$ & $\begin{array}{l}0 \\
0\end{array}$ & $\begin{array}{l}0 \\
0\end{array}$ & $\begin{array}{l}0 \\
0\end{array}$ & 0 & 0 \\
\hline $08: 00 \mathrm{AM}$ & 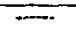 & $08: 15 \mathrm{AM}$ & 0 & 0 & 0 & 0 & 0 & 0 & $\pi$ & 0 & 0 & 0 & $\frac{0}{0}$ & $\frac{0}{0}$ & 0 \\
\hline 08:15 AM & $\ldots$ & 08:30 AM & 0 & 0 & 0 & 0 & 0 & 0 & 0 & 0 & 0 & 0 & 0 & 0 & 0 \\
\hline 08:30 AM & $\ldots$ & 08:45 AM & 0 & 0 & 0 & 0 & 0 & 0 & 0 & 0 & 0 & 0 & 1 & 0 & $i$ \\
\hline 08:45 AM & - & $09: 00 \mathrm{AM}$ & 0 & 0 & 0 & 0 & 0 & 0 & 0 & 0 & 0 & 0 & 0 & 0 & 0 \\
\hline $\begin{array}{l}09: 00 \mathrm{AM} \\
09: 15 \mathrm{AM}\end{array}$ & $\ldots$ & 09:15 AMM & 0 & 0 & 0 & 0 & 8 & 0 & $\begin{array}{l}0 \\
0 \\
0\end{array}$ & 2 & 0 & 0 & 0 & 0 & 2 \\
\hline $09: 30 \mathrm{AM}$ & $=$ & $\begin{array}{l}\text { 09:30 AMM } \\
09: 45 \mathrm{AM}\end{array}$ & $\begin{array}{l}0 \\
0\end{array}$ & $\begin{array}{l}0 \\
0\end{array}$ & $\begin{array}{l}0 \\
0\end{array}$ & $\begin{array}{l}0 \\
0\end{array}$ & 0 & 0 & 0 & 0 & $\begin{array}{l}0 \\
0\end{array}$ & 0 & 1 & 0 & 1 \\
\hline $09: 45$ AM & $=$ & $\begin{array}{l}10.00 \mathrm{AM} \\
1\end{array}$ & 0 & 0 & 0 & 0 & 0 & 0 & 0 & 2 & $\begin{array}{l}0 \\
0 \\
0\end{array}$ & $\begin{array}{l}0 \\
0\end{array}$ & $\begin{array}{l}0 \\
0\end{array}$ & $\begin{array}{l}0 \\
0\end{array}$ & $i$ \\
\hline 10:00 AM & $=$ & $10: 15 \mathrm{AM}$ & 0 & 0 & 0 & 0 & 0 & $\overline{0}$ & 8 & 0 & $\overline{0}$ & 0 & 0 & 10 & $\frac{1}{0}$ \\
\hline 10:15 AM & - & $10: 30 \mathrm{AM}$ & 0 & 0 & 0 & 0 & 0 & 0 & 0 & 1 & 0 & 0 & 2 & 0 & 3 \\
\hline 10:30 AM & $\cdots$ & 10:45 AM & 0 & 0 & 0 & 0 & 0 & 0 & 0 & 0 & 0 & 0 & 1 & 0 & $i$ \\
\hline $10: 45 \mathrm{AM}$ & $-\ldots$ & $11: 00 \mathrm{AM}$ & 0 & 0 & 0 & 0 & 0 & 0 & 0 & 0 & 0 & 0 & 0 & $\therefore$ & 0 \\
\hline $1100 \mathrm{AM}$ & 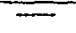 & $11: 15 \mathrm{AM}$ & 0 & 0 & 0 & 0 & 0 & 0 & 0 & 0 & 0 & 0 & 0 & ii & 6 \\
\hline 11:15 AM & $\ldots$ & $11: 30 \mathrm{AM}$ & 0 & 0 & 0 & 0 & 0 & 0 & 0 & 0 & 0 & 0 & 0 & $"$ & 0 \\
\hline $\begin{array}{l}11: 30 \mathrm{AM} \\
11.45 \mathrm{AM}\end{array}$ & $=$ & $\begin{array}{l}11: 45 \mathrm{AM} \\
12: 00 \mathrm{PM}\end{array}$ & $\begin{array}{l}0 \\
0\end{array}$ & $\begin{array}{l}0 \\
0\end{array}$ & $\begin{array}{l}0 \\
0\end{array}$ & $\begin{array}{l}0 \\
0\end{array}$ & $\begin{array}{l}0 \\
0\end{array}$ & $\begin{array}{l}0 \\
0\end{array}$ & 0 & 2 & 0 & 0 & $i$ & $"$ & 3 \\
\hline $12: 00 \mathrm{PM}$ & $\mp$ & $12: 15 \mathrm{PM}$ & 0 & 0 & 0 & 0 & $\hat{0}$ & 0 & 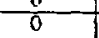 & $\frac{0}{7}$ & $\frac{0}{0}$ & $\frac{0}{0}$ & $g$ & ." & $\because$ \\
\hline 12:15 PM & $\ldots$ & $12: 30 \mathrm{PM}$ & 0 & 0 & 0 & 0 & 0 & 0 & 0 & 1 & 0 & .0 & $n$ & 10 & 1 \\
\hline 12:30 PM & $\ldots$ & 12:45 PM & 0 & 0 & $\begin{array}{l}0 \\
0 \\
0\end{array}$ & $\begin{array}{l}0 \\
0\end{array}$ & 0 & ${ }_{0}^{0}$ & 0 & 0 & 0 & 0 & 0 & " & 0 \\
\hline$\frac{12: 45 \text { PM }}{0.100 \text { PM }}$ & $=$ & $\frac{01: 00 \mathrm{PM}}{0115 \mathrm{PM}}$ & $\frac{0}{0}$ & $\frac{0}{0}$ & $\frac{0}{0}$ & $\overline{0}$ & $\frac{0}{0}$ & $\frac{0}{0}$ & $\frac{0}{0}$ & 0 & 0 & 0 & o & $\because-$ & 0 \\
\hline ol:1S PM & $=$ & $01: 30 \mathrm{PM}$ & 0 & 0 & 0 & 0 & 0 & 0 & 0 & 0 & $\begin{array}{l}0 \\
0\end{array}$ & $\begin{array}{l}0 \\
0\end{array}$ & $\begin{array}{l}1 \\
1\end{array}$ & : & 2 \\
\hline $0130 \mathrm{PM}$ & $\cdots$ & $01: 45 \mathrm{PM}$ & 0 & 0 & 0 & 0 & 0 & 0 & 0 & 0 & 0 & 0 & 0 & " & 0 \\
\hline 01:45 PM & $\cdots$ & 02.00 PM & 0 & 0 & 0 & 0 & 0 & 0 & 0 & $i$ & 0 & 0 & 1 & $\because$ & 2 \\
\hline $02: 00 \mathrm{PM}$ & $\ldots$ & $02.15 P M$ & 0 & 0 & 0 & 0 & 0 & 0 & 0 & 0 & 0 & 0 & $T$ & $\because$ & 1 \\
\hline 02:15 PM & - & 02:30 PM & 0 & 0 & 0 & 0 & 0 & 0 & 0 & 1 & 0 & 0 & 9 & $"$ & $i$ \\
\hline 02:30 PM & $\ldots$ & $02: 45$ PM & 0 & 0 & 0 & 0 & 0 & 0 & 0 & 0 & 0 & 0 & $n$ & $\because$ & 0 \\
\hline 02:45 PM & $\cdots$ & $03.00 \mathrm{PM}$ & 0 & 0 & 0 & 0 & 0 & 0 & 0 & 0 & 0 & 0 & 0 & $\because$ & 0 \\
\hline $03: 00 \mathrm{PM}$ & $\cdots$ & $03: 15 \mathrm{PM}$ & 0 & 0 & 0 & 0 & 0 & 0 & 0 & $T$ & 0 & 0 & a & i. & \\
\hline 03:1S PM & ... & 03:30 PM & 0 & 0 & 0 & 0 & 0 & 0 & 0 & 0 & 0 & 0 & 0 & .. & 0 \\
\hline $03: 30 \mathrm{PM}$ & $\ldots$ & 03:45 PM & 0 & 0 & 0 & 0 & 0 & 0 & 0 & 0 & 0 & 0 & 0 & .. & 0 \\
\hline $03: 45 \mathrm{PM}$ & $\ldots$ & $04: 00$ PM & 0 & 0 & 0 & 0 & 0 & 0 & 0 & 0 & 0 & 0 & 0 & $\because$ & 0 \\
\hline $04: 00 \mathrm{PM}$ & $=$ & $04: 15 \mathrm{PM}$ & 0 & 0 & 0 & 0 & 0 & $\overline{0}$ & 0 & 0 & 0 & 0 & 1 & "is & $T$ \\
\hline 04:15 PM & $\ldots$ & 04:30 PM & 0 & 0 & 0 & 0 & 0 & 0 & 0 & 0 & 0 & 0 & 0 & " & $\theta$ \\
\hline 04:30 PM & $\overline{-}$ & 04:45 PM & $:$ & 0 & $\begin{array}{l}0 \\
0\end{array}$ & $\begin{array}{l}0 \\
0\end{array}$ & $\begin{array}{l}0 \\
0\end{array}$ & $\begin{array}{l}0 \\
0 \\
0\end{array}$ & 0 & 1 & 0 & 0 & 0 & $\because$ & 1 \\
\hline 04:45 PM & $=$ & $05: 00 \mathrm{PM}$ & 0 & 0 & 0 & & $\frac{0}{R L Y}$ & TOTAL & 0 & 0 & 0 & $0=$ & $\underline{0}$ & $\because$ & 0 \\
\hline T00AM & $=$ & $88.00 \mathrm{AM}$ & 0 & 0 & $\sigma$ & $\sigma$ & 0 & 0 & $\sigma$ & 0 & $\sigma$ & $\sigma$ & $\pi$ & (1) & $\gamma$ \\
\hline 07:15 AM & - & 08:15 AM & 0 & 0 & 0 & 0 & 0 & 0 & 0 & 0 & 0 & 0 & , & $"$ & 0 \\
\hline $07: 30 \mathrm{AM}$ & $\ldots$ & OB:30 AM & 0 & 0 & 0 & 0 & 0 & 0 & 0 & 0 & 0 & 0 & $\underline{0}$ & ". & 0 \\
\hline $07.45 \mathrm{AM}$ & $\cdots$ & $08: 45 \mathrm{AM}$ & 0 & 0 & $\overline{0}$ & 0 & jo & 0 & 0 & 0 & 0 & 0 & $T$ & $"{ }^{-}$ & 1 \\
\hline 08:00 AM & - & 09:00 AM & 0 & 0 & 0 & ${ }^{0}$ & 0 & 0 & 0 & 0 & 0 & 0 & 1 & $\because$ & 1 \\
\hline $08: 15 \mathrm{AM}$ & $=$ & $09: 15 \mathrm{AM}$ & 0 & 0 & 0 & 0 & 0 & 0 & 0 & $\frac{2}{7}$ & 0 & 0 & $\frac{1}{3}$ & $\because:$ & 3 \\
\hline $08: 30 \mathrm{AM}$ & $\ldots$ & $09: 30 \mathrm{AM}$ & 0 & 0 & 0 & 0 & 0 & 0 & 0 & $\frac{2}{2}$ & $\overline{0}$ & 0 & 5 &.. & 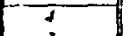 \\
\hline 08:45 AM & - & 09:45 AM & 0 & 0 & 0 & 0 & 0 & 0 & 0 & 2 & 0 & 0 & 1 & ". & 3 \\
\hline $09: 00 \mathrm{AM}$ & $\ldots$ & $10: 00 \mathrm{AM}$ & 0 & 0 & 0 & 0 & 0 & 0 & 0 & 4 & 0 & 0 & $i$ & $"$ & 5 \\
\hline $09: 15 \mathrm{AM}$ & $=$ & $10: 15 \mathrm{AM}$ & 0 & $\overline{0}$ & 0 & 0 & 0 & 0 & 0 & 2 & 0 & 0 & $T$ & $\therefore$ & 3 \\
\hline $09: 30 \mathrm{AM}$ & - & 10:30 AM & 0 & 0 & 0 & 0 & 0 & 0 & 0 & 3 & 0 & 0 & 2 & i. & 5 \\
\hline 09.45 AM & $=$ & $10: 43 \mathrm{AM}$ & 0 & 0 & 0 & 0 & 0 & 0 & 0 & 3 & 0 & 0 & 3 & $:$ & 6 \\
\hline $10: 00 \mathrm{AM}$ & $\cdots$ & $11: 00 \mathrm{AM}$ & 0 & 0 & 0 & $\overline{0}$ & 8 & 0 & 0 & 1 & $\dot{0}$ & 0 & 3 & : & 7 \\
\hline $10: 15 \mathrm{AM}$ & - & $11: 15$ AM & 0 & 0 & 0 & 0 & 0 & 0 & 0 & $i$ & 0 & 0 & 3 & .. & $j$ \\
\hline $10: 30 \mathrm{AM}$ & $=$ & 11:30 AM & 0 & 0 & 0 & 0 & 0 & 0 & 0 & 0 & 0 & 0 & 1 &. & $i$ \\
\hline $10: 45 \mathrm{AM}$ & $-\infty$ & $11: 45 \mathrm{AM}$ & 0 & 0 & 0 & 0 & 0 & 0 & 0 & 2 & 0 & $\overline{0}$ & $\frac{2}{1}$ & $"$ & 3 \\
\hline $11: 00 \mathrm{AM}$ & $\ldots$ & $12: 00 \mathrm{PM}$ & 0 & 0 & 0 & 0 & 0 & 0 & 0 & 2 & 0 & 0 & $i$ & ." & \\
\hline 11:15 AM & $\ldots$ & 12:15 PM & 0 & 0 & 0 & 0 & 0 & 0 & 0 & 9 & 0 & 0 & i. & $\because$ & 10 \\
\hline $11: 30 \mathrm{AM}$ & $-\infty$ & $12: 30 \mathrm{PM}$ & 0 & 0 & 0 & $\overline{0}$ & 0 & 0 & $\overline{0}$ & 10 & 0 & 0 & $T$ & i) & II \\
\hline $11: 45 \mathrm{AM}$ & $\cdots$ & 12:45 9M & $\begin{array}{l}0 \\
0\end{array}$ & $\begin{array}{l}0 \\
0\end{array}$ & 0 & 0 & $\begin{array}{l}0 \\
0\end{array}$ & $\begin{array}{l}0 \\
0\end{array}$ & $\begin{array}{l}0 \\
0\end{array}$ & 8 & $\begin{array}{l}0 \\
0\end{array}$ & $\begin{array}{l}0 \\
0\end{array}$ & $\begin{array}{l}0 \\
0\end{array}$ & $" 1$ & 8 \\
\hline$\frac{12: 00 \mathrm{PM}}{12: 15 \mathrm{PM}}$ & $=$ & $01: 00 \mathrm{PM}$ & $\frac{0}{0}$ & $\frac{0}{0}$ & $\frac{0}{0}$ & $\frac{0}{0}$ & 0 & $\frac{5}{0}$ & $\frac{0}{0}$ & $\frac{8}{2}$ & $\frac{0}{0}$ & $\frac{0}{0}$ & $\frac{0}{1}$ & $\frac{1}{10}$ & $\frac{8}{3}$ \\
\hline $\begin{array}{l}12: 15 \mathrm{PM} \\
12: 30 \mathrm{PM}\end{array}$ & $=$ & $\begin{array}{l}01: 15 \text { PM } \\
01: 30 \mathrm{PM}\end{array}$ & 0 & 0 & 0 & 0 & 0 & 0 & 0 & 1 & 0 & 0 & 2 & "1" & \\
\hline $\begin{array}{l}\text { 12:30 PM } \\
12: 45 \mathrm{PM} \\
\end{array}$ & $=$ & $\begin{array}{l}01: 30 \text { PM } \\
01: 45 \text { PM }\end{array}$ & 0 & 0 & 0 & 0 & 0 & 0 & 0 & $\mathrm{i}$ & 0 & 0 & 2 & 0 & 3 \\
\hline 01:00 PM & $=$ & $02.00 \mathrm{PM}$ & 0 & 0 & \% & 0 & 0 & 0 & \% & 2 & 0 & 0 & 3 & a & 5 \\
\hline 01:15 PM & - & 02:15 PM & 0 & 0 & 0 & 0 & 0 & 0 & 0 & 1 & 0 & 0 & 3 & 0 & $t$ \\
\hline $01: 30$ PM & $=$ & 02:30 PM & 0 & 0 & 0 & 0 & 0 & 0 & 0 & 2 & 0 & 0 & 2 & i) & 4 \\
\hline $01: 15 \mathrm{PM}$ & $=$ & $02: 05$ PM & 0 & 0 & 0 & 0 & 0 & 0 & 0 & 2 & $\overline{0}$ & 0 & 2 & 0 & 7 \\
\hline 02:00 PM & - & $03.00 \mathrm{PM}$ & 0 & 0 & 0 & 0 & 0 & 0 & 0 & 1 & 0 & 0 & I & 0 & 2 \\
\hline $02: 19 \mathrm{PM}$ & - & $03: 15 \mathrm{PM}$ & 0 & 0 & 0 & 0 & 0 & 0 & 0 & 2 & 0 & 0 & 0 & 0 & 2 \\
\hline $02.30 \mathrm{PM}$ & 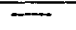 & $03: 30 \mathrm{PM}$ & 0 & 0 & 0 & 0 & 0 & 0 & 0 & $?$ & 0 & 0 & 0 & 0 & ! \\
\hline 02:45 PM & $\ldots$ & $03: 45 \mathrm{PM}$ & 0 & 0 & 0 & 0 & 0 & 0 & 0 & $i$ & 0 & 0 & 0 & i) & $i$ \\
\hline $03: 00 \mathrm{PM}$ & $\cdots$ & $04: 00 \mathrm{PM}$ & 0 & 0 & 0 & 0 & 0 & 0 & 0 & $i$ & 0 & 0 & 0 & 0 & 3 \\
\hline $03: 15 \mathrm{PM}$ & $=$ & 04.15 PM & 0 & 0 & 0 & 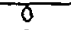 & $\overline{0}$ & 0 & 0 & 0 & 0 & 0 & $T$ & a & 5 \\
\hline 03:30 PM & 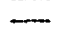 & O4:30 PM & 0 & 0 & 0 & 0 & 0 & 0 & 0 & 0 & 0 & 0 & i & 0 & $i$ \\
\hline 03:45 PM & - & OA:45 PM & 0 & 0 & 0 & 0 & 0 & 0 & 0 & 1 & 0 & 0 & 1 & 0 & 2 \\
\hline $04: 00 \mathrm{PM}$ & $=$ & $05: 00 \mathrm{PM}$ & 0 & 0 & 0 & 0 & 0 & 0 & 0 & 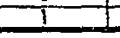 & 0 & 0 & & 0 & 2 \\
\hline
\end{tabular}




\section{Baymetrics Traffic Resources INTERSECTION TURNING MOVEMENT SUMMARY}

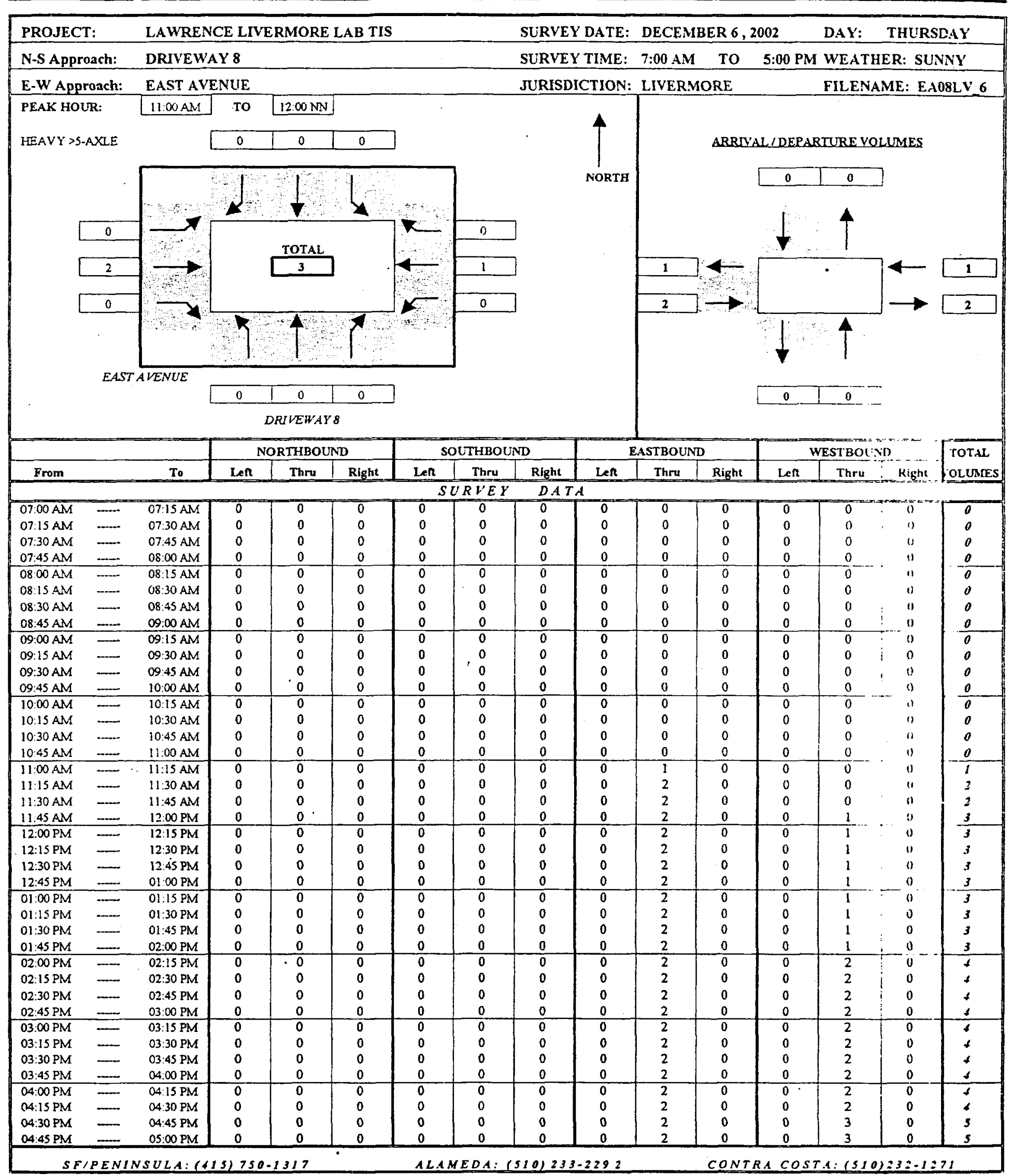




\section{Baymetrics Traffic Resources, INTERSECTION TURNING MOVEMENT SUMMARY}

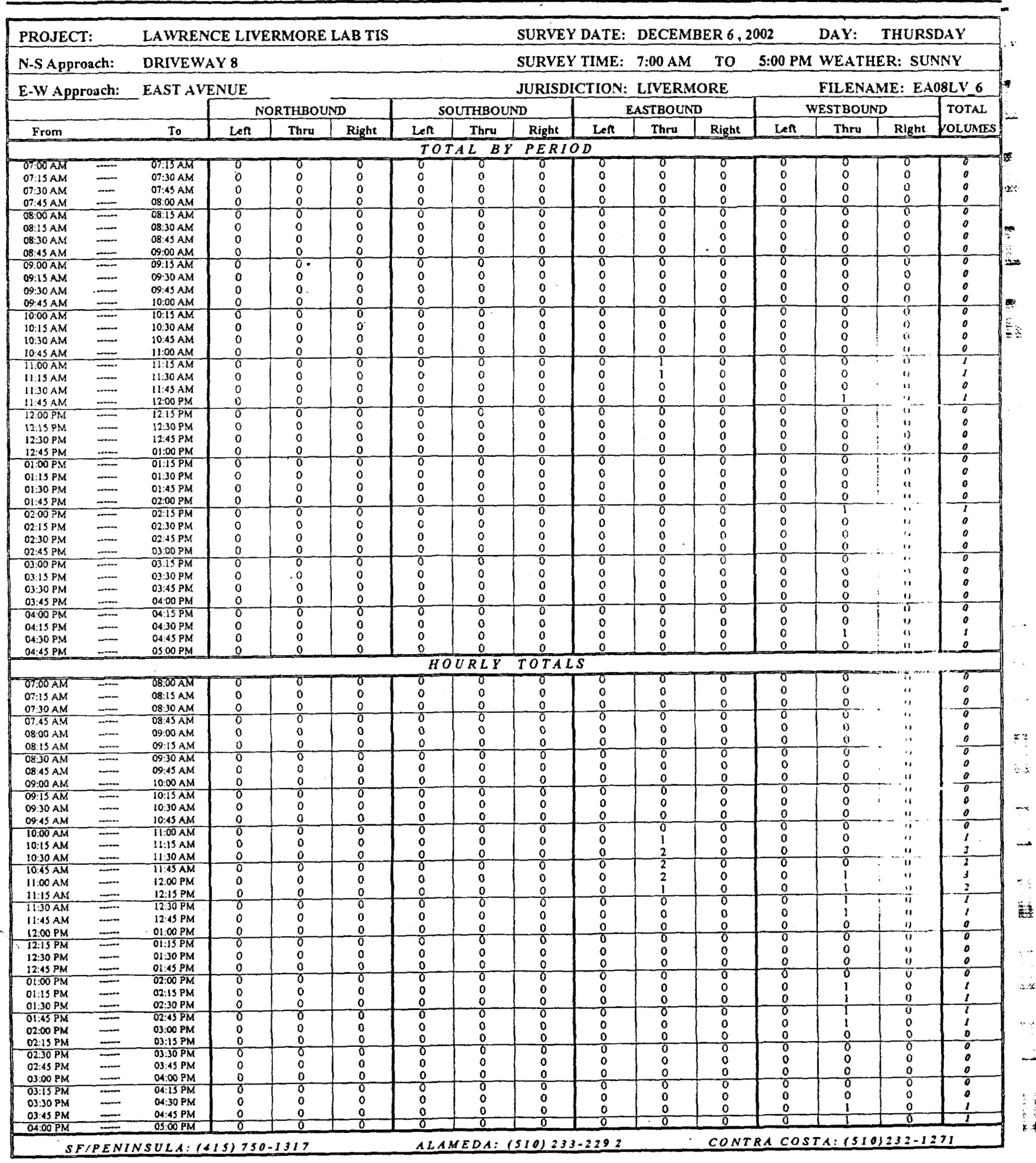


Truck Counts

\section{Locations 7 and 8}

Friday, December 7, 2001 



\section{Baymetrics Traffic Resources INTERSECTION TURNING MOVEMENT SUMMARY}

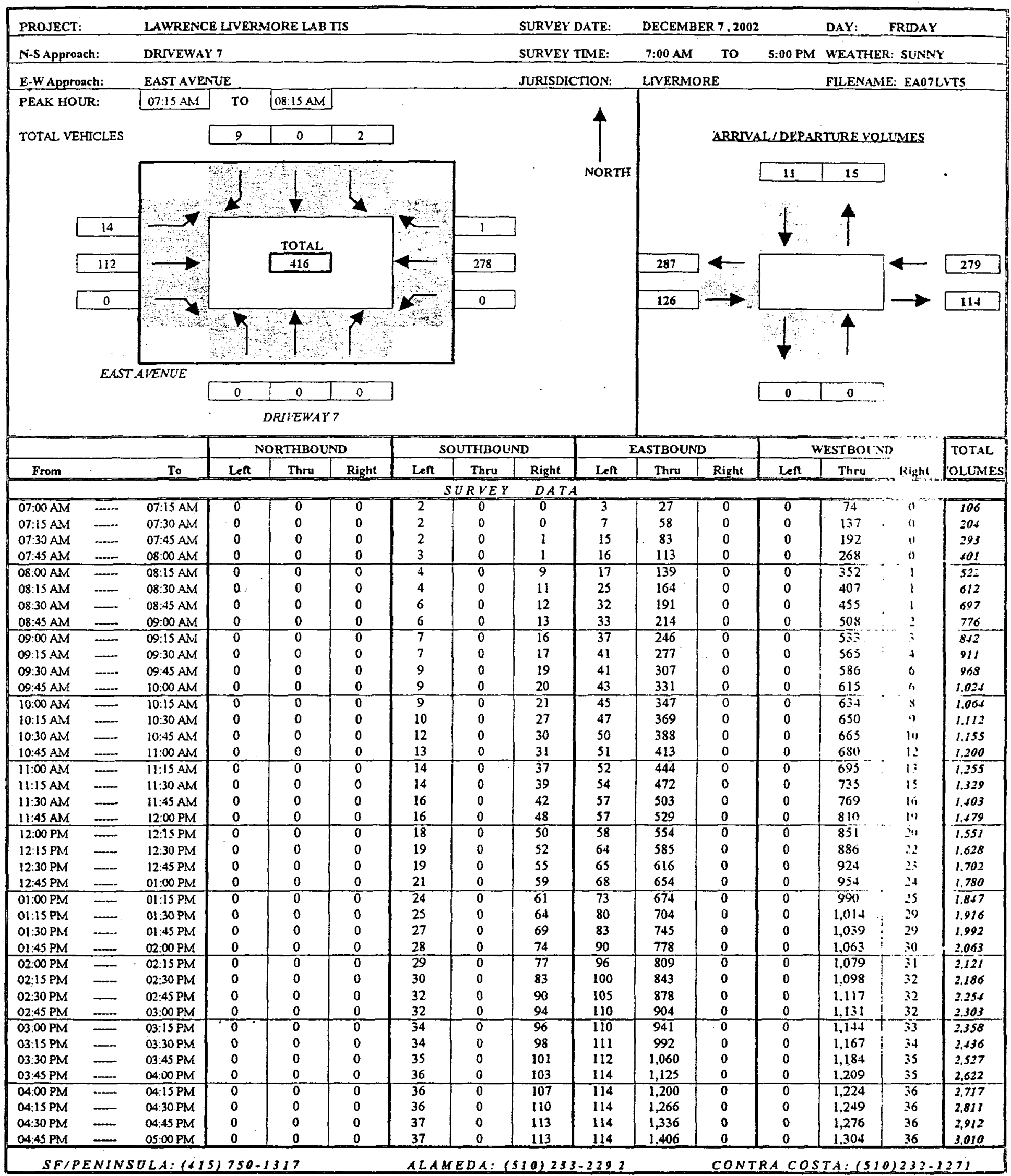




\section{Baymetrics Traffic Resources INTERSECTION TURNING MOVEMENT SUMMARY}

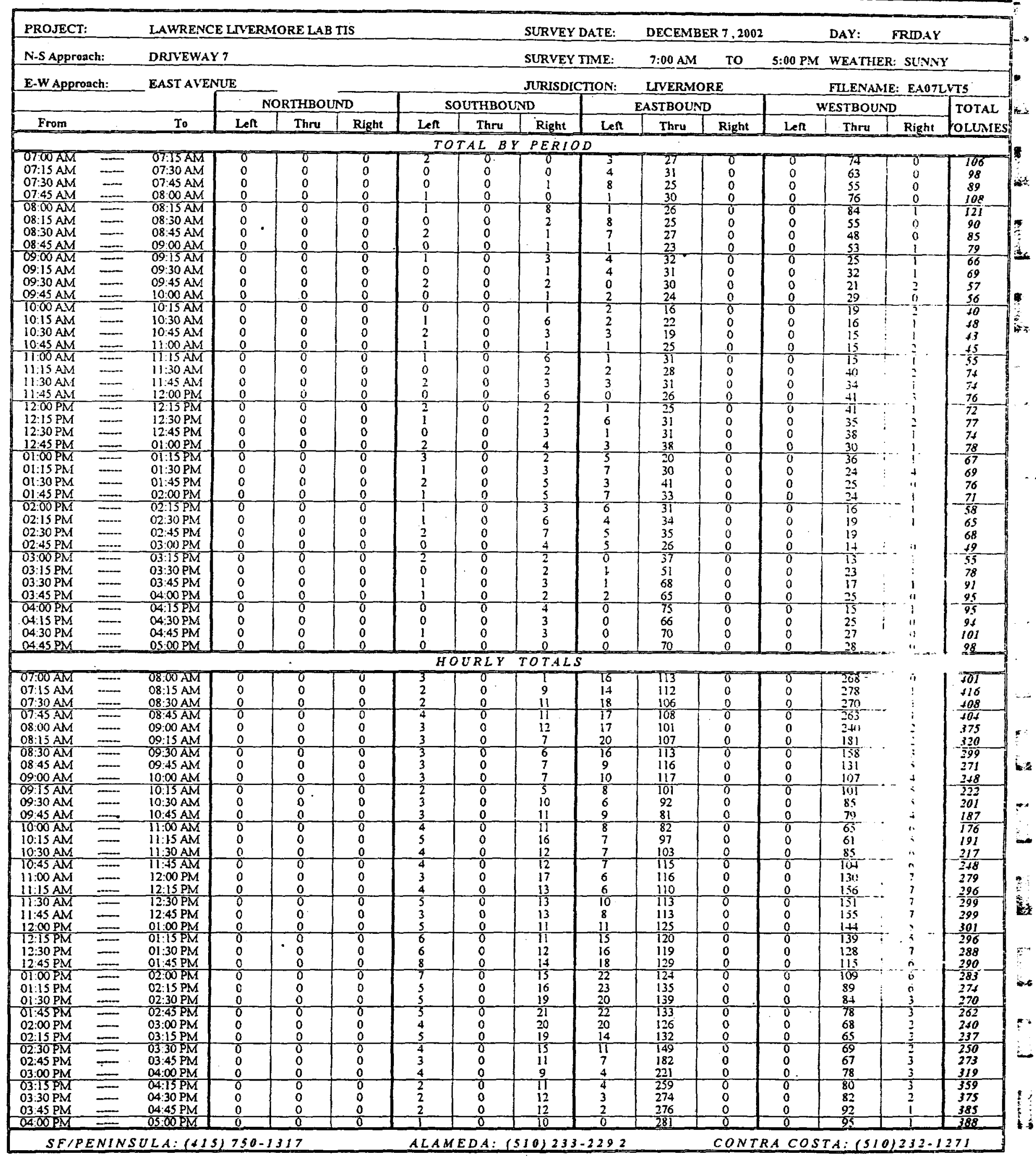




\section{Baymetrics Traffic Resources INTERSECTION TURNING MOVEMENT SUMMARY}

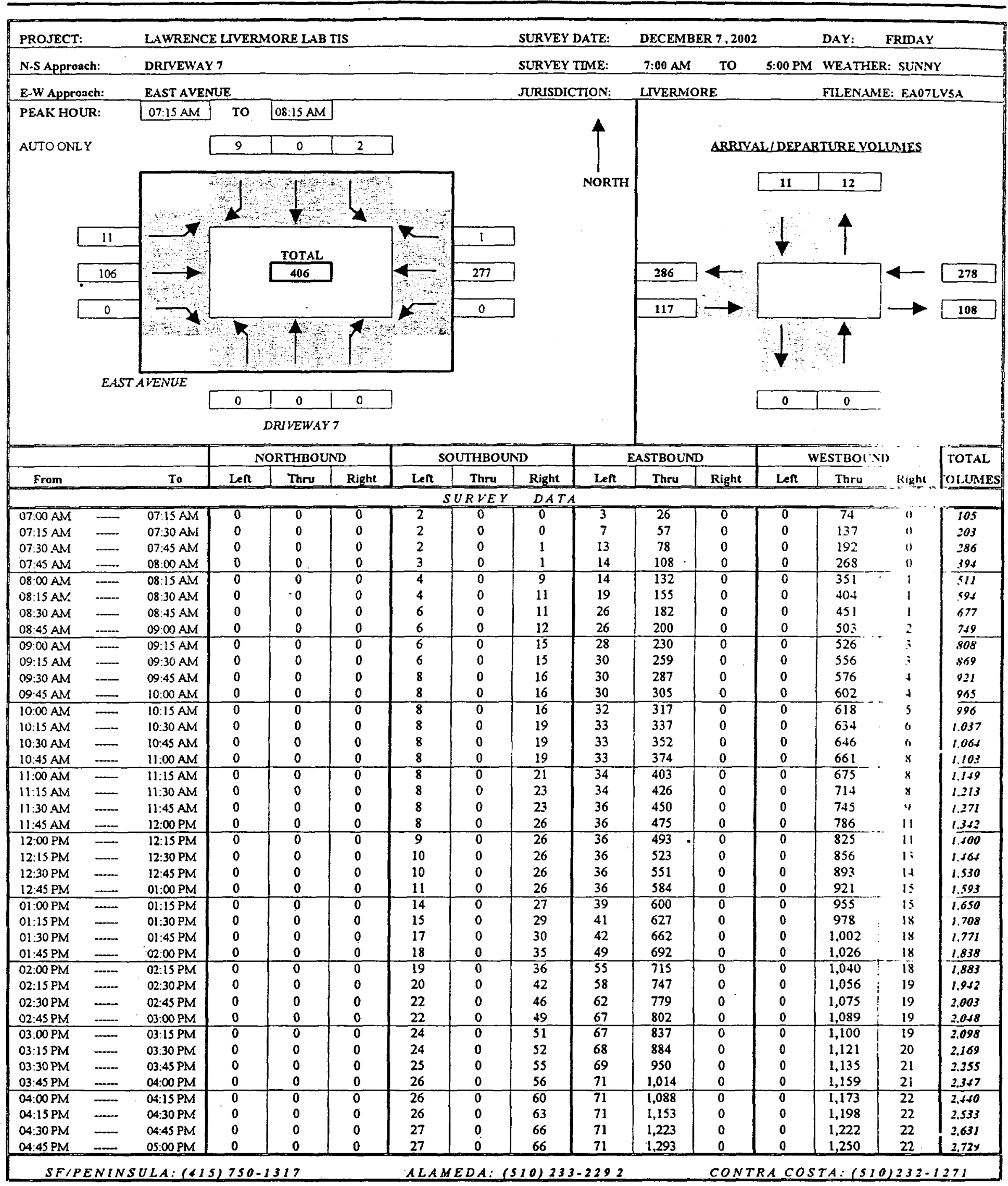




\section{Baymetrics Traffic Resources INTERSECTION TURNING MOVEMENT SUMMARY}

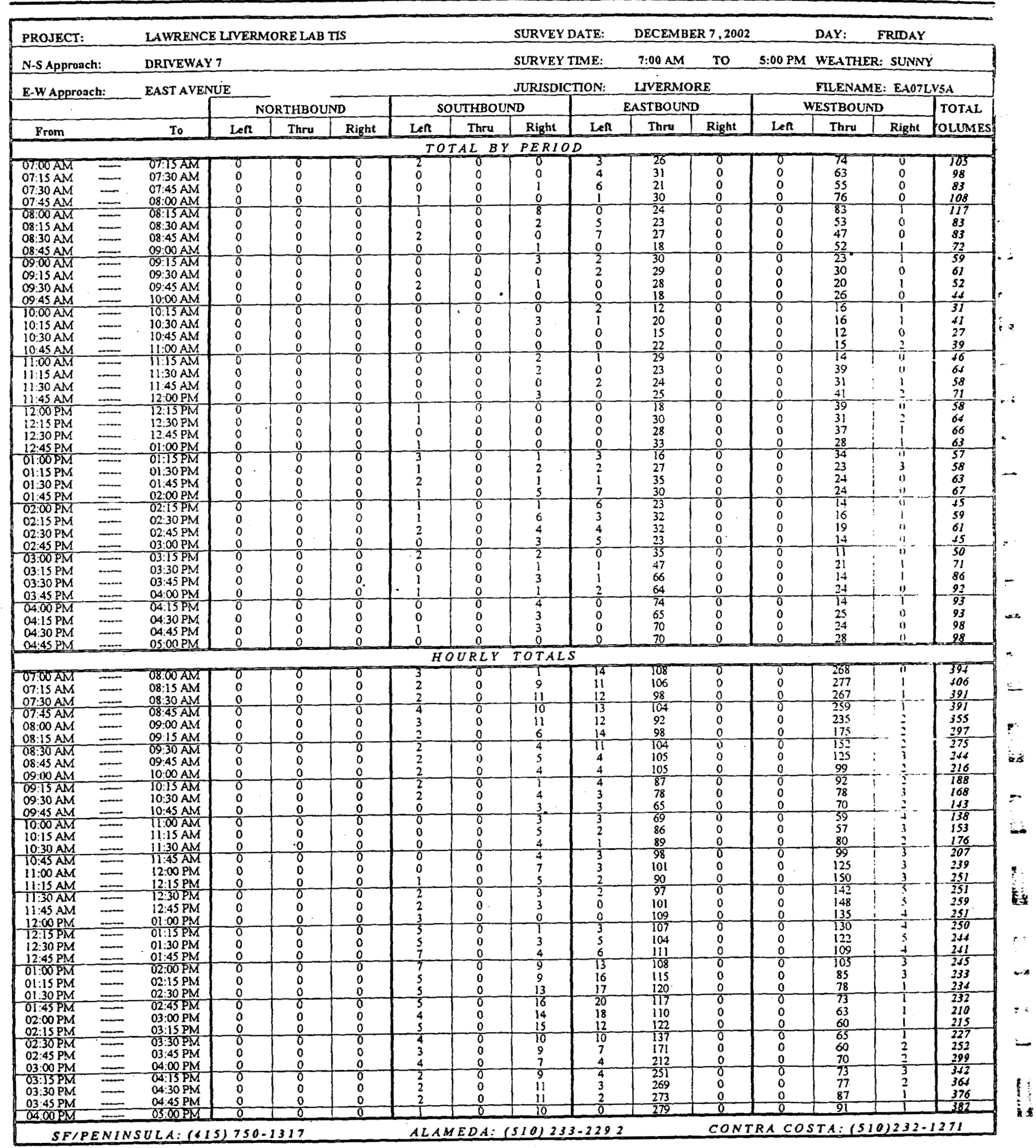




\section{Baymetrics Traffic Resources INTERSECTION TURNING MOVEMENT SUMMARY}

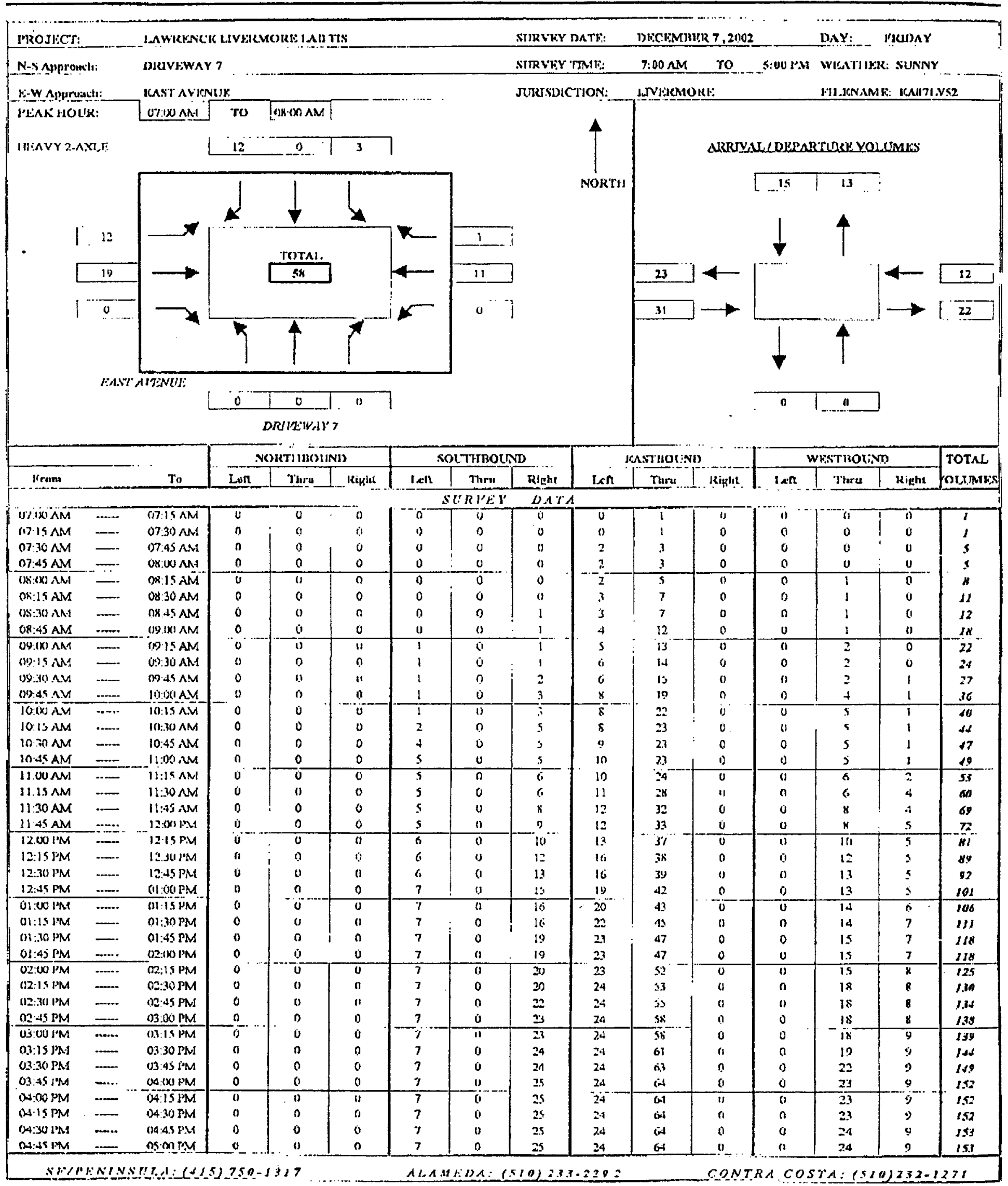




\section{Baymetrics Traffic Resources INTERSECTION TURNING MOVEMENT SUMMARY}

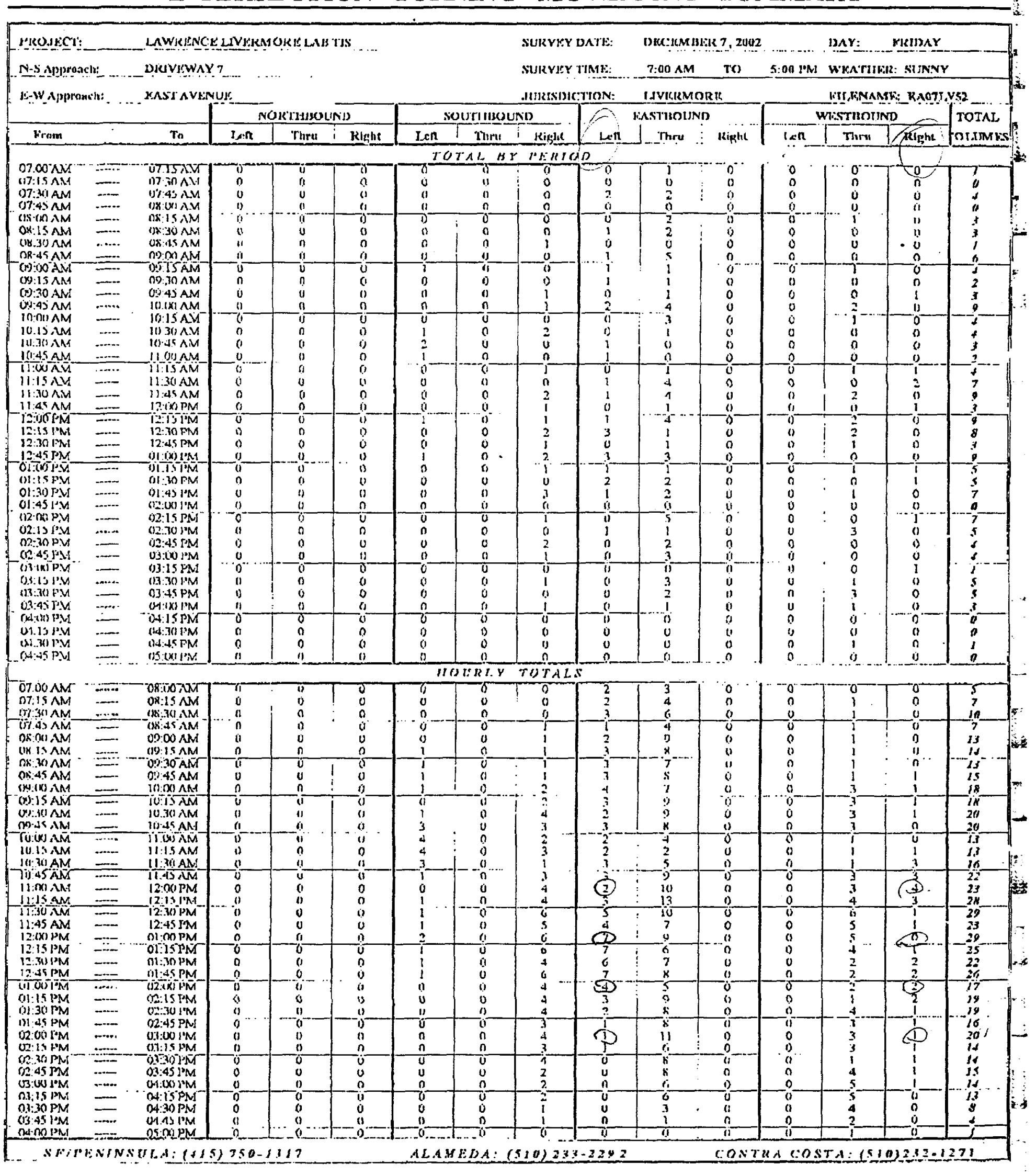


Baymetrics Traffic Resources INTERSECTION TURNING MOVEMENT SUMMARY

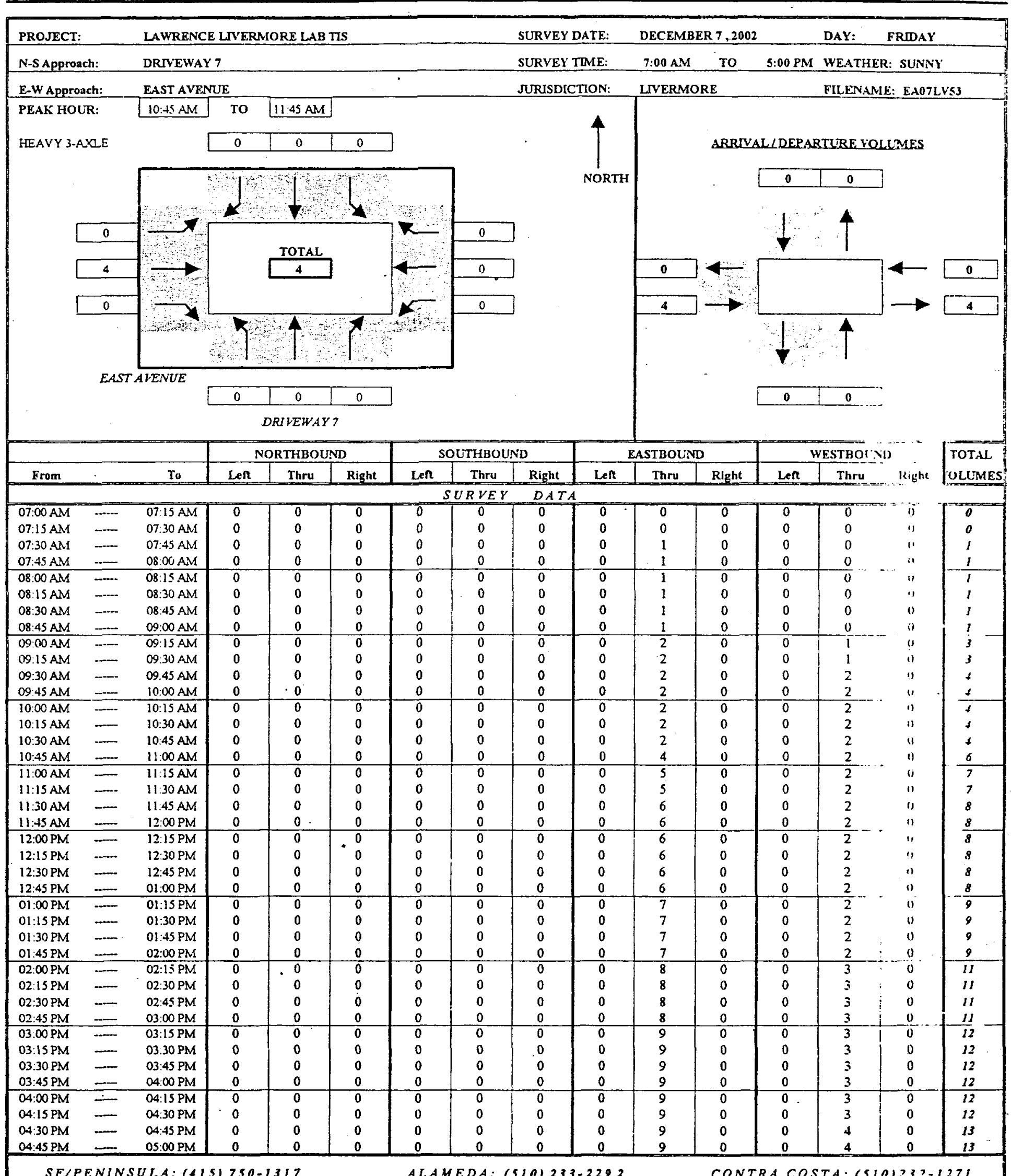




\section{Baymetrics Traffic Resources INTERSECTION TURNING MOVEMENT SUMMARY}

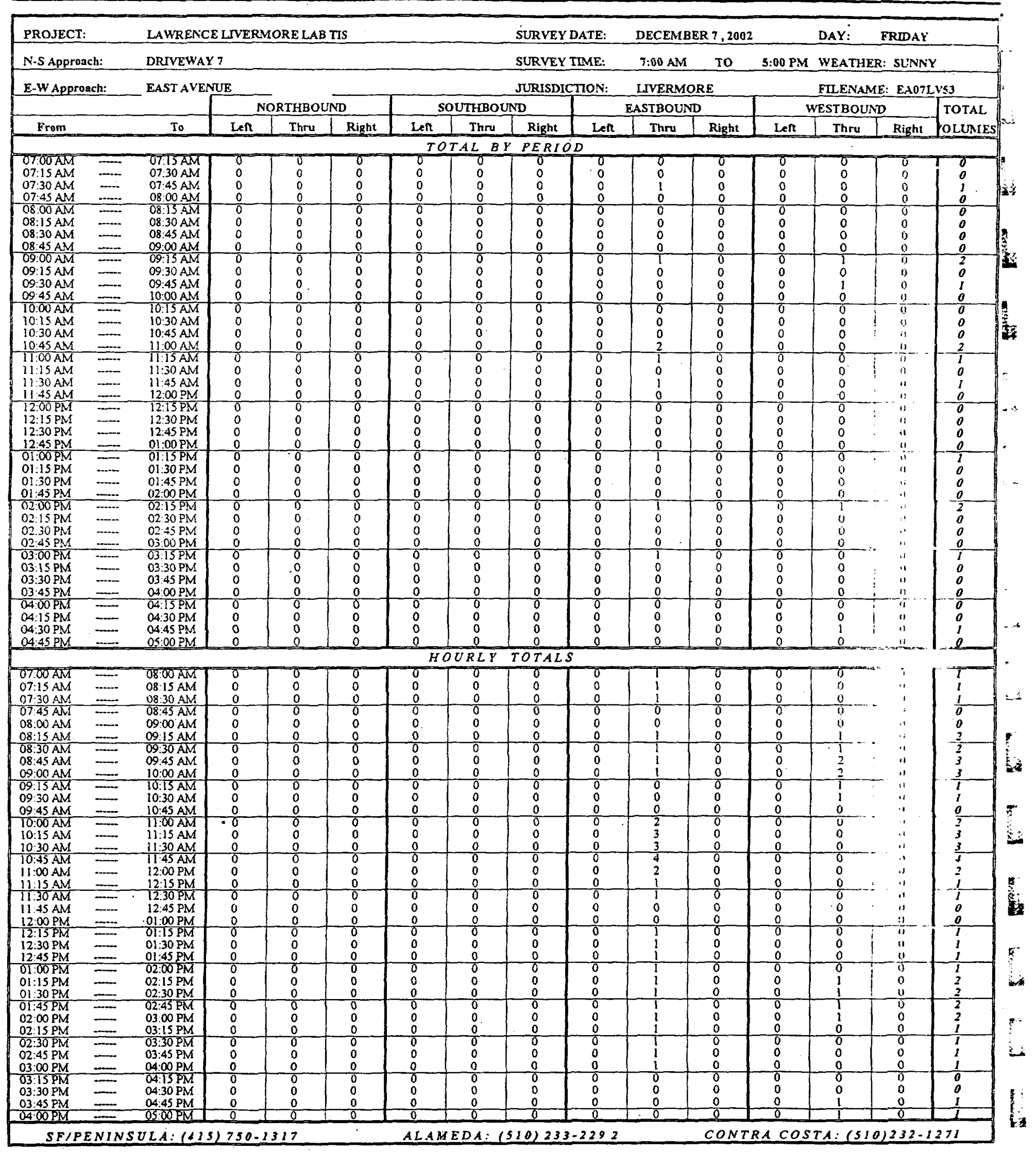




\section{Baymetrics Traffic Resources INTERSECTION TURNING MOVEMENT SUMMARY}

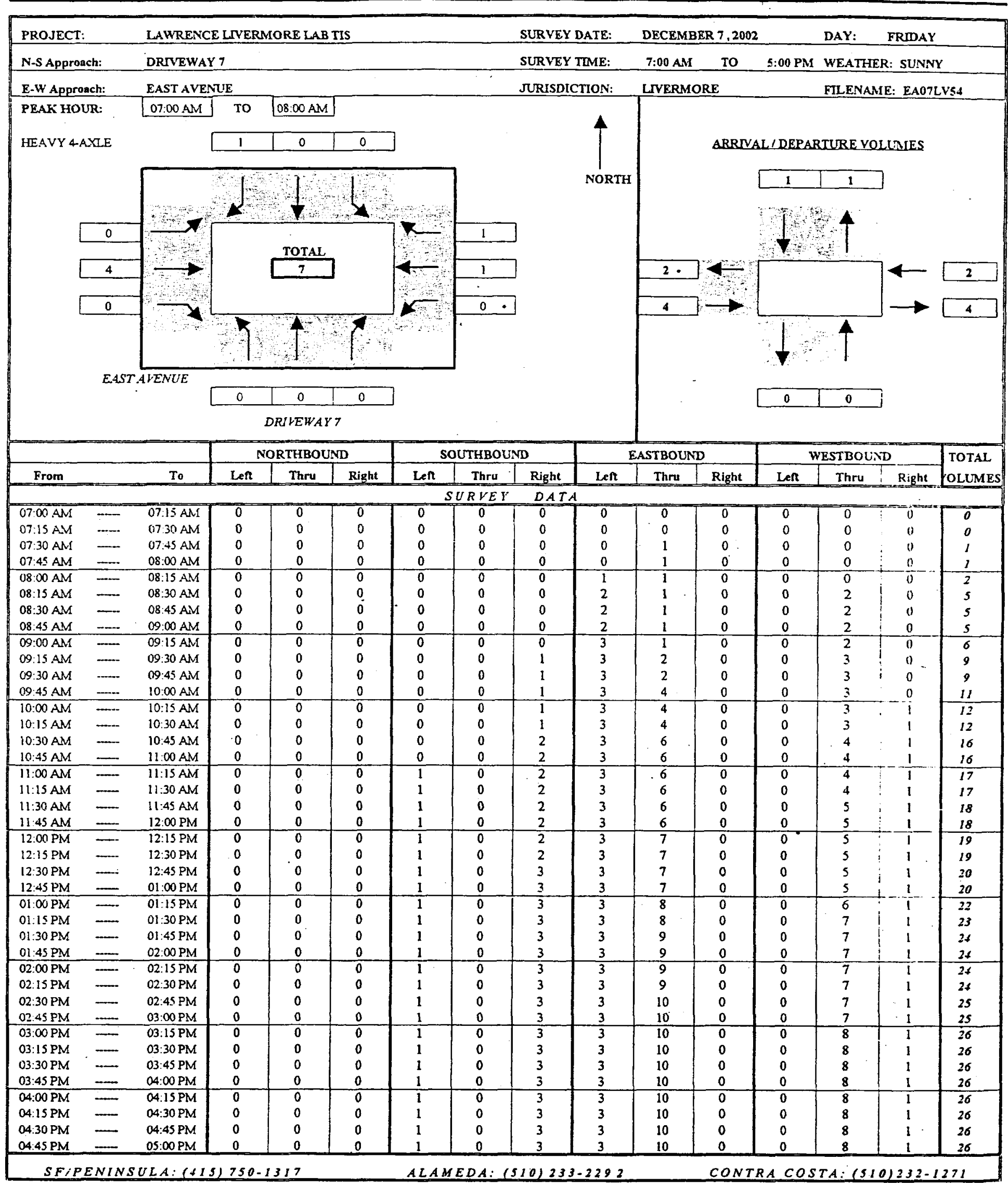




\section{Baymetrics Traffic Resources INTERSECTION TURNING MOVEMENT SUMMARY}

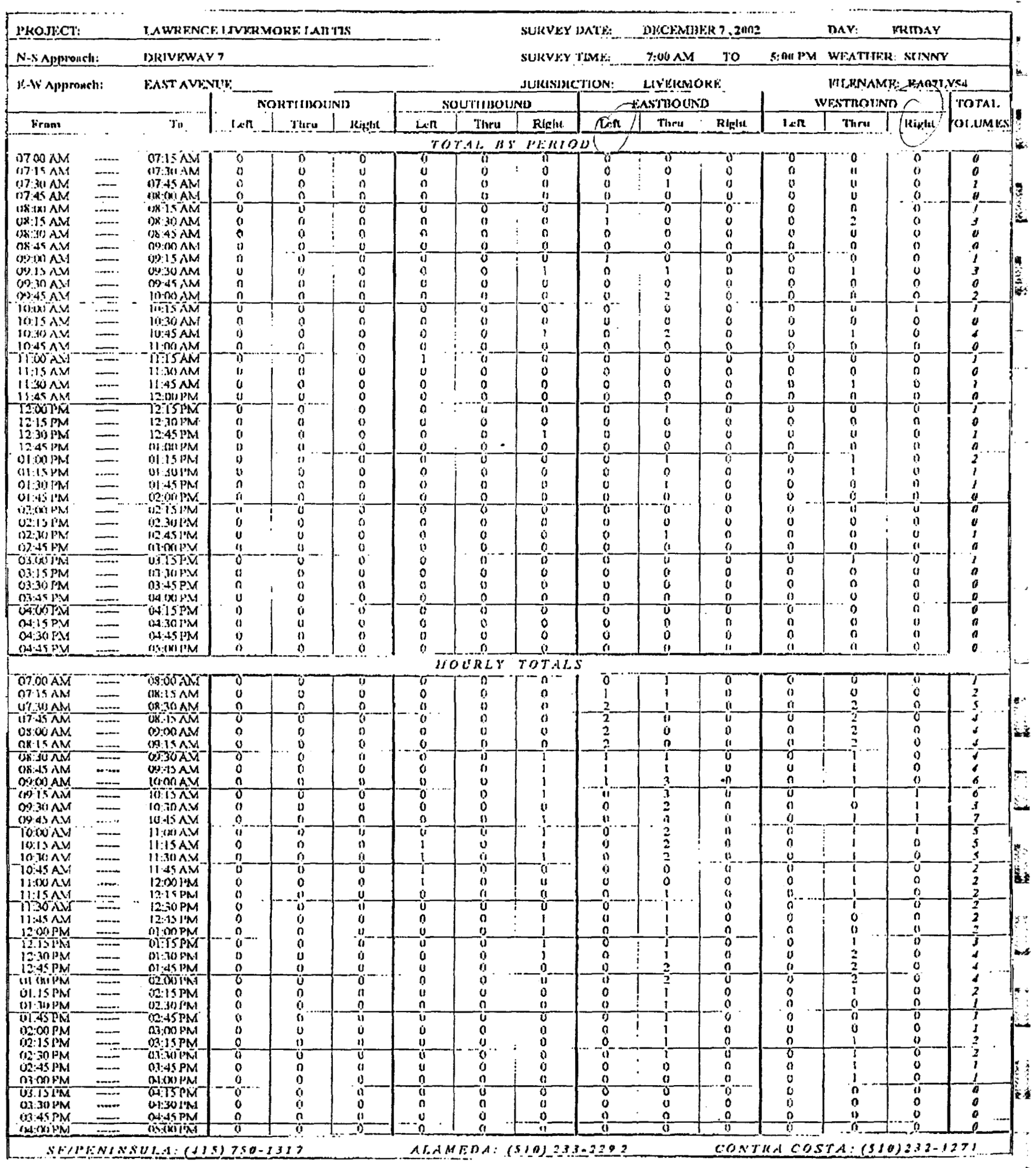




\section{Baymetrics Traffic Resources INTERSECTION TURNING MOVEMENT SUMMARY}

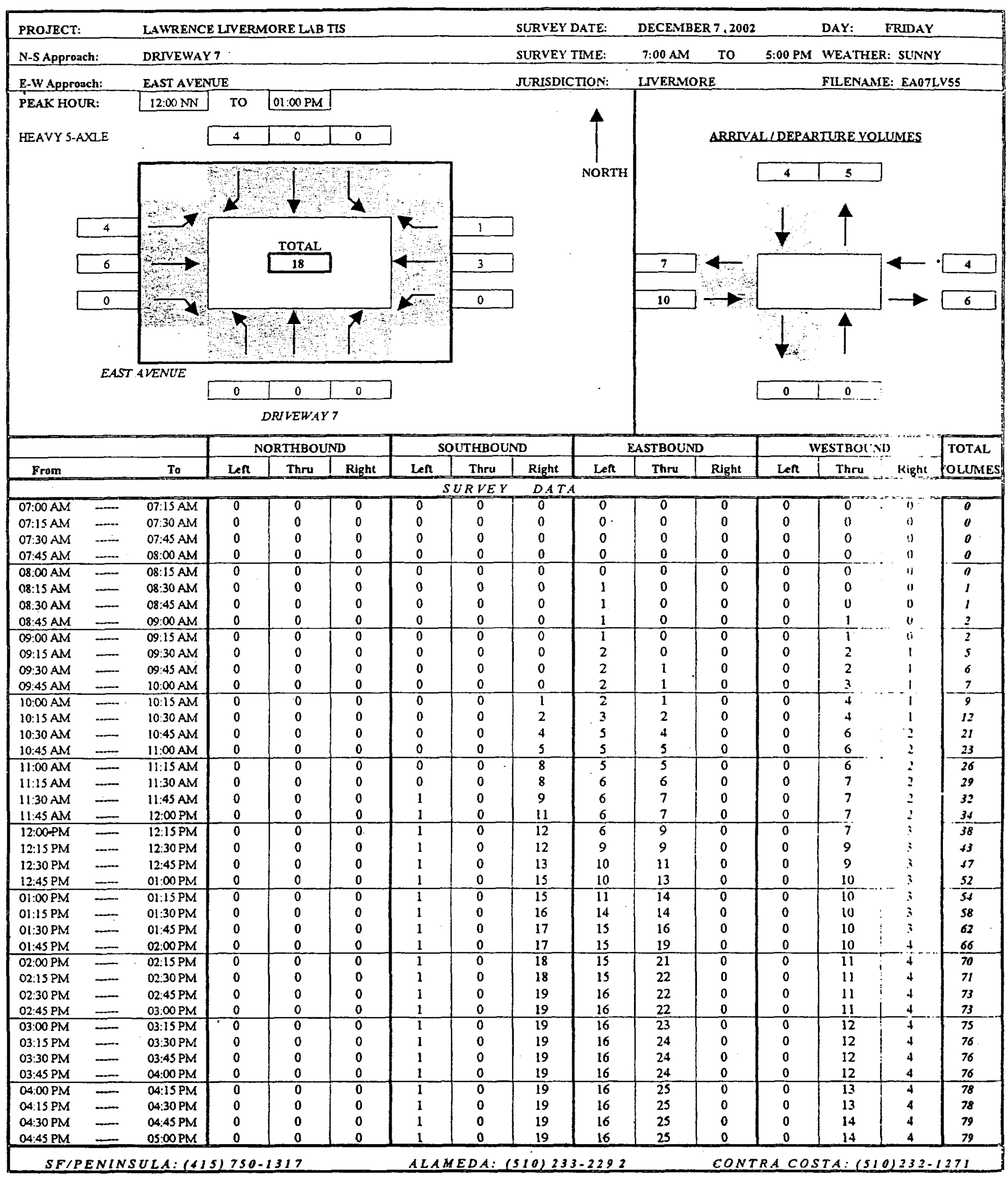




\section{Baymetrics Traffic Resources INTERSECTION TURNING MOVEMENT SUMMARY}

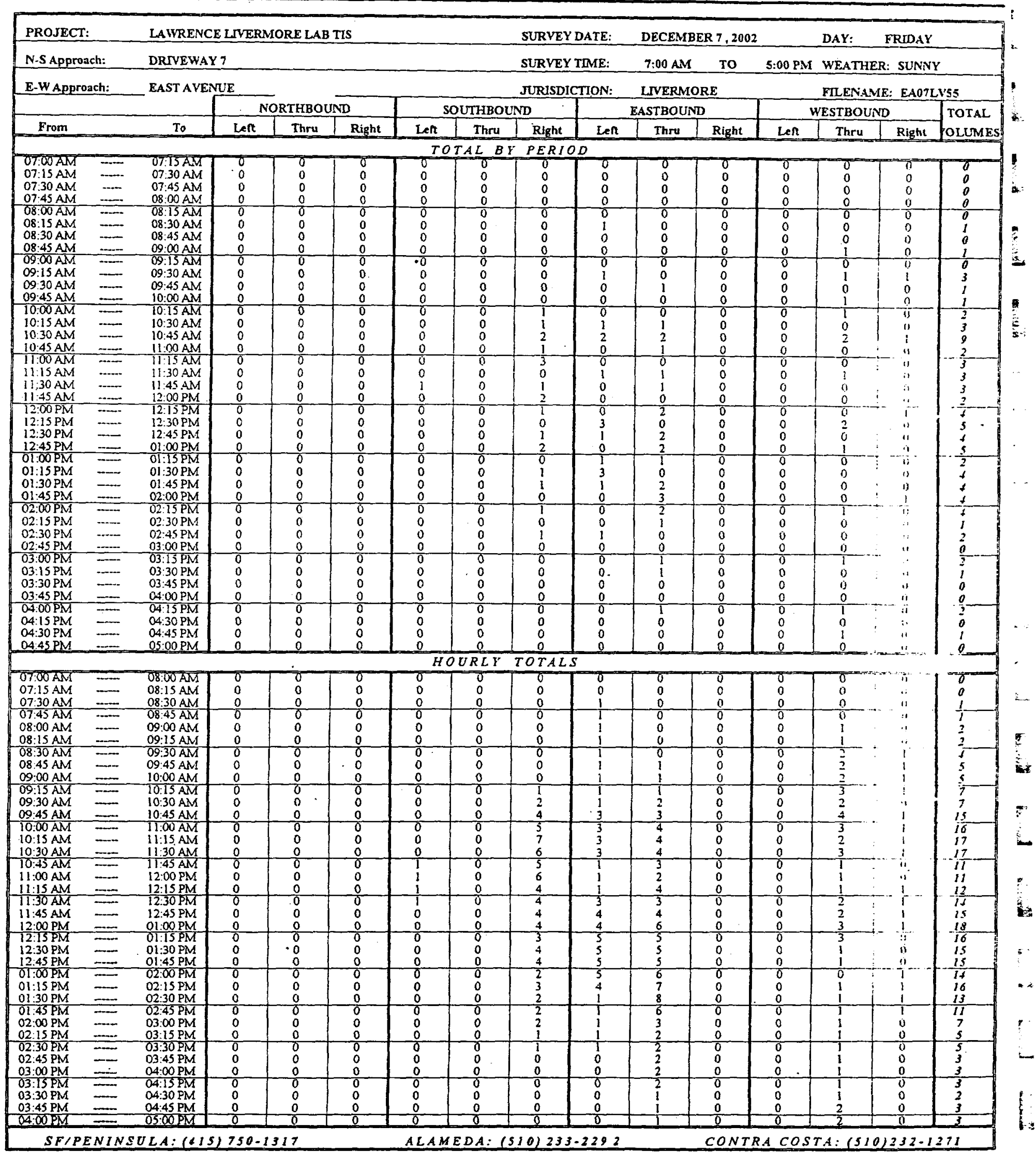




\section{Baymetrics Traffic Resources INTERSECTION TURNING MOVEMENT SUMMARY}

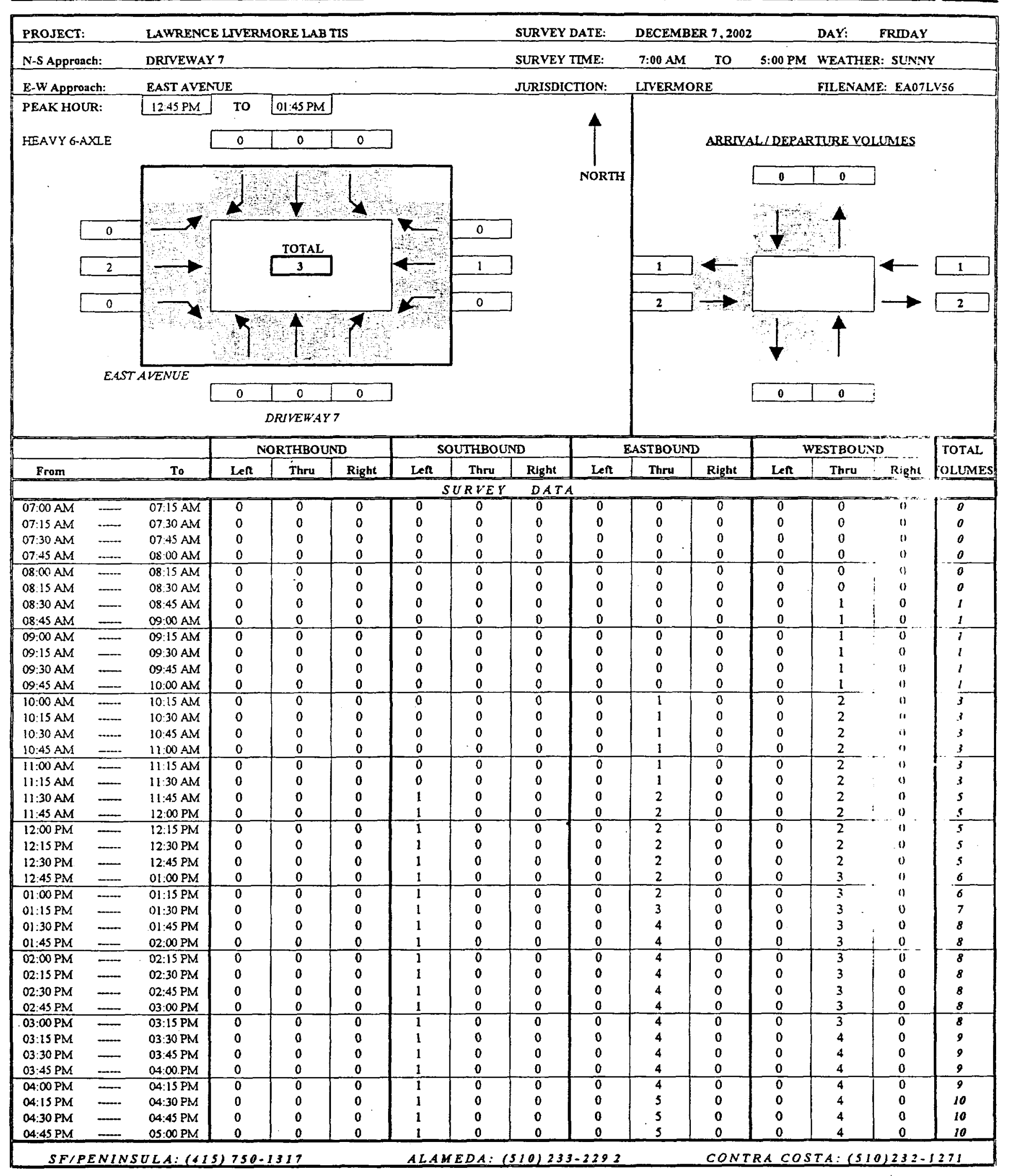




\section{Baymetrics Traffic Resources INTERSECTION TURNING MOVEMENT SUMMARY}

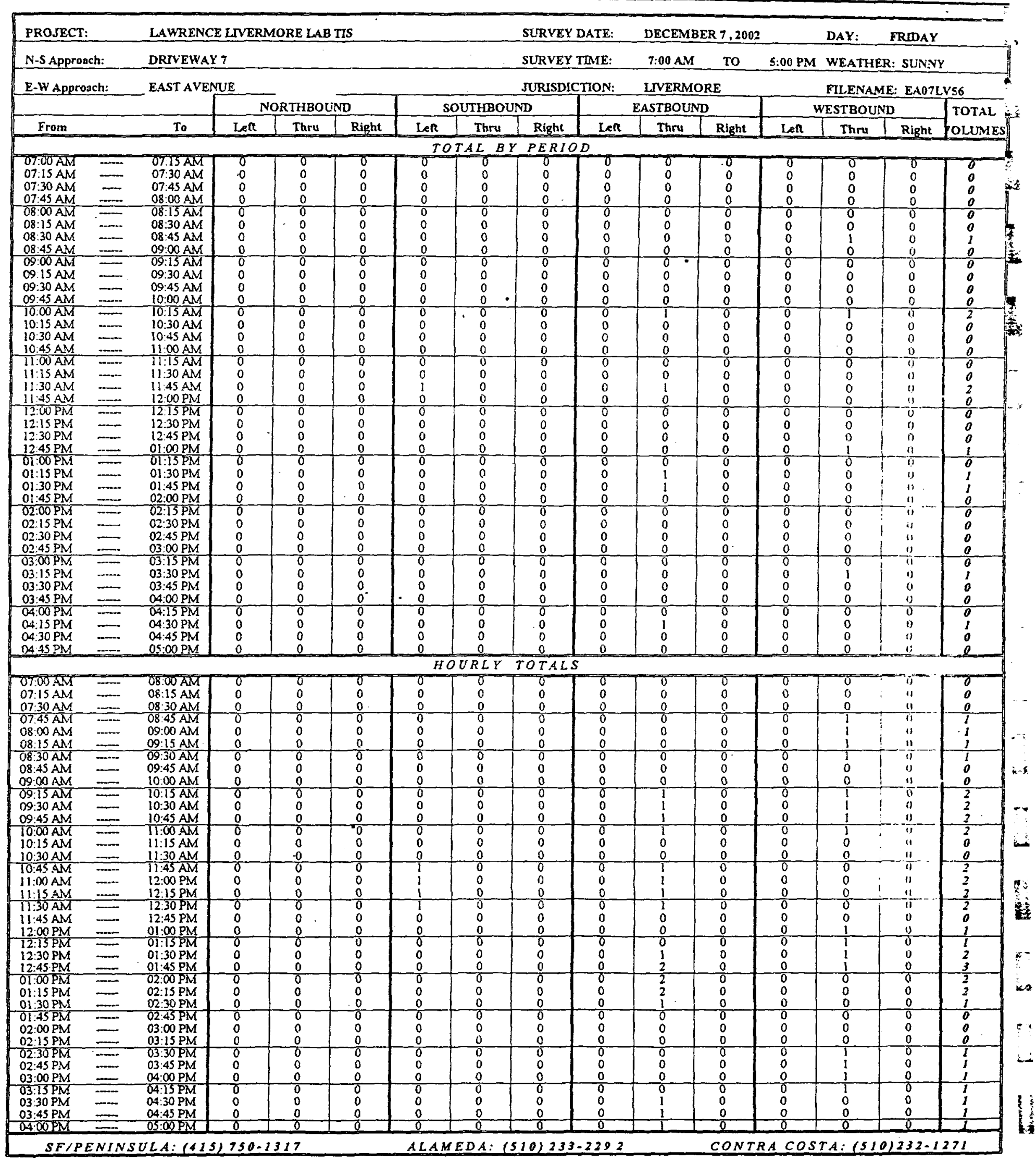




\section{Baymetrics Traffic Resources INTERSECTION TURNING MOVEMENT SUMMARY}

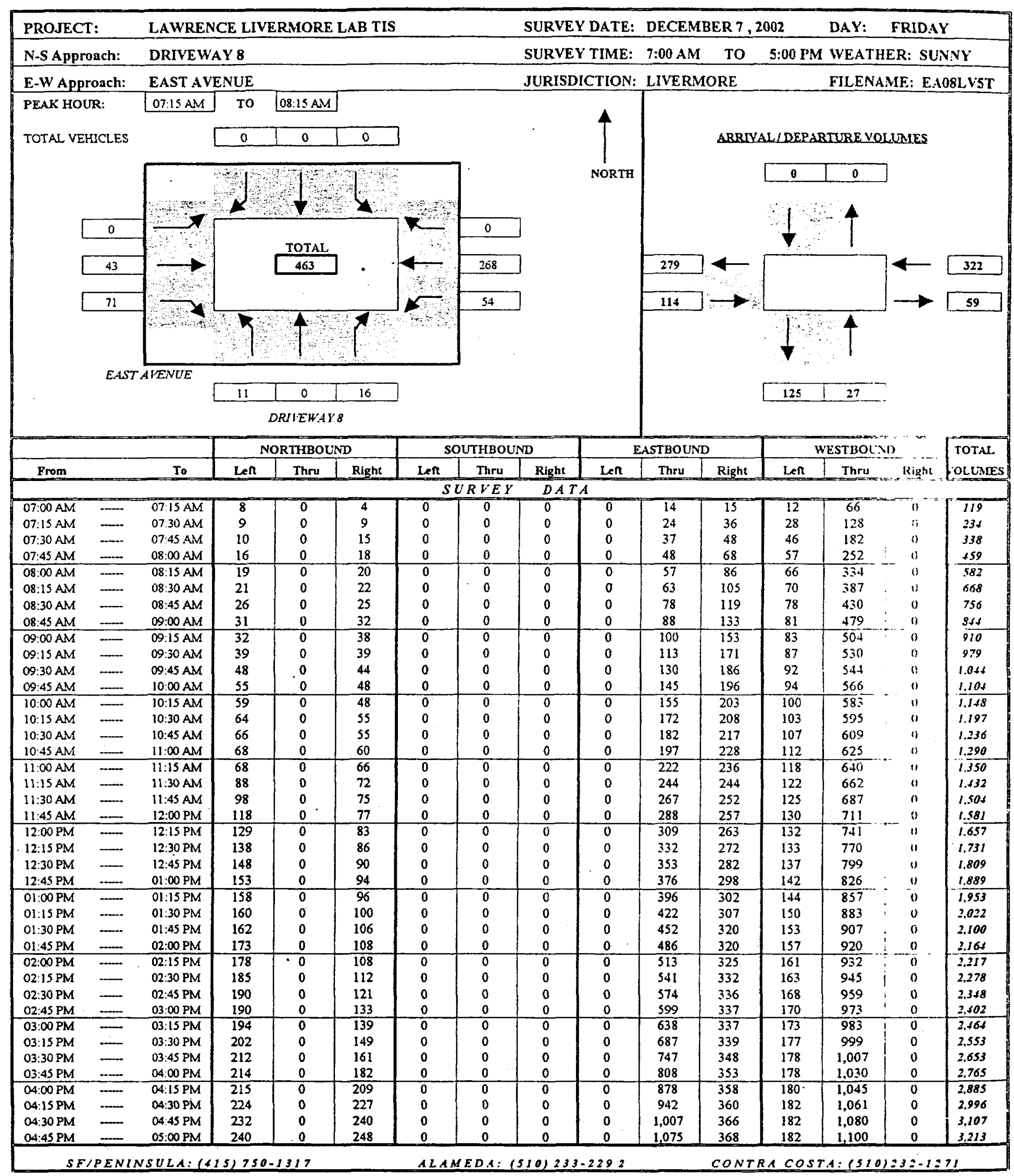




\section{Baymetrics Traffic Resources INTERSECTION TURNING MOVEMENT SUMMARY}

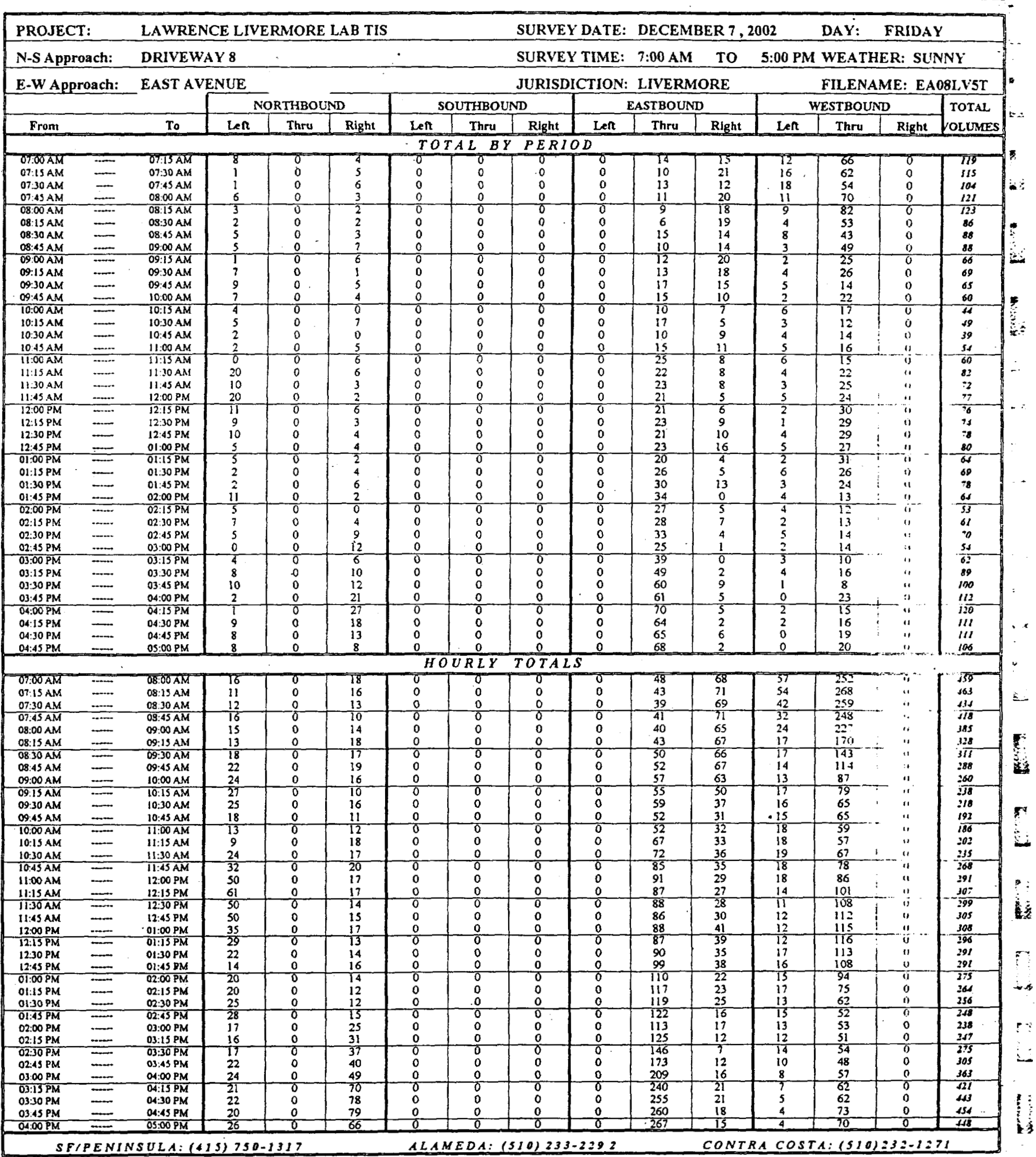




\section{Baymetrics Traffic Resources INTERSECTION TURNING MOVEMENT SUMMARY}

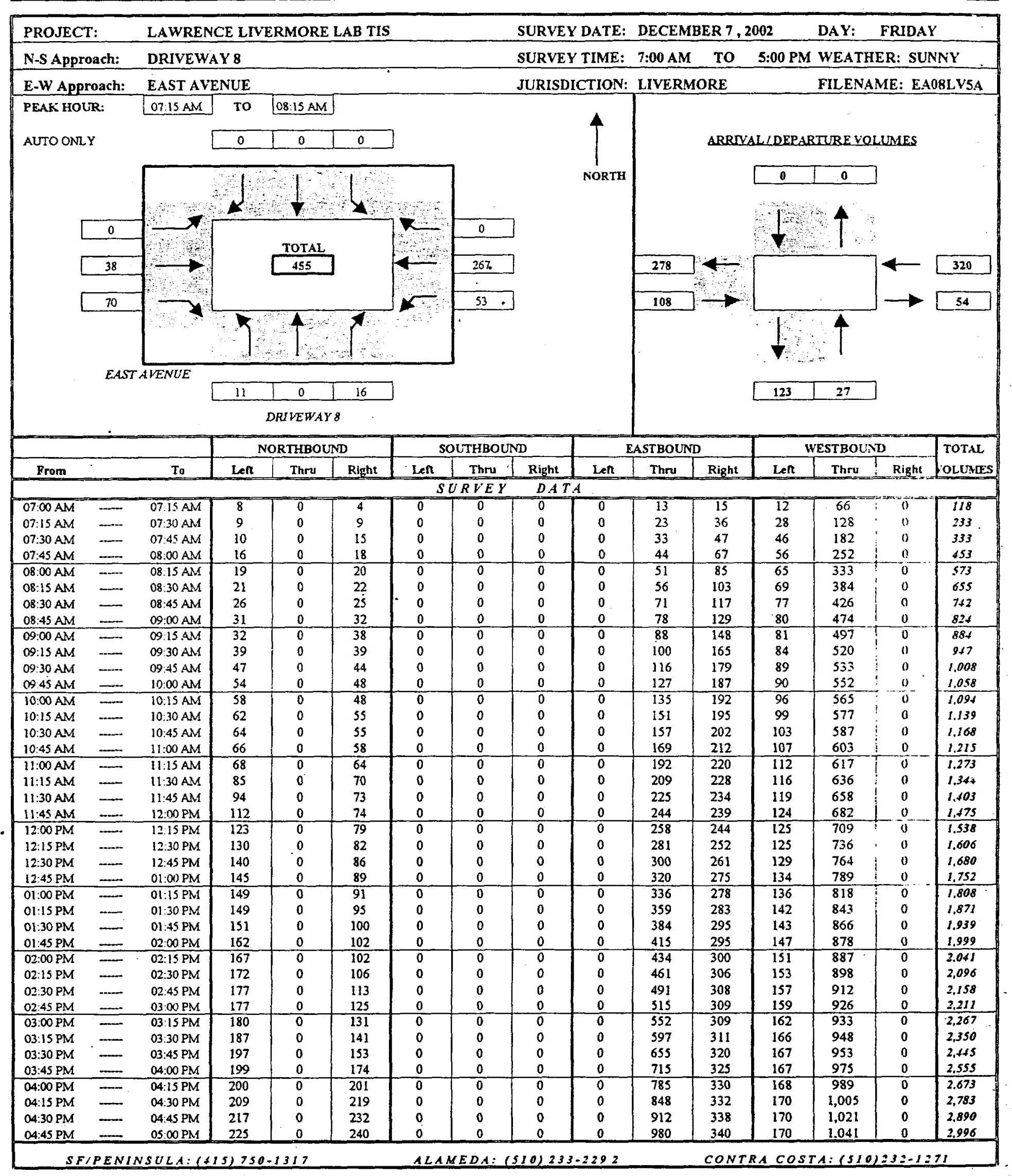




\section{Baymetrics Traffic Resources INTERSECTION TURNING MOVEMENT SUMMARY}

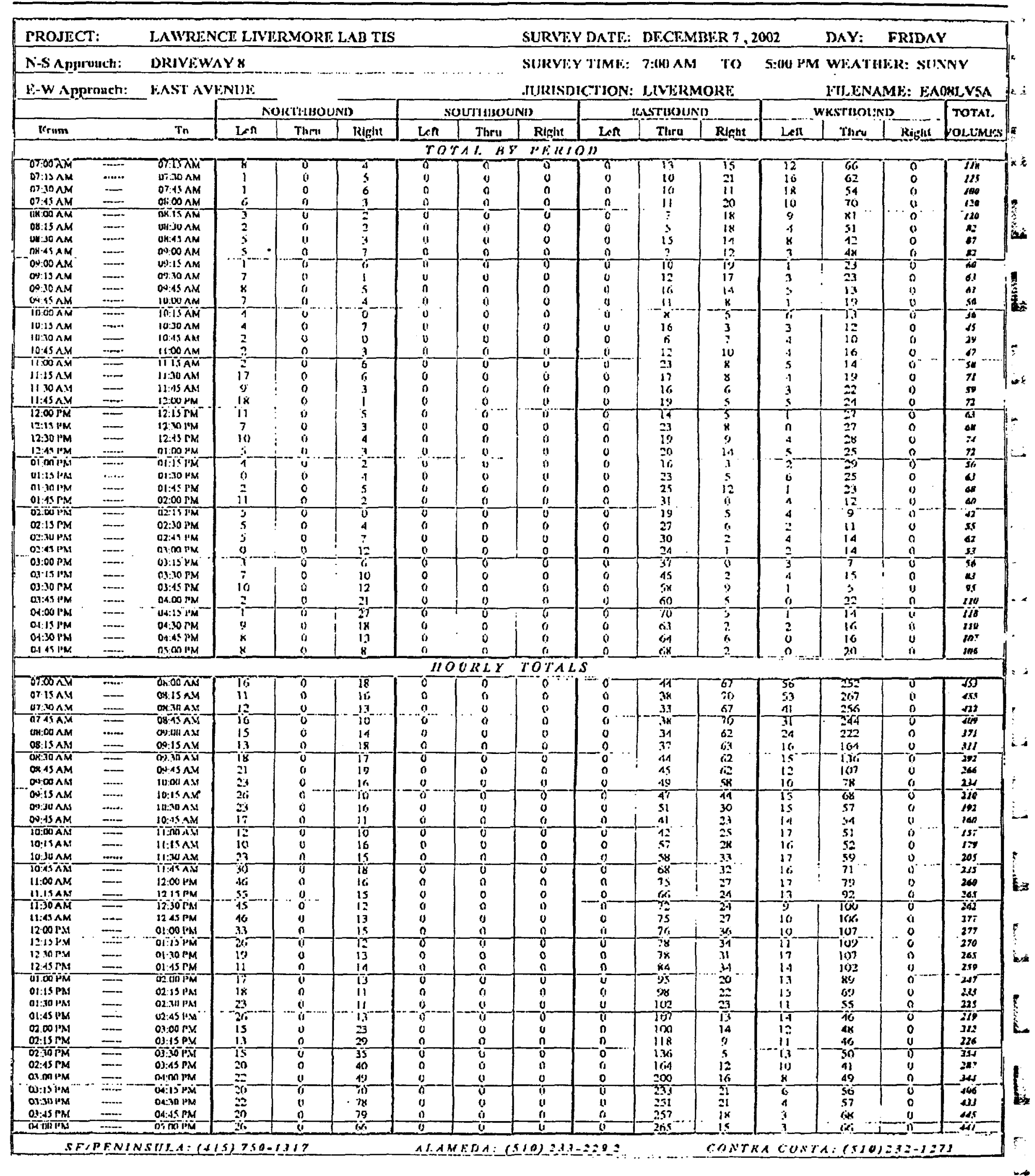




\section{Baymetrics Traffic Resources INTERSECTION TURNING MOVEMENT SUMMARY}

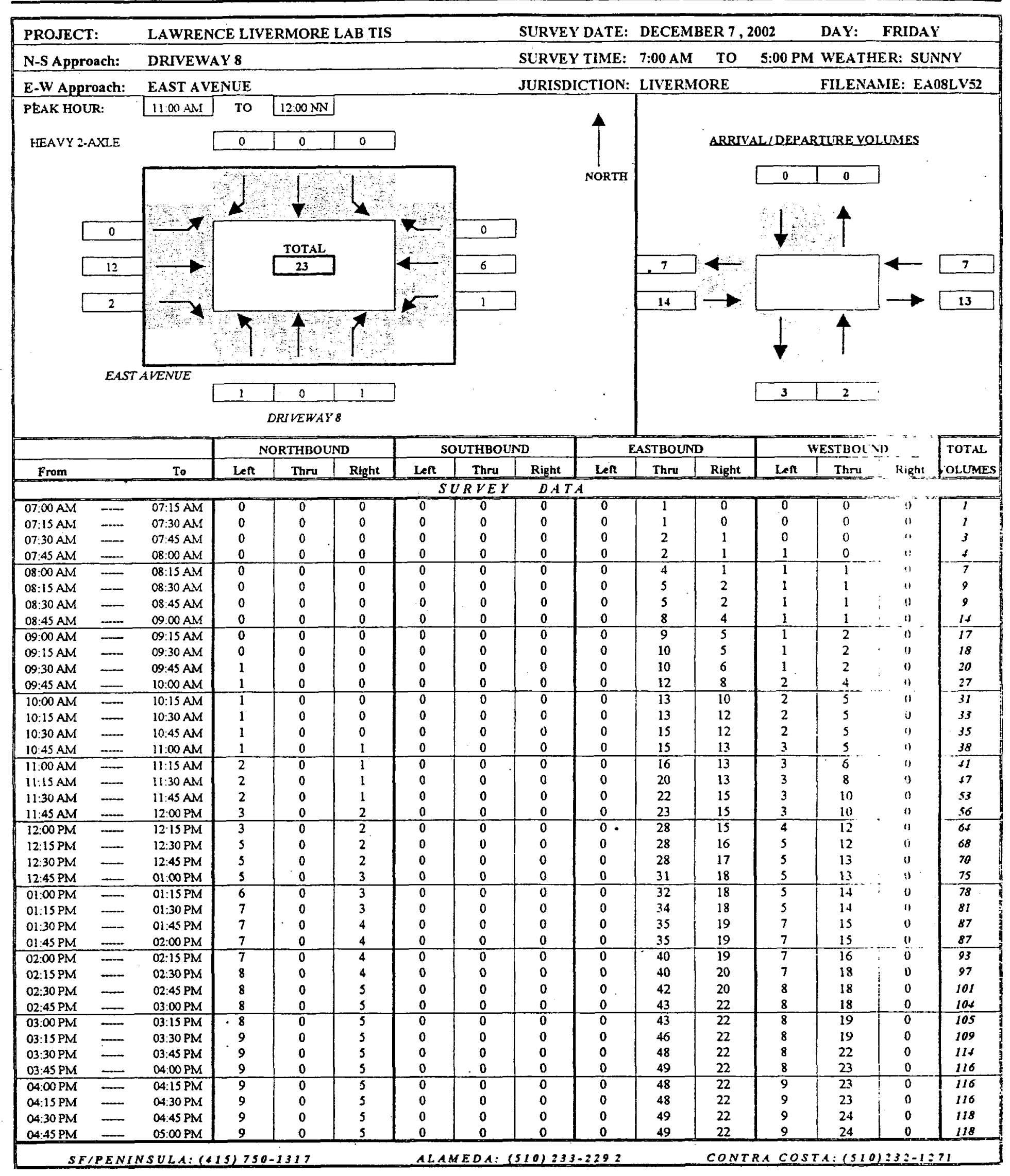




\section{$\frac{\text { Baymetrics Traffic Resources }}{\text { INTERSCTION TURNING MOVEMENT SUMMARY }}$}

\begin{tabular}{|c|c|c|c|c|c|c|c|c|c|c|c|c|c|c|c|c|}
\hline \multirow{2}{*}{\multicolumn{2}{|c|}{$\begin{array}{l}\text { PROJECT: } \\
\text { N-S A pproach: }\end{array}$}} & \multicolumn{6}{|c|}{ LAWRENCE LIVERMORE LAB TIS } & \multicolumn{2}{|c|}{ SURVEY DATE: } & DECEN & ER 7,2 & 002 & DAY: & FRIDA & & \\
\hline & & DRIVEY & & & & & & SURVE & IIME: & 7:00 AM & To & 5:00 Pi & WEAT & ER: SU & NYY & \\
\hline E-W Ap & oach: & EAST A & UE & & & & & JURISD & TION: & LIVER & ORE & & FILEN & ME: EA & $08 \mathrm{LV} 52$ & Fis \\
\hline & & & & THBO & & & THBO & & & ASTBOUI & & & ESTBOL & & TOTAL & \\
\hline From & & To & Len & Thru & Right & Len & Thru & Right & Len & Thru & Right & Len & Thru & Right & KOLUMES & \\
\hline & & & & & & & $L B$ & $P E R I$ & & & & & & & & \\
\hline ग7:00AM & $=$ & OIISAM & 0 & 0 & o & $\sigma$ & 6 & 8 & 0 & $T$ & 0 & 0 & 0 & $\pi$ & 7 & 5 \\
\hline $07.15 \mathrm{AM}$ & - & 07:30 AM & 0 & $\therefore$ & $a$ & 0 & 0 & 0 & 0 & 0 & 0 & 0 & : & 0 & 0 & \\
\hline $\begin{array}{l}07: 30 \mathrm{AM} \\
07: 45 \mathrm{AM}\end{array}$ & $\ldots$ & $\begin{array}{l}\text { 07:45 AM } \\
08: 00 \text { AM }\end{array}$ & $\begin{array}{l}0 \\
0\end{array}$ & $\begin{array}{l}0 \\
0\end{array}$ & $\begin{array}{l}0 \\
0 \\
\end{array}$ & $\begin{array}{l}0 \\
0\end{array}$ & $\begin{array}{l}0 \\
0 \\
\end{array}$ & $\begin{array}{l}0 \\
0\end{array}$ & $\begin{array}{l}0 \\
0 \\
\end{array}$ & $\begin{array}{l}1 \\
0\end{array}$ & 0 & $\begin{array}{l}0 \\
1\end{array}$ & $\begin{array}{l}0 \\
0 \\
\end{array}$ & $\begin{array}{l}0 \\
0\end{array}$ & 2 & in \\
\hline $08: 00 \mathrm{AM}$ & $\square$ & $08: 15$ AM & 0 & 0 & 0 & 0 & 0 & 0 & 0 & $\frac{\pi}{2}$ & 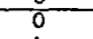 & $\frac{1}{0}$ & 1 & 0 & 3 & \\
\hline 08:15 AM & $\cdots$ & 08:30 AM & 0 & 0 & 0 & 0 & 0 & 0 & 0 & 1 & 2 & 0 & 0 & 0 & 2 & Ha \\
\hline $\begin{array}{l}\text { 08:30 AM } \\
08: 45 \text { AM }\end{array}$ & $=$ & $\begin{array}{l}\text { 08:45 AM } \\
09: 00 \mathrm{AM}\end{array}$ & $\begin{array}{l}0 \\
0\end{array}$ & $\begin{array}{l}0 \\
0\end{array}$ & $\begin{array}{l}0 \\
0\end{array}$ & $\begin{array}{l}0 \\
0\end{array}$ & $\begin{array}{l}0 \\
0\end{array}$ & $\begin{array}{l}0 \\
0\end{array}$ & $\begin{array}{l}0 \\
0\end{array}$ & ${ }_{3}^{0}$ & $\begin{array}{l}0 \\
2\end{array}$ & $\begin{array}{l}0 \\
0\end{array}$ & $\begin{array}{l}0 \\
0\end{array}$ & $\begin{array}{l}0 \\
0\end{array}$ & 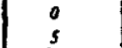 & II \\
\hline $09: 00 \mathrm{AM}$ & $\ldots$ & $09: 15 \mathrm{AM}$ & 0 & 0 & 0 & 0 & 0 & 8 & 0 & 1 & $\frac{1}{1}$ & 0 & $T$ & $i$ & 3 & : \\
\hline 09:15 AM & $\ldots$ & $09: 30 \mathrm{AM}^{\circ}$ & 0 & 0 & 0 & 0 & 0 & 0 & 0 & 1 & 0 & 0 & 0 & 0 & 1 & 12 \\
\hline $\begin{array}{l}09: 30 \mathrm{AM} \\
09: 45 \mathrm{AM}\end{array}$ & $\ldots$ & $\begin{array}{l}09: 45 \mathrm{AM} \\
10: 00 \mathrm{AM}\end{array}$ & $!$ & $\begin{array}{l}0 \\
0\end{array}$ & 0 & 0 & 0 & $\begin{array}{l}0 \\
0\end{array}$ & $\begin{array}{l}0 \\
0\end{array}$ & 2 & $\frac{1}{2}$ & 0 & $\begin{array}{l}0 \\
2\end{array}$ & 0 & 2 & \\
\hline $10: 00 \mathrm{AM}$ & $\ldots$ & $10: 15 \mathrm{AM}$ & -0 & 0 & 0 & 0 & 0 & 0 & 0 & $i$ & $\frac{7}{2}$ & 0 & 1 & $\%$ & 7 & 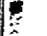 \\
\hline 10:15 AM & $\ldots$ & $10: 30 \mathrm{AM}$ & 0 & 0 & 0 & 0 & 0 & 0 & 0 & 0 & 2 & 0 & 0 & ci & 2 & \\
\hline 10:30 AM & $\cdots$ & $10: 45 \mathrm{AM}$ & 0 & 0 & 0 & 0 & D & 0 & 0 & 2 & 0 & 0 & 0 & 0 & 2 & 4 \\
\hline $10: 45 \mathrm{AM}$ & $\cdots$ & $11: 00 \mathrm{AM}$ & 0 & 0 & 1 & 0 & 0 & 0 & 0 & 0 & 1 & 1 & 0 & u & 3 & \\
\hline $11: 00 \mathrm{AM}$ & $\cdots$ & $11: 15 \mathrm{AM}$ & 1 & 0 & 0 & 0 & 0 & 0 & 0 & 1 & 0 & o & 1 & ii & 3 & \\
\hline $11: 15 \mathrm{AM}$ & $\cdots$ & $11: 30 \mathrm{AM}$ & 0 & 0 & 0 & 0 & 0 & 0 & 0 & 4 & 0 & 0 & 2 & ": & 6 & $\cdots$ \\
\hline $11: 30 \mathrm{AM}$ & - & II: $: 45$ AM & 0 & 0 & 0 & 0 & 0 & 0 & 0 & 2 & 2 & 0 & 2 & $\therefore$ & 6 & \\
\hline 11: $49 \mathrm{AM}$ & $\ldots$ & 12:00 PM & 1 & 0 & 1 & 0 & 0 & 0 & 0 & $i$ & 0 & 0 & 0 & $\because ;$ & 3 & \\
\hline $12: 00 \mathrm{PM}$ & 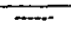 & $12: 15 \mathrm{PM}$ & 0 & $\sigma$ & 0 & 0 & 0 & 0 & 0 & 5 & 0 & $T$ & 2 & $\therefore$ & 8 & $\therefore$ \\
\hline 12.15 PM & $\cdots$ & $12.30 \mathrm{PM}$ & 2 & 0 & 0 & 0 & 0 & 0 & 0 & 0 & 1 & 1 & 0 & !! & s & \\
\hline $12: 30 \mathrm{PM}$ & $\cdots$ & $12: 45 \mathrm{PM}$ & 0 & 0 & 0 & 0 & 0 & 0 & 0 & 0 & $i$ & 0 & i & i1 & 2 & \\
\hline $12: 45 \mathrm{PM}$ & $\ldots$ & $0100 \mathrm{PM}$ & 0 & 0 & 1 & 0 & 0 & 0 & 0 & 3 & $i$ & 0 & () & $\because$ & 5 & \\
\hline $01: 00 \mathrm{PM}$ & $=$ & $01: 15 \mathrm{PM}$ & 1 & 0 & 0 & \% & 0 & 0 & 0 & 1 & $\bar{D}$ & 0 & 1 & $\therefore$ & 3 & \\
\hline 01:15 PM & $\cdots$ & $01: 30 \mathrm{PM}$ & 1 & 0 & 0 & 0 & 0 & 0 & 0 & 2 & 0 & 0 & 0 & ". & 3 & \\
\hline $\begin{array}{l}\text { 01:30 PM } \\
\text { 01:45 PM }\end{array}$ & $\ldots$ & $\begin{array}{l}01: 45 \mathrm{PM} \\
02: 00 \mathrm{PM}\end{array}$ & $\begin{array}{l}0 \\
0\end{array}$ & $\begin{array}{l}0 \\
0\end{array}$ & $\begin{array}{l}1 \\
0\end{array}$ & $\begin{array}{l}0 \\
0\end{array}$ & $\begin{array}{l}0 \\
0\end{array}$ & $\begin{array}{l}0 \\
0\end{array}$ & $\begin{array}{l}0 \\
0\end{array}$ & $\begin{array}{l}1 \\
0\end{array}$ & $\begin{array}{l}1 \\
0\end{array}$ & $\begin{array}{l}2 \\
0\end{array}$ & $\begin{array}{l}1 \\
0\end{array}$ & $\because 1$ & 6 & \\
\hline $02: 00 \mathrm{PM}$ & $=$ & 02:15 PM & 0 & 0 & 0 & 0 & $\frac{\pi}{0}$ & $\frac{0}{0}$ & $\frac{0}{0}$ & $\frac{2}{5}$ & $\frac{\pi}{a}$ & 0 & $\frac{\pi}{1}$ & $\because$ & 6 & \\
\hline $02: 15 \mathrm{PM}$ & $\ldots$ & 02:30 PM & 1 & 0 & 0 & 0 & 0 & 0 & 0 & 0 & 1 & 0 & $=$ & ". & j & \\
\hline $02: 30 \mathrm{PM}$ & $\ldots$ & $02: 45$ PM & 0 & 0 & 1 & 0 & 0 & 0 & 0 & 2 & 0 & 1 & 0 & . & 1 & \\
\hline $02: 45 \mathrm{PM}$ & $\ldots$ & 03:00 PM & 0 & 0 & 0 & 0 & 0 & 0 & 0 & 1 & 2 & 0 & 0 & $\therefore$ & 3 & \\
\hline $03: 00 \mathrm{PM}$ & $\ldots$ & $03: 15 \mathrm{PM}$ & 0 & 0 & 0 & 0 & 0 & 0 & 0 & 0 & 0 & 0 & $T$ & $\therefore$ & $T$ & \\
\hline $03: 15$ PM & $\ldots$ & 03:30 PM & 1 & 0 & 0 & 0 & 0 & 0 & 0 & 3 & 0 & 0. & 0 & .. & $s$ & \\
\hline $01: 30 \mathrm{PM}$ & $\ldots$ & $03: 45 \mathrm{PM}$ & 0 & 0 & 0 & 0 & 0 & o & 0 & 2 & 0 & 0 & 3 & ". & 3 & \\
\hline $03: 45 \mathrm{PM}$ & - & $04: 00 \mathrm{PM}$ & 0 & 0 & 0 & 0 & 0 & 0 & 0 & $i$ & 0 & 0 & $i$ & $\therefore$ & $i$ & \\
\hline $0400 \mathrm{PM}$ & $\cdots$ & Qd.15 PM & 0 & 0 & 0 & 0 & 0 & 0 & 0 & (I) & 0 & 1 & 0 & $\because !$ & 0 & \\
\hline OA:15 PM & 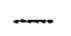 & $04: 30 \mathrm{PM}$ & 0 & 0 & 0 & 0 & 0 & 0 & 0 & 0 & 0 & 0 & 0 & $\because$ & 0 & \\
\hline 04:30 PM & $\cdots$ & 04:45 PM & 0 & 0 & 0 & 0 & 0 & 0 & 0 & 1 & 0 & 0 & $i$ & " & 3 & $\cdots$ \\
\hline 04:45 PM & $=$ & 05:00 PM & $\underline{0}$ & 0 & 0 & $\frac{0}{H}$ & $\frac{0}{R L Y}$ & $\frac{0}{T O T A 1}$ & 0 & 0 & 0 & 0 & 0 & $=\because x$ & +2 & \\
\hline mon & $\ldots$ & $08.00 A M$ & $\pi$ & & & $\frac{H}{O}$ & $\frac{K L 1}{0}$ & 0 & & & & & & $\therefore$ & & * \\
\hline 07:15 AM & $\ldots$ & $08: 15 \mathrm{AM}$ & 0 & 0 & 0 & 0 & 0 & 0 & 0 & 3 & 1 & 1 & $i$ & $\therefore$ & 8 & \\
\hline $07: 30 \mathrm{AM}$ & $\ldots$ & $08: 30 \mathrm{AM}$ & 0 & 0 & 0 & 0 & 0 & 0 & 0 & 4 & 2 & $i$ & $i$ & .. & 8 & $=:$ \\
\hline $07: 45 \mathrm{AM}$ & $\cdots$ & $08: 45 \mathrm{AM}$ & 0 & $\overline{0}$ & 0 & 0 & 0 & 0 & 0 & 3 & 1 & 1 & 1 & ." & 6 & \\
\hline 08:00 AM & $\ldots$ & $09.00 \mathrm{AM}$ & 0 & 0 & 0 & 0 & 0 & 0 & 0 & 6 & 3 & 0 & 1 & $\cdot$ & 10 & \\
\hline OB:IS AM & $\ldots$ & $09: 15 \mathrm{AM}$ & 0 & 0 & 0 & 0 & 0 & 0 & 0 & 5 & 4 & 0 & $i$ &. & 10 & 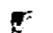 \\
\hline $08: 30 \mathrm{AM}$ & $=$ & $09: 30 \mathrm{AM}$ & 0 & 0 & 0 & 0 & 0 & 0 & o & 5 & 3 & 0 & $T$ &.. & 9 & $\approx$ \\
\hline $\begin{array}{l}\text { 08:45 AM } \\
09: 00 \mathrm{AM}\end{array}$ & $=$ & $\begin{array}{l}09: 45 \mathrm{AM} \\
10: 00 \mathrm{AM}\end{array}$ & 1 & $\begin{array}{l}0 \\
0\end{array}$ & $\begin{array}{l}0 \\
0\end{array}$ & $\begin{array}{l}0 \\
0\end{array}$ & $\begin{array}{l}0 \\
0\end{array}$ & $\begin{array}{l}0 \\
0\end{array}$ & $\begin{array}{l}0 \\
0\end{array}$ & $\begin{array}{l}5 \\
4\end{array}$ & $\begin{array}{l}4 \\
4\end{array}$ & $\begin{array}{l}0 \\
1\end{array}$ & $\frac{1}{3}$ & $\because$ & 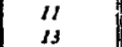 & 5 \\
\hline $09: 15 \mathrm{AM}$ & $\equiv$ & $\frac{10.15 \mathrm{MM}}{10.1}$ & 1 & 0 & 0 & 0 & 0 & 0 & 0 & 4 & 5 & 1 & 3 & & 14 & \\
\hline $09: 30 \mathrm{AM}$ & - & $10: 30 \mathrm{AM}$ & 1 & 0 & 0 & 0 & 0 & 0 & 0 & 3 & 7 & 1 & 3 & $\because$ & is & \\
\hline $09.43 \mathrm{AM}$ & $\rightarrow$ & 10:45 AM & 0 & 0 & 0 & 0 & 0 & 0 & 0 & 5 & 6 & $i$ & 3 & .. & 15 & 5 \\
\hline $10: 00 \mathrm{AM}$ & $=$ & $11: 00 \mathrm{AM}$ & 0 & 0 & $T$ & 0 & 0 & 0 & 0 & 3 & 5 & $T$ & $T$ & $" 1$ & $\pi$ & \\
\hline $10: 19 \mathrm{AM}$ & - & 11:15 AM & 1 & 0 & 1 & 0 & 0 & 0 & 0 & 3 & 3 & $!$ & $\frac{1}{3}$ & $\because$. & 10 & 5 \\
\hline $10: 30 \mathrm{AM}$ & $\ldots$ & $11: 30 \mathrm{AM}$ & 1 & 0 & 1 & 0 & 0 & 0 & 0 & 7 & $\frac{1}{3}$ & $\frac{1}{1}$ & 3 & $\because *$ & -18 & \\
\hline $10.45 \mathrm{AM}$ & $=$ & $11: 45 \mathrm{AM}$ & 1 & 0 & & 0 & 0 & $\overline{0}$ & 0 & 7 & 3 & 1 & 5 & '" & 78 & \\
\hline $11: 00 \mathrm{AM}$ & - & 12:00 PM & 2 & 0 & 1 & 0 & 0 & 0 & 0 & 8 & 2 & 0 & 5 & $"$ & 18 & \\
\hline $11: 15 \mathrm{AM}$ & $\ldots$ & 12:19 PM & 1 & 0 & $i$ & 0 & 0 & 0 & 0 & 12 & 2 & 1 & 6 & 11 & 23 & 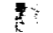 \\
\hline $11: 30 \mathrm{AM}$ & $=$ & $12: 30 \mathrm{PM}$ & 3 & 0 & 1 & 0 & 0 & 0 & 0 & 8 & 3 & 2 & 4 & $"$ & 21 & \\
\hline $11: 45 \mathrm{AM}$ & $-\infty$ & 12:45 PM & 3 & 0 & $i$ & 0 & 0 & 0 & 0 & 6 & 2 & 2 & 3 & " & 17 & 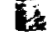 \\
\hline $12: 00 \mathrm{PM}$ & $=$ & $01: 00$ PM & 2 & 0 & 1 & 0 & 0 & 0 & 0 & 8 & 3 & 2 & 3 & 4 & 19 & \\
\hline $12: 15 \mathrm{PM}$ & $=$ & OIISPM & $\frac{3}{2}$ & $\begin{array}{l}0 \\
0\end{array}$ & $\frac{1}{1}$ & 0 & $\begin{array}{l}0 \\
0\end{array}$ & $\begin{array}{l}0 \\
0\end{array}$ & $\begin{array}{l}0 \\
0\end{array}$ & 4 & 3 & $T$ & 2 & "i & $\pi$ & \\
\hline $\begin{array}{l}\text { 12:30 PM } \\
12: 49 \text { PM }\end{array}$ & $=$ & $\begin{array}{l}01: 30 \text { PM } \\
01: 45 \text { PM }\end{array}$ & $\begin{array}{l}2 \\
2\end{array}$ & 0 & $\frac{1}{2}$ & $\begin{array}{l}0 \\
0\end{array}$ & 0 & $\begin{array}{l}0 \\
0\end{array}$ & $\begin{array}{l}0 \\
0\end{array}$ & 9 & $\frac{2}{2}$ & $\begin{array}{l}0 \\
2 \\
\end{array}$ & $\frac{2}{2}$ & 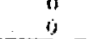 & $\begin{array}{l}13 \\
19\end{array}$ & 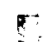 \\
\hline $0100 \mathrm{PM}$ & $=$ & $02: 00 \mathrm{PM}$ & 2 & 0 & 1 & 0 & 0 & & 0 & 4 & 1 & $\frac{1}{2}$ & 2 & i & 12 & \\
\hline 01:15 PM & - & 02:15 PM & 1 & 0 & $i$ & 0 & 0 & 0 & 0 & 8 & i & $\frac{2}{2}$ & 3 & 9 & is & 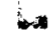 \\
\hline$\frac{01: 30 \mathrm{PM}}{01: 45 \mathrm{PM}}$ & $=$ & $\frac{02: 30 \text { PM }}{02: 45 P M}$ & $\frac{1}{1}$ & $\frac{0}{0}$ & $\frac{1}{1}$ & $\frac{0}{0}$ & $\frac{0}{0}$ & $\frac{0}{0}$ & $\frac{0}{0}$ & $\frac{6}{2}$ & $\frac{2}{1}$ & $\frac{2}{1}$ & $\frac{4}{3}$ & $\frac{0}{0}$ & $\frac{16}{16}$ & \\
\hline $02: 00 \mathrm{PM}$ & $\equiv$ & $\begin{array}{l}02: 45 \mathrm{PM} \\
03: 00 \mathrm{PM}\end{array}$ & $i$ & 0 & 1 & 0 & 0 & 0 & 0 & 8 & 3 & $i$ & 3 & 0 & $n$ & \\
\hline 02:1S PM & - & 03:13 PM & 1 & 0 & 1 & 0 & 0 & 0 & 0 & 3 & 3 & i & 3 & 0 & 12 & ? \\
\hline $02: 30 \mathrm{PM}$ & $=$ & $03: 30 \mathrm{PM}$ & $T$ & 0 & 1 & 0 & 0 & 0 & 0 & 8 & 2 & $T$ & 1 & $\overline{0}$ & 72 & $5:$ \\
\hline $02: 45$ PM & - & 03:45 PM & i & $\stackrel{0}{0}$ & $\begin{array}{l}0 \\
0\end{array}$ & $\therefore$ & $\begin{array}{l}0 \\
0\end{array}$ & $\begin{array}{l}0 \\
0\end{array}$ & 0 & 6 & 2 & 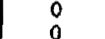 & $\begin{array}{c}4 \\
5\end{array}$ & $\begin{array}{l}0 \\
n\end{array}$ & $\begin{array}{l}13 \\
12\end{array}$ & نسن \\
\hline 03:00 PM & $=$ & $\frac{04: 00 P M}{04 \cdot 15 P M}$ & $\frac{1}{1}$ & $\frac{0}{0}$ & $\frac{0}{0}$ & $\frac{0}{0}$ & $\frac{0}{0}$ & $\frac{0}{0}$ & $\frac{\pi}{0}$ & $\frac{0}{5}$ & 0 & T & 4 & & II & \\
\hline $\begin{array}{l}03: 15 \mathrm{PM} \\
03: 30 \mathrm{PM}\end{array}$ & $=$ & $\begin{array}{l}\text { 04:115M } \\
\text { 04:30 PM }\end{array}$ & 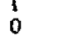 & 0 & 0 & 0 & 0 & 0 & 0 & 2 & 0 & i & 4 & 0 & 7 & 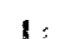 \\
\hline 03:1S PM & $=$ & OA:45 PM & 0 & 0 & $\stackrel{0}{0}$ & 0 & 0 & 0 & 0 & 1 & 0 & 1 & 2 & 0 & 1 & \\
\hline $04: 00 \mathrm{PM}$ & $=$ & $05: 00 \mathrm{PM}$ & 5 & $\pi$ & $x=$ & 0 & 0 & 0 & 0 & 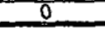 & 0 & & & 0 & 2 & \\
\hline$s$ & $E N I$ & ULA & 17 & 7 & & $A L$ & $D A$ & (a) 2 & 29 & & ON & $1 c$ & 15 & $232=$ & & \\
\hline
\end{tabular}




\section{Baymetrics Traffic Resources INTERSECTION TURNING MOVEMENT SUMMARY}

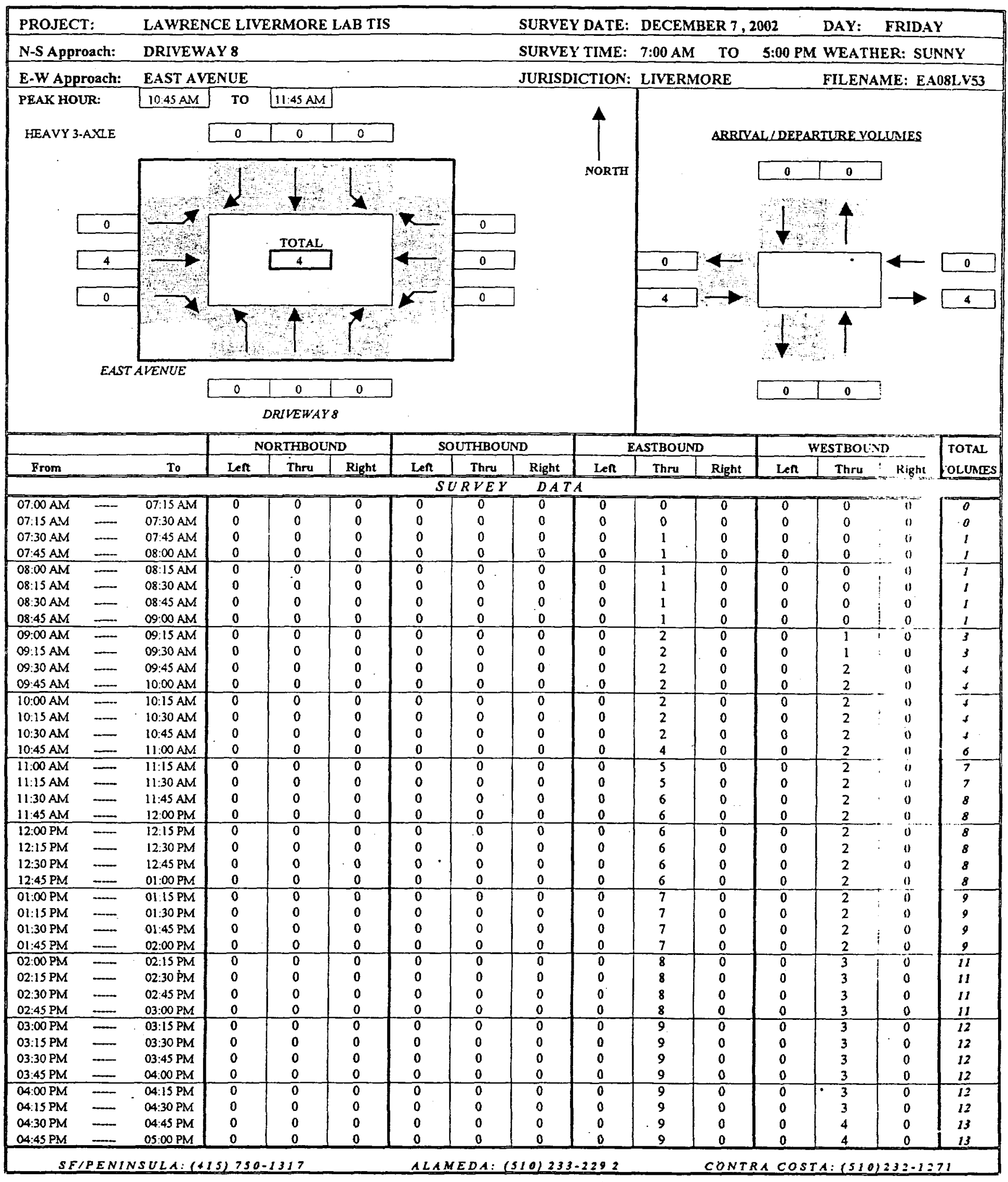




\section{Baymetrics Traffic Resources INTERSECTION TURNING MOVEMENT SUMMARY}

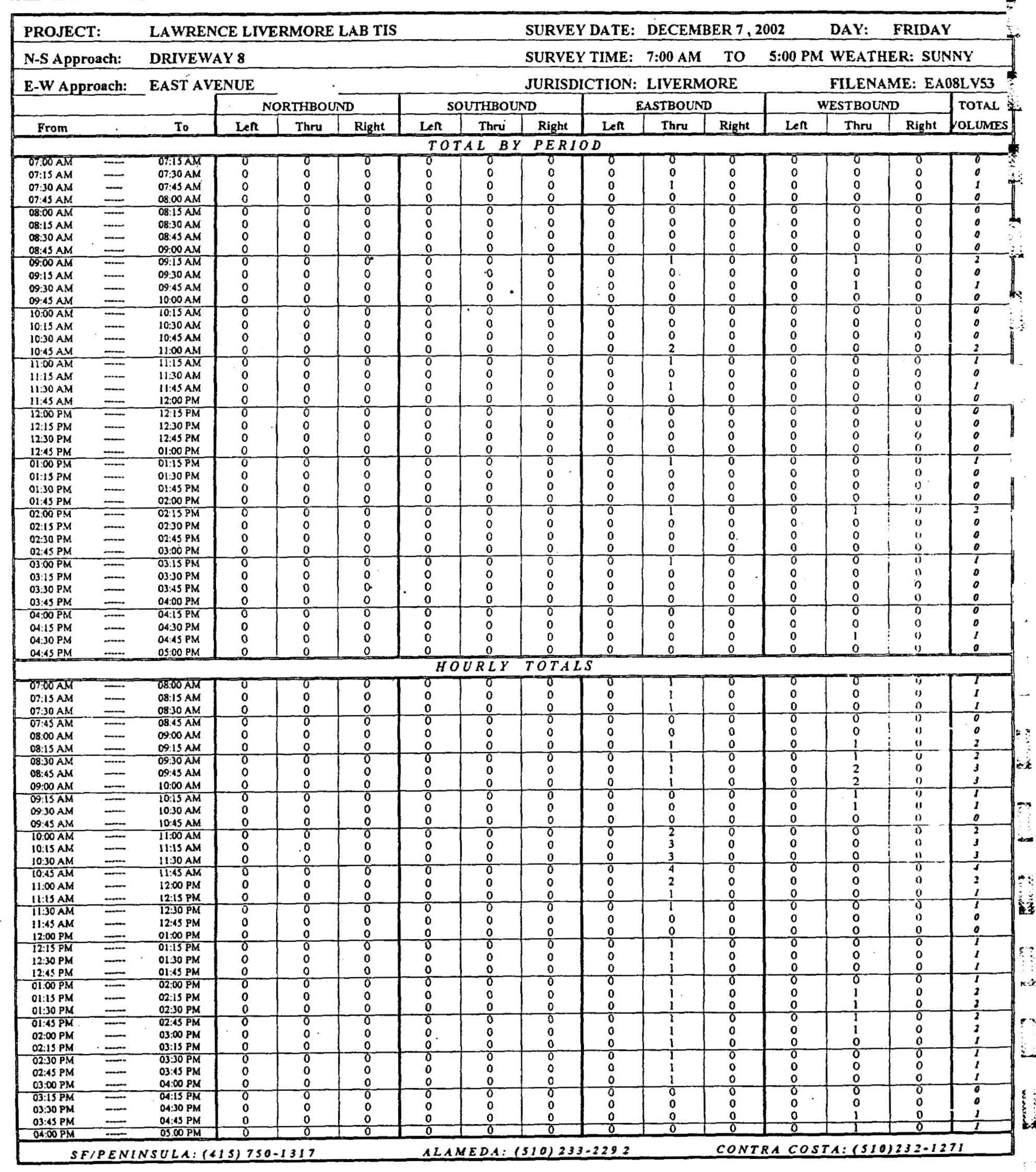




\section{Baymetrics Traffic Resources INTERSECTION TURNING MOVEMENT SUMMARY}

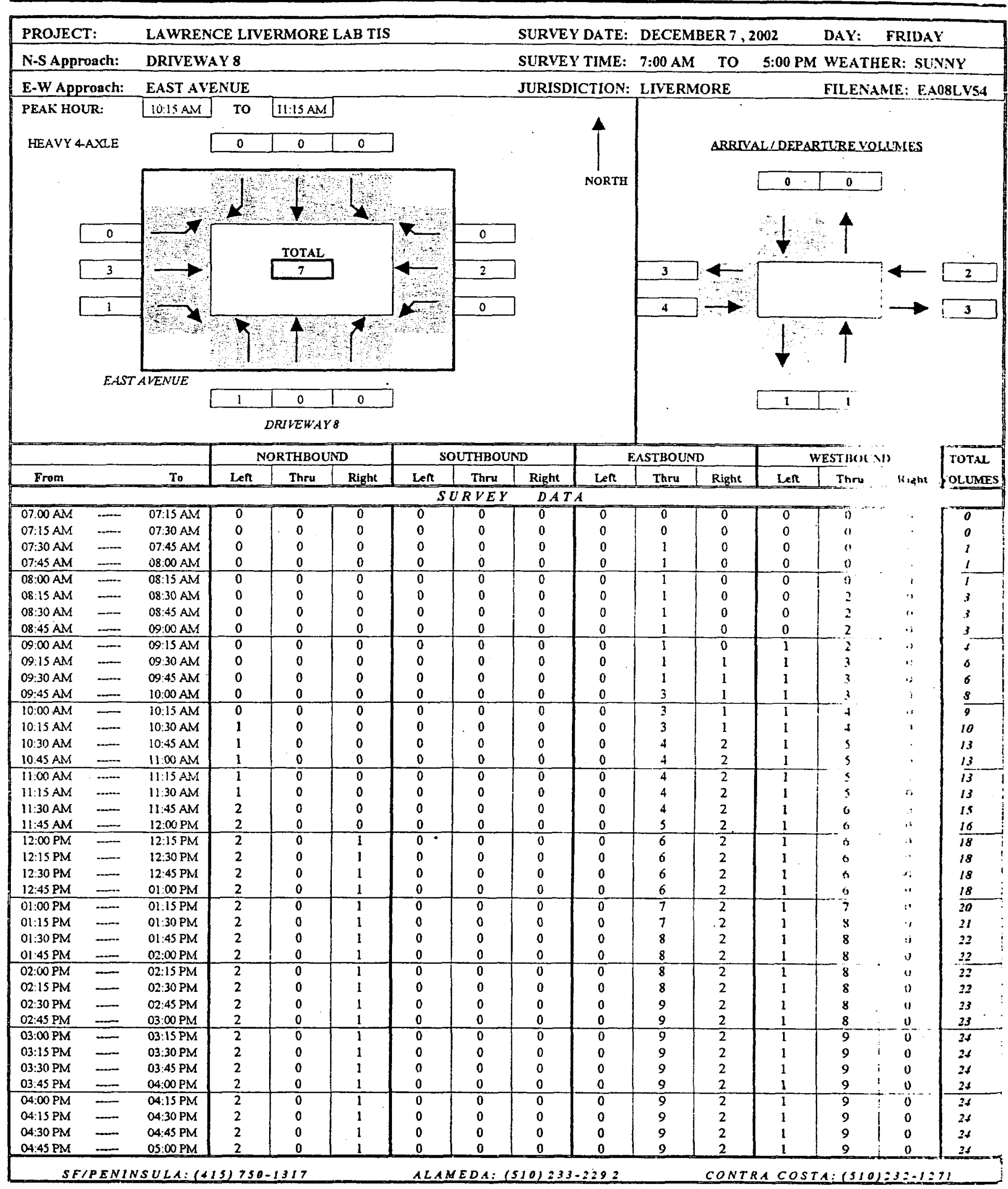




\section{Baymetrics Traffic Resources INTERSECTION TURNING MOVEMENT SUMMARY}

\begin{tabular}{|c|c|c|c|c|c|c|c|c|c|c|c|c|c|c|c|}
\hline \multirow{2}{*}{\multicolumn{2}{|c|}{$\begin{array}{l}\text { PROJECT: } \\
\text { N-S Approach: }\end{array}$}} & \multicolumn{6}{|c|}{ LAWRENCE LIVERMORE LAB TIS } & \multicolumn{5}{|c|}{ SURVEY DATE: DECEMBER 7, 2002} & DAY: & \multicolumn{2}{|c|}{ FRIDAY } \\
\hline & & DRIVE H & & & & & & SURVE & IME: & 7:00 AM & To & 5:00 PM & WEATH & ER: SUI & YNY \\
\hline E-W Ap & ach: & EAST AI & IUE & & & & & JURISD & TION: & LIVERN & RE & & FILENA & ME: EA & 081 V Y4 \\
\hline & & & & THBO & & & THBOI & & & ASTBOUN & & & ESTBOU: & & TOTAL \\
\hline Fram & & To & Len & Thru & Right & Len & Thru & Right & Lent & Thru & Right & Len & Thru & Right & YOLUMES \\
\hline & & & & & & & $L B$ & PERI & & & & & & & \\
\hline 07:00AM & $\overline{-1}$ & O:15AM & 0 & 0 & $\overline{0}$ & 0 & 0 & 0 & 0 & 0 & $\sigma$ & $\sigma$ & $\sigma$ & $\pi$ & 8 \\
\hline $\begin{array}{l}07: 15 \text { AM } \\
07: 30 \text { AM }\end{array}$ & $=$ & $\begin{array}{l}07: 30 \mathrm{AM} \\
07: 45 \mathrm{AM}\end{array}$ & 0 & 0 & 0 & 0 & 0 & $\begin{array}{l}0 \\
0\end{array}$ & 0 & $\begin{array}{l}0 \\
1\end{array}$ & 0 & 0 & 0 & 0 & 0 \\
\hline 07:45 AM & $=$ & $\begin{array}{l}0: 15 \mathrm{AM} \\
08: 00 \mathrm{AM}\end{array}$ & 0 & 0 & 0 & $\begin{array}{l}0 \\
0\end{array}$ & 0 & 0 & $\begin{array}{l}0 \\
0\end{array}$ & $\begin{array}{l}1 \\
0\end{array}$ & $\begin{array}{l}0 \\
0\end{array}$ & $\begin{array}{l}0 \\
0\end{array}$ & $\begin{array}{l}0 \\
0\end{array}$ & $\begin{array}{l}0 \\
0\end{array}$ & 1 \\
\hline $08: 00 \mathrm{AM}$ & $\rightarrow$ & $08: 13 \mathrm{AM}$ & $\overline{0}$ & 0 & 0 & 0 & 0 & 0 & 0 & 0 & 0 & 0 & 0 & 0 & $\div$ \\
\hline $08: 15 \mathrm{AM}$ & - & $08: 30 \mathrm{AM}$ & 0 & 0 & 0 & 0 & 0 & 0 & 0 & 0 & 0 & 0 & 2 & 0 & 2 \\
\hline $\begin{array}{l}\text { 08:30 AM } \\
08: 45 \text { AM }\end{array}$ & $\bar{Z}$ & $\begin{array}{l}\text { 08:45 AM } \\
09: 00 \mathrm{AM}\end{array}$ & 0 & $\begin{array}{l}0 \\
0\end{array}$ & $\begin{array}{l}0 \\
0\end{array}$ & $\begin{array}{l}0 \\
0\end{array}$ & $\begin{array}{l}0 \\
0\end{array}$ & $\begin{array}{l}0 \\
0\end{array}$ & $\begin{array}{l}0 \\
0\end{array}$ & $\begin{array}{l}0 \\
0\end{array}$ & $\begin{array}{l}0 \\
0\end{array}$ & $\begin{array}{l}0 \\
0\end{array}$ & $\begin{array}{l}0 \\
0\end{array}$ & $\begin{array}{l}0 \\
0 \\
0\end{array}$ & 0 \\
\hline $09: 00 \mathrm{AM}$ & 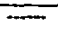 & $09: 15 \mathrm{AM}$ & 0 & 0 & 0 & $\pi$ & 0 & 0 & 0 & 0 & 0 & $\frac{0}{1}$ & 0 & $\frac{0}{0}$ & $\frac{v}{1}$ \\
\hline C9:15 AM & - & $09: 30 \mathrm{AM}$ & 0 & 0 & 0 & 0 & 0 & 0 & 0 & 0 & 1 & 0 & 1 & 0 & 2 \\
\hline $09: 30 \mathrm{AM}$ & $\cdots$ & $09: 45 \mathrm{AM}$ & 0 & 0 & 0 & 0 & 0 & 0 & 0 & 0 & 0 & 0 & 0 & 0 & 0 \\
\hline$\frac{09: 45 \mathrm{AM}}{10.00 \mathrm{AM}}$ & $=$ & $10: 00 \mathrm{AM}$ & 0 & 0 & 0 & 0 & 0 & 0 & 0 & 2 & 0 & 0 & 0 & 0 & 2 \\
\hline $\begin{array}{l}10: 00 \mathrm{AM} \\
10: 15 \mathrm{AM}\end{array}$ & $\bar{I}$ & $10: 15 \mathrm{AM}$ & 0 & 0 & 0 & 0 & 0 & 0 & 0 & 0 & $\overline{0}$ & 0 & $T$ & i & $T$ \\
\hline $10: 30 \mathrm{AM}$ & $=$ & $\begin{array}{l}10.30 \mathrm{AM} \\
10.4 \mathrm{AM}\end{array}$ & $\begin{array}{l}1 \\
0\end{array}$ & $\begin{array}{l}0 \\
0 \\
0\end{array}$ & $\begin{array}{l}0 \\
0\end{array}$ & 0 & $\stackrel{0}{0}$ & $\begin{array}{l}0 \\
0\end{array}$ & 0 & 0 & 0 & 0 & 0 & i & I \\
\hline $10.45 \mathrm{AM}$ & $\ldots$ & $11: 00 \mathrm{AM}$ & 0 & 0 & 0 & $\begin{array}{l}0 \\
0 \\
\end{array}$ & $\begin{array}{l}0 \\
0\end{array}$ & $\begin{array}{l}0 \\
0\end{array}$ & $\begin{array}{l}0 \\
0\end{array}$ & $\begin{array}{l}1 \\
0\end{array}$ & $\begin{array}{l}1 \\
0\end{array}$ & $\begin{array}{l}0 \\
0\end{array}$ & $\begin{array}{l}1 \\
0\end{array}$ & 3 & 3 \\
\hline $11: 00 \mathrm{AM}$ & $=$ & 11:15AM & 0 & 0 & 0 & 0 & 0 & 0 & -0 & 0 & 0 & 0 & 0 & i) & 0 \\
\hline 11:15 AM & $\ldots$ & $11: 30 \mathrm{AM}$ & 0 & 0 & 0 & 0 & 0 & 0 & 0 & 0 & 0 & 0 & 0 & "1 & 0 \\
\hline $11: 30 \mathrm{AM}$ & - & 11:45 AM & $i$ & 0 & 0 & 0 & 0 & 0 & 0 & 0 & 0 & 0 & 3 & 1 & 3 \\
\hline 1I:45 AM & $\ldots$ & $12: 00 \mathrm{PM}$ & 0 & 0 & 0 & 0 & 0 & 0 & 0 & 1 & 0 & 0 & 0 & $\because$ & $i$ \\
\hline $12: 00 \mathrm{PM}$ & 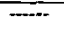 & $12: 15 \mathrm{PM}$ & 0 & 0 & 1 & 0 & 0 & 0 & 0 & $i$ & 0 & 0 & 0 & $\pi$ & 2 \\
\hline $12: 15$ PM & $\ldots$ & 12:30 PM & 0 & 0 & 0 & 0 & 0 & 0 & 0 & 0 & 0 & 0 & 0 & a & 0 \\
\hline 12:30 PM & - & 12:45 PM & 0 & 0 & 0 & 0 & 0 & 0 & 0 & 0 & 0 & 0 & 0 & "1 & 0 \\
\hline 12:45 PM & 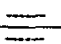 & $\frac{01: 00 \mathrm{PM}}{01: 15 \mathrm{PM}}$ & 0 & 0 & 0 & 0 & 0 & 0 & $\frac{a}{0}$ & $\frac{0}{1}$ & 0 & 0 & 은 & $\therefore$ & 0 \\
\hline $\begin{array}{l}01: 00 \mathrm{PM} \\
01: 15 \mathrm{PM}\end{array}$ & $m$ & $\begin{array}{l}01: 15 \mathrm{PM} \\
01: 30 \mathrm{PM}\end{array}$ & 0 & 0 & $\begin{array}{l}0 \\
0\end{array}$ & $\begin{array}{l}9 \\
0\end{array}$ & 0 & 0 & $\begin{array}{l}0 \\
0 \\
0\end{array}$ & $\begin{array}{l}1 \\
0\end{array}$ & 0 & 0 & $T$ & a & 3 \\
\hline $01: 30 \mathrm{PM}$ & $\ldots$ & $01: 45 \mathrm{PM}$ & 0 & 0 & 0 & 0 & 0 & $\begin{array}{l}0 \\
0\end{array}$ & 0 & 1 & $\begin{array}{l}0 \\
0\end{array}$ & 0 & 1 & "' & 1 \\
\hline $01: 45 \mathrm{PM}$ & $\ldots$ & $02: 00 \mathrm{PM}$ & 0 & 0 & 0 & 0 & 0 & 0 & 0 & $\begin{array}{l}1 \\
0\end{array}$ & 0 & $\begin{array}{l}0 \\
0\end{array}$ & 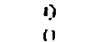 & $\because$ & 1 \\
\hline $02.00 \mathrm{PM}$ & $=$ & $02: 15 \mathrm{PM}$ & $\overline{0}$ & 0 & 0 & 0 & 0 & 0 & 0 & 0 & $\overline{0}$ & $\frac{0}{0}$ & $\frac{a}{10}$ & $\begin{array}{l}1 \\
11\end{array}$ & $\because$ \\
\hline $02.15 \mathrm{RM}$ & - & 02:30 PM & 0 & 0 & 0 & 0 & 0 & 0 & 0 & 0 & 0 & 0 & i. & i: & 0 \\
\hline $02: 30 \mathrm{PM}$ & $\ldots$ & 02:45 PM & 0 & 0 & 0 & 0 & 0 & 0 & 0 & 1 & 0 & 0 & $\ddot{u}$ &. & $i$ \\
\hline $02: 45 \mathrm{PM}$ & $=$ & 03:00 PM & 0 & 0 & 0 & 0 & 0 & 0 & 0 & 0 & 0 & 0 & 0 & $\therefore$ & 0 \\
\hline $03: 00 \mathrm{PM}$ & - & $03: 15 \mathrm{PM}$ & 0 & 0 & 0 & 0 & $\sigma$ & 0 & 0 & 0 & 0 & 0 & $T$ & $\therefore$ & I \\
\hline 03:15 PMM & $\ldots$ & 03:30 PM & 0 & 0 & 0 & 0 & 0 & 0 & 0 & 0 & 0 & 0 & i) & $\therefore$ & 0 \\
\hline 03:30 PM & $\ldots$ & 03:45 PM & 0 & 0 & 0 & 0 & 0 & 0 & 0 & 0 & 0 & 0 & 0 & $"$ & 0 \\
\hline 03:45 PM & $=$ & $04.00 \mathrm{PM}$ & 0 & 0 & 0 & 0 & 0 & 0 & 0 & 0 & 0 & 0 & 0 & $\therefore$ & 0 \\
\hline $0400 \mathrm{PM}$ & $\bar{E}$ & O4:15 PM & 0 & 0 & 0 & 0 & 0 & 0 & 0 & 0 & 0 & 0 & 0 & 11 & 0 \\
\hline $\begin{array}{l}004: 113 \mathrm{PM} \\
04: 30 \mathrm{FM}\end{array}$ & $=$ & $04: 45 \mathrm{PM}$ & 0 & 0 & $\begin{array}{l}0 \\
0\end{array}$ & ${ }_{0}^{0}$ & $\begin{array}{l}0 \\
0\end{array}$ & $\begin{array}{l}0 \\
0\end{array}$ & 0 & 0 & 0 & 0 & 0 & $"$ & 0 \\
\hline O1:15 PM & $=$ & OS:00 PM & 0 & 0 & 0 & 0 & 0 & $\begin{array}{l}0 \\
0\end{array}$ & 0 & $\begin{array}{l}0 \\
0 \\
\end{array}$ & $\begin{array}{l}0 \\
0 \\
\end{array}$ & $\begin{array}{l}0 \\
0\end{array}$ & $\begin{array}{l}0 \\
0\end{array}$ & $":$ & $\begin{array}{l}0 \\
0\end{array}$ \\
\hline & & & & & & & $R L Y$ & TOTAL & & & & & & & \\
\hline 7:00 AM & $=$ & $08: \overline{C O} A M$ & 0 & 0 & 0 & 0 & $\overline{0}$ & 8 & 8 & $T$ & $\overline{0}$ & o & $\pi$ & $\therefore$ & $\pi$ \\
\hline $\begin{array}{l}07: 15 \mathrm{AM} \\
07.30 \mathrm{AM}\end{array}$ & $\overline{-}$ & 08:15 AM & $\begin{array}{l}0 \\
0\end{array}$ & $\begin{array}{l}0 \\
0\end{array}$ & 0 & $\begin{array}{l}0 \\
0\end{array}$ & $\begin{array}{l}0 \\
0\end{array}$ & $\begin{array}{l}0 \\
0\end{array}$ & 0 & 1 & 0 & $\begin{array}{l}0 \\
0\end{array}$ & $\because$ & $" 1$ & 1 \\
\hline $07: 45 A M$ & $\equiv$ & $\frac{08: 30 . A M}{08: 45 A M}$ & $-\frac{u}{0}$ & 0 & $\frac{0}{0}$ & $\frac{0}{0}$ & $\frac{1}{0}$ & 0 & 0 & $\frac{1}{0}$ & $\frac{0}{0}$ & $\frac{0}{0}$ & $\frac{1}{2}$ & "“ & $-\frac{3}{3}$ \\
\hline OB:00 A.M & $\ldots$ & $09: 00 \mathrm{AM}$ & 0 & 0 & 0 & 0 & 0 & 0 & 0 & 0 & 0 & 0 & $\Xi$ & ", & 2 \\
\hline OB:ISAM & $=-$ & $09: 15 \mathrm{AM}$ & 0 & 0 & 0 & 0 & 0 & 0 & 0 & 0 & 0 & 1 & $\vdots$ & $\therefore$ & 3 \\
\hline $0830 \mathrm{AM}$ & $=$ & $09.30 \mathrm{AM}$ & 0 & 0 & 0 & 0 & 0 & 0 & 0 & 0 & $T$ & $T$ & 1 & $\because$ & 3 \\
\hline $08: 45 \mathrm{AM}$ & 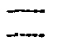 & $\begin{array}{l}09: 45 \mathrm{AM} \\
10: 00 \mathrm{AM}\end{array}$ & $\begin{array}{l}0 \\
0 \\
0\end{array}$ & $\begin{array}{l}0 \\
0\end{array}$ & $\begin{array}{l}0 \\
0\end{array}$ & $\begin{array}{l}0 \\
0\end{array}$ & 0 & 0 & 0 & 0 & 1 & 1 & 1 & $" 1$ & 3 \\
\hline $09: 15 \mathrm{AM}$ & $=$ & $\frac{10: 00 \mathrm{AM}}{10.15 \mathrm{AM}}$ & 0 & 0 & $\frac{0}{0}$ & $\frac{a}{0}$ & - & $\frac{0}{0}$ & $\frac{0}{0}$ & $\frac{2}{2}$ & $\frac{1}{1}$ & $\frac{1}{0}$ & $\frac{1}{2}$ & $\because "$ & $\frac{5}{5}$ \\
\hline $09: 30 \mathrm{AM}$ & $\ldots$ & $10: 30 \mathrm{AM}$ & 1 & 0 & 0 & 0 & 0 & 0 & 0 & $\frac{2}{2}$ & $b_{0}^{1}$ & $\begin{array}{l}0 \\
0\end{array}$ & $\frac{2}{1}$ & .. & t \\
\hline $09: 45 \mathrm{AM}$ & $=$ & $10: 45 \mathrm{AM}$ & 1 & 0 & 0 & 0 & 0 & 0 & 0 & 3 & 1 & 0 & & $\therefore$ & 7 \\
\hline $10: 00 \mathrm{AM}$ & $=$ & $11: 00 \mathrm{AM}$ & & $\sigma$ & 0 & 0 & 0 & 0 & 0 & $T$ & l & 0 & $\frac{2}{2}$ & .. & 5 \\
\hline 10:15 AM & - & HISAM & 1 & 0 & 0 & 0 & 0 & 0 & 0 & $i$ & $i$ & 0 & $i$ & $1:$ & 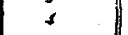 \\
\hline 10:30 AM & $=$ & $11: 30 \mathrm{AM}$ & 0 & 0 & 0 & 0 & 0 & 0 & 0 & $i$ & $\mathrm{i}$ & 0 & $i$ & , & 3 \\
\hline $10.45 \mathrm{AM}$ & $=$ & I1:45 AM & 1 & 0 & 0 & 0 & 0 & $\overline{0}$ & 0 & 0 & 0 & 0 & $i$ & .4 & $\frac{2}{2}$ \\
\hline $11: 00 \mathrm{AM}$ & - & 12:00 PM & 1 & 0 & 0 & 0 & 0 & 0 & 0 & $i$ & 0 & 0 & $i$ & " & $j$ \\
\hline 11:15.AM & $\ldots$ & 12:15 PM & $i$ & 0 & 1 & 0 & 0 & 0 & 0 & 2 & 0 & 0 & i & ". & 3 \\
\hline $11: 30 \mathrm{AM}$ & - & $12: 30 \mathrm{PM}$ & 1 & 0 & $T$ & 0 & 0 & 0 & 0 & 2 & 0 & 0 & $T$ & 11 & 5 \\
\hline $\begin{array}{l}11: 45 \text { AM } \\
12.00 \mathrm{PM}\end{array}$ & $=$ & $\begin{array}{l}\text { 12:45 PM } \\
01: 00 \text { PM }\end{array}$ & $\begin{array}{l}0 \\
0\end{array}$ & $\begin{array}{l}0 \\
0\end{array}$ & 1 & $\begin{array}{l}0 \\
0\end{array}$ & $\begin{array}{l}0 \\
0\end{array}$ & $\begin{array}{l}0 \\
0\end{array}$ & 0 & $\frac{2}{1}$ & $\begin{array}{l}0 \\
0\end{array}$ & $\begin{array}{l}0 \\
0\end{array}$ & 0 & $\ddot{0}$ & 3 \\
\hline $12: 15$ PM & $=$ & $01: 15 P M$ & 0 & 0 & 0 & 0 & 0 & 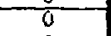 & 8 & 1 & 0 & 0 & $\frac{\pi}{1}$ & 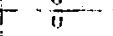 & $\frac{2}{3}$ \\
\hline $\begin{array}{l}12.30 \mathrm{PM} \\
12.45 \mathrm{PM}\end{array}$ & $\overline{ }$ & $\begin{array}{l}01.30 \mathrm{PM} \\
01.45 \mathrm{PM}\end{array}$ & $\stackrel{0}{0}$ & $\begin{array}{l}0 \\
0\end{array}$ & $\begin{array}{l}0 \\
0\end{array}$ & 0 & 0 & 0 & $\stackrel{0}{0}$ & $\frac{1}{2}$ & 0 & 0 & 3 & $"$ & 3 \\
\hline 01:00 PM & $=$ & $.02: 00 \mathrm{PM}$ & $\frac{0}{0}$ & 0 & $\frac{0}{0}$ & $\frac{0}{0}$ & 0 & $\frac{0}{0}$ & $-\frac{0}{0}$ & $\frac{2}{2}$ & $\frac{0}{0}$ & $\frac{0}{0}$ & $\frac{2}{3}$ & $\frac{11}{11}$ & \pm \\
\hline $01: 15 \mathrm{PM}$ & - & $02: 15 \mathrm{PM}$ & 0 & 0 & 0 & 0 & 0 & 0 & 0 & $i$ & 0 & 0 & $i$ & i) & 3 \\
\hline $01: 30$ PM & $=$ & 02:30 PM & 0 & 0 & 0 & 0 & 0 & 0 & 0 & $\mathrm{i}$ & 0 & 0 & 0 & ij & $i$ \\
\hline $01: 45$ PM & - & $02: 45$ PM & 0 & 0 & 0 & 0 & 0 & 0 & 0 & 1 & 0 & 0 & 0 & 0 & I \\
\hline $02: 00$ PM & - & $03: 00 \mathrm{PM}$ & 0 & 0 & 0 & 0 & 0 & 0 & 0 & $i$ & 0 & 0 & 0 & 0 & 1 \\
\hline $02: 15 \mathrm{PM}$ & $=$ & $03: 15$ PM & 0 & 0 & 0 & 0 & 0 & 0 & 0 & 1 & 0 & 0 & 1 & 0 & 2 \\
\hline $02: 30 \mathrm{PM}$ & $-\infty$ & $03: 30 \mathrm{PM}$ & 0 & 0 & 0 & 0 & 0 & 0 & 0 & 1 & 0 & 0 & 1 & 0 & 2 \\
\hline $02: 15 \mathrm{PM}$ & 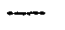 & 03:45 PM & $\begin{array}{l}0 \\
0\end{array}$ & 0 & 0 & $\begin{array}{l}0 \\
0\end{array}$ & $\begin{array}{l}0 \\
0\end{array}$ & $\begin{array}{l}0 \\
0\end{array}$ & $\begin{array}{l}0 \\
0\end{array}$ & $\begin{array}{l}0 \\
0\end{array}$ & $\begin{array}{l}0 \\
0\end{array}$ & $\begin{array}{l}0 \\
0\end{array}$ & 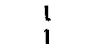 & 0 & 1 \\
\hline $03: 00 \mathrm{PM}$ & $=$ & O4:00 PM & $\frac{0}{0}$ & 0 & 0 & 0 & 0 & 0 & 0 & $\frac{0}{0}$ & 0 & & 1 & 0 & 1 \\
\hline 03:15 PM & $=$ & 04:15 PM & 0 & 0 & 0 & 0 & ? & 0 & 0 & $0_{0}^{0}$ & 0 & 0 & 0 & 0 & 0 \\
\hline 03:30 PM & - & 04:30 PM & $\begin{array}{l}0 \\
0\end{array}$ & $\begin{array}{l}0 \\
0\end{array}$ & $\begin{array}{l}0 \\
0 \\
0\end{array}$ & $\begin{array}{l}0 \\
0 \\
0\end{array}$ & $\begin{array}{l}0 \\
0\end{array}$ & $\begin{array}{l}0 \\
0\end{array}$ & $\begin{array}{l}0 \\
0\end{array}$ & $\begin{array}{l}0 \\
0\end{array}$ & $\begin{array}{l}0 \\
0\end{array}$ & $\begin{array}{l}0 \\
0 \\
0\end{array}$ & ju & 0 & 0 \\
\hline$\frac{03: 45 \mathrm{PM}}{-0400 \mathrm{PM}}$ & $\equiv$ & $\begin{array}{l}04: 45 \mathrm{PM} \\
05: 00 \mathrm{PM} \\
\end{array}$ & 0 & 0 & 0 & 0 & 0 & $\frac{0}{0}$ & 0 & 0 & 0 & $\frac{\pi}{0}$ & 0 & $\frac{0}{0}$ & 0 \\
\hline$S F$ & $E N I$ & $U L A: A$ & 7 & & & . & DA & a) 2 & 292 & & $O_{i}$ & $c$ & 15 & 32. & \\
\hline
\end{tabular}




\section{Baymetrics Traffic Resources INTERSECTION TURNING MOVEMENT SUMMARY}

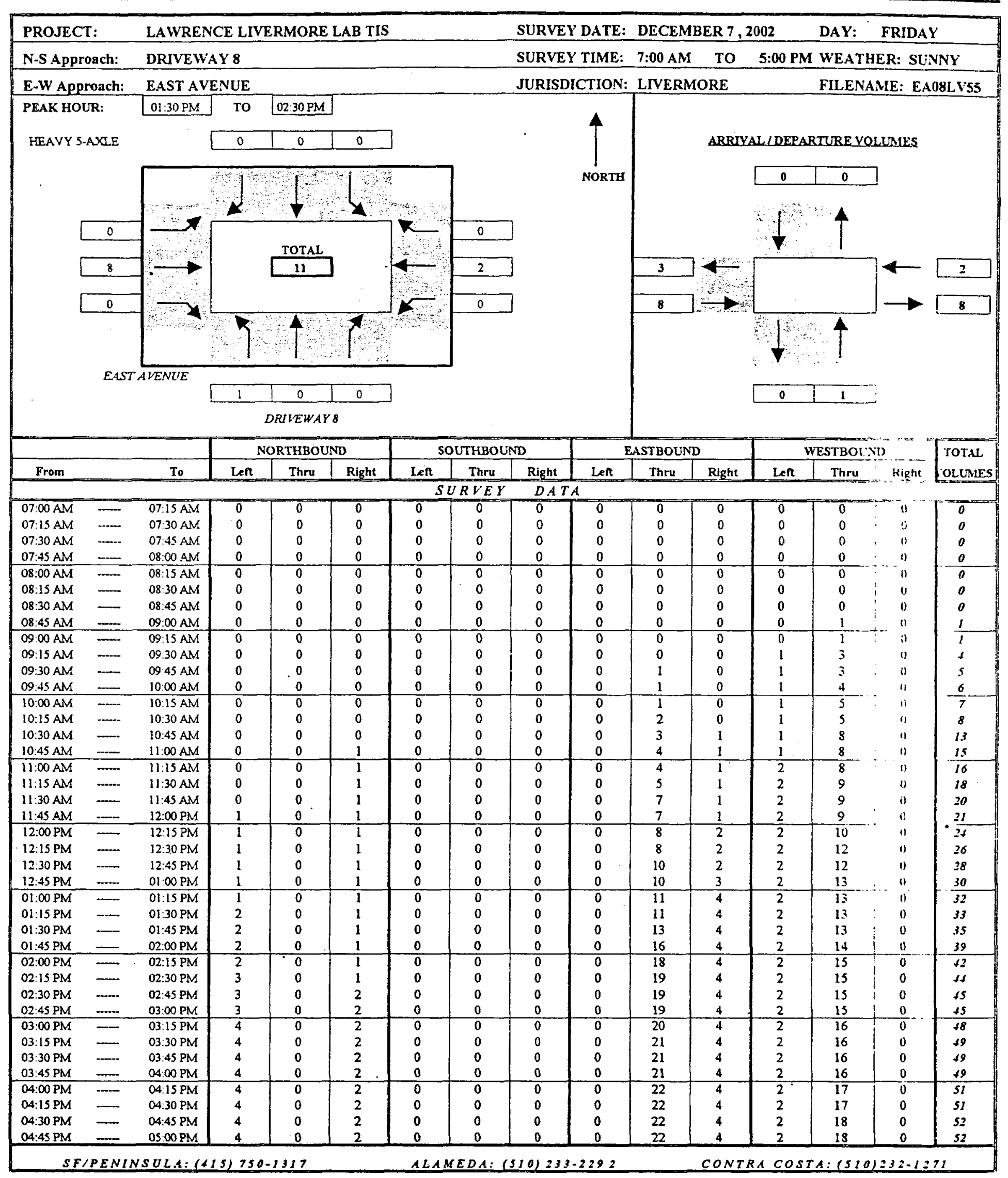




\section{Baymetrics Traffic Resources INTERSECTION TURNING MOVEMENT SUMMARY}

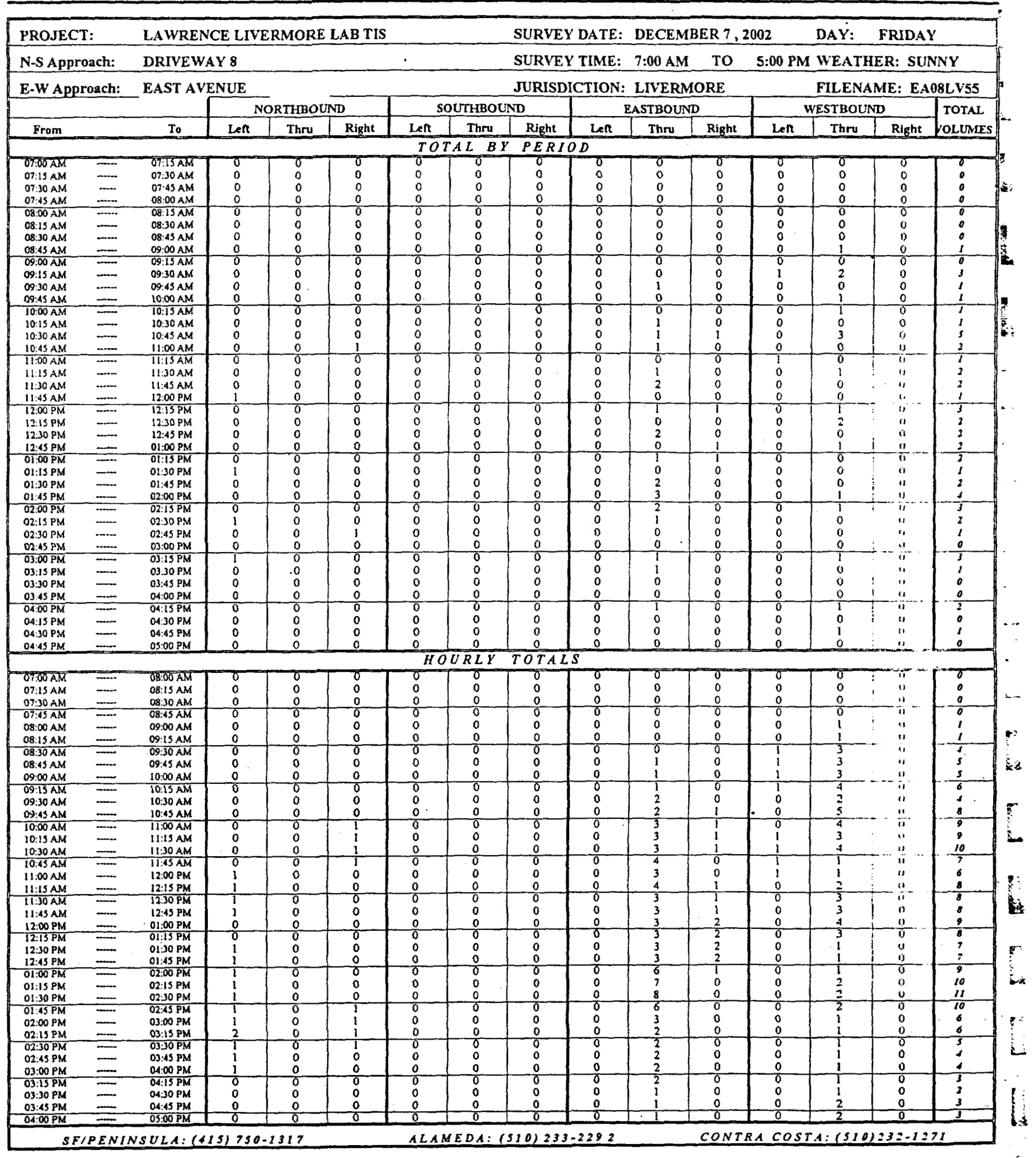




\section{Baymetrics Traffic Resources INTERSECTION TURNING MOVEMENT SUMMARY}

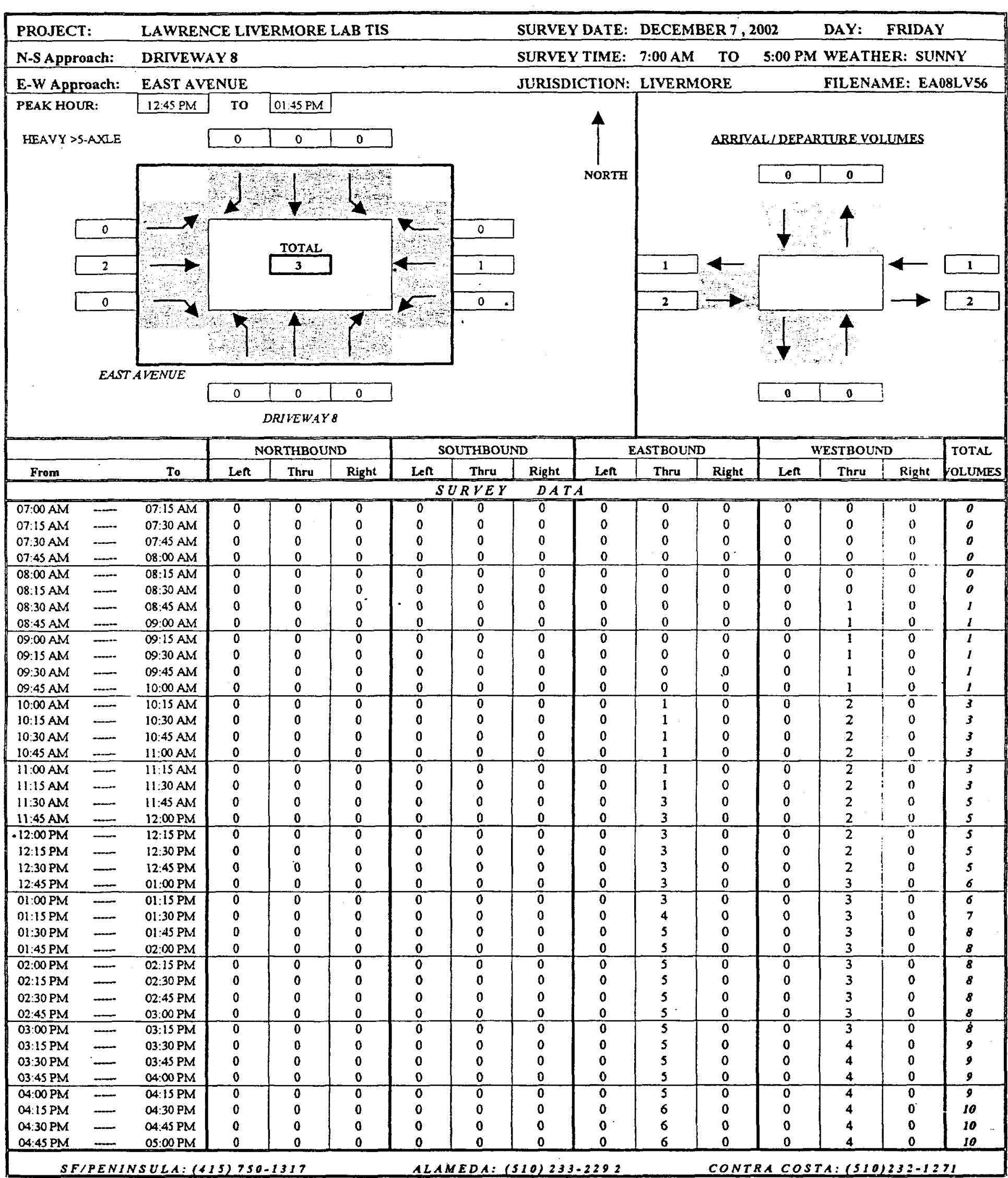




\section{Baymetrics Traffic Resources INTERSECTION TURNING MOVEMENT SUMMARY}

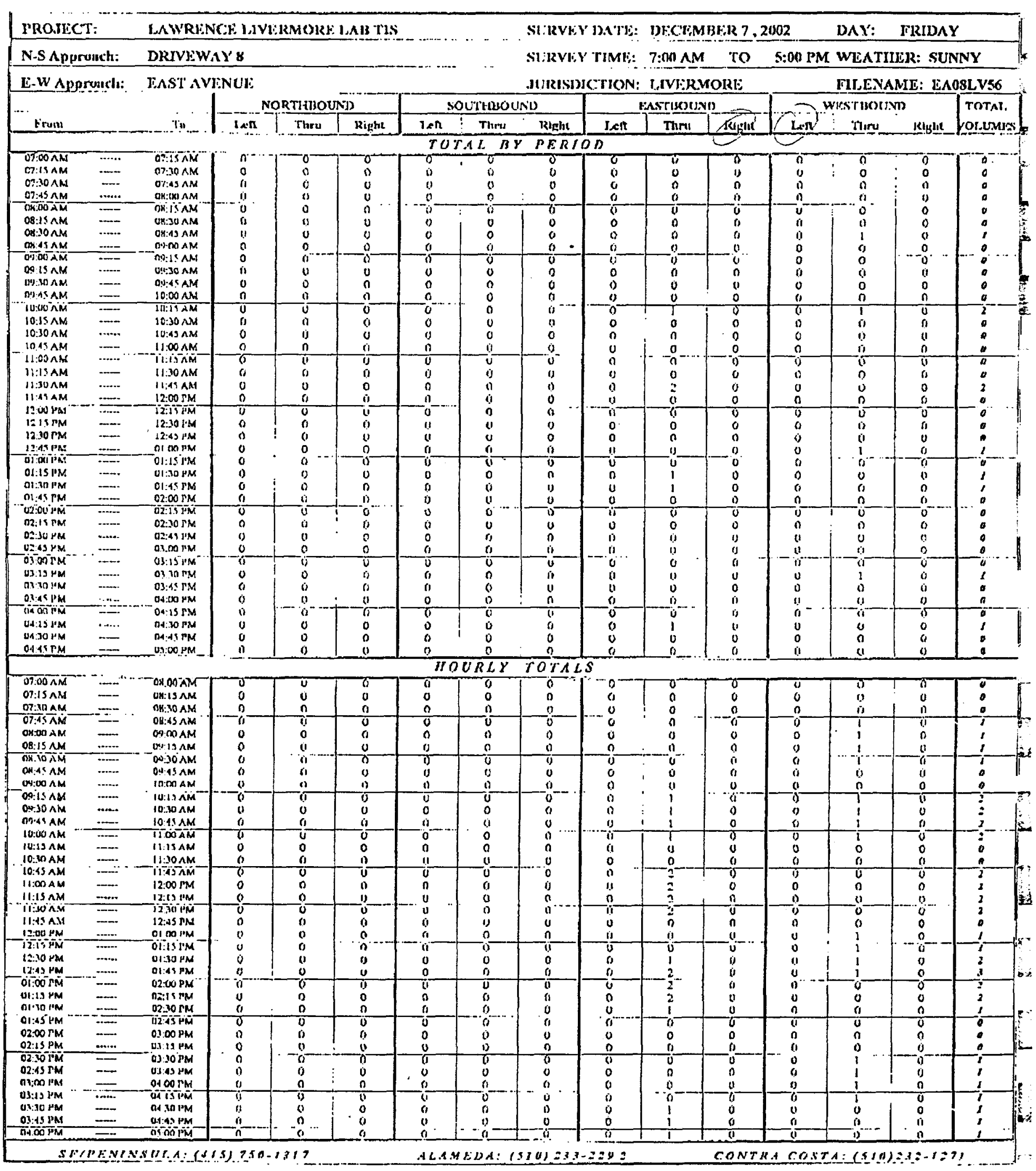




\section{Truck Counts}

\section{Locations 7 and 8}

Monday, December 10, 2001 



\section{Baymetrics Traffic Resources INTERSECTION TURNING MOVEMENT SUMMARY}

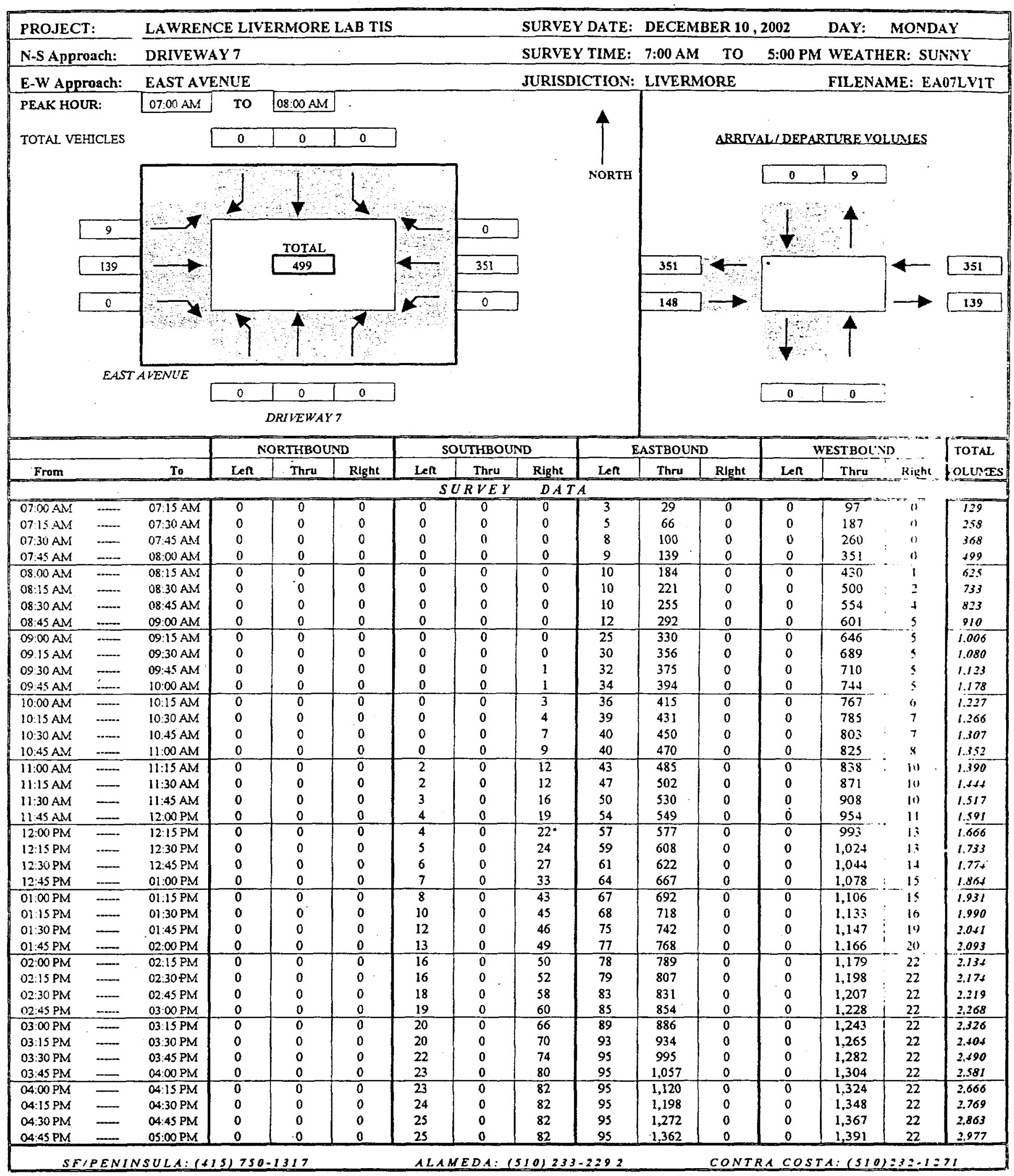




\section{Baymetrics Traffic Resources INTERSECTION TURNING MOVEMENT SUMMARY}

\begin{tabular}{|c|c|c|c|c|c|c|c|c|c|c|c|c|c|c|c|c|}
\hline \multirow{2}{*}{\multicolumn{2}{|c|}{$\begin{array}{l}\text { PROJECT: } \\
\text { N-S Approach: }\end{array}$}} & \multicolumn{6}{|c|}{ LAWRENCE LIVERMORE LAB TIS } & \multicolumn{2}{|c|}{ SURVEY DATE: } & DECEM & ER 10, & 2002 & DAY: & MOND & & \\
\hline & & DRIVEW & & & & & & SURVE & CIME: & $7: 00 \mathrm{AM}$ & TO & 5:00 PM & WEAT & ER: SU & NNY & \\
\hline E-W AP & oach: & EAST AV & VUE & & & & & JURISD & TION: & LIVERA & $R E$ & & FILEN & ME: EA & 97LV1T & \\
\hline & & & & THBO & & & TTHBOU & ND & & ASTBOU: & & & ESTBOU & & TOTAL & 4 \\
\hline From & & To & Left & Thru & Righe & Len & Thru & Right & Len & Thru & Right & Left & Thru & Right & YOLUMES & \\
\hline & & & & & & & $L B$ & $P E R I$ & & & & & & & & \\
\hline O7.00AM & $=$ & Oा:I5AM & \% & 0 & $\pi$ & 0 & 0 & 0 & 3 & 29 & 8 & 0 & 97 & $\overline{5}$ & 729 & \\
\hline $\begin{array}{l}\text { 07:15 AM } \\
07: 30 \mathrm{AM}\end{array}$ & 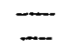 & $\begin{array}{l}07.30 \mathrm{AM} \\
07: 45 \mathrm{AM}\end{array}$ & $\begin{array}{l}0 \\
0 \\
0\end{array}$ & $\stackrel{0}{\circ}$ & $\begin{array}{l}0 \\
0\end{array}$ & $\stackrel{0}{0}$ & $\stackrel{0}{0}$ & $\begin{array}{l}0 \\
0\end{array}$ & $\frac{2}{3}$ & $\begin{array}{l}37 \\
34\end{array}$ & $\begin{array}{l}0 \\
0\end{array}$ & $\begin{array}{l}0 \\
0\end{array}$ & $\begin{array}{l}90 \\
73\end{array}$ & $\begin{array}{l}0 \\
0\end{array}$ & $\begin{array}{l}139 \\
110\end{array}$ & 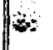 \\
\hline$\frac{07.45 \mathrm{AM}}{08: 00 \mathrm{AM}}$ & + & $\frac{08: 00 \mathrm{AM}}{08: 15 \mathrm{AM}}$ & $\frac{0}{0}$ & $\frac{0}{0}$ & $\frac{0}{0}$ & $\frac{0}{0}$ & $\frac{0}{0}$ & 0 & $\frac{1}{1}$ & $\frac{39}{45}$ & $\frac{0}{0}$ & 0 & $\frac{91}{78}$ & & 33 & \\
\hline $08: 15$ AM & $\overline{-}$ & $\begin{array}{l}\text { 88:15 AMM } \\
08: 30 \mathrm{AM}\end{array}$ & 0 & 0 & 0 & $\begin{array}{l}0 \\
0\end{array}$ & 0 & 0 & $\begin{array}{l}1 \\
0\end{array}$ & $\begin{array}{l}45 \\
37\end{array}$ & $\begin{array}{l}0 \\
0\end{array}$ & $\begin{array}{l}0 \\
0\end{array}$ & $\begin{array}{l}79 \\
70\end{array}$ & 1 & $\begin{array}{l}126 \\
108\end{array}$ & Hs \\
\hline 08:30 AM & - & OB:AS AM & 0 & 0 & 0 & 0 & 0 & 0 & 0 & 34 & 0 & 0 & 54 & 2 & 90 & sis \\
\hline $08: 45 \mathrm{AM}$ & $\cdots$ & $\frac{09: 00 \mathrm{AM}}{09.15 \mathrm{AM}}$ & -0 & -0 & 0 & 0 & $\frac{2}{0}$ & 0 & $\frac{2}{13}$ & 37 & 0 & 0 & 47 & $i$ & 87 & \\
\hline $09.00 \mathrm{AM}$ & $-\cdots$ & $\begin{array}{l}09.15 \mathrm{AM} \\
09.30 \mathrm{AM}\end{array}$ & $\begin{array}{l}0 \\
0\end{array}$ & $\begin{array}{l}0 \\
0\end{array}$ & 0 & 0 & 0 & 0 & 13 & 38 & 0 & 0 & & 0 & 96 & 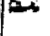 \\
\hline 09:15 AM & $\ldots$ & & $\begin{array}{l}0 \\
0\end{array}$ & $\begin{array}{l}0 \\
0\end{array}$ & 0 & 0 & 0 & 0 & 5 & 26 & 0 & 0 & 43 & 0 & 74 & \\
\hline $09: 30 \mathrm{AM}$ & $\ldots$ & $\begin{array}{l}09: 45 \mathrm{AM} \\
10: 00 \mathrm{AM}\end{array}$ & $\begin{array}{l}0 \\
0\end{array}$ & $\begin{array}{l}0 \\
0\end{array}$ & $\begin{array}{l}0 \\
0\end{array}$ & 0 & 0. & 1 & 2 & $\begin{array}{l}19 \\
19\end{array}$ & $\begin{array}{l}0 \\
0\end{array}$ & $\begin{array}{l}0 \\
0 \\
0\end{array}$ & & 0 & 43 & 4 \\
\hline$\frac{09: 45.4 \mathrm{MM}}{10: 00 \mathrm{AM}}$ & $\ldots$ & $10: 15 \mathrm{AM}$ & 0 & 0 & 0 & $\frac{1}{0}$ & 0 & $\frac{5}{2}$ & $\frac{6}{2}$ & $\frac{19}{21}$ & 0 & 0 & $\frac{34}{23}$ & 1 & $-\sqrt{9}$ & 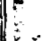 \\
\hline $10: 15 \mathrm{AM}$ & $\ldots$ & $10: 30 \mathrm{AM}$ & 0 & 0 & 0 & 0 & 0 & 1 & 3 & 16 & 0 & 0 & 18 & $i$ & 39 & 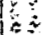 \\
\hline $10: 30 \mathrm{AM}$ & $\underline{-}$ & $10: 45 \mathrm{AM}$ & $\begin{array}{l}0 \\
0\end{array}$ & $\begin{array}{l}0 \\
0\end{array}$ & $\begin{array}{l}0 \\
0\end{array}$ & 0 & 0 & $\begin{array}{l}3 \\
2\end{array}$ & $\begin{array}{l}1 \\
0\end{array}$ & is & 0 & 0 & 18 & $"$ & $4 I$ & \\
\hline$\frac{10: 45 . \mathrm{AM}}{11: 00 \mathrm{AM}}$ & & $\frac{11: 00 \mathrm{AM}}{11: 15 \mathrm{AM}}$ & $\frac{0}{0}$ & 0 & $\frac{0}{0}$ & $\frac{0}{2}$ & $\frac{0}{0}$ & $\frac{2}{3}$ & $\frac{0}{3}$ & $\frac{20}{15}$ & $\frac{0}{0}$ & -0 & $\frac{22}{13}$ & $\frac{1}{2}$ & -45 & \\
\hline $11: 15 \mathrm{AM}$ & $\ldots$ & $11.30 \mathrm{AM}$ & 0 & 0 & 0 & 0 & 0 & 0 & 4 & 17 & 0 & 0 & 33 & i) & st & \\
\hline 11:30 AM & - & $11.45 \mathrm{AM}$ & 0 & $a$ & 0 & 1 & 0 & 4 & 3 & 28 & 0 & 0 & 37 & $"$ & 3 & \\
\hline $11.45 \mathrm{AM}$ & $\ldots$ & $12: 00 \mathrm{PM}$ & 0 & 0 & 0 & 1 & 0 & 3 & 4 & 19 & 0 & 0 & 46 & $\vdots$ & is & \\
\hline $12.6 \mathrm{PM}$ & $\cdots$ & $12.15 \mathrm{PM}$ & 0 & 0 & 0 & 0 & 0 & 3 & 3 & 28 & 0 & $\overline{0}$ & 39 & $=$ & is & \\
\hline 12:15 PM & - & 12:30 PM & 0 & 0 & 0 & 1 & 0 & 2 & 2 & 31 & 0 & 0 & 31 & ii & 67 & \\
\hline 12:30 PM & $\cdots$ & $\begin{array}{l}12.45 \mathrm{PM} \\
0100 \mathrm{PM}\end{array}$ & $\begin{array}{l}0 \\
0\end{array}$ & $\begin{array}{l}0 \\
0\end{array}$ & $\begin{array}{l}0 \\
0\end{array}$ & 1 & 0 & 3 & 2 & 14 & 0 & 0 & 20 & $i$ & II & \\
\hline$-\frac{12: 45 \mathrm{PM}}{01: 00 \mathrm{PM}}$ & $=$ & $\frac{01: 00 \mathrm{PM}}{01: 15 \mathrm{PM}}$ & $\frac{0}{0}$ & 0 & 0 & $-\frac{1}{1}$ & 0 & 6 & $\frac{3}{3}$ & $-\frac{45}{75}$ & 0 & 0 & 34 & $i$ & 90 & \\
\hline $01: 15 \mathrm{PM}$ & $=$ & $\begin{array}{l}01: 15 \mathrm{PM} \\
0.30 \mathrm{PM}\end{array}$ & & 0 & $\begin{array}{l}0 \\
0\end{array}$ & $\begin{array}{l}1 \\
2\end{array}$ & $\begin{array}{l}0 \\
0\end{array}$ & 10 & 3 & 25 & 0 & 0 & 28 & 11 & 67 & \\
\hline $01: 30 \mathrm{PM}$ & 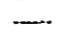 & $01: 45$ PM & 0 & 0 & 0 & 2 & 0 & $\frac{2}{1}$ & 7 & $\begin{array}{l}26 \\
24\end{array}$ & $\begin{array}{l}0 \\
0\end{array}$ & $\begin{array}{l}0 \\
0\end{array}$ & 27 & 3 & 59 & \\
\hline $01: 45$ PM & $\ldots$ & 02:00 PM & 0 & 0 & 0 & 1 & 0 & 3 & 2 & $\begin{array}{r}24 \\
26 \\
\end{array}$ & 0 & 0 & $\begin{array}{l}14 \\
19 \\
\end{array}$ & 3 & $s z$ & \\
\hline $02: 00 \mathrm{PM}$ & $\cdots$ & $02: 15 \mathrm{PM}$ & 0 & 0 & 0 & 3 & 0 & 1 & 1 & 21 & D & 0 & 13 & $=$ & $\pi$ & \\
\hline $02.13 \mathrm{PM}$ & - & $02.30 \mathrm{PM}$ & 0 & 0 & 0 & 0 & 0 & 2 & 1 & 18 & 0 & 0 & 19 & $\therefore$ & so & \\
\hline 02:30 PM & $\cdots$ & 02:45 PM & 0 & 0 & 0 & 2 & 0 & 6 & 4 & 24 & 0 & 0 & 9 & " & 45 & \\
\hline $02: 59 \mathrm{PM}$ & $=$ & $03: 00 \mathrm{PM}$ & 0 & a & 0 & 1 & 0 & 2 & 2 & 23 & 0 & 0 & 21 & $\therefore$ & 49 & \\
\hline $03,00 \mathrm{PM}$ & & $03: 15 \mathrm{PM}$ & 0 & 0 & 0 & 1 & 0 & 6 & 4 & 32 & 0 & 0 & 15 & $i^{-}$ & 58 & \\
\hline 03:1S PMI & $\ldots$ & 03:30 PM & 0 & 0 & 0 & 0 & 0 & 4 & 4 & 48 & 0 & 0 & 22 & $"$ & 8 & \\
\hline $03: 30 \mathrm{PM}$ & - & 03:45 PM & 0 & 0 & 0. & 2 & 0 & 4 & 2 & 61 & 0 & 0 & 17 & 0 & 86 & \\
\hline $03: 45 \mathrm{FM}$ & $\ldots$ & $04: 00 \mathrm{PM}$ & 0 & 0 & $0^{\circ}$ & 1 & 0 & 6 & 0 & 62 & 0 & 0 & 22 & a) & 9 & \\
\hline $04: 00 \mathrm{PM}$ & - & $04: 15 \mathrm{PM}$ & $\overline{0}$ & 0 & 0 & 0 & 0 & 2 & 0 & 63 & 0 & 0 & 20 & 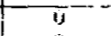 & 85 & \\
\hline OA IS PM & - & $04: 30 \mathrm{PM}$ & 0 & 0 & 0 & 1 & 0 & 0 & 0 & 78 & 0 & 0 & 24 & 0 & 103 & $+\infty$ \\
\hline 04:30 PM & $\ldots$ & 04:45 PM & 0 & 0 & 0 & i & 0 & 0 & 0 & 74 & 0 & 0 & 19 & "1 & Is & 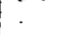 \\
\hline Q4:45 PM & $\ldots$ & $05: 00 \mathrm{PM}$ & 0 & 0 & 0 & 0 & 0 & 0 & 0 & 90 & 0 & 0 & 24 & $\therefore$ & 115 & \\
\hline & & & & & & & $R L Y$ & $T O T A L$ & & & & & & & & " \\
\hline$\overline{07.00 \mathrm{AM}}$ & $=$ & $08600 \mathrm{AM}$ & 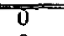 & 0 & $\%$ & $\sigma$ & $\overline{0}$ & 0 & 9 & T39 & 0 & 0 & 35! & $\pi$ & J99 & \\
\hline $07: 15 \mathrm{AM}$ & $\cdots$ & 08:15 AM & 0 & 0 & 0 & 0 & 0 & 0 & 7 & 155 & 0 & 0 & 333 & ! & 396 & $\approx$ \\
\hline $07: 30 \mathrm{AM}$ & $\ldots$ & $08: 30 \mathrm{AM}$ & 0 & 0 & 0 & 0 & 0 & 0 & 5 & $\frac{155}{155}$ & 0 & 0 & 313 & $=$ & $1=5$ & $+\infty$ \\
\hline $07: 45 \mathrm{AM}$ & $=$ & $08.45 \mathrm{AM}$ & 0 & 0 & 0 & 0 & 0 & 0 & 2 & 155 & 0 & 0 & 294 & \pm & is5 & \\
\hline 08:00 AM & $\cdots$ & 09:00.AN. & 0 & 0 & 0 & 0 & 0 & 0 & 3 & 153 & 0 & 0 & 250 & 5 & st! & \\
\hline OS: $15 \mathrm{AM}$ & $\ldots$ & D): 15 AM & 0 & 0 & 0 & 0 & 0 & 0 & 15 & 146 & 0 & 0 & 216 & 1 & 388 & 8 \\
\hline $08: 30 \mathrm{AM}$ & $\cdots$ & $09: 30 \mathrm{AM}$ & -0 & 0 & 0 & 0 & 0 & 0 & 20 & 135 & 0 & 0 & 189 & 3 & 35 & \\
\hline 08:45 AM & - & $09: 45 \mathrm{AM}$ & 0 & 0 & 0 & 0 & 0 & i & 22 & 120 & 0 & 0 & 156 & 1 & 300 & 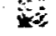 \\
\hline $09: 00 \mathrm{AM}$ & $=$ & $1000 \mathrm{AM}$ & 0 & 0 & 0 & 0 & 0 & $i$ & 22 & 102 & 0 & 0 & 143 & $\because$ & 268 & \\
\hline $09: 15 \overline{A M}$ & $=$ & $10.15 \mathrm{AM}$ & $\overline{0}$ & 0 & o & 0 & 0 & 3 & II & 85 & $\overline{0}$ & 0 & 121 & $T$ & 328 & \\
\hline 09:30 AM & $\cdots$ & $10: 30 \mathrm{AM}$ & 0 & 0 & 0 & 0 & 0 & 4 & 9 & 75 & 0 & 0 & 96 & $\vdots$ & 186 & \\
\hline $09: 45 \mathrm{AM}$ & - & $10: 45 \mathrm{AM}$ & 0 & 0 & 0 & 0 & $a$ & $\frac{6}{8}$ & 8 & 75 & 0 & 0 & 93 & & tos & 8 \\
\hline $10: 00 \mathrm{AM}$ & $=$ & 11:00 AM & $\begin{array}{l}0 \\
0\end{array}$ & $\begin{array}{l}0 \\
0\end{array}$ & $\begin{array}{l}0 \\
0\end{array}$ & $\begin{array}{l}0 \\
2\end{array}$ & 0 & 8 & 6 & $\begin{array}{l}76 \\
70\end{array}$ & $\begin{array}{l}0 \\
0\end{array}$ & $\begin{array}{l}0 \\
0\end{array}$ & 81 & 3 & 174 & 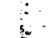 \\
\hline $\begin{array}{l}10: 19 \mathrm{AM} \\
10: 30 \mathrm{AM}\end{array}$ & $=$ & $\begin{array}{l}11: 15 \mathrm{AM} \\
11: 30 \mathrm{AM}\end{array}$ & $\begin{array}{l}0 \\
0 \\
\end{array}$ & $\begin{array}{l}0 \\
.0 \\
\end{array}$ & $\begin{array}{l}0 \\
0 \\
\end{array}$ & $\begin{array}{l}2 \\
2 \\
\end{array}$ & $\begin{array}{l}0 \\
0 \\
\end{array}$ & $\begin{array}{l}9 \\
8 \\
\end{array}$ & $\begin{array}{l}7 \\
8 \\
\end{array}$ & $\begin{array}{l}70 \\
71\end{array}$ & $\begin{array}{l}0 \\
0\end{array}$ & $\begin{array}{l}0 \\
0 \\
\end{array}$ & $\begin{array}{l}11 \\
86 \\
\end{array}$ & 3 & $\begin{array}{l}163 \\
788 \\
9\end{array}$ & 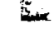 \\
\hline $10: 45 \mathrm{AM}$ & $=$ & $\frac{11: 30 \mathrm{AM}}{11.45 \mathrm{AM}}$ & 0 & 0 & - & 3 & 0 & 9 & 10 & 80 & 0 & 0 & 105 & -3 & 210 & \\
\hline $11: 00 \mathrm{AM}$ & $\cdots$ & $12: 00 \mathrm{PM}$ & 0 & 0 & 0 & 4 & 0 & 10 & 14 & 79 & 0 & 0 & 129 & 3 & 139 & \\
\hline $11: 15 \mathrm{AM}$ & $\ldots$ & 12:15 PM & 0 & 0 & 0 & 2 & 0 & 10 & 14 & 92 & 0 & 0 & 155 & 3 & 276 & 5 \\
\hline $11: 30 \mathrm{AM}$ & $\cdots$ & $12: 30 \overline{\mathrm{PM}}$ & 0 & 0 & 0 & 3 & 0 & 12 & 12 & 106 & 0 & 0 & 153 & 3 & 289 & \\
\hline $11: 45$ AM & - & 12:45 PM & 0 & 0 & $\begin{array}{l}0 \\
0\end{array}$ & 3 & $\begin{array}{l}0 \\
0\end{array}$ & 11 & 11 & $\begin{array}{c}92 \\
118\end{array}$ & $\begin{array}{l}0 \\
0\end{array}$ & $\begin{array}{l}0 \\
0\end{array}$ & $\begin{array}{l}136 \\
124\end{array}$ & 4 & 257 & 3 \\
\hline 12:00 PM & $=$ & O1:00 PM & -0 & $\frac{0}{0}$ & $\frac{0}{0}$ & $\frac{3}{4}$ & $\frac{0}{0}$ & $\frac{14}{21}$ & $\frac{10}{10}$ & $\frac{118}{115}$ & $\frac{0}{0}$ & $\frac{0}{0}$ & $\frac{324}{113}$ & $\frac{4}{2}$ & $\frac{131}{365}$ & \\
\hline $\begin{array}{l}12: 15 \mathrm{PM} \\
12: 10 \mathrm{PM}\end{array}$ & 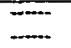 & $\begin{array}{l}01: 15 \mathrm{PM} \\
01: 30 \mathrm{PM}\end{array}$ & $\begin{array}{l}0 \\
0\end{array}$ & $\begin{array}{l}0 \\
0\end{array}$ & $\begin{array}{l}0 \\
0\end{array}$ & 5 & $\begin{array}{l}0 \\
0\end{array}$ & $\begin{array}{l}21 \\
21\end{array}$ & 9 & 110 & 0 & 0 & 109 & $\overline{3}$ & 257 & $r$ \\
\hline $\begin{array}{l}12: 10 \mathrm{PM} \\
12: 45 \mathrm{PM} \\
\end{array}$ & $=$ & $\begin{array}{l}0: 10 \mathrm{PM} \\
0 \mathrm{O}: 4 \mathrm{PM}\end{array}$ & 0 & 0 & 0 & 6 & 0 & 19 & 14 & 120 & 0 & 0 & 103 & $\leq$ & 367 & \\
\hline $01.00 \mathrm{PM}$ & $\infty$ & $02: 00 \mathrm{PM}$ & 0 & $\overline{0}$ & 0 & 6 & 0 & 16 & 13 & 101 & 0 & 0 & 88 & 3 & 219 & \\
\hline 01:15 PM & $\ldots$ & 02:15 PM & 0 & $\therefore$ & $\therefore$ & $\begin{array}{l}8 \\
6\end{array}$ & 0 & $?$ & il & $\begin{array}{l}97 \\
89\end{array}$ & $\begin{array}{l}0 \\
0\end{array}$ & $\begin{array}{l}0 \\
0\end{array}$ & $\begin{array}{l}73 \\
65\end{array}$ & 6 & $\begin{array}{l}203 \\
184\end{array}$ & 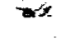 \\
\hline$\frac{01.30 \mathrm{PM}}{01: 45 \mathrm{PM}}$ & $=$ & $\frac{02: 30 \mathrm{PM}}{02: 45 \mathrm{PM}}$ & & $\frac{0}{0}$ & $\frac{0}{0}$ & $\frac{6}{6}$ & $\frac{0}{0}$ & $\frac{7}{12}$ & $\frac{11}{8}$ & $\frac{89}{89}$ & $\frac{0}{0}$ & $\frac{0}{0}$ & $-\frac{63}{60}$ & $\frac{0}{3}$ & $\frac{60}{178}$ & \\
\hline $\begin{array}{l}01: 45 \mathrm{PM} \\
02: 00 \mathrm{PM}\end{array}$ & $=$ & $\begin{array}{l}\text { 02:45 PM } \\
03: 00 \mathrm{PM}\end{array}$ & $\begin{array}{l}0 \\
0\end{array}$ & 0 & $\begin{array}{l}0 \\
0\end{array}$ & $\begin{array}{l}6 \\
6\end{array}$ & 0 & 11 & $\begin{array}{l}8 \\
8\end{array}$ & 86 & 0 & 0 & 62 & 3 & i7s & E: \\
\hline $02: 15 \mathrm{PM}$ & $-\cdots$ & $03: 15$ PM & 0 & 0 & 0 & 4 & 0 & 16 & 11 & 97 & 0 & 0 & 64 & 0 & 192 & 8 \\
\hline $02: 30 \mathrm{PM}$ & $=$ & 03:30 PM & 0 & 0 & 0 & 4 & 0 & 18 & 14 & 127 & 0 & 0 & 67 & 0 & 330 & \\
\hline $02: 45$ PM & - & 03:45 PM & $a$ & 0 & 0 & 4 & $\stackrel{0}{a}$ & 16 & 12 & $\begin{array}{l}164 \\
203\end{array}$ & $\begin{array}{l}0 \\
0\end{array}$ & $\begin{array}{l}0 \\
0\end{array}$ & $\begin{array}{l}75 \\
76\end{array}$ & $\begin{array}{l}0 \\
0\end{array}$ & $\begin{array}{l}371 \\
3 ! 3\end{array}$ & - \\
\hline 03:00 PM & $=$ & 04:00 PM & $\frac{0}{0}$ & $\frac{0}{0}$ & 0 & $\frac{4}{3}$ & $\frac{0}{0}$ & $-\frac{20}{16}$ & $\frac{10}{6}$ & $-\frac{203}{234}$ & 0 & 0 & $\frac{10}{81}$ & & & \\
\hline $03: 15$ PM & $=$ & $\begin{array}{l}04: 13 \text { PM } \\
04.10 P M\end{array}$ & $\begin{array}{l}0 \\
0\end{array}$ & $\begin{array}{l}0 \\
0 \\
0\end{array}$ & $\begin{array}{l}0 \\
0\end{array}$ & $\begin{array}{l}3 \\
4\end{array}$ & $\begin{array}{l}0 \\
0\end{array}$ & 12 & $\begin{array}{l}6 \\
2\end{array}$ & $\begin{array}{l}234 \\
264\end{array}$ & 0 & 0 & 83 & 0 & 365 & \\
\hline $\begin{array}{l}03: 30 \mathrm{PM} \\
03: 45 \mathrm{PM}\end{array}$ & $\overline{-}$ & $\begin{array}{l}\text { 04:30 PM } \\
04: 45 \text { PM }\end{array}$ & 0 & 0 & 0 & 3 & 0 & 8 & 0 & 277 & 0 & 0 & 85 & 0 & $3: 3$ & (3) \\
\hline $04.00 \mathrm{PM}$ & $=$ & $05: 00 \mathrm{PM}$ & 0 & 8 & 0 & 2 & 0 & 2 & $\gamma$ & 305 & 0 & 0 & 87 & 0 & 396 & $\$$ \\
\hline$s$ & $E X$ & ULA: & 18 & 17 & & 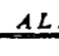 & $D \lambda$ & 1012 & 2292 & & cos & $c$ & $: 15$ & 2 & & \\
\hline
\end{tabular}




\section{Baymetrics Traffic Resources INTERSECTION TURNING MOVEMENT SUMMARY}

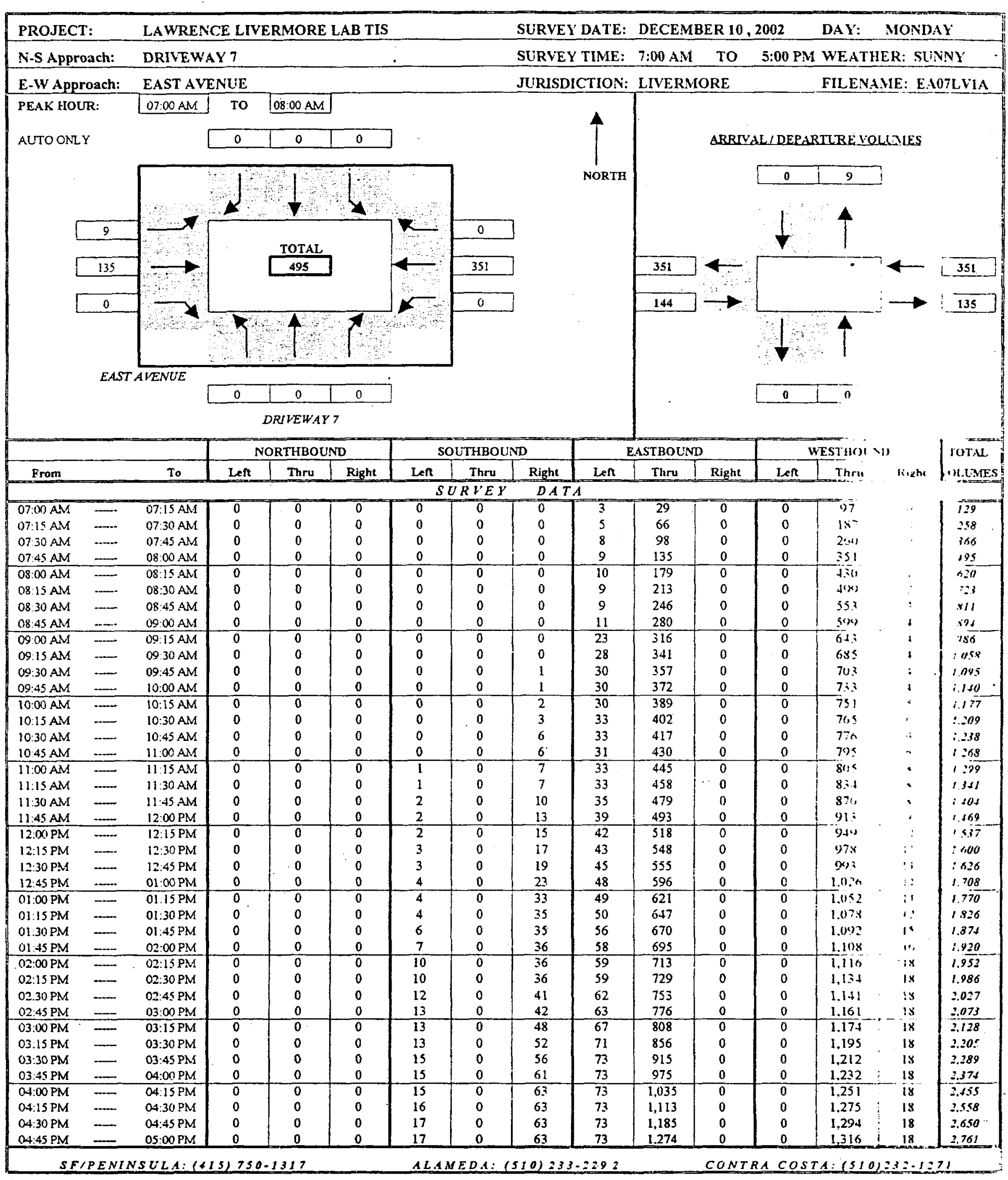




\section{Baymetrics Traffic Resources INTERSECTION TURNING MOVEMENT SUMMARY}

\begin{tabular}{|c|c|c|c|c|c|c|c|c|c|c|c|c|c|c|c|}
\hline \multirow{2}{*}{\multicolumn{2}{|c|}{$\begin{array}{l}\text { PROJECT: } \\
\text { N-S Approach: }\end{array}$}} & \multicolumn{6}{|c|}{ LAWRENCE LIVERMORE LAB TIS } & \multicolumn{2}{|c|}{ SURVEY DATE } & DECEN & ER 10, & 2002 & DAX: & \multicolumn{2}{|c|}{ MONDAY } \\
\hline & & DRIVEY & & & & & & SURVE & CIME: & $7: 00 \mathrm{AN}$ & TO & 5:00 PN & WEAT & IER: SU! & VNY \\
\hline E.W Ap & oach: & EAST AI & IUE & & & & & JURISD & TION: & LIVER: & ORE & & FILEN & ME: EA & 07LV1A \\
\hline & & & & THBO & & & THBO & & & ASTBOU & & & ESTBOU & & TOTAL \\
\hline From & & To & Len & Thru & Right & Left & Thru & Right & Len & Thru & Right & Len & Thru & Right & boluMres \\
\hline & & & & & & & $L B$ & $P E R I$ & & & & & & & \\
\hline D7:00लM & $\ldots$ & OT:ISAM & $\overline{0}$ & $\pi$ & 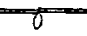 & 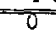 & 0 & 0 & 3 & 29 & 0 & $\pi$ & 97 & $\pi$ & T29 \\
\hline $\begin{array}{l}07: 15 \mathrm{AM} \\
07: 30 \mathrm{AM}\end{array}$ & $\ldots$ & $\begin{array}{l}07: 30 \mathrm{AM} \\
07.45 \mathrm{AM}\end{array}$ & $\begin{array}{l}0 \\
0\end{array}$ & $0_{0}^{0}$ & 0 & $\begin{array}{l}0 \\
0\end{array}$ & 0 & 0 & 2 & 37 & 0 & 0 & 90 & 0 & 129 \\
\hline $\begin{array}{l}07.30 \mathrm{AM} \\
07.45 \mathrm{AM}\end{array}$ & $\ldots$ & $\begin{array}{l}\text { 7:7:45 AM } \\
\text { O8:00 AMM }\end{array}$ & 0 & $\begin{array}{l}0 \\
0 \\
\end{array}$ & 0 & $\begin{array}{l}0 \\
0\end{array}$ & $\begin{array}{l}0 \\
0\end{array}$ & 0 & $\frac{3}{1}$ & $\begin{array}{l}32 \\
37\end{array}$ & $\begin{array}{l}0 \\
0\end{array}$ & $\begin{array}{l}0 \\
0\end{array}$ & $\begin{array}{l}73 \\
9 ?\end{array}$ & $\begin{array}{l}0 \\
0\end{array}$ & ${ }_{x 28}^{208}$ \\
\hline $08.00 \mathrm{AM}$ & $\cdots$ & $08: 15$ AM & 0 & 0 & 0 & 0 & 0 & 0 & 1 & 44 & 0 & 0 & 79 & T & 125 \\
\hline $\begin{array}{l}\text { 08:15 A.M } \\
08: 30 \mathrm{AM}\end{array}$ & $\ldots$ & $\begin{array}{l}\text { 08:30 AM } \\
08: 45 \mathrm{AM}\end{array}$ & $\begin{array}{l}0 \\
0\end{array}$ & $\begin{array}{l}0 \\
0\end{array}$ & $\begin{array}{l}0 \\
0\end{array}$ & $\begin{array}{l}0 \\
0\end{array}$ & $\begin{array}{l}0 \\
0\end{array}$ & $\begin{array}{l}0 \\
0\end{array}$ & 0 & $\begin{array}{l}34 \\
33\end{array}$ & $\begin{array}{l}0 \\
0\end{array}$ & $\begin{array}{l}0 \\
0\end{array}$ & $\begin{array}{l}69 \\
54\end{array}$ & i & $\begin{array}{l}103 \\
88\end{array}$ \\
\hline $08: 45$ AM & $\ldots$ & $0900.4 M$ & 0 & 0 & 0 & 0 & 0 & 0 & 2 & 34 & 0 & $\begin{array}{l}0 \\
0\end{array}$ & 46 & $i$ & 8 \\
\hline $\begin{array}{l}09: 00 \overline{A M} \\
09.15 \mathrm{AM}\end{array}$ & $=$ & $09: 15 \mathrm{AM}$ & 0 & 0 & a & 0 & 0 & 0 & 12 & 36 & 0 & 0 & 44 & 0 & 92 \\
\hline $\begin{array}{l}09: 15 \mathrm{AM} \\
09: 30 \mathrm{AM}\end{array}$ & $\ldots$ & $09: 30 \mathrm{AM}$ & $\begin{array}{l}0 \\
0\end{array}$ & 0 & 0 & $\begin{array}{l}0 \\
0\end{array}$ & 0 & 0 & 5 & 25 & 0 & 0 & 42 & 0 & 2 \\
\hline $09: 45 \mathrm{AM}$ & $=$ & $10: 00 \mathrm{AM}$ & 0 & 0 & $\begin{array}{l}0 \\
0 \\
0\end{array}$ & $\begin{array}{l}0 \\
0\end{array}$ & 0 & $\begin{array}{l}1 \\
0\end{array}$ & $\begin{array}{l}2 \\
0\end{array}$ & $\begin{array}{l}16 \\
15\end{array}$ & $\begin{array}{l}0 \\
0\end{array}$ & $\begin{array}{l}0 \\
0\end{array}$ & $\begin{array}{l}18 \\
30\end{array}$ & $\begin{array}{l}0 \\
0\end{array}$ & $\begin{array}{l}37 \\
45\end{array}$ \\
\hline $10: 00 \mathrm{AN}$ & $=$ & $10.15 \mathrm{AM}$ & 0 & 0 & 0 & 0 & 0 & $\frac{T}{1}$ & 0 & 17 & 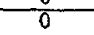 & 0 & 18 & $\frac{1}{1}$ & 37 \\
\hline $10: 15 \mathrm{AM}$ & $\ldots$ & $10: 30 \mathrm{AM}$ & 0 & 0 & 0 & 0 & 0 & i & 3 & 13 & 0 & 0 & 14 & $i$ & 32 \\
\hline $10: 30$ AM & $\ldots$ & $10: 45.9 M$ & 0 & 0 & 0 & 0 & 0 & 3 & 0 & 15 & 0 & 0 & 11 & $\therefore$ & 29 \\
\hline $10: 45 \mathrm{AM}$ & 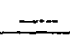 & 11:00 AM & 0 & 0 & 0 & 0 & 0 & 0 & (2) & 13 & 0 & 0 & 19 & $"$ & 30 \\
\hline $11: 00 \mathrm{AM}$ & $=$ & $11: 15 \times M$ & 0 & 0 & 8 & 1 & 0 & $T$ & 2 & -15 & 0 & 0 & 10 & & $3 j$ \\
\hline $11,15 \mathrm{AM}$ & $\ldots$ & $11: 30 \mathrm{AM}$ & 0 & 0 & 0 & 0 & 0 & 0 & 0 & 13 & 0 & 0 & 29 & $\overline{i i}$ & 42 \\
\hline $\begin{array}{l}11.30 \mathrm{AM} \\
11.45 \mathrm{AM}\end{array}$ & $\cdots$ & $11.45 \mathrm{AM}$ & $\begin{array}{l}0 \\
0\end{array}$ & 0 & 8 & 1 & 0 & 3 & 2 & 21 & 0 & 0 & 36 & $\because$ & 63 \\
\hline$\frac{114.49}{12: 00 \mathrm{PM}}$ & $\ldots$ & $\frac{12: 00 \mathrm{PM}}{12: 15 \mathrm{PM}}$ & -0 & $-\frac{0}{0}$ & $\frac{0}{0}$ & 0 & 0 & $\frac{3}{2}$ & $\frac{4}{3}$ & $\frac{14}{25}$ & $\frac{0}{0}$ & $\frac{0}{0}$ & $-\frac{43}{36}$ & $?$ & $\frac{65}{68}$ \\
\hline $12: 15 \mathrm{PM}$ & $\ldots$ & $12: 20 \mathrm{PM}$ & 0 & 0 & 0 & 1 & 0 & 2 & 1 & 30 & 0 & 0 & 29 & $\bar{i}$ & 63 \\
\hline $12: 30 \mathrm{PM}$ & - & $12.45 \mathrm{PM}$ & 0 & 0 & $\begin{array}{l}0 \\
0\end{array}$ & 0 & 0 & $\begin{array}{l}2 \\
4\end{array}$ & 2. & 7 & 0 & 0 & 15 & $\because$ & 26 \\
\hline $01: 00 \mathrm{PM}$ & $=$ & $\frac{0100 \mathrm{MM}}{01: 15 \mathrm{PM}^{-}}$ & 0 & 0 & 0 & $\frac{1}{0}$ & $\frac{0}{0}$ & $\frac{4}{10}$ & $\frac{3}{1}$ & $\frac{41}{25}$ & $\frac{0}{0}$ & $\frac{0}{0}$ & $-\frac{3}{2} 6$ & 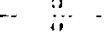 & $\frac{82}{62}$ \\
\hline 01.15 PM & - & $01: 30 \mathrm{PM}$ & 0 & 0 & 0 & 0 & 0 & 2 & 1 & 26 & 0 & 0 & 26 & : & só \\
\hline 01:30 PM & $\ldots$ & 01:45 PM & 0 & 0 & 0 & 2 & 0 & 0 & 6 & 23 & 0 & 0 & 4 & 3 & 48 \\
\hline 01:45 PM & $\ldots$ & 02:00 PM & 0 & 0 & 0 & 1 & 0 & 1 & 2 & 25 & 0 & 0 & 16 & $\vdots$ & 6 \\
\hline $02.00 \mathrm{PM}$ & $\omega$ & $02: 15 \mathrm{PM}$ & 0 & 0 & 0 & 3 & 0 & o & $\frac{2}{1}$ & 18 & 0 & 0 & -8 & $=$ & -5 \\
\hline $02.15 \mathrm{PM}$ & $\ldots$ & $02: 30 \mathrm{PM}$ & 0 & 0 & 0 & 0 & 0 & 0 & 0 & 16 & 0 & 0 & is & $\bar{z}$ & is \\
\hline $02: 30 \mathrm{PM}$ & $\ldots$ & $02: 45 \mathrm{PM}$ & 0 & 0 & 0 & 2 & 0 & $s$ & 3 & 24 & 0 & 0 & & $\therefore$ & Al \\
\hline $02: 45$ PM & $\ldots$ & $03.00 \mathrm{PM}$ & 0 & 0 & 0 & i & 0 & $i$ & 1 & 23 & 0 & -0 & 20 & $\because$ & 16 \\
\hline $03: 00 \mathrm{PM}$ & $\ldots$ & $03: 15 \mathrm{PM}$ & 0 & 0 & 0 & $\frac{1}{0}$ & 0 & $\frac{1}{6}$ & $\frac{1}{4}$ & $\frac{.5}{32}$ & 0 & $=0$ & $T 3$ & $\vdots$ & 55 \\
\hline $03: 15 \mathrm{PM}$ & $\ldots$ & $03: 30 \mathrm{PM}$ & 0 & 0 & 0 & 0 & 0 & 4 & 4 & 48 & 0 & 0 & $=1$ & & $\cdots$ \\
\hline $03: 30$ PM & $\ldots$ & $03.45 \mathrm{PM}$ & 0 & 0 & 0 & 2 & 0 & 4 & 2 & 59 & 0 & 0 & 17 & ." & 84 \\
\hline 03:45 PM & $\ldots$ & $04: 00 \mathrm{PM}$ & 0 & 0 & 0 & 0 & 0 & 5 & 0 & 60 & 0 & 0 & 20 & $\therefore$ & 85 \\
\hline $04.00 \mathrm{PM}$ & $=$ & $04.15 \mathrm{PM}$ & $\frac{1}{0}$ & 0 & 0 & 0 & 0 & 2 & 0 & 60 & 0 & 0 & $\frac{-0}{19}$ & a & 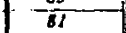 \\
\hline 04:15 PM & $\longrightarrow$ & $04: 30 \mathrm{PM}$ & 0 & 0 & 0 & $i$ & 0 & 0 & 0 & 78 & 0 & 0 & 2.4 & $!$ & 103 \\
\hline 04:30 PM & 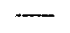 & 04:45 PM & 0 & 0 & 0 & 1 & 0 & 0 & 0 & 72 & 0 & 0 & 19 & $\because$ & 92 \\
\hline $04.45 \mathrm{PM}$ & $=$ & $05: 00 \mathrm{PM}$ & 0 & 0 & 0 & $\frac{0}{4}$ & $\frac{0}{R I Y}$ & $\frac{0}{T O T A}$ & 0 & 89 & 0 & 9 & $=2$ & $\because$ & 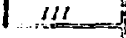 \\
\hline & & & & & & & & TOTAL & & & & & & & \\
\hline $67.00 .9 \mathrm{M}$ & $\cdots$ & $08.00 \mathrm{AN}$ & 8 & 0 & $\overline{0}$ & $\bar{\sigma}$ & 0 & 0 & $\overline{9}$ & $\sqrt{35}$ & 0 & $\pi$ & उड़ा & $\because$ & s3s \\
\hline 07:15 AM & $\ldots$ & $08: 15 \mathrm{AM}$ & 0 & 0 & 0 & 0 & 0 & 0 & 7 & 150 & 0 & 0 & 333 & 1 & 991 \\
\hline $07: 30 \mathrm{AM}$ & $\ldots$ & 08:30 AM & 0 & 0 & 0 & 0 & 0 & 0 & 4 & 147 & 0 & 0 & $31=$ & : & sas \\
\hline $07.45 \mathrm{AM}$ & $\ldots$ & $08.45 \mathrm{AM}$ & 0 & 0 & 0 & 0 & 0 & $\frac{5}{0}$ & 1 & 148 & 0 & $\tilde{0}$ & $-\frac{2}{39} \overline{3}$ & i & 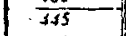 \\
\hline $08: 00 \mathrm{AM}$ & $\ldots$ & $09: 00 \mathrm{AM}$ & 0 & 0 & 0 & 0 & 0 & 0 & 2 & 145 & 0 & 0 & 2.18 & : & 398 \\
\hline 08:15 AM & $\ldots$ & $09: 15$ AM & 0 & 0 & 0 & 0 & 0 & 0 & 13 & 137 & 0 & 0 & 213 & $\vdots$ & 360 \\
\hline $08: 30 \mathrm{AM}$ & $=$ & $09: 30 . \mathrm{AM}$ & 0 & 0 & 0 & 0 & 0 & 0 & 19 & 128 & 0 & -0 & 186 & $:$ & $j 5$ \\
\hline 08:4S AM & $\ldots$ & $09: 45 \mathrm{AM}$ & 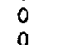 & $\begin{array}{l}0 \\
0\end{array}$ & 0 & $\begin{array}{l}0 \\
0\end{array}$ & $\begin{array}{l}0 \\
0\end{array}$ & 1 & 21 & 111 & $\begin{array}{l}0 \\
0\end{array}$ & $\begin{array}{l}0 \\
0\end{array}$ & isto & $\vdots$ & 284 \\
\hline$\frac{09: 00 \mathrm{AM}}{09: 15 \mathrm{AM}}$ & $=$ & $\frac{10: 00 \mathrm{AM}}{10.15 \mathrm{AM}}$ & -0 & $\frac{0}{0}$ & $\frac{0}{0}$ & $\frac{0}{0}$ & $\frac{0}{0}$ & $\frac{1}{2}$ & $\frac{19}{7}$ & $-\frac{92}{73}$ & $\frac{0}{0}$ & $\frac{0}{0}$ & $\frac{134}{1018}$ & $i$ & $\frac{256}{191}$ \\
\hline $09: 30 \mathrm{AM}$ & - & $10: 30 \mathrm{AM}$ & 0 & 0 & 0 & 0 & 0 & 3 & 5 & 61 & 0 & 0 & 80 & $\vdots$ & ISI \\
\hline 09:45 AM & $\ldots$ & $10: 45 \mathrm{AM}$ & 0 & 0 & 0 & 0 & 0 & $\frac{5}{5}$ & $-\frac{3}{1}$ & $-\frac{60}{58}$ & $\frac{0}{0}$ & $\frac{0}{0}$ & $\frac{73}{62}$ & $\vdots$ & $\frac{183}{128}$ \\
\hline $\begin{array}{l}1000 \mathrm{AMM} \\
1015 \mathrm{AM}\end{array}$ & $=$ & $\begin{array}{l}11: 00 \mathrm{AM} \\
11: 15 \mathrm{AM}\end{array}$ & $\begin{array}{l}0 \\
0\end{array}$ & $\begin{array}{l}0 \\
0\end{array}$ & $\begin{array}{l}0 \\
0\end{array}$ & $\begin{array}{l}0 \\
1\end{array}$ & $\begin{array}{l}0 \\
0\end{array}$ & $\begin{array}{l}5 \\
5\end{array}$ & $\frac{1}{3}$ & $\begin{array}{l}58 \\
56\end{array}$ & 0 & $\begin{array}{l}0 \\
0\end{array}$ & 5.2 & $\vdots$ & $\begin{array}{l}128 \\
123\end{array}$ \\
\hline $10: 30 \mathrm{AM}$ & 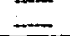 & 11:30 AM & 0 & $\begin{array}{l}0 \\
0\end{array}$ & 0 & 1 & 0 & 4 & 0 & 56 & 0 & 0 & 69 & $:$ & 132 \\
\hline $10: 45 \mathrm{AM}$ & $\ldots$ & $11.45 \mathrm{AM}$ & 0 & 0 & 0 & 2 & 0 & $\frac{7}{4}$ & 2 & 62 & 0 & 0 & 94 & $\vdots$ & 160 \\
\hline $11: 00 \mathrm{AM}$ & $\ldots$ & $12: 00 \mathrm{PM}$ & 0 & 0 & 0 & 2 & 0 & 7 & 8 & 63 & 0 & 0 & 118 & $i$ & 301 \\
\hline $11: 15 \mathrm{AM}$ & $\ldots$ & 12:15 PM & 0 & 0 & 0 & 1 & 0 & 8 & 9 & 73 & 0 & 0 & 114 & $i$ & 38 \\
\hline $11: 30 \mathrm{AM}$ & $\ldots$ & $12: 30 \mathrm{PM}$ & 0 & 0 & 0 & 2 & 0 & 10 & 10 & 90 & 0 & 0 & 1.1.1. & : & 259 \\
\hline $11: 45 \mathrm{AM}$ & $\ldots$ & $12: 45 \mathrm{PM}$ & 0 & 0 & 0 & 1 & 0 & 9 & 10 & 76 & 0 & 0 & $i \geq 3$ & 1 & $=2$ \\
\hline$\frac{12: 00 \mathrm{PM}}{12: 5 \mathrm{PM}}$ & $\ldots$ & $\frac{0100 \mathrm{PM}}{0.15 \mathrm{PM}}$ & $\frac{0}{0}$ & $\frac{0}{0}$ & 0 & $\frac{2}{2}$ & $\frac{0}{0}$ & $\frac{10}{18}$ & -9 & $\frac{103}{103}$ & $\frac{0}{0}$ & $\frac{0}{0}$ & $\frac{113}{103}$ & $\because 1$ & $-\frac{239}{2.33}$ \\
\hline $12.30 \mathrm{PM}$ & $=$ & $01: 30 \mathrm{PM}$ & 0 & 0 & 0 & 1 & $\begin{array}{l}0 \\
0\end{array}$ & $\begin{array}{l}18 \\
18\end{array}$ & $\begin{array}{l}7 \\
7\end{array}$ & $\begin{array}{l}1103 \\
99\end{array}$ & $\begin{array}{l}0 \\
0\end{array}$ & $\begin{array}{l}0 \\
0\end{array}$ & $\begin{array}{l}103 \\
100\end{array}$ & 1 & 236 \\
\hline $12: 43 \mathrm{PM}$ & $\ldots$ & $01.45 \mathrm{PM}$ & 0 & 0 & 0 & 3 & 0 & 16 & 11 & 115 & 0 & 0 & 99 & 4 & 238 \\
\hline$\frac{12.3 \mathrm{PM}}{01: 00 \mathrm{PM}}$ & $=$ & $02: 00 \mathrm{PM}$ & 0 & $\frac{0}{0}$ & 0 & 3 & 0 & 13 & 10 & 99 & 0 & 0 & 82 & 5 & 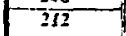 \\
\hline O1:15 PM & $\cdots$ & $02: 15 \mathrm{PM}$ & 0 & 0 & 0 & 6 & 0 & 3 & 10 & 92 & 0 & 0 & 64 & 6 & 182 \\
\hline $01: 30 \mathrm{PM}$ & $=$ & 02:30 PM & 0 & -0 & $\frac{0}{0}$ & 6 & 0 & $\frac{1}{6}$ & $\frac{9}{6}$ & $-\frac{82}{83}$ & $-\frac{0}{0}$ & $\frac{0}{0}$ & $\frac{56}{49}$ & $-\frac{6}{3}$ & $\frac{160}{153}$ \\
\hline $\begin{array}{l}01: 15 \mathrm{PM} \\
02: 00 \mathrm{PM}\end{array}$ & $\overline{-}$ & $\begin{array}{l}02: 45 \mathrm{PM} \\
03: 00 \mathrm{PM}\end{array}$ & $\begin{array}{l}0 \\
0\end{array}$ & $\begin{array}{l}0 \\
0\end{array}$ & $\begin{array}{l}0 \\
0\end{array}$ & $\begin{array}{l}6 \\
6\end{array}$ & $\begin{array}{l}0 \\
0\end{array}$ & $\begin{array}{l}6 \\
6\end{array}$ & $\begin{array}{l}6 \\
5\end{array}$ & $\begin{array}{l}83 \\
81\end{array}$ & $\begin{array}{l}0 \\
0\end{array}$ & $\begin{array}{l}0 \\
0\end{array}$ & 53 & 2 & iss \\
\hline $\begin{array}{l}02: 00 \mathrm{PM} \\
02: 13 \mathrm{PM} \\
\end{array}$ & - & $\begin{array}{l}03: 00 \mathrm{PM} \\
03: 15 \mathrm{PM} \\
\end{array}$ & 0 & 0 & 0 & 3 & 0 & 12 & 8 & 95 & 0 & 0 & 58 & $n$ & 176 \\
\hline $02: 30 \mathrm{PM}$ & $\cdots$ & $03.30 \mathrm{PM}$ & 0 & 0 & 0 & 3 & 0 & 16 & 12 & 127 & 0 & 0 & 61 & $\pi$ & 219 \\
\hline 02:45 PM & $=$ & 03:45 PM & $\begin{array}{l}0 \\
0\end{array}$ & $\begin{array}{l}0 \\
0\end{array}$ & $\begin{array}{l}0 \\
0\end{array}$ & $\frac{3}{2}$ & $\begin{array}{l}0 \\
0\end{array}$ & $\begin{array}{l}15 \\
19\end{array}$ & $\begin{array}{l}11 \\
10\end{array}$ & $\begin{array}{l}162 \\
199\end{array}$ & $\begin{array}{l}0 \\
0\end{array}$ & $\stackrel{0}{0}$ & $\begin{array}{l}31 \\
71\end{array}$ & $\begin{array}{l}0 \\
0 \\
0\end{array}$ & $\begin{array}{l}262 \\
301\end{array}$ \\
\hline$\frac{03: 00 P M}{03: 13 P M}$ & $\equiv$ & $\frac{04: 00 \mathrm{PM}}{0.15 \mathrm{PM}}$ & $\frac{0}{0}$ & $-\frac{1}{0}$ & $\frac{0}{0}$ & $\frac{2}{2}$ & 0 & -19 & $\frac{10}{6}$ & $-\frac{197}{227}$ & 0 & $\frac{0}{0}$ & $\frac{11}{77}$ & ij & $\frac{301}{327}$ \\
\hline $\begin{array}{l}\text { 03:130 PMM } \\
\text { 03: }\end{array}$ & $=$ & 04:30 PM & 0 & 0 & 0 & $\frac{2}{3}$ & 0 & 11 & 2 & 257 & 0 & 0 & 80 & 0 & 353 \\
\hline 03:45 PM & $\longrightarrow$ & $04: 45 \mathrm{PM}$ & 0 & 0 & 0 & 2 & 0 & 7 & 0 & 270 & 0 & 0 & 82 & 0 & 361 \\
\hline $04: 00 \mathrm{PM}$ & $=$ & $05: 00 \mathrm{PM}$ & 0 & 0 & & 2 & 0 & 2 & 0 & 299 & 0 & 0 & 84 & a & 387 \\
\hline si & $E N I$ & $U L A: 1$ & 7 & 7 & & $A L$ & $D A$ & $(0)=$ & 292 & & 01 & $c$ & 15 & $32-$ & \\
\hline
\end{tabular}




\section{Baymetrics Traffic Resources INTERSECTION TURNING MOVEMENT SUMMARY}

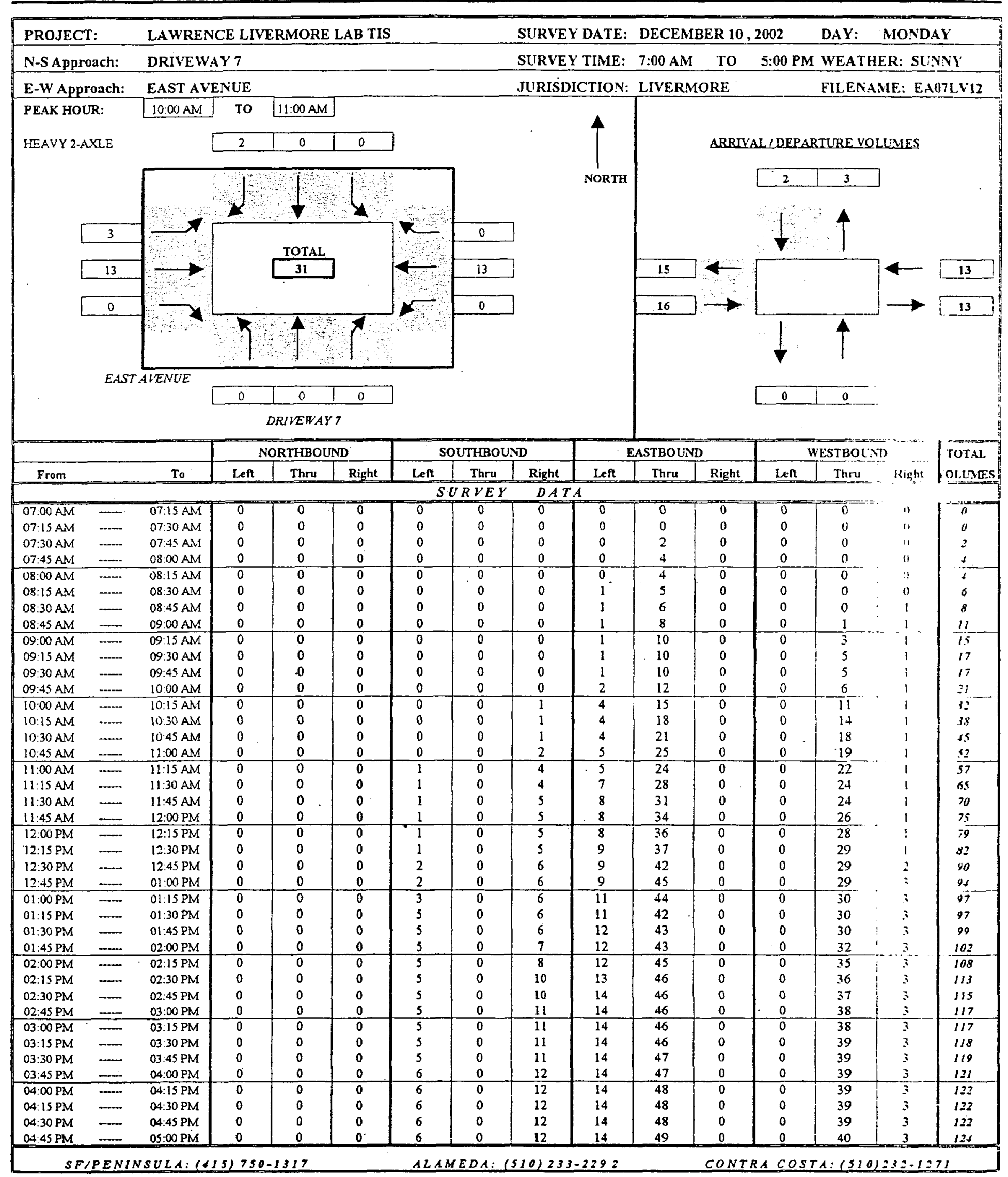




\section{Baymetrics Traffic Resources INTERSECTION TURNING MOVEMENT SUMMARY}

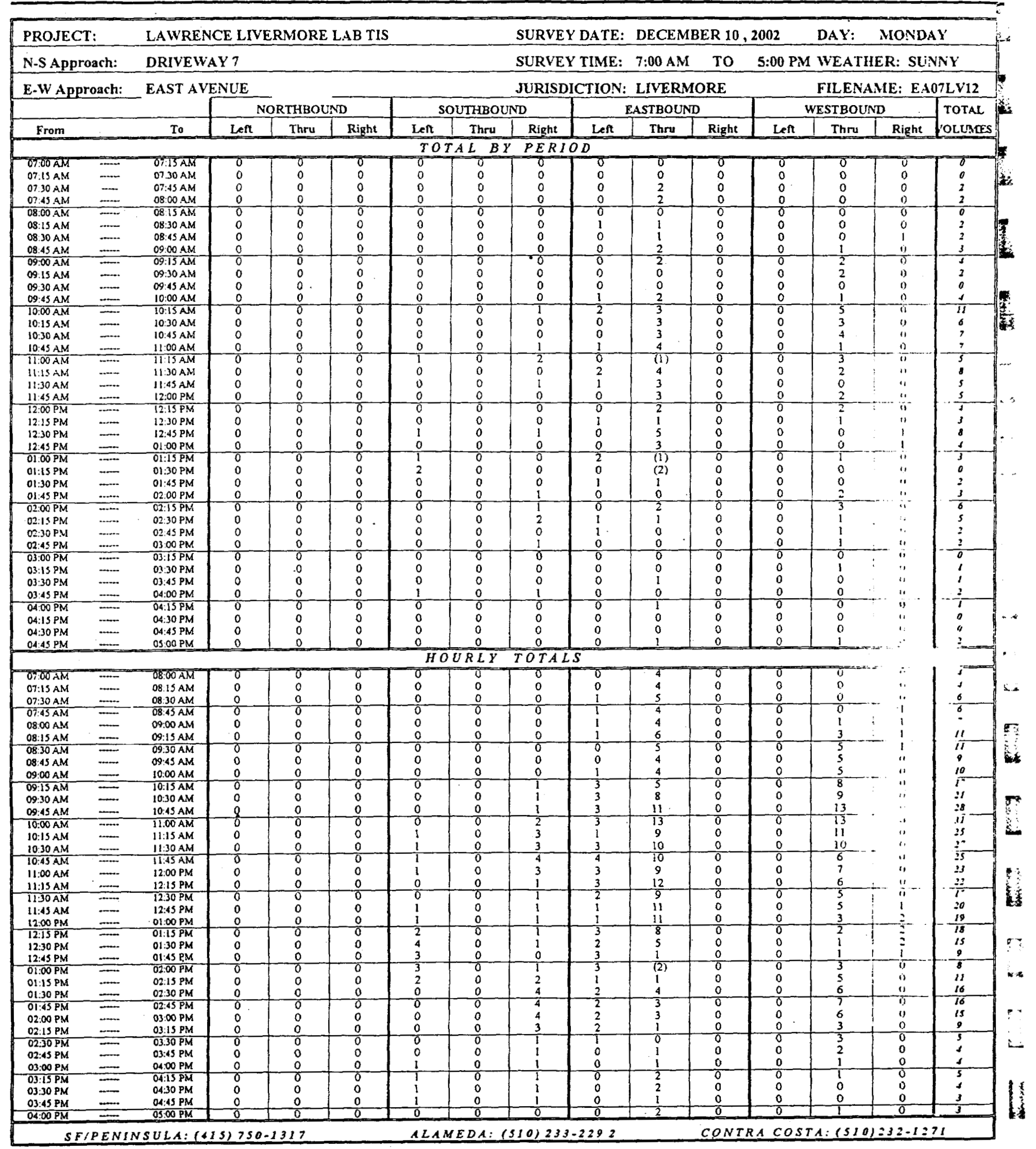




\section{Baymetrics Traffic Resources INTERSECTION TURNING MOVEMENT SUMMARY}

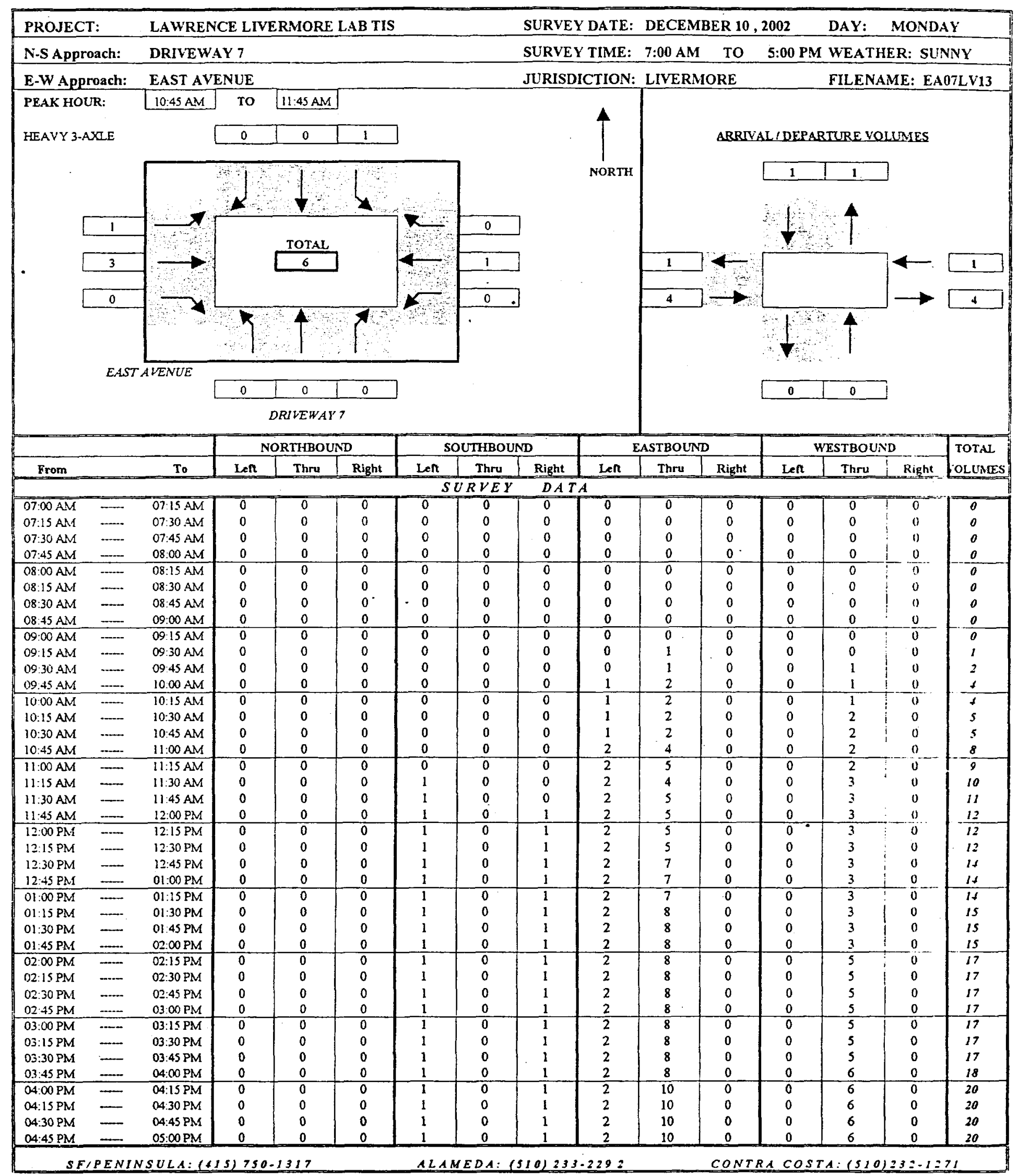




\section{Baymetrics Traffic Resources INTERSECTION TURNING MOVEMENT SUMMARY}

\begin{tabular}{|c|c|c|c|c|c|c|c|c|c|c|c|c|c|c|c|}
\hline PROJEC & $:$ & LAWRE & E LI & MOR & AB TIS & & & SURVE & DATE & DECEN & ER 10 & 2002 & DAY: & MONDA & \\
\hline N-S App & ach: & DRIVEW & & & & & & SURVE & TIME: & $7: 00 \mathrm{AN}$ & TO & 5:00 PM & WEAT & ER: SUS & INY \\
\hline E-W AP & oach: & EAST AY & VUE & & & & & JURISD & TION & LIVER & DRE & & FILEN & ME: E & $7 \mathrm{LV} 13$ \\
\hline & & & & THBO & & & THBO & & & ASTBOU & & & VESTBOL & D. & TOTAL \\
\hline From & & To & Left & Thru & Right & Lef & Thru & Right & Len & Thru & Right & Len & Thru & 1 Right & KOLUMES \\
\hline & & & & & & & $L B$ & $P E R I$ & & & & & & & \\
\hline $0780 \overline{A M}$ & $\ldots$ & काडिA & 0 & $\%$ & 7 & 0 & 0 & 0 & 0 & 0 & 0 & 0 & 8 & it & 8 \\
\hline $\begin{array}{l}07: 15 \mathrm{AM} \\
07.30 \mathrm{AM}\end{array}$ & $\cdots$ & 07.30 AM & 0 & 0 & 0 & 0 & 0 & 0 & 0 & $?$ & 0 & 0 & 0 & $y$ & $\therefore$ \\
\hline $\begin{array}{l}07: 30 \mathrm{AM} \\
07: 45 \mathrm{AM}\end{array}$ & $\ldots$ & $\begin{array}{l}07: 45 \mathrm{AM} \\
08: 00 \mathrm{AM}\end{array}$ & $\begin{array}{l}0 \\
0 \\
0\end{array}$ & $\begin{array}{l}0 \\
0\end{array}$ & $\begin{array}{l}0 \\
0\end{array}$ & $\begin{array}{l}0 \\
0\end{array}$ & $\begin{array}{l}0 \\
0\end{array}$ & $\begin{array}{l}0 \\
0\end{array}$ & $\begin{array}{l}0 \\
0\end{array}$ & $\begin{array}{l}0 \\
0\end{array}$ & $\begin{array}{l}0 \\
0\end{array}$ & 0 & 0 & $"$ & 0 \\
\hline$\frac{07: 45 \mathrm{AM}}{08: 00 \mathrm{AM}}$ & $=$ & $08.15 \overline{\mathrm{AM}}$ & 0 & 0 & 0 & 0 & 0 & 0 & $\frac{1}{0}$ & $\frac{\pi}{0}$ & $\frac{0}{a}$ & $-\frac{0}{0}$ & $\frac{0}{0}$ & $-\frac{9}{11}$ & $\frac{0}{0}$ \\
\hline OB:15 AM & $\cdots$ & 08:30 AM & 0 & 0 & 0 & 0 & 0 & 0 & 0 & 0 & 0 & 0 & 0 & $\because:$ & 0 \\
\hline 08:30 AM & - & 08:45 AM & 0 & 0 & 0 & 0 & 0 & 0 & $\circ$ & 0 & 0 & 0 & 0 & $"$ & 0 \\
\hline 08:45 AM & - & $09.00 \mathrm{AM}$ & 0 & 0 & 0 & 0 & 0 & 0 & 0 & 0 & 0 & 0 & 0 & $\therefore$ & 0 \\
\hline $09: 00 \overline{A M}$ & $=$ & $09: 15 \mathrm{AM}$ & 0 & 0 & 0 & 0 & 0 & 0 & 0 & 0 & 0 & 0 & 0 & $i$ & 0 \\
\hline 09:15 AM & $\cdots$ & 09:30 AM & 0 & 0 & 0 & 0 & 0 & 0 & 0 & 1 & 0 & 0 & 0 & " & 1 \\
\hline 09:30 AM & $\ldots$ & $09: 45 \mathrm{AM}$ & 0 & 0 & 0 & 0 & 0 & 0 & 0 & 0 & 0 & 0 & 1 & $\because$ & $t$ \\
\hline$\frac{09: 45 \mathrm{AM}}{10: 00 \mathrm{AM}}$ & $=$ & $\frac{10: 00 \mathrm{AM}}{10: 15 \mathrm{AM}}$ & $-\frac{0}{0}$ & $\div$ & $\frac{0}{0}$ & $\frac{0}{0}$ & $\frac{0}{0}$ & $\frac{0}{0}$ & $-\frac{1}{0}$ & $-\frac{1}{0}$ & $\frac{0}{0}$ & 0 & a & $\because$ & $-\frac{2}{n}$ \\
\hline $10: 15 \mathrm{AM}$ & $\ldots$ & $10: 30 \mathrm{AM}$ & 0 & 0 & 0 & $\begin{array}{l}0 \\
0 .\end{array}$ & 0 & 0 & 0 & 0 & $\begin{array}{l}0 \\
0\end{array}$ & $\begin{array}{l}0 \\
0\end{array}$ & $i$ & $\therefore$ & $i$ \\
\hline 10:30 AM & $\cdots$ & 10:45 AM & 0 & 0 & 0 & 0 & 0 & 0 & 0 & 0 & 0 & 0 & $\because$ & 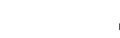 & 0 \\
\hline $10: 45.4 M$ & $\ldots$ & $11: 00 \mathrm{AM}$ & 0 & 0 & 0 & 0 & 0 & 0 & 1 & 2 & 0 & 0 & 0. & . & 3 \\
\hline $1100 \mathrm{AM}$ & - & $1115 \mathrm{AM}$ & 0 & 0 & 0 & 0 & 0 & 0 & 0 & 1 & 0 & 0 & $\because$ & 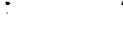 & $T$ \\
\hline 11:15 AM & - & $11: 30 \mathrm{AM}$ & 0 & 0 & 0 & 1 & 0 & 0 & 0 & (l) & 0 & 0 & 1 & & $t$ \\
\hline 11:30 AM & $\ldots$ & $11: 45 \mathrm{AM}$ & 0 & 0 & 0 & 0 & 0 & 0 & 0 & 1 & 0 & 0 & $"$ & & 1 \\
\hline $11: 45 \mathrm{AM}$ & $\cdots$ & $12: 00 \mathrm{PM}$ & 0 & 0 & 0 & 0 & 0 & 1 & 0 & 0. & D) & 0 & $\therefore$ & & $i$ \\
\hline $12: 00 \mathrm{PM}$ & $\ldots$ & $12: 15 \mathrm{PM}$ & 0 & 0 & $\sigma$ & 0 & 0 & 0 & 0 & 0 & 0 & $v$ & $i$ & $\therefore$ & 0 \\
\hline $12: 15 \mathrm{PM}$ & $\ldots$ & 12.30 PM & 0 & 0 & 0 & 0 & 0 & 0 & 0 & 0 & 0 & 0 & i & & 0 \\
\hline $12: 30 \mathrm{PM}$ & - & 12:45 PM & 0 & 0 & 0 & 0 & 0 & 0 & 0 & 2 & 0 & 0 & $\because$ & & 3 \\
\hline $12: 45 \mathrm{PM}$ & - & $01: 00$ PM & 0 & 0 & 0 & 0 & 0 & 0 & 0 & 0 & 0 & 0 & “" & & $\therefore$ \\
\hline $01: 00 \mathrm{PM}$ & $\rightarrow$ & OLIS PM & 0 & 0 & 0 & 0 & 0 & 0 & 0 & 0 & 0 & 0 & 7 & & $-\frac{6}{0}$ \\
\hline $01: 15$ PM & - & 01:30 PM & 0 & 0 & 0 & 0 & 0 & 0 & 0 & 1 & 0 & 0 & ? & & 1 \\
\hline 01:30 PM & $\ldots$ & 01:45 PM & 0 & 0 & 0 & 0 & 0 & 0 & 0 & 0 & 0 & 0 & $" 1$ & & 0 \\
\hline 01.45 PM & $\ldots$ & $02: 00 \mathrm{PM}$ & 0 & 0 & 0 & 0 & 0 & 0 & 0 & 0 & 0 & 0 & ". & & 0 \\
\hline 02.00 PM & -- & $02: 15$ PM & 0 & 0 & 0 & 0 & 0 & 0 & 0 & 0 & 0 & 0 & $z$ & & 2 \\
\hline 02:15 PM & $\cdots$ & 02:30 PM & 0 & 0 & 0 & 0 & 0 & 0 & 0 & 0 & 0 & 0 & $\therefore$ & & 0 \\
\hline 02:30 PM & $\ldots$ & 02:45 PM & 0 & 0 & 0 & 0 & 0 & 0 & 0 & 0 & 0 & 9 & " & & 0 \\
\hline $02.45 \mathrm{PM}$ & $=$ & $03: 00 \mathrm{PM}$ & 0 & 0 & 0 & 0 & 0 & 0 & 0 & 0 & 0 & 0 & $\therefore$ & & $\stackrel{0}{2}$ \\
\hline $03: 00 \mathrm{PM}$ & $\cdots$ & $03: 15 \mathrm{PM}$ & 0 & 0 & 0 & 0 & $\overline{0}$ & 0 & 0 & $\%$ & $a$ & 0 & $\because$ & & 0 \\
\hline $03: 15 \mathrm{PM}$ & $\ldots$ & 03:30 PM & 0 & 0 & 0 & 0 & 0 & 0 & 0 & 0 & 0 & 0 & i: & & $n$ \\
\hline 03:30 PM & $\ldots$ & 03:45 PM & 0 & 0 & 0 & 0 & 0 & 0 & 0 & 0 & 0 & 0 & $\because$ & & $"$ \\
\hline 03:45 PM & $\ldots$ & $04: 00 \mathrm{PM}$ & 0 & 0 & 0 & 0 & 0 & 0 & 0 & 0 & 0 & 0 & $i$ & & $t$ \\
\hline $04: 00 \mathrm{PM}$ & $=$ & $04: 15 \mathrm{PM}$ & 0 & 0 & 0 & 0 & 0 & 0 & 0 & 2 & 0 & 0 & $i$ & & $\because$ \\
\hline 04:15 PM & - & $04: 30 \mathrm{PM}$ & 0 & 0 & 0 & 0 & 0 & 0 & 0 & 0 & 0 & 0 & is & & \\
\hline $04: 30$ PM & $\ldots$ & 04:45 PM & 0 & 0 & 0 & 0 & 0 & 0 & 0 & 0 & 0 & 0 & $u$ & & a \\
\hline 04:45 PM & 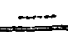 & $05: 00 \mathrm{PM}$ & 0 & 0 & 0 & 0 & 0 & 0 & $a$ & 0 & 0 & 0 & $\because$ & & $a$ \\
\hline & & & & & & & $R L Y$ & TOTAL & & & & & & & \\
\hline $0700 \mathrm{AM}$ & $=$ & $08: 00 \mathrm{AM}$ & 0 & 0 & $\bar{\sigma}$ & $\%$ & $\pi$ & (1) & 8 & 0 & $\pi$ & $\pi$ & $\because$ & & $n$ \\
\hline 07:15 AM & $\cdots$ & $08: 15 \mathrm{AM}$ & 0 & 0 & 0 & 0 & 0 & 0 & 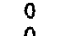 & 0 & 0 & 0 & $\because$ & & 0 \\
\hline $07: 30 \mathrm{AM}$ & $=$ & $\begin{array}{l}08.30 \mathrm{AM} \\
-08.45 .48\end{array}$ & -0 & 0 & $\frac{0}{0}$ & $\frac{0}{0}$ & -0 & 0 & $-\frac{0}{n}$ & $\frac{0}{0}$ & 0 & $\frac{0}{0}$ & $\because$ & & 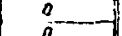 \\
\hline $07: 45 \mathrm{AM}$ & - & $08: 45 \mathrm{AM}$ & 0 & 0 & 0 & 0 & 0 & 0 & 0 & 0 & 0 & 0 & it & & $\bar{a}$ \\
\hline 08:00 AM & $\cdots$ & $09 \cdot 00 \mathrm{AM}$ & 0 & 0 & 0 & 0 & 0 & 0 & 0 & 0 & 0 & 0 & ". & & $n$ \\
\hline 08:15 AM & $\cdots$ & $09: 15 \mathrm{AM}$ & 0 & 0 & 0 & 0 & 0 & 0. & 0 & 0 & 0 & 0 & " & & $\because$ \\
\hline $08.30 \mathrm{AM}$ & $=$ & $09: 30 \overline{A M}$ & 0 & 0. & 0 & 0 & 0 & 0 & 0 & 1 & 0 & 0 & $\because$ & & $i$ \\
\hline 08:45 AM & $\ldots$ & 09:45 AM & 0 & 0 & 0 & 0 & 0 & 0 & 0 & i & 0 & 0 & $i$ & & 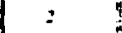 \\
\hline $09: 00 \mathrm{AM}$ & 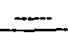 & $1000 \mathrm{AM}$ & 0 & 0 & 0 & 0 & 0 & 0 & 1 & 2 & 0 & 0 & 1 & & \\
\hline $09: 15 A M$ & $=$ & $10.15 . \mathrm{AM}$ & 0 & 0 & 0 & 0 & 0 & 0 & $T$ & 2 & 0 & 0 & $!$ & & $s$ \\
\hline $09: 30 \mathrm{AM}$ & - & $10: 30 \mathrm{AM}$ & 0 & 0 & 0 & 0 & 0 & 0 & $i$ & 1 & 0 & 0 & $=$ & & t \\
\hline $0945 A M$ & $\cdots$ & $10: 45 \mathrm{AM}$ & 0 & 0 & 0 & 0 & 0 & 0 & 1 & 1 & 0 & $a$ & $\therefore$ & & 3 \\
\hline $10: 00 \mathrm{AM}$ & - & $11: 00 \mathrm{AM}$ & 0 & 0 & 0 & 0 & 0 & 0 & $T$ & 2 & $a$ & 0 & $!$ & & 3 \\
\hline 10:15 AM & $\cdots$ & 11:15 AM & 0 & 0 & 0 & 0 & 0 & 0 & i & 3 & 0 & 0 & i & & : \\
\hline 10:30 AM & $=$ & $11: 30 \mathrm{AM}$ & 0 & 0 & 0 & 1 & 0 & 0 & 1 & 2 & 0 & 0 & $:$ & & $\varepsilon$ \\
\hline $10: 45 \mathrm{AM}$ & - & $11: 45 \mathrm{AM}$ & 0 & 0 & 0 & 1 & 0 & 0 & t & 3 & 0 & 0 & 7 & & 5 \\
\hline $11: 00 \mathrm{AM}$ & - & 12:00 PM & 0 & 0 & 0 & 1 & 0 & 1 & 0 & 1 & 0 & 0 & 1 & & $s$ \\
\hline 11:15 AM & $=$ & 12:15 PM & 0 & 0 & 0 & 1 & 0. & 1 & 0 & 0 & 0 & 0 & $-!$ & & 3 \\
\hline $11: 30 \mathrm{AM}$ & $\ldots$ & $12: 30 \mathrm{PM}$ & 0 & 0 & 0 & 0 & 0 & 1 & 0 & $!$ & 0 & a & $\because:$ & & 5 \\
\hline $11: 45$ AM & $\ldots$ & 12:45 PM & 0 & 0 & 0 & 0. & 0 & 1 & 0 & 2 & 0 & 0 & “. & & 1 \\
\hline $12: 00 \mathrm{PM}$ & $=$ & $01: 00 \mathrm{PM}$ & 0 & 0 & 0 & 0. & 0 & 0 & 0 & $\frac{2}{3}$ & 0 & 0 & $\therefore$ & & 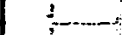 \\
\hline $12.15 \mathrm{PM}$ & $\ldots$ & $01: 15 \mathrm{PM}$ & 0 & 0 & 0 & 0 & $\stackrel{0}{0}$ & 0 & 0 & $\frac{2}{2}$ & 0 & 0 & $i$ & & $z$ \\
\hline 12:30 PM & $\ldots$ & $01: 30 \mathrm{PM}$ & 0 & 0 & ${ }_{0}^{0}$ & 0 & $\begin{array}{l}0 \\
0\end{array}$ & 8 & 0 & 3 & $\begin{array}{l}0 \\
0\end{array}$ & 0 & $\because$ & : & 3 \\
\hline $12: 45 \mathrm{PM}$ & $\cdots$ & 01:45 PM & 0 & $\frac{a}{0}$ & 0 & $\frac{0}{0}$ & $\frac{0}{0}$ & $\frac{0}{0}$ & $\frac{0}{0}$ & -1 & 0 & 0 & $\ddot{0}$ & $\therefore$ & 8 \\
\hline $01: 00 \mathrm{PM}$ & $\cdots$ & $02: \mathrm{CO} P \mathrm{PM}$ & 0 & 0 & 0 & 0 & 0 & 0 & 0 & 1 & 0 & 0 & 0 & 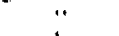 & 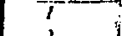 \\
\hline 01:15 PM & $\ldots$ & $02: 15 \mathrm{PM}$ & 0 & 0 & 0 & $D$ & 0 & $\stackrel{0}{0}$ & 0 & 1 & $\stackrel{0}{0}$ & $\stackrel{0}{0}$ & $\frac{2}{9}$ & $\because$ & 3 \\
\hline $01: 30 \mathrm{PM}$ & $\ldots$ & 02:30 PM & 0 & 0 & 0 & 0 & 0 & 0 & 0 & 0 & 0 & $\stackrel{0}{0}$ & 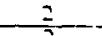 & $\therefore-$ & 2 \\
\hline $01: 45$ PM & $\cdots$ & $02: 45 \mathrm{PM}$ & 0 & 0 & 0 & 0 & 0 & 0 & 0 & 0 & 0 & 0 & 2 & $\because{ }^{-}$ & 2 \\
\hline 02:00 PM & $\ldots$ & $03.00 \mathrm{PM}$ & 0 & 0 & 0 & 0 & $\stackrel{0}{0}$ & 0 & $:$ & ${ }_{0}^{0}$ & 0 & $\stackrel{0}{0}$ & $=$ & $"$ & 2 \\
\hline 02:15 PM & $=$ & 03:15 PM & 0 & 0 & 0 & 0 & 0 & 0 & 0 & 0 & -0 & $\frac{0}{0}$ & 0 & 0 & $\therefore$ \\
\hline $02: 30 \mathrm{PM}$ & - & $03: 30 \mathrm{PM}$ & 0 & 0 & 0 & 0 & 0 & 0 & 0 & $\begin{array}{l}0 \\
0\end{array}$ & 0 & $\begin{array}{l}0 \\
0\end{array}$ & 0 & $a$ & 0 \\
\hline 02:45 PM & - & 03:45 PM & $\begin{array}{l}0 \\
0\end{array}$ & $\begin{array}{l}0 \\
0\end{array}$ & $\begin{array}{l}0 \\
0\end{array}$ & $\begin{array}{l}0 \\
0\end{array}$ & $\begin{array}{l}0 \\
0\end{array}$ & $\begin{array}{l}0 \\
0\end{array}$ & $\begin{array}{l}0 \\
0\end{array}$ & $\begin{array}{l}0 \\
0\end{array}$ & $\begin{array}{l}0 \\
0\end{array}$ & 0 & 1 & $\because$ & i \\
\hline$\frac{03: 00 \mathrm{PM}}{-03: 15 \mathrm{PM}}$ & $=$ & $\frac{04: 00 \mathrm{PM}}{04: 15 \mathrm{PM}}$ & $-\frac{0}{0}$ & 0 & & $\%$ & 0 & 0 & $\overline{0}$ & 2 & $\overline{0}$ & 0 & 1 & ii- & 3 \\
\hline $03: 30 \mathrm{PM}$ & - & $04: 30 \mathrm{PM}$ & 0 & 0 & 0 & 0 & 0 & 0 & 0 & 2 & 0 & 0 & 1 & ") & 3 \\
\hline $03: 4 \leq \mathrm{PM}$ & $\ldots$ & 04:45 PM & 0 & 0 & 0 & 0 & 0 & 0 & 0 & 2 & 0 & 0 & 1 & $\therefore$ & 3 \\
\hline at:00 PM & $=$ & $05: 00 \mathrm{PM}$ & 0 & 0 & 0 & 0 & 0 & 0 & 0 & 3 & 0 & - & 0 & 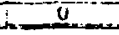 & 2 \\
\hline$s t$ & $P E N$ & ULA: & 7 & & & 11 & $O A$ & a) 2 & 92 & & con & $A C$ & 1 & $z=$ & \\
\hline
\end{tabular}




\section{Baymetrics Traffic Resources INTERSECTION TURNING MOVEMENT SUMMARY}

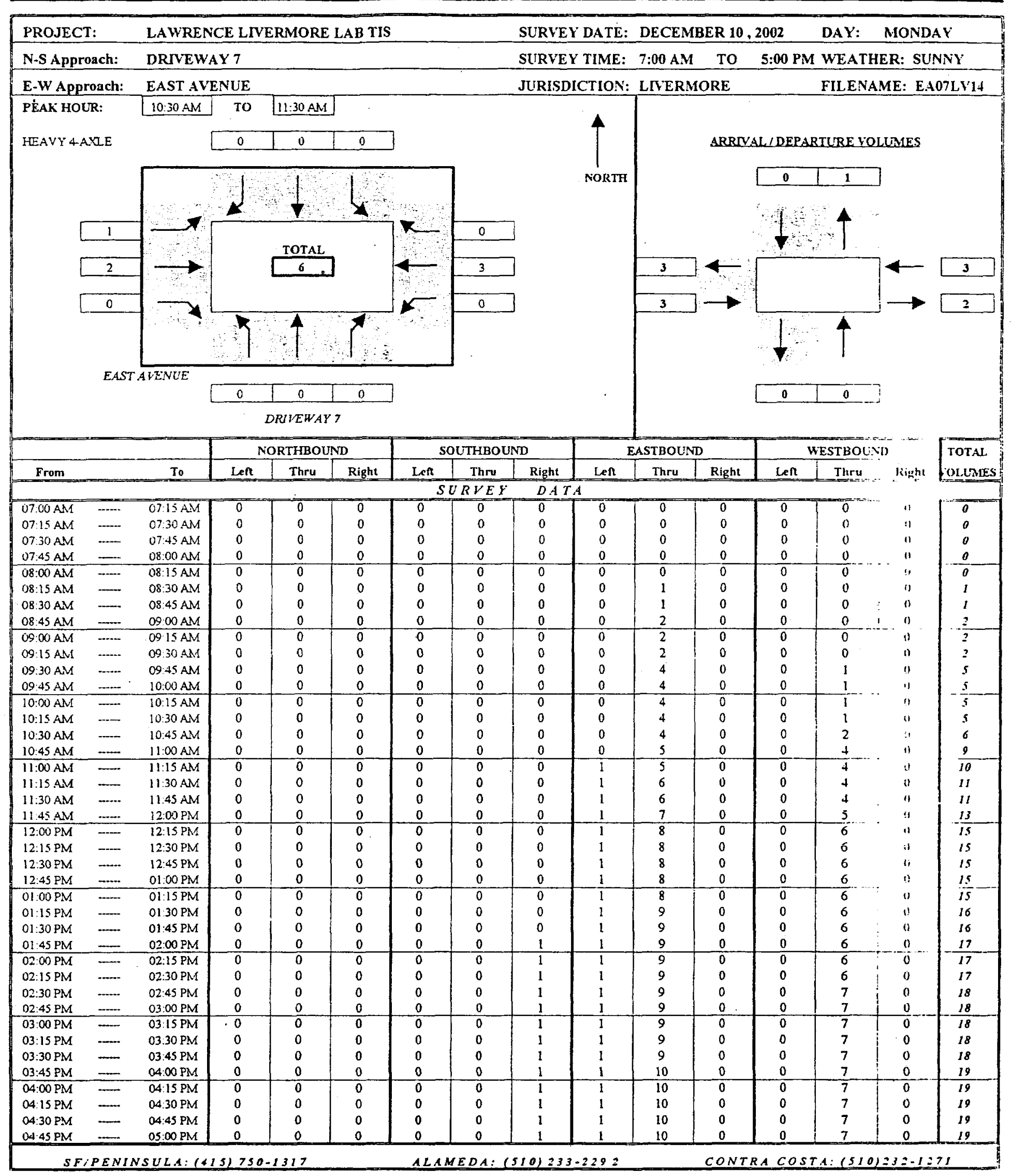




\section{$\frac{\text { Baymetrics Traffic Resources }}{\text { INTERSECTION TURNING MOVEMENT SUMMARY }}$}

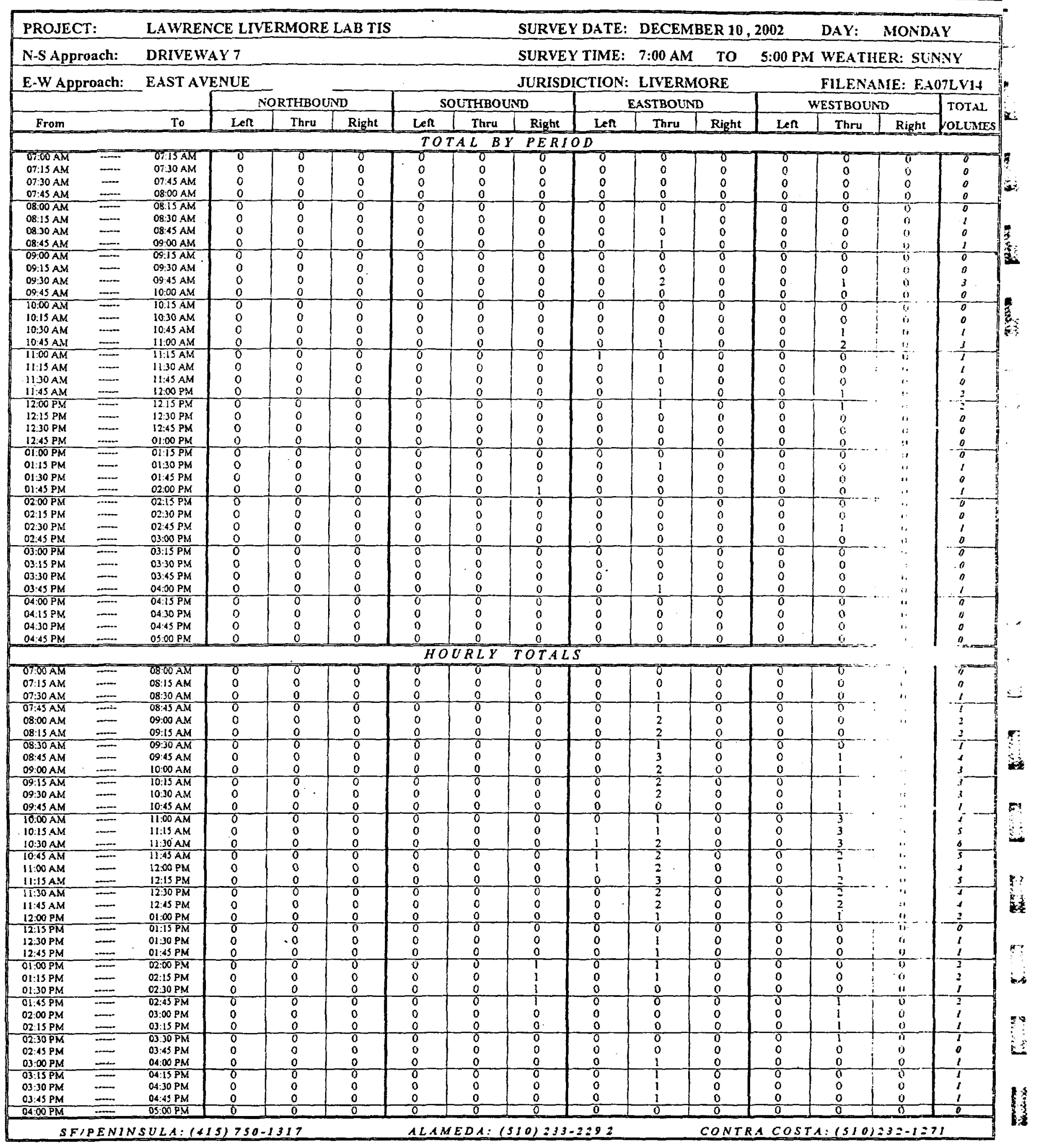




\section{Baymetrics Traffic Resources INTERSECTION TURNING MOVEMENT SUMMARY}

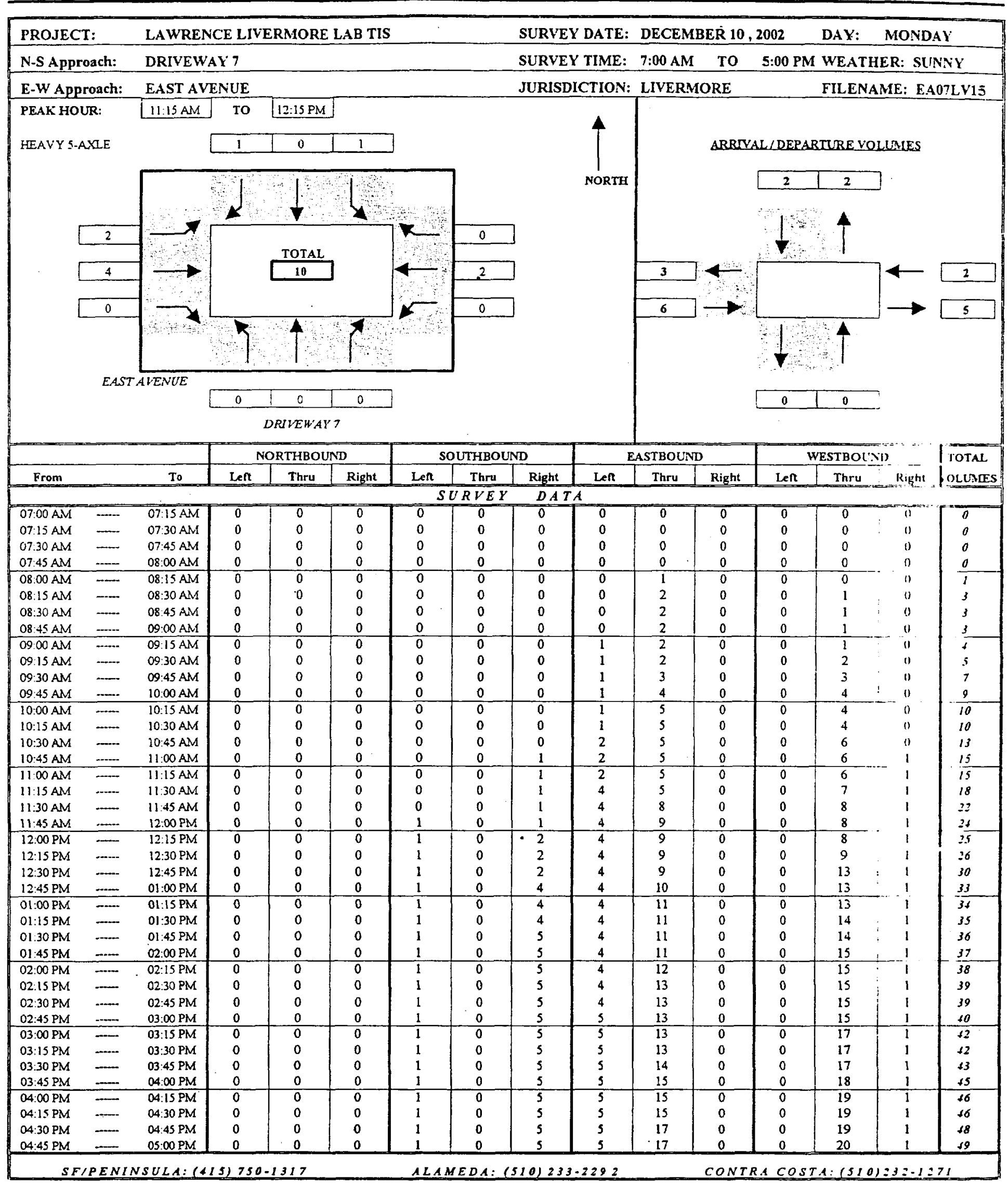




\section{Baymetrics Traffic Resources INTERSECTION TURNING MOVEMENT SUMMARY}

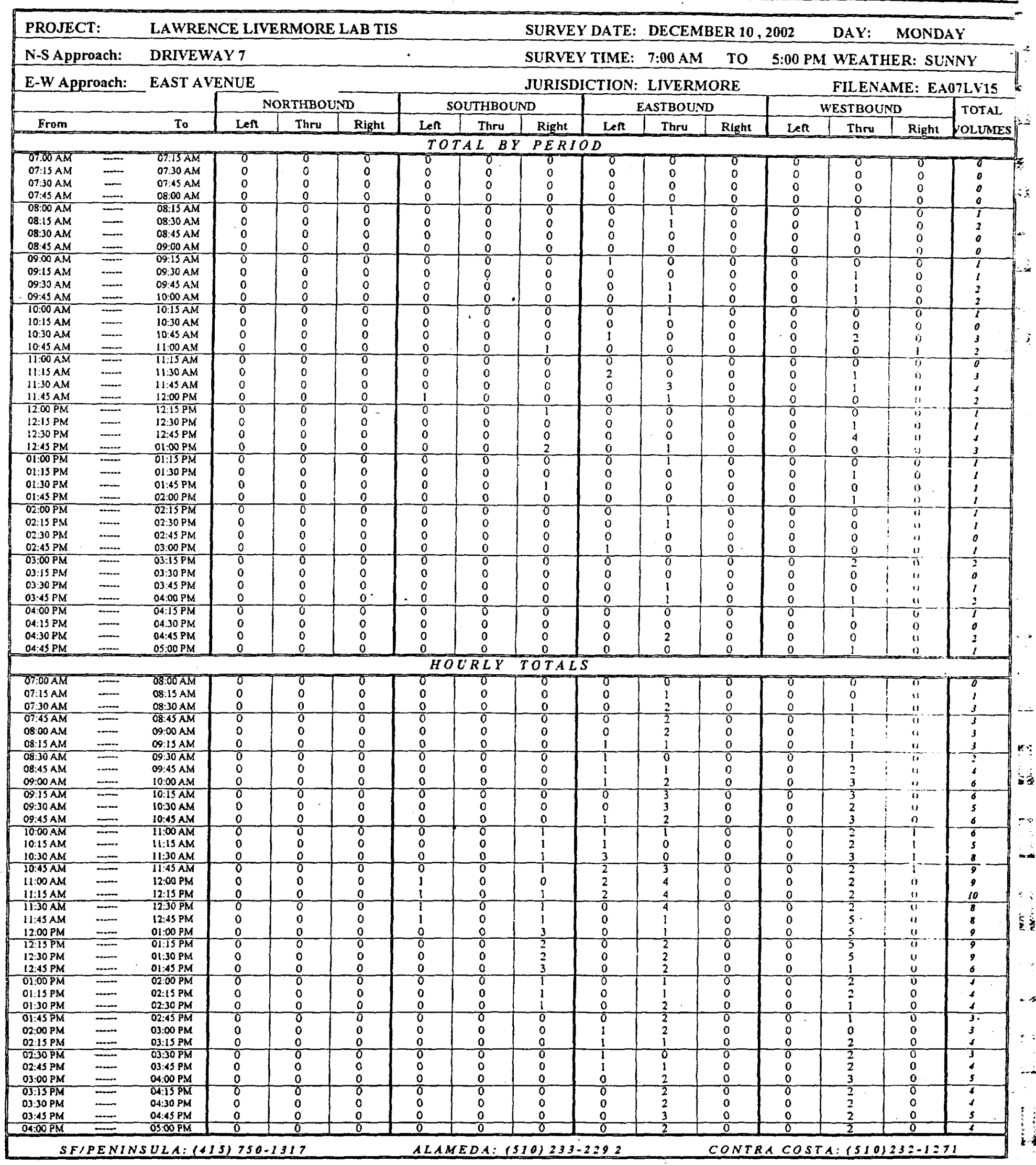




\section{Baymetrics Traffic Resources INTERSECTION TURNING MOVEMENT SUMMARY}

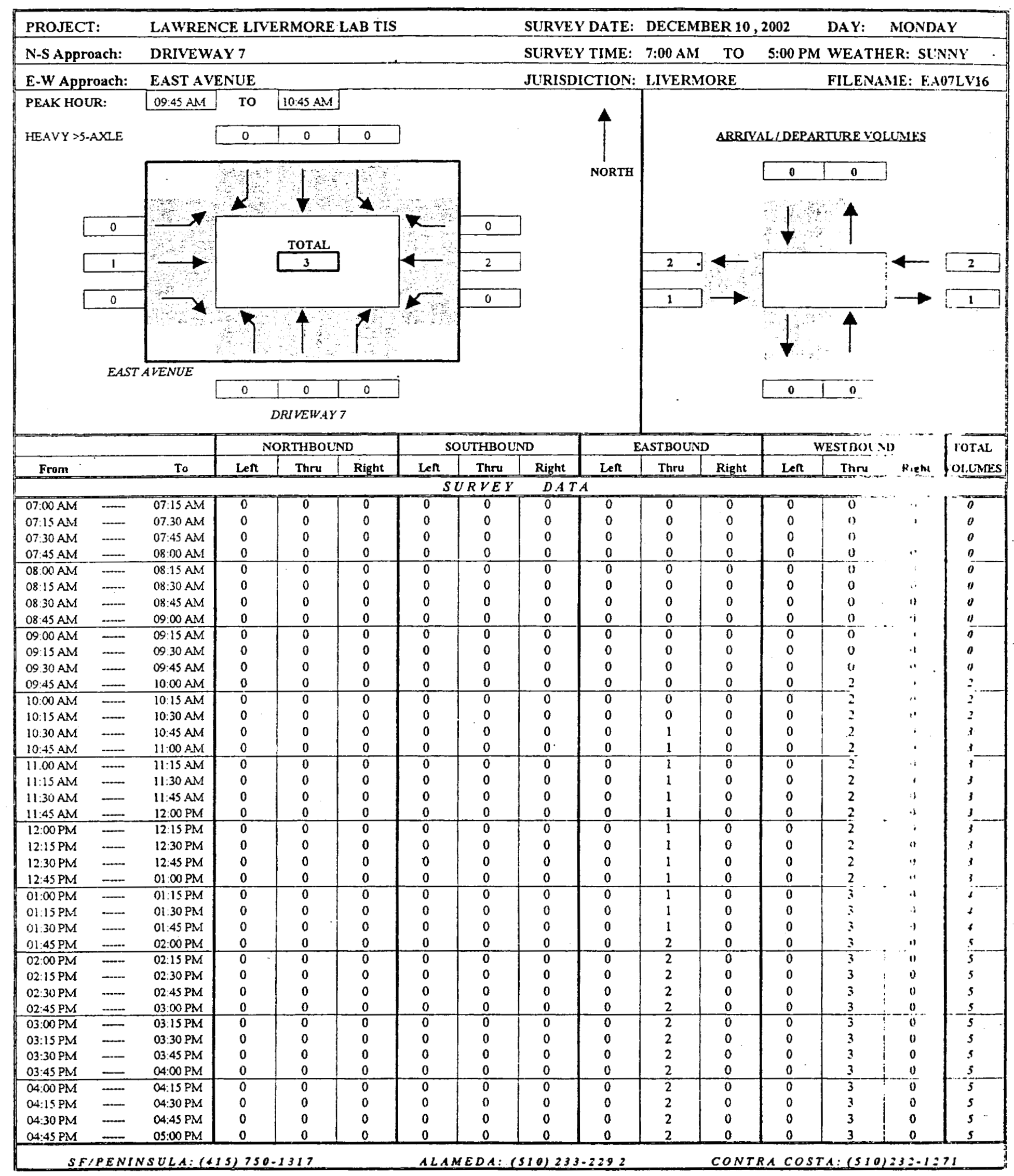




\section{$\frac{\text { B aymetrics Traffic Resources }}{\text { INTERSECTION TURNING MOVEMENT SUMMARY }}$}

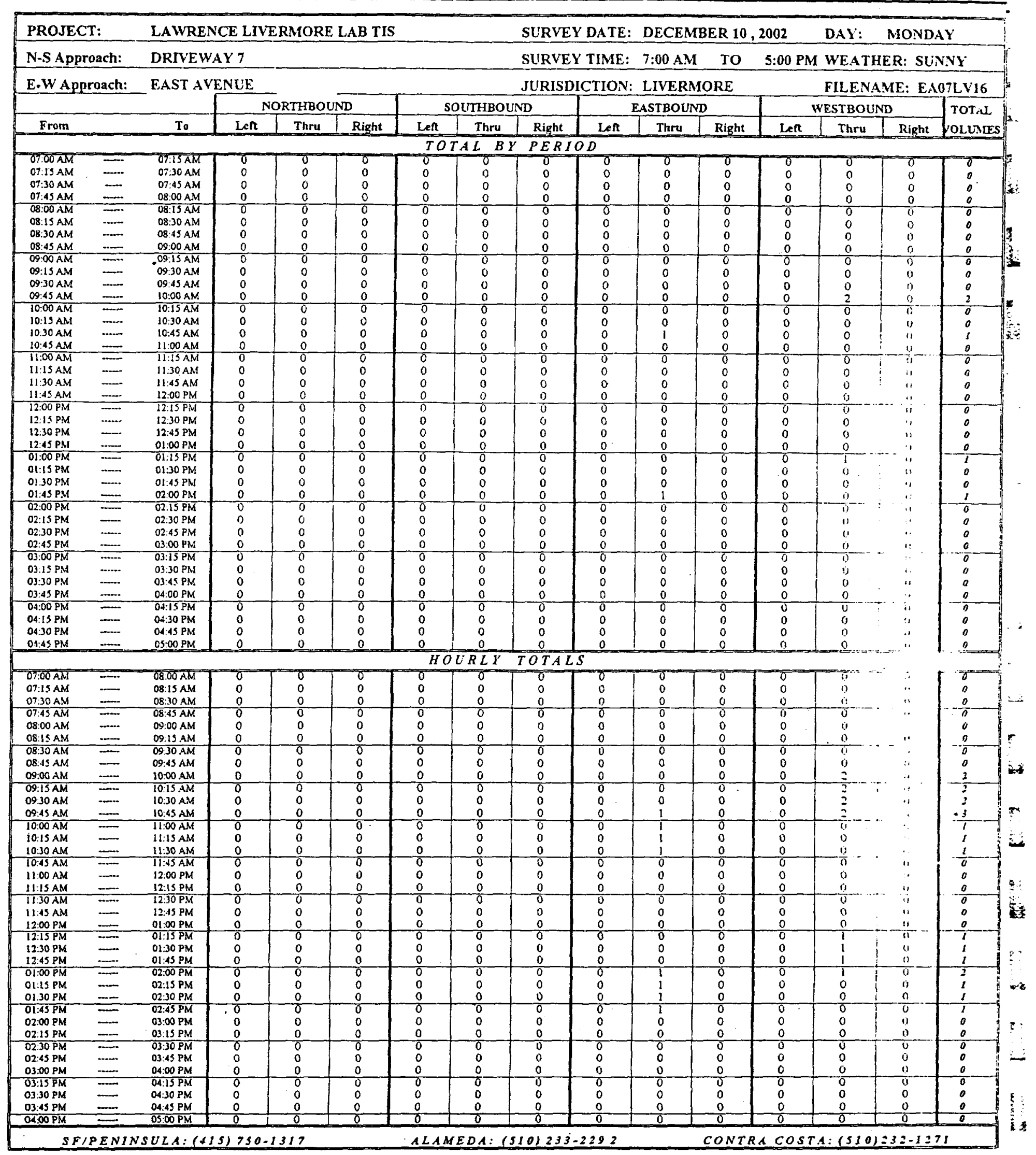




\section{Baymetrics Traffic Resources INTERSECTION TURNING MOVEMENT SUMMARY}

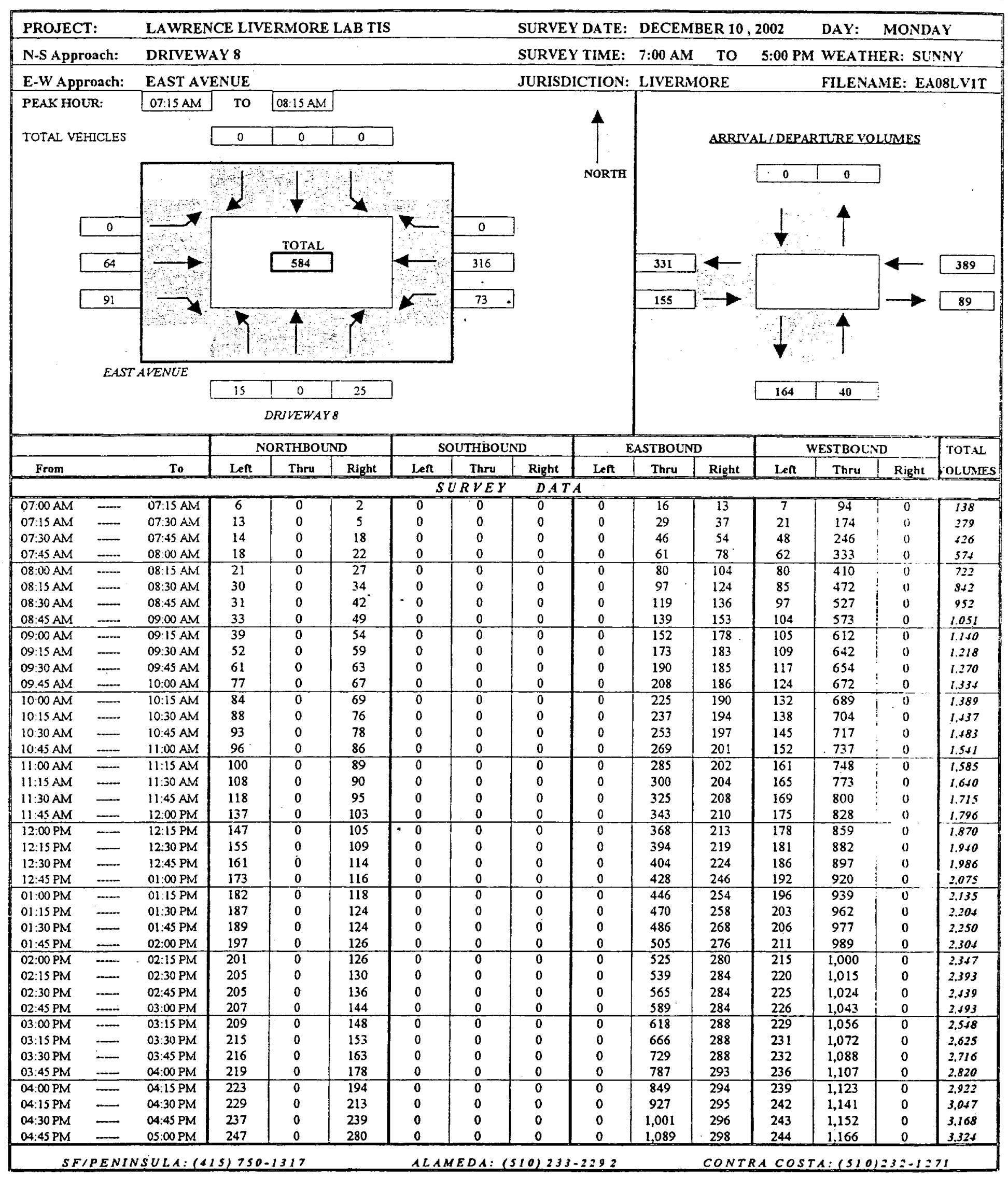




\section{Baymetrics Traffic Resources INTERSECTION TURNING MOVEMENT SUMMARY}

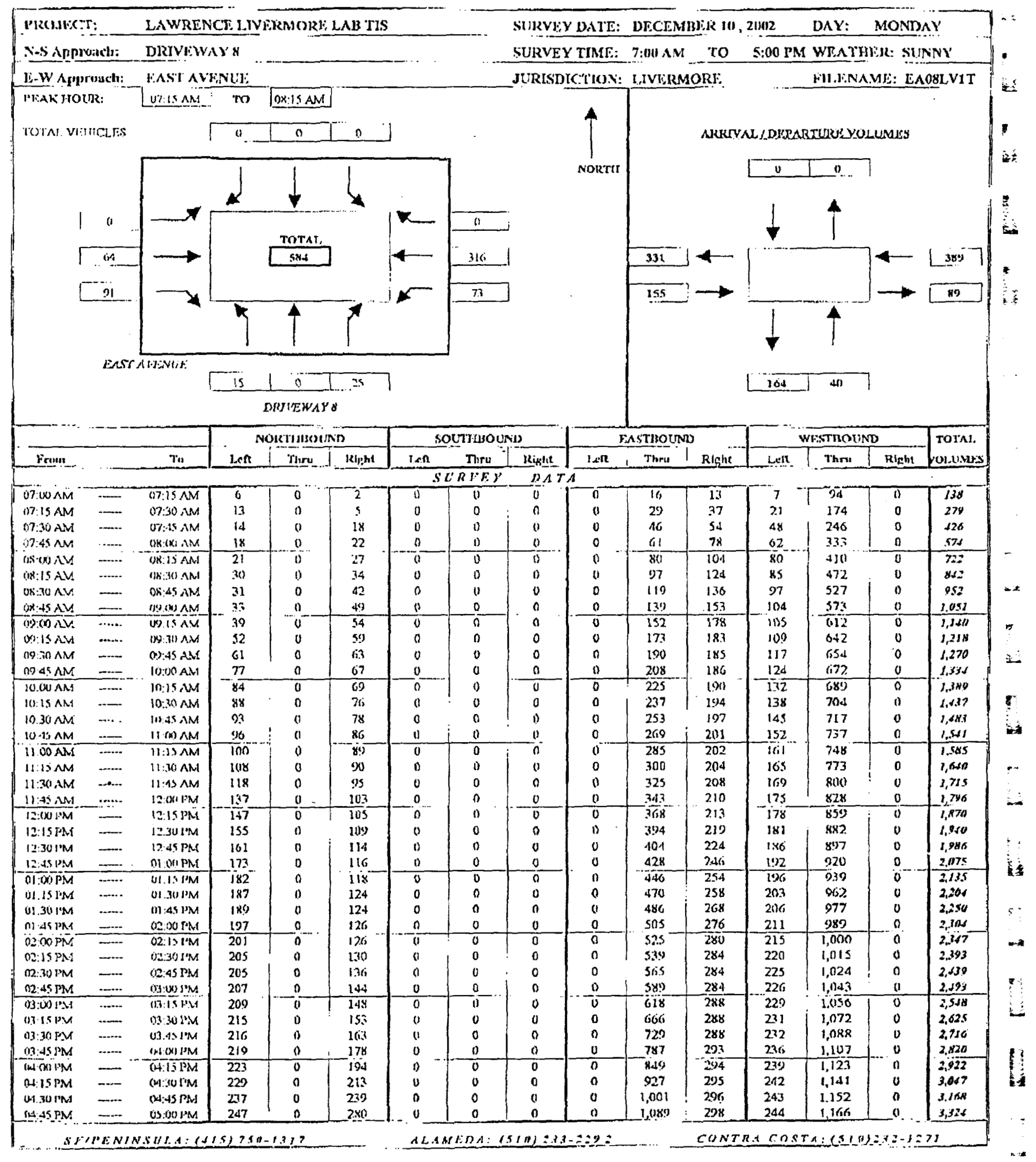




\section{Baymetrics Traffic Resources INTERSECTION TURNING MOVEMENT SUMMARY}

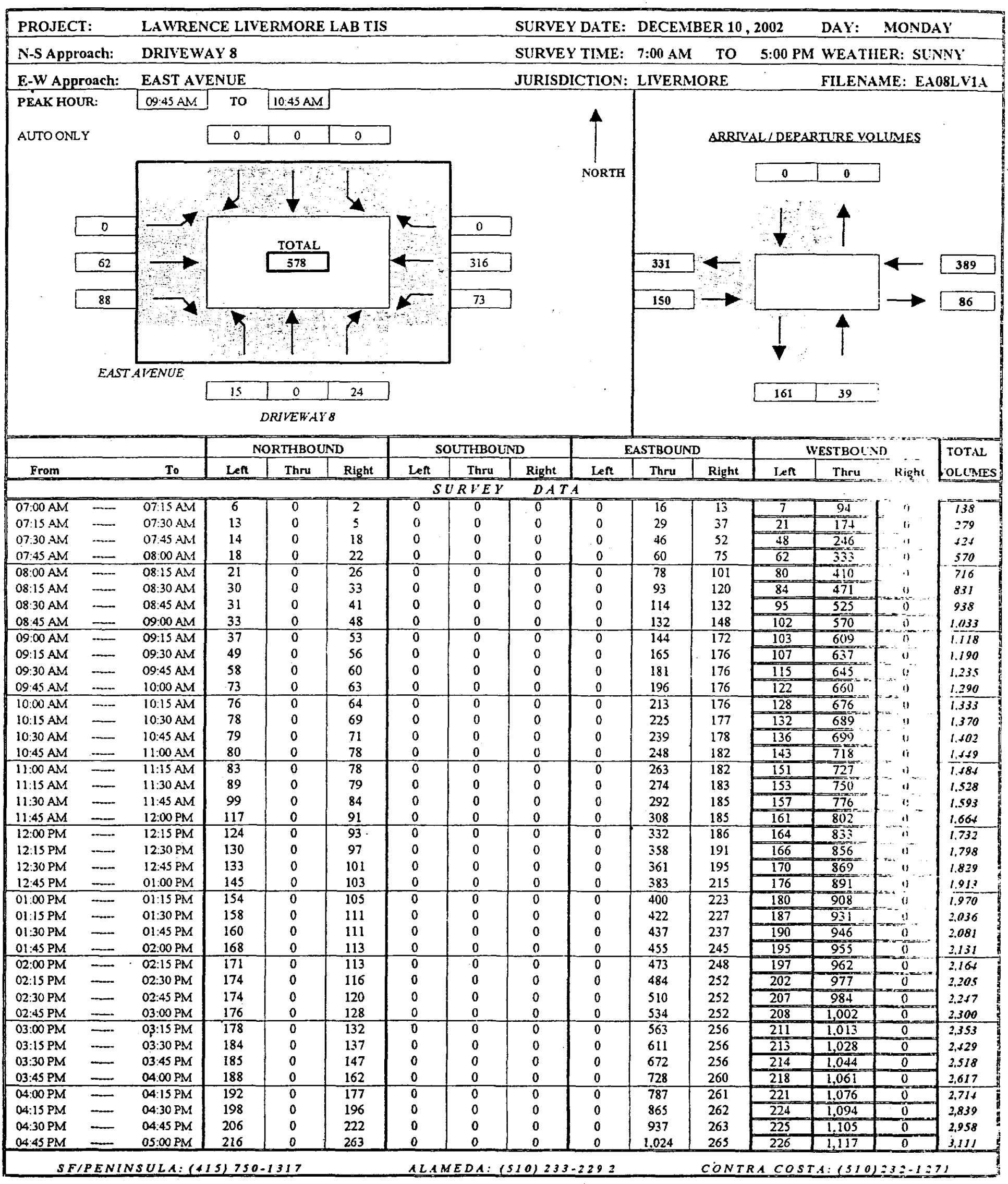




\section{Baymetrics Traffic Resources : INTERSECTION TURNING MOVEMENT SUMMARY}

\begin{tabular}{|c|c|c|c|c|c|c|c|c|c|c|c|c|c|c|c|}
\hline \multirow{2}{*}{\multicolumn{2}{|c|}{$\begin{array}{l}\text { PROJECT: } \\
\text { N-S Approach: }\end{array}$}} & \multicolumn{6}{|c|}{ LAWRENCE LIVERMORE LAB TIS } & SURVE & ATE: & DECEM & ER 10, & 2002 & DAY: & MOND & \\
\hline & & DRIVE V & & & & & & SURVE & IME: & 7:00 AM & To & 5:00 PM & WEAT & ER: SU & NYY \\
\hline E-W ApI & Oach: & EAST A & VUE & & & & & JURISI & TION & LIVERN & REE & & FILEN & ME: EA & $08 \mathrm{LV} 1 \mathrm{~A}$ \\
\hline & & & & THBO & & & THBO & & & ASTBOUN & & & ESTBOL & D & rotal \\
\hline From & & To & Len & Thru & Right & Lent & Thru & Right & Len & Thru & Right & Len & Thru & Right & VOLUALES \\
\hline & & & & & & & $\angle B$ & $P E R I$ & & & & & & & \\
\hline Or:00AM & $=$ & $075 \mathrm{AM}$ & 6 & 8 & 2 & $\sigma$ & 0 & 0 & 0 & 76 & $T 3$ & & 94 & 0 & 138 \\
\hline $\begin{array}{l}07.15 \mathrm{AM} \\
07: 30 \mathrm{AM}\end{array}$ & $\ldots$ & $\begin{array}{l}07: 30 \mathrm{AM} \\
07: 45 \mathrm{AM}\end{array}$ & $\begin{array}{l}7 \\
1\end{array}$ & $\begin{array}{l}0 \\
0\end{array}$ & $\begin{array}{c}3 \\
13\end{array}$ & $\begin{array}{l}0 \\
0\end{array}$ & $\begin{array}{l}0 \\
0\end{array}$ & $\begin{array}{l}0 \\
0\end{array}$ & $\begin{array}{l}0 \\
0\end{array}$ & $\begin{array}{l}13 \\
17\end{array}$ & $\begin{array}{l}24 \\
15\end{array}$ & $\begin{array}{l}14 \\
27\end{array}$ & $\begin{array}{l}80 \\
72\end{array}$ & $\begin{array}{l}2 \\
0\end{array}$ & $\begin{array}{l}\text { INS } \\
\text { IJS }\end{array}$ \\
\hline $07: 49 \mathrm{AM}$ & $\ldots$ & 08:00 AM & 4 & 0 & 4 & 0 & 0 & 0 & 0 & 14 & 23 & 14 & 87 & 0 & \\
\hline $08: 00 \mathrm{AM}$ & $\ldots$ & 08:15 AM & 3 & 0 & 4 & 0 & 0 & 0 & 0 & 18 & 26 & $\frac{14}{18}$ & $-\frac{0}{77}$ & 0 & 186 \\
\hline 08:15 AM & -- & $08.30 \mathrm{AM}$ & 9 & 0 & $?$ & 0 & 0 & 0 & 0 & is & 19 & 4 & 61 & 0 & 115 \\
\hline $\begin{array}{l}\text { 08:30 AM } \\
\text { 08:45 AM }\end{array}$ & $=$ & $\begin{array}{l}\text { 08:45 AM } \\
09: 00 \mathrm{AM}\end{array}$ & $\frac{1}{2}$ & 0. & $\begin{array}{l}8 \\
7\end{array}$ & $\begin{array}{l}0 \\
0\end{array}$ & $\begin{array}{l}0 \\
0\end{array}$ & $\begin{array}{l}0 \\
0\end{array}$ & $\begin{array}{l}0 \\
0\end{array}$ & $\begin{array}{l}21 \\
18\end{array}$ & $\begin{array}{l}12 \\
16\end{array}$ & 11 & $\begin{array}{l}54 \\
45\end{array}$ & 0 & $\begin{array}{l}107 \\
95\end{array}$ \\
\hline $09: 00 \mathrm{AM}$ & $\equiv$ & $09: 15 \mathrm{AM}$ & $\frac{6}{4}$ & $-\frac{\pi}{0}$ & 5 & 0 & 0 & 0 & 0 & $\frac{18}{12}$ & 24 & $\frac{1}{1}$ & $-\frac{43}{39}$ & $\frac{9}{6}$ & $\frac{95}{85}$ \\
\hline $09: 13$ AM & $\ldots$ & $09: 30 \mathrm{AM}$ & 12 & 0 & 3. & 0 & 0 & 0 & 0 & 21 & 4 & 4 & 28 & is & $\eta$ \\
\hline $09: 30 \mathrm{AM}$ & $\cdots$ & $09: 45 \mathrm{AM}$ & 9 & 0 & 4 & 0 & $\begin{array}{l}0 \\
0\end{array}$ & 0 & 0 & 16 & 0 & 8 & 8 & 9 & $\sqrt{s}$ \\
\hline 09.45 AM & $=$ & & $\frac{15}{3}$ & $\frac{0}{0}$ & 3 & $\frac{0}{0}$ & $-\frac{0}{0}$ & 0 & 0 & 15 & 0 & 7 & 15 & +9 & 55 \\
\hline $10: 00 \mathrm{AM}$ & $\ldots$ & $\begin{array}{l}10115 \mathrm{AM} \\
10: 30 \mathrm{AM}\end{array}$ & 3 & 0 & $\frac{1}{5}$ & 0 & 0 & 0 & 0 & 17 & $\overline{0}$ & 6 & 16 & 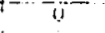 & $\sqrt{3}$ \\
\hline 10:15 AM & $\ldots$ & $\begin{array}{l}10: 30 \mathrm{AM} \\
10: 45 \mathrm{AM}\end{array}$ & 2 & 0 & $\xi$ & $\begin{array}{l}0 \\
0 \\
0\end{array}$ & $\begin{array}{l}0 \\
0\end{array}$ & 0 & 0 & 12 & 1 & 4 & 13 & i: & $3 ?$ \\
\hline $10: 30 \mathrm{AM}$ & $\ldots$ & $\begin{array}{l}10: 45 \mathrm{AM} \\
1100 \mathrm{AM}\end{array}$ & 1 & $\begin{array}{l}0 \\
0\end{array}$ & $\frac{2}{7}$ & $\begin{array}{l}0 \\
0\end{array}$ & $\begin{array}{l}0 \\
0\end{array}$ & 0 & 0 & 14 & 1 & 4 & 10 & $"$ & 32 \\
\hline $10: 45 \mathrm{AM}$ & $\ldots$ & $\frac{1100 \mathrm{AM}}{11: 15 \mathrm{AM}}$ & $\frac{1}{3}$ & 0 & 0 & 0 & 0 & 0 & 0 & 9 & 4 & 7 & 19 & $\because$ & $y$ \\
\hline $1100 \mathrm{AM}$ & $\ldots$ & $\begin{array}{l}\text { T1:15 AM } \\
11.30 \mathrm{AM}\end{array}$ & $\begin{array}{l}3 \\
6\end{array}$ & 0 & i & & & 0 & 0 & 15 & 0 & 8 & 9 & a & -35 \\
\hline $11: 15 \mathrm{AM}$ & $\ldots$ & $\begin{array}{l}11.10 \mathrm{AM} \\
11: 45 \mathrm{AM}\end{array}$ & $\begin{array}{c}6 \\
10\end{array}$ & 0 & & & & 0 & 0 & 11 & 1 & 2 & 23 & $\therefore$ & 4 \\
\hline $11,30 \mathrm{AM}$ & $\ldots$ & & $\begin{array}{l}10 \\
18\end{array}$ & 0 & 5 & & $\begin{array}{l}0 \\
0\end{array}$ & 0 & 0 & 18 & 2 & 4 & 26 & $\because$ & os \\
\hline $11.45 \mathrm{AM}$ & $\cdots$ & $\frac{12: 00 \mathrm{PM}}{12: 15 \mathrm{PM}}$ & $-\frac{18}{9}$ & & 7 & & 0 & 0 & 0 & 16 & 0 & 4 & 26 & $\therefore$ & -1 \\
\hline $12.00 \mathrm{PM}$ & $\cdots$ & $12: 15 \mathrm{PM}$ & 6 & 0 & 2 & 0 & 0 & 0 & 0 & 24 & 1 & 3 & 31 & ". & 68 \\
\hline $12: 15 \mathrm{PM}$ & $\ldots$ & $\begin{array}{l}\text { 12:30 PM } \\
12: 45 \mathrm{PM}\end{array}$ & 6 & 0 & 4 & 0 & 0 & 0 & 0 & 26 & 5 & 2 & 23 & $\therefore$ & 66 \\
\hline $12: 30 \mathrm{PM}$ & $\ldots$ & $\begin{array}{l}12: 45 \mathrm{PM} \\
01: 00 \mathrm{PM}\end{array}$ & 3 & 0 & 4 & 0 & 0 & 0 & 0 & 3 & 4 & 4 & $i 3$ & $\therefore$ & II \\
\hline $12: 45$ PM & & $\frac{01: 00 \mathrm{PM}}{01: 15 \mathrm{PM}}$ & 12 & 0 & 2 & 0 & 0 & 0 & 0 & 22 & 20 & 6 & 22 & $\because$ & 84 \\
\hline $01.00 \mathrm{PM}$ & - & $\begin{array}{l}01: 15 \mathrm{PM} \\
01: 30 \mathrm{PM}\end{array}$ & 9 & 0 & 2 & 0 & 0 & 0 & 0 & $\frac{17}{17}$ & 8 & 4 & 17 & $\because$ & 5 \\
\hline 01:15 PM & $=$ & $\begin{array}{l}01: 30 \mathrm{PM} \\
0.105 \mathrm{PM}\end{array}$ & $\begin{array}{l}4 \\
2\end{array}$ & 0 & 6 & 0 & 0 & 0 & 0 & 22 & 4 & 7 & 23 & '; & 66 \\
\hline $\begin{array}{l}01: 30 \mathrm{PM} \\
01: 45 \mathrm{PM}\end{array}$ & $=$ & $\begin{array}{l}01: 45 \text { PM } \\
02: 00 \text { PM }\end{array}$ & $\begin{array}{l}2 \\
8 \\
\end{array}$ & $\begin{array}{l}0 \\
0\end{array}$ & $\begin{array}{l}0 \\
2\end{array}$ & $\begin{array}{l}0 \\
0\end{array}$ & $\begin{array}{l}0 \\
0\end{array}$ & $\begin{array}{l}0 \\
0\end{array}$ & $\begin{array}{l}0 \\
0\end{array}$ & $\begin{array}{l}15 \\
18\end{array}$ & $\begin{array}{c}10 \\
8\end{array}$ & $\begin{array}{l}3 \\
5\end{array}$ & 15 & $\because$ & $\begin{array}{l}15 \\
50\end{array}$ \\
\hline $02: 00 \mathrm{PM}$ & $\ldots$ & $02: 15 \mathrm{PM}$ & 3 & $\overline{0}$ & $\frac{5}{0}$ & 0 & 0 & 0 & 0 & 18 & 3 & $\frac{2}{2}$ & & $\therefore$ & -13 \\
\hline 02:15 PM & $-\ldots$ & 02:30 PM & 3 & 0 & 3 & 0 & 0 & 0 & 0 & 11 & 4 & 5 & 15 & .. & I \\
\hline 02:30 PM & $\ldots$ & 02:45 PM & 0 & 0 & 4 & ${ }_{0}^{0}$ & 0 & 0 & 0 & 26 & 0 & 5 & 7 & . & 2 \\
\hline $02.45 \mathrm{PM}$ & $\cdots$ & 03:00 PM & $\frac{2}{2}$ & 0 & 8 & $\frac{0}{0}$ & 0 & 0 & 0 & 24 & 0 & $\frac{1}{3}$ & 18 & - & 33 \\
\hline $03: 00 \mathrm{PM}$ & $\ldots$ & $03: 15 \mathrm{PM}$ & 2 & 0 & 4 & 0 & 0 & 0 & 0 & 29 & 4 & 3 & 11 & $\because$ & 5.1 \\
\hline $03: 19 \mathrm{PM}$ & $\ldots$ & $0 \geq: 30$ PM & 6 & 0 & 5 & 0 & 0 & 0 & 0. & 48 & 0 & 2 & 15 & $\therefore$ & -6 \\
\hline 03:30 PM & $\ldots$ & 03.45 PM & 1 & 0 & 10 & 0 & 0 & 0 & 0 & 61 & 0 & i & 16 & $"$ & 89 \\
\hline $03: 45 \mathrm{PM}$ & $\ldots$ & $04: 00 \mathrm{PM}$ & 3 & 0 & 15 & 0 & 0 & 0 & 0 & 56 & 4 & 4 & 17 & a & 99 \\
\hline $04: 00 \mathrm{PM}$ & $\ldots$ & $04: 15 \mathrm{PM}$ & 4 & 0 & -15 & 0 & 0 & 0 & 0 & 39 & $T$ & 3 & 15 & ". & $-\frac{1}{9+}$ \\
\hline O4:15 PM & $\ldots$ & 04:30 PM & 6 & 0 & 19 & 0 & 0 & 0 & 0 & 78 & $i$ & 3 & 18 & " & 125 \\
\hline $04: 30 \mathrm{PM}$ & - & $04: 45 \mathrm{PM}$ & 8 & 0 & 26 & 0 & 0 & 0 & 0 & 72 & $i$ & 1 & i1 & .' & 119 \\
\hline 04:45 PM & $\ldots$ & $05.00 \mathrm{PM}$ & 10 & 0 & 41 & 0 & 0 & 0 & 0 & 87 & 2 & $i$ & i= & , & 13 \\
\hline & & & & & & & $R L Y$ & TOTA & & & & & & & \\
\hline OT:CAM & 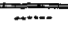 & $08: 00 \mathrm{nM}$ & 78 & 0 & 22 & 8 & $\gamma$ & \% & $\overline{0}$ & 80 & 75 & 62 & $33^{-}$ & . & $=\pi$ \\
\hline $07.15 \mathrm{AM}$ & $\ldots$ & 08:15 AM & 15 & 0 & 24 & 0 & 9 & 0 & 0 & 62 & 88 & 73 & 316 & $\because$ & 58 \\
\hline $07: 30 \mathrm{AM}$ & $=$ & 08:30 AM & $\frac{17}{17}$ & $-\frac{a}{0}$ & $\frac{28}{67}$ & 0 & 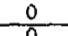 & 0 & 0 & 64 & $\frac{33}{80}$ & $\frac{63}{47}$ & $\frac{29}{190}$ & " & 552 \\
\hline $07: 45 \mathrm{AM}$ & $=$ & 08:45AM & 17 & $\overline{0}$ & 23 & 0 & 0 & 0 & 0 & 68 & 80 & 47 & $\frac{2}{9} 9$ & $\because$ & 514 \\
\hline $08.00 \mathrm{AM}$ & - & 09:00 AM & 15 & 0 & 26 & 0 & 0 & 0 & 0 & 72 & 73 & 40 & 232 & .' & 103 \\
\hline $08: 15 \mathrm{AM}$ & $\ldots$ & $09: 15$ AM & 16 & 0 & 27 & 0 & 0 & 0 & 0 & 65 & 71 & 23 & 199 & , & $\$ m 2$ \\
\hline $08: 30 \mathrm{AM}$ & $\cdots$ & $09: 30 \mathrm{AM}$ & 19 & 0 & 23 & 0 & 0 & 0 & 0 & 72 & 56 & 23 & 160 & 4 & 39 \\
\hline 08:45 AM & - & $09: 45 \mathrm{AM}$ & $\begin{array}{l}27 \\
40\end{array}$ & 0 & 19 & & 0 & $\begin{array}{l}0 \\
0\end{array}$ & $\begin{array}{l}0 \\
0\end{array}$ & 67 & 44 & 20 & 120 & ". & 27 \\
\hline $09: 00 \mathrm{AM}$ & $=$ & $10.00 \mathrm{AM}$ & $\frac{40}{39}$ & 0 & 15 & $\frac{0}{0}$ & $\frac{0}{0}$ & $\frac{0}{0}$ & $\frac{0}{0}$ & 64 & 28 & -20 & 90 & $"$ & $y=$ \\
\hline $\begin{array}{l}09: 15 \mathrm{AM} \\
09: 30 \mathrm{AM}\end{array}$ & $=$ & $10: 15 \mathrm{AM}$ & $\begin{array}{l}39 \\
29\end{array}$ & $\begin{array}{l}0 \\
0\end{array}$ & 11 & $\begin{array}{l}0 \\
0\end{array}$ & $\begin{array}{l}0 \\
0\end{array}$ & $\begin{array}{l}0 \\
0\end{array}$ & $\begin{array}{l}0 \\
0\end{array}$ & $\begin{array}{l}69 \\
60\end{array}$ & 4 & 25 & $6:$ & $\because$ & $\frac{115}{180}$ \\
\hline $\begin{array}{l}09: 30 \mathrm{AM} \\
09: 45 \mathrm{AM}\end{array}$ & $=$ & $\begin{array}{l}10: 30 \mathrm{AM} \\
10: 45 \mathrm{AM}\end{array}$ & $\begin{array}{l}29 \\
21 \\
\end{array}$ & 0 & $\begin{array}{l}13 \\
11\end{array}$ & $\begin{array}{l}0 \\
0\end{array}$ & 0 & 0 & 0 & $\begin{array}{l}60 \\
58 \\
\end{array}$ & $\begin{array}{l}1 \\
2\end{array}$ & $\begin{array}{l}25 \\
2 \mathrm{I}\end{array}$ & $\begin{array}{l}5= \\
54\end{array}$ & $\because$ & $\begin{array}{l}180 \\
16^{\circ}\end{array}$ \\
\hline $10: 00 \mathrm{AM}$ & $=$ & $11: 00 \mathrm{AM}$ & 7 & 0 & 15 & 0 & 5 & 0 & 0 & 52 & 6 & 21 & $s \bar{s}$ & & $1 \sqrt{9}$ \\
\hline -10:15 AM & - & 11:15 AM & 7 & 0 & 14 & 0 & 0 & 0 & 0 & $\begin{array}{l}50 \\
40\end{array}$ & 6 & 23 & $\vdots 1$ & $\therefore$ & ist \\
\hline 10:30 AM & $\ldots$ & $11: 30 \mathrm{AM}$ & 11 & $\frac{0}{n}$ & $\frac{10}{12}$ & 0 & $\frac{0}{0}$ & -0 & -0 & $\frac{49}{5-3}$ & 6 & 21 & $-6 !$ & . & 158 \\
\hline $10.45 \mathrm{AM}$ & $\ldots$ & $11: 15 \mathrm{AM}$ & 20 & 0 & 13 & 0 & 0 & 0 & 0 & 53 & 7 & $2 !$ & & ?. & 991 \\
\hline $11.00 \mathrm{AM}$ & - & 12:00 PM & 37 & 0 & 13 & 0 & 0 & 0 & 0 & 60 & 3 & 18 & 87 & . & Its \\
\hline 11:15 AM & $=$ & 12:15 PM & 41 & 0 & 15 & 0 & 0 & 0 & 0 & 69 & 4 & 13 & 106 & $" 1$ & $\therefore 8$ \\
\hline $11: 30 \mathrm{AM}$ & $\ldots$ & $12: 30 \mathrm{PM}$ & 41 & 0 & 18 & 0 & 0 & 0 & 0 & 84 & 8 & 13 & 106 & ". & 20 \\
\hline $11: 43$ AM & $\ldots$ & 12:45 PM & 34 & 0 & 17 & 0 & 0 & 0 & 0 & 69 & 10 & 13 & 93 & "i & 236 \\
\hline $12: 00 \mathrm{PM}$ & $\ldots$ & $01: 008 \mathrm{M}$ & 28 & 0 & $-\frac{12}{12}$ & -0 & $\frac{0}{0}$ & $\frac{0}{a}$ & $\frac{0}{0}$ & $\frac{75}{68}$ & $\frac{30}{37}$ & $\frac{15}{16}$ & $\frac{89}{95}$ & $\ddot{3}$ & $2 \frac{99}{38}$ \\
\hline $12: 19 \mathrm{PM}$ & $=$ & $01: 15 \mathrm{PM}$. & 30 & 0 & 12 & 0 & 0 & 0 & 0 & 68 & 37 & 16 & 75 & '1 & 238 \\
\hline $12: 30 \mathrm{PM}$ & - & 01:30 PM & 28 & - 0 & 14 & 0 & 0 & 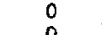 & 0 & $\begin{array}{l}64 \\
76\end{array}$ & 36 & 210 & 75 & 11 & 238 \\
\hline 12:4S PM & $\ldots$ & $01: 45$ PM & 27 & 0 & 10 & 0 & $\frac{0}{0}$ & $\frac{0}{0}$ & 0 & $\frac{76}{72}$ & $\frac{42}{30}$ & $\frac{20}{19}$ & $\frac{37}{64}$ & 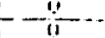 & $-\frac{252}{238}$ \\
\hline $01.00 \mathrm{PM}$ & $=$ & $02: 00 \mathrm{PM}$ & 23 & 0 & 10 & 0 & $\begin{array}{l}0 \\
0\end{array}$ & $\begin{array}{l}0 \\
0\end{array}$ & $\begin{array}{l}0 \\
0\end{array}$ & 72 & $\begin{array}{l}30 \\
25\end{array}$ & 17 & 54 & 0 & $2 / 8$ \\
\hline $\begin{array}{l}01: 15 \mathrm{PM} \\
01.30 \mathrm{PM}\end{array}$ & $=$ & $\begin{array}{l}02: 19 \mathrm{PM} \\
02: 30 \mathrm{PM}\end{array}$ & 17 & 0 & 8 & $\begin{array}{l}0 \\
0\end{array}$ & 0 & 0 & 0 & 62 & 25 & 15 & 46 & 0 & $\begin{array}{l}194 \\
169\end{array}$ \\
\hline$\frac{01: 30 \mathrm{PM}}{01: 45 \mathrm{PM}}$ & $=$ & $\frac{02: 30 \mathrm{PM}}{02: 13 \mathrm{PM}}$ & $\frac{16}{14}$ & $\frac{0}{0}$ & $-\frac{3}{9}$ & 0 & 0 & 0 & 0 & 73 & 15 & 17 & 38 & 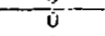 & $\frac{109}{166}$ \\
\hline 02:00 PM & - & $03.00 \mathrm{PM}$ & 8 & 0 & is & 0 & 0 & 0 & 0 & 79 & $?$ & 13 & 47 & 0 & 168 \\
\hline $02: 15 \mathrm{PM}$ & $\ldots$ & 03:15 PM & 7 & 0 & 19 & 0 & 0 & 0 & 0 & 90 & 8 & 14 & $5 i$ & 0 & 189 \\
\hline $02: 30 \mathrm{pM}$ & $=$ & $03: 30 \mathrm{PM}$ & To & 0 & 21 & 0 & 0 & 0 & 0 & 127 & 4 & $\pi$ & 31 & 0 & 238 \\
\hline $02: 45 \mathrm{PM}$ & - & 03:45 PM & 11 & 0 & 27 & 0 & 0 & 0 & 0 & 162 & 4 & 7 & 60 & $u$ & $2 \pi$ \\
\hline $\begin{array}{l}03: 00 \mathrm{PM} \\
0\end{array}$ & $\div$ & $04.00 \mathrm{PM}$ & 12 & 0 & 34 & 0 & 0 & 0 & 0 & 194 & 8 & 10 & 59 & 0 & 31: \\
\hline $03: 15 \mathrm{PM}$ & $=$ & $04: 15 \mathrm{PM}$ & 14 & 0 & 45 & 0 & 0 & 0 & 0 & 224 & 5 & 10 & 63 & 0 & 361 \\
\hline $03: 30 \mathrm{PM}$ & $\longrightarrow$ & $04.30 \mathrm{PM}$ & 14 & 0 & 59 & 0 & 0 & 0 & 0 & 254 & 6 & 11 & 66 & 0 & 510 \\
\hline 03:15 PM & - & 04:45 PM & 21 & 0 & 75 & 0 & 0 & 0 & 0 & 265 & 7 & 11 & 61 & 0 & sso \\
\hline $04: 00 \mathrm{PM}$ & $\equiv$ & $05: 00 \mathrm{PM}$ & 28 & 0 & 101 & 0 & 0 & 0 & 0 & 296 & 5 & 8 & 56 & 0 & 898 \\
\hline
\end{tabular}




\section{Baymetrics Traffic Resources INTERSECTION TURNING MOVEMENT SUMMARY}

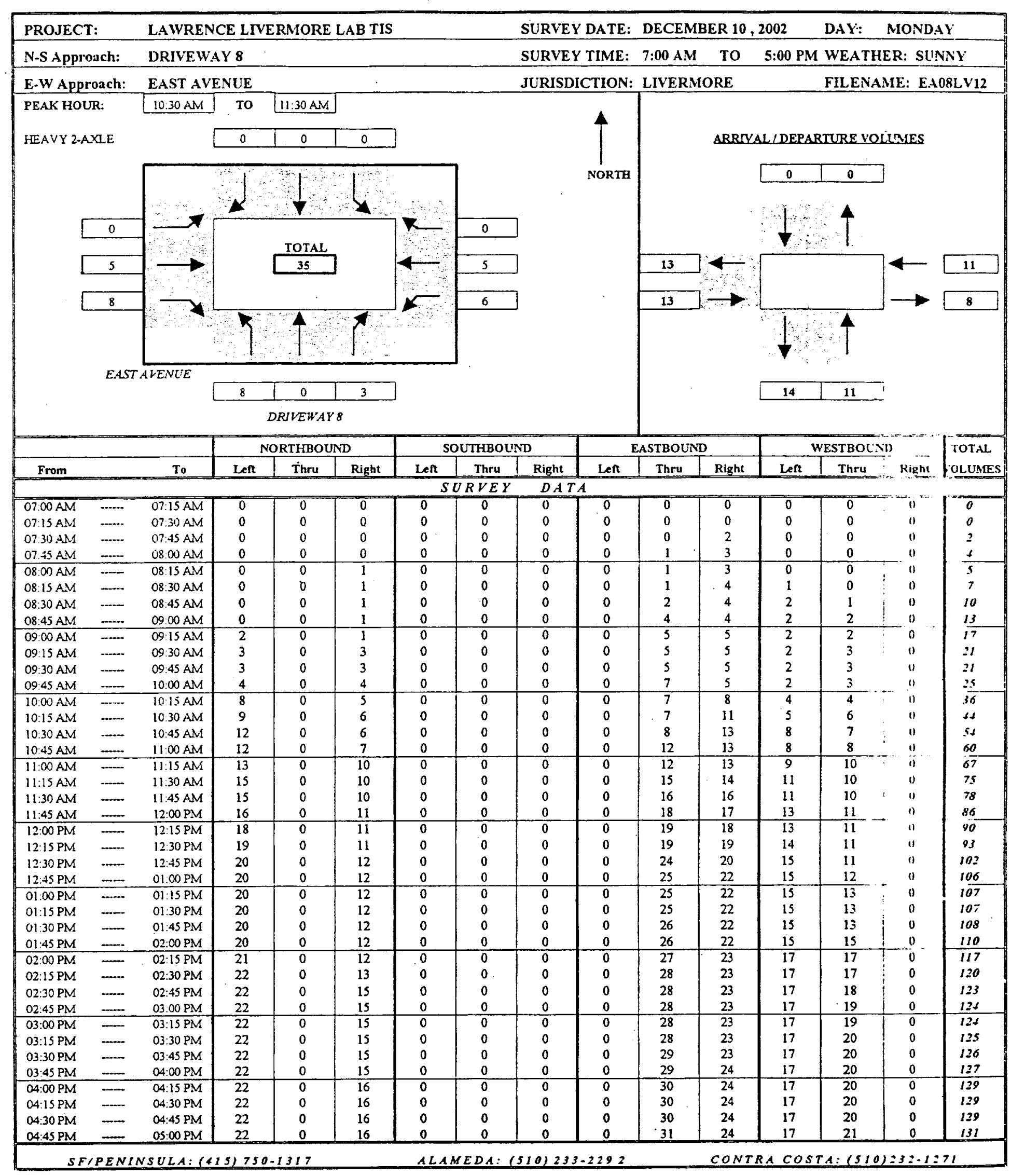




\section{$\frac{\text { Baymetrics Traffic Resources}}{\text { INTERSECTION TURNING MOVEMENT SUMMARY }}$}

\begin{tabular}{|c|c|c|c|c|c|c|c|c|c|c|c|c|c|c|}
\hline PROJECT: & LAWRE & CE LIV & RMORI & LAB TIS & & & SURVE & DATE: & DECEME & BER 10 & 2002 & DAY: & MOND & \\
\hline N-S Approach: & DRRVEK & & & & & & SURVE & TIME: & 7:00 AM & To & S:00 P & M WEAT & IER: SU & \\
\hline E-W Approach: & EAST AI & NUE & & & & & JURISL & CTION: & LIVERM & ORE & & FILEN & MIE: EA & $08 \mathrm{LV}_{12}$ \\
\hline & & & $\frac{\text { IRTHBO }}{T}$ & & & UTHBOU & & & EASTBOUNI & & & WESTBOU & & тот \\
\hline From & To & Lent & Thru & Right & $\frac{\text { Len }}{T}$ & $\frac{\text { Thru }}{A L B Y}$ & $\frac{1 \text { Right }}{P E R I}$ & $\frac{\text { Len }}{D}$ & Thru I & Right & Len & Thru & Right & OLUMES \\
\hline 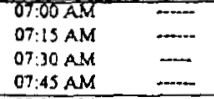 & 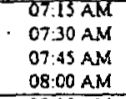 & & $\begin{array}{l}0 \\
0 \\
0 \\
0\end{array}$ & & & & $0_{0}$ & & $\begin{array}{l}0 \\
0 \\
0\end{array}$ & $\therefore$ & $\begin{array}{l}0 \\
0 \\
0 \\
0\end{array}$ & $\begin{array}{l}0 \\
0 \\
0\end{array}$ & $\begin{array}{l}0 \\
0 \\
0 \\
0\end{array}$ & \\
\hline 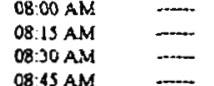 & 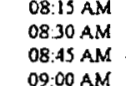 & & $\begin{array}{l}0 \\
0\end{array}$ & & & & 0 & & & & & 0 & 0 & \\
\hline 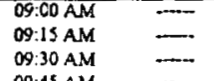 & 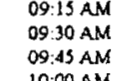 & & 0 & & & & $\begin{array}{l}0 \\
0 \\
0\end{array}$ & & $\begin{array}{l}0 \\
0 \\
0\end{array}$ & & & & 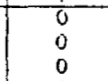 & \\
\hline $\begin{array}{l}10.00 \mathrm{AM} \\
10.1 \mathrm{ANA} \\
10.30 \mathrm{AM}\end{array}$ & 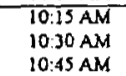 & & $\begin{array}{c}0 \\
0 \\
0\end{array}$ & & 0 & & $\stackrel{0}{a}$ & & $i$ & & & & & \\
\hline 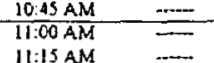 & 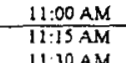 & & & & & & & & & & & & 17 & \\
\hline 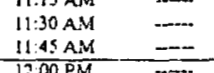 & 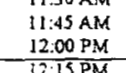 & & & & 0 & 0 & $\begin{array}{l}0 \\
0 \\
0\end{array}$ & $\begin{array}{l}0 \\
0\end{array}$ & 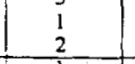 & & 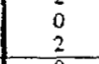 & & ii & \\
\hline $\begin{array}{l}12: 15 \mathrm{PM} \\
12.30 \mathrm{PM}\end{array}=$ & 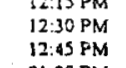 & & $\begin{array}{c}0 \\
0 \\
0\end{array}$ & & 0 & & $\begin{array}{l}0 \\
0 \\
0\end{array}$ & 0 & $\begin{array}{l}1 \\
5 \\
s\end{array}$ & & $i$ & $: 0$ & 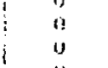 & 3 \\
\hline 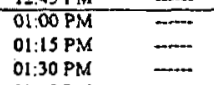 & 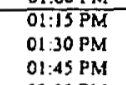 & 0 & $\begin{array}{l}0 \\
0 \\
0\end{array}$ & & 0 & & $\begin{array}{l}0 \\
0 \\
0\end{array}$ & & 0 & & $\begin{array}{l}0 \\
0 \\
0 \\
0\end{array}$ & & : & \\
\hline 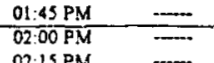 & $\begin{array}{l}02: 200 M \\
02: 15 P M \\
\end{array}$ & & & & & & & & & & & & $-\frac{10}{12}$ & \\
\hline 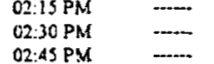 & 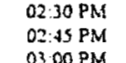 & & $\begin{array}{c}0 \\
0 \\
0\end{array}$ & & $\begin{array}{l}0 \\
0 \\
0\end{array}$ & & $\begin{array}{l}0 \\
0 \\
0\end{array}$ & 0 & $\begin{array}{l}1 \\
0 \\
0\end{array}$ & $\therefore$ & $\begin{array}{l}0 \\
0 \\
0\end{array}$ & & 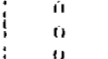 & \\
\hline 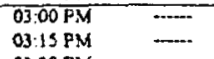 & & & 0 & & $\begin{array}{l}0 \\
0\end{array}$ & & $\begin{array}{l}0 \\
0\end{array}$ & & 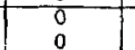 & $\begin{array}{l}0 \\
0 \\
0\end{array}$ & $\begin{array}{l}0 \\
0 \\
0\end{array}$ & & 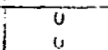 & \\
\hline 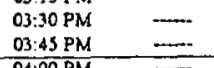 & 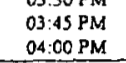 & & & & 0 & & 0 & 0 & & 0 & : & 0 & y & $i$ \\
\hline 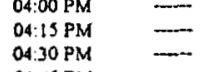 & 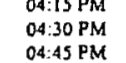 & & $: 0$ & 0 & & & $\begin{array}{l}0 \\
0 \\
0\end{array}$ & $\begin{array}{l}0 \\
0\end{array}$ & $\begin{array}{l}1 \\
0 \\
0\end{array}$ & 0 & $\begin{array}{l}0 \\
0 \\
0\end{array}$ & 0 & "1" & $\frac{2}{0}$ \\
\hline $04: 6$ & & & & & & & $T$ & & & & & & & \\
\hline 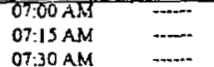 & 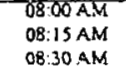 & & $\begin{array}{l}0 \\
0 \\
0\end{array}$ & & & $e_{0}^{0}$ & $\begin{array}{c}0 \\
0 \\
0\end{array}$ & 0 & & & $\begin{array}{l}0 \\
0 \\
1\end{array}$ & 8 & $\because$ & $s$ \\
\hline 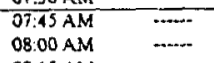 & 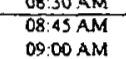 & & 0 & & & & $\begin{array}{l}0 \\
0\end{array}$ & $\begin{array}{c}0 \\
0 \\
0\end{array}$ & & & $\frac{2}{2}$ & & $" 1$ & 8 \\
\hline$\frac{0.815 A M}{0.830 A M}=$ & & & $\div$ & & & & $\frac{0}{0}$ & & & & & & $-i 1$ & $\frac{21}{1 s}$ \\
\hline 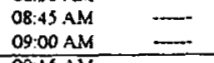 & $\begin{array}{r}09.4 \mathrm{SM} \\
10.0 \mathrm{AM} \\
\end{array}$ & & 0 & & $\begin{array}{l}0 \\
0 \\
\end{array}$ & 0 & $\begin{array}{l}0 \\
0 \\
0\end{array}$ & $\stackrel{0}{0}$ & 3 & & $\begin{array}{l}0 \\
0 \\
\end{array}$ & 2 & $"$ & $\begin{array}{l}11 \\
12 \\
2\end{array}$ \\
\hline 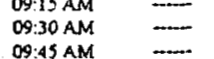 & 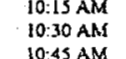 & & $\begin{array}{l}0 \\
0\end{array}$ & & $\begin{array}{l}0 \\
0\end{array}$ & & $\begin{array}{ll}0 \\
0 \\
0\end{array}$ & 0 & $\frac{2}{3}$ & & $\frac{2}{3}$ & 3 & n. & 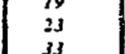 \\
\hline $\begin{array}{l}10.00 \mathrm{AM} \\
10.1 \mathrm{SM}\end{array}$ & $\begin{array}{l}1100 \mathrm{MM} \\
1110 \mathrm{AMM}\end{array}$ & & 0 & & & & 0 & 0 & & & 5 & $\frac{5}{6}$ & "i" & $\begin{array}{l}35 \\
31 \\
15\end{array}$ \\
\hline$\frac{1030 \mathrm{AM}}{104 \mathrm{AM}}=$ & $-11: 30 \mathrm{AM}$ & & 9 & $\frac{4}{4}$ & & & 0 & 0 & $\frac{8}{8}$ & & & & $\frac{10}{0}$ & $\frac{31}{22}$ \\
\hline $\begin{array}{l}11: 00 \mathrm{AM} \\
11: 15 \mathrm{AM}\end{array}=$ & $\begin{array}{l}12.000 \mathrm{MM} \\
-2121.1 \mathrm{PMM} \\
\end{array}$ & & $\begin{array}{l}0 \\
0 \\
0\end{array}$ & & & & $\begin{array}{l}0 \\
0\end{array}$ & $: 0$ & 6 & & $\begin{array}{l}5 \\
4 \\
\end{array}$ & 1 & 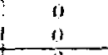 & $\begin{array}{l}26 \\
23 \\
\end{array}$ \\
\hline 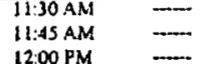 & 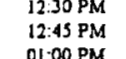 & & $\begin{array}{l}0 \\
0 \\
0\end{array}$ & 2 & & & $\begin{array}{l}0 \\
0 \\
0\end{array}$ & $\begin{array}{l}0 \\
0 \\
0\end{array}$ & $\begin{array}{l}4 \\
8 \\
7\end{array}$ & & $\begin{array}{l}3 \\
2 \\
\end{array}$ & 1 & $\stackrel{8}{a}$ & $\begin{array}{l}38 \\
32 \\
32\end{array}$ \\
\hline $\begin{array}{l}12215 \mathrm{PP} \\
12.30 \mathrm{PM}\end{array}$ & $\begin{array}{l}0.115 \mathrm{sm} \\
01030 \mathrm{PM}\end{array}$ & & $\begin{array}{l}0 \\
0 \\
0\end{array}$ & & 0 & & $\begin{array}{l}0 \\
0 \\
0\end{array}$ & $\begin{array}{l}0 \\
0\end{array}$ & 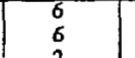 & & & 2 & 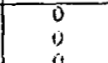 & $\begin{array}{l}17 \\
14\end{array}$ \\
\hline$=$ & 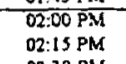 & & 0 & 0 & 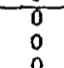 & & 0 & 0 & $\frac{2}{3}$ & & $\frac{2}{2}$ & 4 & 0 & io \\
\hline$\equiv$ & 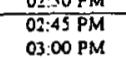 & $\frac{2}{2}$ & 0 & & & & 0 & 0 & 2 & & 2 & 4 & 0 & is \\
\hline$=$ & & -0 & 0 & & & & & & & & 0 & & $\frac{0}{0}$ & \\
\hline 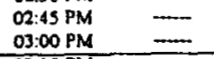 & $\begin{array}{l}0.145 \mathrm{SM} \\
0400 \mathrm{PM}\end{array}$ & $\begin{array}{l}0 \\
0 \\
0 \\
0\end{array}$ & .0 & 年 & & & $\begin{array}{r}0 \\
0 \\
0\end{array}$ & 0 & & & 0 & & 0 & 3 \\
\hline 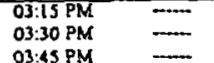 & 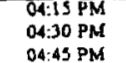 & 0 & $\begin{array}{l}0 \\
0 \\
0\end{array}$ & & $\begin{array}{l}a \\
0\end{array}$ & & $\begin{array}{l}0 \\
0 \\
0\end{array}$ & $\begin{array}{l}0 \\
0\end{array}$ & $\frac{2}{2}$ & & $\begin{array}{l}0 \\
0 \\
0\end{array}$ & $\begin{array}{l}1 \\
0 \\
0\end{array}$ & $\begin{array}{l}0 \\
0 \\
0\end{array}$ & $\begin{array}{l}5 \\
3 \\
3\end{array}$ \\
\hline & & & & & & & & & & & & 1510 & & \\
\hline
\end{tabular}




\section{Baymetrics Traffic Resources INTERSECTION TURNING MOVEMENT SUMMARY}

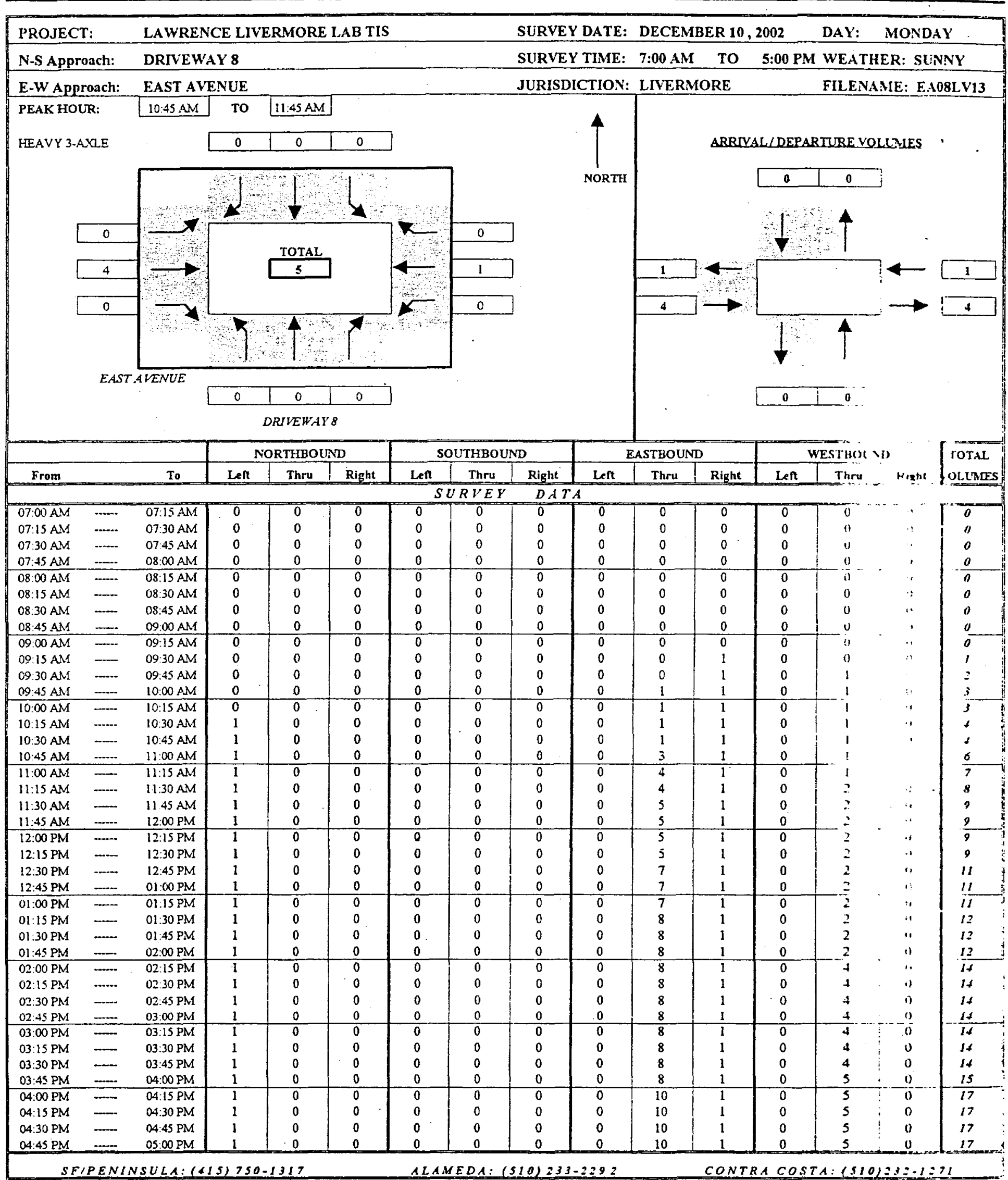




\section{$\frac{\text { Baymetrics Traffic Resources}}{\text { INTERSECTION TURNING MOVEMENT SUMMARY }}$}

\begin{tabular}{|c|c|c|c|c|c|c|c|c|c|c|c|c|c|c|c|}
\hline \multirow{2}{*}{\multicolumn{2}{|c|}{$\begin{array}{l}\text { PROJECT: } \\
\text { N-S Approach: }\end{array}$}} & LAWRE & LIV & MOR & AB TIS & & & SURVE & DATE: & DECE & ER 10, & 2002 & DAY: & MOND & \\
\hline & & DRIVEX & & & & * & & SURVE & TIME: & $7: 00 \mathrm{AN}$ & To & 5:00 PM & WEATH & IER: SLI! & $\mathrm{NNY}$ \\
\hline EW Ap & oach: & EAST A & UE & & & & & JURISD & TION: & LIVER & REE & & FILEN & ME: EA & $08 \mathrm{LV13}$ \\
\hline & & & & THBO & & & THBO & & & ASTBOU & & & ESTBOU & & TOTAL \\
\hline From & & To & Left & Thru & Right & Left & Thru & Right & Lent & Thru & Right & Len & Thru & Righe & YouUNES \\
\hline & & & & & & & $L \quad B$ & PERI & & & & & & & \\
\hline 67:00 AM & $\pi$ & $07: 15 \mathrm{AM}$ & $\sigma$ & 8 & 0 & \% & $\sigma$ & 0 & $\sigma$ & .0 & $\sigma$ & $\sigma$ & $\sigma$ & 0 & 8 \\
\hline $07: 15 \mathrm{AM}$ & $\cdots$ & $07.30 \mathrm{AM}$ & 0 & 0 & 0 & 0 & 0 & 0 & 0 & 0 & 0 & 0 & 0 & 0 & $a$ \\
\hline $07: 30 \mathrm{AM}$ & - & $07: 45 \mathrm{AM}$ & 0 & 0 & 0 & 0 & 0 & 0 & 0 & 0 & 0 & 0 & 0 & 0 & 0 \\
\hline$\frac{07: 45 \mathrm{AM}}{08: 00 \mathrm{AM}}$ & $=$ & $\frac{08: 00 \mathrm{AM}}{08: 15 \mathrm{AM}}$ & 0 & 0 & 0 & 0 & 0 & 0 & 0 & 0 & 0 & 0 & 0 & 0 & 0 \\
\hline $08.15 \mathrm{AM}$ & $\ldots$ & $\begin{array}{l}08: 15 \mathrm{AM} \\
08: 30 \mathrm{AM}\end{array}$ & $\begin{array}{l}0 \\
0\end{array}$ & $\begin{array}{l}0 \\
0\end{array}$ & $\begin{array}{l}0 \\
0\end{array}$ & 0 & 0 & 0 & 0 & 0 & $\begin{array}{l}0 \\
0\end{array}$ & $\begin{array}{l}0 \\
0\end{array}$ & $\begin{array}{l}0 \\
0 \\
0\end{array}$ & 9 & 0 \\
\hline $08: 30 \mathrm{AM}$ & $\ldots$ & $08: 45 \mathrm{AM}$ & 0 & 0 & 0 & 0 & 0 & $\stackrel{0}{0}$ & $\begin{array}{l}0 \\
0 \\
0\end{array}$ & $\begin{array}{l}0 \\
0\end{array}$ & & 0 & D & 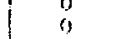 & 0 \\
\hline 08:45 AM & $\cdots$ & $09: 00 \mathrm{AM}$ & 0 & 0 & 0 & 0 & 0 & 0 & 0 & 0 & 0 & 0 & 0 & (1) & $a$ \\
\hline $0900 \mathrm{AM}$ & $\ldots$ & $09: 15 \mathrm{AM}$ & 0 & 0 & 0 & 0 & 0 & 0 & 0 & 0 & 0 & 0 & 0 & $\pi$ & 0 \\
\hline 09:15.AM & $\ldots$ & $09 \cdot 30 \mathrm{AM}$ & 0 & 0 & 0 & 0 & 0 & 0 & 0 & 0 & 1 & 0 & 0 & a & 1 \\
\hline $09: 30 \mathrm{AM}$ & - & $09: 45 \mathrm{AM}$ & 0 & 0 & 0 & 0 & 0 & 0 & 0 & 0 & 0 & 0 & l & 0 & $i$ \\
\hline $09: 45$ AM & $=$ & $10: 00 \mathrm{AM}$ & 0 & 0 & 0 & 0 & 0 & 0 & 0 & 1 & 0 & 0 & 0 & t) & 1 \\
\hline $10: 00 \mathrm{AM}$ & $=$ & $10: 15 \mathrm{AM}$ & 0 & 0 & 0 & 0 & 0 & 0 & $\overline{0}$ & 0 & 0 & 0 & 0 & iv & $a$ \\
\hline $10: 15 \mathrm{AM}$ & $\ldots$ & $10: 30 \mathrm{AM}$ & 1 & 0 & 0 & 0 & 0 & 0 & 0 & 0 & 0 & 0 & 0 & 0 & i \\
\hline $10: 30 \mathrm{AM}$ & $\cdots$ & $10.45 \mathrm{AM}$ & 0 & 0 & 0 & 0 & 0 & 0 & 0 & 0 & 0 & 0 & 0 & 1 & 0 \\
\hline $10: 45 \mathrm{AM}$ & $=$ & $11: 00 \mathrm{AM}$ & 0 & 0 & 0 & 0 & 0 & 0 & 0 & 2 & 0 & 0 & 0 & i. & 2 \\
\hline $11: 00 \mathrm{AM}$ & $\cdots$ & 11:15.AM & 0 & 0 & 0 & 0 & 0 & 0 & 0 & 1 & 0 & 0 & $\overline{0}$ & $\because 7$ & I \\
\hline $11: 15 \mathrm{AM}$ & $\cdots$ & $11: 30 \mathrm{AM}$ & 0 & 0 & 0 & 0 & 0 & 0 & 0 & 0 & 0 & 0 & 1 & $\therefore$ & 1 \\
\hline $11: 30 \mathrm{AM}$ & - & $11: 45$ AM & 0 & 0 & 0 & 0 & 0 & 0 & 0 & 1 & 0 & 0 & 0 & $\because$ & $i$ \\
\hline $11: 45$ AM & $\ldots$ & $12: 00 \mathrm{PM}$ & 0 & 0 & 0 & 0 & 0 & 0 & 0 & 0 & 0 & 0 & 0 & $"$ & 0 \\
\hline $12: \overline{0} \overline{\mathrm{PM}}$ & $=$ & $17: 15 \mathrm{PM}$ & 0 & 0 & 0 & 0 & 0 & 0 & 0 & 0 & 0 & $\overline{0}$ & 0 & " & 0 \\
\hline 12:15 PM & $\cdots$ & 12:30 PM & 0 & 0 & 0 & 0 & 0 & 0 & 0 & 0 & 0 & 0 & 0 & $"$ & 0 \\
\hline $12: 30 \mathrm{PM}$ & $\cdots$ & 12:45 PM & 0 & 0 & 0 & 0 & ) & 0 & 0 & 2 & 0 & o & 0 & $\therefore$ & 2 \\
\hline 12:45 PM & - & $01: 00 \mathrm{PM}$ & 0 & 0 & 0 & 0 & 0 & 0 & 0 & 0 & 0 & 0 & 0 & "י & 0 \\
\hline $01: 00 \mathrm{PM}$ & + & $01: 15$ PM & $\overline{0}$ & 0 & 0 & 0 & o & 0 & 0 & 0 & 0 & 0 & 0 &. & $\because$ \\
\hline or:15 PM & $\ldots$ & $01: 30 \mathrm{PM}$ & 0 & 0 & 0 & 0 & 0 & 0 & 0 & 1 & 0 & 0 & 0 & $\therefore$ & $i$ \\
\hline $01: 30 \mathrm{PM}$ & $\cdots$ & $01.45 \mathrm{PM}$ & 0 & 0 & 0 & 0 & 0 & 0 & 0 & 0 & 0 & 0 & 0 & $\therefore$ & $a$ \\
\hline $01: 45 \mathrm{PM}$ & $\cdots$ & $02: 00 \mathrm{PM}$ & 0 & 0 & 0 & 0 & 0 & 0 & 0 & 0 & 0 & 0 & 0 & $\because$ & 0 \\
\hline $02: 00 \mathrm{PM}$ & $=$ & $02: 15 \mathrm{PM}$ & 0 & 0 & 0 & 0 & 0 & 0 & 0 & 0 & 0 & 0 & 2 & . & 3 \\
\hline 02:15 PM & - & $02: 30 \mathrm{PM}$ & 0 & 0 & 0 & 0 & 0 & 0 & 0 & 0 & 0 & 0 & ") & $\because$ & 0 \\
\hline 02:30 PM & $\ldots$ & $02: 45 \mathrm{PM}$ & 0 & 0 & 0 & 0 & 0 & 0 & 0 & 0 & 0 & 0 & is & $\therefore$ & 0 \\
\hline 02:as PM & $\cdots$ & $03: 00 \mathrm{PM}$ & 0 & 0 & 0 & 0 & 0 & 0 & 0 & 0 & 0 & 0 & 0 & " & 0 \\
\hline $03: 00 \mathrm{PM}$ & $\cdots$ & $03: 15 \mathrm{PM}$ & 0 & 0 & 0 & 0 & 0 & 0 & 0 & 0 & 0 & 0 & $\overline{0}$ & ". & -7 \\
\hline 03:15 PM & $\ldots+$ & 03:30 PM & 0 & 0 & 0 & 0 & 0 & 0 & 0 & 0 & 0 & 0 & 0 & '. & 0 \\
\hline $\begin{array}{l}03: 30 \mathrm{PM} \\
00.15 \mathrm{PM}\end{array}$ & $=$ & $\begin{array}{l}03: 45 \mathrm{PM} \\
00.00 \mathrm{PM}\end{array}$ & $\begin{array}{l}0 \\
0 \\
0\end{array}$ & $\begin{array}{l}0 \\
0\end{array}$ & $\begin{array}{l}0 \\
0\end{array}$ & $\begin{array}{l}0 \\
0 \\
0\end{array}$ & 0 & 0 & 0 & $\begin{array}{l}0 \\
0\end{array}$ & 0 & 0 & 0 & $"$ & 0 \\
\hline$\frac{03: 40 \mathrm{PM}}{04: 00 \mathrm{PM}}$ & 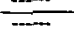 & $04: 15 \mathrm{PM}$ & 0 & 0 & 0 & 0 & 0 & $\frac{0}{0}$ & 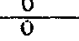 & $\frac{0}{2}$ & $\frac{0}{0}$ & $\frac{0}{0}$ & $\frac{1}{0}$ & $\because$ & $\frac{1}{2}$ \\
\hline 04:15 PM & $\ldots$ & 04:30 PM & 0 & 0 & 0 & 0 & 0 & 0 & 0 & 0 & 0 & 0 & $n$ & $\because 1$ & $i$ \\
\hline 04:30 PM & $\ldots$ & 04:45 PM & 0 & 0 & 0 & 0 & 0 & 0 & 0 & 0 & 0 & 0 & 0 & ". & 0 \\
\hline $04.45 \mathrm{PM}$ & $\ldots$ & $05: 00 \mathrm{PM}$ & 0 & 0 & 0 & 0 & 0 & 0 & 0 & 0 & 0 & 0 & 9 & $\therefore$ & n \\
\hline & & & & & & & $R L Y$ & TOTAL & & & & & & & \\
\hline$\overline{0700 \mathrm{AM}}$ & $\cdots$ & $08.00 \mathrm{AM}$ & 0 & \% & o & 8 & $\overline{0}$ & $\bar{\sigma}$ & $\overline{0}$ & $\overline{0}$ & 8 & $\overline{0}$ & $\pi$ & $\because$ & $\overline{0}$ \\
\hline $07: 15 \mathrm{AM}$ & - & 08:15 AM & 0 & 0 & 0 & 0 & 0 & 0 & 0 & 0 & 0 & 0 & $\therefore$ & $\therefore$ & 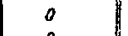 \\
\hline $07: 30 \mathrm{AM}$ & $\equiv$ & $-\frac{08.30 \mathrm{AM}}{08.45 \mathrm{AM}}$ & $\frac{0}{0}$ & $\frac{0}{0}$ & 0 & 0 & 0 & 0 & -0 & 0 & $\frac{0}{0}$ & 0 & i & $\therefore$ & $\theta$ \\
\hline $\begin{array}{l}\text { OB:A5 AM } \\
\text { OB:00 AM }\end{array}$ & $\bar{m}$ & $09: 00 \mathrm{AM}$ & 0 & 0 & 0 & 0 & $\stackrel{0}{0}_{0}^{2}$ & 0 & 0 & $\begin{array}{l}0 \\
0\end{array}$ & 0 & 0 & 0 & $\because$ & 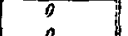 \\
\hline OB: 15 AM & $\ldots$ & 09:15 AM & 0 & 0 & $\begin{array}{l}0 \\
0 \\
\end{array}$ & 0 & 0 & 0 & 0 & 0 & $\begin{array}{l}0 \\
0\end{array}$ & $\begin{array}{l}0 \\
0 \\
\end{array}$ & $\begin{array}{l}0 \\
0\end{array}$ & i: & 0 \\
\hline $08: 30 \mathrm{AM}$ & 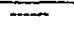 & $09: 30 \mathrm{AM}$ & 0 & 0 & 0 & $\overline{0}$ & $\overline{0}$ & 0 & 0 & 0 & $T$ & 0 & 0 & . & 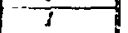 \\
\hline 08:43 AM & $\cdots$ & $09: 45 \mathrm{AM}$ & 0 & 0 & 0 & 0 & 0 & 0 & 0 & 0 & i & 0 & 1 & $" 1$ & $i$ \\
\hline $03: 00 \mathrm{AM}$ & $\ldots$ & $1000 \mathrm{AM}$ & 0 & 0 & 0 & 0 & 0 & 0 & 0 & 1 & $i$ & 0 & $i$ & $\because$ & 3 \\
\hline $09: 15 \mathrm{AM}$ & $\cdots$ & $10: 15 \mathrm{AM}$ & 0 & 0 & 0 & 0 & 0 & 0 & 0 & $T$ & $T$ & 0 & 1 & $\therefore$ & 3 \\
\hline 09:30.9.M & $\ldots$ & $10: 30 \mathrm{AM}$ & 1 & 0 & 0 & 0 & 0 & 0 & 0 & 1 & 0 & 0 & 1 & ". & 3 \\
\hline $09: 45 . \mathrm{AM}$ & $\cdots$ & $10: 45 \mathrm{AM}$ & 1 & .0 & 0 & 0 & 0 & 0 & 0 & 1 & 0 & 0 & "I & .. & 2 \\
\hline $10: 00 \mathrm{AM}$ & $\ldots$ & $11.00 \mathrm{AM}$ & $T$ & 0 & 0 & 0 & $\overline{0}$ & D & 0 & 2 & 0 & 0 & $\pi$ & . & $\pi$ \\
\hline $10: 15 \mathrm{AM}$ & $=$ & I1:15 AM & $i$ & 0 & $\begin{array}{l}0 \\
0\end{array}$ & 0 & $0_{0}^{0}$ & $\begin{array}{l}0 \\
0\end{array}$ & ${ }_{0}^{0}$ & 3 & 0 & 0 & 9 & .1 & \pm \\
\hline$\frac{10: 30 \mathrm{AM}}{10.45 \mathrm{AM}}$ & $=$ & $\frac{11: 30 \mathrm{AM}}{11.45 \mathrm{AM}}$ & $\frac{0}{8}$ & $\frac{0}{a}$ & $\frac{0}{0}$ & $\frac{0}{0}$ & $\frac{0}{0}$ & $\frac{0}{0}$ & $\frac{0}{0}$ & $\frac{3}{4}$ & $\frac{0}{9}$ & $\frac{0}{0}$ & $\frac{1}{1}$ & $\because$ & $-\frac{1}{5}$ \\
\hline $11: 00 \mathrm{AM}$ & $\ldots$ & $12: 00 \mathrm{PM}$ & 0 & 0 & 0 & 0 & 0 & 0 & 0 & 2 & 0 & 0 & $i$ & $\because:$ & \\
\hline 11:1S AM & $\cdots$ & 12:15 PM & 0 & 0 & 0 & 0 & 0 & 0 & 0 & 1 & 0 & 0 & 1 & $\because$ & 2 \\
\hline $11: 30 \mathrm{~A}$ & $\cdots$ & 12 & 0 & 0 & 0 & 0 & 0 & 0 & 0 & $T$ & 0 & 0 & 0 & " & 1 \\
\hline 11:4S AM & $\cdots$ & 12:45 PM & 0 & $\circ$ & 0 & 0 & 0 & 0 & 0 & 2 & 0 & 0 & 0 & 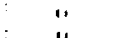 & 2 \\
\hline 12:00 PM & - & $01: 00 \mathrm{PM}$ & 0 & $\frac{a}{a}$ & 0 & $\frac{0}{0}$ & $\frac{0}{0}$ & $\frac{0}{0}$ & 0 & $\frac{2}{3}$ & $\frac{0}{0}$ & 0 & 0 & $\ddot{\prime \prime}$ & $\frac{2}{1}$ \\
\hline 12:15 PM & $=$ & & 0 & 0 & 0 & 0 & 0 & 0 & 0 & 2 & 0 & 0 & 0 & ii & 2 \\
\hline 12:30 PM & $\cdots$ & O1:30 PM & 0 & 0 & a & 0 & $\begin{array}{l}0 \\
0\end{array}$ & $\begin{array}{l}0 \\
0\end{array}$ & 0 & 3 & 0 & 0 & $\begin{array}{l}0 \\
0\end{array}$ & $"$ & 3 \\
\hline$\frac{12: 45 \mathrm{PM}}{01: 00 \mathrm{PM}}$ & $=$ & $01: 45$ PM & 0 & $\begin{array}{l}0 \\
0\end{array}$ & $\frac{0}{0}$ & $\frac{0}{0}$ & $\frac{1}{0}$ & $\frac{0}{0}$ & $\frac{0}{0}$ & $\frac{1}{1}$ & $\frac{9}{0}$ & $\frac{0}{0}$ & $\frac{0}{0}$ & $\frac{1}{0}$ & $\frac{1}{1}$ \\
\hline $\begin{array}{l}01: 00 \mathrm{PM} \\
01: 15 \mathrm{PM}\end{array}$ & $\cdots$ & $\begin{array}{l}\text { 02:00 PM } \\
02: 15 \text { PM }\end{array}$ & $\begin{array}{l}0 \\
0\end{array}$ & $\begin{array}{l}0 \\
0\end{array}$ & 0 & 0 & 0 & 0 & 0 & $i$ & 0 & 0 & 2 & ij & 3 \\
\hline $01: 30 \mathrm{PM}$ & $\ldots$ & $02: 30 \mathrm{PM}$ & 0 & 0 & 0 & 0 & 0 & 0 & 0 & 0 & 0 & 0 & 2 & 0 & 2 \\
\hline $01.45 \mathrm{PM}$ & $=$ & 02:A5 PM & 0 & 0 & D & 0 & 0 & 0 & 6 & 0 & 0 & 0 & 5 & 0 & 2 \\
\hline 02:00 PM & $m$ & 03:00 PM & 0 & 0 & 0 & 0 & 0 & 0 & 0 & 0 & 0 & 0 & 2 & 0 & 2 \\
\hline $02: 19 \mathrm{PM}$ & - & 03:15 PM & 0 & 0 & 0 & 0 & 0 & 0 & 0 & 0 & 0 & 0 & 0 & 0 & 0 \\
\hline $02: 30 \mathrm{PM}$ & $=$ & $03.30 \mathrm{PM}$ & 0 & 0 & $\overline{0}$ & 0 & $\overline{0}$ & 0 & 0 & 0 & 0 & 0 & 0 & (1) & 0 \\
\hline $02: 45$ PM & 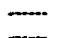 & 03:45 PM & 0 & 0 & 0 & & 0 & $\begin{array}{l}0 \\
0\end{array}$ & $\begin{array}{l}0 \\
0\end{array}$ & $\begin{array}{l}0 \\
0\end{array}$ & 0 & $\begin{array}{l}0 \\
0\end{array}$ & 0 & 0 & 0 \\
\hline $03: 00$ PM & $=$ & O4:00 PM & 0 & 0 & 0 & 0 & 0 & 0 & $\frac{0}{0}$ & $\frac{0}{3}$ & 0 & $\frac{0}{a}$ & $\frac{1}{1}$ & 0 & $I$ \\
\hline $03: 15$ PM & $\Gamma$ & $04: 15 \mathrm{PM}$ & 0 & 0 & 0 & 0 & 3 & 0 & 0 & 2 & 0 & 0 & 1 & 0 & 3 \\
\hline 03:30 PM & - & 04:30 PM & 0 & 0 & $\begin{array}{l}0 \\
0\end{array}$ & ${ }_{0}^{0}$ & $\stackrel{0}{0}$ & 0 & 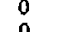 & 2 & 0 & 0 & 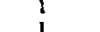 & 0 & 3 \\
\hline 03:45 PM & $=$ & OA:AS PM & 0 & 0 & 0 & 0 & 0 & $\frac{a}{0}$ & $\frac{0}{0}$ & $\frac{2}{3}$ & 0 & 0. & $\frac{1}{0}$ & $\frac{1}{0}$ & 3 \\
\hline $04: 00 \mathrm{PM}$ & $=$ & $05: 00$ PM & 0 & 0 & 0 & 0 & 0 & 0 & 0 & & 0 & 0 & 0 & 0 & 7 \\
\hline$S t$ & PENII & $U \angle A:$ & 0 & 7 & & $A L$ & $D A$ & 0) 2 & 292 & & on & co & 15 & $32=$ & \\
\hline
\end{tabular}




\section{Baymetrics Traffic Resources INTERSECTION TURNING MOVEMENT SUMMARY}

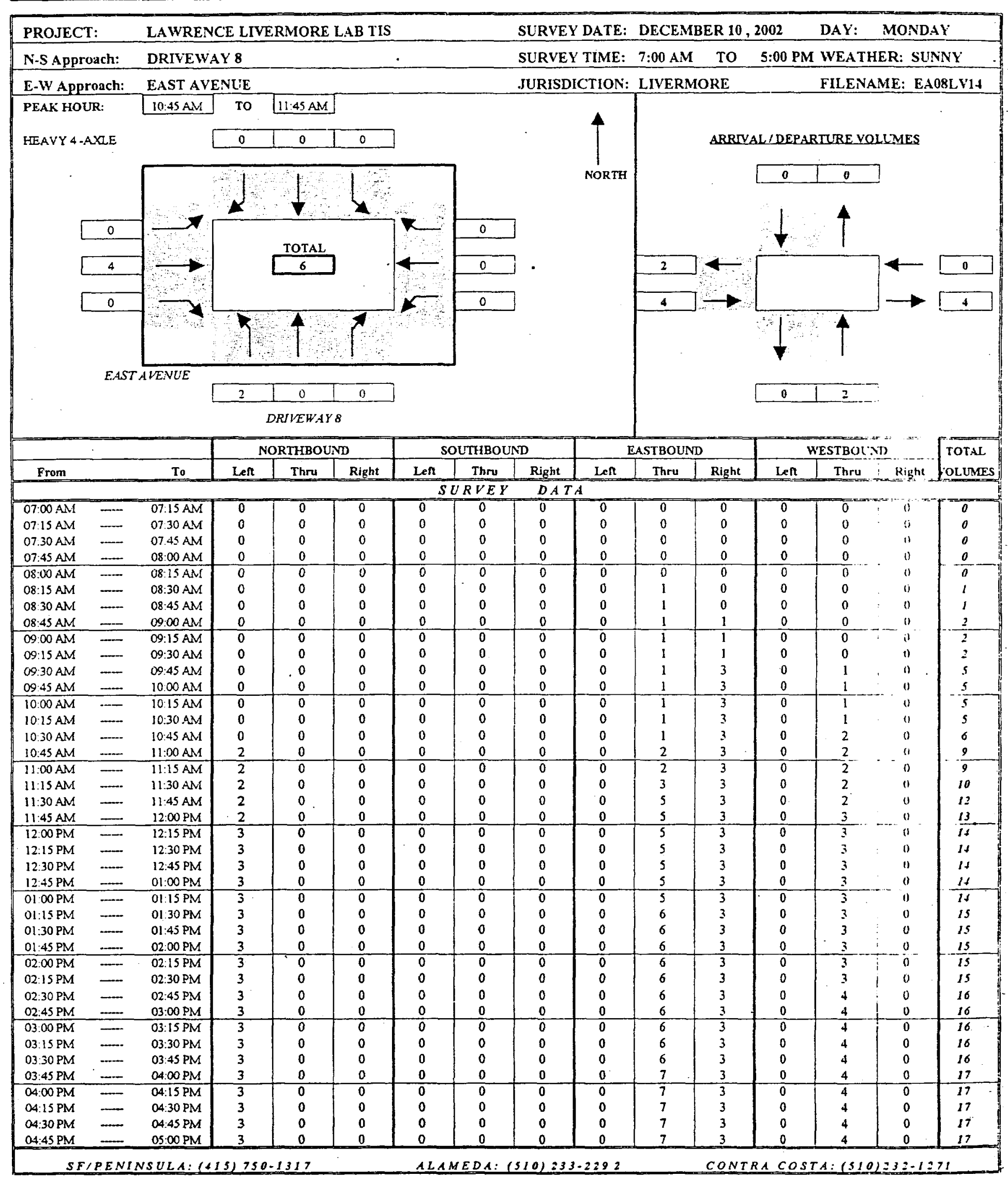




\section{Baymetrics Traffic Resources INTERSECTION TURNING MOVEMENT SUMMARY}

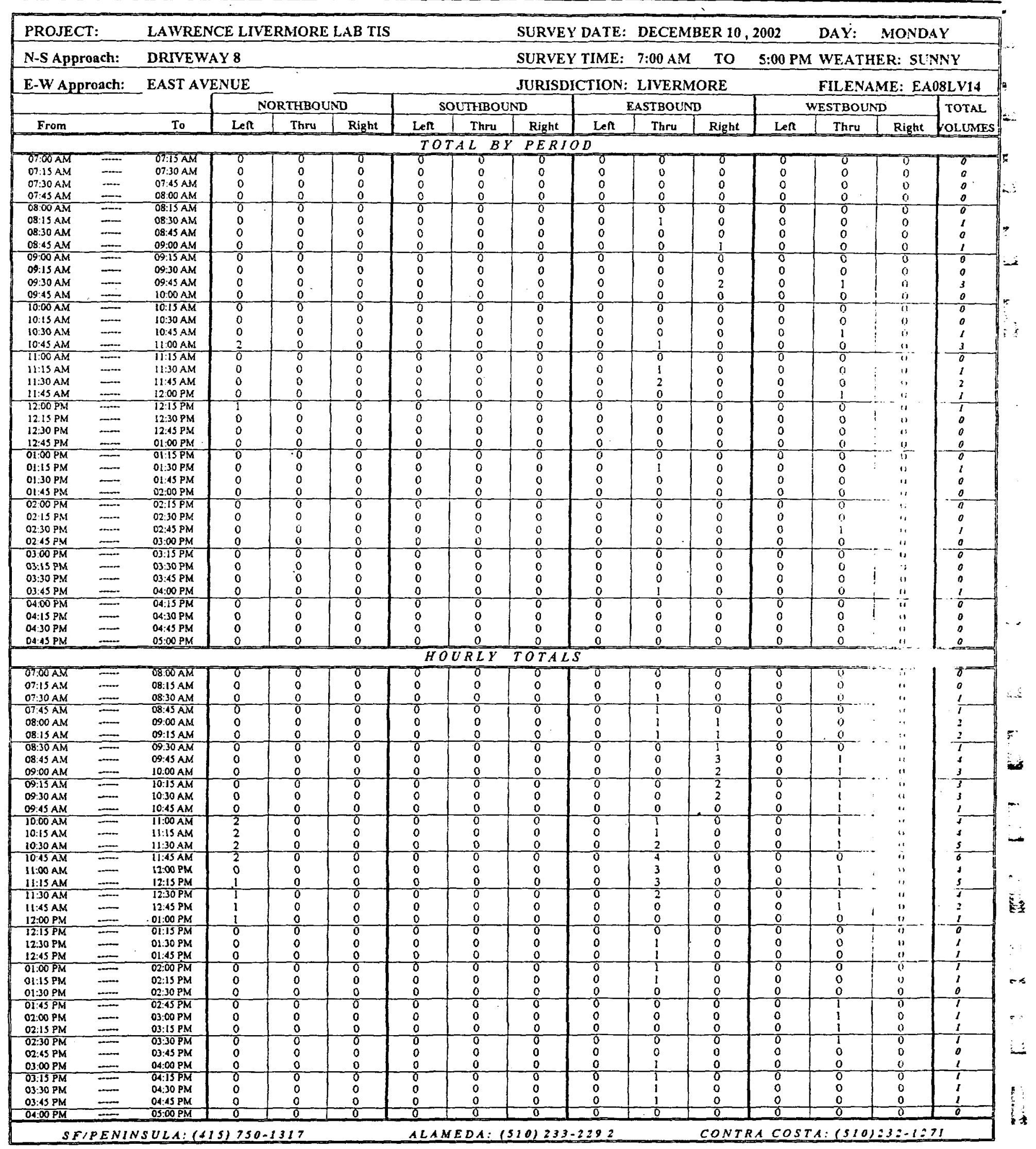




\section{Baymetrics Traffic Resources INTERSECTION TURNING MOVEMENT SUMMARY}

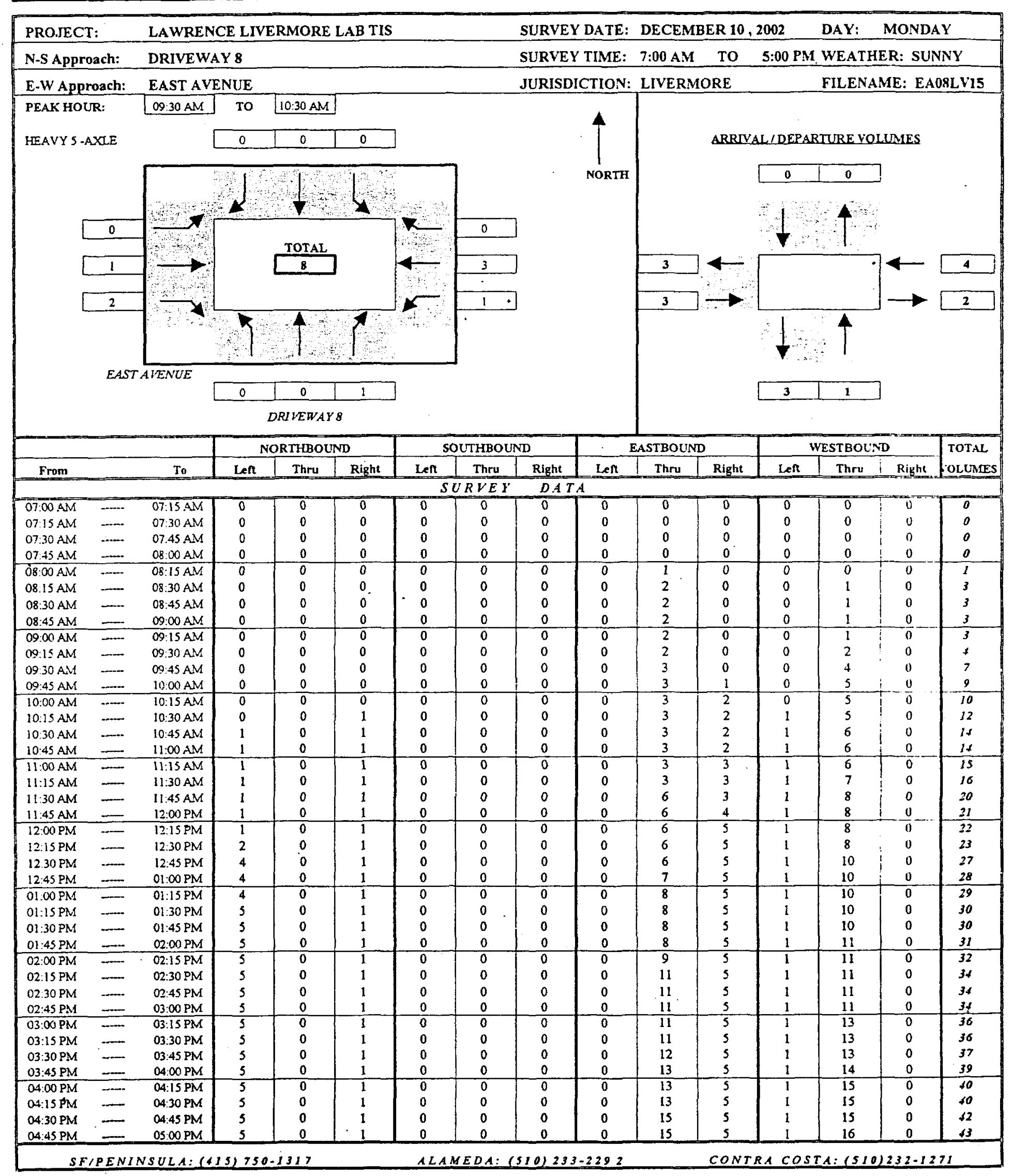




\section{Baymetrics Traffic Resources INTERSECTION TURNING MOVEMENT SUMMARY}

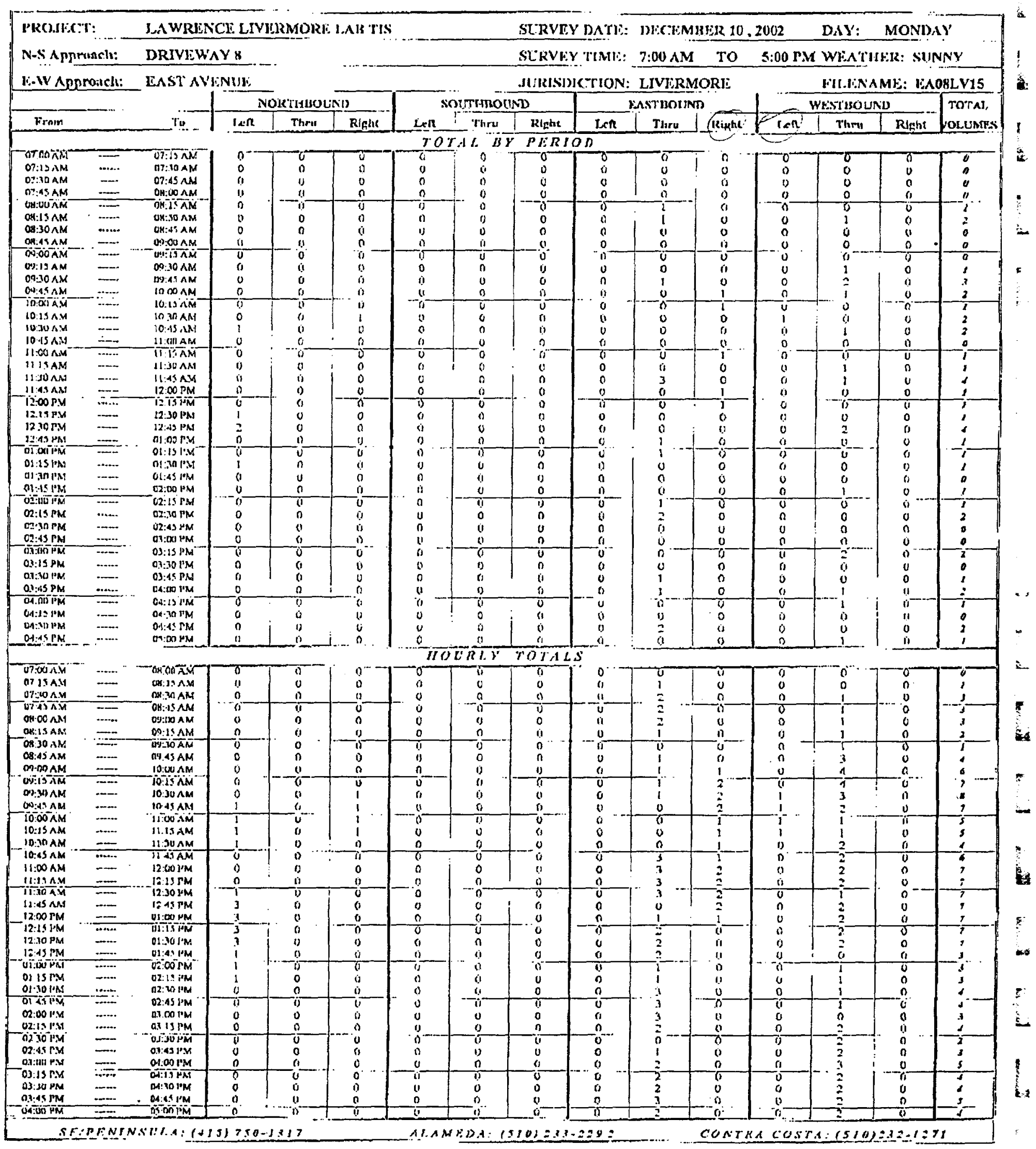




\section{Baymetrics Traffic Resources INTERSECTION TURNING MOVEMENT SUMMARY}

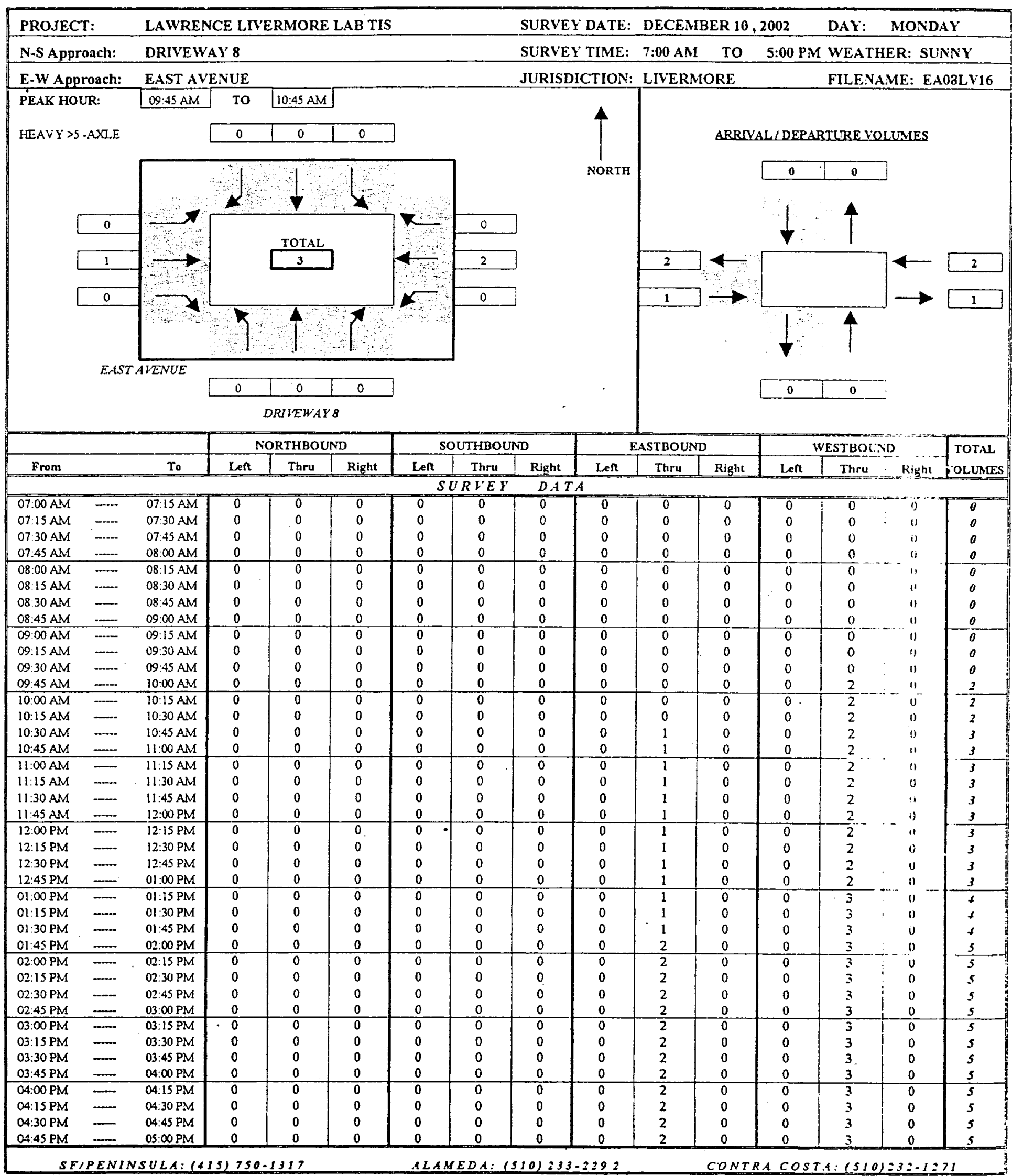




\section{Baymetrics Traffic Resources INTERSECTION TURNING MOVEMENT SUMMARY}

\begin{tabular}{|c|c|c|c|c|c|c|c|c|c|c|c|c|c|c|c|}
\hline \multirow{2}{*}{\multicolumn{2}{|c|}{$\begin{array}{l}\text { PROJECT: } \\
\text { N.S Approach: }\end{array}$}} & \multicolumn{6}{|c|}{ LAWRENCE LIVERMORE LAB TIS } & \multicolumn{5}{|c|}{ SURVEY DATE: DECEMBER 10,2002 } & DAY: & \multicolumn{2}{|c|}{ MONDAY } \\
\hline & & DRIVEV & & & & & & SURVE & IME: & 7:00 AM & To & 5:00 P & WEAT & IER: SLit & NY \\
\hline E-W AP & oach: & EAST A & iUE & & & & & JURISD & TION: & LIVERI & ORE & & FILEN & ME: EA & $38 \mathrm{LV} 16$ \\
\hline & & & & THBC & & & THBO & & & ASTBOUN & & & ESTBOU & & TOTAL \\
\hline From & & To & Left & Thru & Right & Len & Thru & Right & Len & Thru & Right & Len & Thru & Right & Yolumes \\
\hline & & & & & & & $L B$ & $P E R I$ & & & & & & & \\
\hline $\begin{array}{l}\text { T1:00AM } \\
07: 15 \text { AM }\end{array}$ & $-\ldots$ & $\begin{array}{l}07.15 \mathrm{AM} \\
07.30 \mathrm{AM}\end{array}$ & 0 & $\begin{array}{l}0 \\
0\end{array}$ & $\begin{array}{l}0 \\
0\end{array}$ & $\begin{array}{l}\overline{0} \\
0\end{array}$ & $\begin{array}{l}7 \\
0\end{array}$ & $\begin{array}{l}0 \\
0\end{array}$ & $\begin{array}{l}8 \\
0\end{array}$ & $\begin{array}{l}0 \\
0\end{array}$ & 9 & $\begin{array}{l}0 \\
0\end{array}$ & $\begin{array}{l}0 \\
0\end{array}$ & $\begin{array}{l}0 \\
0\end{array}$ & 0 \\
\hline $\begin{array}{l}07: 15 \mathrm{AM} \\
07: 30 \mathrm{AM} \\
07: 45 \mathrm{AM}\end{array}$ & $=$ & $\begin{array}{l}07.30 \mathrm{AM} \\
07.45 \mathrm{AM} \\
08.00 \mathrm{AM}\end{array}$ & $\begin{array}{l}0 \\
0 \\
0\end{array}$ & $\begin{array}{l}0 \\
0 \\
0\end{array}$ & $\begin{array}{l}0 \\
0 \\
0\end{array}$ & $\begin{array}{l}0 \\
0 \\
0\end{array}$ & $\begin{array}{l}0 \\
0 \\
0\end{array}$ & $\begin{array}{l}0 \\
0 \\
0\end{array}$ & $\begin{array}{l}0 \\
0 \\
0\end{array}$ & $\begin{array}{l}0 \\
0 \\
0\end{array}$ & $\begin{array}{l}0 \\
0 \\
0\end{array}$ & $\begin{array}{l}1 \\
0 \\
0\end{array}$ & $\begin{array}{l}0 \\
0 \\
0\end{array}$ & $\begin{array}{l}0 \\
0 \\
0\end{array}$ & 0 \\
\hline $08.00 \mathrm{AM}$ & $=$ & $08: 15 \mathrm{AM}$ & 0 & 0 & 0 & 0 & 0 & 0 & 0 & $-\frac{1}{0}$ & 0 & $\frac{0}{0}$ & $\frac{0}{0}$ & -0 & $\div$ \\
\hline $\begin{array}{l}08: 15 \mathrm{AM} \\
08.30 \mathrm{AM}\end{array}$ & $\overline{-}$ & $\begin{array}{l}08: 30 \mathrm{AM} \\
08.45 \mathrm{AM}\end{array}$ & $\begin{array}{l}0 \\
0\end{array}$ & $\begin{array}{l}0 \\
0\end{array}$ & 0 & $\circ$ & $\stackrel{0}{0}$ & 0 & 0 & 0 & 0 & 0 & 0 & 0 & 0 \\
\hline $08: 45$ AM & $=$ & $0900 \mathrm{AM}$ & 0 & 0 & 0 & $\begin{array}{l}0 \\
0\end{array}$ & $\begin{array}{l}0 \\
0\end{array}$ & $\begin{array}{l}0 \\
0\end{array}$ & $\begin{array}{l}0 \\
0\end{array}$ & $\begin{array}{l}0 \\
0\end{array}$ & $\begin{array}{l}0 \\
0\end{array}$ & $\begin{array}{l}0 \\
0\end{array}$ & $\begin{array}{l}0 \\
0\end{array}$ & 0 & 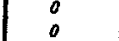 \\
\hline $09: 00 \mathrm{AM}$ & $\ldots$ & $09: 15 \mathrm{AM}$ & 0 & $\overline{0}$ & 0 & 0 & 0. & 0 & 0 & 0 & 0 & 0 & 0 & 0 & $-\frac{1}{0}$ \\
\hline $09: 15 \mathrm{AM}$ & $\ldots$ & $09: 30 \mathrm{AM}$ & 0 & 0 & 0. & 0 & 0 & 0 & 0 & 0 & 0 & 0 & 0 & 0 & $o$ \\
\hline $\begin{array}{l}09: 30 \mathrm{AM} \\
09: 45 \mathrm{AM}\end{array}$ & $\ldots$ & $\begin{array}{l}09: 45 \text { AM } \\
\text { 10:00 AM }\end{array}$ & $\begin{array}{l}0 \\
0\end{array}$ & $\begin{array}{l}0 \\
0\end{array}$ & $\begin{array}{l}0 \\
0\end{array}$ & $\begin{array}{l}0 \\
0\end{array}$ & $\begin{array}{l}0 \\
0\end{array}$ & $\begin{array}{l}0 \\
0\end{array}$ & $\begin{array}{l}0 \\
0\end{array}$ & $\begin{array}{l}0 \\
0\end{array}$ & $\begin{array}{l}0 \\
0\end{array}$ & $\begin{array}{l}0 \\
0\end{array}$ & $\begin{array}{l}0 \\
2\end{array}$ & $\begin{array}{l}0 \\
0\end{array}$ & $?$ \\
\hline $10: 00 \mathrm{AM}$ & $\ldots$ & $10.15 \mathrm{AM}$ & 0 & a & 0 & 0 & 0 & 0 & 0 & $\frac{5}{0}$ & 0 & 0 & $\dot{0}$ & 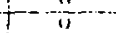 & i \\
\hline $10: 15 \mathrm{AM}$ & $\ldots$ & $10: 30 \mathrm{AM}$ & 0 & 0 & 0 & 0 & 0 & 0 & 0 & 0 & 0 & 0 & 0 & i) & 0 \\
\hline $10.30 \mathrm{AM}$ & $\ldots$ & I0.45 AM & 0 & 0 & 0 & 0 & 0 & 0 & 0 & 1 & 0 & 0 & 0 & $\therefore$ & 1 \\
\hline$\frac{10.45 \mathrm{AM}}{11.00 \mathrm{AM}}$ & $\ldots$ & $1100 \mathrm{AM}$ & 0 & 0 & 0 & 0 & 0 & 0 & 0 & 0 & 0 & 0 & 0 & 11 & 0 \\
\hline $\begin{array}{l}11: 00 \mathrm{AM} \\
11115 \mathrm{AM}\end{array}$ & $\cdots$ & $1115 \mathrm{AM}$ & 0 & 0 & 0 & 0 & 0 & 0 & 0 & 0 & 0 & 0 & 0 & $1 i$ & 0 \\
\hline 11:30 AM & $\ldots$ & $\begin{array}{l}11: 30 \mathrm{AM} \\
11: 45 \mathrm{AM}\end{array}$ & $\begin{array}{l}0 \\
0\end{array}$ & $\begin{array}{l}0 \\
0\end{array}$ & 0 & $\begin{array}{l}0 \\
0\end{array}$ & $\begin{array}{l}0 \\
0\end{array}$ & 0 & 0 & 0 & 0 & 0 & 0 & $\because$ & 0 \\
\hline II:45 AM & $=$ & $12: 00 \mathrm{PM}$ & 0 & 0 & 0 & $\begin{array}{l}0 \\
0 \\
\end{array}$ & 0 & $\begin{array}{l}0 \\
0 \\
\end{array}$ & $\begin{array}{l}0 \\
0\end{array}$ & $\begin{array}{l}0 \\
0\end{array}$ & $\begin{array}{l}0 \\
0\end{array}$ & $\begin{array}{l}0 \\
0\end{array}$ & $\begin{array}{l}0 \\
0\end{array}$ & $\because$ & $\begin{array}{l}0 \\
0\end{array}$ \\
\hline $12: C 0 \mathrm{PM}$ & $\cdots$ & $12: 15 \mathrm{PM}$ & 0 & 0 & 0 & 0 & 0 & 0 & 0 & 0 & 0 & 0 & 0 & $\because$ & -6 \\
\hline $12: 15 \mathrm{PM}$ & - & $12: 30 \mathrm{PM}$ & 0 & 0 & 0 & 0 & 0 & 0 & 0 & 0 & 0 & 0 & 0 & 1! & 0 \\
\hline 12:30 PM & - & 12:45 PM & 0 & 0 & 0 & 0 & 0 & 0 & 0 & 0 & 0 & 0 & 0 & $\because$ & 0 \\
\hline 12:45 PM & $\ldots$ & $01: 00 \mathrm{PM}$ & 0 & 0 & 0 & 0 & $(1$ & 0 & 0 & 0 & 0 & 0 & 0 & " & 0 \\
\hline $01.00 \mathrm{PM}$ & $=$ & $01: 15 \mathrm{PM}$ & 0 & $\delta$ & 0 & 0 & 0 & 0 & 0 & 0 & 0 & 0 & 1 & $\because$ & $\frac{T}{l}$ \\
\hline 01:15 PM & $\ldots . .$. & $01: 30 \mathrm{PM}$ & 0 & 0 & 0 & 0 & 0 & 0 & 0 & 0 & 0 & 0 & 0 & 11 & 0 \\
\hline $01: 30 \mathrm{PM}$ & $\ldots$ & 01:45 PM & 0 & 0 & 0 & 0 & 0 & 0 & 0 & 0 & 0 & 0 & 0 & $\therefore$ & $a$ \\
\hline $01.45 \mathrm{PM}$ & - & 02:00 PM & 0 & 0 & 0 & 0 & 0 & 0 & 0 & 1 & 0 & 0 & 0 & $\because$ & $t$ \\
\hline $02: 00 \mathrm{PM}$ & $\ldots$ & $02.15 \mathrm{PM}$ & 0 & 0 & 0 & 0 & 0 & 0 & 0 & 0 & 0 & 0 & 0 & $\therefore$ & $\frac{1}{0}$ \\
\hline $02: 15 \mathrm{PM}$ & $\ldots$ & $02: 30 \mathrm{PM}$ & 0 & 0 & 0 & 0 & 0 & 0 & $0^{\circ}$ & 0 & 0 & 0 & 0 & $:$ & 0 \\
\hline $02: 30 \mathrm{PM}$ & $\ldots$ & $02: 45 \mathrm{PM}$ & 0 & 0 & 0 & 0 & 0 & 0 & 0 & 0 & 0 & 0 & 0 & : & 0 \\
\hline $02.45 \mathrm{PM}$ & $\ldots$ & 03:00 PM & 0 & 0 & 0 & 0 & 0 & 0 & 0 & 0 & 0 & 0 & 0 & $\therefore$ & 0 \\
\hline $03: 03 \mathrm{PM}$ & $=$ & $03: 15 \mathrm{PM}$ & 0 & 0 & 0 & 0 & 0 & 0 & 0 & 0 & 0 & 0 & 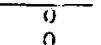 & $\therefore$ & 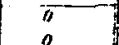 \\
\hline $\begin{array}{l}03: 15 \mathrm{PM} \\
03.30 \mathrm{PM}\end{array}$ & 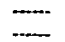 & $\begin{array}{l}03: 30 \mathrm{PM} \\
03.45 \mathrm{PM}\end{array}$ & $\begin{array}{l}0 \\
0\end{array}$ & $\begin{array}{l}0 \\
0\end{array}$ & $\begin{array}{l}0 \\
0\end{array}$ & $\begin{array}{l}0 \\
0\end{array}$ & 0 & $\begin{array}{l}0 \\
0\end{array}$ & $0_{0}^{0}$ & $\begin{array}{l}0 \\
0\end{array}$ & $\stackrel{0}{0}$ & 0 & $\stackrel{n}{0}$ & 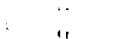 & $\stackrel{0}{a}$ \\
\hline $\begin{array}{l}\text { 03:30 PM } \\
\text { 03:45 PM }\end{array}$ & $\overline{-}$ & 04:00 PM & 0 & 0 & 0 & 0 & 0 & 0 & 0 & 0 & $\begin{array}{l}0 \\
0\end{array}$ & $\begin{array}{l}0 \\
0\end{array}$ & $\begin{array}{l}0 \\
0\end{array}$ & $\because$ & $\stackrel{0}{0}$ \\
\hline $04: 00 \mathrm{PM}$ & $\ldots$ & $04: 15$ PM & 0 & 0 & 0 & 0 & 0 & 0 & 0 & 0 & 0 & 0 & 0 & $\because$ & 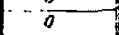 \\
\hline 04:IS PM & $\ldots$ & 04:30 PM & 0 & 0 & 0 & 0 & $a$ & 0 & 0 & 0 & 0 & 0 & 0 & "1 & 0 \\
\hline $\begin{array}{l}\text { 04:30 PM } \\
\text { 04:45 PM }\end{array}$ & $\ldots$ & $\begin{array}{l}\text { 04:45 PM } \\
\text { 05:00 PM }\end{array}$ & $\begin{array}{l}0 \\
0\end{array}$ & $\begin{array}{l}0 \\
0\end{array}$ & $\begin{array}{l}0 \\
0\end{array}$ & $\begin{array}{l}0 \\
0\end{array}$ & $\begin{array}{l}0 \\
0\end{array}$ & $\begin{array}{l}0 \\
0\end{array}$ & $\begin{array}{l}0 \\
0\end{array}$ & $\begin{array}{l}0 \\
0\end{array}$ & $\begin{array}{l}0 \\
0\end{array}$ & 0 & $\begin{array}{l}0 \\
0\end{array}$ & $\because$ & 0 \\
\hline & & & & & & & $R L Y$ & TOTAL & & & & & & 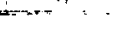 & \\
\hline $0: 00 \mathrm{AM}$ & 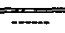 & OS:00 AM & $\overline{0}$ & 0 & 0 & 0 & 0 & 8 & $\overline{0}$ & $\sigma$ & $\overline{0}$ & $\overline{0}$ & 0 & $\therefore$ & 5 \\
\hline $07: 15 \mathrm{AM}$ & $\ldots$ & $08.15 \mathrm{AM}$ & 0 & 0 & 0 & 0 & 0 & 0 & 0 & 0 & 0 & 0 & 0 & $\therefore$ & 0 \\
\hline $07.30 \mathrm{AM}$ & $\ldots$ & $08: 30 \mathrm{AM}$ & 0 & 0 & 0 & 0 & 0 & 0 & 0 & 0 & 0 & 0 & 0 & $\therefore:$ & 0 \\
\hline 07.45 AM & - & $08: 45 \mathrm{AM}$ & 0 & 0 & 0 & 0 & 0 & 0 & 0 & 0 & 0 & 0 & 0 & $\therefore$ & 0 \\
\hline $\begin{array}{l}\text { 08:00 AM } \\
\text { 08:15 AM }\end{array}$ & $\cdots$ & $09: 00 \mathrm{AM}$ & 0 & 0 & 0 & 0 & 0 & 0 & 0 & 0 & 0 & 0 & 0 & " & 0 \\
\hline$\frac{08: 15 \mathrm{AM}}{08: 30 \mathrm{AM}}$ & $\ldots$ & 09:15 AM & 0 & 0 & 0 & 0 & 0 & $\frac{0}{0}$ & 0 & 0 & 0 & a & 0 & 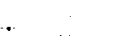 & 0 \\
\hline & $\overline{-}$ & $\begin{array}{l}09: 30 \mathrm{AM} \\
09: 45 \mathrm{AM}\end{array}$ & $\begin{array}{l}0 \\
0\end{array}$ & $\begin{array}{l}0 \\
0\end{array}$ & $\begin{array}{l}0 \\
0\end{array}$ & $\begin{array}{l}0 \\
0\end{array}$ & $\begin{array}{l}0 \\
0\end{array}$ & $\begin{array}{l}0 \\
0\end{array}$ & $\begin{array}{l}0 \\
0\end{array}$ & $\begin{array}{l}0 \\
0\end{array}$ & $\begin{array}{l}0 \\
0\end{array}$ & $\begin{array}{l}0 \\
0\end{array}$ & 0 & $\because$ & 0 \\
\hline 09:00 AM & $\ldots$ & $10: 00 \mathrm{AM}$ & 0 & 0 & 0 & 0 & 0 & 0 & 0 & 0 & 0 & 0 & 2 & $\therefore$ & 2 \\
\hline $09: 15 \mathrm{AM}$ & $=$ & $10: 15 \mathrm{AM}$ & 0 & 0 & 0 & 0 & 0 & 0 & 0 & 0 & 0 & 0 & $\frac{7}{2}$ & ii & i \\
\hline $09: 30 \mathrm{AM}$ & $\ldots$ & $10: 30 \mathrm{AM}$ & 0 & 0 & 0 & 0 & 0 & 0 & 0 & 0 & 0 & 0 & $\Sigma$ & 11 & j \\
\hline 09:45 AM & - & $10: 45 \mathrm{AM}$ & 0 & 0 & 0 & 0 & 0 & 0 & 0 & 1 & 0 & 0 & $\vdots$ & $\because$ & $i$ \\
\hline $10: 00 \mathrm{AM}$ & $=$ & $11: 00$ AM & 0 & 0 & 0 & 0 & 0 & 0 & 0 & $i$ & 0 & 0 & $\overline{0}$ & .. & $i$ \\
\hline 10:IS AM & - & IIIS AM & 0 & 0 & 0 & 0 & 0 & 0 & 0 & $\mathrm{i}$ & 0 & 0 & 0 & ". & $i$ \\
\hline 10:30 AM & $\ldots$ & $11: 30 \mathrm{AM}$ & 0 & 0 & 0 & 0 & 0 & 0 & 0 & 1 & 0 & 0 & 0 & $\therefore$ & l \\
\hline $10.45 \mathrm{AM}$ & $=$ & $11: 45 \mathrm{AM}$ & 0 & 0 & 0 & 0 & 0 & 0 & 0 & 0 & a & 0 & 0 &.. & $\therefore$ \\
\hline $1100 \mathrm{AM}$ & - & $12: 00 \mathrm{PM}$ & 0 & 0 & 0 & 0 & 0 & 0 & 0 & 0 & 0 & 0 & 0 & $\therefore$ & 0 \\
\hline 11:15 AM & $=$ & 12:19 PM & 0 & 0 & 0 & 0 & 0 & 0 & 0 & 0 & 0 & 0 & 0 & " & $o$ \\
\hline $11: 30 \mathrm{AM}$ & + & $12: 30 \mathrm{PM}$ & 0 & 0 & 0 & 0 & 0 & 0 & 0 & 0 & 0 & 0 & 0 & $\therefore$ & $\bar{n}$ \\
\hline $\begin{array}{l}11: 45 \mathrm{AM} \\
12: 00 \mathrm{PM}\end{array}$ & $\ldots$ & 12:45 PM & 0 & $\begin{array}{l}0 \\
0 \\
0\end{array}$ & 0 & 0 & $\begin{array}{l}0 \\
0\end{array}$ & $\begin{array}{l}0 \\
0\end{array}$ & $\begin{array}{l}0 \\
0\end{array}$ & $\begin{array}{l}0 \\
0\end{array}$ & $\begin{array}{l}0 \\
0\end{array}$ & 0 & 0 & $"$ & 0 \\
\hline$-\frac{12: 00 P M}{12: 15 P M}$ & - & $\frac{01.00 \mathrm{PM}}{01: 15 \mathrm{PM}}$ & $\frac{0}{0}$ & $\frac{0}{0}$ & $\frac{0}{0}$ & 0 & $\frac{0}{0}$ & 0 & $\frac{0}{0}$ & 0 & $\frac{0}{0}$ & $\frac{0}{0}$ & $\frac{0}{1}$ & ". & $\frac{0}{1}$ \\
\hline 12:30 PM & - & $01: 30 \mathrm{PM}$ & 0 & .0 & 0 & 0 & 0 & 0 & 0 & 0 & 0 & 0 & $i$ & "a & 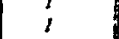 \\
\hline $12: 45$ PM & $\ldots$ & $01: 45 \mathrm{PM}$ & 0 & 0 & 0 & 0 & 0 & 0 & 0 & 0 & 0 & 0 & $i$ & $\because 1$ & 1 \\
\hline $01: 00 \mathrm{PM}$ & $\square$ & $02: 00 \mathrm{PM}$ & 0 & 0 & 0 & 0 & 0 & 0 & 0 & $T$ & 0 & 0 & 1 & 0 & 2 \\
\hline 01:IS PM & - & 02:15 PM & 0 & 0 & 0 & 0 & 0 & a & 0 & l & 0 & 0 & 0 & 0 & 1 \\
\hline$\frac{01: 30 \mathrm{PM}}{-01: 45 \mathrm{PM}}$ & - & 02:30 PM & 0 & $\frac{0}{0}$ & $\frac{0}{0}$ & $\frac{0}{0}$ & $\frac{0}{0}$ & $\frac{0}{0}$ & 0 & $\frac{1}{1}$ & $\frac{0}{0}$ & $\frac{0}{0}$ & -0 & 0 & 1 \\
\hline $\begin{array}{l}01: 45 \mathrm{PM} \\
02: 00 \mathrm{PM}\end{array}$ & 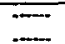 & 02:45 PM & $\begin{array}{l}0 \\
0\end{array}$ & $\begin{array}{l}0 \\
0\end{array}$ & $\begin{array}{l}0 \\
0\end{array}$ & 0 & 0 & 0 & 0 & 0 & $\begin{array}{l}0 \\
0\end{array}$ & $\begin{array}{l}0 \\
0\end{array}$ & $\begin{array}{l}0 \\
0\end{array}$ & $\begin{array}{l}0 \\
0\end{array}$ & 1 \\
\hline $\begin{array}{l}\text { 02:00 PM } \\
\text { 02:15 PMM }\end{array}$ & 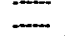 & $\begin{array}{l}\text { 03:00 PM } \\
03: 15 \text { PM }\end{array}$ & 0 & 0 & 0 & 0 & 0 & 0 & 0 & 0 & 0 & 0 & 0 & $a$ & 0 \\
\hline $02: 30 \mathrm{PM}$ & $\ldots$ & $03: 30 \mathrm{PM}$ & 0 & 0 & 0 & 0 & $\%$ & 0 & 0 & 0 & 0 & 0 & o & 0 & 0 \\
\hline 02:15 PM & $\cdots$ & 03:45 PM & 0 & 0 & 0 & 0 & 0 & 0 & 0 & 0 & 0 & 0 & 0 & 0 & 0 \\
\hline 03:00 PM & $\ldots$ & $04: 00 \mathrm{PM}$ & 0 & 0 & 0 & 0 & 0 & 0 & 0 & 0 & 0 & 0 & 0 & 0 & 0 \\
\hline $03: 15 \mathrm{PM}$ & $=$ & $04.15 \mathrm{PM}$ & 0 & 0 & 0 & 0 & 0 & 0 & 0 & 0 & 0 & 0 & 0 & 0 & 0 \\
\hline $03: 30 \mathrm{PM}$ & - & $04: 30 \mathrm{PM}$ & 0 & 0 & 0 & 0 & 0 & 0 & 0 & 0 & 0 & 0 & 0 & 0 & 0 \\
\hline $03.45 \mathrm{PM}$ & - & 04:45 PM & 0 & 0 & 0 & 0 & 0 & 0 & 0 & 0 & 0 & 0 & 0 & 0 & 0 \\
\hline QA:00 PM & $=$ & $05: 00 \mathrm{PM}$ & 0 & 0 & 0 & 0 & 0 & 0 & 0 & 0 & 0 & 0 & 0 & 0 & 0 \\
\hline
\end{tabular}




\section{Truck Counts}

Locations 7 and 8

Tuesday, December 11, 2001 



\section{Baymetrics Traffic Resources INTERSECTION TURNING MOVEMENT SUMMARY}

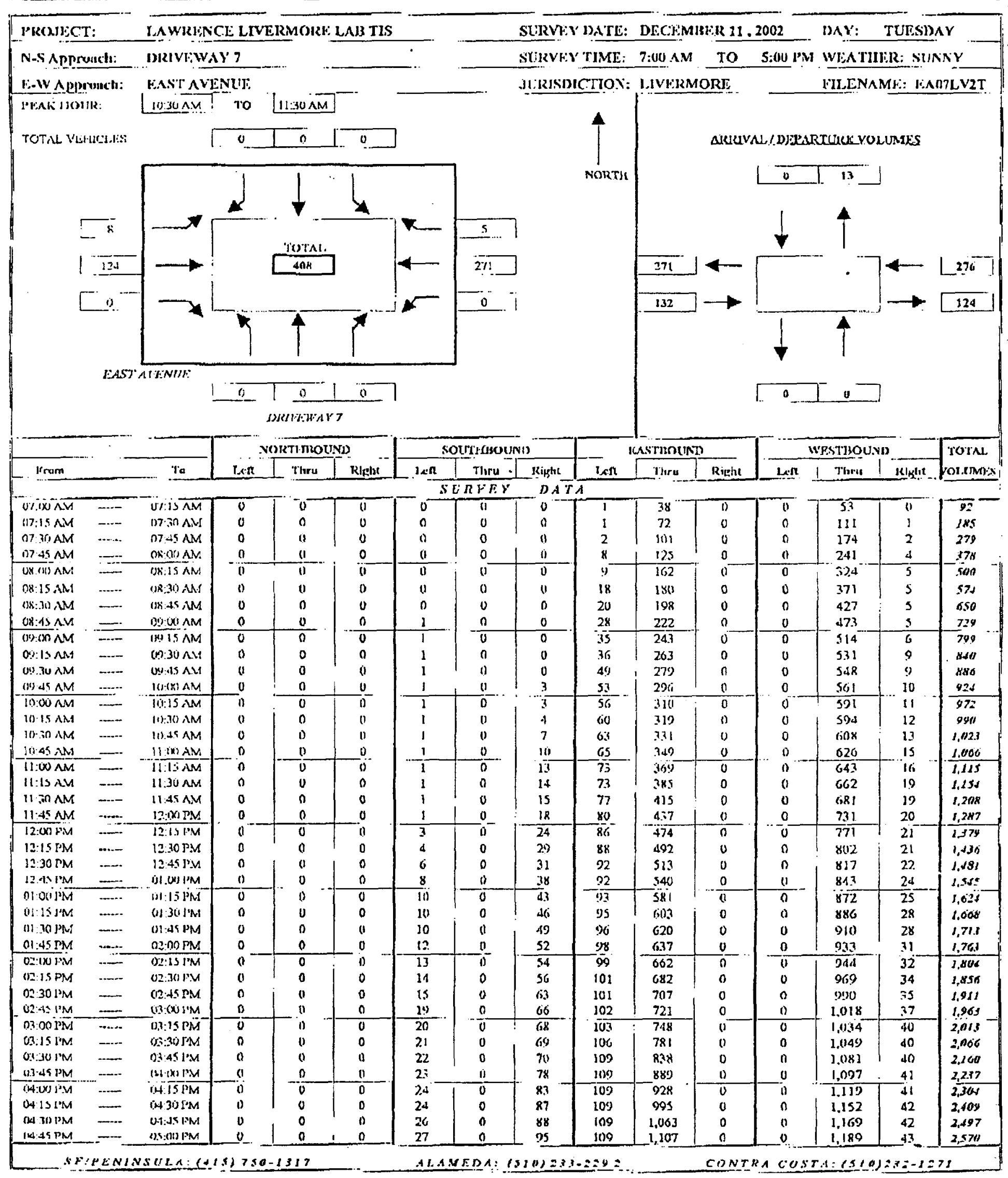




\section{Baymetrics Traffic Resources INTERSECTION TURNING MOVEMENT SUMMARY}

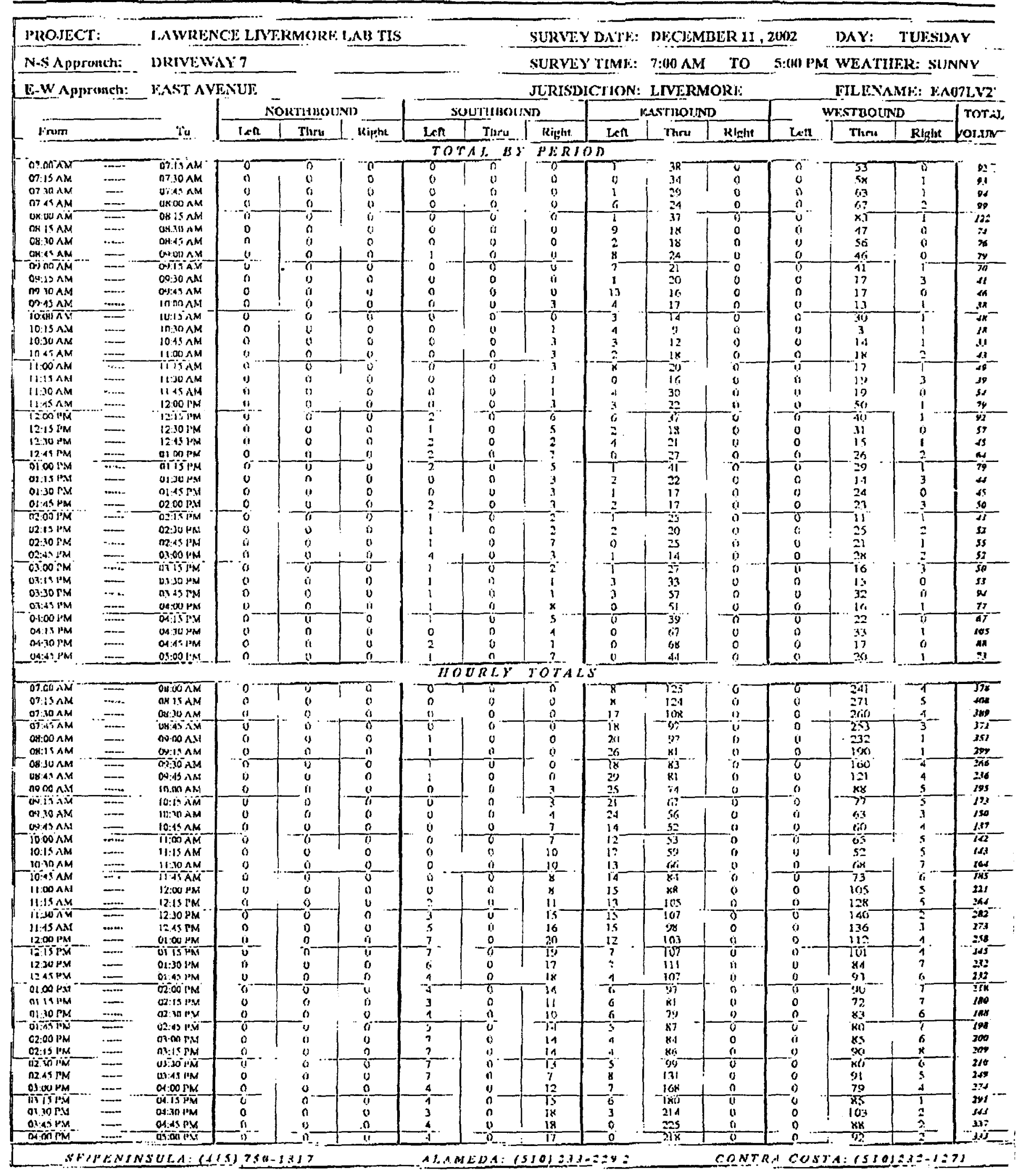




\section{Baymetrics Traffic Resources INTERSECTION TURNING MOVEMENT SUMMARY}

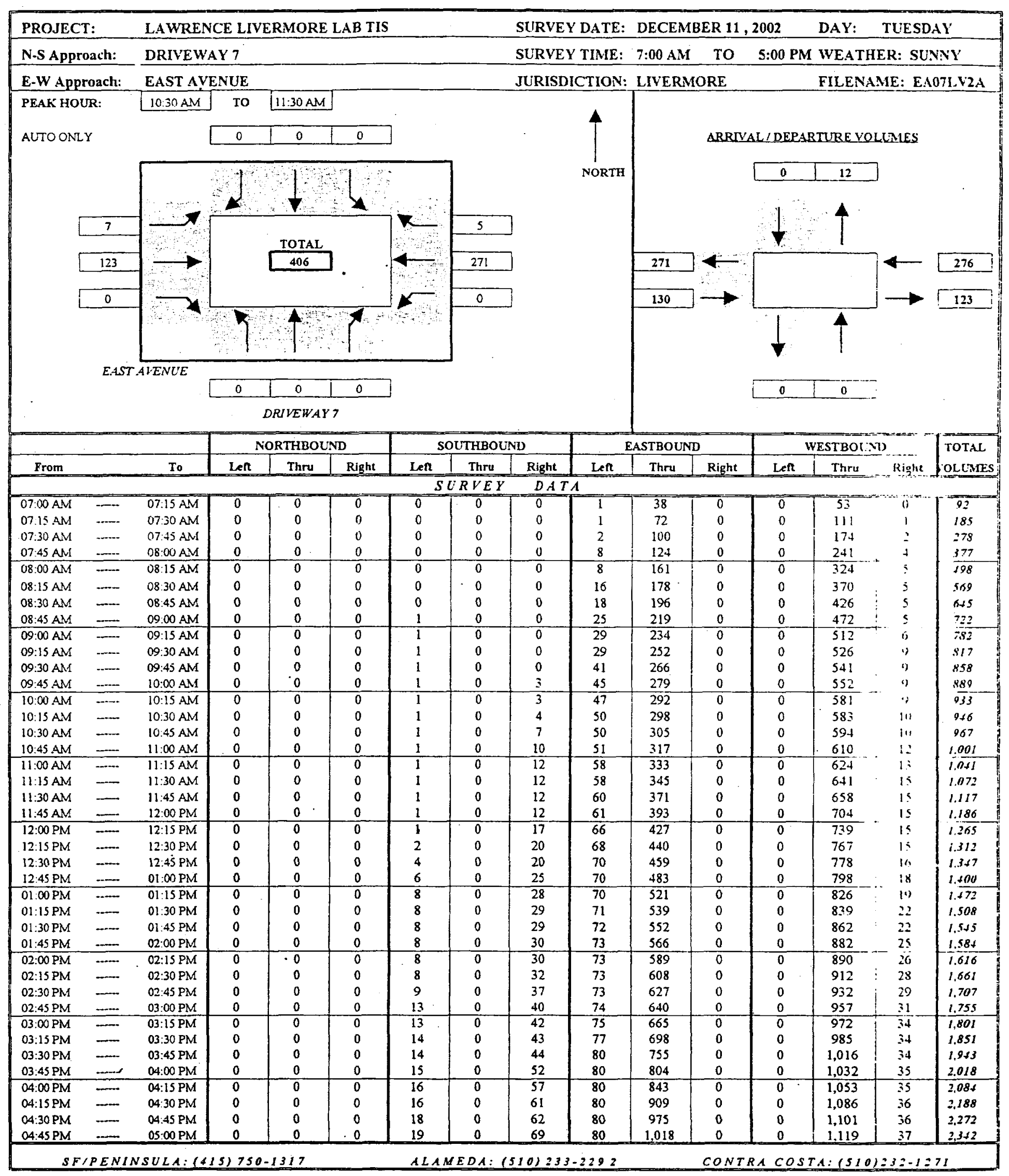




\section{Baymetrics Traffic Resources INTERSECTION TURNING MOVEMENT SUMMARY}

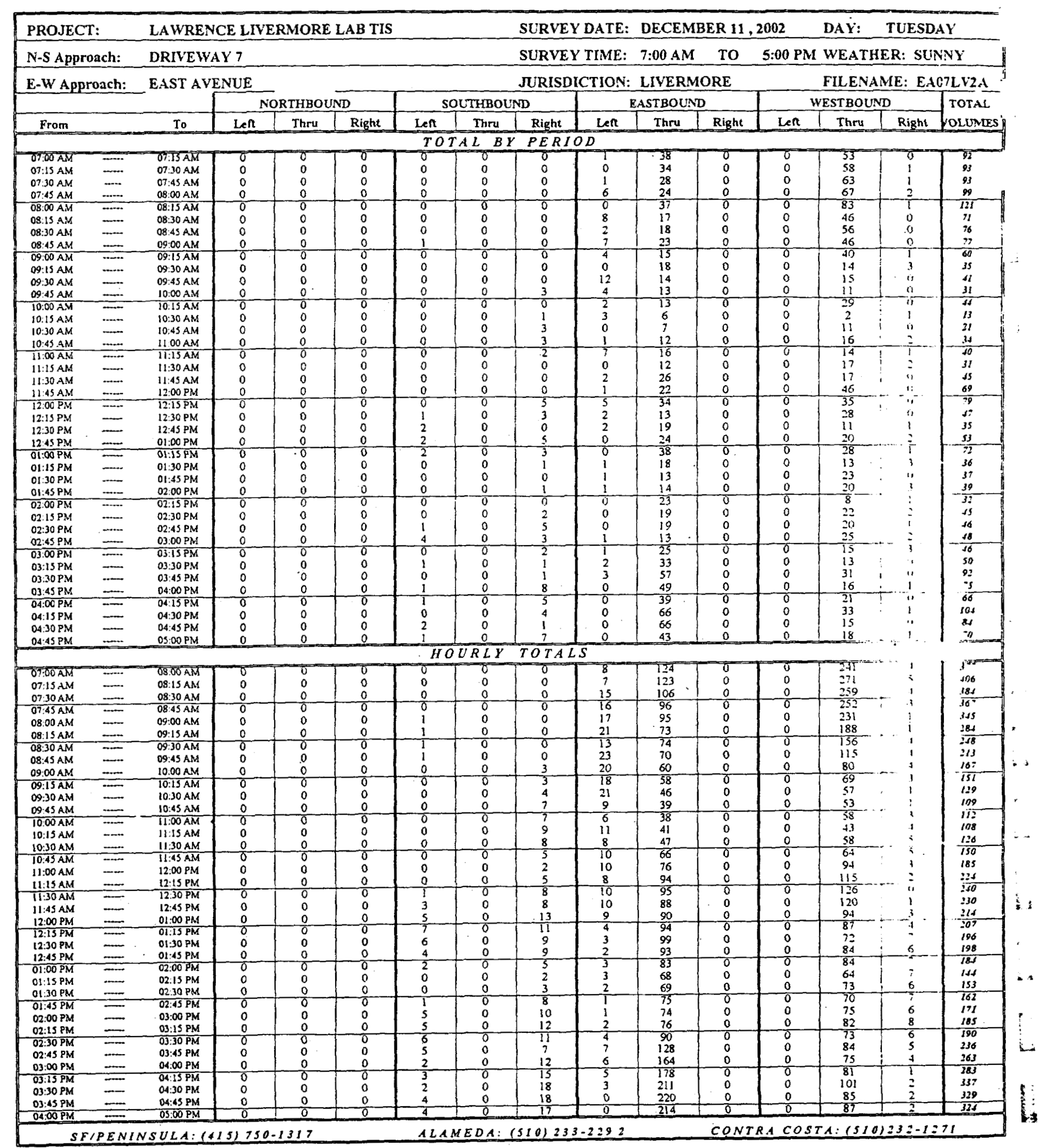




\section{Baymetrics Traffic Resources INTERSECTION TURNING MOVEMENT SUMMARY}

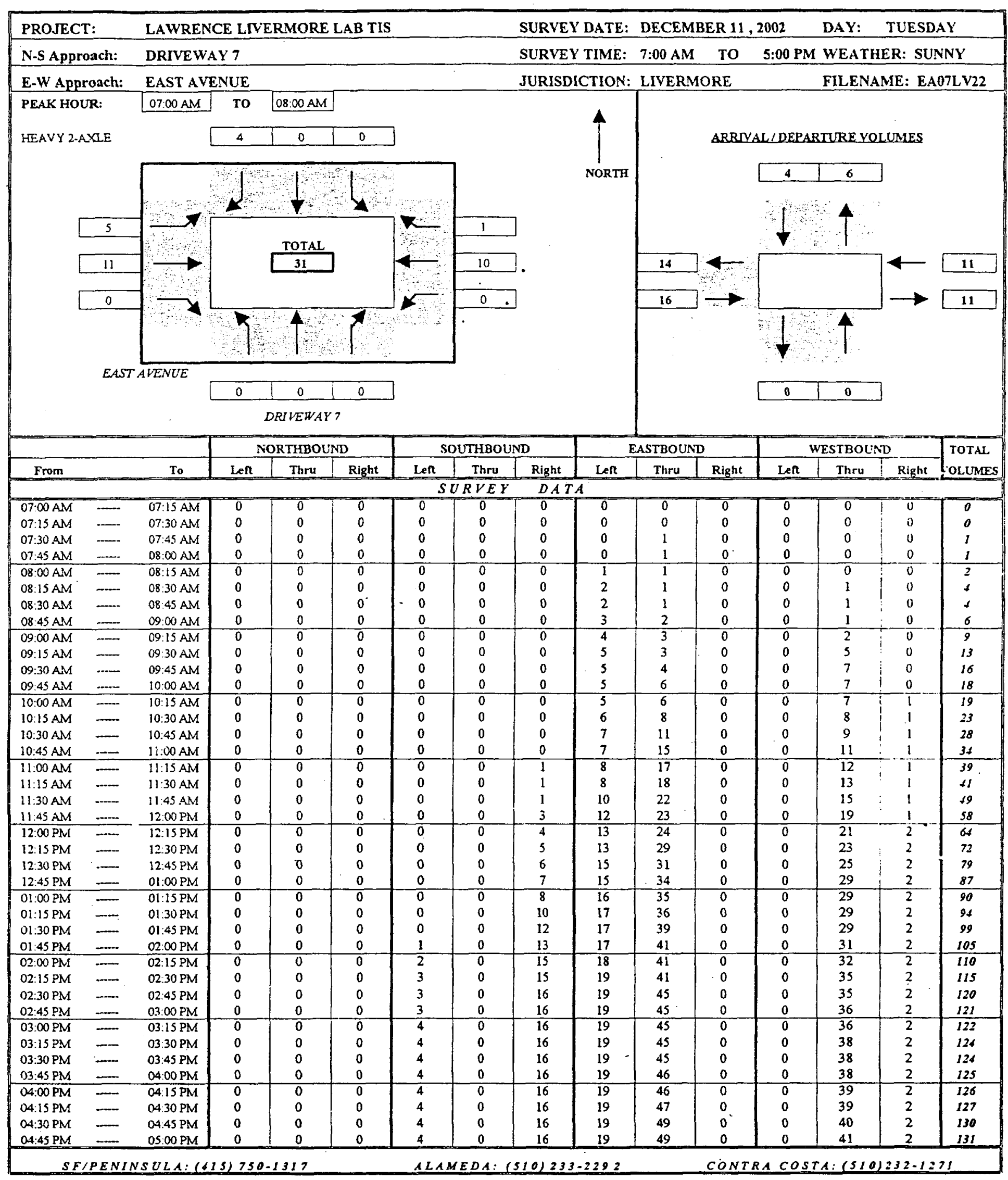




\section{Baymetrics Traffic Resources INTERSECTION TURNING MOVEMENT SUMMARY}

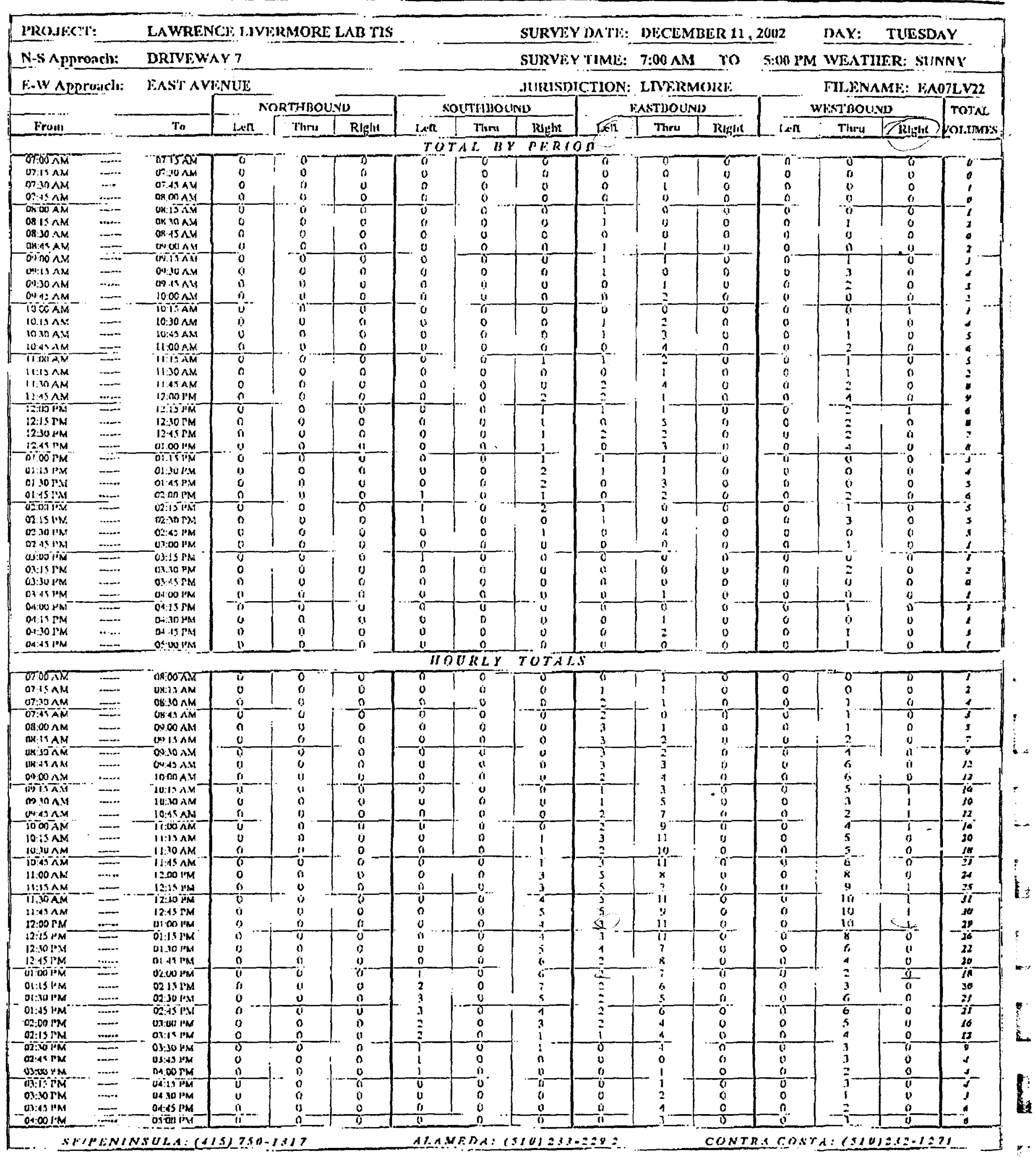




\section{Baymetrics Traffic Resources INTERSECTION TURNING MOVEMENT SUMMARY}

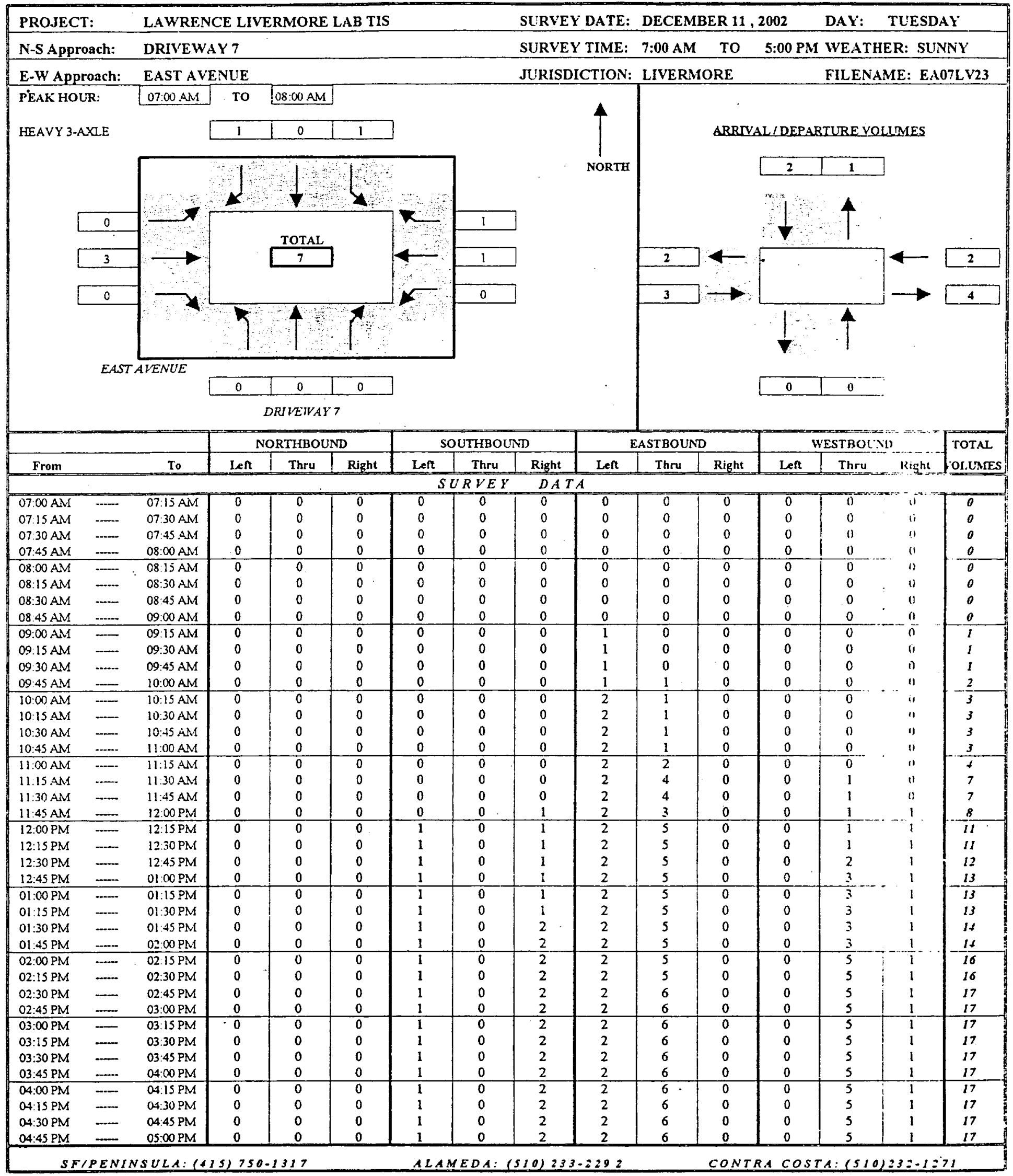




\section{Baymetrics Traffic Resources INTERSECTION TURNING MOVEMENT SUMMARY}

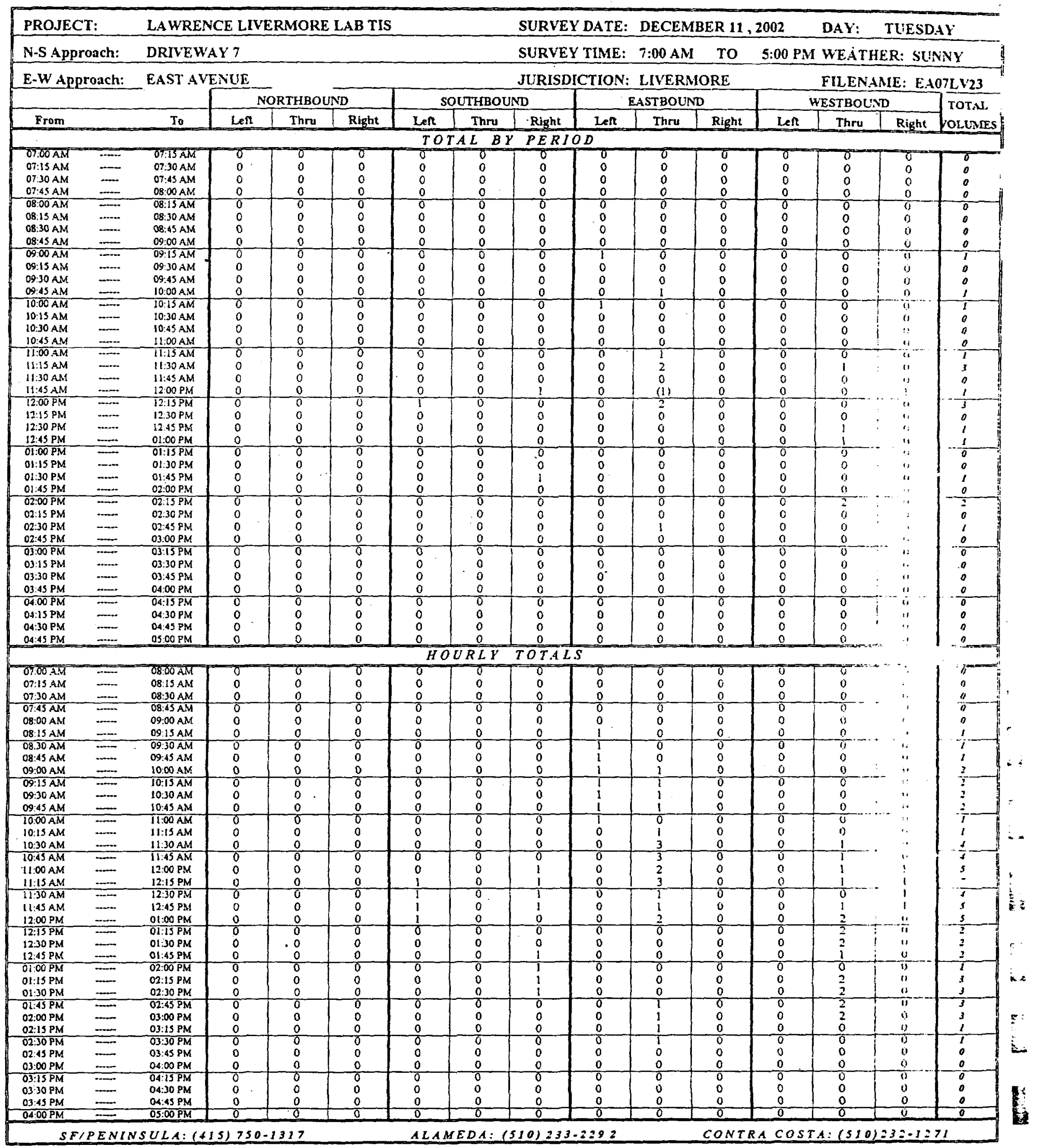




\section{Baymetrics Traffic Resources INTERSECTION TURNING MOVEMENT SUMMARY}

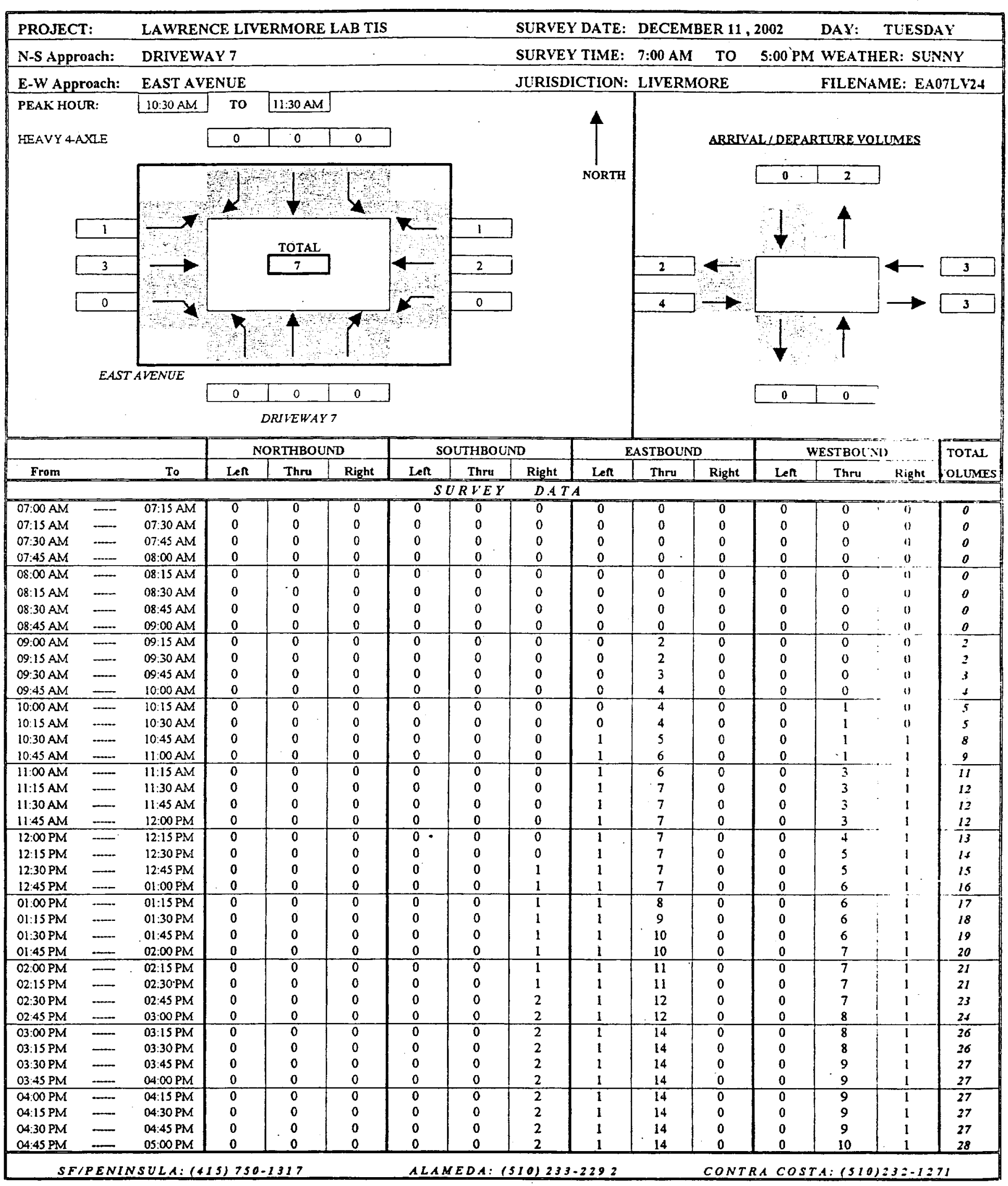




\section{Baymetrics Traffic Resources INTERSECTION TURNING MOVEMENT SUMMARY}

\begin{tabular}{|c|c|c|c|c|c|c|c|c|c|c|c|c|c|c|c|}
\hline \multirow{2}{*}{\multicolumn{2}{|c|}{$\begin{array}{l}\text { PROJECT: } \\
\text { N-S Approach: }\end{array}$}} & \multicolumn{6}{|c|}{ LAWRENCE LIVERMORE LAB TIS } & \multicolumn{5}{|c|}{ SURVEY DATE: DECEMBER 11, 2002 } & DAY: & TUESD & \\
\hline & & DRIVEY & & & & & & SURVE & IME: & 7:00 AM & To & 5:00 P: & WEAT & ER: SU & NY \\
\hline E-W App & Dach: & EAST A & VUE & & & & & JURISD & TION: & LIVERN & DRE & & FILEN & ME: E: & $7 \mathrm{LV} 24$ \\
\hline & & & & THBO & & & THBO & & & ASTBOUN & & & ESTBOT & & TOTAL \\
\hline From & & To & Left & Thru & Right & Len & Thru & Right & Len & Thru & Right & Len & Thru & Right & Lolunies \\
\hline & & & & & & & $L B$ & $P E R I$ & & & & & & & \\
\hline $07.00 \mathrm{AM}$ & $=$ & DभISAM & 0 & 8 & 8 & 0 & 0 & 0 & $\sigma$ & 0 & 8 & 0 & 0 & $\theta$ & 8 \\
\hline $\begin{array}{l}07: 15 \mathrm{AM} \\
07: 30 \mathrm{AM}\end{array}$ & $\ldots$ & $\begin{array}{l}07: 30 \mathrm{AM} \\
07.15 \mathrm{AM}\end{array}$ & $\begin{array}{l}0 \\
0\end{array}$ & $\begin{array}{l}0 \\
0\end{array}$ & $\begin{array}{l}0 \\
0\end{array}$ & $\begin{array}{l}0 \\
0 \\
0\end{array}$ & $\begin{array}{l}0 \\
0\end{array}$ & $\begin{array}{l}0 \\
0\end{array}$ & $\begin{array}{l}0 \\
0\end{array}$ & $\begin{array}{l}0 \\
0\end{array}$ & $\begin{array}{l}0 \\
0\end{array}$ & $\begin{array}{l}0 \\
0\end{array}$ & $\begin{array}{l}0 \\
0\end{array}$ & $\begin{array}{l}0 \\
0\end{array}$ & : \\
\hline 07:45 AM & $=$ & 08:00 AM & 0 & 0 & 0 & 0 & 0 & 0 & 0 & 0 & 0 & 0 & 0 & 0 & 0 \\
\hline $08: 00 \mathrm{AM}$ & $=$ & $08: 15 \mathrm{AM}$ & 0 & 0 & 0 & 0 & 0 & $\overline{0}$ & 0 & 0 & $\overline{0}$ & 0 & 0 & 0 & 8 \\
\hline $\begin{array}{l}\text { OB:15 AM } \\
\text { O8:30 AM }\end{array}$ & $\ldots$ & $\begin{array}{l}\text { 08:30 AM } \\
\text { 08:45 AM }\end{array}$ & $\begin{array}{l}0 \\
0\end{array}$ & $\begin{array}{l}0 \\
0\end{array}$ & $\begin{array}{l}0 \\
0\end{array}$ & $\begin{array}{l}0 \\
0\end{array}$ & $\begin{array}{l}0 \\
0\end{array}$ & $\begin{array}{l}0 \\
0\end{array}$ & $\begin{array}{l}0 \\
0\end{array}$ & 0 & $\begin{array}{l}0 \\
0\end{array}$ & $\begin{array}{l}0 \\
0\end{array}$ & $\begin{array}{l}0 \\
0\end{array}$ & $\begin{array}{l}0 \\
0\end{array}$ & $:$ \\
\hline 08:45 AM & $=$ & 09:00 AM & 0 & 0 & 0 & 0 & 0 & 0 & 0 & 0 & 0 & 0 & 0 & n & 0 \\
\hline $09: 00 \mathrm{AM}$ & $\cdots$ & O9:15AM & 0 & 0 & 0 & 0 & 0 & 0 & 0 & 2 & 0 & 0 & 0 & 0 & 2 \\
\hline 09:15 AM & - & 09:30 AM & 0 & 0 & 0 & 0 & $\therefore$ & 0 & $\begin{array}{l}0 \\
0 \\
0\end{array}$ & 0 & $a$ & 0 & 0 & 0 & 0 \\
\hline $\begin{array}{l}09: 30 \mathrm{AM} \\
09: 45 \mathrm{AM}\end{array}$ & $=$ & $\begin{array}{l}09: 45 \mathrm{AM} \\
10.00 \mathrm{AM} \\
\end{array}$ & $\begin{array}{l}0 \\
0\end{array}$ & $\begin{array}{l}0 \\
0 \\
\end{array}$ & $\begin{array}{l}0 \\
0 \\
\end{array}$ & $\begin{array}{l}0 \\
0\end{array}$ & $\begin{array}{l}0 \\
0 \\
\end{array}$ & $\begin{array}{l}0 \\
0 \\
\end{array}$ & $\begin{array}{l}0 \\
0\end{array}$ & $\begin{array}{l}1 \\
1 \\
\end{array}$ & 0 & 0 & $\begin{array}{l}0 \\
0\end{array}$ & $\begin{array}{l}0 \\
0\end{array}$ & 1 \\
\hline $10: 00 \mathrm{AM}$ & $=$ & 10:15AM & 0 & 0 & 0 & 0 & 0 & 0 & 0 & 0 & 0 & 0 & 1 & 0 & $T$ \\
\hline $\begin{array}{l}10.15 \mathrm{AM} \\
10.30 \mathrm{AM}\end{array}$ & $\overline{-}$ & $\begin{array}{l}10: 30 \mathrm{AM} \\
10.45 \mathrm{AM}\end{array}$ & $\begin{array}{l}0 \\
0\end{array}$ & 0 & 0 & $\begin{array}{l}0 \\
0\end{array}$ & $\begin{array}{l}0 \\
0\end{array}$ & $\begin{array}{l}0 \\
0\end{array}$ & 0 & i & $\begin{array}{l}0 \\
0\end{array}$ & $\begin{array}{l}0 \\
0\end{array}$ & $\stackrel{0}{a}$ & 0 & $a$ \\
\hline $\begin{array}{l}10: 30 \mathrm{AM} \\
10: 45 \mathrm{AM}\end{array}$ & 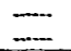 & $\begin{array}{l}10: 45 \mathrm{AM} \\
11: 00 \mathrm{AM}\end{array}$ & $\begin{array}{l}0 \\
0 \\
\end{array}$ & $\begin{array}{l}0 \\
0 \\
0\end{array}$ & $\begin{array}{l}0 \\
0 \\
0\end{array}$ & $\begin{array}{l}0 \\
0\end{array}$ & $\begin{array}{l}0 \\
0\end{array}$ & $\begin{array}{l}0 \\
0\end{array}$ & $\begin{array}{l}1 \\
0\end{array}$ & 1 & $\begin{array}{l}0 \\
0 \\
\end{array}$ & $\begin{array}{l}0 \\
0\end{array}$ & $\begin{array}{l}0 \\
0\end{array}$ & $\begin{array}{l}1 \\
0\end{array}$ & 3 \\
\hline $11: 00 \mathrm{AM}$ & - & $11: 15 \mathrm{AM}$ & 0 & 0 & 0 & 0 & 0 & $\overline{0}$ & 0 & $\frac{1}{0}$ & 0 & 0 & $\frac{5}{2}$ & 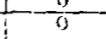 & $\frac{1}{2}$ \\
\hline 11:15 AM & $\rightarrow$ & $11: 30 \mathrm{AM}$ & 0 & 0 & 0 & 0 & 0 & 0 & 0 & 1 & 0 & 0 & 0 & a & $t$ \\
\hline $11: 30 \mathrm{AM}$ & $\ldots$ & $11: 45 \mathrm{AM}$ & 0 & 0 & 0 & 0 & 0 & 0 & 0 & 0 & 0 & 0 & 0 & 0 & 0 \\
\hline II:AS AM & $\ldots$ & $12.00 \mathrm{PM}$ & 0 & 0 & 0 & 0 & 0 & 0 & 0 & 0 & 0 & 0 & 0 & 0 & 0 \\
\hline $12: 00 \mathrm{PM}$ & - & $12: 15 \mathrm{PM}$ & 0 & 0 & 0 & $\overline{0}$ & 0 & 0 & 0 & 0 & 0 & 0 & $T$ & 4 & 1 \\
\hline $12: 15 \mathrm{PM}$ & $\ldots$ & $12: 30 \mathrm{PM}$ & 0 & 0 & 0 & 0 & 0 & 0 & 0 & 0 & 0 & 0 & 1 & $n$ & $t$ \\
\hline $12: 30 \mathrm{PM}$ & $\ldots$ & 12:45 PM & 0 & 0 & 0 & 0 & 0 & 1 & 0 & 0 & 0 & 0 & 0 & ") & 1 \\
\hline $12: 45 \mathrm{PM}$ & $\cdots$ & $01: 00$ PM & 0 & 0 & 0 & 0 & 0 & 0 & 0 & 0 & 0 & 0 & 1 & i & 1 \\
\hline $01: 00 \mathrm{PM}$ & $=$ & $01: 15 \mathrm{PM}$ & 0 & 0 & 0 & 0 & 0 & 0 & 0 & 1 & 0 & 0 & 0 & i) & $i$ \\
\hline $01: 15$ PM & $\ldots$ & $01: 30 \mathrm{PM}$ & 0 & 0 & 0 & 0 & 0 & 0 & 0 & i & 0 & 0 & 0 & 0 & 1 \\
\hline $01.30 \mathrm{PM}$ & $\cdots$ & 01:45 PM & 0 & 0 & 0 & 0 & 0 & 0 & 0 & 1 & 0 & 0 & 0 & 0 & $l$ \\
\hline $01: 45$ FM & $=$ & $0200 \mathrm{PM}$ & 0 & 0 & 0 & 0 & 0 & 0 & 0 & 0 & 0 & 0 & 1 & 11 & 1 \\
\hline $02: 00 \mathrm{PM}$ & $\cdots$ & $02: 15 \mathrm{PM}$ & 0 & 0 & 0 & 0 & 0 & 0 & 0 & $\mathrm{i}$ & 0 & $\overline{0}$ & $\overline{0}$ & 0 & 1 \\
\hline $02: 15 \mathrm{PM}$ & - & $01: 30 \mathrm{PM}$ & 0 & 0 & 0 & 0 & 0 & 0 & 0 & 0 & 0 & 0 & 0 & 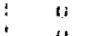 & ; \\
\hline 02:30 PM & $\cdots$ & 02.45 PM & 0 & 0 & 0 & 0 & 0 & 1 & 0 & 1 & 0 & 0 & 0 & 0 & 2 \\
\hline $02: 45$ PM & $=$ & $03: 00 P M$ & 0 & 0 & 0 & 0 & 0 & 0 & 0 & 0 & 3. & 0 & 1 & $i$ & 1 \\
\hline $0300 \mathrm{PM}$ & $\cdots$ & $03: 15$ PM & 0 & 0 & 0 & 0 & 0 & 0 & $\overline{0}$ & 2 & 0 & 0 & 0 & 17 & 3 \\
\hline 03.15 PM & $\ldots$ & $03: 30 \mathrm{PM}$ & 0 & 0 & 0 & 0 & 0 & 0 & 0 & 0 & 0 & 0 & 0 & $" 1$ & 0 \\
\hline 03:30 PM & - & $03: 45 \mathrm{PM}$ & 0 & 0 & 0 & 0 & 0 & 0 & 0 & 0 & 0 & 0 & 1 & $"$ & 1 \\
\hline $03: 45 \mathrm{PM}$ & $=$ & $04: 00 \mathrm{PM}$ & 0 & 0 & 0 & 0 & 0 & 0 & 0 & 0 & 0 & 0 & 0 & 11 & $\therefore$ \\
\hline $04: 00 \mathrm{PM}$ & $E$ & $04: 15 \mathrm{PM}$ & 0 & 0 & 0 & $\%$ & 0 & 0 & 0 & $\overline{0}$ & 0 & 0 & $\overline{0}$ & 5 & $\overline{0}$ \\
\hline $04: 15 \mathrm{PM}$ & - & $04: 30 \mathrm{PM}$ & 0 & 0 & 0 & 0 & 0 & 0 & 0 & 0 & 0 & 0 & 0 & ") & 0 \\
\hline $04: 30 \mathrm{PM}$ & - & $04: 45 \mathrm{PM}$ & 0 & 0 & 0 & 0 & 0 & 0 & 0 & 0 & 0 & 0 & 0 & $"$ & 0 \\
\hline 04.45 PM & $=$ & $0500 \mathrm{PM}$ & 0 & 0 & 0 & 0 & 0 & 0 & 0 & 0 & 0 & 0 & 1 & 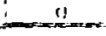 & 1 \\
\hline & & & & & & & $R L Y$ & $T O T A$ & & & & & & & \\
\hline $0.00 \mathrm{AM}$ & $=$ & 08:00AM & $\sigma$ & $\gamma$ & o & $\sigma$ & 8 & $\sigma$ & 0 & 0 & 0 & $\sigma$ & 0 & $\pi$ & 0 \\
\hline 07:15 AM & $\longrightarrow$ & 08:15 AM & 0 & 0 & 0 & B & 0 & 0 & $\begin{array}{l}0 \\
0\end{array}$ & 0 & $\begin{array}{l}0 \\
0\end{array}$ & $\begin{array}{l}0 \\
0\end{array}$ & $\begin{array}{l}0 \\
0\end{array}$ & $\begin{array}{l}0 \\
0 \\
0\end{array}$ & 0 \\
\hline $07: 30 \mathrm{AM}$ & $=$ & $08: 30 . \mathrm{AM}$ & 0 & 0 & 0 & $\frac{0}{n}$ & $\frac{0}{0}$ & 0 & $\frac{0}{0}$ & 0 & 0 & 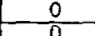 & 0 & 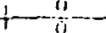 & $\therefore$ \\
\hline $07.45 \mathrm{AM}$ & - & $08: 45 \mathrm{AM}$ & 0 & 0 & 0 & 0 & 0 & 0 & 0 & 0 & 0 & 0 & 0 & i) & 0 \\
\hline 08:00 AM & - & $09: 00 \mathrm{AM}$ & 0 & 0 & 0 & 0 & 0 & 0 & 0 & 0 & 0 & 0 & 0 & 11 & ; \\
\hline 08:15 AM & $\ldots$ & $09: 15 \mathrm{AM}$ & 0 & 0 & 0 & 0 & 0 & 0 & 0 & 2 & 0 & 0 & a & 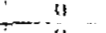 & $\frac{2}{2}$ \\
\hline $08: 30$ AMI & & $09: 30 \mathrm{AM}$. & 0 & 0 & 0 & 0 & 0 & 0 & 0 & 2 & 0 & 0 & 0 & "i & 3 \\
\hline $08: 45$ AM & $\longrightarrow$ & 09:45 AM & 0 & 0 & 0 & 0 & 0 & 0 & 0 & 3 & 0 & 0 & $\begin{array}{l}0 \\
0 \\
0\end{array}$ & $"$ & 3 \\
\hline$\frac{09: 00 \mathrm{AM}}{09: 15 \mathrm{AM}}$ & $\equiv$ & $\frac{10: 00 \mathrm{AM}}{10: 15 \mathrm{AM}}$ & 0 & $\frac{0}{0}$ & $\frac{0}{0}$ & $\frac{0}{0}$ & $\frac{0}{0}$ & $\frac{0}{0}$ & $\frac{0}{0}$ & $\frac{4}{2}$ & $\frac{0}{0}$ & 0 & 1 & $-\frac{10}{11}$ & $\frac{4}{3}$ \\
\hline $09: 30 \mathrm{AM}$ & - & & 0 & 0 & 0 & 0 & 0 & 0 & 0 & 2 & D & 0 & 1 & $"$ & 3 \\
\hline $09: 45$ AM & $\Longrightarrow$ & $10: 43 \mathrm{AM}$ & 0 & 0 & 0 & 0 & 0 & 0 & 1 & 2 & 0 & 0 & 1 & 1 & 5 \\
\hline & $=$ & $11: 00 \mathrm{AM}$ & 0 & 0 & $\overline{0}$ & 0 & 0 & 0 & $T$ & 2 & $\overline{0}$ & 0 & 1 & $T$ & 5 \\
\hline $10: 15 \mathrm{AM}$ & - & $11: 15 \mathrm{AM}$ & 0 & 0 & 0 & 0 & 0 & 0 & 1 & 2 & 0 & 0 & 2 & i & 6 \\
\hline $10.30 \mathrm{AM}$ & $=$ & $\frac{11: 30 \mathrm{AM}}{1.5 \mathrm{AM}}$ & $\frac{0}{0}$ & $\frac{.0}{0}$ & $\frac{0}{0}$ & 0 & $-\frac{a}{0}$ & $\frac{0}{0}$ & $\frac{1}{0}$ & $\frac{3}{2}$ & $\frac{0}{0}$ & $\frac{0}{0}$ & $\frac{2}{3}$ & $\frac{1}{11}$ & \\
\hline 10:45 AM & $\ldots$ & $\begin{array}{l}11: 45 \mathrm{AM} \\
1200 \mathrm{PM}\end{array}$ & $\begin{array}{l}0 \\
0\end{array}$ & 0 & $\begin{array}{l}0 \\
0\end{array}$ & $\begin{array}{l}0 \\
0 \\
0\end{array}$ & $\begin{array}{l}0 \\
0\end{array}$ & $\begin{array}{l}0 \\
0\end{array}$ & $\begin{array}{l}0 \\
0\end{array}$ & 3 & $\begin{array}{l}0 \\
0\end{array}$ & $\begin{array}{l}0 \\
0\end{array}$ & $\frac{2}{2}$ & () & 3 \\
\hline $\begin{array}{l}11: 00 \text { AM } \\
11115 \text { AM }\end{array}$ & $\ldots$ & $\begin{array}{l}12: 00 \text { PM } \\
12: 15 \text { PM }\end{array}$ & 0 & $\begin{array}{l}0 \\
0\end{array}$ & $\begin{array}{l}0 \\
0\end{array}$ & $\begin{array}{l}0 \\
0\end{array}$ & $\begin{array}{l}0 \\
0\end{array}$ & $\begin{array}{l}0 \\
0\end{array}$ & $\begin{array}{l}0 \\
0\end{array}$ & 1 & o & a & $\frac{2}{1}$ & in & 2 \\
\hline$\frac{11: 35 \mathrm{AM}}{11,3 \mathrm{~A}}$ & $=$ & $12: 30 \mathrm{PM}$ & 0 & a & 0 & 0 & 0 & 0 & 0 & 0 & 0 & 0 & 2 & $\pi$ & 3 \\
\hline $11: 44 \mathrm{AM}$ & $\ldots$ & 12:45 PM & 0 & 0 & 0 & 0 & 0 & I & 0 & 0 & 0 & 0 & $\frac{2}{3}$ & 0 & 3 \\
\hline $12.00 \mathrm{PM}$ & $=$ & $01: 00$ PM & $\therefore$ & $\frac{0}{0}$ & $\frac{0}{0}$ & $\frac{0}{0}$ & $\frac{0}{0}$ & $\frac{1}{1}$ & $\frac{0}{0}$ & $\frac{0}{1}$ & 0 & $\frac{0}{0}$ & $\frac{3}{2}$ & & $\frac{1}{8}$ \\
\hline $13: 15 \mathrm{PM}$ & $\ldots$ & $01: 15 \mathrm{PM}$ & 0 & 0 & 0 & $\overline{0}$ & 0 & 1 & 0 & $\frac{1}{2}$ & 0 & $\begin{array}{l}0 \\
0\end{array}$ & 2 & $\begin{array}{l}0 \\
0\end{array}$ & 3 \\
\hline 12:30 PM & $\ldots$ & 01:30 PM & 0 & $:$ & 0 & $\begin{array}{l}0 \\
0\end{array}$ & $\begin{array}{l}0 \\
0\end{array}$ & $\begin{array}{l}1 \\
0\end{array}$ & $\begin{array}{l}0 \\
0\end{array}$ & 3 & $\begin{array}{l}0 \\
0 \\
0\end{array}$ & $\begin{array}{l}0 \\
0\end{array}$ & 1 & $\begin{array}{l}0 \\
0\end{array}$ & + \\
\hline$\frac{12: 45 \mathrm{PM}}{01.00 \mathrm{PM}}$ & $\approx$ & $01: 45 \mathrm{PM}$ & 0 & 0 & 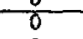 & 0 & 0 & 0 & 0 & -3 & 0 & 0 & $T$ & $\pi$ & 8 \\
\hline Ol:15 PM & $\ldots$ & 02:15 PM & 0 & 0 & 0 & 0 & $\begin{array}{l}0 \\
0\end{array}$ & 0 & $:$ & 3 & $\begin{array}{l}0 \\
0\end{array}$ & $\stackrel{0}{0}$ & $\frac{1}{1}$ & 8 & 3 \\
\hline O1:30 PM & $=$ & 02:30 PM & 0 & $\stackrel{0}{0}$ & 0 & 0 & $\stackrel{0}{0}$ & $\frac{a}{1}$ & $\frac{0}{0}$ & $\frac{2}{2}$ & 0 & 0 & & & 5 \\
\hline $01: 45 \mathrm{PM}$ & $=$ & $02: 45 \% \mathrm{M}$ & 0 & 0 & 0 & $\overline{0}$ & 0 & 1 & 0 & 2 & 0 & 0 & 1 & 0 & 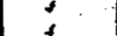 \\
\hline $02: 00 \mathrm{PM}$ & - & 03:00 PM & 0 & 0 & 0 & 0 & $0_{0}^{0}$ & 1 & $\begin{array}{l}0 \\
0\end{array}$ & 2 & 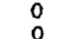 & $\stackrel{0}{0}$ & t & $\begin{array}{l}0 \\
0\end{array}$ & 5 \\
\hline $02: 15 \mathrm{PM}$ & $=$ & 03:15 PM & 0 & 0 & 0 & 0 & $\therefore$ & & $\frac{0}{0}$ & 3 & 0 & $\frac{0}{0}$ & & 0 & $\frac{5}{5}$ \\
\hline $02: 30 \mathrm{PM}$ & $=$ & $03: 30 P M$ & $\begin{array}{l}0 \\
0\end{array}$ & 0 & 0 & 0 & 0 & 1 & 0 & $\frac{3}{2}$ & $\begin{array}{l}0 \\
0\end{array}$ & $\begin{array}{l}0 \\
0 \\
0\end{array}$ & $\frac{1}{2}$ & $\begin{array}{l}0 \\
0\end{array}$ & 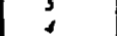 \\
\hline $\begin{array}{l}02: 45 \text { PM } \\
03: 00 \mathrm{PM}\end{array}$ & $=$ & $\begin{array}{l}\text { 03:45 PM } \\
\text { 04:00 PM }\end{array}$ & 0 & $\begin{array}{l}0 \\
0\end{array}$ & 0 & 0 & $\begin{array}{l}0 \\
0\end{array}$ & 0 & 0 & 2 & 0 & 0 & 1 & 0 & 3 \\
\hline $03: 13 \mathrm{PM}$ & $=$ & $\frac{04: 15 \mathrm{PM}}{0.03}$ & 0 & 0 & 0 & $\frac{1}{0}$ & 0 & 0 & 0 & 0 & 0 & 0 & $T$ & 0 & $T$ \\
\hline $03: 30 \mathrm{PM}$ & - & $04: 30 \mathrm{PM}$ & 0 & 0 & 0 & 0 & 0 & 0 & 0 & 0 & 0 & 0 & 1 & 0 & 1 \\
\hline 03:45 PM & - & 04:45 PM & 0 & 0 & 0 & 0 & 0 & 0 & 0 & 0 & 0 & 0 & 0 & 0 & 0 \\
\hline $01: 00 \mathrm{PM}$ & $=$ & $05: 00 \mathrm{pM}$ & 0 & 0 & 0 & 0 & 0 & 0 & 0 & $\sigma$ & 0 & 0 & & 0 & \\
\hline$S E$ & ENI & OLA: & & & & 41 & $D A$ & 0) & 29 & & Co & $A C$ & 1 & 32 & \\
\hline
\end{tabular}




\section{Baymetrics Traffic Resources INTERSECTION TURNING MOVEMENT SUMMARY}

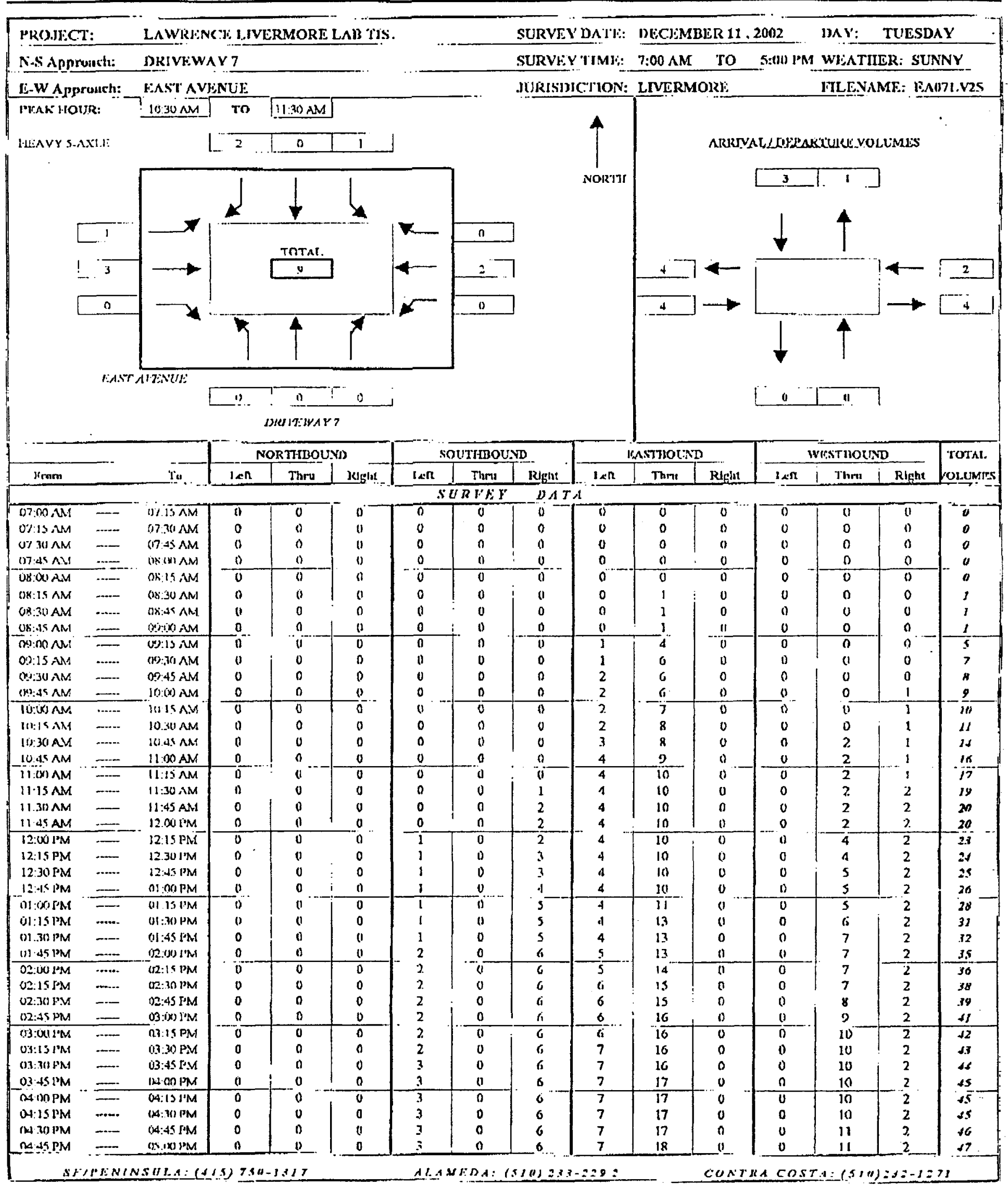




\section{Baymetrics Traffic Resources INTERSECTION TURNING MOVEMENT SUMMARY}

\begin{tabular}{|c|c|c|c|c|c|c|c|c|c|c|c|c|c|c|c|}
\hline \multirow{2}{*}{\multicolumn{2}{|c|}{$\begin{array}{l}\text { PROJECT: } \\
\text { N-S Approach: }\end{array}$}} & \multicolumn{6}{|c|}{ LAWRENCE LIVERMORE LAB TIS } & \multicolumn{2}{|c|}{ SURVEY DATE } & DECEN & ER 11, & 2002 & DAY: & TUESD & \\
\hline & & DRIVEY & & & & & & SURVE & CIME: & $7: 00 \mathrm{AM}$ & TO & 5:00 PM & WEATI & ER: SUI & VNY \\
\hline$E_{-}-\mathrm{W}$ AP & oach: & EAST A & VUE & & & & & JLIRISD & TION: & LIVERI & RE & & & ME: EA & $07 L V 25$ \\
\hline & & & & THBO & & & THBO & & & ASTBOUN & & & ESTBOUT & & TOTAL. \\
\hline From & & To & Lent & Thru & Right & Left & Thru & Right & Left & Thru & Right & Len & Thru & Right & LOLUMES \\
\hline & & & & & & & $L \quad B$ & PERI & & & & & & & \\
\hline $07: 15 \mathrm{AM}$ & $=$ & $\begin{array}{l}\text { O7.15 AM } \\
07: 30 \mathrm{AM}\end{array}$ & $\begin{array}{l}0 \\
0\end{array}$ & $\begin{array}{l}\sigma \\
0\end{array}$ & $\begin{array}{l}0 \\
0\end{array}$ & $\begin{array}{l}0 \\
0\end{array}$ & $\begin{array}{l}8 \\
0\end{array}$ & $\begin{array}{l}0 \\
0\end{array}$ & $\begin{array}{l}8 \\
0\end{array}$ & $\begin{array}{l}0 \\
0\end{array}$ & $\begin{array}{l}0 \\
0\end{array}$ & $\begin{array}{l}0 \\
0\end{array}$ & 0 & 0 & 8 \\
\hline $07: 30 \mathrm{AM}$ & - & $07: 45$ AM & 0 & 0 & 0 & o & 0 & 0 & 0 & 0 & 0 & $\begin{array}{l}0 \\
0\end{array}$ & $\begin{array}{l}0 \\
0\end{array}$ & 0 & $\begin{array}{l}0 \\
0\end{array}$ \\
\hline$\frac{07: 45 \mathrm{AM}}{08: 00 \mathrm{AM}}$ & $=$ & $\frac{08: 00 \text { AM }}{08: 15 \text { AM }}$ & $-\frac{0}{0}$ & $\frac{0}{0}$ & 0 & $\frac{0}{0}$ & 0 & 0 & 0 & 0 & 0 & 0 & 0 & 0 & 0 \\
\hline O8:15 AM & $\ldots$ & O8:30 AM & 0 & 0 & 0 & 0 & 0 & 0 & $\begin{array}{l}0 \\
0\end{array}$ & $\begin{array}{l}0 \\
i\end{array}$ & $\begin{array}{l}0 \\
0\end{array}$ & 0 & 0 & $n$ & 0 \\
\hline 08:30 AN & $\cdots$ & $08: 45 \mathrm{AM}$ & 0 & 0 & 0 & 0 & 0 & 0 & 0 & 0 & $\begin{array}{l}0 \\
0\end{array}$ & o & $\begin{array}{l}0 \\
0\end{array}$ & $\begin{array}{l}0 \\
0\end{array}$ & $\begin{array}{l}1 \\
0\end{array}$ \\
\hline$\frac{08: 45 \mathrm{AM}}{09: 00 \mathrm{AM}}$ & $=$ & $\frac{09: 00 \mathrm{AM}}{09: 13 \mathrm{AM}}$ & -0 & $\frac{0}{0}$ & $\frac{0}{0}$ & $\frac{0}{0}$ & $-\frac{0}{0}$ & $\frac{0}{0}$ & 0 & $\frac{0}{3}$ & $\frac{0}{n}$ & $\frac{0}{0}$ & 0 & 0 & 0 \\
\hline $09: 15 \mathrm{AM}$ & $\ldots$ & $08: 30 \mathrm{AM}$ & 0 & 0 & 0 & 0 & 0 & 0 & 0 & $\cdot 3$ & $\begin{array}{l}0 \\
0\end{array}$ & $\begin{array}{l}0 \\
0\end{array}$ & $\begin{array}{l}0 \\
0\end{array}$ & 0 & 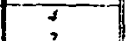 \\
\hline 09:30 AM & - & $09.45 \mathrm{AM}$ & 0 & 0 & 0 & 0 & 0 & 0 & 1 & 0 & 0 & 0 & $\begin{array}{l}0 \\
0\end{array}$ & $?$ & $?$ \\
\hline $09: 45 . \mathrm{AM}$ & + & 10:00 AM & 0 & 0 & 0 & 0 & 0 & 0 & 0 & 0 & 0 & 0 & 0 & 1 & $i$ \\
\hline $10: 00 \mathrm{AM}$ & $\ldots$ & $10: 15 \mathrm{AM}$ & 0 & 0 & 0 & 0 & 0 & 0 & 0 & 1 & 0 & 0 & 0 & i) & 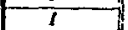 \\
\hline 10:15 AM & - & 10:30 AM & 0 & 0 & 0 & 0 & 0 & 0 & 0 & $i$ & 0 & 0 & 0 & is & $i$ \\
\hline $10: 30 . \mathrm{AM}$ & - & 10:45 AM & 0 & 0 & 0 & 0 & 0 & 0 & 1 & 0 & 0 & 0 & 2 & i) & $j$ \\
\hline 10:45 AM & $\cdots$ & $11: 00 \mathrm{AM}$ & 0 & 0 & 0 & 0 & 0 & 0 & 1 & 1 & 0 & 0 & 0 & 9 & 2 \\
\hline $11: 00 \mathrm{AM}$ & $\ldots$ & T1:15AM & 0 & 0 & 0 & 0 & 0 & 0 & $\overline{0}$ & 1 & 0 & 0 & 0 & 13 & 1 \\
\hline 11:15 A.M & $\cdots$ & 11:30 AM & 0 & 0 & 0 & 0 & 0 & 1 & 0 & 0 & 0 & 0 & 0 & 1 & 2 \\
\hline $\begin{array}{l}11: 30 \text { AM } \\
11: 45 \text { AM }\end{array}$ & $\ldots$ & $\begin{array}{l}\text { 11:45 AM } \\
12: 00 \text { PM }\end{array}$ & $\begin{array}{l}0 \\
0\end{array}$ & $\begin{array}{l}0 \\
0\end{array}$ & $\begin{array}{l}0 \\
0\end{array}$ & $\begin{array}{l}0 \\
0\end{array}$ & 0 & 1 & $\begin{array}{l}0 \\
0\end{array}$ & $\begin{array}{l}0 \\
0\end{array}$ & $\begin{array}{l}0 \\
0\end{array}$ & $\begin{array}{l}0 \\
0\end{array}$ & 9 & ". & $t$ \\
\hline $1200 \mathrm{PM}$ & $\ldots$ & $12: 15 \mathrm{PM}$ & 0 & 0 & 0 & $\frac{2}{1}$ & 0 & 0 & 0 & 0 & 0 & 0 & $\frac{\pi}{2}$ & i) & $-\frac{0}{3}$ \\
\hline 12:15 PM & - & $12: 30 \mathrm{PM}$ & 0 & 0 & 0 & 0 & 0 & 1 & 0 & 0 & 0 & 0 & 0 & :1 & 1 \\
\hline 12:30 PM & $\cdots$ & $12: 45 \mathrm{PM}$ & 0 & 0 & 0 & 0 & 0 & 0 & 0 & 0 & 0 & 0 & 1 & "s & $i$ \\
\hline 12:45 PM & $\cdots$ & $01: 00 \mathrm{PM}$ & 0 & 0 & 0 & 0 & 0 & 1 & 0 & 0 & 0 & 0 & 0 & a & $i$ \\
\hline $01: 00 \mathrm{PM}$ & - & $01: 15 \mathrm{PM}$ & 0 & 0 & 0 & 0 & 0 & 1 & 0 & 1 & D & 0 & a & 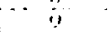 & $-\frac{1}{3}$ \\
\hline OI:15 PM & $\ldots$ & 01:30 PM & 0 & 0 & 0 & 0 & 0 & 0 & 0 & 2 & 0 & 0 & $i$ & $\therefore$ & j \\
\hline 01:30 PM & $\cdots$ & $01: 45$ PM & 0 & 0 & 0 & 0 & 0 & 0 & 0 & 0 & 0 & 0 & $i$ & $:$ & 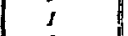 \\
\hline Ol:49 PM & $\ldots$ & $02.00 \mathrm{PM}$ & 0 & 0 & 0 & 1 & 0 & 1 & 1 & 0 & 0 & 0 & 0 & $"$ & 3 \\
\hline $02: 00 \mathrm{PM}$ & $-\cdots$ & $02: 15 \mathrm{PM}$ & 0 & 0 & 0 & 0 & 0 & 0 & 0 & $T$ & 0 & 0 & ij & $\because$ & I \\
\hline 02:15 PM & $\cdots$ & 02:30 PM & 0 & 0 & 0 & 0 & 0 & 0 & 1 & i & 0 & 0 & 0 & "1 & 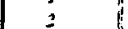 \\
\hline 02:30 PM & $\cdots$ & $02: 45$ PM & 0 & 0 & 0 & $\dot{0}$ & 0 & 0 & 0 & 0 & 0 & 0 & 1 & .. & $j$ \\
\hline $02.45 \mathrm{PM}$ & - & $0300 \mathrm{PM}$ & 0 & 0 & 0 & 0 & 0 & 0 & 0 & $\mathrm{I}$ & 0 & 0 & I & $\therefore$ & 3 \\
\hline $03: 00 \mathrm{PM}$ & 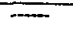 & $03: 15 \mathrm{PM}$ & 0 & 0 & 0 & 0 & 0 & 0 & 0 & 0 & 0 & $\overline{0}$ & $i^{-}$ & $\because$ & $T$ \\
\hline $\begin{array}{l}\text { 03:15 PM } \\
0.30 \mathrm{PMM}\end{array}$ & $=$ & $\begin{array}{l}03: 30 \mathrm{PM} \\
03.45 \mathrm{PM}\end{array}$ & $\stackrel{0}{0}$ & $\begin{array}{l}0 \\
0\end{array}$ & $\begin{array}{l}0 \\
0\end{array}$ & 0 & 8 & $\begin{array}{l}0 \\
0\end{array}$ & 1 & 0 & 0 & $\begin{array}{l}0 \\
0\end{array}$ & 0 & $\therefore$ & 1 \\
\hline $03: 45 \mathrm{PM}$ & $\ldots$ & $04: 00 \mathrm{PM}$ & 0 & 0 & 0 & 0 & $\begin{array}{l}0 \\
0\end{array}$ & 最 & $\begin{array}{l}0 \\
0\end{array}$ & $\begin{array}{l}0 \\
1\end{array}$ & $\begin{array}{l}0 \\
0\end{array}$ & $\begin{array}{l}0 \\
0\end{array}$ & $\begin{array}{l}0 \\
0\end{array}$ & $"$ & I \\
\hline $04: 00 \mathrm{PM}$ & $=$ & $04: 15 \mathrm{PM}$ & 0 & 0 & 0 & 0 & 0 & 0 & 0 & 0 & 0 & 0 & & $\ddot{n}-$ & 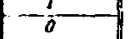 \\
\hline 04:15 PM & - & $0430 \mathrm{PM}$ & 0 & 0 & 0 & 0 & 0 & 0 & 0 & 0 & 0 & 0 & $a$ & $"$ & 0 \\
\hline 04:30 PM & $\bar{T}$ & 04:45 PM & 0 & 0 & 0 & 0 & 0 & 0 & 0 & 0 & 0 & 0 & $!$ & $"$ & 1 \\
\hline $04: 45 \mathrm{PM}$ & $\ldots$ & $05: 00 \mathrm{PM}$ & 0 & 0 & 0 & 0 & 0 & 0 & 0 & 1 & 0 & 0 & 0 & $\because$ & 1 \\
\hline & & & & & & & $R L Y$ & TOTAL & & & & & & & \\
\hline 8T:00AM & -1 & $08.00 \mathrm{AM}$ & 0 & 0 & $\overline{0}$ & 0 & 70 & $\overline{0}$ & $\bar{\sigma}$ & $\bar{u}$ & 0 & 0 & i) & $\pi$ & $\overline{0}$ \\
\hline $\begin{array}{l}07: 15 \mathrm{AM} \\
07: 30 . \mathrm{MM}\end{array}$ & $\cdots$ & $\begin{array}{l}08: 15 \mathrm{AM} \\
08: 30 \mathrm{AM}\end{array}$ & $\begin{array}{l}0 \\
0\end{array}$ & $\begin{array}{l}0 \\
0\end{array}$ & $\begin{array}{l}0 \\
0\end{array}$ & $\begin{array}{l}0 \\
0\end{array}$ & $\begin{array}{l}0 \\
0\end{array}$ & $\begin{array}{l}0 \\
0\end{array}$ & $\begin{array}{l}0 \\
0\end{array}$ & $i$ & $\begin{array}{l}0 \\
0\end{array}$ & $\begin{array}{l}0 \\
0\end{array}$ & 0 & $\ddot{~}$ & i \\
\hline $07: 45 \mathrm{AM}$ & $=$ & $08.45 \mathrm{AM}$ & 0 & 0 & 0 & 0 & 0 & 0 & 0 & $i$ & 0 & 0 & 8 & $\ddot{i}$ & $\frac{1}{8}$ \\
\hline 08:00 AM & - & $09: 00 \mathrm{AM}$ & 0 & 0 & 0 & 0 & 0 & 0 & 0 & 1 & 0 & 0 & 0 & & 1 \\
\hline 08:15AM & $\cdots$ & $09: 15$ AM & $-\frac{0}{0}$ & 0 & 0 & $\frac{0}{0}$ & 0 & $\frac{0}{0}$ & 1 & 4 & 0 & 0 & $y$ & $\because$ & 5 \\
\hline 08:30 AM & - & $09: 30 \mathrm{AM}$ & 0 & 0 & 0 & 0 & 0 & o & 1 & 5 & $\overline{0}$ & 0 & 5 & $\therefore$ & 6 \\
\hline 08:45 AM & $\ldots$ & $09: 45 \mathrm{AM}$ & & $\begin{array}{l}0 \\
0\end{array}$ & $\begin{array}{l}0 \\
0\end{array}$ & 0 & 0 & 0 & 2 & $\begin{array}{l}5 \\
5\end{array}$ & $\begin{array}{l}0 \\
0\end{array}$ & $\begin{array}{l}0 \\
0\end{array}$ & $\begin{array}{l}0 \\
0\end{array}$ & "1 & $\dot{8}$ \\
\hline $09: 00 \mathrm{AM}$ & $\cdots$ & $10: 00 \mathrm{AM}$ & $\frac{0}{0}$ & 0 & $\frac{0}{0}$ & $\frac{0}{0}$ & 0 & 0 & 2 & $\frac{5}{3}$ & $\frac{0}{0}$ & $\frac{0}{0}$ & $\frac{D}{0}$ & 3 & 8 \\
\hline $\begin{array}{l}09: 15 \mathrm{AM} \\
09: 30 \mathrm{AM}\end{array}$ & $=$ & $\begin{array}{l}10: 15 \overline{A M} \\
10: 30 \mathrm{AM}\end{array}$ & 0 & 0 & 0 & 0 & $\begin{array}{l}0 \\
0\end{array}$ & $\begin{array}{l}0 \\
0\end{array}$ & $\frac{1}{1}$ & 2 & 0 & $\begin{array}{l}0 \\
0\end{array}$ & $\begin{array}{l}0 \\
0\end{array}$ & $i$ & 3 \\
\hline 09:45 AM & $\ldots$ & 10:45 AM & 0 & 0 & 0 & 0 & 0 & 0 & $i$ & 2 & 0 & 0 & $=$ & $i$ & 6 \\
\hline $10: 00 \mathrm{AN}$ & $=$ & $11: 00 \mathrm{AM}$ & 0 & 0 & 0 & 0 & 0 & 0 & 2 & 3 & 0 & 0 & $=$ & $\because$ & $?$ \\
\hline $\begin{array}{l}10: 15 \mathrm{AM} \\
10: 30 \mathrm{AM}\end{array}$ & $\bar{z}$ & $\begin{array}{l}11: 15 \mathrm{AM} \\
11: 30 \mathrm{AM}\end{array}$ & $\begin{array}{l}0 \\
0\end{array}$ & $\begin{array}{l}0 \\
0\end{array}$ & $\begin{array}{l}0 \\
0\end{array}$ & $\begin{array}{l}0 \\
0\end{array}$ & $\begin{array}{l}0 \\
0\end{array}$ & $\begin{array}{l}0 \\
1\end{array}$ & $\frac{2}{2}$ & $\begin{array}{l}3 \\
2\end{array}$ & $\begin{array}{l}0 \\
0\end{array}$ & $\begin{array}{l}0 \\
0\end{array}$ & 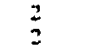 & $\dddot{~}$ & $\dot{s}$ \\
\hline $10: 45 \mathrm{AM}$ & $\cdots$ & $\frac{1.5 \mathrm{AM}}{11.45 \mathrm{AM}}$ & 0 & 0 & 0 & 0 & 0 & 2 & 1 & 2 & 0 & 0 & $\check{0}$ & $i$ & $\frac{6}{6}$ \\
\hline $11: 00 \mathrm{AM}$ & $\ldots$ & $12: 00 \mathrm{PM}$ & 0 & 0 & 0 & 0 & 0 & 2 & 0 & $i$ & 0 & 0 & 0 & $\vdots$ & 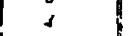 \\
\hline $11: 15 \mathrm{AM}$ & $\ldots$ & 12:15 PM & 0 & 0 & 0 & 1 & 0 & 2 & 0 & 0 & 0 & 0 & $=$ & $!$ & 6 \\
\hline 1130 AM & $-\infty$ & $12.30 \mathrm{PM}$ & 0 & 0 & 0 & 1 & 0 & 2 & 0 & 0 & 0 & 0 & 2 & $i$ & 5 \\
\hline 11:45 AM & - & 12:45 PM & 0 & $:$ & 0 & 1 & 0 & 1 & 0 & 0 & $\stackrel{0}{0}$ & 0 & 3 & 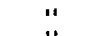 & s \\
\hline 12:00 PM & $=$ & & 0 & $\stackrel{0}{0}$ & 0 & 1 & 0 & $\frac{2}{3}$ & 0 & $\underline{0}$ & 0 & 0 & 3 & $\because$ & $\therefore$ \\
\hline $12: 15 \mathrm{PM}$ & $=$ & OLIIS PM & 0 & 0 & 0 & 0 & 0 & 3 & 0 & 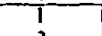 & 0 & 0 & I & 7 & 5 \\
\hline $\begin{array}{l}\text { 12:30 PM } \\
\text { 10.4PMM }\end{array}$ & $=$ & $\begin{array}{l}01: 30 \text { PM } \\
00.45 \text { PM }\end{array}$ & 0 & 0 & $0_{0}^{0}$ & $0_{0}^{0}$ & $\stackrel{0}{0}$ & $\frac{2}{2}$ & 0 & 3 & 0 & $a_{0}^{0}$ & 2 & 8 & ; \\
\hline 12:45 PM & $=$ & Ol: & $\frac{0}{0}$ & $-\frac{0}{0}$ & $\frac{0}{0}$ & $\frac{0}{1}$ & $\frac{0}{0}$ & $\frac{2}{2}$ & $\frac{0}{1}$ & $\frac{3}{3}$ & $\frac{0}{0}$ & $\frac{0}{0}$ & $\frac{2}{2}$ & $\frac{0}{8}$ & $\frac{3}{9}$ \\
\hline $\begin{array}{l}\text { 01:00 PM } \\
\text { o1:15 PM }\end{array}$ & $\bar{Z}$ & $\begin{array}{l}02: 00 \mathrm{PM} \\
02: 15 \mathrm{PM}\end{array}$ & 0 & 0 & 0 & $i$ & 0 & i & 1 & 3 & 0 & 0 & $\frac{2}{2}$ & 0 & 8 \\
\hline $01: 30 \mathrm{PM}$ & - & $02: 30 \mathrm{PM}$ & 0 & 0 & 0 & 1 & 0 & $i$ & 2 & 2 & 0 & 0 & $i$ & 0 & ; \\
\hline $01: 45 \mathrm{PM}$ & $\cdots$ & $02.45 \mathrm{PM}$ & 0 & 0 & 0 & $T$ & 0 & $T$ & 2 & 2 & 0 & 0 & 1 & 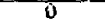 & 7 \\
\hline 02:00 PM & - & 03:00 PM & 0 & 0 & 0 & 0 & 0 & 0 & 1 & 3 & 0 & 0 & 2 & 0 & 6 \\
\hline 02:15 PM & - & $03: 15 \mathrm{PM}$ & 0 & 0 & 0 & 0 & 0 & 0 & $i$ & 2 & 0 & 0 & 3 & 0 & 6 \\
\hline $02: 30 \mathrm{PM}$ & $=$ & $03: 30 \mathrm{PM}$ & 0 & 0 & 0 & 0 & 0 & 0 & 1 & 1 & 0 & 0 & 3 & a & 5 \\
\hline 02:45 PM & - & $03: 45 P M$ & 0 & 0 & 0 & 1 & 0 & 0 & 1 & 1 & 0 & 0 & 2 & 0 & 5 \\
\hline $03: 00 \mathrm{PM}$ & $\ldots$ & 04:00 PM & 0 & 0 & 0 & $\mathrm{i}$ & 0 & 0 & $i$ & 1 & 0 & 0 & $i$ & i) & 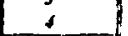 \\
\hline $03: 15$ PM & $=$ & $04: 158 M$ & 0 & 0 & 0 & I & 0 & 0 & $\mathrm{~T}$ & 8 & $\tilde{0}$ & 0 & 0 & 0 & 3 \\
\hline 03:30 PM & $\cdots$ & $04: 30 \mathrm{PM}$ & 0 & 0 & 0 & j & 0 & 0 & 0 & i & & 0 & 0 & 0 & 3 \\
\hline 03:45 PM & $\ldots$ & 04:45 PM & 0 & 0 & 0 & 0 & 0 & 0 & 0 & 1 & 0 & 0 & 1 & 0 & 2 \\
\hline 04:00 PM & $=$ & $05.00 \mathrm{PM}$ & 0 & 0 & 0 & 0 & 0 & 0 & 0 & & 0 & 0 & & 0 & 8 \\
\hline$s$ & $E N I$ & ULA: & 75 & & & $A L$ & D.A: & o) 2 & 29 & & $O \Lambda$ & c & 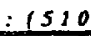 & $2=$ & \\
\hline
\end{tabular}




\section{Baymetrics Traffic Resources INTERSECTION TURNING MOVEMENT SUMMARY}

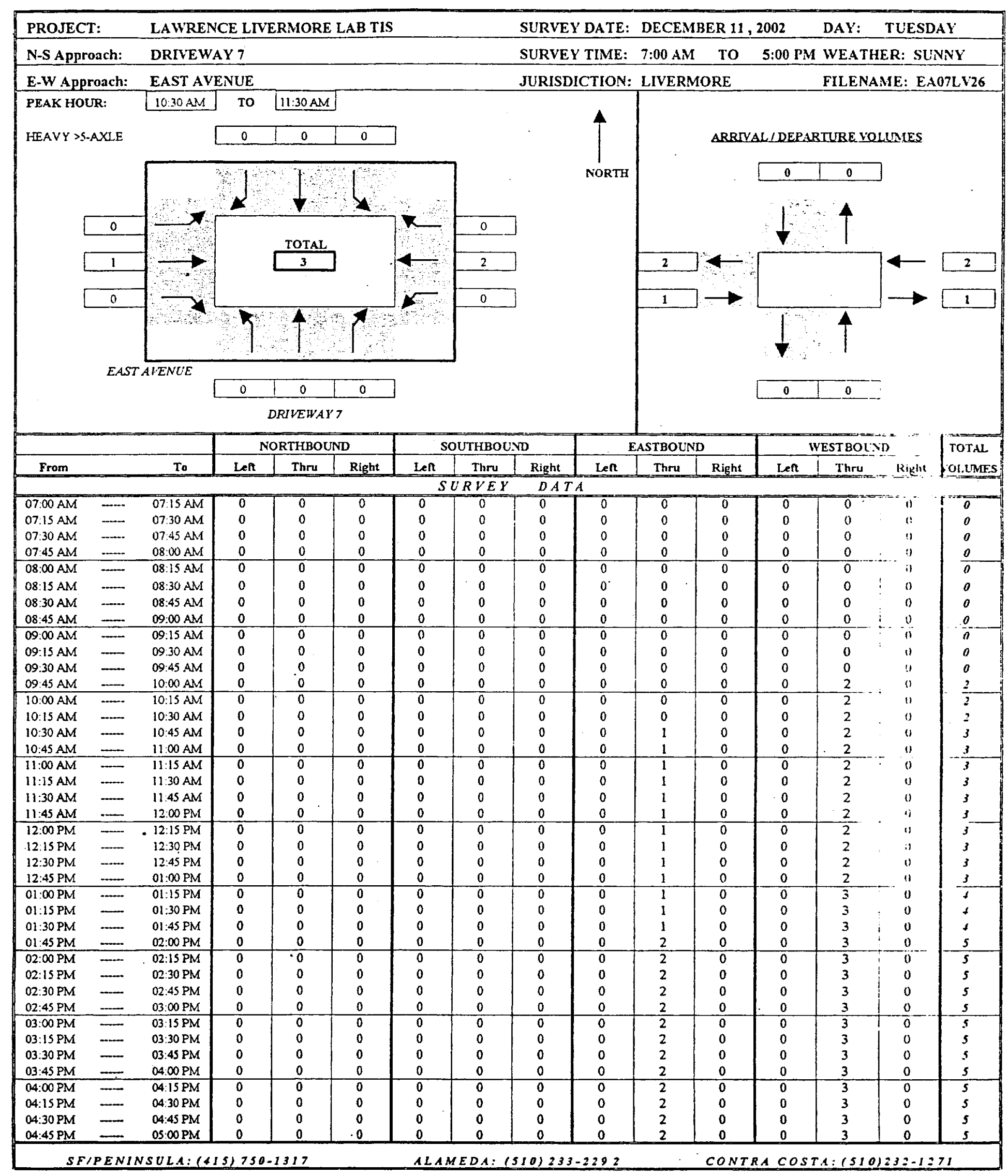




\section{Baymetrics Traffic Resources INTERSECTION TURNING MOVEMENT SUMMARY}

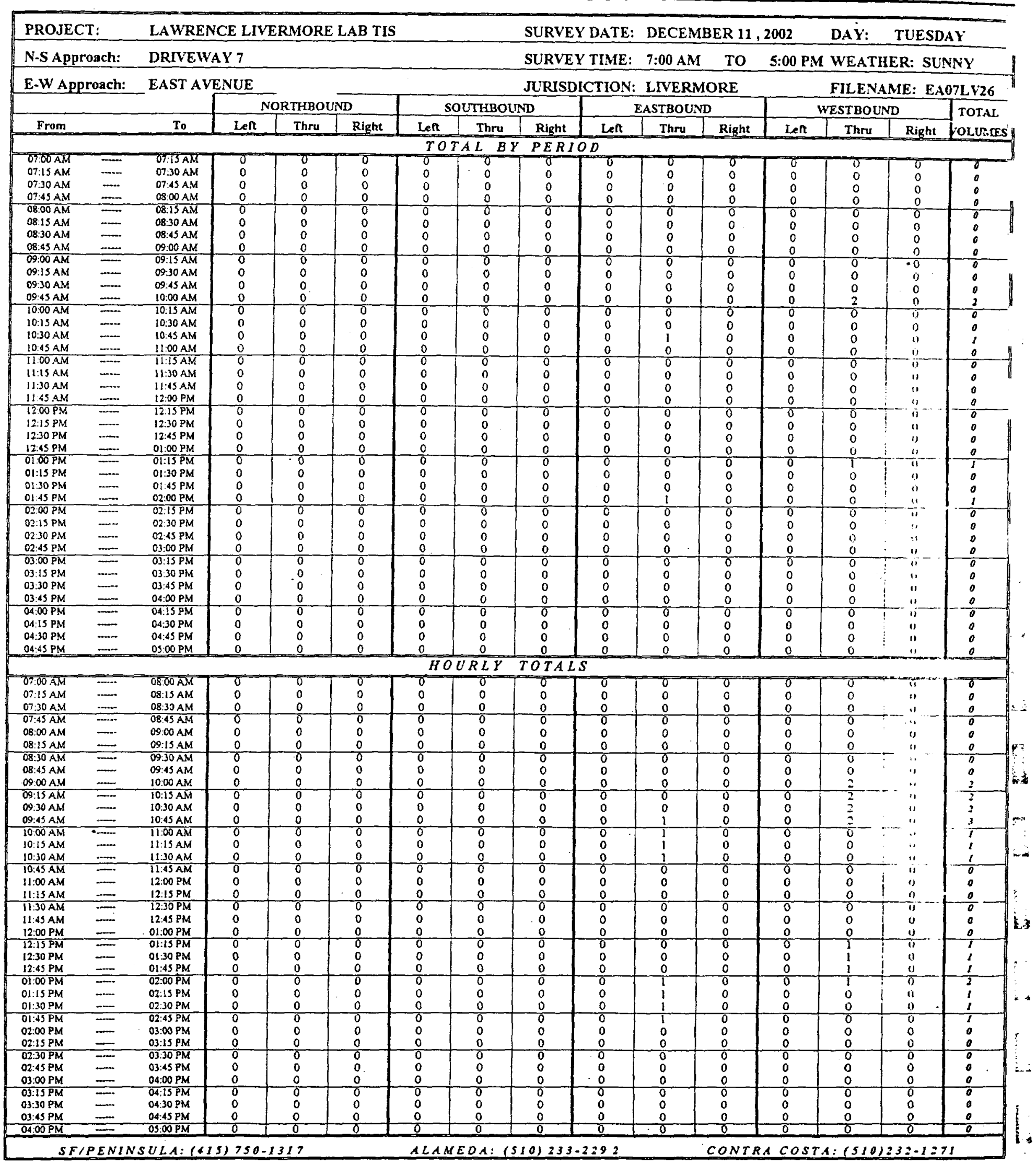




\section{Baymetrics Traffic Resources INTERSECTION TURNING MOVEMENT SUMMARY}

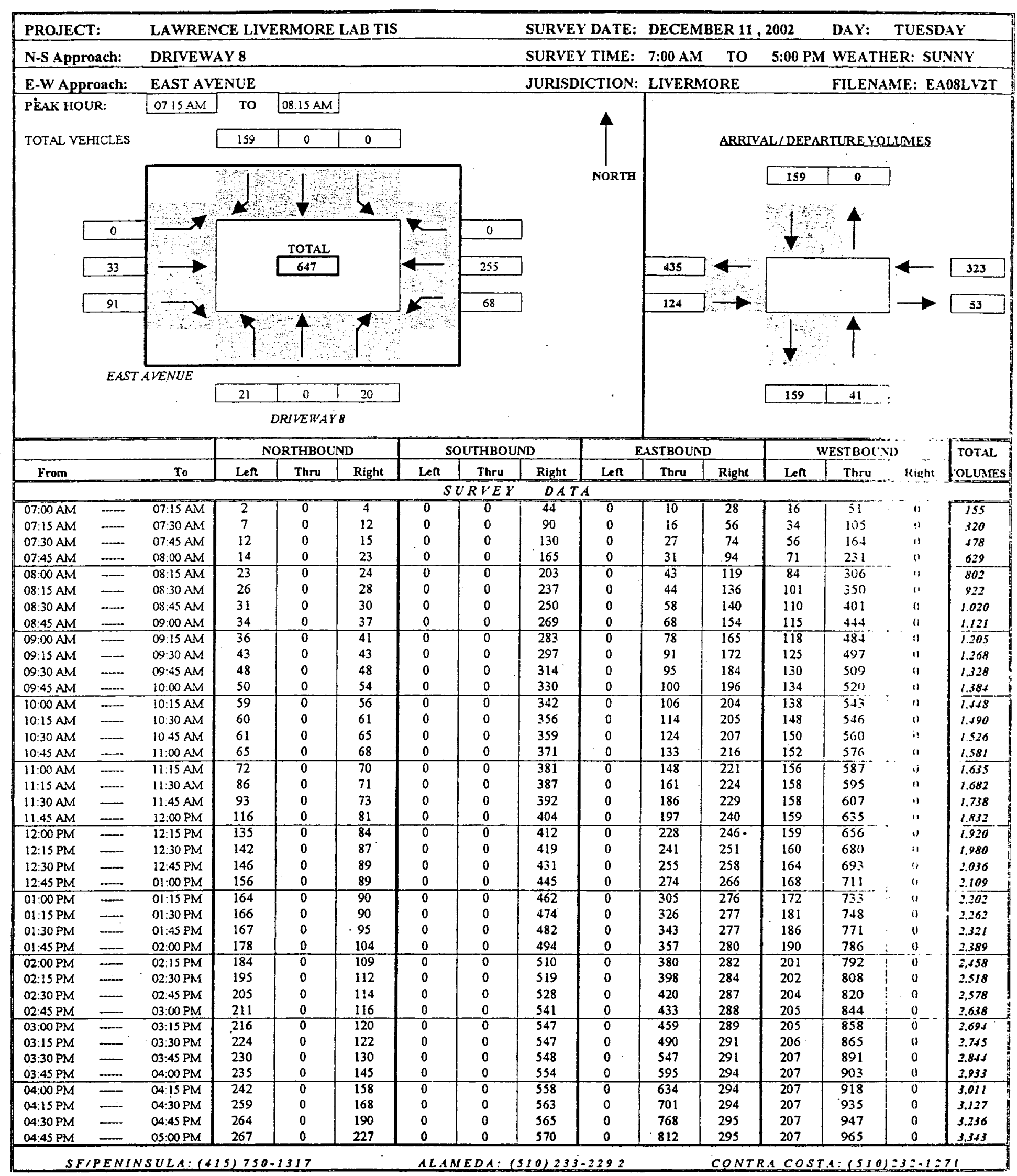




\section{Baymetrics Traffic Resources INTERSECTION TURNING MOVEMENT SUMMARY}

\begin{tabular}{|c|c|c|c|c|c|c|c|c|c|c|c|c|c|c|c|}
\hline \multirow{2}{*}{\multicolumn{2}{|c|}{$\begin{array}{l}\text { PROJECT: } \\
\text { N-S Approach: }\end{array}$}} & \multicolumn{6}{|c|}{ LAWRENCE LIVERMORE LAB TIS } & \multicolumn{5}{|c|}{ SURVEY DATE: DECEMBER 11,2002} & DAY: & \multicolumn{2}{|c|}{ TUESDAY } \\
\hline & & DRIVEY & & & & & & SURVE & IME: & $7: 00 \mathrm{AM}$ & To & 5:00 P & WEAT & ER: St: & $\mathrm{iNY}$ \\
\hline E-W Ap & oach: & EAST A & UE & & & & & JURISD & TION: & LIVER & RE & & FILEN & ME: EA & $18 \mathrm{LV} 2 \mathrm{~T}$ \\
\hline & & & & THBO & & & THBO & & & ASTBOU: & & & ESTBOU & & TOTAL \\
\hline From & & To & Left & Thru & Right & Lent & Thru & Right & Left & Thru & Right & Lent & Thru & Right & LoLInES \\
\hline & & & & & & & $L B$ & $P E R I$ & & & & & & & \\
\hline $\begin{array}{l}\text { 07:00 AM } \\
\text { 07:15 AM }\end{array}$ & $\ldots$ & $\begin{array}{l}0715 \mathrm{AM} \\
07: 30 \mathrm{AM}\end{array}$ & 5 & 0 & $\begin{array}{l}4 \\
8\end{array}$ & $\begin{array}{l}0 \\
0\end{array}$ & 0 & $\begin{array}{l}44 \\
46\end{array}$ & 0 & $\begin{array}{c}10 \\
6\end{array}$ & $\begin{array}{l}\frac{23}{28} \\
28\end{array}$ & $\begin{array}{l}16 \\
18\end{array}$ & $\begin{array}{l}51 \\
54\end{array}$ & 0 & $\begin{array}{l}755 \\
165\end{array}$ \\
\hline $\begin{array}{l}07: 30 \mathrm{AM} \\
07.43 \mathrm{AM}\end{array}$ & $\ldots$ & $\begin{array}{l}07: 45 \mathrm{AM} \\
08: 00 \mathrm{AM}\end{array}$ & $\begin{array}{l}5 \\
2\end{array}$ & 0 & $\begin{array}{l}0 \\
3 \\
8\end{array}$ & 0 & 0 & $\begin{array}{l}40 \\
35\end{array}$ & $\begin{array}{l}0 \\
0\end{array}$ & $\underset{4}{11}$ & $\begin{array}{l}18 \\
20\end{array}$ & $\begin{array}{l}22 \\
15\end{array}$ & $\begin{array}{l}59 \\
67\end{array}$ & $\begin{array}{l}0 \\
0\end{array}$ & $\begin{array}{l}\text { iss } \\
15 t\end{array}$ \\
\hline $08.00 \mathrm{AM}$ & $\ldots$ & $08.15 A N$ & 9 & 0 & $i$ & $\frac{0}{0}$ & 0 & -38 & 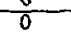 & 12 & 25 & 13 & 75 & 0 & $\frac{281}{133}$ \\
\hline 08:15 AM & $\cdots$ & 08:30 AM & 3 & 0 & 4 & 0 & 0 & 34 & 0 & 1 & 17 & 17 & 44 & i & 120 \\
\hline $\begin{array}{l}08: 30 \text { AM } \\
08: 45 \text { AM }\end{array}$ & $\ldots$ & $\begin{array}{l}08: 45 \mathrm{AM} \\
09: 00 \mathrm{AM}\end{array}$ & $\begin{array}{l}5 \\
3\end{array}$ & $\begin{array}{l}0 \\
0\end{array}$ & $\frac{2}{7}$ & 0 & 0 & 13 & 0 & 14 & 4 & 9 & 51 & i) & 98 \\
\hline $09: 00 \mathrm{AM}$ & $=$ & $09: 15 \mathrm{AM}$ & $-\frac{3}{2}$ & $\frac{0}{0}$ & $\frac{1}{4}$ & $\frac{0}{0}$ & $\frac{0}{0}$ & $\frac{19}{14}$ & $\frac{0}{0}$ & $\frac{10}{10}$ & $\frac{14}{11}$ & $\frac{5}{3}$ & $\frac{43}{40}$ & $\frac{1}{0}$ & $\frac{101}{81}$ \\
\hline 09:15 AM & $\ldots$ & - $09: 30 \mathrm{AM}$ & 7 & 0 & 2. & 0 & 0 & 14 & 0 & 13 & 7 & 7 & 13 & $\because$ & 63 \\
\hline 09:30 AM & $\cdots$ & $09: 45 \mathrm{AM}$ & 5 & 0 & 5 & 0 & 0 & 17 & 0 & 4 & 12 & 5 & 12 & $i$ & 60 \\
\hline $09: 45 \mathrm{AM}$ & $\ldots$ & $10.00 \mathrm{AM}$ & 2 & 0 & 6 & 0 & 0 & 16 & 0 & 5 & 12 & 4 & 11 & 1 & 56 \\
\hline $10: 00 \mathrm{AM}$ & $\infty$ & 10:15 AM & 9 & 0 & 2 & 0 & 0 & 12 & 0 & 6 & 8 & 4 & 23 & $\pi$ & os \\
\hline $\begin{array}{l}10: 15 \mathrm{AM} \\
10: 30 \mathrm{AM}\end{array}$ & $=$ & $\begin{array}{l}10: 30 \mathrm{AM} \\
10: 45 \mathrm{AM}\end{array}$ & 1 & $\begin{array}{l}0 \\
0\end{array}$ & 5 & $\begin{array}{l}0 \\
0\end{array}$ & $\begin{array}{l}0 \\
0\end{array}$ & $\begin{array}{c}14 \\
3\end{array}$ & $\begin{array}{l}0 \\
0\end{array}$ & $\begin{array}{l}8 \\
10\end{array}$ & $\begin{array}{l}1 \\
2\end{array}$ & $\begin{array}{c}10 \\
2\end{array}$ & 14 & :: & 32 \\
\hline 10:45 AM & $\ldots$ & $11: 00 \mathrm{AM}$ & 4 & 0 & 3 & 0 & 0 & 12 & 0 & 9 & 9 & $\frac{2}{2}$ & $\begin{array}{l}17 \\
16\end{array}$ & $\because$ & s5 \\
\hline HIOCAM & $=$ & 11:15 AM & 7 & 0 & 2 & 0 & 0 & 10 & 0 & 15 & 5 & 4 & 11 & $\therefore$ & 54 \\
\hline 11:15 AM & $\cdots$ & $11: 30 \mathrm{AM}$ & 14 & 0 & 1 & 0 & 0 & 6 & 0 & 13 & 3 & 2 & 8 & i: & 4 \\
\hline 11:30 AM & $\cdots$ & $11: 45 \mathrm{AM}$ & 7 & 0 & 2 & 0 & 0 & 5 & 0 & 25 & 5 & 0 & 12 & $\therefore$ & 16 \\
\hline $11: 45 \mathrm{AM}$ & ...... & $12: 00 \mathrm{PM}$ & 23 & 0 & 8 & 0 & 0 & 12 & 0 & 11 & 11 & 1 & $2 \pi$ & $\therefore$ & 94 \\
\hline $12.00 \mathrm{PM}$ & $\cdots$ & $12: 15 \mathrm{PM}$ & 19 & 0 & 3 & 0 & 0 & 8 & 0 & 31 & 6 & $\pi$ & 31 & $" 1$ & 88 \\
\hline $\begin{array}{l}12: 15 \mathrm{PM} \\
12.30 \mathrm{PM}\end{array}$ & $\cdots$ & $\begin{array}{l}12: 30 \mathrm{PM} \\
12.45 \mathrm{PM}\end{array}$ & 7 & $\begin{array}{l}0 \\
0\end{array}$ & $\frac{3}{2}$ & $0_{0}^{0}$ & ? & 7 & 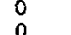 & 13 & $\frac{5}{7}$ & 1 & $=1$ & $\because$ & 60 \\
\hline $\begin{array}{l}12: 30 \mathrm{PM} \\
12: 45 \mathrm{PM}\end{array}$ & $=$ & $\begin{array}{l}2: 25) \mathrm{PM} \\
0100 \mathrm{PM}\end{array}$ & 40 & 0 & 0 & 0 & 0 & 14 & $\begin{array}{l}0 \\
0\end{array}$ & $\begin{array}{l}14 \\
19\end{array}$ & 8 & 4 & $\begin{array}{l}13 \\
18\end{array}$ & 14 & $\begin{array}{l}56 \\
-3\end{array}$ \\
\hline $01: 00 \mathrm{PM}$ & $=$ & $01: 15 \mathrm{PM}$ & 8 & 0 & 1 & 0 & 0 & 17 & 0 & 31 & 10 & 4 & $2=$ & $\because \cdots$ & 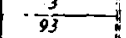 \\
\hline 01:15 PM & $\rightarrow$ & $01: 30 \mathrm{PM}$ & 2 & 0 & 0 & 0 & 0 & 12 & 0 & 21 & 1 & 9 & 15 & $\because$ & oo \\
\hline $01 \cdot 30 \mathrm{PM}$ & $\cdots$ & $01: 45 \mathrm{PM}$ & 1 & $\begin{array}{l}0 \\
0 \\
0\end{array}$ & 5 & $\begin{array}{l}0 \\
0 \\
0\end{array}$ & 8 & 8 & 8 & 17 & 0 & 5 & 23 & $\ddot{0}$ & 59 \\
\hline$\frac{01.45 \mathrm{PM}}{02: 00 \mathrm{PM}}$ & $\ldots$ & $\begin{array}{l}02: 00 \mathrm{PM} \\
02: 15 \mathrm{PM}\end{array}$ & $\frac{\pi}{6}$ & 0 & $\frac{9}{5}$ & 0 & $\frac{0}{0}$ & $\frac{12}{16}$ & -0 & $\frac{14}{23}$ & 3 & 4 & 15 & " & 68 \\
\hline $02: 15$ PM & $\ldots$ & $02: 30 \mathrm{PM}$ & 11 & 0 & 3 & 0 & 0 & $\begin{array}{c}16 \\
9\end{array}$ & 0 & $\begin{array}{l}23 \\
18\end{array}$ & $\begin{array}{l}2 \\
2\end{array}$ & $\pi$ & $\begin{array}{l}6 \\
16\end{array}$ & $\because$ & 69 \\
\hline 02:30 PM & $\ldots$ & 02:45 PM & 10 & 0 & 2 & 0 & 0 & 9 & 0 & 22 & 3 & 2 & 12 & $\therefore$ & $\begin{array}{l}60 \\
60\end{array}$ \\
\hline $0 \leq: 45 \mathrm{PM}$ & $\ldots$ & $03: 00 \mathrm{PM}$ & 6 & 0 & 2 & 0 & 0 & 13 & 0 & 13 & $i$ & 1 & 24 & ' & 60 \\
\hline $03: 00 \mathrm{PM}$ & $\cdots$ & $03: 15 \mathrm{PM}$ & 5 & 0 & 4 & 0 & 0 & 6 & 0 & 26 & $\mathrm{~T}$ & 0 & 14 & .. & 56 \\
\hline 03:15 PM & $\ldots$ & $03: 30 \mathrm{PM}$ & 8 & 0 & 2 & 0 & 0 & 0 & 0 & 31 & 2 & 1 & 7 & .. & 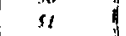 \\
\hline $03: 30 \mathrm{PM}$ & $\ldots$ & $03: 45$ PM & 6 & 0 & 8 & 0 & 0 & 1 & $0^{\circ}$ & 57 & $\overline{0}$ & $\mathrm{i}$ & 26 & . & $2 y$ \\
\hline 03:45 PM & $\ldots$ & 04:00 PM & 5 & 0 & 15 & 0 & 0 & 6 & 0 & 48 & 3 & 0 & 10 &. & 88 \\
\hline $04: 00 \mathrm{PM}$ & $=$ & $04: 15$ PM & $\frac{7}{7}$ & 0 & 13 & 0 & 0 & $\frac{5}{4}$ & 0 & 39 & 0 & 0 & 15 & $\therefore$ & 8 \\
\hline $04: 15 \mathrm{PM}$ & $\cdots$ & 04:30 PM & 17 & 0 & 10 & 0 & 0 & 5 & 0 & 67 & 0 & 0 & 17 & " & $1 i 6$ \\
\hline $04: 30 \mathrm{PM}$ & $\cdots$ & 04:45 PM & 5 & 0 & 22 & 0 & 0 & 2 & 0 & 67 & $i$ & 0 & $1=$ & $\because$ & 109 \\
\hline 06:45 PM & $\ldots$ & $0900 \mathrm{PM}$ & 3 & 0 & 37 & 0 & $\underline{0}$ & 5 & 0 & 44 & 0 & 0 & 18 & ". & $m=$ \\
\hline & & & & & & & $R L \bar{Y}$ & $T O T A L$ & & & & & & & \\
\hline O7:00AM & -1 & 68:00 AM & 78 & 0 & 23 & 0 & $\sigma$ & 765 & 0 & उ1 & 94 & $\pi$ & 331 & $\therefore$ & Sक \\
\hline $07: 15 \mathrm{AM}$ & $\ldots$ & DB:IS AM & $2 \mathrm{I}$ & 0 & 20 & 0 & 0 & 159 & 0 & 33 & 91 & 68 & 255 & $\therefore$ & $x^{\circ}$ \\
\hline $07: 30 \mathrm{AM}$ & $\ldots$ & $08: 30 \mathrm{AM}$ & 19 & 0 & 16 & 0 & 0 & 147 & 0 & 28 & 80 & 67 & 245 & ". & $B D Z$ \\
\hline $07: 45 . \mathrm{AM}$ & $\cdots$ & $08: 45 \overline{A M}$ & 19 & 0 & 15 & 0 & 0 & 120 & 0 & 31 & 66 & 54 & 33 & & $\sqrt{2}$ \\
\hline 08:00 AM & $\cdots$ & $09: 00 \mathrm{AM}$ & 20 & 0 & 14 & 0 & 0 & 104 & 0 & 37 & 60 & 44 & 21.3 & & 102 \\
\hline 08:15 AM & - & $09: 15 \mathrm{AM}$ & 13 & 0 & 17 & 0 & 0 & 80 & 0 & 35 & 46 & 34 & 178 & $\therefore$ & in: \\
\hline $08: 30 \mathrm{AM}$ & $\omega$ & $09: 30 \mathrm{AM}$ & 17 & 0 & 15 & 0 & 0 & 60 & 0 & 47 & 36 & 24 & $11 \%$ & . & 180 \\
\hline $08: 45 \mathrm{AM}$ & $\cdots$ & $09: 45 \mathrm{AM}$ & 17 & 0 & 18 & 0 & 0 & 6.4 & 0 & 37 & 44 & 20 & 108 &.. & 308 \\
\hline $09: 00 \mathrm{AM}$ & $=$ & $10: 00 \mathrm{AM}$ & 16 & 0 & 17 & 0 & 0 & 61 & 0 & 32 & 42 & 19 & 76 & & 26.1 \\
\hline $09: 15 \mathrm{AM}$ & - & $10: 15 \mathrm{AM}$ & 23 & 0 & 15 & $\overline{0}$ & $\delta$ & $\$ 9$ & 0 & 28 & 39 & 20 & इ9 & . & 2J \\
\hline $09: 30 \mathrm{AM}$ & $\ldots$ & $10: 30 \mathrm{AM}$ & 17 & 0 & 18 & 0 . & 0 & 59 & 0 & 23 & 33 & 23 & 49 & $\cdot$ & $\because 2$ \\
\hline O9:45 AM & $\ldots$ & $10: 45 \mathrm{AM}$ & 13 & 0 & $\begin{array}{l}10 \\
17\end{array}$ & 0 & 0 & 45 & 0 & 29 & 23 & 20 & s) & , & $39 \pi$ \\
\hline $10: 00 \mathrm{AM}$ & - & $11: 00 \mathrm{AM}$ & 15 & 0 & 14 & 0 & 0 & .41 & 0 & 33 & 20 & 18 & 56 & & $190^{\circ}$ \\
\hline $10: 15 \mathrm{AM}$ & $\ldots$ & $11: 15 \mathrm{AM}$ & 13 & 0 & 14 & 0 & 0 & 39 & 0 & $\sqrt{2}$ & 17 & 18 & 4.1 & $\therefore$ & $18^{-}$ \\
\hline $10: 30 \mathrm{AM}$ & $\ldots$ & 11:30 AM & 26 & 0 & 10 & 0 & 0 & 31 & 0 & 47 & 19 & 10 & 49 & .. & $19 ?$ \\
\hline $10: 45 \mathrm{AM}$ & $\cdots$ & 11.45 AM & 32 & 0 & 8 & 0 & 0 & 33 & 0 & 62 & 22 & 8 & 47 &. & 212 \\
\hline $11: 00 \mathrm{AM}$ & $\cdots$ & $12: 00 \mathrm{PM}$ & 51 & 0 & 13 & 0 & 0 & 33 & 0 & 64 & 24 & 7 & 59 & $\because$ & 258 \\
\hline IIIISAM & $\therefore$ & 12:13 PM & 63 & 0 & 14 & 0 & 0 & 31 & 0 & 80 & 25 & 3 & 69 & $\therefore$ & $2 n s$ \\
\hline $11: 30 \mathrm{AM}$ & $\ldots$ & $12: 30 \mathrm{PM}$ & 56 & 0 & 16 & 0 & 0 & 32 & 0 & 80 & 27 & 2 & 85 & " & .98 \\
\hline $11: 45 \mathrm{AM}$ & - & $\begin{array}{l}\text { 12:45 PM } \\
01.00 \mathrm{PM}\end{array}$ & 53 & $\begin{array}{l}0 \\
0\end{array}$ & $\begin{array}{l}16 \\
8\end{array}$ & $\begin{array}{l}0 \\
0\end{array}$ & $\begin{array}{l}0 \\
0\end{array}$ & $\begin{array}{l}39 \\
41\end{array}$ & 0 & $\begin{array}{l}69 \\
77\end{array}$ & $\begin{array}{l}29 \\
26\end{array}$ & $\begin{array}{l}6 \\
9\end{array}$ & $\begin{array}{l}86 \\
76\end{array}$ & "y & 98 \\
\hline$\frac{12: 00 \mathrm{PM}}{12: 15 \mathrm{PM}}$ & $=$ & $\frac{01: 00 \mathrm{PM}}{01: 15 \mathrm{PM}}$ & $\frac{40}{29}$ & $\frac{5}{0}$ & $\frac{8}{6}$ & $\frac{0}{0}$ & $\frac{0}{0}$ & $\frac{41}{50}$ & $\frac{0}{0}$ & $\frac{11}{7 \gamma}$ & -30 & $\frac{9}{13}$ & 77 & “i & 2 \\
\hline $12: 30 \mathrm{PM}$ & - & ol:30 PM & 24 & .0 & 3 & 0 & 0 & 55 & 0 & 85 & 26 & 21 & 68 & $" 1$ & 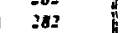 \\
\hline $12: 45 \mathrm{PM}$ & $\cdots$ & $01.45 \mathrm{PM}$ & 21 & 0 & 6 & 0 & 0 & 51 & 0 & 88 & 19 & 22 & 78 & $"$ & 285 \\
\hline $01: 00 \mathrm{PM}$ & $\cdots$ & $02: 00 \mathrm{PM}$ & 22 & 0 & 15 & 0 & 0 & 49 & 0 & 83 & 74 & 22 & $\begin{array}{l}75 \\
50\end{array}$ & $1 i$ & 280 \\
\hline $\begin{array}{l}01: 15 \mathrm{PM} \\
01: 30 \mathrm{PM}\end{array}$ & $=$ & $\begin{array}{l}02: 15 \mathrm{PM} \\
02: 30 \text { PM }\end{array}$ & $\begin{array}{l}20 \\
29\end{array}$ & $\begin{array}{l}0 \\
0\end{array}$ & $\begin{array}{l}19 \\
22\end{array}$ & $\begin{array}{l}0 \\
0\end{array}$ & $\begin{array}{l}0 \\
0\end{array}$ & $\begin{array}{l}48 \\
45\end{array}$ & $\begin{array}{l}0 \\
0\end{array}$ & $\begin{array}{l}75 \\
72\end{array}$ & $\begin{array}{l}6 \\
7\end{array}$ & $\begin{array}{l}29 \\
21\end{array}$ & $\begin{array}{l}59 \\
60\end{array}$ & $"$ & $\begin{array}{l}256 \\
2.18\end{array}$ \\
\hline$\frac{01: 30 \mathrm{PM}}{01: 45 \mathrm{PM}}$ & $=$ & $\frac{02.3 \mathrm{PM}}{02: 45 \mathrm{PM}}$ & $-\frac{29}{38}$ & 0 & $\frac{22}{19}$ & $\frac{u}{0}$ & 0 & $\frac{42}{46}$ & 0 & $\frac{12}{77}$ & 10 & 18 & 49 & $1 i$ & $\frac{15}{25}$ \\
\hline 02:00 PM & $\cdots$ & $03: 00 \mathrm{PM}$ & 33 & 0 & 12 & 0 & 0 & 47 & 0 & 76 & 8 & $\begin{array}{l}10 \\
15\end{array}$ & 58 & 0 & 349 \\
\hline $02: 15$ PM & $\ldots$ & $03: 15 \mathrm{PM}$ & $\frac{32}{30}$ & 0 & 11 & 0 & 0 & 37 & 0 & 79 & $j$ & 4 & 66 & 0 & 236 \\
\hline $02: 30 \mathrm{PM}$ & $=$ & $03: 30 \mathrm{PM}$ & 29 & 0 & 10 & 0 & $\begin{array}{l}0 \\
0\end{array}$ & 28 & 0 & 92 & $?$ & 4 & 57 & 9 & 237 \\
\hline $\begin{array}{l}\text { 02:15 PM } \\
\text { 03:00 PM }\end{array}$ & $\cdots$ & $\begin{array}{l}03: 45 \mathrm{PM} \\
04: 00 \mathrm{PM}\end{array}$ & $\begin{array}{l}25 \\
24\end{array}$ & $\begin{array}{l}0 \\
0\end{array}$ & $\begin{array}{l}16 \\
29\end{array}$ & $\begin{array}{l}0 \\
0\end{array}$ & $\begin{array}{l}0 \\
0\end{array}$ & $\begin{array}{l}20 \\
13\end{array}$ & $\begin{array}{l}0 \\
0\end{array}$ & $\begin{array}{l}127 \\
162\end{array}$ & $\begin{array}{l}4 \\
6 \\
\end{array}$ & $\frac{3}{2}$ & $\begin{array}{l}11 \\
59\end{array}$ & $\begin{array}{l}0 \\
0\end{array}$ & $\begin{array}{l}266 \\
295\end{array}$ \\
\hline $03: 15$ PM & $=-\infty$ & $04: 15 \mathrm{PM}$ & $-\frac{26}{26}$ & 0 & 38 & 0 & 0 & 11 & 0 & 175 & $s$ & $\frac{\pi}{2}$ & 60 & 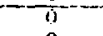 & $\pi$ \\
\hline 03:30 PM & $\cdots$ & $04: 30 \mathrm{PM}$ & 35 & 0 & 46 & 0 & 0 & 16 & $\therefore$ & 211 & 3 & 1 & 70 & 0 & 382 \\
\hline 03:45 PM & $\ldots$ & Q4:45 PM & -34 & 0 & 60 & $\frac{0}{0}$ & 0 & $\frac{17}{16}$ & -0 & $\frac{221}{312}$ & 4 & $\frac{0}{0}$ & $\frac{56}{62}$ & $\frac{0}{0}$ & 392 \\
\hline$\frac{04: 00 P M}{s}$ & $\overline{E N}$ & $\frac{05: 00 \mathrm{PM}}{U L A:(1}$ & 32 & 0 & & $A$ & $D A$ & a) & 92 & & 06 & $\underline{0}$ & 02 & 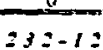 & \\
\hline
\end{tabular}




\section{Baymetrics Traffic Resources INTERSECTION TURNING MOVEMENT SUMMARY}

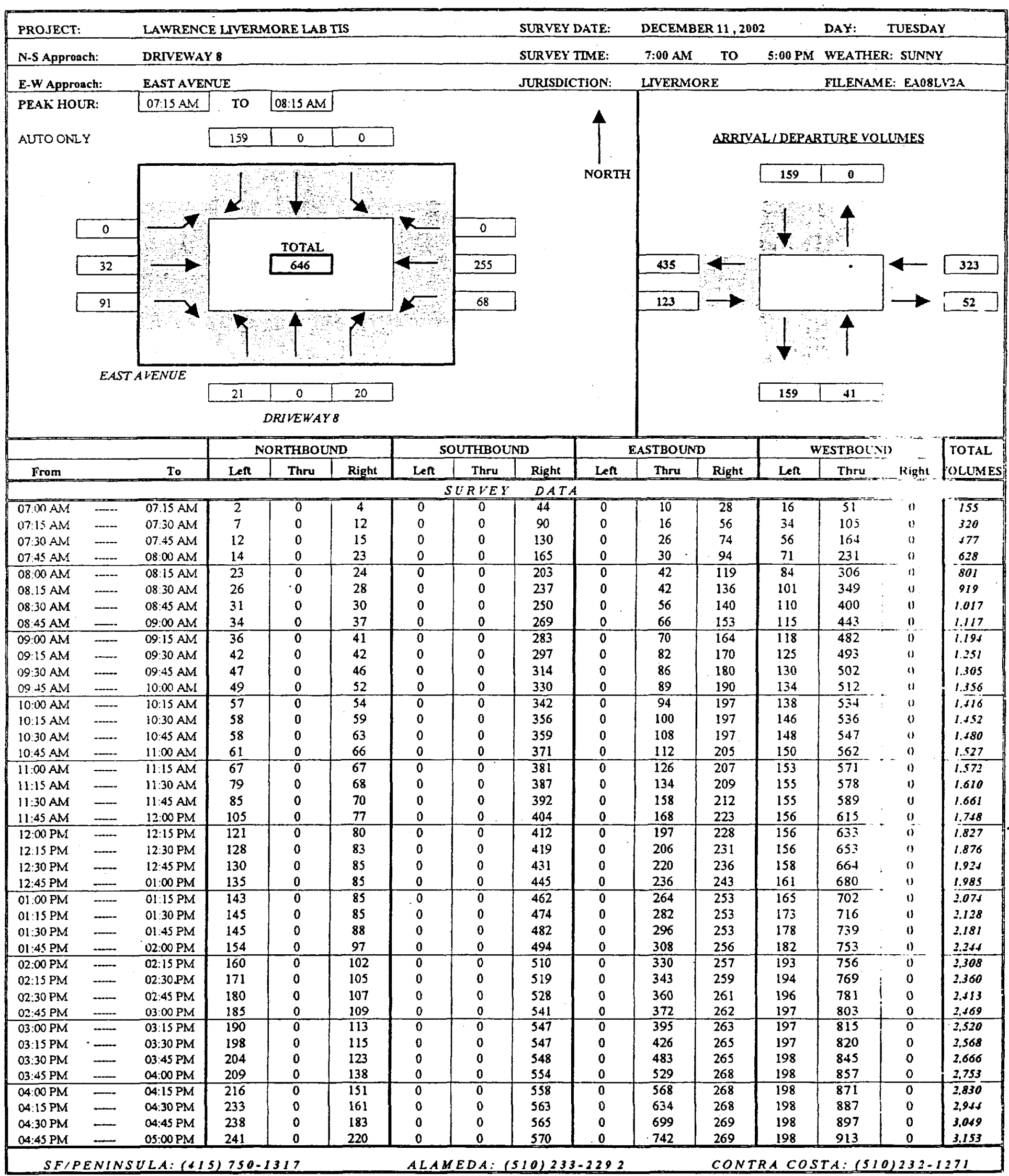




\section{Baymetrics Traffic Resources INTERSECTION TURNING MOVEMENT SUMMARY}

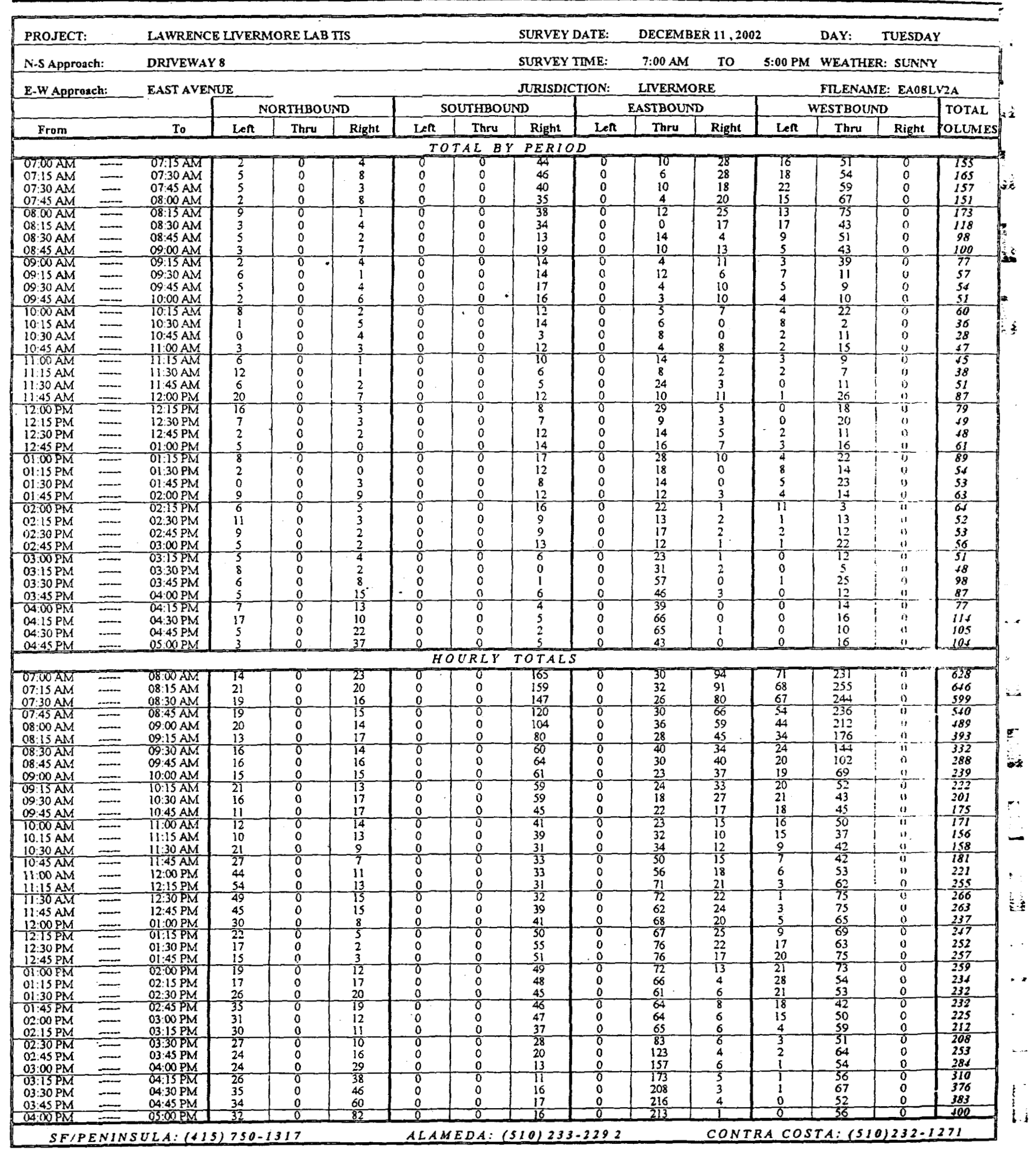




\section{Baymetrics Traffic Resources INTERSECTION TURNING MOVEMENT SUMMARY}

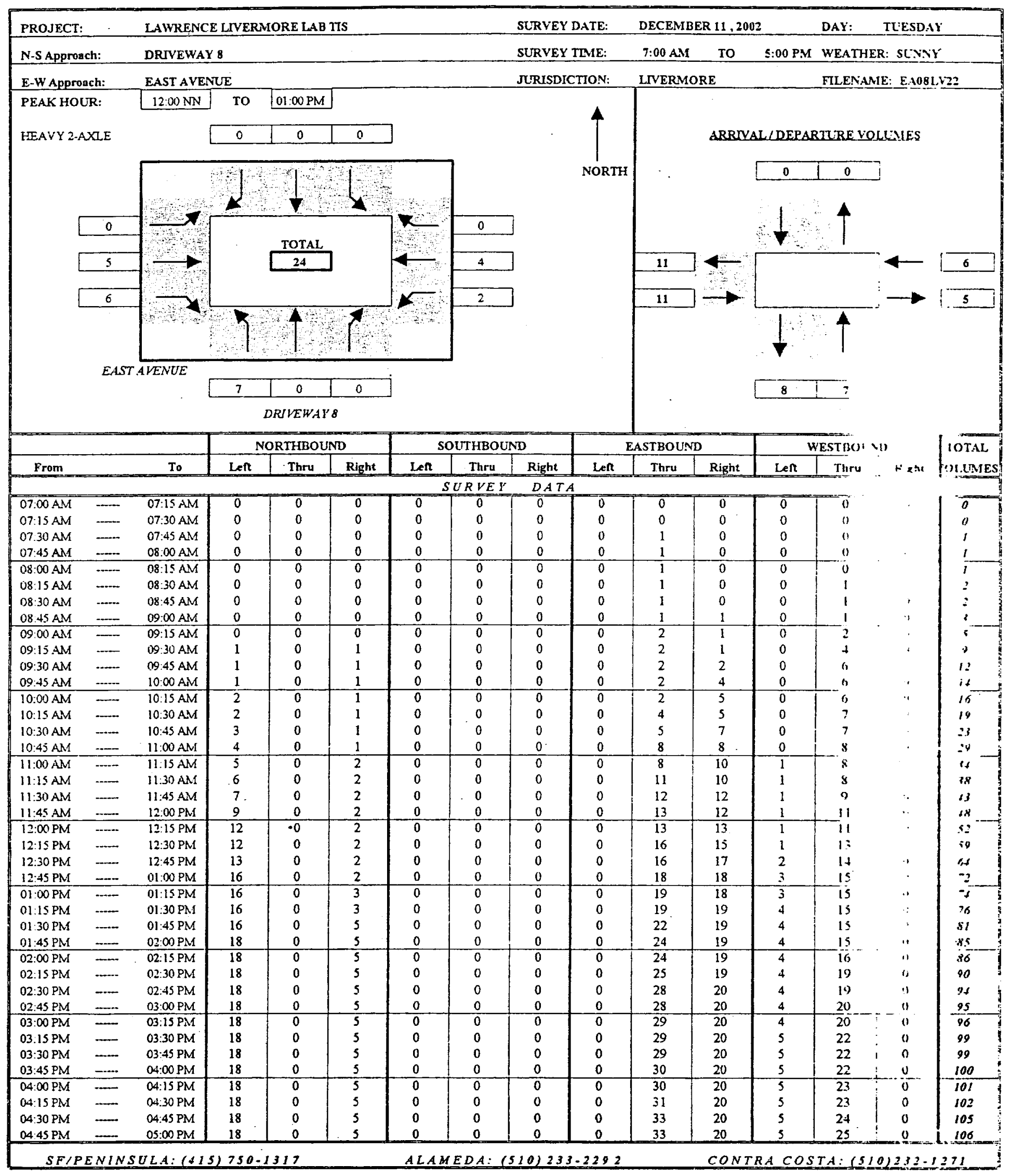




\section{Baymetrics Traffic Resources INTERSECTION TURNING MOVEMENT SUMMARY}

\begin{tabular}{|c|c|c|c|c|c|c|c|c|c|c|c|c|c|c|c|}
\hline \multicolumn{2}{|c|}{ PROJECT: } & \multicolumn{6}{|c|}{ LAWRENCE LTVERMORE LAR TIS } & \multicolumn{2}{|c|}{ SURYEY DATE: } & \multicolumn{3}{|c|}{ DECEMBER 11,2002} & DAY: & \multicolumn{2}{|c|}{ TUESDAY } \\
\hline N-S Appro & & DRTVEWA & & & & & & SLRVEY & IE: & 7:00 AM & To & 5:00 PM & WEATH & R: SUNN & \\
\hline E-W Appr & & EASTAVE & & & & & & JURISDI & ON: & LNERM & & & FILENA & 1E: E, 1081 & 122 \\
\hline & & & & THBO & & & THBO & & & ASTBOU & & & ESTBOU & & TOTAL \\
\hline From & & To & Left & Thru & Right & Len & Thru & Right & Len & Thru & Right & Len & Thru & Right & boLUMES \\
\hline & & & & & & & $4 L \quad B$ & PERIC & & & & & & & \\
\hline $\begin{array}{l}\text { OT.00 AM } \\
0715 \mathrm{AM}\end{array}$ & $\ldots$ & $\begin{array}{l}07.15 \mathrm{AM} \\
07.30 \mathrm{AM}\end{array}$ & $\begin{array}{l}\pi \\
0\end{array}$ & $\begin{array}{l}0 \\
0 \\
0\end{array}$ & $\begin{array}{l}0 \\
0\end{array}$ & 0 & 0 & $\begin{array}{l}0 \\
0\end{array}$ & 0 & To & 0 & 0 & 8 & a & 8 \\
\hline $07: 30 \mathrm{AM}$ & - & $0745 \mathrm{AM}$ & 0 & 0 & 0 & 0 & 0 & 0 & 0 & 1 & 0 & 0 & $\begin{array}{l}0 \\
0 \\
0\end{array}$ & $\begin{array}{l}0 \\
0\end{array}$ & $\begin{array}{l}0 \\
I\end{array}$ \\
\hline $08.00 \mathrm{AM}$ & $=$ & $\frac{08: 00 \mathrm{AM}}{08: 15 \mathrm{AM}}$ & -0 & $\frac{\pi}{0}$ & 0 & $\frac{\pi}{0}$ & 0 & $\frac{0}{0}$ & $\frac{0}{0}$ & $\frac{0}{0}$ & $\frac{0}{0}$ & 0 & $\frac{0}{0}$ & $\frac{0}{0}$ & 0 \\
\hline $08: 15 \mathrm{AM}$ & 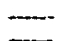 & $08.30 \mathrm{AM}$ & 0 & 0 & 0 & 0 & 0 & 0 & 0 & 0 & 0 & 0 & 1 & $\begin{array}{l}0 \\
0\end{array}$ & $\stackrel{0}{1}$ \\
\hline $\begin{array}{l}08: 30 \mathrm{AM} \\
08: 45 \mathrm{AM}\end{array}$ & 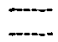 & $\begin{array}{l}08: 45 \mathrm{AM} \\
09.00 \mathrm{AM}\end{array}$ & $\begin{array}{l}0 \\
0\end{array}$ & $\begin{array}{l}0 \\
0 \\
0\end{array}$ & $\begin{array}{l}0 \\
0\end{array}$ & $\begin{array}{l}0 \\
0\end{array}$ & $\begin{array}{l}0 \\
0\end{array}$ & $\begin{array}{l}0 \\
0\end{array}$ & $\begin{array}{l}0 \\
0\end{array}$ & $\begin{array}{l}0 \\
0\end{array}$ & 0 & $\begin{array}{l}0 \\
0\end{array}$ & $\begin{array}{l}0 \\
0\end{array}$ & 0 & o \\
\hline $09: 00 \mathrm{AM}$ & $=$ & $09.15 \mathrm{AM}$ & $\%$ & 0 & 0 & 0 & $\frac{0}{0}$ & 0 & 0 & $\frac{0}{1}$ & $\frac{1}{0}$ & 0 & $\frac{0}{1}$ & $\frac{0}{11}$ & $-\frac{1}{2}$ \\
\hline $09: 15$ AM & - & $09: 30 \mathrm{AM}$ & 1 & 0 & 1 & 0 & 0 & 0 & 0 & 0 & 0 & 0 & 2 & 0 & 4 \\
\hline $\begin{array}{l}09: 30 \mathrm{AM} \\
09: 45 \mathrm{AM}\end{array}$ & $=$ & $\begin{array}{l}09: 45 \mathrm{AM} \\
10: 00 \mathrm{AM}\end{array}$ & $\begin{array}{l}0 \\
0 \\
0\end{array}$ & $\begin{array}{l}0 \\
0\end{array}$ & $\begin{array}{l}0 \\
0 \\
\end{array}$ & $\begin{array}{l}0 \\
0\end{array}$ & $\begin{array}{l}0 \\
0\end{array}$ & $\begin{array}{l}0 \\
0\end{array}$ & $\begin{array}{l}0 \\
0\end{array}$ & $\begin{array}{l}0 \\
0\end{array}$ & $\frac{1}{2}$ & $\begin{array}{l}0 \\
0\end{array}$ & $\frac{2}{0}$ & $\begin{array}{l}0 \\
i\end{array}$ & $\begin{array}{l}3 \\
2\end{array}$ \\
\hline $1000 \mathrm{AM}$ & $\cdots$ & $10: 15 \mathrm{AM}$ & 1 & 0 & 0 & 0 & 0 & 0 & 0 & 0 & $T$ & 0 & 0 & 0 & 3 \\
\hline $10: 15 \mathrm{AM}$ & $\ldots$ & $1030 \mathrm{AM}$ & 0 & 0 & 0 & 0 & 0 & 0 & 0 & 2 & 0 & 0 & 1 & i) & 3 \\
\hline 10:30 AM & $\cdots$ & 10:45 AM & 1 & 0 & 0 & 0 & 0 & 0 & 0 & 1 & 2 & 0 & 0 & "1 & $t$ \\
\hline$\frac{10: 45 \mathrm{AM}}{11: 0 \mathrm{AM}}$ & $\cdots$ & $\frac{11: 00 \mathrm{AM}}{11: 15 \mathrm{AM}}$ & $-\frac{1}{1}$ & $\frac{0}{0}$ & $\frac{0}{1}$ & $\frac{0}{0}$ & 0 & $\frac{0}{0}$ & $\frac{0}{0}$ & 3 & $\frac{1}{2}$ & $\frac{0}{1}$ & $\frac{1}{0}$ & 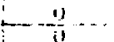 & 6 \\
\hline II:15 AM & ...... & $11: 30 \mathrm{AM}$ & $i$ & 0 & 0 & 0 & 0 & 0 & 0 & 3 & 0 & 0 & 0 & i. & 3 \\
\hline $11: 30, A M$ & -... & $1145 \mathrm{AM}$ & I & 0 & 0 & 0 & 0 & 0 & 0. & 1 & 2 & 0 & 1 & ii & 5 \\
\hline $11: 45 \mathrm{AM}$ & $\ldots$ & $12: 00 \mathrm{PM}$ & 2 & 0 & 0 & 0 & 0 & 0 & 0 & 1 & 0 & 0 & -2 & $\therefore$ & 5 \\
\hline $1200 \mathrm{PM}$ & $\ldots$ & $12: 15 \mathrm{PM}$ & 3 & 0 & 0 & 0 & 0 & 0 & 0 & 0 & $\frac{1}{3}$ & 0 & ? & $" 1$ & 7 \\
\hline 12:15 PM & $\ldots$ & $\begin{array}{l}12: 30 \mathrm{PM} \\
12.45 \mathrm{PM}\end{array}$ & $\stackrel{0}{1}$ & o & $\begin{array}{l}0 \\
0\end{array}$ & $\begin{array}{l}0 \\
0\end{array}$ & $\begin{array}{l}0 \\
0\end{array}$ & $\begin{array}{l}0 \\
0 \\
0\end{array}$ & 0 & 0 & $\frac{2}{2}$ & i & j & "1 & $\begin{array}{l}7 \\
5\end{array}$ \\
\hline $12: 45 \mathrm{PM}$ & $\therefore$ & o1 $00 \mathrm{PM}$ & 3 & 0 & 0 & 0 & 0 & 0 & 0 & 2 & & 1 & $i$ & $n$ & 8 \\
\hline $01: 00 \mathrm{PM}$ & $\cdots$ & $01: 15 \mathrm{PM}$ & 0 & 0 & 1 & 0 & 0 & 0 & 0 & $T$ & 0 & 0 & 0 & $i$ & $\frac{2}{2}$ \\
\hline 01:15 PM & $\overline{5}$ & 01:30 PM & $\begin{array}{l}0 \\
0\end{array}$ & $\begin{array}{l}0 \\
0\end{array}$ & 2 & 0 & 0 & ${ }_{0}^{0}$ & 0 & 0 & ! & 1 & 0 & $" 1$ & 2 \\
\hline 01:30 PM & $\bar{\ldots}$ & $\begin{array}{l}01: 45 \mathrm{PM} \\
02: 00 \mathrm{PM}\end{array}$ & $\frac{u}{2}$ & 0 & 0 & $\begin{array}{l}0 \\
0\end{array}$ & 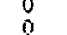 & 0 & 0 & $\frac{3}{2}$ & $0_{0}^{0}$ & 0 & 0 & $\therefore$ & 5 \\
\hline$\frac{01.45 \mathrm{PM}}{0200 \mathrm{PM}}$ & $=$ & $02.15 \mathrm{PM}$ & $\frac{2}{0}$ & 0 & 0 & $\frac{1}{0}$ & 0 & 0 & 0 & $\frac{2}{0}$ & $\frac{0}{0}$ & $\frac{0}{0}$ & $\frac{1}{1}$ & $\because$ & $-\frac{1}{1}$ \\
\hline $02: 15$ PM & $\ldots$ & $0230 \mathrm{PMI}$ & 0 & 0 & 0 & 0 & 0 & 0 . & 0 & 1 & 0 & 0 & $\frac{1}{3}$ & . & $e^{7}$ \\
\hline $02: 30 \mathrm{PM}$ & $\ldots$ & $02: 45 \mathrm{PM}$ & 0 & 0 & 0 & 0 & 0 & 0 & 0 & 3 & i & 0 & $a$ & .1 & 4 \\
\hline $0245 \mathrm{PM}$ & $=$ & $03.00 \mathrm{PM}$ & 0 & 0 & $\ddot{0}$ & 0 & 0 & 0 & 0 & 0 & 0 & 0 & i & $\because$ & 1 \\
\hline $03.00 \overline{P M}$ & $\ldots$ & $03: 15 \overline{\mathrm{PM}}$ & 0 & 0 & 0 & 0 & 0 & 0 & 0 & $T$ & 0 & 0 & 0 & $\therefore$ & $\pi$ \\
\hline $03: 15 \mathrm{PM}$ & $\ldots$ & $03.30 \mathrm{PM}$ & 0 & 0 & 0 & 0 & 0 & 0 & 0 & 0 & 0 & 1 & 2 & $\therefore$ & 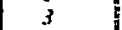 \\
\hline $03: 30 \mathrm{PM}$ & $\ldots$ & $03: 45 \mathrm{PM}$ & 0 & 0 & 0 & 0 & 0 & 0 & 0 & 0 & 0 & 0 & 0 & ". & a \\
\hline $03.45 \mathrm{PM}$ & $=$ & $0400 \mathrm{PM}$ & 0 & 0 & 0 & 0 & 0 & 0 & 0 & 1 & 0 & 0 & 0 & $\therefore$ & \\
\hline $04: 00 \mathrm{PM}$ & $=$ & $04: 15 \mathrm{PM}$ & 0 & 0 & 0 & 0 & $\overline{0}$ & 0 & 0 & 0 & 0 & 0 & $T$ & -11 & $T$ \\
\hline $04: 15 \mathrm{PM}$ & $\cdots$ & $04.30 \mathrm{PM}$ & 0 & 0 & 0 & 0 & 0 & 0 & 0 & 1 & 0 & 0 & 0 & .1 & 1 \\
\hline $\begin{array}{l}04: 30 \text { PM } \\
04.45 \text { PM }\end{array}$ & $\ldots$ & 04:45 PM & $\begin{array}{l}0 \\
0\end{array}$ & $\begin{array}{l}0 \\
0\end{array}$ & $\begin{array}{l}0 \\
0\end{array}$ & $\begin{array}{l}0 \\
0\end{array}$ & $\begin{array}{l}0 \\
0\end{array}$ & $\begin{array}{l}0 \\
0\end{array}$ & $\begin{array}{l}0 \\
0\end{array}$ & $\begin{array}{l}2 \\
0\end{array}$ & $\begin{array}{l}0 \\
0\end{array}$ & $\begin{array}{l}0 \\
0\end{array}$ & $!$ & $\because "$ & 3 \\
\hline & & & & & & & $R L Y$ & TOTAL & & & & & & & \\
\hline $07: 00 \mathrm{AM}$ & 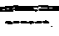 & $08: 00 \mathrm{AM}$ & 0 & 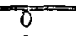 & 0 & 0 & 8 & C & 0 & $T$ & $\sigma$ & 0 & $\pi$ & $\because$ & $T$ \\
\hline $07: 15 \mathrm{AM}$ & - & $08: 15 \mathrm{AM}$ & 0 & 0 & 0 & 0 & 0 & 0 & 0 & $i$ & 0 & 0 & ) & $\cdot 1$ & i \\
\hline $07: 30 \mathrm{AM}$ & $=$ & $08: 30 \mathrm{AM}$ & 0 & 0 & 0 & 0 & 0 & 0 & 0 & 1 & 0 & 0 & 1 & .1 & \\
\hline $07.45 \mathrm{AM}$ & $\ldots$ & $08.45 \mathrm{AM}$ & 0 & 0 & 0 & 0 & 0 & 0 & 0 & $\overline{0}$ & 0 & 0 & $T$ & ." & $t$ \\
\hline $08: 00 \mathrm{AM}$ & -.... & $09: 00 \mathrm{AM}$ & 0 & $\begin{array}{l}0 \\
0\end{array}$ & 0 & 0 & 0 & 0 & 0 & 0 & 1 & a & 1 &. & 2 \\
\hline$\frac{08.15 \mathrm{AM}}{08.30 \mathrm{AM}}$ & 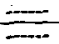 & $09: 15 \mathrm{AM}$ & $\frac{0}{1}$ & $\frac{0}{0}$ & $\frac{0}{1}$ & $\frac{0}{0}$ & $\frac{0}{0}$ & $\frac{0}{0}$ & $\frac{0}{0}$ & $\frac{1}{1}$ & $\frac{1}{1}$ & $\frac{0}{0}$ & $-\frac{2}{3}$ & $\therefore$ & $\frac{4}{7}$ \\
\hline $08: 45 \mathrm{AM}$ & 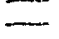 & $09: 45 \mathrm{AM}$ & $i$ & 0 & 1 & 0 & 0 & 0 & 0 & $\mathrm{i}$ & 2 & 0 & 5 & $\because$ & 10 \\
\hline $09: 00 \mathrm{AM}$ & $=$ & $10: 90 \mathrm{AM}$ & $\frac{1}{2}$ & $\frac{0}{0}$ & 1 & 0 & 0 & 0 & 0 & 1 & 3 & 0 & 5 & $\therefore$ & 11 \\
\hline $\begin{array}{l}09: 15 \mathrm{AM} \\
0930 \mathrm{AM}\end{array}$ & $=$ & $\begin{array}{l}10.15 \mathrm{AM} \\
10.30 \mathrm{AM}\end{array}$ & $?_{1}^{2}$ & $\begin{array}{l}0 \\
0\end{array}$ & $\begin{array}{l}1 \\
0\end{array}$ & $\begin{array}{l}0 \\
0\end{array}$ & $\begin{array}{l}0 \\
0\end{array}$ & $\begin{array}{l}0 \\
0\end{array}$ & $\begin{array}{l}0 \\
0\end{array}$ & 0 & $\frac{4}{4}$ & $\begin{array}{l}0 \\
0\end{array}$ & $\frac{4}{3}$. & $\therefore$ & $\frac{\pi 1}{10}$ \\
\hline $09.45 \mathrm{AM}$ & $=$ & $10: 45 \mathrm{AM}$ & 2 & 0 & 0 & 0 & 0 & 0 & 0 & 3 & 5 & 0 & $\therefore$ & $\therefore$ & 11 \\
\hline $10.00 \mathrm{AM}$ & 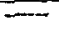 & $1100 \mathrm{AM}$ & 3 & 0 & 0 & 0 & 0 & 0 & 0 & 6 & 4 & $\%$ & $\frac{2}{3}$ & , & 15 \\
\hline 10:15 AMS & 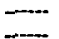 & $11: 15 \mathrm{AM}$ & $\begin{array}{l}3 \\
4\end{array}$ & $\begin{array}{l}0 \\
0\end{array}$ & i & $\begin{array}{l}0 \\
0\end{array}$ & $\begin{array}{l}0 \\
0\end{array}$ & $\begin{array}{l}0 \\
0\end{array}$ & $\begin{array}{l}0 \\
0\end{array}$ & 6 & $\begin{array}{l}5 \\
5\end{array}$ & 1 & 3 & . & 18 \\
\hline$\frac{1.30 \mathrm{AM}}{10.45 \mathrm{AM}}$ & $\equiv$ & $11.45 \mathrm{AM}$ & 4 & 0 & $\frac{1}{b}$ & $\frac{0}{0}$ & $\frac{0}{0}$ & $\frac{0}{0}$ & $\frac{0}{0}$ & $\frac{1}{9}$ & $\frac{3}{5}$ & $\frac{1}{1}$ & $\frac{2}{2}$ &.. & $\frac{19}{20}$ \\
\hline $11: 00 \mathrm{AM}$ & $\ldots$ & $12:$ & 5 & 0 & 1 & 0 & 0 & 0 & 0 & 5 & 4 & $i$ & $\overline{3}$ & $\therefore$ & ig \\
\hline 11:15 AMA & 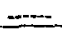 & $12: 15 \mathrm{PM}$ & 7 & 0 & 0 & 0 & 0 & 0 & 0 & 5 & 3 & 0 & 3 & $\because$ & 18 \\
\hline $1130 \mathrm{AM}$ & 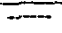 & $1230 \mathrm{PM}$ & 6 & 0 & 0 & 0 & 0 & $\overline{0}$ & 0 & 5 & 5 & 0 & 5 & "i & 21 \\
\hline $11.45 \mathrm{AM}$ & - & $12: 45 \mathrm{PM}$ & 6 & 0 & 0 & 0 & 0 & 0 & 0 & 4 & 5 & 1. & 5 & 1 & 21 \\
\hline & $=$ & $01: 00 \mathrm{PM}$ & 7 & 0 & 0 & 0 & & & 0 & 5 & 6 & & & & 24 \\
\hline 12:15 PM & $\ldots$ & $01.15 \mathrm{PM}$ & 4 & $\overline{0}$ & $T$ & 0 & $\overline{0}$ & 0 & 0 & 6 & 5 & 2 & 4 & ii & 25 \\
\hline $12: 30 \mathrm{PM}$ & $\ldots$ & $01: 30 P M$ & 4 & 0 & 1 & 0 & 0 & 0 & 0 & 3 & 4 & 3 & 2 & "1 & 17 \\
\hline $12: 45 \mathrm{PM}$ & $\ldots$ & $01.45 \mathrm{PM}$ & 3 & 0 & 3 & 0 & 0 & 0 & 0 & 6 & 2 & 2 & 1 & j & 17 \\
\hline $01: 00 \mathrm{PM}$ & - & $02: 00 \mathrm{PM}$ & 2 & 0 & 3 & 0 & 0 & 0 & 0 & 6 & 1 & 1 & 0 & 0 & 13 \\
\hline $01: 15 \mathrm{PM}$ & - & 02:15 PM & 2 & 0 & $\frac{2}{2}$ & 0 & 0 & 0 & 0 & 5 & 1 & 1 & 1 & y & 12 \\
\hline $01: 30 \mathrm{PM}$ & $\cdots$ & $02: 30 \mathrm{PM}$ & $\frac{2}{2}$ & 0 & 2 & 0 & 0 & a & 0 & & 0 & & & 0 & It \\
\hline $01: 45 \mathrm{PM}$ & $=$ & $\overline{02} .45 \mathrm{PM}$ & 2 & 0 & 0 & $\bar{\sigma}$ & 0 & 0 & 0 & $\overline{6}$ & 1 & 0 & 4 & i) & 13 \\
\hline $02.00 \mathrm{PM}$ & - & 03:00 PM & 0 & 0 & 0 & 0 & 0 & 0 & 0 & 4 & 1 & $?$ & 5 & 0 & 10 \\
\hline & $=$ & & $\frac{0}{0}$ & 0 & $\frac{0}{0}$ & $\frac{0}{0}$ & $\frac{0}{0}$ & $\frac{0}{0}$ & $-\frac{0}{0}$ & $\frac{5}{4}$ & $\frac{1}{1}$ & $\frac{0}{1}$ & $\frac{4}{3}$ & $\frac{0}{0}$ & $\frac{10}{8}$ \\
\hline $02: 30 \mathrm{PM}$ & $=$ & $\begin{array}{l}03: 30 \mathrm{PM} \\
03: 45 \mathrm{PM}\end{array}$ & $\begin{array}{l}0 \\
0\end{array}$ & $\begin{array}{l}0 \\
0\end{array}$ & $0_{0}^{0}$ & $\begin{array}{l}0 \\
0\end{array}$ & 0 & $\begin{array}{l}0 \\
0\end{array}$ & $\begin{array}{l}0 \\
0\end{array}$ & $\frac{4}{1}$ & $t_{0}^{1}$ & 1 & $\begin{array}{l}3 \\
3\end{array}$ & 6 & 5 \\
\hline $03: 00 \mathrm{PM}$ & $\overline{-}$ & $04: 00 \mathrm{PM}$ & 0 & 0 & 0 & 0 & 0 & 0 & 0 & $i$ & 0 & i & 2 & 0 & 5 \\
\hline $03: 15 \mathrm{PM}$ & $=$ & $04.15 \mathrm{PM}$ & 0 & 0 & 0 & 0 & 0 & 0 & 0 & 1 & 0 & $T$ & 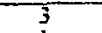 & 0 & 5 \\
\hline 03:30 PM & - & $04: 30 \mathrm{PM}$ & 0 & 0 & 0 & 0 & 0 & 0 & 0 & 2 & 0 & 0 & 1 & 0 & 3 \\
\hline$\frac{03.45 \mathrm{PM}}{0400 \mathrm{PM}}$ & $=$ & $\frac{04: 45 \mathrm{PM}}{05: 20 \mathrm{MM}}$ & $\frac{0}{0}$ & $\frac{0}{0}$ & $\frac{0}{0}$ & $\frac{0}{0}$ & $\frac{0}{0}$ & $\frac{0}{0}$ & $\frac{0}{0}$ & $\frac{4}{3}$ & $\frac{0}{0}$ & $\frac{0}{0}$ & $\frac{2}{3}$ & $\frac{1}{0}$ & $\frac{6}{6}$ \\
\hline$S F / I$ & $N I N$ & $\| L A:(\ll 1$ & 75 & & & 12 & $A$ & 0) & 29 & & $=0$ & $c$ & $4: 1$ & 232 & 71 \\
\hline
\end{tabular}




\section{Baymetrics Traffic Resources INTERSECTION TURNING MOVEMENT SUMMARY}

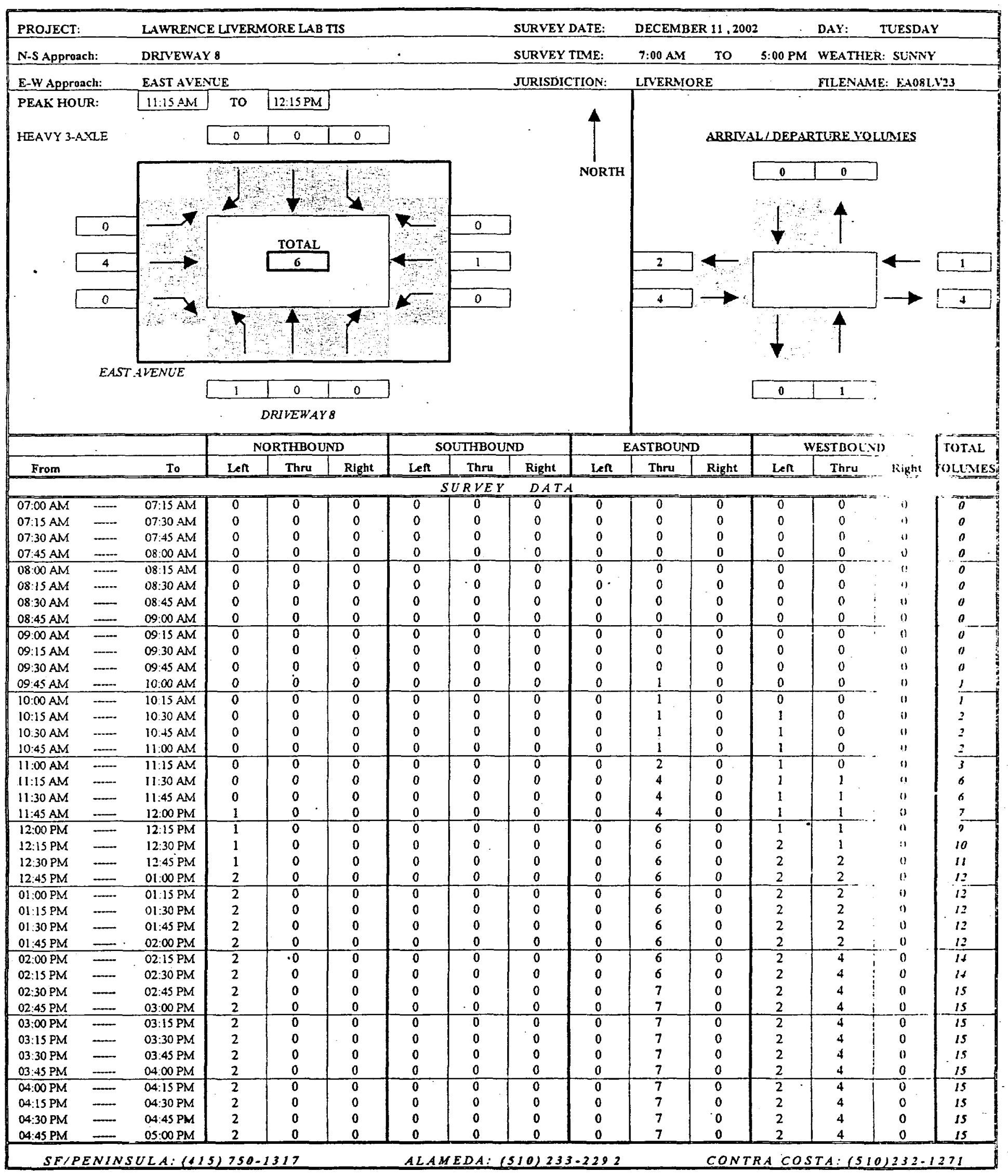




\section{Baymetrics Traffic Resources INTERSECTION TURNING MOVEMENT SUMMARY}

\begin{tabular}{|c|c|c|c|c|c|c|c|c|c|c|c|c|c|c|c|}
\hline \multirow{2}{*}{\multicolumn{2}{|c|}{$\begin{array}{l}\text { PROJECT: } \\
\text { N-S Approach: }\end{array}$}} & \multicolumn{6}{|c|}{ LAWRENCE LVVERMORE LAB TIS } & \multicolumn{2}{|c|}{ SURVEY DATE: } & \multicolumn{3}{|c|}{ DECEMBER 11,2002} & DAY: & \multicolumn{2}{|c|}{ TUESDAY } \\
\hline & & \multicolumn{6}{|c|}{ DRIVEWAY 8} & SURVEY & ME: & $7: 00 \mathrm{AM}$ & To & 5:00 PM & WEATH & R: SUNNY & \\
\hline E-W Appro & & EAST AVE & & & & & & JURISDIC & ON: & LVVERMC & & & FILENA & 1E: EA08L & $\sqrt{23}$ \\
\hline & & & & THBO & & & THBO & & & ASTBOUN & & & ESTBOU & & TOTAL \\
\hline From & & To & Left & Thru & Right & Lent & Thru & Right & Len & Thru & Right & Lent & Thru & Right & LOLUMES \\
\hline & & & & & & & $L \quad B$ & PERIC & & & & & & & \\
\hline O7.00AM & - & OIISAM & 0 & $\sigma$ & 0 & 0 & $\frac{\pi}{0}$ & 0 & 0 & o & 0 & 0 & 0 & o & \\
\hline 07:15 AM & $\ldots$ & $07: 30$ AM & $\begin{array}{l}0 \\
0\end{array}$ & $\begin{array}{l}0 \\
0\end{array}$ & 0 & $\begin{array}{l}0 \\
0\end{array}$ & 0 & 0 & 0 & 0 & 0 & 0 & 0 & 0 & $a$ \\
\hline $07.45 \mathrm{AM}$ & $=$ & $\begin{array}{l}07: 45 \text { AM } \\
08: 00 \text { AM }\end{array}$ & 0 & 0 & $\begin{array}{l}0 \\
0\end{array}$ & 0 & $\begin{array}{l}0 \\
0\end{array}$ & $\begin{array}{l}0 \\
0\end{array}$ & 0 & $a$ & 0 & 0 & 0 & $\begin{array}{l}0 \\
0\end{array}$ & $\begin{array}{l}a \\
0\end{array}$ \\
\hline $08: 00 \mathrm{ANI}$ & $\cdots$ & $08: 15 \mathrm{AM}$ & 0 & 0 & 0 & 0 & 0 & 0 & 0 & 0 & 0 & $\overline{0}$ & 0 & 0 & 0 \\
\hline 08:15 AM & 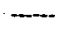 & 08:30 AM & 0 & 0 & 0 & 0 & 0 & 0 & 0 & 0 & 0 & 0 & 0 & $a$ & 0 \\
\hline $\begin{array}{l}\text { 08:30 AM } \\
08: 45 \mathrm{AM}\end{array}$ & $\ldots$ & $\begin{array}{l}08: 45 \mathrm{AM} \\
09: 00 \mathrm{AM}\end{array}$ & $\begin{array}{l}0 \\
0\end{array}$ & $\begin{array}{l}0 \\
0\end{array}$ & $\begin{array}{l}0 \\
0\end{array}$ & $\begin{array}{l}0 \\
0\end{array}$ & $\begin{array}{l}0 \\
0\end{array}$ & $\begin{array}{l}0 \\
0\end{array}$ & $\begin{array}{l}0 \\
0\end{array}$ & $\begin{array}{l}0 \\
0\end{array}$ & $\begin{array}{l}0 \\
0\end{array}$ & $\begin{array}{l}0 \\
0\end{array}$ & $\begin{array}{l}0 \\
0\end{array}$ & 0 & $a$ \\
\hline $09: 00 \mathrm{AM}$ & $\ldots$ & $09: 15 . \mathrm{AM}$ & 0 & 0 & 0 & 0 & 0 & 0 & 0 & 0 & $0^{\circ}$ & 0 & 0 & $\overline{1}$ & 0 \\
\hline $09: 15 \mathrm{AM}$ & $\cdots$ & $09: 30 \mathrm{AM}$ & 0 & 0 & 0 & 0 & 0 & 0 & 0 & 0 & 0 & 0 & 0 & 0 & 0 \\
\hline $\begin{array}{l}09: 30 \mathrm{AM} \\
09.45 \mathrm{AM}\end{array}$ & $\ldots$ & $\begin{array}{l}09.45 \mathrm{AM} \\
10: 00 \mathrm{AM}\end{array}$ & $\begin{array}{l}0 \\
0\end{array}$ & $\begin{array}{l}0 \\
0\end{array}$ & $\begin{array}{l}0 \\
0\end{array}$ & $\begin{array}{l}0 \\
0\end{array}$ & $\begin{array}{l}0 \\
0\end{array}$ & $\begin{array}{l}0 \\
0\end{array}$ & $\begin{array}{l}0 \\
0\end{array}$ & $\begin{array}{l}0 \\
1\end{array}$ & $\begin{array}{l}0 \\
0\end{array}$ & $\begin{array}{l}0 \\
0\end{array}$ & $\begin{array}{l}0 \\
0\end{array}$ & 0 & 0 \\
\hline $30: 00 \mathrm{AM}$ & $=$ & $10: 15 \mathrm{AM}$ & $\overline{0}$ & 0 & 0 & 0 & 0 & 0 & 0 & 10 & 0 & 0 & $\frac{0}{0}$ & $\frac{1}{0}$ & $\frac{1}{0}$ \\
\hline $10: 15 \mathrm{AM}$ & - & $10: 30 \mathrm{AM}$ & 0 & 0 & 0 & 0 & 0 & 0 & 0 & 0 & 0 & 1 & 0 & 0 & 1 \\
\hline $\begin{array}{l}10: 30 \mathrm{AM} \\
10.45 \mathrm{AM}\end{array}$ & $m$ & $\begin{array}{l}10: 45 \mathrm{AM} \\
11: 00 \mathrm{AM}\end{array}$ & $\begin{array}{l}0 \\
0\end{array}$ & $\begin{array}{l}0 \\
0\end{array}$ & $\begin{array}{l}0 \\
0\end{array}$ & $\begin{array}{l}0 \\
0\end{array}$ & $\begin{array}{l}0 \\
0\end{array}$ & $\begin{array}{l}0 \\
0\end{array}$ & $\begin{array}{l}0 \\
0\end{array}$ & $\begin{array}{l}0 \\
0\end{array}$ & $\begin{array}{l}0 \\
0\end{array}$ & $\begin{array}{l}0 \\
0\end{array}$ & $\begin{array}{l}0 \\
0 \\
\end{array}$ & 0 & $\begin{array}{l}0 \\
0\end{array}$ \\
\hline $11.00 \mathrm{AM}$ & $\cdots$ & $11: 15 \mathrm{AM}$ & 0 & 0 & 0 & - & 0 & 0 & 0 & î & 0 & 0 & 0 & i & $i$ \\
\hline 11:15 AM & $\cdots$ & 11:30 AM & 0 & 0 & 0 & 0 & 0 & 0 & 0 & 2 & 0 & 0 & $i$ & $" 1$ & 3 \\
\hline $\begin{array}{l}11: 30 \mathrm{AM} \\
11: 45 \mathrm{AM}\end{array}$ & $\cdots$ & $\begin{array}{l}11: 45 \mathrm{AM} \\
12: 00 \mathrm{PM}\end{array}$ & 1 & 0 & 0 & $\begin{array}{l}0 \\
0\end{array}$ & $\begin{array}{l}0 \\
0\end{array}$ & $\begin{array}{l}0 \\
0\end{array}$ & $\begin{array}{l}0 \\
0\end{array}$ & $\begin{array}{l}0 \\
0\end{array}$ & $\begin{array}{l}0 \\
0\end{array}$ & $\begin{array}{l}0 \\
0\end{array}$ & $\begin{array}{l}0 \\
0\end{array}$ & $\because 4$ & 0 \\
\hline $1200 \mathrm{PM}$ & $\cdots$ & $12: 15$ PM & $\overline{0}$ & 0 & 0 & 0 & 0 & 0 & 0 & 2 & 0 & 0 & 0 & "1 & $\frac{1}{2}$ \\
\hline 12:15 PM & $\cdots$ & $12: 30 \mathrm{PM}$ & 0 & 0 & 0 & 0 & 0 & 0 & 0 & 0 & 0 & 1 & 0 & is & $i$ \\
\hline $\begin{array}{l}12: 30 \mathrm{PM} \\
12: 45 \mathrm{PM}\end{array}$ & $\ldots$ & $\begin{array}{l}12: 45 \mathrm{PM} \\
0100 \mathrm{PM}\end{array}$ & $\begin{array}{l}0 \\
1\end{array}$ & $\begin{array}{l}0 \\
0\end{array}$ & $\begin{array}{l}0 \\
0\end{array}$ & $\begin{array}{l}0 \\
0\end{array}$ & $\begin{array}{l}0 \\
0\end{array}$ & $\begin{array}{l}0 \\
0\end{array}$ & $\begin{array}{l}0 \\
0\end{array}$ & $\begin{array}{l}0 \\
0\end{array}$ & $\begin{array}{l}0 \\
0\end{array}$ & $\begin{array}{l}0 \\
0\end{array}$ & ! & $\ddot{11}$ & I \\
\hline$\frac{1}{01.00 \mathrm{PM}}$ & $\ldots$ & $01: 15 \mathrm{PM}$ & 0 & 0 & 0 & $-\overline{0}$ & 0 & $\frac{\pi}{0}$ & 0 & 0 & 0 & 0 & $0^{-}$ & $\frac{11}{11}$ & $\frac{1}{0}$ \\
\hline $01: 15 \mathrm{PM}$ & $\ldots$ & $01.30 \mathrm{PM}$ & 0 & 0 & 0 & 0 & 0 & 0 & 0 & 0 & 0 & 0 & 0 & 11 & 0 \\
\hline $01: 30$ PM & - & $01: 45 \mathrm{PM}$ & 0 & 0 & 0 & 0 & 0 & 0 & 0 & 0 & 0 & 0 & 0 & $a$ & 0 \\
\hline$\frac{01: 45 \mathrm{PM}}{02: 00 \mathrm{PM}}$ & $=$ & $\frac{02: 00 \mathrm{PM}}{02: 15 \mathrm{PM}}$ & $-\frac{0}{0}$ & $\frac{0}{0}$ & $\frac{0}{0}$ & $\frac{0}{0}$ & 0 & $\frac{0}{0}$ & 0 & $\frac{0}{0}$ & $\frac{0}{0}$ & $\frac{0}{0}$ & $\frac{n}{2}$ & $\because 1$ & $-\frac{0}{2}$ \\
\hline $02: 15$ PM & $\ldots$ & $02: 30 \mathrm{PM}$ & 0 & 0 & 0 & 0 & 0 & 0 & 0 & 0 & 0 & 0 & 0 & $\therefore$ & 0 \\
\hline $02: 30$ & $\ldots$ & $02: 45 \mathrm{PM}$ & 0 & 0 & 0 & 0 & 0 & 0 & 0 & 1 & 0 & 0 & 0 & $\because$ & $i$ \\
\hline $02: 15 \mathrm{PM}$ & $\ldots$ & $03: 00 \mathrm{PM}$ & 0 & 0 & 0 & 0 & 0 & 0 & 0 & 0 & 0 & 0 & 0 & $\because:$ & $a$ \\
\hline $03.00 \mathrm{PM}$ & $\cdots$ & $03.15 \mathrm{PM}$ & 0 & 0 & 0 & 0 & 0 & 0 & 0 & 0 & 0 & 0 & 0 & $\therefore$ & 0 \\
\hline 03:15 PM & $\cdots$ & 03:30 PM & 0 & 0 & 0 & 0 & 0 & 0 & 0 & 0 & 0 & 0 & 0 & $\because$ & 0 \\
\hline 03:30 PM & $\cdots$ & 03:45 PM & 0 & ${ }_{0}^{0}$ & ${ }_{0}^{0}$ & 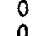 & 0 & 0 & 0 & 0 & 0 & 0 & ${ }_{0}^{0}$ & "1 & 0 \\
\hline $04: 00 \mathrm{PM}$ & $=$ & $04: 15 \mathrm{PM}^{2}$ & 0 & $\frac{\pi}{0}$ & $\frac{\pi}{0}$ & 0 & 0 & $\frac{\pi}{0}$ & 0 & 0 & 0 & 0 & $\frac{0}{0}$ & $\overline{11}-$ & $\frac{\pi}{0}$ \\
\hline $04: 15 \mathrm{PM}$ & $\ldots$ & $04: 30 \mathrm{PM}$ & 0 & 0 & 0 & 0 & 0 & 0 & 0 & 0 & 0 & 0 & 0 & $" 1$ & 0 \\
\hline $04: 30 \mathrm{PM}$ & - & $04: 45 \mathrm{PM}$ & 0 & 0 & 0 & 0 & 0 & 0 & 0 & 0 & 0 & 0 & 0 & $" 1$ & $a$ \\
\hline $0445 \mathrm{PM}$ & - & $05: 00 \mathrm{PM}$ & 0 & 0 & 0 & 0 & $\frac{0}{R L Y}$ & $\frac{0}{C O T A L}$ & 0 & 0 & 0 & 0 & 0 & $\therefore$ & 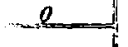 \\
\hline $0700 \mathrm{aM}$ & $=$ & & $\sigma$ & $\overline{0}$ & $\overline{0}$ & 6 & $\%$ & 8 & $\overline{0}$ & $\sigma$ & $\overline{0}$ & $\sigma$ & & $\pi$ & $=8$ \\
\hline $07: 15 \mathrm{AM}$ & 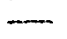 & $08: 15$ AM & 0 & 0 & 0 & 0 & 0 & 0 & 0 & 0 & 0 & 0 & 0 & $\|$ & 0 \\
\hline $07: 30 \mathrm{AM}$ & $=$ & $08: 30 \mathrm{AM}$ & 0 & 0. & 0 & 0 & 0 & 0 & 0 & 0 & 0 & 0 & 0 & $\because$ & 0 \\
\hline $\begin{array}{l}07.45 \mathrm{AM} \\
08: 00 \mathrm{AM}\end{array}$ & $\ldots$ & $\begin{array}{l}08: 45 \mathrm{AM} \\
09: 00 \mathrm{AM}\end{array}$ & 0 & $\begin{array}{l}0 \\
0\end{array}$ & $\begin{array}{l}0 \\
0\end{array}$ & $\begin{array}{l}0 \\
0\end{array}$ & $\begin{array}{l}0 \\
0\end{array}$ & $\begin{array}{l}0 \\
0\end{array}$ & 0 & $\begin{array}{l}0 \\
0\end{array}$ & $\begin{array}{l}0 \\
0\end{array}$ & $\begin{array}{l}0 \\
0\end{array}$ & 0 & $"$ & 0 \\
\hline $\begin{array}{l}08: 00 \mathrm{AM} \\
08: 15 \mathrm{AM}\end{array}$ & $=$ & $\begin{array}{l}\text { 09:00 AM } \\
09: 15 \mathrm{AM}\end{array}$ & 0 & 0 & 0 & 0 & 0 & 0 & 0 & 0 & 0 & 0 & 0 &. & $\begin{array}{l}0 \\
0\end{array}$ \\
\hline $08: 30 \mathrm{AM}$ & $=$ & $09: 30 \mathrm{AM}$ & 0 & 0 & 0 & 0 & 0 & 0 & 0 & 0 & 0 & 0 & 0 & $\because "$ & $\overline{0}$ \\
\hline $08.45 \mathrm{AM}$ & 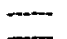 & $09: 45 \mathrm{AM}$ & 0 & 0 & 0 & $\begin{array}{l}0 \\
0\end{array}$ & 0 & 0 & 0 & 0 & 0 & $\begin{array}{l}0 \\
0\end{array}$ & 0 & 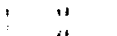 & 0 \\
\hline$\frac{09: 00 \mathrm{AM}}{09.15 \mathrm{AM}}$ & $\equiv$ & $\frac{10: 00 \mathrm{AM}}{10.15 \mathrm{AM}}$ & $-\frac{0}{0}$ & $\frac{0}{0}$ & $\frac{0}{0}$ & $\frac{0}{0}$ & -0 & $\frac{0}{0}$ & $-\frac{9}{0}$ & $\frac{1}{1}$ & $\frac{0}{0}$ & $\frac{0}{0}$ & $\frac{0}{0}$ & $-\cdots$ & $\frac{1}{1}$ \\
\hline $09: 30 \mathrm{AM}$ & $\ldots$ & $10: 30 \mathrm{AM}$ & 0 & 0 & 0 & 0 & 0 & 0 & 0 & i & 0 & I & 0 & $" 1$ & $\frac{1}{2}$ \\
\hline $09: 45 \mathrm{AM}$ & $=$ & $10.45 \mathrm{AM}$ & 0 & 0 & 0 & 0 & 0 & 0 & 0 & i & 0 & 1 & 0 & .1 & $\underline{2}$ \\
\hline $1000 \mathrm{AM}$ & $\ldots$ & $1100 \mathrm{AM}$ & 0 & 0 & 0 & 0 & 0 & 0 & 0 & 0 & 0 & $T$ & 0 & i' & $i$ \\
\hline $10: 15 \mathrm{AM}$ & - & $11: 15 \mathrm{AM}$ & 0 & 0 & 0 & 0 & 0 & 0 & 0 & 1 & 0 & $i$ & 0 & 12 & 2 \\
\hline $10: 30 \mathrm{AM}$ & $\ldots$ & $11: 30 \mathrm{AM}$ & 0 & 0 & 0 & 0 & 0 & 0 & 0 & 3 & 0 & 0 & 1 & $\therefore$ & $i$ \\
\hline $10: 45 \mathrm{AM}$ & $\ldots$ & $11: 45 \mathrm{AM}$ & 0 & 0 & 0 & 0 & 0 & 0 & 0 & 3 & 0 & 0 & $i$ & “ & \\
\hline $11: 00 \mathrm{AM}$ & $\ldots$ & $12: 00 \mathrm{PM}$ & l & 0 & 0 & 0 & 0 & 0 & 0 & 3 & 0 & 0 & $i$ & $"$ & 5 \\
\hline & $\ldots$ & & $i$ & 0 & 0 & 0 & 0 & 0 & 0 & 4 & 0 & 0 & & 11 & \\
\hline $11: 30 \mathrm{AM}$ & $\cdots$ & $12: 30 \mathrm{PM}$ & 1 & $\overline{0}$ & 0 & 0 & 0 & 0 & 0 & 2 & 0 & $T$ & $\sigma$ & 11 & 8 \\
\hline $\begin{array}{l}11: 45 \mathrm{AM} \\
12: 00 \mathrm{PM}\end{array}$ & 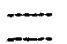 & $\begin{array}{l}12: 45 \mathrm{PM} \\
01: 00 \mathrm{PM}\end{array}$ & 1 & $\begin{array}{l}0 \\
0\end{array}$ & $\begin{array}{l}0 \\
0\end{array}$ & $\begin{array}{l}0 \\
0\end{array}$ & $\begin{array}{l}0 \\
0\end{array}$ & $\begin{array}{l}0 \\
0\end{array}$ & $\begin{array}{l}0 \\
0\end{array}$ & $\frac{2}{2}$ & $\begin{array}{l}0 \\
0\end{array}$ & 1 & $\begin{array}{l}1 \\
1\end{array}$ & $\begin{array}{l}11 \\
0\end{array}$ & 5 \\
\hline$\frac{1}{12: 15 \mathrm{PM}}$ & $=$ & $\frac{1.00 P M}{01: 15 P M}$ & 1 & 8 & $\frac{0}{0}$ & -0 & 0 & 0 & 0 & $\frac{1}{0}$ & 0 & $\frac{1}{1}$ & 1 & is & $\frac{3}{3}$ \\
\hline 12:30 PM & {[} & $0130 \mathrm{PM}$ & $i$ & 0 & 0 & 0 & 0 & 0 & 0 & 0 & 0 & 0 & 1 & 0 & 2 \\
\hline$\frac{12.45 \mathrm{PM}}{01.00 \mathrm{PM}}$ & $\equiv$ & $\frac{01: 45 \mathrm{PM}}{02.00 \mathrm{PM}}$ & $-\frac{1}{0}$ & $\frac{0}{0}$ & $\frac{0}{0}$ & 0 & $\frac{0}{0}$ & $\frac{0}{0}$ & $\frac{0}{0}$ & $\frac{0}{0}$ & $\frac{0}{0}$ & $\frac{0}{0}$ & 0 & $\theta$ & \\
\hline $01: 15 \mathrm{PM}$ & $\ldots$ & 02:15 PM & 0 & 0 & 0 & 0 & 0 & 0 & 0 & 0 & $\begin{array}{l}0 \\
0\end{array}$ & $\begin{array}{l}0 \\
0\end{array}$ & $\begin{array}{l}0 \\
2\end{array}$ & 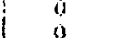 & 0 \\
\hline $01: 30 \mathrm{PM}$ & $=$ & $02: 30 \mathrm{PM}$ & 0 & 0 & 0 & 0 & 0 & 0 & 0 & 0 & $\begin{array}{l}0 \\
0\end{array}$ & 0 & 2 & 0 & 2 \\
\hline $01: 45 \mathrm{PM}$ & $\ldots$ & $02: 45 \mathrm{PM}$ & 0 & 0 & 0 & 0 & 0 & 0 & 0 & 1 & 0 & 0 & 2 & 0 & 3 \\
\hline $02: 00 \mathrm{PM}$ & $\ldots$ & & 0 & 0 & $\stackrel{0}{0}$ & 0 & $\begin{array}{l}0 \\
0\end{array}$ & $\begin{array}{l}0 \\
0\end{array}$ & $\begin{array}{l}0 \\
0\end{array}$ & 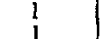 & ${ }_{0}^{0}$ & $\begin{array}{l}0 \\
0\end{array}$ & 2 & 0 & 3 \\
\hline & $\equiv$ & & $-\frac{0}{0}$ & 0 & $\frac{0}{0}$ & $\frac{0}{0}$ & $\frac{0}{0}$ & 0 & $\frac{0}{0}$ & $\frac{1}{1}$ & $\frac{0}{0}$ & $\frac{0}{0}$ & $\frac{0}{0}$ & & \\
\hline $\begin{array}{l}02: 30 \mathrm{PM} \\
02: 45 \mathrm{PM}\end{array}$ & $-\ldots$ & 03:45 PM & 0 & 0 & 0 & 0 & 0 & 0 & 0 & 0 & 0 & 0 & 0 & 0 & $a$ \\
\hline $03: 00 \mathrm{PM}$ & $=$ & $04: 00$ & 0 & 0 & 0 & 0 & 0 & 0 & 0 & 0 & 0 & 0 & 0 & 0 & 0 \\
\hline $03: 15 \mathrm{PM}$ & $=$ & $04: 15$ PM & 0 & 0 & 0 & 0 & 0 & 0 & 0 & 0 & 0 & 0 & $\overline{0}$ & 0 & 0 \\
\hline $03: 30 \mathrm{PM}$ & - & $04: 30 \mathrm{PM}$ & 0 & 0 & 0 & 0 & 0 & 0 & 0 & 0 & 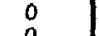 & 0 & 0 & 0 & $a$ \\
\hline$\frac{03: 45 \mathrm{PM}}{04: 00 \mathrm{PM}}$ & $\Rightarrow$ & $\frac{04: 45 \mathrm{PM}}{05: 00 \mathrm{PM}}$ & $\begin{array}{l}0 \\
0 \\
0\end{array}$ & $\frac{0}{0}$ & $\frac{a}{0}$ & $\frac{0}{0}$ & $\frac{0}{0}$ & $\frac{0}{0}$ & 0 & 0 & $\frac{a}{0}$ & $\frac{0}{0}$ & $\frac{0}{0}$ & $\frac{0}{0}$ & $\frac{0}{0}$ \\
\hline SF & NIN & $U L A:(\$ 1$ & 73 & & & $A L A$ & $D A$ & $0) 2$ & 292 & & col & $A C$ & A: & 2232 & 271 \\
\hline
\end{tabular}




\section{Baymetrics Traffic Resources INTERSECTION TURNING MOVEMENT SUMMARY}

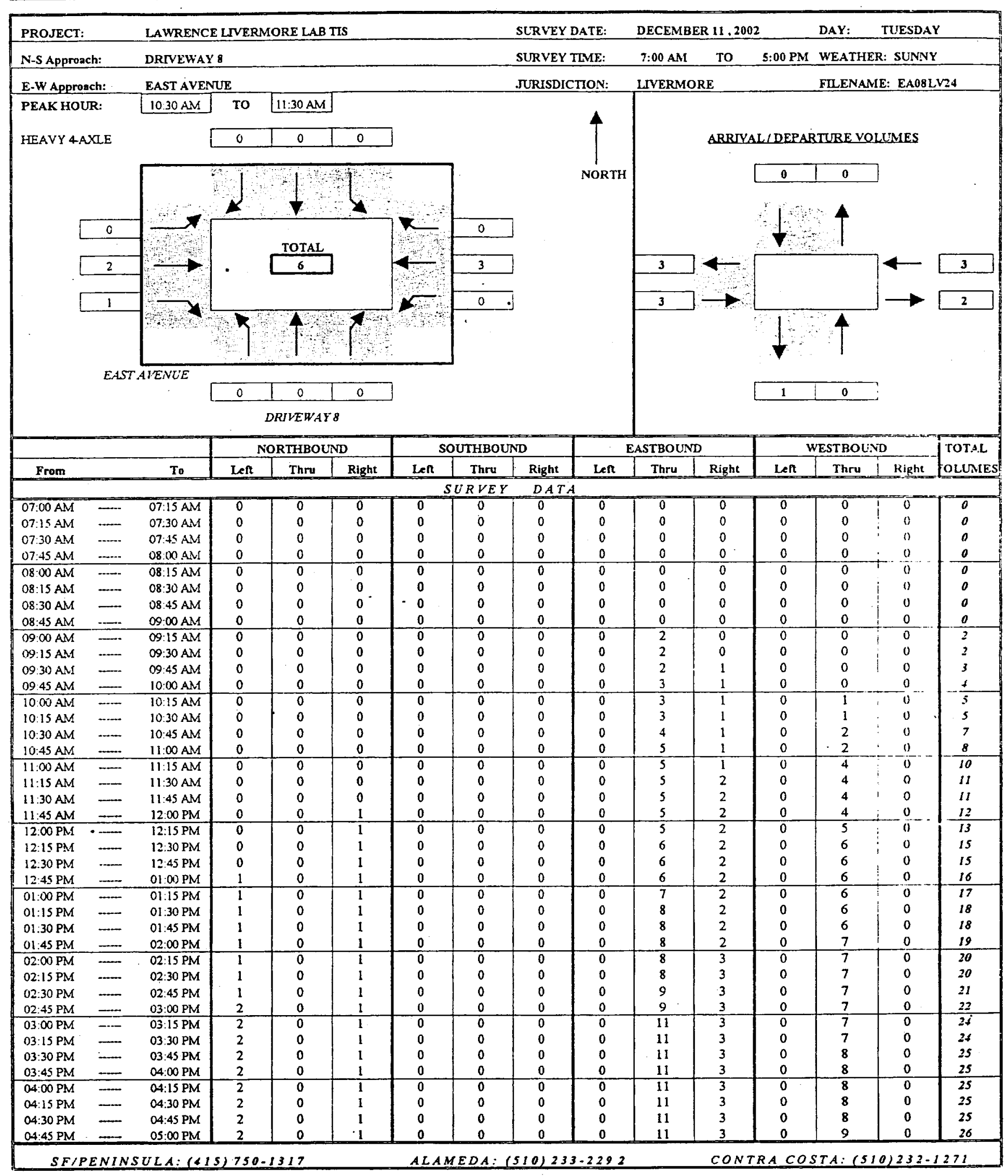




\section{Baymetrics Traffic Resources INTERSECTION TURNING MOVEMENT SUMMARY}

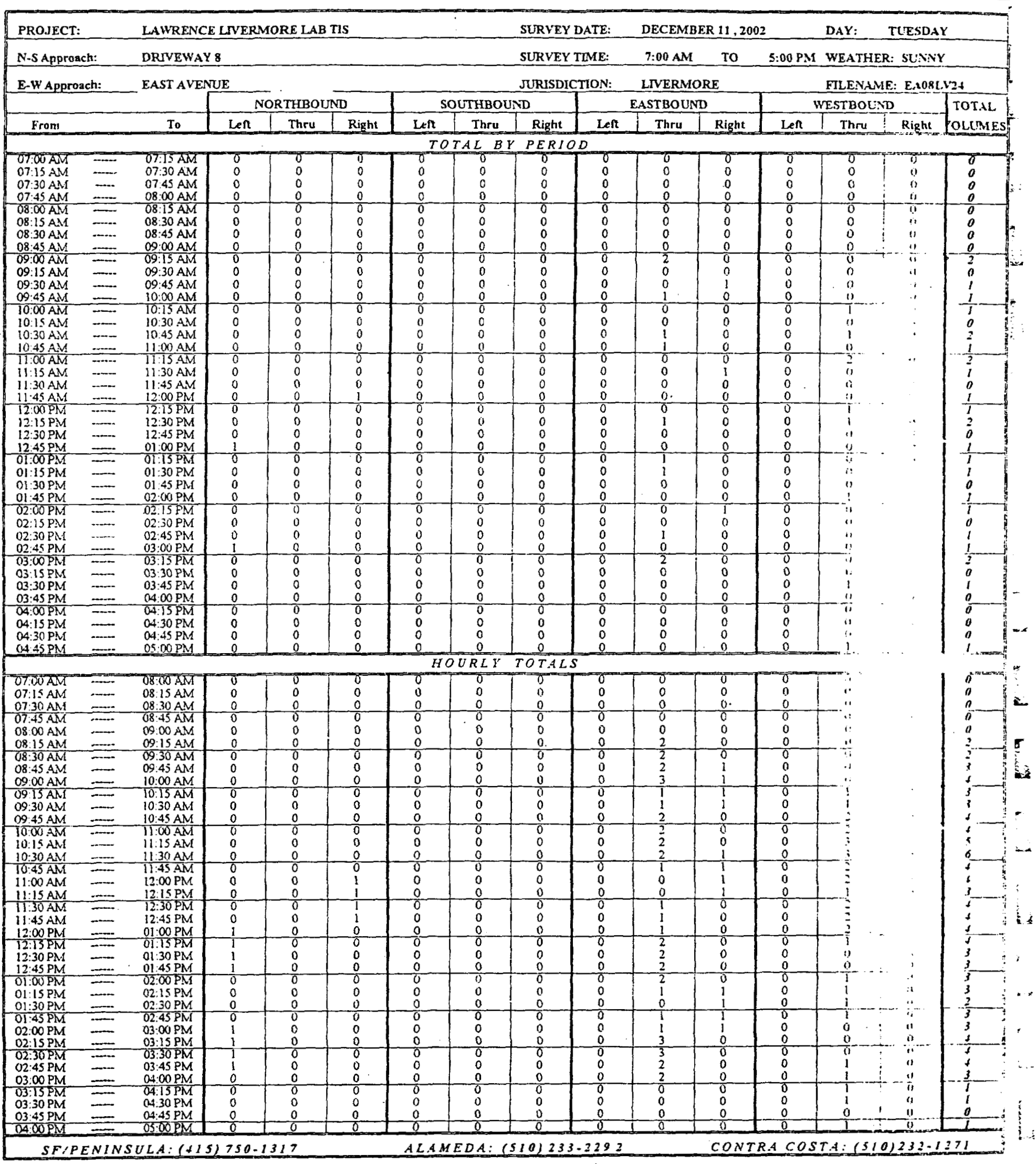




\section{Baymetrics Traffic Resources INTERSECTION TURNING MOVEMENT SUMMARY}

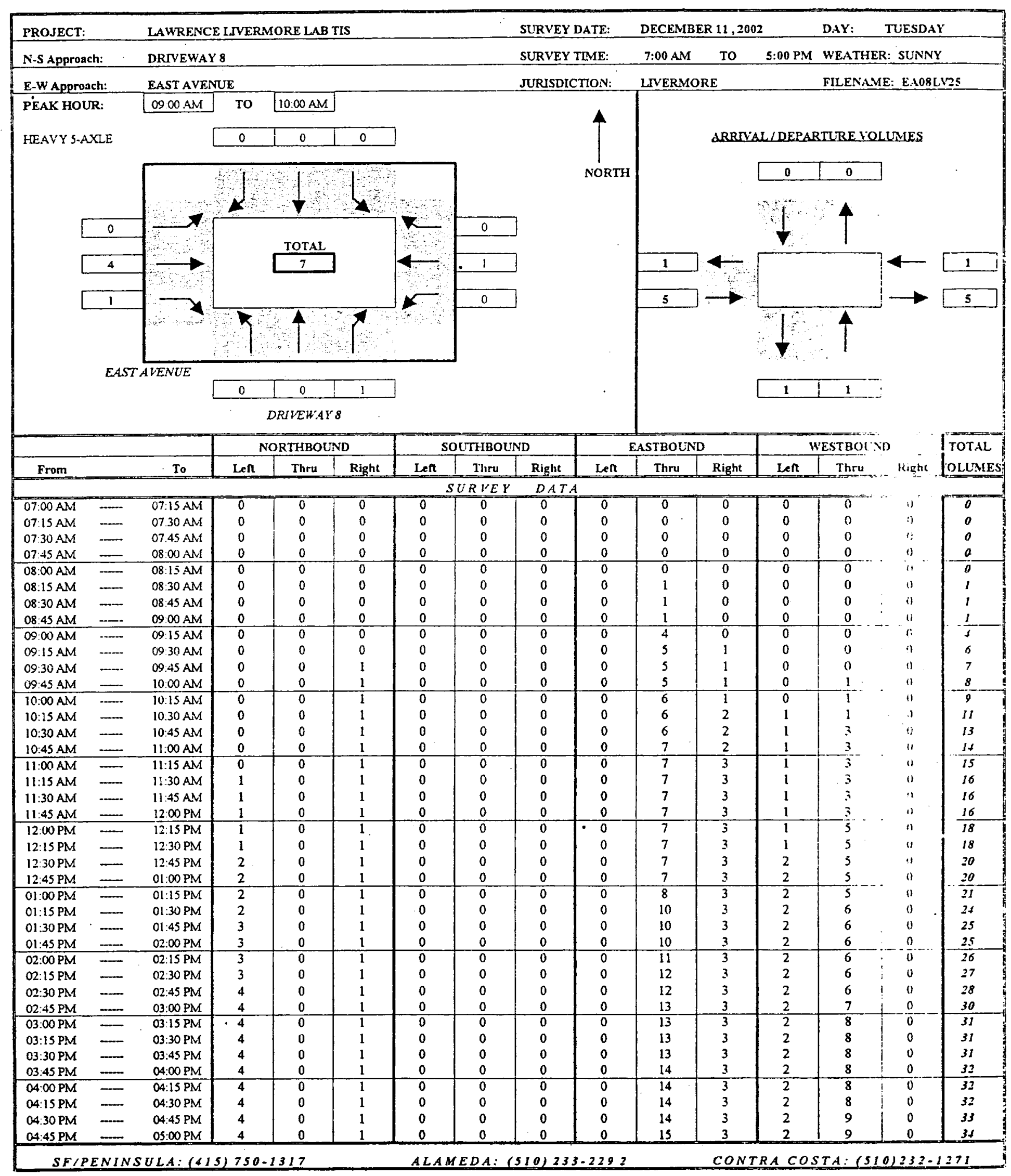




\section{Baymetrics Traffic Resources INTERSECTION TURNING MOVEMENT SUMMARY}

\begin{tabular}{|c|c|c|c|c|c|c|c|c|c|c|c|c|c|c|c|}
\hline \multirow{2}{*}{\multicolumn{2}{|c|}{$\begin{array}{l}\text { PROJECT: } \\
\text { N.S Approach: }\end{array}$}} & \multicolumn{6}{|c|}{ LAWRENCE LNVERMORE LAB TIS } & \multicolumn{2}{|c|}{ SURVEY DATE: } & \multicolumn{3}{|c|}{ DECEMBER 11,2002} & DAY: & \multicolumn{2}{|c|}{ TLESDAY } \\
\hline & & \multicolumn{6}{|c|}{ DRVVEWAY 8} & SURVEY & ME: & 7:00 ANI & ro & 5:00 PM & WEATH & R: SL'NNY & \\
\hline E-W_Appr & & EAST AVE & & & & & & JURISDIC & ON: & CVERMC & & & FILENA & E: EAO8L & 25 \\
\hline & & & & IHBO & & & THBO & & & STBOUN & & & ESTBOU & & TOTAL \\
\hline Erom & & To & Len & Thru & Right & Len & Thru & Right & Len & Thru & Right & Len & Ihru & Right & OLLUMES: \\
\hline & & & & & & & $A L B$ & PERIO & & & & & & & \\
\hline $\begin{array}{l}07.00 \mathrm{AM} \\
07.15 \mathrm{AM}\end{array}$ & $\ldots$ & $0715 \mathrm{AM}$ & $\begin{array}{l}0 \\
0\end{array}$ & 0 & 0 & 0 & 0 & 8 & $\sigma_{0}^{\circ}$ & 0 & $\begin{array}{l}0 \\
0\end{array}$ & 8 & $\begin{array}{l}0 \\
0\end{array}$ & $\overline{0}$ & 0 \\
\hline 07:30 AM & $\ldots$ & $07: 45 \mathrm{AM}$ & 0 & 0 & 0 & 0 & 0 & 0 & 0 & 0 & $\begin{array}{l}0 \\
0\end{array}$ & 0 & o & $\begin{array}{l}0 \\
0\end{array}$ & $\begin{array}{l}0 \\
0\end{array}$ \\
\hline$\frac{07.45 \mathrm{AM}}{08.00 \mathrm{AM}}$ & $\ldots$ & $\frac{0800 \mathrm{AM}}{08.15 \mathrm{AM}}$ & $\frac{0}{0}$ & $-\frac{0}{0}$ & 0 & 0 & 0 & 0 & 0 & 0 & 0 & 0 & 0 & 0 & \\
\hline $\begin{array}{l}08: 00 \mathrm{AM} \\
08: 15 \mathrm{AM}\end{array}$ & $\ldots$ & $\begin{array}{l}08: 13 \mathrm{AM} \\
08: 30 \mathrm{AM}\end{array}$ & $\begin{array}{l}0 \\
0\end{array}$ & 0 & $\begin{array}{l}0 \\
0\end{array}$ & $\begin{array}{l}0 \\
0\end{array}$ & $\begin{array}{l}0 \\
0\end{array}$ & $\begin{array}{l}0 \\
0\end{array}$ & $\begin{array}{l}0 \\
0\end{array}$ & $\begin{array}{l}0 \\
1\end{array}$ & $\begin{array}{l}0 \\
0\end{array}$ & $\begin{array}{l}0 \\
0\end{array}$ & $\begin{array}{l}0 \\
0\end{array}$ & $\begin{array}{l}1 \\
0\end{array}$ & i \\
\hline $0830 \mathrm{AM}$ & $=$ & $\begin{array}{l}08: 45 \mathrm{AM} \\
09.00 \mathrm{AM}\end{array}$ & 0 & $\begin{array}{l}0 \\
0\end{array}$ & 0 & 0 & 0 & 0 & 0 & 0 & $a$ & 0 & 0 & 0 & $a$ \\
\hline$\frac{08.45 \mathrm{AM}}{09: 00 \mathrm{AM}}$ & $\ldots$ & $\frac{09.00 \mathrm{AM}}{09.15 \mathrm{AM}}$ & 0 & $=$ & $\frac{0}{0}-$ & $\frac{0}{0}$ & $\frac{0}{0}$ & 0 & $\frac{0}{0}$ & $\frac{0}{3}$ & o & $\frac{0}{0}$ & $\frac{0}{0}$ & $\frac{1}{2}$ & 3 \\
\hline $09: 15 \mathrm{AM}$ & ....- & $09: 30 \mathrm{AM}$ & 0 & 0 & 0 & 0 & 0 & 0 & 0 & 1 & 1 & 0 & 0 & 0 & 3 \\
\hline $09: 30$ AM & $-\infty$ & $09: 45$ AM & 0 & 0 & 1 & 0 & 0 & 0 & 0 & 0 & 0 & 0 & 0 & i) & 3 \\
\hline $10: 00 \mathrm{AM}$ & $=$ & $\frac{1000 \mathrm{AM}}{10: 15 \mathrm{AM}}$ & $\frac{0}{0}$ & $\frac{0}{0}$ & $\frac{0}{0}$ & $\frac{0}{0}$ & $-\frac{0}{0}$ & $\frac{0}{0}$ & $\frac{0}{0}$ & $\frac{0}{1}$ & $\frac{0}{0}$ & $\frac{0}{0}$ & $\frac{1}{0}$ & $\frac{g}{4}$ & $I$ \\
\hline 10:15 AM & $-\infty$ & 10:30 AM & 0 & 0 & 0 & 0 & 0 & 0 & 0 & 0 & l & l & 0 & ii & 2 \\
\hline 10:30 AM & $\cdots$ & 10:45 AM & 0 & 0 & 0 & 0 & 0 & 0 & 0 & 0 & 0 & 0 & 2 & 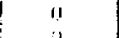 & 2 \\
\hline$\frac{10.45 \mathrm{AM}}{1100 \mathrm{AM}}$ & $=$ & $\frac{11: 00 \mathrm{AM}}{11.15 \mathrm{AM}}$ & $-\frac{0}{0}$ & $\frac{0}{0}$ & $\frac{0}{0}$ & $\frac{0}{0}$ & $\frac{0}{0}$ & $\frac{0}{0}$ & $\frac{0}{0}$ & $\frac{1}{0}$ & $\frac{0}{1}$ & 0 & & 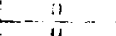 & 1 \\
\hline $11: 15 \mathrm{AM}$ & $\ldots$ & $11: 30 \mathrm{AM}$ & 1 & 0 & 0 & 0 & 0 & 0 & 0 & 0 & 0 & 0 & 0 & " & $i$ \\
\hline $11 ; 30 \mathrm{AM}$ & $\cdots$ & $11: 45 \mathrm{AM}$ & 0 & 0 & 0 & 0 & 0 & 0 & 0 & 0 & 0 & 0 & 0 & ; & 0 \\
\hline$\frac{11: 45 \mathrm{AM}}{12.00 \mathrm{PM}}$ & $\cdots$ & $\frac{12: 00 \mathrm{PM}}{12.15 \mathrm{PM}}$ & $\frac{0}{0}$ & $\frac{0}{0}$ & $\frac{0}{0}$ & $\frac{0}{0}$ & 0 & 0 & 0 & $\frac{a}{0}$ & $\frac{0}{2}$ & 9 & 0 & $\therefore$ & 0 \\
\hline 12:15 PM & $\cdots$ & $12: 30 \mathrm{PM}$ & 0 & 0 & 0 & 0 & $\begin{array}{l}0 \\
0\end{array}$ & $\begin{array}{l}0 \\
0\end{array}$ & 0 & $\begin{array}{l}0 \\
0\end{array}$ & $\begin{array}{l}0 \\
0 \\
0\end{array}$ & $\begin{array}{l}0 \\
0\end{array}$ & $0^{2}$ & $" 1$ & 2 \\
\hline 12:30 PM & ....- & 12:45 PM & 1 & 0 & 0 & 0 & 0 & 0 & 0 & 0 & 0 & 1 & 0 & 4 & 2 \\
\hline 12:45 PM & $\ldots$ & $0100 \mathrm{PM}$ & 0 & 0 & 0 & 0 & 0 & 0 & 0 & 0 & 0 & i & 0 & $\because$ & 0 \\
\hline $0100 \mathrm{PM}$ & $\cdots$ & $0115 \mathrm{PM}$ & 0 & 0 & 0 & 0 & 0 & 0 & 0 & 1 & 0 & 0 & 0 & $\because$ & $t$ \\
\hline 01:15 PM & $\cdots$ & $01: 30 \mathrm{PM}$ & 0 & 0 & 0 & 0 & 0 & 0 & 0 & 2 & 0 & 0 & 1 & i) & 3 \\
\hline 01:30 PM & -...- & $01.45 \mathrm{PM}$ & 1 & 0 & 0 & 0 & 0 & 0 & 0 & 0 & 0 & 0 & 0 & •; & 1 \\
\hline $01: 45 \mathrm{PM}$ & $\cdots$ & $02: 00 \mathrm{PM}$ & 0 & 0 & 0 & 0 & 0 & 0 & 0 & 0 & 0 & 0 & 0 & $\therefore$ & 0 \\
\hline $02: 00 \mathrm{PM}$ & $\cdots$ & $02: 15$ PM & 0 & 0 & 0 & 0 & 0 & 0 & 0 & $T$ & $\overline{0}$ & $\overline{0}$ & D & :: & $i$ \\
\hline $02: 15 \mathrm{PM}$ & $\ldots$ & 02:30 PM & 0 & 0 & 0 & 0 & 0 & 0 & 0 & 1 & 0 & 0 & 0 & $\because$ & \\
\hline $\begin{array}{l}02: 30 \mathrm{PM} \\
02.45 \mathrm{PM}\end{array}$ & $\ldots$ & $\begin{array}{l}02.45 \mathrm{PM} \\
03: 00 \mathrm{PM}\end{array}$ & $\begin{array}{l}1 \\
0\end{array}$ & $\begin{array}{l}0 \\
0\end{array}$ & $\begin{array}{l}0 \\
0\end{array}$ & $\begin{array}{l}0 \\
0\end{array}$ & $\begin{array}{l}0 \\
0\end{array}$ & $\begin{array}{l}0 \\
0\end{array}$ & $\begin{array}{l}0 \\
0\end{array}$ & i & $\begin{array}{l}0 \\
0\end{array}$ & 0 & i & $\therefore$ & ! \\
\hline $03: 00 \mathrm{PM}$ & - & $03.15 \mathrm{PM}$ & 0 & 0 & 0 & 0 & 0 & 0 & $\overline{0}$ & 0 & $\frac{\pi}{0}$ & $\frac{0}{0}$ & 1 & & $\because$ \\
\hline 03:15 PM & $\cdots$ & $03: 30 \mathrm{PM}$ & 0 & 0 & 0 & 0 & 0 & 0 & 0. & 0 & 0 & 0 & 0 & & \\
\hline $\begin{array}{l}03: 30 \mathrm{PM} \\
03.45 \mathrm{PM}\end{array}$ & $\ldots$ & $03: 45$ PM & $\begin{array}{l}0 \\
0\end{array}$ & $\begin{array}{l}0 \\
0\end{array}$ & $\begin{array}{l}0 \\
0\end{array}$ & $\begin{array}{l}0 \\
0\end{array}$ & 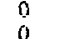 & 0 & $0_{0}^{0}$ & 0 & $0_{0}^{0}$ & 0 & 0 & $\because$ & 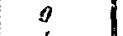 \\
\hline $0400 \mathrm{PM}$ & $\ldots$ & $04: 15 \mathrm{PM}$ & 0 & 0 & $\frac{\pi}{0}$ & 0 & $\frac{0}{0}$ & $\frac{0}{0}$ & $\frac{0}{0}$ & $\frac{1}{0}$ & $\frac{0}{0}$ & $\frac{0}{0}$ & $\frac{0}{v}$ & ") & $\frac{1}{0}$ \\
\hline $04: 15 \mathrm{PM}$ & $\ldots$ & $04: 30 \mathrm{PM}$ & 0 & 0 & 0 & 0 & 0 & 0 & 0 & 0 & 0 & 0 & 0 & "' & 0 \\
\hline $04: 30 \mathrm{PM}^{2}$ & +- & $04: 45 \mathrm{PM}$ & 0 & 0 & 0 & 0 & 0 & 0 & 0 & 0 & 0 & 0 & i & $:$ & $i$ \\
\hline $0445 \mathrm{PM}$ & $\ldots$ & $0500 \mathrm{PM}$ & 0 & 0 & 0 & 0 & 0 & 0 & 0 & 1 & 0 & $\underline{0}$ & 0 & $\therefore$ & \\
\hline & & & & & & & $R L Y$ & TOTAL & & & & & & & \\
\hline $07.00 \mathrm{AM}$ & - & 08:00 AM & 8 & 8 & 0 & 0 & 0 & 8 & 0 & $\overline{0}$ & 0 & $\overline{0}$ & 0 & $\therefore$ & 0 \\
\hline $\begin{array}{l}07: 15 \mathrm{AM} \\
07.30 \mathrm{AM}\end{array}$ & $=$ & $\begin{array}{l}08: 15 \text { AM } \\
08: 30 \mathrm{AM}\end{array}$ & $\begin{array}{l}0 \\
0\end{array}$ & $\begin{array}{l}0 \\
0 \\
\end{array}$ & $\begin{array}{l}0 \\
0 \\
0\end{array}$ & $\begin{array}{l}0 \\
0 \\
\end{array}$ & $\begin{array}{l}0 \\
0\end{array}$ & $\begin{array}{l}0 \\
0 \\
\end{array}$ & $\begin{array}{l}0 \\
0\end{array}$ & $\begin{array}{l}0 \\
1\end{array}$ & $\begin{array}{l}0 \\
0\end{array}$ & $\begin{array}{l}0 \\
0\end{array}$ & $\begin{array}{l}0 \\
0\end{array}$ & $\because "$ & 0 \\
\hline $07: 45 \mathrm{AM}$ & $=$ & $08: 45 \mathrm{AM}$ & 0 & 0 & 0 & 0 & 0 & 0 & 0 & $T$ & to & 0 & 0 & ; & $\frac{\pi}{3}$ \\
\hline 08:00 AM & $=$ & $09: 00 \mathrm{AM}$ & 0 & $0_{0}^{0}$ & $\begin{array}{l}0 \\
0\end{array}$ & 0 & 0. & 0 & 0 & 1 & 0 & 0 & 0 & $\because$ & 1 \\
\hline$\frac{08.15 \mathrm{AM}}{08.30 \mathrm{AM}}$ & $=$ & $\frac{09.15 \mathrm{AM}}{09 \cdot 30 \mathrm{AM}}$ & $\frac{0}{0}$ & $\frac{0}{0}$ & $\frac{0}{0}$ & $\frac{0}{0}$ & $\frac{0}{0}$ & 0 & 0 & $\frac{4}{4}$ & $\frac{0}{1}$ & 0 & $\frac{0}{1}$ & & \\
\hline $08: 45 \mathrm{AM}$ & 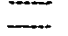 & $09.45 \mathrm{AM}$ & 0 & 0 & 1 & 0 & 0 & 0 & 0 & $\frac{7}{4}$ & 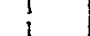 & 0 & 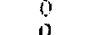 & “: & 5 \\
\hline $09: 00 \mathrm{AM}$ & $\ldots$ & $1000) \mathrm{AM}$ & 0 & 0 & 1 & 0 & 0 & 0 & 0 & 4 & .1 & $\begin{array}{l}0 \\
0\end{array}$ & $i$ & $\ddot{.1}$ & $\frac{6}{7}$ \\
\hline $09.15 \mathrm{AM}$ & $\ldots$ & $10: 15 \mathrm{AM}$ & 0 & 0 & 1 & 0 & 0 & 0 & 0 & 2 & 1 & 0 & $T$ & . & 5 \\
\hline 09:30 AM & - & 10:30 AM & 0 & 0 & 1 & 0 & 0 & 0 & & 1 & i & 1 & I & .. & $s$ \\
\hline $09: 45 \mathrm{AM}$ & $=$ & $10: 45 \mathrm{AM}$ & 0 & $a$ & 0 & 0 & 0 & 0 & 0 & & 1 & 1 & 3 & $: 1$ & $\because "$ \\
\hline $10: 00 \mathrm{AM}$ & $\ldots$ & $11: 00 \overline{\mathrm{AM}}$ & 0 & 0 & 0 & 0 & 0 & $\overline{0}$ & 0 & 2 & 1 & $T$ & 2 & .. & $\bar{\sigma}$ \\
\hline $\begin{array}{l}10: 15 \mathrm{AM} \\
10: 30 \mathrm{AM}\end{array}$ & $\bar{E}$ & $\begin{array}{l}11: 15 \mathrm{AM} \\
11: 30 \mathrm{AM}\end{array}$ & $\begin{array}{l}0 \\
1\end{array}$ & 0 & $\begin{array}{l}0 \\
0\end{array}$ & $\begin{array}{l}0 \\
0\end{array}$ & $\begin{array}{l}0 \\
0\end{array}$ & $\begin{array}{l}0 \\
0\end{array}$ & $\begin{array}{l}0 \\
0\end{array}$ & 1 & $\frac{2}{1}$ & $\begin{array}{l}1 \\
0\end{array}$ & 2 & ، & $\begin{array}{l}6 \\
5\end{array}$ \\
\hline $10: 45 \mathrm{AM}$ & 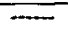 & $11: 45 \mathrm{AM}$ & $i$ & 0 & 0 & $\overline{0}$ & 0 & $\overline{0}$ & 0 & 3 & $i$ & 0 & 0 & $\ddot{i}$ & $\frac{3}{3}$ \\
\hline 11:00 AM & $\ldots$ & $12: 00 \mathrm{PM}$ & 1 & 0 & $\begin{array}{l}0 \\
0\end{array}$ & 0 & $\begin{array}{l}0 \\
0\end{array}$ & $\begin{array}{l}0 \\
0\end{array}$ & 0 & $\begin{array}{l}0 \\
0\end{array}$ & 1 & 0 & 0 & :" & $?$ \\
\hline$\frac{11: 15 \mathrm{AM}}{11.30 \mathrm{AM}}$ & $\equiv$ & $\frac{12: 15 \mathrm{PM}}{12 \cdot 30 \mathrm{PM}}$ & $\frac{1}{0}$ & $\frac{0}{0}$ & $\frac{0}{0}$ & $\frac{0}{0}$ & $\frac{0}{0}$ & $\frac{0}{0}$ & $\frac{0}{0}$ & $\frac{0}{0}$ & $\frac{0}{0}$ & $\frac{0}{0}$ & $\frac{2}{2}$ & $\ddot{3}$ & $\frac{3}{7}$ \\
\hline $11: 45 \mathrm{AM}$ & - & $12: 45 \mathrm{PM}$ & 1 & 0 & 0 & 0 & 0 & 0 & 0 & 0 & 0 & i & 2 & $"$ & 3 \\
\hline $12: 00 \mathrm{PM}$ & $\ldots$ & $0100 \mathrm{PM}$ & 1 & 0 & 0 & 0 & 0 & $\frac{0}{1}$ & 0 & 0 & $\underline{0}$ & $\frac{1}{1}$ & & 11. & f \\
\hline $12: 15 \mathrm{PM}$ & $-\infty$ & $01: 15 \mathrm{PM}$ & 1 & 0 & 0 & 0 & $a$ & $\overline{0}$ & 0 & 1 & 0 & 1 & $\overline{0}$ & $\pi$ & 3 \\
\hline $\begin{array}{l}\text { 12:30 PM } \\
12.45 \text { PM }\end{array}$ & $\cdots$ & $\begin{array}{l}01: 30 \mathrm{PM} \\
01.45 \mathrm{PM}\end{array}$ & 1 & $\begin{array}{l}0 \\
0 \\
0\end{array}$ & $\begin{array}{l}0 \\
0\end{array}$ & $\begin{array}{l}0 \\
0\end{array}$ & $\begin{array}{l}0 \\
0\end{array}$ & $\begin{array}{l}0 \\
0\end{array}$ & $\begin{array}{l}0 \\
0\end{array}$ & $\frac{3}{3}$ & $\begin{array}{l}0 \\
0\end{array}$ & $\begin{array}{l}1 \\
0\end{array}$ & 1 & "1 & 6 \\
\hline$\frac{124 \mathrm{SM}}{01: 00 \mathrm{PM}}$ & $=$ & $02: 00 \mathrm{PM}$ & 1 & 0 & $\frac{5}{0}$ & 0 & 0 & 0 & 0 & $\frac{\pi}{3}$ & 0 & 0 & 1 & 6 & $\frac{3}{5}$ \\
\hline $01: 15$ PM & $\cdots$ & 02:15 PM & 1 & $\stackrel{0}{0}-1$ & 0 & 0 & 0 & $\begin{array}{l}0 \\
0\end{array}$ & 0 & 3 & 0 & 0 & 1 & 11 & 5 \\
\hline 01:30 PM & - & & 1 & 0 & $\frac{0}{0}$ & $\frac{0}{0}$ & 0 & $\frac{0}{0}$ & $=0$ & $\frac{2}{2}$ & $\frac{0}{0}$ & 0 & $\frac{0}{10}$ & a & 3 \\
\hline $01: 45$ PM & - & $02: 45 \mathrm{PM}$ & 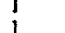 & $0_{0}^{0}$ & o & $\begin{array}{l}0 \\
0\end{array}$ & 0 & $\begin{array}{l}0 \\
0 \\
0\end{array}$ & 0 & 3 & ${ }_{0}^{0}$ & 0 & 0 & ij & 3 \\
\hline $\begin{array}{l}\text { 02:00 PM } \\
02: 15 \mathrm{PM}\end{array}$ & $=$ & $\begin{array}{l}\text { 03:00 PM } \\
03: 15 \text { PM }\end{array}$ & $\begin{array}{l}1 \\
1\end{array}$ & $\begin{array}{l}0 \\
0\end{array}$ & 0 & 0 & $\begin{array}{l}0 \\
0\end{array}$ & $\begin{array}{l}D_{0} \\
0\end{array}$ & 0 & $\frac{3}{2}$ & $\begin{array}{l}0 \\
0\end{array}$ & 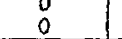 & $\frac{1}{2}$ & $\begin{array}{l}0 \\
0\end{array}$ & $\begin{array}{l}5 \\
3\end{array}$ \\
\hline $02: 30 \mathrm{PM}$ & $=$ & $03: 30 \mathrm{PM}$ & 1 & 0 & 0 & 0 & 0 & 0 & 0 & $T$ & 0 & 0 & $\overline{2}$ & 0 & 7 \\
\hline 02:45 PM & $\cdots$ & 03:45 PM & 0 & 0 & 0 & 0 & 0 & 0 & 0 & i & 0 & 0 & 2 & 0 & 3 \\
\hline & 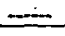 & 04:00 FM & 0 & 0 & $a$ & $\frac{0}{0}$ & 0 & 0 & 0 & 1 & 0 & 0 & 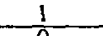 & 0 & 9 \\
\hline $03: 15 \mathrm{PM}$ & - & $04: 15 \mathrm{PM}$ & 0 & 0 & 0 & 0 & 0 & 0 & 0 & 1 & 0 & 0 & 0 & 0 & 1 \\
\hline 03:30 PM & - & 04:30 PM & 0 & $\begin{array}{l}0 \\
0 \\
0\end{array}$ & $\begin{array}{l}0 \\
0\end{array}$ & $\begin{array}{l}0 \\
0 \\
0\end{array}$ & $0_{0}^{0}$ & $\begin{array}{l}0 \\
0 \\
0\end{array}$ & $\begin{array}{l}0 \\
0\end{array}$ & $\begin{array}{l}1 \\
1\end{array}$ & ${ }_{0}^{0}$ & ${ }_{0}^{0}$ & $\begin{array}{l}0 \\
1\end{array}$ & $\begin{array}{l}0 \\
0\end{array}$ & 3 \\
\hline$\frac{03: 45 \mathrm{PM}}{04: 00 \mathrm{PM}}$ & $\Rightarrow$ & $\frac{04: 45 P M}{05.00 P M}$ & $\frac{0}{0}$ & 0 & 0 & 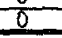 & 0 & 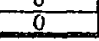 & 0 & & 0 & o & & 0 & \\
\hline$S F / F$ & $N I N$ & $U L A:(4)$ & 5 & & & $A L$ & $2 A$ & $0) 2$ & 29 & & $\mathrm{CO}$ & $c$ & 1: & 232 & 81 \\
\hline
\end{tabular}




\section{Baymetrics Traffic Resources INTERSECTION TURNING MOVEMENT SUMMARY}

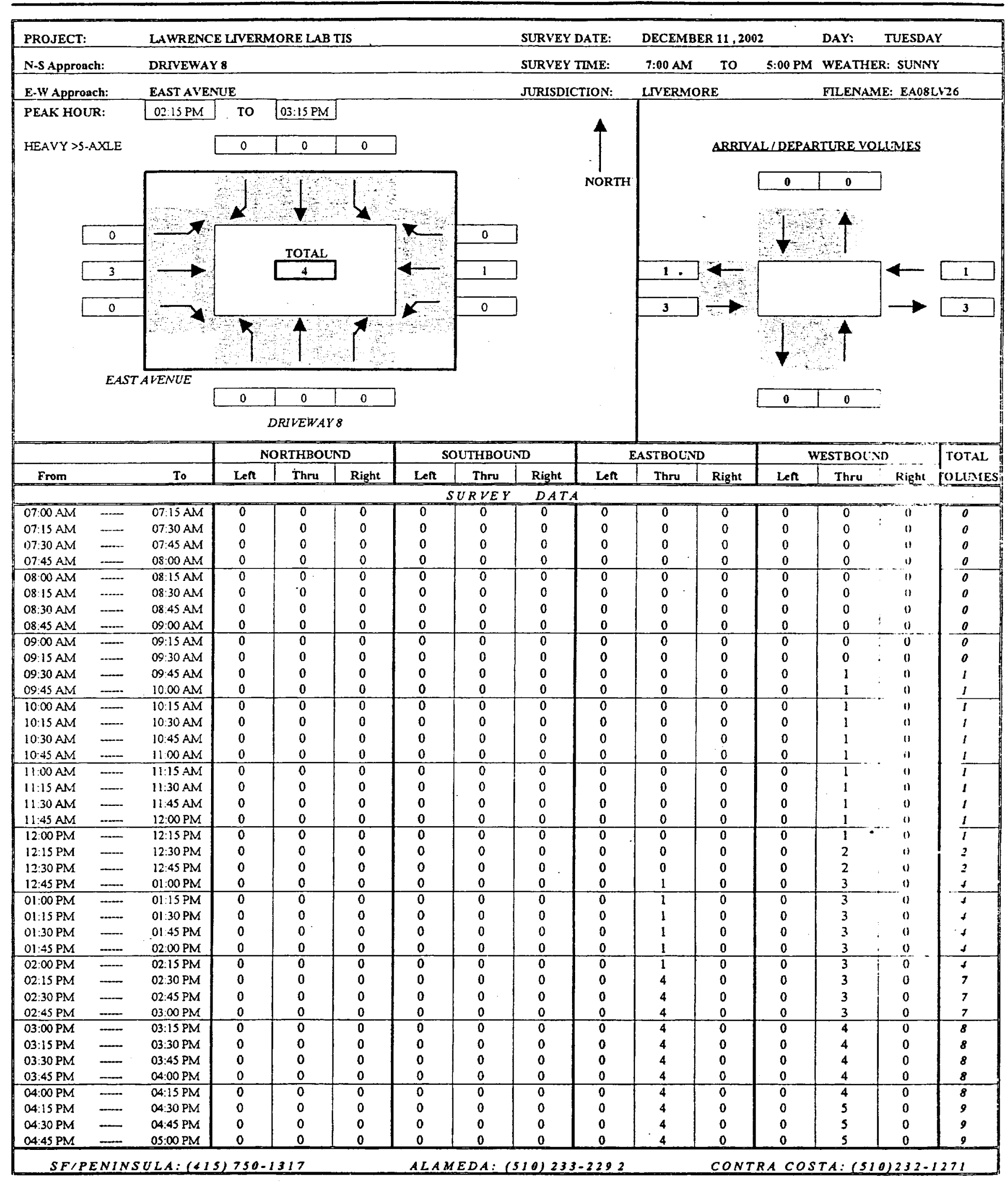




\section{Baymetrics Traffic Resources INTERSECTION TURNING MOVEMENT SUMMARY}

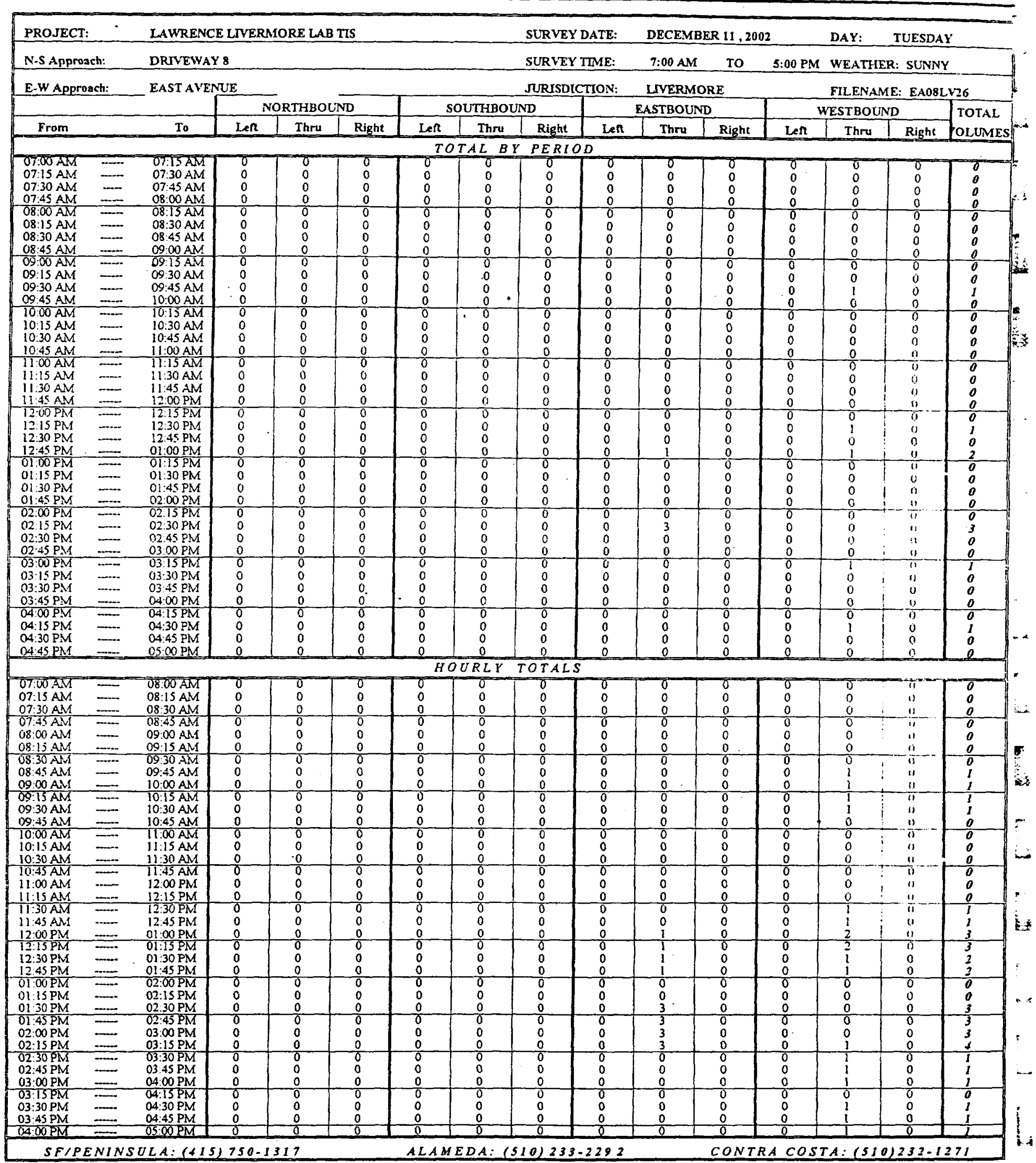




\section{Truck Counts}

Locations 7 and 8

Wednesday, December 12, 2001 



\section{Baymetrics Traffic Resources INTERSECTION TURNING MOVEMENT SUMMARY}

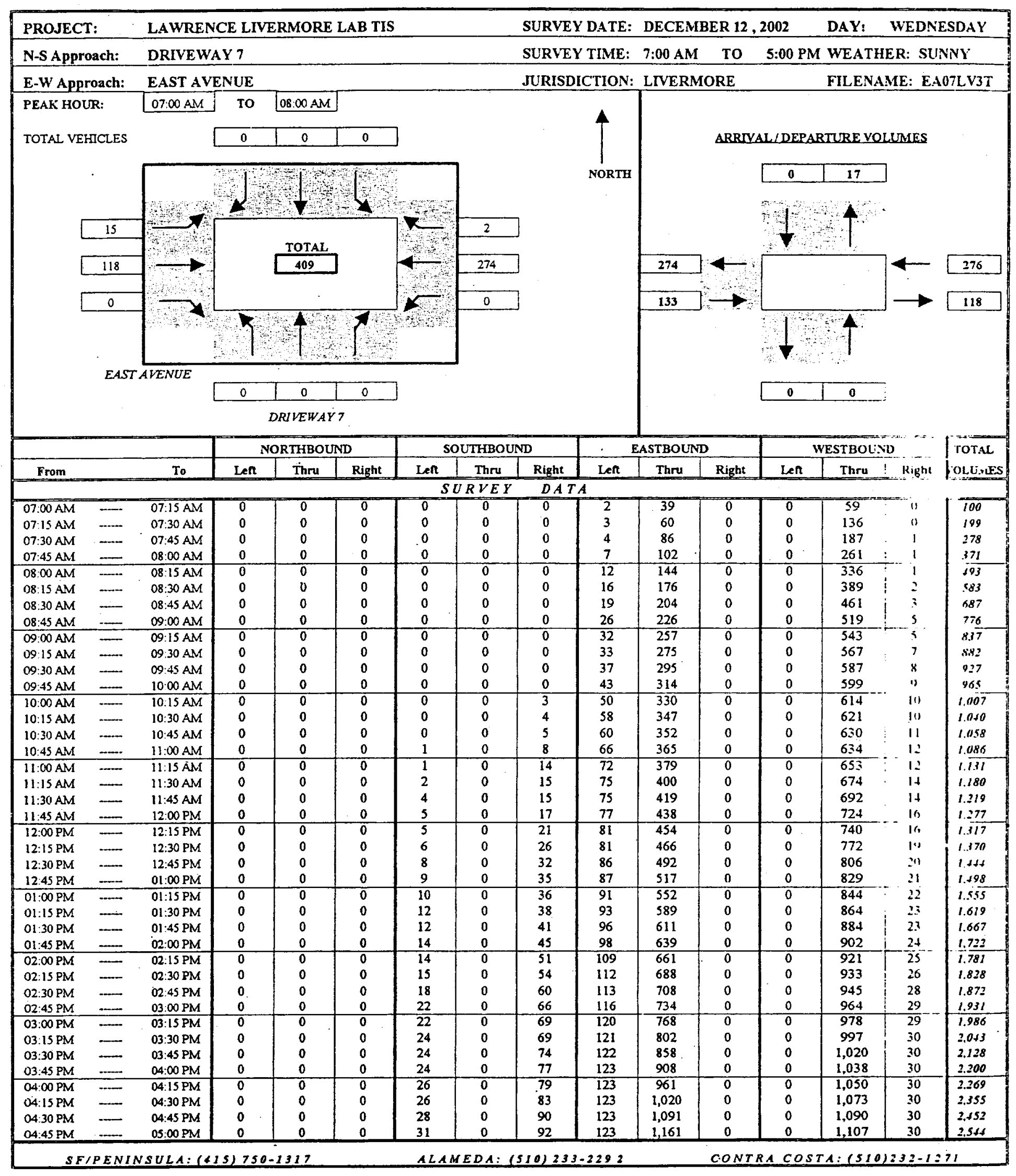




\section{Baymetrics Traffic Resources INTERSECTION TURNING MOVEMENT SUMMARY}

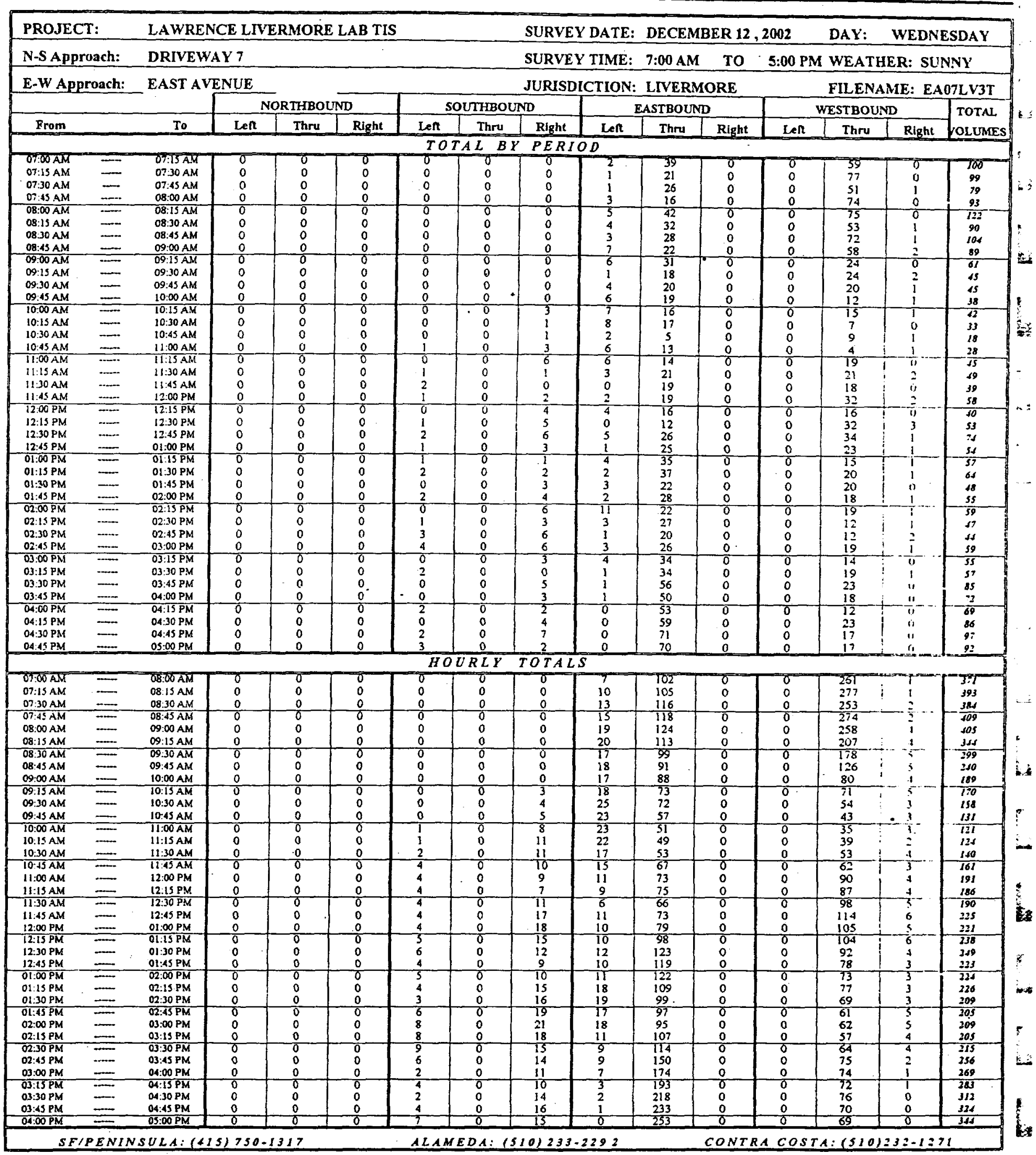




\section{Baymetrics Traffic Resources INTERSECTION TURNING MOVEMENT SUMMARY}

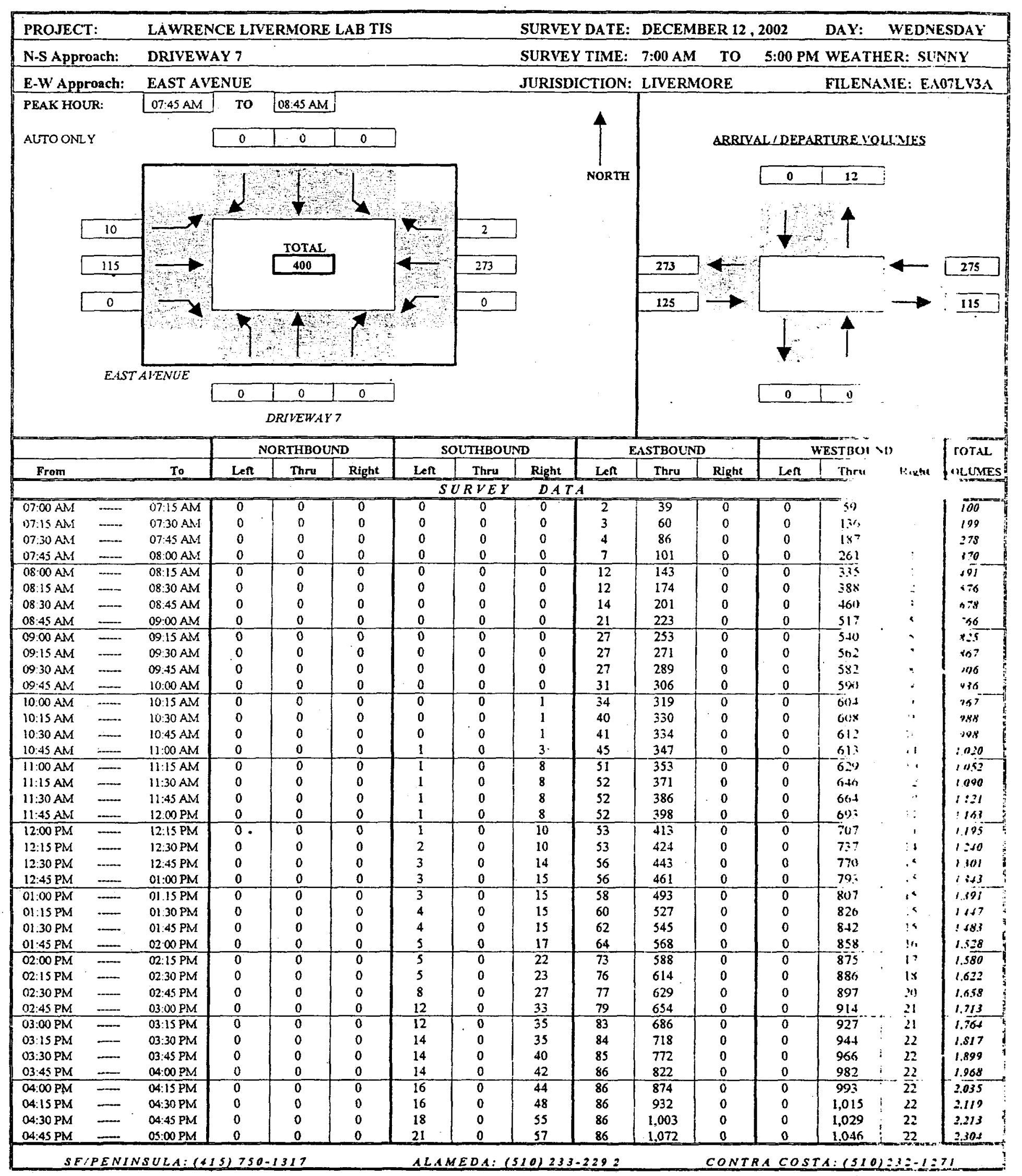




\section{$\frac{\text { Baymetrics Traffic Resources }}{\text { INTERSECTION TURNING MOVEMENT SUMMARY }}$}

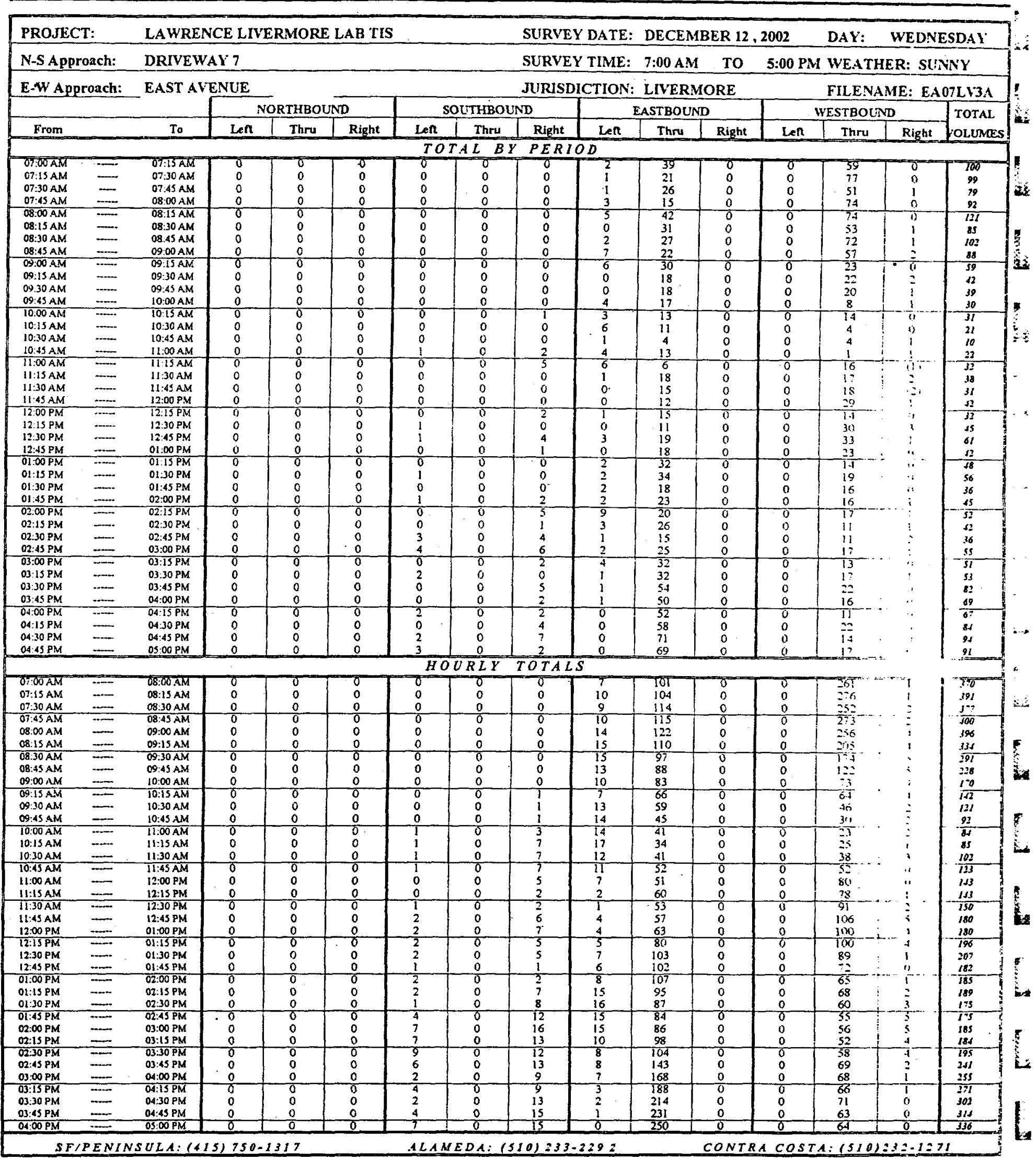




\section{Baymetrics Traffic Resources INTERSECTION TURNING MOVEMENT SUMMARY}

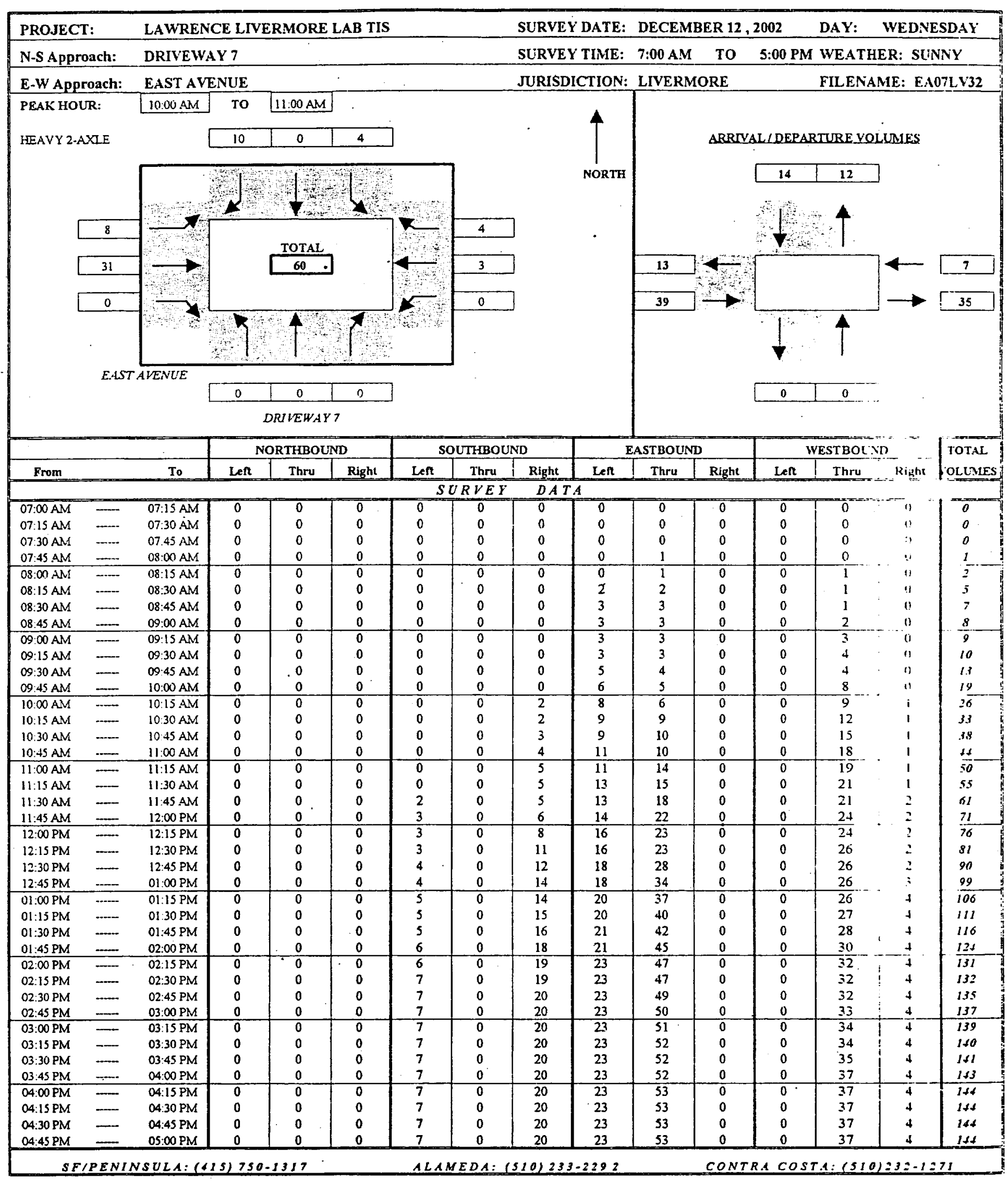




\section{Baymetrics Traffic Resources INTERSECTION TURNING MOVEMENT SUMMARY}

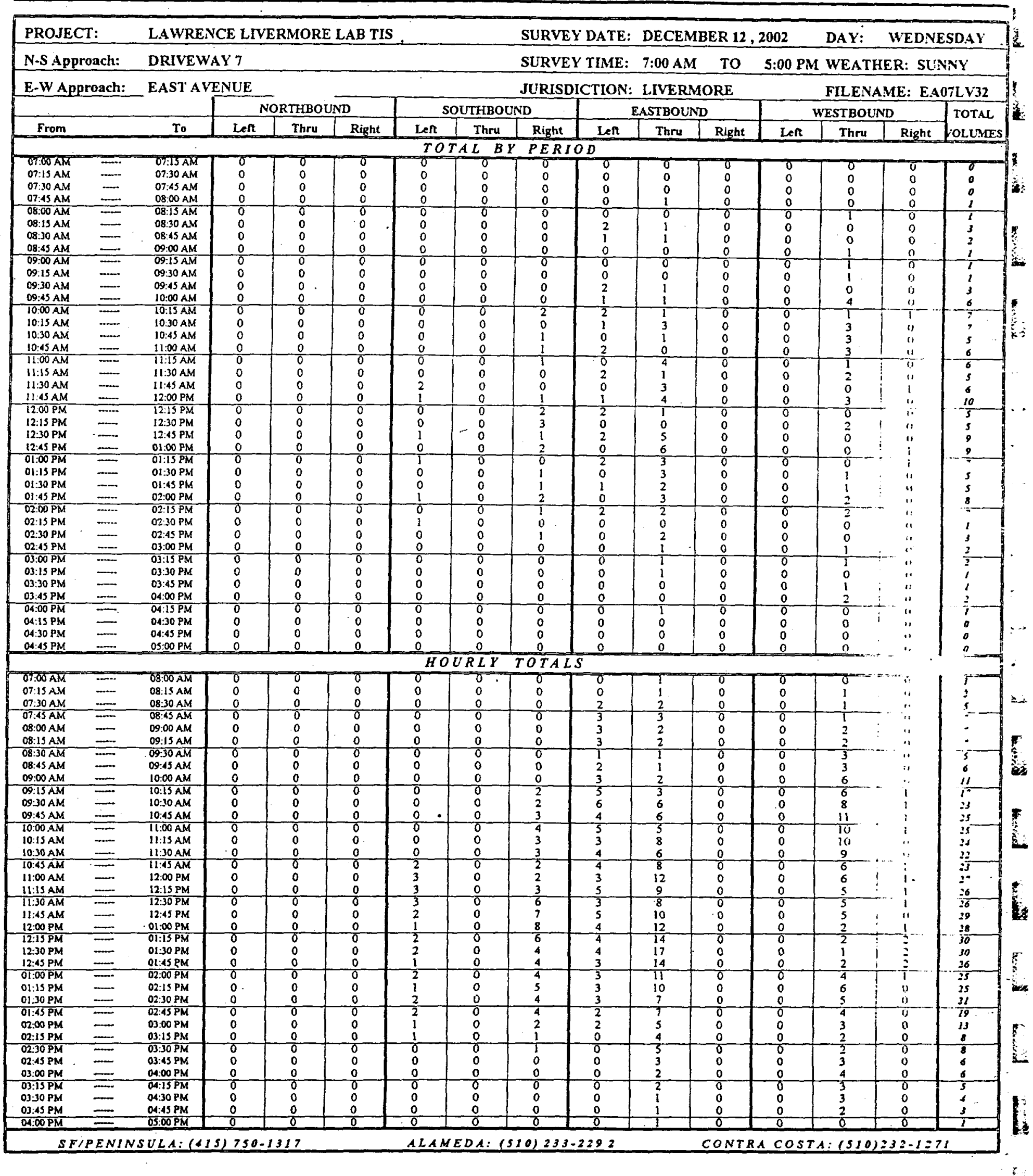




\section{Baymetrics Traffic Resources INTERSECTION TURNING MOVEMENT SUMMARY}

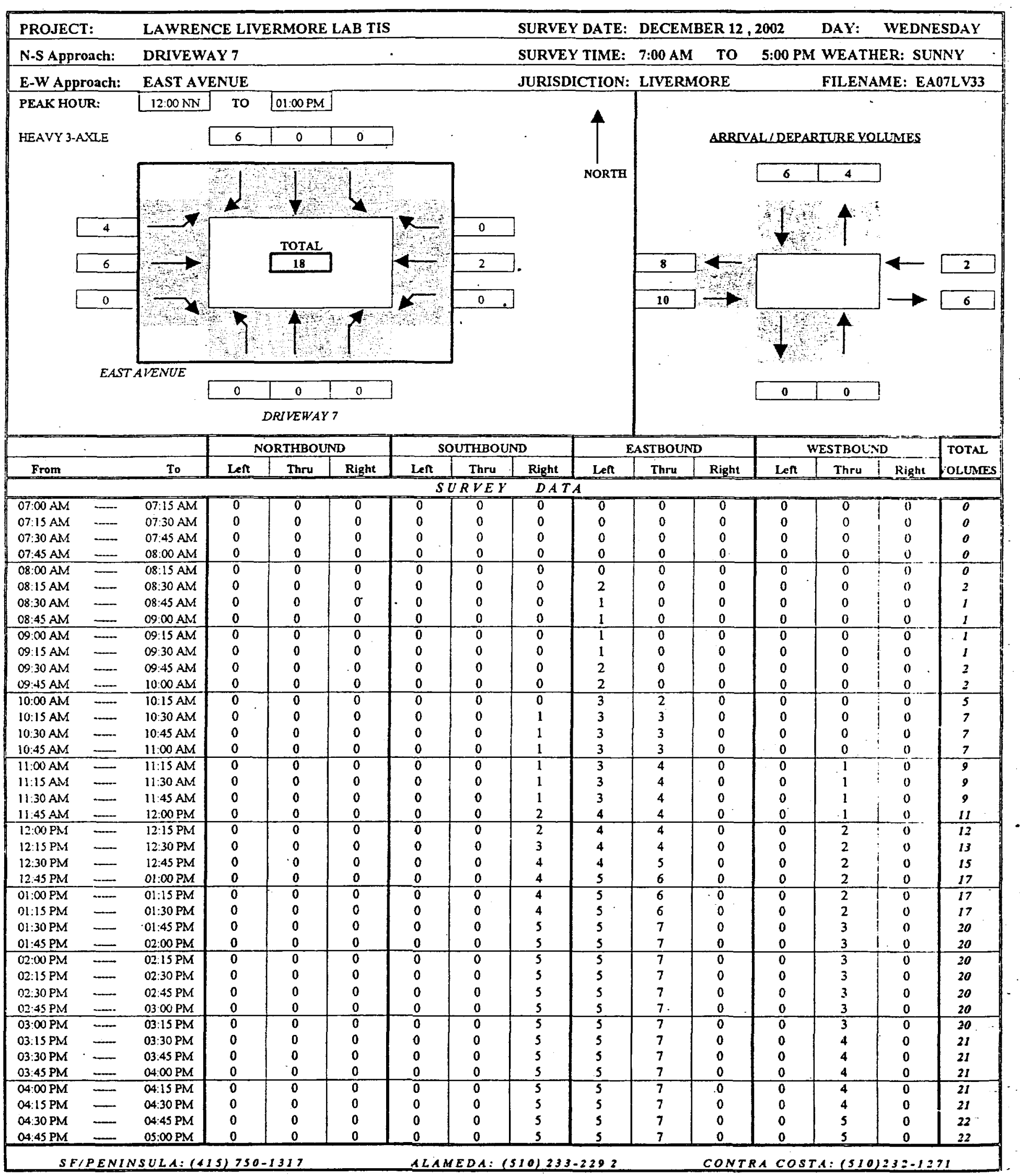




\section{$\frac{\text { B a ymetrics Traffic Resources }}{\text { INTERSECTION TURNING MOVEMENT SUMMARY }}$}

\begin{tabular}{|c|c|c|c|c|c|c|c|c|c|c|c|c|c|c|c|}
\hline \multirow{2}{*}{\multicolumn{2}{|c|}{$\begin{array}{l}\text { PROJECT: } \\
\text { N-S Approach: }\end{array}$}} & \multicolumn{6}{|c|}{ LAWRENCE LIVERMORE LAB TIS } & \multicolumn{5}{|c|}{ SURVEY DATE: DECEMBER 12, 2002} & DAY: & WEDN & SDAY \\
\hline & & DRIVEY & & & & & & SURVEY & rIME: & $7: 00 \mathrm{AM}$ & To & 5:00 PM & WEAT & ER: SL: & $\mathbf{N Y}$ \\
\hline E-W Ap & ach: & EAST AI & UE & & & & & JURISD & TION & LIVERN & RE & & FILEN & ME: E: & $7 \mathrm{l}, \mathrm{V33}$ \\
\hline & & & & THBO & & & THBOI & & & ASTBOUN & & & ESTBOL & & TOTAL \\
\hline From & & To & Left & Thru & Right & Left & Thru & Right & Len & Thru & Right & Len & Thru & Right & LOLUACES \\
\hline & & & & & & $T O$ & $L \quad B$ & $P E R I$ & & & & & & & \\
\hline $\begin{array}{l}\text { O7.00AM } \\
07: 15 \mathrm{AM}\end{array}$ & $\ldots$ & $\begin{array}{l}\text { 7.15 AM } \\
07: 30 \mathrm{AM}\end{array}$ & 0 & $\begin{array}{l}0 \\
0\end{array}$ & $\begin{array}{l}0 \\
0\end{array}$ & $\begin{array}{l}\sigma \\
0\end{array}$ & 0 & o & $\begin{array}{l}0 \\
0\end{array}$ & 0 & $\begin{array}{l}0 \\
0\end{array}$ & $\begin{array}{l}0 \\
0\end{array}$ & 0 & i & 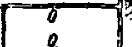 \\
\hline $07: 30 \mathrm{AM}$ & $\ldots$ & $07: 45 \mathrm{AM}$ & 0 & 0 & 0 & 0 & 0 & 0 & 0 & 0 & 0 & 0 & 0 & a) & 0 \\
\hline$\frac{07.45 \mathrm{AM}}{08.00 \mathrm{AM}}$ & $=$ & $\frac{08: 00 \mathrm{AM}}{08: 15 \mathrm{AM}}$ & $\frac{0}{0}$ & $\frac{0}{0}$ & $\frac{0}{0}$ & $\frac{0}{0}$ & $\frac{0}{0}$ & 0 & 0 & $\frac{0}{a}$ & 0 & $\frac{0}{0}$ & $\frac{0}{0}$ & 8 & $\therefore$ \\
\hline 08.15 AM & - & $08: 30 \mathrm{AM}$ & 0 & 0 & 0 & 0 & 0 & $\begin{array}{l}0 \\
0\end{array}$ & $\frac{0}{2}$ & $\begin{array}{l}0 \\
0\end{array}$ & 0 & 年 & 0 & 0 & 3 \\
\hline $\begin{array}{l}\text { O8:30 AM } \\
08.45 \mathrm{AM}\end{array}$ & $\equiv$ & $\begin{array}{l}08: 45 \mathrm{AM} \\
09: 00 \mathrm{AM}\end{array}$ & $\begin{array}{l}0 \\
0\end{array}$ & $\begin{array}{l}0 \\
0\end{array}$ & $\begin{array}{l}0 \\
0\end{array}$ & $\begin{array}{l}0 \\
0\end{array}$ & $\begin{array}{l}0 \\
0\end{array}$ & 0 & (i) & 0 & 0 & $\begin{array}{l}0 \\
0\end{array}$ & $\begin{array}{l}0 \\
0\end{array}$ &. & (d) \\
\hline $09: 00 \mathrm{AM}$ & $=$ & $09: 15 \mathrm{AM}$ & 0 & 0 & 0 & 0 & 0 & 0 & $\frac{0}{0}$ & $\frac{0}{0}$ & 0 & 0 & $\frac{u}{u}$ & $\ddot{i}$ & $\frac{\pi}{0}$ \\
\hline 0:15 AM & $\cdots$ & $09: 30 \mathrm{AM}$ & 0 & 0 & 0 & 0 & 0 & 0 & 0 & 0 & 0 & 0 & a & $\because$ & 0 \\
\hline $09: 30 \mathrm{AM}$ & $\cdots$ & 09:45 AM & 0 & 0 & 0 & 0 & 0 & 0 & 1 & 0 & 0 & 0 & 0 & ". & 1 \\
\hline 09:45 AM & $=$ & $10: 00 \mathrm{AM}$ & 0 & 0 & 0 & 0 & 0 & 0 & 0 & 0 & 0 & 0 & a & $\cdot 3$ & 0 \\
\hline $10.00 \mathrm{AM}$ & $\cdots$ & $10: 15 \mathrm{AM}$ & 0 & 0 & 0 & 0 & 0 & 0 & 1 & 2 & 0 & 0 & $0^{\circ}$ & . & 3 \\
\hline 10.15 AM & $\cdots$ & $10: 30 \mathrm{AM}$ & 0 & 0 & 0 & 0 & 0 & 1 & 0 & 1 & 0 & 0 & 0 & $\therefore$ & 2 \\
\hline $10: 30 \mathrm{AM}$ & $\cdots$ & $10: 45$ AM & 0 & 0 & 0 & 0 & 0 & 0 & 0 & 0 & 0 & 0 & 0 & & 0 \\
\hline $10: 45 \mathrm{AM}$ & $\ldots$ & $11: 00 \mathrm{AM}$ & 0 & 0 & 0 & 0 & 0 & 0 & 0 & 0 & 0 & 0 & $\underline{a}$ & & 0 \\
\hline $11: 00 \mathrm{AM}$ & $\cdots$ & $11: 15 . \bar{M}$ & 0 & 0 & 0 & 0 & 0 & 0 & 0 & 1 & $\overline{0}$ & 0 & 1 & - & $\frac{2}{2}$ \\
\hline $11: 15 \mathrm{AM}$ & 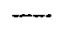 & 11:30 AMS & 0 & 0 & 0 & 0 & 0 & 0 & 0 & 0 & 0 & 0 & " & & 0 \\
\hline $11: 30 \mathrm{AM}$ & $\cdots$ & $11: 45 \mathrm{AM}$ & 0 & 0 & 0 & 0 & 0 & 0 & 0 & 0 & 0 & 0 & ii & & $a$ \\
\hline$\frac{11: 45 \mathrm{AM}}{12: 00 \mathrm{PM}}$ & $=$ & $\frac{12: 00 \mathrm{PM}}{12: 15 \mathrm{PM}}$ & 0 & 0 & 0 & 0 & $\frac{0}{n}$ & $\frac{1}{0}$ & 1 & $0^{\circ}$ & 0 & $\frac{0}{0}$ & $\because$ & . & 2 \\
\hline 12:15 PM & $\ldots$ & $\begin{array}{l}\text { 12:15 PM } \\
12: 30 \mathrm{PM}\end{array}$ & $\begin{array}{l}0 \\
0\end{array}$ & $\begin{array}{l}0 \\
0\end{array}$ & $\begin{array}{l}0 \\
0\end{array}$ & $\begin{array}{l}0 \\
0\end{array}$ & $\begin{array}{l}0 \\
0 \\
0\end{array}$ & $\begin{array}{l}0 \\
1\end{array}$ & $\begin{array}{l}0 \\
0\end{array}$ & $\begin{array}{l}0 \\
0 \\
0\end{array}$ & $\begin{array}{l}0 \\
0\end{array}$ & : & $\vdots$ & & $i$ \\
\hline 12:30 PM & $\ldots$ & $12.45 \mathrm{PM}$ & 0 & 0 & 0 & 0 & 0 & 1 & 0 & $i$ & 0 & 0 & $\because$ & & 2 \\
\hline 12:45 PM & $=$ & $01: 00 \mathrm{PM}$ & 0 & 0 & 0 & 0 & 0 & 0 & 1 & 1 & 0 & 0 & $\therefore$ & & 2 \\
\hline $0 !: 00 \mathrm{PM}$ & $\cdots$ & $01: 15 \mathrm{PM}$ & 0 & 0 & 0 & 0 & 0 & 0 & 0 & 0 & 0 & 0 & ii & & 0 \\
\hline 01:15 PM & - & $01: 30$ PM & 0 & 0 & 0 & 0 & 0 & 0 & 0 & 0 & 0 & 0 & 11 & & $"$ \\
\hline 01:30 PM & ... & $01: 45$ PM & 0 & 0 & 0 & 0 & 0 & 1 & 0 & 1 & 0 & 0 & 1 & & 3 \\
\hline 01:45 PM & $\cdots$ & $02: 00 \mathrm{PM}$ & 0 & 0 & 0 & $\frac{0}{0}$ & 0 & 0 & 0 & 0 & 0 & 0 & $\because$ & & $n$ \\
\hline $02: 00 \mathrm{PM}$ & $=$ & $02: 159 \mathrm{PM}$ & 0 & 0 & 0 & 0 & 0 & 0 & 0 & 0 & 0 & 0 & $\because$ & & $\bar{n}$ \\
\hline $02: 15 \mathrm{PM}$ & - & 02:30 PM & 0 & 0 & 0 & 0 & 0 & 0 & 0 & 0 & 0 & 0 & s. & & $a$ \\
\hline 02:30 PM & $\cdots$ & 02:45 PM & 0 & 0 & 0 & 0 & 0 & 0 & o & 0 & 0 & 0 & ". & & $n$ \\
\hline $02: 45$ PM & $\ldots$ & $03: 00 \mathrm{PM}$ & 0 & 0 & 0 & 0 & 0 & 0 & 0 & 0 & 0 & 0 & $\therefore$ & & $?$ \\
\hline$\overline{03.00 \mathrm{PM}}$ & $=$ & $03: 15 \mathrm{PM}$ & 0 & 0 & 0 & 0 & 0 & 0 & 0 & 0 & 0 & 0 & $\because$ & . & $"$ \\
\hline $03: 15 \mathrm{PM}$ & $-\infty$ & 03:30 PM & 0 & 0 & 0 & 0 & $\dot{0}$ & 0 & 0 & 0 & 0 & 0 & 1 & & 1 \\
\hline 03:30 PM & - & 03:45 PM & 0 & 0 & 0 & 0 & 0 & 0 & 0 & 0 & 0 & 0 & i) & & 0 \\
\hline 03:45 PM & - & $04: 00 \mathrm{PM}$ & 0 & 0 & 0 & 0 & 0 & 0 & 0 & 0 & 0 & 0 & :i & & 0 \\
\hline $04.00 \mathrm{PM}$ & $=$ & $04: 15 \mathrm{PM}$ & 0 & 0 & 0 & 0 & 0 & 0 & 0 & $\gamma$ & 0 & 0 & 7 & $\cdot$ & 0 \\
\hline $\begin{array}{l}04: 15 \text { PM } \\
04: 30 \mathrm{PMM}\end{array}$ & $\cdots$ & $\begin{array}{l}04: 30 \mathrm{PM} \\
04: 45 \mathrm{PM}\end{array}$ & 0 & $\begin{array}{l}0 \\
0\end{array}$ & $\begin{array}{l}0 \\
0\end{array}$ & $\begin{array}{l}0 \\
0\end{array}$ & $\begin{array}{l}0 \\
0\end{array}$ & 0 & 0 & $\begin{array}{l}0 \\
0 \\
0\end{array}$ & $\begin{array}{l}0 \\
0\end{array}$ & $\begin{array}{l}0 \\
0\end{array}$ & "z: & . & $i$ \\
\hline $\begin{array}{l}04: 30 \mathrm{PM} \\
\text { 04:49: PM } \\
\end{array}$ & $\ldots$ & $\begin{array}{l}\text { O4:5S PM } \\
\text { 05:00 PM } \\
\end{array}$ & $\begin{array}{l}0 \\
0 \\
\end{array}$ & $\begin{array}{l}0 \\
0 \\
\end{array}$ & $\begin{array}{l}0 \\
0 \\
\end{array}$ & $\begin{array}{l}0 \\
0 \\
\end{array}$ & $\begin{array}{l}0 \\
0\end{array}$ & $\begin{array}{l}0 \\
0 \\
\end{array}$ & 0 & 0 & 0 & 0 & $\therefore$ & ' & 1 \\
\hline & & & & & & & $R L Y$ & TOTAL & & & & & & & \\
\hline गDणAM & $=$ & 88:00 AM & $\sigma$ & $\gamma$ & o & 0 & 8 & $\overline{0}$ & $\sigma$ & 0 & 0 & $\overline{0}$ & 8 & & \\
\hline 07:15 AM & $\cdots$ & 08:15 AM & 0 & 0 & 0 & 0 & 0 & 0 & 0 & 0 & 0 & 0 & '. & & $?$ \\
\hline$\frac{07.30 \mathrm{AM}}{07.45 \mathrm{AM}}$ & 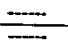 & $\frac{08: 30 \mathrm{AM}}{08: 45 \mathrm{AM}}$ & $-\frac{0}{0}$ & $-\frac{0}{0}$ & $\frac{0}{0}$ & $\frac{0}{0}$ & $\frac{0}{0}$ & $\frac{0}{0}$ & $-\frac{2}{1}$ & $\frac{0}{0}$ & $\frac{0}{0}$ & $\frac{0}{0}$ & $\because$ & & $\vdots$ \\
\hline $08: 00 \mathrm{AM}$ & $\ldots$ & $09: 00 \mathrm{AM}$ & 0 & 0 & 0 & 0 & 0 & 0 & $i$ & 0 & 0 & 0 & $\therefore$ & & \\
\hline OB: IS AM & $\ldots$ & $09: 15 \mathrm{AM}$ & 0 & 0 & 0 & 0 & 0 & 0. & $i$ & 0 & 0 & 0 & $\because$ & & 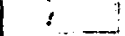 \\
\hline $08: 30 \mathrm{AM}$ & $=$ & $09: 30 \mathrm{AM}$ & 0 & 0 & 0 & 0 & 0 & 0 & (1) & $\overline{0}$ & 0 & 0 & $\therefore$ & & iij \\
\hline 08:45 AM & $=$ & $09: 45 \mathrm{AM}$ & 0 & $\begin{array}{l}0 \\
0\end{array}$ & $\begin{array}{l}0 \\
0\end{array}$ & $\begin{array}{l}0 \\
0\end{array}$ & $\begin{array}{l}0 \\
0\end{array}$ & 0 & 1 & $\begin{array}{l}0 \\
0\end{array}$ & $\begin{array}{l}0 \\
0\end{array}$ & $\begin{array}{l}0 \\
0\end{array}$ & $" \because$ & & $i$ \\
\hline$\frac{09: 00 \mathrm{AM}}{09.15 \mathrm{AM}}$ & $-\infty$ & $\frac{10: 00 \mathrm{AM}}{10.15 \mathrm{AM}}$ & $\frac{0}{0}$ & $\frac{0}{0}$ & $\frac{0}{0}$ & $\frac{9}{0}$ & $-\frac{0}{0}$ & $\frac{0}{0}$ & $\frac{1}{2}$ & $\frac{\pi}{2}$ & $\frac{1}{0}$ & $\frac{\pi}{0}$ & $\because$. & & $\because$ \\
\hline $09: 30 \mathrm{AM}$ & - & $10: 30 \mathrm{AM}$ & 0 & 0 & 0 & 0 & 0 & 1 & 2 & 3 & 0 & 0 & $\therefore$ & & \\
\hline $09.45 A M$ & $=$ & $10: 43 \mathrm{AM}$ & 0 & 0 & 0 & 0 & 0 & $\mathrm{i}$ & 1 & 3 & 0 & 0 & " & & 5 \\
\hline $1000 \mathrm{AM}$ & $\ldots$ & $11: 00 \overline{A M}$ & 0 & 0 & 0 & 0 & 0 & 1 & $T$ & 3 & 0 & 0 & 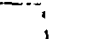 & $\cdot$ & 5 \\
\hline 10:15 AM & $\cdots$ & 11:15 AMA & 0 & 0 & 0 & 0 & 0 & 1 & 0 & 2 & 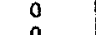 & 0 & ! & & 1 \\
\hline$\frac{10.30 \mathrm{AM}}{1}$ & $=$ & $11: 30 . \mathrm{M}$ & a & $\stackrel{0}{0}$ & $\frac{0}{0}$ & 0 & $\frac{0}{0}$ & $\frac{0}{0}$ & $\frac{0}{0}$ & $\frac{1}{1}$ & $\frac{0}{0}$ & $\frac{0}{0}$ & $\vdots$ & . & $\because$ \\
\hline $10: 45 \mathrm{AM}$ & $\square$ & $11: 45 \mathrm{AM}$ & 0 & 0 & 0 & ${ }_{0}^{0}$ & 0 & 0 & 0 & $!$ & 0 & 0 & 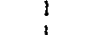 & & $\therefore$ \\
\hline 11:00 AM & 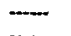 & 12:00 PM & ${ }_{0}^{0}$ & 0 & 0 & 0 & 0 & $!$ & 1 & 1 & 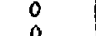 & 0 & 1 & & i \\
\hline 11:15 AM & $\cdots$ & 12:15 PM & 0 & 0 & 0 & 0 & 0 & $\frac{1}{1}$ & 1 & 0 & 0 & 0 & -1 & & 3 \\
\hline 11:30 AM & $=$ & 12:30 PM & 0 & 0 & 0 & 0 & 0 & 2 & $T$ & 0 & 0 & 0 & 1 & & 3 \\
\hline 11:45 AM & - & 12:45 PM & 0 & 0 & 0 & 0 & 0 & 3 & 1 & 1 & 0 & 0 & ! & & 6 \\
\hline $12: 00 \mathrm{PM}$ & $\Longrightarrow$ & $01: 00 \mathrm{PM}$ & 0 & 0 & 0 & 0 & 0 & 2 & $i$ & 2 & 0 & 0 & $i$ & & 6 \\
\hline $12: 15 \mathrm{PM}$ & $\mp$ & $01: 15 \mathrm{PM}$ & $\overline{0}$ & 0 & 0 & 0 & 0 & 2 & 1 & 2 & 0 & 0 & iv & & $s$ \\
\hline 12:30 PM & $\ldots$ & $01: 30$ PM & 0 & 0 & 0 & 0 & 0 & 1 & 1 & 2 & 0 & 0 & $\because$ & & 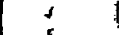 \\
\hline $12: 45 \mathrm{PM}$ & $=$ & $0145 \mathrm{PM}$ & 0 & 0 & 0 & 0 & 0 & $i$ & $\mathrm{i}$ & 2 & 0 & 0 & $i$ & & 5 \\
\hline $0100 \mathrm{PM}$ & $-\infty$ & $02: 009 M$ & 0 & 0 & 0 & 0 & 0 & 1 & $\overline{0}$ & 1 & 0 & 0 & $i$ & ' & 3 \\
\hline Ol:IS PM & - & 02:15 PM & 0 & 0 & 0 & 0 & 0 & 1 & 0 & 1 & 0 & 0 & I & & 3 \\
\hline $01: 30 \mathrm{PM}$ & $=$ & $02: 30 \mathrm{PM}$ & 0 & 0 & 0 & 0 & 0 & $\mathrm{i}$ & 0 & $i$ & 0 & 0 & $\mathrm{i}$ & $\because$ & 3 \\
\hline $01: 45 \mathrm{PM}$ & - & $02: 45 \mathrm{PM}$ & 0 & 0 & 0 & 0 & 0 & 0 & 0 & 0 & $\overline{0}$ & 0 & $\overline{0}$ & $\because$ & 0 \\
\hline $\begin{array}{l}02: 00 \mathrm{PM} \\
02: 15 \mathrm{PM}\end{array}$ & + & $\begin{array}{l}03: 00 \text { PM } \\
03: 15 \text { PM }\end{array}$ & $\begin{array}{l}0 \\
0\end{array}$ & $\begin{array}{l}0 \\
0\end{array}$ & $\begin{array}{l}0 \\
0\end{array}$ & $\begin{array}{l}0 \\
0\end{array}$ & $\begin{array}{l}0 \\
0\end{array}$ & $\begin{array}{l}0 \\
0\end{array}$ & $\begin{array}{l}0 \\
0\end{array}$ & $\begin{array}{l}0 \\
0\end{array}$ & $\begin{array}{l}0 \\
0\end{array}$ & $\begin{array}{l}0 \\
0\end{array}$ & $\begin{array}{l}0 \\
0\end{array}$ & $\because "$ & $\stackrel{o}{a}$ \\
\hline$-\frac{02: 15 \mathrm{PM}}{02: 30 \mathrm{PM}}$ & $\equiv$ & $\begin{array}{l}033113 \mathrm{PM} \\
09: 30 \mathrm{PM}\end{array}$ & 0 & 0 & o & $\frac{5}{0}$ & 0 & $\frac{0}{0}$ & 0 & 0 & 0 & 0 & $\frac{T}{1}$ & $a$ & $\frac{\pi}{1}$ \\
\hline $\begin{array}{l}02: 30 \mathrm{PM} \\
02: 45 \mathrm{PM}\end{array}$ & $\ldots$ & $03.45 \mathrm{PM}$ & 0 & 0 & 0 & 0 & 0 & 0 & 0 & 0 & 0 & 0 & $i$ & $"$ & $i$ \\
\hline 03:00 PM & $=$ & $04: 00 \mathrm{PM}$ & 0 & 0 & 0 & 0 & 0 & 0 & 0 & 0 & 0 & 0 & i & $"$ & 2 \\
\hline $03: 15 \mathrm{PM}$ & - & 04:15 PM & 0 & 0 & 0 & 0 & 0 & 0 & 0 & 0 & $\overline{0}$ & 0 & $T$ & i) & 1 \\
\hline 03:30 PM & $\overline{-}$ & 04:30 PM & 0 & $\begin{array}{l}0 \\
0 \\
0\end{array}$ & o & $\begin{array}{l}0 \\
0 \\
0\end{array}$ & $\begin{array}{l}0 \\
0 \\
0\end{array}$ & 青 & $\begin{array}{l}0 \\
0 \\
0\end{array}$ & $\begin{array}{l}0 \\
0\end{array}$ & $\begin{array}{l}0 \\
0 \\
0\end{array}$ & $\begin{array}{l}0 \\
0\end{array}$ & $?$ & : & 3 \\
\hline 03:45 PM & $=$ & $04: 49 \mathrm{PM}$ & 0 & $\frac{0}{0}$ & 0 & $\frac{\partial}{0}$ & 0 & $\frac{a}{0}$ & $\frac{8}{0}$ & $\frac{0}{0}$ & $\frac{0}{0}$ & $\frac{0}{0}$ & & 10 & \\
\hline OA:00 PM & $\cdots$ & $09: 00 \mathrm{PM}$ & & $\underline{\underline{0}}$ & & 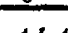 & & 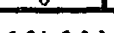 & & 0 & & & & & \\
\hline$s$ & $E N I$ & ULA: & 2 & & & 12 & $D A$ & (0)2 & 292 & & an & 10 & $: f 5$ & $=3$ & \\
\hline
\end{tabular}




\section{Baymetrics Traffic Resources INTERSECTION TURNING MOVEMENT SUMMARY}

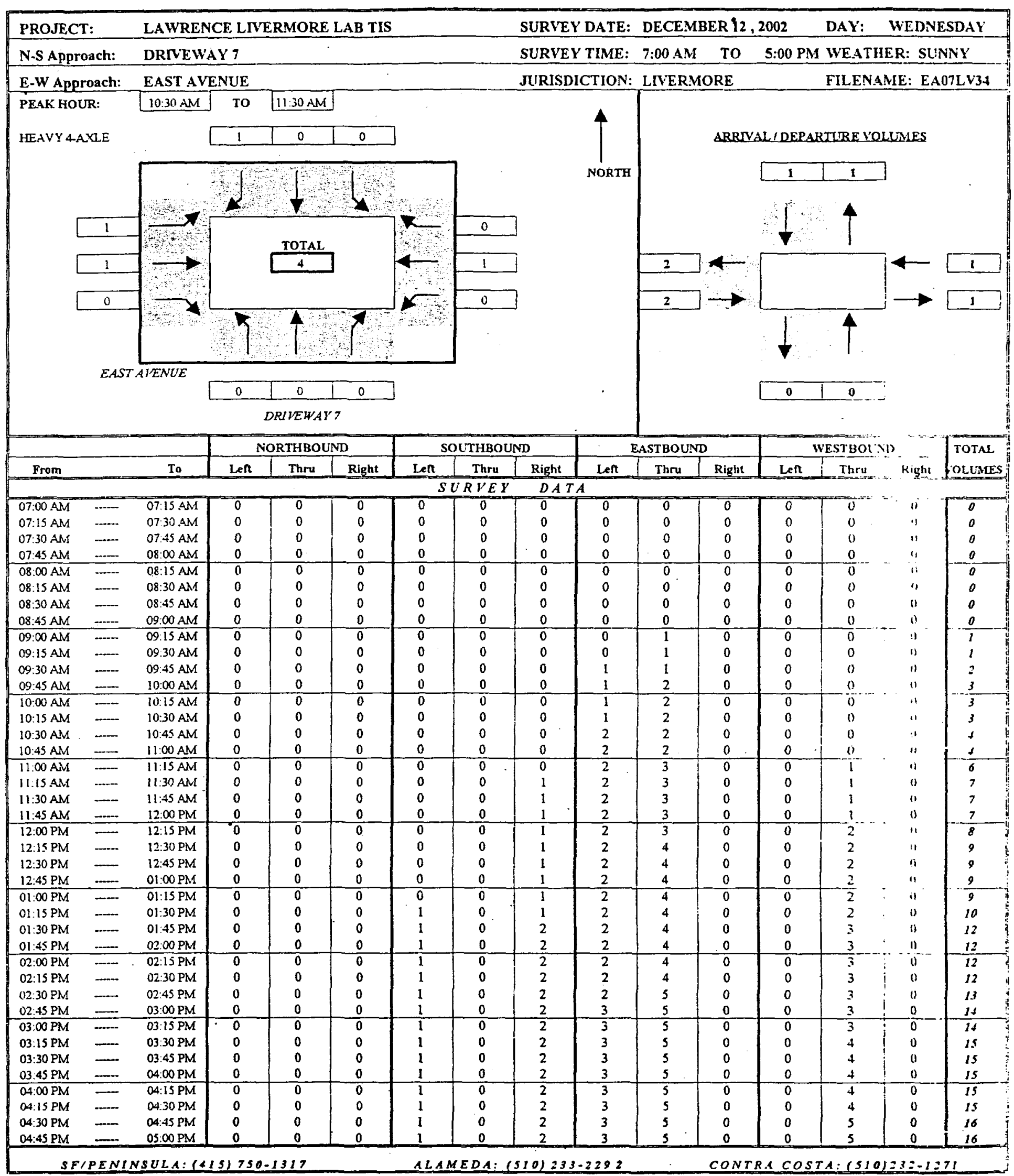




\section{Baymetrics Traffic Resources INTERSECTION TURNING MOVEMENT SUMMARY}

\begin{tabular}{|c|c|c|c|c|c|c|c|c|c|c|c|c|c|c|c|}
\hline \multirow{2}{*}{\multicolumn{2}{|c|}{$\begin{array}{l}\text { PROJECT: } \\
\text { N-S Approach: }\end{array}$}} & \multicolumn{6}{|c|}{ LAWRENCE LIVERMORE LAB TIS } & \multicolumn{2}{|c|}{ SURVEY DATE } & DECEN & ER 12, & 2002 & Ax: & WEDNF & SDAY \\
\hline & & DRIVEW & & & & & & SURVE & IME: & 7:00 AM & To & 5:00 PN & WEAT & IER: SLS & VNY \\
\hline E.W Ap & ach: & EAST AV & IUE & & & & & JURISD & TION & LIVERA & RE & & FILEN & ME: FAI & $07 L v 34$ \\
\hline & & & & Trsio & & & IHBOI & & & ASTBOUI & & & STRBOT & $\mathrm{VD}$ & TOTAL \\
\hline From & & To & Len & Thru & Right & Len & Thru & Right & Len & Thru & Right & Len & Thru & Right & Kolunes \\
\hline & & & & & & TO & $\angle B$ & $P E R I$ & & & & & & & \\
\hline $\begin{array}{l}\text { Tr:00 AM } \\
07: 15 \mathrm{AM}\end{array}$ & $=$ & $\begin{array}{l}\text { DI:ISAM } \\
07: 30 \mathrm{AM}\end{array}$ & $\begin{array}{l}0 \\
0 \\
0\end{array}$ & $\begin{array}{l}0 \\
0\end{array}$ & 0 & $\begin{array}{l}0 \\
0\end{array}$ & $\begin{array}{l}0 \\
0\end{array}$ & $\begin{array}{l}8 \\
0 \\
0\end{array}$ & $\begin{array}{l}0 \\
0 \\
0\end{array}$ & $\begin{array}{l}8 \\
0 \\
0\end{array}$ & $\begin{array}{l}0 \\
0\end{array}$ & $\begin{array}{l}0 \\
0 \\
0\end{array}$ & $\begin{array}{l}0 \\
0 \\
0\end{array}$ & $\begin{array}{l}0 \\
0\end{array}$ & 0 \\
\hline $\begin{array}{l}07: 30 \mathrm{AM} \\
07.05 \mathrm{AM}\end{array}$ & $\ldots$ & $\begin{array}{l}\text { 07:AS AM } \\
\text { 08:00 AM }\end{array}$ & $\begin{array}{l}0 \\
0\end{array}$ & $\begin{array}{l}0 \\
0\end{array}$ & $\begin{array}{l}0 \\
0\end{array}$ & $\begin{array}{l}0 \\
0\end{array}$ & $\begin{array}{l}0 \\
0\end{array}$ & $\begin{array}{l}0 \\
0\end{array}$ & $\begin{array}{l}0 \\
0\end{array}$ & $\begin{array}{l}0 \\
0\end{array}$ & $\begin{array}{l}0 \\
0\end{array}$ & $\begin{array}{l}0 \\
0\end{array}$ & $\begin{array}{l}0 \\
0\end{array}$ & $\begin{array}{l}0 \\
0 \\
0\end{array}$ & 0 \\
\hline $08: 00 \mathrm{AM}$ & $=$ & $08: 15 \mathrm{AM}$ & 0 & 0 & 0 & 0 & 0 & 0 & $\frac{0}{0}$ & 0 & 0 & 0 & $\frac{0}{0}$ & $\frac{9}{0}$ & $\frac{0}{0}$ \\
\hline OB:IS AM & $=$ & O8:30 AM & 0 & 0 & $\begin{array}{l}0 \\
0\end{array}$ & $\begin{array}{l}0 \\
0\end{array}$ & 0 & 0 & 0 & 0 & 0 & 0 & 0 & $\theta$ & 0 \\
\hline $\begin{array}{l}08: 30 \mathrm{AM} \\
08: 45 \mathrm{AM}\end{array}$ & $\overline{-}$ & $08: 45$ AM & $\begin{array}{l}0 \\
0\end{array}$ & 0 & 0 & $\begin{array}{l}0 \\
0\end{array}$ & 0 & $\begin{array}{l}0 \\
0\end{array}$ & 0 & $\begin{array}{l}0 \\
0\end{array}$ & $\begin{array}{l}0 \\
0\end{array}$ & 0 & $\begin{array}{l}0 \\
0\end{array}$ & ") & 0 \\
\hline $09.00 \mathrm{AM}$ & $=$ & $08.15 A M$ & 0 & 0 & $\frac{0}{0}$ & 0 & 0 & 0 & $\frac{0}{0}$ & $\frac{0}{1}$ & 0 & $\frac{0}{0}$ & $\frac{2}{0}$ & $\frac{0}{3}-$ & $\frac{e}{1}$ \\
\hline 09:15 AN4 & $\cdots$ & 09:30 AM. & 0 & 0 & 0. & 0 & 0 & 0 & 0 & 0 & 0 & 0 & 0 & 11 & 0 \\
\hline $09: 30 \mathrm{AM}$ & - & 09:45 AM & 0 & 0 & 0 & 0 & 0 & 0 & 1 & 0 & 0 & 0 & 0 & $" 1$ & 1 \\
\hline $09: 45 \mathrm{AM}$ & $\cdots$ & $10: 00 \mathrm{AM}$ & 0 & 0 & 0 & 0 & 0 & 0 & 0 & 1 & 0 & 0 & 0 & (1) & $i$ \\
\hline $10: 00 \mathrm{AM}$ & 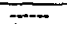 & 10:15AM & 0 & 0 & 0 & 0 & 0 & 0 & 0 & 0 & 0 & 0 & 0 & $\pi$ & 6 \\
\hline 10:15 AM & - & $10: 30 \mathrm{AM}$ & 0 & 0 & 0 & 0 & 0 & 0 & 0 & 0 & 0 & 0 & $\dot{0}$ & $\because$ & $a$ \\
\hline $10: 30 \mathrm{AM}$ & 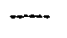 & 10:45 AM & 0 & 0 & 0 & 0 & 0 & 0 & 1 & 0 & 0. & 0 & 0 & $\because 1$ & 1 \\
\hline 10:45 AM & $\ldots$ & $11: 00 \mathrm{AM}$ & 0 & 0 & 0 & 0 & 0 & 0 & 0 & 0 & 0 & $D$ & 9 & $\because$ & 0 \\
\hline$\overline{11}: 00 \mathrm{AM}$ & $E$ & 11:15 AM & 0 & $\overline{0}$ & 0 & 0 & 0 & 0 & 0 & 1 & $\begin{array}{l}0 \\
0\end{array}$ & 是 & $\begin{array}{l}1 \\
0\end{array}$ & $\because:$ & 2 \\
\hline $\begin{array}{l}1115 \mathrm{AMM} \\
\text { 11:30 AM }\end{array}$ & $\equiv$ & $\begin{array}{l}11: 30 \mathrm{AM} \\
11: 45 \mathrm{AM}\end{array}$ & $\begin{array}{l}0 \\
0\end{array}$ & $\begin{array}{l}0 \\
0\end{array}$ & $\begin{array}{l}0 \\
0\end{array}$ & $\begin{array}{l}0 \\
0\end{array}$ & 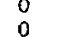 & $\begin{array}{l}1 \\
0\end{array}$ & $\begin{array}{l}0 \\
0 \\
0\end{array}$ & $\begin{array}{l}0 \\
0 \\
0\end{array}$ & $\begin{array}{l}0 \\
0\end{array}$ & 0 & 0 & $\therefore$ & 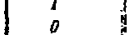 \\
\hline 11.4S AM & $\ldots$ & $12: 00 \mathrm{PM}$ & 0 & 0 & 0 & 0 & 0 & 0 & 0 & 0 & 0 & 0 & 0 & 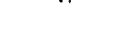 & $a$ \\
\hline $12.00 \mathrm{PM}$ & $\ldots$ & $1215 \mathrm{PM}$ & 0 & 0 & 0 & $\overline{0}$ & 0 & 0 & 0 & 0 & 0 & 0 & $T$ & $\because$ & 3 \\
\hline 12:15 PM & $\cdots$ & $12: 30$ PM & 0 & 0 & 0 & $0_{0}^{0}$ & 0 & 0 & 0 & 1 & 9 & 0 & 0 & $"$ & 1 \\
\hline 12:30 PM & - & 12:45 PM & $\stackrel{0}{0}$ & 0 & 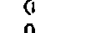 & 0 & 0 & 0 & 0 & 0 & 0 & 0 & 0 & •. & a \\
\hline$\frac{12: 45 P M}{P A}$ & 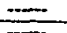 & $01: 00 \mathrm{PM}$ & 0 & 0 & 0 & 0 & 0 & $\frac{a}{a}$ & 0 & 0 & 0 & 0 & 9 & " & $\underline{a}$ \\
\hline $01: 00 \mathrm{PM}$ & $=$ & OU:15 PM & 0 & 0 & 0 & 0 & $\pi$ & $\overline{0}$ & 0 & $\overline{0}$ & 0 & 0 & 6 & ". & 0 \\
\hline 01:15 PM & - & 01:30 PM & 0 & 0 & 0 & 1 & 0 & 0 & 0 & 0 & 0 & 0 & 0 & "' & 1 \\
\hline 01:30 PM & - & $01: 45$ PM & 0 & 0 & 0 & 0 & 0 & 1 & 0 & 0 & 0 & 0 & 1 & " & 2 \\
\hline 01:45 PM & $\ldots$ & 02:00 PM & 0 & 0 & 0 & 0 & 0 & 0 & 0 & 0 & 0 & 0 & a. & .. & $n$ \\
\hline $02: 00 \mathrm{PM}$ & $\ldots$ & $02: 15 \mathrm{PM}$ & 0 & 0 & 0 & $\overline{0}$ & 0 & $\overline{0}$ & 0 & 0 & 0 & 0 & $\overline{0}$ & '. & 0 \\
\hline 02:15 PM & 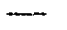 & 02:30 PM & 0 & 0 & 0 & 0 & 0 & 0 & 0 & 0 & 0 & 0 & 0 & & 0 \\
\hline 02:30 PM & - & 02:45 PM & 0 & 0 & 0 & 0 & 0 & 0 & 0 & I & 0 & 0 & i) & . & 1 \\
\hline 02:15 PM & $\ldots$ & $03: 00 \mathrm{PM}$ & 0 & 0 & 0 & 0 & 0 & 0 & 1 & $\dot{0}$ & 0 & 0 & a & $\therefore$ & $i$ \\
\hline $03: 00 \mathrm{PM}$ & $=$ & $03: 15 \mathrm{PM}$ & $\overline{0}$ & 0 & 0 & 0 & 0 & 0 & 0 & 0 & $a$ & 0 & 0 & ". & $\pi$ \\
\hline $03: 15 \mathrm{PM}$ & $\ldots$ & 03:30 PM & 0 & 0 & 0 & 0 & 0 & 0 & 0. & 0 & 0 & 0 & $i$ & $\therefore$ & 1 \\
\hline 03:30 PM & - & 03:45 PM & 0 & 0 & 0 & 0 & 0 & 0 & $0^{\circ}$ & 0 & 0 & 0 & 8 & $"$ & 0 \\
\hline $03: 45 \mathrm{PM}$ & $\ldots$ & $04: 00 \mathrm{PM}$ & 0 & 0 & 0 & 0 & 0 & 0 & 0 & 0 & 0 & 0 & 0 & .1 & 0 \\
\hline $04: 00 \mathrm{PM}$ & $=$ & $04: 13 \mathrm{PM}$ & 0 & 0 & 0 & 0 & 0 & 0 & 0 & 0 & 0 & 0 & 0 & ., & 6 \\
\hline 04:15 PM & - & $04: 30 \mathrm{PM}$ & 0 & 0 & 0 & 0 & 0 & 0 & 0 & 0 & 0 & 0 & 0 & $"$ & $n$ \\
\hline 04:30 PM & $\cdots$ & 04:45 PM & 0 & 0 & 0 & 0 & 0 & 0 & 0 & 0 & 0 & 0 & 1 & ". & 1 \\
\hline 04:45 PM & $\ldots$ & $05: 00 \mathrm{PM}$ & 0 & 0 & 0 & 0 & 0 & $\underline{0}$ & 0 & 0 & 0 & 0 & 0 & ' & $\stackrel{0}{=\rightarrow-1}$ \\
\hline & & & & & & & $R L Y$ & TOTAL & & & & & & & \\
\hline $0700 \mathrm{MM}$ & $=$ & $08000 \mathrm{AM}$ & 0 & $\sigma$ & 0 & $\sigma$ & $\pi$ & 0 & O & 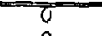 & t) & 0 & 8 & $:$ & \\
\hline 07:15 AM & 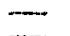 & 08:15 AM & 0 & 0 & $\stackrel{0}{0}$ & 0 & 0 & 0 & 0 & 0 & 0 & 0 & 0 & & 0 \\
\hline 07:30 AM & $=$ & 08:30 AM & 0 & 0 & 0 & 0 & $=0$ & 0 & 0 & 0 & 0 & $\frac{0}{1}$ & $\frac{0}{10}$ & . & $\frac{a}{0}$ \\
\hline $07: 45$ AM & 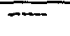 & 08:45 AM & 0 & 0 & 0 & 0 & $\overline{0}$ & $\overline{0}$ & 0 & $\sigma$ & 0 & $\sigma$ & 5 & . & 8 \\
\hline 08:00 AM & $\rightarrow$ & 09:00 AM & 0 & 0 & 0 & 0 & 0 & 0 & 0 & 0 & 0 & 0 & 0 & & i \\
\hline $08: 15$ AM & $\cdots$ & 08:15 AM & 0 & 0 & 0 & 0 & 0 & 0 & 0 & 1 & 0 & 0 & 0 & . & $\therefore$ \\
\hline $08: 30 \mathrm{AM}$ & $=$ & $09: 30 \mathrm{AM}$ & 0 & 0 & 0 & 0 & 0 & 0 & 0 & 1 & 0 & 0 & 8 & $\therefore$ & $t$ \\
\hline $\begin{array}{l}08: 45 \mathrm{AM} \\
09: 00 \mathrm{AM}\end{array}$ & $=$ & $\begin{array}{l}09: 45 \mathrm{AM} \\
10: 00 \mathrm{AM}\end{array}$ & $\begin{array}{l}0 \\
0\end{array}$ & $\begin{array}{l}0 \\
0\end{array}$ & $\begin{array}{l}0 \\
0\end{array}$ & $\begin{array}{l}0 \\
0\end{array}$ & $\begin{array}{l}0 \\
0\end{array}$ & $\begin{array}{l}0 \\
0\end{array}$ & 1 & $\frac{1}{2}$ & $\begin{array}{l}0 \\
0 \\
\end{array}$ & $\begin{array}{l}0 \\
0\end{array}$ & 0 & $\because$ & $\frac{2}{3}$ \\
\hline $0 Q: 15 \mathrm{AM}$ & 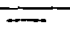 & $10: 15 \mathrm{AM}$ & 0 & 0 & 0 & $\sigma$ & 0 & 0 & 1 & 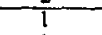 & 0 & 0 & $\overline{\hat{o}}$ & : & $!$ \\
\hline $09: 30 \mathrm{AM}$ & 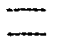 & 10:30 AM & 0 & 0 & $\begin{array}{l}0 \\
0\end{array}$ & $\begin{array}{l}0 \\
0\end{array}$ & $\begin{array}{l}0 \\
0\end{array}$ & $\begin{array}{l}0 \\
0\end{array}$ & 1 & 1 & $\begin{array}{l}0 \\
0\end{array}$ & $\begin{array}{l}0 \\
0\end{array}$ & 0 & $:$ & 2 \\
\hline $09: 45 \mathrm{AM}$ & & 10:45 AM & 0 & 0 & 0 & $\frac{0}{0}$ & 0 & 0 & $\frac{1}{1}$ & $\frac{1}{0}$ & 0 & 0 & $\frac{1}{10}$ & $"$ & $\vdots-$ \\
\hline $10.00 \mathrm{AM}$ & 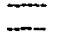 & $11: 00 \mathrm{AM}$ & 0 & 0 & 0 & 0 & 0 & $0_{0}^{0}$ & 1 & 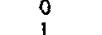 & $\begin{array}{l}0 \\
0 \\
0\end{array}$ & 0 & $?$ & "̈ & $!$ \\
\hline $\begin{array}{l}\text { 10:15 AM } \\
\text { 10:30 AM }\end{array}$ & $\ldots$ & $\begin{array}{l}11: 15 \mathrm{AM} \\
11: 30 \mathrm{AM}\end{array}$ & $\begin{array}{l}0 \\
0\end{array}$ & $\begin{array}{l}0 \\
0\end{array}$ & $\begin{array}{l}0 \\
0\end{array}$ & $\begin{array}{l}0 \\
0\end{array}$ & $\begin{array}{l}0 \\
0\end{array}$ & 0 & 1 & 1 & 要 & $\begin{array}{l}0 \\
0 \\
0\end{array}$ & 1 & $"$ & 3 \\
\hline & $\ldots$ & $\frac{11: 30 \mathrm{AM}}{11: 45 \mathrm{AM}}$ & $\frac{0}{0}$ & 0 & 0 & 0 & $\frac{5}{0}$ & $\frac{1}{1}$ & 0 & 1 & 0 & 0 & $\frac{1}{1}$ & 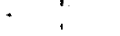 & $\frac{3}{3}$ \\
\hline $\begin{array}{l}10.45 \mathrm{AM} \\
11: 00 \mathrm{AM}\end{array}$ & - & 17:00 PM & 0 & 0 & 0 & 0 & 0 & 1 & 0 & $i$ & 0 & 0 & 1 & " & \\
\hline $\begin{array}{l}11: 00 \mathrm{AM} \\
11: 15 \mathrm{AM} \\
\end{array}$ & $=$ & 12:15 PM & 0 & 0 & 0 & 0 & 0 & $i$ & 0 & 0 & 0 & 0 & 1. &.. & 3 \\
\hline $1130 \mathrm{AM}$ & 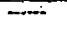 & $12: 30$ & 0 & 0 & 0 & 0 & 0 & 0 & 0 & 1 & 0 & 0 & $T$ & ". & 2 \\
\hline 11:4S AM & - & 12:45 PM & 0 & 0 & 0 & 0 & $\stackrel{0}{0}$ & 0 & 0 & 1 & 0 & $0_{0}^{0}$ & 1 & $\because$ & 2 \\
\hline 12:00 PM & $=$ & $01: 00$ PM & 0 & $\stackrel{0}{0}$ & $\frac{0}{0}$ & 0 & -0 & $\frac{0}{0}$ & 0 & 1 & $\frac{0}{0}$ & 0 & $\frac{1}{10}$ & ". & 2 \\
\hline $12: 15 \mathrm{PM}$ & $=$ & $01: 13$ & 0 & 0 & 0 & 0 & 0 & 0 & $\bar{\sigma}$ & $T$ & $\overline{0}$ & 0 & $\overline{0}$ & $\because$ & $T$ \\
\hline 12:30 PM & $\ldots$ & $01: 30 \mathrm{PM}$ & 0 & 0 & 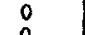 & 1 & & 0 & 0 & 0 & 0 & 0 & 0 & $\because$ & 3 \\
\hline$\frac{12: 45 P M}{01: 00 P M}$ & $\equiv$ & $\frac{01: 45 \text { PM }}{02: 00 \mathrm{PM}}$ & $\frac{0}{0}$ & $\frac{0}{0}$ & $\frac{0}{0}$ & $\frac{1}{1}$ & $\frac{0}{0}$ & $\frac{1}{1}$ & $\frac{0}{0}$ & $\frac{0}{0}$ & $\frac{0}{0}$ & $\frac{0}{0}$ & $\frac{1}{1}$ & 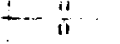 & $\frac{3}{3}$ \\
\hline $01: 15$ PM & - & 0:15 PM & 0 & 0 & 0 & $\mathrm{i}$ & 0 & 1 & 0 & 0 & 0 & 0 & 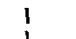 & "1" & 3 \\
\hline $01: 30 \mathrm{PM}$ & $=$ & 02:30 PM & 0 & 0 & 0 & $\stackrel{0}{0}$ & $\frac{0}{0}$ & $\frac{1}{6}$ & 0 & 0 & 0 & 0 & $\frac{1}{1}$ & $\because-$ & 2 \\
\hline $01.45 \mathrm{PM}$ & $=$ & $02: 45$ PM & 0 & 0 & 0 & 0 & 0 & 0 & 0 & 1 & 0 & 0 & o & $i^{-1}$ & $!$ \\
\hline 02:00 PM & $=$ & $\begin{array}{l}03.00 \mathrm{PM} \\
03: 15 \mathrm{PM}\end{array}$ & 0 & ${ }_{0}^{0}$ & 0 & 0 & $: 0$ & $\begin{array}{l}0 \\
0\end{array}$ & 1 & 1 & $\begin{array}{l}0 \\
0\end{array}$ & $\begin{array}{l}0 \\
0 \\
0\end{array}$ & 0 & 3 & 2 \\
\hline & $\equiv$ & $\frac{03: 15 \mathrm{PM}}{03: 30 \mathrm{PM}}$ & $\frac{0}{0}$ & $\frac{0}{0}$ & $\frac{0}{0}$ & $\frac{0}{0}$ & -0 & $\frac{0}{\partial}$ & 1 & $\frac{1}{1}$ & $\frac{\pi}{0}$ & $\frac{0}{0}$ & $\frac{0}{1}$ & it & $\frac{2}{3}$ \\
\hline $\begin{array}{l}02: 30 \mathrm{PM} \\
02: 43 \mathrm{PM}\end{array}$ & $+\cdots$ & $\begin{array}{l}\text { 03:30 PM } \\
\text { 03:45 PM }\end{array}$ & 0 & 0 & 0 & 0 & 0 & 0 & 1 & 0 & 0 & 0 & 1 & 8) & 2 \\
\hline $03: 00 \mathrm{PM}$ & $\div$ & $04: 00 \mathrm{PM}$ & 0 & 0 & 0 & 0 & 0 & 0 & 0 & 0 & 0 & 0 & 1 & 0 & 1 \\
\hline $03: 15 \mathrm{PM}$ & $=$ & $04: 15 \mathrm{PM}$ & 0 & 0 & 0 & 0 & 0 & 0 & 0 & 0 & 0 & 0 & 1 & 0 & $I$ \\
\hline 03:30 9M & $\ldots$ & O4:30 PM & 0 & 0 & 0 & 0 & 0 & 0 & 0 & 0 & 0 & 0 & 0 & 0 & 0 \\
\hline 03:15 PM & $=$ & $04: 45 \mathrm{PM}$ & 0 & 0 & 0 & 0 & 0 & 0 & 0 & 0 & 0 & 0 & 1 & D & 1 \\
\hline $0400 \mathrm{PM}$ & $=$ & $03: 00 \mathrm{PM}$ & 0 & 0 & 0 & 0 & 0 & 0 & 0 & 0 & 0 & o & $T$ & 0 & 1 \\
\hline & ENI & LA: & 7 & & & & 24 & 1 & 9 & & $\underline{\theta}$ & $c$ & cs $s$ & $2-$ & \\
\hline
\end{tabular}




\section{Baymetrics Traffic Resources INTERSECTION TURNING MOVEMENT SUMMARY}

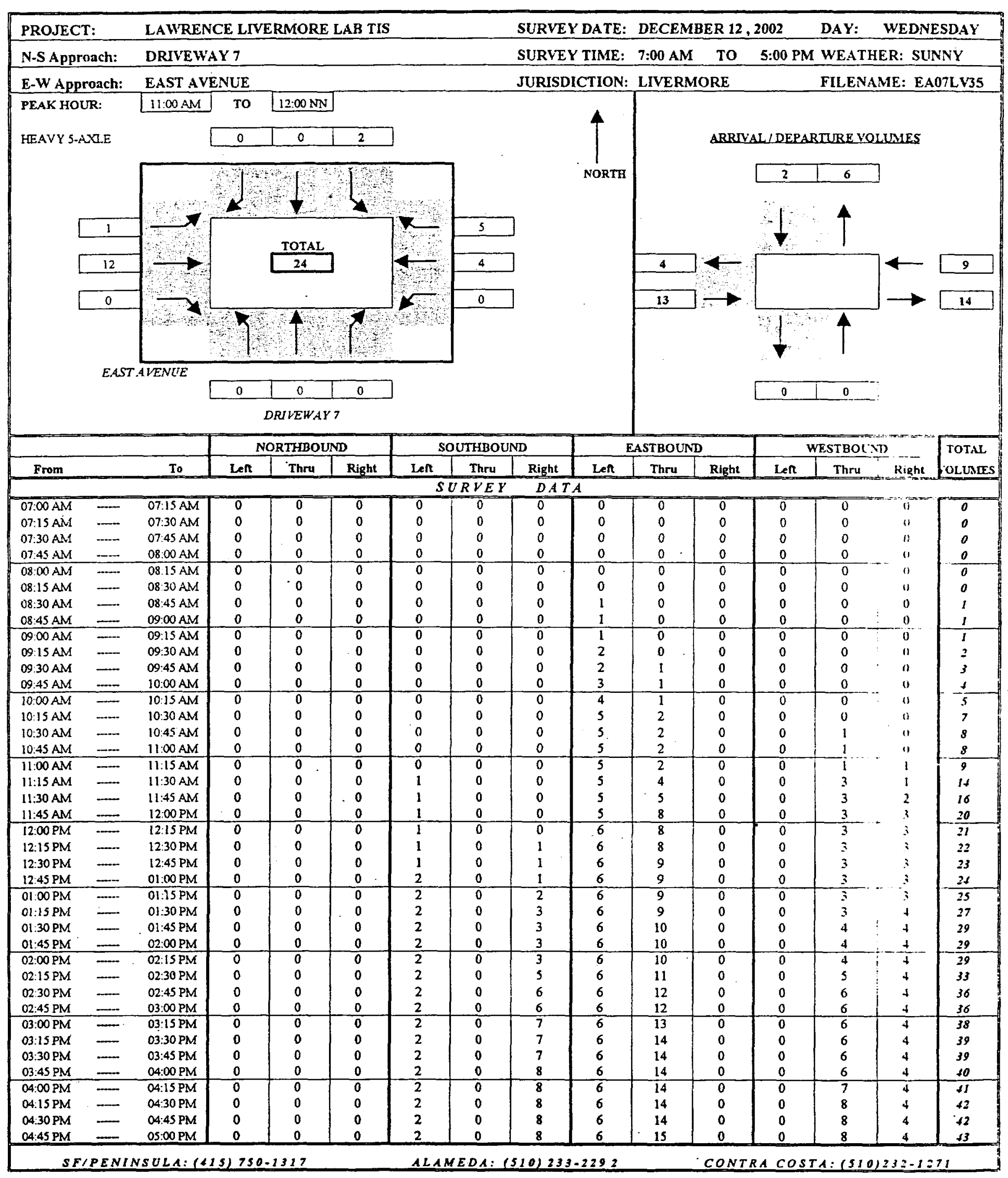




\section{Baymetrics Traffic Resources INTERSECTION TURNING MOVEMENT SUMMARY}

\begin{tabular}{|c|c|c|c|c|c|c|c|c|c|c|c|c|c|c|c|}
\hline \multirow{2}{*}{\multicolumn{2}{|c|}{$\begin{array}{l}\text { PROJECT: } \\
\text { N-S Approach: }\end{array}$}} & \multicolumn{6}{|c|}{ LAWRENCE LIVERMORE LAB TIS } & \multicolumn{2}{|c|}{ SURVEY DATE: } & DECEI & BER 12, & 2002 & DAY: & \multicolumn{2}{|c|}{ WEDNESDAY } \\
\hline & & \multicolumn{6}{|c|}{ DRIVEWAY 7} & \multicolumn{2}{|c|}{ SURVEY TIME: } & 7:00 As & To & 5:00 Ps & WEAT & IER: SU: & iNY \\
\hline E-W Ap & oach: & EAST A & NUE & & & & & JURISDI & TION & LIVER & ORE & & FILEN & ME: EA & $07 L v 35$ \\
\hline & & & & THBO & & & THBOT & & & ASTBOU & & & VESTBOU & & TOTAL \\
\hline From & & To & Len & Thru & Right & Len & Thru & Rught & Left & Thru & Right & Lent & Thru & Right & lolunas \\
\hline & & & & & & $T O$ & $\angle \quad B$ & $P E R I$ & & & & & & & \\
\hline $\begin{array}{l}07,00 \mathrm{AM} \\
07: 15 \mathrm{AM} \\
07: 30 \mathrm{AM}\end{array}$ & $\bar{E}$ & $\begin{array}{l}\text { 07T5AM } \\
07: 30 \mathrm{AM} \\
07: 45 \mathrm{AM}\end{array}$ & $\begin{array}{l}0 \\
0 \\
0\end{array}$ & $\begin{array}{l}0 \\
0 \\
0\end{array}$ & $\begin{array}{l}0 \\
0 \\
0\end{array}$ & $\begin{array}{l}0 \\
0 \\
0\end{array}$ & $\begin{array}{l}0 \\
0 \\
0\end{array}$ & $\begin{array}{l}0 \\
0 \\
0\end{array}$ & $\begin{array}{l}0 \\
0 \\
0\end{array}$ & $\begin{array}{l}0 \\
0 \\
0\end{array}$ & $\begin{array}{l}0 \\
0 \\
0\end{array}$ & $\begin{array}{l}0 \\
0 \\
0\end{array}$ & $\begin{array}{l}\gamma \\
0 \\
0\end{array}$ & $\begin{array}{l}0 \\
0 \\
0\end{array}$ & $\begin{array}{l}0 \\
0 \\
0\end{array}$ \\
\hline $07: 45 \mathrm{AM}$ & $=$ & $\frac{08: 00 \mathrm{AM}}{08.15 \mathrm{AM}}$ & $\frac{0}{0}$ & $\div$ & $\frac{0}{0}$ & $\frac{0}{0}$ & $\begin{array}{l}0 \\
0 \\
0\end{array}$ & $\frac{0}{0}$ & 0 & 0 & 0 & 0 & 0 & $\begin{array}{l}0 \\
0 \\
\end{array}$ & 0 \\
\hline OB: $15 A M$ & - & 08:30 AM & 0 & 0 & 0 & 0 & 0 & $\begin{array}{l}0 \\
0\end{array}$ & $\begin{array}{l}0 \\
0\end{array}$ & & $\begin{array}{l}0 \\
0\end{array}$ & $\begin{array}{l}0 \\
0\end{array}$ & $\begin{array}{l}0 \\
0\end{array}$ & $\begin{array}{l}0 \\
0\end{array}$ & 0 \\
\hline $\begin{array}{l}\text { 08:30 AM } \\
08: 45 \mathrm{AM}\end{array}$ & $=$ & 08:45 AM & 0 & 0 & 0 & 0 & 0 & 0 & 1 & 0 & 0 & 0 & 0 & 0 & 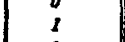 \\
\hline $09: 00.4 \mathrm{M}$ & $\equiv$ & $09: 15 A M$ & $\frac{0}{0}$ & $\frac{0}{0}$ & 0 & $\frac{0}{0}$ & $\begin{array}{l}0 \\
.0\end{array}$ & $\frac{0}{0}$ & $\frac{0}{0}$ & $\frac{0}{0}$ & $\frac{0}{0}$ & $\frac{0}{0}$ & $\frac{a}{0}$ & $\frac{0}{0}$ & 0 \\
\hline $09: 15 \mathrm{AM}$ & $\cdots$ & 09:30 AM & 0 & 0 & 0 & 0 & 0 & 0 & 1 & 0 & 0 & 0 & 0 & 0 & $t$ \\
\hline $09: 30 \mathrm{AM}$ & - & $09: 45 \mathrm{AM}$ & 0 & 0 & 0 & 0 & 0 & 0 & 0 & 1 & 0 & 0 & 0 & 0 & 3 \\
\hline $09: 45$ AM & $\ldots$ & $10: 00 \mathrm{AM}$ & 0 & 0 & 0 & 0 & 0 & 0 & 1 & $\begin{array}{l}1 \\
0\end{array}$ & 0 & 0 & 0 & 0 & 1 \\
\hline $10: 00 \mathrm{AM}$ & $=$ & $10: 15 \mathrm{AM}$ & 0 & 0 & 0 & 0 & 0 & 0 & $i$ & 0 & 0 & 0 & 0 & 11 & $t$ \\
\hline $\begin{array}{l}10: 15 \mathrm{AM} \\
10: 30 \mathrm{AM}\end{array}$ & $=$ & $\begin{array}{l}10.30 \mathrm{AM} \\
10.45 \mathrm{AM}\end{array}$ & 0 & 0 & 0 & $\begin{array}{l}0 \\
0\end{array}$ & $\begin{array}{l}0 \\
0\end{array}$ & 0 & 1 & $\frac{1}{0}$ & 0 & 0 & 0 & a & 3 \\
\hline 10:45 AM & $\bar{z}$ & $\begin{array}{l}10.45 \mathrm{AM} \\
11.00 \mathrm{AM}\end{array}$ & $\begin{array}{l}0 \\
0\end{array}$ & $\begin{array}{l}0 \\
0 \\
0\end{array}$ & $\begin{array}{l}0 \\
0\end{array}$ & $\begin{array}{l}0 \\
0\end{array}$ & 0 & $\begin{array}{l}0 \\
0\end{array}$ & 0 & $\begin{array}{l}0 \\
0\end{array}$ & $\begin{array}{l}0 \\
0\end{array}$ & $\begin{array}{l}0 \\
0\end{array}$ & 1 & $" 1$ & 1 \\
\hline $11: 00 \mathrm{AM}$ & $=$ & $11: 15 \mathrm{AM}$ & 0 & 0 & 0 & $\overline{0}$ & 0 & 0 & 0 & 0 & $\frac{0}{0}$ & $\frac{0}{0}$ & $\frac{0}{0}$ & $\frac{9}{1}$ & $\frac{0}{1}$ \\
\hline 11:15 AM & - & $11: 30 \mathrm{AM}$ & 0 & 0 & 0 & 1 & 0 & 0 & 0 & 2 & 0 & 0 & 2 & $\dot{v}$ & $s$ \\
\hline $11: 30 \mathrm{AM}$ & - & $11: 45 \mathrm{AM}$ & 0 & 0 & 0 & 0 & 0 & 0 & 0 & 1 & 0 & 0 & 0 & i & 2 \\
\hline 11:45 AM & $=$ & $\frac{12: 00 \mathrm{PM}}{12.15 \mathrm{PM}}$ & 0 & 0 & 0 & 0 & 0 & 0 & 0 & 3 & 0 & 0 & 0 & $i$ & 8 \\
\hline $12: 00 \mathrm{PM}$ & $\ldots$ & $\begin{array}{l}\text { 12:15 PM } \\
12: 30 \mathrm{PM}\end{array}$ & 0 & 0 & 0 & 0 & 0 & 0 & 1 & 0 & 0 & 0 & 0 & i) & $T$ \\
\hline & $=$ & $\begin{array}{l}\text { 12:30 PM } \\
12: 45 \mathrm{PM}\end{array}$ & 0 & 0 & 0 & 0 & 0 & 1 & 0 & 0 & 0 & 0 & 0 & 0 & $i$ \\
\hline $\begin{array}{l}12: 30 \mathrm{PM} \\
12: 45 \mathrm{PM}\end{array}$ & $=$ & $\begin{array}{l}\text { ot:00 PM } \\
\text { of: }\end{array}$ & $\begin{array}{l}0 \\
0\end{array}$ & $\begin{array}{l}0 \\
0\end{array}$ & $\begin{array}{l}0 \\
0\end{array}$ & $\begin{array}{l}0 \\
1\end{array}$ & $\begin{array}{l}0 \\
0\end{array}$ & $\begin{array}{l}0 \\
0\end{array}$ & $\begin{array}{l}0 \\
0\end{array}$ & 1 & $\begin{array}{l}0 \\
0\end{array}$ & $\begin{array}{l}0 \\
0\end{array}$ & $\begin{array}{l}0 \\
0\end{array}$ & 0 & 1 \\
\hline $01: 00 \mathrm{PM}$ & $=$ & $01: 15 \mathrm{PM}$ & 0 & 0 & 0 & 0 & 0 & 1 & 0 & 0 & $\frac{\pi}{0}$ & 0 & $\frac{0}{0}$ & $\frac{1}{i j}$ & $i$ \\
\hline 01:15 PM & $\cdots$ & $01: 30 \mathrm{PM}$ & 0 & 0 & 0 & 0 & 0 & $i$ & 0 & 0 & 0 & 0 & 0 & 1 & 2 \\
\hline $01: 30 \mathrm{PM}$ & 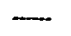 & $01: 45$ PM & 0 & 0 & 0 & 0 & 0 & 0 & 0 & 1 & 0 & 0 & 1 & $"$ & 2 \\
\hline $01: 45 \mathrm{PM}$ & 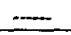 & $02: 00 \mathrm{PM}$ & 0 & 0 & 0 & 0 & 0 & 0 & 0 & 0 & $a$ & 0 & 0 & $\therefore$. & 0 \\
\hline $\begin{array}{l}02: 00 \mathrm{PM} \\
02.15 \mathrm{PM}\end{array}$ & 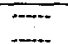 & $02: 15 \mathrm{PAT}$ & 0 & 0 & $\overline{0}$ & 0 & 0 & 0 & 0 & 0 & $\overline{0}$ & 0 & 0 & i" & 0 \\
\hline $\begin{array}{l}02: 15 \mathrm{PM} \\
02: 30 \mathrm{PM}\end{array}$ & $\ldots$ & $\begin{array}{l}\text { 02:30 PM } \\
02: 45 \mathrm{PM}\end{array}$ & $\begin{array}{l}0 \\
0\end{array}$ & $\begin{array}{l}0 \\
0\end{array}$ & $\stackrel{0}{0}$ & $\stackrel{0}{0}$ & 0 & 2 & 0 & 1 & 0 & 0 & I & $\because$ & 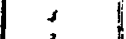 \\
\hline $02.45 \mathrm{PM}$ & $=$ & $03: 00$ PM & 0 & 0 & 0 & 0 & 0 & $\begin{array}{l}1 \\
0 \\
\end{array}$ & $\begin{array}{l}0 \\
0\end{array}$ & $\begin{array}{l}1 \\
0\end{array}$ & $\begin{array}{l}0 \\
0\end{array}$ & $\begin{array}{l}0 \\
0\end{array}$ & $\begin{array}{l}1 \\
0\end{array}$ & $"$ & 3 \\
\hline $03: 00 \mathrm{PM}$ & 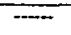 & $03: 15 \mathrm{PM}$ & 0 & 0 & 0 & 0 & 0 & 1 & 0 & 1 & 0 & 0 & 0 & $71^{-}$ & 2 \\
\hline 03:15 PM & $\cdots$ & 03:30 PM & 0 & 0 & 0 & 0 & 0 & 0 & 0 & 1 & 0 & 0 & 0 & 0 & 1 \\
\hline 03:30 PM & $\ldots$ & 09.45 PM & $\therefore$ & 0 & 0. & 0 & 0 & 0 & 0 & 0 & 0 & 0 & 0 & i) & $\theta$ \\
\hline 03:45 PM & - & $04: 00$ PM & -0 & $-\frac{0}{0}$ & $\frac{0}{0}$ & 0 & 0 & $\frac{1}{n}$ & 0 & 0 & 0 & 0 & 0 & $a$ & 1 \\
\hline $\begin{array}{l}04: 00 \mathrm{PM} \\
04: 19 \mathrm{PM}\end{array}$ & $=$ & $\begin{array}{l}04: 15 \mathrm{PM} \\
04: 30 \mathrm{PM}\end{array}$ & $\begin{array}{l}0 \\
0\end{array}$ & $\begin{array}{l}0 \\
0\end{array}$ & $\begin{array}{l}0 \\
0\end{array}$ & $\begin{array}{l}0 \\
0\end{array}$ & $\begin{array}{l}0 \\
0\end{array}$ & $\begin{array}{l}0 \\
0\end{array}$ & $\begin{array}{l}0 \\
0\end{array}$ & $\begin{array}{l}0 \\
0\end{array}$ & $\begin{array}{l}0 \\
0\end{array}$ & 0 & 1 & $\overline{0}$ & ! \\
\hline 04:30 PM & - & $04: 45 \mathrm{PM}$ & 0 & 0 & 0 & 0 & 0 & 0 & 0 & 0 & $\begin{array}{l}0 \\
0\end{array}$ & $\begin{array}{l}0 \\
0\end{array}$ & l & ") & 1 \\
\hline 04:45 PM & $=$ & $05: 00 \mathrm{PM}$ & 0 & 0 & 0 & 0 & 0 & 0 & 0 & 1 & 0 & 0 & 0 & 4 & 1 \\
\hline & & & & & & & $R L Y$ & TOTAL & & & & & & & \\
\hline סः:00 & $=$ & - $8800 \mathrm{AM}$ & 8 & 0 & 0 & 7 & 8 & 0 & r & 0 & $\bar{U}$ & 0 & $\sigma$ & $i$ & 8 \\
\hline 07.15 AM & $\ldots$ & $\begin{array}{l}\text { 08:15 AM } \\
08: 30 \mathrm{AM}\end{array}$ & $\begin{array}{l}0 \\
0\end{array}$ & $\begin{array}{l}0 \\
0\end{array}$ & $\begin{array}{l}0 \\
0\end{array}$ & $\begin{array}{l}0 \\
0\end{array}$ & 0 & 0 & 0 & 0 & 0 & 0 & 0 & $"$ & 0 \\
\hline 07.45 AN & $=$ & $08.45 \mathrm{AM}$ & 0 & 0 & 0 & 0 & $\frac{\pi}{a}$ & $\frac{0}{0}$ & $\frac{0}{1}$ & $\frac{0}{0}$ & $\frac{0}{0}$ & $\frac{0}{0}$ & $\frac{0}{0}$ & $\frac{11}{6}$ & $\frac{0}{l}$ \\
\hline 08:00 AM & - & 09:00 AM & 0 & 0 & 0 & 0 & 0 & 0 & $i$ & 0 & 0 & 0 & 0 & s & $i$ \\
\hline 08:15 AM & - & 09:15 AM & 0 & 0 & 0 & 0 & 0 & 0 & $i$ & 0 & 0 & 0 & 0 & 0 & 1 \\
\hline $08: 30 \mathrm{AM}$ & $=$ & $09: 30 \mathrm{AM}$ & 0 & 0 & 0 & 0 & 0 & 0 & 2 & 0 & 0 & 0 & 0 & $i$ & 2 \\
\hline 08:45 AM & - & 09:45.AM & 0 & 0 & 0 & 0 & 0 & 0 & $i$ & $i$ & 0 & 0 & 0 & 1 & 3 \\
\hline $09: 00 \mathrm{AM}$ & $=$ & $10: 00 \mathrm{AM}$ & 0 & 0 & 0 & 0 & 0 & $a$ & 2 & 1 & 0. & 0 & 0 & 0 & 3 \\
\hline $\begin{array}{l}09: 15 \mathrm{AM} \\
09: 30 \mathrm{AM}\end{array}$ & $=$ & $10: \overline{1 S A} \bar{M}$ & 0 & 0 & 0 & 0 & 0 & 0 & 3 & $\frac{1}{2}$ & 0 & 0 & 0 & ii & 4 \\
\hline $\begin{array}{l}09: 30 \text { AM } \\
09: 45 \text { AM }\end{array}$ & $\bar{E}$ & $\begin{array}{l}10: 30 \mathrm{AM} \\
10: 45 \mathrm{AM}\end{array}$ & $\begin{array}{l}0 \\
0\end{array}$ & $\begin{array}{l}0 \\
0\end{array}$ & $\begin{array}{l}0 \\
0\end{array}$ & $\begin{array}{l}0 \\
0\end{array}$ & $\begin{array}{l}0 \\
0\end{array}$ & $\begin{array}{l}0 \\
0\end{array}$ & $\begin{array}{l}3 \\
3\end{array}$ & $\begin{array}{l}2 \\
1\end{array}$ & - 0 & 0 & $\begin{array}{l}0 \\
1\end{array}$ & $" 1$ & $s$ \\
\hline $10: 00 \mathrm{AM}$ & $=$ & $11: 00 \mathrm{AM}$ & 8 & 6 & 0 & $\frac{0}{0}$ & 0 & 0 & 2 & 1 & 0 & 0 & 1 & 16 & $\frac{1}{2}$ \\
\hline $\begin{array}{l}10: 15 \text { AM } \\
10: 30 \text { AM }\end{array}$ & $\overline{ }$ & 11:15 AMI & $\begin{array}{l}0 \\
0\end{array}$ & .0 & $\begin{array}{l}0 \\
0\end{array}$ & 0 & $\begin{array}{l}0 \\
0\end{array}$ & $\begin{array}{l}0 \\
0\end{array}$ & 1 & 1 & 0 & 0 & 1 & 1 & \{ \\
\hline & $=$ & $\frac{11: 30 \mathrm{AM}}{11: 15 \mathrm{AM}}$ & $\frac{0}{0}$ & 0 & $\frac{0}{0}$ & $\frac{1}{1}$ & $\frac{0}{0}$ & $\frac{0}{0}$ & & $-\frac{2}{3}$ & 0 & 0 & $\frac{3}{7}$ & $!$ & 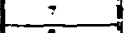 \\
\hline $\begin{array}{l}10: 43 \mathrm{AM} \\
11: 00 \mathrm{AM}\end{array}$ & $\ldots$ & 12:00 PM & 0 & 0 & 0 & 1 & 0 & $\begin{array}{l}0 \\
0\end{array}$ & $\begin{array}{l}0 \\
0\end{array}$ & $\begin{array}{l}3 \\
6\end{array}$ & $\begin{array}{l}0 \\
0\end{array}$ & $\begin{array}{l}0 \\
0\end{array}$ & 2 & $\vdots$ & 8 \\
\hline IIIS AMM & $=$ & 12:15 PM & 0 & 0 & 0 & 1 & 0 & 0 & 1 & $\begin{array}{l}0 \\
6\end{array}$ & 0 & $\begin{array}{l}0 \\
0 \\
0\end{array}$ & 2 & $\frac{1}{2}$ & $\begin{array}{l}13 \\
12\end{array}$ \\
\hline $11: 30 \mathrm{AM}$ & $=$ & $12: 30 \mathrm{P}$ & 0 & 0 & 0 & 0 & 0 & 1 & $T$ & 4 & 0 & 0 & $\overline{0}$ & $\Sigma$ & 8 \\
\hline 11:4S AM & $\ldots$ & 12:45 PM & 0 & 0 & 0 & 0 & 0 & 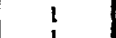 & 1 & 4 & 0 & 0 & 0 & 1 & , \\
\hline $12: 00$ & $\ldots$ & -01 & $-\frac{0}{0}$ & $-\frac{0}{0}$ & $\frac{0}{0}$ & $\frac{1}{1}$ & 0 & $\frac{1}{2}$ & 1 & $\frac{1}{1}$ & $\frac{0}{0}$ & 0 & $\frac{0}{2}$ & ü & \pm \\
\hline $12: 15 \mathrm{PM}$ & $=$ & 01 & $\begin{array}{l}0 \\
0\end{array}$ & $\begin{array}{l}0 \\
0\end{array}$ & $\begin{array}{l}0 \\
0\end{array}$ & 1 & $\begin{array}{l}0 \\
0\end{array}$ & $\frac{2}{2}$ & $\stackrel{0}{0}$ & $\begin{array}{l}1 \\
1\end{array}$ & 0 & $\begin{array}{l}0 \\
0 \\
0\end{array}$ & $\overline{0}$ & ii & 8 \\
\hline $\begin{array}{l}\text { 12:30 PM } \\
12: 45 \mathrm{PM}\end{array}$ & $=$ & $\begin{array}{l}01: 30 \mathrm{PM} \\
01: 45 \mathrm{PM}\end{array}$ & $\begin{array}{l}0 \\
0 \\
\end{array}$ & 0 & 0 & 1 & $\begin{array}{l}0 \\
0\end{array}$ & $\frac{1}{2}$ & $\begin{array}{l}0 \\
0\end{array}$ & 1 & $\begin{array}{l}0 \\
0\end{array}$ & $\begin{array}{l}0 \\
0\end{array}$ & $\begin{array}{l}0 \\
1\end{array}$ & 1 & 5 \\
\hline $01: 00$ & $\cdots$ & 02 & 0 & 0 & 0 & 0 & 0 & 2 & 0 & $i$ & 0 & $\overline{0}$ & $T$ & $T$ & 5 \\
\hline $01: 19$ PM & & $02: 15$ PM & 0 & 0 & 0 & 0 & 0 & 1 & 0 & 1 & 0 & 0 & $i$ & i & $s$ \\
\hline & $\div$ & 02 & 0 & 0 & 0 & 0 & 0 & 2 & 0 & 2 & 0 & 0 & $i$ & $\dot{0}$ & 6 \\
\hline 01:45 PM & $=$ & $02: 45$ & 0 & 0 & $\delta$ & 0 & 0 & 3 & 0 & 2 & 0 & 0 & 2 & 0 & 9 \\
\hline $02: 00 \mathrm{PM}$ & $\ldots$ & 03:00 PM & 0 & 0 & 0 & 0 & 0 & 3 & 0 & 2 & 0 & 0 & 2 & 0 & 7 \\
\hline $02: 15 \mathrm{PM}$ & $=$ & $03: 15 \mathrm{PM}$ & 0 & 0 & 0 & 0 & 0 & 4 & 0 & 3 & 0 & 0 & 2 & 0 & $?$ \\
\hline $02: 30 \mathrm{PM}$ & + & $03: 30 \mathrm{PM}$ & 8 & $\sigma$ & 0 & 0 & 0 & 2 & 0 & 3 & 0 & 0 & $i$ & 0 & 8 \\
\hline 02.45 PM & 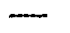 & 03:45 PM & 0 & 0 & 0 & 0 & 0 & 1. & 0 & 2 & 0 & 0 & 0 & 0 & 3 \\
\hline $03: 00 \mathrm{PM}$ & - & O4:00 PM & 0 & 0 & 0 & 0 & 0 & 2 & 0 & 2 & 0 & 0 & 0 & 0 & 1 \\
\hline $03.15 \mathrm{PM}$ & 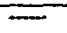 & $04: 15 \mathrm{PM}$ & 0 & 0 & 0 & 0 & 0 & 1 & 0 & 1 & 0 & 0 & 1 & 0 & 3 \\
\hline $03: 30 \mathrm{PM}$ & $=$ & $\begin{array}{l}04: 30 \mathrm{PM} \\
04.45 \mathrm{PM}\end{array}$ & 0 & $\begin{array}{l}0 \\
0\end{array}$ & 0 & $\begin{array}{l}0 \\
0\end{array}$ & $\begin{array}{l}0 \\
0\end{array}$ & i & : & $\begin{array}{l}0 \\
0\end{array}$ & $\begin{array}{l}0 \\
0 \\
0\end{array}$ & $\stackrel{0}{0}$ & 2 & 0 & 3 \\
\hline$\frac{03: 45 \mathrm{PM}}{-04: 00 \mathrm{PM}}$ & $\equiv$ & $\frac{04: 45 \text { PM }}{05: 00 P M}$ & $\frac{0}{0}$ & 0 & 0 & $\frac{0}{0}$ & $\frac{0}{0}$ & $\frac{1}{0}$ & 0 & 0 & 0 & $\frac{0}{0}$ & & $\frac{0}{0}$ & $\frac{3}{3}$ \\
\hline
\end{tabular}




\section{Baymetrics Traffic Resources INTERSECTION TURNING MOVEMENT SUMMARY}

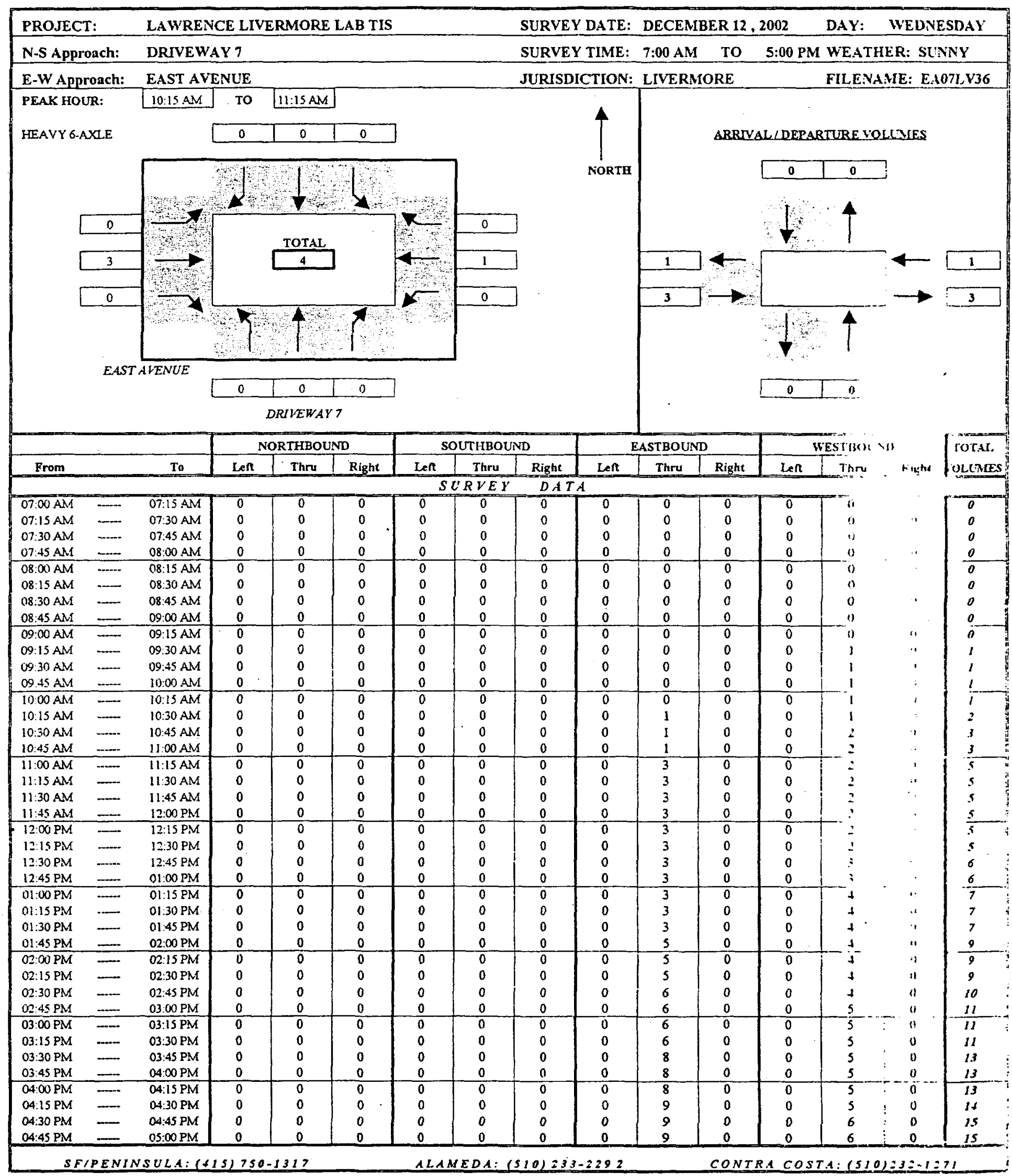




\section{Baymetrics Traffic Resources INTERSECTION TURNING MOVEMENT SUMMARY}

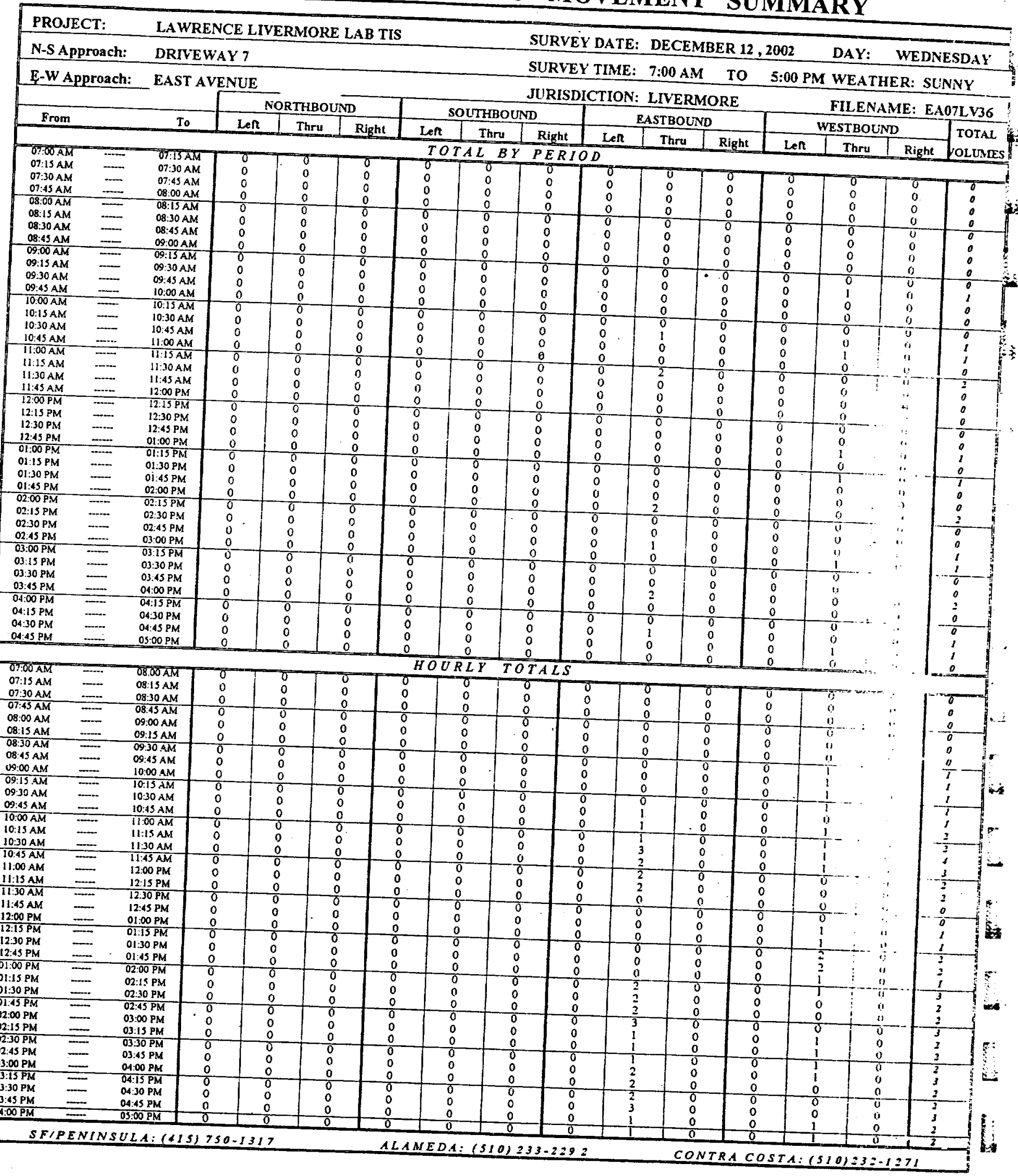




\section{Baymetrics Traffic Resources INTERSECTION TURNING MOVEMENT SUMMARY}

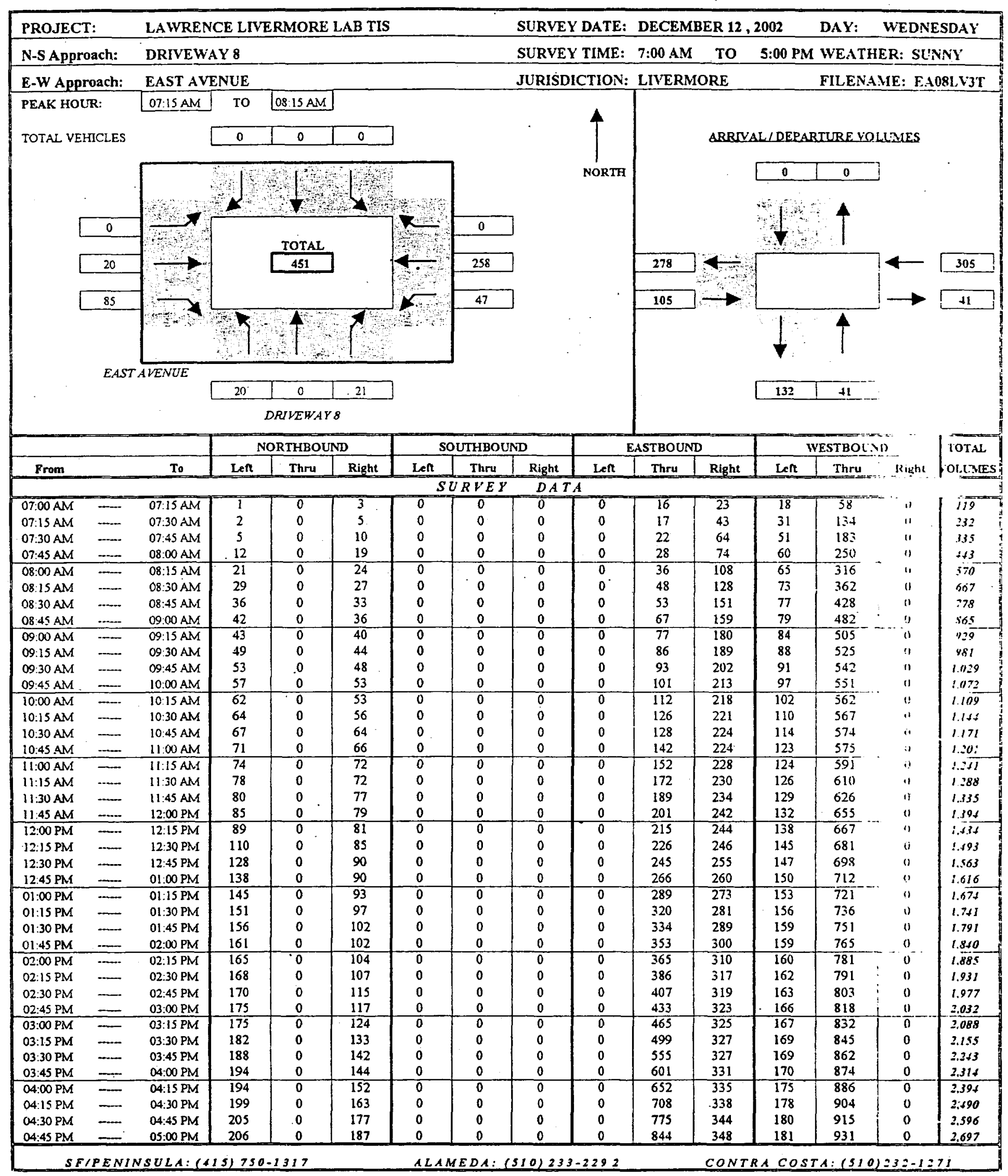




\section{Baymetrics Traffic Resources INTERSECTION TURNING MOVEMENT SUMMARY}

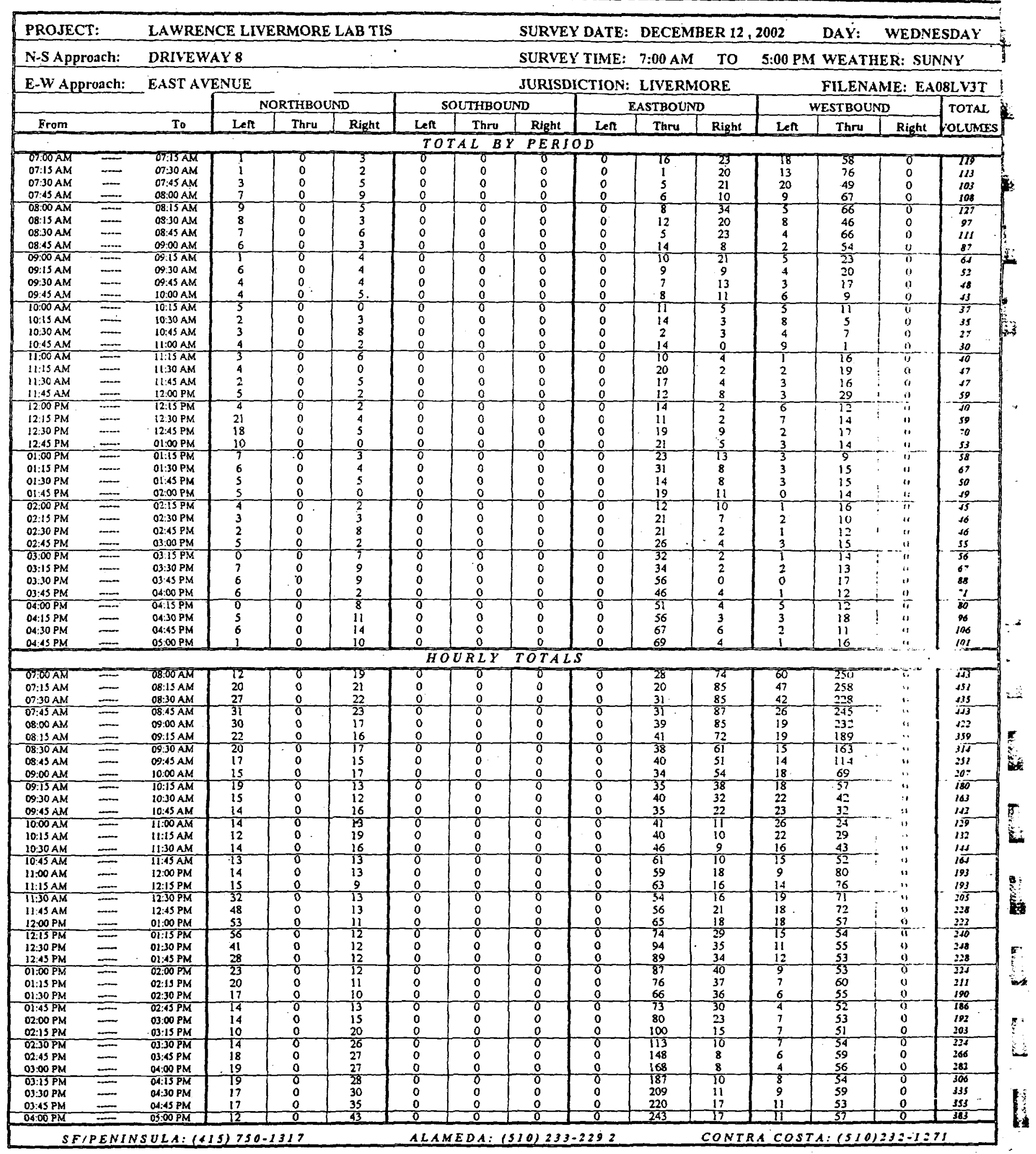




\section{Baymetrics Traffic Resources. INTERSECTION TURNING MOVEMENT SUMMARY}

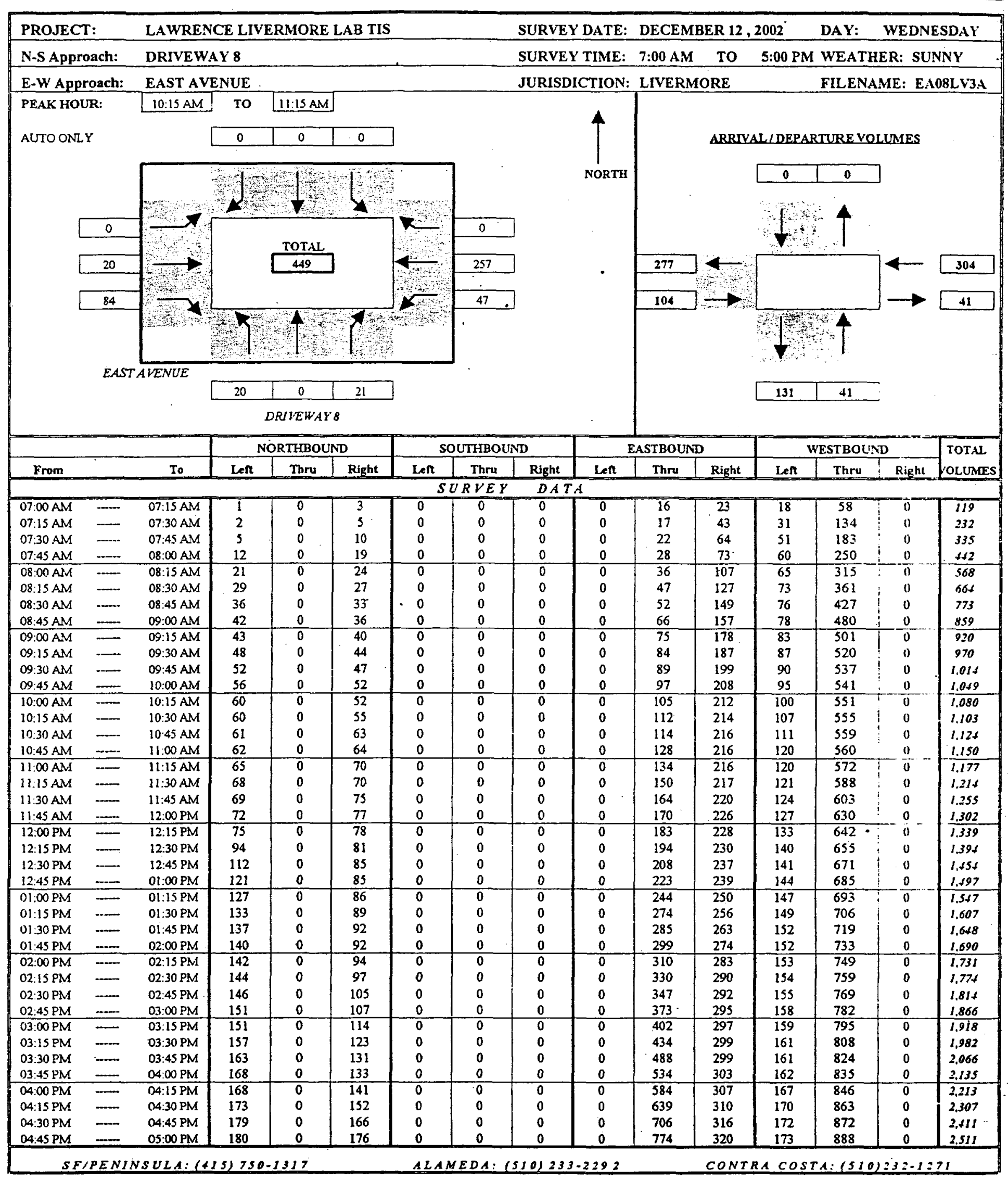




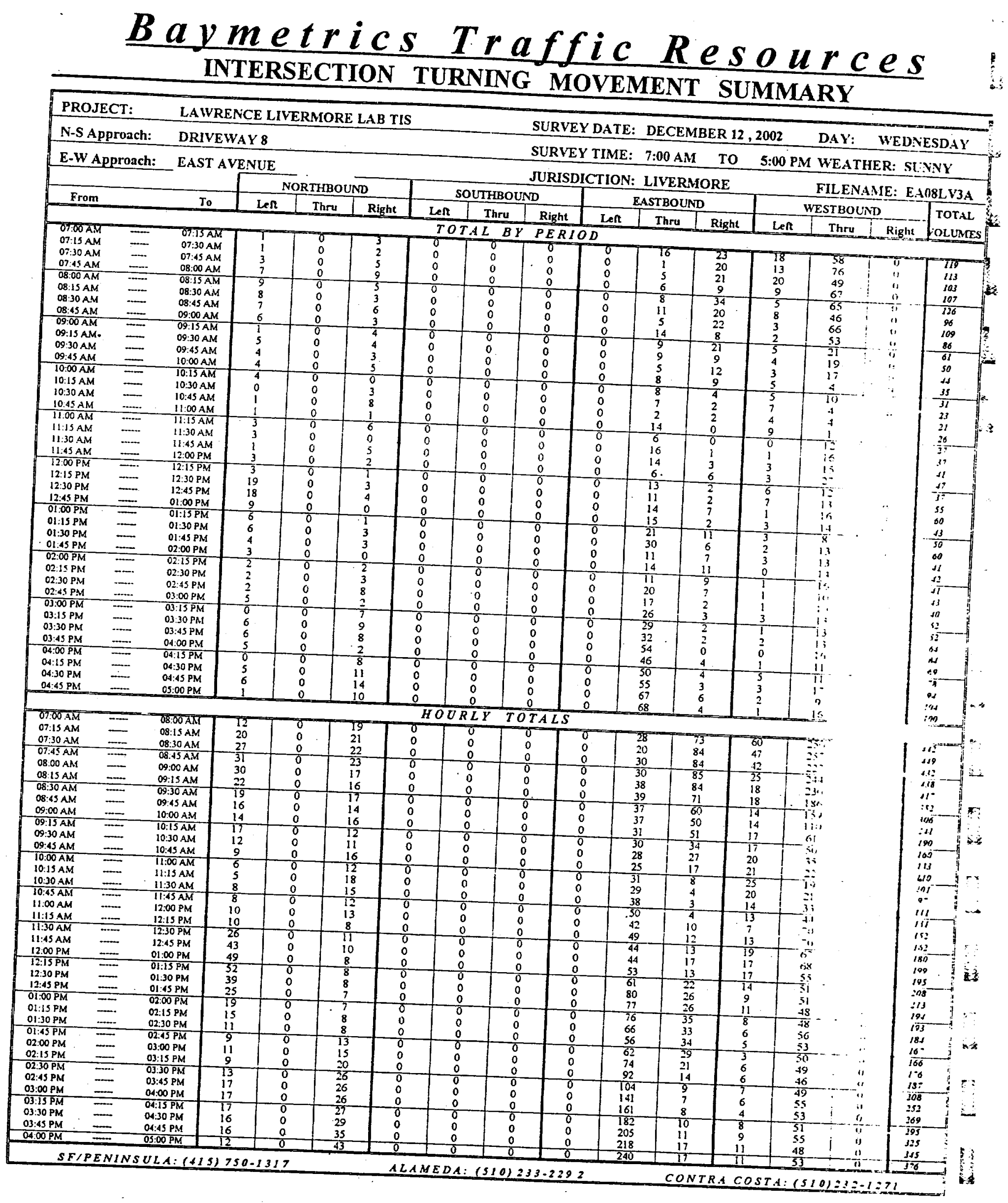




\section{Baymetrics Traffic Resources INTERSECTION TURNING MOVEMENT SUMMARY}

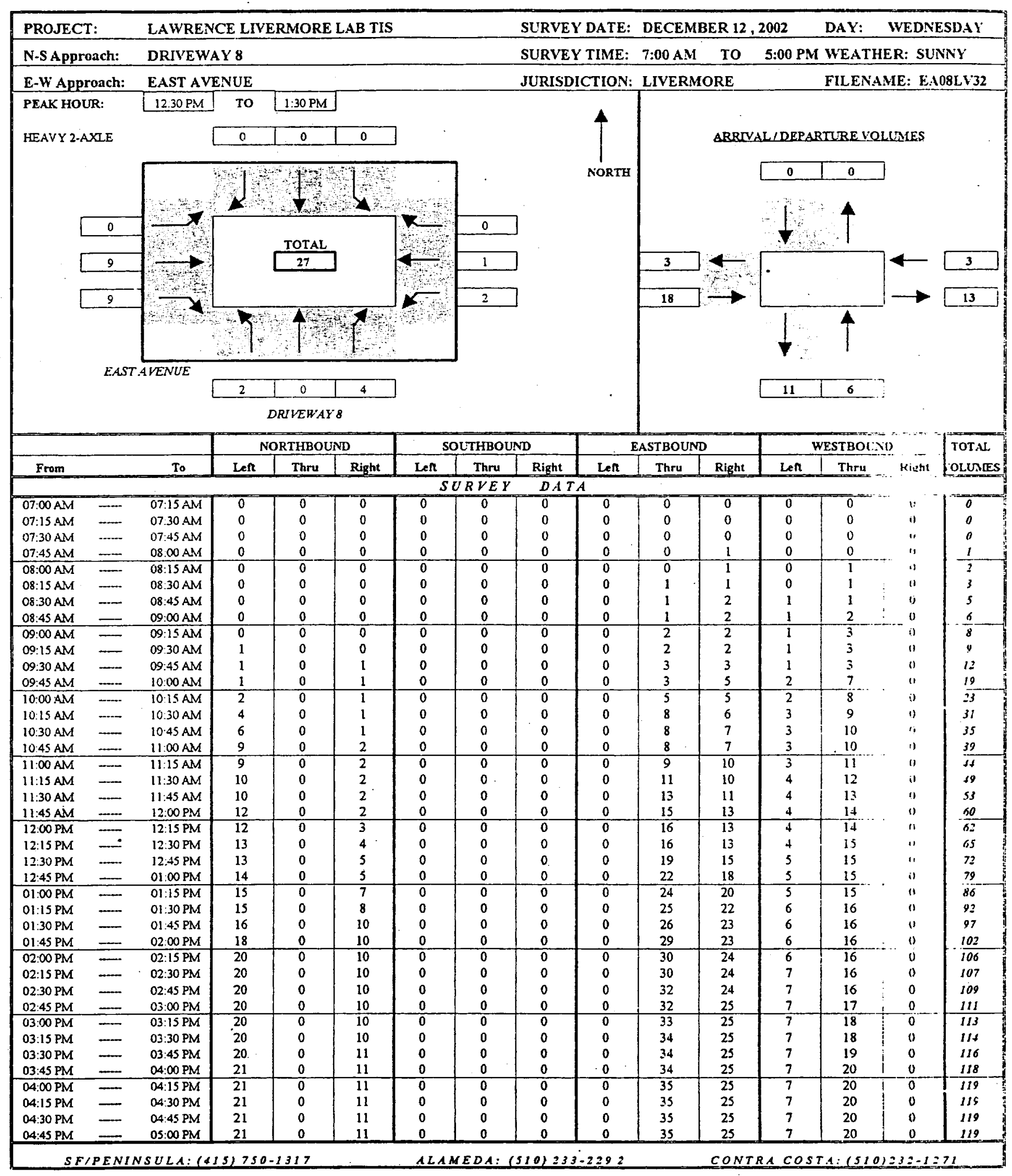




\section{Baymetrics Traffic Resources}

\begin{tabular}{|c|c|c|c|c|c|c|c|c|c|c|c|c|c|c|c|}
\hline \multirow{2}{*}{\multicolumn{2}{|c|}{$\begin{array}{l}\text { PROJECT: } \\
\text { N-S Approach: }\end{array}$}} & \multicolumn{6}{|c|}{ LAWRENCE LIVERMORE LAB TIS } & SURVE & ATE: & DECEMI & ER 12, & 2002 & DAY: & WEDN & SDAY \\
\hline & & DRIVEU & & & & & & SURVE & IME: & $7: 00 \mathrm{AM}$ & To & 5:00 PM & WEAT & ER: SL: & iNY \\
\hline E-W AP & oasch: & EAST AV & VUE & & & & & JURISD & TION: & LIVERM & RE & & FIL_EN & ME: EA & $8 \mathrm{LV} 32$ \\
\hline & & & & THBO & & & TIBBO & & & ASTBOUN & & & ESTBOI & D & TOTAL \\
\hline From & & To & Len & Thru & Right & Len & Thru & Right & Len & Thru & Right & Len & Thru & Right & LoLUMES \\
\hline & & & & & & & $L B$ & $P E R I$ & & & & & & & \\
\hline O7:06 AM & $=$ & $6715 \mathrm{AM}$ & 0 & 0 & 0 & $\sigma$ & 0 & 9 & 0 & 0 & 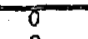 & 8 & \% & $\pi$ & 8 \\
\hline $07: 15 \mathrm{AM}$ & $\cdots$ & $07: 30 \mathrm{AM}$ & 0 & 0 & 0 & 0 & 0 & 0 & 0 & 0 & 0 & 0 & 0. & 0 & 0 \\
\hline $\begin{array}{l}07: 30 \mathrm{AM} \\
07: 43 \mathrm{AM} \\
\end{array}$ & $=$ & $\begin{array}{l}07: 5.5 \mathrm{AM} \\
08: 00 \mathrm{AM}\end{array}$ & $\begin{array}{l}0 \\
0 \\
\end{array}$ & $\begin{array}{l}0 \\
0 \\
\end{array}$ & $\begin{array}{l}0 \\
0 \\
\end{array}$ & $\begin{array}{l}0 \\
0 \\
\end{array}$ & $\begin{array}{l}0 \\
0\end{array}$ & $\begin{array}{l}0 \\
0 \\
\end{array}$ & $\begin{array}{l}0 \\
0 \\
\end{array}$ & $\begin{array}{l}0 \\
0 \\
\end{array}$ & $\begin{array}{l}0 \\
1 \\
\end{array}$ & $\begin{array}{l}0 \\
0 \\
\end{array}$ & $\begin{array}{l}0 \\
0\end{array}$ & $\begin{array}{l}0 \\
D \\
D\end{array}$ & $\begin{array}{l}0 \\
1 \\
\end{array}$ \\
\hline $08: 00 \mathrm{AM}$ & $=$ & $08: 15 \mathrm{AM}$ & 8 & 0 & 0 & 0 & 0 & 0 & 0 & 0 & 0 & 0 & 1 & 0 & $T$ \\
\hline $08: 15 \mathrm{AM}$ & - & $08: 30 \mathrm{AM}$ & 0 & 0 & 0 & 0 & 0 & 0 & 0 & 1 & 0 & $?$ & 0 & g & 1 \\
\hline $\begin{array}{l}08.30 \mathrm{AM} \\
08.45 \mathrm{AM}\end{array}$ & 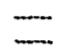 & $\begin{array}{l}08: 45 \mathrm{AM} \\
09: 00 \mathrm{AM}\end{array}$ & 0 & 0 & $\begin{array}{l}0 \\
0\end{array}$ & $\begin{array}{l}0 \\
0\end{array}$ & $\begin{array}{l}0 \\
0\end{array}$ & $\begin{array}{l}0 \\
0\end{array}$ & 0 & 0 & 1 & 1 & 0 & 0 & 3 \\
\hline $09: 00 \mathrm{AN}$ & $=$ & $\frac{090 \mathrm{AM}}{09: 15 \mathrm{AM}}$ & 0 & 0 & $\frac{0}{0}$ & 0 & $\frac{0}{0}$ & 0 & 0 & $\frac{0}{1}$ & $\frac{1}{0}$ & 0 & $\frac{1}{1}$ & o- & $\frac{2}{2}$ \\
\hline $09: 15 \mathrm{AM}$ & $-\ldots$ & $09: 10 \mathrm{AM}$ & 1 & 0 & 0 & 0 & 0 & 0 & 0 & 0 & 0 & 0 & 0 & n & 1 \\
\hline $09.30 \mathrm{AM}$ & - & $09: 45 \mathrm{AM}$ & 0 & 0 & i. & 0 & 0 & 0 & 0 & 1 & 1 & 0 & 0 & 0 & 3 \\
\hline $09: 45$ AM & $=$ & $10.00 \mathrm{AM}$ & 0 & 0 & 0 & 0 & 0 & 0 & 0 & 0 & 2 & 1 & 4. & 0 & 3 \\
\hline $10: 00 \mathrm{AM}$ & $=$ & $10: 15 \mathrm{AM}$ & 1 & 0 & 0 & 0 & 0 & 0 & 0 & & 0 & 0 & $T$ & 6 & $\pi$ \\
\hline 10:15 AM & - & $10: 30 \mathrm{AM}$ & 2 & 0 & 0 & 0. & 0 & 0 & 0 & 3 & 1 & 1 & i & 0 & 8 \\
\hline $\begin{array}{l}10: 30 \mathrm{AM} \\
10.45 \mathrm{AM}\end{array}$ & $=$ & $10.45 \mathrm{AM}$ & 2 & & 0 & & 0 & 0 & 0 & 0 & $i$ & 0 & 1 & $"$ & 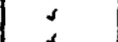 \\
\hline $11: 00 \mathrm{AM}$ & $=$ & $=\frac{11: 00 \mathrm{AM}}{11: 15 \mathrm{AM}}$ & $\frac{3}{0}$ & $\frac{0}{0}$ & $\frac{1}{0}$ & $\frac{0}{0}$ & $\frac{0}{0}$ & $\begin{array}{l}0 \\
0\end{array}$ & $\begin{array}{l}0 \\
0\end{array}$ & $\frac{0}{1}$ & $\frac{0}{3}$ & $\frac{0}{0}$ & $\frac{0}{1}$ & ;i. & $\frac{4}{5}$ \\
\hline 11:15 AM & - & $11: 30 \mathrm{AM}$ & 1 & 0 & 0 & 0 & 0 & 0 & 0 & 2 & 0 & $i$ & 1 & $\because$ & 2 \\
\hline $11: 30 \mathrm{AM}$ & - & $11: 45 \mathrm{AM}$ & 0 & $\begin{array}{l}0 \\
0\end{array}$ & 0 & 0 & 0 & 0 & 0 & 2 & 1 & 0 & $i$ & $\because$ & 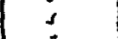 \\
\hline$\frac{11: 43 \mathrm{AM}}{12: 00 \mathrm{PM}}$ & $=$ & $\frac{12: 00 \mathrm{PM}}{12: 15 \mathrm{PM}}$ & $\frac{2}{0}$ & $\frac{1}{0}$ & $\frac{0}{1}$ & $\frac{0}{0}$ & $\frac{0}{0}$ & $\frac{0}{0}$ & 0 & $\frac{2}{1}$ & $\frac{2}{0}$ & $\frac{0}{0}$ & $\frac{1}{0}$ & $\because-$ & 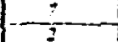 \\
\hline $12: 15 \mathrm{PM}$ & $\ldots$ & $12: 30 \mathrm{PM}$ & 1 & 0 & 1 & 0 & 0 & 0 & $\begin{array}{l}0 \\
0\end{array}$ & 0 & $\begin{array}{l}0 \\
0\end{array}$ & 0 & 1 & $" 1$ & 3 \\
\hline $12: 30 \mathrm{PM}$ & - & 12:45 PM & 0 & 0 & $i$ & 0 & 0 & 0 & 0 & 3 & 2 & $i$ & 9 & $\because$ & . \\
\hline $12: 45$ PM & $=$ & 01.00 PM & 1 & 0 & $\frac{0}{2}$ & 0 & 0 & 0 & 0 & $\frac{3}{2}$ & $-\frac{3}{2}$ & $\frac{0}{0}$ & 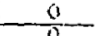 & $\because$. & $\therefore$ \\
\hline $01: 00 \mathrm{PM}$ & $=$ & $01: 15 \mathrm{PM}$ & 1 & 0 & 2 & 0 & 0 & $\overline{0}$ & 0 & 2 & $\frac{2}{2}$ & 0 & 0 & $"$ & \\
\hline $0: 13$ PM & $\cdots$ & $01: 30 \mathrm{PM}$ & 0 & 0 & 1 & 0 & 0 & 0 & 0 & $!$ & 2 & 1 & 1 & " & 6 \\
\hline 01:30 PM & - & 01:45 PM & 1 & 0 & 2 & 0 & 0 & 0 & 0 & 1 & 1 & 0 & 9 & "' & 3 \\
\hline O1: $\triangle S$ PM & $\cdots$ & 22:00 PM & $-\frac{2}{2}$ & 0 & 0 & 0 & 0 & 0 & 0 & 3 & 0 & 0 & 9 & . & $s$ \\
\hline $02: 00 \mathrm{PM}$ & $=$ & $02: 15 \mathrm{PM}$ & $\frac{2}{6}$ & 0 & 0 & 0 & 0 & 0 & 0 & 1 & $t$ & 0 & 1 & ' & 7 \\
\hline 02:15 PM & - & $02: 30 \mathrm{PM}$ & 0 & 0 & 0 & 0 & 0 & 0 & 0 & 0 & 0 & 1 & 0 & .' & 1 \\
\hline $02.30 \mathrm{PM}$ & $\ldots$ & $02.45 \mathrm{PM}$ & 0 & 0 & 0 & 0 & 0 & 0 & 0 & 2 & 0 & 0 & 0 & "' & 2 \\
\hline $02: 45 \mathrm{PM}$ & $-\infty$ & $03: 00 \mathrm{PM}$ & 0 & 0 & 0 & 0 & 0 & 0 & 0 & 0 & 1 & 0 & 1 & $\therefore$ & 2 \\
\hline $03.00 \mathrm{PM}$ & $\ldots$ & $03: 15 \mathrm{PM}$ & 0 & 0 & 0 & 0 & $\sigma$ & 0 & 0 & $T$ & 0 & a & 1 & "1 & 2 \\
\hline 03:15 PM & $\ldots$ & $03: 30 \mathrm{PM}$ & 0 & 0 & 0 & 0 & 0 & 0 & 0 & 1 & 0 & 0 & 0 & 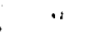 & 1 \\
\hline $03: 30 \mathrm{PM}$ & - & $03: 45 \mathrm{PM}$ & 0 & 0 & 1 & 0 & 0 & 0 & 0. & 0 & 0 & 0 & 1 & ". & $:$ \\
\hline $09: 45 \mathrm{PM}$ & $\ldots$ & $04: 00 \mathrm{PM}$ & 1 & 0 & 0 & 0 & 0 & 0 & 0 & 0 & 0 & 0 & 1 & $\because$ & $\therefore$ \\
\hline $04: 00$ PM & $=$ & $04: 15 \mathrm{PM}$ & 0 & 0 & 0 & 0 & 0 & 0 & 0 & 1 & 0 & 0 & o & ii & $T$ \\
\hline $04: 15$ PM & - & $04: 30 \mathrm{PM}$ & 0 & 0 & 0 & 0 & 0 & 0 & 0 & 0 & 0 & 0 & 0 & $\because$ & $a$ \\
\hline 04:30 PM & - & $04: 45 \mathrm{PM}$ & 0 & 0 & 0 & 0 & 0 & 0 & 0 & 0 & 0 & 0 & $a$ & $"$ & 0 \\
\hline $04: 45 \mathrm{PM}$ & $\ldots$ & $05: 00 \mathrm{PM}$ & $a$ & 0 & 0 & 0 & 0 & 0 & 0 & 0 & 0 & 0 & 0 & 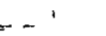 & $?$ \\
\hline & & & & & & & $R L Y$ & TOTA & & & & & & & \\
\hline O:00AM & $\cdots$ & $08: 06.9 M$ & $\sigma$ & $\sigma$ & \% & 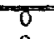 & $\overline{0}$ & 8 & $\overline{0}$ & $\pi$ & $T$ & $\sigma$ & $\pi$ & $\because$ & $?$ \\
\hline $07: 15 \mathrm{AM}$ & - & $08.15 \mathrm{AM}$ & 0 & 0 & 0 & 0 & 0 & 0 & 0 & 0 & 1 & 0 & $!$ & $\because$ & $?$ \\
\hline $07: 30 \mathrm{AM}$ & $=$ & 08:30 AM & 0 & 0 & 0 & 0 & 0 & 0 & 0 & 1 & 1 & $\underline{0}$ & 1 & . & 3 \\
\hline $07: 45 \mathrm{AM}$ & $\cdots$ & $08: 45 \mathrm{AM}$ & $\overline{0}$ & 0 & 0 & 0 & 0 & 0 & 0 & 1 & 2 & $T$ & 1 & $\because$ & 5 \\
\hline $08: 00 \mathrm{AM}$ & $\ldots$ & 09:00 AM & 0 & 0 & 0 & 0 & 0 & 0 & 0 & 1 & 1 & 1 & 2 & :' & 5 \\
\hline O8:15 AM & $=$ & $09: 15 \mathrm{AM}$ & 0 & $-\frac{0}{2}$ & 0 & 0 & 0 & 0 & 0 & 2 & 1 & 1 & $=$ & $\therefore$ & $\therefore$ \\
\hline $0830 \mathrm{AM}$ & $=$ & $09: 30 \mathrm{AM}$ & $T$ & $\overline{0}$ & $\sigma$ & 0 & 0 & 0 & 0 & $T$ & 1 & 1 & 3 & "i & 6 \\
\hline $08: 45$ AM & - & $09: 45 \mathrm{AM}$ & 1 & 0 & 1 & 0 & 0 & 0 & 0 & 2 & 1 & 0 & 2 & " & is \\
\hline $09.00 \mathrm{AM}$ & $=$ & $10: 00 \mathrm{AM}$ & 1 & 0 & 1 & 0 & 0 & 0 & 0 & 2 & 3 & 1 & 5 & $::$ & 3 \\
\hline $09.15 \mathrm{AM}$ & $=$ & $10: 15 \mathrm{AM}$ & 2 & 0 & 1 & 0 & 0 & $\delta$ & 0 & 3 & 3 & $T$ & 5 & "' & 75 \\
\hline $09: 30 \mathrm{AM}$ & $\ldots$ & $10: 30 \mathrm{AM}$ & 3 & 0 & 1 & 0 & 0 & 0 & 0 & 6 & 4 & 2 & 6 & $\cdot \cdot$ & 23 \\
\hline $09: 45$ AM & $-\infty$ & 10:45 AM & 5 & 0 & 0 & 0 & 0 & 0 & 0 & 5 & 4 & 2 & 3 & . & 23 \\
\hline $10: 00 \mathrm{AM}$ & $=$ & $11: 00 \mathrm{AM}$ & 8 & $\sigma$ & $T$ & 0 & 0 & 0 & 0 & 5 & 2 & 1 & 3 & . & 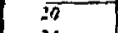 \\
\hline 10:15 AM & $\ldots$ & 11.15 AM & 7 & 0 & i & 0 & 0 & 0 & 0 & $A$ & 5 & 1 & 3 & . & 3 \\
\hline $10.30 \mathrm{AM}$ & $\ldots$ & $11: 30 \mathrm{AM}$ & 6 & 0 & $i$ & 0 & 0 & 0 & 0 & 3 & 4 & $\frac{1}{1}$ & 3 & & 18 \\
\hline $10: 45 \mathrm{AM}$ & $\ldots$ & $11: 45 \mathrm{AM}$ & 4 & 0 & $T$ & 0 & $\bar{\sigma}$ & $\overline{0}$ & 0 & 5 & 4 & 1 & $\sqrt{3}$ & ' & at \\
\hline $11000 \mathrm{AM}$ & - & $12: 00 \mathrm{PM}$ & 3 & 0 & 0 & 0 & 0 & 0 & 0 & 7 & 6 & 1 & 4 & $:$ & 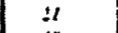 \\
\hline I1:19 AM & - & 12:15 PM & 3 & 0 & 1 & 0 & 0 & 0 & 0 & 1 & 3 & $i$ & 3 & "، & 18 \\
\hline $11: 30 \mathrm{AM}$ & $=$ & $12: 30 \mathrm{PM}$ & 3 & 0 & 2 & 0 & 0 & 0 & 0 & 5 & 3 & 0 & 3 & “' & $\overline{16}$ \\
\hline II:45 AM & $\cdots$ & 12:45 PM & 3 & 0 & 3 & 0 & 0 & 0 & 0 & 6 & 4 & 1 & 2 & 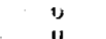 & 89 \\
\hline$\frac{12: 00 \mathrm{PM}}{12.15 \mathrm{PM}}$ & $=$ & $\frac{01: 00 P M}{01.15 P M}$ & $\frac{2}{3}$ & $\frac{0}{0}$ & $\frac{3}{4}$ & $\frac{0}{0}$ & $\frac{0}{0}$ & $\frac{0}{0}$ & $\frac{a}{0}$ & $\frac{7}{8}$ & $\frac{5}{7}$ & $T$ & $\frac{1}{1}$ & $\frac{3}{11}$ & \\
\hline $\begin{array}{l}12: 15 \mathrm{PM} \\
12: 30 \mathrm{PM}\end{array}$ & $=$ & $\begin{array}{l}01: 15 \mathrm{PM} \\
01 \cdot 30 . P M\end{array}$ & $\begin{array}{l}3 \\
2\end{array}$ & 0 & $\begin{array}{l}4 \\
4\end{array}$ & 8 & $\begin{array}{l}0 \\
0\end{array}$ & $\begin{array}{l}0 \\
0\end{array}$ & $\begin{array}{l}0 \\
0\end{array}$ & ${ }_{9}^{8}$ & $\begin{array}{l}7 \\
9\end{array}$ & 2 & 1 & ، & 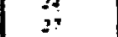 \\
\hline $12: 45 \mathrm{PM}$ & $=$ & Di: $25 \mathrm{PM}$ & 3 & .0 & 5 & 0 & 0 & 0 & 0 & 7 & 8 & 1 & 1 & a & 35 \\
\hline $01: 00 \mathrm{PM}$ & $=$ & $02: 00 \mathrm{PM}$ & 4 & 0 & 5 & 0 & 0 & $\overline{0}$ & 0 & 7 & 5 & & $T$ & $\overline{0}$ & 23 \\
\hline $01: 15 \mathrm{PM}$ & - & $02: 15 \mathrm{PM}$ & 5 & 0 & 3 & 0 & 0 & 0 & 0 & 6 & 4 & 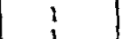 & 1 & 8 & 20 \\
\hline $01: 30 \mathrm{PM}$ & $=$ & 02:30 PM & 5 & $\frac{0}{0}$ & $\frac{2}{0}$ & $\frac{a}{a}$ & $\frac{0}{0}$ & $\frac{0}{0}$ & $\frac{0}{0}$ & $\frac{5}{6}$ & 2 & $\frac{1}{1}$ & $\frac{0}{0}$ & $\frac{a}{0}-$ & $\frac{d S}{d 2}$ \\
\hline $\begin{array}{l}01: 45 \mathrm{PM} \\
02: 00 \mathrm{PM}\end{array}$ & \pm & $02.75 \mathrm{PM}$ & 4 & 0 & 0 & 0 & $\begin{array}{l}0 \\
0\end{array}$ & $\begin{array}{l}0 \\
0\end{array}$ & $\begin{array}{l}0 \\
0\end{array}$ & ${ }_{3}^{6}$ & $\frac{1}{2}$ & 1 & 1 & $\begin{array}{l}0 \\
0\end{array}$ & 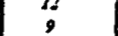 \\
\hline $\begin{array}{l}\text { 02:200 PMM } \\
\text { 02:19 PM }\end{array}$ & $=$ & $\begin{array}{l}\text { 03:00 PM } \\
03: 15 \mathrm{PM}\end{array}$ & 0 & $\begin{array}{l}0 \\
0 .\end{array}$ & 0 & 0 & 0 & 0 & 0 & 3 & 1 & 1 & $\frac{1}{2}$ & 0 & 7 \\
\hline$\frac{0.15 \mathrm{SM}}{02: 30 \mathrm{PM}}$ & $=$ & $03.30 \mathrm{PM}$ & 0 & 8 & 0 & 0 & $\sigma$ & $\gamma$ & 0 & 4 & 1 & 0 & 5 & 0 & \\
\hline 0z:c5 PM & $=$ & $03: 45 \mathrm{PM}$ & 0 & 0 & l & 0 & 0 & 0 & 0 & 2 & i & 0 & 3 & 0 & $?$ \\
\hline 01:00 PM & $\ldots$ & $04: 00 \mathrm{PM}$ & 1 & 0 & $i$ & 0 & 0 & 0 & 0 & 2 & 0 & 0 & 3 & $\underline{0}$ & $\therefore$ \\
\hline & $=$ & OA:15 PM & $i$ & & & 0 & 0 & 0 & 0 & 2 & 0 & 0 & 2 & 0 & 6 \\
\hline 03:30 PM & $=$ & 01:30 PM & 1 & 0 & i & 0 & 0 & 0 & 0 & $i$ & 0 & 0 & 2 & 0 & 5 \\
\hline 03:43 PM & $\ldots$ & OA: $45 \mathrm{PM}$ & I & 0 & 0 & 0. & 0 & 0 & 0 & 1 & 0 & 0 & 1 & $\underline{u}$ & 3 \\
\hline $04: 00 \mathrm{PM}$ & $=$ & $05: 00 \mathrm{PM}$ & 8 & 0 & 0 & 0 & 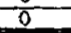 & 0 & 0 & & 0 & & 0 & 0 & \\
\hline
\end{tabular}




\section{Baymetrics Traffic Resources INTERSECTION TURNING MOVEMENT SUMMARY}

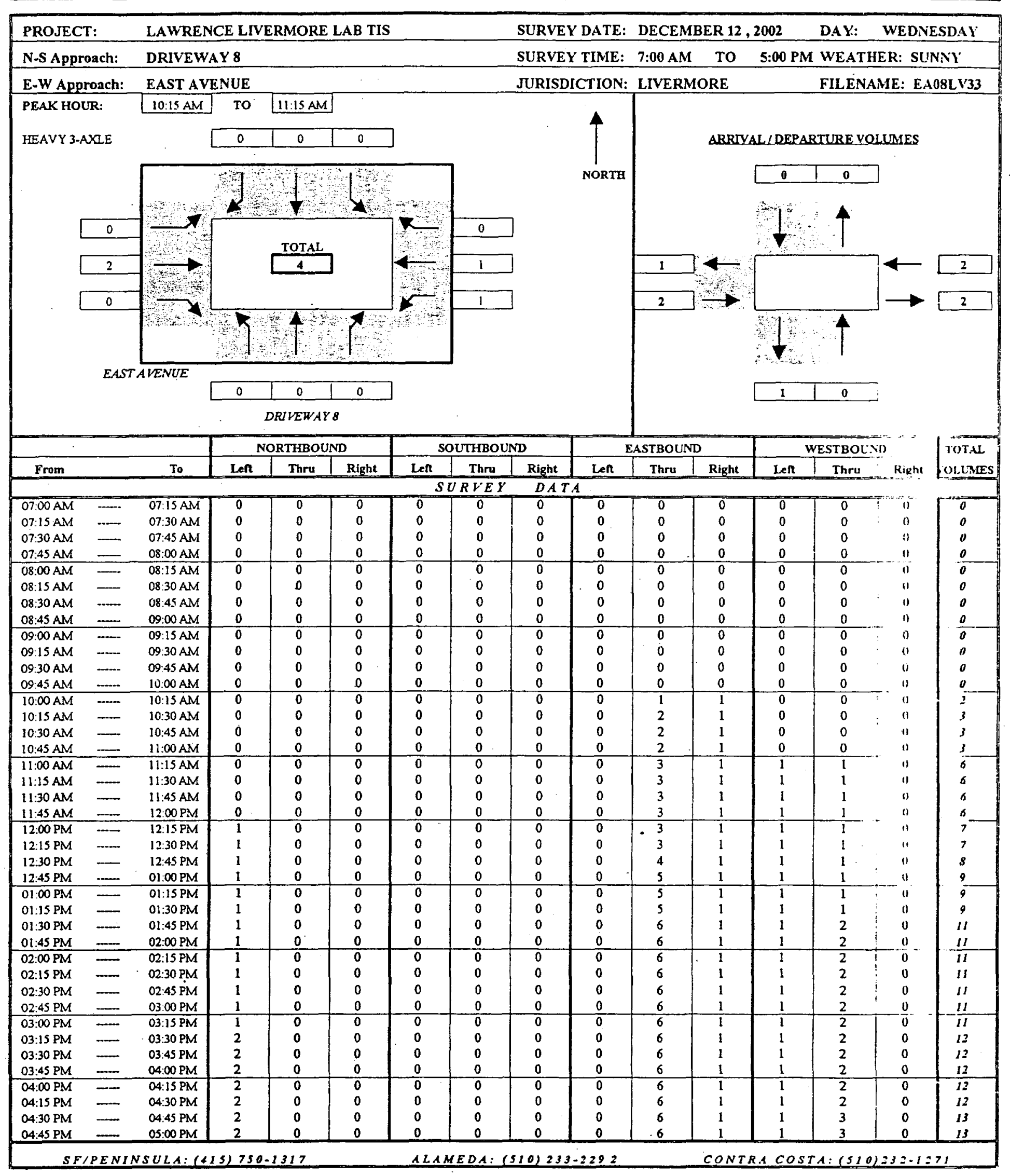




\section{Baymetrics Traffic Resources, INTERSECTION TURNING MOVEMENT SUMMARY}

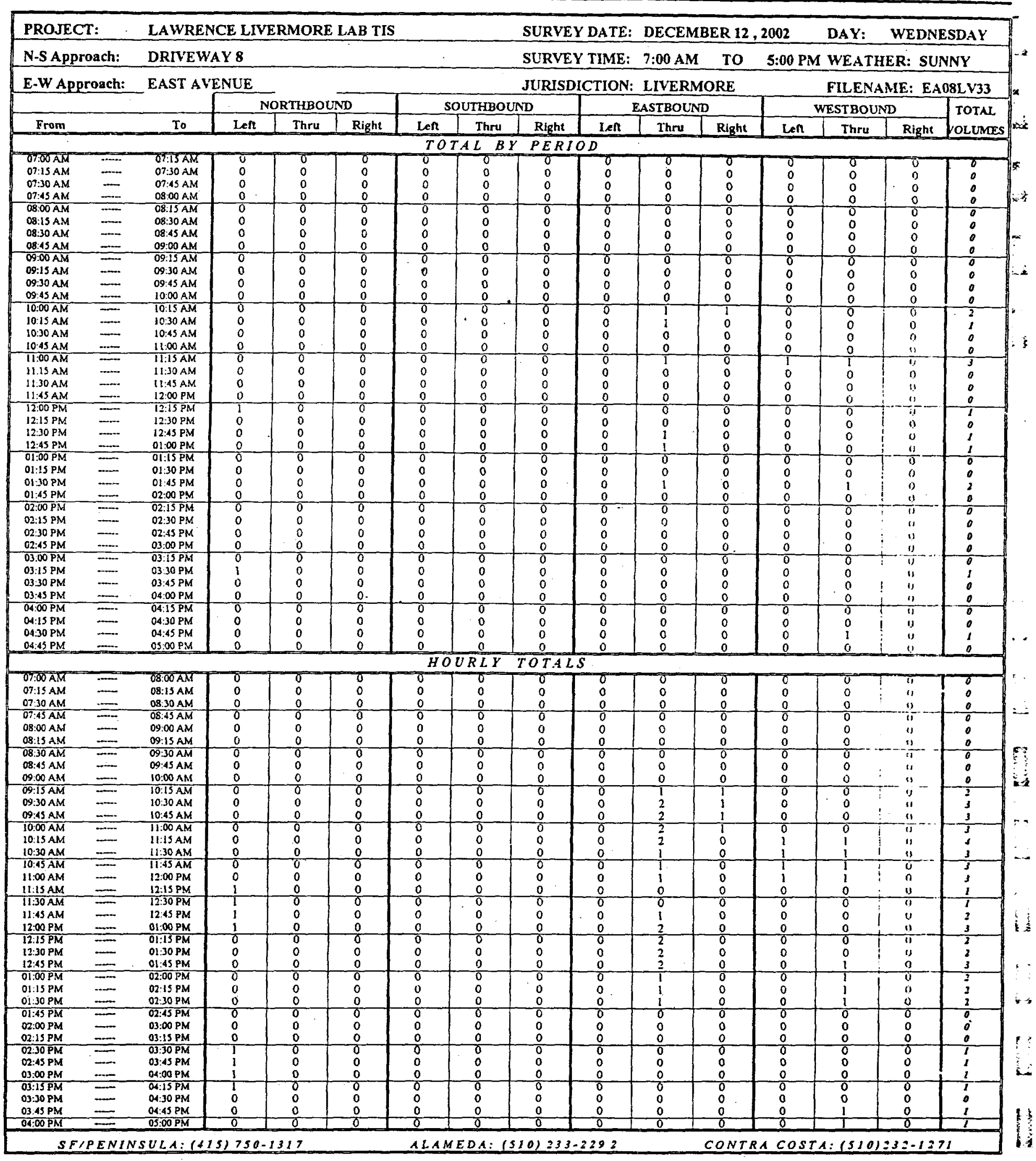




\section{Baymetrics Traffic Resources INTERSECTION TURNING MOVEMENT SUMMARY}

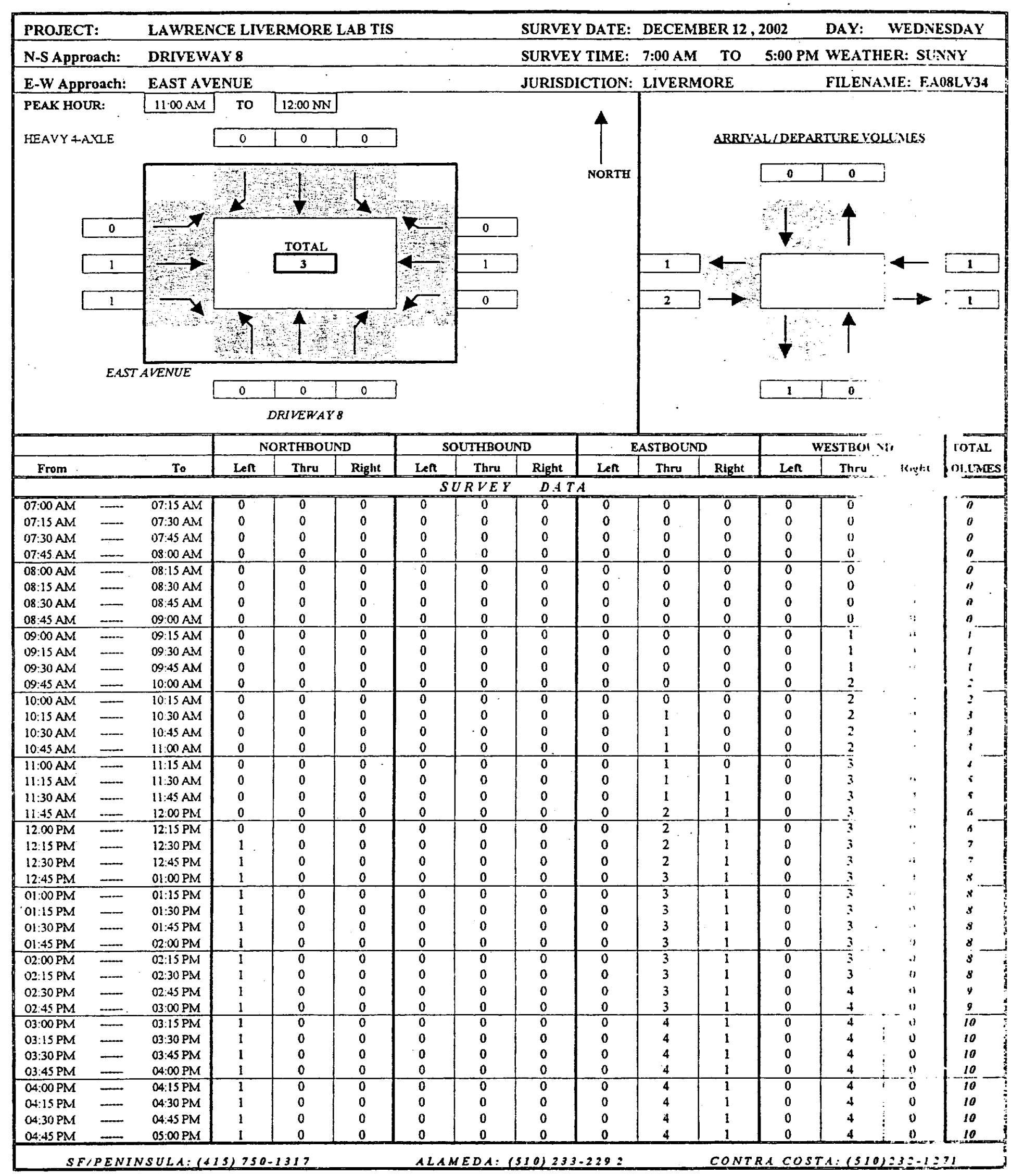




\section{Baymetrics Traffic Resources INTERSECTION TURNING MOVEMENT SUMMARY}

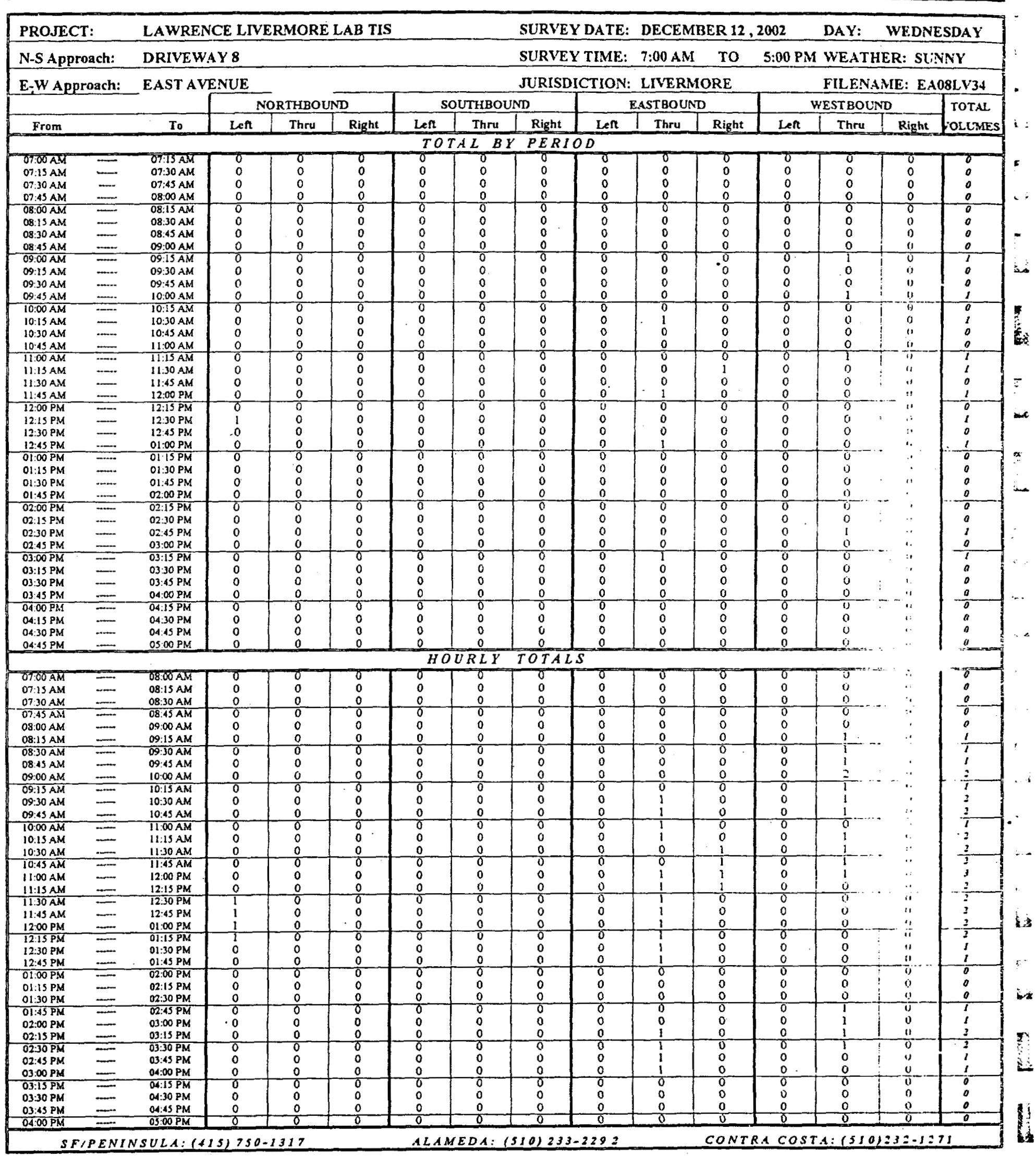




\section{Baymetrics Traffic Resources INTERSECTION TURNING MOVEMENT SUMMARY}

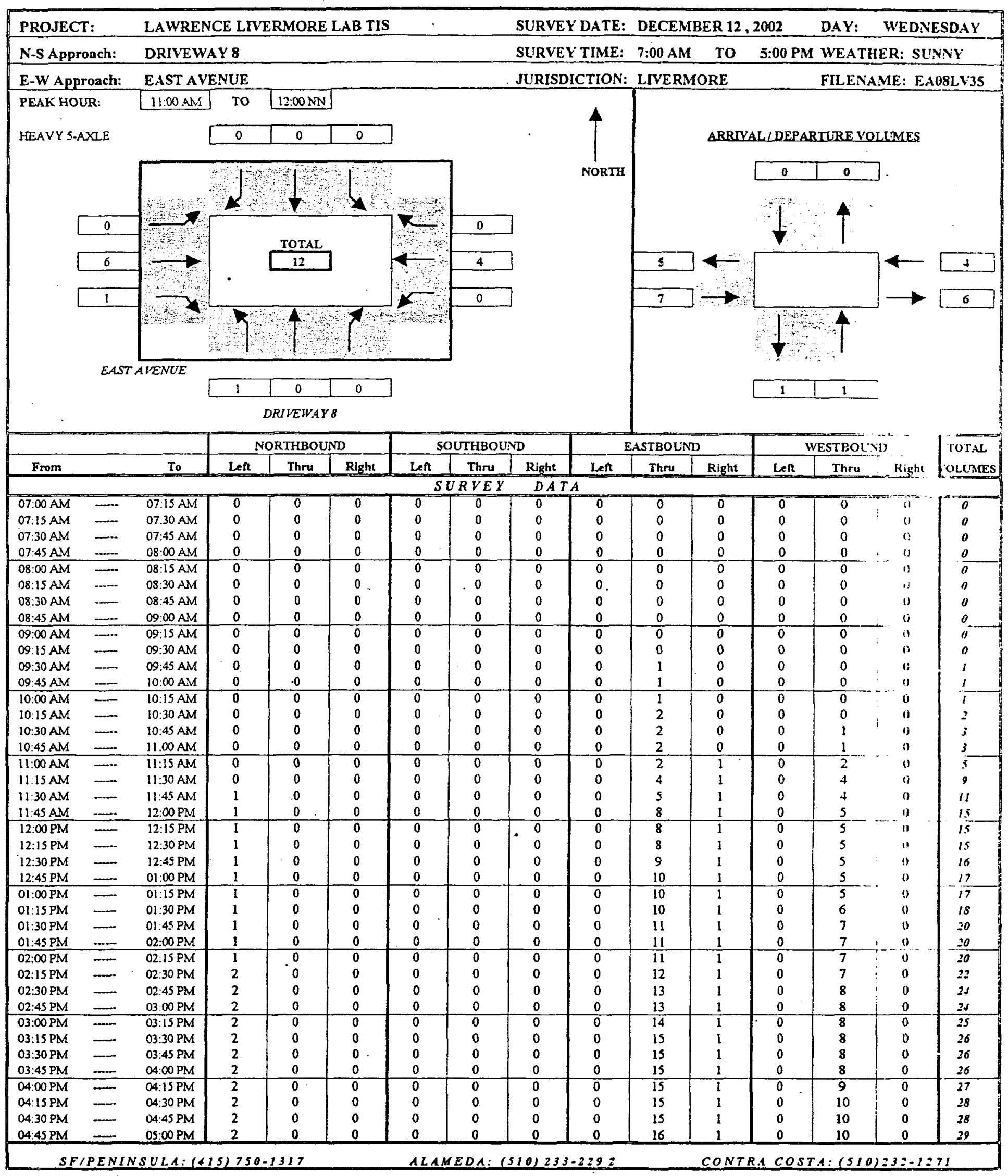




\section{$\frac{\text { Baymetrics Traffic Resources}}{\text { INTERSECTION TURNING MOVEMENT SUMMARY }}$}

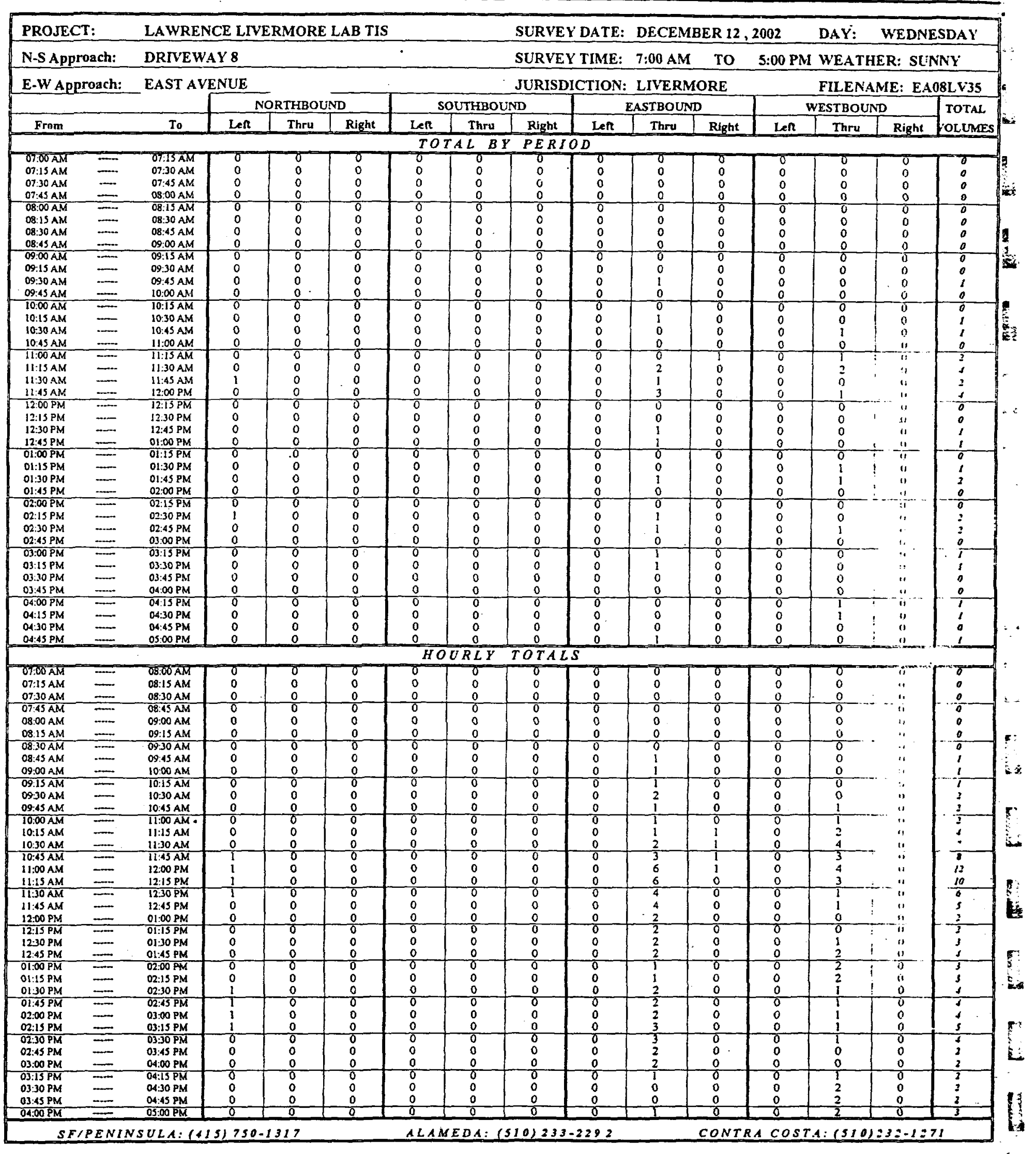




\section{Baymetrics Traffic Resources INTERSECTION TURNING MOVEMENT SUMMARY}

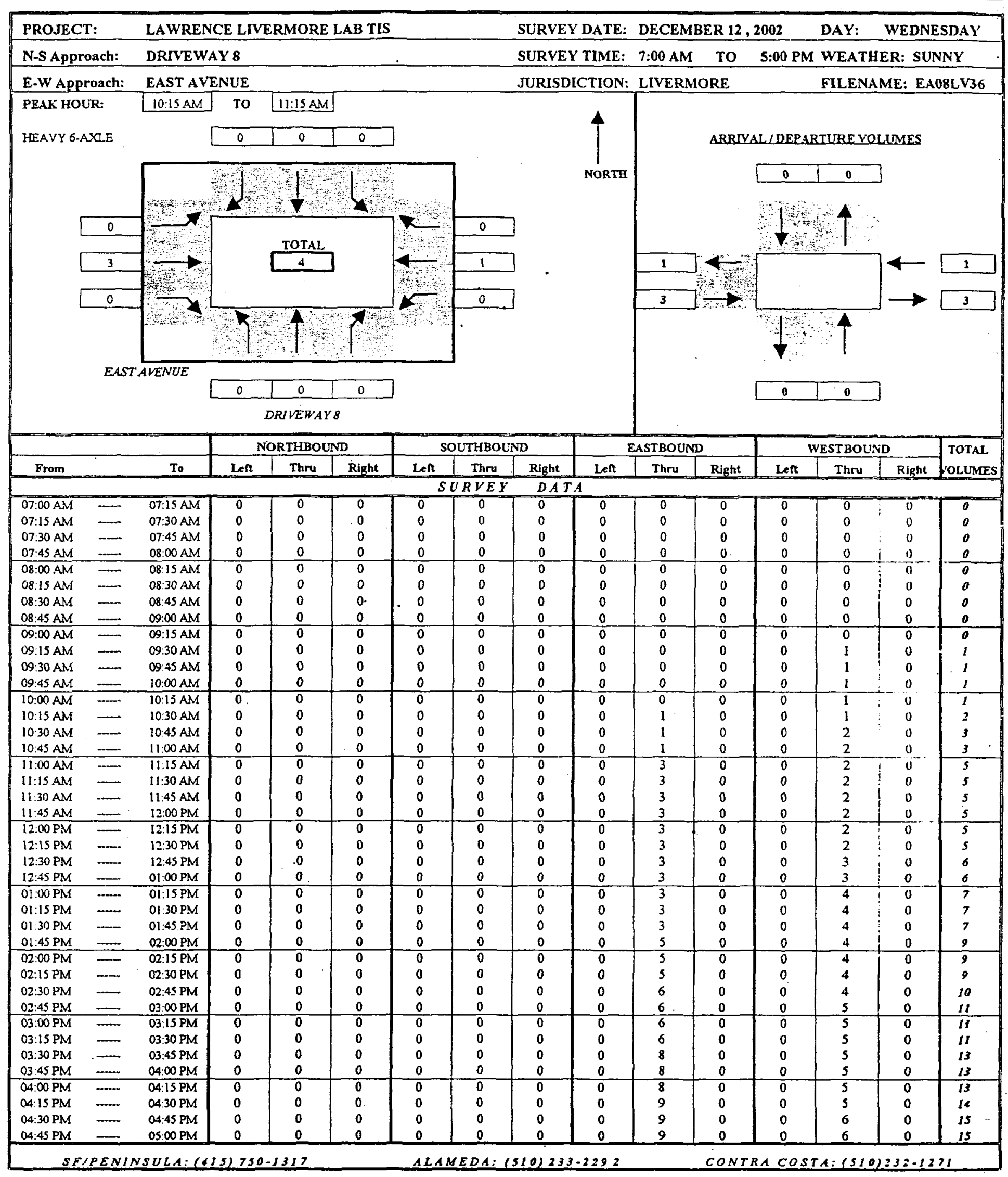




\section{Baymetrics Traffic Resources INTERSECTION TURNING MOVEMENT SUMMARY}

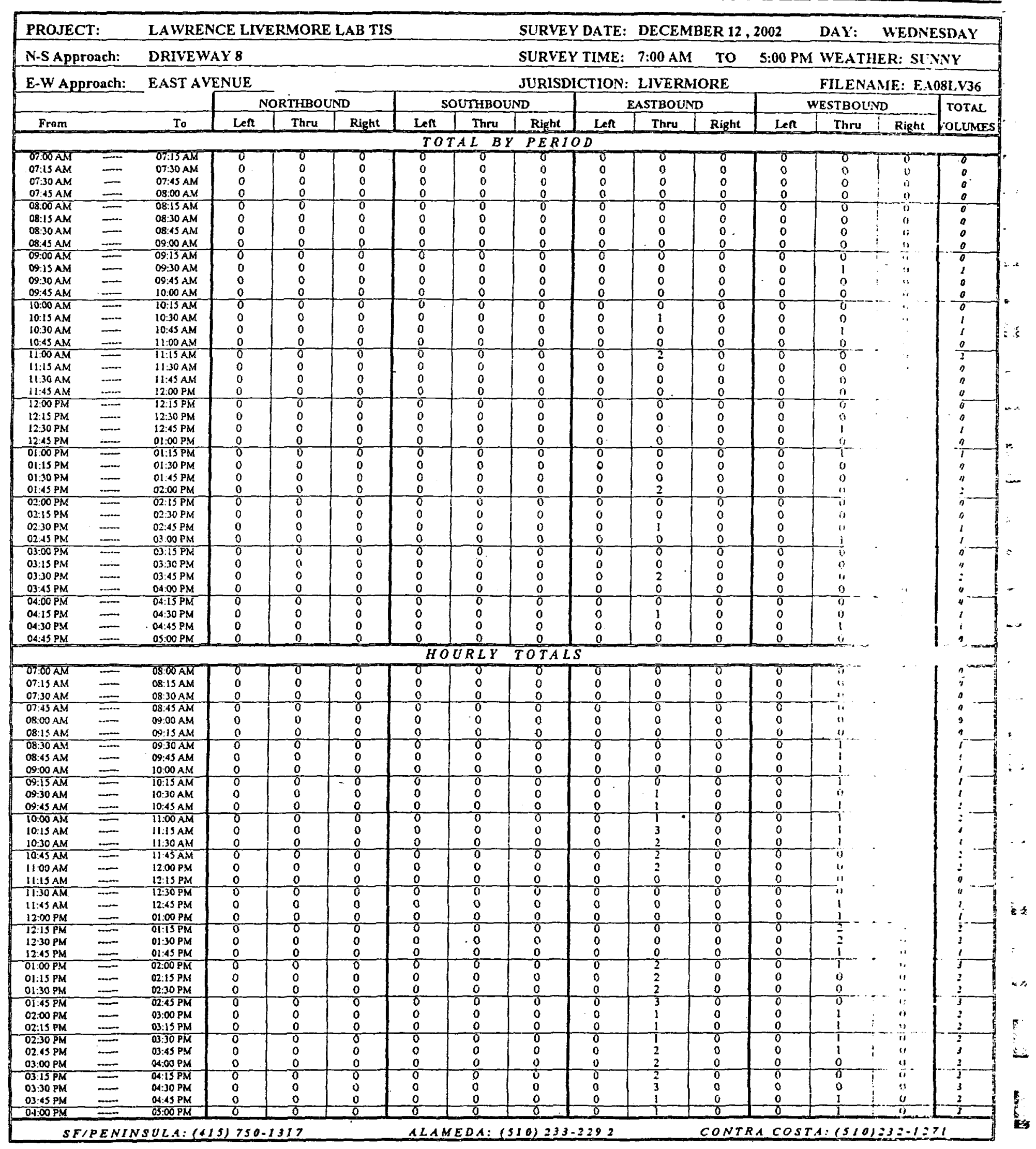


D. TRAFFIC ANALYSIS

Existing Conditions 



$\rightarrow \rightarrow r+4+1+b+1$

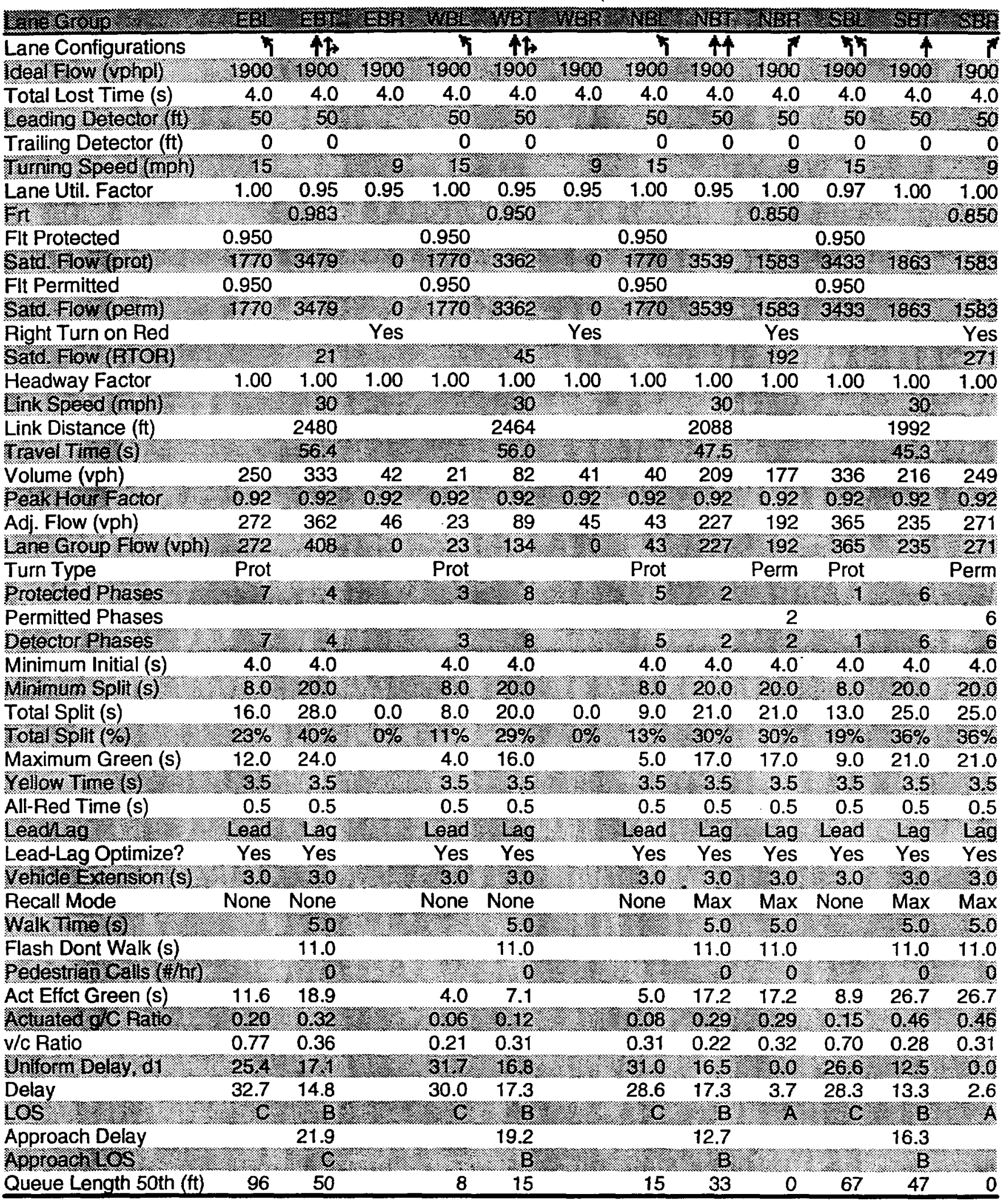




\section{$\rightarrow \rightarrow r+1+1+1+1$}

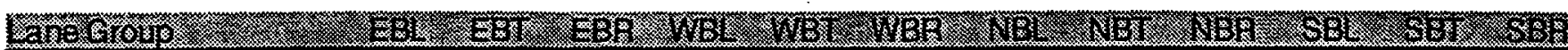

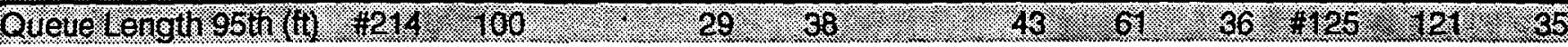
Internal Link Dist ( $\mathrm{ft}$ )

23842008

1912

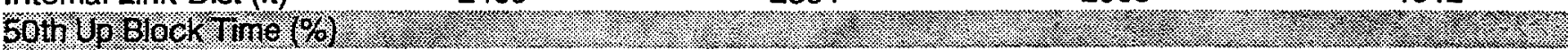
95th Up Block Time (\%)

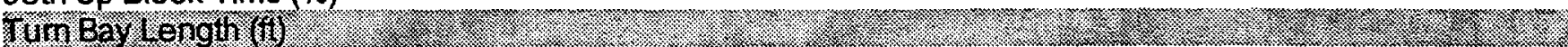
50 th Bay Block Time \%

G5th Bay Block Trne \% . Queuing Penalty (veh)

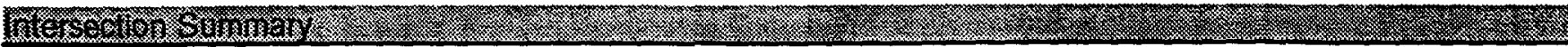

Area Type:

Other

C.cle Length 7 O .

Actuated Cycle Length: 58.6

Natural Gycle 70 :

Control Type: Semi Act-Uncoord

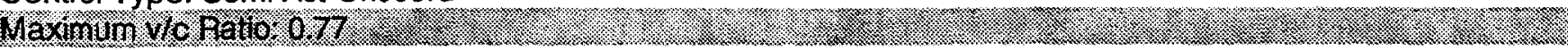
Intersection Signal Delay: 17.5

Intersection LOS: B

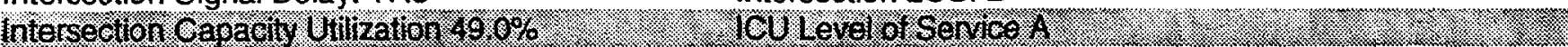
\# 95th percentile volume exceeds capacity, queue may be longer.

Orere shown Is naximumafter wo ajcles.

Splits and Phases: 1: East Ave \& Vasco Road

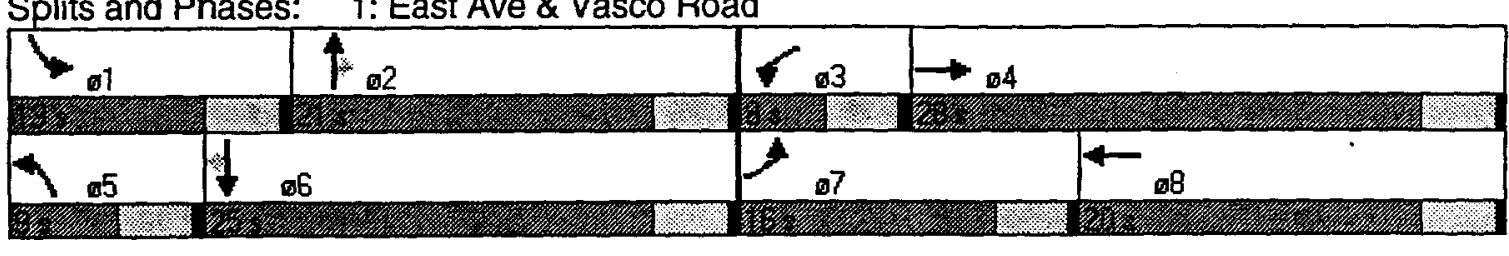




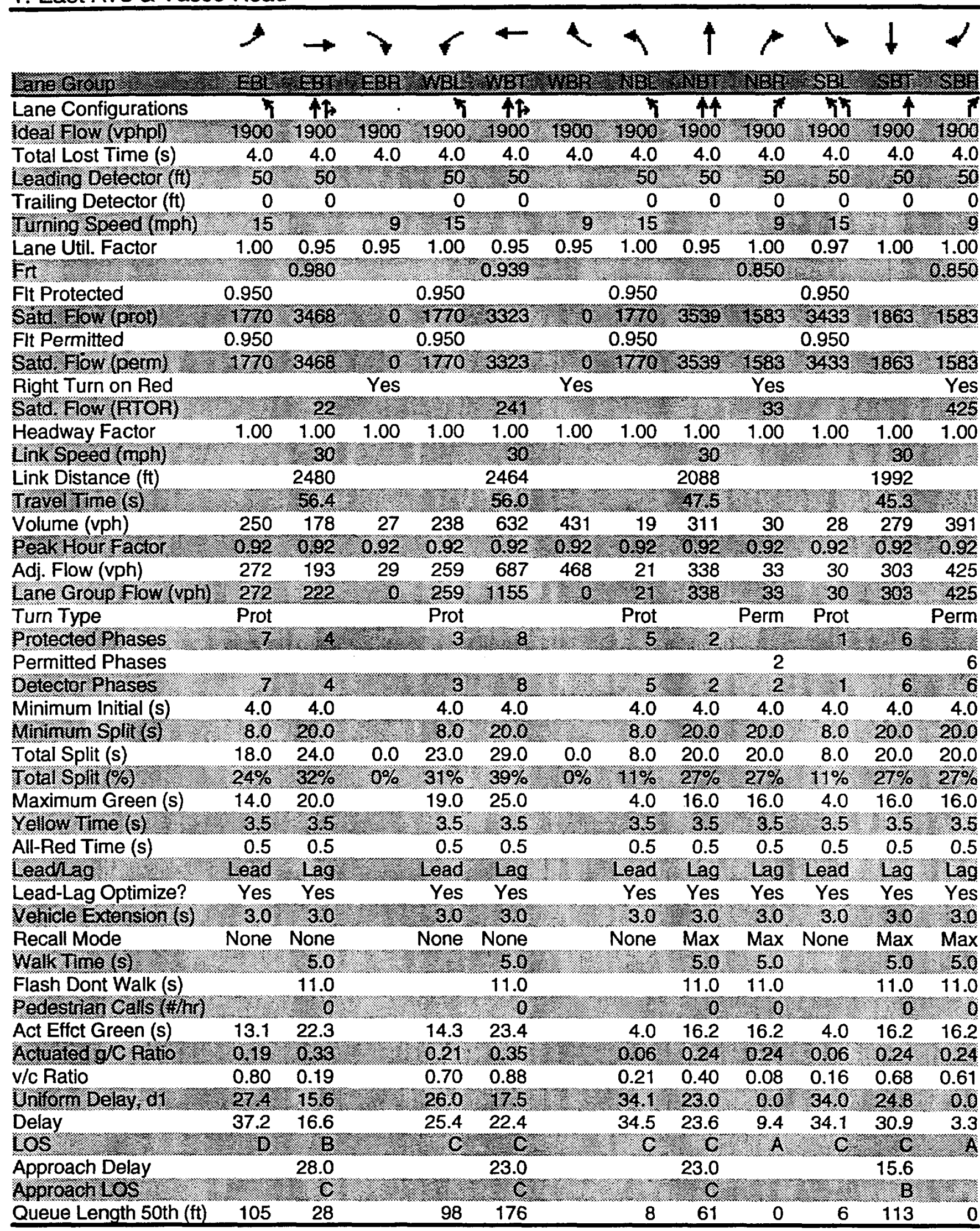

\section{Existing PM}

Korve Engineering

KORVEBAYL4-FF51 


\section{$\rightarrow \rightarrow r \leftarrow+\uparrow+\downarrow+\downarrow$}

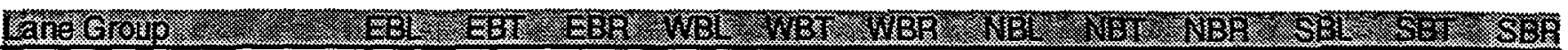

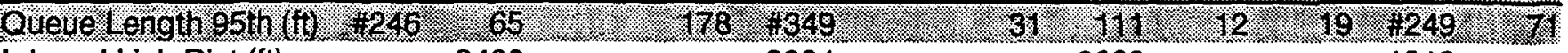

Internal Link Dist (ft)

2400

2384

2008

1912

50 th Up Bieck Tine (\%)

95th Up Block Time (\%)

Jum Bay lergut (f)

50th Bay Block Time \%

G5th Bay Block Tine \% : Queuing Penalty (veh)

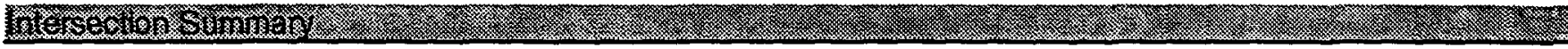

Area Type: Other

C.ele Length 75 . Actuated Cycle Length: 67.8

Natural Gycle ?5.

Control Type: Semi Act-Uncoord

Maxinum Ve hato 0.88

Intersection Signal Delay: 21.9

Intersection LOS: C

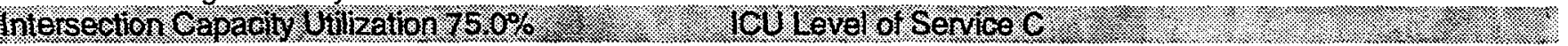
\# 95th percentile volume exceeds capacity, queue may be longer.

Queva shown is maximimater wo gactes.

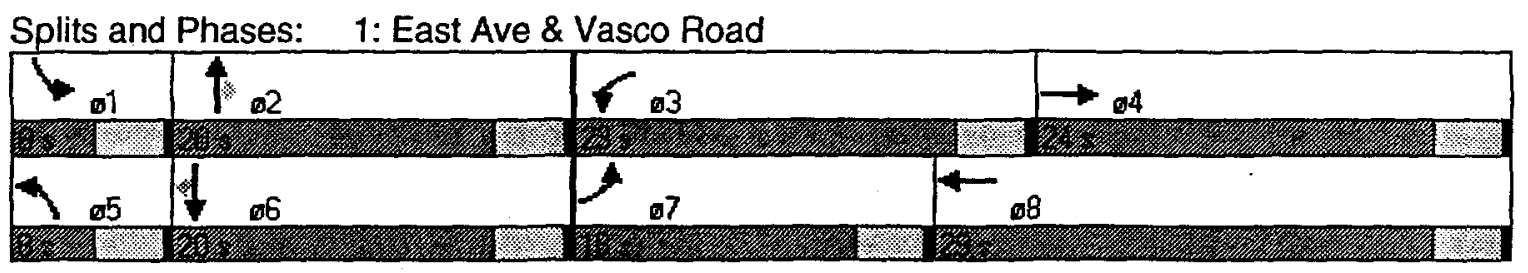


inalyst:

igency/Co.:

late Performed: 1/21/02

inalysis Time Period: AM Peak

Intersection:

East Ave \& SW Entrance (Location \#4)

Jurisdiction:

tnalysis Year:

project ID: Lawrence

iast/West Street:

Livermore National Laboratory

Jorth/South Street:

East Ave

SW Entrance/West Perimeter Dr

Intersection Orientation: EW

Study period (hrs): 1.00

Vehicle Volumes and Adjustments

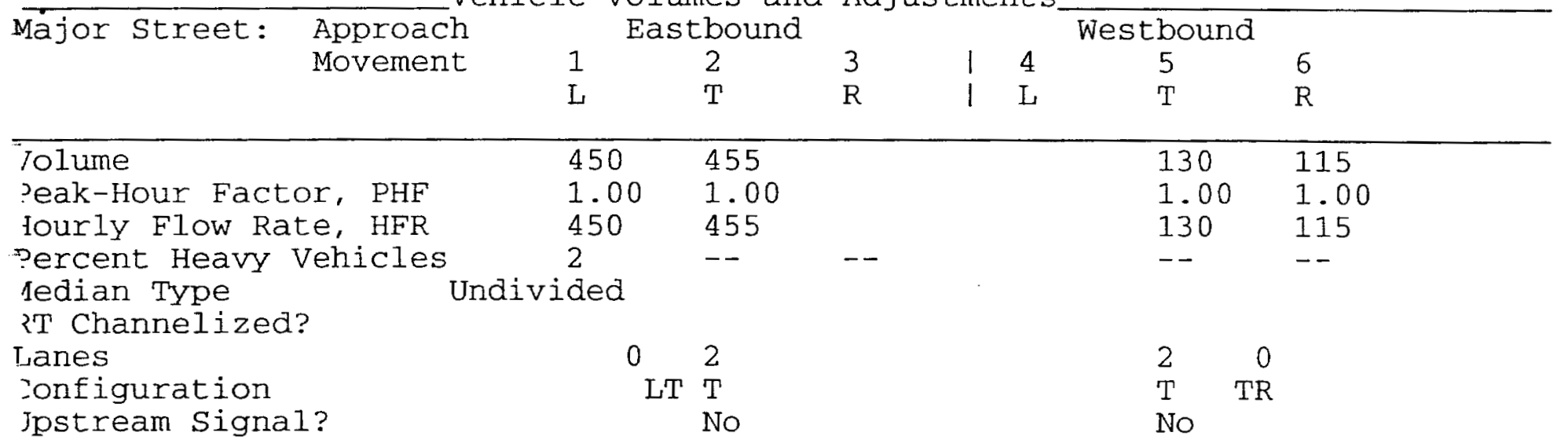

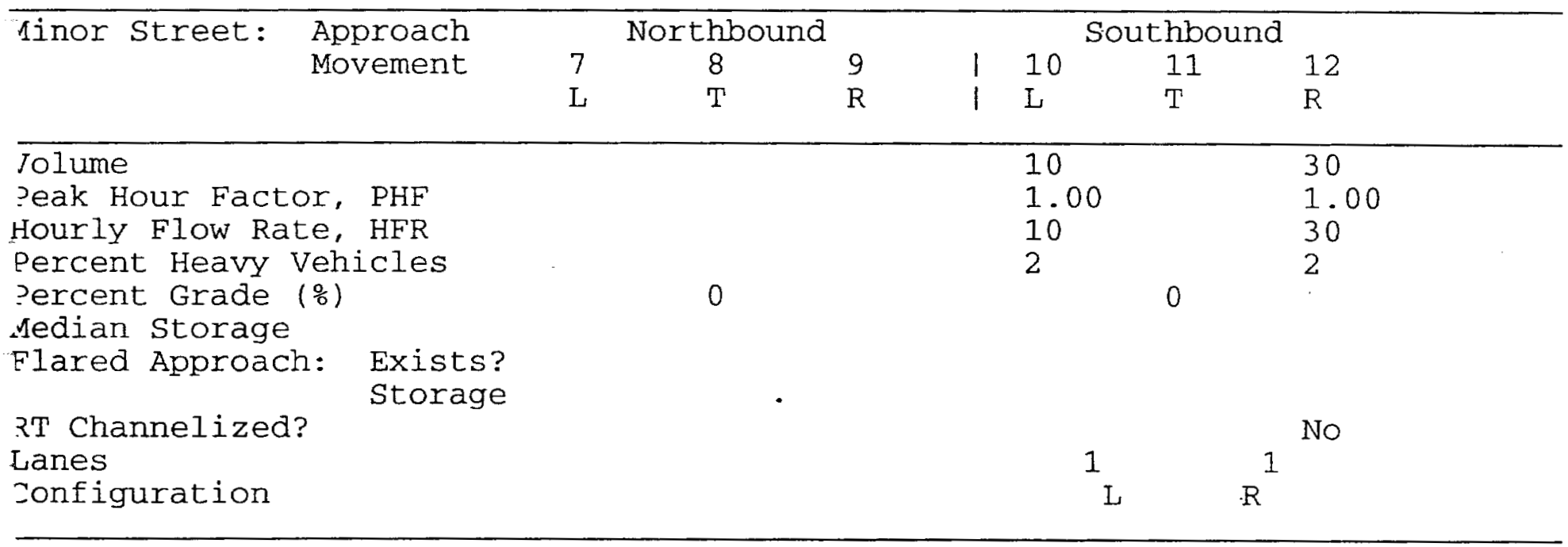

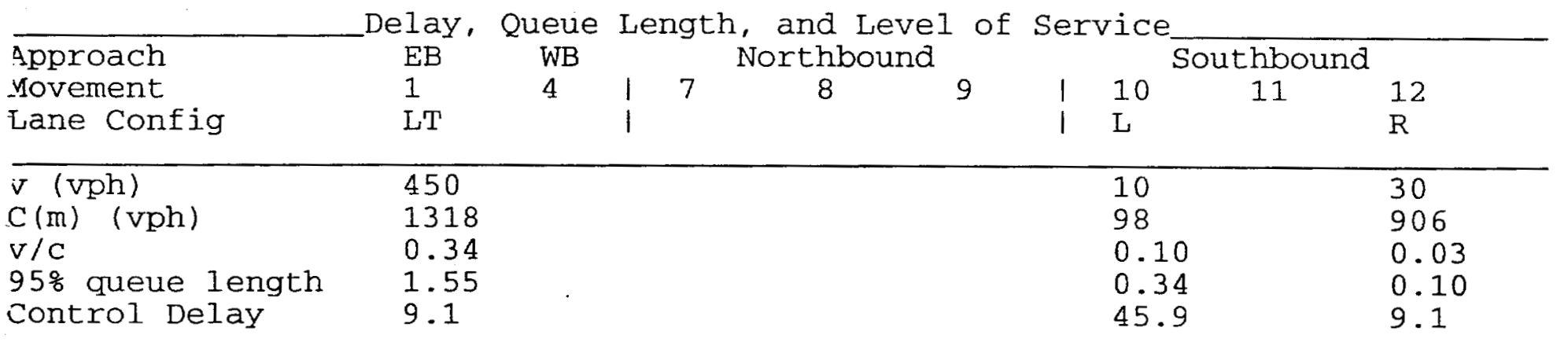


HCS2000: Unsignalized Intersections Release 4.1

Korve Engineering, Inc.

Phone:

Fax:

E-Mai1:

TWO-WAY STOP CONTROL (TWSC) ANALYSIS

Analyst:

Agency/Co.:

Date Performed: 1/21/02

Analysis Time Period: AM Peak

Intersection: East Ave \& SW Entrance

Jurisdiction:

Analysis Year:

2001

Project ID: Lawrence Livermore National Laboratory

East/west Street: East Ave

North/South Street: SW Entrance/West Perimeter Dr

Intersection orientation: EW $\quad$ Study period (hrs): 1.00

Vehicle Volumes and Adjustments

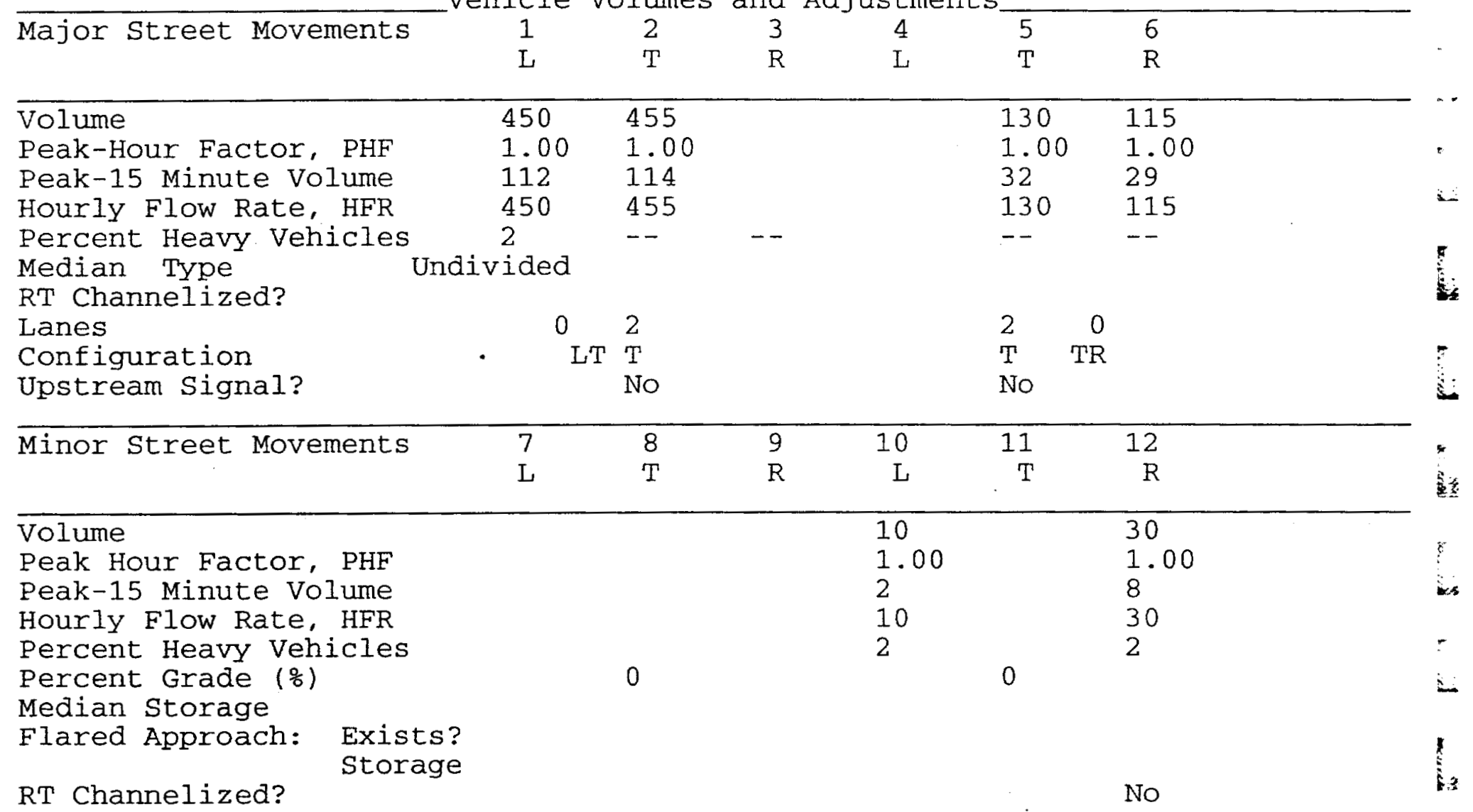


Pedestrian Volumes and Adjustments

\begin{tabular}{lllll}
\hline Movements & 13 & 14 & 15 & 16 \\
\hline Flow (ped/hr) & & & & \\
Lane Width (ft) & 0 & 0 & 0 & 0 \\
Walking Speed (ft/sec) & 12.0 & 12.0 & 12.0 & 12.0 \\
Percent Blockage & 4.0 & 4.0 & 4.0 & 4.0 \\
\end{tabular}

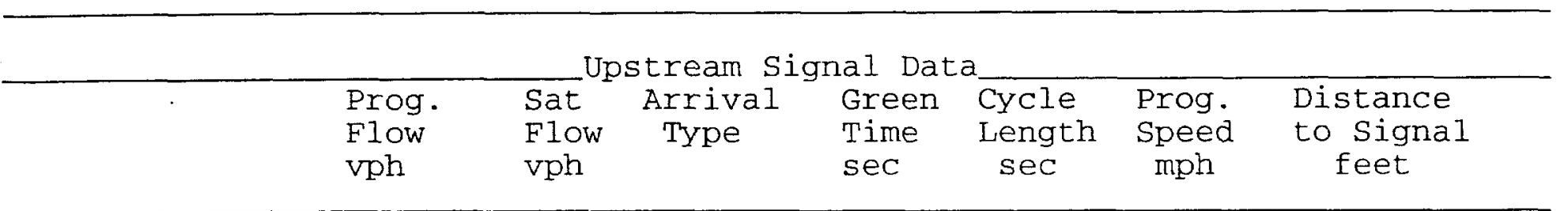

\begin{tabular}{ll}
\hline s2 & Left-Turn \\
& Through \\
s5 & Left-Turn \\
& Through
\end{tabular}

Norksheet 3-Data for Computing Effect of Delay to Major Street Vehicles

Movement 2 Movement 5

Shared In volume, major th vehicles:

Shared In volume, major rt vehicles:

sat flow rate, major th vehicles:

sat flow rate, major rt vehicles:

Number of major street through lanes:

0

1700

1700

2

vorksheet 4-Critical Gap and Follow-up Time Calculation

\begin{tabular}{|c|c|c|c|c|c|c|c|c|c|}
\hline \multicolumn{10}{|c|}{ Iritical Gap Calculation } \\
\hline Movement & & I & $\begin{array}{l}4 \\
\mathrm{~L}\end{array}$ & $\begin{array}{l}7 \\
L\end{array}$ & $\begin{array}{l}8 \\
\mathrm{~T}\end{array}$ & $\begin{array}{l}9 \\
\mathrm{R}\end{array}$ & $\begin{array}{r}10 \\
\mathrm{~L}\end{array}$ & $\begin{array}{r}11 \\
\mathrm{~T}\end{array}$ & $\begin{array}{r}12 \\
\mathrm{R}\end{array}$ \\
\hline $\begin{array}{l}=(c, \text { base }) \\
=(c, h v) \\
P(h v) \\
=(c, g) \\
\text { Irade } / 10 \\
t(3, I t) \\
\pm(c, T): \\
=(c)\end{array}$ & $\begin{array}{l}0 \\
1 \text {-stage } \\
2 \text {-stage } \\
1 \text {-stage } \\
2 \text {-stage }\end{array}$ & $\begin{array}{l}0.00 \\
0.00 \\
0.00 \\
4.1\end{array}$ & $\begin{array}{l}0.00 \\
0.00\end{array}$ & $\begin{array}{l}2.00 \\
0.20 \\
0.00 \\
0.00 \\
1.00\end{array}$ & $\begin{array}{l}2.00 \\
0.20 \\
0.00 \\
0.00 \\
1.00\end{array}$ & $\begin{array}{l}2.00 \\
0.10 \\
0.00 \\
0.00 \\
0.00\end{array}$ & $\begin{array}{l}7.5 \\
2.00 \\
2 \\
0.20 \\
0.00 \\
0.70 \\
0.00 \\
1.00 \\
6.8\end{array}$ & $\begin{array}{l}2.00 \\
0.20 \\
0.00 \\
0.00 \\
1.00\end{array}$ & $\begin{array}{l}6.9 \\
2.00 \\
2 \\
0.10 \\
0.00 \\
0.00 \\
0.00 \\
0.00 \\
6.9\end{array}$ \\
\hline $\begin{array}{l}\text { Follow-Ur } \\
\text { Movement }\end{array}$ & Time C & $\begin{array}{c}\operatorname{arcula} \\
1 \\
L\end{array}$ & $\begin{array}{c}\text { ions } \\
4 \\
\text { L }\end{array}$ & $\begin{array}{l}7 \\
\mathrm{~L}\end{array}$ & $\begin{array}{l}8 \\
\mathrm{~T}\end{array}$ & $\begin{array}{l}9 \\
R\end{array}$ & $\begin{array}{r}10 \\
\mathrm{~L}\end{array}$ & $\begin{array}{r}11 \\
\mathrm{~T}\end{array}$ & $\begin{array}{r}12 \\
\mathrm{R}\end{array}$ \\
\hline $\begin{array}{l}E(\mathrm{f}, \text { base }) \\
E(\mathrm{E}, \mathrm{HV}) \\
\mathrm{P}(\mathrm{HV}) \\
=(\mathrm{E})\end{array}$ & & $\begin{array}{l}2.20 \\
1.00 \\
2 \\
2.2\end{array}$ & 1.00 & 1.00 & 1.00 & 1.00 & $\begin{array}{l}3.50 \\
1.00 \\
2 \\
3.5\end{array}$ & 1.00 & $\begin{array}{l}3.30 \\
1.00 \\
2 \\
3.3\end{array}$ \\
\hline
\end{tabular}


Worksheet 5-Effect of Upstream Signals

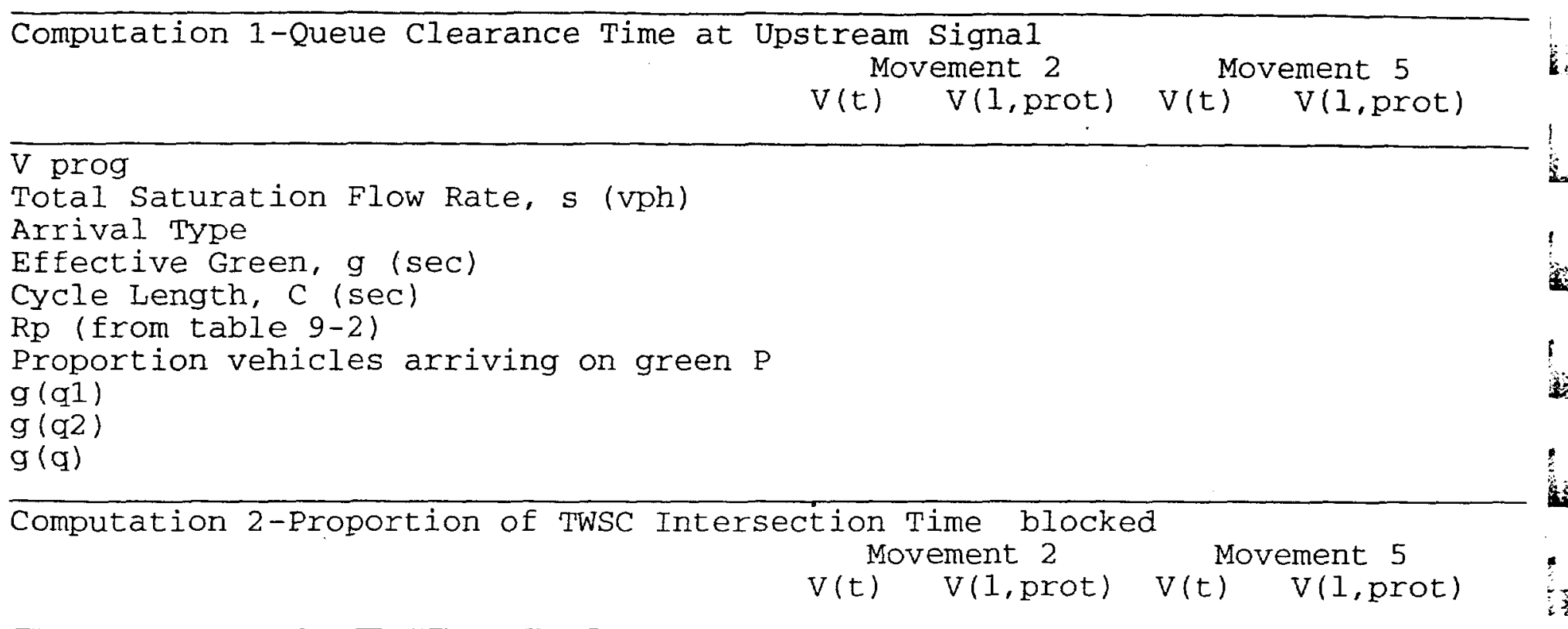

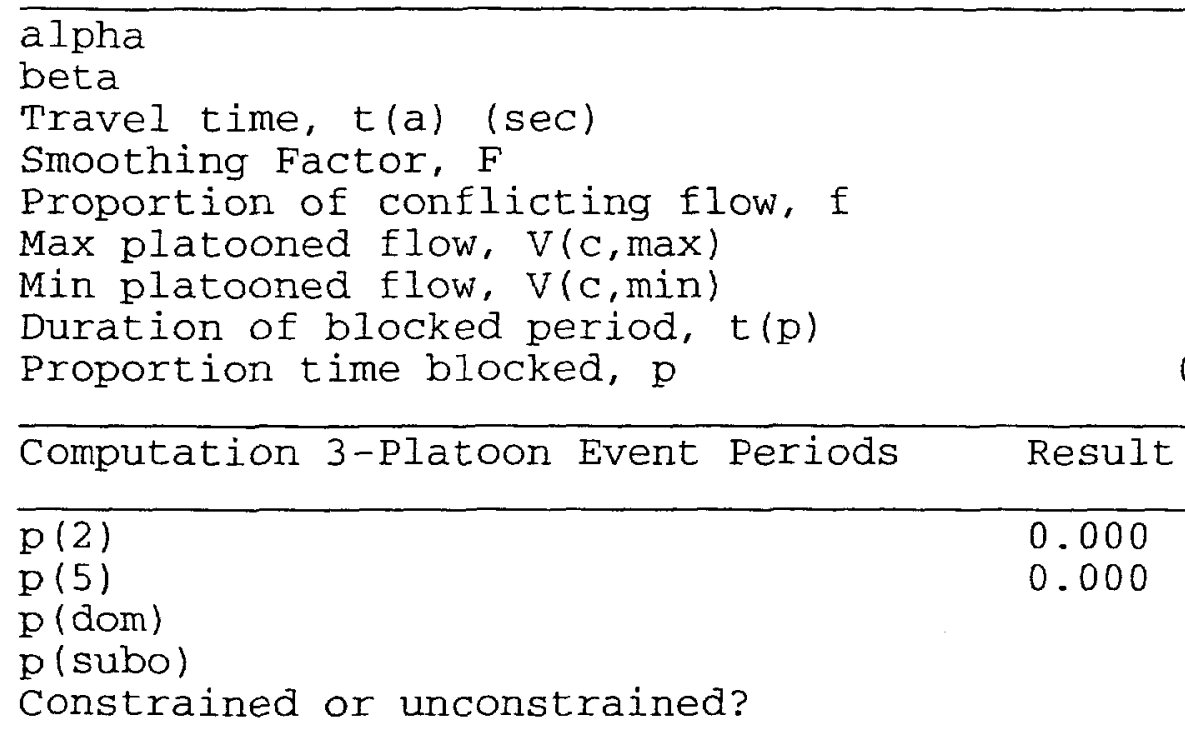

\begin{tabular}{lc}
\hline Computation 3-Platoon Event Periods & Result \\
\hline$p(2)$ & 0.000 \\
$\mathrm{p}(5)$ & 0.000 \\
$\mathrm{p}(\mathrm{dom})$ & \\
$\mathrm{p}($ subo) & \\
Constrained or unconstrained? &
\end{tabular}

$0.000 \quad 0.000$

\section{Proportion}

unblocked

for minor

movements, $\mathrm{p}(\mathrm{x})$

single-stage Process

$p(1)$
$p(4)$
$p(7)$
$p(8)$
$p(9)$
$p(10)$
$p(11)$
$p(12)$

Computation 4 and 5 Single-stage Process Movement

$\begin{array}{lll}1 & 4 & 7 \\ \mathrm{~L} & \mathrm{~L} & \mathrm{~L}\end{array}$

(3)

Two-Stage Process Stage I Stage. II 


$$
\sqrt{(c, x)}
$$

; (x)

Iorksheet 6-Impedance and Capacity Equations

\begin{tabular}{|c|c|c|}
\hline 3tep 1: RT from Minor St. & 9 & 12 \\
\hline $\begin{array}{l}\text { :onflicting Flows } \\
\text { Potential Capacity } \\
\text { 'edestrian Impedance Factor } \\
\text { lovement Capacity } \\
\text { probability of Queue free St. }\end{array}$ & $\begin{array}{l}1.00 \\
1.00\end{array}$ & $\begin{array}{l}122 \\
906 \\
1.00 \\
906 \\
0.97\end{array}$ \\
\hline itep 2: LT from Major St. & 4 & 1 \\
\hline $\begin{array}{l}\text { Conflicting Flows } \\
\text { 'otential Capacity } \\
\text { 'edestrian Impedance Factor } \\
\text { Movement Capacity } \\
\text { 'robability of Queue free St. } \\
\text { laj L-Shared Prob Q free St. }\end{array}$ & $\begin{array}{l}1.00 \\
1.00\end{array}$ & $\begin{array}{l}245 \\
1318 \\
1.00 \\
1318 \\
0.66 \\
0.66\end{array}$ \\
\hline itep 3: TH from Minor st. & 8 & 11 \\
\hline $\begin{array}{l}\text { 'onflicting Flows } \\
\text { Potential Capacity } \\
\text { 'edestrian Impedance Factor } \\
\text { :ap. Adj. factor due to Impeding mvmnt } \\
\text { Movement Capacity } \\
\text { 'robability of Queue free St. }\end{array}$ & $\begin{array}{l}1.00 \\
0.66 \\
1.00\end{array}$ & $\begin{array}{l}1.00 \\
0.66 \\
1.00\end{array}$ \\
\hline step 4: LT from Minor st. & 7 & 10 \\
\hline $\begin{array}{l}\text { :onflicting Flows } \\
\text { 'otential Capacity } \\
\text { Pedestrian Impedance Factor } \\
\text { laj. L, Min T Impedance factor } \\
\text { laj. I, Min T Adj. Imp Factor. } \\
\text { Cap. Adj. factor due to Impeding mumnt } \\
\text { lovement Capacity }\end{array}$ & $\begin{array}{l}1.00 \\
0.66 \\
0.73 \\
0.71\end{array}$ & $\begin{array}{l}1315 \\
149 \\
1.00\end{array}$ \\
\hline
\end{tabular}




\begin{tabular}{l}
\hline Step 3: TH from Minor St. \\
\hline Part 1 - First Stage \\
Conflicting Flows \\
Potential Capacity \\
Pedestrian Impedance Factor \\
Cap. Adj. factor due to Impeding mvint \\
Movement Capacity \\
Probability of Queue free st.
\end{tabular}

Part 2 - Second Stage

Conflicting Flows

Potential Capacity

Pedestrian Impedance Factor

Cap. Adj. factor due to Impeding mumnt

Movement Capacity

Part 3 - Single stage

Conflicting Flows

Potential Capacity

Pedestrian Impedance Factor

Cap. Adj. factor due to Impeding mvmnt

1.00

1.00

Movement Capacity

Result for 2 stage process:

a

Y

C $t$

Probability of Queue free St.

$1.00 \quad 1.00$

Step 4: LT from Minor st.

7 10

Part 1 - First stage

Conflicting Flows

Potential Capacity

Pedestrian Impedance Factor

Cap. Adj. factor due to Impeding mvmnt

Movement Capacity

Part 2 - Second Stage

Conflicting Flows

Potential Capacity

Pedestrian. Impedance Factor

cap. Adj. factor due to Impeding mvmnt

Movement Capacity

Part 3 - Single stage

Conflicting Flows

Potential Capacity

Pedestrian Impedance Factor

Maj. L, Min T Impedance factor

0.66

1.00

Maj. L, Min T Adj. Imp Factor.

0.73

Cap. Adj. factor due to Impeding mvmnt

0.71

0.66

Movement Capacity

Results for Two-stage process:

a

$y$ 
Norksheet 8-Shared Lane Calculations

\begin{tabular}{|c|c|c|c|c|c|c|}
\hline Movement & $\begin{array}{l}7 \\
\mathrm{~L}\end{array}$ & $\begin{array}{l}8 \\
T\end{array}$ & $\begin{array}{l}9 \\
\mathrm{R}\end{array}$ & $\begin{array}{r}10 \\
\mathrm{~L}\end{array}$ & $\begin{array}{r}11 \\
\mathrm{~T}\end{array}$ & $\begin{array}{r}12 \\
\mathrm{R}\end{array}$ \\
\hline $\begin{array}{l}\text { Volume (vph) } \\
\text { Movement Capacity (vph) } \\
\text { Shared Lane Capacity (vph) }\end{array}$ & & & & $\begin{array}{l}10 \\
98\end{array}$ & & $\begin{array}{l}30 \\
906\end{array}$ \\
\hline
\end{tabular}

Norksheet 9-Computation of Effect of Flared Minor street Approaches

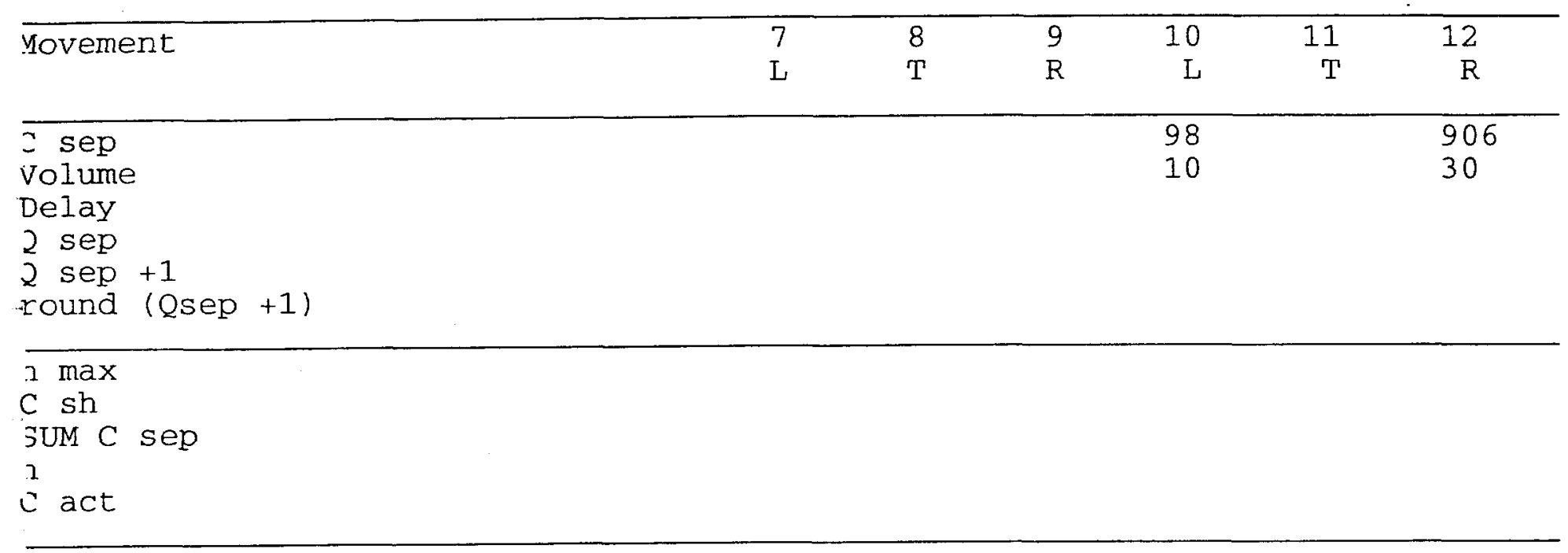

Norksheet 10-Delay, Queue Length, and Level of Service

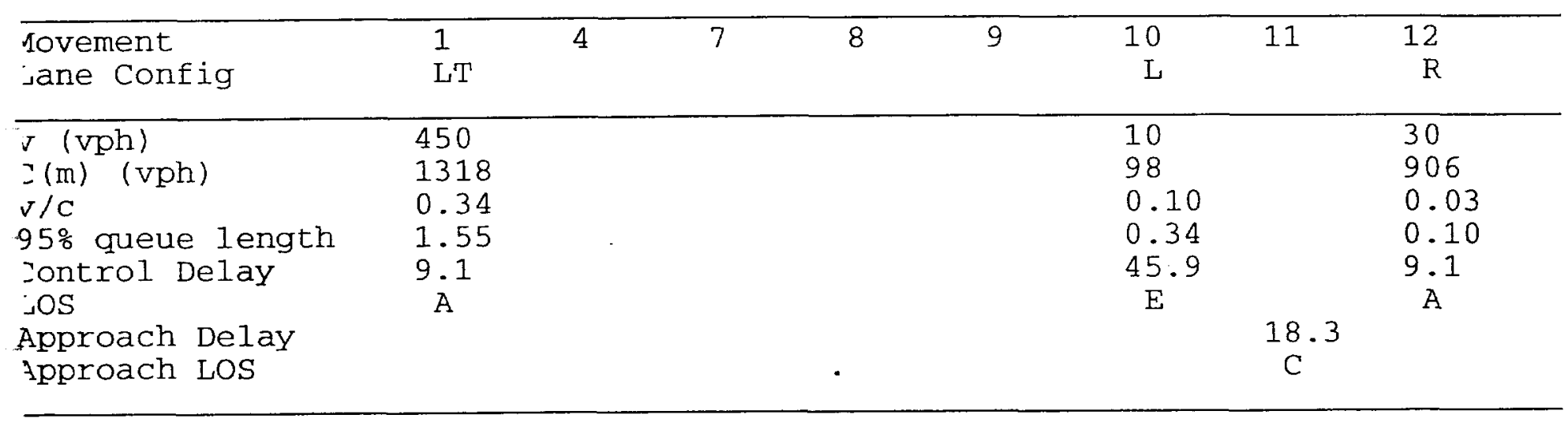

Norksheet 11-Shared Major LT Impedance and Delay

\begin{tabular}{|c|c|c|}
\hline & Movement 2 & Movement 5 \\
\hline 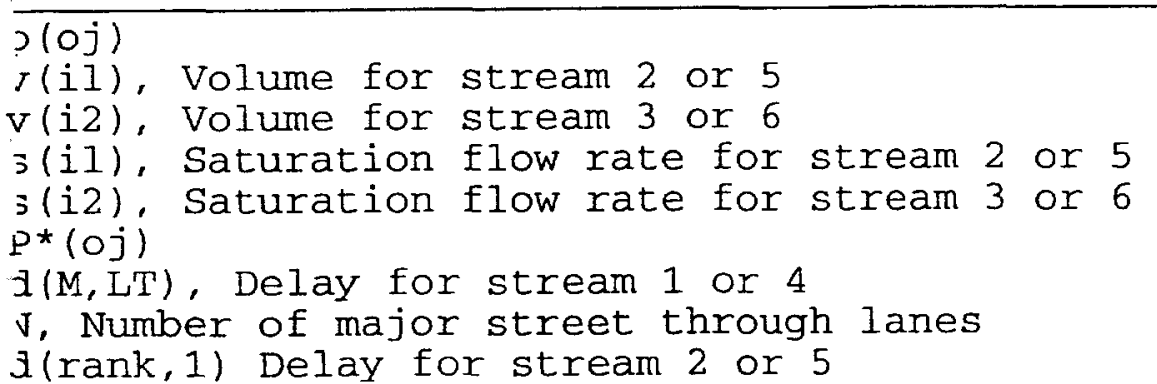 & $\begin{array}{l}0.66 \\
0 \\
0 \\
1700 \\
1700 \\
0.66 \\
9.1 \\
2\end{array}$ & 1.00 \\
\hline
\end{tabular}




\section{Analyst:}

Agency/Co.:

Date Performed: 1/21/02

Analysis Time Period: PM Peak

Intersection:

East Ave \& SW Entrance (Locction \#4)

Jurisdiction:

Analysis Year:

2001

Project ID: Lawrence Livermore National Laboratory

East/West Street: East Ave

North/South Street: SW Entrance/West Perimeter Dr

Intersection orientation: EW $\quad$ Study period (hrs): 1.00

Vehicle Volumes and Adjustments

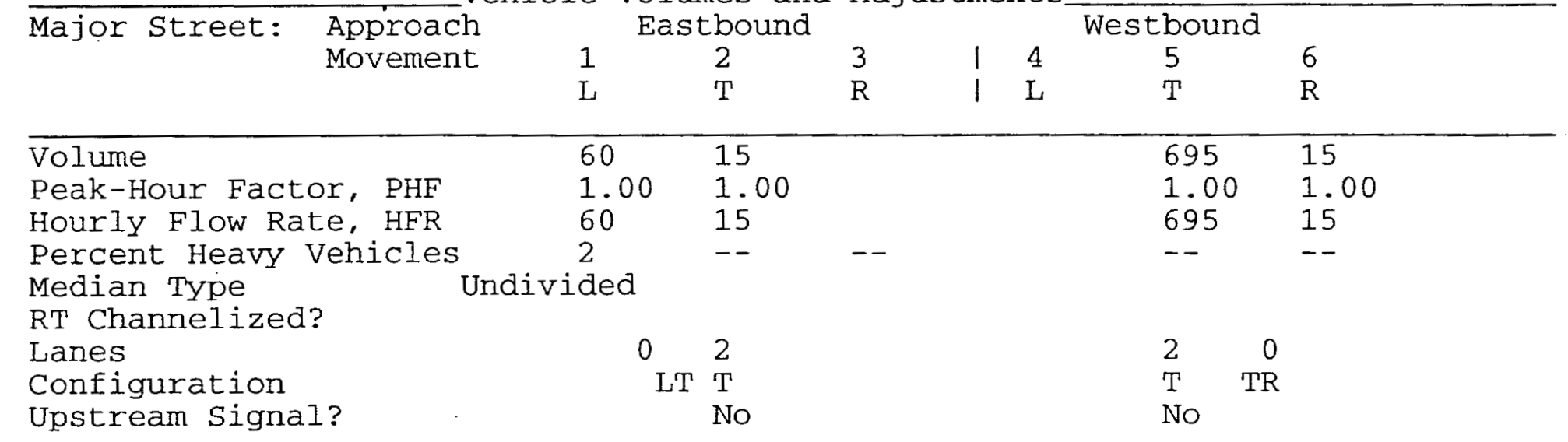

\begin{tabular}{lllcllll}
\hline Minor street: & Approach & \multicolumn{3}{c}{ Northbound } & \multicolumn{3}{c}{ Southbound } \\
& Movement & 7 & 8 & 9 & 10 & 11 & 12 \\
& & $\mathrm{~L}$ & $\mathrm{~T}$ & $\mathrm{R}$ & $\mathrm{L}$ & $\mathrm{T}$ & $\mathrm{R}$
\end{tabular}

Volume

Peak Hour Factor, PHF

Hourly Flow Rate, HFR

Percent Heavy Vehicles

Percent Grade (\%)

Median Storage

Flared Approach: Exists?

Storage

RT Channelized?

Lanes

Configuration

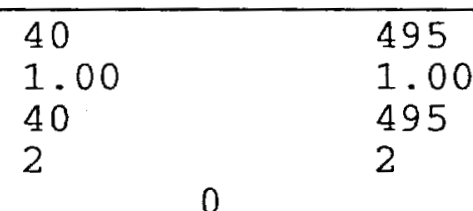

0

12

0

$1 \quad \mathrm{R}^{1^{\text {No }}}$

Delay, Queue Length, and Level of Service

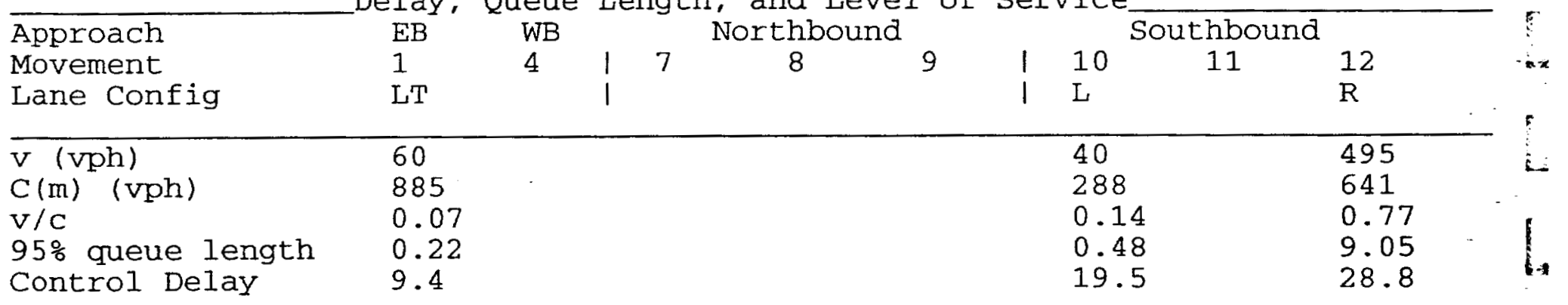


Fax:

;-Mai1:

TWO-WAY STOP CONTROL (TWSC) ANALYSIS

Anailyst:

igency/Co.:

late Performed: 1/21/02

nnalysis Time Period: PM Peak

Tntersection:

East Ave \& SW Entrance

urisdiction:

nalysis Year:

2001

Project ID: Lawrence Livermore National Laboratory

iast/West Street: East Ave

lorth/South Street: SW Entrance/West Perimeter Dr

Tntersection orientation: EW

Study period (hrs): 1.00

Vehicle Volumes and Adjustments

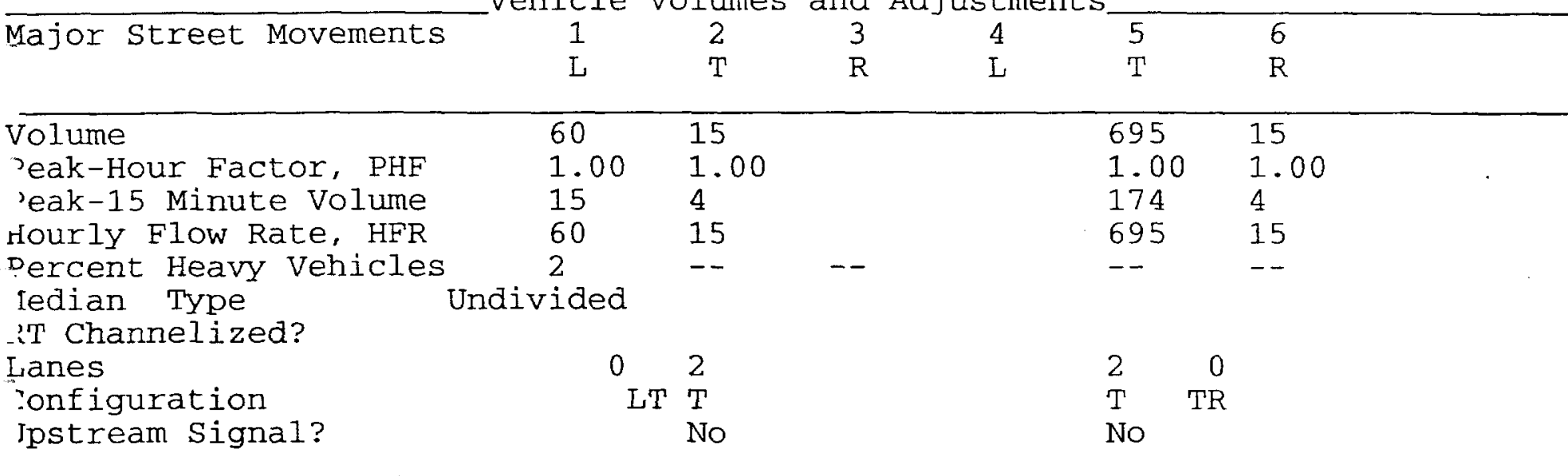

- inor street Movements

$\begin{array}{ll}7 & 8 \\ \mathrm{~L} & \mathrm{~T}\end{array}$

$\begin{array}{ll}8 & 9 \\ T & R\end{array}$

R $\quad 10$

\section{Volume}

seak Hour Factor, PHF

seak-15 Minute Volume

Hourly Flow Rate, HFR

Jercent Heavy Vehicles

'ercent Grade (\%)

Median Storage

mlared Approach: Exists?

¿2T Channelized?

Storage

$\begin{array}{rrr}10 & 11 & 12 \\ \mathrm{~L} & \mathrm{~T} & \mathrm{R}\end{array}$

$\begin{array}{ll}40 & 495 \\ 1.00 & 1.00 \\ 10 & 124 \\ 40 & 495 \\ 2 & 2\end{array}$


Pedestrian Volumes and Adjustments

\begin{tabular}{lllll}
\hline Movements & 13 & 14 & 15 & 16 \\
\hline Flow (ped/hr) & 0 & 0 & 0 & 0 \\
Lane Width (ft) & 12.0 & 12.0 & 12.0 & 12.0 \\
Walking Speed (ft/sec) & 4.0 & 4.0 & 4.0 & 4.0 \\
Percent Blockage & 0 & 0 & 0 & 0
\end{tabular}

Upstream Signal Data

$\begin{array}{lllllll}\text { Prog. } & \text { Sat } & \text { Arrival } & \text { Green } & \text { Cycle } & \text { Prog. } & \text { Distance } \\ \text { Flow } & \text { Flow } & \text { Type } & \text { Time } & \text { Length } & \text { Speed } & \text { to Signal } \\ \text { voh } & \text { vph } & & \text { sec } & \text { sec } & \text { mph } & \text { feet }\end{array}$

S2 Left-Turn

Through

S5 Left-Turn

Through

Worksheet 3-Data for Computing Effect of Delay to Major street Vehicles Movement 2 Movement 5

Shared In volume, major th vehicles:

Shared In volume, major rt vehicles:

Sat flow rate, major th vehicles:

Sat flow rate, major rt vehicles:

Number of major street through lanes:

0
1700
1700
2

Worksheet 4-Critical Gap and Follow-up Time Calculation

\begin{tabular}{|c|c|c|c|c|c|c|c|c|c|c|}
\hline $\begin{array}{l}\text { Critical } \\
\text { Movement }\end{array}$ & Gap Calc & $\begin{array}{l}\text { ulati } \\
1 \\
L\end{array}$ & $\begin{array}{l}4 \\
L\end{array}$ & $\begin{array}{l}7 \\
\mathrm{~L}\end{array}$ & $\begin{array}{l}8 \\
\mathrm{~T}\end{array}$ & $\begin{array}{l}9 \\
\mathrm{R}\end{array}$ & $\begin{array}{r}10 \\
\mathrm{~L}\end{array}$ & $\begin{array}{r}11 \\
\mathrm{~T}\end{array}$ & $\begin{array}{r}12 \\
\mathrm{R}\end{array}$ & \\
\hline $\begin{array}{l}t(c, \text { base }) \\
t(c, h v) \\
P(h v) \\
t(c, g) \\
G r a d e / 100 \\
t(3,1 t) \\
t(c, T): \\
t(c)\end{array}$ & $\begin{array}{l}1 \text {-stage } \\
\text { 2-stage } \\
1 \text {-stage } \\
\text { 2-stage }\end{array}$ & $\begin{array}{l}0.00 \\
0.00 \\
0.00 \\
4.1\end{array}$ & $\begin{array}{l}0.00 \\
0.00\end{array}$ & $\begin{array}{l}2.00 \\
0.20 \\
0.00 \\
0.00 \\
1.00\end{array}$ & $\begin{array}{l}2.00 \\
0.20 \\
0.00 \\
0.00 \\
1.00\end{array}$ & $\begin{array}{l}2.00 \\
0.10 \\
0.00 \\
0.00 \\
0.00\end{array}$ & $\begin{array}{l}7.5 \\
2.00 \\
2 \\
0.20 \\
0.00 \\
0.70 \\
0.00 \\
1.00 \\
6.8\end{array}$ & $\begin{array}{l}2.00 \\
0.20 \\
0.00 \\
0.00 \\
1.00\end{array}$ & $\begin{array}{l}6.9 \\
2.00 \\
2 \\
0.10 \\
0.00 \\
0.00 \\
0.00 \\
0.00 \\
6.9\end{array}$ & • \\
\hline $\begin{array}{l}\text { Follow-Up } \\
\text { Movement }\end{array}$ & Time $\mathrm{Ca}$ & $\begin{array}{c}1 \text { cula } \\
1 \\
\mathrm{~L}\end{array}$ & $\begin{array}{c}\text { Lons } \\
4 \\
\text { L }\end{array}$ & $\begin{array}{l}7 \\
\mathrm{~L}\end{array}$ & $\begin{array}{l}8 \\
\mathrm{~T}\end{array}$ & $\begin{array}{l}9 \\
\mathrm{R}\end{array}$ & $\begin{array}{r}10 \\
\mathrm{~L}\end{array}$ & $\begin{array}{r}11 \\
T\end{array}$ & $\begin{array}{r}12 \\
\mathrm{R}\end{array}$ & \\
\hline $\begin{array}{l}t(\mathrm{f}, \text { base }) \\
t(\mathrm{f}, \mathrm{HV}) \\
P(\mathrm{HV}) \\
t(\mathrm{f})\end{array}$ & & $\begin{array}{l}2.20 \\
1.00 \\
2 \\
2.2\end{array}$ & 1.00 & 1.00 & 1.00 & 1.00 & $\begin{array}{l}3.50 \\
1.00 \\
2 . \\
3.5\end{array}$ & 1.00 & $\begin{array}{l}3.30 \\
1.00 \\
2 \\
3.3\end{array}$ & \\
\hline
\end{tabular}


worksheet 5-Effect of Upstream Signals

Iomputation 1-Queue Clearance Time at Upstream Signal

Movement 2

Movement 5

$V(t) \quad V(1$, prot $) \quad V(t) \quad V(1$, prot $)$

\section{i prog}

Total Saturation Flow Rate, s (vph)

Arrival Type

iffective Green, $g$ (sec)

iycle Length, $\mathrm{C}$ (sec)

Rp (from table 9-2)

?roportion vehicles arriving on green $\mathrm{P}$

I (q1)

$g(q 2)$

$j(q)$

Computation 2-Proportion of TWSC Intersection Time blocked Movement 2 Movement 5

$V(t) \quad V(1$, prot $) \quad V(t) \quad V(1$, prot $)$

alpha
seta
'ravel time, t(a) (sec)
Smoothing Factor, F
'roportion of conflicting flow, f
lax platooned flow, V(c,max)
Min platooned flow, V(c,min)
Duration of blocked period, t(p)
'roportion time blocked, p
Computation 3-Platoon Event Periods
'(2) 0.000
(5)
(dom)
'(subo)
constrained or unconstrained?

'ropor
inblock
for min
lovemen
p(1)
(4)
(7)
$p(8)$
$p(9)$
(10)
(11)
$p(12)$

(1)

Single-stage -Process
0.000

0.000

omputation 4 and 5 single-stage process Movement

$\begin{array}{rrrrrrrr}1 & 4 & 7 & 8 & 9 & 10 & 11 & 12 \\ \mathrm{~L} & \mathrm{~L} & \mathrm{~L} & \mathrm{~T} & \mathrm{R} & \mathrm{L} & \mathrm{T} & \mathrm{R}\end{array}$




\section{$\mathrm{V}(\mathrm{c}, \mathrm{x})$}

$S$

$\mathrm{P}(\mathrm{x})$

3400

$V(c, u, x)$

$\mathrm{C}(r, \mathrm{x})$

$C(p l a t, x)$

Worksheet 6-Impedance and Capacity Equations

\begin{tabular}{|c|c|c|}
\hline Step 1: RT from Minor St. & 9 & 12 \\
\hline $\begin{array}{l}\text { Conflicting Flows } \\
\text { Potential Capacity } \\
\text { Pedestrian Impedance Factor } \\
\text { Movement Capacity } \\
\text { Probability of queue free st. }\end{array}$ & $\begin{array}{l}1.00 \\
1.00\end{array}$ & $\begin{array}{l}355 \\
641 \\
1.00 \\
641 \\
0.23\end{array}$ \\
\hline Step 2: LT from Major St. & 4 & 1 \\
\hline $\begin{array}{l}\text { Conflicting Flows } \\
\text { Potential Capacity } \\
\text { Pedestrian Impedance Factor } \\
\text { Movement Capacity } \\
\text { Probability of Queue free St. } \\
\text { Maj L-Shared Prob Q free St. }\end{array}$ & $\begin{array}{l}1.00 \\
1.00\end{array}$ & $\begin{array}{l}710 \\
885 \\
1.00 \\
885 \\
0.93 \\
0.93\end{array}$ \\
\hline Step 3: TH from Minor st. & 8 & 11 \\
\hline $\begin{array}{l}\text { Conflicting Flows } \\
\text { Potential Capacity } \\
\text { Pedestrian Impedance Factor } \\
\text { Cap. Adj. factor due to Impeding mvmnt } \\
\text { Movement Capacity } \\
\text { Probability of Queue Eree St. }\end{array}$ & $\begin{array}{l}1.00 \\
0.93 \\
1.00\end{array}$ & $\begin{array}{l}1.00 \\
0.93 \\
1.00\end{array}$ \\
\hline Step 4: LT from Minor St. & 7 & 10 \\
\hline $\begin{array}{l}\text { Conflicting Flows } \\
\text { Potential Capacity } \\
\text { Pedestrian Impedance Factor } \\
\text { Maj. L, Min T Impedance factor } \\
\text { Maj. L, Min T Adj. Imp Factor. } \\
\text { Cap. Adj. factor due to Impeding mvmnt } \\
\text { Movement Capacity }\end{array}$ & $\begin{array}{l}1.00 \\
0.93 \\
0.95 \\
0.22\end{array}$ & $\begin{array}{l}829 \\
309 \\
1.00\end{array}$ \\
\hline
\end{tabular}


Norksheet 7-Computation of the Effect of Two-stage Gap Acceptance

\begin{tabular}{lcc}
\hline Step 3: TH from Minor St. & 8 & 11 \\
\hline Part 1 - First Stage & \\
Conflicting Flows & & \\
Potential Capacity & \\
Pedestrian Impedance Factor & \\
Cap. Adj. factor due to Impeding mvmnt \\
Movement Capacity
\end{tabular}

Part 2 - Second stage

conflicting Flows

Potential Capacity

Pedestrian Impedance Factor

Cap. Adj. factor due to Impeding mumnt

Yovement Capacity

Part 3 - Single stage

conflicting Flows

?otential Capacity

Pedestrian Impedance Factor $\quad 1.00 \quad 1.00$

Cap. Adj. factor due to Impeding mvmnt 0.93

vovement Capacity

Result for 2 stage process:

$\exists$

C $t$

Probability of Queue free St. $1.00 \quad 1.00$

\begin{tabular}{llll}
\hline Step 4: LT from Minor St. & 70
\end{tabular}

?art 1 - First Stage

zonflicting Flows

Potential Capacity

Pedestrian Impedance Factor

zap. Adj. factor due to Impeding mvmnt

Movement Capacity

?art 2 - Second Stage

zonflicting Flows

Potential Capacity

Pedestrian Impedance Factor

aap. Adj. factor due to Impeding mvmnt

Movement Capacity

?art 3 - Single Stage

Conflicting Flows

Potential Capacity

?edestrian Impedance Factor

vaj. L, Min T Impedance factor

Maj. L, Min T Adj. Imp Factor.

zap. Adj. Factor due to Impeding mvmnt

novement Capacity

0.93

0.22 
Worksheet 8-Shared Lane Calculations

\begin{tabular}{|c|c|c|c|c|c|c|}
\hline Movement & $\begin{array}{l}7 \\
\mathrm{~L}\end{array}$ & $\begin{array}{l}8 \\
T\end{array}$ & $\begin{array}{l}9 \\
R\end{array}$ & $\begin{array}{r}10 \\
\mathrm{~L}\end{array}$ & $\begin{array}{r}11 \\
\mathrm{~T}\end{array}$ & $\begin{array}{r}12 \\
\mathrm{R}\end{array}$ \\
\hline $\begin{array}{l}\text { Volume (vph) } \\
\text { Movement Capacity (vph) } \\
\text { Shared Lane Capacity (vph) }\end{array}$ & & & & $\begin{array}{l}40 \\
288\end{array}$ & & $\begin{array}{l}495 \\
641\end{array}$ \\
\hline
\end{tabular}

Worksheet 9-Computation of Effect of Flared Minor street Approaches

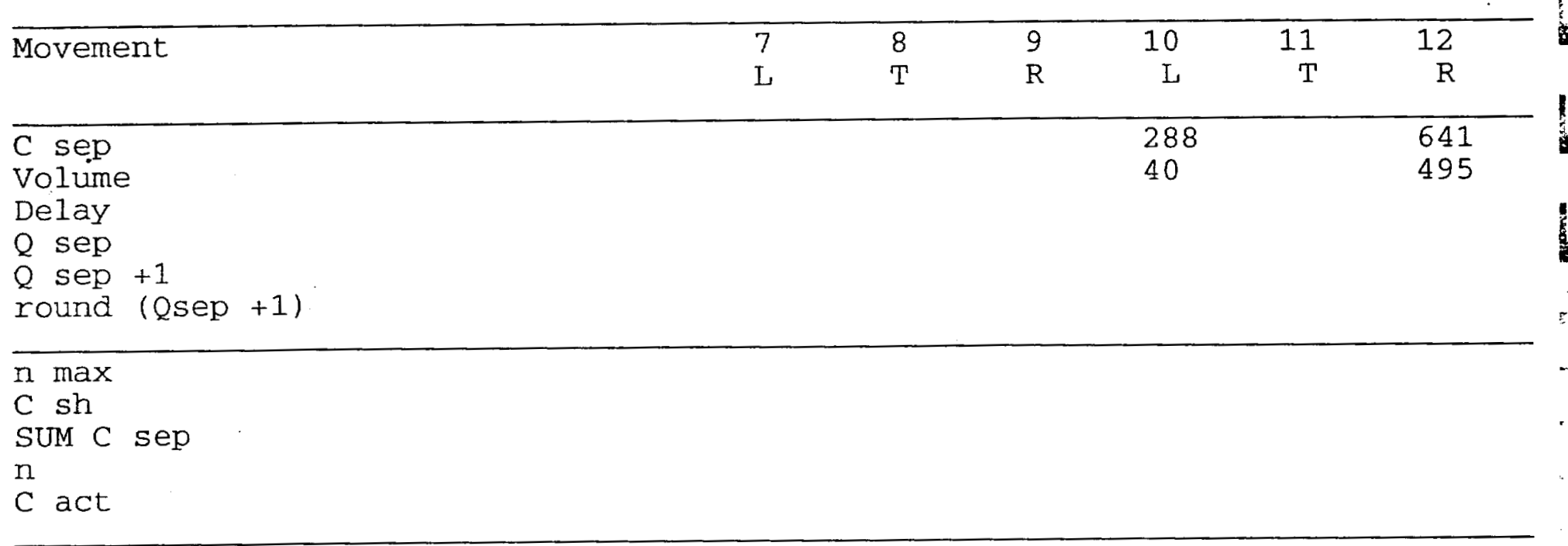

Worksheet 10-Delay, Queue Length, and Level of Service

\begin{tabular}{|c|c|c|c|c|c|c|c|c|}
\hline $\begin{array}{l}\text { Movement } \\
\text { Lane Config }\end{array}$ & $\begin{array}{l}1 \\
\mathrm{LT}\end{array}$ & 4 & 7 & 8 & 9 & $\begin{array}{r}10 \\
L\end{array}$ & 11 & $\begin{array}{r}12 \\
\mathrm{R}\end{array}$ \\
\hline $\begin{array}{l}\text { V (vph) } \\
C(m) \text { (vph) } \\
\text { V/C } \\
95 \% \text { queue length } \\
\text { Control Delay } \\
\text { LOS } \\
\text { Approach Delay } \\
\text { Approach LOS }\end{array}$ & $\begin{array}{l}60 \\
885 \\
0.07 \\
0.22 \\
9.4 \\
\text { A }\end{array}$ & & & & • & $\begin{array}{l}40 \\
288 \\
0.14 \\
0.48 \\
19.5 \\
C\end{array}$ & $\begin{array}{c}28.1 \\
D\end{array}$ & $\begin{array}{c}495 \\
641 \\
0.77 \\
9.05 \\
28.8 \\
D\end{array}$ \\
\hline
\end{tabular}

Worksheet 11-Shared Major LT Impedance and Delay

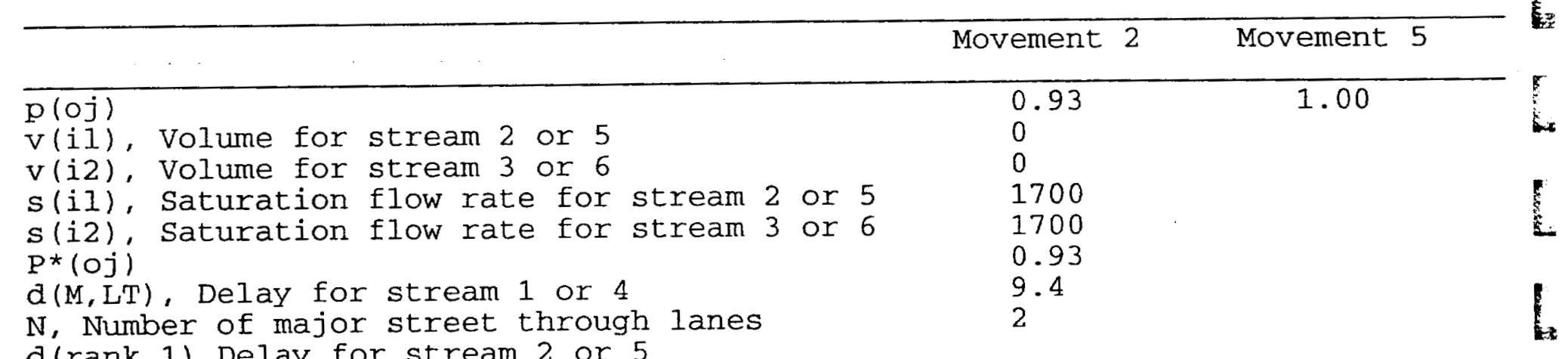




\section{Analyst:}

rency/Co.:

ite Performed: 1/21/02

inalysis Time Period: PM Peak

- itersection:

East Ave \& W Entrance (Location \# 5)

risdiction:

2001

: talysis Year:

sroject ID: Lawrence Livermore National Laboratory st/West Street: East Ave

irth/South Street: West Entrance to Sandia Labs

t.tersection Orientation: EW Study period (hrs): 1.00

Vehicle Volumes and Adjustments

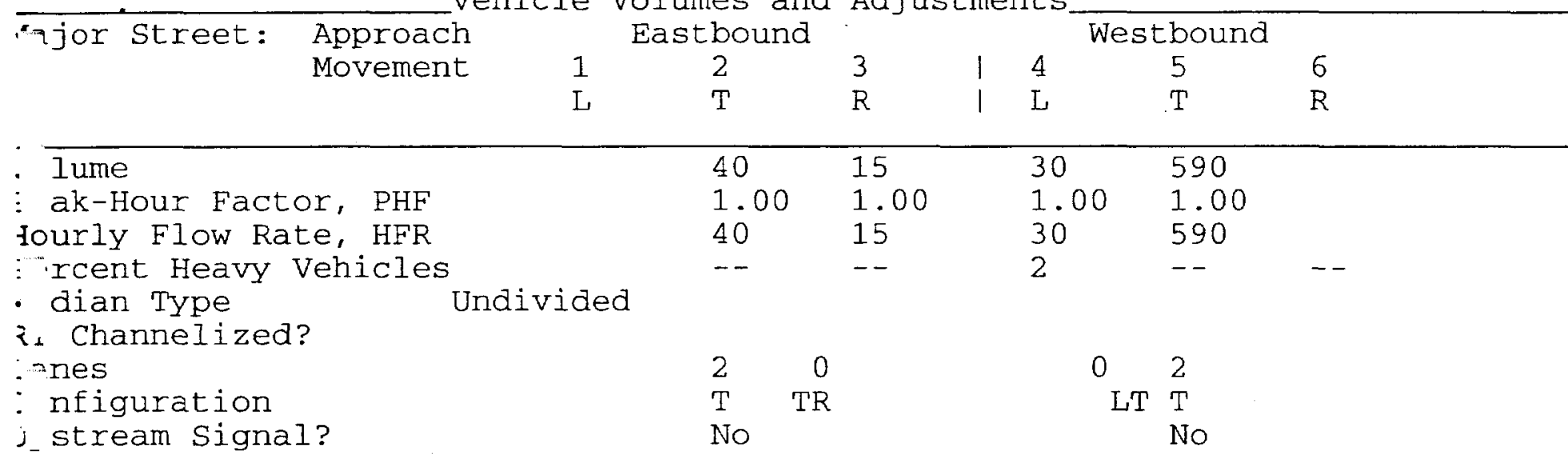

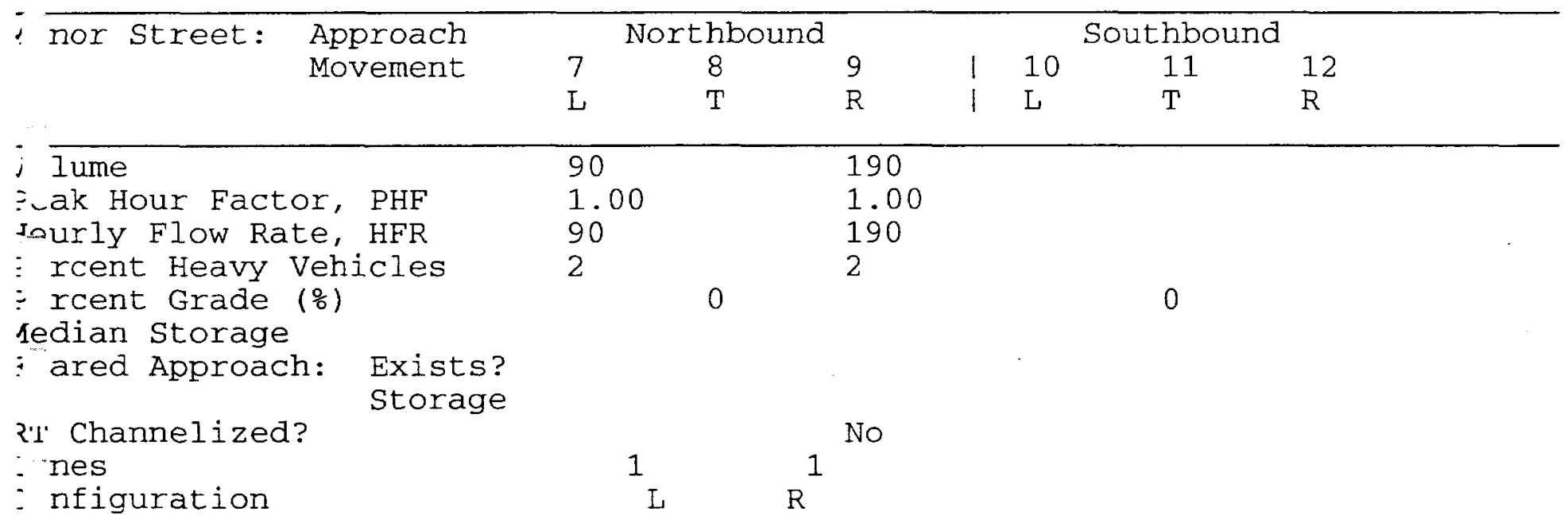

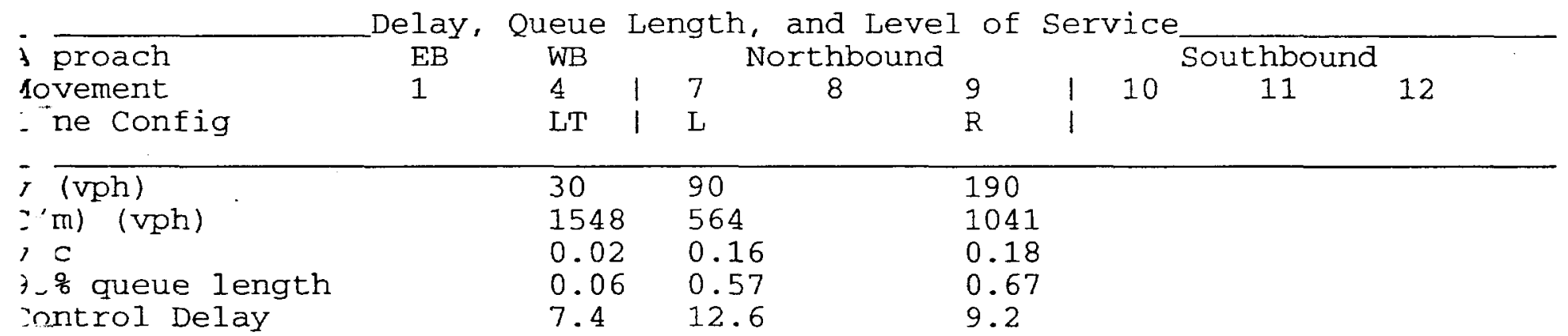


OS

pproach Delay pproach LOS
A

B

10.3

A

HCS2000: Unsignalized Intersections Release 4.1

orve Engineering, Inc.

hone:

Fax:

-Mail:

TWO-WAY STOP CONTROL (TWSC) ANALYSIS

nalyst:

gency/Co.:

ate Performed: 1/21/02

nalysis Time Period: PM Peak

ntersection: East Ave \& W Entrance

urisdiction:

nalysis Year: 2001

roject ID: Lawrence Livermore National Laboratory

ast/West Street: East Ave

orth/South Street: West Entrance to Sandia Labs

ntersection orientation: EW study period (hrs): 1.00

Vehicle Volumes and Adjustments

\begin{tabular}{lccccccc}
\hline ajor street Movements & 1 & 2 & 3 & 4 & 5 & 6
\end{tabular}

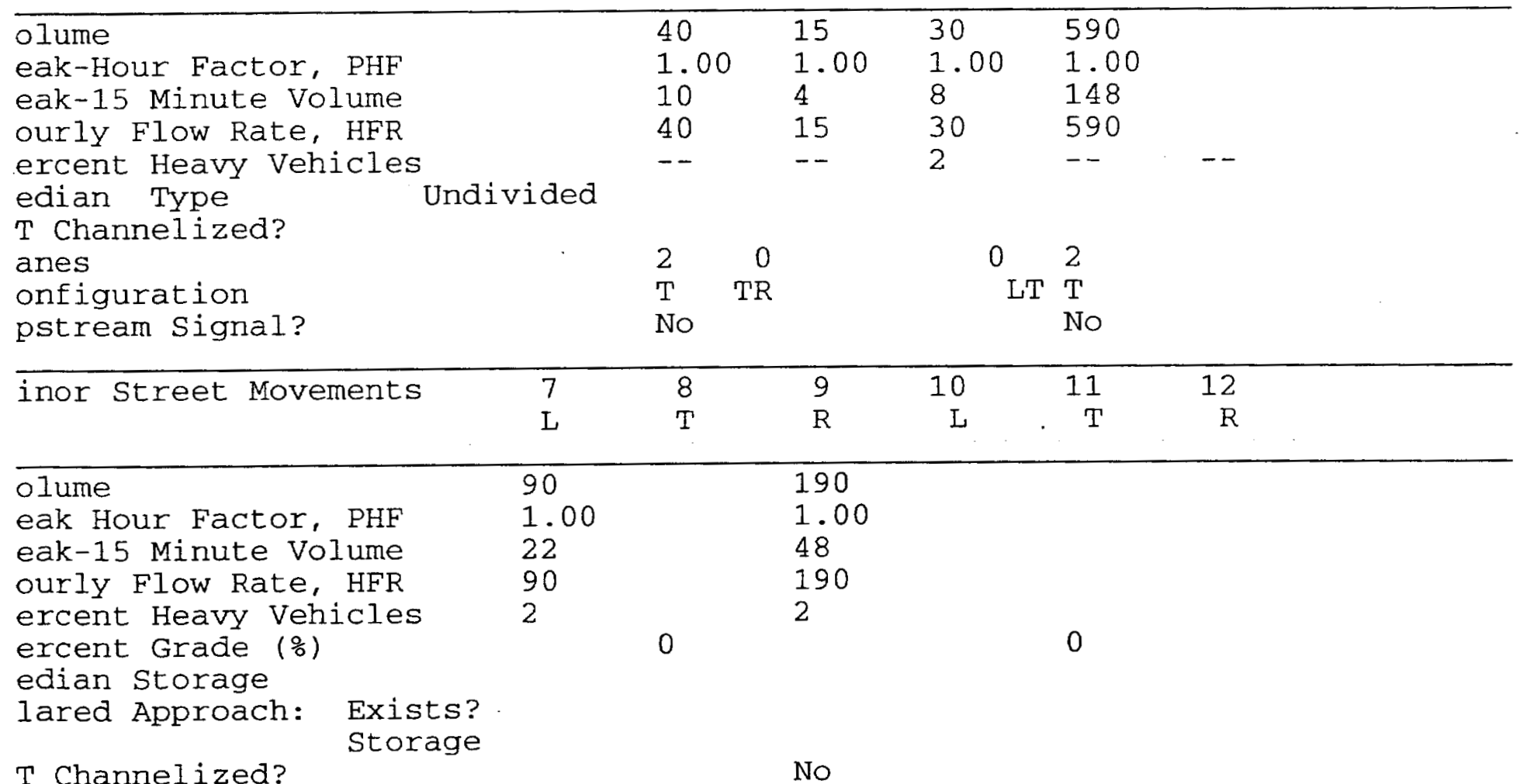




$\begin{array}{lcc}\text { ines } & 1 & 1 \\ \text { snfiguration } & \mathrm{L} & \mathrm{R}\end{array}$

Pedestrian Volumes and Adjustments

\begin{tabular}{lllll}
\hline yovements & 13 & 14 & 15 & 16 \\
\hline .Ow (ped/hr) & 0 & 0 & 0 & 0 \\
sane Width (ft) & 12.0 & 12.0 & 12.0 & 12.0 \\
ilking Speed (ft/sec) & 4.0 & 4.0 & 4.0 & 4.0 \\
?rcent Blockage & 0 & 0 & 0 & 0
\end{tabular}

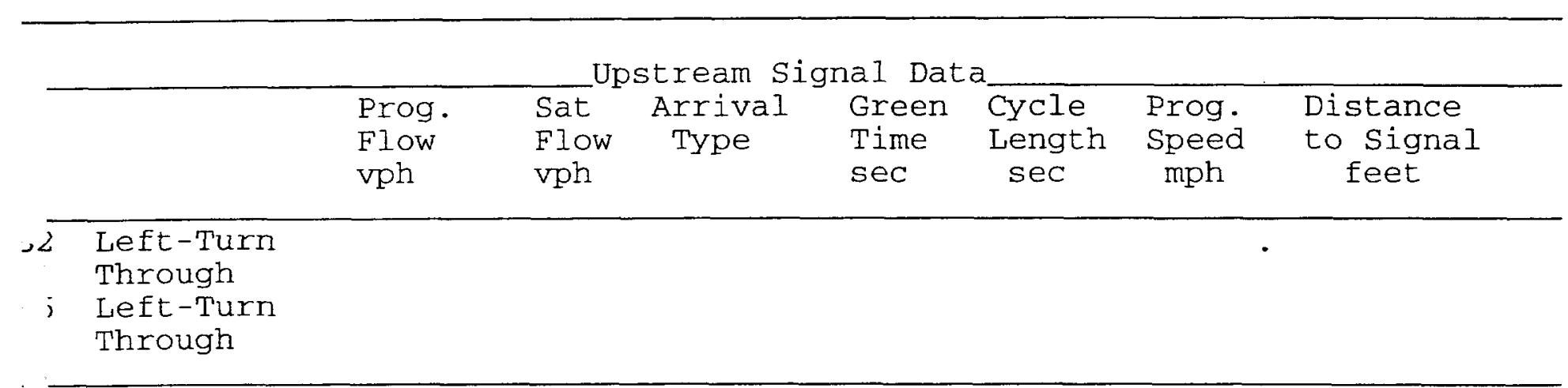

rrksheet 3-Data for Computing Effect of Delay to Major Street Vehicles

\begin{tabular}{|c|c|c|}
\hline & Movement 2 & Movement 5 \\
\hline $\begin{array}{l}\text { Hared In volume, major th vehicles: } \\
\text { "lared In volume, major rt vehicles: } \\
\text { it flow rate, major th vehicles: } \\
\vdots \text { it flow rate, major rt vehicles: } \\
\text { vumber of major street through lanes: }\end{array}$ & & $\begin{array}{l}0 \\
0 \\
1700 \\
1700 \\
2\end{array}$ \\
\hline
\end{tabular}

vorksheet 4-Critical Gap and Follow-up Time Calculation

\begin{tabular}{|c|c|c|c|c|c|c|c|c|c|}
\hline $\begin{array}{l}\text { itical } \\
\text {-uvement }\end{array}$ & Gap Cal & $\begin{array}{c}\text { culati } \\
1 \\
\mathrm{~L}\end{array}$ & $\begin{array}{l}4 \\
\mathrm{~L}\end{array}$ & $\begin{array}{l}7 \\
\mathrm{~L}\end{array}$ & $\begin{array}{l}8 \\
\mathrm{~T}\end{array}$ & $\begin{array}{l}9 \\
\mathrm{R}\end{array}$ & $\begin{array}{r}10 \\
\mathrm{~L}\end{array}$ & $\begin{array}{r}11 \\
\mathrm{~T}\end{array}$ & $\begin{array}{r}12 \\
\mathrm{R}\end{array}$ \\
\hline $\begin{array}{l}\text { (c, base) } \\
(c, h v) \\
\text { (hv) } \\
\text { c,g) } \\
\text { irade }(100 \\
(3,1 t) \\
\text { c, T): } \\
\text { (c) }\end{array}$ & $\begin{array}{l}\text { 1-stage } \\
2 \text {-stage } \\
1 \text {-stage } \\
\text { 2-stage }\end{array}$ & $\begin{array}{l}0.00 \\
0.00\end{array}$ & $\begin{array}{l}0.00 \\
0.00 \\
0.00 \\
4.1\end{array}$ & $\begin{array}{l}7.5 \\
2.00 \\
2 \\
0.20 \\
0.00 \\
0.70 \\
0.00 \\
1.00 \\
6.8\end{array}$ & $\begin{array}{l}2.00 \\
0.20 \\
0.00 \\
0.00 \\
1.00\end{array}$ & $\begin{array}{l}6.9 \\
2.00 \\
2 \\
0.10 \\
0.00 \\
0.00 \\
0.00 \\
0.00 \\
6.9\end{array}$ & $\begin{array}{l}2.00 \\
0.20 \\
0.00 \\
0.00 \\
1.00\end{array}$ & $\begin{array}{l}2.00 \\
0.20 \\
0.00 \\
0.00 \\
1.00\end{array}$ & $\begin{array}{l}2.00 \\
0.10 \\
0.00 \\
0.00 \\
0.00\end{array}$ \\
\hline $\begin{array}{l}\text { ollow-Up } \\
\text { ivement }\end{array}$ & Time C & $\begin{array}{c}\text { alcula } \\
\frac{1}{L}\end{array}$ & $\begin{array}{c}\text { ions } \\
4 \\
\text { L }\end{array}$ & $\begin{array}{l}7 \\
\mathrm{~L}\end{array}$ & $\begin{array}{l}8 \\
\mathrm{~T}\end{array}$ & $\begin{array}{l}9 \\
\mathrm{R}\end{array}$ & $\begin{array}{r}10 \\
\mathrm{~L}\end{array}$ & $\begin{array}{r}11 \\
\mathrm{~T}\end{array}$ & $\begin{array}{r}12 \\
\mathrm{R}\end{array}$ \\
\hline $\begin{aligned} & \text { (f, base) } \\
& \text { f,HV) } \\
& \vdots \text { HV) } \\
&-(\mathrm{f})\end{aligned}$ & & 1.00 & $\begin{array}{l}2.20 \\
1.00 \\
2 \\
2.2\end{array}$ & $\begin{array}{l}3.50 \\
1.00 \\
2 \\
3.5\end{array}$ & 1.00 & $\begin{array}{l}3.30 \\
1.00 \\
2 \\
3.3\end{array}$ & 1.00 & 1.00 & 1.00 \\
\hline
\end{tabular}


orksheet 5-Effect of Upstream Signals

omputation 1-Queue Clearance Time at Upstream Signal

Movement 2

Movement 5

$V(t) \quad V(1$, prot $) \quad V(t) \quad V(1$, prot $)$

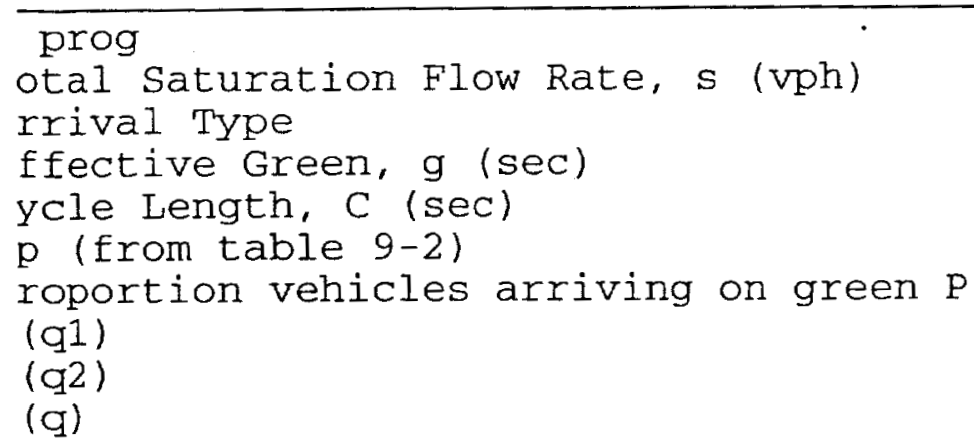

\section{Ipha}

eta

ravel time, $t(a)$ (sec)

moothing Factor, $F$

roportion of conflicting flow, $f$

ax platooned flow, $\mathrm{V}(\mathrm{c}, \mathrm{max})$

in platooned flow, $\mathrm{V}(\mathrm{c}, \mathrm{min})$

uration of blocked period, $t(p)$

roportion time blocked, $\mathrm{p}$

0.000

0.000

omputation 3-Platoon Event Periods Result

\begin{tabular}{ll}
\hline$(2)$ & 0.000 \\
$(5)$ & 0.000
\end{tabular}

(com)

(subo)

onstrained or unconstrained?

\begin{tabular}{|c|c|c|}
\hline $\begin{array}{l}\text { roportion } \\
\text { nblocked }\end{array}$ & (1) & (2) \\
\hline or minor & Single-stage & Two-Stage Process \\
\hline ovements, $p(x)$ & Process & Stage II \\
\hline
\end{tabular}

(1)

(4)

(7)

(8)

(9)

(10)

(11)

(12)

omputation 4 and 5

ingle-stage Process

ovement

\begin{tabular}{rrrrrrrr}
1 & 4 & 7 & 8 & 9 & 10 & 11 & 12 \\
$\mathrm{~L}$ & $\mathrm{~L}$ & $\mathrm{~L}$ & $\mathrm{~T}$ & $\mathrm{R}$ & $\mathrm{L}$ & $\mathrm{T}$ & $\mathrm{R}$ \\
\hline
\end{tabular}


$\mathrm{Px}$

$c, u, x$

$=r, x$

plat, $x$

wo-Stage Process

Stage1 ${ }^{7}$ Stage2 stage1 ${ }^{8}$ stage2 stage1 $^{10}$ stage2 $^{\text {stage1 }}{ }^{11}$ stage2

$\bar{r}(\mathrm{C}, \mathrm{x})$

( : $x)$

$v(c, u, x)$

$r, x$

-iplat, $x$ )

sksheet 6-Impedance and Capacity Equations

-ep 1: RT from Minor St.

9

12

jonflicting Flows

tential Capacity

:destrian Impedance Factor

suvement Capacity

robability of Queue free st.

$\begin{array}{ll}28 & \\ 1041 & \\ 1.00 & 1.00 \\ 1041 & 1.00 \\ 0.82 & \end{array}$

: ep 2: LT from Major st.

4

-nflicting Flows

tential Capacity

?edestrian Impedance Factor

jvement Capacity

obability of Queue free St.

aj L-Shared Prob Q free St.

\section{5}

1548

1.00

1548

0.98

0.98

8
1.00

1.00

ep 3: TH from Minor st.

zonflicting Flows

-tential Capacity

destrian Impedance Factor

-ap. Adj. factor due to Impeding mvmnt

1.00

0.98

1.00

Jvement Capacity

obability of Queue free st.

1.00

1.00

\begin{tabular}{lll}
\hline itep 4: LT from Minor St. & 7 & 10 \\
\hline nflicting Flows & 403 & 575 \\
?otential Capacity & 1.00 & 1.00 \\
destrian Impedance Factor & & 0.98 \\
j. L, Min T Impedance factor . & 0.98 & 0.99 \\
haj. L, Min T Adj. Imp Factor. & 564 & 0.81 \\
ap. Adj. factor due to Impeding mvmnt & & \\
vement Capacity &
\end{tabular}


orksheet 7-Computation of the Effect of Two-stage Gap Acceptance

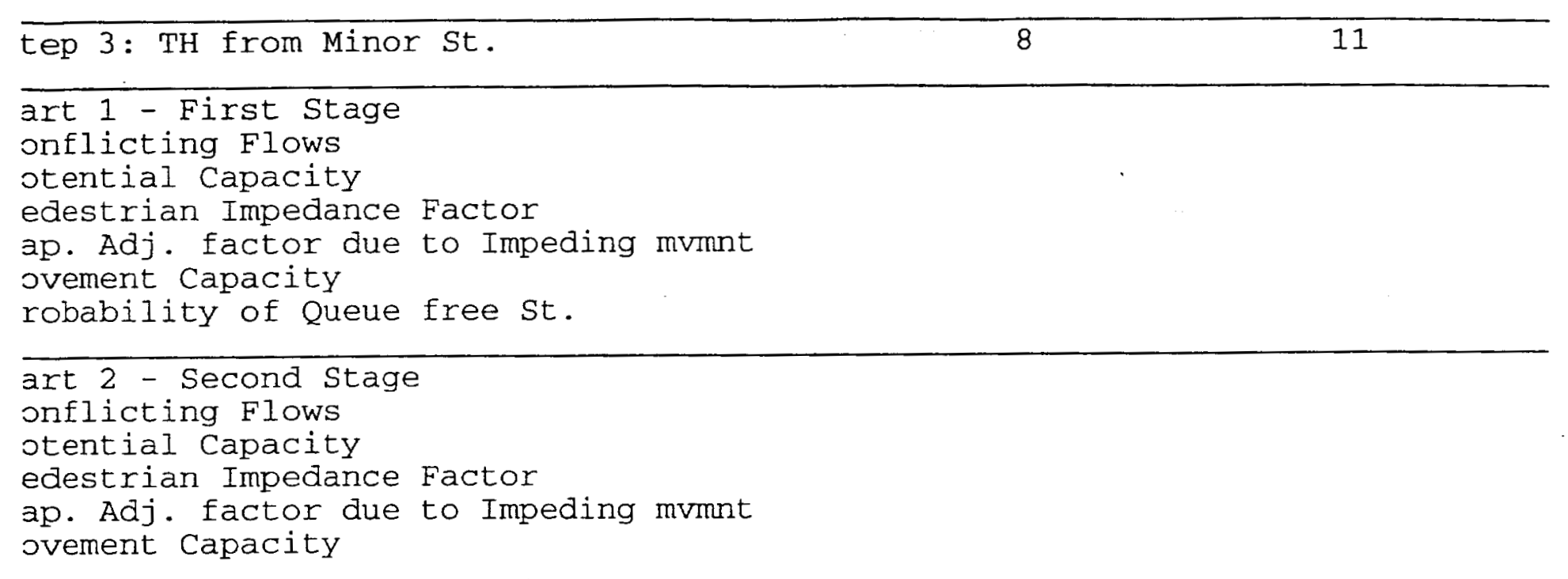

art 3 - single stage

onflicting Flows

otential Capacity

edestrian Impedance Factor

ap. Adj. factor due to Impeding mvmnt

1.00

1.00

ovement Capacity

esult for 2 stage process:

$t$

robability of queue free st.

1.00

1.00

tep 4: LT from Minor st.

7

10

art 1 - First Stage

onflicting Flows

otential Capacity

edestrian Impedance Factor

ap. Adj. factor due to Impeding mvmnt

ovement Capacity

art 2 - Second stage

onflicting Flows

otential Capacity

edestrian Impedance Factor

ap. Adj. factor due to Impeding mvmnt

svement Capacity

art 3 - Single Stage

onflicting flows

otential Capacity

edestrian Impedance Factor

403

575

1.00

1.00

. $L$ Min $T$ Impedance factor

aj. L, Min T Adj. Imp Factor.

ap. Adj. factor due to Impeding mvmnt

ovement Capacity

0.98

0.98

0.99

564

0.81

esults for Two-stage process: 
rksheet 8-Shared Lane Calculations

\begin{tabular}{|c|c|c|c|c|c|c|}
\hline Lovement & $\begin{array}{l}7 \\
L\end{array}$ & $\begin{array}{l}8 \\
\mathrm{~T}\end{array}$ & $\begin{array}{l}9 \\
R\end{array}$ & $\begin{array}{r}10 \\
\mathrm{~L}\end{array}$ & $\begin{array}{r}11 \\
\mathrm{~T}\end{array}$ & $\begin{array}{r}12 \\
\mathrm{R}\end{array}$ \\
\hline $\begin{array}{l}\text { Jume (vph) } \\
\text { vement Capacity (vph) } \\
\text { ared Lane Capacity (vph) }\end{array}$ & $\begin{array}{l}90 \\
564\end{array}$ & & $\begin{array}{l}190 \\
1041\end{array}$ & & & \\
\hline
\end{tabular}

- rksheet 9-Computation of Effect of Flared Minor Street Approaches

\begin{tabular}{|c|c|c|c|c|c|c|}
\hline lovement & $\begin{array}{l}7 \\
\mathrm{~L}\end{array}$ & $\begin{array}{l}8 \\
\mathrm{~T}\end{array}$ & $\begin{array}{l}9 \\
\mathrm{R}\end{array}$ & $\begin{array}{r}10 \\
\mathrm{~L}\end{array}$ & $\begin{array}{r}11 \\
\mathrm{~T}\end{array}$ & $\begin{array}{r}12 \\
\mathrm{R}\end{array}$ \\
\hline 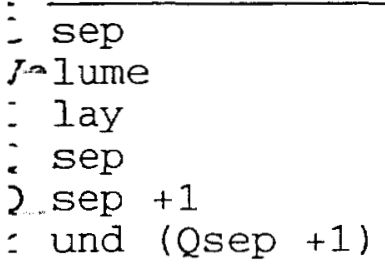 & $\begin{array}{l}564 \\
90\end{array}$ & & $\begin{array}{l}1041 \\
190\end{array}$ & & & \\
\hline $\begin{array}{l}2 \max \\
\vdots \text { sh } \\
\vdots \mathrm{MC} \text { sep } \\
\vdots \text { act }\end{array}$ & & & & & & \\
\hline
\end{tabular}

vorksheet 10-Delay, Queue Length, and Level of Service

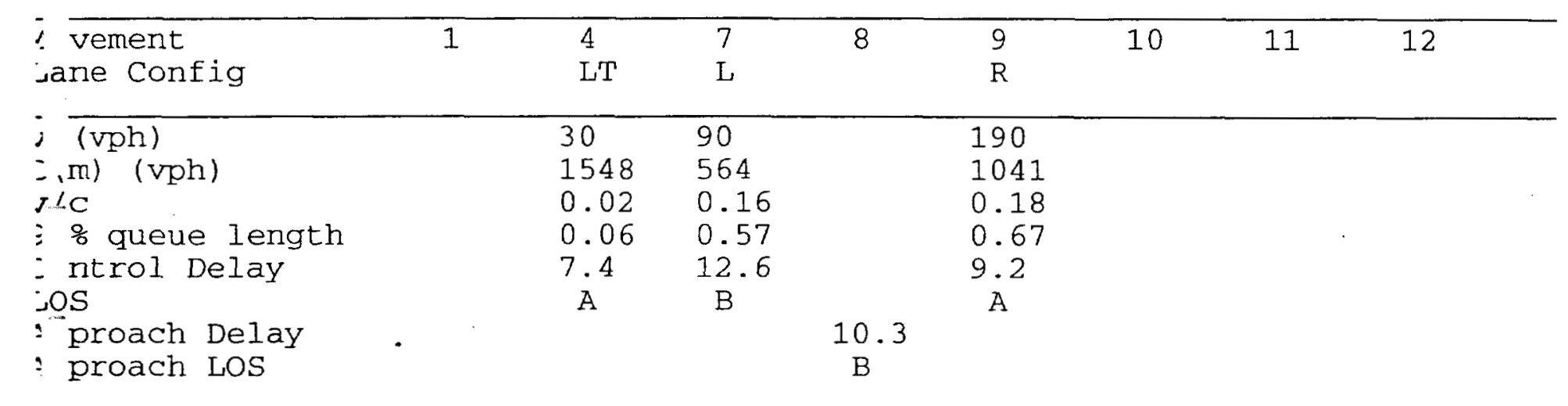

1 rksheet 11-Shared Major LT Impedance and Delay

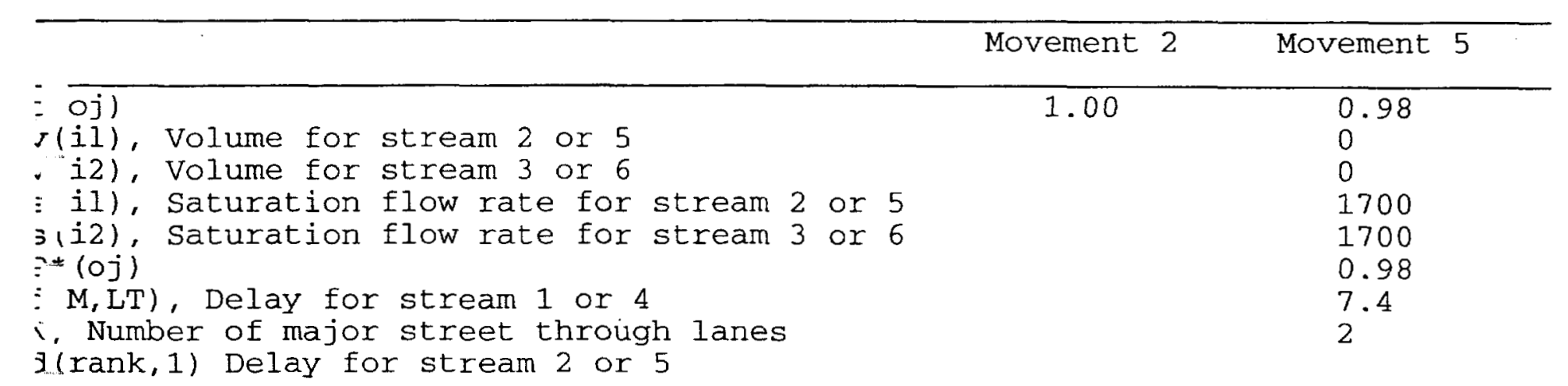


nalyst:

Jency/Co.:

ate Performed: $\quad 1 / 21 / 02$

nalysis Time Period: AM Peak

ntersection:

East Ave \& W Entrance (Location \#5)

urisdiction:

nalysis Year:

2001

roject ID: Lawrence Livermore National Laboratory

ast/West Street: East Ave

orth/South Street: West Entrance to Sandia Labs

ntersection Orientation: EW

Study period (hrs): 1.00

Vehicle Volumes and Adjustments

ajor Street: Approach Eastbound

\begin{tabular}{cccc|cccc} 
Movement & 1 & 2 & 3 & & 4 & 5 & 6 \\
& $\mathrm{~L}$ & $\mathrm{~T}$ & $\mathrm{R}$ & $\mathrm{I}$ & $\mathrm{T}$ & $\mathrm{R}$
\end{tabular}

Dlume

$\begin{array}{llll}265 & 200 & 140 & 90\end{array}$

eak-Hour Factor, PHF

ourly Flow Rate, HFR

ercent Heavy Vehicles

edian Type

Undivided

$\begin{array}{llll}1.00 & 1.00 & 1.00 & 1.00\end{array}$

$\begin{array}{llll}265 & 200 & 140 & 90\end{array}$

T Channelized?

anes

onfiguration

pstream Signal?

20

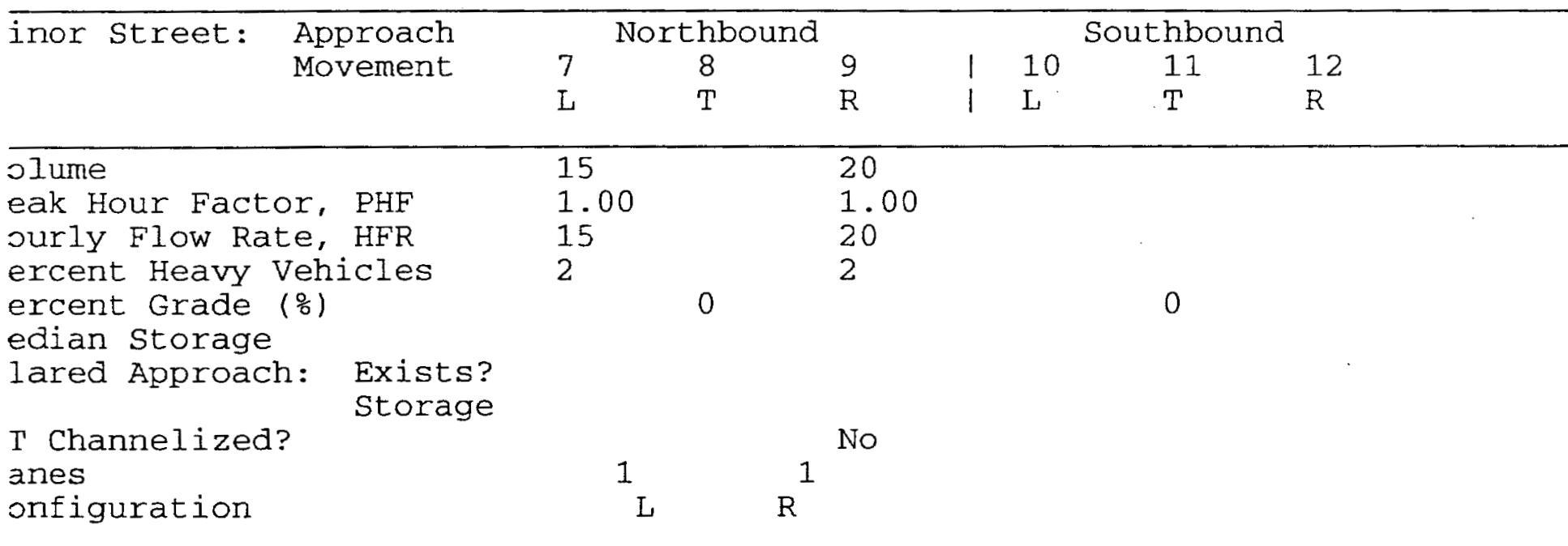

$\begin{array}{lrrl}2 & 0 & 0 & 2 \\ T & T R & \text { LT } & \text { T } \\ \text { No } & & \text { No }\end{array}$

Delay, Queue Length, and Level of Service.

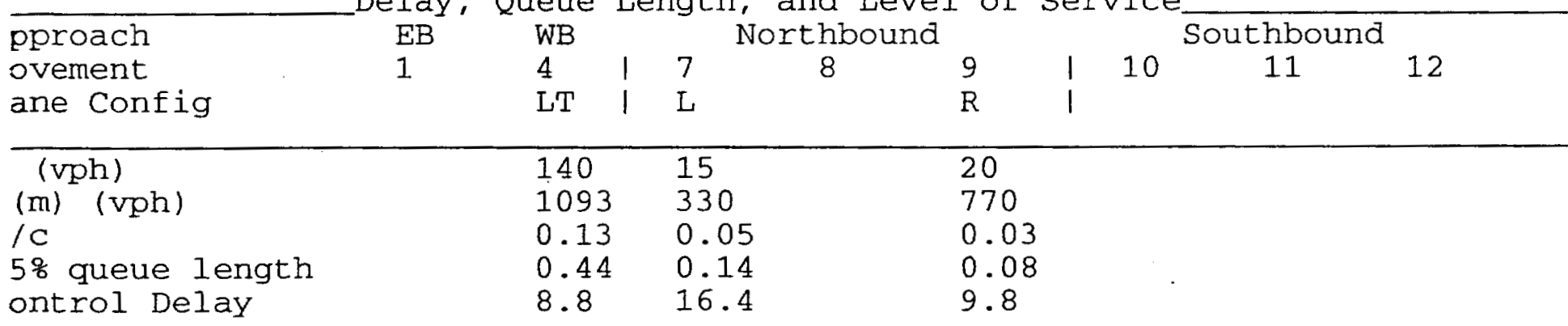


HCS2000: Unsignalized Intersections Release 4.1

rve Engineering, Inc.

one:

Fax :

I-Mail: TWO-WAY STOP CONTROL (TWSC) ANALYSIS

välyst:

ency/Co.:

we Performed: 1/21/02

inalysis Time Period: AM Peak

tersection: East Ave \& W Entrance

risdiction:

inalysis Year:

oject ID: Lawrence Livermore National Laboratory

st/West Street: East Ave

jorth/South Street: West Entrance to Sandia Labs

I tersection Orientation: EW $\quad$ Study period (hrs): 1.00

\begin{tabular}{|c|c|c|c|c|c|c|}
\hline jor street Movements & $\begin{array}{l}1 \\
\mathrm{~L}\end{array}$ & $\begin{array}{l}2 \\
\mathrm{~T}\end{array}$ & $\begin{array}{l}3 \\
\mathrm{R}\end{array}$ & $\begin{array}{l}4 \\
\mathrm{~L}\end{array}$ & $\begin{array}{l}5 \\
\mathrm{~T}\end{array}$ & $\begin{array}{l}6 \\
\mathrm{R}\end{array}$ \\
\hline $\begin{array}{l}\text {-lume } \\
\text { ak-Hour Factor, PHF } \\
\text { ¿ak-15 Minute Volume } \\
\text { tourly Flow Rate, HFR } \\
\text { rcent Heavy Vehicles } \\
\text { dian Type } \\
\text { 2T Channelized? } \\
\text { nes } \\
\text { nfiguration } \\
\text { Jpstream Signal? }\end{array}$ & Undivided & $\begin{array}{l}265 \\
1.00 \\
66 \\
265 \\
--\end{array}$ & $\begin{array}{l}200 \\
1.00 \\
50 \\
200 \\
--\end{array}$ & $\begin{array}{l}140 \\
1.00 \\
35 \\
140 \\
2\end{array}$ & $\begin{array}{l}90 \\
1.00 \\
22 \\
90 \\
--\end{array}$ & -- \\
\hline nor Street Movements & $\begin{array}{l}7 \\
\mathrm{~L}\end{array}$ & $\begin{array}{l}8 \\
T\end{array}$ & $\begin{array}{l}9 \\
R\end{array}$ & $\begin{array}{r}10 \\
\mathrm{~L}\end{array}$ & $\begin{array}{r}11 \\
\mathrm{~T}\end{array}$ & $\begin{array}{r}12 \\
\mathrm{R}\end{array}$ \\
\hline $\begin{array}{l}\text { lume } \\
\text { ak Hour Factor, PHF } \\
\text { ?eak-15 Minute Volume } \\
\text { urly Flow Rate, HFR } \\
\text { rcent Heavy Vehicles } \\
\text { ?ercent Grade (\%) } \\
\text { codian Storage } \\
\text { ared Approach: Exis } \\
\text { iT Channelized? }\end{array}$ & $\begin{array}{l}15 \\
1.00 \\
4 \\
15 \\
2\end{array}$ & 0 & $\begin{array}{l}20 \\
1.00 \\
5 \\
20 \\
2\end{array}$ & & 0 & \\
\hline
\end{tabular}


Pedestrian Volumes and Adjustments

\begin{tabular}{lllll}
\hline svements & 13 & 14 & 15 & 16 \\
\hline Low (ped/hr) & & & & \\
zne Width (ft) & 0 & 0 & 0 & 0 \\
ilking Speed (ft/sec) & 12.0 & 12.0 & 12.0 & 12.0 \\
ərcent Blockage & 4.0 & 4.0 & 4.0 & 4.0 \\
\end{tabular}

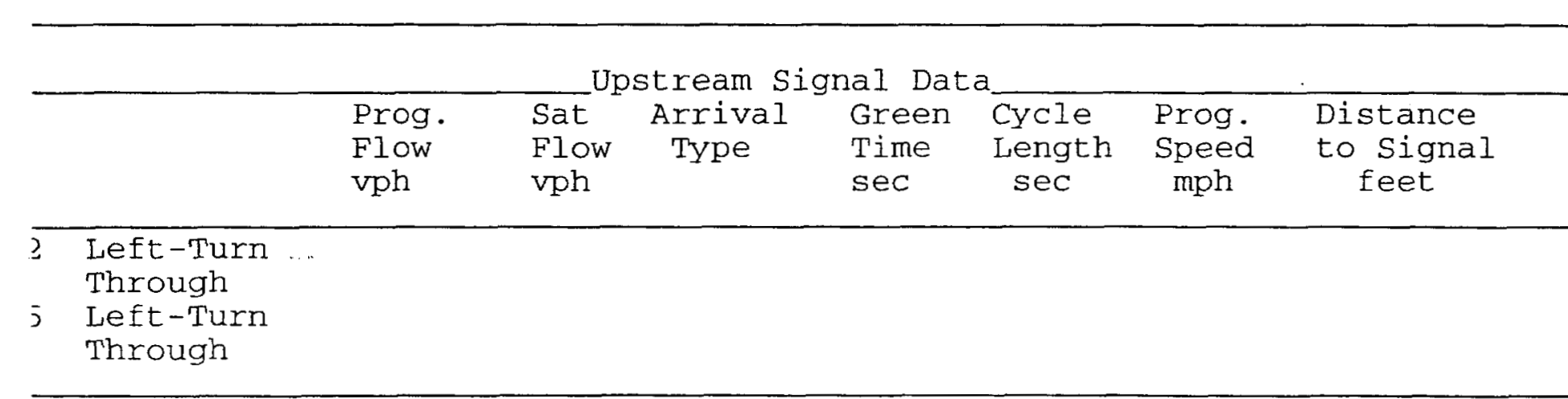

srksheet 3-Data for Computing Effect of Delay to Major street Vehicles

\begin{tabular}{lcc}
\hline & Movement 2 & Movement 5 \\
\hline lared In volume, major th vehicles: & \\
lared In volume, major rt vehicles: & 0 \\
at flow rate, major th vehicles: & 1700 \\
at flow rate, major rt vehicles: & 1700 \\
dmber of major street through lanes: & 2
\end{tabular}

srksheet 4-Critical Gap and Follow-up Time Calculation

\begin{tabular}{|c|c|c|c|c|c|c|c|c|c|}
\hline $\begin{array}{l}\text { =itical } \\
\text { Jvement }\end{array}$ & Gap Cal & $\begin{array}{c}\text { culat } \\
1 \\
L\end{array}$ & $\begin{array}{r}4 \\
-\mathrm{L}\end{array}$ & $\begin{array}{l}7 \\
\mathrm{~L}\end{array}$ & $\begin{array}{l}8 \\
\mathrm{~T}\end{array}$ & $\begin{array}{l}9 \\
\mathrm{R}\end{array}$ & $\begin{array}{r}10 \\
\mathrm{~L}\end{array}$ & $\begin{array}{r}11 \\
\mathrm{~T}\end{array}$ & $\begin{array}{r}12 \\
\mathrm{R}\end{array}$ \\
\hline $\begin{array}{l}\text { (c, base) } \\
(\mathrm{c}, \mathrm{hv}) \\
(\mathrm{hv}) \\
(\mathrm{c}, \mathrm{g}) \\
\text { rade/100 } \\
(3, \mathrm{lt}) \\
(\mathrm{c}, \mathrm{T}): \\
(\mathrm{c})\end{array}$ & $\begin{array}{l}\text { 1-stage } \\
\text { 2-stage } \\
1 \text {-stage } \\
\text { 2-stage }\end{array}$ & $\begin{array}{l}0.00 \\
0.00\end{array}$ & $\begin{array}{l}4.1 \\
2.00 \\
2\end{array}$ & $\begin{array}{l}7.5 \\
2.00 \\
2 . \\
0.20 \\
0.00 \\
0.70 \\
0.00 \\
1.00 \\
6.8\end{array}$ & $\begin{array}{l}2.00 \\
0.20 \\
0.00 \\
0.00 \\
1.00\end{array}$ & $\begin{array}{l}6.9 \\
2.00 \\
2 \\
0.10 \\
0.00 \\
0.00 \\
0.00 \\
0.00 \\
6.9\end{array}$ & $\begin{array}{l}2.00 \\
0.20 \\
0.00 \\
0.00 \\
1.00\end{array}$ & $\begin{array}{l}2.00 \\
0.20 \\
0.00 \\
0.00 \\
1.00\end{array}$ & $\begin{array}{l}2.00 \\
0.10 \\
0.00 \\
0.00 \\
0.00\end{array}$ \\
\hline $\begin{array}{l}\text { J11ow-Up } \\
\text { svement }\end{array}$ & Time C & $\begin{array}{c}\text { alcula } \\
1 \\
L\end{array}$ & $\begin{array}{c}\text { ions } \\
4 \\
L\end{array}$ & $\begin{array}{l}7 \\
\mathrm{~L}\end{array}$ & $\begin{array}{l}8 \\
\mathrm{~T}\end{array}$ & $\begin{array}{l}9 \\
R\end{array}$ & $\begin{array}{r}10 \\
\mathrm{~L}\end{array}$ & $\begin{array}{r}11 \\
\mathrm{~T}\end{array}$ & $\begin{array}{r}12 \\
\mathrm{R}\end{array}$ \\
\hline $\begin{array}{l}\text { (f, base) } \\
(\mathrm{f}, \mathrm{HV}) \\
(\mathrm{HV}) \\
\text { (f) }\end{array}$ & & 1.00 & $\begin{array}{l}2.20 \\
1.00 \\
2 \\
2.2\end{array}$ & $\begin{array}{l}3.50 \\
1.00 \\
2 \\
3.5\end{array}$ & 1.00 & $\begin{array}{l}3.30 \\
1.00 \\
2 \\
3.3\end{array}$ & 1.00 & 1.00 & 1.00 \\
\hline
\end{tabular}


$\mathrm{x}$

$c, u, x$

$r, x$

plat, $x$

wo-Stage Process

$7 \quad 8 \quad 10 \quad 11$

Stage1 Stage2 stage1 Stage2 stage1 Stage2 Stage1 Stage2

$(c, x)$

(x)

3400

$(c, u, x)$

$(r, x)$

(plat, $x)$

srksheet 6-Impedance and Capacity Equations

\begin{tabular}{|c|c|c|}
\hline tep 1: RT from Minor St. & 9 & 12 \\
\hline $\begin{array}{l}\text { onflicting Flows } \\
\text { stential Capacity } \\
\text { odestrian Impedance Factor } \\
\text { ovement Capacity } \\
\text { robability of Queue free st. }\end{array}$ & $\begin{array}{l}232 \\
770 \\
1.00 \\
770 \\
0.97\end{array}$ & $\begin{array}{l}1.00 \\
1.00\end{array}$ \\
\hline Eep 2: LT from Major st. & 4 & 1 \\
\hline $\begin{array}{l}\text { Jnflicting Flows } \\
\text { stential Capacity } \\
\text { adestrian Impedance Factor } \\
\text { svement Capacity } \\
\text { cobability of Queue free St. } \\
\text { aj L-Shared Prob Q free st. }\end{array}$ & $\begin{array}{l}465 \\
1093 \\
1.00 \\
1093 \\
0.87 \\
0.87\end{array}$ & $\begin{array}{l}1.00 \\
1.00\end{array}$ \\
\hline zep 3: TH from Minor st. & 8 & 11 \\
\hline $\begin{array}{l}\text { onflicting Flows } \\
\text { stential Capacity } \\
\text { ədestrian Impedance Factor } \\
\text { ap. Adj. factor due to Impeding mumnt } \\
\text { svement Capacity } \\
\text { robability of Queue free st. }\end{array}$ & $\begin{array}{l}1.00 \\
0.87 \\
1.00\end{array}$ & $\begin{array}{l}1.00 \\
0.87 \\
1.00\end{array}$ \\
\hline zep 4: LT from Minor st. & 7 & 10 \\
\hline $\begin{array}{l}\text { onflicting Flows } \\
\text { stential Capacity } \\
\text { ədestrian Impedance Factor } \\
\text { aj. L, Min T Impedance factor } \\
\text { aj. L, Min T Adj. Imp Factor. } \\
\text { ap. Adj. factor due to Impeding mvmnt } \\
\text { svement Capacity }\end{array}$ & $\begin{array}{l}690 \\
379 \\
1.00 \\
0.87 \\
330\end{array}$ & $\begin{array}{l}1.00 \\
0.87 \\
0.90 \\
0.88\end{array}$ \\
\hline
\end{tabular}


rrksheet 7-Computation of the Effect of Two-stage Gap Acceptance

\begin{tabular}{|c|c|c|}
\hline *ep 3: TH from Minor st. & 8 & 11 \\
\hline $\begin{array}{l}\text { zrt } 1 \text { - First stage } \\
\text { onflicting Flows } \\
\text { stential Capacity } \\
\text { ədestrian Impedance Factor } \\
\text { aap. Adj. factor due to Impeding mvmnt } \\
\text { jvement Capacity } \\
\text { cobability of Queue free st. }\end{array}$ & & \\
\hline $\begin{array}{l}\text { art } 2 \text { - Second stage } \\
\text { snflicting Flows } \\
\text { stential Capacity } \\
\text { Pedestrian Impedance Factor } \\
\text { ap. Adj. factor due to Impeding mvmnt } \\
\text { svement Capacity }\end{array}$ & & \\
\hline $\begin{array}{l}\text { art } 3 \text { - Single Stage } \\
\text { snflicting Flows } \\
\text { stential Capacity } \\
\text { nodestrian Impedance Factor } \\
\text { ap. Adj. factor due to Impeding mvmnt } \\
\text { svement Capacity }\end{array}$ & $\begin{array}{l}1.00 \\
0.87\end{array}$ & $\begin{array}{l}1.00 \\
0.87\end{array}$ \\
\hline $\begin{array}{l}t \\
\text { cobability of Queue free st. }\end{array}$ & 1.00 & 1.00 \\
\hline step 4: LT from Minor st. & 7 & 10 \\
\hline $\begin{array}{l}\text { art } 1 \text { - First Stage } \\
\text { zonflicting Flows } \\
\text { stential Capacity } \\
\text { zdestrian Impedance Factor } \\
\text {-ap. Adj. factor due to Impeding mumnt } \\
\text {-ovement Capacity }\end{array}$ & & \\
\hline $\begin{array}{l}\text { - art } 2 \text { - Second Stage } \\
\text { Jonflicting Flows } \\
\text { stential Capacity } \\
\text { ədestrian Impedance Factor } \\
\text { ap. Adj. factor due to Impeding munnt } \\
\text { svement Capacity }\end{array}$ & & \\
\hline $\begin{array}{l}\text { rart } 3 \text { - Single stage } \\
\text {-onflicting Flows } \\
\text { stential Capacity } \\
\text { - ədestrian Impedance Factor } \\
\text { Vaj. L, Min T Impedance factor } \\
\text { aj. L, Min T Adj. Imp Factor. } \\
\text { ap. Adj. factor due to Impeding mvmnt } \\
\text { Movement Capacity }\end{array}$ & $\begin{array}{l}690 \\
379 \\
1.00 \\
0.87 \\
330\end{array}$ & $\begin{array}{l}1.00 \\
0.87 \\
0.90 \\
0.88\end{array}$ \\
\hline
\end{tabular}

эsults for Two-stage process: 
orksheet 8-Shared Lane Calculations

\begin{tabular}{|c|c|c|c|c|c|c|}
\hline ovement & $\begin{array}{l}7 \\
\mathrm{~L}\end{array}$ & $\begin{array}{l}8 \\
\mathrm{~T}\end{array}$ & $\begin{array}{l}9 \\
R\end{array}$ & $\begin{array}{r}10 \\
\mathrm{I}\end{array}$ & $\begin{array}{r}11 \\
\mathrm{~T}\end{array}$ & $\begin{array}{r}12 \\
\mathrm{R}\end{array}$ \\
\hline $\begin{array}{l}\text { olume (vph) } \\
\text { ovement Capacity (vph) } \\
\text { hared Lane capacity (vph) }\end{array}$ & $\begin{array}{l}15 \\
330\end{array}$ & & $\begin{array}{l}20 \\
770\end{array}$ & & & \\
\hline
\end{tabular}

orksheet 9-Computation of Effect of Flared Minor street Approaches

\begin{tabular}{|c|c|c|c|c|c|c|}
\hline ovement & $\begin{array}{l}7 \\
\mathrm{~L}\end{array}$ & $\begin{array}{l}8 \\
\mathrm{~T}\end{array}$ & $\begin{array}{l}9 \\
R\end{array}$ & $\begin{array}{r}10 \\
\mathrm{~L}\end{array}$ & $\begin{array}{r}11 \\
\mathrm{~T}\end{array}$ & $\begin{array}{r}12 \\
\mathrm{R}\end{array}$ \\
\hline $\begin{array}{l}\text { s.ep } \\
\text { olume } \\
\text { elay } \\
\text { sep } \\
\text { sep }+1 \\
\text { ound (Qsep +1) }\end{array}$ & $\begin{array}{l}330 \\
15\end{array}$ & & $\begin{array}{l}770 \\
20\end{array}$ & & & \\
\hline $\begin{array}{l}\max \\
\text { sh } \\
\text { JM C sep } \\
\text { act }\end{array}$ & & & & & & \\
\hline
\end{tabular}

srksheet 10-Delay, Queue Length, and Level of Service

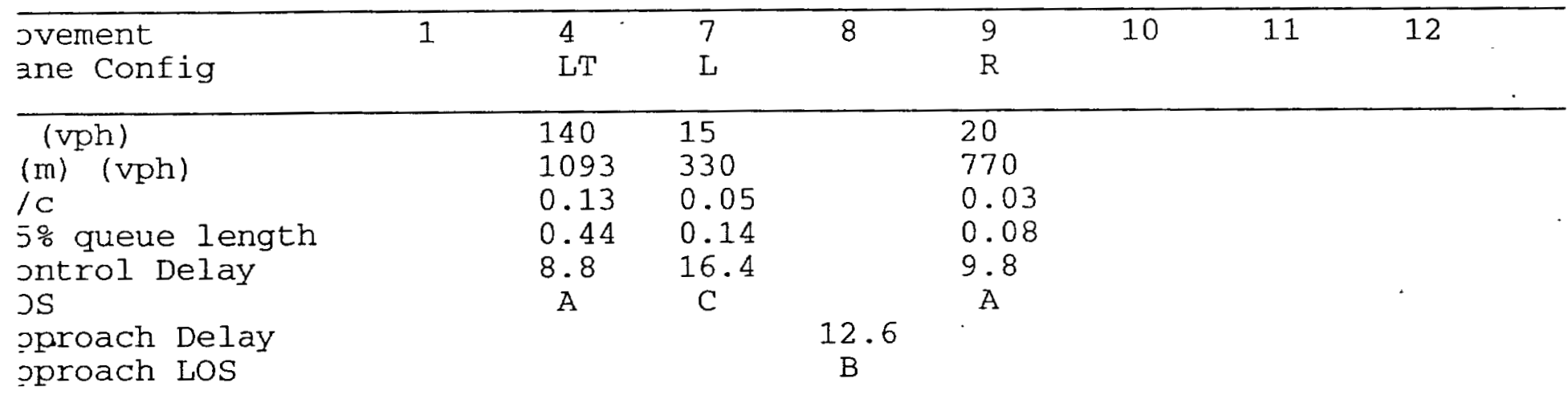

srksheet 11-Shared Major LT Impedance and Delay

Movement 2 Movement 5

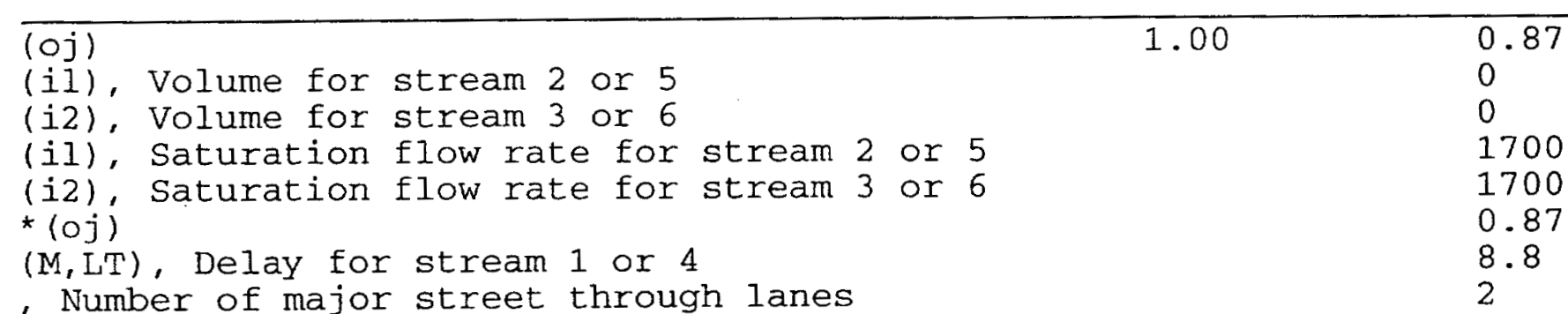

(rank, 1) Delay for stream 2 or 5 
Lalyst:

ency/Co.:

te Performed: 1/21/02

inalysis Time Period: AM Peak

- tersection:

East Ave \& S Gate (Location \#6)

risdiction:

malysis Year:

2001

Pxoject ID: Lawrence Livermore National Laboratory

i st/West street: East Ave

: rth/South Street: South Gate

( tersection Orientation: EW Study period (hrs): 1.00

Vehicle Volumes and Adjustments

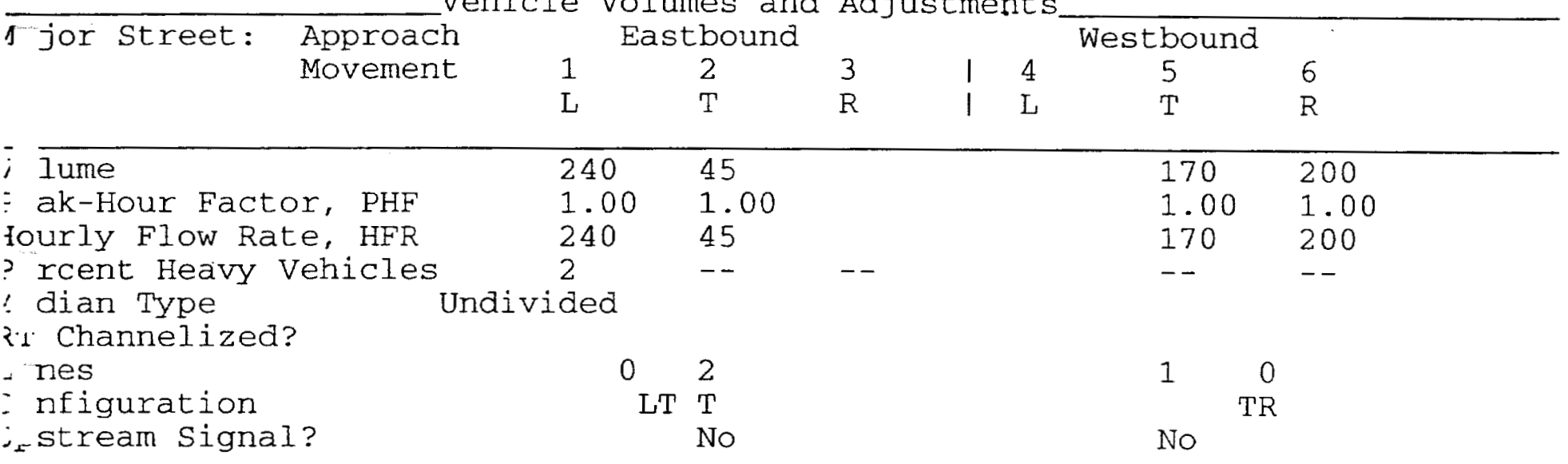

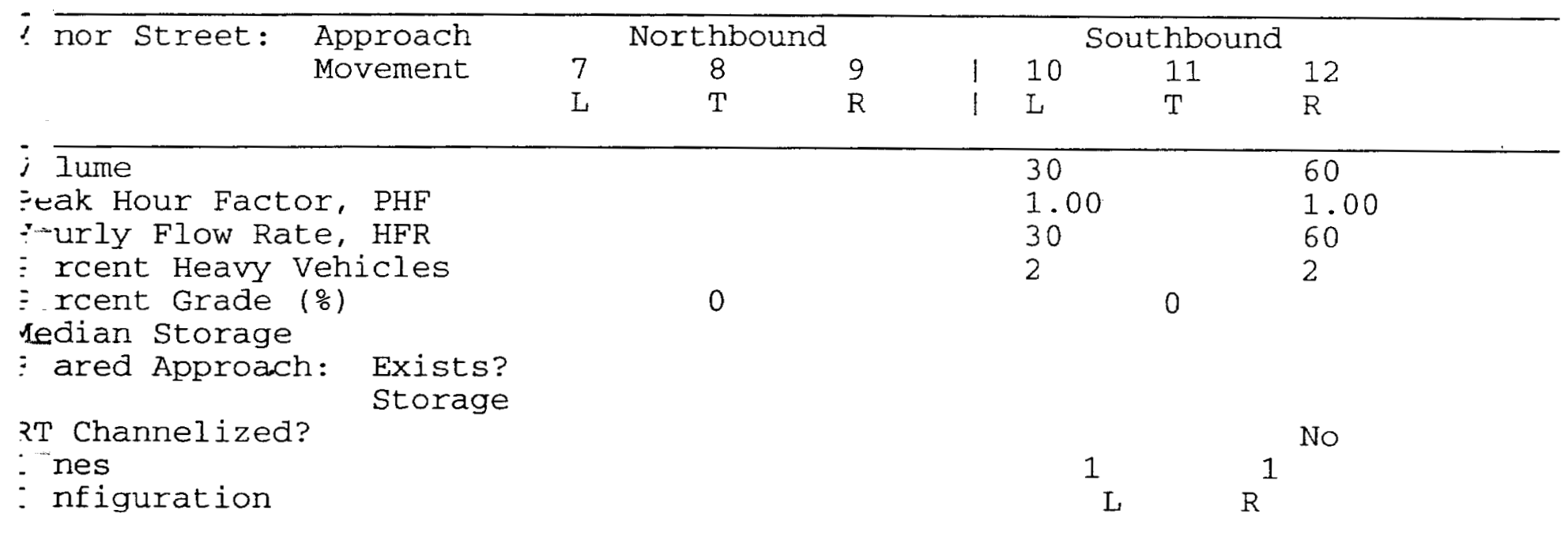

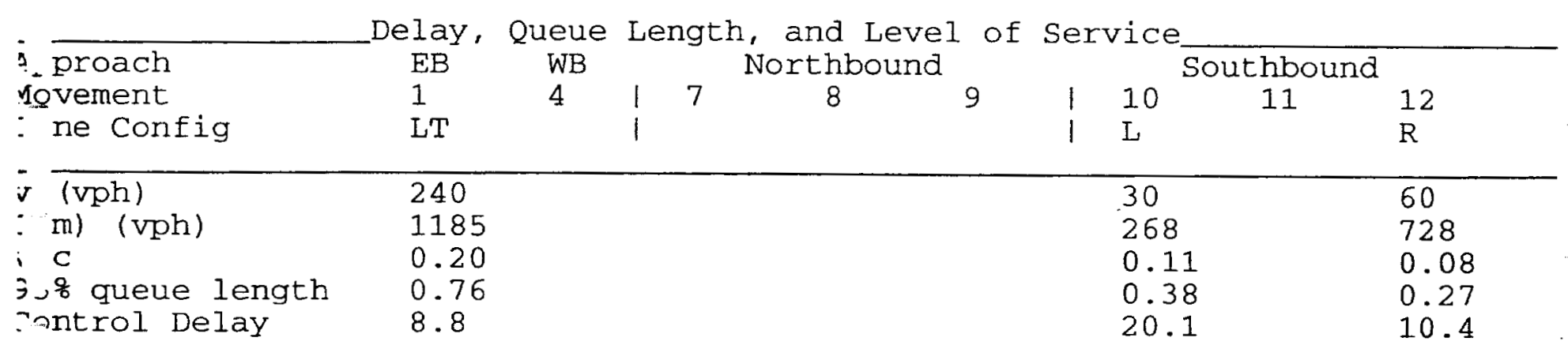


HCS2000: Unsignalized Intersections Release 4.1

orve Engineering, Inc.

hone:

-Mail:

Fax:

TWO-WAY STOP CONTROL (TWSC) ANALYSIS

nalyst :

gency/Co.:

ate Performed: 1/21/02

nalysis Time Period: AM Peak

ntersection:

East Ave \& $S$ Gate

urisdiction:

nalysis Year: 2001

roject ID: Lawrence Livermore National Laboratory

ast/West Street: East Ave

orth/South Street: South Gate

ntersection Orientation: EW

Study period (hrs): 1.00

Vehicle Volumes and Adjustments

$\begin{array}{llllllll}\text { ajor Street Movements } & 1 & 2 & 3 & 4 & 5 & 6 \\ & \mathrm{~L} & \mathrm{~T} & \mathrm{R} & \mathrm{L} & \mathrm{T} & \mathrm{R}\end{array}$

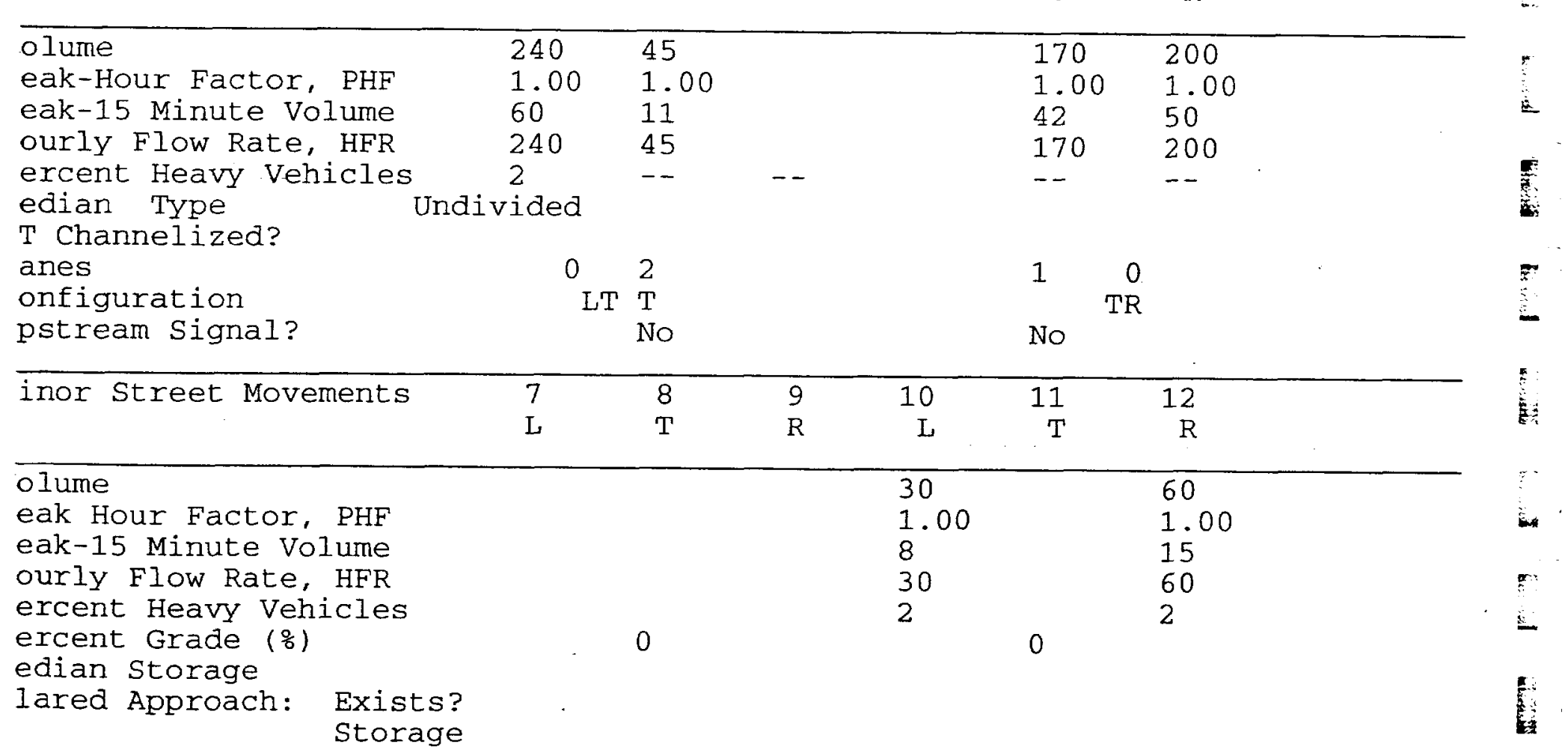

T Channelized? 
Pedestrian Volumes and Adjustments

\begin{tabular}{lllll}
\hline 1ovements & 13 & 14 & 15 & 16 \\
\hline Low (ped/hr) & & & & \\
Jane Width (ft) & 12.0 & 12.0 & 12.0 & 12.0 \\
alking Speed (ft/sec) & 4.0 & 4.0 & 4.0 & 4.0 \\
arcent Blockage & 0 & 0 & 0 & 0
\end{tabular}

\begin{tabular}{|c|c|c|c|c|c|c|c|c|}
\hline \multicolumn{9}{|c|}{ Dat } \\
\hline & & $\begin{array}{l}\text { Prog. } \\
\text { Flow } \\
\text { vph }\end{array}$ & $\begin{array}{l}\text { Sat } \\
\text { Flow } \\
\text { vph }\end{array}$ & $\begin{array}{c}\text { Arrival } \\
\text { Type }\end{array}$ & $\begin{array}{l}\text { Green } \\
\text { Time } \\
\text { sec }\end{array}$ & $\begin{array}{l}\text { Cycle } \\
\text { Length } \\
\text { sec }\end{array}$ & $\begin{array}{l}\text { Prog. } \\
\text { Speed } \\
\text { mph }\end{array}$ & $\begin{array}{c}\text { Distance } \\
\text { to Signal } \\
\text { feet }\end{array}$ \\
\hline 25 & $\begin{array}{l}\text { Left-Turn } \\
\text { Through } \\
\text { Left-Turn } \\
\text { Through }\end{array}$ & & & & & & & . \\
\hline
\end{tabular}

Irksheet 3-Data for Computing Effect of Delay to Major street Vehicles

\begin{tabular}{lll}
\hline & Movement 2 & Movement 5 \\
\hline suared In volume, major th vehicles: & 0 \\
'Tared In volume, major rt vehicles: & 0 \\
:t flow rate, major th vehicles: & 1700 & \\
$\vdots$ t flow rate, major rt vehicles: & 1700 & \\
jumber of major street through lanes: & 2 \\
\hline
\end{tabular}

vorksheet 4-Critical Gap and Follow-up Time Calculation

\begin{tabular}{|c|c|c|c|c|c|c|c|c|c|}
\hline $\begin{array}{l}\text { itical } \\
\text { suvement }\end{array}$ & Gap Cal & $\begin{array}{c}\text { culatj } \\
1 \\
L\end{array}$. & $\begin{array}{l}4 \\
\mathrm{~L}\end{array}$ & $\begin{array}{l}7 \\
L\end{array}$ & $\begin{array}{l}8 \\
\mathrm{~T}\end{array}$ & $\begin{array}{l}9 \\
\mathrm{R}\end{array}$ & $\begin{array}{r}10 \\
\mathrm{~L}\end{array}$ & $\begin{array}{r}11 \\
\mathrm{~T}\end{array}$ & $\begin{array}{r}12 \\
\mathrm{R}\end{array}$ \\
\hline $\begin{array}{l}\text { (c, base) } \\
=(c, h v) \\
\text { 'hv) } \\
=c, g) \\
\text { irade/100 } \\
\text { (3, lt) } \\
\text { c,T): } \\
-(c)\end{array}$ & $\begin{array}{l}\text { 1-stage } \\
\text { 2-stage } \\
1 \text {-stage } \\
\text { 2-stage }\end{array}$ & $\begin{array}{l}0.00 \\
0.00 \\
0.00 \\
4.1\end{array}$ & $\begin{array}{l}0.00 \\
0.00\end{array}$ & $\begin{array}{c}2.00 \\
. \\
0.20 \\
0.00 \\
0.00 \\
1.00\end{array}$ & $\begin{array}{l}2.00 \\
0.20 \\
0.00 \\
0.00 \\
1.00\end{array}$ & $\begin{array}{l}2.00 \\
0.10 \\
0.00 \\
0.00 \\
0.00\end{array}$ & $\begin{array}{l}7.5 \\
2.00 \\
2 \\
0.20 \\
0.00 \\
0.70 \\
0.00 \\
1.00 \\
6.8\end{array}$ & $\begin{array}{l}2.00 \\
0.20 \\
0.00 \\
0.00 \\
1.00\end{array}$ & $\begin{array}{l}6.9 \\
2.00 \\
2 \\
0.10 \\
0.00 \\
0.00 \\
0.00 \\
0.00 \\
6.9\end{array}$ \\
\hline $\begin{array}{l}\text { Sollow-Up } \\
\text { ivement }\end{array}$ & Time $\mathrm{C}$ & $\begin{array}{c}\operatorname{alcula} \\
1 \\
L\end{array}$ & $\begin{array}{c}\text { Lons } \\
4 \\
\text { L }\end{array}$ & $\begin{array}{l}7 \\
\mathrm{~L}\end{array}$ & $\begin{array}{l}8 \\
\mathrm{~T}\end{array}$ & $\begin{array}{l}9 \\
\mathrm{R}\end{array}$ & $\begin{array}{r}10 \\
\mathrm{~L}\end{array}$ & $\begin{array}{r}11 \\
\mathrm{~T}\end{array}$ & $\begin{array}{r}12 \\
\mathrm{R}\end{array}$ \\
\hline $\begin{array}{l}\text { (f,base) } \\
f, H V) \\
\vdots \text { (HV) } \\
(f)\end{array}$ & & $\begin{array}{l}2.20 \\
1.00 \\
2 \\
2.2\end{array}$ & 1.00 & 1.00 & 1.00 & 1.00 & $\begin{array}{l}3.50 \\
1.00 \\
2 \\
3.5\end{array}$ & 1.00 & $\begin{array}{l}3.30 \\
1.00 \\
2 \\
3.3\end{array}$ \\
\hline
\end{tabular}


Jrksheet 5-Effect of Upstream Signals

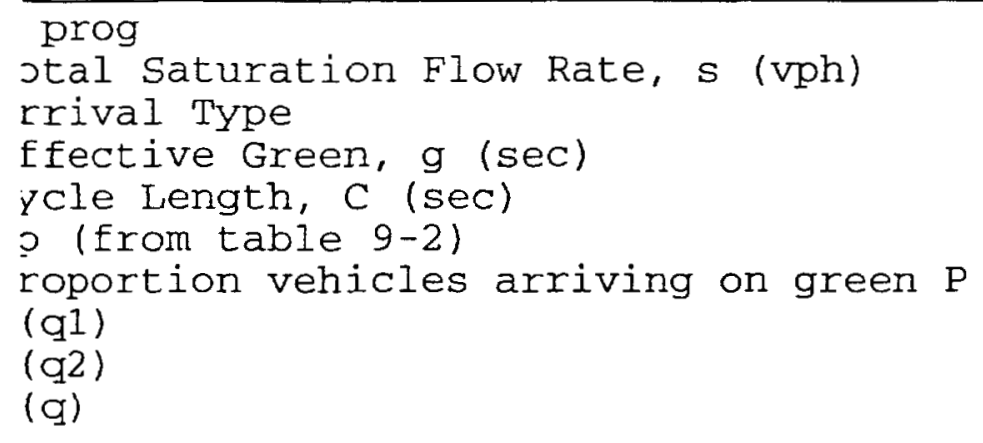

(subo)

onstrained or unconstrained?

\section{roportion}

nblocked

or minor

ovements, $\mathrm{p}(\mathrm{x})$
(2)

Single-stage . Two-Stage Process

Process Stage I Stage II
(1)
(4)
(7)
(8)
(9)
(11)
(12)

omputation 4 and 5 ingle-stage Process ovement

\begin{tabular}{rrrrrrrr}
1 & 4 & 7 & 8 & 9 & 10 & 11 & 12 \\
$\mathrm{~L}$ & $\mathrm{~L}$ & $\mathrm{~L}$ & $\mathrm{~T}$ & $\mathrm{R}$ & $\mathrm{L}$ & $\mathrm{T}$ & $\mathrm{R}$ \\
\hline
\end{tabular}


$c, u, x$

$r, x$

plat, $x$

No-Stage Process

stage1 ${ }^{7}$ stage2 stage1 ${ }^{8}$ stage2 stage1 $^{10}$ stage2 stagel $^{11}$ stage2

$(\mathrm{C}, \mathrm{x})$

$\mathrm{x})$

3400

$r(\mathrm{c}, \mathrm{u}, \mathrm{x})$

$r, x)$

$:($ plat,$x)$

-rksheet 6-Impedance and Capacity Equations

\begin{tabular}{llll}
\hline ep 1: RT from Minor St. & 9 & 12
\end{tabular}

jonflicting Flows

: tential Capacity

destrian Impedance Factor

luvement Capacity

:-sobability of Queue free st.

270

728

$1.00 \quad 1.00$

$\begin{array}{ll}1.00 & 728 \\ 1.00 & 0.92\end{array}$

; ep 2: LT from Major St.

4

: nflicting Flows

: tential Capacity

sedestrian Impedance Factor

- vement Capacity

: obability of Queue free st.

raj L-Shared Prob Q free st.

$\begin{array}{ll} & 370 \\ .00 & 1185 \\ & 1.00 \\ .00 & 1185 \\ & 0.80 \\ & 0.80\end{array}$

\begin{tabular}{|c|c|c|}
\hline ep 3: TH from Minor st. & 8 & 11 \\
\hline $\begin{array}{l}\text { Oonflicting Flows } \\
\text { tential Capacity } \\
\text { destrian Impedance Factor } \\
\text { :ap. Adj. factor due to Impeding mumnt } \\
\text { "vement Capacity } \\
\text { obability of Queue free st. }\end{array}$ & $\begin{array}{l}1.00 \\
0.80 \\
1.00\end{array}$ & $\begin{array}{l}1.00 \\
0.80 \\
1.00\end{array}$ \\
\hline tep 4: LT from Minor st. & 7 & 10 \\
\hline $\begin{array}{l}\text { nflicting Flows } \\
\text { potential Capacity } \\
\text { destrian Impedance Factor } \\
\text { j. L, Min T Impedance factor } \\
\text { aj. L, Min T Adj. Imp Factor. } \\
\text { p. Adj. factor due to Impeding mvmnt } \\
\text { vement Capacity }\end{array}$ & $\begin{array}{l}1.00 \\
0.80 \\
0.84 \\
0.77\end{array}$ & $\begin{array}{l}772 \\
336 \\
1.00\end{array}$ \\
\hline
\end{tabular}


Jrksheet 7-Computation of the Effect of Two-stage Gap Acceptance

zep 3: TH from Minor st. $\quad 8 \quad 811$

zrt 1 - First Stage

onflicting Flows

tential Capacity

edestrian Impedance Factor

ap. Adj. factor due to Impeding mvmnt

svement Capacity

robability of Queue free St.

art 2 - Second stage

onflicting Flows

stential Capacity

edestrian Impedance Factor

ap. Adj. factor due to Impeding mvmnt

svement Capacity

art 3 - Single stage

onflicting Flows

stential Capacity

edestrian Impedance Factor

ap. Adj. factor due to Impeding mumnt

1.00

0.80

1.00

ovement Capacity

esult for 2 stage process:

$t$

robability of Queue free st.

1.00

1.00

tep 4: LT from Minor st.

7

10

art 1 - First stage

onflicting Flows

otential Capacity

edestrian Impedance Factor

ap. Adj. factor due to Impeding mvmnt

ovement Capacity

art 2 - Second stage

onflicting Flows

otential Capacity

edestrian Impedance Factor

ap. Adj. factor due to Impeding mumnt

ovement Capacity

art 3 - Single stage

onflicting Flows

otential Capacity

772

edestrian Impedance Factor

336

aj. L, Min T Impedance factor

aj. L, Min T Adj. Imp Factor.

ap. Adj. factor due to Impeding mvmnt

ovement Capacity

1.00

1.00

0.80

0.84

$\begin{array}{ll}0.77 & 0.80\end{array}$

esults for Two-stage process:

268 
Jrksheet 8-Shared Lane Calculations

\begin{tabular}{|c|c|c|c|c|c|c|}
\hline Tovement & $\begin{array}{l}7 \\
\mathrm{~L}\end{array}$ & $\begin{array}{l}8 \\
\mathrm{~T}\end{array}$ & $\begin{array}{l}9 \\
\mathrm{R}\end{array}$ & $\begin{array}{r}10 \\
\mathrm{~L}\end{array}$ & $\begin{array}{r}11 \\
\mathrm{~T}\end{array}$ & $\begin{array}{r}12 \\
\mathrm{R}\end{array}$ \\
\hline $\begin{array}{l}\text { Jolume (vph) } \\
\text { jvement Capacity (vph) } \\
\text { lared Lane Capacity (vph) }\end{array}$ & & & & $\begin{array}{l}30 \\
268\end{array}$ & & $\begin{array}{l}60 \\
728\end{array}$ \\
\hline
\end{tabular}

srksheet 9-Computation of Effect of Flared Minor Street Approaches

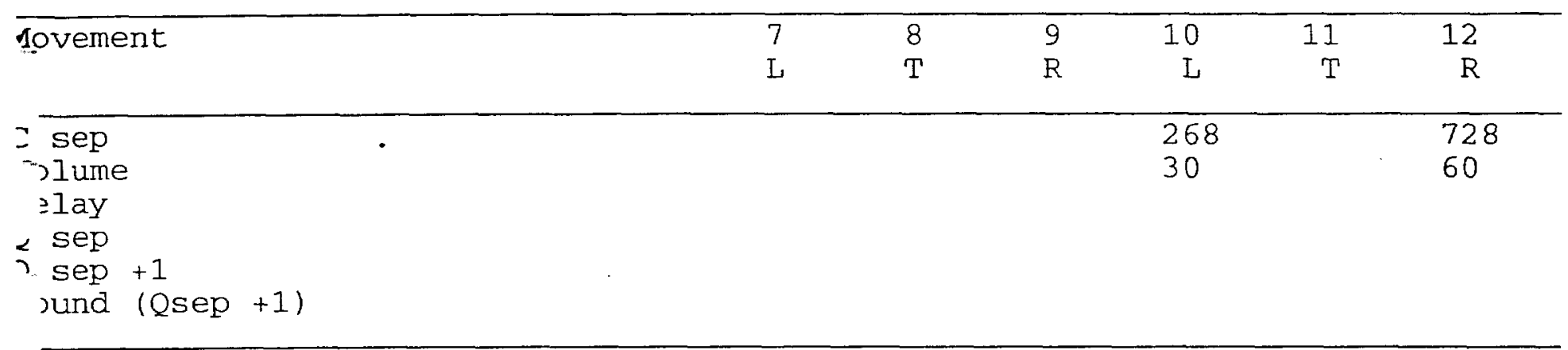

\footnotetext{
I $\max$

sh

JM C sep

.

act
}

Norksheet 10-Delay, Queue Length, and Level of Service

\begin{tabular}{|c|c|c|c|c|c|c|c|c|}
\hline $\begin{array}{l}\text { Jvement } \\
\text { Lane Config }\end{array}$ & $\begin{array}{l}1 \\
\mathrm{LT}\end{array}$ & 4 & 7 & 8 & 9 & $\begin{array}{r}10 \\
\mathrm{~L}\end{array}$ & 11 & $\begin{array}{r}12 \\
\mathrm{R}\end{array}$ \\
\hline $\begin{aligned} & \text { (vph) } \\
& \text { (m) (vph) } \\
& \text { / } / \mathrm{C} \\
& \text {;\% queue length } \\
& \text { ontrol Delay } \\
& \text { sOS } \\
& \text { sproach Delay. } \\
& \text { sproach LOS }\end{aligned}$ & $\begin{array}{l}240 \\
1185 \\
0.20 \\
0.76 \\
8.8 \\
A\end{array}$ & & & & & $\begin{array}{l}30 \\
268 \\
0.11 \\
0.38 \\
20.1 \\
C\end{array}$ & $\begin{array}{r}13 \\
\mathrm{~B}\end{array}$ & $\begin{array}{l}60 \\
728 \\
0.08 \\
0.27 \\
10.4 \\
B\end{array}$ \\
\hline
\end{tabular}

srksheet 11-Shared Major LT Impedance and Delay

\begin{tabular}{|c|c|c|}
\hline & Movement 2 & Movement 5 \\
\hline $\begin{array}{l}\text { (oj) } \\
\text { v(il), Volume for stream } 2 \text { or } 5 \\
\quad(i 2) \text {, Volume for stream } 3 \text { or } 6 \\
\quad(i l) \text {, Saturation flow rate for stream } 2 \text { or } 5 \\
\text { s(i2), Saturation flow rate for stream } 3 \text { or } 6 \\
\quad *(o j) \text {. } \\
\quad(M, L T) \text {, Delay for stream } 1 \text { or } 4 \\
\text { iv, Number of major street through lanes } \\
\text { d(rank, 1) Delay for stream } 2 \text { or } 5\end{array}$ & $\begin{array}{l}0.80 \\
0 \\
0 \\
1700 \\
1700 \\
0.80 \\
8.8 \\
2\end{array}$ & 1.00 \\
\hline
\end{tabular}


unalyst :

jency/Co.:

ite Performed:

$1 / 21 / 02$

inalysis Time Period: PM Peak

itersection:

East Ave \& S Gate (Location \#6)

irisdiction:

walysis Year:

2001

rroject ID: Lawrence Livermore National Laboratory

ist/West Street: East Ave

srth/South Street: South Gate

itersection Orientation: EW

Study period (hrs): 1.00

Vehicle Volumes and Adjustments

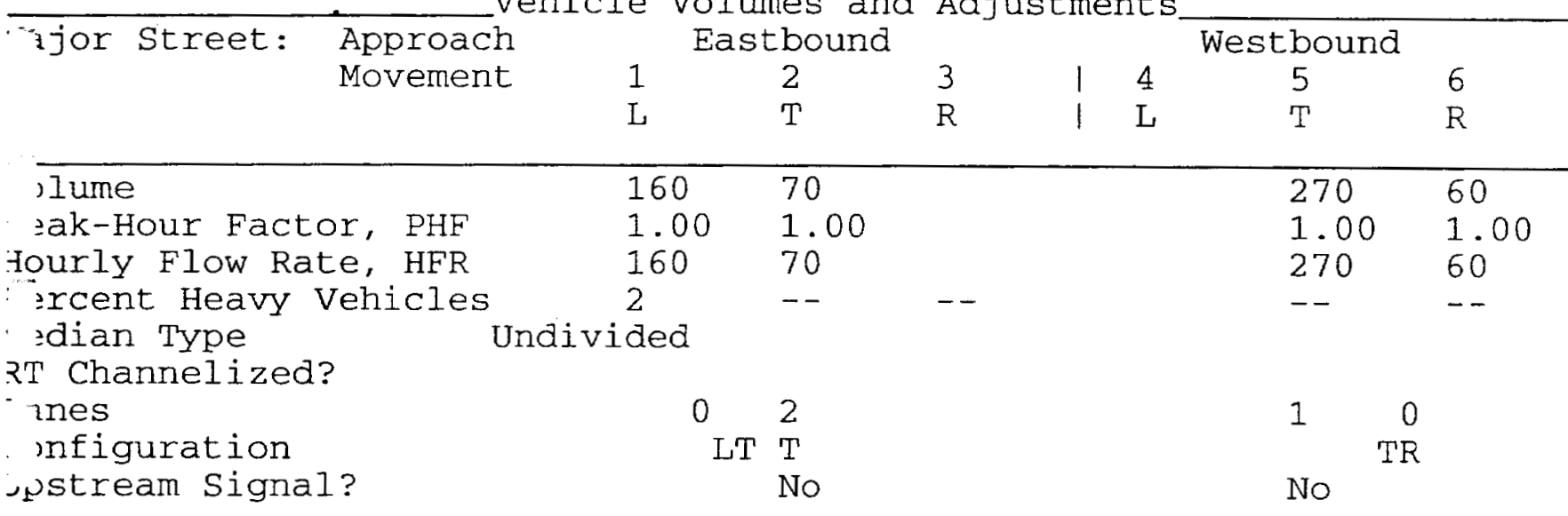

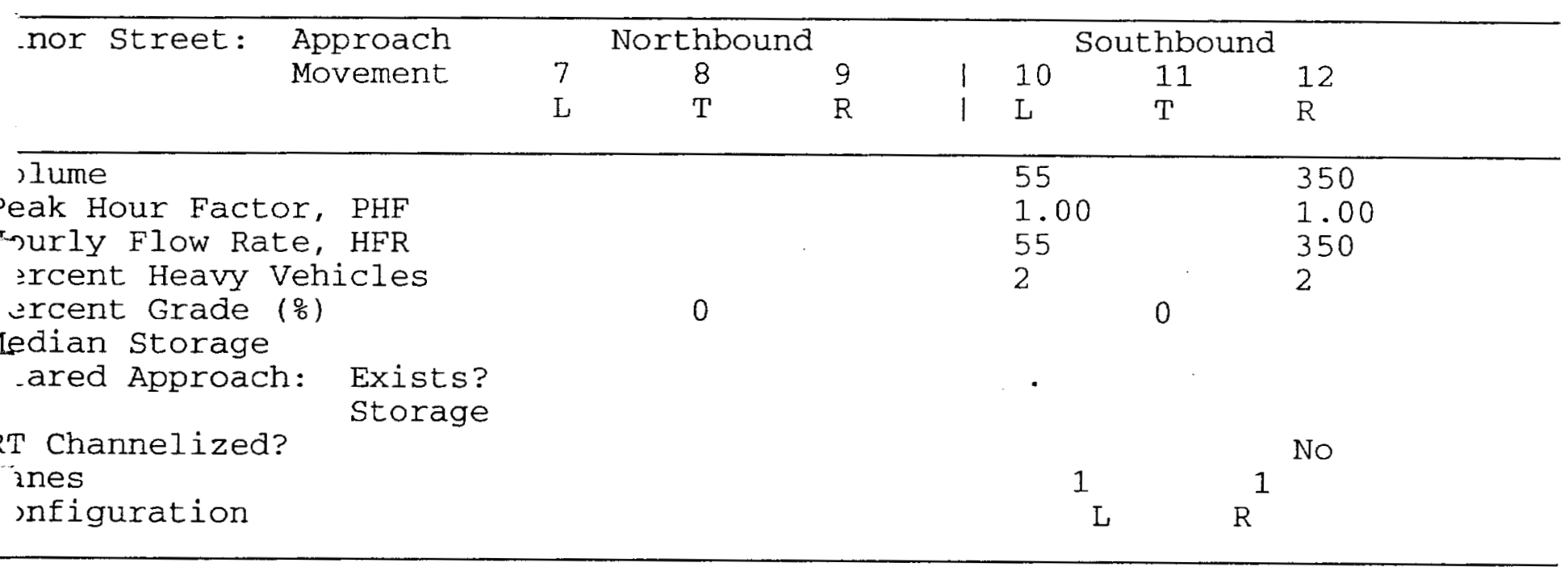

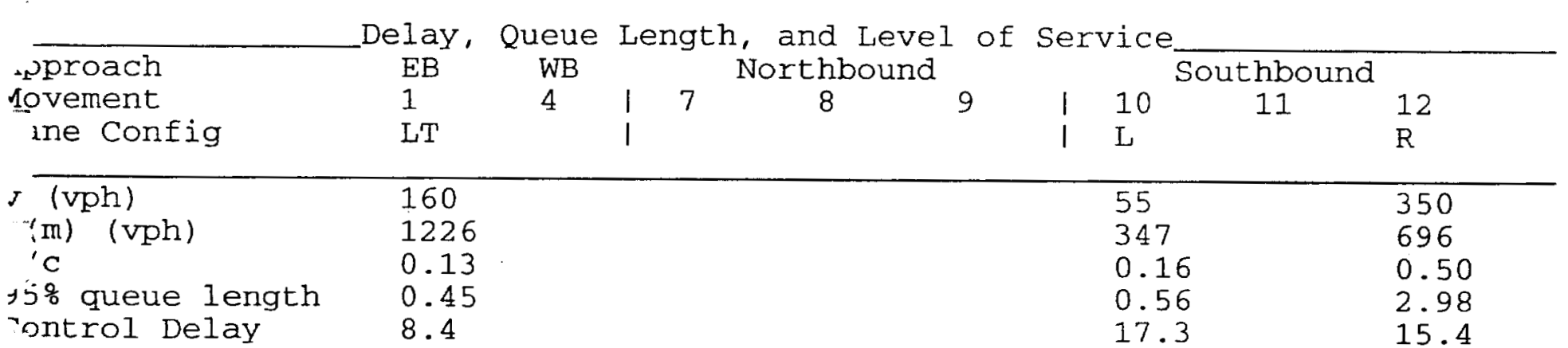


HCS2000: Unsignalized Intersections Release 4.1

orve Engineering, Inc.

hone:

Fax:

-Mail: TWO-WAY STOP CONTROL (TWSC) ANALYSIS

nalyst :

Jency/Co.:

ate Performed: 1/21/02

nalysis Time Period: PM Peak

ntersection: East Ave \& S Gate

urisdiction:

lalysis Year: 2001

roject ID: Lawrence Livermore National Laboratory

ast/West Street: East Ave

srth/South Street: South Gate

atersection Orientation: EW

study period (hrs): 1.00

Vehicle Volumes and Adjustments

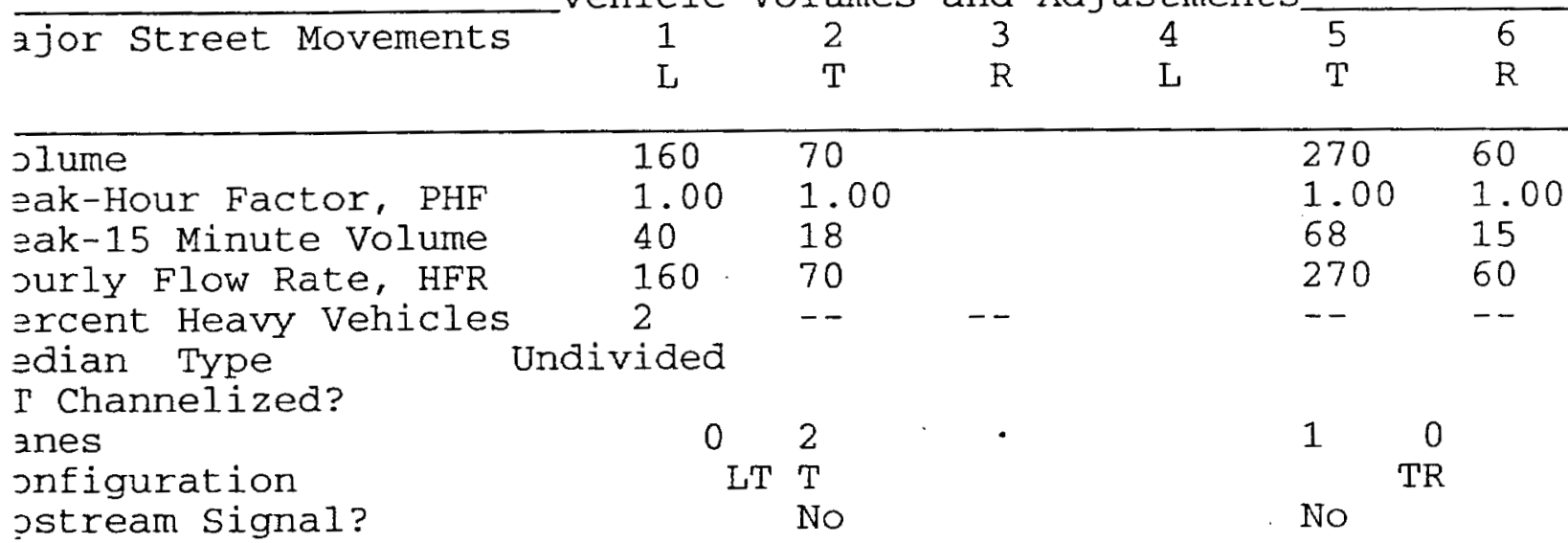

\begin{tabular}{|c|c|c|c|c|c|c|}
\hline inor Street Movements & $\begin{array}{l}7 \\
L\end{array}$ & $\begin{array}{l}8 \\
T\end{array}$ & $\begin{array}{l}9 \\
\mathrm{R}\end{array}$ & $\begin{array}{r}10 \\
\mathrm{~L}\end{array}$ & $\begin{array}{r}11 \\
\mathrm{~T}\end{array}$ & $\begin{array}{r}12 \\
\mathrm{R}\end{array}$ \\
\hline $\begin{array}{l}\text { Jlume } \\
\text { eak Hour Factor, PHF } \\
\text { əak-15 Minute Volume } \\
\text { surly Flow Rate, HFR } \\
\text { ərcent Heavy Vehicles } \\
\text { ercent Grade (\%) }\end{array}$ & & 0 & & $\begin{array}{l}55 \\
1.00 \\
14 \\
55 \\
2\end{array}$ & 0 & $\begin{array}{l}350 \\
1.00 \\
88 \\
350 \\
2\end{array}$ \\
\hline
\end{tabular}

sian storage

lared Approach: Exists?

T Channelized? 
Pedestrian Volumes and Adjustments

\begin{tabular}{lllll}
\hline 1ovements & 13 & 14 & 15 & 16 \\
\hline ow (ped/hr) & & & & \\
sane Width (ft) & 12.0 & 12.0 & 12.0 & 12.0 \\
l.lking Speed (ft/sec) & 4.0 & 4.0 & 4.0 & 4.0 \\
rcent Blockage & 0 & 0 & 0 & 0
\end{tabular}

\begin{tabular}{|c|c|c|c|c|c|c|c|c|}
\hline \multicolumn{9}{|c|}{ Upstream Signal Data } \\
\hline & & $\begin{array}{l}\text { Prog. } \\
\text { Flow } \\
\text { vph }\end{array}$ & $\begin{array}{l}\text { Sat } \\
\text { Flow } \\
\text { vph }\end{array}$ & $\begin{array}{l}\text { Arrival } \\
\text { Type }\end{array}$ & $\begin{array}{l}\text { Green } \\
\text { Time } \\
\text { sec }\end{array}$ & $\begin{array}{l}\text { Cycle } \\
\text { Length } \\
\text { sec }\end{array}$ & $\begin{array}{l}\text { Prog. } \\
\text { Speed } \\
\text { mph }\end{array}$ & $\begin{array}{c}\text { Distance } \\
\text { to Signal } \\
\text { feet }\end{array}$ \\
\hline 32 & $\begin{array}{l}\text { Left-Turn } \\
\text { Through } \\
\text { Left-Turn } \\
\text { Through }\end{array}$ & & & & $\cdot$ & & & \\
\hline
\end{tabular}

- rksheet 3-Data for Computing Effect of Delay to Major Street Vehicles

\begin{tabular}{lll}
\hline & Movement 2 & Movement 5 \\
\hline inared ln volume, major th vehicles: & 0 \\
"lared ln volume, major rt vehicles: & 0 \\
$t$ flow rate, major th vehicles: & 1700 & \\
i.t flow rate, major rt vehicles: & 1700 & \\
jumber of major street through lanes: & 2 & \\
\hline
\end{tabular}

Jorksheet 4-Critical Gap and Follow-up Time Calculation

\begin{tabular}{|c|c|c|c|c|c|c|c|c|c|}
\hline 1ovement & & $\begin{array}{l}1 \\
\mathrm{~L}\end{array}$ & $\begin{array}{l}4 \\
\mathrm{~L}\end{array}$ & $\begin{array}{l}7 \\
\mathrm{~L}\end{array}$ & $\begin{array}{l}8 \\
T\end{array}$ & $\begin{array}{l}9 \\
\mathrm{R}\end{array}$ & $\begin{array}{r}10 \\
\mathrm{~L}\end{array}$ & $\begin{array}{r}11 \\
\mathrm{~T}\end{array}$ & $\begin{array}{r}12 \\
\mathrm{R}\end{array}$ \\
\hline 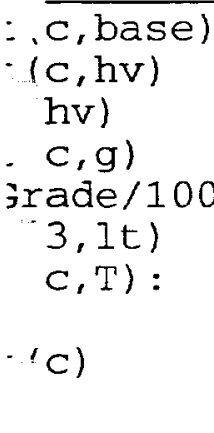 & $\begin{array}{l}1 \text {-stage } \\
2 \text {-stage } \\
1 \text {-stage } \\
2 \text {-stage }\end{array}$ & $\begin{array}{l}0.00 \\
0.00 \\
0.00 \\
4.1\end{array}$ & $\begin{array}{l}0.00 \\
0.00\end{array}$ & $\begin{array}{l}2.00 \\
0.20 \\
0.00 \\
0.00 \\
1.00\end{array}$ & $\begin{array}{l}2.00 \\
0.20 \\
0.00 \\
0.00 \\
1.00\end{array}$ & $\begin{array}{l}2.00 \\
0.10 \\
0.00 \\
0.00 \\
0.00\end{array}$ & $\begin{array}{l}7.5 \\
2.00 \\
2 \\
0.20 \\
0.00 \\
0.70 \\
0.00 \\
1.00 \\
6.8\end{array}$ & $\begin{array}{l}2.00 \\
0.20 \\
0.00 \\
0.00 \\
1.00\end{array}$ & $\begin{array}{l}6.9 \\
2.00 \\
2 \\
0.10 \\
0.00 \\
0.00 \\
0.00 \\
0.00 \\
6.9\end{array}$ \\
\hline $\begin{array}{l}\text { Pollow-Up } \\
\text { vement }\end{array}$ & Time $\mathrm{Ca}$ & $\begin{array}{l}\operatorname{arcula} \\
1 \\
\mathrm{~L}\end{array}$ & $\begin{array}{l}\text { Lons } \\
4 \\
L\end{array}$ & $\begin{array}{l}7 \\
L\end{array}$ & $\begin{array}{l}8 \\
\mathrm{~T}\end{array}$ & $\begin{array}{l}9 \\
\mathrm{R}\end{array}$ & $\begin{array}{r}10 \\
\mathrm{~L}\end{array}$ & $\begin{array}{r}11 \\
\mathrm{~T}\end{array}$ & $\begin{array}{r}12 \\
\mathrm{R}\end{array}$ \\
\hline $\begin{aligned} & \mathrm{f}, \text { base) } \\
& \mathrm{f}, \mathrm{HV}) \\
= & (\mathrm{HV}) \\
& (\mathrm{f})\end{aligned}$ & & $\begin{array}{l}2.20 \\
1.00 \\
2 \\
2.2\end{array}$ & 1.00 & 1.00 & 1.00 & 1.00 & $\begin{array}{l}3.50 \\
1.00 \\
2 \\
3.5\end{array}$ & 1.00 & $\begin{array}{l}3.30 \\
1.00 \\
2 \\
3.3\end{array}$ \\
\hline
\end{tabular}


orksheet 5-Effect of Upstream Signals

$\begin{array}{rr}\text { omputation 1-Queue Clearance Time at Upstream Signal } & \text { Movement } 5 \\ \text { Movement } 2 & V(t) \quad V(1, p r o t) \\ & V(t) \quad V(1, p r o t)\end{array}$

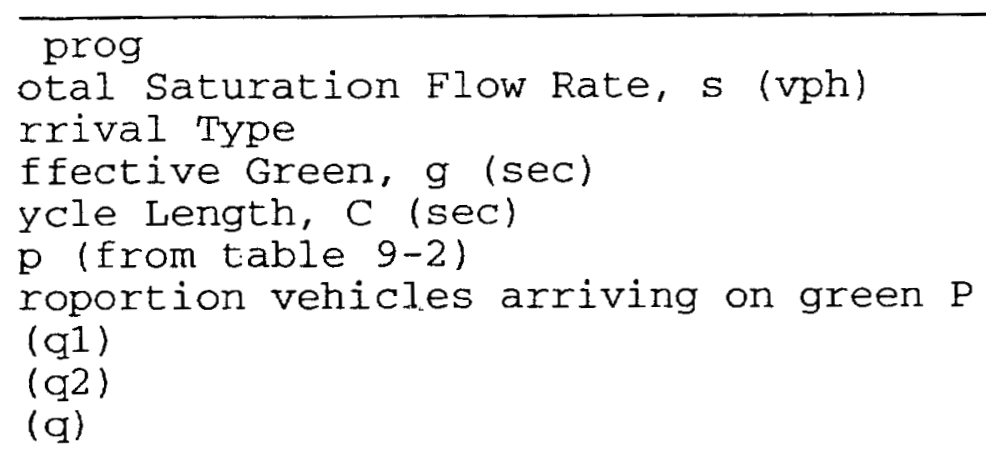

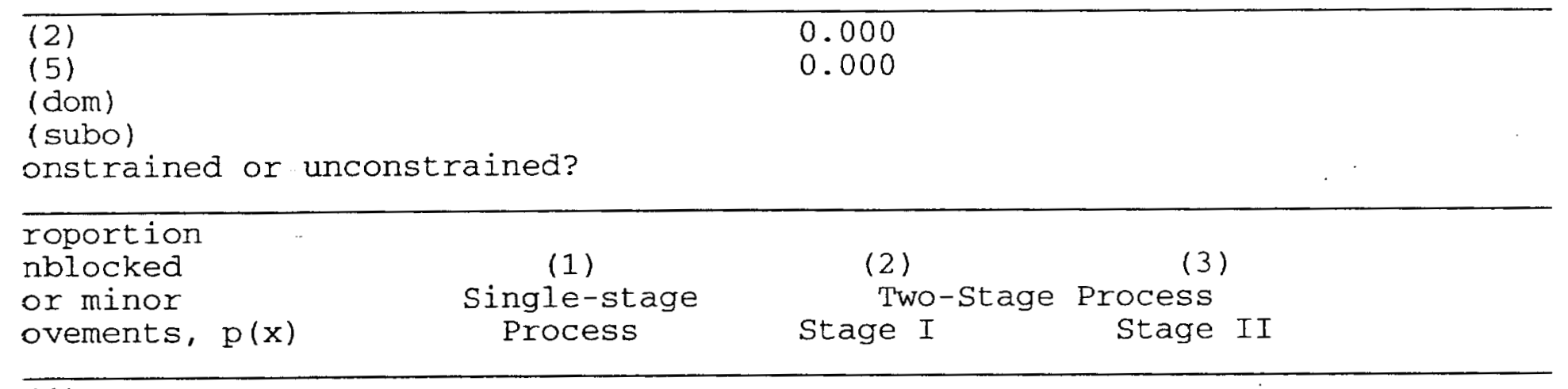
(1)
(4)
(7)
(8)
(9)
(10)
(11)
(12)

omputation 4 and 5

ingle-stage Process ovement

\begin{tabular}{ll}
4 & 7 \\
\hline &
\end{tabular}

8

$9 \quad 10$


PY.

$\mathrm{C}, \mathrm{u}, \mathrm{x}$

$r, x$

plat, $x$

wo-Stage Process

Stage1 ${ }^{7}$ stage2 stage1 ${ }^{8}$ stage2 stage $^{10}$ stage2 stagel $^{11}$ stage2

$r(\mathrm{C}, \mathrm{X})$

$x)$

$J(\mathrm{c}, \mathrm{u}, \mathrm{x})$

$(x, x)$

$\because($ plat, $\mathrm{x})$

:urksheet 6-Impedance and Capacity Equations

\begin{tabular}{lcl}
\hline ep 1: RT from Minor St. & 9 & 12 \\
\hline :onflicting Flows & & 300 \\
$\vdots$ tential Capacity & 696 \\
destrian Impedance Factor & 1.00 & 1.00 \\
novement Capacity & 696 \\
:-obability of Queue free St. & 1.00 & 0.50 \\
:-ep 2: LT from Major St. & 4 & 1 \\
$\vdots$ nflicting Flows & 1.00 & 330 \\
$\vdots$ tential Capacity & & 1226 \\
;edestrian Impedance Factor & 1.00 & 1.00 \\
$\vdots$ vement Capacity & & 0.87 \\
labj L-Shared Prob Q free St. & & 0.87
\end{tabular}

$\vdots$ ep 3: TH from Minor St.

8

11

zonflicting Flows

tential Capacity

destrian Impedance Factor

:ap. Adj. factor due to Impeding mumnt

3400

-vement Capacity

obability of Queue free St.

$\begin{array}{ll}1.00 & 1.00 \\ 0.87 & 0.87\end{array}$

沬ep 4: LT from Minor st.

$1.00 \quad 1.00$

nflicting Flows

sotential Capacity

destrian Impedance Factor

j. L, Min T Impedance factor

1aj. L, Min T Adj. Imp Factor.

- p. Adj. factor due to Impeding mumnt

vement capacity

$7 \quad 10$

1.00

0.87

0.90

0.45

399

1.00

0.87

347 
orksheet 7-Computation of the Effect of Two-stage Gap Acceptance

\begin{tabular}{|c|c|}
\hline tep 3: TH from Minor St. & 11 \\
\hline $\begin{array}{l}\text { art } 1 \text { - First Stage } \\
\text { onflicting Flows } \\
\text { otential Capacity } \\
\text { edestrian Impedance Factor } \\
\text { ap. Adj. factor due to Impeding mumnt } \\
\text { ovement Capacity } \\
\text { robability of Queue free st. }\end{array}$ & \\
\hline
\end{tabular}

art 2 - Second stage

onflicting Flows

otential Capacity

edestrian Impedance Factor

ap. Adj. factor due to Impeding mvmnt

ovement Capacity

art 3 - single stage

onflicting Flows

otential Capacity

edestrian Impedance Factor

ap. Adj. factor due to Impeding mvmnt

1.00

1.00

ovement Capacity

esult for 2 stage process:

$t$

robability of Queue free st.

1.00

1.00

tep 4: LT from Minor St.

7

10

art 1-First stage

onflicting Flows

otential Capacity

edestrian Impedance Factor

ap. Adj. factor due to Impeding mvmnt

ovement Capacity

art 2 - Second stage

onflicting Flows

otential Capacity

edestrian Impedance Factor

ap. Adj. factor due to Impeding mvmnt

ovement Capacity

art 3 - Single stage

onflicting Flows

otential Capacity

edestrian Impedance Factor

əj. I, Min T Impedance factor

aj. L, Min T Adj. Imp Factor.

ap. Adj. factor due to Impeding mvmnt

ovement Capacity

$\begin{array}{ll} & 655 \\ 1.00 & 399 \\ 0.87 & 1.00 \\ 0.90 & \\ 0.45 & 0.87 \\ & 347\end{array}$

esults for Two-stage process: 
HCS2000: Unsignalized Intersections Release 4.1

TWO-WAY STOP CONTROL SUMMARY

nalyst:

gency/Co.:

ate Performed: 1/21/02

nalysis Time Period: PM Peak

ntersection:

East Ave \& E Entrance (Locaticn \# 8)

urisdiction:

nalysis Year: 2001

roject ID: Lawrence Livermore National Laboratory

ast/West Street: East Ave

orth/South Street: East Entrance

ntersection Orientation: EW Study period (hrs): 1.00

Vehicle Volumes and Adjustments.

ajor street: Approach Eastbound Westbound

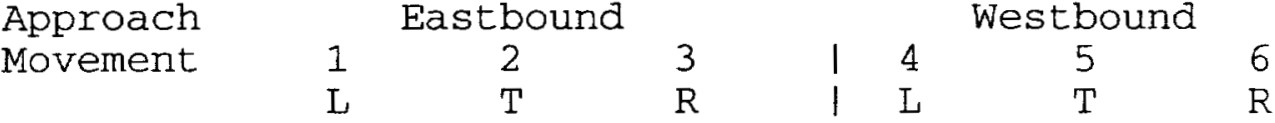

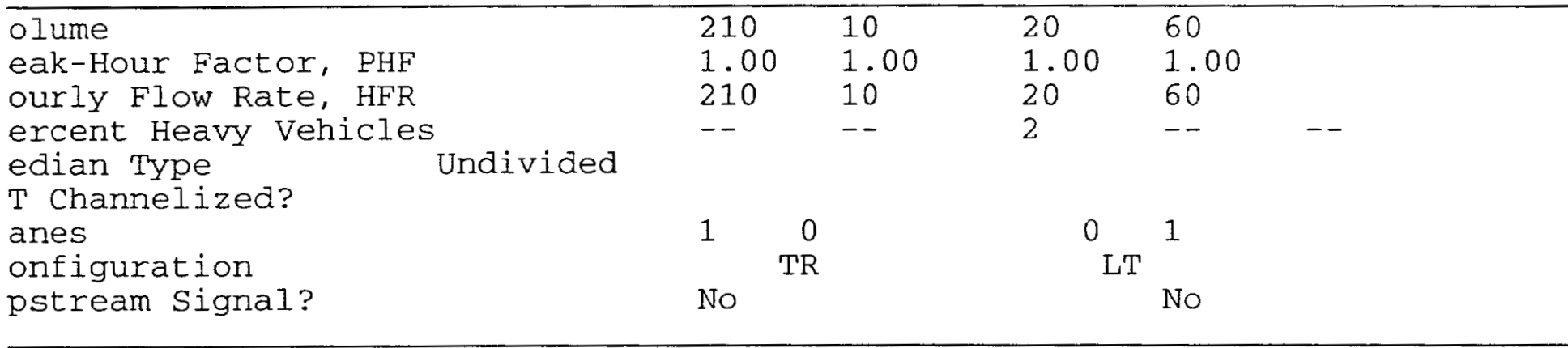

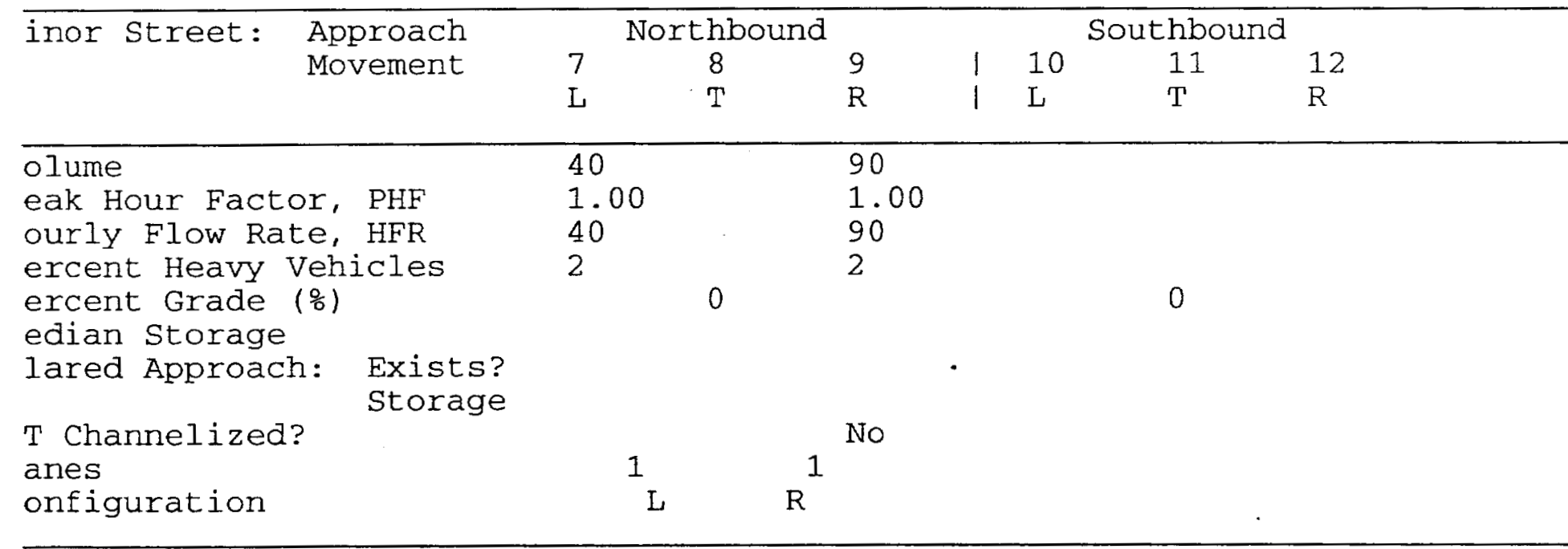

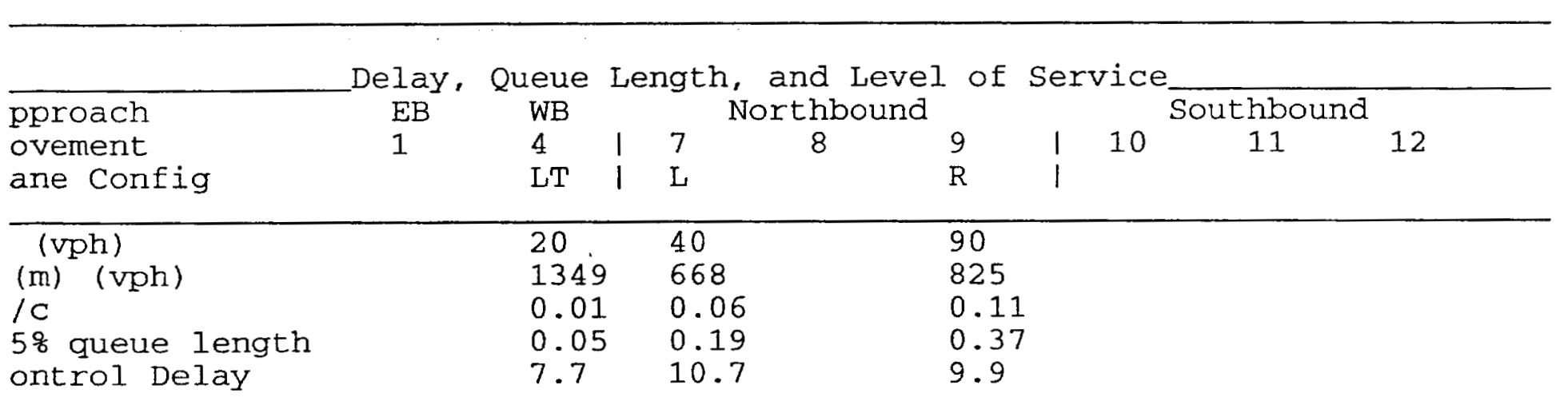


HCS2000: Unsignalized Intersections Release 4.1

rve Engineering, Iric.

. lone:

Fax :

I-Mail:

TWO-WAY STOP CONTROL (TWSC) ANALYSIS

- alyst:

,ency/Co.:

Jate Performed: 1/21/02

- alysis Time Period: PM Peak

tersection:

East Ave \& E Entrance

- drisdiction:

inalysis Year:

oject ID: Lawrence Livermore National Laboratory

ist/West Street:

vorth/South Street:

East Ave

East Entrance

tersection orientation: EW

study period (hrs): 1.00

Vehicle Volumes and Adjustments

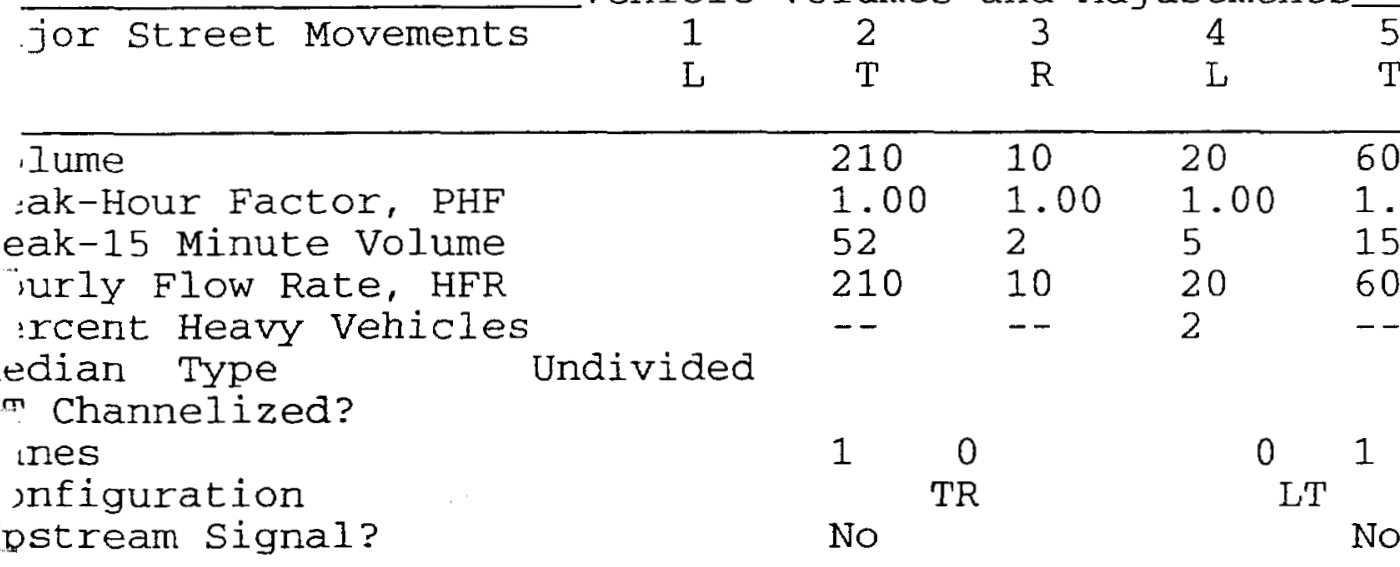

\begin{tabular}{|c|c|c|c|c|c|c|}
\hline nor street Movements & $\begin{array}{l}7 \\
\mathrm{~L}\end{array}$ & $\begin{array}{l}8 \\
\mathrm{~T}\end{array}$ & $\begin{array}{l}9 \\
R\end{array}$ & $\begin{array}{r}10 \\
\mathrm{~L}\end{array}$ & $\begin{array}{r}11 \\
T\end{array}$ & $\begin{array}{r}12 \\
\mathrm{R}\end{array}$ \\
\hline $\begin{array}{l}\text { Ilume } \\
\text { eak Hour Factor, PHF } \\
\text { eak-15 Minute Volume } \\
\text { surly Flow Rate, HFR } \\
\text { 2rcent Heavy Vehicles } \\
\text { ercent Grade ( } 8) \\
\text { zdian Storage } \\
\text {-ared Approach: Exists? } \\
\end{array}$ & $\begin{array}{l}40 \\
1.00 \\
10 \\
40 \\
2\end{array}$ & 0 & $\begin{array}{l}90 \\
1.00 \\
22 \\
90 \\
2\end{array}$ & & 0 & \\
\hline
\end{tabular}

Channelized? 


\begin{tabular}{lllll}
\hline ovements & 13 & 14 & 15 & 16 \\
\hline low (ped/hr) & 0 & 0 & 0 & 0 \\
ane Width (ft) & 12.0 & 12.0 & 12.0 & 12.0 \\
alking Speed (ft/sec) & 4.0 & 4.0 & 4.0 & 4.0 \\
ercent Blockage & 0 & 0 & 0 & 0
\end{tabular}

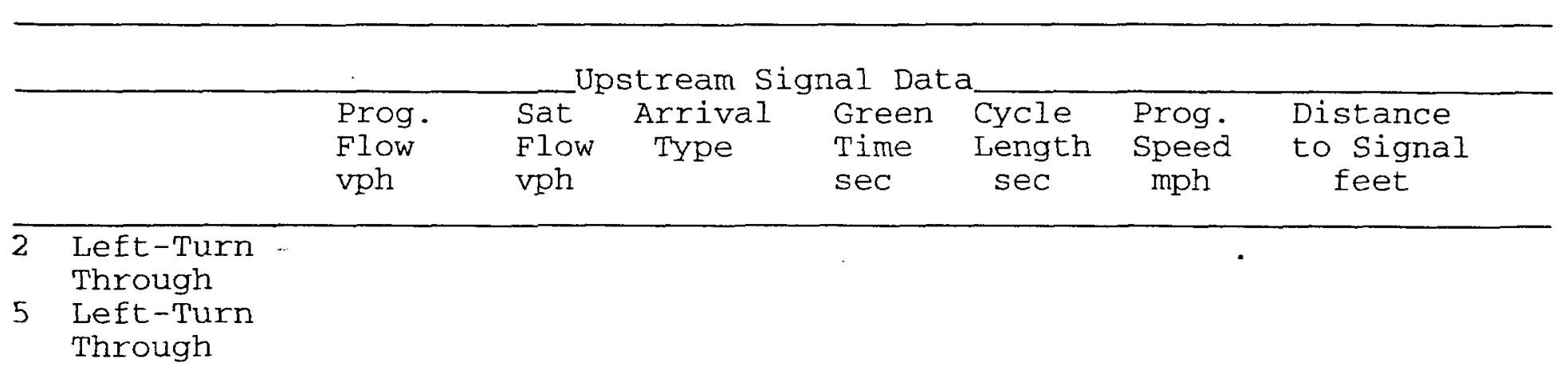

orksheet 3-Data for Computing Effect of Delay to Major Street Vehicles Movement 2 Movement 5

hared in volume, major th vehicles:

hared In volume, major rt vehicles:

at flow rate, major th vehicles:

at flow rate, major rt vehicles:

umber of major street through lanes:

60
0
1700
1700
1

1

orksheet 4-Critical Gap and Follow-up Time Calculation

\begin{tabular}{|c|c|c|c|c|c|c|c|c|c|}
\hline $\begin{array}{l}\text { ritical } \\
\text { ovement }\end{array}$ & Gap Calc & $\begin{array}{c}\text { culati } \\
1 \\
\mathrm{~L}\end{array}$ & $\begin{array}{l}4 \\
\mathrm{~L}\end{array}$ & $\begin{array}{l}7 \\
\mathrm{~L}\end{array}$ & $\begin{array}{l}8 \\
T\end{array}$ & $\begin{array}{l}9 \\
\mathrm{R}\end{array}$ & $\begin{array}{r}10 \\
\mathrm{~L}\end{array}$ & $\begin{array}{r}11 \\
\mathrm{~T}\end{array}$ & $\begin{array}{r}12 \\
\mathrm{R}\end{array}$ \\
\hline $\begin{array}{l}(\mathrm{c}, \text { base }) \\
(\mathrm{c}, \mathrm{hv}) \\
(\mathrm{hv}) \\
(\mathrm{c}, \mathrm{g}) \\
\text { rade } / 100 \\
(3,1 \mathrm{t}) \\
(\mathrm{c}, \mathrm{T}): \\
(\mathrm{c})\end{array}$ & $\begin{array}{l}\text { 1-stage } \\
\text { 2-stage } \\
1 \text {-stage } \\
\text { 2-stage }\end{array}$ & $\begin{array}{l}0.00 \\
0.00\end{array}$ & $\begin{array}{l}0.00 \\
0.00 \\
0.00 \\
4.1\end{array}$ & $\begin{array}{l}7.1 \\
1.00 \\
2 \\
0.20 \\
0.00 \\
0.70 \\
0.00 \\
1.00 \\
6.4\end{array}$ & $\begin{array}{l}1.00 \\
0.20 \\
0.00 \\
0.00 \\
1.00\end{array}$ & $\begin{array}{l}6.2 \\
1.00 \\
2 . \\
0.10 \\
0.00 \\
0.00 \\
0.00 \\
0.00 \\
6.2\end{array}$ & $\begin{array}{l}1.00 \\
0.20 \\
0.00 \\
0.00 \\
1.00\end{array}$ & $\begin{array}{l}1.00 \\
0.20 \\
0.00 \\
0.00 \\
1.00\end{array}$ & $\begin{array}{l}1.00 \\
0.10 \\
0.00 \\
0.00 \\
0.00\end{array}$ \\
\hline $\begin{array}{l}\text { ollow-Up } \\
\text { ovement }\end{array}$ & Time $\mathrm{Ca}$ & $\begin{array}{l}\text { alcula } \\
1 \\
L\end{array}$ & $\begin{array}{c}\text { ions } \\
4 \\
L\end{array}$ & $\begin{array}{l}7 \\
L\end{array}$ & $\begin{array}{l}8 \\
\mathrm{~T}\end{array}$ & $\begin{array}{l}9 \\
\mathrm{R}\end{array}$ & $\begin{array}{r}10 \\
\mathrm{~L}\end{array}$ & $\begin{array}{r}11 \\
\mathrm{~T}\end{array}$ & $\begin{array}{r}12 \\
\mathrm{R}\end{array}$ \\
\hline $\begin{array}{l}(f, \text { base }) \\
(f, H V) \\
(H V) \\
(f)\end{array}$ & & 0.90 & $\begin{array}{l}2.20 \\
0.90 \\
2 \\
2.2\end{array}$ & $\begin{array}{l}3.50 \\
0.90 \\
2 \\
3.5\end{array}$ & 0.90 & $\begin{array}{l}3.30 \\
0.90 \\
2 \\
3.3\end{array}$ & 0.90 & 0.90 & 0.90 \\
\hline
\end{tabular}


Norksheet 5-Effect of Upstream Signals

mputation 1-Queue Clearance Time at Upstream Signal

Movement 2 Movement 5

$\mathrm{V}(\mathrm{t}) \quad \mathrm{V}(1$, prot $) \quad \mathrm{V}(\mathrm{t}) \quad \mathrm{V}(1$, prot $)$

prog

I tal Saturation Flow Rate, s (vph)

- rival Type

¿.fective Green, $g$ (sec)

zxcle Length, C (sec)

(from table 9-2)

oportion vehicles arriving on green $\mathrm{P}$

$\exists(\mathrm{q} 1)$

$\equiv \mathrm{q} 2)$

q)

-mputation 2-Proportion of TWSC Intersection Time blocked

Movement 2 Movement 5

$v(t) \quad V(1$, prot $) \quad V(t) \quad V(1$, prot $)$

: pha

z ta

[ravel time, $t(a)$ (sec)

"oothing Factor, F

oportion of conflicting flow, f

1ax platooned flow, $V(c, m a x)$

"n platooned flow, $V(c, m i n)$

- ration of blocked period, $t(p)$

?-oportion time blocked, p

0.000

0.000

mputation 3-Platoon Event Periods Result

(2) 0.000

5)

0.000

(dom)

(subo)

anstrained or unconstrained?

sportion

inblocked

$r$ minor

. vements, $\mathrm{p}(\mathrm{x})$

Single-stage

Process

(2)

Two-stage Proces

Stage I Stage II

1)

4)

, (7)

(8)

9)

, .10)

(11)

12)

Eomputation 4 and 5

- ngle-stage Process

vement

1

4

7

8

9
$\mathrm{R}$

10

11

12 
$\mathrm{X}$

$\mathrm{C}, \mathrm{u}, \mathrm{x}$

$r, \mathrm{x}$

plat, $x$

wo-Stage Process

$7 \quad 8 \quad 10$

11

Stagel Stage2 Stage1 Stage2 Stagel Stage2 Stage1 Stage2

$(c, x)$

$(x)$

1700

$(\mathrm{C}, \mathrm{u}, \mathrm{x})$

$(r, x)$

$($ plat, $x)$.

orksheet 6-Impedance and Capacity Equations

tep 1: RT from Minor st.

9

onflicting Flows

otential Capacity

edestrian Impedance Factor

ovement capacity

robability of Queue free st.

\begin{tabular}{lll}
\hline tep 2: LT from Major St. & 4 & 1 \\
\hline snflicting Flows & 220 & 1349 \\
stential Capacity & 1.00 & 1.00 \\
adestrian Impedance Factor & 1349 & 0.99 \\
svement Capacity & 0.98 & 1.00 \\
robability of Queue free St. & & \\
zj L-Shared Prob Q free St. &
\end{tabular}

Lep 3: TH from Minor St.

215

825

1.00

825

0.89

1.00

1.00

8

11

onflicting Flows

otential Capacity .

ədestrian Impedance Factor

ap. Adj. factor due to Impeding mvmnt

svement Capacity

robability of Queue free $\mathrm{St}$.

1.00

1.00

0.98

0.98

Eep 4: LT from Minor st.

1.00

1.00

onflicting Flows

stential Capacity

ədestrian Impedance Factor

aj. L, Min T. Impedance factor

aj. L, Min T Adj. Imp Factor.

æp. Adj. factor due to Impeding mvmnt

Jvement Capacity

7

315

678

1.00

0.99

668

10

1.00

0.98

$0.9 \cdot 9$

10

0.88 
orksheet 8-Shared Lane Calculations

\begin{tabular}{llllrrr}
\hline ovement & 7 & 8 & 9 & 10 & 11 & 12 \\
R & L & T & R & L & & \\
\hline olume (vph) & & 40 & & 90 & & \\
ovement Capacity (vph) & 668 & & & & \\
hared Lane Capacity (vph) & & & & \\
\hline
\end{tabular}

orksheet 9-Computation of Effect of Flared Minor Street Approaches

\begin{tabular}{llllrrr}
\hline ovement & 7 & 8 & 9 & 10 & 11 & 12 \\
$R$ & L & T & R & L & T & \\
\hline sep & & 668 & & 825 & & \\
olume & 40 & & 90 & & \\
elay & & & & & \\
sep & & & & & \\
sep +1 & & & & & \\
ound (Qsep +1) & & & &
\end{tabular}

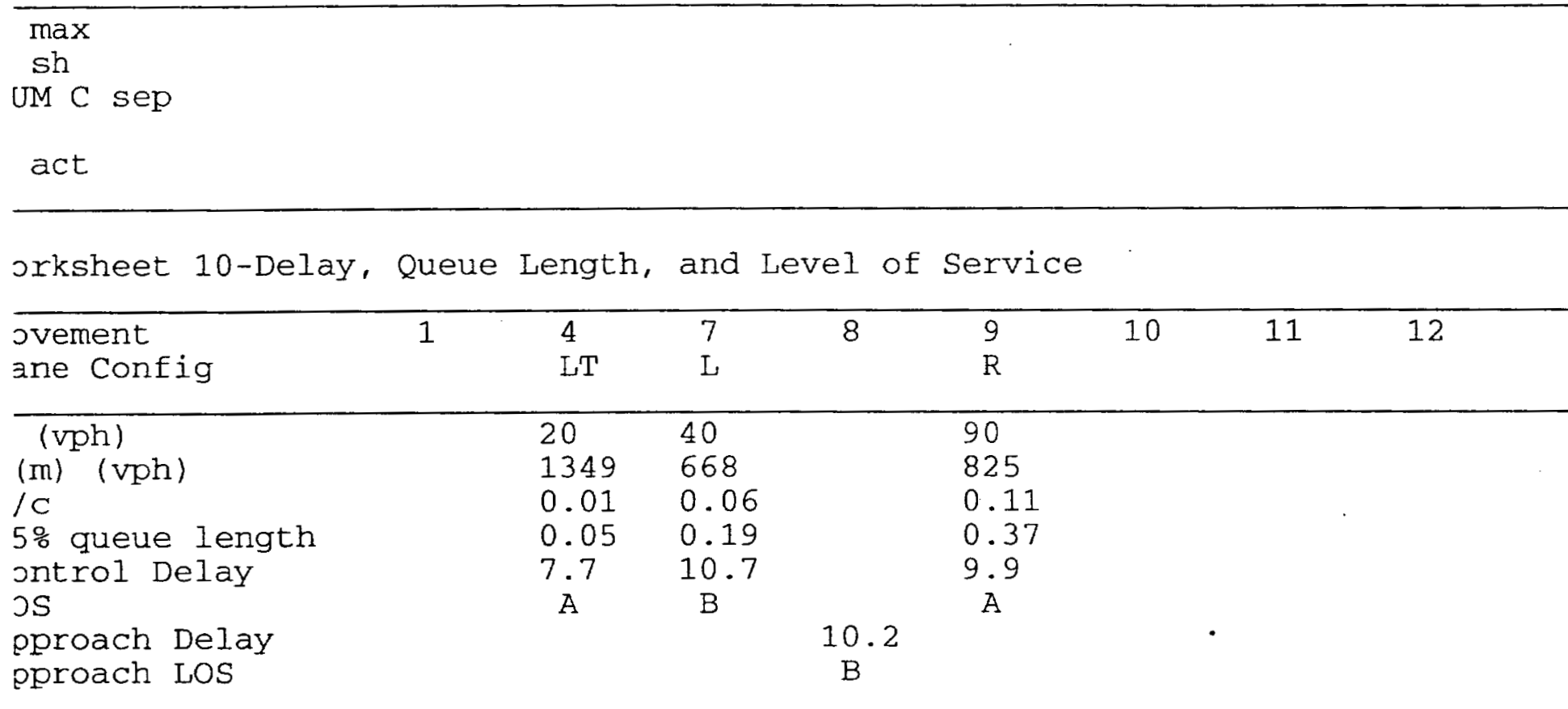

orksheet 11-Shared Major LT Impedance and Delay

\begin{tabular}{lcl}
\hline & Movement 2 & Movement 5 \\
\hline (oj) & 1.00 & 0.99 \\
(i1), Volume for stream 2 or 5 & & 60 \\
(i2), Volume for stream 3 or 6 & & 0 \\
(il), Saturation flow rate for stream 2 or 5 & & 1700 \\
* (oj) Saturation flow rate for stream 3 or 6 & & 0.98 \\
(M, LT), Delay for stream 1 or. 4 & & 7.7 \\
(Number of major street through lanes & & 1 \\
(rank, 1) Delay for stream 2 or 5 & & 0.1
\end{tabular}


Analyst:

lency/co.:

ite Performed:

$1 / 16 / 02$

Analysis Time Period: Existing AM

; itersection:

East Ave \& Greenville Ra

Irisdiction:

malysis Year:

2001

roject ID: Lawrence Livermore Lab Traffic Impact Analysis

st/West Street: East Avenue

irth/South Street: Greenville Road

itersection Orientation: NS

Study period (hrs): 0.25

Vehicle Volumes and Adjustments

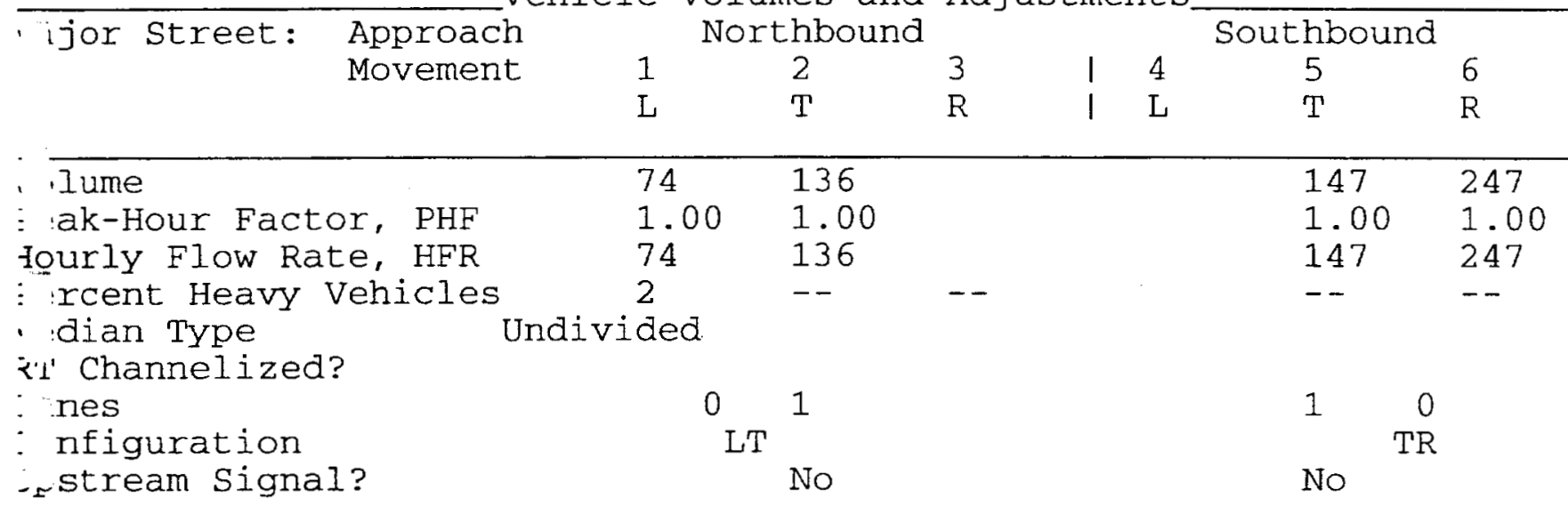

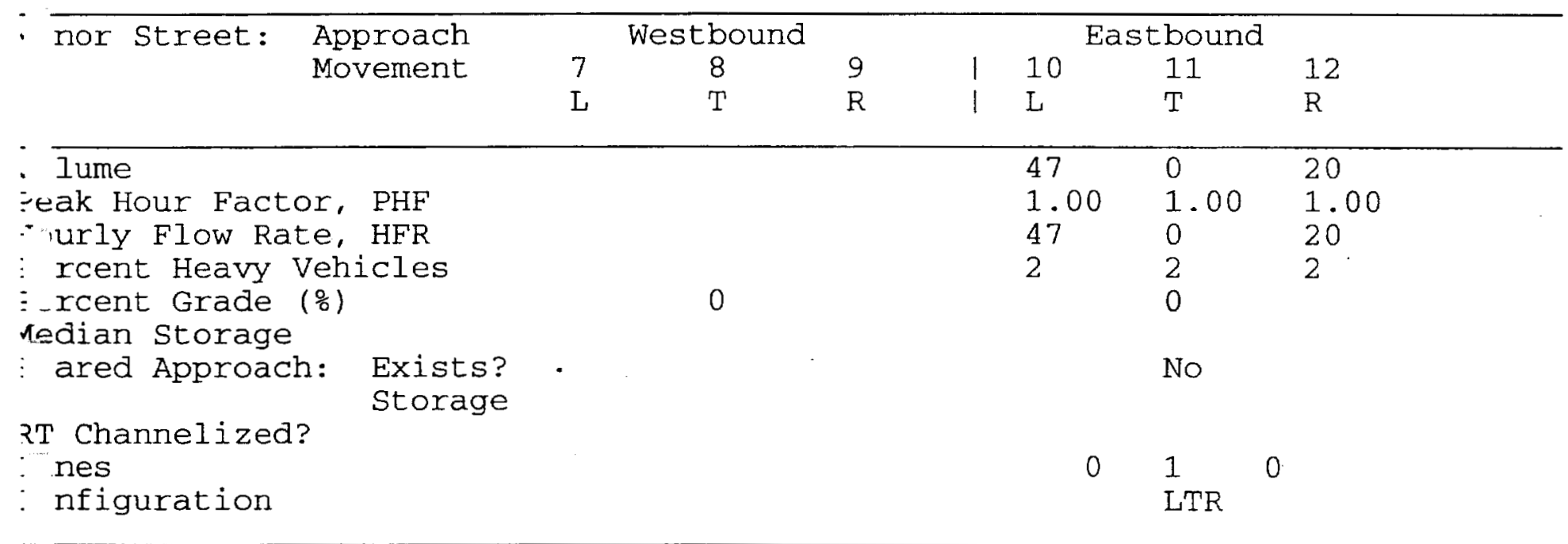

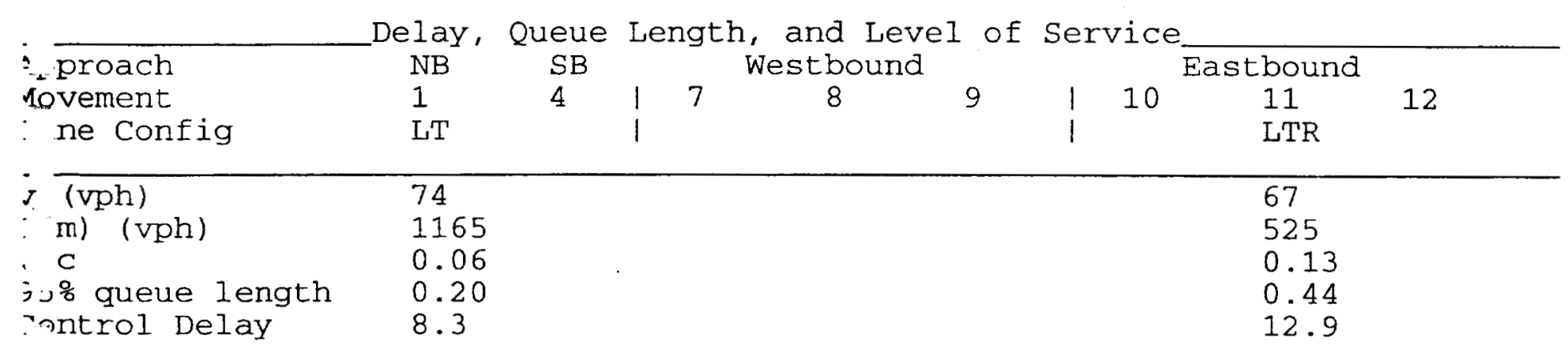


HCS2000: Unsignalized Intersections Release 4.1

srve Engineering, Inc.

rone:

Fax:

-Mail:

TWO-WAY STOP CONTROL (TWSC) ANALYSIS

ralyst:

Jency/Co.:

the Performed:

$1 / 16 / 02$

lalysis Time Period: Existing AM

atersection:

East Ave \& Greenville Rd

irisdiction:

lalysis Year:

2001

roject ID: Lawrence Livermore Lab Traffic Impact Analysis

ast/West Street: East Avenue

orth/South Street: Greenville Road

atersection Orientation: NS

Study period (hrs): 0.25

Vehicle Volumes and Adjustments

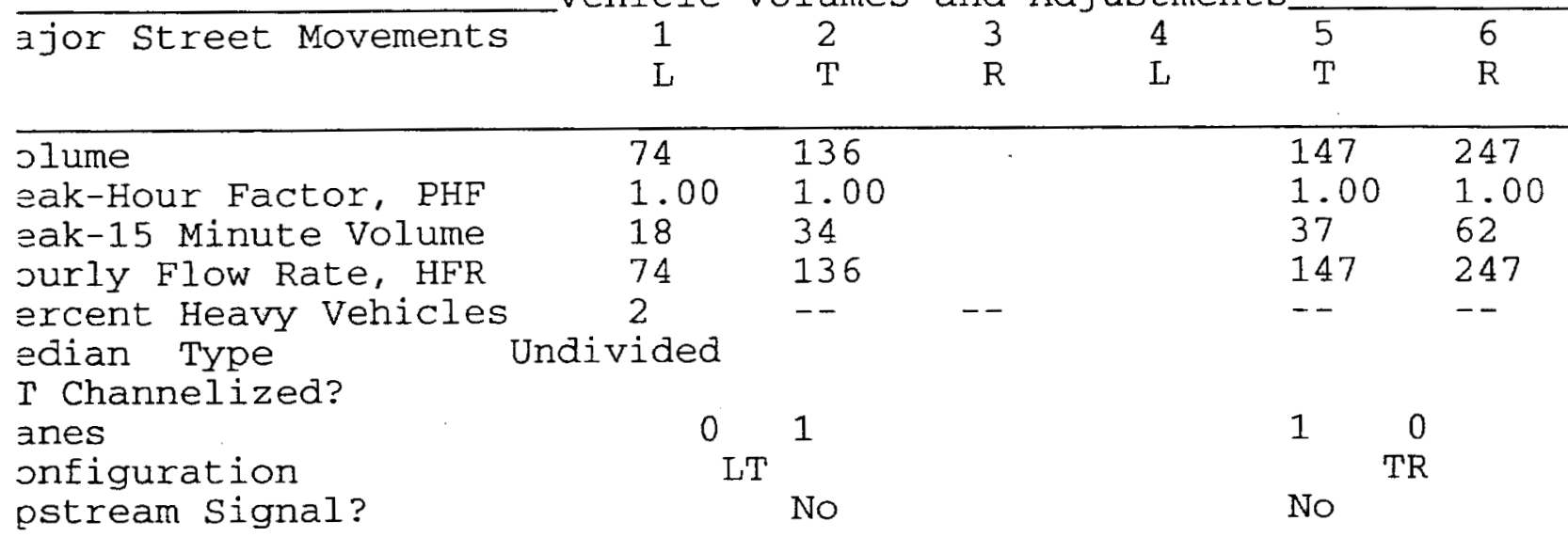

pstream Signale 9

\begin{tabular}{|c|c|c|c|c|c|c|}
\hline inor Street Movements & $\begin{array}{l}7 \\
\mathrm{I}\end{array}$ & $\begin{array}{l}8 \\
T\end{array}$ & $\begin{array}{l}9 \\
R\end{array}$ & $\begin{array}{r}10 \\
\mathrm{~L}\end{array}$ & $\begin{array}{r}11 \\
\mathrm{~T}\end{array}$ & $\begin{array}{r}12 \\
\mathrm{R}\end{array}$ \\
\hline $\begin{array}{l}\text { olume } \\
\text { eak Hour Factor, PHF } \\
\text { eak-15 Minute Volume } \\
\text { ourly Flow Rate, HFR } \\
\text { ercent Heavy Vehicles } \\
\text { ercent Grade }\end{array}$ & & 0 & & $\begin{array}{l}47 \\
1.00 \\
12 \\
47 \\
2\end{array}$ & $\begin{array}{l}0 \\
1.00 \\
0 \\
0 \\
2 \\
0\end{array}$ & $\begin{array}{l}20 \\
1.00 \\
5 \\
20 \\
2\end{array}$ \\
\hline
\end{tabular}

ercent Grade (\%)

edian storage

lared Approach: Exists?

T Channelized?

Storage

No 
Pedestrian Volumes and Adjustments

\begin{tabular}{lllll}
\hline & 13 & 14 & 15 & 16 \\
\hline ow (ped/hr) & 0 & 0 & 0 & 0 \\
sane Width (ft) & 12.0 & 12.0 & 12.0 & 12.0 \\
I Iking Speed (ft/sec) & 4.0 & 4.0 & 4.0 & 4.0 \\
rcent Blockage & 0 & 0 & 0 & 0
\end{tabular}

\begin{tabular}{|c|c|c|c|c|c|c|c|c|}
\hline & & $\begin{array}{l}\text { Prog. } \\
\text { Flow } \\
\text { vph }\end{array}$ & $\begin{array}{l}\text { Sat } \\
\text { Flow } \\
\text { vph }\end{array}$ & $\begin{array}{l}\text { Arrival } \\
\text { Type }\end{array}$ & $\begin{array}{l}\text { Green } \\
\text { Time } \\
\text { sec }\end{array}$ & $\begin{array}{l}\text { Cycle } \\
\text { Length } \\
\text { sec }\end{array}$ & $\begin{array}{l}\text { Prog. } \\
\text { Speed } \\
\text { mph }\end{array}$ & $\begin{array}{c}\text { Distance } \\
\text { to Signal } \\
\text { feet }\end{array}$ \\
\hline 32 & $\begin{array}{l}\text { Left-Turn } \\
\text { Through } \\
\text { Left-Turn } \\
\text { Through }\end{array}$ & & & & & . & & \\
\hline
\end{tabular}

rksheet 3-Data for Computing Effect of Delay to Major street Vehicles

Movement 2 Movement 5

$\begin{array}{ll}\text { inared In volume, major th vehicles: } & 136 \\ \text { 'ared In volume, major rt vehicles: } & 0 \\ t \text { flow rate, major th vehicles: } & 1700 \\ \text { jat flow rate, major rt vehicles: } & 1700 \\ \text { rmber of major street through lanes: } & 1\end{array}$

worksheet 4-Critical Gap and Follow-up Time Calculation

\begin{tabular}{|c|c|c|c|c|c|c|c|c|c|}
\hline $\begin{array}{r}\text { itical } \\
\text { lovement }\end{array}$ & Gap Cal & $\begin{array}{c}\text { culats } \\
1 \\
\mathrm{~L}\end{array}$ & $\begin{array}{l}4 \\
\mathrm{~L}\end{array}$ & $\begin{array}{l}7 \\
\mathrm{~L}\end{array}$ & $\begin{array}{l}8 \\
\mathrm{~T}\end{array}$ & $\begin{array}{l}9 \\
\mathrm{R}\end{array}$ & $\begin{array}{r}10 \\
\mathrm{~L}\end{array}$ & $\begin{array}{r}11 \\
\mathrm{~T}\end{array}$ & $\begin{array}{r}12 \\
\mathrm{R}\end{array}$ \\
\hline $\begin{array}{c}\text { (c, base) } \\
\text { (c, hv) } \\
\text { hv) } \\
c, g) \\
\text { irade/100 } \\
\text { 3, lt) } \\
\text { c, T): } \\
\text { (c) }\end{array}$ & $\begin{array}{l}1 \\
1 \text {-stage } \\
2 \text {-stage } \\
1 \text {-stage } \\
2 \text {-stage }\end{array}$ & $\begin{array}{l}0.00 \\
0.00 \\
0.00 \\
4.1\end{array}$ & $\begin{array}{l}0.00 \\
0.00\end{array}$ & $\begin{array}{l}1.00 \\
0.20 \\
0.00 \\
0.00 \\
1.00\end{array}$ & $\begin{array}{l}1.00 \\
0.20 \\
0.00 \\
0.00 \\
1.00\end{array}$ & $\begin{array}{l}1.00 \\
0.10 \\
0.00 \\
0.00 \\
0.00\end{array}$ & $\begin{array}{l}7.1 \\
1.00 \\
2 \\
0.20 \\
0.00 \\
0.70 \\
0.00 \\
1.00 \\
6.4\end{array}$ & $\begin{array}{l}6.5 \\
1.00 \\
2 \\
0.20 \\
0.00 \\
0.00 \\
0.00 \\
1.00 \\
6.5\end{array}$ & $\begin{array}{l}6.2 \\
1.00 \\
2 \\
0.10 \\
0.00 \\
0.00 \\
0.00 \\
0.00 \\
6.2\end{array}$ \\
\hline $\begin{array}{l}\text { osllow-Up } \\
\text { ivement }\end{array}$ & Time 0 & $\begin{array}{c}\text { alcula } \\
1 \\
\text { L }\end{array}$ & $\begin{array}{c}\text { ions } \\
4 \\
\text { L }\end{array}$ & $\begin{array}{l}7 \\
\mathrm{~L}\end{array}$ & $\begin{array}{l}8 \\
\mathrm{~T}\end{array}$ & $\begin{array}{l}9 \\
\mathrm{R}\end{array}$ & $\begin{array}{r}10 \\
\mathrm{~L}\end{array}$ & $\begin{array}{r}11 \\
\mathrm{~T}\end{array}$ & $\begin{array}{r}12 \\
\mathrm{R}\end{array}$ \\
\hline $\begin{array}{l}\text { (f, base) } \\
\text { f, HV) } \\
(\mathrm{HV}) \\
\text { (f) }\end{array}$ & & $\begin{array}{l}2.20 \\
0.90 \\
2 . \\
2.2\end{array}$ & 0.90 & 0.90 & 0.90 & 0.90 & $\begin{array}{l}3.50 \\
0.90 \\
2 \\
3.5\end{array}$ & $\begin{array}{l}4.00 \\
0.90 \\
2 \\
4.0\end{array}$ & $\begin{array}{l}3.30 \\
0.90 \\
2 \\
3.3\end{array}$ \\
\hline
\end{tabular}


orksheet 5-Effect of Upstream Signals

omputation 1-Queue Clearance Time at Upstream Signal

Movement 2

Movement 5

$V(t) \quad V(1$, prot $) \quad V(t) \quad V(1$, prot $)$

\section{prog}

otal Saturation Flow Rate, s (vph)

rrival Type

ffective Green, g (sec)

ycle Length, $\mathrm{C}$ (sec)

$\mathrm{p}$ (from table 9-2)

roportion vehicles arriving on green $P$

(q1)

(q2)

(g)

omputation 2-Proportion of TWSC Intersection Time blocked Movement 2 Movement 5 $V(t) \quad V(1$, prot $) \quad V(t) \quad V(1$, prot $)$

Ipha
eta
ravel time, $t(a)$ (sec)
noothing Factor, $F$.
roportion of conflicting flow, f
ax platooned flow, $V(c, m a x)$
in platooned flow, $v(c, m i n)$
uration of blocked period, $t(p)$
roportion time blocked, p
Jmputation 3-Platoon Event Periods
(2)

0.000

0.000

(dom)

(subo)

onstrained or unconstrained?

\begin{tabular}{lccc}
\hline $\begin{array}{l}\text { roportion } \\
\text { nblocked }\end{array}$ & $(1)$ & $(2)$ & (3) \\
or minor & Single-stage & Two-stage Process \\
ovements, $p(x)$ & Process & Stage I & Stage II
\end{tabular}
(1)
(4)
(7)
(8)
(9)
(11)
(12)

omputation 4 and 5 ingle-stage process ovement

\begin{tabular}{rrrrrrrr}
1 & 4 & 7 & 8 & 9 & 10 & 11 & 12 \\
$\mathrm{~L}$ & $\mathrm{~L}$ & $\mathrm{~L}$ & $\mathrm{~T}$ & $\mathrm{R}$ & $\mathrm{L}$ & $\mathrm{T}$ & $\mathrm{R}$ \\
\hline
\end{tabular}


P.x.

$c, u, x$

$=r, x$

plat, $x$

iwo-Stage Process

Stage1 ${ }^{7}$ stage2 stage1 ${ }^{8}$ stage2 stagel $^{10}$ stage2 stage1 $^{11}$ Stage2

$T(\mathrm{C}, \mathrm{X})$

$\vdots x)$

1700

1700

$J(c, u, x)$

r, X)

$\because($ plat,$x)$.

-urksheet 6-Impedance and Capacity Equations

\begin{tabular}{llll}
\hline ep 1: RT from Minor St. & 9 & 12
\end{tabular}

zonflicting Flows

tential Capacity

destrian Impedance Factor

vovement Capacity

? obability of Queue free st.

$\begin{array}{ll} & 270 \\ 1.00 & 769 \\ & 1.00 \\ 1.00 & 769 \\ & 0.97\end{array}$

ఏ_ep 2: LT from Major St.

41

nflicting Flows

tential Capacity

Pedestrian Impedance Factor

vement Capacity

obability of Queue free st.

raj L-Shared Prob $Q$ free st.

\begin{tabular}{lll}
\hline ep 3: TH from Minor St. & 8 & 11 \\
\hline Sonflicting Flows & & 554 \\
tential Capacity & 440 \\
destrian Impedance Factor & 1.00 & 1.00 \\
zap. Adj. factor due to Impeding mvmnt & 0.93 & 0.93 \\
vvement Capacity & & 410 \\
obability of Queue free St. & 1.00 & 1.00 \\
\hline step 4: LT from Minor St. & 70 \\
-nflicting Flows & & 554 \\
Potential Capacity & 1.00 & 493 \\
destrian Impedance Factor & 0.93 & 1.00 \\
j. L, Min T Impedance factor & 0.95 & 0.92 \\
Maj. L, Min T Adj. Imp Factor. & & 462
\end{tabular}


orksheet 7-Computation of the Effect of Two-stage Gap Acceptance

tep 3: TH from Minor st.

8

11

art 1 - First stage

onflicting Flows

otential Capacity

edestrian Impedance Factor

ap. Adj. factor due to Impeding mvmnt

ovement Capacity

robability of Queue free st.

art 2 - Second stage

onflicting Flows

otential Capacity

edestrian Impedance Factor

ap. Adj. factor due to Impeding mvmnt

ovement Capacity

art 3 - Single stage

onflicting Flows

otential Capacity

edestrian Impedance Factor

ap. Adj. factor due to Impeding mvmnt

ovement Capacity

554

440

1.00

1.00

0.93

0.93

410

esult for 2 stage process:

$t$

robability of Queue free st.

1.00

tep 4: IT from Minor st.

7

10

art 1 - First stage

onflicting Flows

otential Capacity

edestrian Impedance Factor

ap. Adj. factor due to Impeding mumnt

ovement Capacity

art 2 - Second Stage

onflicting Flows

otential Capacity

edestrian Impedance Factor

ap. Adj. factor due to Impeding mvmnt

ovement Capacity

art 3 - Single stage

onflicting Flows

stential Capacity

edestrian Impedance Factor

aj. L, Min T Impedance factor

aj. L, Min T Adj. Imp Factor.

ap. Adj. factor due to Impeding mvmnt

ovement Capacity

0.95

0.92

0.94

462

esults for Two-stage process: 
ralyst:

yency/Co.:

ate Performed: 1/16/02

lalysis Time Period: Existing PM

atersection: East Ave \& Greenville Rd

urisdiction:

lalysis Year: 2001

roject ID: Lawrence Livermore Lab Traffic Impact Analysis

ast/West Street: East Avenue

srth/South Street: Greenville Road

atersection orientation: NS

study period (hrs): 0.25

Vehicle Volumes and Adjustments

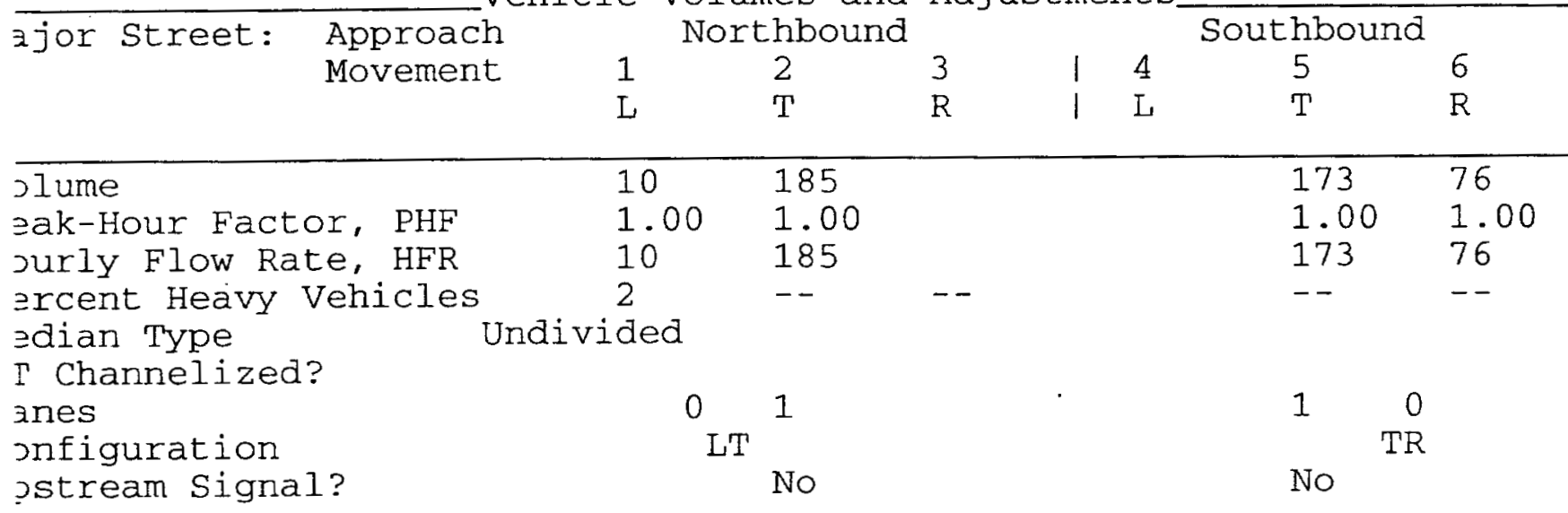

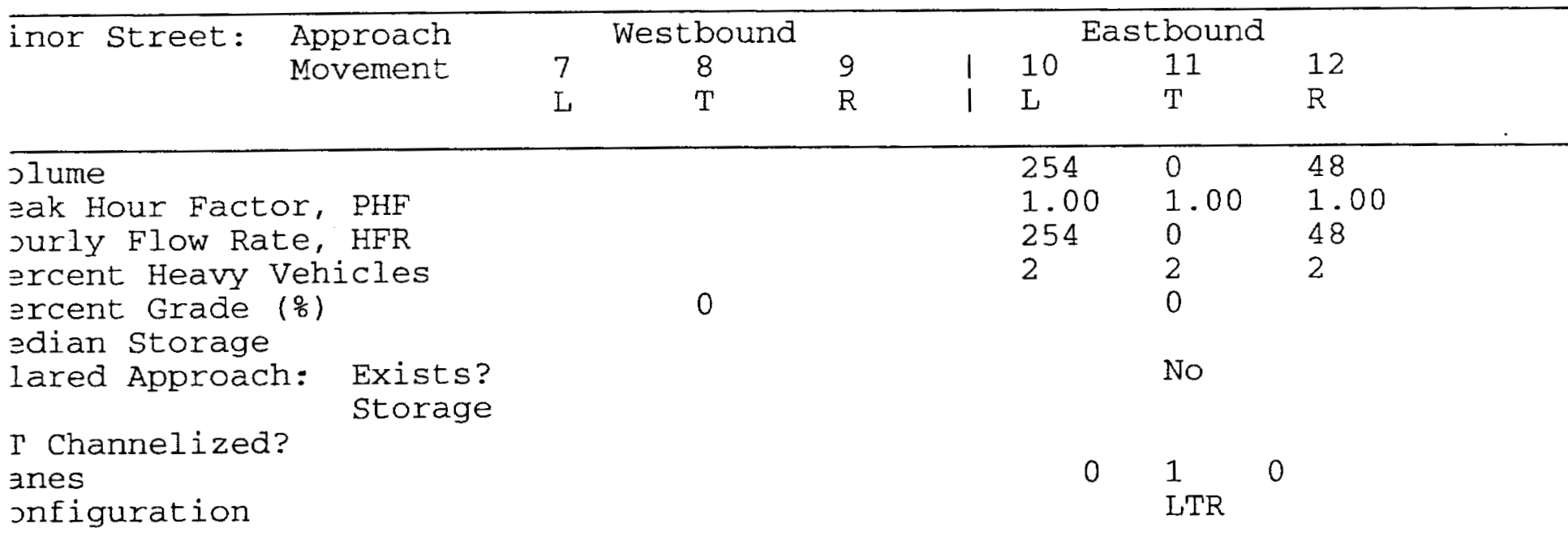

Delay, Queue Length, and Level of Service

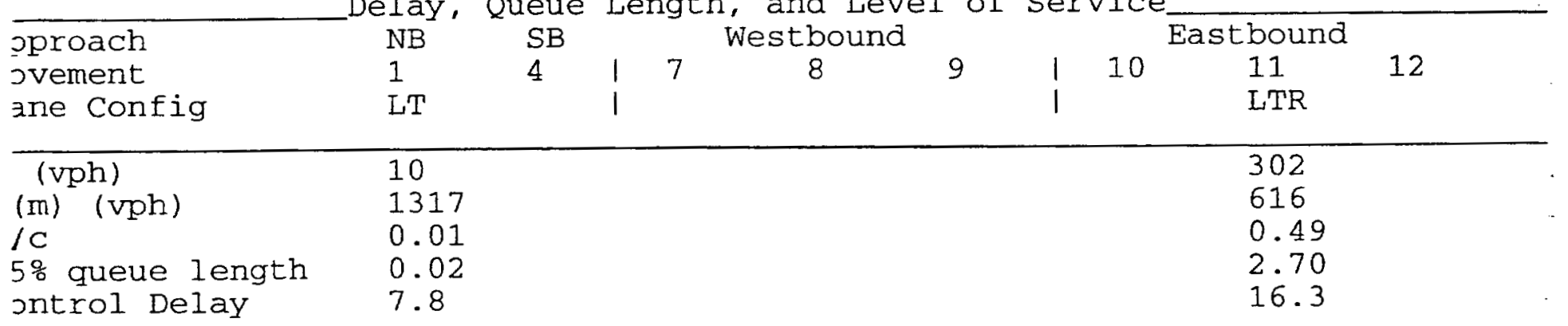




\section{- ralyst:}

jency/Co.:

the Performed:

$1 / 16 / 02$

1nalysis Time Period: Existing PM

itersection:

irisdiction:

Analysis Year:

East Ave \& Greenville Rd

-oject ID: Lawrence Livermore Lab Traffic Impact Analysis

: ist/West Street:

East Avenue

ivuth/South street:

Greenville Road

ttersection Orientation: NS

study period (hrs): 0.25

Vehicle Volumes and Adjustments

jor Street Movements $\begin{array}{ll}1 & 2 \\ \mathrm{~L} & \mathrm{~T}\end{array}$

$\begin{array}{ll}3 & 4 \\ \mathrm{R} & \mathrm{L}\end{array}$

\begin{tabular}{ll}
\hline 5 & 6 \\
$T$ & $\mathrm{R}$
\end{tabular}

"lume

sak-15 Minute Volume

rourly Flow Rate, HFR

rrcent Heavy Vehicles

' dian Type

रT Channelized?

ines

- mfiguration

jpstream Signal?

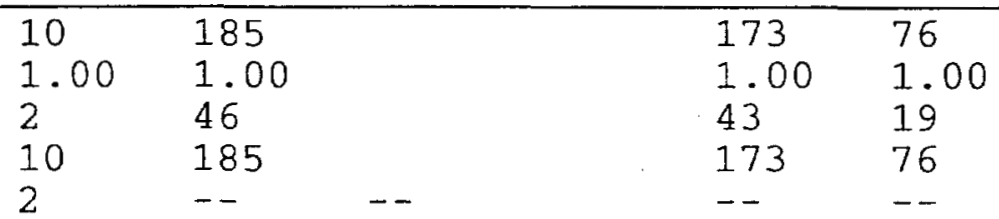

Undivided

$\begin{array}{lllr}0 & 1 & 1 & 0 \\ \text { LT } & & & \text { TR } \\ & \text { No } & \text { No } & \end{array}$.

nor street Movements

7

$\begin{array}{ll}8 & 9 \\ T & R\end{array}$

\section{lume}

: ak Hour Factor, PHF

seak-15 Minute Volume

iurly flow Rate, HFR

ircent Heavy Vehicles

jercent Grade ( $z$ )

¿dian Storage

ared Approach: Exists?

2T Channelized?

Storage

$\begin{array}{rrr}10 & 11 & 12 \\ \mathrm{~L} & \mathrm{~T} & \mathrm{R}\end{array}$

$\begin{array}{lll}254 & 0 & 48 \\ 1.00 & 1.00 & 1.00 \\ 64 & 0 & 12 \\ 254 & 0 & 48 \\ 2 & 2 & 2 \\ & 0 . & \end{array}$

No 


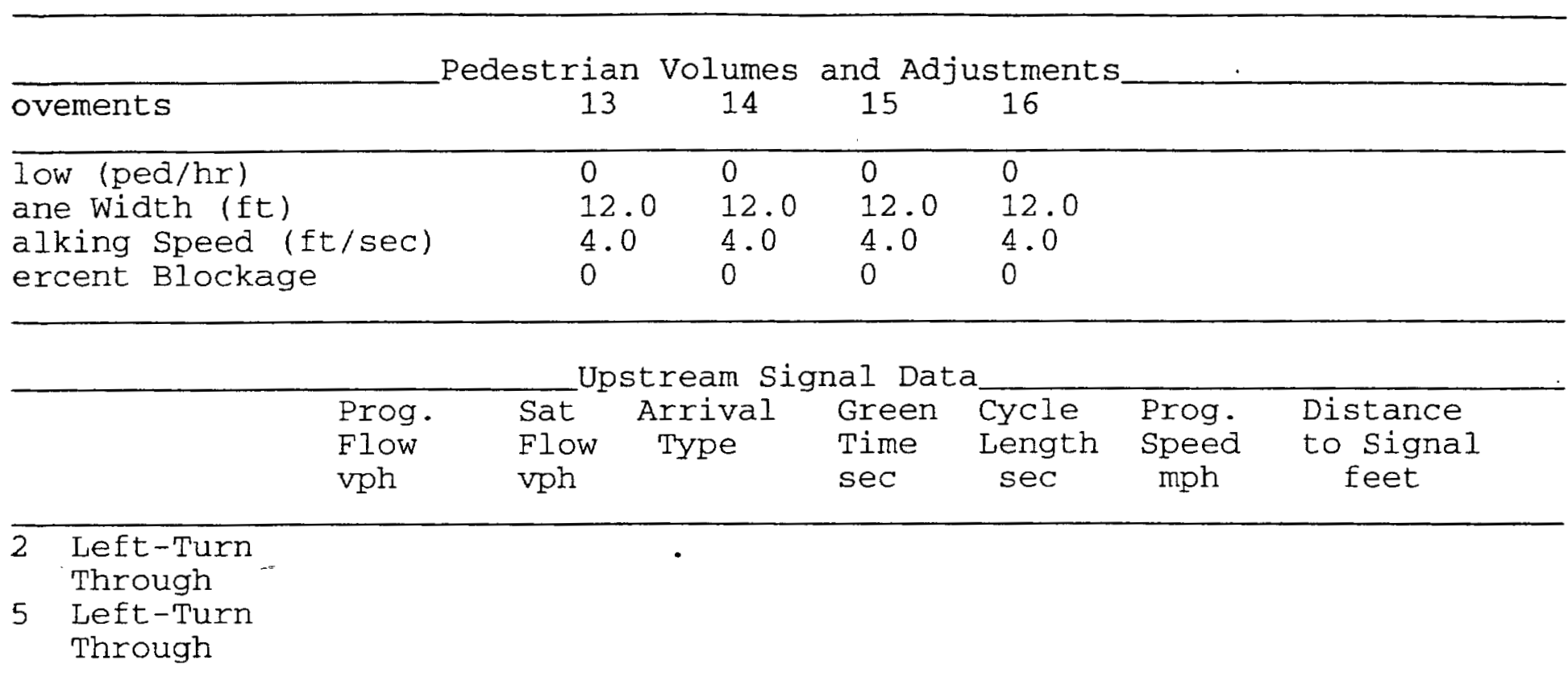

orksheet 3-Data for Computing Effect of Delay to Major street Vehicles

\begin{tabular}{lll}
\hline & Movement 2 & Movement 5 \\
\hline hared In volume, major th vehicles: & 185 \\
hared In volume, major rt vehicles: & 0 \\
at flow rate, major th vehicles: & 1700 \\
at flow rate, major rt vehicles: & 1700 \\
umber of major street through lanes: & 1 & \\
\hline
\end{tabular}

orksheet 4-Critical Gap and Follow-up Time Calculation

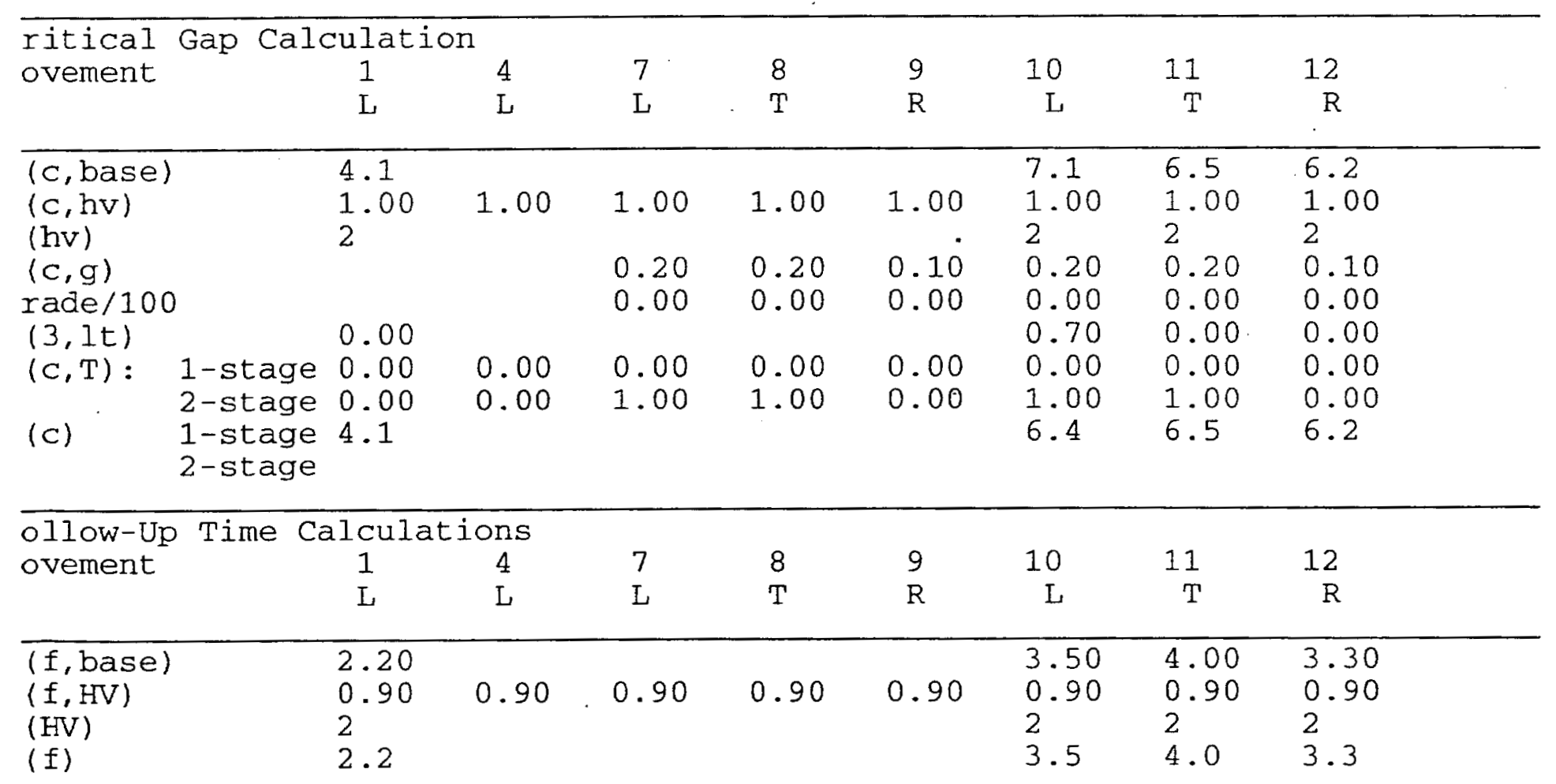




\section{T-rksheet 5-Effect of Upstream Signals}

mputation 1-Queue Clearance Time at Upstream Signal

\section{Movement 2}

$V(t) \quad V(1$, prot $) \quad V(t) \quad V(1$, prot $)$

\section{i prog}

l tal Saturation Flow Rate, s (vph)

: rival Type

isfective Green, g (sec)

Cle Length, C (sec)

(from table 9-2)

oportion vehicles arriving on green $P$

x (q1)

q2)

q)

mputation 2-Proportion of TWSC Intersection Time blocked Movement $2 \quad$ Movement 5 $V(t) \quad V(1$, prot $) \quad V(t) \quad V(1$, prot $)$

$\vdots$ pha

: ta

Travel time, $t(a)$ (sec)

oothing Factor, $F$

oportion of conflicting flow, $f$

lax platooned flow, $V(c, \max )$

"n platooned flow, $V(c, m i n)$

ration of blocked period, $t(p)$

$\vdots$ oportion time blocked, p

0.000

0.000

: mputation 3-Platoon Event Periods Result

$\begin{array}{ll}\supset(2) & 0.000 \\ \vdots 5) & 0.000\end{array}$

dom)

ว (subo)

- onstrained or unconstrained?

\section{E: oportion \\ inblocked}

ir minor

i vements, $p(x)$
(1)

Single-stage

Process
(2) Stage I
(3)

Stage II

\begin{tabular}{|c|c|c|c|c|c|c|c|c|}
\hline \begin{tabular}{r}
$(1)$ \\
$4)$ \\
$5(7)$ \\
$(8)$ \\
91 \\
\hdashline$(10)$ \\
$5(11)$ \\
$12)$
\end{tabular} & & & & & & & & \\
\hline $\begin{array}{l}\text { amputation } 4 \text { and } 5 \\
\text { ingle-Stage Process } \\
\text { ivement }\end{array}$ & $\begin{array}{l}1 \\
\mathrm{~L}\end{array}$ & $\begin{array}{l}4 \\
\mathrm{~L}\end{array}$ & $\begin{array}{l}7 \\
L\end{array}$ & $\begin{array}{l}8 \\
\mathrm{~T}\end{array}$ & $\begin{array}{l}9 \\
R\end{array}$ & $\begin{array}{r}10 \\
\mathrm{~L}\end{array}$ & $\begin{array}{r}11 \\
T\end{array}$ & $\begin{array}{r}12 \\
\mathrm{R}\end{array}$ \\
\hline
\end{tabular}


$\mathrm{x}$

$c, u, x$

$r, x$

plat, $\mathrm{x}$

wo-Stage Process

$7 \quad 8 \quad 10$

11

Stage1 Stage2 Stage1 Stage2 Stage1 stage2 Stage1 Stage2

$(c, x)$

(x)

1700

1700

(c, $u, x)$

$(x, x)$

(plat, $x$ )

orksheet 6-Impedance and Capacity Equations

\begin{tabular}{|c|c|c|}
\hline tep 1: RT from Minor st. & 9 & 12 \\
\hline $\begin{array}{l}\text { onflicting Flows } \\
\text { otential Capacity } \\
\text { edestrian Impedance Factor } \\
\text { ovement Capacity } \\
\text { robability of queue free st. }\end{array}$ & $\begin{array}{l}1.00 \\
1.00\end{array}$ & $\begin{array}{l}211 \\
829 \\
1.00 \\
829 \\
0.94\end{array}$ \\
\hline Lep 2: LT from Major st. & 4 & 1 \\
\hline $\begin{array}{l}\text { onflicting Flows } \\
\text { otential Capacity } \\
\text { edestrian Impedance Factor } \\
\text { ovement Capacity } \\
\text { robability of Queue free st. } \\
\text { aj L-Shared Prob Q free St. }\end{array}$ & $\begin{array}{l}1.00 \\
1.00\end{array}$ & $\begin{array}{l}249 \\
1317 \\
1.00 \\
1317 \\
0.99 \\
0.99\end{array}$ \\
\hline tep 3: TH from Minor st. & 8 & 11 \\
\hline $\begin{array}{l}\text { onflicting Flows } \\
\text { stential Capacity } \\
\text { adestrian Impedance Factor } \\
\text { ap. Adj. factor due to Impeding mumnt } \\
\text { ovement Capacity } \\
\text { robability of Queue free st. }\end{array}$ & $\begin{array}{l}1.00 \\
0.99 \\
1.00\end{array}$ & $\begin{array}{l}416 \\
527 \\
1.00 \\
0.99 \\
523 \\
1.00\end{array}$ \\
\hline tep 4: LT from Minor st. & 7 & 10 \\
\hline $\begin{array}{l}\text { onflicting Flows } \\
\text { otential Capacity } \\
\text { edestrian Impedance Factor } \\
\text { aj. L, Min T Impedance factor } \\
\text { aj. L, Min T Adj. Imp Factor. } \\
\text { ap. Adj. factor due to Impeding mvmnt } \\
\text { ovement Capacity }\end{array}$ & $\begin{array}{l}1.00 \\
0.99 \\
0.99 \\
0.94\end{array}$ & $\begin{array}{l}416 \\
593 \\
1.00\end{array}$ \\
\hline
\end{tabular}




\begin{tabular}{|c|c|c|}
\hline Step 3: TH from Minor St. & 8 & 11 \\
\hline $\begin{array}{l}\text {.rt } 1 \text { - First Stage } \\
\text { onflicting Flows } \\
\vdots \text { itential Capacity } \\
\vdots \text { destrian Impedance Factor } \\
\text {-dp. Adj. factor due to Impeding mumnt } \\
\text { : vement Capacity } \\
\text { obability of Queue free st. }\end{array}$ & & \\
\hline $\begin{array}{l}\text { Part } 2 \text { - Second Stage } \\
\text { nflicting Flows } \\
\text { t tential Capacity } \\
\text { ?edestrian Impedance Factor } \\
\text { p. Adj. factor due to Impeding mvmnt } \\
\text { vement Capacity }\end{array}$ & & \\
\hline $\begin{array}{l}\text {-rt } 3 \text { - Single Stage } \\
\text { nflicting Flows } \\
\text { - tential Capacity } \\
\text { ?edestrian Impedance Factor } \\
\text { p. Adj. factor due to Impeding mumnt } \\
\text { vement Capacity }\end{array}$ & $\begin{array}{l}1.00 \\
0.99\end{array}$ & $\begin{array}{l}416 \\
527 \\
1.00 \\
0.99 \\
523\end{array}$ \\
\hline 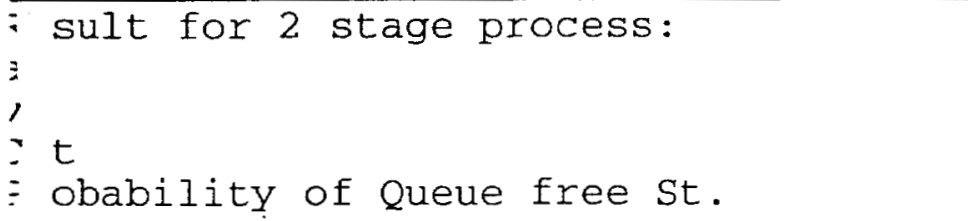 & 1.00 & $\begin{array}{l}523 \\
1.00\end{array}$ \\
\hline 3tep 4: LT from Minor St. & 7 & 10 \\
\hline $\begin{array}{l}\text { rt } 1 \text { - First Stage } \\
\text { onflicting Flows } \\
\text { tential Capacity } \\
\text { testrian Impedance Factor } \\
\text { ap. Adj. factor due to Impeding mvmnt } \\
\text { Movement Capacity. }\end{array}$ & & \\
\hline $\begin{array}{l}\text { rt } 2 \text { - Second Stage } \\
\text { conflicting Flows } \\
\text { tential Capacity } \\
\text { destrian Impedance Factor } \\
\text { ap. Adj. factor due to Impeding mumnt } \\
\text { vement Capacity }\end{array}$ & & \\
\hline $\begin{array}{l}\text { jurt } 3 \text { - Single stage } \\
\text { onnficting Flows } \\
\text { ’ tential Capacity } \\
\text { destrian Impedance Factor } \\
\text { 1aj. L. Min T Impedance factor } \\
\text { j. L, Min T Adj. Imp Factor. } \\
\text { p. Adj. factor due to Impeding mvmnt } \\
\text { lovement Capacity }\end{array}$ & $\begin{array}{l}1.00 \\
0.99 \\
0.99 \\
0.94\end{array}$ & $\begin{array}{l}416 \\
593 \\
1.00\end{array}$ \\
\hline
\end{tabular}

\section{sults for Two-stage process:}




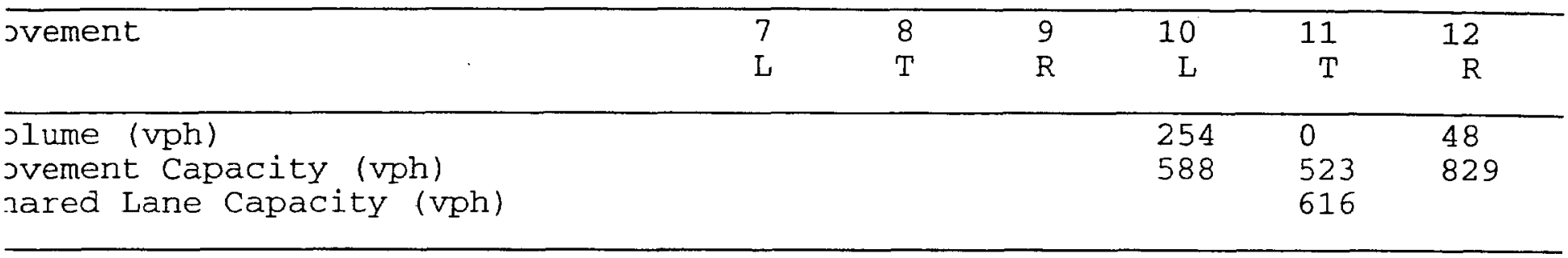

srksheet 9-Computation of Effect of Flared Minor street Approaches

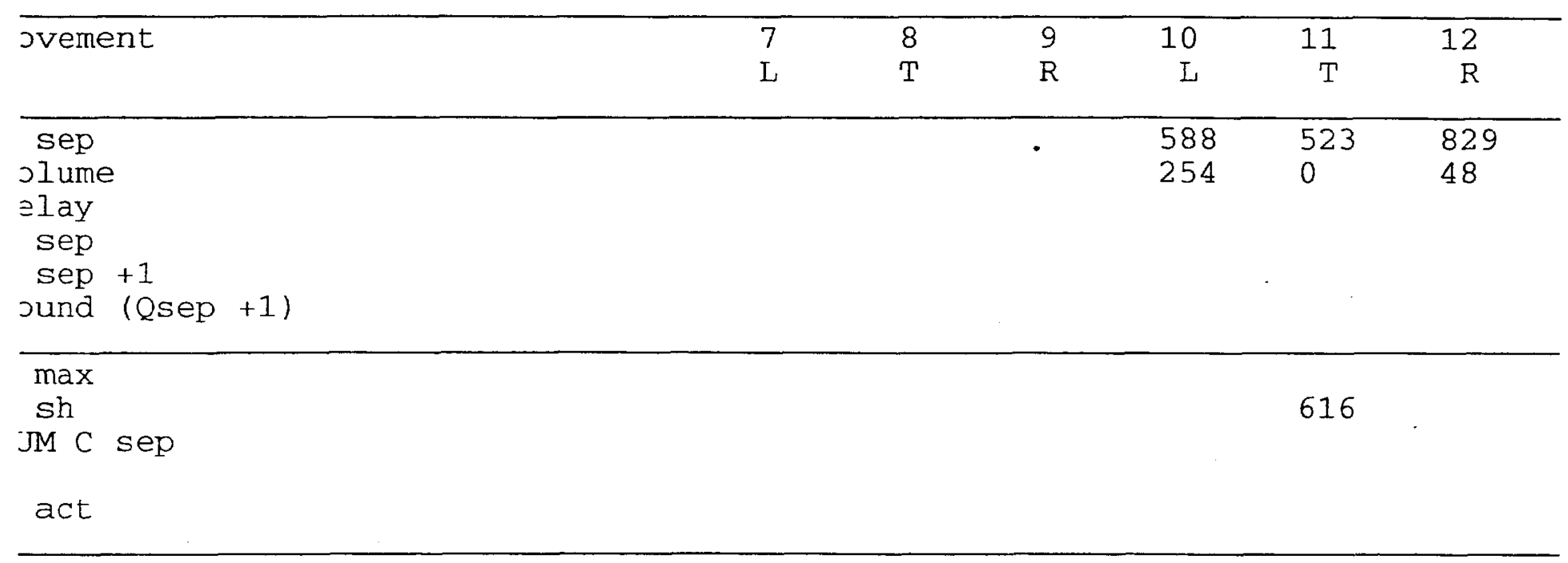

orksheet 10-Delay, Queue Length, and Level of Service

\begin{tabular}{|c|c|c|c|c|c|c|c|c|}
\hline $\begin{array}{l}\text { ovement } \\
\text { ane Config }\end{array}$ & $\begin{array}{l}1 \\
\mathrm{LT} T\end{array}$ & 4 & 7 & 8 & 9 & 10 & $\begin{array}{l}11 \\
\text { LTR }\end{array}$ & 12 \\
\hline $\begin{array}{l}\text { (vph) } \\
\text { (m) (vph) } \\
\text { /C } \\
\text { 5\% queue length } \\
\text { ontrol Delay } \\
\text { os } \\
\text { pproach Delay } \\
\text { pproach Los }\end{array}$ & $\begin{array}{l}10 \\
1317 \\
0.01 \\
0.02 \\
7.8 \\
\mathrm{~A}\end{array}$ & . & & & & & $\begin{array}{l}302 \\
616 \\
0.49 \\
2.70 \\
16.3 \\
C \\
16.3 \\
C\end{array}$ & \\
\hline
\end{tabular}

orksheet 11-Shared Major LT Impedance and Delay

\begin{tabular}{|c|c|c|}
\hline & Movement 2 & Movement 5 \\
\hline $\begin{array}{l}\text { (oj) } \\
\text { (il), Volume for stream } 2 \text { or } 5 \\
\text { (i2), Volume for stream } 3 \text { or } 6 \\
\text { (il), Saturation flow rate for stream } 2 \text { or } 5 \\
\text { (i2); Saturation flow rate for stream } 3 \text { or } 6 \\
\text { * (oj) } \\
\text { (M, LT), Delay for stream } 1 \text { or } 4 \\
\text { Number of major street through lanes } \\
\text { (rank, 1) Delay for stream } 2 \text { or } 5\end{array}$ & $\begin{array}{l}0.99 \\
185 \\
0 \\
1700 \\
1700 \\
0.99 \\
7.8 \\
1 \\
0.1\end{array}$ & 1.00 \\
\hline
\end{tabular}


E. TRAFFIC ANALYSIS

Future Conditions - Year 2020 



$\rightarrow \rightarrow+\infty+1+1+1+1$

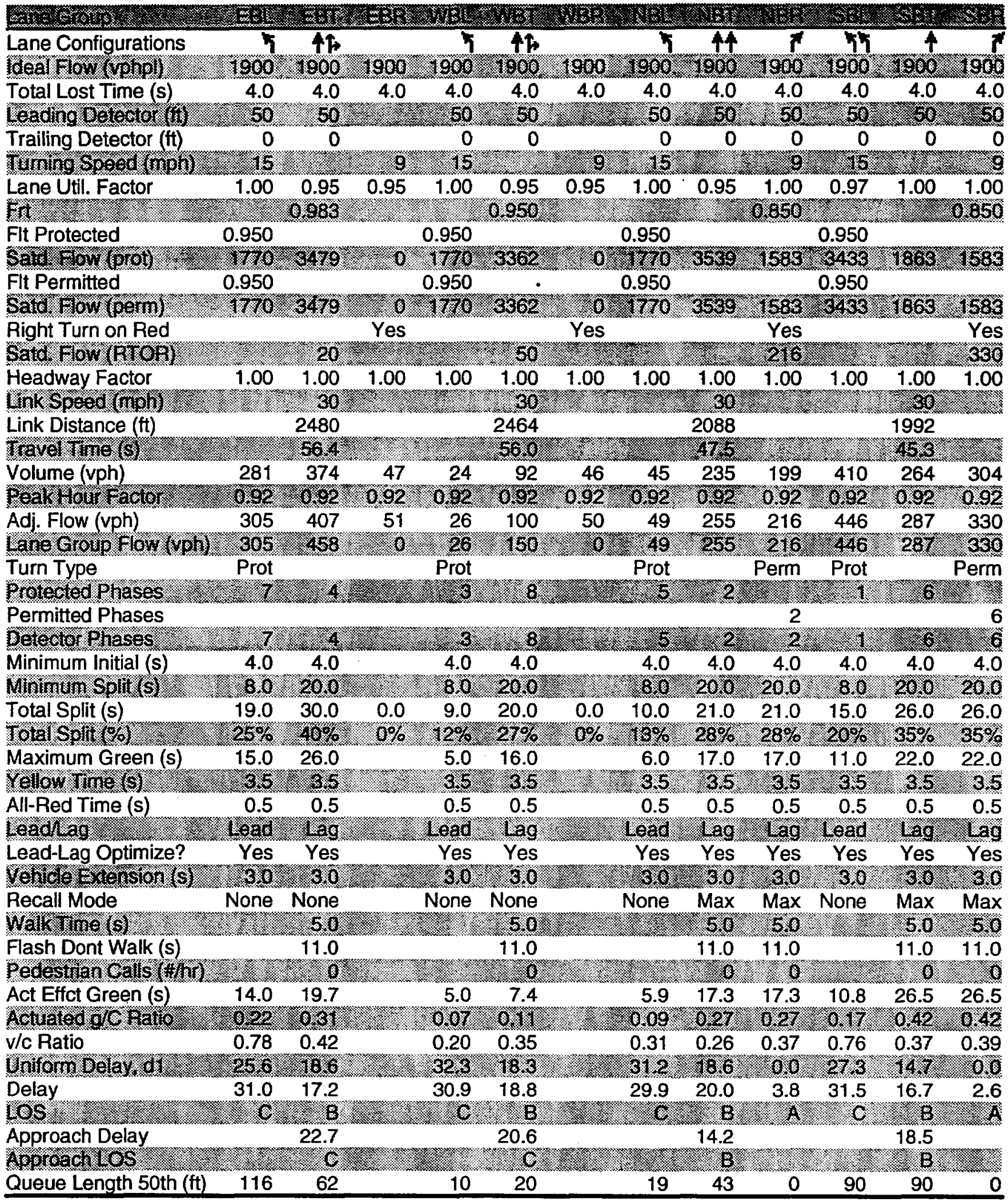




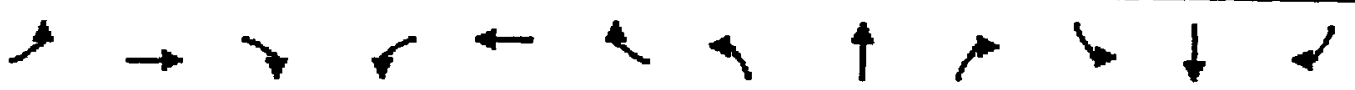

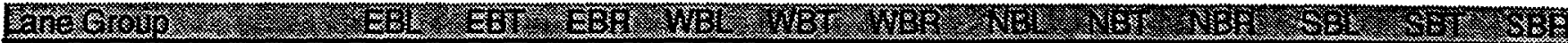

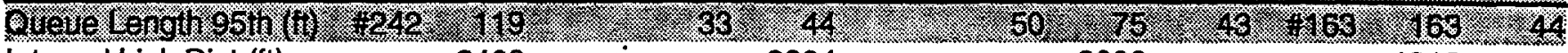

Internal Link Dist ( $\mathrm{ft}$ ) 2400

2384

2008

1912

50 UP B BOCK TIme $(\%)$. 95th Up Block Time (\%)

TumBay Lengi $(\mathrm{T})$ ) 50 th Bay Block Time \%

S5f Bay Block TIMe \% Queuing Penalty (veh)

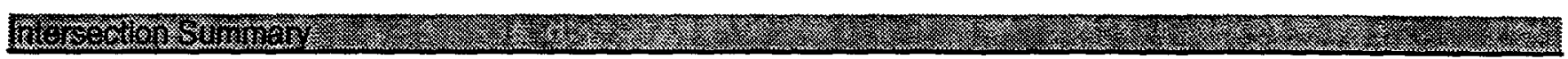

Area Type: Other

Grcle Length 75 . Actuated Cycle Length: 63.3

Natural Cyce 75 . Control Type: Semi Act-Uncoord

Maxinun We Ratio: 076 . Intersection Signal Delay: 19.0 Intersection LOS: B

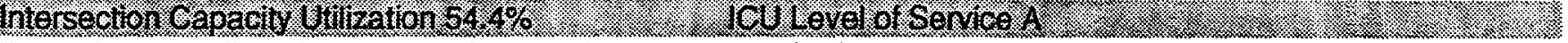
\# 95th percentile volume exceeds capacity, queue may be longer.

Ouene shown is maxinum atter two cyoles

Splits and Phases: 1: East Ave \& Vasco Road

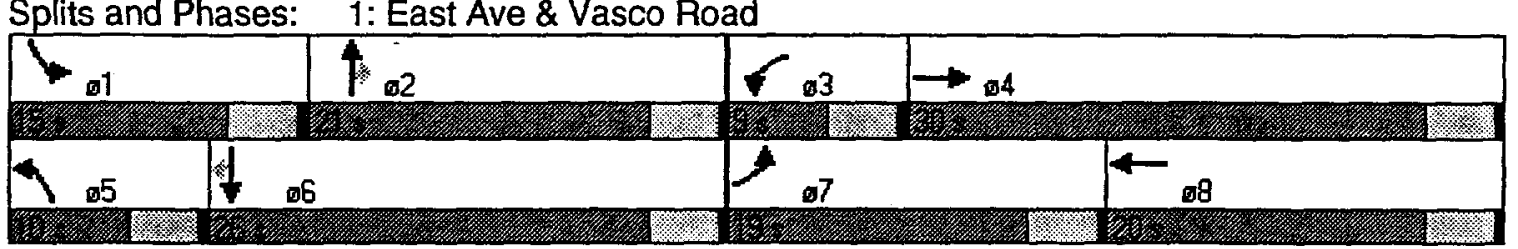




\section{$t \rightarrow \gamma+4+1+b+1$}

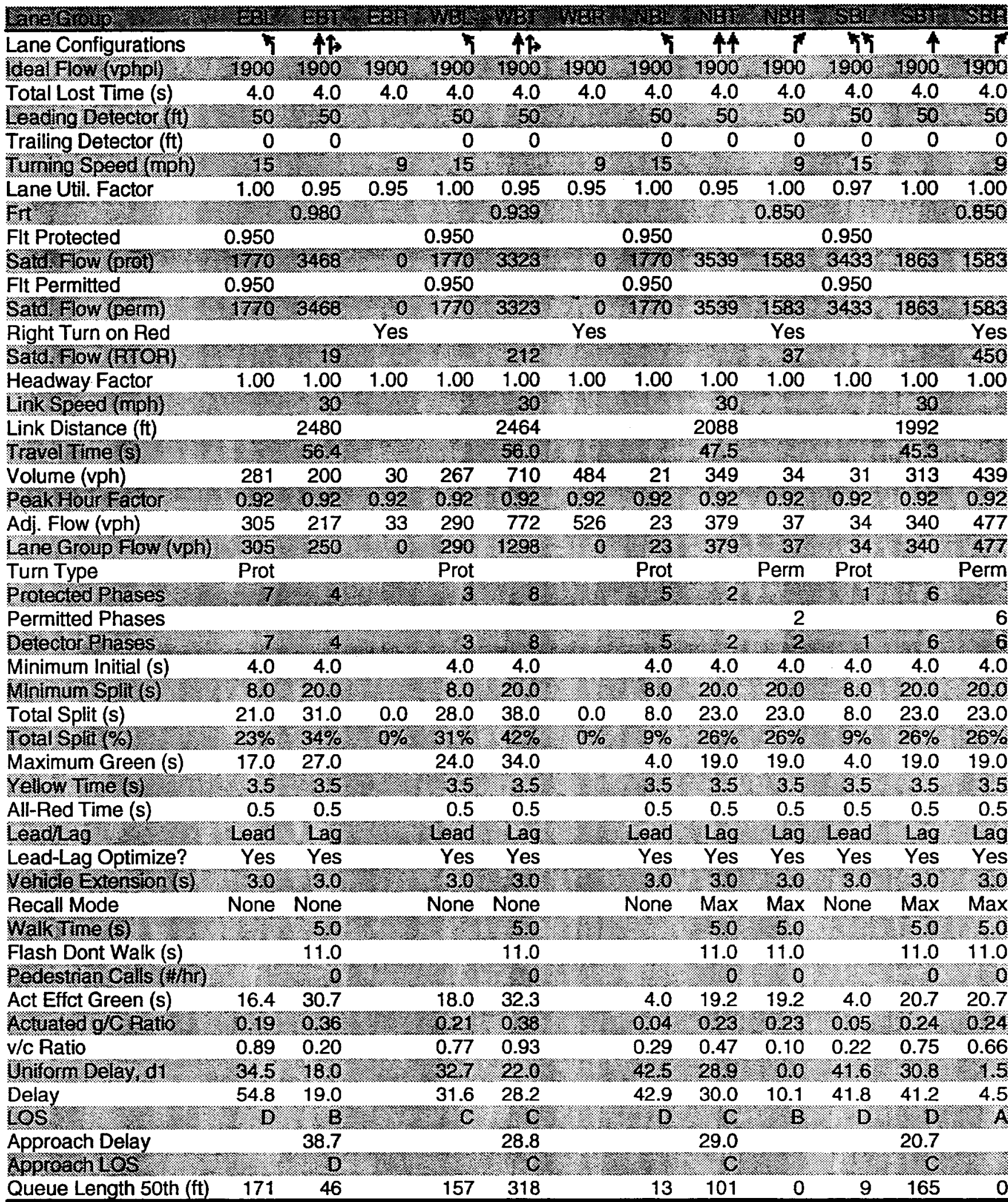

2020 PM Peak $\quad$ Synchro 5 Report

KORVEBAYL4-FF51 


\section{$\rightarrow \rightarrow r+1+1+1+1$}

Lane G.0Y

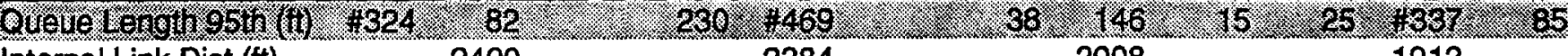

Internal Link Dist (tt) 2400 2384

50hT UP Block TIme $(\%)$. 95th Up Block Time (\%)

Tim Bay Lengf (t) 50 th Bay Block Time \%

gsth Bay Block Tline o Queuing Penalty (veh)

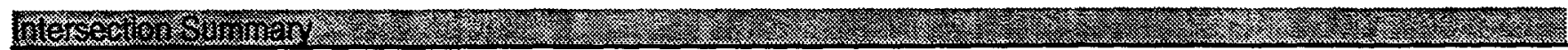

Area Type: Other

Giclelengh 90 : Actuated Cycle Length: 84.5

Natural cycle 90 . Control Type: Semi Act-Uncoord

Maxinum Vo RatG G.93 : Intersection Signal Delay: 28.4 Intersection LOS: C

Intersection Capacily Utilization $83.0 \%$. Ul Level of Serice D \# 95th percentile volume exceeds capacity, queue may be longer.

Queue stown is naxinum after wo cycles.

Splits and Phases: 1: East Ave \& Vasco Road

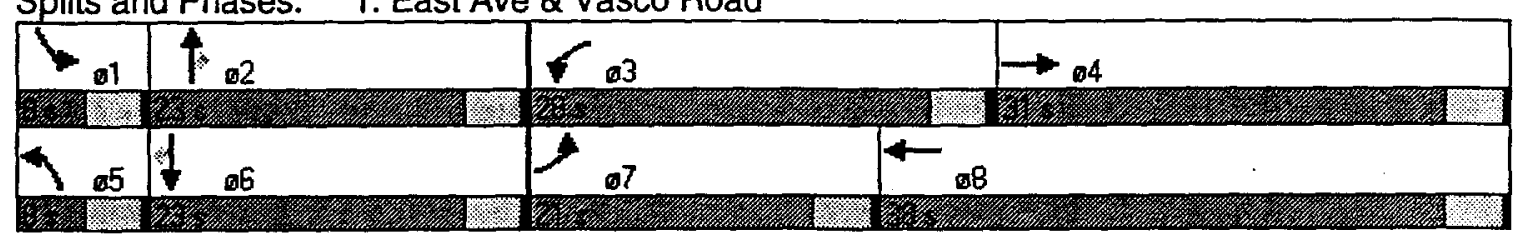


Inalyst:

Agency/Co.:

Jate Performed:

$1 / 21 / 02$

inalysis Time Period: AM Peak

intersection:

East Ave \& SW Entrance (Location \#4)

Jurisdiction:

tnalysis Year:

rroject ID: Lawrence Livermore National Laboratory

East/West Street: East Ave

Jorth/South Street: SW Entrance/West Perimeter Dr

intersection orientation: EW $\quad$ study period (hrs): 1.00

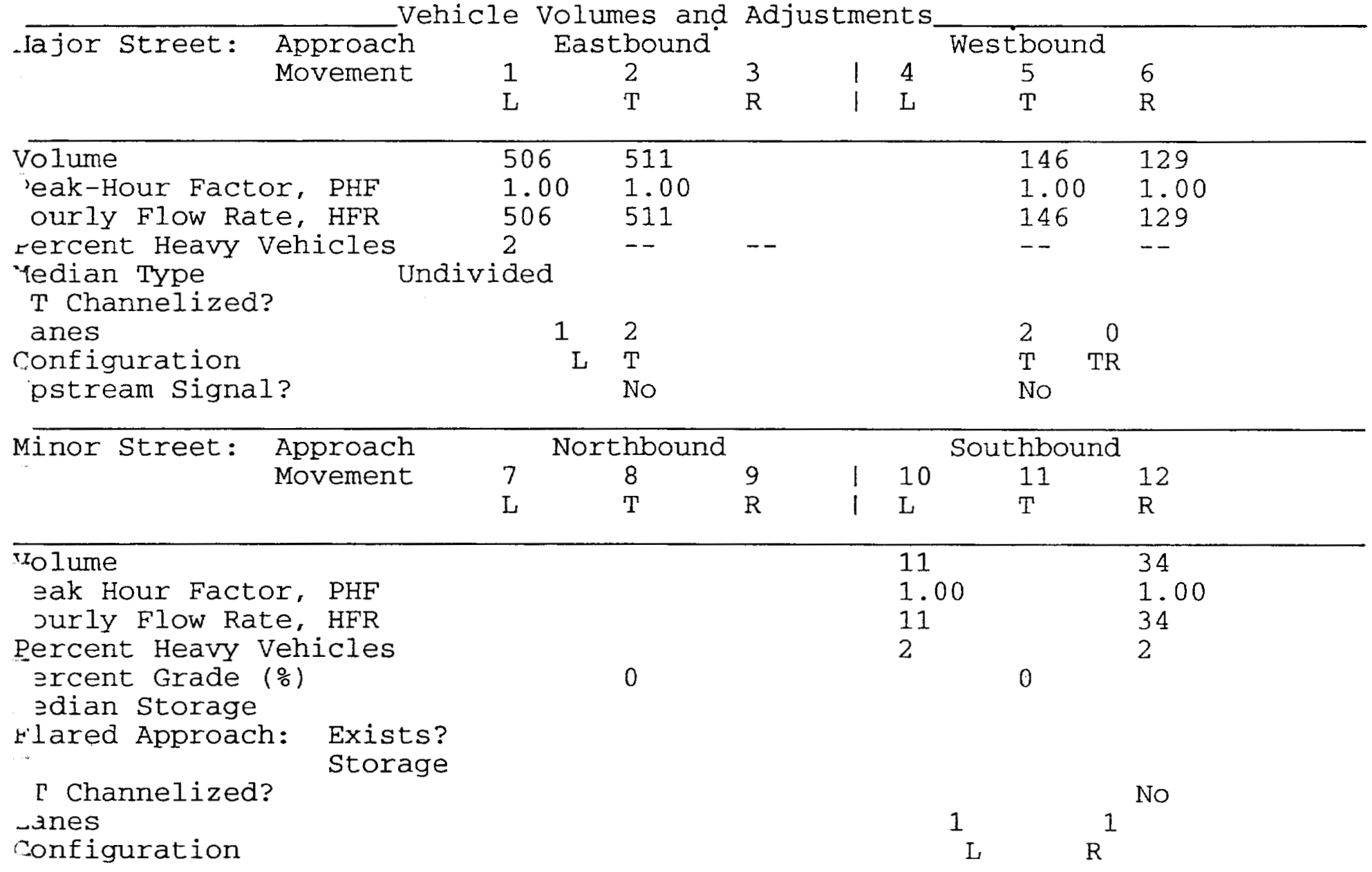

Delay, Queue Length, and Level of Service

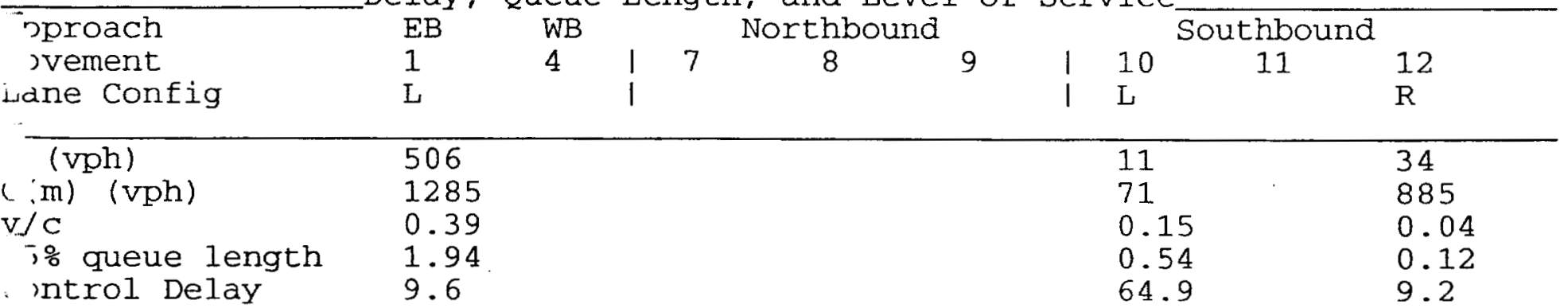




\section{HCS2000: Unsignalized Intersections Release 4.1}

Korve Engineering, Inc.

Phone:

Fax:

E-Mail:

TWO-WAY STOP CONTROL(TWSC) ANALYSIS

Analyst :

Agency/Co.:

Date Performed: 1/21/02

Analysis Time Period: AM Peak

Intersection: East Ave \& SW Entrance

Jurisdiction:

Analysis Year: 2020

Project ID: Lawrence Livermore National Laboratory

East/West Street: East Ave

North/South Street: SW Entrance/West Perimeter Dr

Intersection orientation: EW Study period (hrs): 1.00

Vehicle Volumes and Adjustments.

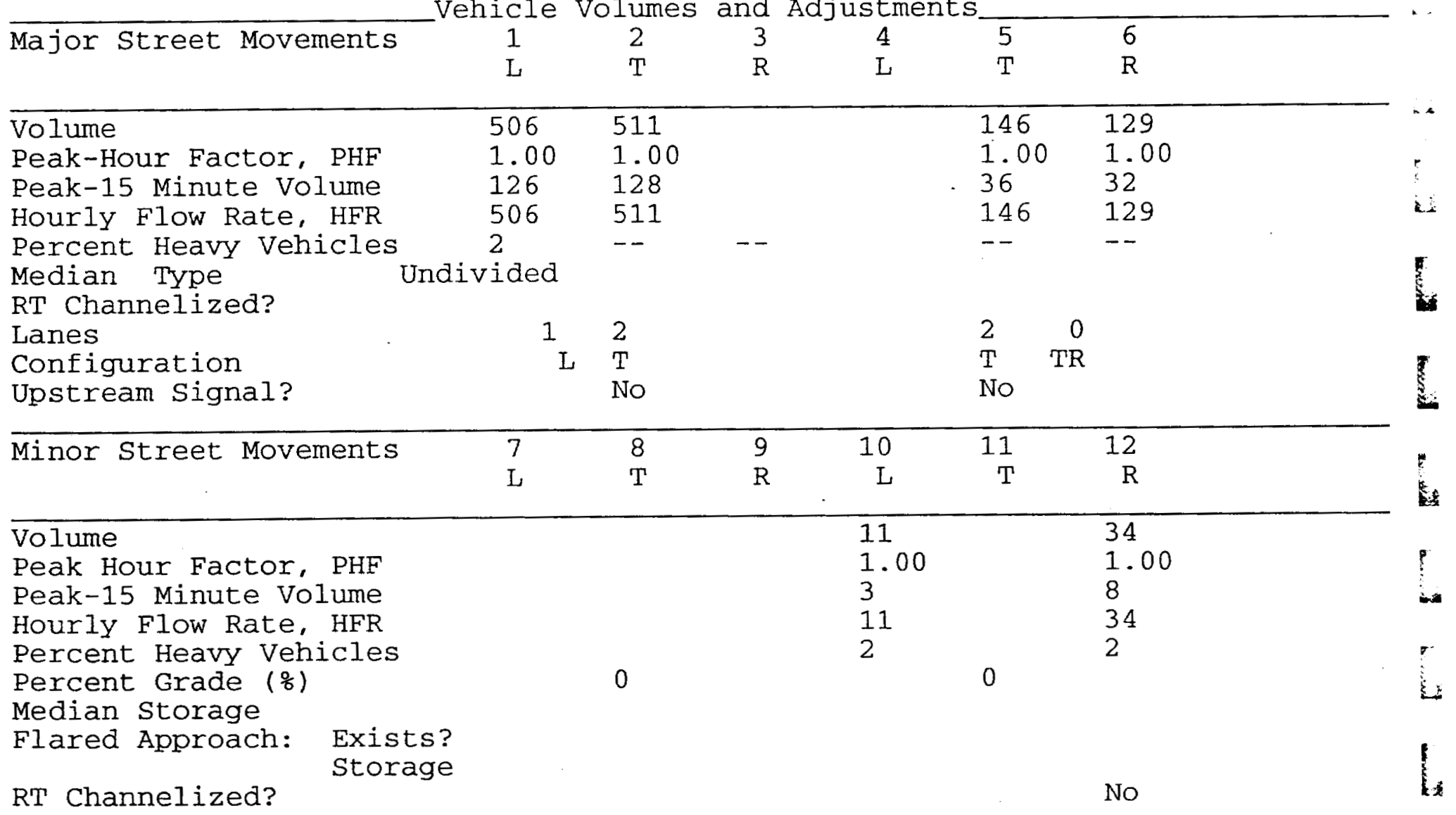


Pedestrian Volumes and Adjustments

\begin{tabular}{lllll}
\hline Movements & 13 & 14 & 15 & 16 \\
\hline Flow (ped/hr) & & & & \\
Jane Width (ft) & 12.0 & 0 & 0 & 0 \\
Nalking Speed (ft/sec) & $4.0 \cdot$ & 4.0 & 4.0 & 4.0 \\
Percent Blockage & 0 & 0 & 0 & 0
\end{tabular}

\begin{tabular}{llllll}
\hline \multicolumn{1}{l}{ Upstream Signal Data } \\
\hline Prog. & Sat Arrival Green Cycle & Prog. & Distance \\
Flow & Flow Type & Time & Length & Speed & to Signal \\
vph & vph & sec & sec & mph & feet
\end{tabular}

\section{i2 Left-Turn \\ Through \\ S5 Left-Turn \\ Through}

Torksheet 3-Data for Computing Effect of Delay to Major Street Vehicles

Movement 2 Movement 5

hared In volume, major th vehicles:

-hared In volume, major rt vehicles:

sat flow rate, major th vehicles:

at flow rate, major rt vehicles:

umber of major street through lanes:

orksheet 4-Critical Gap and Follow-up Time Calculation

\begin{tabular}{|c|c|c|c|c|c|c|c|c|c|}
\hline $\begin{array}{l}\text { sritical } \\
\text { ovement }\end{array}$ & Gap Cal & $\begin{array}{l}\mathrm{L} \text { lat } \\
\mathrm{L}\end{array}$ & $\begin{array}{l}4 \\
\mathrm{~L}\end{array}$ & $\begin{array}{l}7 \\
\text { L }\end{array}$ & $\begin{array}{l}8 \\
\mathrm{~T}\end{array}$ & $\begin{array}{l}9 \\
\mathrm{R}\end{array}$ & $\begin{array}{r}10 \\
\mathrm{~L}\end{array}$ & $\begin{array}{r}11 \\
\mathrm{~T}\end{array}$ & $\begin{array}{r}12 \\
\mathrm{R}\end{array}$ \\
\hline $\begin{array}{l}\text { (c, base) } \\
(\mathrm{c}, \mathrm{hv}) \\
\mathrm{p}(\mathrm{hv}) \\
(\mathrm{c}, \mathrm{g}) \\
\text { rade } / 100 \\
(3, \mathrm{t}) \\
\mathrm{t}(\mathrm{c}, \mathrm{T}): \\
\text { (c) }\end{array}$ & $\begin{array}{l}\text { 1-stage } \\
\text { 2-stage } \\
1 \text {-stage } \\
\text { 2-stage }\end{array}$ & $\begin{array}{l}0.00 \\
0.00 \\
0.00 \\
4.1\end{array}$ & $\begin{array}{l}0.00 \\
0.00\end{array}$ & $\begin{array}{l}2.00 \\
0.20 \\
0.00 \\
0.00 \\
1.00\end{array}$ & $\begin{array}{l}2.00 \\
0.20 \\
0.00 \\
0.00 \\
1.00\end{array}$ & $\begin{array}{l}2.00 \\
0.10 \\
0.00 \\
0.00 \\
0.00\end{array}$ & $\begin{array}{l}7.5 \\
2.00 \\
2 \\
0.20 \\
0.00 \\
0.70 \\
0.00 \\
1.00 \\
6.8\end{array}$ & $\begin{array}{l}2.00 \\
0.20 \\
0.00 \\
0.00 \\
1.00\end{array}$ & $\begin{array}{l}6.9 \\
2.00 \\
2 \\
0.10 \\
0.00 \\
0.00 \\
0.00 \\
0.00 \\
6.9\end{array}$ \\
\hline $\begin{array}{l}\text { ollow-Up } \\
\text { Movement }\end{array}$ & Time C & $\begin{array}{l}1 \mathrm{cul} \\
1 \\
\mathrm{~L}\end{array}$ & $\begin{array}{c}\text { Ons } \\
4 \\
\text { L }\end{array}$ & $\begin{array}{l}7 \\
\mathrm{~L}\end{array}$ & $\begin{array}{l}8 \\
\mathrm{~T}\end{array}$ & $\begin{array}{l}9 \\
\mathrm{R}\end{array}$ & $\begin{array}{r}10 \\
\mathrm{~L}\end{array}$ & $\begin{array}{r}11 \\
\mathrm{~T}\end{array}$ & $\begin{array}{r}12 \\
\mathrm{R}\end{array}$ \\
\hline $\begin{aligned}- & (\mathrm{f}, \text { base }) \\
t & (\mathrm{f}, \mathrm{HV}) \\
& (\mathrm{HV}) \\
& (\mathrm{f})\end{aligned}$ & & $\begin{array}{l}2.20 \\
1.00 \\
2 \\
2.2\end{array}$ & 1.00 & 1.00 & 1.00 & 1.00 & $\begin{array}{l}3.50 \\
1.00 \\
2 \\
3.5\end{array}$ & 1.00 & $\begin{array}{l}3.30 \\
1.00 \\
2 \\
3.3\end{array}$ \\
\hline
\end{tabular}


Worksheet 5-Effect of Upstream Signals

Computation 1-Queue Clearance Time at Upstream Signal

Movement 2

$V(t) \quad V(1$, prot $) \quad V(t) \quad V(1$, prot $)$

$\mathrm{V}$ prog

Total Saturation Flow Rate, s (vph)

Arrival Type

Effective Green, g (sec)

Cycle Length, C (sec)

Rp (from table 9-2)

Proportion vehicles arriving on green $P$

$g(q 1)$

$g(q 2)$

$g(q)$

Computation 2-Proportion of TWSC Intersection Time blocked $\begin{array}{ccc}\text { Movement } 2 & \text { Movement } 5 \\ V(t) \quad V(1, \text { prot }) & V(t) \quad V(1, \text { prot })\end{array}$

\section{a)pha}

beta

Travel time, $t(a)$ (sec)

Smoothing Factor, $\mathrm{F}$

Proportion of conflicting flow, $f$

Max platooned flow, $V(c, \max )$

Min platooned flow, $V(c, \min )$

Duration of blocked period, $t(p)$

Proportion time blocked, p

0.000

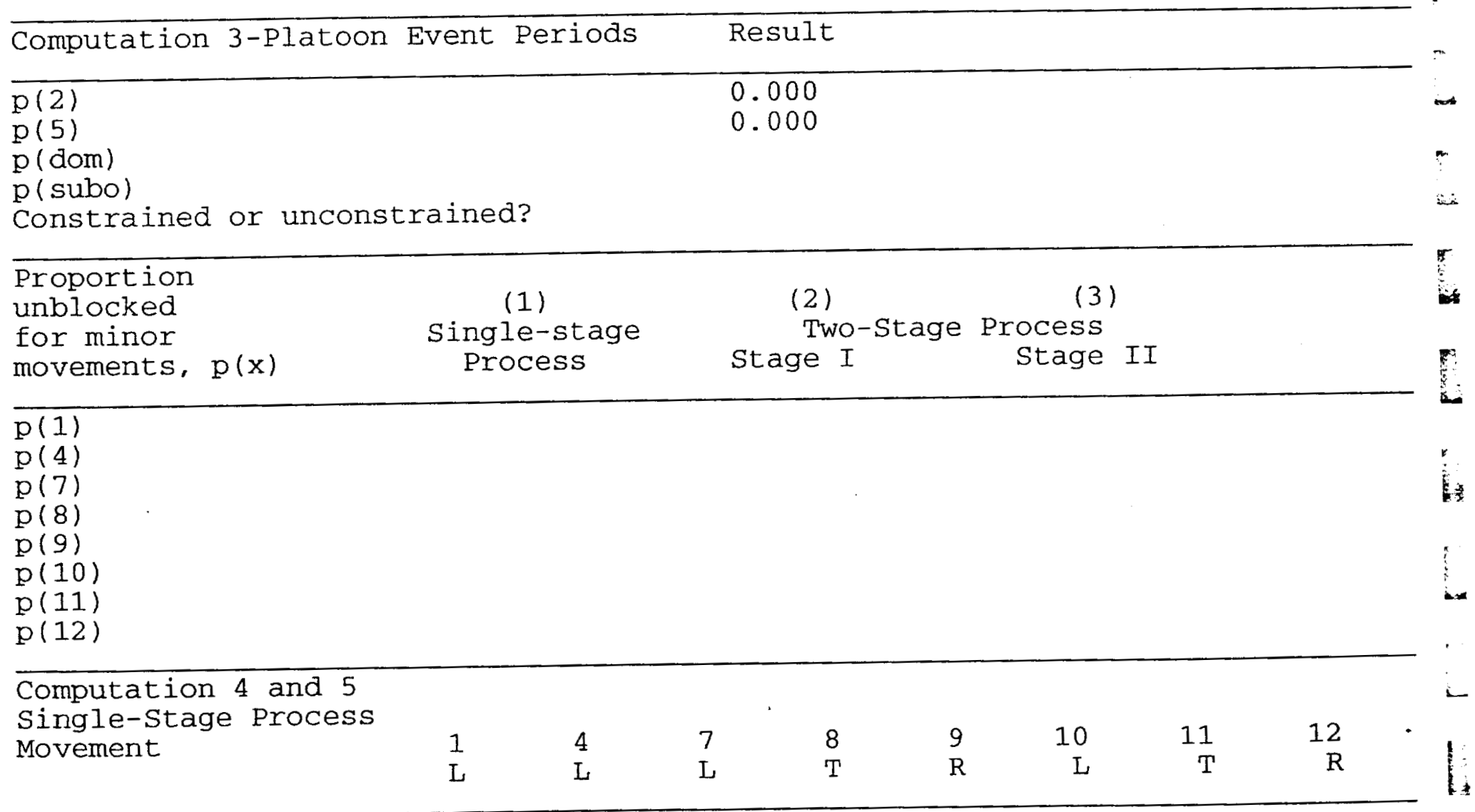




\section{Iwo-Stage Process}

$7 \quad 8 \quad 10$

11

Stage1 Stage2 Stage1 Stage2 stage1 Stage2 stage1 Stage2

$J(c, x)$

$\mathrm{s}$

$?(\mathrm{x})$

3400

$I(\mathrm{c}, \mathrm{u}, \mathrm{x})$

$\overline{Y(r, x)}$

$?($ plat,$x)$

Jorksheet 6-Impedance and Capacity Equations

\begin{tabular}{|c|c|c|}
\hline Step 1: RT from Minor st. & 9 & 12 \\
\hline $\begin{array}{l}\text { Sonflicting Flows } \\
\text { Potential Capacity } \\
\text { 'edestrian Impedance Factor } \\
\text { lovement Capacity } \\
\text { 'robability of queue free st. }\end{array}$ & $\begin{array}{l}1.00 \\
1.00\end{array}$ & $\begin{array}{l}138 \\
885 \\
1.00 \\
885 \\
0.96\end{array}$ \\
\hline itep 2: LT from Major st. & 4 & 1 \\
\hline $\begin{array}{l}\text { Conflicting Flows } \\
\text { 'otential Capacity } \\
\text { 'edestrian Impedance Factor } \\
\text { rovement Capacity } \\
\text { 'robability of Queue free St. } \\
\text { aj L-Shared Prob o free St. }\end{array}$ & $\begin{array}{l}1.00 \\
1.00\end{array}$ & $\begin{array}{l}275 \\
1285 \\
1.00 \\
1285 \\
0.61\end{array}$ \\
\hline
\end{tabular}

\begin{tabular}{lcc}
\hline Step 3: TH from Minor St. & 8 & 11 \\
\hline onflicting Flows & & \\
Potential Capacity & & 1.00 \\
'edestrian Impedance Factor & 1.00 & 0.61 \\
ap. Adj. factor due to Impeding mvmnt & 0.61 & 1.00 \\
.ovement Capacity & 1.00 & 10 \\
Probability of Queue free St. & 7 & 1477 \\
tep 4: LT from Minor St. & 117 \\
-onflicting Flows & 1.00 \\
otential Capacity & 1.00 & 0.61 \\
redestrian Impedance Factor & 0.69 & 0.61 \\
aj. L, Min T Impedance factor & 0.67 & 71 \\
-ap. Adj. factor due to Impeding mvmnt & & \\
Movement Capacity &
\end{tabular}


Part 1 - First Stage

Conflicting Flows

Potential Capacity

Pedestrian Impedance Factor

Cap. Adj. factor due to Impeding mumnt

Movement Capacity

Probability of Queue free $\mathrm{St}$.

Part 2 - Second Stage

Conflicting Flows

Potential Capacity

Pedestrian Impedance Factor

Cap. Adj. factor due to Impeding mvmnt

Movement Capacity

Part 3 - Single stage

Conflicting Flows

Potential Capacity

Pedestrian Impedance Factor

Cap. Adj. factor due to Impeding mvmnt

1.00

1.00

Movement Capacity

Result for 2 stage process:

a

Y

C $t$

Probability of Queue free St.

1.00

1.00

Step 4: LT from Minor st.

7

10

Part 1 - First Stage

Conflicting Flows

Potential Capacity

Pedestrian Impedance Factor

Cap. Adj. Factor due to Impeding mumnt

Movement Capacity

Part 2 - Second stage

Conflicting Flows

Potential Capacity

Pedestrian Impedance Factor

Cap. Adj. factor due to Impeding mumnt

Movement Capacity

Part 3 - Single stage

Conflicting Flows

Potential Capacity

Pedestrian Impedance Factor

117

Maj. L, Min T Impedance factor

Maj. L, Min T Adj. Imp Factor.

1.00

1.00

Cap. Adj. factor due to Impeding mvmnt

0.61

0.69

Movement Capacity

0.67

0.61

71

Results for Two-stage process:

a

y 
Worksheet 8-Shared Lane Calculations

\begin{tabular}{|c|c|c|c|c|c|c|}
\hline sovement & $\begin{array}{l}7 \\
\mathrm{~L}\end{array}$ & $\begin{array}{l}8 \\
T\end{array}$ & $\begin{array}{l}9 \\
R\end{array}$ & $\begin{array}{r}10 \\
\mathrm{~L}\end{array}$ & $\begin{array}{r}11 \\
\mathrm{~T}\end{array}$ & $\begin{array}{r}12 \\
\mathrm{R}\end{array}$ \\
\hline $\begin{array}{l}\text { Tolume (vph) } \\
\text { Hovement Capacity (vph) } \\
\text { Thared Lane Capacity (vph) }\end{array}$ & & & & $\begin{array}{l}11 \\
71\end{array}$ & & $\begin{array}{l}34 \\
885\end{array}$ \\
\hline
\end{tabular}

Worksheet 9-Computation of Effect of Flared Minor Street Approaches

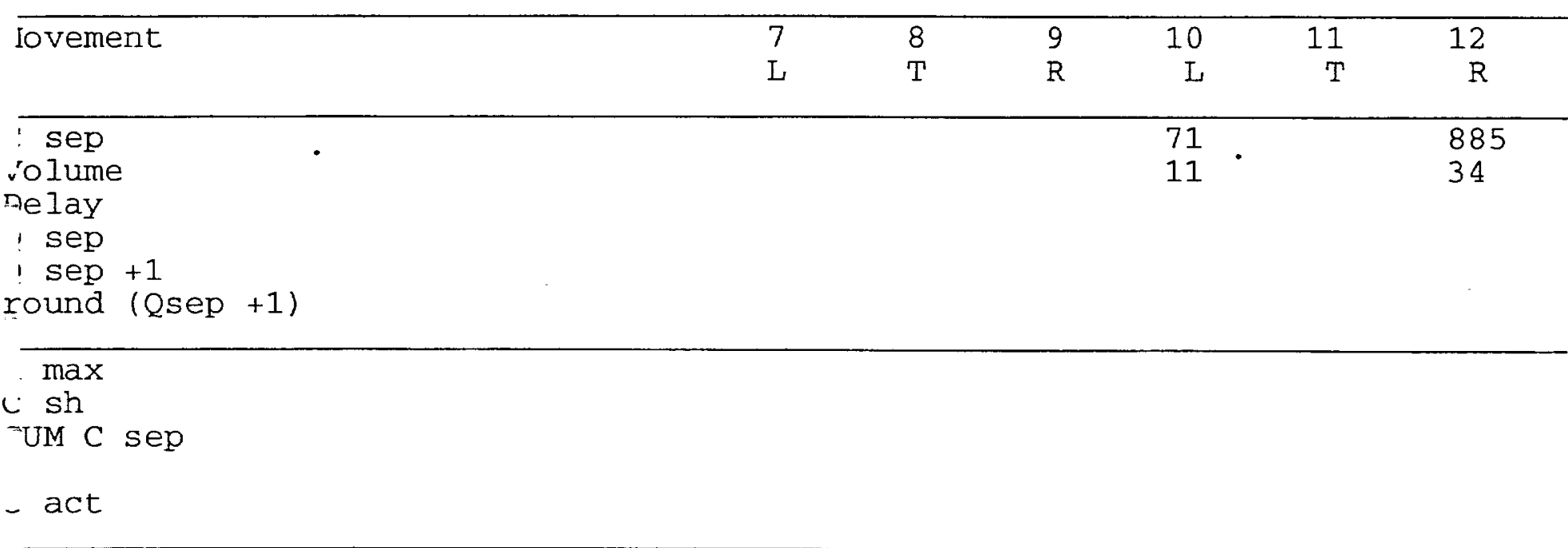

orksheet 10-Delay, Queue Length, and Level of Service

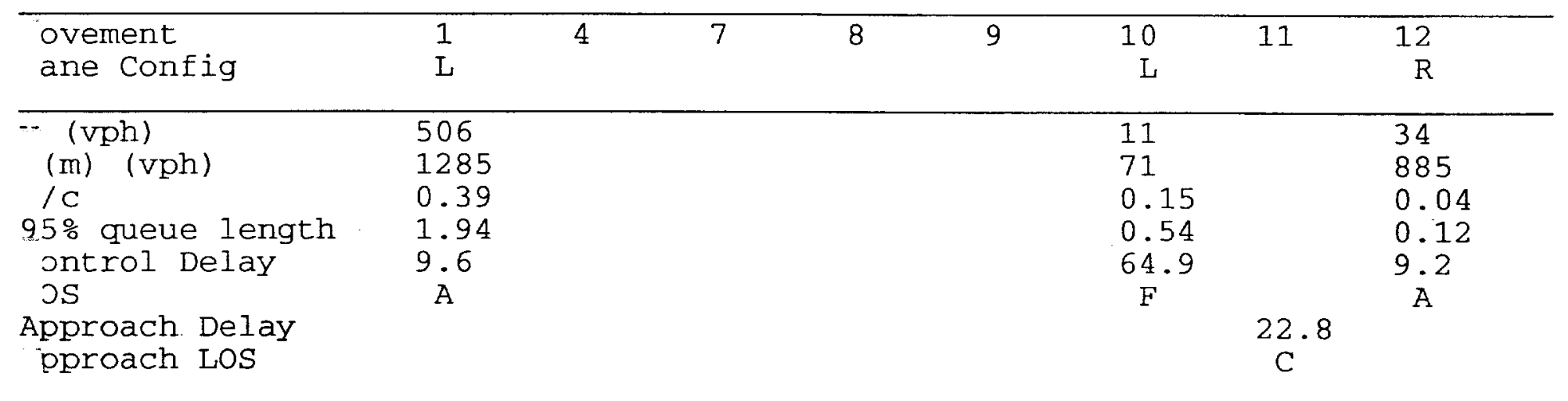

rarksheet 11-Shared Major LT Impedance and Delay

Movement 2 Movement 5

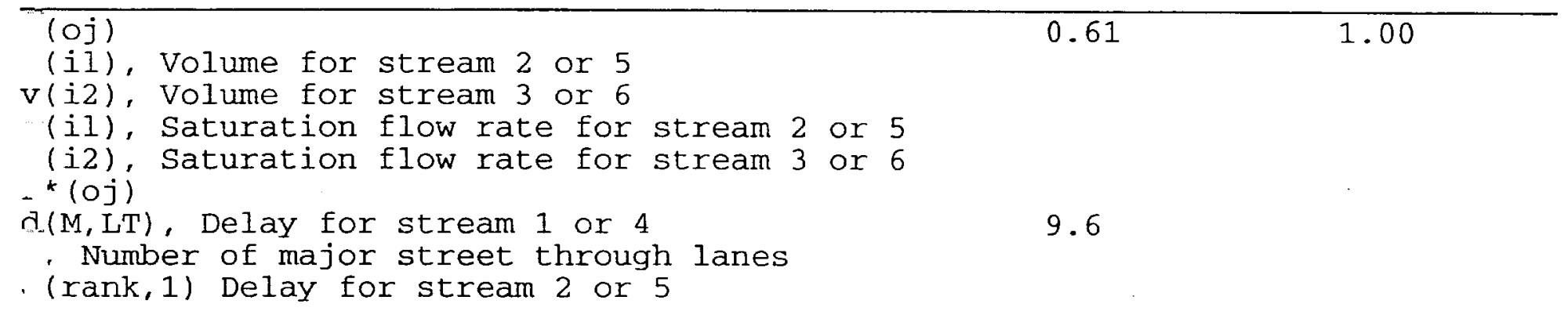


Analyst :

Agency/Co.:

Date Performed: 1/21/02

Analysis Time Period: PM Peak

Intersection:

East Ave \&.SW Entrance (Location \#4)

Jurisdiction:

Analysis Year: 2020

Project ID: Lawrence Livermore National Laboratory

East/West Street: East Ave

North/South Street: SW Entrance/West Perimeter Dr

Intersection Orientation: EW

study period (hrs): 1.00

Vehicle Volumes and Adjustments

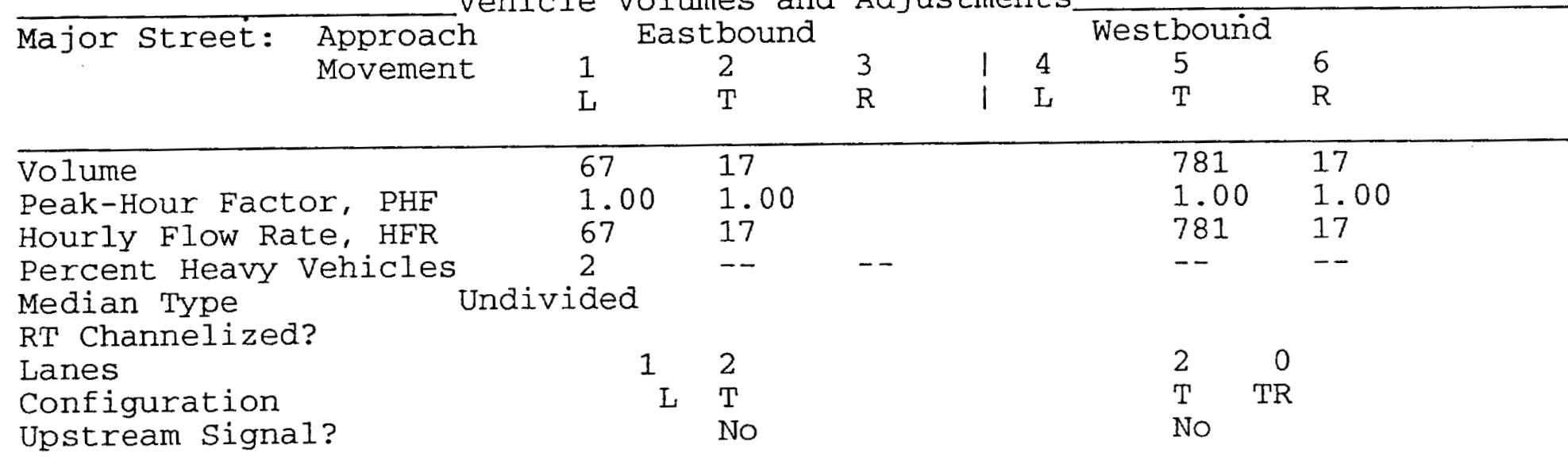

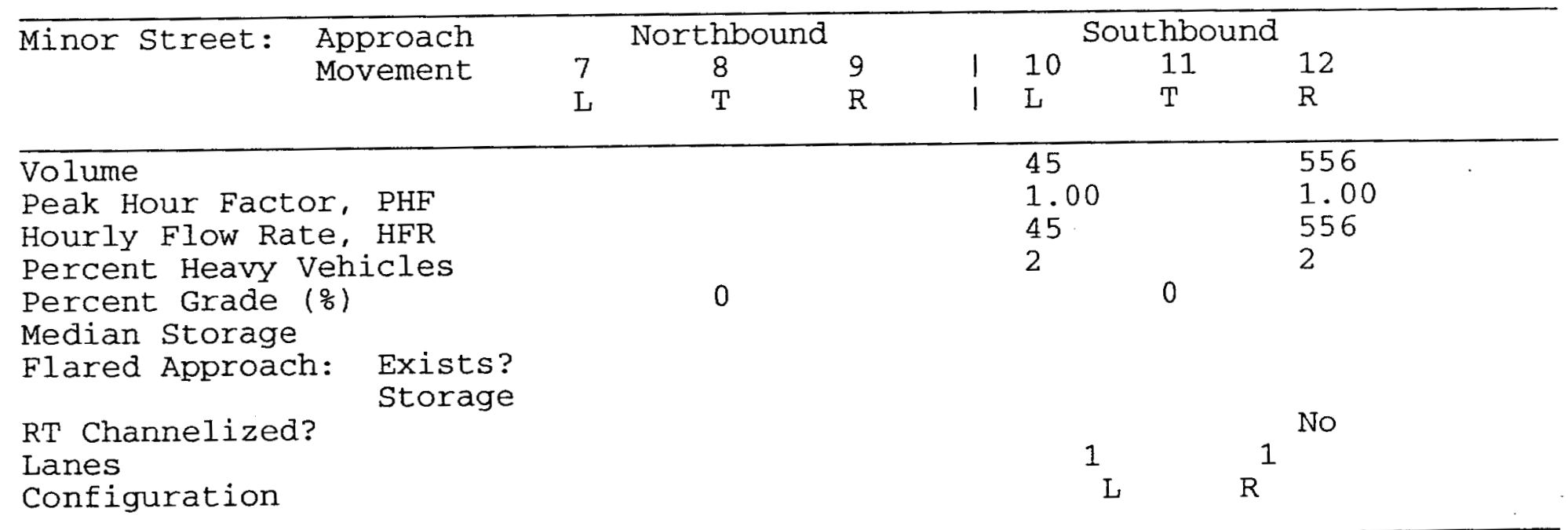

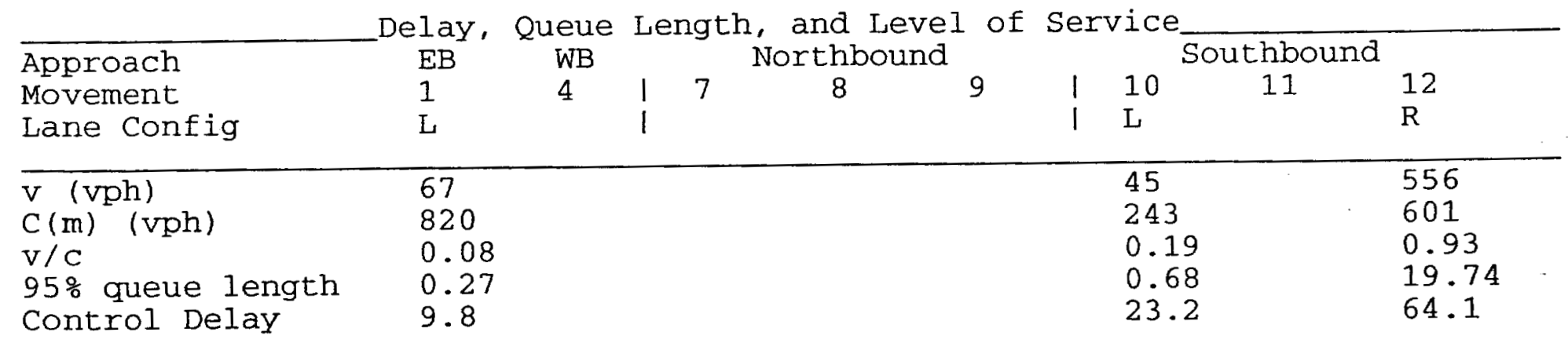




\section{HCS2000: Unsignalized Intersections Release 4.1}

Korve Engineering, Inc.

shone:

Fax:

i-Mail:

TWO-WAY STOP CONTROL(TWSC) ANALYSIS

inalyst :

Agency/CO.:

late Performed: 1/21/02

nalysis Time Period: PM Peak

Intersection: East Ave \& SW Entrance

"urisdiction:

nalysis Year:

2020

project ID: Lawrence Livermore National Laboratory

mast/West street: East Ave

lorth/South Street: SW Entrance/West Perimeter Dr

Tntersection orientation: EW Study period (hrs): 1.00

Vehicle Volumes and Adjustments

Major street Movements

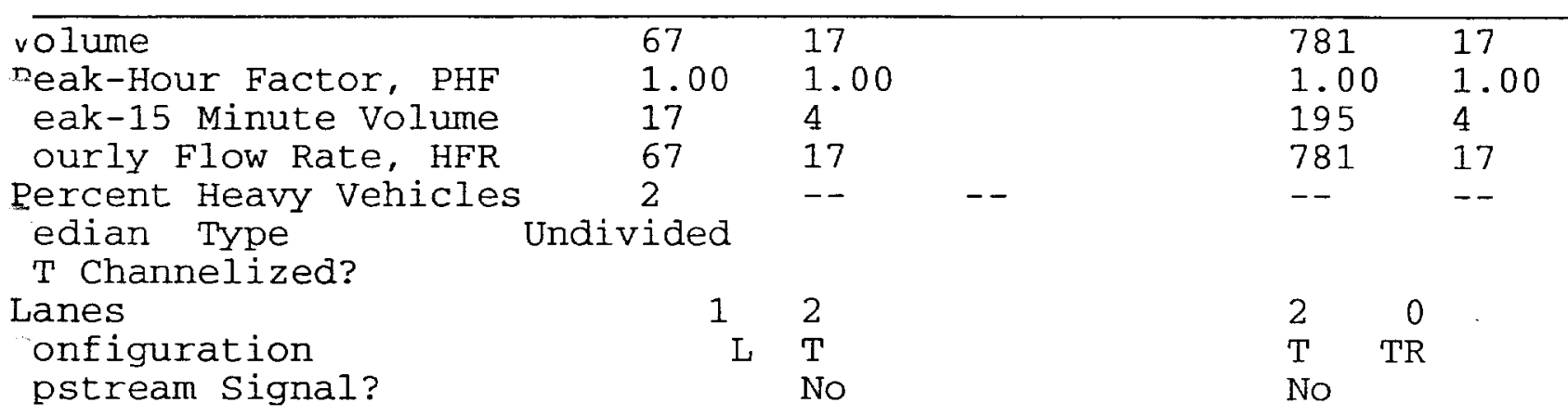

\begin{tabular}{|c|c|c|c|c|c|c|}
\hline Minor Street Movements & $\begin{array}{l}7 \\
\mathrm{~L}\end{array}$ & $\begin{array}{l}8 \\
T\end{array}$ & $\begin{array}{l}9 \\
\mathrm{R}\end{array}$ & $\begin{array}{r}10 \\
\mathrm{~L}\end{array}$ & $\begin{array}{r}11 \\
\mathrm{~T}\end{array}$ & $\begin{array}{r}12 \\
\mathrm{R}\end{array}$ \\
\hline $\begin{array}{l}\text { Volume } \\
\text { eak Hour Factor, PHF } \\
\text { eak-15 Minute Volume } \\
\text { Hourly Flow Rate, HFR } \\
\text { ercent Heavy Vehicles } \\
\text { ercent Grade ( } \%)\end{array}$ & & 0 & & $\begin{array}{l}45 \\
1.00 \\
11 \\
45 \\
2\end{array}$ & 0 & $\begin{array}{l}556 \\
1.00 \\
139 \\
556 \\
2\end{array}$ \\
\hline
\end{tabular}

. adian Storage

Flared Approach: Exists? 
Pedestrian Volumes and Adjustments.

\begin{tabular}{lllll}
\hline Movements & 13 & 14 & 15 & 16 \\
\hline Flow (ped/hr) & 0 & 0 & 0 & 0 \\
Lane Width (ft) & 12.0 & 12.0 & 12.0 & 12.0 \\
Walking Speed (ft/sec) & 4.0 & 4.0 & 4.0 & 4.0 \\
Percent Blockage & 0 & 0 & 0 & 0
\end{tabular}

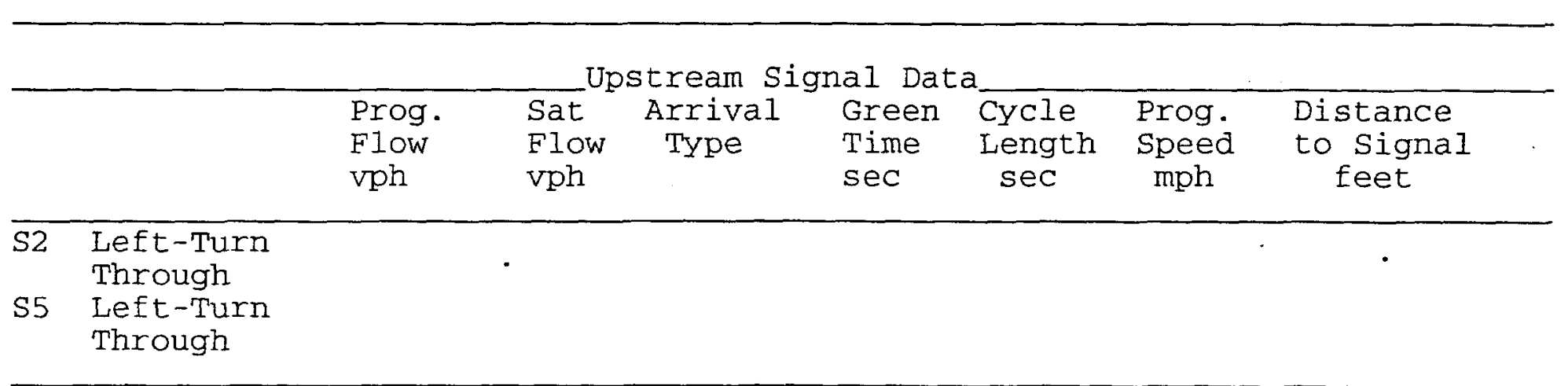

Worksheet 3-Data for Computing Effect of Delay to Major Street Vehicles

\section{Movement 2 Movement 5}

Shared In volume, major th vehicles:

Shared In volume, major rt vehicles:

Sat flow rate, major th vehicles:

Sat flow rate, major rt vehicles:

Number of major street through lanes:

Worksheet 4-Critical Gap and Follow-up Time Calculation

\begin{tabular}{|c|c|c|c|c|c|c|c|c|c|}
\hline $\begin{array}{l}\text { Critical } \\
\text { Movement }\end{array}$ & Gap Calc & $\begin{array}{c}\text { culati } \\
1 \\
\mathrm{~L}\end{array}$ & $\begin{array}{l}4 \\
L\end{array}$ & $\begin{array}{l}7 \\
\mathrm{~L}\end{array}$ & $\begin{array}{l}8 \\
\mathrm{~T}\end{array}$ & $\begin{array}{l}9 \\
\mathrm{R}\end{array}$ & $\begin{array}{r}10 \\
\mathrm{~L}\end{array}$ & $\begin{array}{r}11 \\
\mathrm{~T}\end{array}$ & $\begin{array}{r}12 \\
\mathrm{R}\end{array}$ \\
\hline $\begin{array}{l}t(c, \text { base }) \\
t(c, h v) \\
P(h v) \\
t(c, g) \\
\text { Grade/100 } \\
t(3,1 t) \\
t(c, T): \\
t(c)\end{array}$ & $\begin{array}{l}\text { 1-stage } \\
\text { 2-stage } \\
1 \text {-stage } \\
\text { 2-stage }\end{array}$ & $\begin{array}{l}0.00 \\
0.00 \\
0.00 \\
4.1\end{array}$ & $\begin{array}{l}0.00 \\
0.00\end{array}$ & $\begin{array}{l}2.00 \\
0.20 \\
0.00 \\
0.00 \\
1.00\end{array}$ & $\begin{array}{l}2.00 \\
0.20 \\
0.00 \\
0.00 \\
1.00\end{array}$ & $\begin{array}{l}2.00 \\
0.10 \\
0.00 \\
0.00 \\
0.00\end{array}$ & $\begin{array}{l}7.5 \\
2.00 \\
2 \\
0.20 \\
0.00 \\
0.70 \\
0.00 \\
1.00 \\
6.8\end{array}$ & $\begin{array}{l}2.00 \\
0.20 \\
0.00 \\
0.00 \\
1.00\end{array}$ & $\begin{array}{l}6.9 \\
2.00 \\
2 \\
0.10 \\
0.00 \\
0.00 \\
0.00 \\
0.00 \\
6.9\end{array}$ \\
\hline $\begin{array}{l}\text { Follow-Up } \\
\text { Movement }\end{array}$ & Time $\mathrm{Ca}$ & $\begin{array}{c}\text { a cula } \\
1 \\
\mathrm{~L}\end{array}$ & $\begin{array}{c}\text { ions } \\
4 \\
\text { L }\end{array}$ & $\begin{array}{l}7 \\
L\end{array}$ & $\begin{array}{l}8 \\
\mathrm{~T}\end{array}$ & $\begin{array}{l}9 \\
\mathrm{R}\end{array}$ & $\begin{array}{r}10 \\
I\end{array}$ & $\begin{array}{r}11 \\
\mathrm{~T}\end{array}$ & $\begin{array}{r}12 \\
\mathrm{R}\end{array}$ \\
\hline $\begin{array}{l}t(f, \text { base }) \\
t(f, H V) \\
P(H V) \\
t(f)\end{array}$ & & $\begin{array}{l}2.20 \\
1.00 \\
2 \\
2.2\end{array}$ & 1.00 & 1.00 & 1.00 & 1.00 & $\begin{array}{l}3.50 \\
1.00 \\
2 \\
3.5\end{array}$ & 1.00 & $\begin{array}{l}3.30 \\
1.00 \\
2 \\
3.3\end{array}$ \\
\hline
\end{tabular}


Vorksheet 5-Effect of Upstream Signals

\begin{tabular}{cc}
\hline Zomputation 1-Queue Clearance Time at Upstream Signal & Movement 5 \\
Movement 2 & $V(t) \quad \mathrm{V}(1, \mathrm{prot}) \quad \mathrm{V}(\mathrm{t}) \quad \mathrm{V}(1, \mathrm{prot})$
\end{tabular}

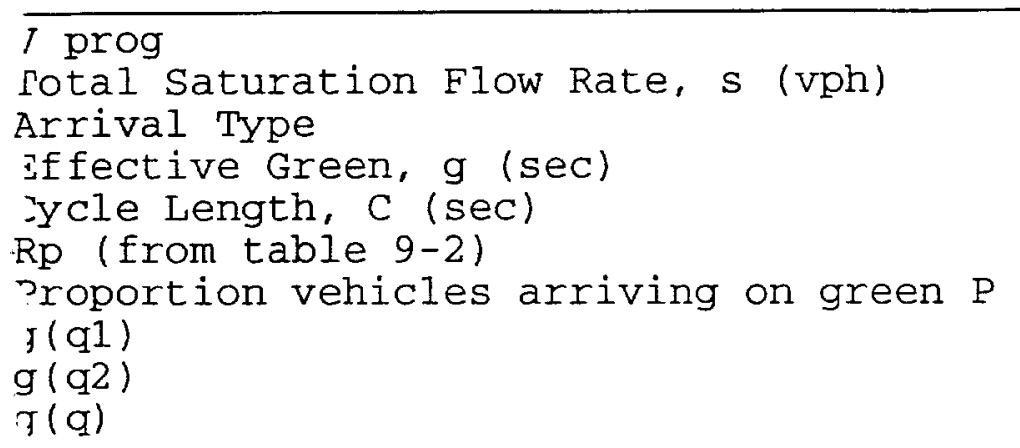

(1)

Single-stage Process
(2)

Two-Stage Process

(1)

)$(4)$

(7)

(8)

o(9)

- (10)

(11)

$\mathrm{p}(12)$

omputation 4 and 5 -ingle-stage Process Movement

$\begin{array}{rrrrrrrr}1 & 4 & 7 & 8 & 9 & 10 & 11 & 12 \\ \mathrm{~L} & \mathrm{~L} & \mathrm{~L} & \mathrm{~T} & \mathrm{R} & \mathrm{L} & \mathrm{T} & \mathrm{R}\end{array}$


$\mathrm{Px}$

$\mathrm{V}, \mathrm{c}, \mathrm{u}, \mathrm{x}$

$\mathrm{C} r, \mathrm{x}$

C plat, $x$

Two-Stage Process

7 8

11

Stage1 Stage2 Stage1 stage2 stagel stage2 Stage1 Stage2

$\mathrm{V}(\mathrm{C}, \mathrm{x})$

$S$

$P(x)$

3400

$\mathrm{V}(\mathrm{c}, \mathrm{u}, \mathrm{x})$

$\mathrm{C}(\mathrm{r}, \mathrm{x})$

$c(p l a t, x)$

Worksheet 6-Impedance and Capacity Equations

Step 1: RT from Minor st.

9

Conflicting Flows

Potential Capacity

Pedestrian Impedance Factor

Movement Capacity

Probability of Queue free st.

1.00

1.00

1.00

601

0.07

Step 2: LT from Major st.

4

Conflicting Flows

Potential Capacity

Pedestrian Impedance Factor

Movement Capacity

Probability of Queue free st.

Maj L-Shared Prob Q free St.

$\begin{array}{ll} & 798 \\ 1.00 & 820 \\ 1.00 & 1.00 \\ & 820 \\ & 0.92\end{array}$

Step 3: TH from Minor St.

8

11

\section{Conflicting Flows}

Potential Capacity

Pedestrian Impedance Factor

Cap. Adj. factor due to Impeding mvmnt

Movement Capacity

Probability of Queue free st.

1.00

1.00

0.92

0.92

Step 4: LT from Minor st.

1.00

1.00

\section{Conflicting Flows}

Potential Capacity

Pedestrian Impedance Factor

Maj. L, Min T Impedance factor

Maj. L, Min T Adj. Imp Factor.

Cap. Adj. factor due to Impeding mvmnt

Movement Capacity

7

10

932

265

1.00

1.00

0.92

0.94

$0.07 \quad 0.92$

243

1 \\ 1}

20 
lorksheet 7-Computation of the Effect of Two-stage Gap Acceptance

itep 3: TH from Minor St.
'art 1 - First Stage
:onflicting Flows
potential Capacity
'edestrian Impedance Factor
'ap. Adj. factor due to Impeding mumnt
'Iovement Capacity
robability of Queue free st.

'robability of Queue free st.

art 2 - second stage

onflicting Flows

otential Capacity

'edestrian Impedance Factor

Cap. Adj. factor due to Impeding mumnt

ovement Capacity

art 3 - Single stage

onflicting Flows

otential Capacity

edestrian Impedance Factor

$1.00 \quad 1.00$

rap. Adj. factor due to Impeding mvmnt $\quad 0.92 \quad 0.92$

ovement Capacity

kesult for 2 stage process:

$-t$

"robability of Queue free st.

1.00

1.00

\begin{tabular}{llll}
\hline tep 4: LT from Minor St. & 70
\end{tabular}

art 1 - First Stage

onflicting Flows

rotential Capacity

edestrian Impedance Factor

ap. Adj. factor due to Impeding mvmnt

ovement Capacity

art 2 - Second stage

onflicting Flows

Potential Capacity

edestrian Impedance Factor

ap. Adj. factor due to Impeding mvmnt

novement Capacity

art 3 - Single stage

onflicting Flows

potential Capacity

edestrian Impedance Factor

aj. L, Min T Impedance factor

Maj. L, Min T Adj. Imp Factor.

ap. Adj. factor due to Impeding mvmnt

svement Capacity

932

265

1.00

1.00

0.92

0.94

$0.07 \quad 0.92$

pesults for Two-stage process: 
Worksheet 8-Shared Lane Calculations

\begin{tabular}{|c|c|c|c|c|c|c|}
\hline Movement & $\begin{array}{l}7 \\
\mathrm{~L}\end{array}$ & $\begin{array}{l}8 \\
\mathrm{~T}\end{array}$ & $\begin{array}{l}9 \\
R\end{array}$ & $\begin{array}{r}10 \\
\mathrm{~L}\end{array}$ & $\begin{array}{r}11 \\
\mathrm{~T}\end{array}$ & $\begin{array}{r}12 \\
\mathrm{R}\end{array}$ \\
\hline $\begin{array}{l}\text { Volume (vph) } \\
\text { Movement Capacity (vph) } \\
\text { Shared Lane Capacity (vph) }\end{array}$ & & & & $\begin{array}{l}45 \\
243\end{array}$ & & $\begin{array}{l}556 \\
601\end{array}$ \\
\hline
\end{tabular}

Worksheet 9-Computation of Effect of Flared Minor street Approaches

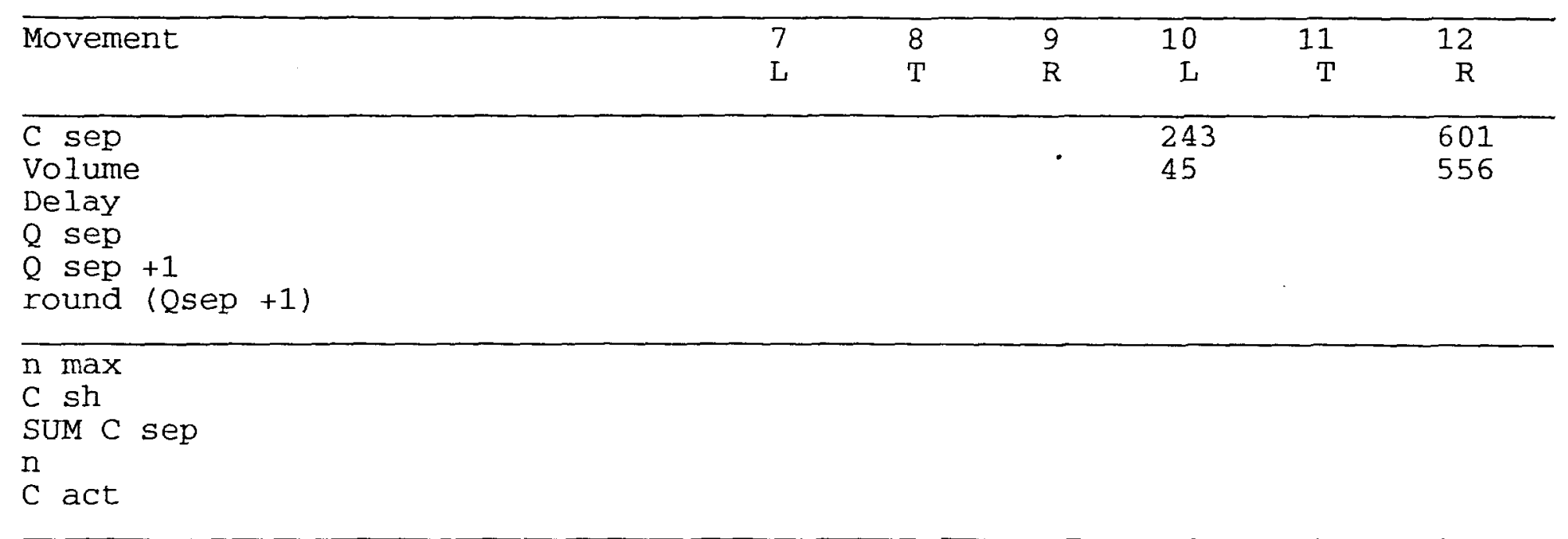

Worksheet 10-Delay, Queue Length, and Level of Service

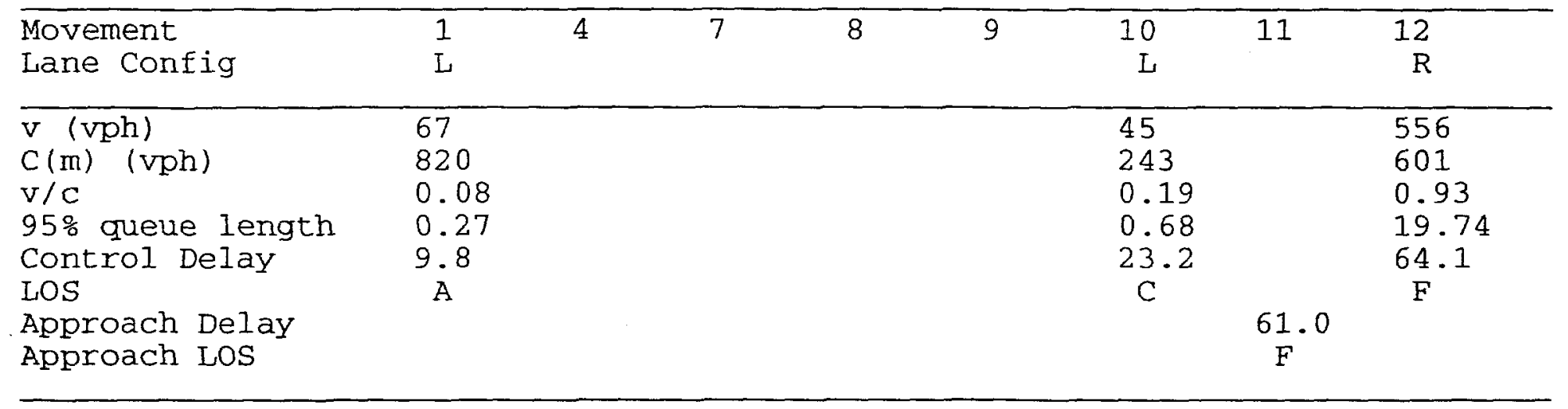

Worksheet 11-Shared Major LT Impedance and Delay

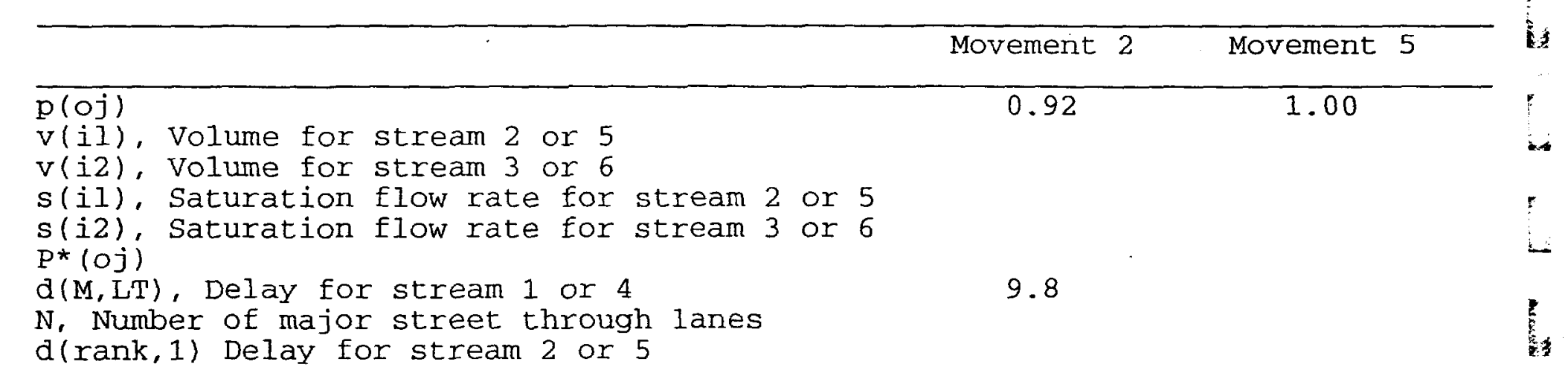




\section{inalyst:}

Agency/Co.:

late Performed:

$1 / 21 / 02$

inalysis Time Period: AM Peak

intersection:

East Ave \& W Entrance (Location \# 5)

Turisdiction:

nalysis Year:

2020

'roject ID: Lawrence Livermore National Laboratory

East/West Street: East Ave

lorth/South Street: West Entrance to Sandia Labs

Intersection Orientation: EW Study period (hrs): 1.00

Vehicle Volumes and Adjustments

ajor Street: Approach Eastbound

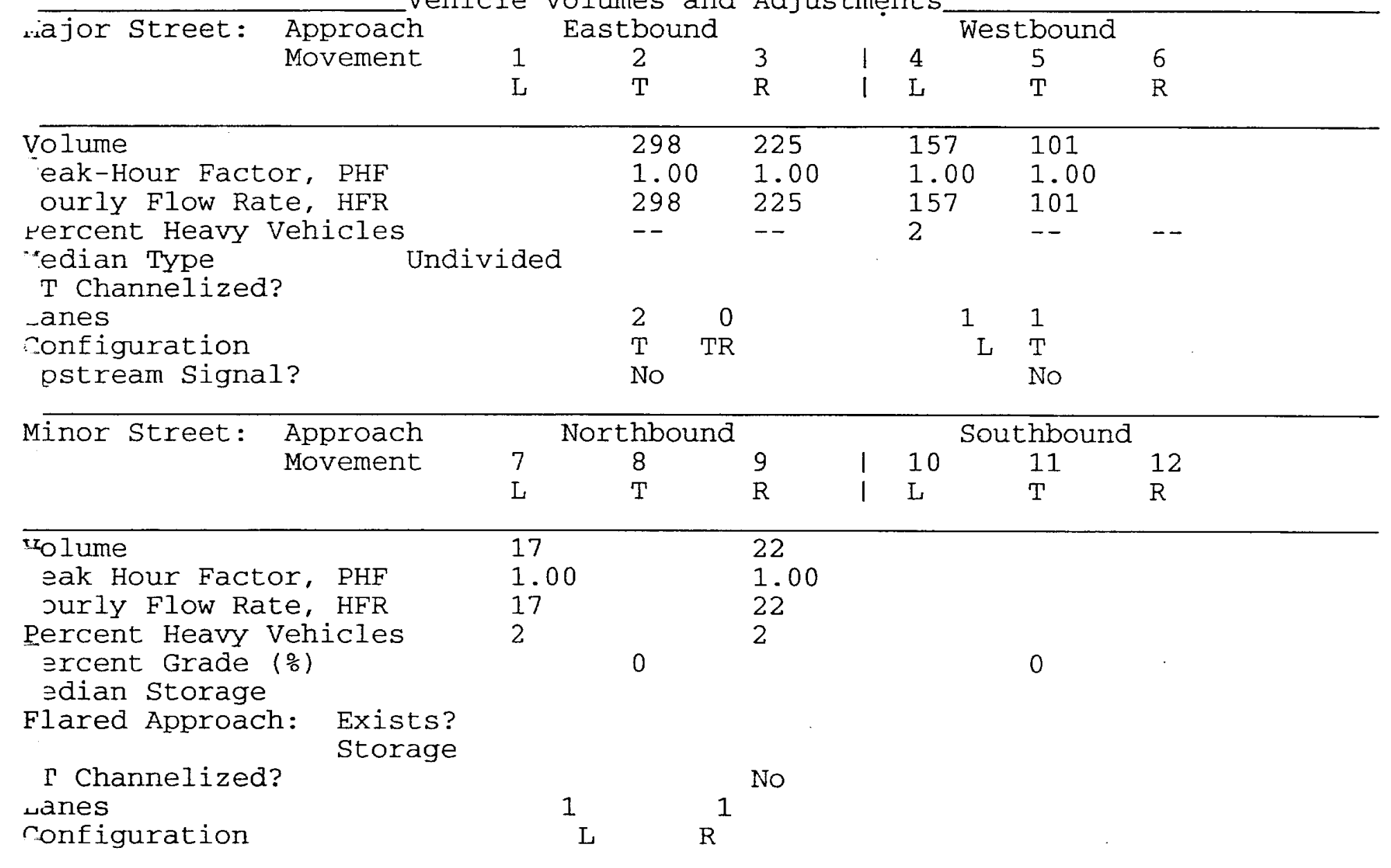

Delay, Queue Length, and Level of Service

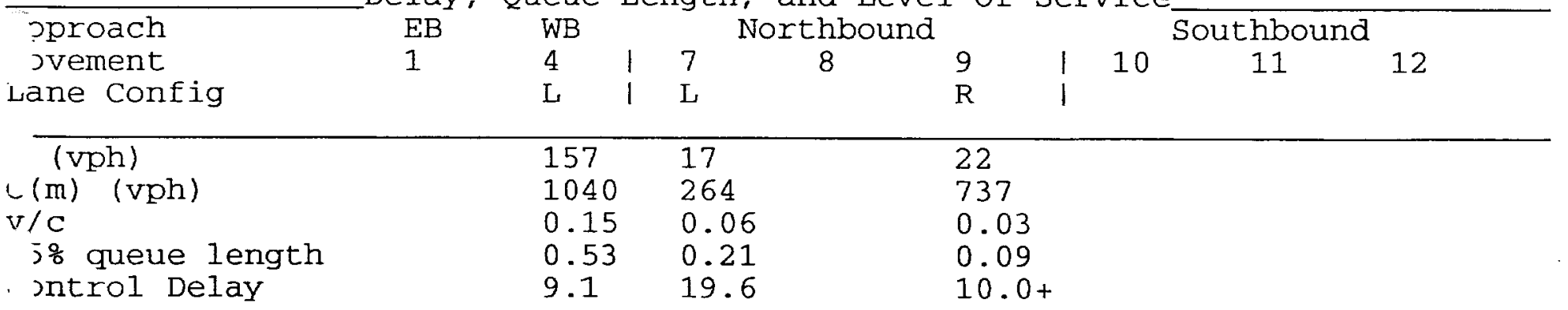




\section{HCS2000: Unsignalized Intersections Release 4.1}

Korve Engineering, Inc.

Phone:

Fax:

E-Mail:

TWO-WAY STOP CONTROL(TWSC) ANALYSIS

Analyst:

Agency/Co.:

Date Performed: $\quad 1 / 21 / 02$

Analysis Time Period: AM Peak

Intersection:

East Ave \& W Entrance

Jurisdiction:

Analysis Year: $\quad 2020$

Project ID: Lawrence Livermore National Laboratory

East/West Street: East Ave

North/South Street: West Entrance to Sandia Labs

Intersection Orientation: EW

Study period (hrs): 1.00

Vehicle Volumes and Adjustments

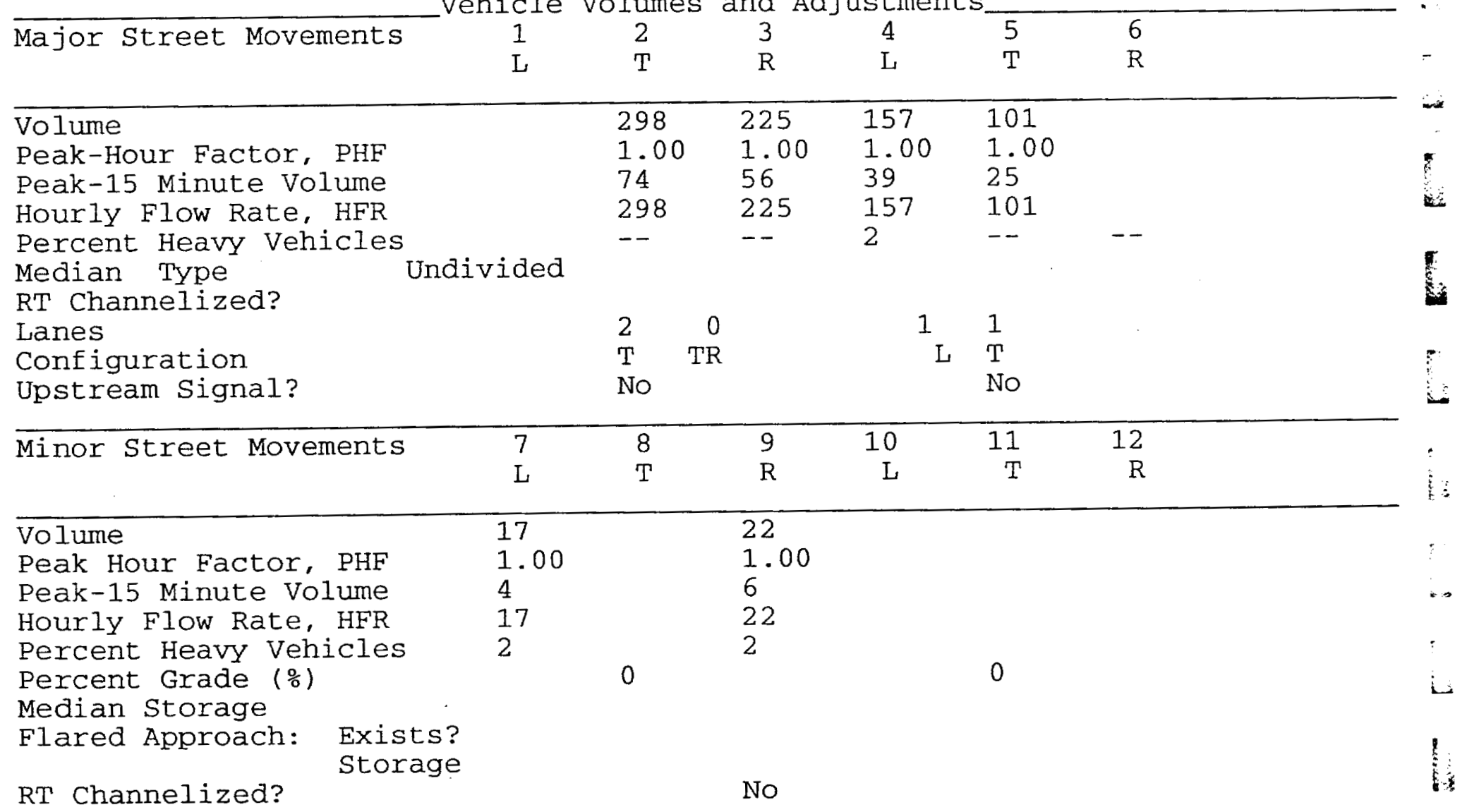




\begin{tabular}{lllll} 
& Pedestrian Volumes & and Adjustment \\
Movements & 13 & 14 & 15 & 16 \\
\hline Plow (ped/hr) & 0 & 0 & 0 & 0 \\
Jane Width (ft) & 12.0 & 12.0 & 12.0 & 12.0 \\
Walking Speed (ft/sec) & 4.0 & 4.0 & 4.0 & 4.0 \\
?ercent Blockage & 0 & 0 & 0 & 0
\end{tabular}

\begin{tabular}{|c|c|c|c|c|c|c|c|c|}
\hline & & $\begin{array}{l}\text { Prog. } \\
\text { Flow } \\
\text { vph }\end{array}$ & $\begin{array}{l}\text { Sat } \\
\text { Flow } \\
\text { vph }\end{array}$ & $\begin{array}{l}\text { Arrival } \\
\text { Type }\end{array}$ & $\begin{array}{l}\text { Green } \\
\text { Time } \\
\text { sec }\end{array}$ & $\begin{array}{l}\text { Cycle } \\
\text { Length } \\
\text { sec }\end{array}$ & $\begin{array}{l}\text { Prog. } \\
\text { Speed } \\
\text { mph }\end{array}$ & $\begin{array}{c}\text { Distance } \\
\text { to Signal } \\
\text { feet }\end{array}$ \\
\hline 32 & $\begin{array}{l}\text { Left-Turn } \\
\text { Through } \\
\text { Left-Turn } \\
\text { Through }\end{array}$ & - & & & & & & \\
\hline
\end{tabular}

lorksheet 3-Data for Computing Effect of Delay to Major Street Vehicles Movement 2 Movement 5

ihared In volume, major th vehicles:

whared In volume, major rt vehicles:

sat flow rate, major th vehicles:

at flow rate, major rt vehicles:

umber of major street through lanes:

orksheet 4-Critical Gap and Follow-up Time Calculation

\begin{tabular}{|c|c|c|c|c|c|c|c|c|c|}
\hline $\begin{array}{l}\text { ritical } \\
\text { ovement }\end{array}$ & Gap Calc & $\begin{array}{c}\text { ulati } \\
1 \\
L\end{array}$ & $\begin{array}{l}4 \\
\mathrm{~L}\end{array}$ & $\begin{array}{l}7 \\
\mathrm{~L}\end{array}$ & $\begin{array}{l}8 \\
\mathrm{~T}\end{array}$ & $\begin{array}{l}9 \\
\mathrm{R}\end{array}$ & $\begin{array}{r}10 \\
\mathrm{~L}\end{array}$ & $\begin{array}{r}11 \\
\mathrm{~T}\end{array}$ & $\begin{array}{r}12 \\
\mathrm{R}\end{array}$ \\
\hline $\begin{array}{l}\text { (c, base) } \\
(\mathrm{c}, \mathrm{hv}) \\
\mathrm{P}(\mathrm{hv}) \\
(\mathrm{c}, \mathrm{g}) \\
\text { rade/100 } \\
(3,1 \mathrm{t}) \\
+(\mathrm{c}, \mathrm{T}): \\
\\
\text { (c) }\end{array}$ & $\begin{array}{l}\text { 1-stage } \\
\text { 2-stage } \\
1 \text {-stage } \\
2 \text {-stage }\end{array}$ & $\begin{array}{l}0.00 \\
0.00\end{array}$ & $\begin{array}{l}0.00 \\
0.00 \\
0.00 \\
4.1\end{array}$ & $\begin{array}{l}7.5 \\
2.00 \\
2 \\
0.20 \\
0.00 \\
0.70 \\
0.00 \\
1.00 \\
6.8\end{array}$ & $\begin{array}{l}2.00 \\
0.20 \\
0.00 \\
0.00 \\
1.00\end{array}$ & $\begin{array}{l}6.9 \\
2.00 \\
2 \\
0.10 \\
0.00 \\
0.00 \\
0.00 \\
0.00 \\
6.9\end{array}$ & $\begin{array}{l}2.00 \\
0.20 \\
0.00 \\
0.00 \\
1.00\end{array}$ & $\begin{array}{l}2.00 \\
0.20 \\
0.00 \\
0.00 \\
1.00\end{array}$ & $\begin{array}{l}2.00 \\
0.10 \\
0.00 \\
0.00 \\
0.00\end{array}$ \\
\hline $\begin{array}{l}\text { ollow-Up } \\
\text { Movement }\end{array}$ & Time $\mathrm{Ca}$ & $\begin{array}{c}\text { 1cul } \\
1 \\
\mathrm{~L}\end{array}$ & $\begin{array}{c}\text { Lons } \\
4 \\
\text { L }\end{array}$ & $\begin{array}{l}7 \\
L\end{array}$ & $\begin{array}{l}8 \\
\mathrm{~T}\end{array}$ & $\begin{array}{l}9 \\
\mathrm{R}\end{array}$ & $\begin{array}{r}10 \\
\mathrm{~L}\end{array}$ & $\begin{array}{r}11 \\
\mathrm{~T}\end{array}$ & $\begin{array}{r}12 \\
\mathrm{R}\end{array}$ \\
\hline $\begin{aligned} & (\mathrm{f}, \text { base }) \\
+ & (\mathrm{f}, \mathrm{HV}) \\
& (\mathrm{HV}) \\
& (\mathrm{f})\end{aligned}$ & & 1.00 & $\begin{array}{l}2.20 \\
1.00 \\
2 \\
2.2\end{array}$ & $\begin{array}{l}3.50 \\
1.00 \\
2 \\
3.5\end{array}$ & 1.00 & $\begin{array}{l}3.30 \\
1.00 \\
2 \\
3.3\end{array}$ & 1.00 & 1.00 & 1.00 \\
\hline
\end{tabular}




\section{alpha}

beta

Travel time, $t(a)$ (sec)

Smoothing Factor, F

Proportion of conflicting flow, $f$

Max platooned flow, $V(c, \max )$

Min platooned flow, $V(c, m i n)$

Duration of blocked period, $t(p)$

Proportion time blocked, $p$

0.000

0.000

Computation 3-Platoon Event Periods Result

\begin{tabular}{ll}
\hline$p(2)$ & 0.000 \\
$p(5)$ & 0.000
\end{tabular}

$\mathrm{p}$ (dom)

$p$ (subo)

Constrained or unconstrained?

\begin{tabular}{|c|c|c|c|c|c|c|c|c|}
\hline $\begin{array}{l}\text { Proportion } \\
\text { unblocked } \\
\text { for minor } \\
\text { movements, } p(x)\end{array}$ & $\begin{array}{r}\text { Sin } \\
P\end{array}$ & $\begin{array}{l}\text { stage } \\
\text { ss }\end{array}$ & & $\begin{array}{l}\text { 2) } \\
\text { Two- } \\
\text { re I }\end{array}$ & ge & $\begin{array}{l}\text { (3) } \\
\text { cess } \\
\text { tage }\end{array}$ & II & \\
\hline $\begin{array}{l}p(1) \\
p(4) \\
p(7) \\
p(8) \\
p(9) \\
p(10) \\
p(11) \\
p(12)\end{array}$ & & & & . & & & & \\
\hline $\begin{array}{l}\text { Computation } 4 \text { and } 5 \\
\text { Single-Stage Process } \\
\text { Movement }\end{array}$ & $\begin{array}{l}1 \\
\mathrm{~L}\end{array}$ & $\begin{array}{l}4 \\
\mathrm{~L}\end{array}$ & $\begin{array}{l}7 \\
\mathrm{~L}\end{array}$ & $\begin{array}{l}8 \\
\mathrm{~T}\end{array}$ & $\begin{array}{l}9 \\
R\end{array}$ & $\begin{array}{r}10 \\
\mathrm{~L}\end{array}$ & $\begin{array}{r}11 \\
\mathrm{~T}\end{array}$ & $\begin{array}{r}12 \\
\mathrm{R}\end{array}$ \\
\hline
\end{tabular}


C plat, $x$

\section{'wo-Stage Process}

$7 \quad 8 \quad 10$

11

Stage1 Stage2 Stage1 Stage2 Stage1 Stage2 Stage1 Stage2

$$
(c, x)
$$

$a$

$(x)$

1700

$(\mathrm{c}, \mathrm{u}, \mathrm{x})$

$(r, x)$

(plat, $x)$

orksheet 6-Impedance and Capacity Equations

\begin{tabular}{lcc}
\hline Step 1: RT from Minor St. & 9 & 12 \\
\hline onflicting Flows & 262 & \\
potential Capacity & 737 & 1.00 \\
edestrian Impedance Factor & 1.00 & 137 \\
ovement Capacity & 0.97 & 1.00 \\
robability of Queue free St. & 4 & \\
\hline tep 2: LT from Major St. & 523 & 1.00 \\
Conflicting Flows & 1040 & 1.00 \\
otential Capacity & 1.00 & \\
edestrian Impedance Factor & 0.85 & \\
movement Capacity & & \\
-robability of Queue free St. & & \\
aj L-Shared Prob Q free St. &
\end{tabular}

\begin{tabular}{|c|c|c|}
\hline Step 3: TH from Minor st. & 8 & 11 \\
\hline $\begin{array}{l}\text { onflicting Flows } \\
\text { Potential Capacity } \\
\text { 'edestrian Impedance Factor } \\
\text { ap. Adj. factor due to Impeding mvmnt } \\
\text { rovement Capacity } \\
\text { Drobability of Queue free st. }\end{array}$ & $\begin{array}{l}1.00 \\
0.85 \\
1.00\end{array}$ & $\begin{array}{l}1.00 \\
0.85 \\
1.00\end{array}$ \\
\hline Eep 4: LT from Minor St. & 7 & 10 \\
\hline $\begin{array}{l}\text { 'onflicting Flows } \\
\text { stential Capacity } \\
\text { Pedestrian Impedance Factor } \\
\text { "Taj. L, Min T Impedance factor } \\
\text { zj. L, Min T Adj. Imp Factor. } \\
\text { cap. Adj. factor due to Impeding mvinnt } \\
\text { Movement Capacity }\end{array}$ & $\begin{array}{l}825 \\
311 \\
1.00\end{array}$ & $\begin{array}{l}1.00 \\
0.85 \\
0.88 \\
0.86\end{array}$ \\
\hline
\end{tabular}




\begin{tabular}{|c|c|}
\hline Step 3: TH from Minor St. & 11 \\
\hline $\begin{array}{l}\text { Part } 1 \text { - First Stage } \\
\text { Conflicting Flows } \\
\text { Potential Capacity } \\
\text { Pedestrian Impedance Factor } \\
\text { Cap. Adj. factor due to Impeding mvmnt } \\
\text { Movement Capacity } \\
\text { Probability of Queue free st. }\end{array}$ & \\
\hline
\end{tabular}

Part 2 - Second stage

Conflicting Flows

Potential Capacity

Pedestrian Impedance Factor

Cap. Adj. factor due to Impeding mvint

Movement Capacity

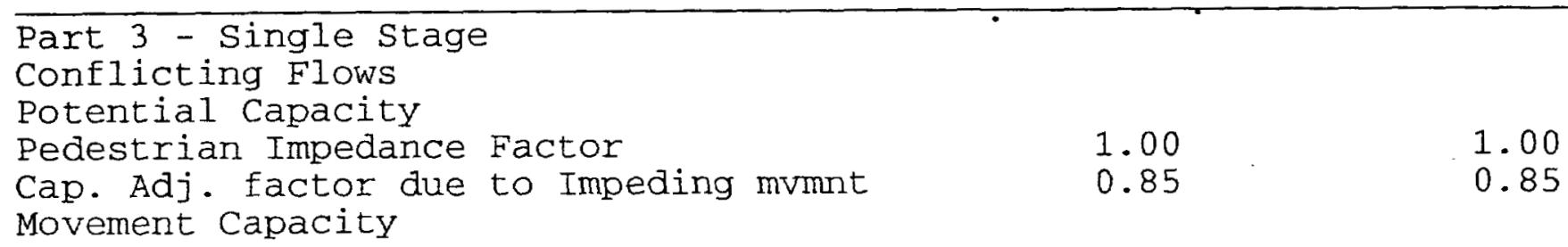

Result for 2 stage process:

a

Y

$c t$

Probability of Queue free St.

$1.00 \quad 1.00$

Step 4: LT from Minor St.

7

10

Part 1 - First Stage

Conflicting Flows

Potential Capacity

Pedestrian Impedance Factor

Cap. Adj. factor due to Impeding mvmnt

Movement Capacity

\begin{abstract}
Part 2 - Second stage
Conflicting Flows

Potential Capacity

Pedestrian Impedance Factor

Cap. Adj. Eactor due to Impeding mumnt

Movement Capacity
\end{abstract}

\begin{tabular}{lll}
\hline Part 3 - Single Stage & & \\
Conflicting Flows & 825 & 1.00 \\
Potential Capacity & 311 & 0.85 \\
Pedestrian Impedance Factor & 1.00 & 0.88 \\
Maj. L, Min T Impedance factor & & 0.86 \\
Maj. L, Min T Adj. Imp Factor. & 0.85 & \\
Cap. Adj factor due to Impeding mvmnt & 264 & \\
Movement Capacity &
\end{tabular}

Results for Two-stage process: a

y 
worksheet 8-Shared Lane Calculations

\begin{tabular}{|c|c|c|c|c|c|c|}
\hline lovement & $\begin{array}{l}7 \\
\mathrm{~L}\end{array}$ & $\begin{array}{l}8 \\
\mathrm{~T}\end{array}$ & $\begin{array}{l}9 \\
R\end{array}$ & $\begin{array}{r}10 \\
\mathrm{~L}\end{array}$ & $\begin{array}{r}11 \\
\mathrm{~T}\end{array}$ & $\begin{array}{r}12 \\
\mathrm{R}\end{array}$ \\
\hline $\begin{array}{l}\text { Jolume (vph) } \\
\text { Movement Capacity (vph) } \\
\text { ihared Lane Capacity (vph) }\end{array}$ & $\begin{array}{l}17 \\
264\end{array}$ & & $\begin{array}{l}22 \\
737\end{array}$ & & & \\
\hline
\end{tabular}

worksheet 9-Computation of Effect of Flared Minor Street Approaches

\begin{tabular}{|c|c|c|c|c|c|c|}
\hline lovement & $\begin{array}{l}7 \\
\mathrm{~L}\end{array}$ & $\begin{array}{l}8 \\
\mathrm{~T}\end{array}$ & $\begin{array}{l}9 \\
\mathrm{R}\end{array}$ & $\begin{array}{r}10 \\
\mathrm{~L}\end{array}$ & $\begin{array}{r}11 \\
\mathrm{~T}\end{array}$ & $\begin{array}{r}12 \\
\mathrm{R}\end{array}$ \\
\hline $\begin{array}{l}\text { sep } \\
\text { Jolume } \\
\text { Telay } \\
\text { ' sep } \\
\text { ! sep +1 } \\
\text { round (Qsep +1) }\end{array}$ & $\begin{array}{ll}\cdot \quad 264 \\
17\end{array}$ & & $\begin{array}{l}737 \\
22\end{array}$ & & & \\
\hline $\begin{array}{l}\max \\
\text { Sh C sep } \\
\text { UM act }\end{array}$ & & & & & & \\
\hline
\end{tabular}

orksheet 10-Delay, Queue Length, and Level of Service

\begin{tabular}{|c|c|c|c|c|c|c|c|c|}
\hline $\begin{array}{l}\text { ovement } \\
\text { ane config }\end{array}$ & 1 & $\begin{array}{l}4 \\
\mathrm{~L}\end{array}$ & $\begin{array}{l}7 \\
L\end{array}$ & 8 & $\begin{array}{l}9 \\
\mathrm{R}\end{array}$ & 10 & 11 & 12 \\
\hline $\begin{array}{l}\text { (vph) } \\
\text { (m) (vph) } \\
\text { /C } \\
\text { a5\% queue length } \\
\text { ontrol Delay } \\
\text { os }\end{array}$ & & $\begin{array}{l}157 \\
1040 \\
0.15 \\
0.53 \\
9.1 \\
\text { A }\end{array}$ & $\begin{array}{l}17 \\
264 \\
0.06 \\
0.21 \\
19.6 \\
C\end{array}$ & & $\begin{array}{l}22 \\
737 \\
0.03 \\
0.09 \\
10.0+ \\
B\end{array}$ & & & \\
\hline $\begin{array}{l}\text { Approach Delay } \\
\text { pproach LoS }\end{array}$ & & & & $\frac{14.2}{B}$ & & & & \\
\hline
\end{tabular}

"orksheet 11-Shared Major LT Impedance and Delay

Movement 2 Movement 5

\section{(oj)}

(i1), Volume for stream 2 or 5

$1.00 \quad 0.85$

$v(i 2)$, Volume for stream 3 or 6

(il). Saturation flow rate for stream 2 or 5

(i2). Saturation flow rate for stream 3 or 6

$-\star(0 j)$

خ(M,LT), Delay for stream 1 or 4

9.1

- Number of major street through lanes

(rank, 1) Delay for stream 2 or 5 
Analyst:

Agency/Co.:

Date Performed: 1/21/02

Analysis Time Period: PM Peak

Intersection:

East Ave \& W Entrance (Location \#5)

Jurisdiction:

Analysis Year:

2020

Project ID: Lawrence Iivermore National Laboratory

East/West Street:

East Ave

North/South Street:

West Entrance to Sandia Labs

Intersection Orientation: $\mathrm{EW}$

study period (hrs): 1.00

Vehiçle Volumes and Adjustments

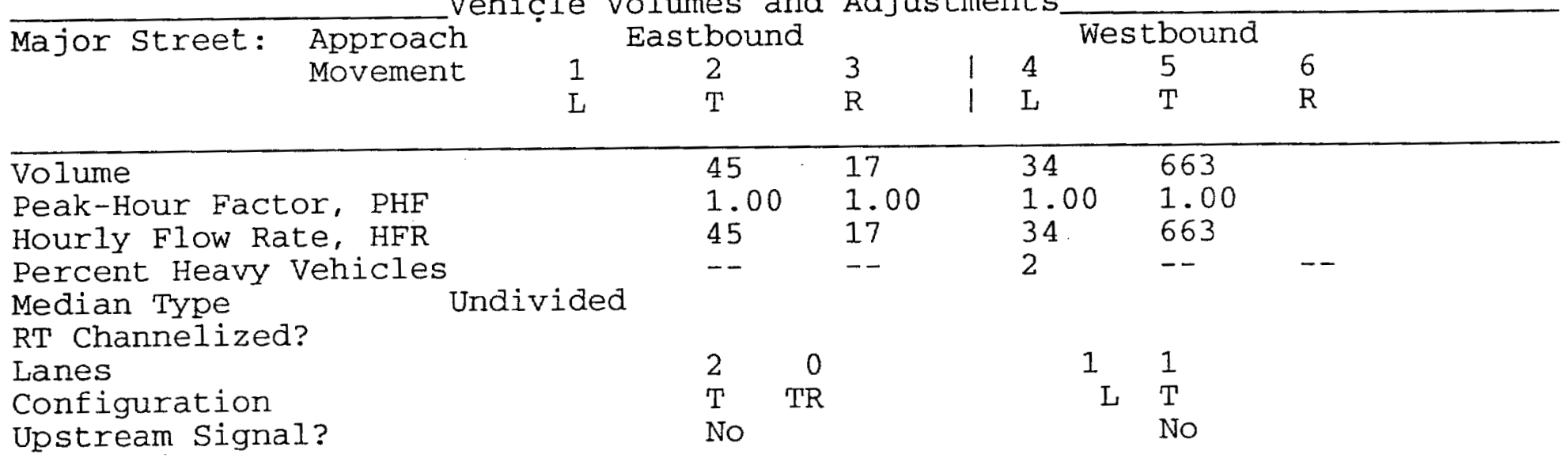

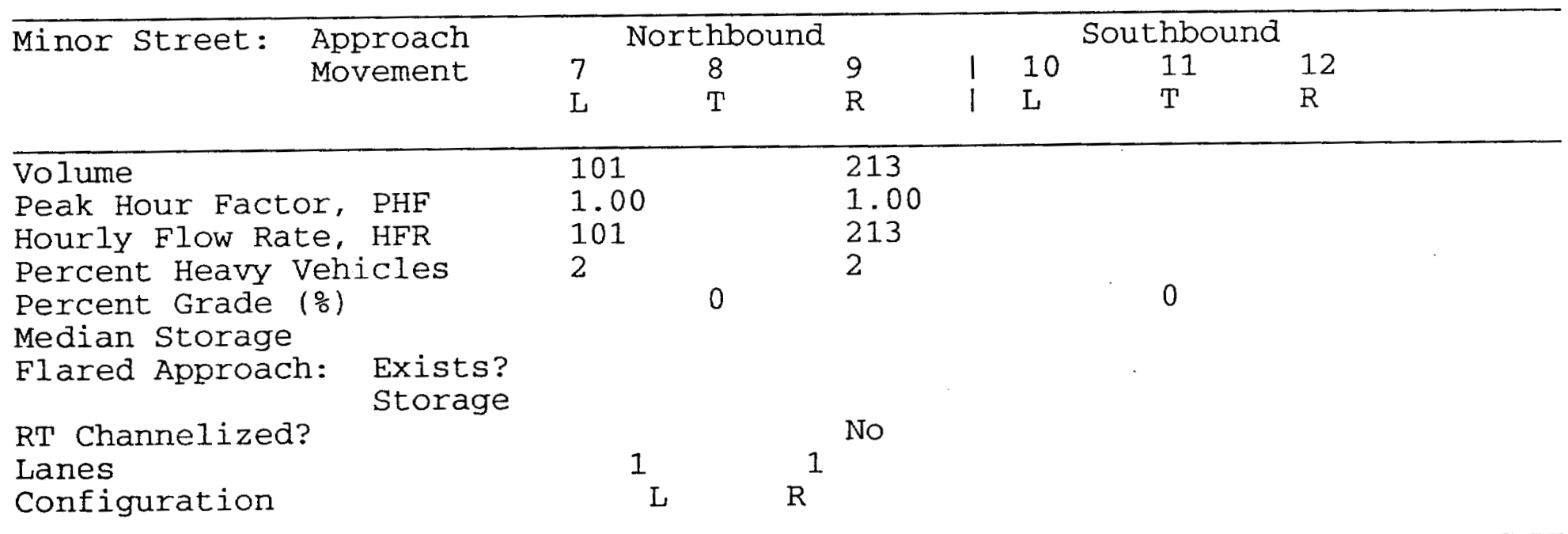

Delay, Queue Length, and Level of Service

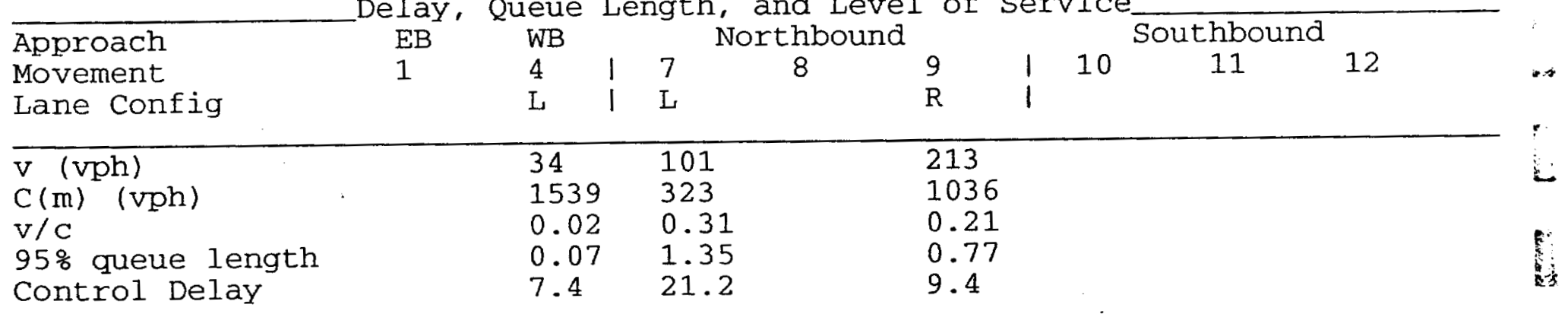


HCS2000: Unsignalized Intersections Release 4.1

Korve Engineering, Inc.

?hone:

Fax:

i-Mail: TWO-WAY STOP CONTROL(TWSC) ANALYSIS

Analyst :

Agency/CO.:

late Performed:

$1 / 21 / 02$

inalysis Time Period: PM Peak

Intersection:

East Ave \& W Entrance

'urisdiction:

nalysis Year: 2020

Project ID: Lawrence Livermore National Laboratory

Tast/West Street: East Ave

loxth/South street: West Entrance to Sandia Labs

Tntersection orientation: EW Study period (hrs): 1.00

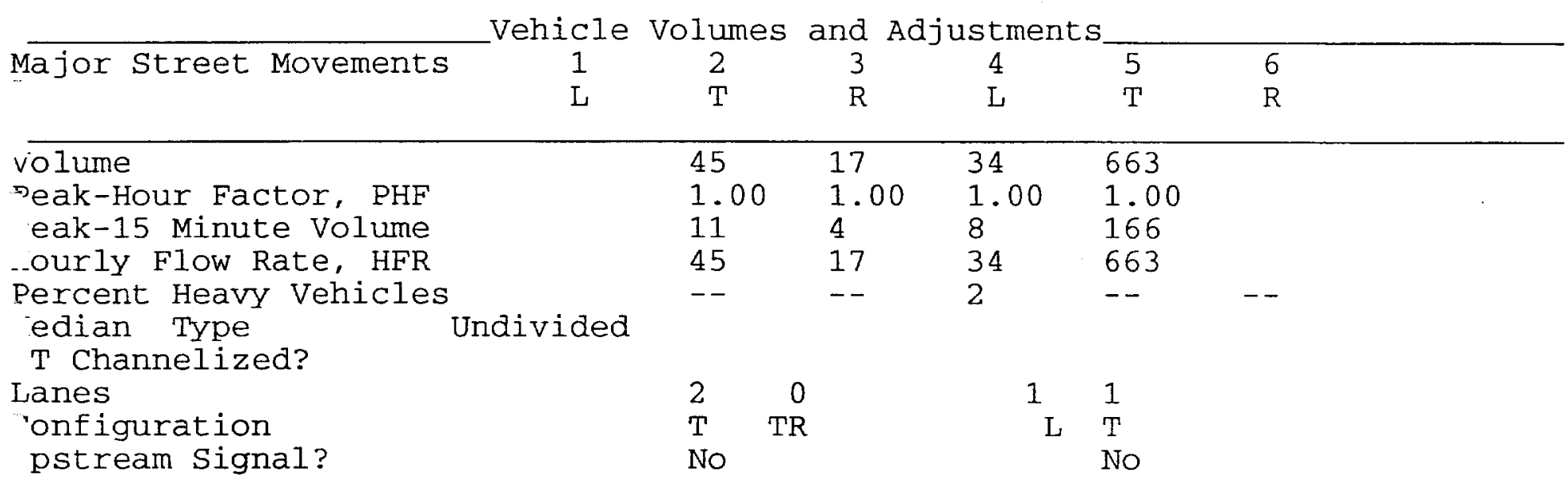

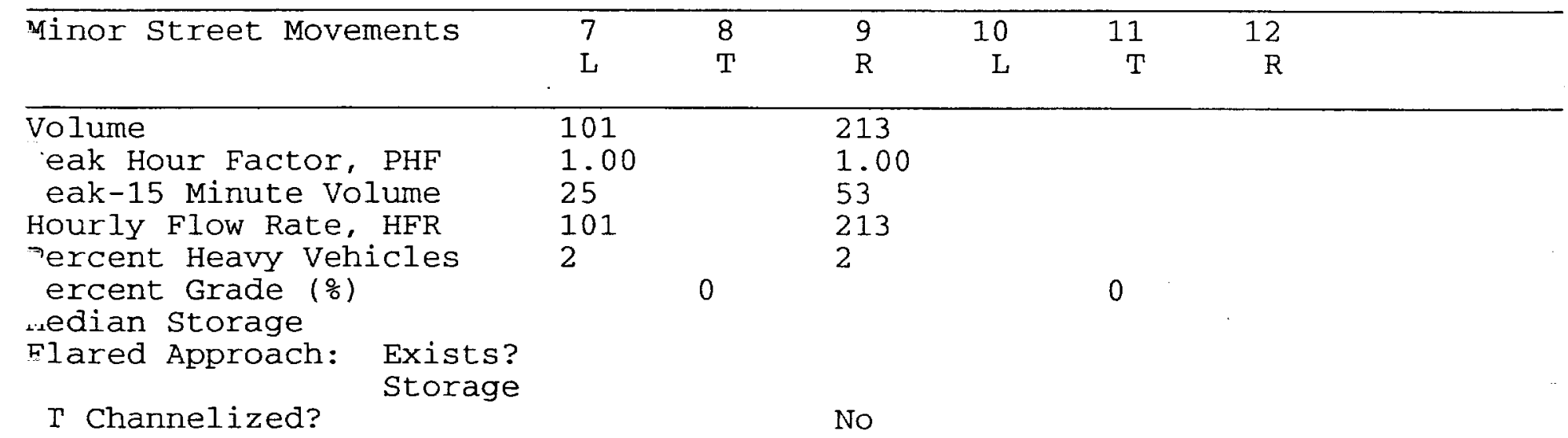




$\begin{array}{lcc}\text { Lanes } & 1 & R^{1} \\ \text { Configuration } & \mathrm{L} & \mathrm{R}\end{array}$

\begin{tabular}{lllll} 
& Pedestrian & Volumes & \multicolumn{3}{c}{ and Adjustment } \\
\cline { 1 - 4 } & 13 & 14 & 15 & 16 \\
\hline Flow (ped/hr) & 0 & 0 & 0 & 0 \\
Lane Width (ft) & 12.0 & 12.0 & 12.0 & 12.0 \\
Walking Speed (ft/sec) & 4.0 & 4.0 & 4.0 & 4.0 \\
Percent Blockage & 0 & 0 & 0 & 0
\end{tabular}

\begin{tabular}{|c|c|c|c|c|c|c|c|c|}
\hline & & $\begin{array}{l}\text { Prog. } \\
\text { Flow } \\
\text { vph }\end{array}$ & $\begin{array}{l}\text { Sat } \\
\text { Flow } \\
\text { vph }\end{array}$ & $\begin{array}{l}\text { Arrival } \\
\text { Type }\end{array}$ & $\begin{array}{l}\text { Green } \\
\text { Time } \\
\text { sec }\end{array}$ & $\begin{array}{l}\text { Cycle } \\
\text { Length } \\
\text { sec }\end{array}$ & $\begin{array}{l}\text { Prog. } \\
\text { Speed } \\
\text { mph }\end{array}$ & $\begin{array}{l}\text { Distance } \\
\text { to Signal } \\
\text { feet }\end{array}$ \\
\hline$S 2$ & $\begin{array}{l}\text { Left-Turn } \\
\text { Through }\end{array}$ & & & & & & & - \\
\hline S5 & $\begin{array}{l}\text { Left-Turn } \\
\text { Through }\end{array}$ & & & & & & & \\
\hline
\end{tabular}

Worksheet 3-Data for Computing Effect of Delay to Major street Vehicles Movement 2 Movement 5

Shared In volume, major th vehicles:
Shared In volume, major rt vehicles:
Sat flow rate, major th vehicles:
Sat flow rate, major rt vehicles:
Number of major street through lanes:

Worksheet 4-Critical Gap and Follow-up Time Calculation

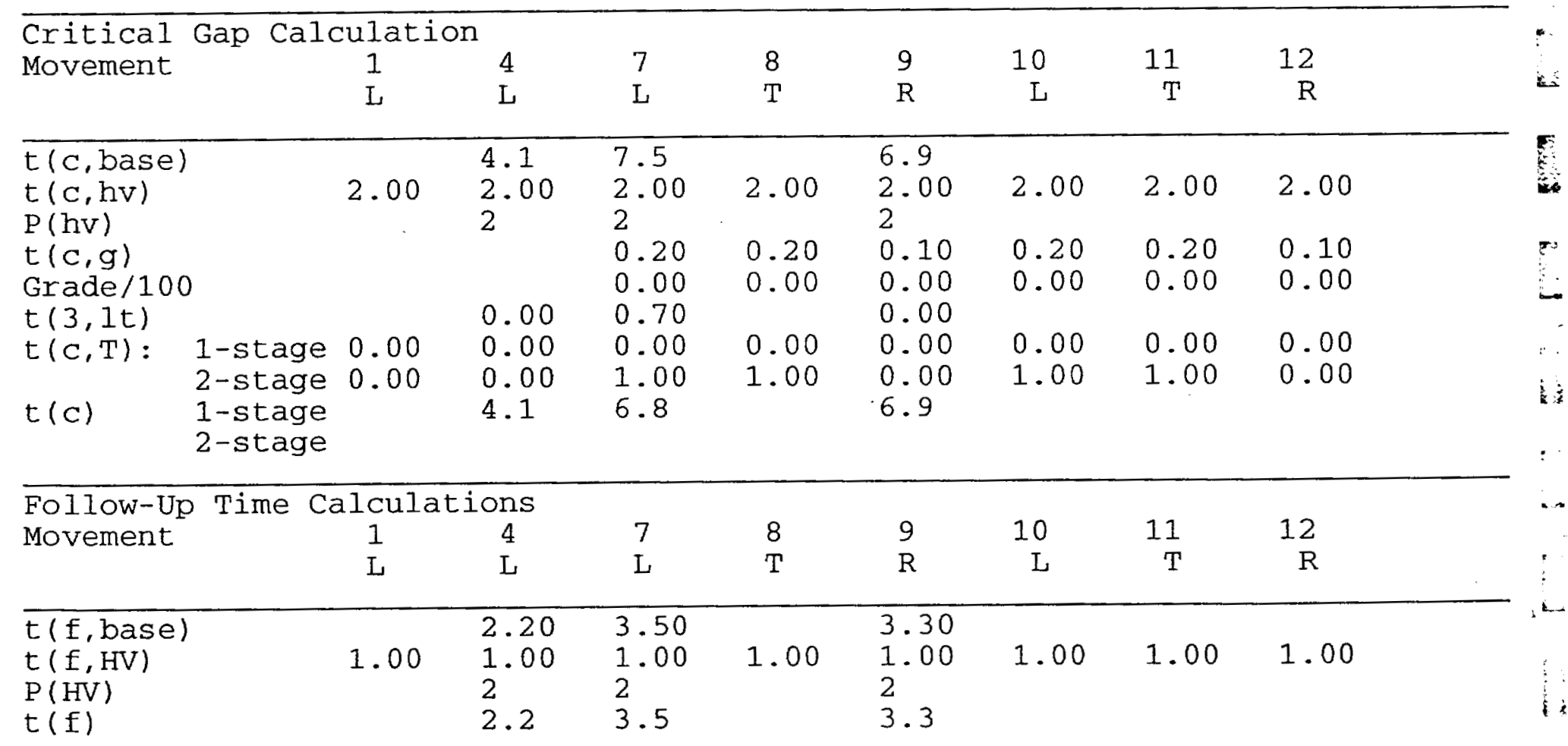


lorksheet 5-Effect of Upstream Signals

$\begin{array}{cc}\text { Somputation 1-Queue Clearance Time at Upstream Signal } & \text { Movement } 5 \\ \text { Movement } 2 & \mathrm{~V}(\mathrm{t}) \quad \mathrm{V}(1, \mathrm{prot}) \mathrm{V}(\mathrm{t}) \quad \mathrm{V}(1, \mathrm{prot})\end{array}$

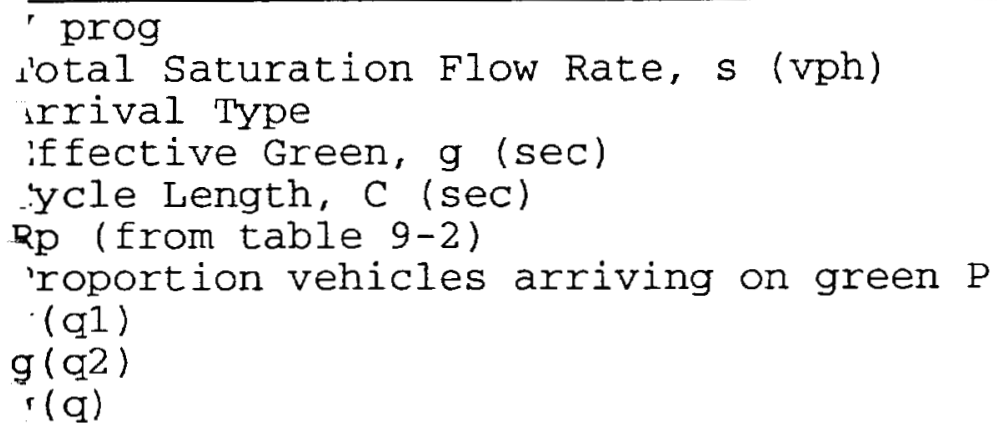

\begin{tabular}{ll}
\hline$(2)$ & 0.000 \\
$\mathrm{p}(5)$ & 0.000
\end{tabular}

, (dom)

.000

(subo)

-onstrained or unconstrained?

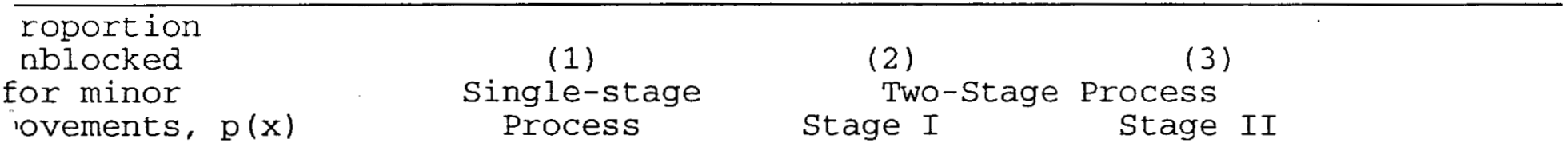

i

$\rightarrow(4)$

(7)

$-(8)$

$D(9)$

(10)

(11)

$p(12)$

mputation 4 and 5 ringle-stage Process sovement

1

4
$\mathrm{~L}$

7
$\mathrm{~L}$

8

$\begin{array}{rrrr}9 & 10 & 11 & 12 \\ \mathrm{R} & \mathrm{L} & \mathrm{T} & \mathrm{R}\end{array}$




\section{$\mathrm{V}(\mathrm{c}, \mathrm{x})$}

$S$

$\mathrm{P}(\mathrm{X})$

$\mathrm{V}(\mathrm{c}, \mathrm{u}, \mathrm{x})$

$C(r, x)$

$C(p l a t, x)$

Worksheet 6-Impedance and Capacity Equations

\begin{tabular}{|c|c|c|}
\hline Step 1: RT from Minor St. & 9 & 12 \\
\hline $\begin{array}{l}\text { Conflicting Flows } \\
\text { Potential Capacity } \\
\text { Pedestrian Impedance Factor } \\
\text { Movement Capacity } \\
\text { Probability of Queue free st. }\end{array}$ & $\begin{array}{l}31 \\
1036 \\
1.00 \\
1036 \\
0.79\end{array}$ & $\begin{array}{l}1.00 \\
1.00\end{array}$ \\
\hline Step 2: LT from Major st. & 4 & 1 \\
\hline $\begin{array}{l}\text { Conflicting Flows } \\
\text { Potential Capacity } \\
\text { Pedestrian Impedance Factor } \\
\text { Movement Capacity } \\
\text { Probability of Queue free St. } \\
\text { Maj L-Shared Prob Q free St. }\end{array}$ & $\begin{array}{l}62 \\
1539 \\
1.00 \\
1539 \\
0.98\end{array}$ & $\begin{array}{l}1.00 \\
1.00\end{array}$ \\
\hline Step 3: TH from Minor st. & 8 & 11 \\
\hline $\begin{array}{l}\text { Conflicting Flows } \\
\text { Potential Capacity } \\
\text { Pedestrian Impedance Factor } \\
\text { Cap. Adj. factor due to Impeding mvmnt } \\
\text { Movement Capacity } \\
\text { Probability of Queue free st. }\end{array}$ & $\begin{array}{l}1.00 \\
0.98 \\
1.00\end{array}$ & $\begin{array}{l}1.00 \\
0.98 \\
1.00\end{array}$ \\
\hline Step $4:$ LT from Minor St. & 7 & 10 \\
\hline $\begin{array}{l}\text { Conflicting Flows } \\
\text { Potential Capacity } \\
\text { Pedestrian Impedance Factor } \\
\text { Maj. L, Min T Impedance factor } \\
\text { Maj. L, Min T Adj. Imp Factor. } \\
\text { Cap. Adj. factor due to Impeding mvmnt } \\
\text { Movement Capacity }\end{array}$ & $\begin{array}{l}785 \\
330 \\
1.00\end{array}$ & $\begin{array}{l}1.00 \\
0.98 \\
0.98 \\
0.78\end{array}$ \\
\hline
\end{tabular}


'orksheet 7-Computation of the Effect of Two-stage Gap Acceptance

\begin{tabular}{|c|c|}
\hline jtep 3: TH from Minor St. & 11 \\
\hline $\begin{array}{l}\text { 'art } 1 \text { - First Stage } \\
\text { anflicting Flows } \\
\text { Potential Capacity } \\
\text { edestrian Impedance Factor } \\
\text { ap. Adj. factor due to Impeding mvmnt } \\
\text { Movement Capacity } \\
\text { robability of Queue free st. }\end{array}$ & \\
\hline
\end{tabular}

iart 2 - second stage

conflicting Flows

otential Capacity

'edestrian Impedance Factor

cap. Adj. factor due to Impeding mvmnt

ovement Capacity

\begin{tabular}{ll}
\hline rart 3 - Single stage & \\
onflicting Flows & \\
otential Capacity & 1.00 \\
edestrian Impedance Factor & 0.98 \\
rap. Adj. factor due to Impeding mvmnt & 0.98 \\
ovement Capacity &
\end{tabular}

Result for 2 stage process:

$-t$

robability of Queue free st.

1.00

1.00

tep 4: LT from Minor st.

7

10

art 1 - First stage

onflicting Flows

rotential Capacity

edestrian Impedance Factor

ap. Adj. factor due to Impeding mvmnt

.ovement Capacity

art 2 - Second stage

onflicting Flows

Potential Capacity

edestrian Impedance Factor

ap. Adj. factor due to Impeding mvmnt

movement Capacity

\begin{tabular}{lll}
\hline art 3 - Single Stage & \\
Jnflicting Flows & 785 & \\
Dotential Capacity & 330 & 1.00 \\
ədestrian Impedance Factor & 1.00 & 0.98 \\
aj. L, Min T Impedance factor & & 0.98 \\
Maj. L, Min T Adj Imp Factor. & 0.98 \\
ap. Adj. factor due to Impeding mvmnt & 323 & \\
Jvement Capacity & &
\end{tabular}

nesults for Two-stage process: 
Worksheet 8-Shared Lane Calculations

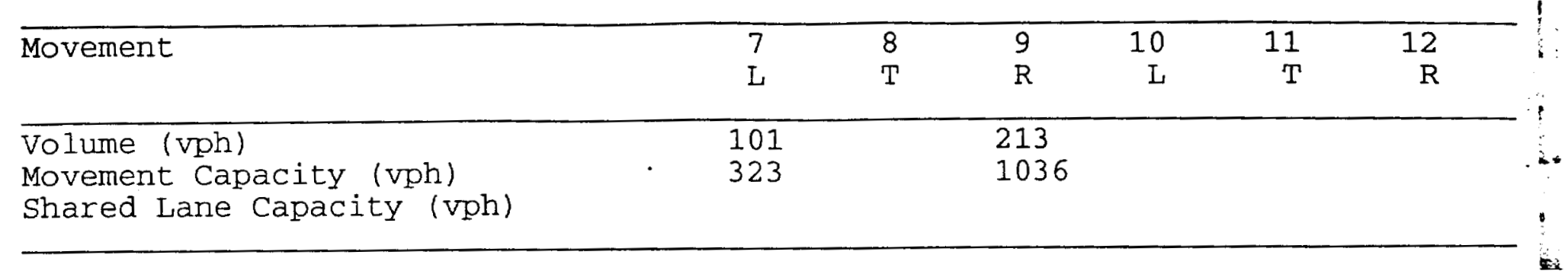

Worksheet 9-Computation of Effect of Flared Minor Street Approaches

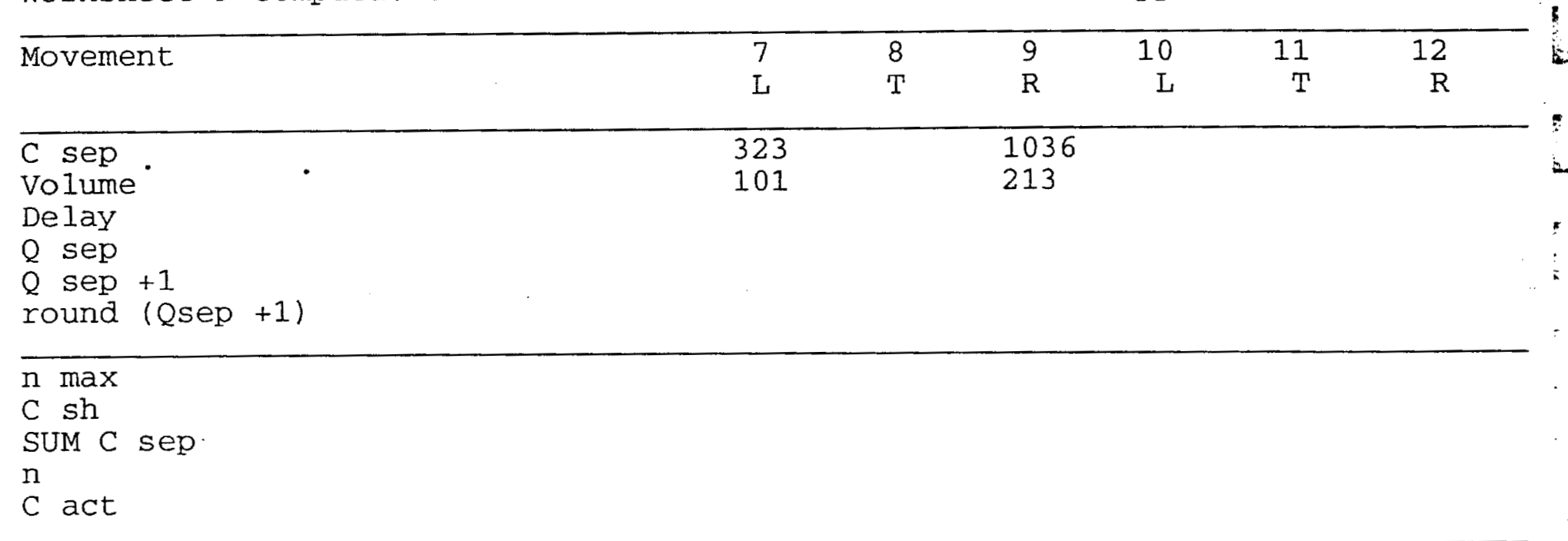

Worksheet 10-Delay, Queue Length, and Level of Service

\begin{tabular}{|c|c|c|c|c|c|c|c|c|}
\hline $\begin{array}{l}\text { Movement } \\
\text { Lane Config }\end{array}$ & 1 & $\begin{array}{l}4 \\
\mathrm{~L}\end{array}$ & $\begin{array}{l}7 \\
\mathrm{~L}\end{array}$ & 8 & $\begin{array}{l}9 \\
\mathrm{R}\end{array}$ & 10 & 11 & 12 \\
\hline $\begin{array}{l}\text { (vph) } \\
\text { C (m) (vph) } \\
\text { v/C } \\
95 \% \text { queue length } \\
\text { Control Delay } \\
\text { LOS } \\
\text { Approach Delay } \\
\text { Approach Los }\end{array}$ & & $\begin{array}{l}34 \\
1539 \\
0.02 \\
0.07 \\
7.4 \\
\text { A }\end{array}$ & $\begin{array}{c}101 \\
323 \\
0.31 \\
1.35 \\
21.2 \\
C\end{array}$ & $\begin{array}{c}13.2 \\
B\end{array}$ & $\begin{array}{l}213 \\
1036 \\
0.21 \\
0.77 \\
9.4 \\
A\end{array}$ & & & \\
\hline
\end{tabular}

Worksheet 11-Shared Major LT Impedance and Delay

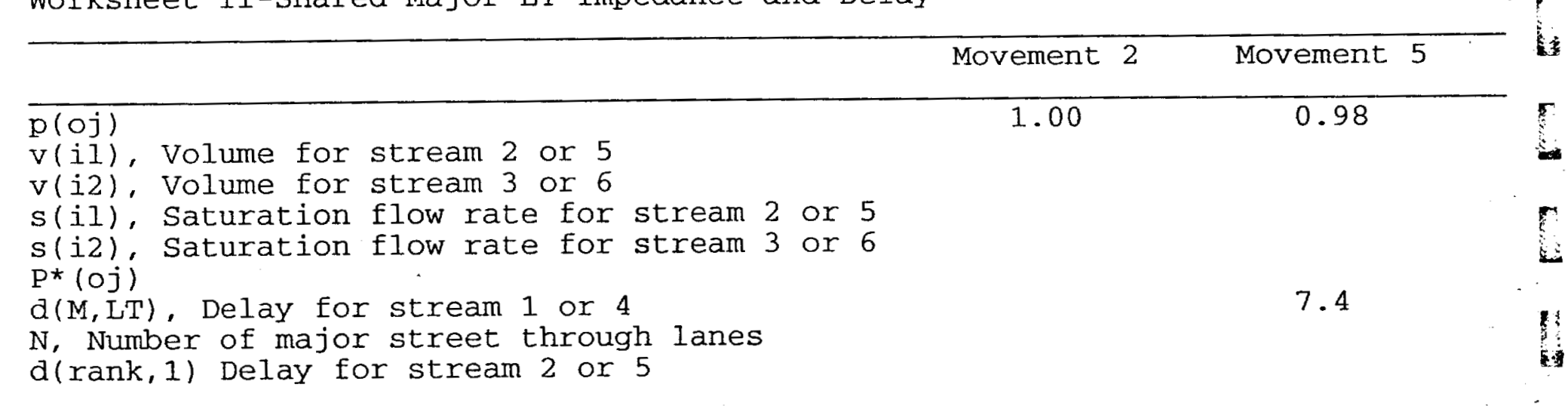


Korve Engineering, Inc.

Phone:

Fax:

E-Mail:

TWO-WAY STOP CONTROL (TWSC) ANALYSIS

Analyst:

Agency/Co.:

Date Performed: 1/21/02

Analysis Time Period: AM Peak

Intersection:

East Ave \& 5 Gate (Locction \#6)

Jurisdiction:

Analysis Year:

2020

Project ID: Lawrence Livermore National Laboratory

East/West Street: East Ave

North/South Street: South Gate

Intersection Orientation: EW

Study period (hrs): 1.00

Vehicle volumes and Adjustments

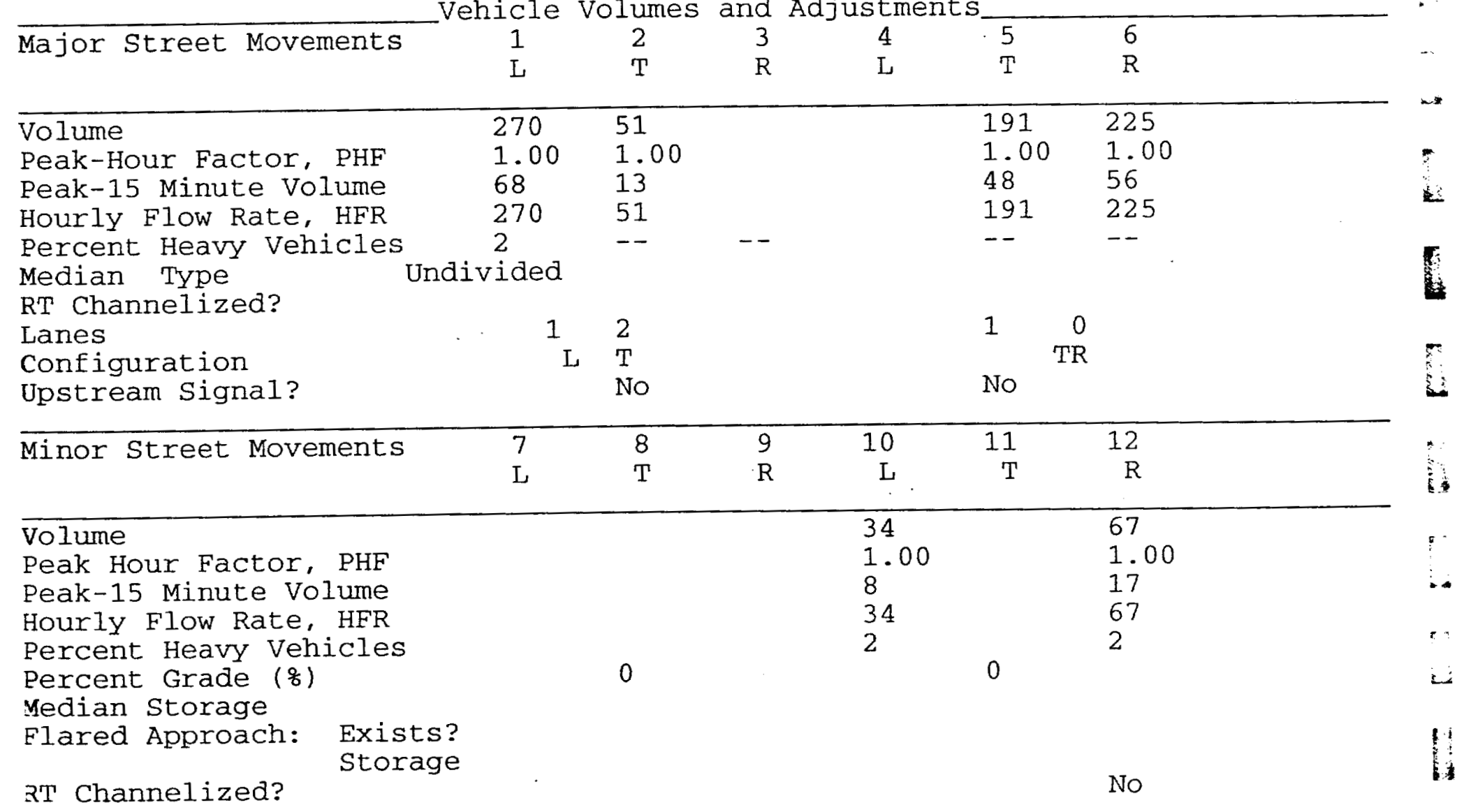


Pedestrian Volumes and Adjustments

\begin{tabular}{lllll}
\hline iovements & 13 & 14 & 15 & 16
\end{tabular}

\begin{tabular}{lllll}
\hline low (ped/hr) & 0 & 0 & 0 & 0 \\
ane width (ft) & 12.0 & 12.0 & 12.0 & 12.0 \\
walking Speed (ft/sec) & 4.0 & 4.0 & 4.0 & 4.0 \\
ercent Blockage & 0 & 0 & 0 & 0
\end{tabular}

ercent Blockage

Upstream Signal Data

$\begin{array}{lllllll}\text { Prog. } & \text { Sat } & \text { Arrival } & \text { Green } & \text { Cycle } & \text { Prog. } & \text { Distance } \\ \text { Flow } & \text { Flow } & \text { Type } & \text { Time } & \text { Length } & \text { Speed } & \text { to Signal } \\ \text { vph } & \text { vph } & & \text { sec } & \text { sec } & \text { mph } & \text { feet }\end{array}$

2 Left-Turn

Through

-5 Left-Turn

Through

orksheet 3-Data for Computing Effect of Delay to Major street Vehicles

Movement 2 Movement 5

hared In volume, major th vehicles:

wared in volume, major rt vehicles:

cat flow rate, major th vehicles:

at flow rate, major rt vehicles:

umber of major street through lanes:

srksheet 4-Critical Gap and Follow-up Time Calculation

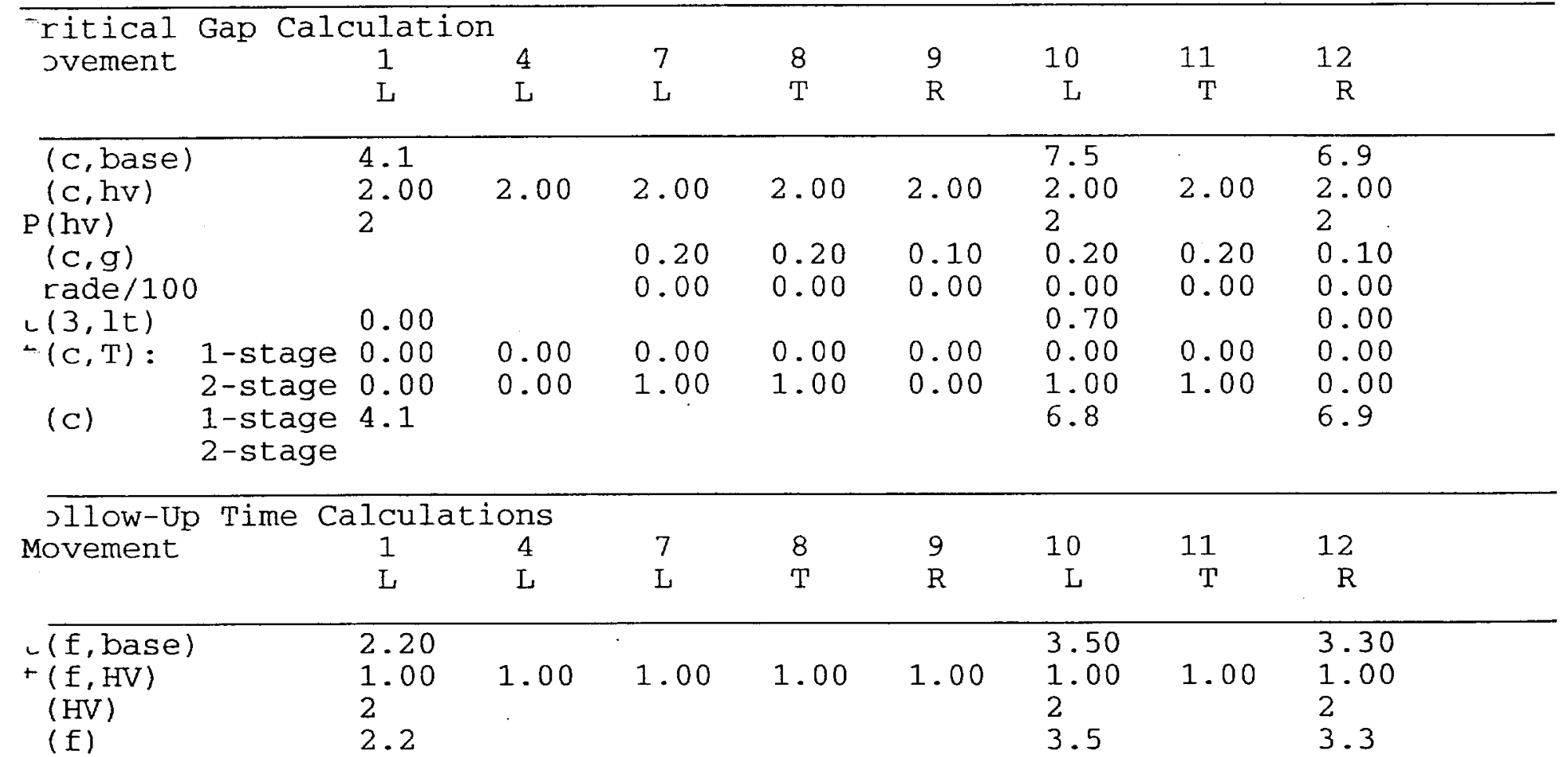




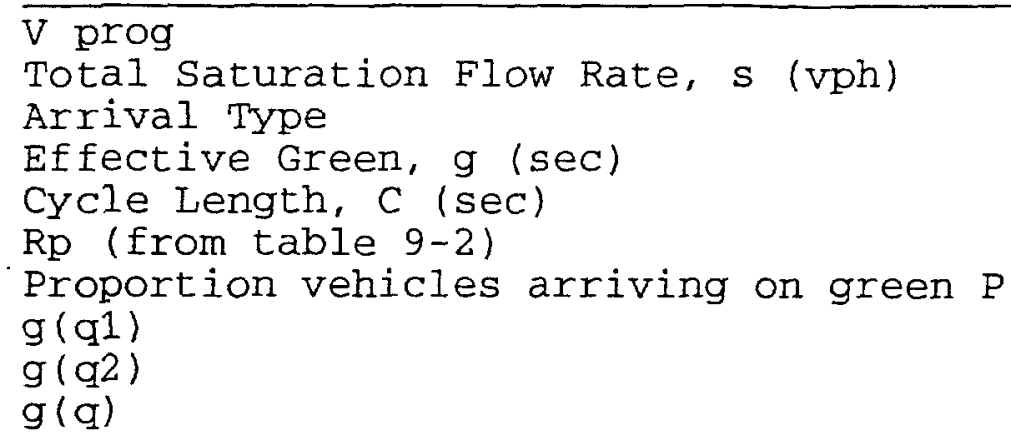

\begin{tabular}{ll}
\hline $\mathrm{p}(2)$ & 0.000 \\
$\mathrm{p}(5)$ & 0.000
\end{tabular}

0.000

$\mathrm{p}$ (dom)

$\mathrm{p}$ (subo)

Constrained or unconstrained?

\begin{tabular}{lccc}
\hline Proportion & & $(1)$ & $(2)$ \\
unblocked & Single-stage & Two-Stage Process \\
for minor & Process & Stage I & Stage II \\
movements, $\mathrm{p}(\mathrm{x})$ & & &
\end{tabular}

$\mathrm{p}(1)$

$\mathrm{p}(4)$

$\mathrm{p}(7)$

$\mathrm{p}(8)$

$\mathrm{p}(9)$

$\mathrm{p}(10)$

$\mathrm{p}(11)$

$\mathrm{p}(12)$

Computation 4 and 5 Single-stage process Movement

\begin{tabular}{rrrrrrrr}
1 & 4 & 7 & 8 & 9 & 10 & 11 & 12 \\
$\mathrm{~L}$ & $\mathrm{~L}$ & $\mathrm{~L}$ & $\mathrm{~T}$ & $\mathrm{R}$ & $\mathrm{L}$ & $\mathrm{T}$ & $\mathrm{R}$ \\
\hline
\end{tabular}


$T \mathrm{C}, \mathrm{u}, \mathrm{x}$

$r, x$

C plat, $x$

Wo-Stage Process

Stage1 ${ }^{7}$ Stage2 stage1 ${ }^{8}$ stage2 stagel $^{10}$ Stage2 stage1 $^{11}$ Stage2

$(\mathrm{C}, \mathrm{x})$

3

'(x)

$r(\mathrm{c}, \mathrm{u}, \mathrm{x})$

$(r, x)$

$\because($ plat,$x)$

'orksheet 6-Impedance and Capacity Equations

\begin{tabular}{lcc}
\hline Step 1: RT from Minor St. & 9 & 12 \\
\hline onflicting Flows & & 304 \\
Potential Capacity & 692 \\
edestrian Impedance Factor & 1.00 \\
ovement Capacity & 1.00 \\
robability of Queue free St. & 1.00 & 0.90 \\
\hline tep 2: LT from Major St. & 4 & 1 \\
conflicting Flows & 1.00 & 116 \\
otential Capacity & 1.00 & 1.00 \\
edestrian Impedance Factor & 139 \\
Movement Capacity & & 0.76 \\
robability of Queue free St. & &
\end{tabular}

\begin{tabular}{lcc}
\hline ctep 3: TH from Minor St. & 8 & 11 \\
\hline onflicting Flows & & \\
Potential Capacity & & 1.00 \\
edestrian Impedance Factor & 1.00 & 0.76 \\
ap. Adj. factor due to Impeding mvmnt & 0.76 & 1.00 \\
rovement Capacity & 1.00 & 10 \\
robability of Queue free St. & 7 & 869 \\
- tep 4: LT from Minor St. & 291 \\
onflicting Flows & 1.00 \\
otential Capacity & 1.00 & 0.76 \\
Pedestrian Impedance Factor & 0.82 & 0.76 \\
aj. L, Min T Impedance factor & 0.74 & 222 \\
aj. L, Min T Adj. Imp Factor. & & \\
cap. Adj. factor due to Impeding mvmnt & & \\
ovement Capacity & &
\end{tabular}


Worksheet 7-Computation of the Effect of Two-stage Gap Acceptance

\begin{tabular}{lll}
\hline Step 3: TH from Minor St. & 8 & 11 \\
\hline Part 1 - First Stage & & \\
Conflicting Flows & & \\
Potential Capacity & & \\
Pedestrian Impedance Factor & \\
Cap. Adj. factor due to Impeding mvmnt & \\
Movement Capacity &
\end{tabular}

Part 2 - Second Stage

Conflicting Flows

Potential Capacity

Pedestrian Impedance Factor

Cap. Adj. factor due to Impeding mvmnt

Movement Capacity

\section{Part 3 - Single stage}

Conflicting Flows

Potential Capacity

Pedestrian Impedance Factor

Cap. Adj. factor due to Impeding mvmnt

1.00

1.00

Movement Capacity

$0.76 \quad 0.76$

Result for 2 stage process:

a

y

C $t$

Probability of Queue free st.

1.00

1.00

Step 4: LT from Minor St.

7

10

Part 1 - First Stage

Conflicting Flows

Potential Capacity

Pedestrian Impedance Factor

Cap. Adj. factor due to Impeding mvmnt

Movement Capacity

Part 2 - Second stage

Conflicting Flows

Potential Capacity

Pedestrian Impedance Factor

Cap. Adj. factor due to Impeding mvmnt

Movement Capacity

Part 3 - Single stage

Conflicting Flows

Potential Capacity

869

Pedestrian Impedance Factor

291

Maj. L, Min T Impedance factor

1.00

1.00

Maj. L, Min T Adj. Imp Factor.

Cap. Adj. factor due to Impeding mvmnt

0.82

0.74

0.76

Movement Capacity

222

Results for Two-stage process:

a

y 
worksheet 8-Shared Lane Calculations

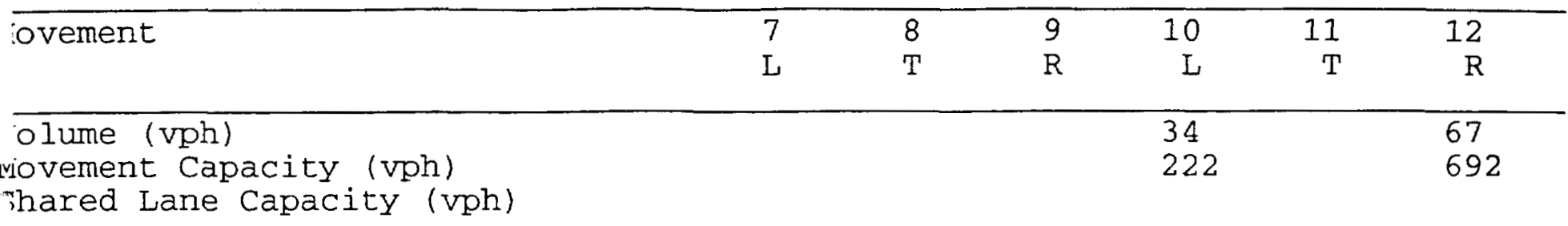

Worksheet 9-Computation of Effect of Flared Minor street Approaches

\begin{tabular}{|c|c|c|c|c|c|c|}
\hline ovement & $\begin{array}{l}7 \\
\text { L }\end{array}$ & $\begin{array}{l}8 \\
\mathrm{~T}\end{array}$ & $\begin{array}{l}9 \\
\mathrm{R}\end{array}$ & $\begin{array}{r}10 \\
\mathrm{~L}\end{array}$ & $\begin{array}{r}11 \\
\mathrm{~T}\end{array}$ & $\begin{array}{r}12 \\
\mathrm{R}\end{array}$ \\
\hline $\begin{array}{l}\text { sep } \\
\text { volume } \\
\text { gelay } \\
\text { sep } \\
\text { sep }+1 \\
\text { round (Qsep }+1)\end{array}$ & & & & $\begin{array}{l}222 \\
34\end{array}$ & & $\begin{array}{l}692 \\
67\end{array}$ \\
\hline
\end{tabular}

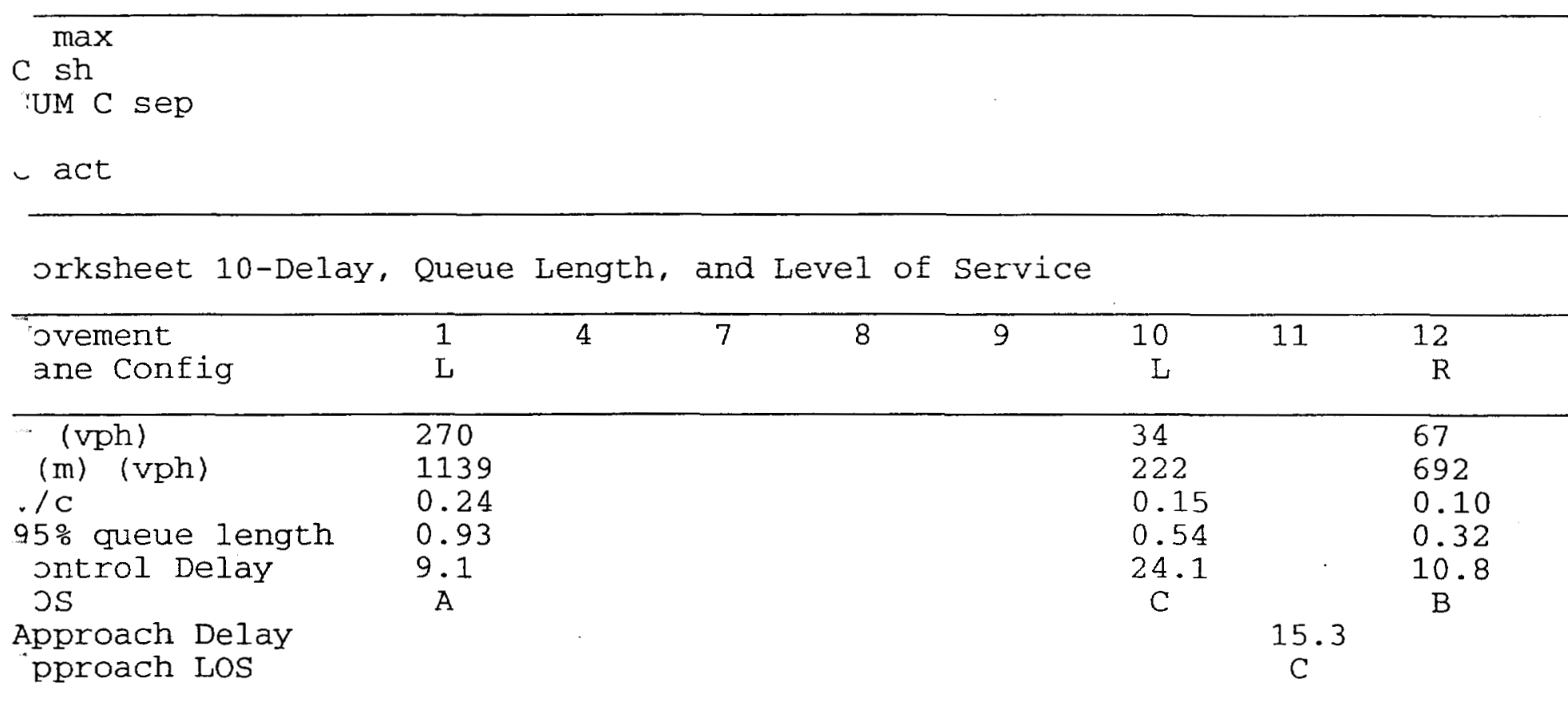

rqorksheet 11-Shared Major LT Impedance and Delay

\begin{tabular}{|c|c|c|}
\hline & Movement 2 & Movement 5 \\
\hline $\begin{array}{l}\text { (oj) } \\
\text { (i1), Volume for stream } 2 \text { or } 5 \\
v(i 2) \text {, Volume for stream } 3 \text { or } 6 \\
\text { (il), Saturation flow rate for stream } 2 \text { or } 5 \\
\text { (i2); Saturation flow rate for stream } 3 \text { or } 6 \\
r^{*}(o j) \\
\text { d(M, LT), Delay for stream } 1 \text { or } 4 \\
\text { Number of major street through lanes } \\
\text { (rank, 1) Delay for stream } 2 \text { or } 5\end{array}$ & 0.76 & 1.00 \\
\hline
\end{tabular}




\section{Analyst:}

Agency/Co.:

Date Performed: $\quad 1 / 21 / 02$

Analysis Time Period: PM Peak

Intersection:

East Ave \& $S$ Gate

\section{(Location \#6)}

Jurisdiction:

Analysis Year:

Project ID: Lawrence Livermore National Laboratory

East/West Street:

East Ave

North/South Street: South Gate

Intersection Orientation: EW

Study period (hrs): 1.00

Major Street: $\begin{aligned} & \text { Approach } \\ & \text { Movement }\end{aligned}$

Vehicle Volumes and Adjustments

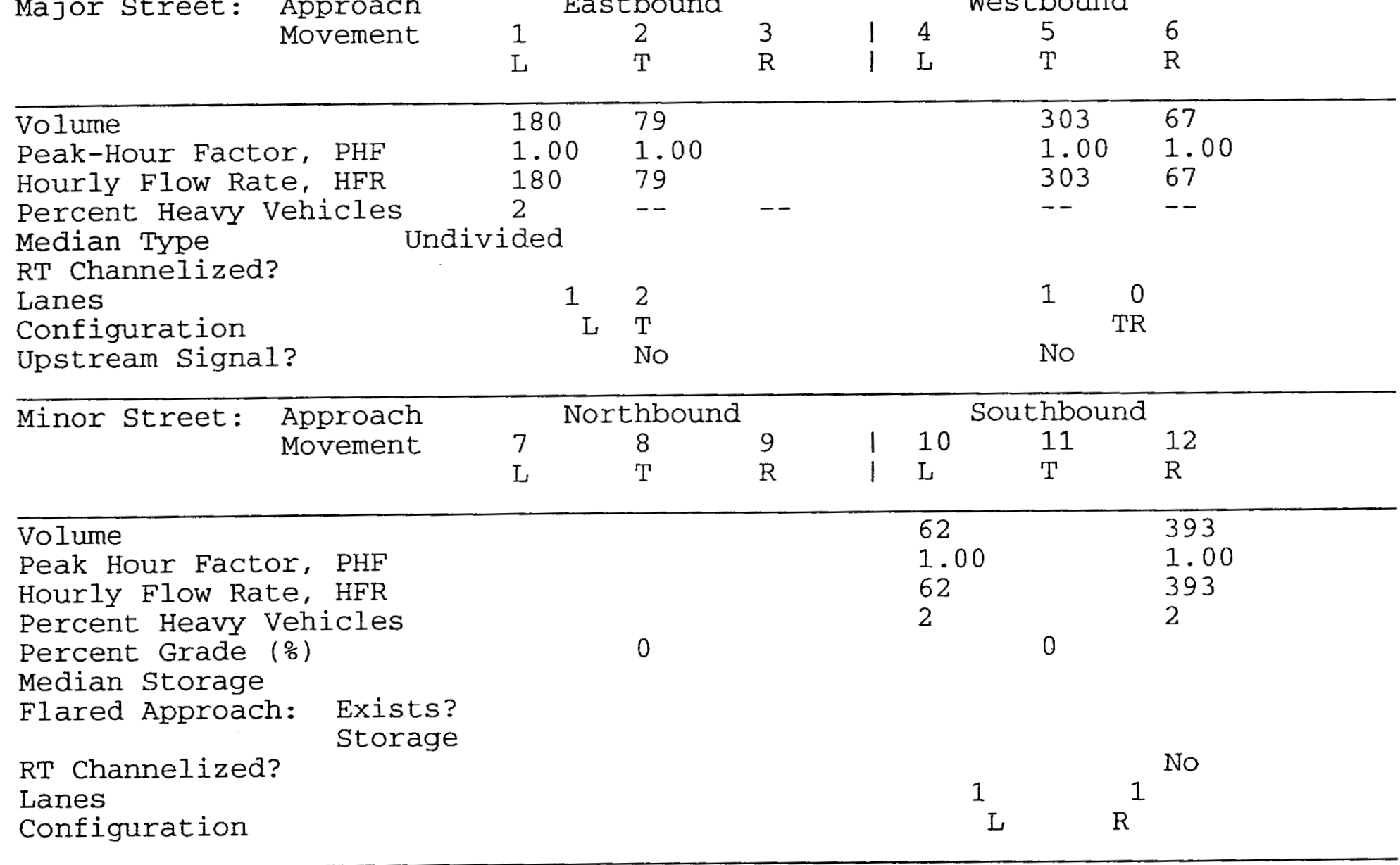

\section{Approach}

Movement

Lane Config

v (vph)

$\mathrm{C}(\mathrm{m})$ (vph)

$\mathrm{V} / \mathrm{C}$

95\% queue length

Control Delay
Delay, Queue Length, and Level of Service

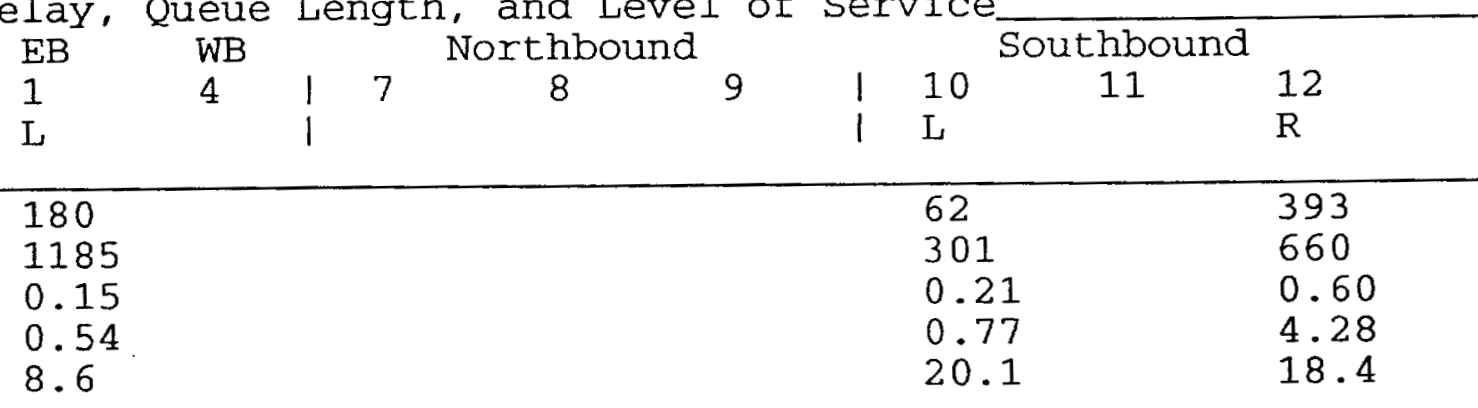


Torve Engineering, Inc.

horie:

Fax:

-Mail:

TWO-WAY STOP CONTROL(TWSC) ANALYSIS

malyst:

agency/Co.:

ate Performed: 1/21/02

nalysis Time Period: PM Peak

Intersection:

East Ave \& S Gate

urisdiction:

nalysis Year: 2020

Project ID: Lawrence Livermore National Laboratory

ast/West Street: East Ave

orth/South Street: South Gate

Intersection Orientation: EW Study period (hrs): 1.00

Vehicle Volumes and Adjustments

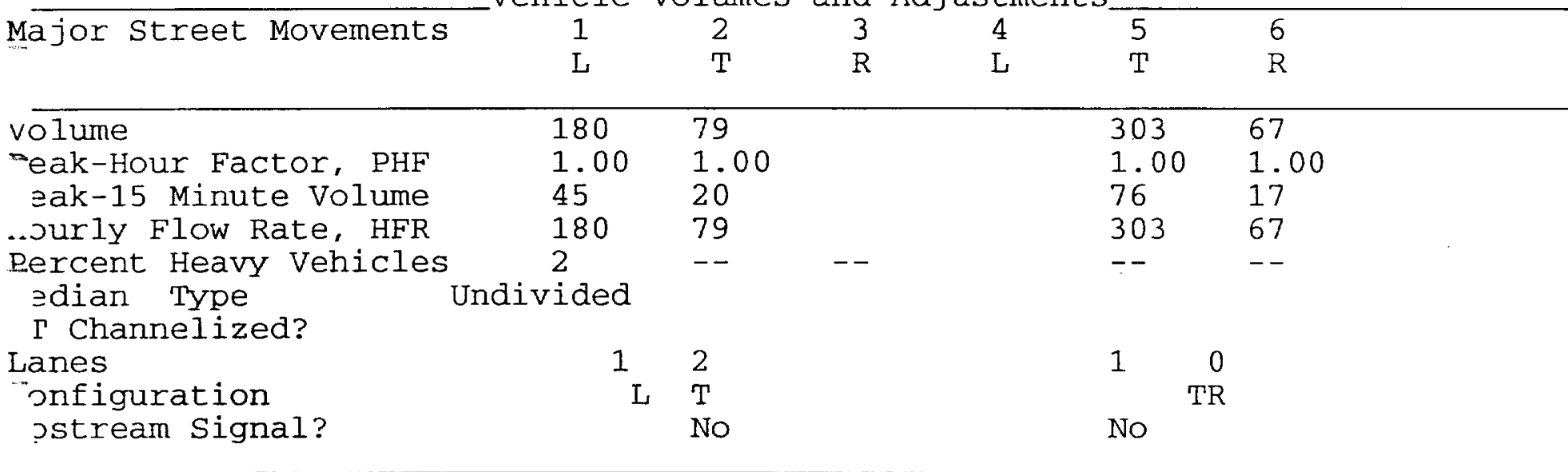

Minor Street Movements

$\begin{array}{llllll}7 & 8 & 9 & 10 & 11 & 12 \\ \mathrm{~L} & \mathrm{~T} & \mathrm{R} & \mathrm{L} & \mathrm{T} & \mathrm{R} \\ & & 62 & & 393 \\ & & 1.00 & & 1.00 \\ & & 16 & & 98 \\ & & 62 & & 393 \\ & & 2 & 0 & 2\end{array}$

Volume
əak Hour Factor, PHF
əak-15 Minute Volume
Hourly Flow Rate, HFR
ercent Heavy Vehicles
ərcent Grade ( $\%$ )

l.edian storage

Elared Approach: Exists?

c Channelized? 
Pedestrian Volumes and Adjustments

\begin{tabular}{lllll}
\hline Movements & 13 & 14 & 15 & 16 \\
\hline Flow (ped/hr) & 0 & 0 & 0 & 0 \\
Lane Width (ft) & 12.0 & 12.0 & 12.0 & 12.0 \\
Walking Speed (ft/sec) & 4.0 & 4.0 & 4.0 & 4.0 \\
Percent Blockage & 0 & 0 & 0 & 0
\end{tabular}

\begin{tabular}{|c|c|c|c|c|c|c|c|c|}
\hline \multicolumn{9}{|c|}{ Upstream Signal Data } \\
\hline & & $\begin{array}{l}\text { Prog. } \\
\text { Flow } \\
\text { vph }\end{array}$ & $\begin{array}{l}\text { Sat } \\
\text { Flow } \\
\text { vph }\end{array}$ & $\begin{array}{l}\text { Arrival } \\
\text { Type }\end{array}$ & $\begin{array}{l}\text { Green } \\
\text { Time } \\
\text { sec }\end{array}$ & $\begin{array}{l}\text { Cycle } \\
\text { Length } \\
\text { sec }\end{array}$ & $\begin{array}{l}\text { Prog } \\
\text { Speed } \\
\text { mph }\end{array}$ & $\begin{array}{l}\text { Distance } \\
\text { to Signal } \\
\text { feet }\end{array}$ \\
\hline S2 & $\begin{array}{l}\text { Left-Turn } \\
\text { Through }\end{array}$ & & - & & & - & & \\
\hline S5 & $\begin{array}{l}\text { Left-Turn } \\
\text { Through }\end{array}$ & & & & & & & \\
\hline
\end{tabular}

Worksheet 3-Data for Computing Effect of Delay to Major Street Vehicles

Movement 2 Movement 5

Shared In volume, major th vehicles:

Shared in volume, major rt vehicles:

Sat flow rate, major th vehicles:

Sat flow rate, major rt vehicles:

Number of major street through lanes:

Worksheet 4-Critical Gap and Follow-up Time Calculation

\begin{tabular}{|c|c|c|c|c|c|c|c|c|c|}
\hline $\begin{array}{l}\text { Critical } \\
\text { Movement }\end{array}$ & Gap Calc & $\begin{array}{l}\text { Lulati } \\
1 \\
\mathrm{~L}\end{array}$ & $\begin{array}{l}4 \\
\mathrm{~L}\end{array}$ & $\begin{array}{l}7 \\
\mathrm{~L}\end{array}$ & $\begin{array}{l}8 \\
T\end{array}$ & $\begin{array}{l}9 \\
\mathrm{R}\end{array}$ & $\begin{array}{r}10 \\
\mathrm{~L}\end{array}$ & $\begin{array}{r}11 \\
\mathrm{~T}\end{array}$ & $\begin{array}{r}12 \\
\mathrm{R}\end{array}$ \\
\hline $\begin{array}{l}t(c, \text { base }) \\
t(c, h v) \\
P(h v) \\
t(c, g) \\
\text { Grade/100 } \\
t(3,1 t) \\
t(c, T): \\
t(c)\end{array}$ & $\begin{array}{l}0 \\
1 \text {-stage } \\
\text { 2-stage } \\
1 \text {-stage } \\
\text { 2-stage }\end{array}$ & $\begin{array}{l}0.00 \\
0.00 \\
0.00 \\
4.1\end{array}$ & $\begin{array}{l}0.00 \\
0.00\end{array}$ & $\begin{array}{l}2.00 \\
0.20 \\
0.00 \\
0.00 \\
1.00\end{array}$ & $\begin{array}{l}2.00 \\
0.20 \\
0.00 \\
0.00 \\
1.00\end{array}$ & $\begin{array}{l}2.00 \\
0.10 \\
0.00 \\
0.00 \\
0.00\end{array}$ & $\begin{array}{l}7.5 \\
2.00 \\
2 \\
0.20 \\
0.00 \\
0.70 \\
0.00 \\
1.00 \\
6.8\end{array}$ & $\begin{array}{l}2.00 \\
0.20 \\
0.00 \\
0.00 \\
1.00\end{array}$ & $\begin{array}{l}6.9 \\
2.00 \\
2 \\
0.10 \\
0.00 \\
0.00 \\
0.00 \\
0.00 \\
6.9\end{array}$ \\
\hline $\begin{array}{l}\text { Follow-Up } \\
\text { Movement }\end{array}$ & p Time $\mathrm{Ca}$ & $\begin{array}{l}\text { alcula } \\
1 \\
\text { L }\end{array}$ & $\begin{array}{c}\text { Ions } \\
4 \\
\text { L }\end{array}$ & $\begin{array}{l}7 \\
\mathrm{~L}\end{array}$ & $\begin{array}{l}8 \\
\mathrm{~T}\end{array}$ & $\begin{array}{l}9 \\
\mathrm{R}\end{array}$ & $\begin{array}{r}10 \\
\mathrm{~L}\end{array}$ & $\begin{array}{r}11 \\
T\end{array}$ & $\begin{array}{r}12 \\
\mathrm{R}\end{array}$ \\
\hline $\begin{array}{l}t(f, \text { base }) \\
t(f, H V) \\
P(H V) \\
t(f)\end{array}$ & & $\begin{array}{l}2.20 \\
1.00 \\
2 \\
2.2\end{array}$ & 1.00 & 1.00 & 1.00 & 1.00 & $\begin{array}{l}3.50 \\
1.00 \\
2 \\
3.5\end{array}$ & 1.00 & $\begin{array}{l}3.30 \\
1.00 \\
2 \\
3.3\end{array}$ \\
\hline
\end{tabular}


$\therefore$ orksheet 5-Effect of Upstream Signals

omputation 1-Queue Clearance Time at Upstream Signal

Movement 2

Movement 5

$\mathrm{V}(\mathrm{t}) \quad \mathrm{V}(1, \mathrm{prot}) \quad \mathrm{V}(\mathrm{t}) \quad \mathrm{V}(1$, prot $)$

prog

l'otal Saturation Flow Rate, s (vph)

Irrival Type

ffective Green, g (sec)

$-y c l e$ Length, $\mathrm{C}$ (sec)

$\mathrm{Rp}$ (from table 9-2)

roportion vehicles arriving on green $\mathrm{P}$

(q1)

$g(q 2)$

(q)

Lomputation 2-Proportion of TWSC Intersection Time blocked

\section{Movement $2 \quad$ Movement 5}

$V(t) \quad V(1$, prot $) \quad V(t) \quad V(1$, prot $)$

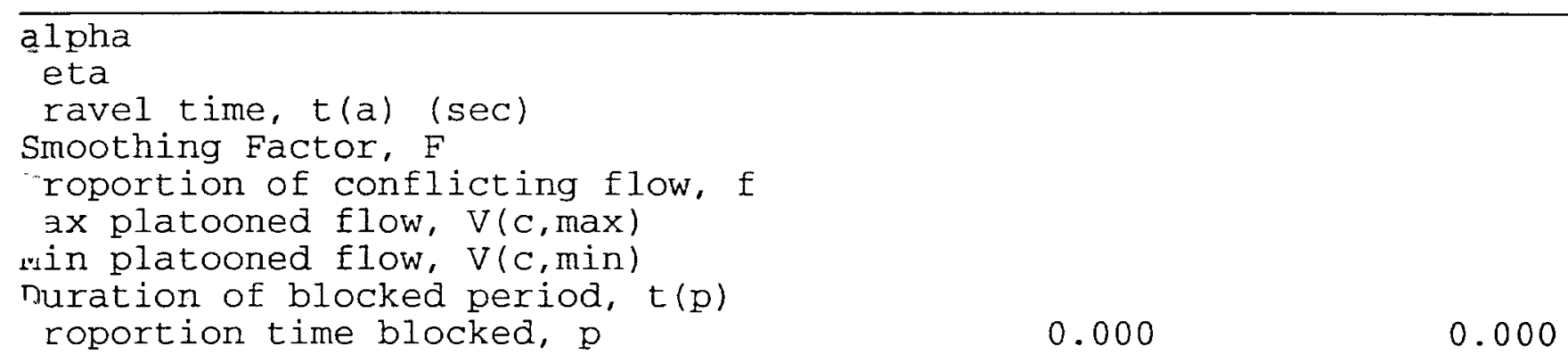

\begin{tabular}{|c|c|}
\hline Computation 3-Platoon Event Periods & Result \\
\hline $\begin{array}{l}(2) \\
p(5) \\
\quad(\text { dom }) \\
\quad \text { (subo) } \\
\text { wonstrained or unconstrained? }\end{array}$ & $\begin{array}{l}0.000 \\
0.000\end{array}$ \\
\hline $\begin{array}{l}\text { roportion } \\
\text { lblocked } \\
\text { for minor } \\
\text { ovements, } p(x)\end{array}$ & $\begin{array}{cc}(2) & (3) \\
\text { Two-Stage } & \text { Process } \\
\text { Stage I } & \text { Stage II }\end{array}$ \\
\hline
\end{tabular}

$\mathrm{p}(1)$

$n(4)$

(7)

$-(8)$

$\mathrm{p}(9)$

(10)

(11)

$\mathrm{p}(12)$

mputation 4 and 5

single-Stage Process

Movement

$\begin{array}{rrrrrrrr}1 & 4 & 7 & 8 & 9 & 10 & 11 & 12 \\ \mathrm{~L} & \mathrm{~L} & \mathrm{~L} & \mathrm{~T} & \mathrm{R} & \mathrm{L} & \mathrm{T} & \mathrm{R}\end{array}$


Stage1 Stage2 stage1 Stage2 stagel Stage2 stage1 ${ }^{11}$ stage2

$\overrightarrow{V(c, x)}$

$\mathrm{P}(\mathrm{x})$

$\mathrm{V}(\mathrm{c}, \mathrm{u}, \mathrm{x})$

$\mathrm{C}(\mathrm{r}, \mathrm{x})$

C (plat, $x)$

Worksheet 6-Impedance and Capacity Equations

\begin{tabular}{|c|c|c|}
\hline Step 1: RT from Minor st. & 9 & 12 \\
\hline $\begin{array}{l}\text { Conflicting Flows } \\
\text { Potential Capacity } \\
\text { Pedestrian Impedance Factor } \\
\text { Movement Capacity } \\
\text { Probability of Queue free st. }\end{array}$ & $\begin{array}{l}1.00 \\
1.00\end{array}$ & $\begin{array}{l}336 \\
660 \\
1.00 \\
660 \\
0.40\end{array}$ \\
\hline Step 2: LT from Major St. & 4 & 1 \\
\hline $\begin{array}{l}\text { Conflicting Flows } \\
\text { Potential Capacity } \\
\text { Pedestrian Impedance Factor } \\
\text { Movement Capacity } \\
\text { Probability of Queue free st. } \\
\text { Maj L-Shared Prob Q free st. }\end{array}$ & $\begin{array}{l}1.00 \\
1.00\end{array}$ & $\begin{array}{l}370 \\
1185 \\
1.00 \\
1185 \\
0.85\end{array}$ \\
\hline Step 3: TH from Minor St. & 8 & 11 \\
\hline $\begin{array}{l}\text { Conflicting Flows } \\
\text { Potential Capacity } \\
\text { Pedestrian Impedance Factor } \\
\text { Cap. Adj. factor due to Impeding mvmnt } \\
\text { Movement Capacity } \\
\text { Probability of Queue free st. }\end{array}$ & $\begin{array}{l}1.00 \\
0.85 \\
1.00\end{array}$ & $\begin{array}{l}1.00 \\
0.85 \\
1.00\end{array}$ \\
\hline Step 4: LT from Minor st. & 7 & 10 \\
\hline $\begin{array}{l}\text { Conflicting Flows } \\
\text { Potential Capacity } \\
\text { Pedestrian Impedance Factor } \\
\text { Maj. L, Min T Impedance factor } \\
\text { Maj. L, Min T Adj. Imp Factor. } \\
\text { Cap. Adj. factor due to Impeding mvmnt } \\
\text { Movement Capacity }\end{array}$ & $\begin{array}{l}1.00 \\
0.85 \\
0.88 \\
0.36\end{array}$ & $\begin{array}{l}735 \\
355 \\
1.00\end{array}$ \\
\hline
\end{tabular}


lorksheet 7-Computation of the Effect of Two-stage Gap Acceptance

\begin{tabular}{llll}
\hline tep 3: TH from Minor St. & 8 & 11
\end{tabular}

art 1 - First stage

onflicting Flows

potential Capacity

edestrian Impedance Factor

ap. Adj. factor due to Impeding mvint

Movement Capacity

'robability of Queue free st.

art 2 - Second Stage

onflicting Flows

otential Capacity

edestrian Impedance Factor

cap. Adj. factor due to Impeding mvmnt

'ovement Capacity

rart 3 - Single stage

onflicting Flows

otential Capacity

edestrian Impedance Factor

$1.00 \quad 1.00$

cap. Adj. factor due to Impeding mvmnt $\quad 0.85 \quad 0.85$

ovement Capacity

Result for 2 stage process:

$\checkmark t$

-robability of Queue free St.

1.00

1.00

tep 4: LT from Minor st.

10

art 1 - First stage

onflicting Flows

Potential Capacity

edestrian Impedance Factor

ap. Adj. factor due to Impeding mvmnt

rovement Capacity

art 2 - Second stage

onflicting Flows

Potential Capacity

edestrian Impedance Factor

ap. Adj. factor due to Impeding mvmnt

Movement Capacity

art 3 - Single stage

-onflicting Flows

Dotential Capacity

edestrian Impedance Factor

эj. L, Min T Impedance factor

Maj. L, Min T Adj. Imp Factor.

0.85

1.00

ap. Adj. factor due to Impeding mvmnt

0.88

ovement Capacity

$0.36 \quad 0.85$

mesults for Two-stage process: 
worksheet 8-Shared Lane Calculations

\begin{tabular}{|c|c|c|c|c|c|c|}
\hline Movement & $\begin{array}{l}7 \\
\mathrm{~L}\end{array}$ & $\begin{array}{l}8 \\
T\end{array}$ & $\begin{array}{l}9 \\
R\end{array}$ & $\begin{array}{r}10 \\
\mathrm{~L}\end{array}$ & $\begin{array}{r}11 \\
\mathrm{~T}\end{array}$ & $\begin{array}{r}12 \\
\mathrm{R}\end{array}$ \\
\hline $\begin{array}{l}\text { Volume (vph) } \\
\text { Movement Capacity (vph) } \\
\text { Shared Lane Capacity (vph) }\end{array}$ & & & & $\begin{array}{l}62 \\
301\end{array}$ & & $\begin{array}{l}393 \\
660\end{array}$ \\
\hline
\end{tabular}

Worksheet 9-Computation of Effect of Flared Minor Street Approaches

\begin{tabular}{|c|c|c|c|c|c|c|}
\hline Movement & $\begin{array}{l}7 \\
L\end{array}$ & $\begin{array}{l}8 \\
\mathrm{~T}\end{array}$ & $\begin{array}{l}9 \\
R\end{array}$ & $\begin{array}{r}10 \\
\mathrm{~L}\end{array}$ & $\begin{array}{r}11 \\
\mathrm{~T}\end{array}$ & $\begin{array}{r}12 \\
\mathrm{R}\end{array}$ \\
\hline $\begin{array}{l}\text { C sep } \\
\text { Volume } \\
\text { Delay } \\
Q \text { sep } \\
Q \text { sep }+1 \\
\text { round (Qsep +1) }\end{array}$ & & & & $\begin{array}{l}301 \\
62\end{array}$ & & $\begin{array}{r}660 \\
393\end{array}$ \\
\hline $\begin{array}{l}\text { n max } \\
\text { C sh } \\
\text { SUM } C \text { sep } \\
\text { n } \\
C \text { act }\end{array}$ & & & & & & \\
\hline
\end{tabular}

Worksheet 10-Delay, Queue Length, and Level of Service

\begin{tabular}{|c|c|c|c|c|c|c|c|c|}
\hline $\begin{array}{l}\text { Movement } \\
\text { Lane Config }\end{array}$ & $\begin{array}{l}1 \\
L\end{array}$ & 4 & 7 & 8 & 9 & $\begin{array}{r}10 \\
\mathrm{~L}\end{array}$ & 11 & $\begin{array}{r}12 \\
\mathrm{R}\end{array}$ \\
\hline $\begin{array}{l}\text { V (vph) } \\
\text { C(m) (vph) } \\
\text { v/C } \\
95 \% \text { queue length } \\
\text { Control Delay } \\
\text { LOS } \\
\text { Approach Delay } \\
\text { Approach LOS }\end{array}$ & $\begin{array}{l}180 \\
1185 \\
0.15 \\
0.54 \\
8.6 \\
\text { A }\end{array}$ & & & & & $\begin{array}{l}62 \\
301 \\
0.21 \\
0.77 \\
20.1 \\
C\end{array}$ & . & $\begin{array}{c}393 \\
660 \\
0.60 \\
4.28 \\
18.4 \\
C\end{array}$ \\
\hline
\end{tabular}

Worksheet 11-Shared Major LT Impedance and Delay

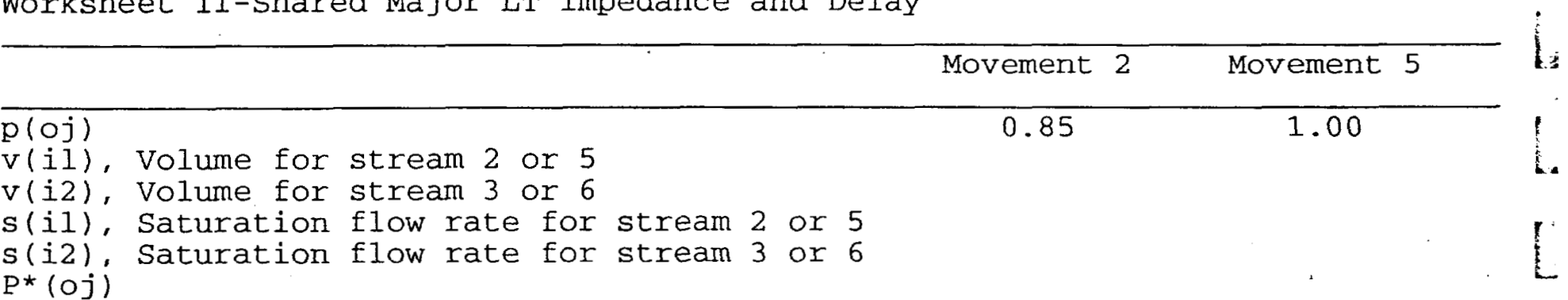

$\mathrm{d}(\mathrm{M}, \mathrm{LT})$, Delay for stream 1 or 4

8.6

$N$, Number of major street through lanes

d(rank, 1) Delay for stream 2 or 5 
inalyst :

rency/Co.

te Performed:

$1 / 21 / 02$

ualysis Time Period: AM Peak

ๆtersection:

East Ave \& E Entrance (Locatim \# 8)

risdiction:

alysis Year:

2020

Project ID: Lawrence Livermore National Laboratory

st/West Street: East Ave

rth/South Street: East Entrance

itersection orientation: EW $\quad$ Study period (hrs): 1.00

Vehicle Volumes and Adjustments

\begin{tabular}{lllclllll}
\hline rajor Street: & Approach & \multicolumn{3}{c}{ Eastbound } & \multicolumn{3}{c}{ Westbound } \\
& Movement & $\mathrm{I}$ & 2 & 3 & 4 & 5 & 6 \\
& & $\mathrm{~L}$ & $\mathrm{~T}$ & $\mathrm{R}$ & $\mathrm{L}$ & $\mathrm{T}$ & $\mathrm{R}$
\end{tabular}

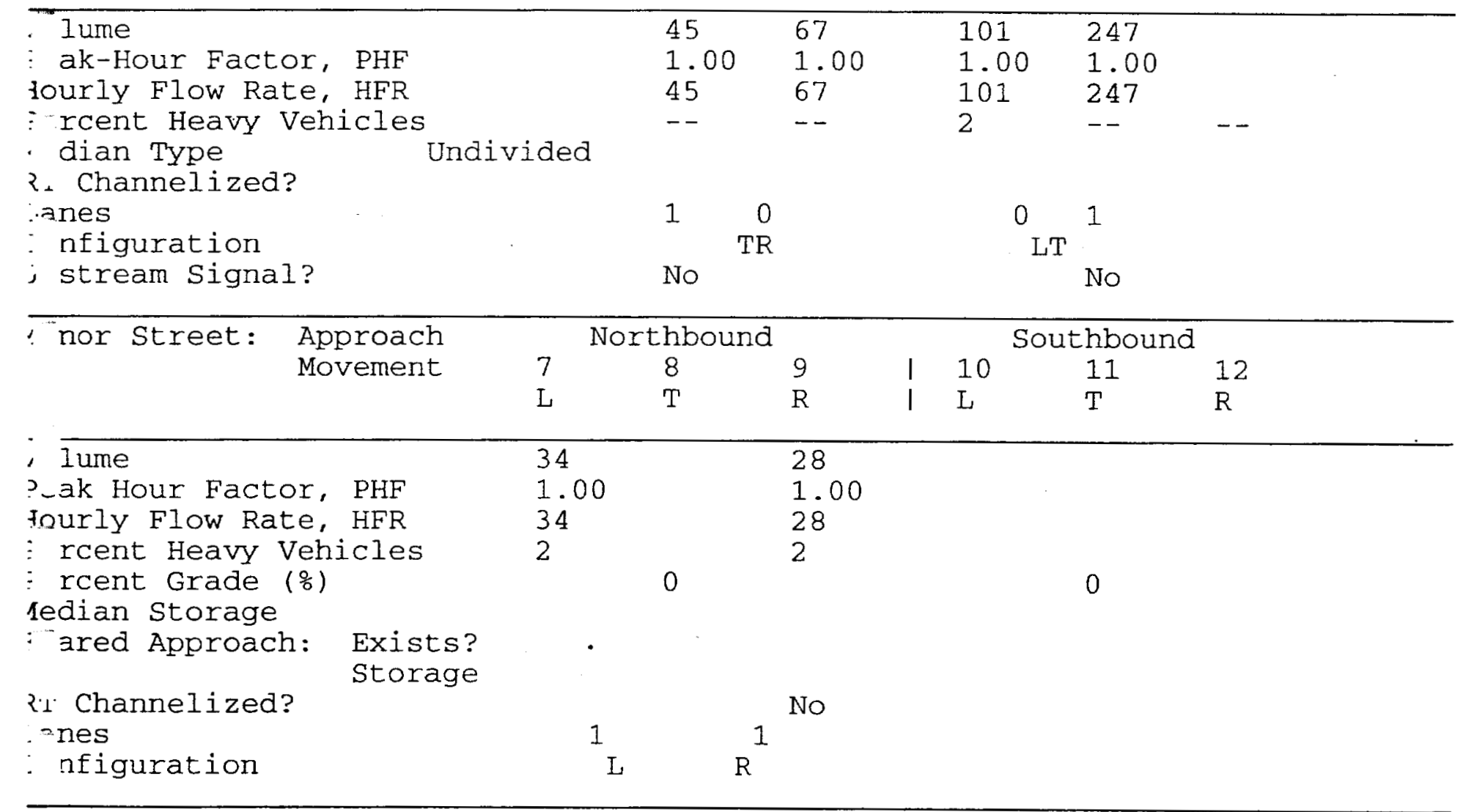

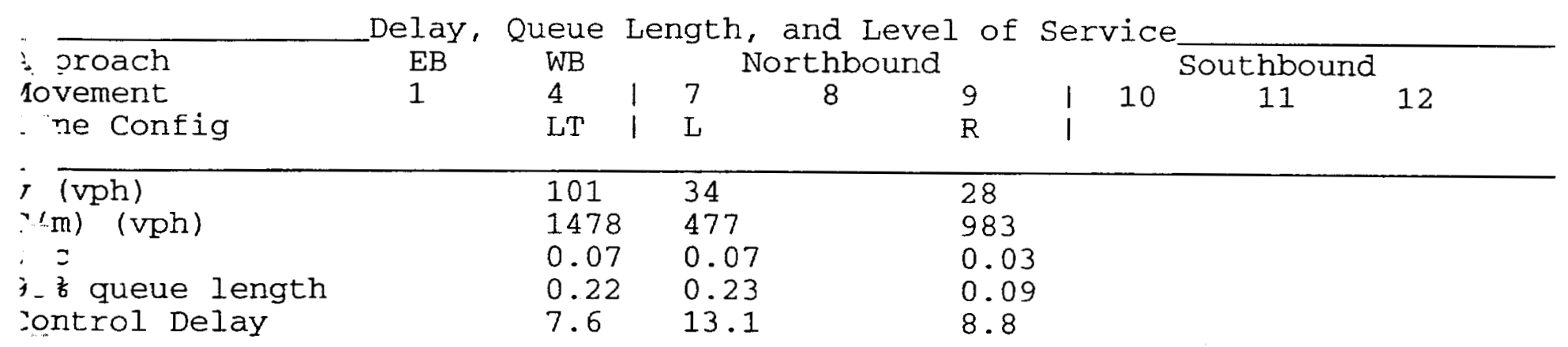


HCS2000: Unsignalized Intersections Release 4.1

orve Engineering, Inc.

hone:

Fax :

-Mail:

TWO-WAY STOP CONTROL(TWSC) ANALYSIS

nalyst:

gency/Co.:

ate Performed: 1/21/02

nalysis Time Period: AM Peak

ntersection:

East Ave \& E Entrance

urisdiction:

nalysis Year:

2020

roject ID: Lawrence Livermore National Laboratory

ast/West Street: East Ave

orth/South Street: East Entrance

ntersection Orientation: EW

Study period (hrs): 1.00

Vehicle Volumes and Adjustments

\begin{tabular}{lllllll}
\hline ajor Street Movements & 1 & 2 & 3 & 4 & 5 & 6 \\
\cline { 5 - 7 } & $\mathrm{L}$ & $\mathrm{T}$ & $\mathrm{R}$ & $\mathrm{L}$ & $\mathrm{T}$ & $\mathrm{R}$
\end{tabular}

\begin{tabular}{lllll}
\hline olume & 45 & 67 & 101 & 247 \\
eak-Hour Factor, PHF & 1.00 & 1.00 & 1.00 & 1.00 \\
eak-15 Minute Volume & 11 & 17 & 25 & 62 \\
ourly Flow Rate, HFR & 45 & 67 & 101 & 247 \\
ercent Heavy Vehicles & -- & -- & 2 & --
\end{tabular}

edian Type Undivided

$\mathrm{T}$ Channelized?

onfiguration

pstream Signal?

$\begin{array}{crcc}1 & 0 & 0 & 1 \\ & \text { TR } & & \text { LT } \\ & & & \text { No }\end{array}$

\begin{tabular}{llllrrr}
\hline inor street Movements & 7 & 8 & 9 & 10 & 11 & 12 \\
& $\mathrm{~L}$ & $\mathrm{~T}$ & $\mathrm{R}$ & $\mathrm{L}$ & $\mathrm{T}$ & $\mathrm{R}$
\end{tabular}

olume

eak Hour Factor, PHF

eak-15 Minute Volume

ourly Flow Rate, HFR

excent Heavy Vehicles

ercent Grade (\%)

$\begin{array}{ll}34 & 28 \\ 1.00 & 1.00 \\ 8 & 7 \\ 34 & 28 \\ 2 & 2\end{array}$

edian Storage

0

28

lared Approach: Exists?

$\mathrm{T}$ Channelized?

Storage 

nes
infiguration
1
1
$\mathrm{R}$

Pedestrian Volumes and Adjustments

\begin{tabular}{lllll}
\hline 1ovements & 13 & 14 & 15 & 16 \\
\hline Ow (ped/hr) & 0 & 0 & 0 & 0 \\
sane Width (ft) & 12.0 & 12.0 & 12.0 & 12.0 \\
".lking Speed (ft/sec) & 4.0 & 4.0 & 4.0 & 4.0 \\
rrcent Blockage & 0 & 0 & 0 & 0
\end{tabular}

\begin{tabular}{|c|c|c|c|c|c|c|c|c|}
\hline \multicolumn{9}{|c|}{$\because$} \\
\hline & & $\begin{array}{l}\text { Prog. } \\
\text { Flow } \\
\text { vph }\end{array}$ & $\begin{array}{l}\text { Sat } \\
\text { Flow } \\
\text { vph }\end{array}$ & $\begin{array}{l}\text { Arrival } \\
\text { Type }\end{array}$ & $\begin{array}{l}\text { Green } \\
\text { Time } \\
\text { sec }\end{array}$ & $\begin{array}{l}\text { Cycle } \\
\text { Length } \\
\text { sec }\end{array}$ & $\begin{array}{l}\text { Prog. } \\
\text { Speed } \\
\text { mph }\end{array}$ & $\begin{array}{c}\text { Distance } \\
\text { to Signal } \\
\text { feet }\end{array}$ \\
\hline & $\begin{array}{l}\text { Left-Turn } \\
\text { Through } \\
\text { Left-Turn } \\
\text { Through }\end{array}$ & & & & - & & & \\
\hline
\end{tabular}

rksheet 3-Data for Computing Effect of Delay to Major street Vehicles

\begin{tabular}{lll}
\hline & Movement 2 & Movement 5 \\
\hline iıared In volume, major th vehicles: & & 247 \\
shared In volume, major rt vehicles: & & 1700 \\
$\vdots$ t flow rate, major th vehicles: & 1700 \\
$j$ t flow rate, major rt vehicles: & 1 \\
jumber of major street through lanes: & \\
\hline
\end{tabular}

vorksheet 4-Critical Gap and Follow-up Time Calculation

\begin{tabular}{|c|c|c|c|c|c|c|c|c|c|}
\hline iv vement & tap & 1 & $\begin{array}{l}4 \\
L\end{array}$ & $\begin{array}{l}7 \\
\mathrm{~L}\end{array}$ & $\begin{array}{l}8 \\
\mathrm{~T}\end{array}$ & $\begin{array}{l}9 \\
\mathrm{R}\end{array}$ & $\begin{array}{r}10 \\
L\end{array}$ & $\begin{array}{r}11 \\
\mathrm{~T}\end{array}$ & $\begin{array}{r}12 \\
\mathrm{R}\end{array}$ \\
\hline $\begin{array}{l}=\text { c, base) } \\
=(c, h v) \\
: \text { hv })\end{array}$ & & 1.00 & $\begin{array}{l}4.1 \\
1.00 \\
2\end{array}$ & $\begin{array}{l}7.1 \\
1.00 \\
2\end{array}$ & 1.00 & $\begin{array}{l}6.2 \\
1.00 \\
2 .\end{array}$ & 1.00 & 1.00 & 1.00 \\
\hline $\begin{array}{l}(c, g) \\
\text { irade/100 } \\
-(3,1 t)\end{array}$ & & & 0.00 & $\begin{array}{l}0.20 \\
0.00 \\
0.70\end{array}$ & $\begin{array}{l}0.20 \\
0.00\end{array}$ & $\begin{array}{l}0.10 \\
0.00 \\
0.00\end{array}$ & $\begin{array}{l}0.20 \\
0.00\end{array}$ & $\begin{array}{l}0.20 \\
0.00\end{array}$ & $\begin{array}{l}0.10 \\
0.00\end{array}$ \\
\hline $\begin{aligned} & C, T): \\
= & (C)\end{aligned}$ & $\begin{array}{l}1 \text {-stage } \\
2 \text {-stage } \\
1 \text {-stage } \\
2 \text {-stage }\end{array}$ & $\begin{array}{l}0.00 \\
0.00\end{array}$ & $\begin{array}{l}0.00 \\
0.00 \\
4.1\end{array}$ & $\begin{array}{l}0.00 \\
1.00 \\
6.4\end{array}$ & $\begin{array}{l}0.00 \\
1.00\end{array}$ & $\begin{array}{l}0.00 \\
0.00 \\
6.2\end{array}$ & $\begin{array}{l}0.00 \\
1.00\end{array}$ & $\begin{array}{l}0.00 \\
1.00\end{array}$ & $\begin{array}{l}0.00 \\
0.00\end{array}$ \\
\hline $\begin{array}{l}\text { ollow-Up } \\
\text { vement }\end{array}$ & Time $\mathrm{C}$ & $\begin{array}{c}\text { alcula } \\
1 \\
\mathrm{~L}\end{array}$ & $\begin{array}{c}\text { ions } \\
4 \\
\text { L }\end{array}$ & $\begin{array}{l}7 \\
\mathrm{~L}\end{array}$ & $\begin{array}{l}8 \\
\mathrm{~T}\end{array}$ & $\begin{array}{l}9 \\
\mathrm{R}\end{array}$ & $\begin{array}{r}10 \\
\mathrm{~L}\end{array}$ & $\begin{array}{r}11 \\
\mathrm{~T}\end{array}$ & $\begin{array}{r}12 \\
\mathrm{R}\end{array}$ \\
\hline $\begin{array}{l}- \text { (f, base) } \\
\text { f, HV) } \\
=(H V) \\
=(\mathrm{fl})\end{array}$ & & 0.90 & $\begin{array}{l}2.20 \\
0.90 \\
2 \\
2.2\end{array}$ & $\begin{array}{l}3.50 \\
0.90 \\
2 \\
3.5\end{array}$ & 0.90 & $\begin{array}{l}3.30 \\
0.90 \\
2 \\
3.3\end{array}$ & 0.90 & 0.90 & 0.90 \\
\hline
\end{tabular}


orksheet 5-Effect of Upstream Signals

omputation 1-Queue Clearance Time at Upstream Signal

Movement 2

Movement 5

$V(t) \quad V(1$, prot $) \quad V(t) \quad V(1$, prot $)$

\section{prog}

otal Saturation Flow Rate, s (vph)

rrival Type

ffective Green, $g$ (sec)

ycle Length, C (sec)

$\mathrm{p}$ (from table 9-2)

roportion vehicles arriving on green $P$

(q1)

(q2)

(q)

omputation 2-Proportion of TWSC Intersection Time blocked

Movement 2 Movement 5

$V(t) \quad V(1$, prot $) \quad V(t) \quad V(1$, prot $)$

\begin{tabular}{|c|c|c|}
\hline $\begin{array}{l}\text { lpha } \\
\text { eta } \\
\text { ravel time, t(a) (sec) } \\
\text { moothing Factor, } F \\
\text { roportion of conflicting flow, f } \\
\text { ax platooned flow, } V(c, m a x) \\
\text { in platooned flow, } V(c, m i n) \\
\text { uration of blocked period, } t(p) \\
\text { roportion time blocked, p }\end{array}$ & 0.000 & 0.000 \\
\hline omputation 3-Platoon Event Periods & Result & \\
\hline $\begin{array}{l}(2) \\
(5) \\
\text { (dom) } \\
\text { (subo) } \\
\text { onstrained or unconstrained? }\end{array}$ & $\begin{array}{l}0.000 \\
0.000\end{array}$ & \\
\hline
\end{tabular}

roportion

nblocked

or minor

ovements, $\mathrm{p}(\mathrm{x})$
(1)

Single-stage Process
(2)

Two-stage Process

Stage I Stage II

(1)

(4)

(7)

(8)

(9)

(10)

(11)

(12)

omputation 4 and 5

ingle-stage Process ovement

1

$\begin{array}{ll}4 & 7 \\ L & \text { L }\end{array}$

8

$\mathrm{T}$

$\begin{array}{rr}9 & 10 \\ R & L\end{array}$

$\begin{array}{rr}11 & 12 \\ \mathrm{~T} & \mathrm{R}\end{array}$


Px

$c, u, x$

$=r, x$

plat, $x$

Iwo-Stage Process

$7 \quad 8 \quad 10$

11

Stage1 Stage2 Stage1 Stage2 Stage1 Stage2 Stage1 Stage2

$\overline{v(c, x)}$

$(x)$

1700

$v(c, u, x)$

$r, x)$

$=($ plat,$x)$

n rksheet 6-Impedance and Capacity Equations

\begin{tabular}{|c|c|c|}
\hline ep 1: RT from Minor St & 9 & 12 \\
\hline $\begin{array}{l}\text { Eonflicting Flows } \\
\text { : tential Capacity } \\
\vdots \text { destrian Impedance Factor } \\
\text { 1uvement Capacity } \\
\text { ?robability of Queue free st. }\end{array}$ & $\begin{array}{l}78 \\
983 \\
1.00 \\
983 \\
0.97\end{array}$ & $\begin{array}{l}1.00 \\
1.00\end{array}$ \\
\hline$\overline{\mathrm{ep}}$ 2: LT from Major St. & 4 & 1 \\
\hline $\begin{array}{l}\text { "nflicting Flows } \\
\text { tential Capacity } \\
\text { ?edestrian Impedance Factor } \\
\text { ravement Capacity } \\
\text { Oobability of Queue free St. } \\
\eta_{u j} \text { L-Shared Prob Q free St. }\end{array}$ & $\begin{array}{l}112 \\
1478 \\
1.00 \\
1478 \\
0.93 \\
0.92\end{array}$ & $\begin{array}{l}1.00 \\
1.00\end{array}$ \\
\hline
\end{tabular}

\begin{tabular}{|c|c|c|}
\hline ep 3: TH from Minor St. & 8 & 11 \\
\hline $\begin{array}{l}\text { Eonflicting Flows } \\
\text { 'tential Capacity } \\
\text { : destrian Impedance Factor } \\
\text { ap. Adj. factor due to Impeding mvmnt } \\
\text { svement Capacity } \\
\text { obability of Queue free St. }\end{array}$ & $\begin{array}{l}1.00 \\
0.92 \\
1.00\end{array}$ & $\begin{array}{l}1.00 \\
0.92 \\
1.00\end{array}$ \\
\hline itep 4: LT from Minor St. & 7 & 10 \\
\hline $\begin{array}{l}\text { nflicting Flows } \\
\text { ?otential Capacity } \\
\text { destrian Impedance Factor } \\
j . \text { L, Min T Impedance factor } \\
\text { Iaj. L, Min T Adj. Imp Factor. } \\
\text { ap. Adj. factor due to Impeding mvmnt } \\
\text { vement Capacity }\end{array}$ & $\begin{array}{l}527 \\
512 \\
1.00\end{array}$ & $\begin{array}{l}1.00 \\
0.92 \\
0.94 \\
0.91\end{array}$ \\
\hline
\end{tabular}


orksheet 7-Computation of the Effect of Two-stage Gap Acceptance

tep 3: TH from Minor St. $\quad 8 \quad 11$

art 1 - First stage

onflicting Flows

otential Capacity

edestrian Impedance Factor

ap. Adj. factor due to Impeding mumnt

ovement Capacity

robability of Queue free st.

art 2 - Second stage

onflicting Flows

otential Capacity

edestrian Impedance Factor

ap. Adj. Eactor due to Impeding mvmnt

ovement Capacity

art 3 - Single stage

onflicting Flows

otential Capacity

edestrian Impedance Factor

ap. Adj. factor due to Impeding mvmnt

1.00

0.92

1.00

ovement Capacity

esult for 2 stage process:

$t$

robability of Queue free St.

1.00

1.00

tep 4: LT from Minor st.

7

art 1 - First stage

onflicting Flows

otential Capacity

edestrian Impedance Factor

ap. Adj. Eactor due to Impeding mumnt

ovement Capacity

art 2 - Second stage

onflicting Flows

otential Capacity

edestrian Impedance Factor

ap. Adj. factor due to Impeding mvmnt

ovement Capacity

art 3 - single stage

onflicting Flows

otential Capacity

edestrian Impedance Factor

527

512

1.00

1.00

aj. L, Min T Impedance factor

aj. L, Min T Adj. Imp Factor.

0.92

ap. Adj. factor due to Impeding mvmnt

ovement Capacity

0.93

0.94

477

0.91

esults for Two-stage process: 
; wksheet 8-Shared Lane Calculations

\begin{tabular}{|c|c|c|c|c|c|c|}
\hline Movement & $\begin{array}{l}7 \\
\mathrm{~L}\end{array}$ & $\begin{array}{l}8 \\
\mathrm{~T}\end{array}$ & $\begin{array}{l}9 \\
R\end{array}$ & $\begin{array}{r}10 \\
\mathrm{~L}\end{array}$ & $\begin{array}{r}11 \\
\mathrm{~T}\end{array}$ & $\begin{array}{r}12 \\
\mathrm{R}\end{array}$ \\
\hline $\begin{array}{l}\text { ivlume (vph) } \\
\because \text { vement Capacity (vph) } \\
\vdots \text { lared Lane Capacity (vph) }\end{array}$ & $\begin{array}{l}34 \\
477\end{array}$ & & $\begin{array}{l}28 \\
983\end{array}$ & & & \\
\hline
\end{tabular}

; rrksheet 9-Computation of Effect of Flared Minor Street Approaches

\begin{tabular}{|c|c|c|c|c|c|c|}
\hline Movement & $\begin{array}{l}7 \\
\mathrm{~L}\end{array}$ & $\begin{array}{l}8 \\
T\end{array}$ & $\begin{array}{l}9 \\
\mathrm{R}\end{array}$ & $\begin{array}{r}10 \\
\mathrm{~L}\end{array}$ & $\begin{array}{r}11 \\
\mathrm{~T}\end{array}$ & $\begin{array}{r}12 \\
\mathrm{R}\end{array}$ \\
\hline $\begin{array}{l}\vdots \text { sep } \\
i \text { lume } \\
\vdots \text { lay } \\
\text { sep } \\
j \text { sep }+1 \\
i \text { und (Qsep +1) }\end{array}$ & $\begin{array}{l}477 \\
34\end{array}$ & & $\begin{array}{l}983 \\
28\end{array}$ & & & \\
\hline $\begin{array}{l}I \max \\
\vdots \text { sh } \\
\vdots \text { M C sep } \\
\text { I } \\
=\text { act }\end{array}$ & & & & & & \\
\hline
\end{tabular}

Norksheet 10-Delay, Queue Length, and Level of Service

\begin{tabular}{|c|c|c|c|c|c|c|c|c|}
\hline $\begin{array}{l}\text { vement } \\
\text { tane Config }\end{array}$ & 1 & $\begin{array}{l}4 \\
\mathrm{LT}\end{array}$ & $\begin{array}{l}7 \\
\mathrm{~L}\end{array}$ & 8 & $\begin{array}{l}9 \\
\mathrm{R}\end{array}$ & 10 & 11 & 12 \\
\hline 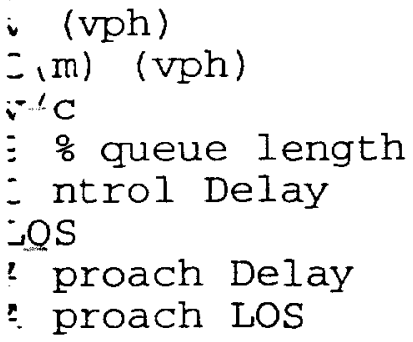 & & $\begin{array}{l}101 \\
1478 \\
0.07 \\
0.22 \\
7.6 \\
\text { A }\end{array}$ & $\begin{array}{l}34 \\
477 \\
0.07 \\
0.23 \\
13.1 \\
B\end{array}$ & $\underset{B}{11.2}$ & $\begin{array}{l}28 \\
983 \\
0.03 \\
0.09 \\
8.8 \\
\text { A }\end{array}$ & & & \\
\hline
\end{tabular}

- rksheet 11-Shared Major LT Impedance and Delay

\begin{tabular}{|c|c|c|}
\hline & Movement 2 & Movement 5 \\
\hline 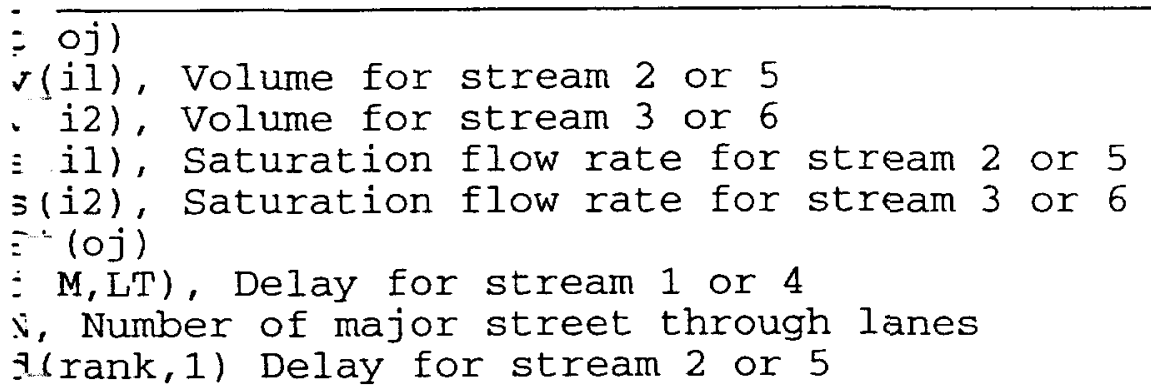 & 1.00 & $\begin{array}{l}0.93 \\
247 \\
0 \\
1700 \\
1700 \\
0.92 \\
7.6 \\
1 \\
0.6\end{array}$ \\
\hline
\end{tabular}


nalyst :

gency/Co.:

ate Performed:

$1 / 21 / 02$

nalysis Time Period: PM Peak

ntersection:

East Ave \& E Entrance (Location \#8)

urisdiction:

nalysis Year:

2020

roject ID: Lawrence Livermore National Laboratory

ast/West Street: East Ave

orth/South Street: East Entrance

ntersection Orientation: EW

Study period (hrs): 1.00

Vehicle Volumes and Adjustments

ajor Street: Approach Movement

Eastbound

\begin{tabular}{lll|l}
1 & 2 & 3 & 4 \\
$\mathrm{~L}$ & $\mathrm{~T}$ & $\mathrm{R}$ & $\mathrm{L}$
\end{tabular}

Westbound

\section{olume}

$236 \quad 11$

eak-Hour Factor, PHF

ourly Flow Rate, HFR

ercent Heavy Vehicles

edian Type

$\begin{array}{llll}1.00 & 1.00 & 1.00 & 1.00\end{array}$

T Channelized?

anes

onfiguration

pstream Signal?

Undivided

$\begin{array}{llll}236 & 11 & 22 & 67\end{array}$

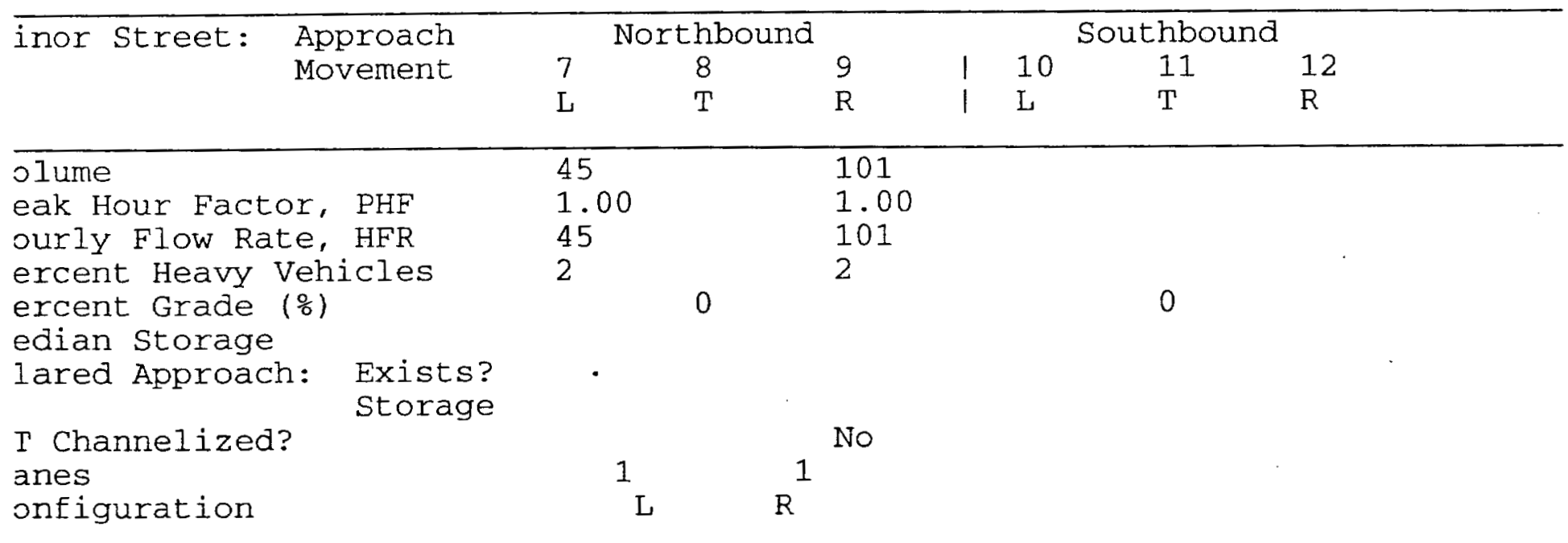

Delay, Queue Length, and Level of Service

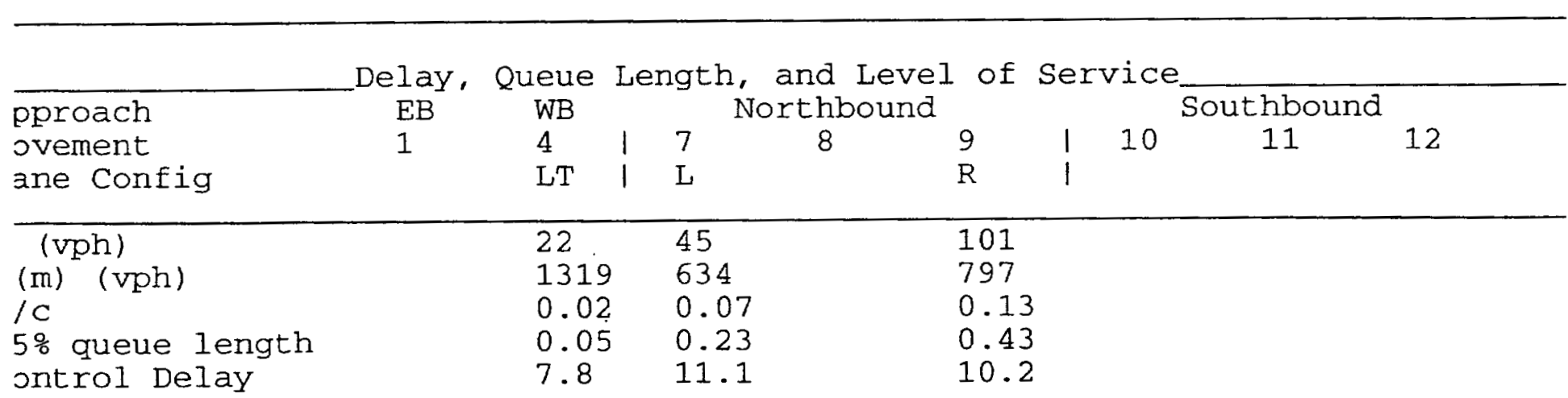

$\begin{array}{lrll}1 & 0 & 0 & 1 \\ \text { No } & \text { TR } & \text { LTT } & \text { NO }\end{array}$


HCS2000: Unsignalized Intersections Release 4.1

rve Engineering, Inc.

Fax :

I-Mail: TWO-WAY STOP CONTROL (TWSC) ANALYSIS

- lalyst :

ency/Co.:

$\because$ te Performed:

$1 / 21 / 02$

tnalysis Time Period: PM Peak

! tersection:

East Ave \& E Entrance

- risdiction:

inalysis Year:

2020

oject ID: Lawrence Livermore National Laboratory

st/West Street: East Ave

Jurth/South Street: East Entrance

? tersection orientation: EW

Study period (hrs): 1.00

Vehicle Volumes and Adjustments

$\begin{array}{cccccccc}\text { jor Street Movements } & 1 & 2 & 3 & 4 & 5 & 6 \\ & \mathrm{~L} & \mathrm{~T} & \mathrm{R} & \mathrm{L} & \mathrm{T} & \mathrm{R}\end{array}$

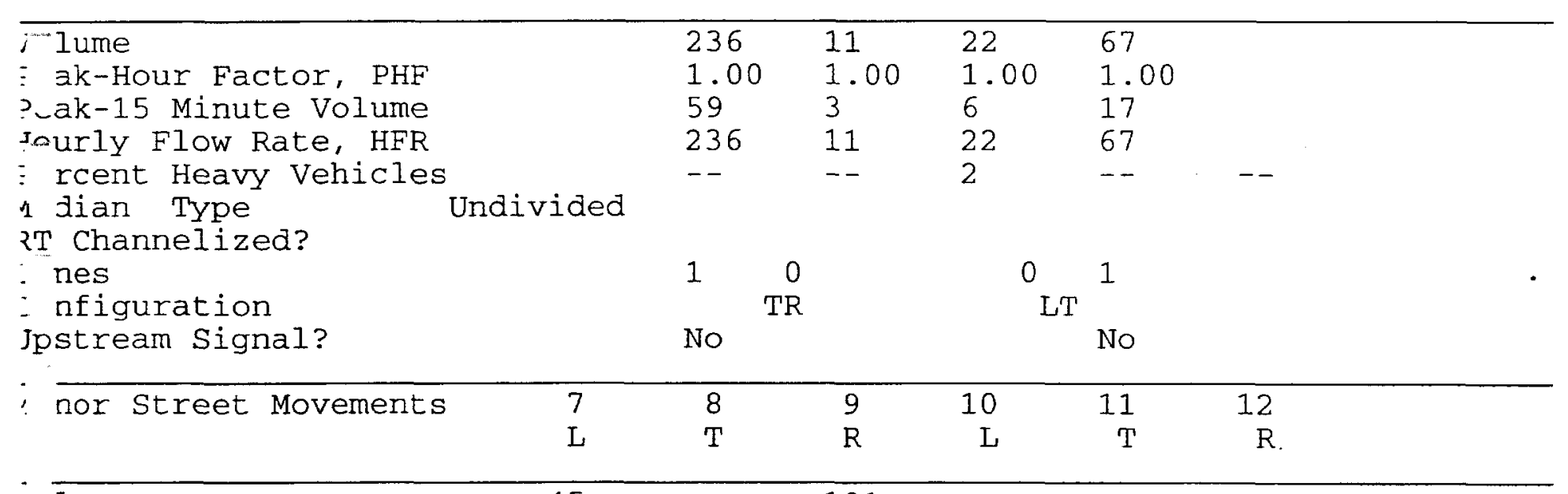

Iume

ak Hour Factor, PHF

seak-15 Minute Volume

urly Flow Rate, HFR

rcent Heavy Vehicles

?ercent Grade (\%)

dian Storage

: ared Approach: Exists?

2T Channelized?

$\begin{array}{ll}45 & 101 \\ 1.00 & 1.00 \\ 11 & 25 \\ 45 & 101 \\ 2 & 2\end{array}$

0

0

Storage

No 
Pedestrian Volumes and Adjustments

\begin{tabular}{lllll}
\hline ovements & 13 & 14 & 15 & 16 \\
\hline low (ped/hr) & 0 & 0 & 0 & 0 \\
ane Width (ft) & 12.0 & 12.0 & 12.0 & 12.0 \\
alking Speed (ft/sec) & 4.0 & 4.0 & 4.0 & 4.0 \\
ercent Blockage & 0 & 0 & 0 & 0
\end{tabular}

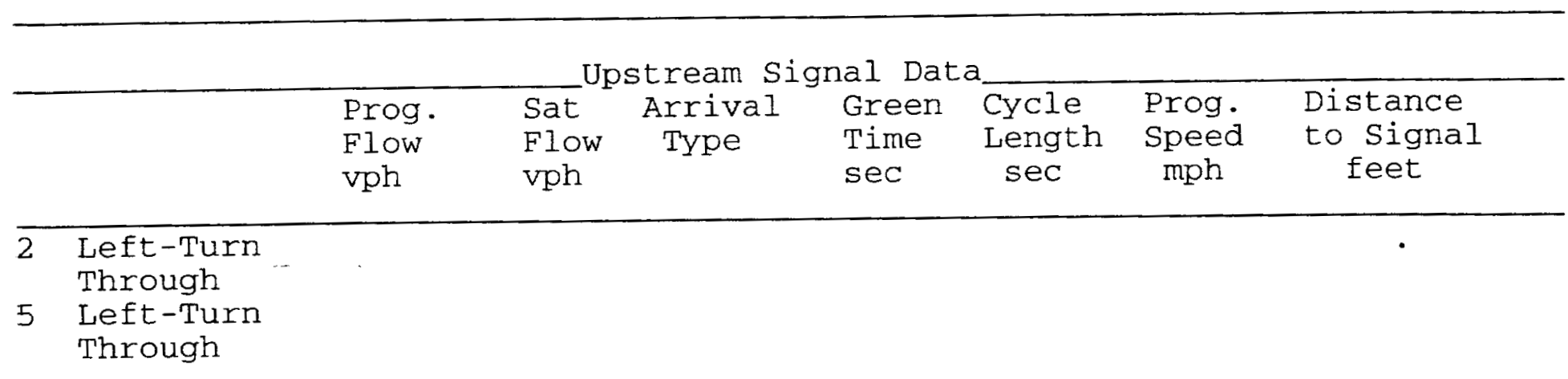

orksheet 3-Data for Computing Effect of Delay to Major street Vehicles

\begin{tabular}{lcc}
\hline & Movement 2 & Movement 5 \\
\hline hared In volume, major th vehicles: & 67 \\
hared In volume, major rt vehicles: & 0 \\
at flow rate, major th vehicles: & 1700 \\
at flow rate, major rt vehicles: & 1700 \\
umber of major street through lanes: & 1 \\
\hline
\end{tabular}

orksheet 4-Critical Gap and Follow-up Time Calculation

\begin{tabular}{|c|c|c|c|c|c|c|c|c|c|}
\hline $\begin{array}{l}\text { ritical } \\
\text { ovement }\end{array}$ & Gap Calc & $\begin{array}{l}\text { culation } \\
1 \\
\mathrm{~L}\end{array}$ & $\begin{array}{l}4 \\
\mathrm{~L}\end{array}$ & $\begin{array}{l}7 \\
\text { L }\end{array}$ & $\begin{array}{l}8 \\
\mathrm{~T}\end{array}$ & $\begin{array}{l}9 \\
\mathrm{R}\end{array}$ & $\begin{array}{r}10 \\
\mathrm{~L}\end{array}$ & $\begin{array}{r}11 \\
\mathrm{~T}\end{array}$ & $\begin{array}{r}12 \\
\mathrm{R}\end{array}$ \\
\hline $\begin{array}{l}\text { (c, base) } \\
(\mathrm{c}, \mathrm{hv}) \\
(\mathrm{hv}) \\
(\mathrm{c}, \mathrm{g}) \\
\text { rade/100 } \\
(3, \mathrm{tt}) \\
(\mathrm{c}, \mathrm{T}): \\
(\mathrm{c})\end{array}$ & $\begin{array}{l}1 \\
1 \text {-stage } \\
\text { 2-stage } \\
1 \text {-stage } \\
\text { 2-stage }\end{array}$ & $\begin{array}{l}0.00 \\
0.00\end{array}$ & $\begin{array}{l}0.00 \\
0.00 \\
0.00 \\
4.1\end{array}$ & $\begin{array}{l}7.1 \\
1.00 \\
2 \\
0.20 \\
0.00 \\
0.70 \\
0.00 \\
1.00 \\
6.4\end{array}$ & $\begin{array}{l}1.00 \\
0.20 \\
0.00 \\
0.00 \\
1.00\end{array}$ & $\begin{array}{l}6.2 \\
1.00 \\
2 . \\
0.10 \\
0.00 \\
0.00 \\
0.00 \\
0.00 \\
6.2\end{array}$ & $\begin{array}{l}1.00 \\
0.20 \\
0.00 \\
0.00 \\
1.00\end{array}$ & $\begin{array}{r}1.00 \\
0.20 \\
0.00 \\
0.00 \\
1.00\end{array}$ & $\begin{array}{l}1.00 \\
0.10 \\
0.00 \\
0.00 \\
0.00\end{array}$ \\
\hline
\end{tabular}

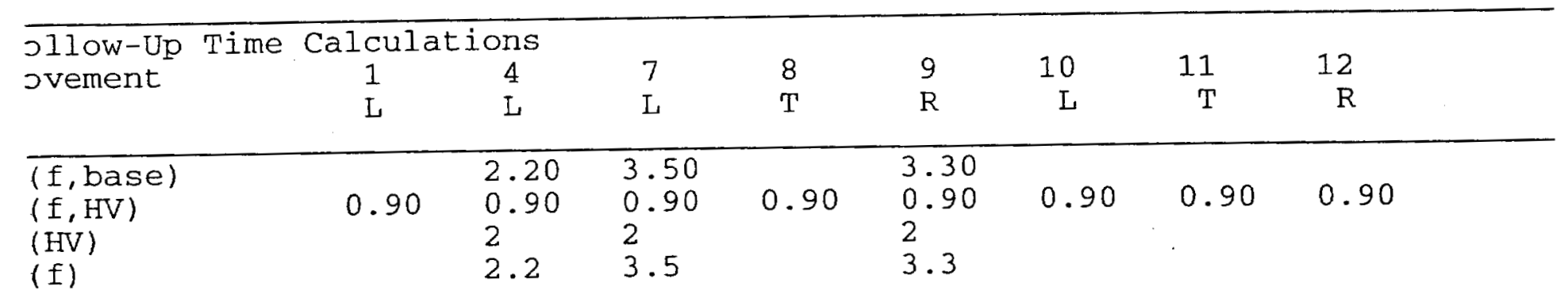


orksheet 5-Effect of Upstream Signals

nputation 1-Queue Clearance Time at Upstream Signal

Movement 2

$V(t) \quad V(1$, prot $) \quad V(t) \quad V(1$, prot $)$

\section{prog}

'tal Saturation Flow Rate, s (vph)

๖. -ival Type

$\because$ Eective Green, g (sec)

vcle Length, C (sec)

3 (from table 9-2)

: sportion vehicles arriving on green $P$

( q1)

(2)

1)

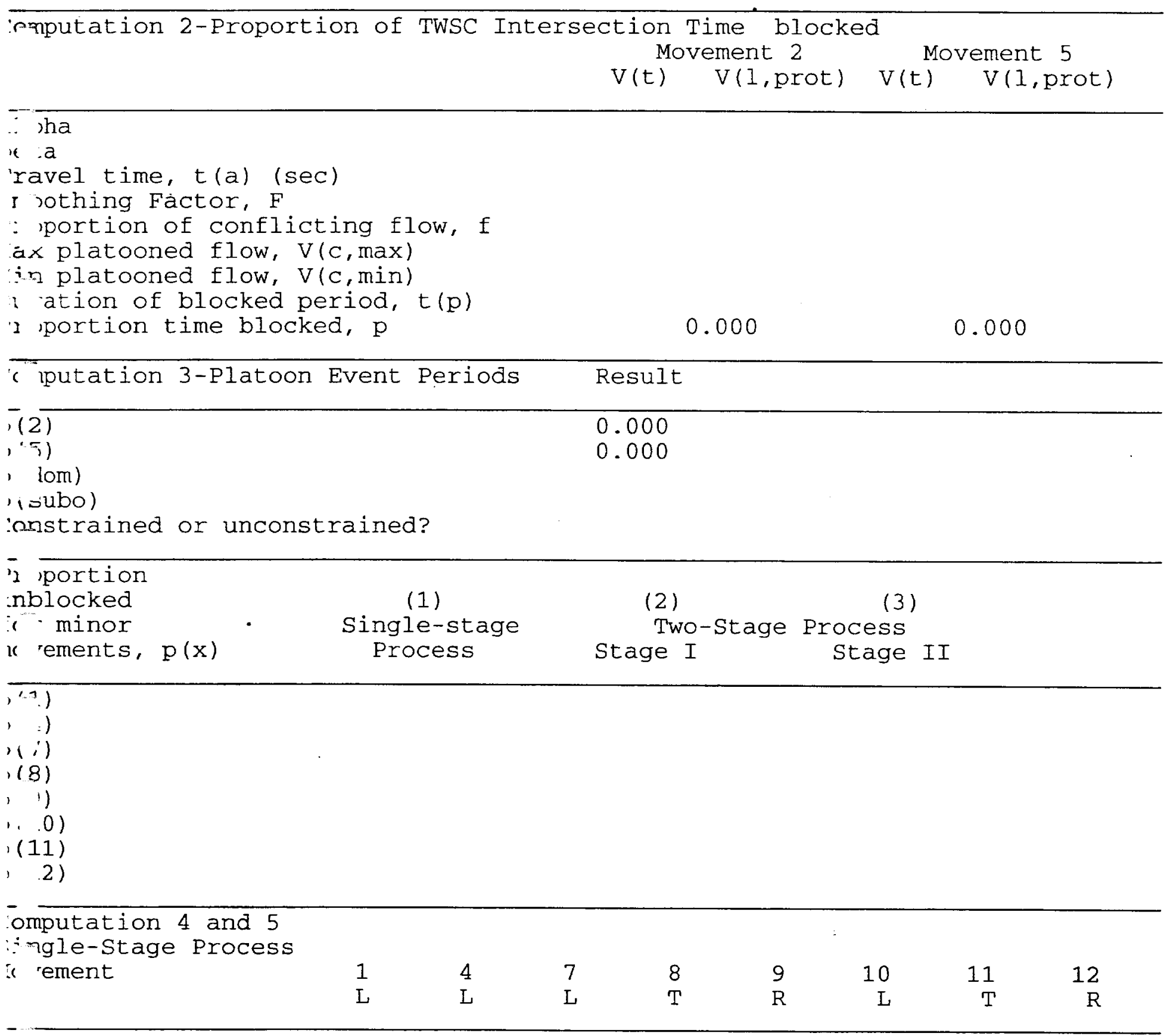


$x$

$c, u, x$

$r, \mathrm{x}$

plat, $x$

wo-Stage Process

7

8

Stage1 ${ }^{7}$ stage2 stage1 ${ }^{8}$ Stage2 stagel $^{10}$ stage2 $^{\text {stagel }}{ }^{11}$ Stage2

11

$(\mathrm{C}, \mathrm{x})$

(x)

1700

$(\mathrm{c}, \mathrm{u}, \mathrm{x})$

$(r, x)$

(plat, $x)$

orksheet 6-Impedance and Capacity Equations

tep 1: RT from Minor St.

9

12

onflicting Flows

stential Capacity

edestrian Impedance Factor

ovement Capacity

robability of Queue free st.

242

797

1.00

797

0.87

1.00

1.00

tep 2: LT from Major St.

4

1

onflicting Flows

stential Capacity

edestrian Impedance Factor

Jvement Capacity

robability of Queue free St.

aj L-Shared Prob Q free St.

247

1319

1.00

1319

0.98

0.98

Eep 3: TH from Minor St.

8

11

onflicting Flows

stential Capacity

edestrian Impedance Factor

ap. Adj. factor due to Impeding mvmnt

svement Capacity

robability of Queue free st.

1.00

1.00

0.98

0.98

Eep 4: LT from Minor st.

1.00

1.00

onflicting Flows

tential Capacity

edestrian Impedance Factor

aj. L, Min T Impedance factor

7

10

aj. L, Min T Adj. Imp Factor.

353
645

645

1.00

1.00

ap. Adj. factor due to Impeding mvmnt

0.98

0.98

ovement Capacity

634

0.99

0.86

1


irt 1 - First Stage

conflicting Flows

itential Capacity

! sestrian Impedance Factor

-up. Adj. factor due to Impeding mumnt

Movement Capacity

; :obability of Queue free st.

Part 2 - Second stage

: inflicting Flows

1 tential Capacity

Pedestrian Impedance Factor

rp. Adj. factor due to Impeding mumnt

: vement Capacity

\begin{tabular}{|c|c|c|}
\hline $\begin{array}{l}\text { Part } 3 \text { - Single stage } \\
\text { nflicting Flows } \\
\text { E tential Capacity } \\
\text { Pedestrian Impedance Factor } \\
\text { p. Adj. factor due to Impeding mumnt } \\
\text { vement Capacity }\end{array}$ & $\begin{array}{l}1.00 \\
0.98\end{array}$ & $\begin{array}{l}1.00 \\
0.98\end{array}$ \\
\hline $\begin{array}{l}\text { sult for } 2 \text { stage process: } \\
\equiv \\
\\
E \text { t obability of queue free st. }\end{array}$ & 1.00 & 1.00 \\
\hline step 4: LT from Minor St. & 7 & 10 \\
\hline $\begin{array}{l}\bar{E} \text { rt } 1 \text { - First Stage } \\
\text {-unflicting Flows } \\
\text { ? } \rightarrow \text { tential Capacity } \\
\text { E destrian Impedance Factor } \\
\text {-.p. Adj. factor due to Impeding mumnt } \\
\text { Lovement Capacity }\end{array}$ & & \\
\hline 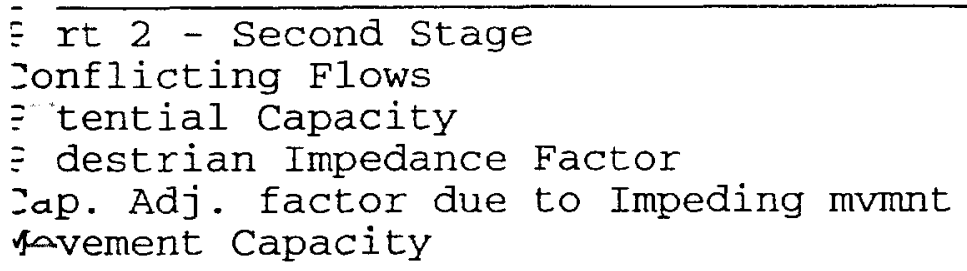 & - & \\
\hline 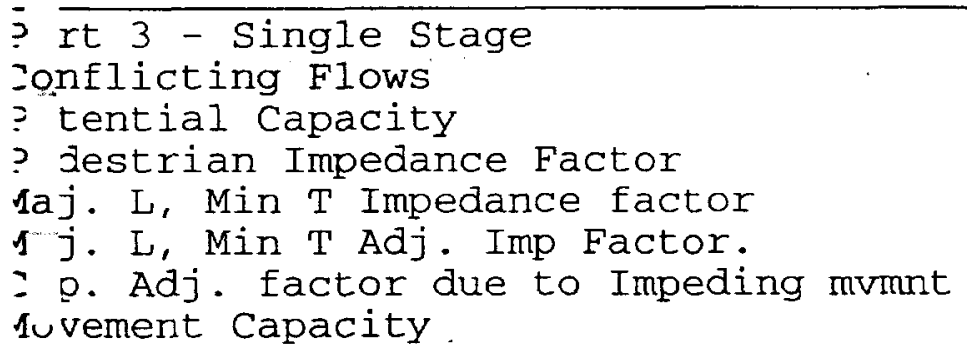 & $\begin{array}{l}353 \\
645 \\
1.00\end{array}$ & $\begin{array}{l}1.00 \\
0.98 \\
0.99 \\
0.86\end{array}$ \\
\hline
\end{tabular}

$\bar{z}$ sults for Two-stage process: 
orksheet 8-Shared Lane Calculations

\begin{tabular}{|c|c|c|c|c|c|c|}
\hline ovement & $\begin{array}{l}7 \\
\mathrm{~L}\end{array}$ & $\begin{array}{l}8 \\
T\end{array}$ & $\begin{array}{l}9 \\
R\end{array}$ & $\begin{array}{r}10 \\
I\end{array}$ & $\begin{array}{r}11 \\
T\end{array}$ & $\begin{array}{r}12 \\
\mathrm{R}\end{array}$ \\
\hline $\begin{array}{l}\text { olume (vph) } \\
\text { ovement Capacity (vph) } \\
\text { hared Lane Capacity (vph) }\end{array}$ & $\begin{array}{l}45 \\
634\end{array}$ & & $\begin{array}{l}101 \\
797\end{array}$ & & & \\
\hline
\end{tabular}

orksheet 9-Computation of Effect of Flared Minor Street Approaches

\begin{tabular}{|c|c|c|c|c|c|c|}
\hline ovement & $\begin{array}{l}7 \\
\mathrm{~L}\end{array}$ & $\begin{array}{l}8 \\
T\end{array}$ & $\begin{array}{l}9 \\
\mathrm{R}\end{array}$ & $\begin{array}{r}10 \\
\mathrm{~L}\end{array}$ & $\begin{array}{r}11 \\
\mathrm{~T}\end{array}$ & $\begin{array}{r}12 \\
\mathrm{R}\end{array}$ \\
\hline $\begin{array}{l}\text { sep } \\
\text { olume } \\
\text { elay } \\
\text { sep } \\
\text { sep }+1 \\
\text { ound (Qsep }+1 \text { ) }\end{array}$ & $\begin{array}{l}634 \\
45\end{array}$ & & $\begin{array}{l}797 \\
101\end{array}$ & & & \\
\hline $\begin{array}{l}\max \\
\text { sh } \\
\text { UM C sep } \\
\text { act }\end{array}$ & & & & & & \\
\hline
\end{tabular}

orksheet 10-Delay, Queue Length, and Level of Service

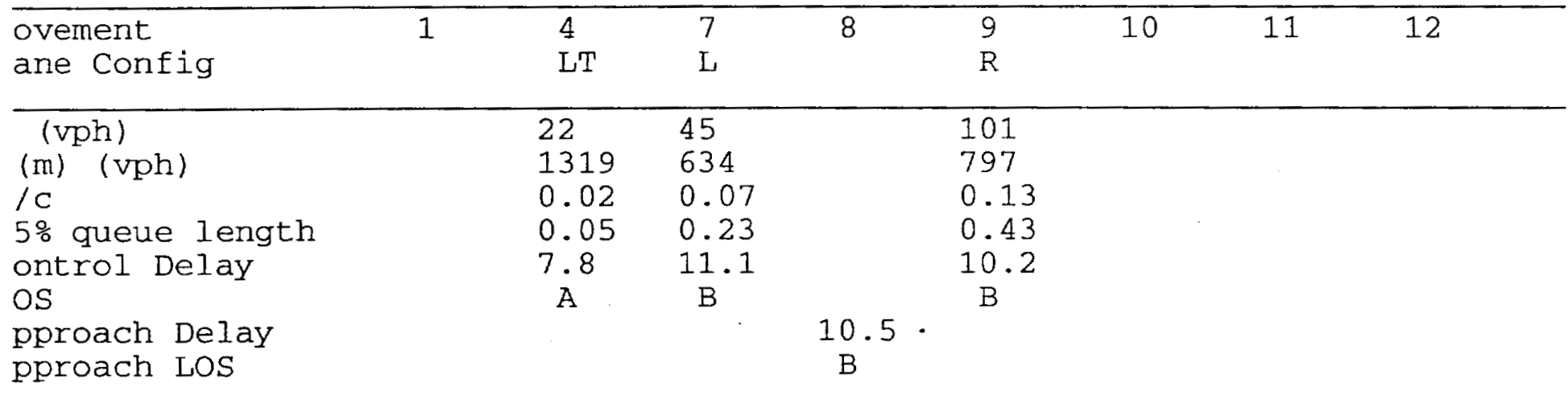

orksheet 11-Shared Major LT Impedance and Delay

\begin{tabular}{|c|c|c|}
\hline & Movement 2 & Movement 5 \\
\hline $\begin{array}{l}\text { (oj) } \\
\text { (i1), Volume for stream } 2 \text { or } 5 \\
\text { (i2), Volume for stream } 3 \text { or } 6 \\
\text { (i1), Saturation flow rate for stream } 2 \text { or } 5 \\
\text { (i2); Saturation flow rate for stream } 3 \text { or } 6 \\
\text { * (oj) } \\
\text { (M,LT), Delay for stream } 1 \text { or } 4 \\
\text { Number of major street through lanes } \\
\text { (rank, 1) Delay for stream } 2 \text { or } 5\end{array}$ & 1.00 & $\begin{array}{l}0.98 \\
67 \\
0 \\
1700 \\
1700 \\
0.98 \\
7.8 \\
1 \\
0.1\end{array}$ \\
\hline
\end{tabular}


tnalyst:

tency/Co.:

: te Performed:

tualysis Time Period: Existing AM

[-tersection:

j risdiction:

? alysis Year:

?roject ID: Lawrence Livermore Lab Traffic Impact Analysis

ミst/West street:

East Avenue

$\therefore$ rth/South street:

Greenville Road

[ tersection Orientation: NS

Study period (hrs): 1.00

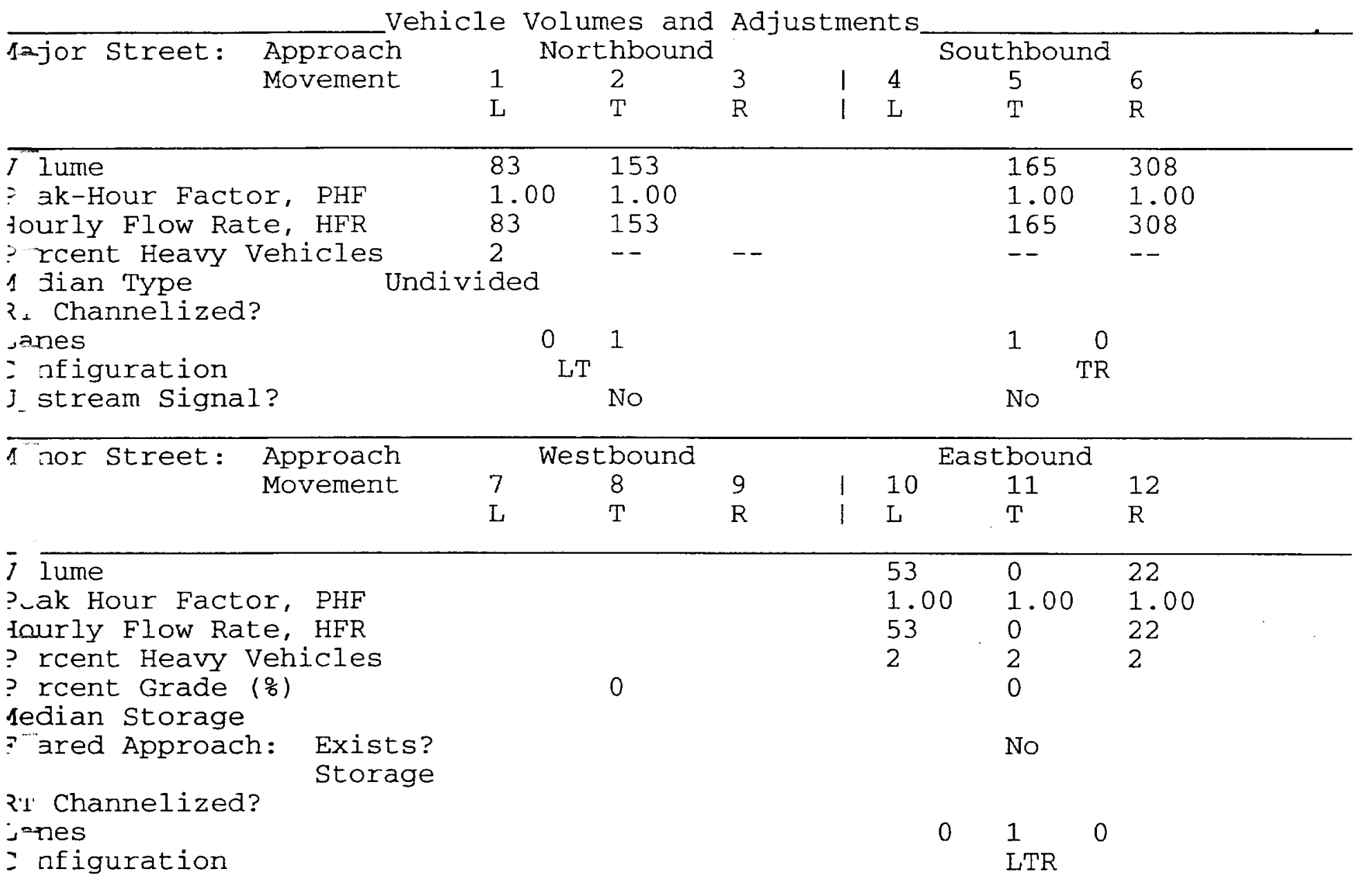

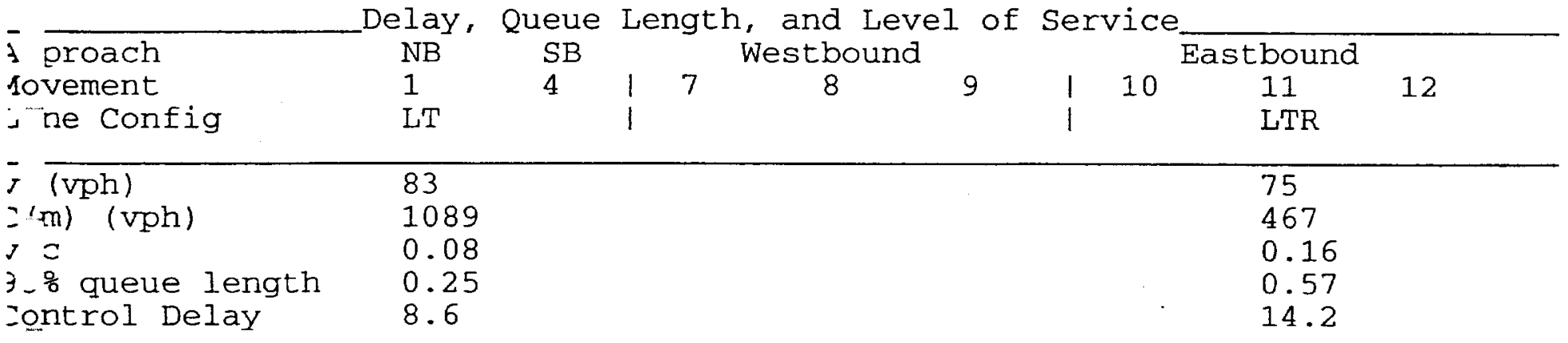


HCS2000: Unsignalized Intersections Release 4.1

orve Engineering, Inc.

hone:

-Mail:

Fax :

TWO-WAY STOP CONTROL (TWSC) ANALYSIS

nalyst :

gency/Co.:

ate Performed: 1/16/02

nalysis Time Period: Existing AM

ntersection: East Ave \& Greenville Rd

urisdiction:

nalysis Year: 2020

roject ID: Lawrence Livermore Lab Traffic Impact Analysis

ast/West Street: East Avenue

orth/South Street: Greenville Road

ntersection Orientation: NS Study period (hrs): 1.00

Vehicle Volumes and Adjustments

\begin{tabular}{llcccccc}
\hline ajor Street & Movements & 1 & 2 & 3 & 4 & 5 & 6 \\
& $\mathrm{~L}$ & $\mathrm{~T}$ & $\mathrm{R}$ & $\mathrm{L}$ & $\mathrm{T}$ & $\mathrm{R}$
\end{tabular}

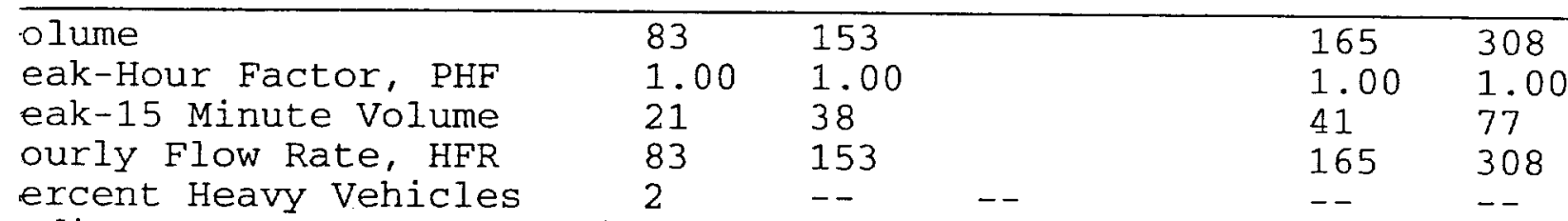

edian Type Undivided

T Channelized?

anes

onfiguration

pstream Signal?

$\begin{array}{lllr}0 & 1 & 1 & 0 \\ \text { LT } & & & \text { TR } \\ & \text { No } & \text { No } & \end{array}$

inor street Movements

$\begin{array}{rrrrrr}7 & 8 & 9 & 10 & 11 & 12 \\ \mathrm{~L} & \mathrm{~T} & \mathrm{R} & \mathrm{L} & \mathrm{T} & \mathrm{R}\end{array}$

olume

eak Hour Factor, PHF

eak-15 Minute Volume

ourly Flow Rate, HFR

ercent Heavy Vehicles

ercent Grade (\%)

edian Storage

lared Approach: Exists?

$\begin{array}{lll}53 & 0 & 22 \\ 1.00 & 1.00 & 1.00 \\ 13 & 0 & 6 \\ 53 & 0 & 22 \\ 2 & 2 & 2\end{array}$

T Channelized?

Storage

No 
Pedestrian Volumes and Adjustments

\begin{tabular}{lllll}
\hline & 13 & 14 & 15 & 16 \\
\hline Movements & & & & \\
\hline OW (ped/hr) & 0 & 0 & 0 & 0 \\
Jane Width (ft) & 12.0 & 12.0 & 12.0 & 12.0 \\
+ Iking Speed (ft/sec) & 4.0 & 4.0 & 4.0 & 4.0 \\
srcent Blockage & 0 & 0 & 0 & 0
\end{tabular}

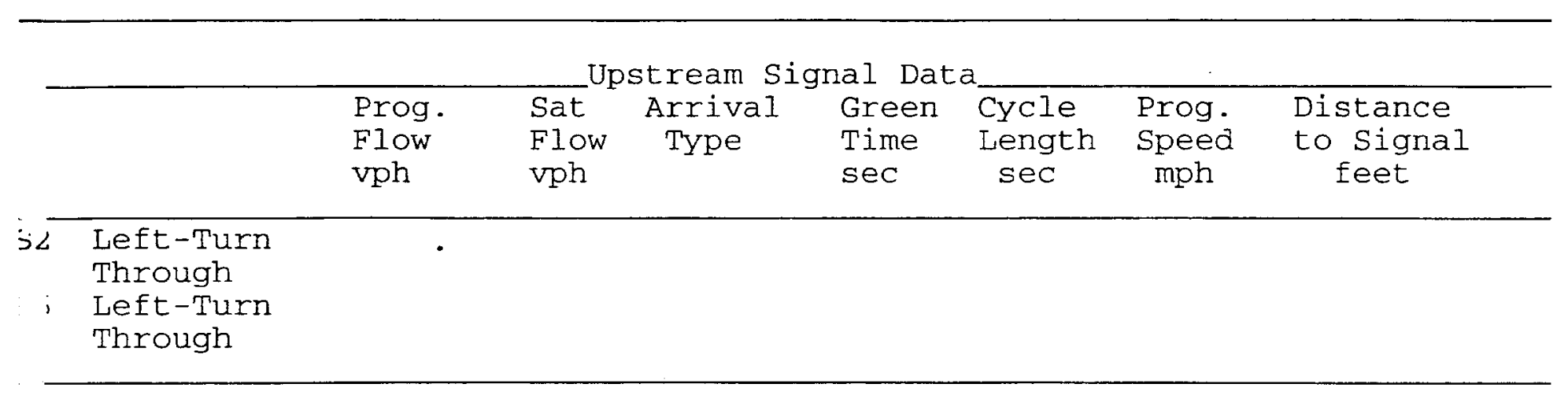

- rrksheet 3-Data for Computing Effect of Delay to Major Street Vehicles

\begin{tabular}{lll}
\hline & Movement 2 & Movement 5 \\
\hline snared ln volume, major th vehicles: & 153 & \\
shared In volume, major rt vehicles: & 0 & \\
$\vdots$.t flow rate, major th vehicles: & 1700 & \\
$\vdots-t$ flow rate, major rt vehicles: & 1700 & \\
Number of major street through lanes: & 1 & \\
\hline
\end{tabular}

Norksheet 4-Critical Gap and Follow-up Time Calculation

\begin{tabular}{|c|c|c|c|c|c|c|c|c|c|}
\hline $\begin{array}{l}\text { itical } \\
\text { luvement }\end{array}$ & Gap Ca & $\begin{array}{l}1 \\
L\end{array}$ & $\begin{array}{l}4 \\
\mathrm{~L}\end{array}$ & $\begin{array}{l}7 \\
L\end{array}$ & $\begin{array}{l}8 \\
\mathrm{~T}\end{array}$ & $\begin{array}{l}9 \\
\mathrm{R}\end{array}$ & $\begin{array}{r}10 \\
L\end{array}$ & $\begin{array}{r}11 \\
\mathrm{~T}\end{array}$ & $\begin{array}{r}12 \\
\mathrm{R}\end{array}$ \\
\hline $\begin{array}{l}\text { (. c, base) } \\
t(c, h v) \\
\text { 'hv) } \\
\text { c,g) } \\
\text { srade/100 } \\
(3,1 t) \\
\quad c, T): \\
+(c)\end{array}$ & $\begin{array}{l}1 \text {-stage } \\
2 \text {-stage } \\
1 \text {-stage } \\
2 \text {-stage }\end{array}$ & $\begin{array}{l}0.00 \\
0.00 \\
0.00 \\
4.1\end{array}$ & 1.00 & $\begin{array}{l}1.00 \\
0.20 \\
0.00 \\
0.00 \\
1.00\end{array}$ & $\begin{array}{l}1.00 \\
0.20 \\
0.00 \\
0.00 \\
1.00\end{array}$ & $\begin{array}{l}1.00 \\
0.10 \\
0.00 \\
0.00 \\
0.00\end{array}$ & $\begin{array}{l}7.1 \\
1.00 \\
2 \\
0.20 \\
0.00 \\
0.70 \\
0.00 \\
1.00 \\
6.4\end{array}$ & $\begin{array}{l}6.5 \\
1.00 \\
2 \\
0.20 \\
0.00 \\
0.00 \\
0.00 \\
1.00 \\
6.5\end{array}$ & $\begin{array}{l}6.2 \\
1.00 \\
2 \\
0.10 \\
0.00 \\
0.00 \\
0.00 \\
0.00 \\
6.2\end{array}$ \\
\hline $\begin{array}{l}\text { Follow-Up } \\
\text { t ivement }\end{array}$ & Time $\mathrm{C}$ & $\begin{array}{c}\text { alcula } \\
1 \\
L\end{array}$ & $\begin{array}{l}\text { ions } \\
4 \\
\text { L }\end{array}$ & $\begin{array}{l}7 \\
\mathrm{~L}\end{array}$ & $\begin{array}{l}8 \\
\mathrm{~T}\end{array}$ & $\begin{array}{l}9 \\
\mathrm{R}\end{array}$ & $\begin{array}{r}10 \\
\mathrm{~L}\end{array}$ & $\begin{array}{r}11 \\
\mathrm{~T}\end{array}$ & $\begin{array}{r}12 \\
\mathrm{R}\end{array}$ \\
\hline $\begin{array}{l}F(f, \text { base) } \\
f(H, H) \\
F, H V) \\
t(f)\end{array}$ & & $\begin{array}{l}2.20 \\
0.90 \\
2 \\
2.2\end{array}$ & 0.90 & 0.90 & 0.90 & 0.90 & $\begin{array}{l}3.50 \\
0.90 \\
2 \\
3.5\end{array}$ & $\begin{array}{l}4.00 \\
0.90 \\
2 \\
4.0\end{array}$ & $\begin{array}{l}3.30 \\
0.90 \\
2 \\
3.3\end{array}$ \\
\hline
\end{tabular}




\section{orksheet 5-Effect of Upstream Signals}

omputation 1-Queue Clearance Time at Upstream Signal

Movement 2

Movement 5

$V(t) \quad V(1$, prot $) \quad V(t) \quad V(1$, prot $)$

\section{prog}

otal Saturation Flow Rate, s (vph)

rrival Type

ffective Green, $g$ (sec)

ycle Length, $C$ (sec)

p (from table 9-2)

roportion vehicles arriving on green $P$

(q1)

(q2)

(q)

omputation 2-Proportion of TWSC Intersection Time blocked

Movement 2 Movement 5

$V(t) \quad V(1$, prot $) \quad V(t) \quad V(1$, prot $)$

\section{Ipha}

eta

ravel time, $t$ (a) (sec)

moothing Factor, $F$

roportion of conflicting flow, $f$

ax platooned flow, $V(c, \max )$

in platooned flow, $V(c, m i n)$

uration of blocked period, $t(p)$

roportion time blocked, $\mathrm{p}$

0.000

0.000

omputation 3-Platoon Event Periods

Result

(2)

(5)

0.000

(dom)

0.000

(subo)

onstrained or unconstrained?

roportion

nblocked

or minor

ovements, $\mathrm{p}(\mathrm{x})$
(1)

Single-stage Process
(2)

(3) Stage I
(3)

Stage II
(1)
(4)
(7)
(8)
(9)
(10)
(11)
(12)

omputation 4 and 5

ingle-stage Process

ovement

$\begin{array}{rrrrrrrr}1 & 4 & 7 & 8 & 9 & 10 & 11 & 12 \\ \mathrm{~L} & \mathrm{~L} & \mathrm{~L} & \mathrm{~T} & \mathrm{R} & \mathrm{L} & \mathrm{T} & \mathrm{R}\end{array}$


$c, u, x$

\section{$r, x$}

plat, $x$

I wo-Stage Process

Stage1 Stage2 Stage1 Stage2 stage1 stage2 stagel ${ }^{11}$ stage2

$\bar{T}(\mathrm{C}, \mathrm{x})$

$\vdots \mathrm{x})$

$J(c, u, x)$

$: \overline{r, x)}$

$=($ plat,$x)$

i rksheet 6-Impedance and Capacity Equations

\begin{tabular}{|c|c|c|}
\hline ep 1: RT from Minor st. & 9 & 12 \\
\hline $\begin{array}{l}\text { Zonflicting Flows } \\
\text { tential Capacity } \\
\text { ' destrian Impedance Factor } \\
\text { 1uvement Capacity } \\
\text { obability of Queue free st. }\end{array}$ & $\begin{array}{l}1.00 \\
1.00\end{array}$ & $\begin{array}{l}319 \\
722 \\
1.00 \\
722 \\
0.97\end{array}$ \\
\hline $\bar{\xi}$ ep 2: LT from Major st. & 4 & 1 \\
\hline $\begin{array}{l}\text { nflicting Flows } \\
\text { tential Capacity } \\
\text { ?edestrian Impedance Factor } \\
1 \text { vement Capacity } \\
\text { obability of Queue free St. } \\
\text { 1uj L-Shared Prob Q free St. }\end{array}$ & $\begin{array}{l}1.00 \\
1.00\end{array}$ & $\begin{array}{l}473 \\
1089 \\
1.00 \\
1089 \\
0.92 \\
0.92\end{array}$ \\
\hline$\vdots$ ep 3: TH from Minor st. & 8 & 11 \\
\hline $\begin{array}{l}\text { Enflicting Flows } \\
\text { tential Capacity } \\
\text { destrian Impedance Factor } \\
\text { ap. Adj. factor due to Impeding mvmnt } \\
\text { : vement Capacity } \\
\text { obability of Queue free st. }\end{array}$ & $\begin{array}{l}1.00 \\
0.92 \\
1.00\end{array}$ & $\begin{array}{l}638 \\
394 \\
1.00 \\
0.92 \\
361 \\
1.00\end{array}$ \\
\hline ;-ep 4: LT from Minor St. & 7 & 10 \\
\hline $\begin{array}{l}\text { nflicting Flows } \\
\text { ?otential Capacity } \\
\text { destrian Impedance Factor } \\
1 \text { j. L, Min T Impedance factor } \\
\text { 1aj. L, Min T Adj. Imp Factor. } \\
\text { : p. Adj. factor due to Impeding mvmnt } \\
1 \text { vement Capacity }\end{array}$ & $\begin{array}{l}1.00 \\
0.92 \\
0.94 \\
0.91\end{array}$ & $\begin{array}{l}638 \\
441 \\
1.00\end{array}$ \\
\hline
\end{tabular}


srksheet 7-Computation of the Effect of Two-stage Gap Acceptance

\begin{tabular}{lllll}
\hline tep 3: TH from Minor St. & 8 & 11
\end{tabular}

art 1 - First Stage

onflicting Flows

otential Capacity

edestrian Impedance Factor

ap. Adj. factor due to Impeding mvmnt

ovement Capacity

robability of Queue free st.

irt 2 - Second stage

onflicting Flows

stential Capacity

edestrian Impedance Factor

ap. Adj. factor due to Impeding mvmnt

ovement Capacity

art 3 - Single stage

onflicting Flows

stential Capacity

edestrian Impedance Factor

ap. Adj. factor due to Impeding mvmnt

ovement Capacity

0.92

1.00

0.92

361

esult for 2 stage process:

$t$

robability of Queue free St.

1.00

361

1.00

tep 4: LT from Minor st.

7

10

art 1 - First stage

onflicting Flows

otential Capacity

edestrian Impedance Factor

ap. Adj. factor due to Impeding mvmnt

ovement Capacity

art 2 - Second Stage

onflicting Flows

otential Capacity

edestrian Impedance Factor

ap. Adj. factor due to Impeding mvmnt

ovement Capacity

art 3 - Single stage

onflicting Flows

otential Capacity

edestrian Impedance Factor

aj. L, Min $T$ Impedance factor

aj. L, Min T Adj. Imp Factor.

ap. Adj. factor due to Impeding mvmnt

ovement Capacity

$\begin{array}{ll} & 638 \\ 1.00 & 441 \\ 0.92 & 1.00 \\ 0.94 & \\ 0.91 & 0.92 \\ & 407\end{array}$

esults for Two-stage process: 
nalyst:

gency/Co.:

ate Performed:

$1 / 16 / 02$

nalysis Time Period: Existing PM

ntersection:

East Ave \& Greenville Rd

urisdiction:

nalysis Year: 2020

roject ID: Lawrence Livermore Lab Traffic Impact Analysis

ast/West Street: East Avenue

orth/South Street: Greenville Road

ntersection Orientation: NS

Study period (hrs): 1.00

Vehicle Volumes and Adjustments

ajor Street: $\begin{aligned} & \text { Approach } \\ & \text { Movement }\end{aligned}$ Northbound

1

23

$\begin{array}{lllllll}1 & 2 & 3 & 1 & 4 & 5 & 6 \\ \mathrm{~L} & \mathrm{~T} & \mathrm{R} & \mathrm{I} & \mathrm{L} & \mathrm{T} & \mathrm{R}\end{array}$

Southbound

olume

11208

$1.00 \quad 1.00$

11208

surly Flow Rate, HFR

ercent Heavy Vehicles

edian Type

208

$194 \quad 85$

I Channelized?

anes

onfiguration

?stream Signal?

$\operatorname{liT}_{\text {LT }} 1$

$1.00 \quad 1.00$

Undivided

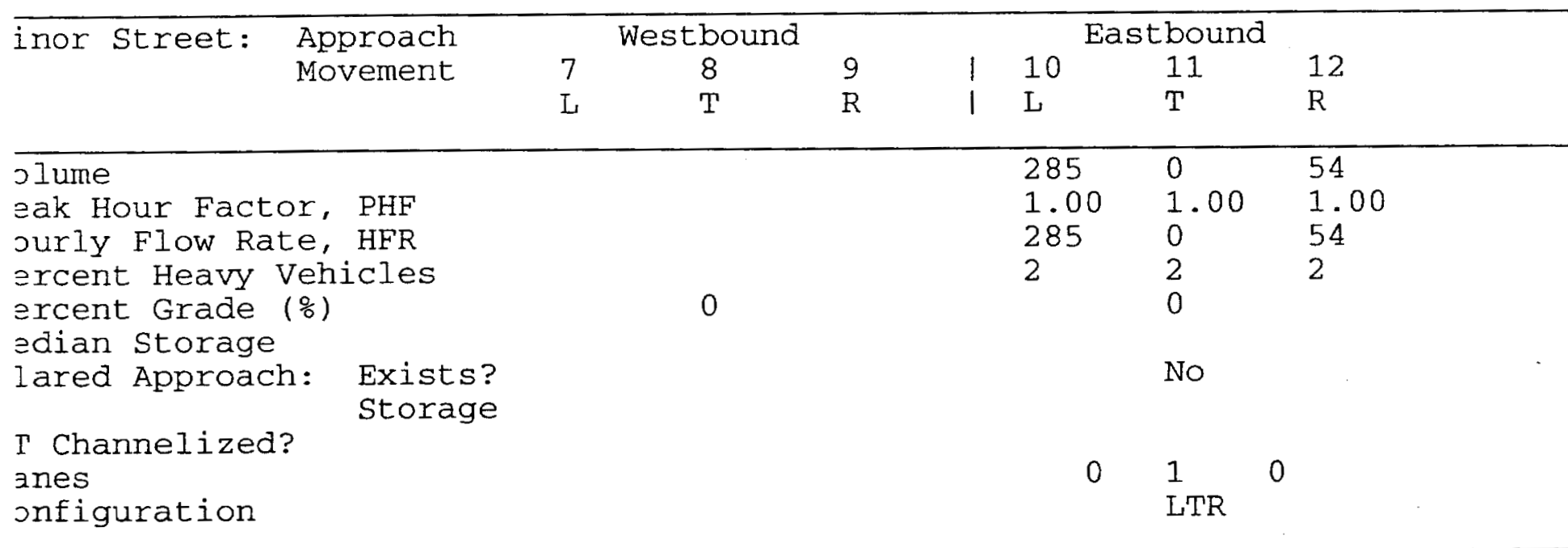

Delay, Queue Length, and Level of Service

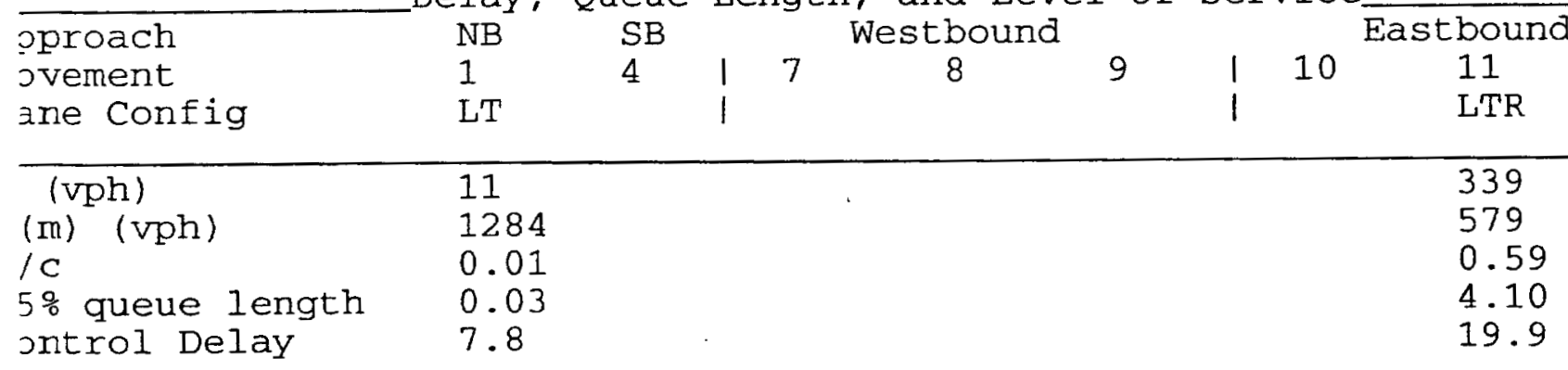


HCS2000: Unsignalized Intersections Release 4.1

rve Engineering, Inc.

Fax:

j-Mail: TWO-WAY STOP CONTROL (TWSC) ANALYSIS

Inalyst:

$:$ ency/Co.:

: te Performed: 1/16/02

łnalysis Time Period: Existing PM

[ tersection:

East Ave \& Greenville Rd

J risdiction:

inalysis Year:

2020

¿ oject ID: Lawrence Livermore Lab Traffic Impact Analysis

I st/West Street: East Avenue

irth/South Street: Greenville Road

[ tersection orientation: NS Study period (hrs): 1.00

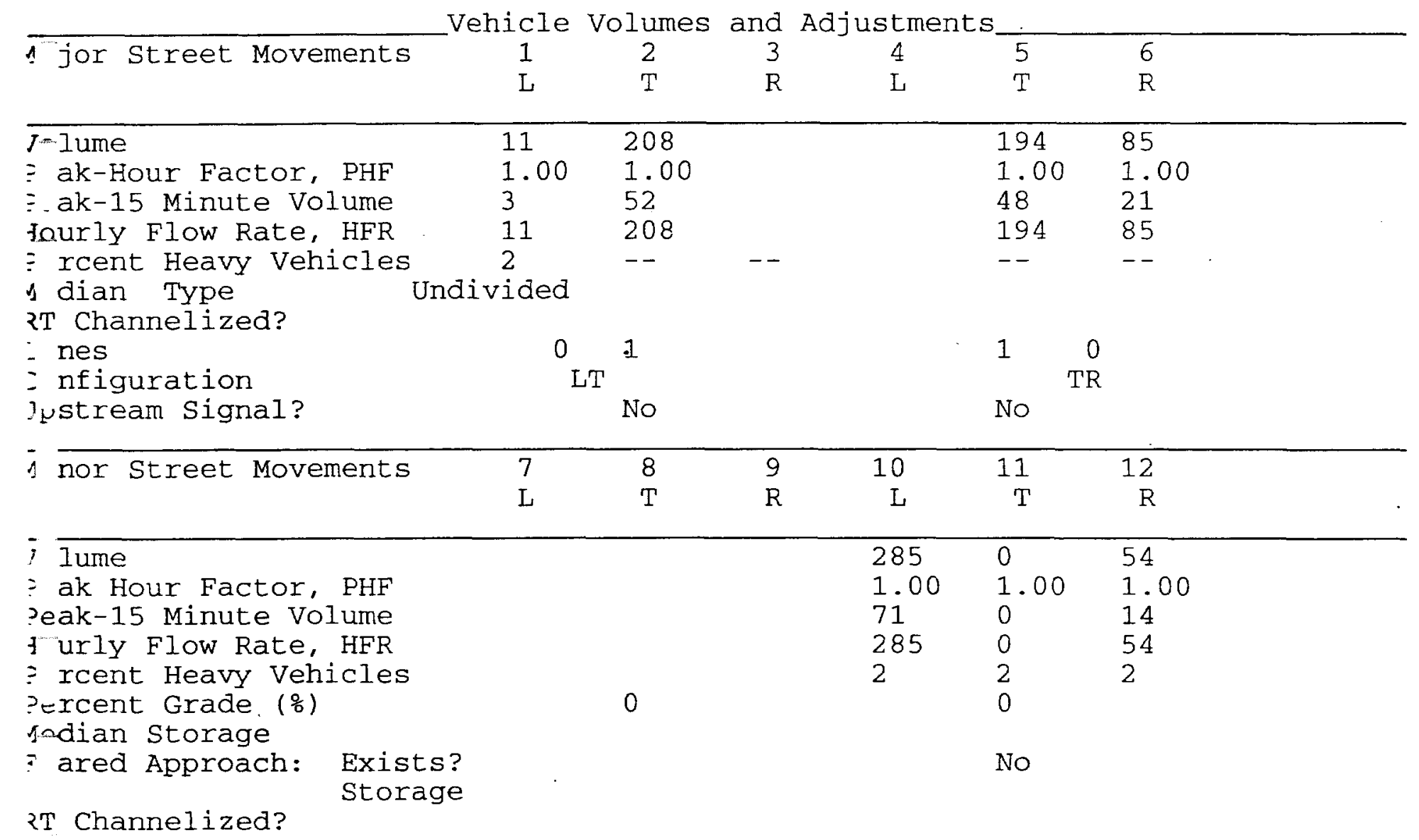


Pedestrian Volumes and Adjustments

\begin{tabular}{lllll}
\hline ovements & 13 & 14 & 15 & 16 \\
\hline low (ped/hr) & 0 & 0 & 0 & 0 \\
ane Width (ft) & 12.0 & 12.0 & 12.0 & 12.0 \\
alking Speed (ft/sec) & 4.0 & 4.0 & 4.0 & 4.0 \\
ercent Blockage & 0 & 0 & 0 & 0
\end{tabular}

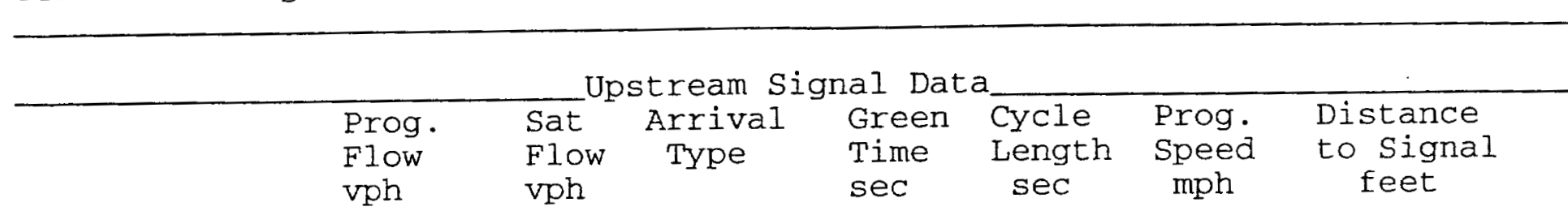

\begin{aligned} \hline 2 & Left-Turn \\ & Through \\ & Left-Turn \\ & Through \end{aligned}

orksheet 3-Data for Computing Effect of Delay to Major street Vehicles

\begin{tabular}{|c|c|c|}
\hline & Movement 2 & Movement 5 \\
\hline $\begin{array}{l}\text { hared In volume, major th vehicles: } \\
\text { hared In volume, major rt vehicles: } \\
\text { at flow rate, major th vehicles: } \\
\text { at flow rate, major rt vehicles: } \\
\text { umber of major street through lanes: }\end{array}$ & $\begin{array}{l}208 \\
0 \\
1700 \\
1700 \\
1\end{array}$ & \\
\hline
\end{tabular}

orksheet 4-Critical Gap and Follow-up Time Calculation

\begin{tabular}{|c|c|c|c|c|c|c|c|c|c|}
\hline $\begin{array}{l}\text { ritical } \\
\text { ovement }\end{array}$ & Gap Calc & $\begin{array}{c}\text { culatic } \\
I \\
I\end{array}$ & 4 & $\begin{array}{l}7 \\
L\end{array}$ & $\begin{array}{l}8 \\
T\end{array}$ & $\begin{array}{l}9 \\
R\end{array}$ & $\begin{array}{r}10 \\
\mathrm{~L}\end{array}$ & $\begin{array}{r}11 \\
\mathrm{~T}\end{array}$ & $\begin{array}{r}12 \\
\mathrm{R}\end{array}$ \\
\hline $\begin{array}{l}\text { (c, base) } \\
(\mathrm{c}, \mathrm{hv}) \\
(\mathrm{hv}) \\
(\mathrm{c}, \mathrm{g}) \\
\text { rade/100 } \\
(3, \mathrm{It}) \\
(\mathrm{c}, \mathrm{T}): \\
(\mathrm{c})\end{array}$ & $\begin{array}{l}\text { 1-stage } \\
\text { 2-stage } \\
1 \text {-stage } \\
\text { 2-stage }\end{array}$ & $\begin{array}{l}4.1 \\
1.00 \\
2 \\
\\
0.00 \\
0.00 \\
0.00 \\
4.1\end{array}$ & $\begin{array}{l}0.00 \\
0.00\end{array}$ & $\begin{array}{l}1.00 \\
0.20 \\
0.00 \\
0.00 \\
1.00\end{array}$ & $\begin{array}{l}1.00 \\
0.20 \\
0.00 \\
0.00 \\
1.00\end{array}$ & $\begin{array}{l}1.00 \\
0.10 \\
0.00 \\
0.00 \\
0.00\end{array}$ & $\begin{array}{l}7.1 \\
1.00 \\
2 \\
0.20 \\
0.00 \\
0.70 \\
0.00 \\
1.00 \\
6.4\end{array}$ & $\begin{array}{l}6.5 \\
1.00 \\
2 \\
0.20 \\
0.00 \\
0.00 \\
0.00 \\
1.00 \\
6.5\end{array}$ & $\begin{array}{l}6.2 \\
1.00 \\
2 \\
0.10 \\
0.00 \\
0.00 \\
0.00 \\
0.00 \\
6.2\end{array}$ \\
\hline $\begin{array}{l}\text { ollow-Up } \\
\text { ovement }\end{array}$ & Time $\mathrm{C}$ & $\begin{array}{c}\text { alculat } \\
1 \\
I\end{array}$ & $\begin{array}{c}\text { ions } \\
4 \\
L\end{array}$ & $\begin{array}{l}7 \\
\mathrm{~L}\end{array}$ & $\begin{array}{l}8 \\
T\end{array}$ & $\begin{array}{l}9 \\
\mathrm{R}\end{array}$ & $\begin{array}{r}10 \\
\mathrm{~L}\end{array}$ & $\begin{array}{r}11 \\
\mathrm{~T}\end{array}$ & $\begin{array}{r}12 \\
\mathrm{R}\end{array}$ \\
\hline $\begin{array}{l}(f, \text { base }) \\
(f, H V) \\
(H V) \\
(f)\end{array}$ & & $\begin{array}{l}2.20 \\
0.90 \\
2 \\
2.2\end{array}$ & 0.90 & 0.90 & 0.90 & 0.90 & $\begin{array}{l}3.50 \\
0.90 \\
2 \\
3.5\end{array}$ & $\begin{array}{l}4.00 \\
0.90 \\
2 \\
4.0\end{array}$ & $\begin{array}{l}3.30 \\
0.90 \\
2 \\
3.3\end{array}$ \\
\hline
\end{tabular}


Worksheet 5-Effect of Upstream Signals

imputation 1-Queue Clearance Time at Upstream Signal

Movement 2

$\mathrm{V}(\mathrm{t}) \quad \mathrm{V}(1$, prot $) \quad \mathrm{V}(\mathrm{t}) \quad \mathrm{V}(1$, prot $)$

$i$ prog

Total Saturation Flow Rate, s (vph)

? rival Type

E fective Green, g (sec)

Ivcle Length, C (sec)

, (from table 9-2)

E oportion vehicles arriving on green $P$

$\exists(q 1)$

"q2)

q)

Emputation 2-Proportion of TWSC Intersection Time blocked Movement 2

Movement 5

$\mathrm{V}(\mathrm{t}) \quad \mathrm{V}(1$, prot $) \quad \mathrm{V}(\mathrm{t}) \quad \mathrm{V}(1$, prot $)$

$\equiv$ pha

= ta

I'ravel time, $t(a)$ (sec)

E.oothing Factor, F

oportion of conflicting flow, $\mathrm{f}$

ux platooned flow, $V(c, \max )$

vin platooned flow, $\mathrm{V}(\mathrm{c}, \mathrm{min})$

= ration of blocked period, $t(p)$

oportion time blocked, p

0.000

0.000

\begin{tabular}{ll}
\hline mputation 3-Platoon Event Periods & Result \\
\hline$(2)$ & 0.000 \\
$=(2)$ & 0.000 \\
$=$ dom) & \\
$?$. subo) &
\end{tabular}

Innstrained or unconstrained?

$\equiv$ sportion

inblocked

E $r$ minor

$r$ vements, $\mathrm{p}(\mathrm{x})$
(1)

Single-stage Process
(2)

Two-Stage Process

Stage I Stage II

-11)

$=4)$

ว . 7)

ว (8)

$=9)$

ว 10$)$

$?(11)$

ว 12$)$

Eumputation 4 and 5

jingle-Stage Process

1 vement

$\begin{array}{rrrrrrrr}1 & 4 & 7 & 8 & 9 & 10 & 11 & 12 \\ \mathrm{~L} & \mathrm{~L} & \mathrm{~L} & \mathrm{~T} & \mathrm{R} & \mathrm{L} & \mathrm{T} & \mathrm{R}\end{array}$


$X$

$\mathrm{C}, \mathrm{u}, \mathrm{x}$

$r, x$

plat, $x$

wo-Stage Process

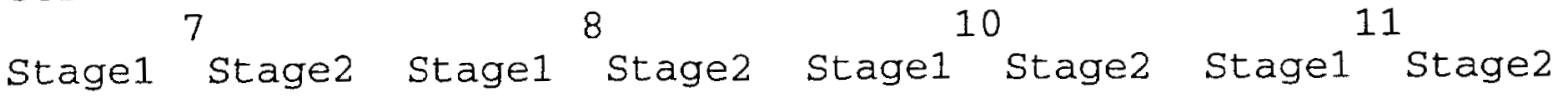

\begin{tabular}{lcc}
\hline$(c, x)$ & 1700 & 1700 \\
$(x)$ & & \\
$(c, u, x)$ & &
\end{tabular}

$(r, x)$

(plat, $x)$

orksheet 6-Impedance and Capacity Equations

\begin{tabular}{|c|c|c|}
\hline tep 1: RT from Minor St. & 9 & 12 \\
\hline $\begin{array}{l}\text { onflicting Flows } \\
\text { otential Capacity } \\
\text { edestrian Impedance Factor } \\
\text { ovement Capacity } \\
\text { robability of Queue free st. }\end{array}$ & $\begin{array}{l}1.00 \\
1.00\end{array}$ & $\begin{array}{l}236 \\
803 \\
1.00 \\
803 \\
0.93\end{array}$ \\
\hline tep 2: LT from Major St. & 4 & 1 \\
\hline $\begin{array}{l}\text { onflicting Flows } \\
\text { otential Capacity } \\
\text { edestrian Impedance Factor } \\
\text { ovement Capacity } \\
\text { robability of Queue free } S t . \\
\text { aj L-Shared Prob Q free St. }\end{array}$ & $\begin{array}{l}1.00 \\
1.00\end{array}$ & $\begin{array}{l}279 \\
1284 \\
1.00 \\
1284 \\
0.99 \\
0.99\end{array}$ \\
\hline tep 3: TH from Minor St. & 8 & 11 \\
\hline $\begin{array}{l}\text { Onflicting Flows } \\
\text { otential Capacity } \\
\text { edestrian Impedance Factor } \\
\text { ap. Adj. factor due to Impeding mumnt } \\
\text { ovement Capacity } \\
\text { robability of queue free } \mathrm{St} .\end{array}$ & $\begin{array}{l}1.00 \\
0.99 \\
1.00\end{array}$ & $\begin{array}{l}466 \\
494 \\
1.00 \\
0.99 \\
489 \\
1.00\end{array}$ \\
\hline tep 4: IT from Minor St. & 7 & 10 \\
\hline $\begin{array}{l}\text { onflicting Flows } \\
\text { otential Capacity } \\
\text { edestrian Impedance Factor } \\
\text { aj. L, Min T Impedance factor } \\
\text { aj. L, Min T Adj. Imp Factor. } \\
\text { ap. Adj. factor due to Impeding mumnt } \\
\text { ovement Capacity }\end{array}$ & $\begin{array}{l}1.00 \\
0.99 \\
0.99 \\
0.93\end{array}$ & $\begin{array}{l}466 \\
555 \\
1.00\end{array}$ \\
\hline
\end{tabular}


; rksheet 7-Computation of the Effect of Two-stage Gap Acceptance

\begin{tabular}{|c|c|}
\hline Step 3: TH from Minor St. & 11 \\
\hline $\begin{array}{l}\text { It } 1 \text { - First Stage } \\
\text { Conflicting Flows } \\
\text { i itential Capacity } \\
\text { I destrian Impedance Factor } \\
\text { ap. Adj. factor due to Impeding mvmnt } \\
\text { Mnvement Capacity } \\
\text { E obability of Queue free st. }\end{array}$ & \\
\hline
\end{tabular}

Part 2 - Second Stage

nflicting Flows

E tential Capacity

Pedestrian Impedance Factor

: p. Adj. factor due to Impeding mumnt

! vement Capacity

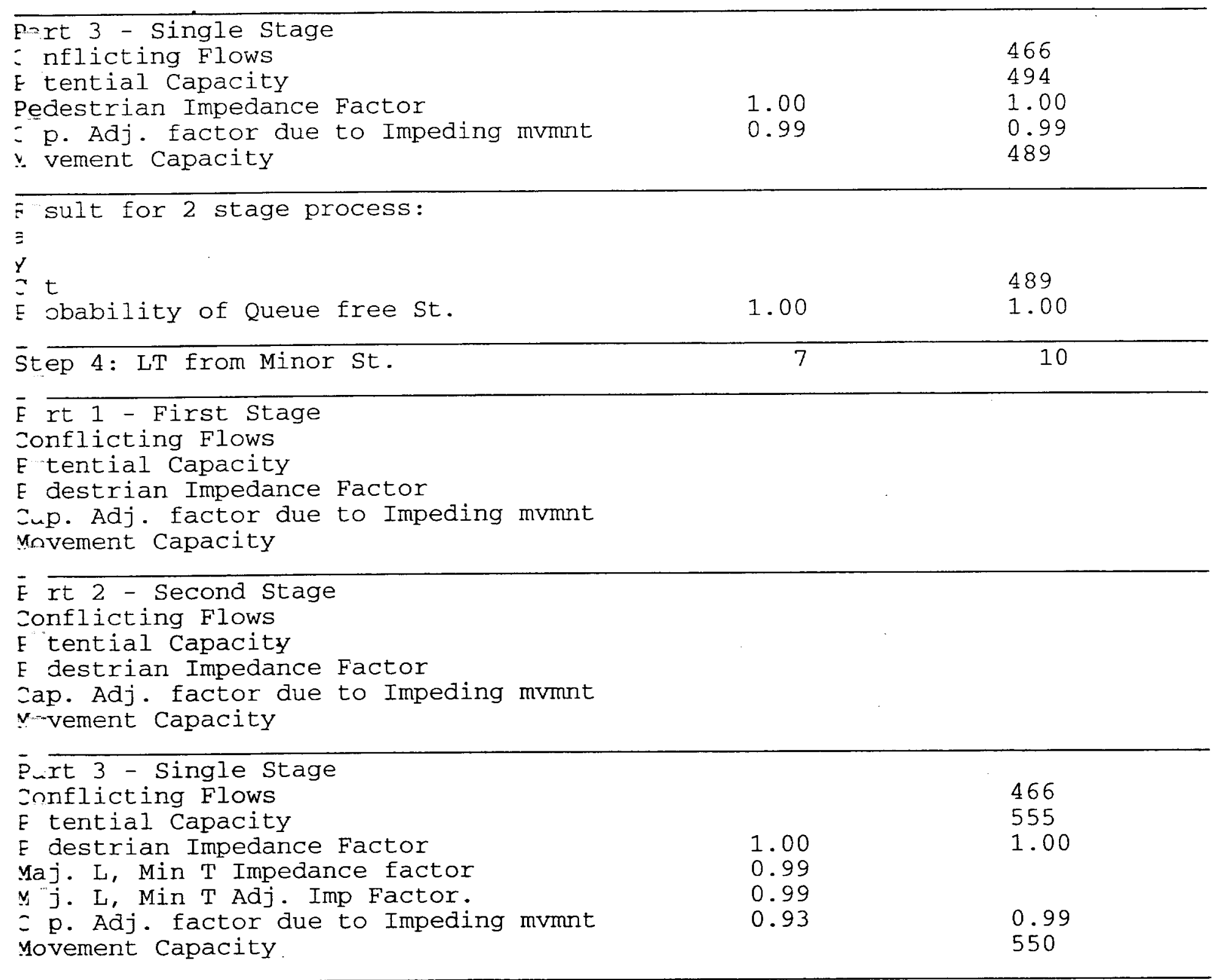

$\bar{R}$ sults for Two-stage process: 
orksheet 8-Shared Lane Calculations

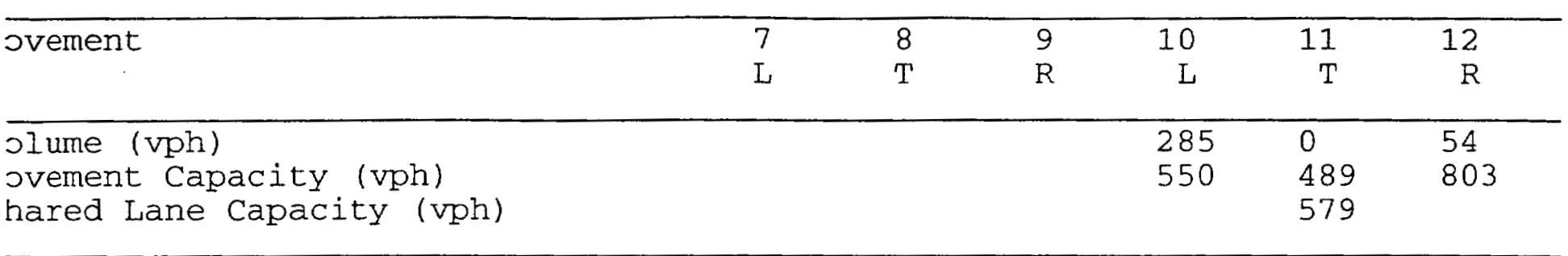

orksheet 9-Computation of Effect of Flared Minor street Approaches

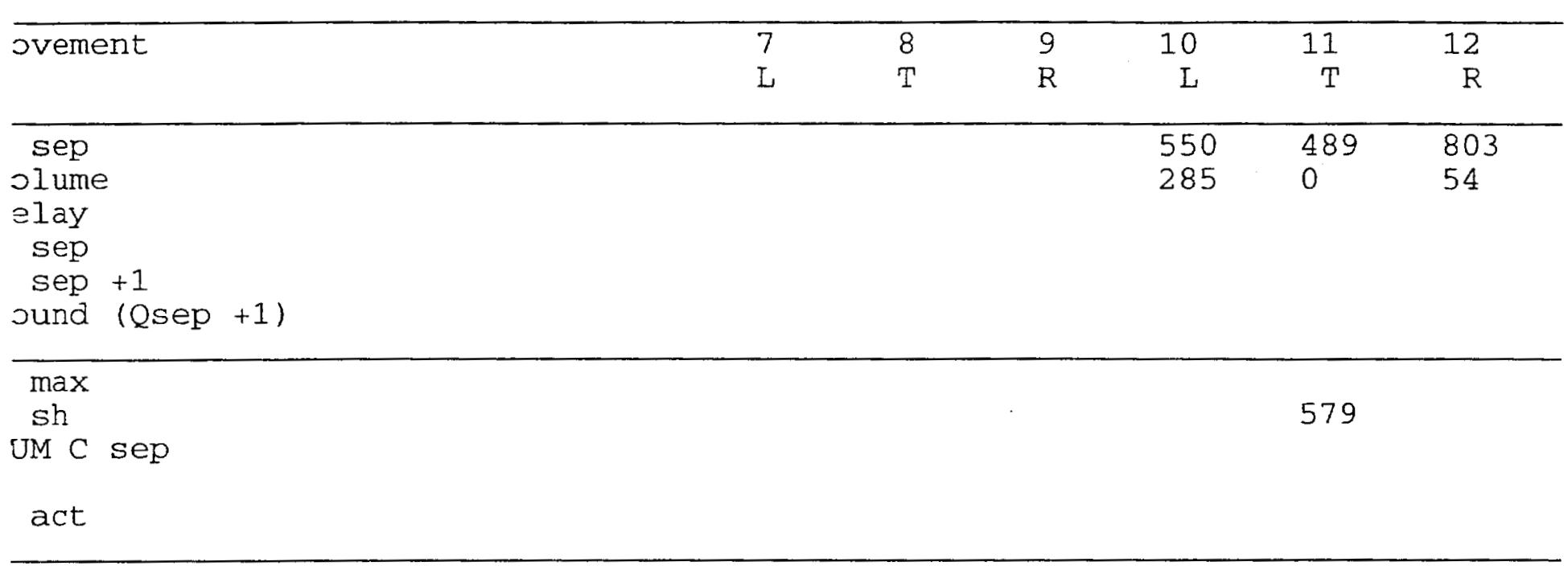

orksheet 10-Delay, Queue Length, and Level of Service

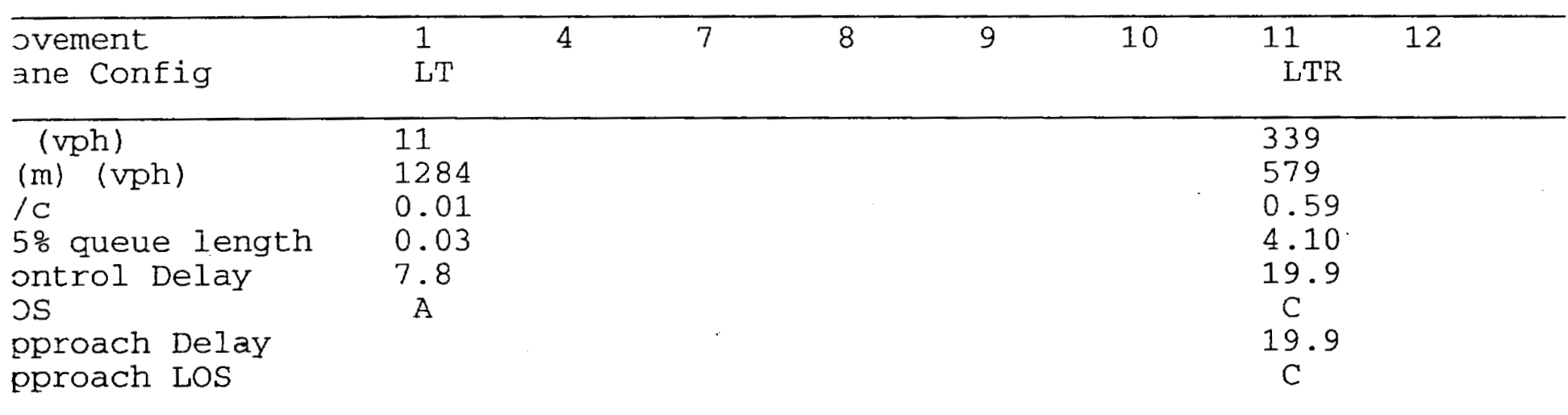

orksheet 11-Shared Major LT Impedance and Delay

\begin{tabular}{|c|c|c|}
\hline & Movement 2 & Movement 5 \\
\hline $\begin{array}{l}\text { (oj) } \\
\text { (i1), Volume for stream } 2 \text { or } 5 \\
\text { (i2), Volume for stream } 3 \text { or } 6 \\
\text { (i1), Saturation flow rate for stream } 2 \text { or } 5 \\
\text { (i2), Saturation flow rate for stream } 3 \text { or } 6 \\
\text { ^(oj) } \\
\text { (M, LT), Delay for stream } 1 \text { or } 4 \\
\text { Number of major street through lanes } \\
\text { (rank, 1) Delay for stream } 2 \text { or } 5\end{array}$ & $\begin{array}{l}0.99 \\
208 \\
0 \\
1700 \\
1700 \\
0.99 \\
7.8 \\
1 \\
0.1\end{array}$ & 1.00 \\
\hline
\end{tabular}

